

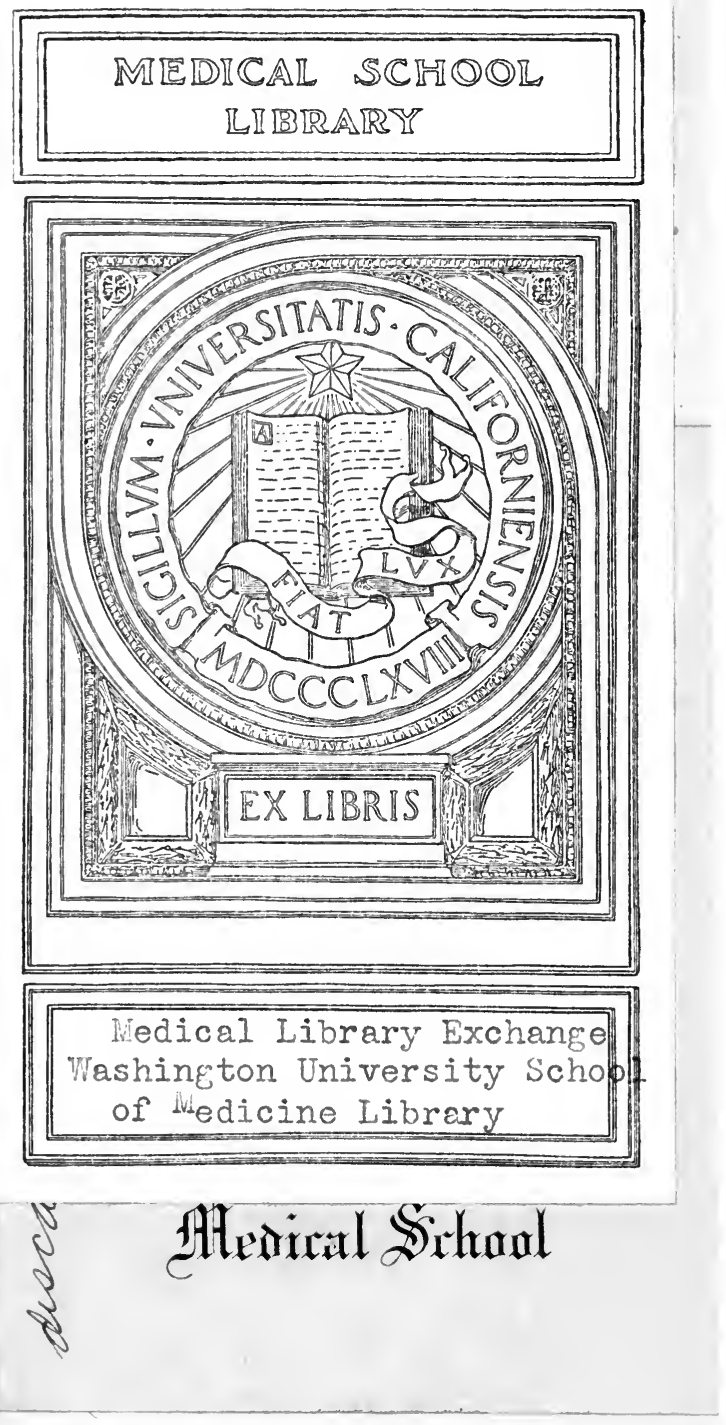




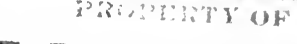

I. I I TS A IE I (Rec'd 1908:

MEDICALDEPT?

Watibistonls 
Digitized by the Internet Archive in 2007 with funding from Microsoft Corporation 
$x^{2}+x^{2}=$
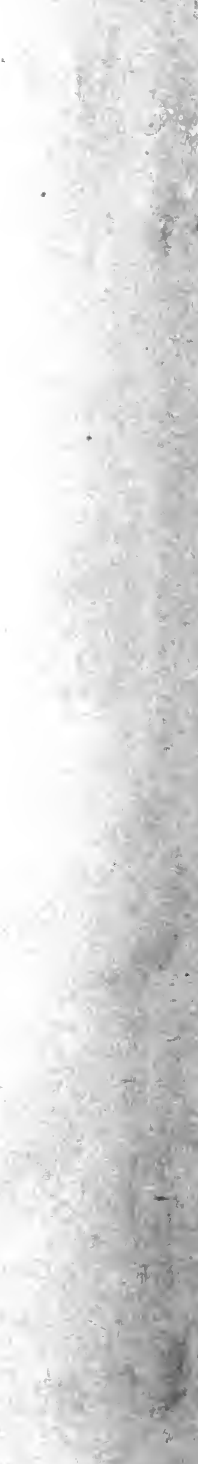


\title{
A LABORATORY MANUAL
}

\author{
OF
}

\section{HUMAN}

ANATOMY

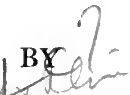

LEWELLYS F. BARKER, M.B. ToR.

PROFESSOR AND HEAD OF THE DEPARTMENT OF ANATOMY IN THE UNIVERSITY OF CHICAGO AND RUSH MEDICAL COLLEGE
\end{abstract}

ASSISTED BY

DEAN DE WiTt LEWIS, A.B., M.D. ANd DANIEL GRAiSberRY REVELl, A.B., M.B. INSTRUCTORS IN ANATOMY IN THE UNIVERSITY OF CHICAGO

ILLUSTRATED

PHILADELPHIA AND LONDON

J. B. LI PPINCOTT COMPANY 
Copyright, 1904

BY

J. B. Lippincott Company

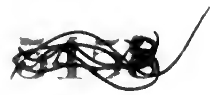

ELECTROTYPED ANO PRINTED BY J. B. LIPPINCOTT COMPANY, PHILADELPHIA, U. S.A. 


\section{PREFACE}

MaNy instructors in anatomy have for some time felt the need of more systematic, more orderly, and more thorough work in the dissecting-room. Anatomy, one of the first sciences to be taught by the laboratory method, has not always kept pace with her younger sister sciences in the advance of methods of instruction. A number of laboratory manuals are, it is true, available; but it has been complained that even the best of these is unsatisfactory, partly on account of nomenclature, partly because so much is included that the student tends to lean entirely upon his manual, rather than to observe at first hand for himself and to consult larger text-books and atlases. So convinced have some anatomists become of the inadequacy of present manuals that they have banished all dissecting guides from their laboratories, and insisted upon their students working with no guide whatever except large atlases and text-books and occasional hints from the instructor. This latter plan has worked very well in some instances, especially where men entering upon the study of medicine had previously undergone rigid laboratory training in physics, chemistry, and biology. But even such men had to pass through a period of doubt, perplexity, and discouragement before they became independent dissectors, and some of them completely failed to do so. Students without such preliminary training in science are very apt to be utterly lost if thrown entirely or almost entirely upon their own resources, especially where classes are large and more than ten students have to be cared for by one instructor. It is believed that the use of the Laboratory Manual here presented will enable the good student to become an independent worker much more quickly than when be is left without such guidance; he will be able to do his work in less time and can assure himself that he is doing it thoroughly, using the Manual, if he prefer, merely as a control of his work; at the same time the student who is less well-equipped will be gradually led into independent work, to which he might otherwise never attain.

There should be also a marked saving of time and energy for the instructor, a matter of no little importance if he is to make 
contributions to knowledge by original investigation in his department. There is no intention, however, to make " word of mouth" instruction superfluous. On the contrary, it is hoped that the use of the Manual will make it possible for the instructor to concentrate his attention upon, and to give better than before, that particular help to the student which comes alone through the personal encounter.

Whether the effort to find the golden mean between the " spoon-feeding" guide, on the one hand, and insufficient guidance, on the other, has been successful must be left to anatomical teachers to decide. The compilers have used the Manual in mimeograph form during the past year in the Anatomical Laboratory of the University of Chicago; instructors and students both feel that it has been distinctly helpful.

The sequence of dissection and the methods of exposing the various parts are those almost universally adopted in the better English and American laboratories. They represent the experience and traditions of more than a hundred years of careful anatomical work. The influence of the Edinburgh school of anatomists has become widespread, and the directions of one of the principal representatives of that school, Professor D. J. Cunningham, of Edinburgh, as embodied in his admirable Manual of Dissection, have been closely followed herein.

The Laboratory Manual is not intended to take the place of a descriptive text-book (see p. 30); on the contrary, it is hoped and expected that it will act as a stimulant to the student not only to use in connection with it the best descriptive text-book and atlas available, but also to refer to various atlases and textbooks, as well as original articles in the literature. Though it is realized that the average medical student has not the time to range far afield, it would seem highly desirable that he should at least gain an idea of the wide extent of the subject and that he should learn that it is not all included between the covers of a dissecting manual.

The nomenclature employed is that which was formulated by the German Society of Anatomists and which is rapidly being adopted in many of the best English and American laboratories. There seems to be no doubt that this nomenclature will be the prevalent one for at least many years to come. The magnificent atlases of Spalteholz and Toldt, which have recently been published, employ it exclusively, and the student will find it a great convenience to have the terms used in his laboratory manual identical with those of his atlas. Unfortunately, only one or 
two of the most recent descriptive text-books of systematic human anatomy by English writers have yet adopted this nomenclature. It is earnestly to be hoped that an advance will quickly be made in this direction and that text-books will employ it uniformly. Special attention is drawn to the fact that in the following Laboratory Manual the old terms, when different from the new, have been added in parentheses, so that the student need never be embarrassed in finding his way even in a descriptive text-book or atlas in which only the old terms are employed. He is advised, however, when purchasing a textbook to give preference to one in which the new nomenclature [BNA] is consistently employed.

Indulgence is asked for misprints and errors almost certain to occur in the first edition of a work including such an enormous number of names as is involved in a subject like human anatomy. Should the Manual find enough friends to make a second edition desirable, many improvements can doubtless be made, and suggestions from instructors and students of anatomy will be cordially welcomed.

The Manual contains about 300 illustrations, of which a special index is given. The drawings and plates represent in a very satisfactory way the most important structures of the body. These illustrations have been chosen to serve as a valuable atlas to students who feel that they cannot afford to buy one of the more expensive atlases like those of Spalteholz and Toldt. On the other hand, students who own Spalteholz's Atlas, the one especially recommended for use in connection with this Manual, will find that the illustrations have been selected with the special aim of supplementing those of Spalteholz, and that they will prove a valuable addition to the latter.

The Anatomical Atlas of Toldt, from which many of the illustrations in this Manual have been borrowed, can be very highly recommended; every student who can afford to do so should purchase it for his library.

Chicago, October 1, 1904. 



\section{TABLE OF CONTENTS INTRODUCTION}

.

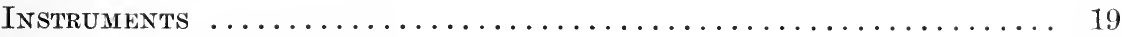

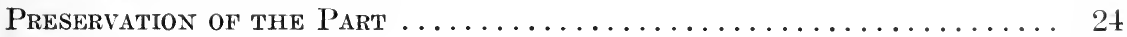

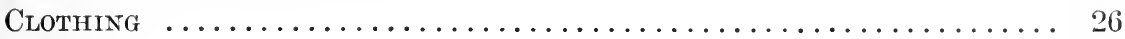

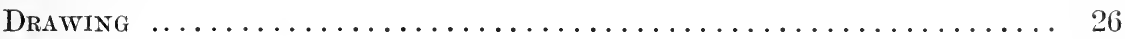

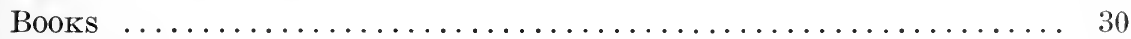

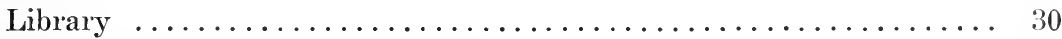

Division of the Cadaver into Parts .................... 34

Anatomical Terms of Position and Direction ................ 34

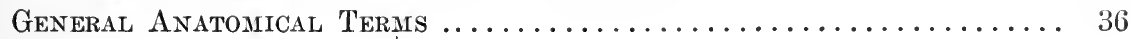

\section{Part I.}

UPPER EXTREMITY.

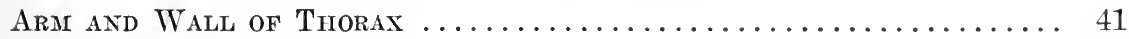

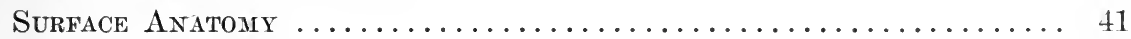

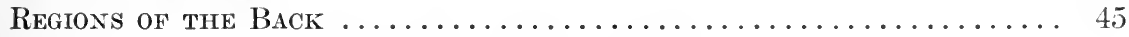

Anterior Thoracic Region and Axillaky Fossa .............. 51

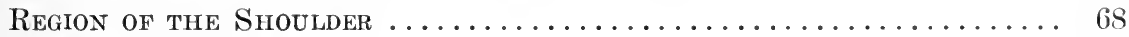

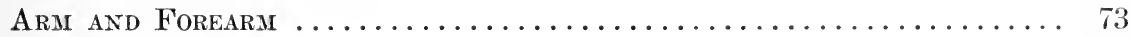

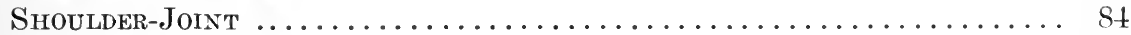

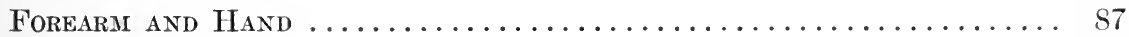

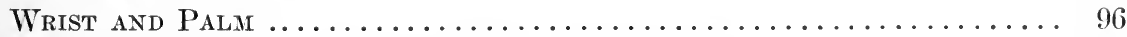

Dorsal Surface and Radial Margin of Forearm .............. 104

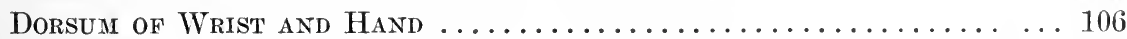

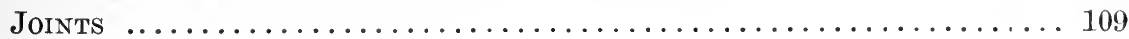

\section{Part II.}

\section{LOWER EXTREMITY.}

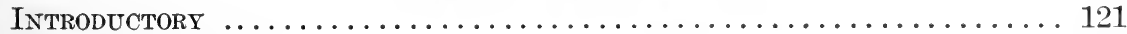

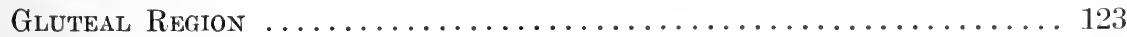

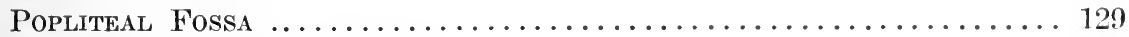

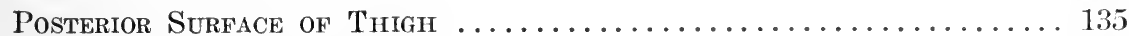

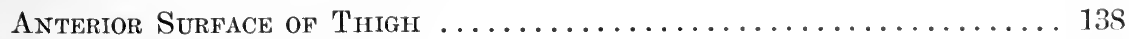

Medial Surface of Thigh ............................ 152

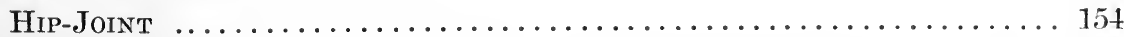

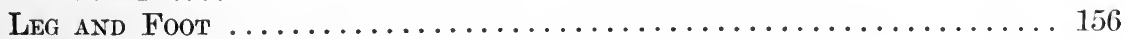




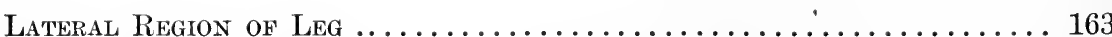

Medial Region of Leg ........................... 165

Posterior Region of Leg and Heel ......................... 165

Plantar Region of Foot ................................ 174

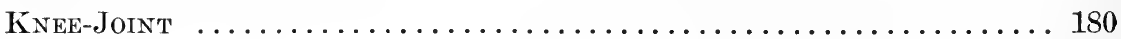

Tibiofibular Joints ................................. 184

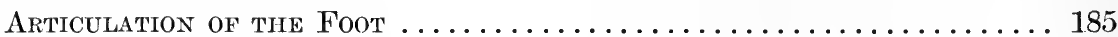

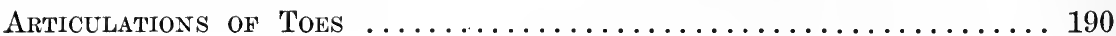

\section{Part III.}

\section{HEAD AND NECK AND DORSUM OF TRUNK.}

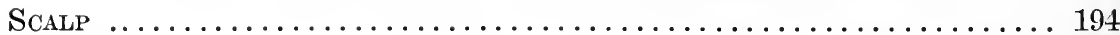

Opening the Cavity of the Skull . . . . . . . . . . . . . . . . . 197

Removal of Bratn .............................. 199

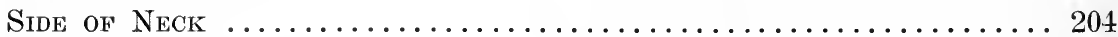

Median Line of Neck .................................. 222

Back of Head, Neck, and Trunk ......................... 228

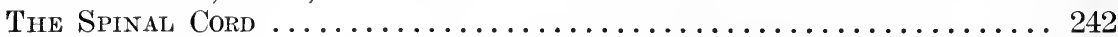

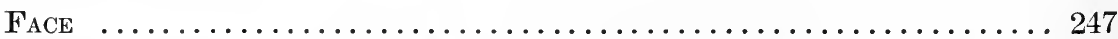

Temporal Region and Retromandibular Fossa ................. 260

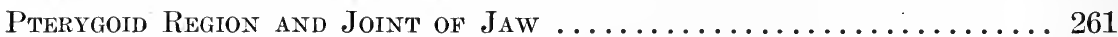

Mandibular Canal and Submaxillaky Region .................. 264

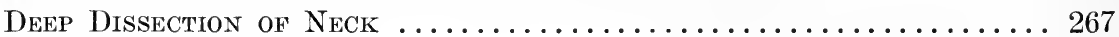

Middle Cranial Fossa ............................ 272

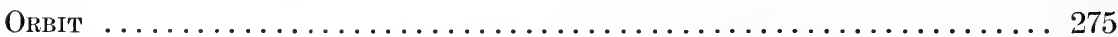

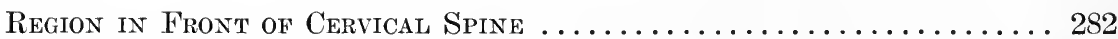

Ligaments of Vertebral Column and Skull $\ldots \ldots \ldots \ldots \ldots \ldots \ldots \ldots . \ldots 286$

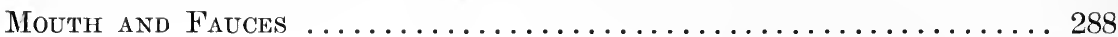

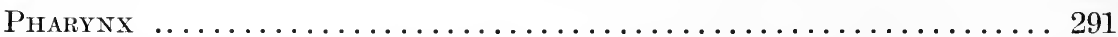

Carotid and Infraorbital Canals ...................... 297

Sagittal Section of Forepart of Head near Median Plane ......... 298

Otic Ganglion ..................................... 299

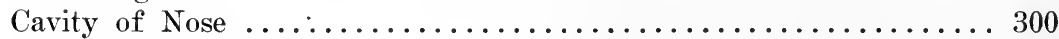

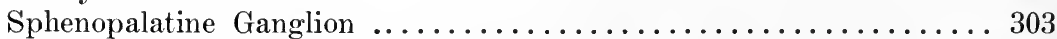

Intraosseous Course of some Cerebral Nerves ............. . . . . 305

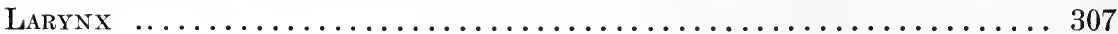

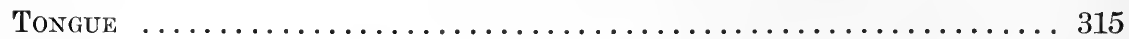

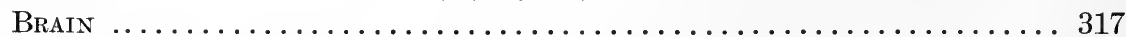

Membranes of Brain ....................................... 317

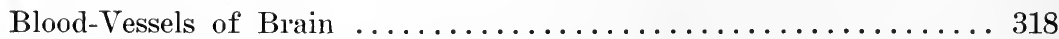

Removal of Meninges and Superficial Blood-Vessels ........... 322

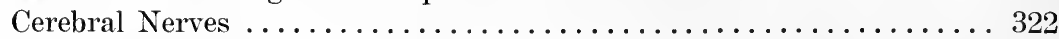

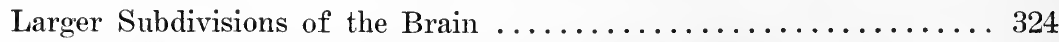

External Morphology of Rhomboid Brain and Midbrain ......... 326

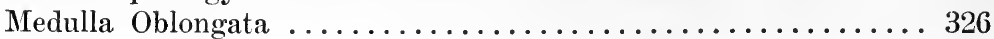

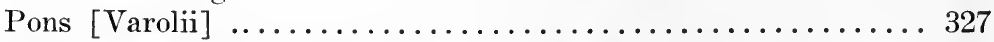

Isthmus of Rhombencephalon $\ldots \ldots \ldots \ldots \ldots \ldots \ldots \ldots \ldots \ldots \ldots \ldots \ldots \ldots \ldots$

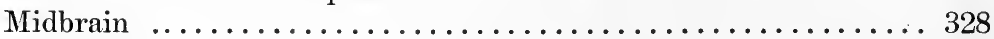

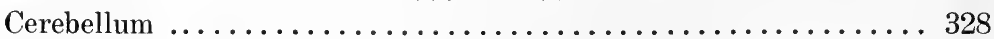

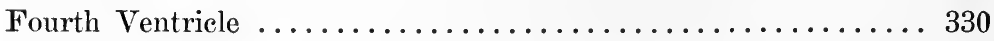


BraIN :

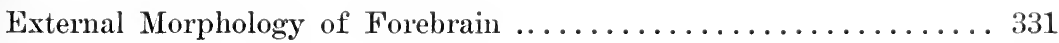

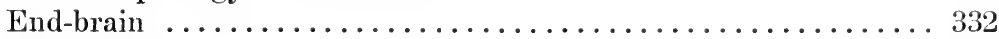

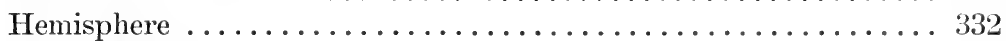

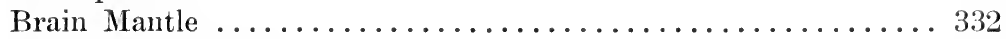

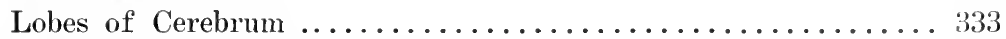

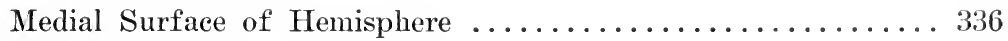

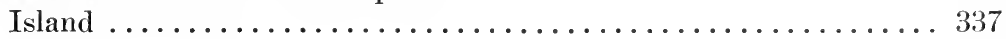

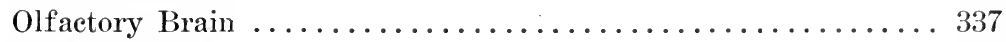

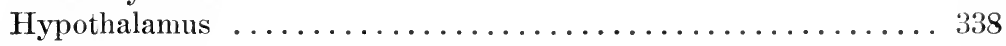

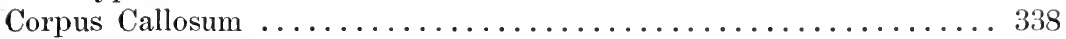

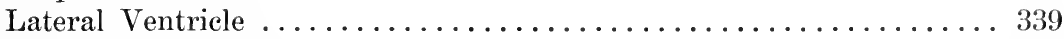

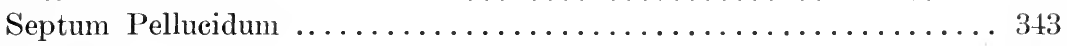

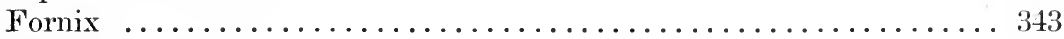

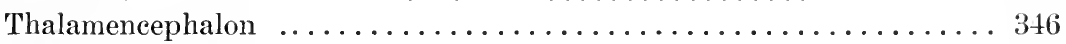

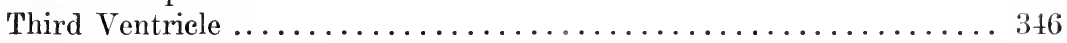

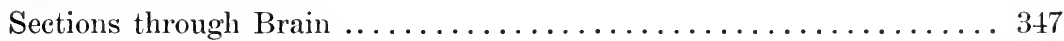

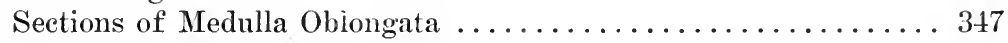

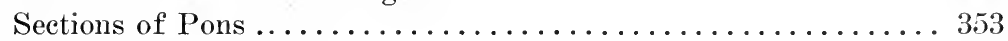

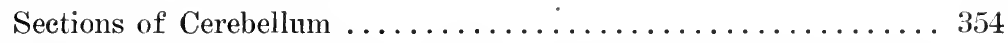

Section of Rhombencephalic Istlmus, Corpora Quadrigemina, and

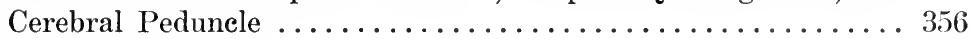

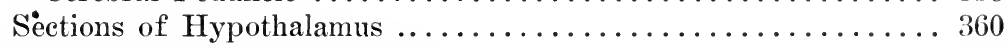

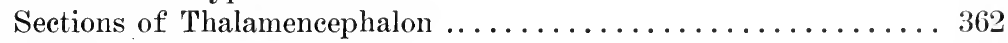

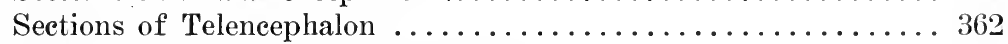

Conduction Paths of the Nervous System ........................ 364

Descending and Ascending Fibre Systems in Spinal Cord ...... 365

Fibre Systems of Cerebral Nerves .................. 365

Conduction Paths of Cerebellar Peduncles ............... 369

Conduction Paths of Diencephalon and Telencephalon ........ 372

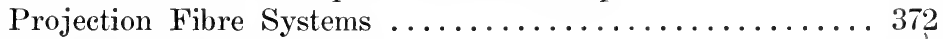

Associative Fibre Systems ....................... 373

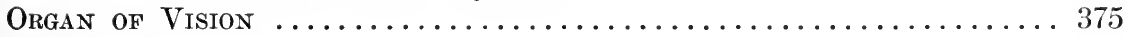

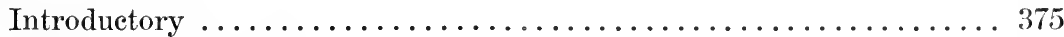

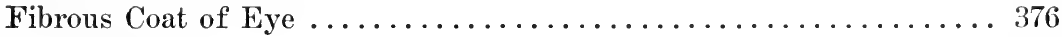

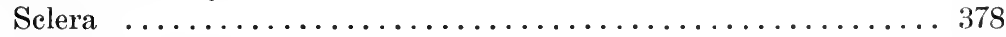

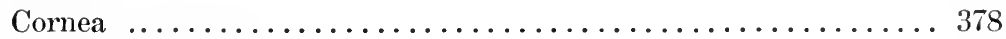

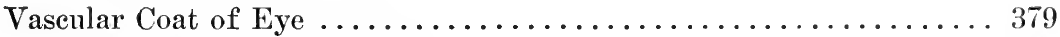

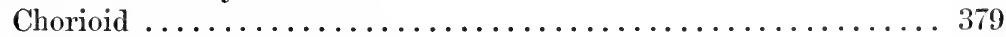

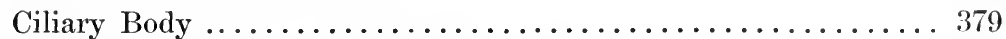

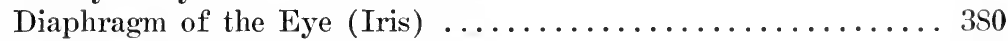

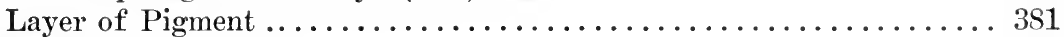

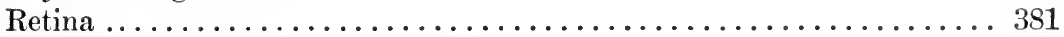

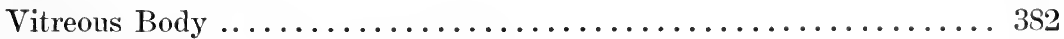

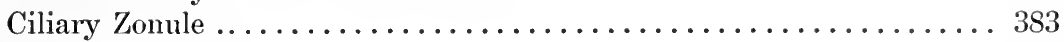

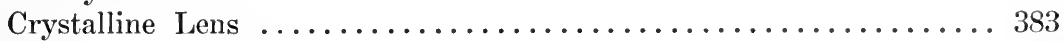

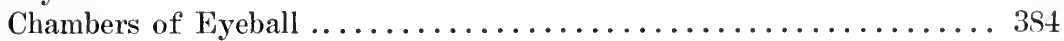

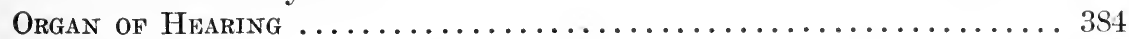

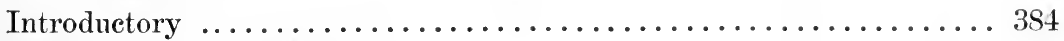

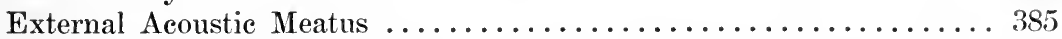

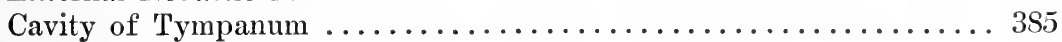


Organ of Hearing:

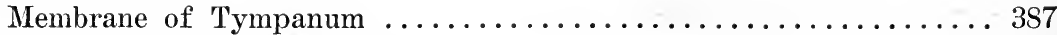

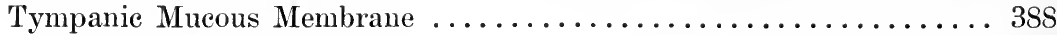

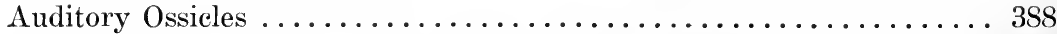

Joints and Ligaments of the Auditory Ossicles ............... 390

Muscles of Auditory Bones ......................... 391

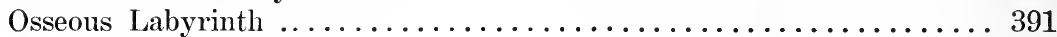

Membranous Labyrinth ............................. 394

\section{Part IV.}

\section{THORAX.}

\section{(WALLS AND VISCERA.)}

INTRODUCTORY . . . . . . . . . . . . . . . . . . . . . . . . . . 399

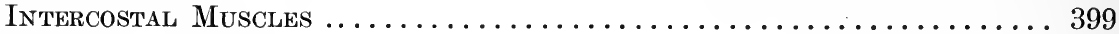

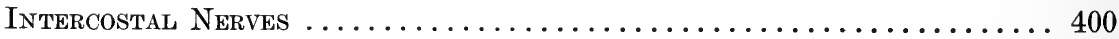

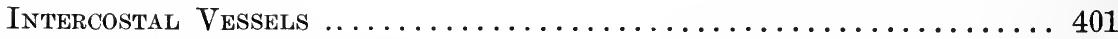

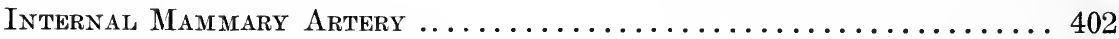

Transverse Muscle of Thorax . . . . . . . . . . . . . . . . . . 402

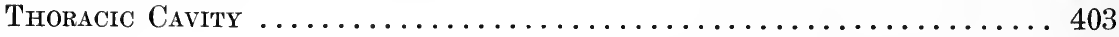

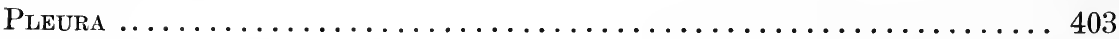

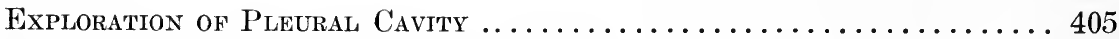

Removal of Central Portion of Sternum ................ 407

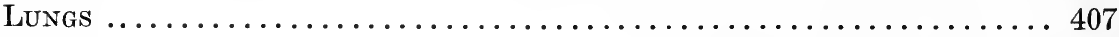

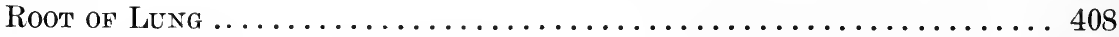

Phrenic Nerve and Cardiac Plexus ......................... 409

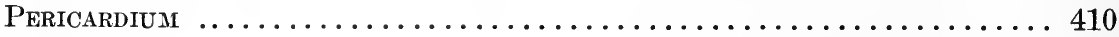

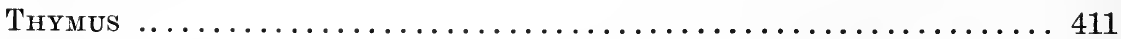

Great Veins of Thorax and their Tributaries ............... 411

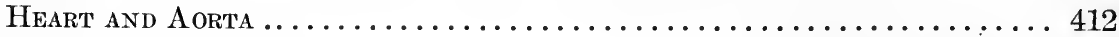

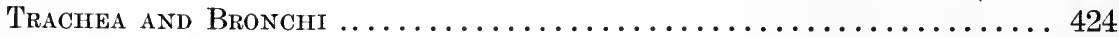

Posterior Mediastinal Cavity and its Contents ............... 424

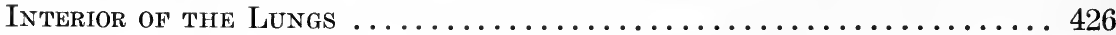

Thoracic Portion of Sympathetic Nervous System .............. 428

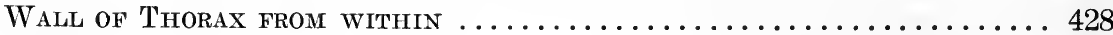

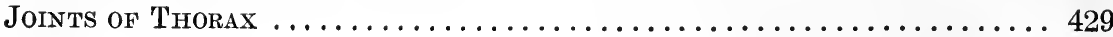

\section{Part V.}

\section{ABDOMEN AND PELVIS.}

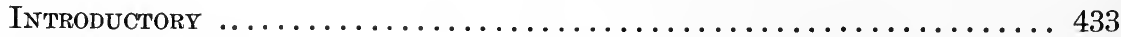

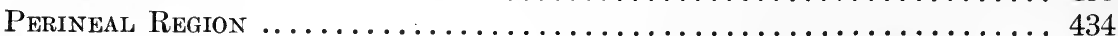

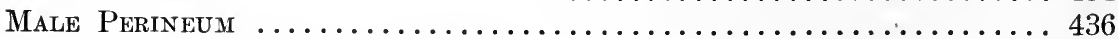

Urogenital Region in the Female .......................... 444

Anal Region and Diaphragm of Pelvis in botrh Sexes ............. 449

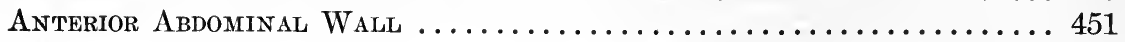


Peritoneum and Abdominal Viscera ..................... 462

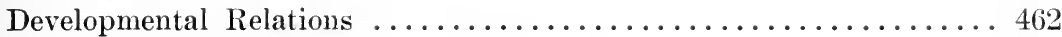

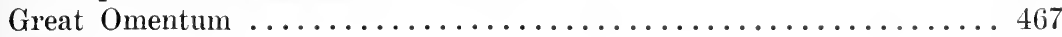

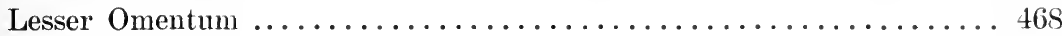

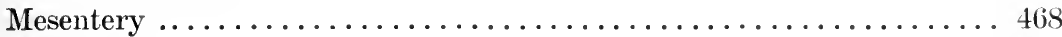

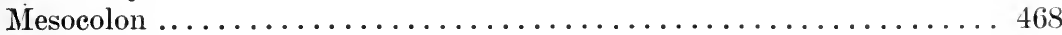

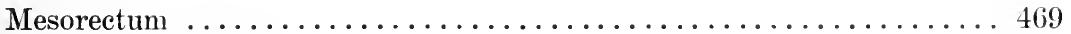

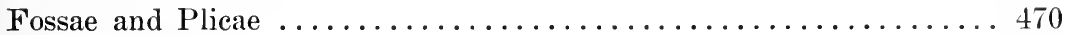

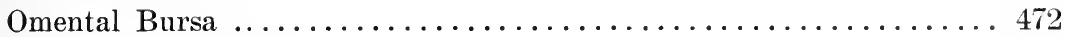

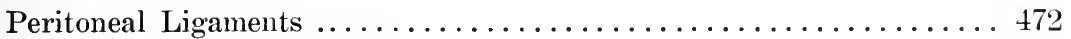

Superior Mesenteric Artery ........................ 474

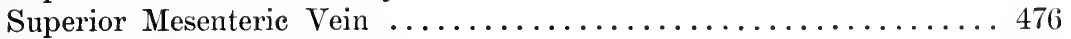

Superior Mesenteric Plexus ...................... 476

Inferior Mesenteric Vessels and Nerves ................... 477

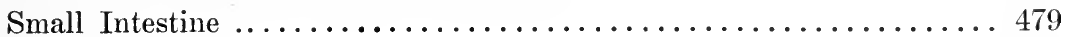

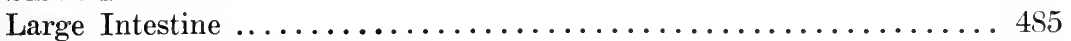

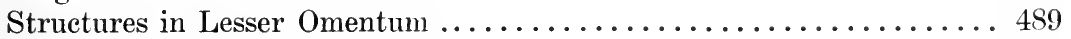

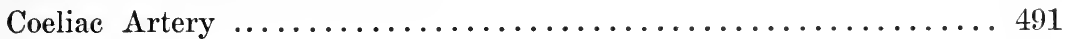

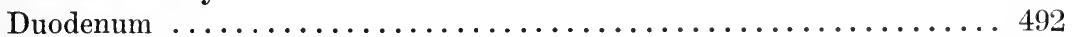

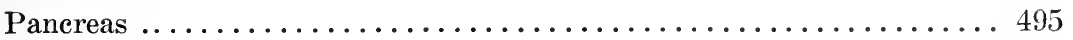

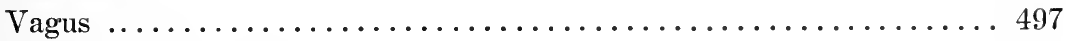

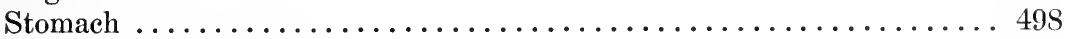

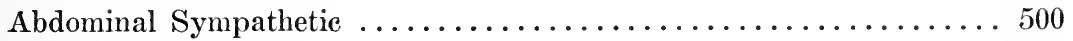

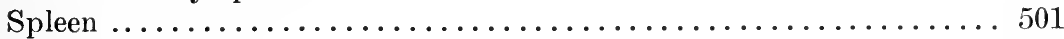

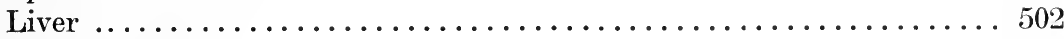

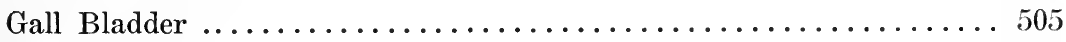

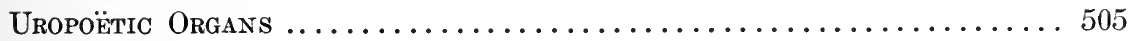

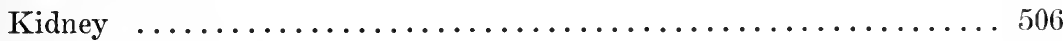

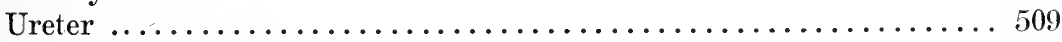

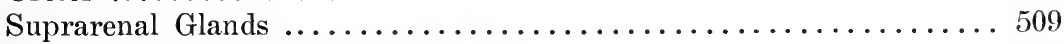

Muscles and Vessels in the Abdonen .................... 511

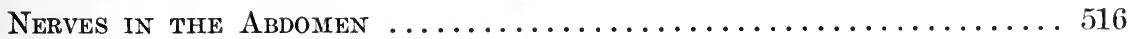

Dissection of the Male Pelvis ......................... 519

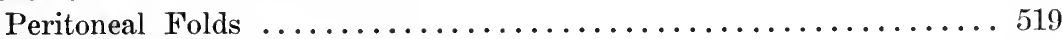

Hypogastric Plexus ................................ 519

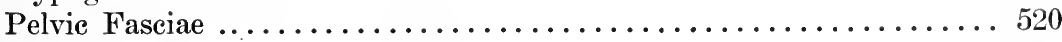

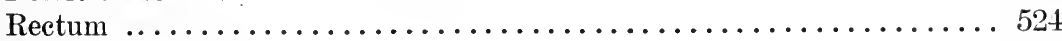

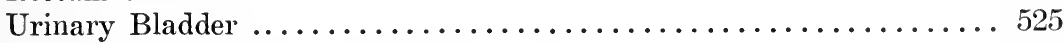

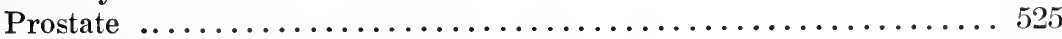

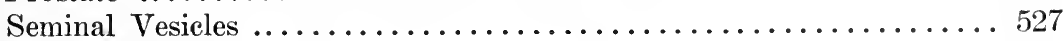

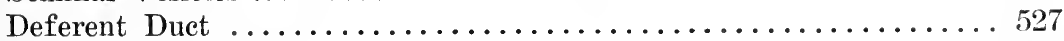

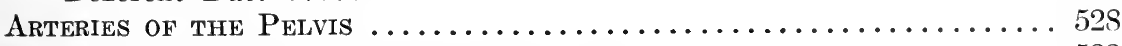

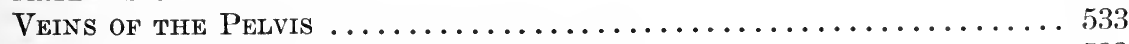

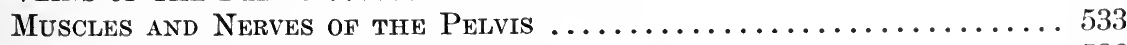

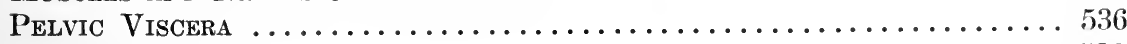

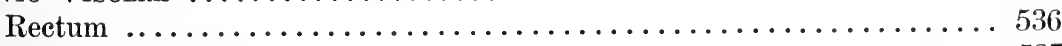

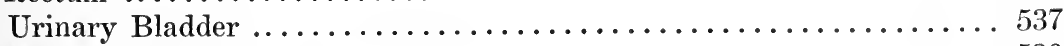

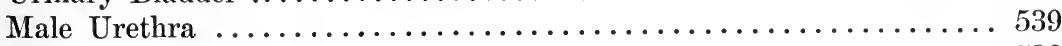

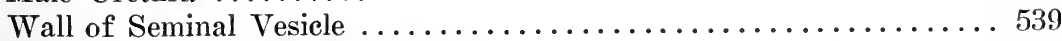

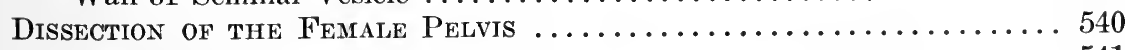

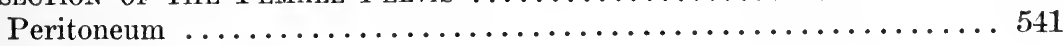


Dissection of the Female Pelvis:

Hypogastric Plexus ............................ 541

Pelvic Fascia ............................... 541

Vessels and Nerves ........................... 542

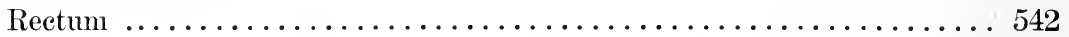

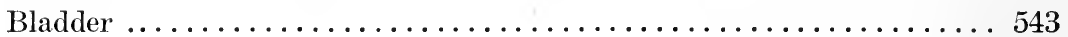

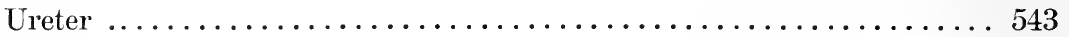

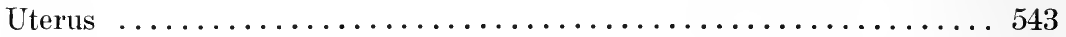

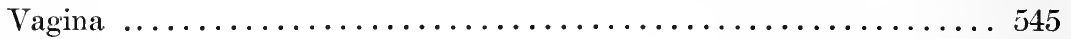

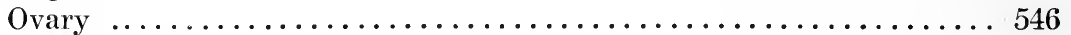

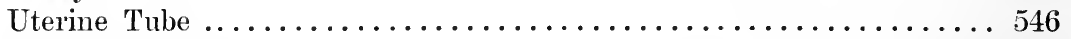

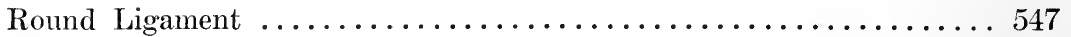

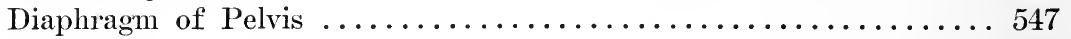

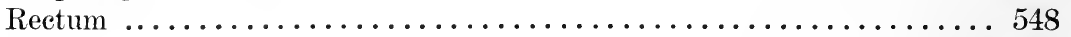

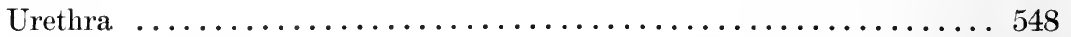

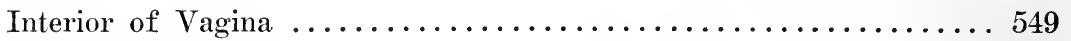

Interior of Uterus ............................. 549

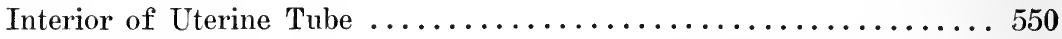

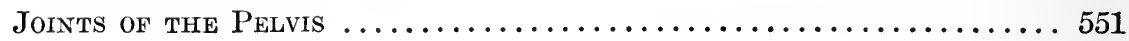




\section{INTRODUCTION}

THE anatomical laboratory has the longest history of all the medical laboratories. The student has the experience of hundreds of years to draw upon. The best methods of isolating the various structures have been worked out and certain rules for dissection have been formulated; the student will do well to familiarize himself with these as early in his course as possible.

Careful, practical work in the dissection-room is the only means the medical student has of obtaining an independent view of the gross structures of which the human body is composed. Text-books, atlases, demonstrations, and lectures are useful aids, helpful in exciting interest, and favorable to the acquisition of powers of description and illustration; but the main part of the work in anatomy should consist of the student's personal observation. He should learn to see what comes under his eye. He should try by his own independent activity to see all that is there, for the student who learns to see only what he is told to see or what is pointed out to him will be spoiled for the study and practice of medicine.

Many students in the past have entered the dissecting-room with an utter lack of independent power of observation, of examination, and of description, and yet these three qualities are absolutely necessary for the man who is to engage successfully in the practice of medicine. While it is to be hoped that the advance in the requirements for admission to professional schools, leading as it does to observational work in physics, chemistry, and biology preliminary to the work of the medical course, will bring the students to our laboratories of anatomy better prepared than hitherto for the independent observation of anatomical structures, there can still be but little doubt that many students will learn how really to study and observe first in the dissecting-room. How important it is that the habits formed at this early period of the professional course should be such as will be of value to the student later, such as he would desire to have throughout his professional career! If he have learned to be exact and steady, systematic and thorough, cleanly 
and artistic, in his work in the dissecting-room, how much better for him than if at the outset of his career he become satisfied with superficial observation, with interrupted and irregular activity, with disorder and uncleanliness! The habits formed in the anatomy building have their effect upon the whole life of the physician.

Moreover, the very first dissection may be all-important as regards the subsequent dissections. He who fails in his earliest dissection to acquire the habit of working according to a definite method seldom learns later to become an exact, quick, and careful dissector. It is almost hopeless to try to make a man do exact and clean work in the dissecting-room after he has once formed inaccurate and dirty habits. The student in the beginning should aim not so much at rapidity as at method. When he has once formed the habit of proceeding always according to a careful method, the rapidity will quickly follow.

It is sometimes asked, "Why should the medical student spend so much time and take so much pains in making a careful and beautiful dissection? Is it not time wasted to work out the smaller branches of the nerves and blood-vessels?" Experienced teachers who require thorough work are not disturbed by such inquiries. Those who have had the best opportunity for judging assert that it is only when a man succeeds in making a fine dissection that he gains an interest and pleasure in an occupation which may not at first be attractive to him. Further, the importance of working out the finer structures lies not always so much in the actual knowledge of these structures gained by the student, as in the acquisition of the habit of thoroughness of observation and investigation. The medical student who in the dissecting-room dissects out only the main trunks of the vessels and nerves, or only the structures which at the moment seem to physicians and surgeons of greatest practical import, is likely as a clinician to be satisfied with the detection of the more obvious and superficial symptoms, to the overlooking of the less prominent symptoms and the more obscure physical signs. The thorough dissector is much more likely later to become a finely discriminating physician and an effective progressive surgeon. Again, if a dissector permit himself to expose structures roughly, to isolate them incompletely, and to clean them imperfectly, so that his dissection becomes indistinct, badsmelling, and repulsive-looking, he will quickly lose interest in his work, slice the part desperately, neglect it, stay away from it, and fail utterly to benefit by it. Each student should, therefore, 
at the beginning, err, if at all, upon the side of over-exactness and over-carefulness in the use of instruments, in the methods of handling anatomical objects, and in the isolation of the details of structure. He should at first force himself to follow established rules and regulations rigidly; if desirable, later on in his work he may allow himself more latitude. He should aim to make his dissection such that he may at any moment and without embarrassment turn it over to an instructor who may wish to deliver a lecture on the part; he should aim to do his work so carefully that an artist may satisfactorily make a drawing of it to illustrate a text-book.

While a large portion of the student's time in the early part of the dissection is occupied with the technique of the work, learning how to find the various structures and to demonstrate them properly, he should not forget that the mere mechanical side of the work, important as it is, is not the only side. It is necessary that he should also study the structures carefully, learn their names, examine their relations, and, above all, understand their significance. Atlases, text-books, models, and moist and dry preparations should be assiduously used in connection with the dissection of the part, if the student is to acquire in the anatomical laboratory the knowledge and skill which it is desired that that laboratory shall give him at the beginning as a basis for his other medical studies.

\section{INSTRUMENTS NEEDED.}

Each student should provide himself with a good set of dissecting instruments made by a reliable manufacturer. They should be made of good steel, properly tempered and well ground. It is false economy to buy cheap instruments or to secure a set at second hand. The quality of the student's work will be much influenced by the kinds of instruments he uses and the state in which he keeps them. A good set of dissecting instruments will not cease to be valuable to the medical student when he finishes his dissection, but will be found convenient for many purposes during his subsequent practical life.

The sets supplied by the manufacturers vary greatly in extent and quality, and the student may find it advantageous to make up a set for himself, choosing the individual instruments from stock, rather than to purchase any regular dissecting-case offered. As a minimum the student should possess: 
1. One cartilage-knife (with bellied blade).

2. Three dissecting scalpels with bellied blades, one large, one of medium size, and one small.

3. Two dissecting scalpels with straight edges and sharp points, one long and one short.

4. One pair of medium-sized scissors.

5. One pair of small scissors.

6. One blunt probe (Mall's).

7. One large dissecting forceps.

8. One tubulus or blowpipe.

9. One sound (fishbone or metal).

10. One good curved needle and sutures.

11. One good razor-strop.

12. A medium-sized sponge.

Other instruments, including mallet and chisels, saws, rhachiotome, myelotome, enterotomes, and bronchial scissors, will be supplied by the laboratory.

The student may find it desirable to have more scalpels than are required above; if so, he will find it best to have more bellybladed knives than knives with straight edges.

When purchasing forceps, the greatest care should be exercised, for it is rare to find properly constructed samples. The branches should be relatively strong, not too flimsy; on closing they should come together almost with the weight of the thumb and finger, as nothing grows more tiresome than forceps which are too stiff to close easily. Forceps with well-worked-out points should be chosen, so that they will grasp fine structures with certainty. The points should not slide over one another on closing.

\section{CARE OF INSTRUMENTS.}

The best instruments are quickly ruined where no care is taken to prevent injury or deterioration. If scalpels and forceps are permitted to fall frequently to the floor, or if cutting instruments are thrown carelessly in contact with other instruments, their value as dissecting implements rapidly diminishes.

Knives should be kept sharp and smooth-cutting. This is absolutely necessary if the student is to do good work, for it is impossible to dissect neatly and quickly with dull knives. The strop, if used at short intervals,- - say, every ten or fifteen minutes,-will keep a smooth-cutting edge on a sharp knife and increase its power of penetration. A knife properly sharpened and preserved from gross injury may be kept in good condition for a long time without honing or grinding if it be properly and frequently stropped. 
When a knife becomes dulled and stropping is no longer sufficient to renew its edge, it should be honed. So few men learn how properly to hone a knife that it has been questioned whether students had better attempt it themselves or turn their scalpels over to an instrument-maker to be honed. Still, as one needs also to know how to keep a microtome-knife in repair, it is an advantage to learn to do the honing one's self. A good oil-stone or Arkansas stone is necessary. The knife, held obliquely, is drawn to and fro with the edge in advance, the stroke extending from the heel along the whole length to the toe of the blade. Experience under some one who is skilled is very desirable, that one may know the exact angle of inclination to employ, and the point when the blade has been sufficiently honed, for after a time a continuance of the honing only does harm. Much skill and experience are required to get exactly the right thinness of edge. It is to be borne in mind that dissecting knives ought not to be so thin as a surgical knife, where quick penetration is all-important and the knife is to be used only for a short period, for such knives are quickly dulled.

After repeated honing a knife may lose all its thin edge; it should then be ground.

The student should avoid injuring his scalpels by cutting against bone or the end of the forceps. It is well to keep one coarse knife especially for cutting near the bone or through calcified tissues.

All instruments should be cleaned and rubbed thoroughly dry before they are put away at the end of a day's work. If a dissection has been completed and the instruments are to be laid aside for some time, they should be boiled for ten minutes in a one per cent. solution of sodium carbonate, thoroughly dried, and lightly coated with clean vaseline. This sterilizes them completely and helps to prevent rust.

\section{USE OF INSTRUMENTS.}

Scalpel.-One of the most important things for the student to learn in technique is the use of the convex or belly-bladed scalpel. This blade has a long cutting edge, and is employed for making large cuts or for cutting large surfaces; it is unsuited for small, careful cuts or for dissecting in angles or in the depth. The convex scalpel is used chiefly in dissecting the skin, fascia, and muscles, and in general where large incisions are to be made and the work is relatively gross. In the dissection of vessels and nerves, on the other hand, the larger belly-bladed 
scalpels should never be used; even the smallest ones must be very carefully employed for such dissection, or important structures will be injured.

The scalpel with the straight edge and sharp point has a use entirely different from that of the belly-bladed knife. Although the blade of the knife with the straight edge may be quite long, one uses only the point of the knife in dissection; the action of such a knife is, therefore, much more limited than is that of the belly-bladed scalpel, and it should be used in making only the smaller, less extensive cuts. Students are frequently seen trying unsuccessfully to make large incisions with such knives, especially when their convex blades are dull. The scalpel with straight edge and sharp point is especially valuable in the dissection of the viscera, sense organs, blood-vessels, and nerves. It may well be employed whenever a small, careful cut is to be made, and it will be found of the greatest convenience when the student is working in corners or in deep fossae.

The dissector should early learn the right way to hold a scalpel. The manner of holding in the dissecting-room is quite different from that in the autopsy-room of the pathologist. In the former case and in surgical work the knife is to be held "like a pen," between the thumb and the first two fingers, the handle of the scalpel pointing obliquely upward. In the post-mortem room the pathologist usually holds his knife " in his fist," the handle of the scalpel being raised only slightly above the level of the blade and resting in the palm of the hand. The only time the dissector in the laboratory of human anatomy is permitted to hold the knife " in his fist", is when he makes a long incision through tough skin; in all other circumstances the knife should be held " like a pen."

The thumb, index and middle fingers should alone be used in holding the knife " like a pen." The ring-finger should not be permitted to touch the knife, no matter how much the dissector is inclined thereto. Only by following this rule can the anatomical workman secure the necessary freedom and acquire the delicacy of movement requisite to good work. The ring-finger and the little finger will be found very useful in making tissues tense, in retracting tissues, or in displacing them to the side.

The scalpel should be grasped by the thumb and fingers at about the junction of blade and handle, certainly not high up on the handle, certainly not far down on the blade. In making careful cuts the hand should be supported upon its ulnar margin. This support permits of greater precision; the cut desired may 
be made with sureness and steadiness and without anxiety. The student should keep his finger-joints nimble, and should practise to acquire increased mobility of these joints. The skill gained in the use of instruments in the dissecting-room may be of great value in subsequent surgical practice.

The importance of having several belly-bladed knives and several knives with straight edges has been referred to above. No good dissector cares to use the same knife for working out skin, vessels, muscles, nerves, etc. There should be a "division of labor"' among scalpels. Hard, firm, tough tissues should be cut through only with the cartilage-knife or with a duller scalpel.

Forceps.-These should be held in the left hand, "like a pen," between the thumb and first two fingers, the ring-finger and little finger being reserved for the support of the hand and never permitted to touch the instrument. The forceps should never be held " in the fist." They should be grasped about the middle, not too high up, and the fingers should not be cramped, otherwise the hand tires too quickly. The support of the hand on its ulnar margin by means of the little finger and the ringfinger is important, as it helps to make the work more exact and more certain.

Scissors.-These should be grasped by the thumb and middle finger. The scissors are generally used where the parts to be cut through are soft, yielding, and easily displaceable and do not offer much resistance to the edge of the knife. Scissors are much employed by many dissectors in the study of the viscera and of the blood-vessels. They are especially valuable in following out through loose connective tissue or fat the finer branches of arteries and veins, which might be more easily cut if the knife were used. Like the straight-edged scalpel with a sharp point, scissors are useful in cutting in angles or corner's and in deep places. Fat held in the forceps can be most easily removed with scissors. Very fine scissor's are useful in the dissection of delicate nerves and of the small muscles of the face and orbit, but in general scissors are little suited to the dissection of muscles and nerves.

Probe.-The flimsy probes usually supplied in dissecting sets are of but little use and should be discarded. The firm probe devised for use in Baltimore by Professor Mall is the best one available. Many students do a very large part of their dissection with this probe. It is especially useful for the beginner, who is afraid of destroying important structures with the knife. The "blunt dissection" which the probe permits of insures the 
working out of many structures which would otherwise be cut away. In using the dissecting-probe the least force necessary to the purpose should be brought into play, the strength of the tissues being, as it were, continually tested, and the loose tissue between the definite structures being gently removed by repeated light strokes instead of by a few strong sweeps of the probe. "Wear away rather than tear away!" This will avoid giving the muscles a ragged appearance and will also preserve many small and delicate structures which would otherwise be lost.

There is danger, however, of over-use of the method of blunt dissection with the probe. It is highly important that the student should gain skill with the scalpel, and this he can do only by constant practice with it. Again, where the probe is used instead of the scalpel, it is not possible to make so clean a dissection as the student should require of himself. The structures are left ragged, and the artistic sense of the dissector remains undeveloped, or, if developed, is offended.

\section{PRESERVATION OF THE PART.}

A large portion of the pleasure in his work and of the benefit to be derived from it will be lost if the student neglects to take proper care of the part while he is dissecting it and during the intervals between the periods of dissection. If a part be allowed to become dirty, if it be permitted to dry up, or if it become foul, the student will lose interest in it, will tire of it, and will inevitably neglect it. If the part has been well preserved in the first place, its preservation after arrival in the dissection-room depends entirely upon the student. If he handle it properly and protect it carefully, he can keep it in prime condition until the dissection has been completed.

Drying of the part is the greatest of all dangers. The moment the tissues become dry, knives are dulled in dissecting them and it quickly becomes impossible to isolate the finer structures, which, moreover, lose their natural color and form. In addition, the part grows repulsive to sight.

The skin of the cadaver is one of the best protectives against drying. Skin when removed should always be kept as a covering for the part. When the dissection is put away at the end of a working period, it should be wrapped carefully in the integument, which should be securely fastened by tying or pinning it in place. Some careful dissectors take the trouble to secure cutaneous flaps in position by stitches each time the part is put away. 
In addition, the part should be thoroughly wrapped in gauze or cheese-cloth saturated with a three per cent. solution of carbolic acid or other preserving fluid and held in place by twine. It is convenient to have a large quantity of the preserving fluid in a tub in the dissecting-room, out of which the wrappings may be wrung by hand or by a laundry wringer. The gauze or cheesecloth should be folded into a convenient shape and size.-a few inches longer than the part to be covered and about eighteen inches wide. After being moistened in the preservative fluid, it may be wrapped about the part and secured by tapes or twine. Such a covering is readily and quickly removed when the part is required for work. It is also very useful for eovering such portions as are not immediately under dissection or study. As an additional protection against drying, a piece of oil-cloth, about a foot square, is advisable for the hand, foot, and face.

As soon as possible after the cadaver is placed on the table, the student should take charge of his part, wrap it up, and attach to it a label bearing his name.

Not only in the intervals between the dissection periods, but also during working hours, care should be taken to prevent drying. Parts not in actual use should be kept covered, for even two hours' exposure causes drying which deteriorates the material. The portion of the dissection actually being worked upon should be moistened occasionally with a wet sponge. The best results are attainable only if the material be kept in good condition; it is not possible to restore dried-out material to as good condition as it was in before drying or partial drying. Especial care should be taken to protect parts belonging to students not present.

The cadaver should be kept raised on dissecting-blocks, so as to allow free drainage away of the fluids which otherwise cause the under parts to become macerated and offensive. The drainpipe in the centre of the table must be kept unobstructed.

Cleanliness about the dissection-table should be cultivated. Bits of fat and connective tissue which are removed should never be allowed to contaminate the wrappings of the part or the table itself. They should be collected on a piece of paper or cloth and then thrown into the refuse-pail. The student should leave his dissection and the dissection-table in perfect order and condition at the close of each day's work. A little care exercised in the formation of orderly and cleanly habits at this period of his development will be of great service to the student in his subsequent work. 


\section{CLOTHING.}

The better methods of preserving the material for dissection prevalent nowadays have removed some of the unpleasant features of the occupation. It is not possible, however, to avoid all odor or all contamination, and the student should, therefore, go to the dissecting-room clad in a manner which will permit him to work freely and without constant worry lest his clothing come into contact with the material. Hence it is necessary to have an old coat to be kept for use in the dissecting-room only, and it is preferable to change the whole suit on entering and on leaving the room. As a roomful of men garbed in old coats is not a pleasing spectacle, it is the custom in modern anatomical laboratories to wear white cotton gowns over all. The student should, therefore, provide himself with three such gowns, so that he may always have the soiled ones laundered while the clean one is being worn. Gowns other than white should be avoided, as should the various oil-cloth sleeves and aprons formerly so much used. No inconsiderable part of the depressing influences of the old-time dissecting-room consisted of the dirty floors, the untidy tables, the tobacco-smoke, the spittoons, the oil-cloth sleeves and aprons, and the multicolored or checked gowns.

Dissection is a serious task, requiring much energy and endurance. Under the best conditions the student tires all too quickly. It is important, therefore, that the sanitary conditions of the room be the best possible, that as comfortable a position as practicable be assumed in dissecting, that the dress be one which while externally uniform throughout the room will permit of the greatest freedom in work, and that all depressing influences be as far as possible removed.

\section{DRAWING IN GROSS ANATOMY.}

The student should not try to draw all that he sees, but only so much as is important in the stage represented. He should limit the contents of each picture to what can be shown clearly, and try to avoid unimportant detail.

The drawing is to be made large enough to show clearly all that it contains. In general, it should be as large as the size of the drawing-paper will permit, allowing for the legend.' It is well to make the drawing on a definite scale, and to record on it the proportion which the size of the picture bears to the natural size, using a fraction to express this,-e.g., $1 / 5,1 / 2,1 /{ }_{1}, 2 / 1$, or whatever the scale is. 
The drawing should always be "placed" carefully, so that it will not be too near either side, the top, or the bottom of the page. Space must be reserved for the legend. The drawings ought not to be crowded: one on a page is usually all there is room for, and only one side of the paper should be used. ${ }^{1}$

The gross features should first be attended to, the general direction of the lines which bound the structures, representing position, size, general shape, and proportions, being sketched in. These lines ought to be made as near right as possible without taking an undue amount of time. The finished drawing will be correct and satisfactory relatively to the accuracy of this preliminary, general sketch.

The details, proceeding from larger to smaller, are to be drawn next. Usually much detail is not only unnecessary but even objectionable. The endeavor to get too much detail, and to get it without first attending to the general features, is almost the sole cause for the common remark, "I can't draw and never shall be able to!', Any one who can write can draw, and, by observing the right method and the proper sequence, can make drawings that are useful and valuable.

The pencil should be medium soft; $\mathrm{HB}$ is the most useful grade. The lines should be drawn on, not into, the paper, and this requires lightness of touch. The pencil point is to be kept long and sharp, ${ }^{2}$ so that it will mark readily and make a fine line that can be seen while it is being made. The strength (that is, width and blackness) of the various lines in the finished drawing should be proportionate to the relative importance of the structures which they represent. The term "relative importance", has reference here not to anatomical or physiological value, but to interest in the picture: the things in the picture that give it a name are the things of greatest relative value in that picture.

In labelling " print" the names neatly, preferably with ink. If there be difficulty in making the words straight and horizontal, light, horizontal lines may be ruled for guides; these can afterwards be erased. No attempt should be made to form the letters exactly like printed (press) type, but a free style is to be used.

The pointing lines, either dotted or unbroken, should be ruled with a sharp pencil. This differentiates them from the lines of

${ }^{1}$ The right side of the drawing-paper should always be used. The wrong side (when only one side is for use) is dimpled so as to look as if impressed with cotton or linen.

${ }^{2}$ A piece of sand-paper glued to a strip of wood is handy for this. 
the drawing. It is sometimes well to mark the exact termination of the line by a small cross.

The name of the drawing in larger letters may be placed at the head or at the foot of the page. The view-point, scale, and date should also be given. Each sheet of the drawings should be initialed for identification, the initials being placed where they cannot be clipped off.

Actual representation of an object is limited to the two dimensions of a plane. The third dimension of solid bodies is expressed by perspective, which is made up of several factors, chiefly $(a)$ binocular vision, $(b)$ aerial perspective, $(c)$ foreshortening, $(d)$ sequence of objects, $(e)$ chiaroscuro, or relation of light and shade.

The part played by binocular vision is of use only in stereoscopic pictures. Laboratory drawing is limited almost wholly to representation of things as seen by one eye.

Foreshortening refers to apparent size and shape of objects (or surfaces) as dependent on position and distance. Ordinarily we do not take cognizance of it at all, or only partially. It may be made evident in several ways, - as, by inverting the head and looking at things upside down; by looking at them in a mirror and regarding the images as situated on its surface; or by interposing a transparent plane object (wire netting or glass-e.g., window-pane) between the eye and the object, perpendicular to the line of vision, and regarding the lines as projected forward and drawn on this surface in one plane. To get a foreshortened drawing it is, then, only necessary to copy on paper the lines as thus seen; or they may be traced with crayon or India ink on the mirror, net, or glass itself.

Objects that are partly hidden by nearer objects are thereby shown to be more distant than the latter.

Aerial perspective, affecting color, distinctness of detail, etc., is of importance only when the distances are considerable, as in landscape, and need not be discussed here.

The shading is self-evident. It is due to the amount of light reflected to the eye from the various surfaces, or areas of a surface, which have different directions, and therefore receive and reflect different amounts of light. For the purposes of drawing, the object may be regarded, and represented, as illuminated by light coming from a source above to the left. This simplifies shading and gives it a constant significance in expressing direction of surfaces. (It is to be noted that shading can also be used, as in black and white pictures, to represent color-value or rela- 
tive brightness of objects of different colors.) All surfaces so directed as to receive and reflect most of this light to the observer's eye will be brightest (unshaded, or having " high lights"); those directed away from the light will be darkest (shaded, or in shadow); while surfaces having an intermediate direction will be shaded less or more according as they approach one or other of the above extremes.

A plane surface will have uniform light or shading. Rounded surfaces will have graduated shading; areas separated by sharp edges will have abrupt differences of shading, the contrast being greater the smaller the angle between the adjacent areas, or, in other words, the greater the difference in their direction. In rounded objects the highest light and the deepest shade are situated close to, but not at, the edge of the outline. All the shading should be kept right in relation to the deepest shadow.

The student should examine book-illustrations and observe how shading is represented. (a) Some degree of depth may be given in a mere outline drawing by making the lines on the lower right side of the objects heaviest. (b) Plain parallel lines are used for shading in "line" drawings. The depth of shading is varied by the heaviness of the lines, their distance apart, and by " cross-hatching" or putting additional sets of shade-lines over the first at very acute angles of crossing. ${ }^{1}(c)$ The pencil may be held obliquely and rubbed most on the parts to be shaded, giving a photographic effect. $(d)$ Some of the lead of the pencil may be scraped off with a knife and applied to the drawing-paper by means of a paper stump, giving a means of rapid working, but one that is apt to give a smudgy effect. (e) Wash-drawings are shaded either by repeating the wash or by using a stronger wash on the shaded parts. $(f)$ A softer pencil may be used for the deeper shading. Finally, it is well to "pick out" the high lights with a soft eraser. $(g)$ Dots may be used instead of lines, as in stippled drawings, Ross-board drawings, and half-tone illustrations.

Colors may be utilized to differentiate structures,-e.g., red for arteries, blue for veins, brown for muscles, green or orange (or plain black) for nerves. Good crayons are useful here. Higgins's inks of various colors may be used in pen work. Washes are very effective also. As a rule, all color should be used very sparingly, and so should shading.

${ }^{1}$ Sometimes the shading lines are curved in conformity to curvatures of the surfaces depicted, as may be seen in old wood-prints, steel engravings, and mechanical drawings. 
To prevent smudging of soft pencil work, the drawings may be "fixed" by spraying them lightly with a fixative, such as bleached shellac dissolved in absolute alcohol or the regular solution that may be obtained, together with a spray-pipe, from dealers in artists' supplies.

\section{BOOKS.}

In addition to this Laboratory Manual, the student of gross human anatomy is advised to provide himself with certain necessary text-books, concerning which he should consult his instructor. In the University of Chicago the following are recommended:

(1) A good atlas of the human body, preferably W. Spalteholz's " Hand Atlas of Human Anatomy" (Barker's translation), or C. Toldt's "Anatomischer Atlas."

(2) A good systematic text-book of human anatomy, preferably (at time of writing) the "Systematic Text-Book of Human Anatomy" edited by D. J. Cunningham or Quain's "Elements of Anatomy" edited by Schäfer and Thane.

(3) A text-book of embryology, preferably (at present) McMurrich's "Human Embryology" or C. S. Minot's "Embryology" or (if the student can read German) J. Kollmann's "Lehrbuch der Entwickelungsgeschichte des Menschen."

(4) A text-book on neurology, preferably Barker's "The Nervous System and its Constituent Neurones," or Van Gehuchten's text-book if a French, or Obersteiner's or Edinger's if a German, author be desired.

\section{LIBRARY.}

Students beginning to study medicine often have difficulty in learning how to use libraries and how to find the bibliography of a subject in which they are interested. Each student should at the earliest opportunity request an instructor or a librarian to teach him how to use the "Index-Catalogue of the SurgeonGeneral's Library" and the "Index Medicus," the keys to medical literature. For convenience, a list of some of the more important reference-books and journals to be consulted in connection with anatomical studies is here inserted. The student will find these books and journals in the college library. If any of them are lacking, he should use his influence to have them added to the library collection as soon as possible. The list is by no means exhaustive, but includes some of the books and journals likely to be frequently consulted; it does not consider the literature of microscopic anatomy and histology. 


\section{General Works on Human Anatomy.}

Allen, H. A System of Human Anatomy. Including its Medical and Surgical Relations. 4to. Philadelphia, 1882.

v. Bardeleben, C. Handbuch der Anatomie des Menschen. \& vols. Jena, 1902.

Gegenbaur, C. Lehrbuch der Anatomie des Menschen. 7 Aufl. 2 vols. Sro. Leipzig, 1898.

Gerrish, F. H. A Text-book of Anatomy by American Authors. 2d ed. Phila. and New York, 1902.

Gray, H. Anatomy, Descriptive and Surgical. Ed. by T. Pickering Pick and R. Howden. Phila. and New York, 1901.

Huntington, G. The Anatomy of the Human Peritoneum and Abdominal Cavity, considered from the Stand-point of Development and Comparative Anatomy. New York, 1903.

Krause, W. Handbuch der Anatomie des Menschen, mit einem Synonymenregister. Auf Grundlage der neuen Baseler anatomiseher Nomenklatur unter Mitwirkung von W. His und W. Waldeyer und unter Verweisung auf den Handatlas von W. Spalteholz bearbeitet. Leipzig, 1898.

von Langer, C. Lehrbuch der systematischen und topographisehen Anatomie. 5 Aufl. 8vo. Wien und Leipzig, 1893.

Morris, H. Human Anatomy. A Complete Systematic Treatise by Various Authors, including a Special Section on Surgical and Topographical Anatomy. Last edition. Philadelphia.

Poirier, P., et A. Charpy. Traité d'Anatomie humaine. 5 vols. Paris, 1901.

Quain, J. Elements of Anatomy. Ed. by Schäfer and Thane. 10th ed. 3 vols. London and New York, 1892.

Rauber, A. Lehrbuch der Anatomie des Menschen. Leipzig.

Sappey (M.-P.-C.). Traité d'Anatomie descriptive. 3 vols. 12mo. Paris, 1850-1864.

Testut, L. Traité d'Anatomie humaine. 2 vols. royal 8vo. Paris, 1889-1891.

\section{Anatomical Atlases.}

Anatomischer Atlas für Studirende und Aerzte unter Mitwirkung von Professor Dr. Alois Dalla Rosa. Hrsg. von Carl Toldt. Wien und Leipzig, 2 Aufl., 1900.

Broesike, G. Anatomischer Atlas des gesamten menschlichen Körpers. Berlin, 1899.

Sobotta, J. Atlas der Anatomie des menschlichen Körpers. München, 1904.

Spalteholz, W. Hand Atlas of Human Anatomy. Translated by L. F. Barker from the 3d German edition. 3 vols. Leipzig, 1901-1903.

[The descriptive text accompanying the plates of this atlas is a model of conciseness; some students prefer it to that of the larger systematic text-books.]

\section{Manuals of Dissection.}

v. Bischoff, Th. L. W. Führer bei den Präparirübungen bearbeitet von Dr. N. Ruedinger. München, 1859.

Braune, W., and His, W. Manuel de Dissection. Trad. par le Dr. G. Foettinger. 8vo. Bruxelles, 1887.

Campbell, W. A. Outlines of Anatomy for Students. A Guide to Dissection, based on Morris's Text-book of Anatomy. 8vo. Philadelphia, 1895. 
Cooke, T., and Cooke, F. G. H. Tablets of Anatomy. 11th ed. 3 vols. London, 1898.

Cunningham, D. J. Manual of Practical Anatomy. Philadelphia and London, 1903.

Eckley, W. T., and Eckley, C. D. A Manual of Dissection and Practical Anatomy. Philadelphia, 1903.

Heath, C. Practical Anatomy; a Manual of Dissections. 8th ed. 12mo. Ed. by W. Anderson. Philadelphia, 1893.

Holden's Anatomy. A Manual of Dissection of the Human Body. 7th ed., revised by A. Hewson. 2 vols. Philadelphia, 1901.

Ruge, G. Anleitungen zu den Präparirübungen an der menschlichen Leiche (1 und 2 Theile). 8vo. Leipzig, 1888.

\section{Anatomy of the Nervous System.}

Barker, L. F. The Nervous System and its Constituent Neurones. New York, 1900.

Bruce, A. A Topographical Atlas of the Human Spinal Cord. Edinburgh, 1901. Dejerine, J. Anatomie des centres nerveux. 2 vols. 1895.

Donaldson, H. H. The Growth of the Brain. New York and London, 1895.

Edinger, L. Anatomy of Central Nervous System of Man and of Vertebrates in General. Transl. by W. S. Hall. Philadelphia, 1899.

Flatau, E. Atlas des menschlichen Gehirns und Faserverlauf. Berlin, 1899.

v. Gehuchten, A. Anatomie du Système nerveux de l'homme. 3d ed. Louvain, 1900.

Gordinier, H. C. The Gross and Minute Anatomy of the Central Nervous System. 1900.

Hardesty, I. Neurological Technique. Chicago, 1902.

v. Koelliker, A. Handbuch der Gewebelehre. Bd. ii. Leipzig, 1896.

v. Lenhossék, M. Der feinere Bau des Nervensystems im Lichte neure Forschungen. Berlin, 1895.

Obersteiner, H. The Anatomy of the Central Nervous Organs in Health and in Disease. Transl. by A. Hill. London, 1900.

Sabin, F. R. An Atlas of the Medulla and Midbrain: A Laboratory Manual. Edited by H. McE. Knower. Baltimore, 1901.

Whitaker, J. R. Anatomy of the Brain and Spinal Cord. London, 1899.

Whitehead. Anatomy of the Brain. Detroit, 1900.

Ziehen, Th. Nervensystem, in Bardeleben's Handbuch der Anatomie des Menschen.

\section{Surgical and Topographical Anatomy.}

v. Bardeleben, K., H. Häckel, und F. Frohse. Atlas der topographischen Anatomie des Menschen. 2 Aufl. Jena, 1901.

Deaver, J. B. Surgical Anatomy. 3 vols. Philadelphia, 1900-1903.

Joessel, G. Lehrbuch der topographisch-chirurgischen Anatomie mit Einschluss der Operationsübungen an der Leiche. Bearbeitet von W. Waldeyer. Bonn, 1898.

MeClellan. Regional Anatomy. Philadelphia, 189-.

Merkel, F. Handbuch der topographischen Anatomie. Braunschweig, 1898.

Rüdinger, N. Cursus der topographischen Anatomie. 4 Aufl. München, 1899.

Treves, F. Surgical Applied Anatomy. 4th ed. London, 1889.

Zuckerkandl, G. Atlas der topographischen Anatomie des Menschen. Wien, 1900. 
Anatomy for Artists.

Fritsch, G. Die Gestalt des Menschen. Stuttgart, 1899.

Pfeiffer, L. Handbuch der angewandten Anatomie. Leipzig, 1899.

Stratz, C. H. Die Schönheit des weiblichen Körpers. Stuttgart, 1899.

Thompson, A. Handbook of Anatomy for Art Students. 2d ed. London, 1899.

\section{Comparative Anatomy of Vertebrates.}

Claus, C. Text-book of Zoology. 2d Eng. ed. London, 1890.

Gegenbaur, C. Vergleichende Anatomie der Wirbelthiere mit Berücksichtigung der Wirbellosen. Leipzig, 1898-1900.

Jayne, H. Mammalian Anatomy as a Preparation for Human and Comparative Anatomy. Philadelphia, 1898.

Parker, T. J., and W. A. Haswell. A Text-book of Zoology. 2 vols. London and New York, 1897.

Wiedersheim, R. Grundriss der vergleichende Anatomie der Wirbelthiere. Jena, 1898.

The Cambridge Natural History. 1895 on.

\section{Embryology.}

Balfour, F. M. A Treatise on Comparative Embryology. 2 vols. 1880-1881. Duval, M. Atlas d'Embryologie. Paris.

Heisler, J. C. A Text-book of Embryology. Philadelphia, 1899.

Hertwig, O. Lehrbuch der Embryologie.

His, W. Anatomie mensehlichen Embryonen. Leipzig.

Kollmann, J. Lehrbuch der Entwickelungsgeschichte des Menschen.

MeMurrich. Text-book of Human Embryology. Philadelphia, 1903.

Minot, C. S. Human Embryology. New York.

\section{Anomalies.}

Le Double, A. Traité de variations du système musculaire de l'homme et de leur signification au point de vue de l'anthropologie zoologique. Tomes i., ii. Paris, 1897.

Le Double, A. F. Traité des variations des os du crâne de l'homme. Paris, 1903.

Testut, Léo. Les anomalies musculaires chez l'homme expliquées par l'anatomie comparée, leur importance en anthropologie. Paris, 1884.

\section{Current Literature of Gross Anatomy.}

American Journal of Anatomy. Baltimore.

Anatomischer Anzeiger. Jena.

Archiv für Anatomie und Entwickelungsgeschichte. Leipzig.

Internationale Monatschrift für Anatomie und Physiologie.

Jahresberichte über die Fortschritte der Anatomie und Entwickelungsgeschichte. Hrsg. von G. Schwalbe. Jena.

Journal of Anatomy and Physiology. London.

Merkel-Bonnet's Ergebnisse der Anatomie und Entwickelungsgeschichte. Wiesbaden.

Zeitschrift für Morphologie und Anthropologie. Hrsg. von Prof. Dr. Schwalbe. Stuttgart. 


\section{DIVISION OF THE CADAVER INTO PARTS.}

The number of men assigned to a given cadaver and the part that each will dissect will vary in different laboratories, partly according to the supply of material, partly according to the particular plan favored by the instructor. Very satisfactory methods of division have been agreed upon, among others the following:

A. For ten students to a cadaver, five on each side.

1. Arm and wall of thorax (extremitas superior).

2. Lower extremity (extremitas inferior).

3. Head, neck, and dorsum of trunk (caput, collum, et dorsum trunci).

4. Thorax.

5. Abdomen and pelvis (abdomen et pelvis).

B. For six students to a cadaver, three on each side.

1. Arm and wall of thorax (extremitas superior).

2. Lower extremity, abdomen, and pelvis (extremitas inferior, abdomen, et pelvis).

3. Head, neck, and upper part of trunk (caput, collum, dorsum trunci, et thorax).

C. For four students to a cadaver, two on each side.

1. Upper half of body (caput, collum, dorsum trunci, thorax, et extremitas superior).

2. Lower half of body (extremitas inferior, abdomen, et pelvis).

\section{ANATOMICAL TERMS INDICATING THE POSITION AND DIRECTION OF PARTS OF THE BODY.}

In descriptive anatomy certain technical terms, derived chiefly from the Latin and Greek, are necessary for the designation of parts of the body and for specifying exactly their spatial relations.

In descriptions the human body is usually thought of as standing upright, with the arms hanging at the sides,-i.e., alive; the older anatomists always represented the volar surface of the forearm and the palm as looking forward, but at present no definite position in space is ascribed to the forearm. The body, so nearly symmetrical, is thought of as being halved by a perpendicular plane, the so-called median (medianus) plane, into a right and a left half. The direction towards this median plane is designated as medial (medialis); that away from it, lateral (lateralis). By a vertical direction (verticalis) is meant one corresponding to the long axis of the median plane; a plane or line at right angles to the median plane, if it be parallel to the 
surface of the earth, is spoken of as being horizontal (horizontalis); if it merely run across the body or one of its parts at right angles to the axis of the body, it is said to be transversal (transversalis). The term transverse (transversus) means across the long axis of the organ concerned. Lines or planes which run parallel to the median plane are called sagittal (sagittalis); those parallel to the surface of the forehead, frontal (frontalis).

The terms inner (intermus) and outer (externus) have been very loosely used in English text-books, often being employed in the sense of medial and lateral, as well as indicating the direction in relation to the interior of organs, cavities of the body, or regions of the body; in the nomenclature used in this book the terms are restricted exclusively to the latter meaning. In describing the dimensions of organs length or longitudinal direction (longitudinalis), breadth or transverse direction (transversus), and thickness are the terms employed to indicate the largest, middle, and smallest dimensions. Particular attention is called to the distinction between transversus and transversalis, the latter being reserved for a direction transverse to that of the median plane.

The terms upper (superior) and lower (inferior) refer to the directions towards the vertex and towards the sole of the foot respectively, while the terms superficial (superficialis) and deep (profundus) indicate a position of less or greater separation from the external surface of a part or organ. The term middle (medius) is used to indicate a position between superior and inferior or between externus and internus, but, since medius is easily confused by the beginning student with medialis, especially when abbreviated, the term intermediate (intermedius) is employed to indicate a position midway between lateralis and medialis.

In order to have terms, independent of the position of the body in space, for the directions towards the head-end or the tail-end of the body, the designations cranial (cranialis) and caudal (caudalis) have been introduced. In the head itself the continuance of the cranial direction can no longer very satisfactorily be designated as cranial; accordingly, this direction as far as the mouth or the nose is spoken of as rostral (rostralis). When the body is in the upright position, the direction towards the front is spoken of as anterior, that towards the back as posterior; the same directions in the body thought of as independent of its position in space, are spoken of as ventral (ven- 
tralis) and dorsal (dorsalis). The adjectives right (dexter) and left (sinister) have their ordinary meaning.

Besides these general terms for the body as a whole, certain special terms for the extremities (termini ad extremitates spectantes) are found convenient. In the extremities especially it is desirable to have designations independent of the position in space. The direction towards the trunk is, therefore, spoken of as proximal (proximalis); that away from the trunk, as distal (distalis). In the forearm and hand the direction towards the thumb side is spoken of as radial (radialis); that towards the little-finger side, as ulnar (ulnaris); similarly, in the leg the direction towards the great-toe side is spoken of as tibial (tibialis) and that towards the little-toe side as fibular (fibularis). Furthermore, in both upper and lower extremities, for the extensor surfaces the expression dorsal (dorsalis) is used; while for the flexor surfaces the term used in the forearm and hand is volar (volaris); that in the foot, plantar (plantaris).

\section{GENERAL ANATOMICAL TERMS (TERMINI GENERALES).}

A list of certain general terms, with the English synonyms, employed in gross anatomy is here included. It is not intended that the student should begin his work by memorizing these words. On the contrary, he will learn them best by adding them gradually to his vocabulary as the dissection proceeds.

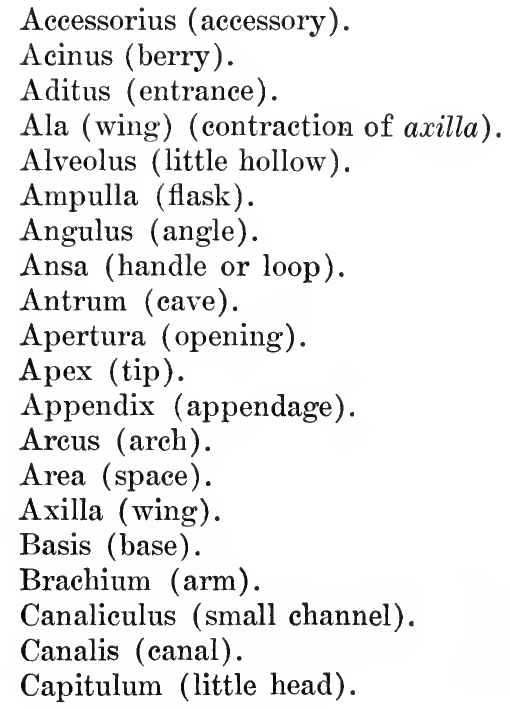

Capsula (eapsule).

Caput (head).

Cartilago (cartilage).

Caruncula (small piece of flesh).

Cauda (tail).

Caverna (cavern).

Cavum (hole or cavity).

Cellula (little chamber or cell).

Circulus (circle).

Cisterna (cistern).

Collum (neck).

Columna (eolumn or pillar).

Commissura (connection or commissure).

Cornu (horn).

Corona (wreath, garland, or crown).

Corpus (body).

Corpusculum (little body or corpuscle).

Crista (erest). 
Crus (leg or limb).

Decussatio (decussation or erossing).

Dorsum (back).

Ductulus (little duct).

Ductus (duet).

Eminentia (eminence or protuberance).

Endothelium (endothelium).

Epithelium (epithelium).

Extremitas (extremity).

Facies (face or surface).

Fascia (bandage or band).

Fasciculus (a little bundle or packet).

Fibra (fibre or filament).

Fibrocartilago (fibrocartilage).

Filum (thread).

Fissura (fissure or cleft).

Flexura (bending).

Folium (leaf).

Folliculus (little sac or bag).

Foramen (hole, aperture, opening).

Formatio (formation).

Fornix (arch or vault).

Fossa (ditch or trench).

Fossula (little fossa).

Fovea (pit).

Foveola (little pit).

Frenulum (cord or rein).

Fundus (bottom).

Funiculus (thin rope, cord, or string).

Geniculum (little knee or knot).

Genu (knee).

Glandula (gland).

Glomerulus (little skein).

Glomus (skein).

Hilus (hilus).

Humor (liquid or fluid).

Junctura (joint).

Impressio (impression).

Incisura (incision or noteh).

Infundibulum (funnel).

Intestinum (intestine or inward).

Isthmus (isthmus).

Labium (lip).

Lacuna (gap, defect).

Lamina (plate or layer).

Latus (broad; flank).

Ligamentum (ligament).

Limbus (border or fringe).

Limen (threshold or boundary).

Linea (line).
Liquor (fluid or liquid).

Lobulus (a little lobe).

Lobus (lobe).

Macula (spot).

Margo (margin).

Massa (mass).

Meatus (way or passage).

Medulla (marrow).

Membrana (membrane).

Membrum (limb or member).

Mucus (mucus).

Musculus (musele).

Nervus (nerve).

Nodulus (nodule).

Nucleus (nucleus or kernel).

Organon (organ).

Orificium (orifice).

Os, oris (mouth).

Os, ossis (bone).

Ostium (entrance).

Papilla (papilla or nipple).

Parenchyma (parenchyma).

Paries (wall).

Perichondrium (perichondrium).

Periosteum (periosteum).

Plexus (plexus).

Plica (fold).

Polus (pole).

Processus (process).

Prominentia (prominence or projection).

Punctum (point or small puncture).

Radix (root).

Ramulus (little branch or twig).

Ramus (branch).

Raphe (raphe or seam).

Recessus (recess).

Regio (region or territory).

Rete (net or net-work).

Rima (slit or fissure).

Rudimentum (rudiment).

Septulum (little septum).

Septum (partition).

Sinus (sinus).

Spatium (space).

Spina (spine or thorn).

Stratum (layer or covering).

Stria (furrow, stripe, or ridge).

Stroma (stroma, or bed).

Substantia (substance).

Succus (juice). 
Sulcus (sulcus or furrow).

Tænia (ribbon; tapeworn).

Tegmen (a cover).

Tela (web).

Tela conjunctiva (eonnecting web).

Tela elastica (elastic web).

Torus (round swelling or protuberance).

Trabecula (little beam).

Tractus (tract).

Trigonum (trigone or triangle).

Trochlea (pulley).

Truncus (trunk).

Tuber (swelling or hump).

Tuberculum (tubercle).

Tubulus (tubule or little tube).

Tunica (coat or covering).

Tunica propria (proper coat).
Umbo (boss or prominence).

Uvula (little cluster or bunch).

Vagina (sheath).

Vallecula (crevice).

Vallum (wall or fortification).

Valvula (valve).

Vas (vessel).

Velum (sail, covering, or curtain).

Vertex (crown of head).

Vesica (bladder).

Vesicula (vesicle or little bladder).

Vestibulum (vestibule or antechamber).

Villus (shaggy hair).

Viscera (entrails, viscera).

Viscus (organ, internal organ).

Vortex (whirlpool).

Zona (girdle or zone). 


\section{Part I}

\section{DISSECTION OF THE UPPER EXTREMITY}





\section{LABORATORY MANUAL}

\section{OF \\ HUMAN ANATOMY}

\section{UPPER EXTREMITY}

MAKE four drawings: $(a)$ anterior view of the upper extremity and the wall of the thorax, $(b)$ posterior view of the same, $(c)$ lateral view of the upper extremity, $(d)$ medial view of the same.

In the drawings show that the upper extremity (extremitas superior) is attached to the chest (thorax) in the clavicular and scapular regions. Note the prominence of the shoulder (axilla $)^{1}$ and the projection in its upper lateral part (acromion). Continuous with the shoulder is the rounded upper arm (brachium); beneath it, between the brachium and the thorax, is the axillary fossa (fossa axillaris). Draw in its anterior and posterior bounding folds (plica axillaris anterior, plica axillaris posterior). Show how the forearm (antibrachium) joins the brachium at the elbow (cubitus); illustrate the change in size and shape of the antibrachium distalward. In the drawings of the brachium label the anterior surface (facies anterior), posterior surface (facies posterior), lateral surface (facies lateralis), and medial surface (facies medialis), also the sulcus bicipitalis lateralis and the sulcus bicipitalis medialis; in the forearm distinguish the radial side or border (margo radialis), the ulnar side (margo ulnaris), the dorsal surface (facies dorsalis), and the volar surface (facies volaris).

Note how the hand (manus) is attached to the antibrachium by the narrower wrist (carpus). Distinguish the proximal portion of the hand (metacarpus) from the subdivided distal portion, the fingers (digiti manus). Number the digits from the thumb side. The thumb is the first (digitus I., or pollex), the index-finger is the second (digitus II., or index), the middle

"This is the proper usage of "axilla." In English texts it is often used as synonymous with " fossa axillaris." 
finger is the third (digitus III., or digitus medius), the ringfinger is fourth (digitus IV., or digitus annularis), and the little finger is fifth (digitus $V$., or digitus minimus). Notice the relative lengths of the digits, especially the relation of the length of the index-finger to that of the ring-finger. Is this constant? In the hand make careful drawings of the "back of the hand" (dorsum manus) and of the flattened "hollow of the hand" (vola manus, s. palma), also of the "thumb side" of the hand (margo radialis) and of the "little finger side" (margo ulnaris). In each digit distinguish the facies dorsalis from the facies volaris and the margo radialis from the margo ulnaris. In the upper extremity what is meant by the "proximal direction" or a " proximal part"? what by the " distal direction" or a "distal part"'?

In the drawings of the upper part of the trunk or chest (thorax) show its relation to the belly (abdomen). In the back (dorsum) look at and feel the spines of the vertebral column (columna vertebralis). Number these in your drawing and pay especial attention to the exact level of each and the relations of other parts (e.g., scapula) to their levels. Designate the spine of vertebra C. VII. separating the neck from the back. Illustrate the position of the projections corresponding to the ribs (costae) and number them. Note especially the rib-levels of the papilla mammae and of various portions of the scapula when the arm is in different positions. Draw the breast and show the depression between the two breasts, the so-called bosom (sinus). Below the bosom, just under the sternum, indicate the flattened so-called "heart fossa" (scrobiculus cordis). Show the limits of the areola mammae about the nipple, and, if Montgomery's glands (glandulae areolares [Montgomerii]) are visible, picture them.

Draw in certain lines useful in topographical anatomy:

(1) Anterior median line (linea mediana anterior).

(2) Posterior median line (linea mediana posterior).

(3) Sternal line (linea sternalis), along the lateral margin of the sternum.

(4) Mammillary line (linea mammillaris), falling perpendicularly through the nipple.

(5) Parasternal line (linea parasternalis), midway between sternal and mammillary lines.

(6) Costo-articular line (linea costo-articularis), from the sternoclavicular articulation to the tip of the eleventh rib.

(7) Axillary line (linea axillaris), perpendicularly from the centre of the fossa axillaris.

(8) Scapular line (linea scapularis), perpendicularly through the lower angle of the scapula. 
FIG. 1.

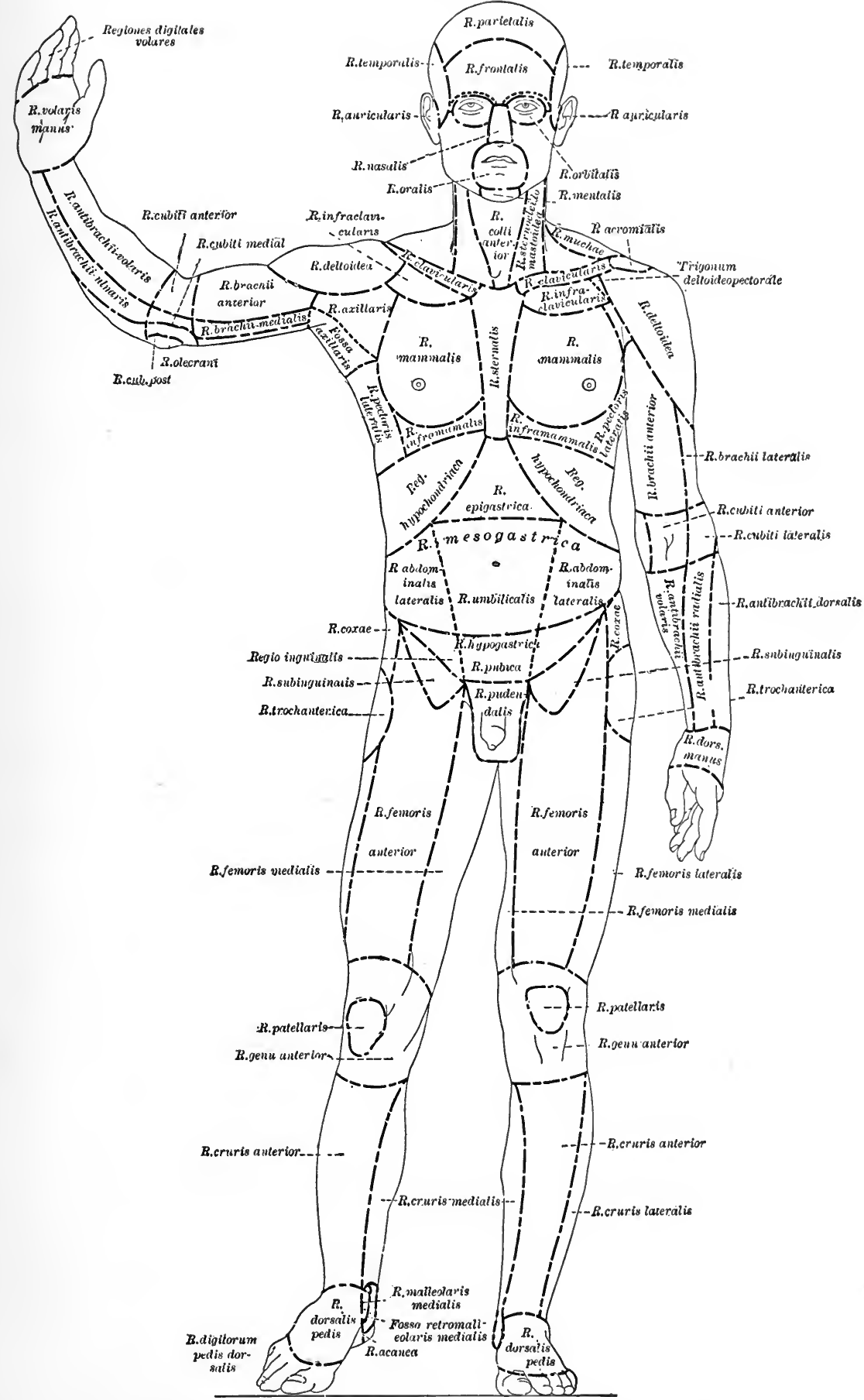

The regions of the anterior surface of the body. (After His, Anatomische Nomenclatur, Leipzig, 1895, Plate I.) 
FIG. 2.

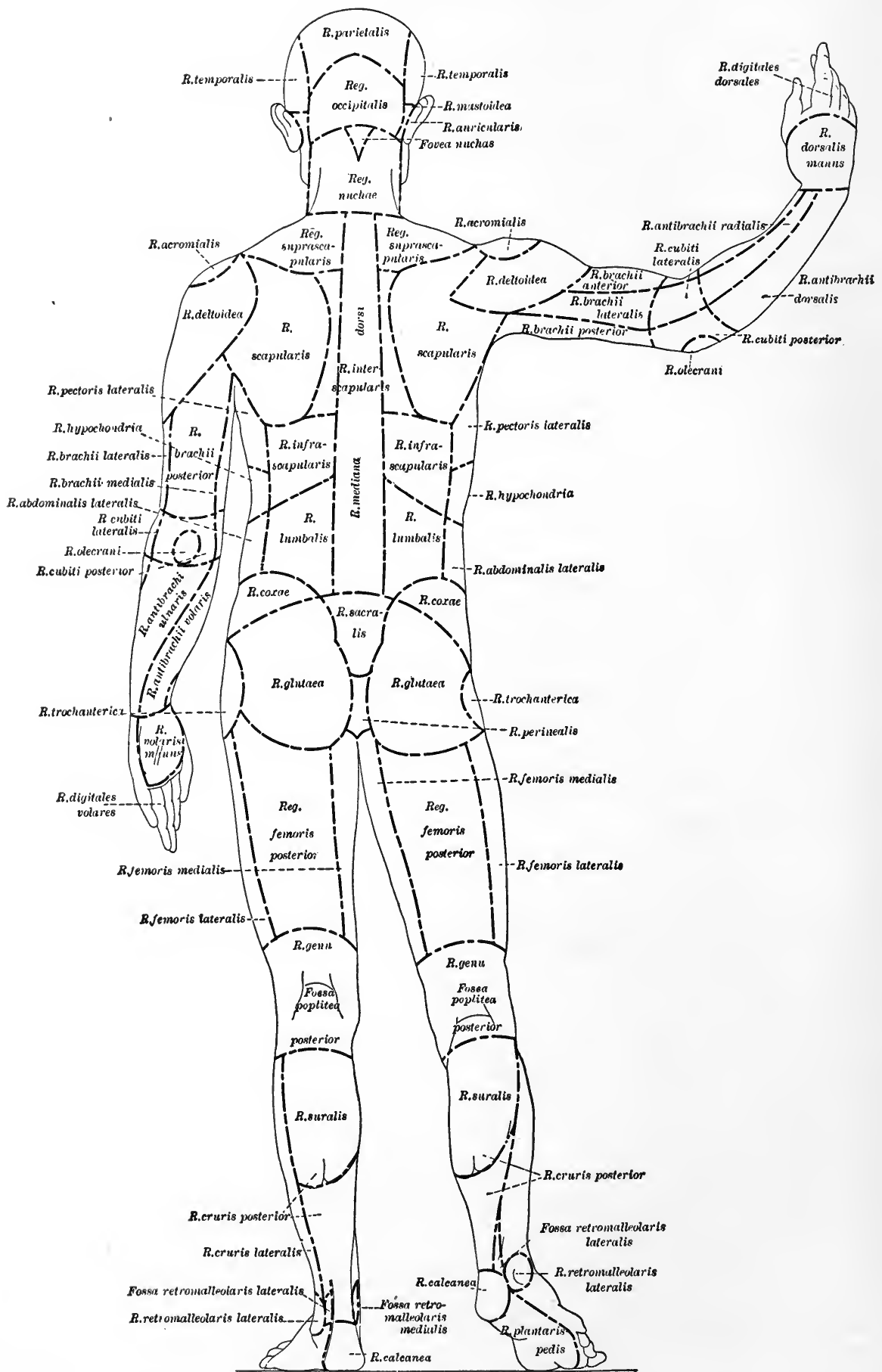

The regions of the posterior surface of the body. (After His, Anatomische Nomenclatur, Leipzig, 1895, Plate II. ) 
Make four additional outline sketches and insert the "regions" of the upper extremity and thoracic wall, having first marked them out accurately with the end of the probe on the body. Use Figs. 1 and 2 as a guide. Ask yourself the reason for the name of each region.

\section{REGIONS OF THE BACK (REGIONES DORSI).}

\section{Surface Anatomy.}

In the median region of the back (regio mediana dorsi) palpate the spinous processes (processus spinosi) of the vertebrae. Are they all in one plane? Find the spine of the seventh cervical vertebra (vertebra prominens). Are any other spines especially noticeable? Enumerate the spines consecutively. Is there any marked kyphosis, lordosis, or scoliosis present? ${ }^{1}$ Palpate the scapula and ascertain its general outline. Move the arm in various directions and note accompanying changes in position of scapula. With the arm of the cadaver by the side, establish the spinal level and the rib-level of the angulus medialis and of the angulus inferior of the scapula. Run the finger along the spina scapulae and the acromion and note that both are subcutaneous. Establish exactly the bony point corresponding to the junction of the lower border of the spina scapulae with the lateral margin of the acromion. (Surgeons measure the arm from this point, the tape being carried down to the epicondylus lateralis of the humerus.)

\section{Superficial Structures.}

Make skin incisions (1) along the linea mediana posterior from the vertebra prominens to the tip of the coccyx, (2) from the tip of the coccyx to the posterior superior iliac spine, then along the crista iliaca to near the anterior superior iliac spine, (3) from the vertebra prominens to the medial edge of the acromion, and (4) from the processus spinosus vertebrae lumbalis $\mathrm{I}$. to the lateral margin of the acromion. Reflect the two triangular flaps of skin, taking none of the fat of the superficial fascia with it. Have your atlases open before you. Having ascertained the region in which the medial set of cutaneous vessels and nerves is likely to be found, with the knife make a small cut through the superficial fascia down to the deep

${ }^{1}$ When a word is met with in this manual (or in any text-book) which is new to the student, he should look up its meaning at once in a good medical dictionary. 
fascia. With the special heavy probe recommended, separate an area of the superficial fascia until you encounter the trunk of a blood-vessel or a nerve coming through the deep fascia from below to enter the superficial fascia. It is common to find a nerve, an artery, and a vein together. Carefully separate the structure or structures found from the superficial fascia, tracing

Fig. 3.

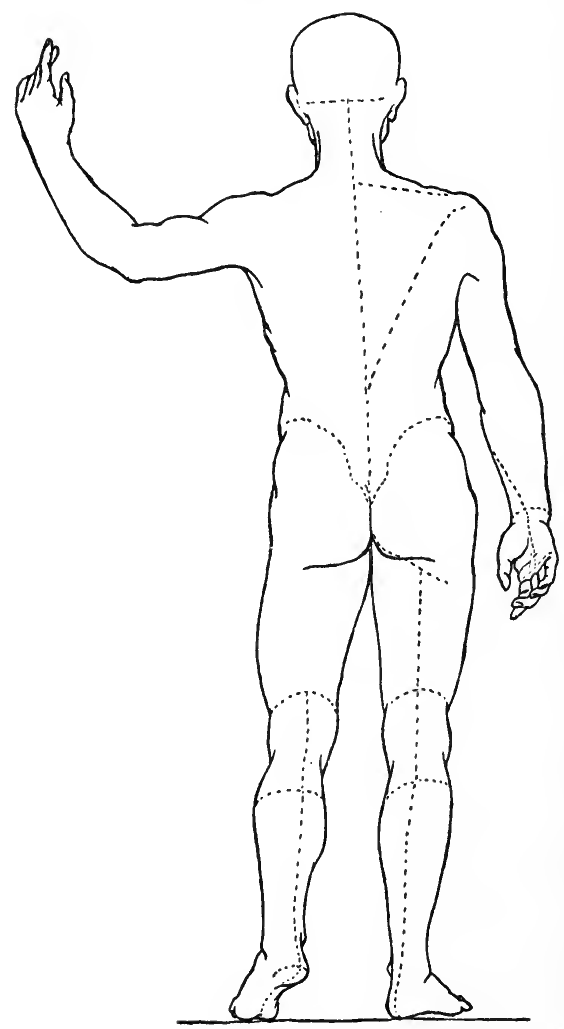

Skin incisions on the posterior surfaces of the body. the blood-vessel or nerve as far as possible and cleaning it thoroughly. In this way, isolate all the superficial blood-vessels and nerves in the medial and lateral areas in which they occur. The nerves and blood-vessels will be found emerging through the deep fascia. Their course and ramifications in the superficial fascia are to be traced out without removing the fat or disturbing the relations of the structures any more than is necessary in exposing them. It should be borne in mind that it is only the natural relations that are of importance, and not the artificial relations due to dissection. Hence each structure should be studied as it is worked out, instead of its study being deferred until after it is isolated and cleaned. When the cutaneous nerves and vessels and superficial glands have been thus exposed, a drawing of these should be made; or the drawing may be made concurrently with the dissection, being then an aid to the determination of the exact relations.

The deep fascia should be cleaned (by careful removal of the superficial fascia by blunt dissection) and its extent, nature, and attachments studied. The fasciae should then be removed piecemeal. In cleaning the surface of the muscles, place the body in such a position that the muscle-fibres will be rendered tense and carry the knife in the direction which permits removal of the fascia with the least disturbance of the underlying muscle. Care 
FIG. 4.

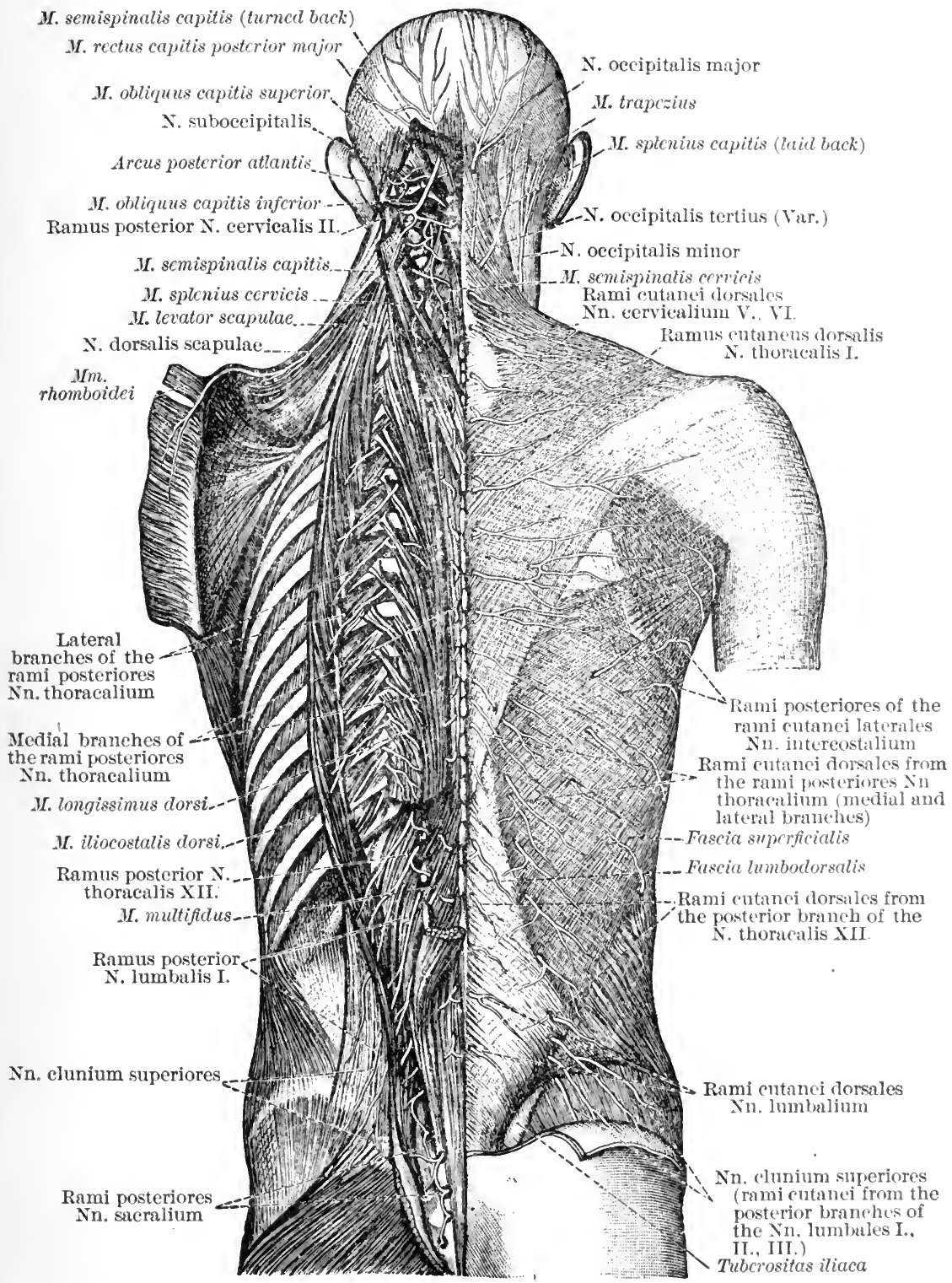

The distribution of the posterior branches, rami posteriores, of the spinal nerres, $\mathrm{Nn}$. spinales. (On the right side of the body the cutaneous branches are shown; on the left side, the muscle-branches; and in part the course of the posterior branches of these are shown.) (After Toldt, Anat. Atlas, Wien, 1903, 3 Aufl., p. 813, Fig. 1245.) 
should be taken to avoid giving a " ragged" appearance to the surface of the muscle. As soon as the trunk of a nerve or bloodvessel is found, ascertain its name from a study of the illustration in your atlas. Look up this name in the index to your Systematic Human Anatomy and refer to the author's description. When handling a nerve, blood-vessel, or indeed any structure, form the habit of repeating to yourself its name; this practice is extremely helpful in fixing the association between the structure and the name which is the sign for it. Also, when reading write down every new term met with and learn its meaning, both literal or etymological and applied or derived.

\section{Nerves. (Fig. 4.)}

(a) Cutaneous branches of posterior rami of thoracic nerves (rami cutanei Rr. post. Nn. thoracalium).

(b) Posterior rami of lateral cutaneous rami of anterior rami of intercostal nerves (rami posteriores Rr. cutan. lat. Rr. ant. Nn. intercostalium).

(c) Medial rami of posterior rami of lumbar, sacral, and coccygeal nerves (rami mediales Rr. post. Nn. lumb., sacral., et coccyg.).

(d) Lateral rami of posterior rami of lumbar nerves (rami laterales Arteries. $R r$. post. $N n$. lumbalium $=N n$. clunium superiores $)$.

(a) Medial cutaneous rami of posterior rami of intercostal arteries (rami cutanei mediales $R r$. post. Aa. intercostalium).

(b) Lateral cutaneous rami of posterior rami of intereostal arteries (rami cutanei laterales $R r$. post. Aa. intercostalium).

(c) Dorsal cutaneous rami of lumbar arteries (rami cutanei dorsales Aa. lumbalium).

(d) Posterior rami of lateral cutaneous rami of anterior rami of intercostal arteries (rami posteriores Rr. cutan. lat. Rr. ant. Aa. intercostalium).

Veins. (Cf. Toldt, Fig. 1036.)

(a) Dorsal rami of intercostal veins (rami dorsales $V v$. intercostalium).

(b) Branches of lumbar veins ( $V v$. lumbales).

\section{Broad Muscles of the Back (First Layer). (Fig. 5.)}

(a) Trapezius muscle (M. trapezius).

(b) Broadest muscle of back (M. latissimus dorsi).

Study the form and position of each, and establish its exact origin and insertion. Test the action of the fibres of the different parts of the muscles. Find their innervation now or later. To what other structures are these muscles related? Examine the boundaries and floor of the "triangle of auscultation." Why is it so called? Examine the boundaries and floor of Petit's triangle (trigonum lumbale [Petiti]). 
Fig. 5.

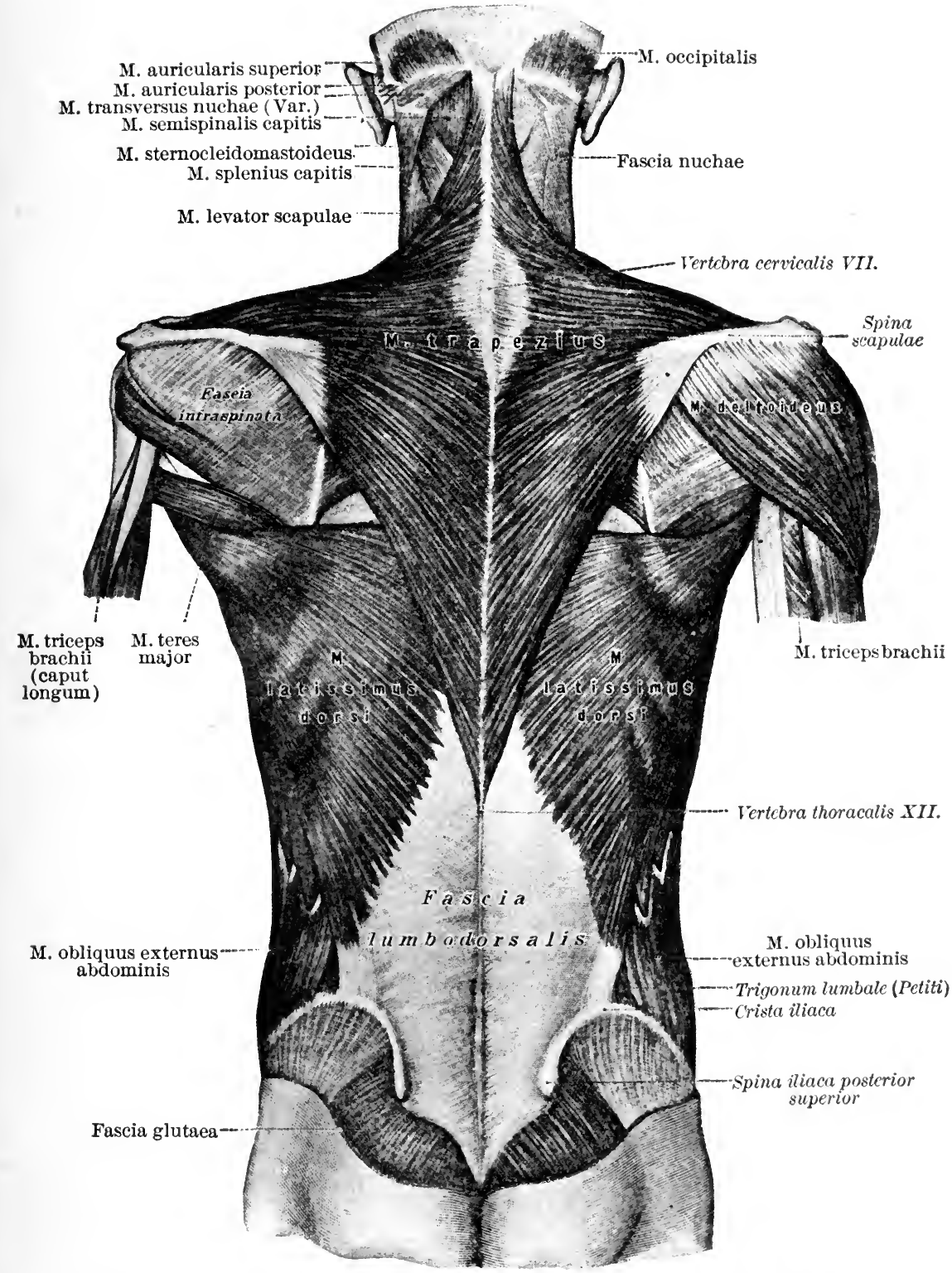

First (superficial) layer of the broad muscles of the back. (After Toldt, Anat. Atlas, Wien, 1900, 2 Aufl., p. 266, Fig. 504.) 
Cut through the M. trapezius by a vertical incision five centimetres lateralward from the median plane, avoiding injury to the muscles of the second layer. Dissect out, on its deep surface, the following structures:

FIG. 6.

Processus costarius vertebrac lumbalis $I V$.

M. iliocostalis

Fascia lumbodorsalis

Ligamentum lumbocostale

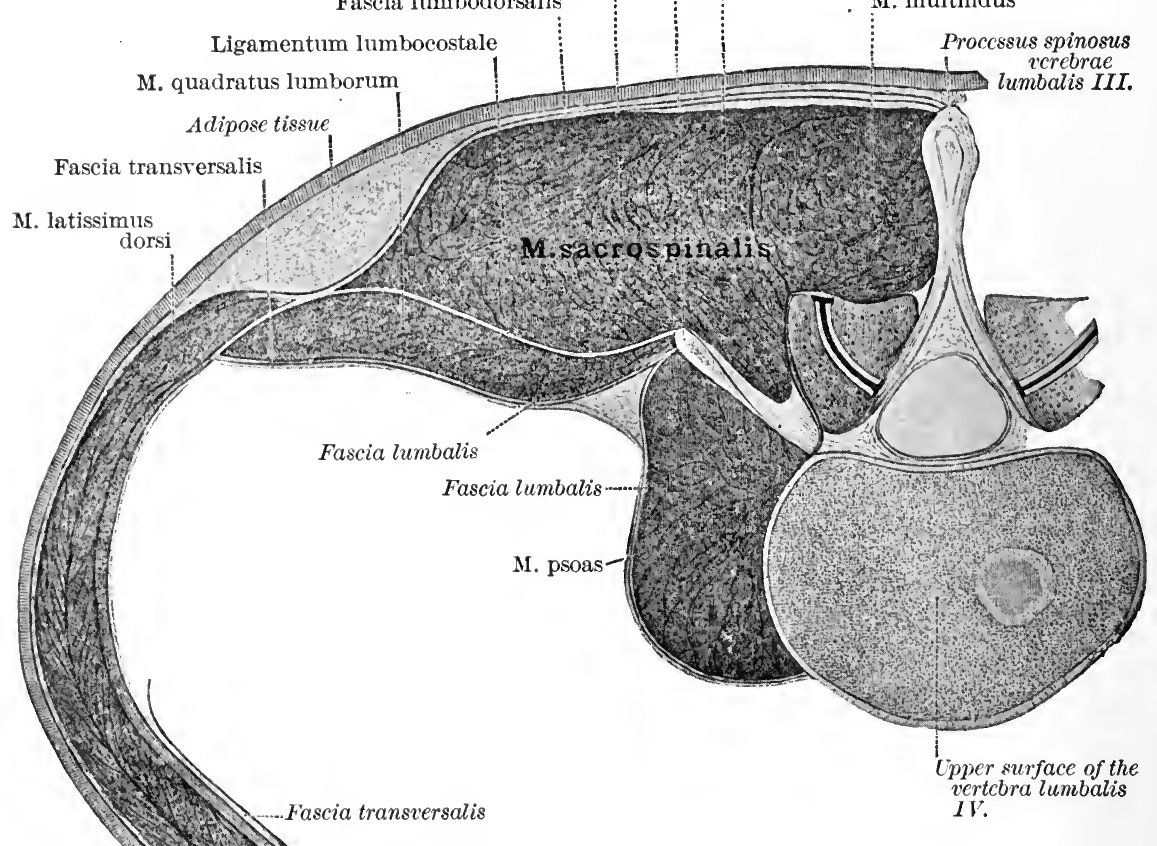

M. transversus abdominis

M. obliquus internus abdominis

M. obliquus externus abdominis

Fascia superficialis Integumentum commune

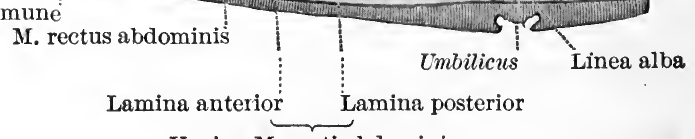

Vagina M. recti abdominis

The muscles and fasciac in a transverse section of the right half of the wall of the trunk. (After Toldt, Anat. Atlas, Wien, 1900, 2 Aufl., p. 281, Fig. 520. )

(a) External ramus of accessory nerve (ramus externus $N$. accessorii).

(b) Muscular rami from cervical nerves (rami musculares Nn. cervicalium). 


\section{Broad Muscles of the Back (Second Layer).}

Make the rhomboid muscles tense and clean their surfaces carefully. Find the trunk of the dorsal nerve of the scapula ( $N$. dorsalis scapulae) (O. 'T. nerve to the rhomboids) and the descending ramus of the transverse artery of the neck (A.transversa colli) in the interval between the M. levator scapulae and the M. rhomboideus minor.

(a) Greater rhomboid muscle ( $M$. rhomboideus major).

(b) Lesser rhomboid muscle (M. rhomboideus minor).

(c) Levator muscle of scapula (M. levator scapulae) (O. T. levator anguli scapulae).

Establish the form, position, origin, insertion, action, and innervation of each. Find the two rami of the cervical nerve on the surface of the M. levator scapulae and then cut the muscle in two in the middle. Reflect the lower portion lateralward. Cut through the attachments of the $\mathrm{Mm}$. rhomboidei near the median plane and reflect the muscles lateralward. Dissect out carefully-

(a) Dorsal nerve of seapula ( $N$. dorsalis scapulae).

(b) Descending ramus of transverse artery of neck (ramus descendens A. transversae colli) (O. T. posterior scapular artery).

Near the superior margin of the scapula expose the inferior belly of the omohyoid muscle (venter inferior $M$. omohyoidei). Define its exact attachment to the scapula. Note the relations of the A. transversa scapulae (O. T. suprascapular artery) and of the $\mathrm{N}$. suprascapularis to the Lig. transversum scapulae superius.

The dissector of the arm may next assist the dissector of the head in making out the muscles of the back. Study the form, position, origin, insertion, action, and innervation of each.

\section{ANTERIOR THORACIC REGION AND AXILLARY FOSSA.}

\section{Surface Anatomy.}

Examine-

(a) Outline of clavicle.

(aa) Shaft of clavicle.

(ab) Sternal extremity (extremitas sternalis).

(ac) Acromial extremity (extremitas acromialis). 
(b) Outline of sternum.

Compare the distance between the acromion and the sternal extremity of the clavicle on the two sides. Why would such a comparison be of importance in surgical diagnosis?

(ba) Manubrium sterni.

Jugular notch (incisura jugularis) (O. T. suprasternal notch).

(bb) Corpus sterni (O. T. gladiolus).

Angle of sternum (angulus sterni) (O. T. angulus Ludovici, angle of Louis), an important landmark in counting ribs and intercostal spaces.

(bc) Processus xiphoideus (O. T. ensiform process).

(c) Costal arches I.-XII.

(ca) Ribs (costae I.-XII.).

( $c b)$ Costal cartilages (cartilagines costales).

Is a " rickety rosary" present?

(d) Coracoid process of scapula (processus coracoideus scapulae). Note its relation to the infraclavicular fossa (fossa infraclavicularis or Mohrenheim's fossa) and the relation of the clavicular origins of the M. pectoralis major and the M. deltoideus to the latter.

(e) Breast (mamma).

(ea) Papilla mammae, or nipple of the breast.

Note its exact level and its distance from the anterior median line.

(eb) Body of breast (corpus mammae).

Note its dimensions.

(ec) Areola mammae.

(1) Sebaceous glands (Gl. sebaceae).

(2) Areolar glands (Gl. areolares [Montgomerii]).

(ed) Accessory breasts (mammae accessoriae).

Are any present? What is the direction of the so-called "milk-line"? Note that of the two rows of milk-glands running from the forelimb to the inguinal fold, the headward portions are preserved in primates, the tailward portions in ruminants. What are meant by the terms polymastism, polythelism, and atavism?

( $f$ ) Boundaries of axillary fossa (upper extremity abducted).

(fa) Anterior axillary fold (plica axillaris anterior).

To what is it due?

( $f b)$ Posterior axillary fold (plica axillaris posterior).

To what is it due? Which fold extends to the lower level?

(g) Upper extremity of humerus.

Palpate, in the axillary fossa, the medial surface of the surgical neck (collum chirurgicum) and feel the head of the humerus (caput humeri) rotate when the arm is rotated.

\section{Skin and Superficial Fascia, with Vessels and Nerves.}

Make the following incisions in the skin (cf. Fig. 7) :

(a) In the linea mediana anterior from the fossa jugularis to the tip of the processus xiphoideus. 
(b) From the processus xiphoideus lateralward to the back.

(c) From the upper end of the first incision lateralward along the clavicle to the tip of the acromion.

(d) From the processus xiphoideus obliquely upward and lateralward along the plica axillaris anterior as far as the brachium.

With a sharp knife dissect up the two triangular flaps marked out by the incisions mentioned. Keep the knife close to the white corium so that none of the fat of the superficial fascia is removed with the skin.

Compare the amount of fat in the superficial fascia in your subject with that in neighboring subjects. Find the fibres of the platysma streaming down into it over the clavicle. Note that the mammary gland is situated in the superficial fascia. Cut boldly through the superficial fascia down to the deep fascia in the middle line in front, and then with the blunt probe dissect up the superficial fascia, passing gradually lateralward. Find the cutaneous blood-vessels and nerves passing into the superficial fascia from the depth. When the trunks of these are found, follow each carefully out as far as possible into the superficial fascia, cleaning it carefully. Avoid injury to the superficial nerves which pass down over the clavicle. Keep your atlases open before you as Fig. 7.

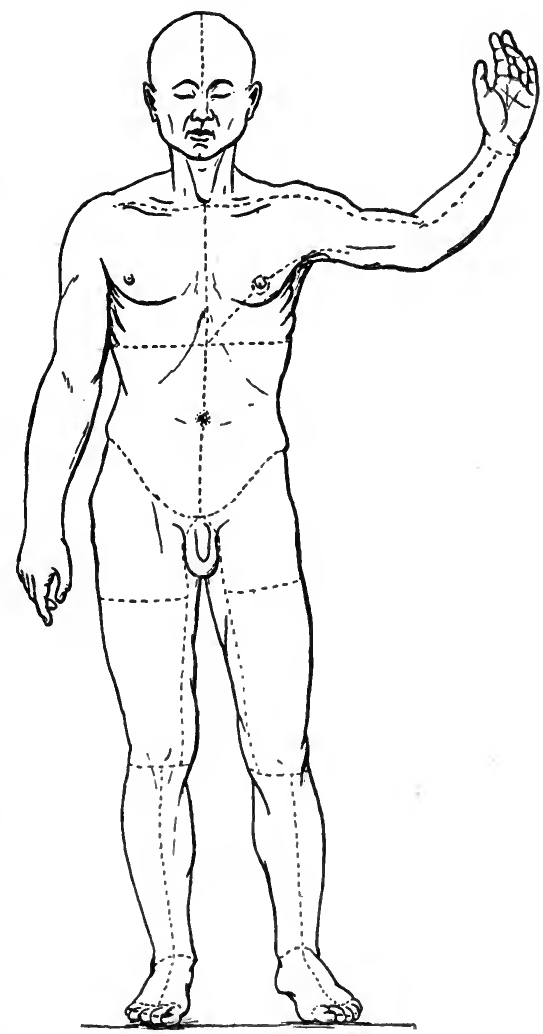

Skin incisions on the anterior surfaces of the body a guide to the location of the various structures. As soon as a nerve or blood-vessel is found, identify it, getting the name from the atlas. Find a description of it in your systematic text-book of anatomy (using the index of the latter) and compare the actual findings in your subject with the text-book description. Note especially any differences between your objective findings and the printed descriptions. 
Fig. 8.

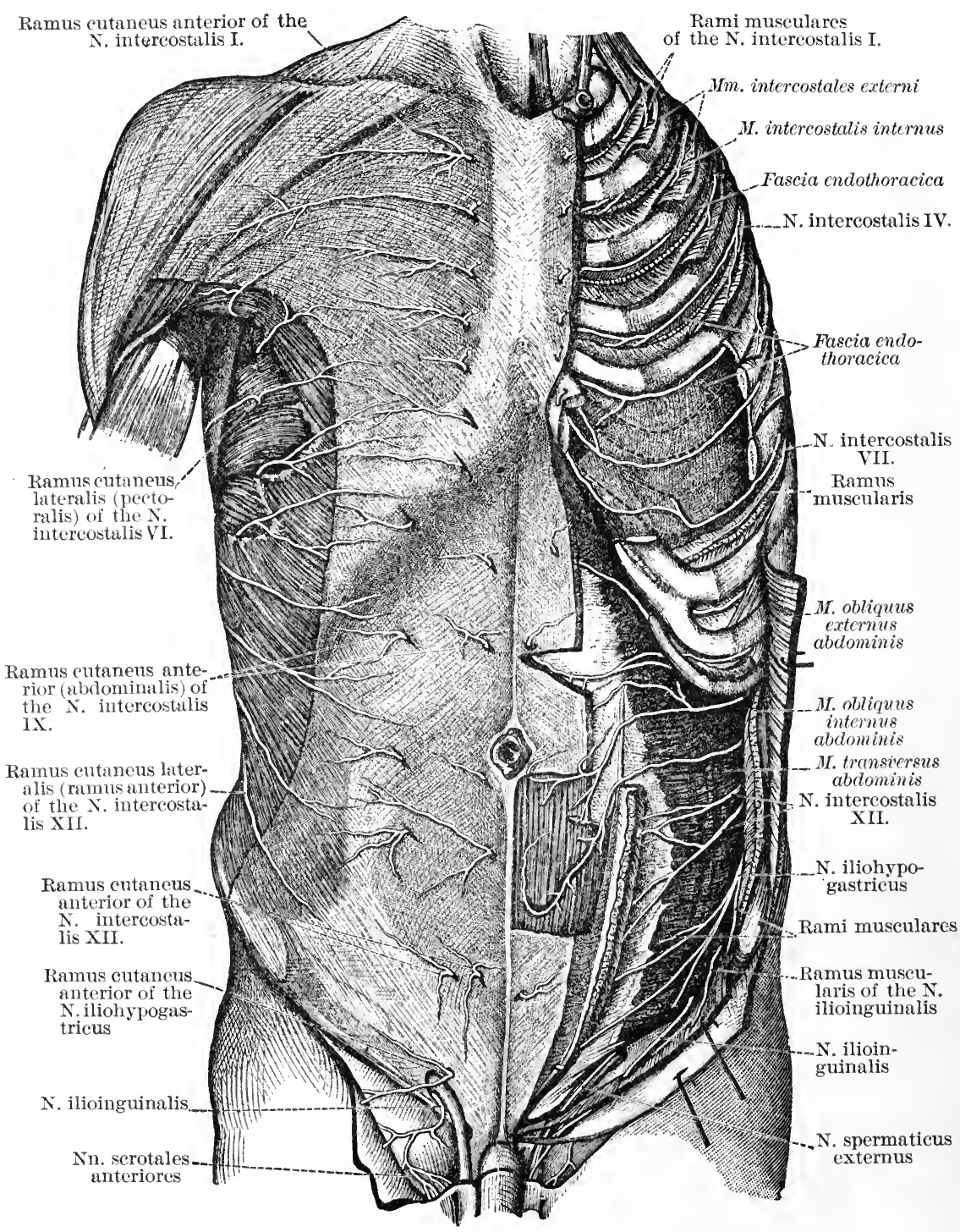

The cutaneous nerves of the anterior side of the trunk. (After Toldt, Anat. Atlas, Wien, 1903, 3 Aufl, p. 814, Fig. 1246.) 


\section{Study the following:}

Superficial Nerves. (Fig. 8.)

(a) Supraclavicular nerves (Nn. supraclaviculares) (O. T. descending cutaneous nerves).

(aa) Anterior (Nn. supraclaviculares anteriores) (O. T. suprasternal branches).

(ab) Middle (Nn. supraclaviculares medii) (O. T. supraclaricular branches).

(ac) Posterior (Nn. supraclaviculares posteriores) (O. T. supraacromial branches).

What are their relations to the platysma?

(b) Anterior cutaneous rami of the intereostal nerves (rami cutanei anteriores $\mathrm{Nn}$. intercostalium).

(ba) Medial mammary rami (rami mammarii mediales).

(c) Lateral cutaneous rami of the intercostal nerves (rami cutanei laterales $N n$. intercostalium), dividing beneath the M. serratus magnus into-

(ca) Posterior rami (rami posteriores).

(cb) Anterior rami (rami anteriores).

Note that the latter give off the lateral mammary rami (rami mammarii laterales).

Arteries. (Fig. 9.)

(a) Perforating rami of internal mammary artery (rami perforantes A. mammarii internae).

(aa) Mammary rami (rami mammarii).

(ab) Muscular rami (rami musculares).

(ac) Cutaneous rami (rami cutanei).

(b) Anterior cutaneous rami of anterior rami of intercostal arteries ( $R r$. cutanei anteriores $R r$. ant. Aa. intercostalium), in fourth and sixth intereostal spaces only.

(ba) Medial mammary rami ( $R r$. mammarii mediales).

(c) Lateral cutaneous rami of anterior rami of intercostal arteries ( $R r$. cutanei laterales $R r$. ant. Aa. intercostalium).

(ca) Posterior ramus (ramus posterior).

(cb) Anterior ramus (ramus anterior).

(cba) Lateral mammary' rami ( $R r$. mammarii laterales).

(d) Lateral thoracic artery (A. thoracalis lateralis).

Veins.

(da) External mammary rami ( $R r$. mammarii externi).

Tributaries of the axillary vein.

(a) Lateral thoracic vein ( $V$. thoracalis lateratis).

(b) Thoraco-epigastric vein ( $V$. thoraco-epigastrica), receiving many of the Vv. cutaneae pectoris.

Tributaries of the $\mathrm{V}$. anonyma.

(a) Tributaries of internal mammary vein.

(aa) Perforating rami (rami perforantes).

$(a b)$ Intercostal veins ( $V v$. intercostales) (sternal extremities).

Tributaries of V. azygos.

(a) Tributaries of interestal veins (vertebralward).

(aa) Cutaneous veins of chest (Vv. cutaneae pectoris). 
Fig. 9.

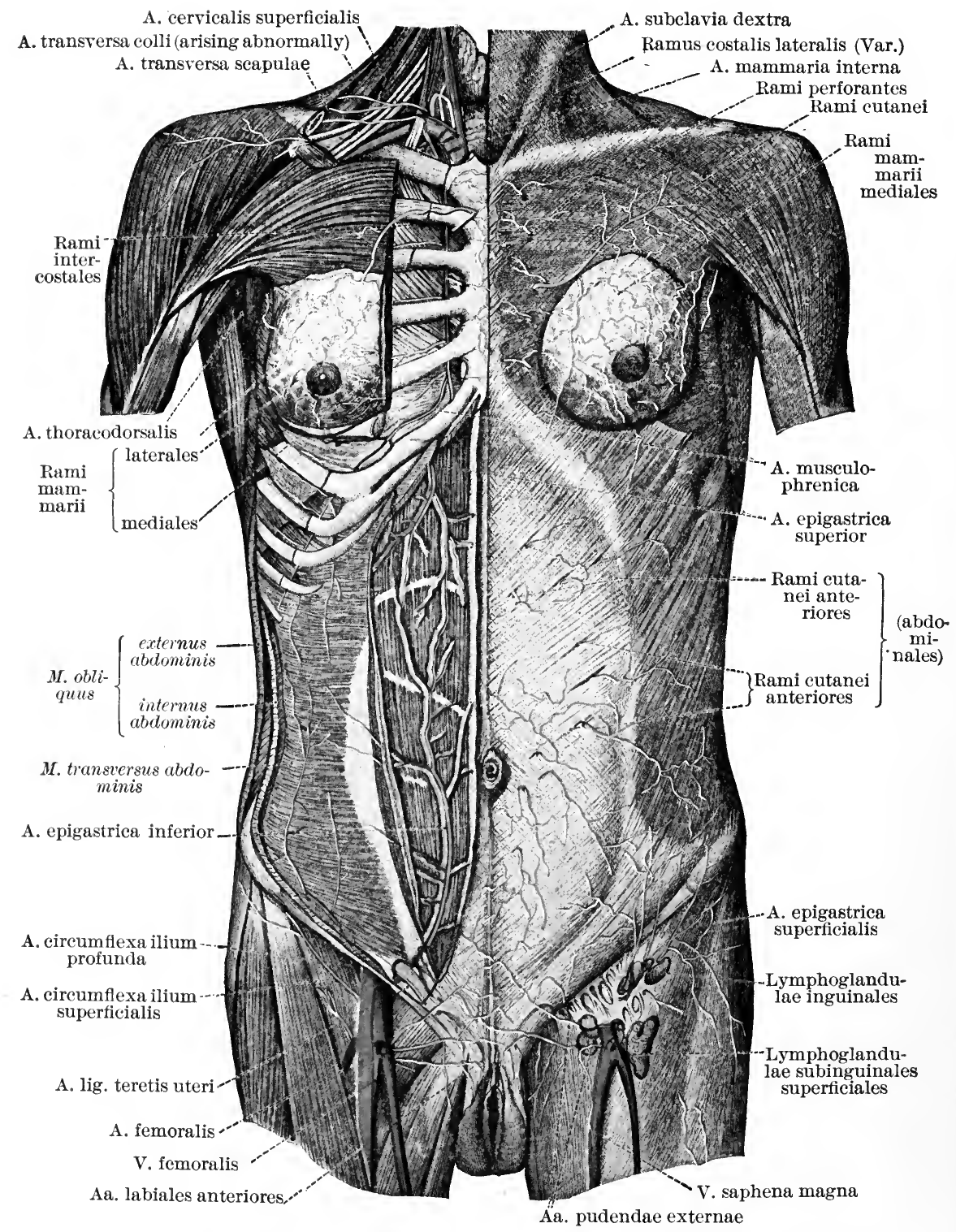

Arteries of the anterior body-wall. (After Toldt, Anat. Atlas, Wien, 1903, 3 Aufl., p. 581, Fig. 957.) 


\section{Mammary Gland (Mamma). (Fig. Io.)}

Compare the gland in your subject with one from a subject of the opposite sex. If the breast is well developed, dissect the gland under water and make out-

(a) Lobes of breast (lobi mammae).

(aa) Lobules of breast (lobuli mammae).

(b) Milk-ducts (ductus lactiferi).

(ba) Milk-sinuses or ampullae (sinus lactiferi).

FIG. 10.

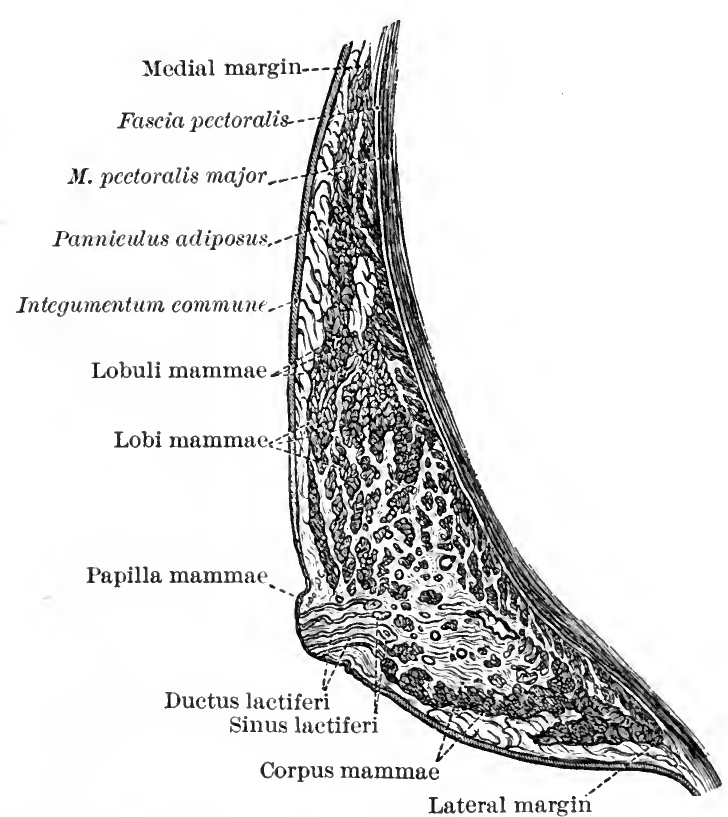

Horizontal eut through the female breast. (After Toldt, Anat. Atlas, Wien, 1900, 2 Aufl., p. 501, Fig. 860.)

Review the blood-supply of the mamma. Study illustrations of the lymphatic channels leading from the breast to the pectoral lymph-glands (lymphoglandulae pectorales), the axillary lymphglands (lymphoglandulae axillares), and the sternal lymphglands (lymphoglandulae sternales). (Cf. Figs. 15, 86, and 93.) Note that where the breasts are very large the lymphatic channels of one breast may communicate with those of the breast of the opposite side across the median line.

\section{Fascia Pectoralis or Deep Fascia.}

Note its relations above, below, medialward, and lateralward, and the process from it to the fascia coracoclavicularis in the fossa infraclavicularis. 
Greater Pectoral Muscle (M. pectoralis major).

Make the muscle tense by abducting the upper extremity. Remove the fascia pectoralis from its surface and the deltoid portion of the fascia brachii from the anterior margin of the M. deltoideus. Stop at the plica axillaris anterior before reaching the fascia axillaris. Avoid injury to the vena cephalica and the acromial ramus of the arteria thoraco-acromialis $(\mathrm{O}, \mathrm{T}$. humeral thoracic artery).

FIG. 11.

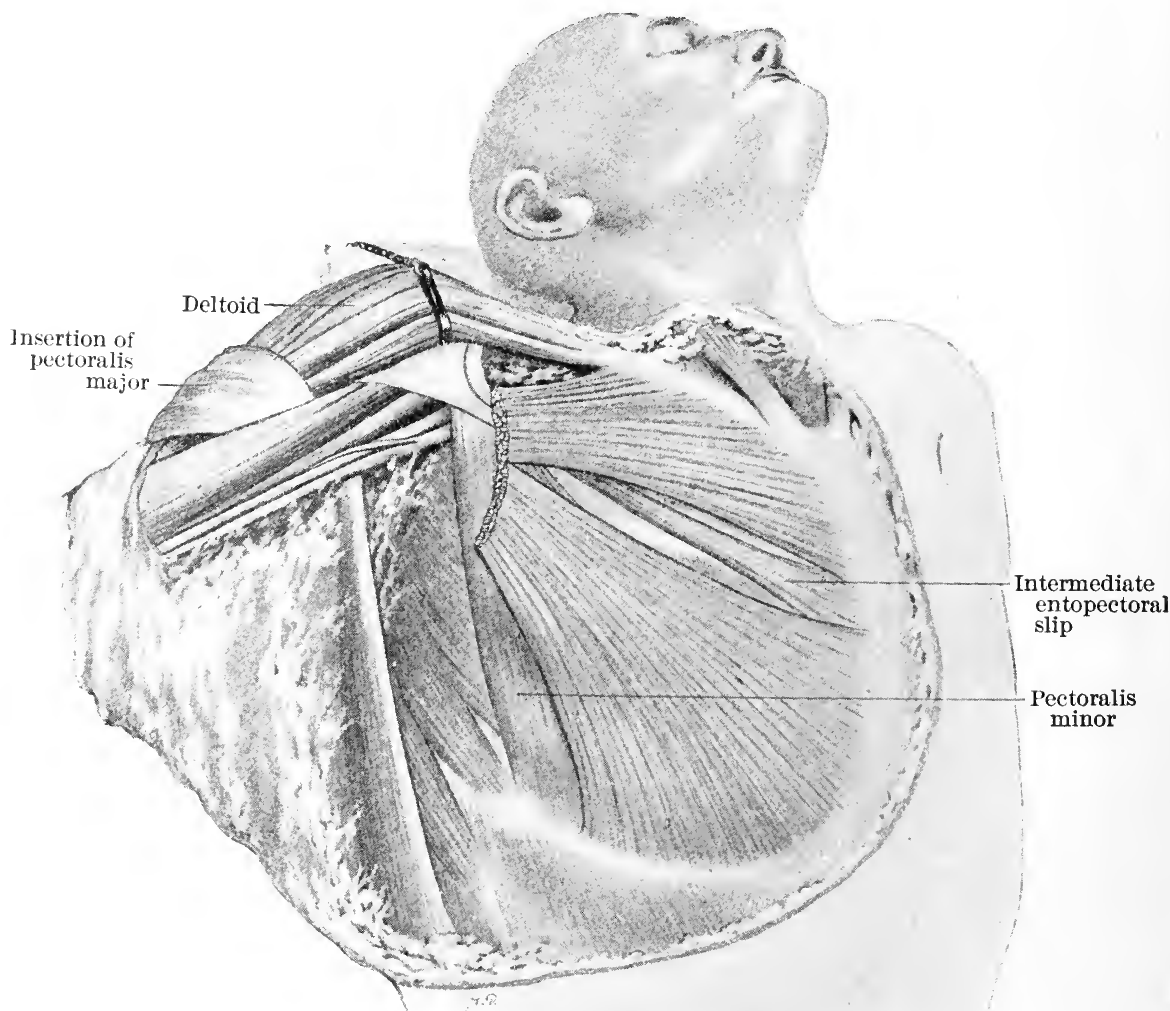

M. pectoralis major with deficiency of sternocostal portion, and resulting production of an atypical displaced intermediatc pectoral muscle (M. tensor semivaginae articulationis humero-scapularis, Gruber, I. pectoralis minimus). Adult human subject. From a fresh dissection. (After G. S. Huntington, Amer. Jour. of Anat., Baltimore, 1903, vol. ii., No. 2, Plate I., Fig. 1.)

Note in connection with the M. pectoralis major-

(a) Its form.

(b) Its position. 
(c) Its origin.

(ca) Pars elavicularis.

( $c b)$ Pars sternocostalis.

(cc) Pars abdominalis.

(d) Its insertion.

(e) Its imnervation ( $N n$. thoracales anteriores).

$(f)$ The bursa $M$. pectoralis majoris.

Look up an article upon the torsion of the tendon of this muscle by Dr. Warren Lewis, in the American Journal of Anatomy.

FIG. 12.

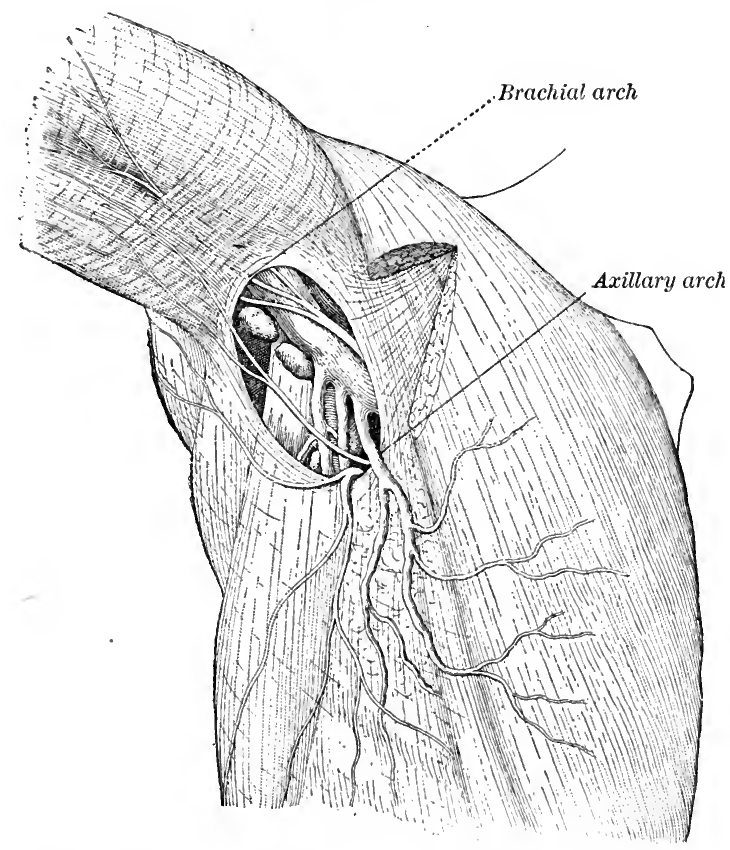

The brachial and axillary arches. (From Poirier et Charpy, Traité d'Alat. hum., Paris, 1901, 2 ed., p. 164, Fig. 126.)

\section{Axillary Fascia (Fascia axillaris). (Figs. I2 and I3.)}

Carefully remove the superficial fascia over the base of the axillary fossa so as to expose the deep or axillary fascia.

Note-

(a) An apparent oval opening between the concave aponeurotic bands; the lower lateral concave band is the brachial arch (Armbogen of Langer); the upper medial concave band is the axillary arch (Achselbogen of Langer). If the dissection be made very carefully, a delicate, much-perforated membrane can be made out, extending from the brachial arch to the axillary arch, so that the bundle of vessels and nerves seen through the oval opening is not really subcutaneous. (Poirier.) Note the striking resemblance of 
this oval opening to the fossa ovalis of the thigh, of the thin membrane covering it to the cribriform fascia, of the axillary arch to the cornu superius of the margo falciformis, and of the brachial arch to the cornu inferius of the margo falciformis.

(b) The continuity of the fascia axillaris lateralward with the fascia brachii and with the fascia enclosing the M. latissimus dorsi and $\mathrm{Mm}$. teretes major et minor; it becomes inserted into the axillary margin of the scapula.

(c) The continuity of the fascia axillaris medialward, not with the fascia pectoralis, but with the fascia coracoclavicularis, which encloses the M. pectoralis minor. (Fig. 13.) The membrane extending from the axillary fascia to the lower border of the M. pectoralis minor was formerly called the suspensory ligament of the axilla (ligament suspenseur of Gerdy).

FIG. 13.

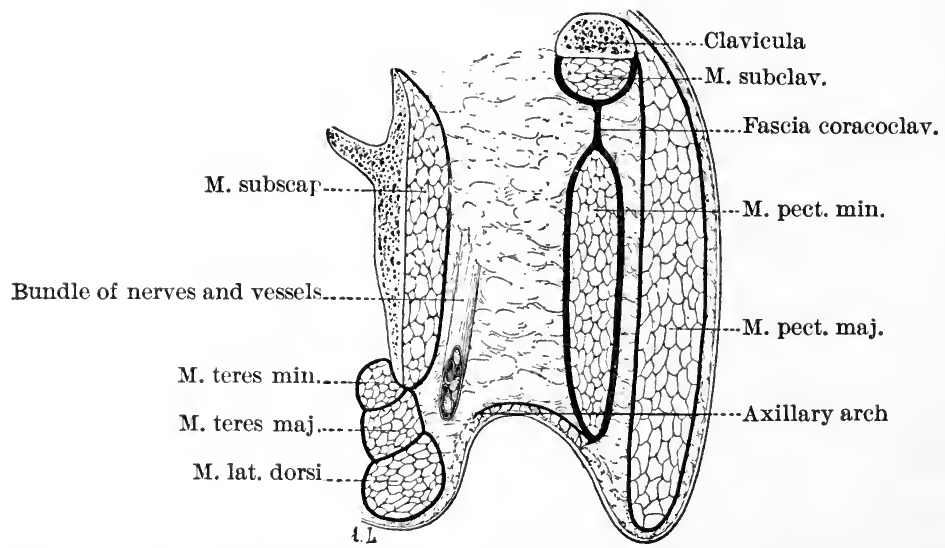

Sagittal section of the axillary fossa. (Very schematic.) (From Poirier et Charpy, Traité d'Anat. hum., Paris, 1901, t. ii. p. 165 , Fig. 128.)

(d) The passage through the oval opening (and the cribriform fascia covering it) of the intercostobrachial nerves ( $N n$. intercostobrachiales) ( $\mathrm{O} . \mathrm{T}$. intercostohumeral nerves). These represent the lateral cutaneous rami of the second and third intercostal nerves (rami cutanei laterales $N n$. intercostalium II. et III.). It is interesting that the vena basilica may occasionally bear the same relation to the oval opening that the vena saphena magna bears to the fossa ovalis in the thigh; the usual condition, however, is for the $\mathrm{V}$. basilica to pass beneath the deep fascia a little above the elbow.

(e) Later in the dissection, the reflections of the axillary fascia upon the arteries and nerves going to the forearm.

\section{Structures in the Fossa Axillaris. (See Figs. I4, I5, and I6.)}

The fascia axillaris may now be reflected backward, care being taken to dissect out carefully the intercostobrachial nerves. The structures in the axillary fossa are to be isolated and 
cleaned, the loose areolar tissue and fat in which they are embedded being gradually removed. Secure first the subscapular artery and the thoracodorsal nerve along the lower border of the M. subscapularis, then the lateral thoracic artery (lower border of M. pectoralis minor), next the long thoracic nerve (lying on M. serratus magnus). The various lymph-glands are

FIG. 14.

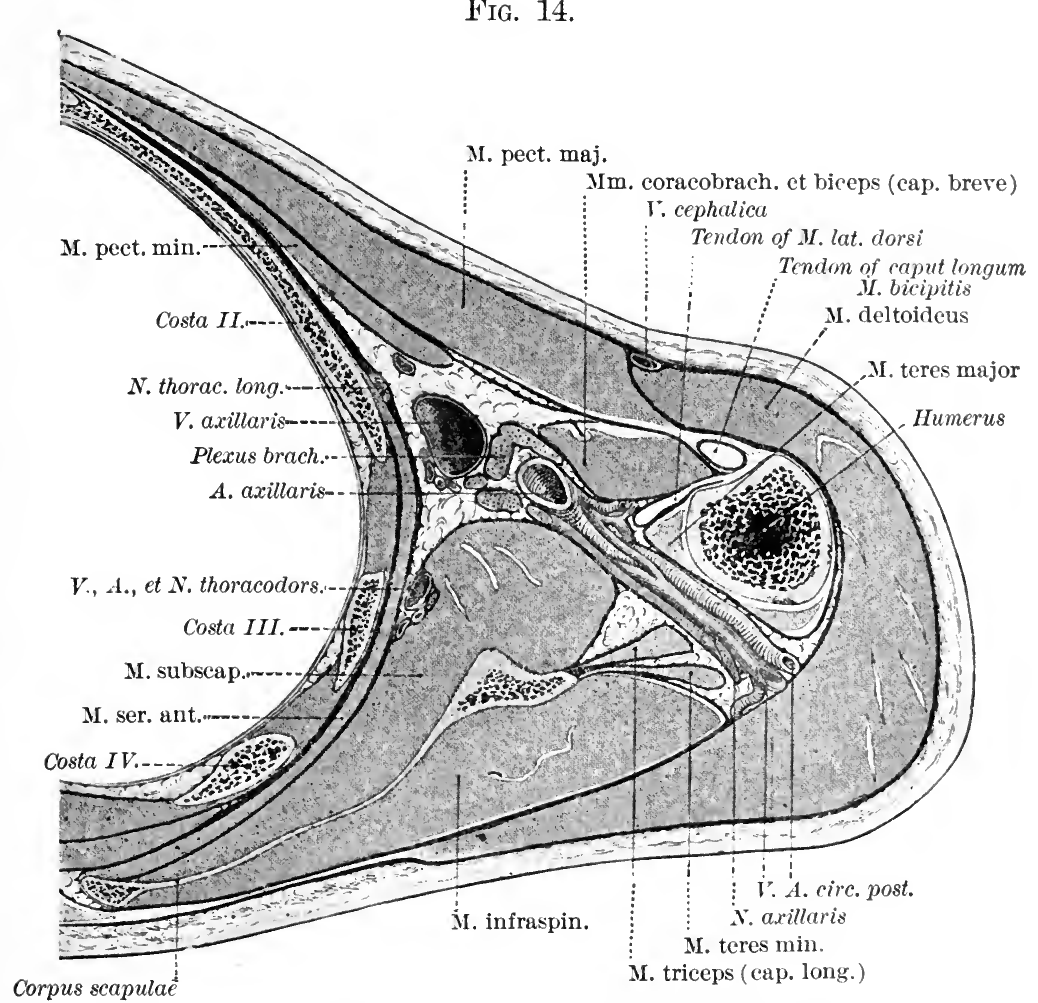

Cross-section of the shoulder above the origin of the circumflex arteries, right side, segment distal to the cut. Subject fixed in formalin chromic acid. The fat over the V. and A. circumflex posterior and the $\mathrm{N}$. axillaris has been removed to show the course of these structures. The $\mathrm{N}$. radialis is seen medial to the N. axillaris. The $\mathbf{N}$. ulnaris lies anterior to the $\mathrm{N}$. radialis. The two heads of the median have just united and the $\mathrm{N}$. musculocutaneus is separating off. (After Poirier et Charpy, Traité d'Anat. hum., Paris, 1901, 2 ed., t. ii. p. 93, Fig. 87̦.)

to be studied carefully as they are exposed and may afterwards be removed. The following structures are to be isolated and studied, some of them being best seen after the dissection of the M. pectoralis minor and the fascia coracoclavicularis.

Lymphatic Glands. (Cf. Figs. 15, S6, and 93.)

(a) Axillary lymph-glands (lymphoglandulae axillares).

(b) Pectoral lymph-glands (lymphoglandulae pectorales). 
FIG. 15.

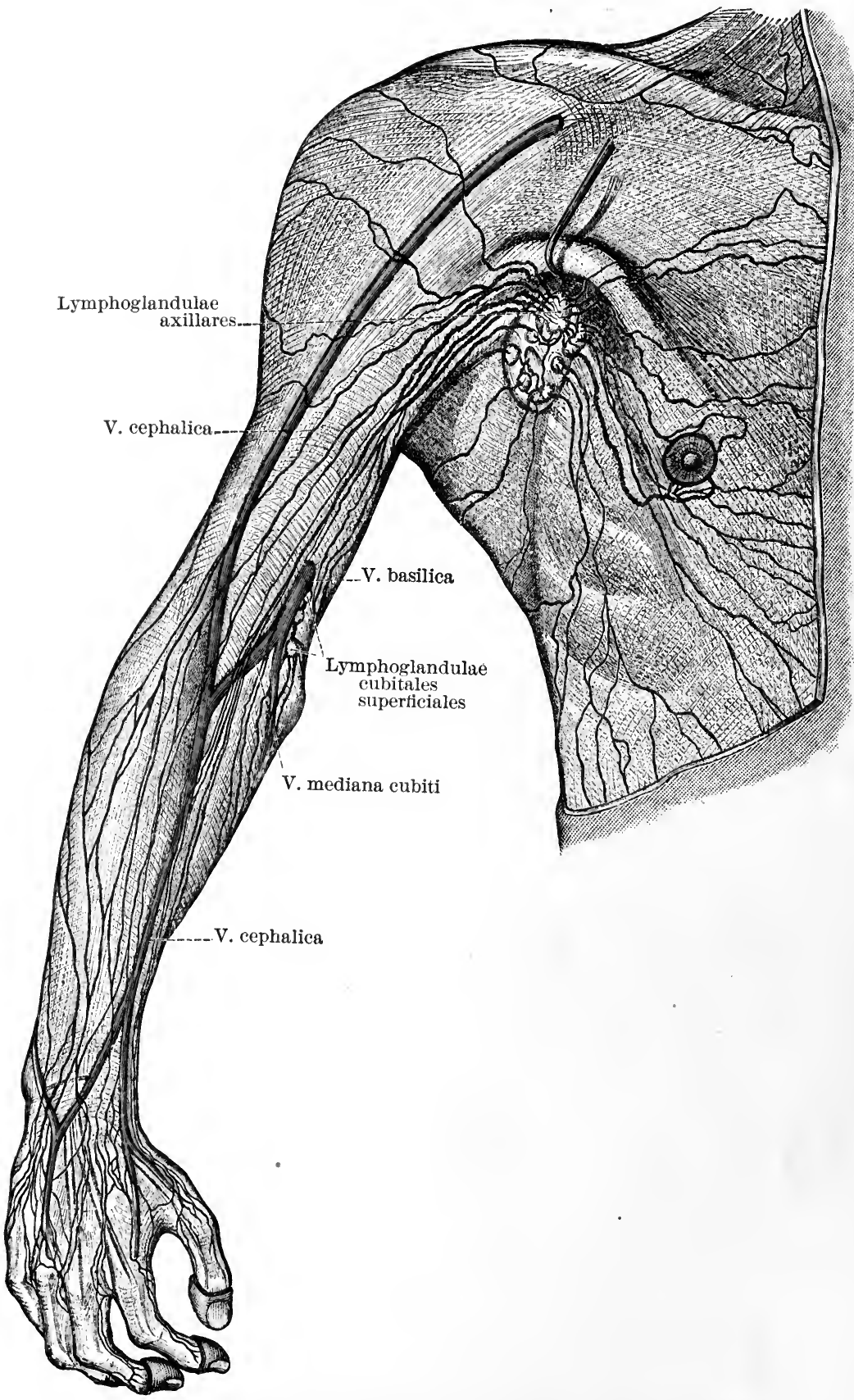

Lymphatics of the arm and of the anterior and lateral sides of the thorax. (From Toldt, Anat. Atlas, Wien, 1900, 2 Aufl., p. 704, Fig. 1086.) 
(c) Subseapular lymph-glands (lymphoglandulae subscapulares).

Count the number of lymph-glands found in each of these three cliains. Whence do the glands of each of these three chains draw lymph? Note especially the intimate relations of the glands of the middle chain to the axillary vein.

FIG. 16.

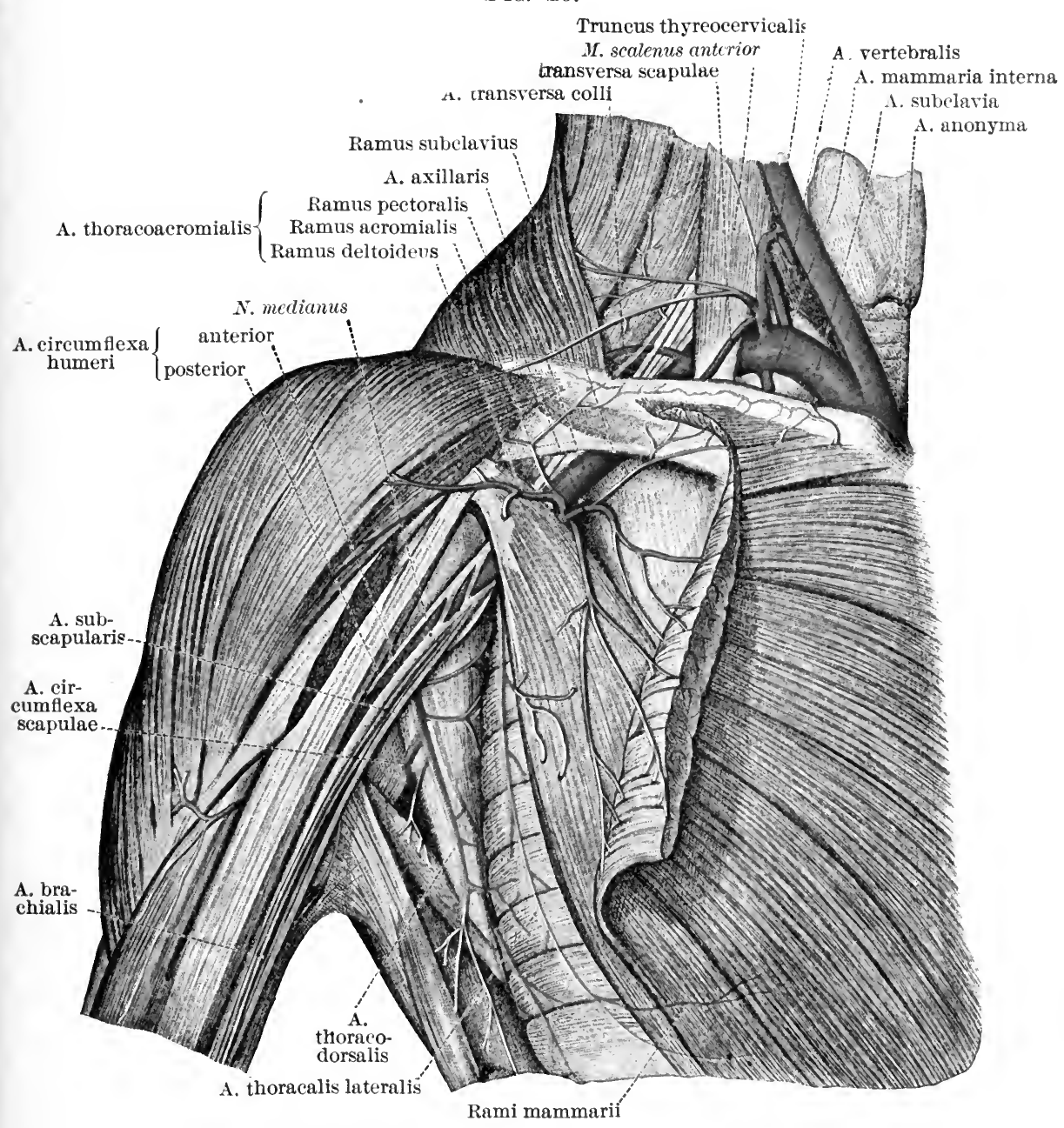

The subclavian and the axillary artery and their relation to the plexus brachialis.

(After Toldt, Anat. Atlas, Wien, 1900, 2 Aufl., p. 610, Fig. 986.)

Arteries. (Fig. 16.)

(a) Axillary artery (A. axillaris).

(aa) Highest thoracic artery (A. thoracalis suprema) (O. T. superior thoracic artery). 
(ab) Thoraco-acromial artery (A. thoraco-acromialis) (O. T. acromiothoracie or thoracic axis).

(ac) Lateral thoracic artery (A. thoracalis lateralis) (O. T. long thoracic).

(aca) External mammary rami (Rr. mammarii externi).

(ad) Subseapular artery (A. subscapularis).

(ada) Thoracodorsal artery (A. thoracodorsalis).

$(a d b)$ Circumflex artery of scapula (A. circumflexa scapulae) (O. T. dorsalis scapulae).

(ae) Anterior circumflex artery of humerus (A. circumflexa humeri anterior).

(af) Posterior circumflex artery of humerus (A. circumflexa

Veins. humeri posterior).

(a) Axillary vein (V. axillaris). (Spalteholz, Fig. 492.)

(aa) Lateral thoracic vein ( $V$. thoracalis lateralis).

(aaa) Thoraco-epigastric vein ( $V$. thoraco-epigastrica). $(a a b)$ Costo-axillary veins ( $V v$. costo-axillares).

(ab) Brachial veins (Vv. brachiales).

(ac) Cephalic vein ( $V$. cephalica).

(aca) Thoraco-acromial vein ( $V$. thoraco-acromiatis).

(ad) Other tributaries corresponding to branches of axillary artery.

Nerves.

At this stage of the dissection the relation of a group of nerves to the A. axillaris should be closely observed, the full study of the brachial plexus and its branches being made a little later. Note then the relations of-

(a) Ulnar nerve (N. ulnaris).

(b) Medial cutaneous nerve of upper arm ( $N$. cutaneus brachii medialis) ( $\mathrm{O} . \mathrm{T}$. lesser internal cutaneous, or nerve of Wrisberg).

(c) Medial cutaneous nerve of forearm (N. cutaneus antibrachii medialis) (O. T. internal cutaneous).

(d) Radial nerve (N. radialis) (O. T. musculospiral).

(e) Axillary nerve (N. axillaris) (O. T. circumflex).

( $f$ ) Median nerve ( $N$. medianus), with its two heads.

(g) Musculocutaneous nerve (N. musculocutaneus).

\section{Shape and Boundaries of Fossa Axillaris.}

Describe the form of the fossa. What is the position of its apex? How many walls has it? What structures help to form the anterior wall ? What structures enter into the formation of the posterior wall? Examine carefully the structures constituting respectively the medial and lateral walls of the fossa.

\section{Coracoclavicular Fascia (Fascia coracoclavicularis).}

Divide the pars clavicularis of the M. pectoralis major close to the clavicle (leaving the pars sternocostalis for the present intact) and reflect it downward and lateralward, avoiding injury 
to the vessels and nerves beneath. The coracoclavicular fascia is now exposed. What are its attachments? How does it form a sheath for the M. subclavius? Divide the anterior layer of the sheath transversely close to the clavicle and expose the M. subclavius; observe the N. subclavius entering the deep surface of the latter; with the handle of the scalpel demonstrate the attachment of the posterior layer of the sheath behind the muscle. The portion of the fascia extending between the clavicle and the M. pectoralis minor is often called the "costocoracoid membrane." Piercing this portion of the fascia find the following:

(a) Acromiothoracic artery (A. acromiothoracalis) (O. T. thoracic axis). (aa) Acromial ramus (ramus acromialis).

(ab) Acromial network (rete acromiale).

(ac) Deltoid ramus ( $R$. deltoideus) (O. T. humeral branch).

(ad) Pectoral rami ( $R r$. pectorales).

(b) Acromiothoracic vein ( $V$. acromiothoracalis).

(c) Cephalic vein (V. cephalica).

(d) Lateral anterior thoracic nerve ( $N$. thoracalis anterior lateralis).

Divide the pars sternocostalis of the M. pectoralis major about its middle and reflect it lateralward and medialward. Preserve the branches of the anterior thoracic nerves entering the deep surface of the muscle; some of them have already passed through the M. pectoralis minor.

Note that the sheath of the M. pectoralis minor is formed by a splitting of the fascia coracoclavicularis and that the fascia is continued from the lower margin of the muscle to become continuous with the fascia axillaris.

Smaller Pectoral Muscle (M. pectoralis minor).

Clean the external surface of the muscle by removing the anterior layer of the investing fascia. Examine carefully its form, position, origin, insertion, action, and innervation, but do not reflect the muscle until later.

\section{Axillary Blood-vessels.}

Remove the so-called costocoracoid membrane,-viz., that portion of the fascia coracoclavicularis which extends between the clavicle and the upper border of the M. pectoralis minor. The fossa axillaris may now be entered from above. Remove any visible areolar tissue and fat and study carefully in this situation the relations of the axillary artery and vein to one another and to the large bundle of nerves. Note that all are 
enclosed in a tough sheath (prolongation of fascia praevertebralis).

Clean the axillary blood-vessels carefully and study them. Into what three parts is it customary arbitrarily to subdivide

\section{Frg. 17.}

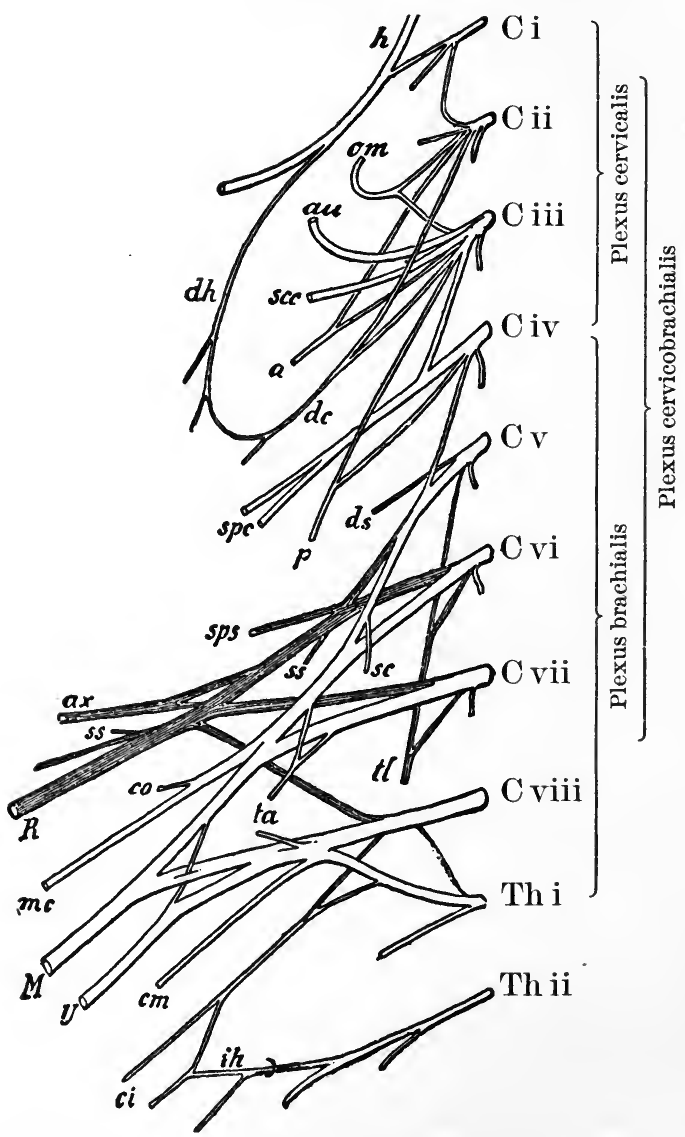

Plexus cervicobrachialis. (After P. Eisler, from Rauber's Text-Book.) Ventral view.

$h$, N. hypoglossus; $d h$, ramus descendens $\mathrm{N}$. hypoglossi, which, along with $d c$, the ramus descendens cervicalis, forms the ansa hypoglossi ; om, N. occipitalis minor; $a u, \mathrm{~N}$. auricularis magnus; $s c c$, N. cutaneus colli ; $a$, to N. accessorius; $s p c$, Nn. supraclaviculares ; $p, \mathrm{~N}$. phrenicus ; $d s, \mathrm{~N}$. dorsalis scapulae; sps, N. suprascapularis; ss, Nn. subscapulares; sc, N. subclavius; ax, N. axillaris; $c o, \mathrm{~N}$. to M. coracobrachialis; $R, \mathrm{~N}$. radialis; $m c, \mathrm{~N}$. musculocutaneus; $M, \mathrm{~N}$. medianus; $t a$, Nn. thoracales anteriores; $t, \mathrm{~N}$. thoracalis longus; $U, \mathrm{~N}$. ulnaris ; $\mathrm{cm}$, N. cutaneus antibrachii medialis; $c i$, N. cutaneus brachii medialis; ih, N. intercostobrachialis. (From Barker, The Nervous System, New York, 1899, p. 324, Fig. 186.)

the axillary artery? Study the relations of each of these three portions. What branches come from the first portion? What from the second? What from the third? Clean each of these 
branches carefully and follow them to their terminations, comparing their behavior with the text-book descriptions. Deal similarly with the axillary vein and its tributaries.

\section{Subclavius Muscle (M. subclavius).}

Clean it. Examine its form, position, origin, insertion, action, and innervation.

With a small hand-saw excise the middle third of the clavicle. Reflect the M. subclavius.

\section{Brachial Plexus (Plexus brachialis). (Fig. I7.)}

Divide the M. pectoralis minor four centimetres from the coracoid process and reflect the two portions lateralward and medialward respectively, avoiding injury to the medial anterior thoracic nerve. Examine the continuity of the axillary artery and vein with the subclavian artery and vein. Ligature the artery in two places at level of clavicle and cut between. Cut vein similarly. Turn axillary vessels downward. Clean thoroughly the nerves making up the brachial plexus. Study carefully the formation of the plexus, using the cadaver before you, Tramond's models, and text-book descriptions. Does the plexus in your cadaver differ from the type ordinarily described? If so, how? Make a careful drawing of the plexus in your subject, with its various branches, labelling each neatly. Note that the plexus can be divided into a supraclavicular portion (pars supraclavicularis) and an infraclavicular portion (pars infraclavicularis). All the short branches are counted as belonging to the former, even though they do not actually come off until the trunks are below the clavicle.

In conjunction with the dissector of the head and neck, study the

Pars supraclavicularis.

(a) Posterior thoracic nerves (Nn. thoracales posteriores).

(aa) Dorsal nerve of scapula (N. dorsalis scapulae) (O. T. nerve to rhomboids).

(ab) Long thoracic nerve ( $N$. thoracalis longus) (O. T. external respiratory nerve of Bell).

(b) Anterior thoracic nerves ( $N n$. thoracales anteriores).

(ba) Lateral (O. T. external).

(bb) Medial (O. T. internal).

(c) Subclavian nerve (N. subclavius).

(d) Suprascapular nerve (N. suprascapularis).

(e) Subscapular nerves (Nn. subscapulares).

(f) Thoracodorsal nerve ( $N$. thoracodorsalis) (O. T. middle or long subscapular).

(g) Axillary nerve ( $N$. axillaris) (O. T. cireumflex). 
The dissector of the arm next studies the

Pars infraclavicularis.

(a) Lateral cord (fasciculus lateralis) (O. T. outer cord).

(aa) Musculocutaneous nerve ( $N$. musculocutaneus).

$(a b)$ Lateral or upper head of median nerve ( $N$. medianus).

(b) Medial cord (fasciculus medialis) (O. T. inner cord).

(ba) Medial or lower head of median nerve (N. medianus).

(bb) Ulnar nerve ( $N$. ulnaris).

(bc) Medial cutaneous nerve of forearm ( $N$. cutaneus antibrachii medialis) (O. T. internal cutaneous).

(bd) Medial cutaneous nerve of upper arm ( $N$. cutaneus brachii medialis) (O. T. lesser internal cutaneous, or nerve of Wrisberg).

(c) Posterior cord (fasciculus posterior).

(ca) Axillary nerve (N. axillaris) (O. T. circumflex, really supraclavicular).

$(c b)$ Radial nerve (N. radiatis) (O. T. musculospiral).

In order to get a good view of the posterior cord and of the Nn. subscapulares, the lateral and medial cords should be divided and reflected downward.

\section{Anterior Serratus Muscle (M. serratus anterior) (O. T. Serratus Magnus).}

Divide the posterior cord of the brachial plexus. Drag the arm forcibly from the body so as to put the M. serratus anterior on the stretch. Clean this muscle thoroughly and study carefully the exact course and distribution of the N. thoracalis longus and the A. thoracalis lateralis. Study the exact form, position, origin, insertion, action, and innervation of the M. serratus anterior. (Spalteholz, Fig. 311.)

[When the student has completed this dissection of the anterior thoracic region and axillary fossa and has dissected that part of the back to which he is entitled, he may remove the upper extremity from the body and continue the dissection at a sidetable. Cut through the Mm. serratus anterior, omohyoideus, and latissimus dorsi, the transverse and dorsal arteries of the scapula, and the Nn. suprascapularis et dorsalis scapulae.]

\section{REGION OF THE SHOULDER.}

\section{Skin and Superficial Fascia.}

Place a block in the axillary fossa, and, beginning in front, dissect the skin off the anterior and lateral aspects of the shoulder as far down as the insertion of the M. deltoideus. Remove no fat with the skin. In the superficial fascia find the following: 
FIG. 18.

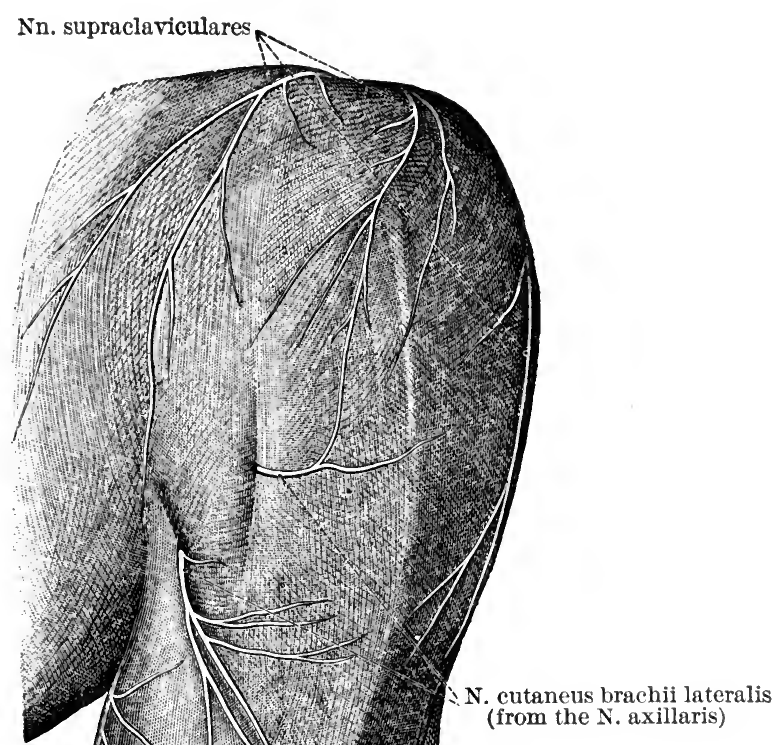

N. cutaneus brachii medialis

N. cutaneus brachii posteriol (from the N. radialis)

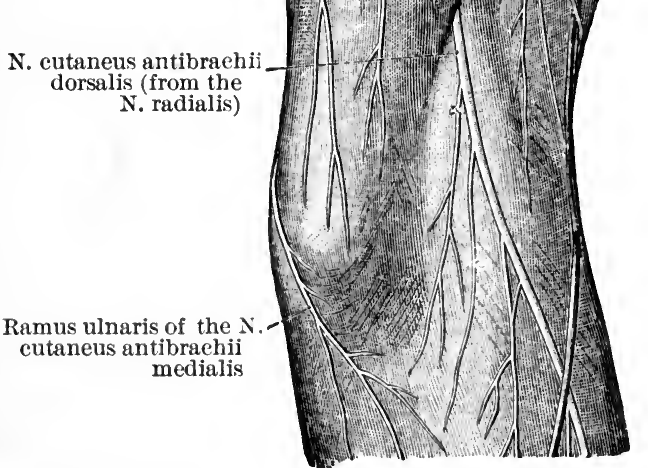

The cutaneous nerves of the posterior surface of the shoulder and of the arm. (After Toldt, Anat. Atlas, Wien., 1903, 3 Aufl., p. 831, Fig. 1264.) 
Arteries.

(a) Cutaneous rami of ramus acromialis and ramus deltoideus of $\mathrm{A}$. thoraco-acromialis.

Veins.

(a) Tributaries of cephalic vein.

Nerves. (Fig. 18.)

(a) Posterior supraclavicular nerves (Nn. supraclaviculares posteriores) (O. T. supra-acromial branches).

(b) Lateral cutaneous nerve of the upper arm ( $N$. cutaneus brachii lateralis) (O. T. cutaneous branch of circumflex). Look for the trunk of this hooking around the posterior margin of M. deltoideus five centimetres above its insertion; dissect it carefully throughout its course in the superficial fascia.

\section{Deep Fascia.}

Remove the superficial fascia and study the appearance and attachments of-

(a) Deltoid fascia (fascia deltoidea).

(b) Subscapular fascia (fascia subscapularis).

(c) Supraspinous fascia (fascia supraspinata).

(d) Infraspinous fascia (fascia infraspinata).

Deltoid Muscle (M. deltoideus) and the So-called Quadrilateral and Triangular Spaces.

Hold the scapula down with hooks to make the fibres of the deltoid muscle tense, and carefully remove the deep fascia from its surface. Study carefully the form, position, origin, insertion, action, and innervation of the muscle. Note especially the three parts of the muscle corresponding to the three distinct muscles in the cat,-deltoclavicular, delto-acromial, and deltospinal of Strauss-Durckheim. How does the texture of the middle portion (delto-acromial) differ from that of the anterior and posterior portions? (Cf. Poirier et Charpy, Fig. 83.) Look for a subcutaneous acromial bursa (bursa subcutanea acromialis). (Cf. Spalteholz, Fig. 349.) Does one exist in your cadaver?

Place the posterior surface of the limb on the table, and examine the area through which the A. circumflexa humeri posterior and the $\mathrm{N}$. axillaris pass to the back part of the shoulder. How is this area, "quadrilateral space," bounded above, below, lateralward, and medialward? Clean the surfaces and edges of the muscles of this region. What is the so-called "triangular space?" How is it bounded above, below, and lateralward? Follow the A. circumflexa scapulae (O. T. dorsalis scapulae) through this space.

Place the anterior surface of the limb on the table, and examine the boundaries of the quadrilateral and triangular spaces from behind. 
Divide the M. deltoideus close to its origin and reflect it downward, avoiding injury to the ramus acromialis of the A. thoraco-acromialis. Examine carefully the subdeltoid bursa (bursa subdeltoidea) and the subacromial bursa (bursa subacromialis).

Beneath the deltoid dissect out carefully-

(a) Posterior circumflex artery of humerus (A. circumflexa humeri posterior).

(b) Anterior circumflex artery of humerus (A. circumflexa humeri anterior).

(c) Veins corresponding to these arteries.

(d) Axillary nerve (N. axillaris) (O. T. eircumflex).

(da) Muscular rami (rami musculares).

(daa) N. teres minor.

(dab) Nn. deltoidei.

(db) Lateral cutaneous nerve of the upper arm (N. cutanevs brachii lateralis).

Teres Major Muscle (M. teres major) ("Larger Round Muscle”).

Study carefully its form, position, origin, insertion, action, and innervation. Note especially the torsion the muscle undergoes. Review the relation of this muscle to the quadrilateral and triangular spaces. Between the tendon of insertion and the bone find the bursa of this muscle (bursa $M$. teretis majoris). Between the tendon of the M. teres major and that of the M. latissimus dorsi find the bursa of the latter muscle (bursa M. latissimi dorsi). Do you find any trace of a M. dorsi-epitrochlearis? Examine carefully at this stage the exact mode of termination of the tendons of the Mm. pectoralis major and latissimus dorsi.

\section{Ligaments of Shoulder-blade and Acromioclavicular Articulation.}

Ligaments of the shoulder-blade. Study-

(a) Coraco-acromial ligament (ligamentum coraco-acromiale).

(b) Superior transverse ligament of scapula (Lig. transversum scapulae inferius).

(The latter will be seen at a later stage of the dissection.)

Acromioclavicular articulation (articulatio acromioclavicularis). Study-

(a) Joint-capsule (capsula articulariş).

(b) Acromioclavicular ligament (ligamentum acromioclaviculare).

(c) Interealated disk of fibrocartilage (discus articularis). Is one present?

(d) Coracoclavieular ligament (ligamentum coracoclaviculare).

(da) Anterior, lateral, quadrangular part, or trapezoid ligament (Lig. trapezoideum). 
(db) Posterior, medial, triangular part, or conoid ligament (Lig. conoideum).

Between $(d a)$ and $(d b)$ look for the bursa of the coracoclavicular ligament (bursa ligamenti coracoclavicularis).

\section{Deep Muscles of the Shoulder.}

Saw through the acromion at its junction with the spina scapulae. Cut through the fascia covering the M. teres minor and reflect it medialward; the septum passing from the fascia infraspinata between the M. teres minor and the M. infraspinatus will be easily found and is the guide in the separation of

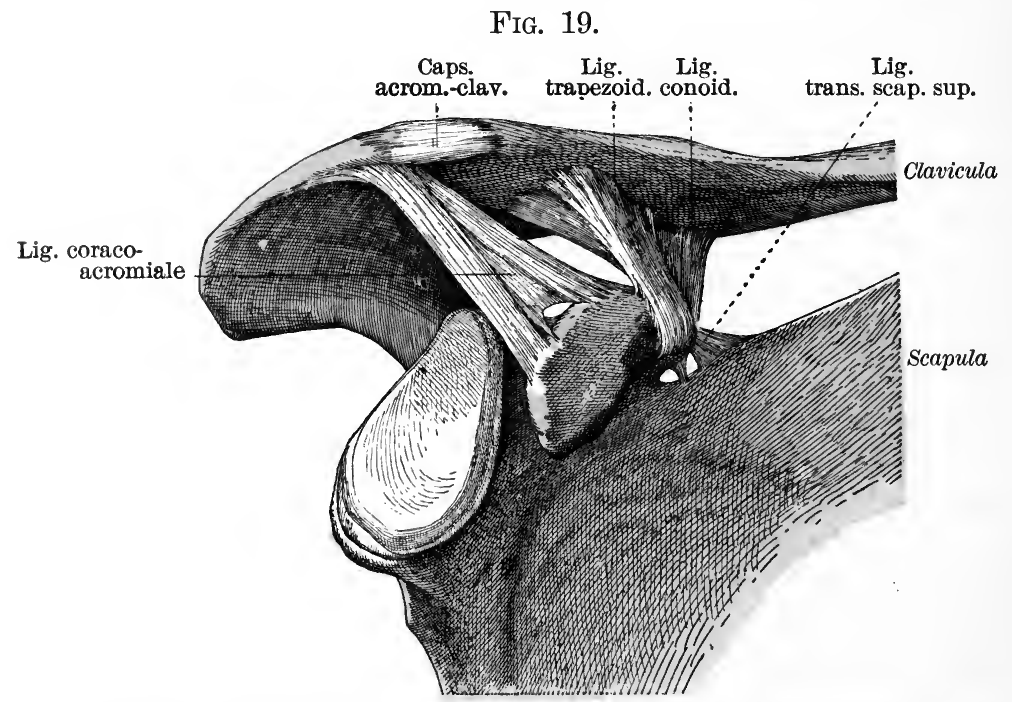

Connection of the clavicle with the scapula ; anterior view. (From Poirier et Charpy, Traité d'Anat. hum., Paris, 1899, 2 ed., t. i. p. 610, Fig. 627.)

the two muscles. Avoid injury to the A. circumflexa scapulae between the M. teres minor and the scapula. Clean the following muscles :

(a) Supraspinous muscle (M. supraspinatus).

(b) Smaller round muscle (M. teres minor).

(c) Infraspinous muscle ( $M$. infraspinatus).

(d) Subscapular muscle (M. subscapularis).

Study the form, position, origin, insertion, action, and innervation of each. Find the bursa M. subscapularis and with a probe demonstrate its continuity with the synovial cavity of the shoulder-joint. In front of the upper part of the tendon of the M. subscapularis, look for the bursa M. coracobrachialis. 


\section{Vessels and Nerves behind the Scapula.}

Cut through the M. infraspinatus three centimetres from its insertion into the tuberculum majus humeri, avoiding injury to vessels beneath. Examine the bursa $M$. infraspinati between the tendon of insertion of the muscle and the articular capsule of the shoulder-joint. Reflect the main body of the muscle cautiously backward and medialward. Cut through the M. supraspinatus in a similar way and reflect it medialward. Study-

(a) Transverse artery of scapula (A. transversa scapulae) (O. T. supraseapular).

Does it pass over or under the Lig. transversum scapulae superius? How is the artery distributed? Find-

(aa) Acromial ramus ( $R$. acromialis) going through M. trapezius to rete acromiale.

(b) Transverse vein of scapula ( $V$. transversa scapulae).

Of what vein is it a tributary?

(c) Supraseapular nerve (N. suprascapularis).

Does it pass over or under the Lig. transversum scapulae superius? Review it to its origin from the supraclavicular portion of the brachial plexus. Note especially the branches-

(ca) N. supraspinatus.

( $c b$ ) N. infraspinatus.

(d) Circumflex artery of scapula (A. circumflexa scapulae) (O. T. dorsalis scapulae artery). Study its exact course from origin to termination. Note the opportunities for anastomoses about the scapula among its three main arteries-

(1) A. transversa colli.

(2) A. transversa scapulae.

(3) A. subscapularis.

Since (1) and (2) come from the A. subclavia (first portion) and (3) comes from the A. axillaris (third portion), the establishment of a collateral circulation after ligation of the subclavian or axillary between the origins of the arteries mentioned is seen to be easily possible.

\section{DISSECTION OF ARM AND FOREARM.}

\section{Surface Anatomy.}

The principal features were studied at the beginning of the dissection. The student should now pay attention to certain special points, using his own arm or that of a companion as a control.

(a) With the help of the photographs and accompanying key in Gerrish's Anatomy (2d edition, Figs. 971 to 979 ), identify the surface prominences due to the various muscles of the arm and forearm.

(b) Note that the vena cephalica runs in the sulcus bicipitalis lateralis and the vena basilica in the sulcus bicipitalis medialis. 
(c) Follow the medial margin (margo medialis) downward to the medial epicondyle (epicondylus medialis), and the lateral margin (margo lateralis) downward to the lateral epicondyle (epicondylus lateralis). Note that, though the margo lateralis is more salient than the margo medialis, the epicondylus medialis is more prominent than the epicondylus lateralis. Feel through the skin behind the medial epicondyle for the groove for the ulnar nerve (sulcus $N$. ulnaris). Press upon the nerve in your own arm ("funny bone," or " crazy bone"). Whence does the sensation seem to come?

(d) Observe the prominence due to the olecranon (1) when the forearm is extended, (2) when the forearm is flexed. Standing in front of a living body, take the two elbow-joints in the palms of the two hands, with the forefinger resting on the tip of the olecranon, the thumb on the lateral epicondyle, and the middle finger on the medial epicondyle. Ask the person to flex and extend the forearms, and notice changes in relative position of bony points. Note the relative distance between the level of the olecranon and the epicondyles. The oleeranon is a little nearer to the medial than to the lateral epicondyle, especially in children. Observe the free movement of the skin over the olecranon, due to a subcutaneous bursa. To what extent is the posterior surface of the ulna subcutaneous? Feel for the margins of the semilunar notch (incisura semilunaris).

(e) Extend the forearm. Place the thumb of the left hand on the lateral epicondyle of the humerus; seize the hand with your right hand and rotate gently. Feel the rounded head of the radius (capitulum radii) rotating immediately below the epicondyle.

( $f$ ) In the forearm palpate-

( $f a)$ Dorsal margin of ulna (margo dorsalis ulnae).

( $f b)$ Styloid process of ulna (processus styloideus ulnae).

( $f c$ ) Rounded head of ulna (capitulum ulnae).

( $f d)$ Styloid process of radius (processus styloideus radii).

(fe) Radial artery (A. radialis).

(ff) The tendons about the wrist.

\section{Skin and Superficial Fascia.}

Cut through the skin in the middle line of the anterior surface of the upper arm and the volar surface of the forearm as far as the wrist. Make a circular incision around the forearm just proximal to the wrist-joint. Remove the skin, dissecting lateralward and medialward, but take none of the fat of the superficial fascia with it. Preserve the skin, as it makes the best primary wrapping for the extremity in the intervals between dissection-periods. In the superficial fascia dissect out carefully the following:

Nerves. (Cf. Fig. 20, and Spalteholz, Figs. 792 and 793.)

(a) Intereostobrachial nerves (Nn. intercostobrachiales) (O. T. intercostohumeral). 
FIG. 20.

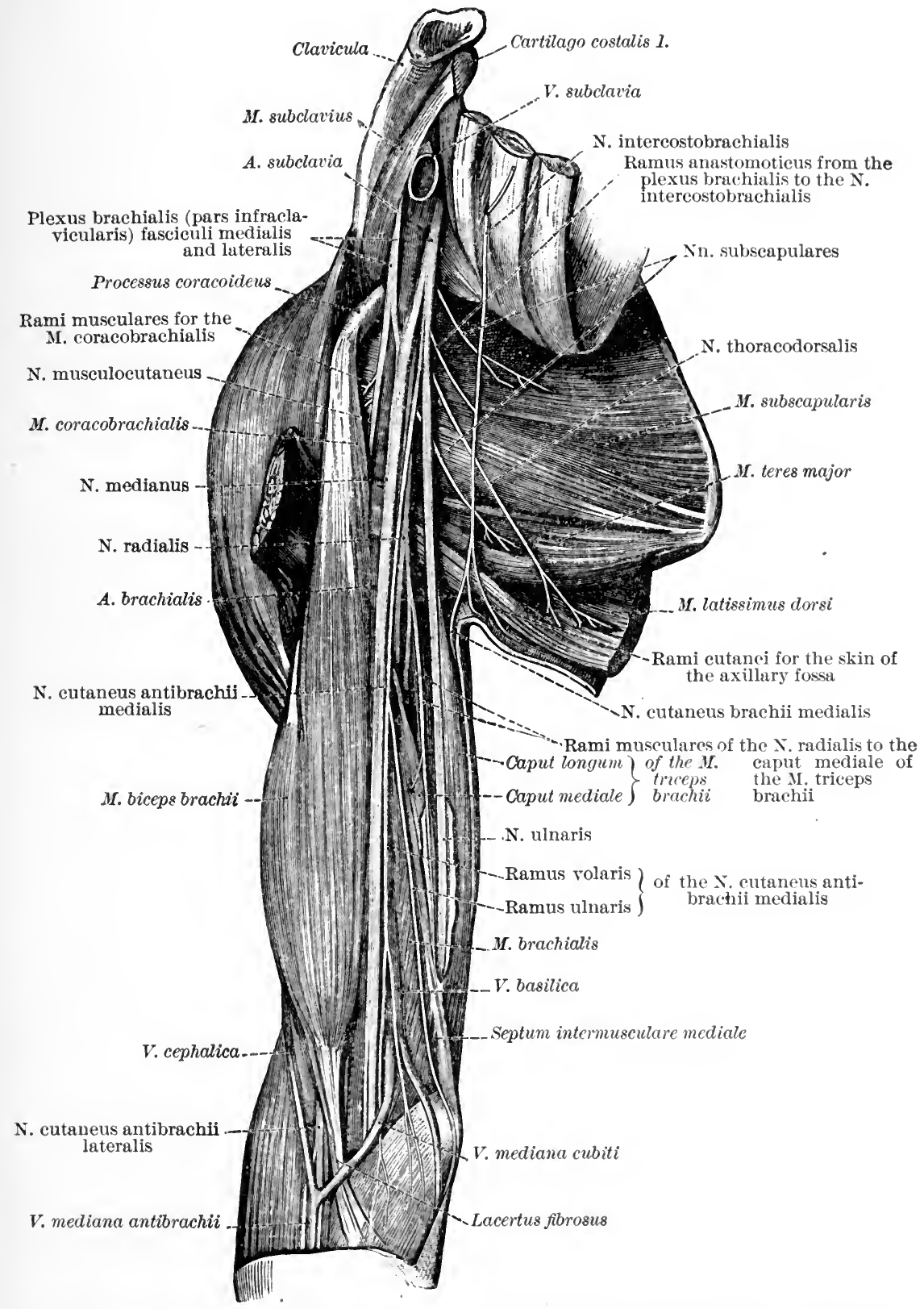

The deep nerves of the shoulder and arm seen from the anterior and medial side. (From Toldt, Anat. Atlas, Wien, 1903, 3 Aufl., p. 822, Fig. 1254.) 
(b) Medial nerve of forearm (N. cutaneus antibrachii medialis) (O. T. internal cutaneous). (See Fig. 24, p. 81.)

(ba) Anterior cutaneous rami to upper arm (rami cutanei brachii anteriores).

(bb) Ulnar ramus (ramus ulnaris) (O. T. posterior branch).

(bc) Volar ramus (ramus volaris) (O. T. anterior branch).

(c) Medial nerve of upper arm ( $N$. cutaneus brachii medialis) (O. T. lesser internal cutaneous, or nerve of Wrisberg).

FIG. 21.

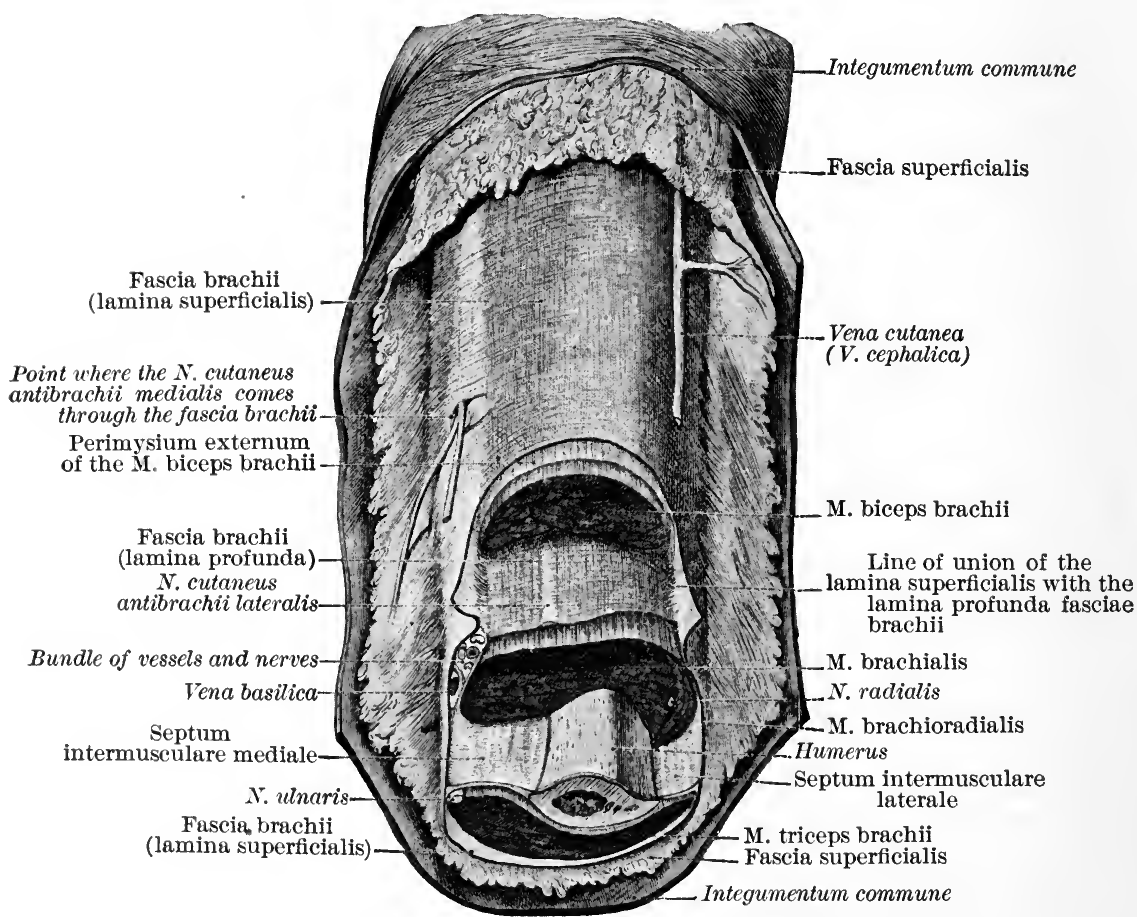

The structures in the lower third of the arm cut across like stairs.

(After Toldt, Anat. Atlas, Wien, 1900, 2 Aufl., p. 264, Fig. 502.)

(d) Radial nerve ( $N$. radialis) (O. T. musculospiral). Only superficial branches of this nerve are seen as yet.

(da) Posterior cutaneous nerve of upper arm (N. cutaneus brachii posterior) (O. T. upper external cutaneous branch of musculospiral).

(db) Posterior cutaneous nerve of forearm ( $N$. cutaneus antibrachii dorsalis) (O. T. lower external cutaneous branch of musculospiral).

(e) Musculocutaneous nerve (N. musculocutaneus).

(ea) Lateral cutaneous nerve of forearm ( $N$. cutaneus antibrachii lateralis) (O. T. terminal cutaneous branch). (See Fig. 24.) 
Veins. (See also Fig. 24.)

(a) Basilic vein ( $V$. basilica). At what point does it pierce the fascia brachii?

(b) Cephalie vein ( $V$. cephalica).

(c) Median vein of elbow ( $V$. mediana cubiti), from V. cephalica obliquely upward to $\mathrm{V}$. basilica.

Instead of $(c)$ there may be a-

(d) Median vein of forearm ( $V$. mediana antibrachii), bifureating at head of elbow into-

(da) Basilic median vein ( $V$. mediana basilica).

$(d b)$ Cephalic median vein ( $V$. mediana cephalica).

In phlebotomy either the V. mediana cubiti or the V. mediana basilica is selected. Note the relations, to these veins, of the lacertus fibrosus, the arteria brachialis, and the ramus volaris of the N. cutaneus antibrachii medialis.

FIG. 22.

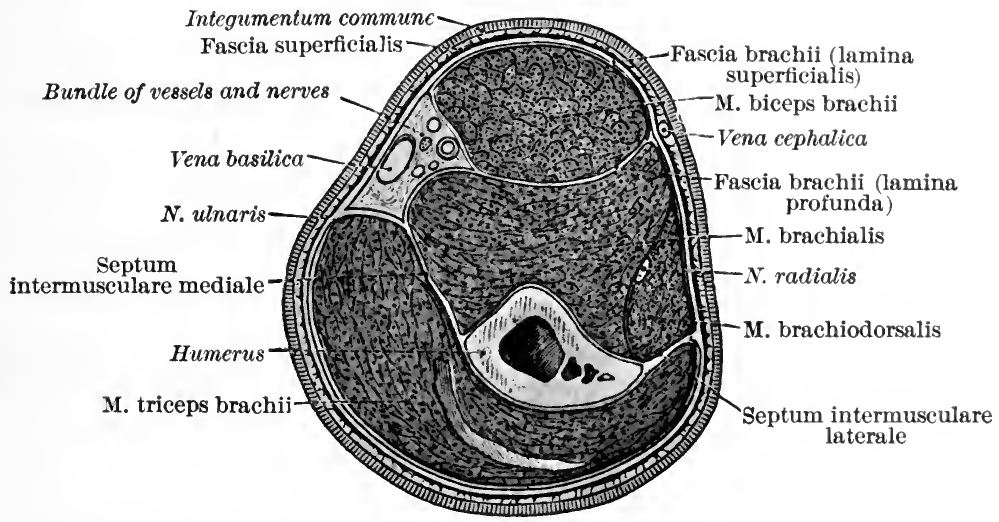

Cross-section of the right arm near its lower end, to show the grouping of the muscles and their relation to muscle-sheaths or fasciae. Half schematic. (After Toldt, Anat. Atlas, Wien, 1900, 2 Aufl, p. 264, Fig. 503.)

\section{Lymphatics.}

(a) Superficial lymph-glands of elbow (lymphoglandulae cubitales superficiales).

They may be enlarged in infection of the hand or fingers.

\section{Deep Fascia of Arm (Fascia brachii) (O. T. Brachial Aponeurosis). (Figs. 2I and 22.)}

Having isolated the vessels and nerves of the superficial fascia, remove all the fat so as to clean the surface of the deep fascia. Note-

(a) Its continuity with the deltoid and axillary fascia above and with the deep fascia of the forearm below.

(b) That it is multiply perforated for the passage of vessels and nerves, and

(c) The lacertus fibrosus. 
Make an incision through the fascia brachii along the middle line of the front of the upper arm and a circular incision proximal to the lacertus fibrosus. Reflect the fascia brachii from the front of the arm in two flaps, medial and lateral, until the strong lateral and medial intermuscular septa are reached,-

(a) Medial intermuscular septum (septum intermusculare [humeri] mediale) (O. T. internal intermuscular septum).

(b) Lateral intermuscular septum (septum intermusculare [humeri] laterale) (O. T. external intermuscular septum).

\section{Structures in Front of the Intermuscular Septa (Anterior Compart- ment of Arm). (See also Fig. 20.)}

Arteries.

Arrange the axillary artery and vein and the divided nerves of the brachial plexus in their proper order and tie them to a small stick, four centimetres long, placed transversely. Fasten this with a loop of cord to the processus coracoideus. Clean the brachial artery carefully throughout its whole extent and study its relations.

(a) Brachial artery (A. brachialis). At what point does it begin? Where and how does it end? With what veins, nerves, and muscles does it enter into relation in the various parts of its course? Study the mode of origin, course, and distribution of the following branches:

(aa) Deep artery of upper arm (A. profunda brachii) (O. T. superior profunda). Note its relation to the radial nerve. Its branches and general distribution will be studied later.

(ab) Superior ulnar collateral artery (A. collateralis ulnaris superior) (O. T. inferior profunda). Note its relation to the ulnar nerve. How does it arise?

(ac) Inferior ulnar collateral artery (A. collateralis ulnaris inferior) (O. T. anastomotica magna). Note its anterior and posterior divisions.

Veins.

(a) Brachial veins ( $V v$. brachiales).

(aa) Radial veins ( $V v$. radiales).

(ab) Ulnar veins ( $V v$. ulnares).

(ac) Basilic veins ( $V v$. basilicae), portions beneath fascia brachii.

(b) Cephalic vein ( $V$. cephalica), portion in a duplicature of the fascia brachii.

Nerves.

(a) Medial cutaneous nerve of upper arm (N. cutaneus brachii medialis) (O. T. lesser internal cutaneous, or nerve of Wrisberg).

(b) Medial cutaneous nerve of forearm ( $N$. cutaneus antibrachii medialis) (O. T. internal cutaneous).

These nerves $(a)$ and $(b)$ have been studied before, with the exception of their parts subjacent to the fascia brachii. 
(c) Median nerve (N. medianus). How does it arise? Note its changing relations as regards the $\mathrm{A}$. brachialis. Does it give off any branches above the elbow?

(d) Ulnar nerve (N. ulnaris). Study its course in the upper part of the arm. Where and how does it leave the anterior compartment of the upper arm?

Fig. 23.

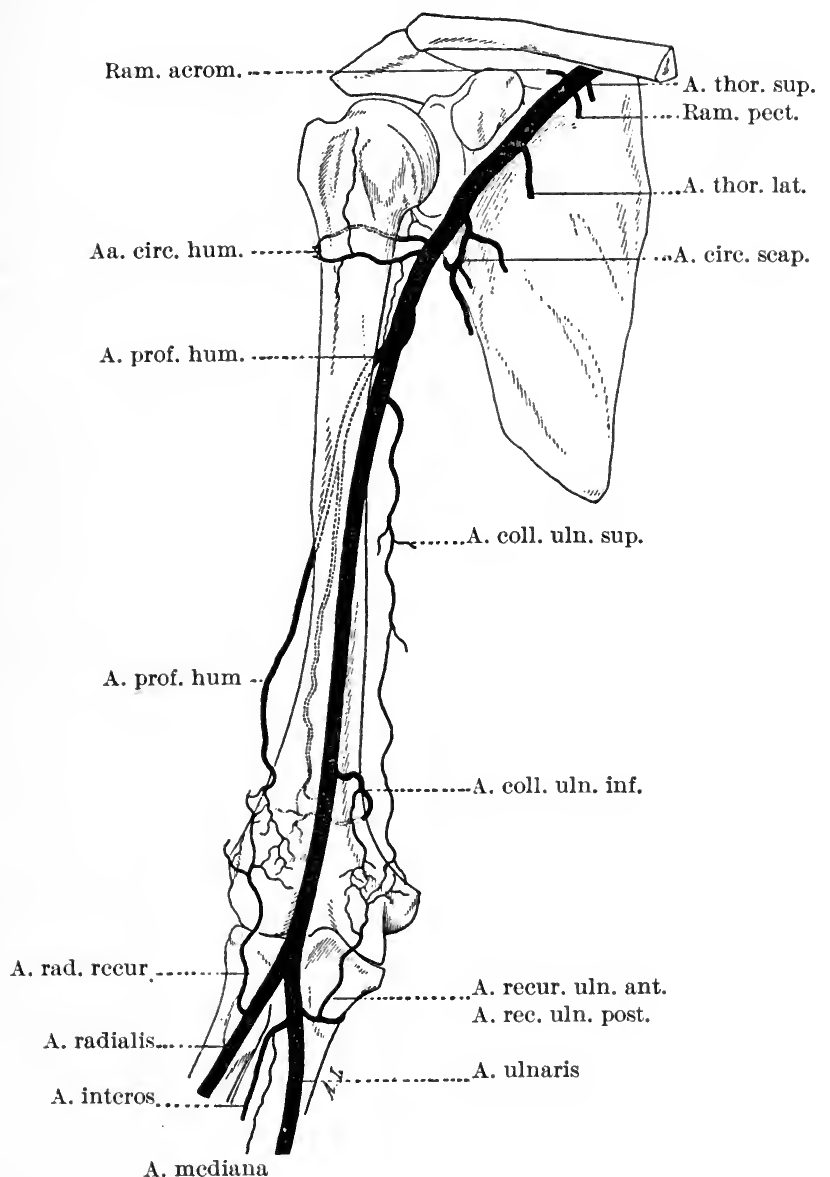

A. mediana

Scheme of the branches of the brachial artery. (From Poirier et Charpy, Traite d'Anat. hum., Paris, 1901, t. ii. p. 732, Fig. 416.)

(e) Musculocutaneous nerve (N. musculocutaneus). Carefully dissect out this nerve and its branches. How does it arise from the plexus brachialis? Study its exact course throughout the upper arm.

(ea) Muscular rami (rami musculares). (eaa) Nerve to M. coracobrachialis. 
Muscles.

(eab) Nerve to M. biceps brachii.

(eac) Nerve to M. brachialis.

(eb) Lateral cutaneous nerve of forearm ( $N$. cutaneus antibrachii lateralis), already studied. (See Fig. 24.) Note the eutaneous ramus given off above the elbow to supply the skin over the lateral region of the elbow.

The muscles of the anterior compartment should now be thoroughly cleaned. Study the form, position, origin, insertion, action, and innervation of each.

(a) Biceps musele of upper arm (M. biceps brachii).

(aa) Long head (caput longum). The exact origin will be examined later. Observe the prolongation of the synovial membrane of the shoulder-joint in the sulcus intertubercularis. It is called the intertubercular mucous sheath (vagina mucosa intertubercularis).

(ab) Short head (caput breve). The insertion, the lacertus fibrosus, and the bursae related to the tendon of insertion will be examined a little further on.

Comparative anatomy indicates that the M. biceps brachii is really a quadrigeminal muscle,-coracoradial, coracocubital, glenoradial, and glenocubital (Krause).

(b) Coracobrachial muscle (M. coracobrachialis).

Find the bursa $M$. coracobrachialis. Note that the M. coracobrachialis really consists of two distinet bundles; these fuse at their two extremities, but not in the middle, thus forming a tunnel three centimetres long for the N. musculocutaneus. Note that the M. coracobrachialis in the arm corresponds to the adductor system of muscles in the thigh.

Find the fibrous arch extending from the insertion of the tendon of the muscle to the inferior border of the tuberculum minus (areh of Struthers).

(c) Brachial muscle ( $M$. brachialis) (O. T. brachialis anticus).

How is it related to the medial and lateral bicipital sulci?

\section{Bend of the Elbow (Cubital Fossa). (Fig. 24.)}

Dissect in the depth between the M. brachialis and the M. brachioradialis for the radial nerve and the radial recurrent artery. Find the branch of the latter which perforates the septum intermusculare laterale to join the rete articulare cubiti, thus establishing an anastomosis with the A. profunda brachii. Seek the branches from the radial nerve to the M. brachioradialis and the M. extensor carpi radialis longus.

Compare the bend of the elbow with the popliteal space. Study the lacertus fibrosus (O. T. semilunar fascia) carefully and examine its relations. Why are these of surgical importance? What is the shape of the space in front of the elbow? How is it bounded? In this space examine the following: 
F'IG. 24.

Y. cutaneus f Ramus ulnaris antibrachii medialis $\{$ Ramus volaris

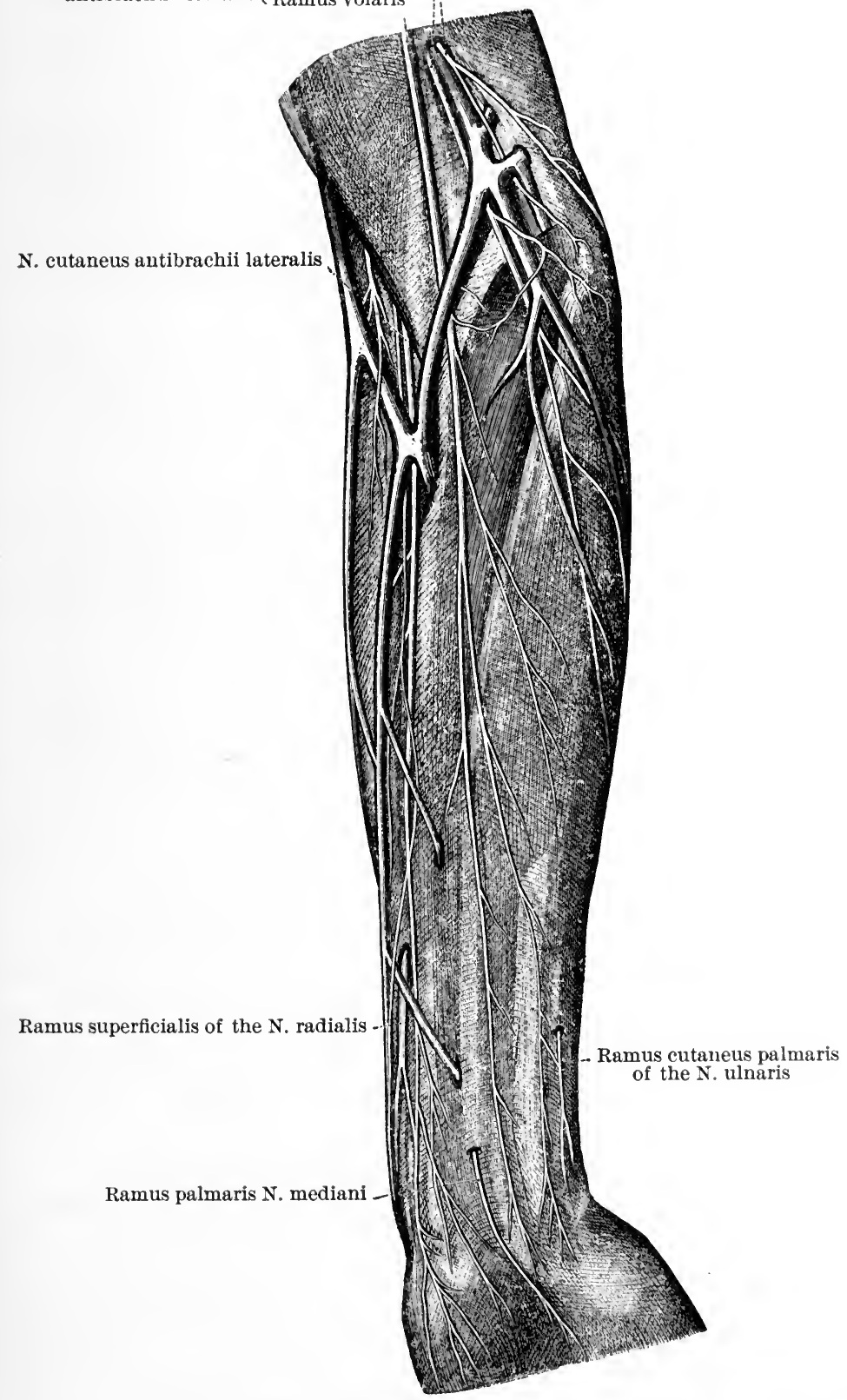

The cutaneous nerves of the volar surface of the forearm. (After Toldt, Anat. Atlas, Wien, 1903, 3 Aufl., p. 830, Fig. 1263.) 
(a) Brachial artery (A. brachialis).

(aa) Radial artery (A. radialis).

(ab) Ulnar artery (A. ulnaris).

(b) Tendon of M. biceps brachii. Find-

(ba) Bursa bicipitoradialis.

(bb) Bursa cubitalis interossea.

(c) Median nerve ( $N$. medianus).

Remove the fat and clean the structures so as to examine in the floor of the space-

(a) Brachial musele (M. brachialis).

(b) Supinator muscle (M. supinator) (O. T. supinator brevis).

Divide the lacertus fibrosus (O. T. semilunar fascia or bicipital fascia), pull the M. brachioradialis far lateralward and the M. pronator teres far medialward. Examine carefully-

(a) Radial nerve ( $N$. radialis).

(aa) Deep ramus (ramus profundus).

(ab) Superficial ramus (ramus superficialis).

(b) Radial recurrent artery (A. radialis recurrens).

(c) Inferior ulnar collateral artery (A. collateralis ulnaris inferior) ( 0. T. anastomotica magna).

(d) Anterior ulnar recurrent artery (A. recurrens ulnaris anterior).

(e) Epitrochlear lymph-gland.

\section{Structures behind the Intermuscular Septa (Posterior Compartment of Arm).}

Dissect the fascia brachii off the M. triceps, cleaning its three heads thoroughly. This will be facilitated by putting the muscle on the stretch (flex the forearm and raise the inferior angle of the scapula as far as possible). Dissect out the A. profunda brachii and the N. radialis and their branches. Study-

(a) Triceps muscle (M. triceps brachii).

Study its form, position, origin, insertion, action, and innervation.

(aa) Long head (caput longum).

(ab) Lateral head (caput laterale) (O. T. outer head).

(ac) Medial head (caput mediale) (O. T. inner head).

Compare the M. triceps with the M. quadriceps femoris. Is a M. subanconaeus present?

(b) Radial nerve (N. radialis) (O. T. musculospiral nerve).

Insert a grooved director or the handle of a scalpel beneath the lateral head of the triceps muscle along the sulcus $\mathrm{N}$. radialis (O. T. musculospiral groove). With this as a guide, cut through the lateral head of the triceps and reflect it medialward and downward. Study the rela- 
Fig. 25.

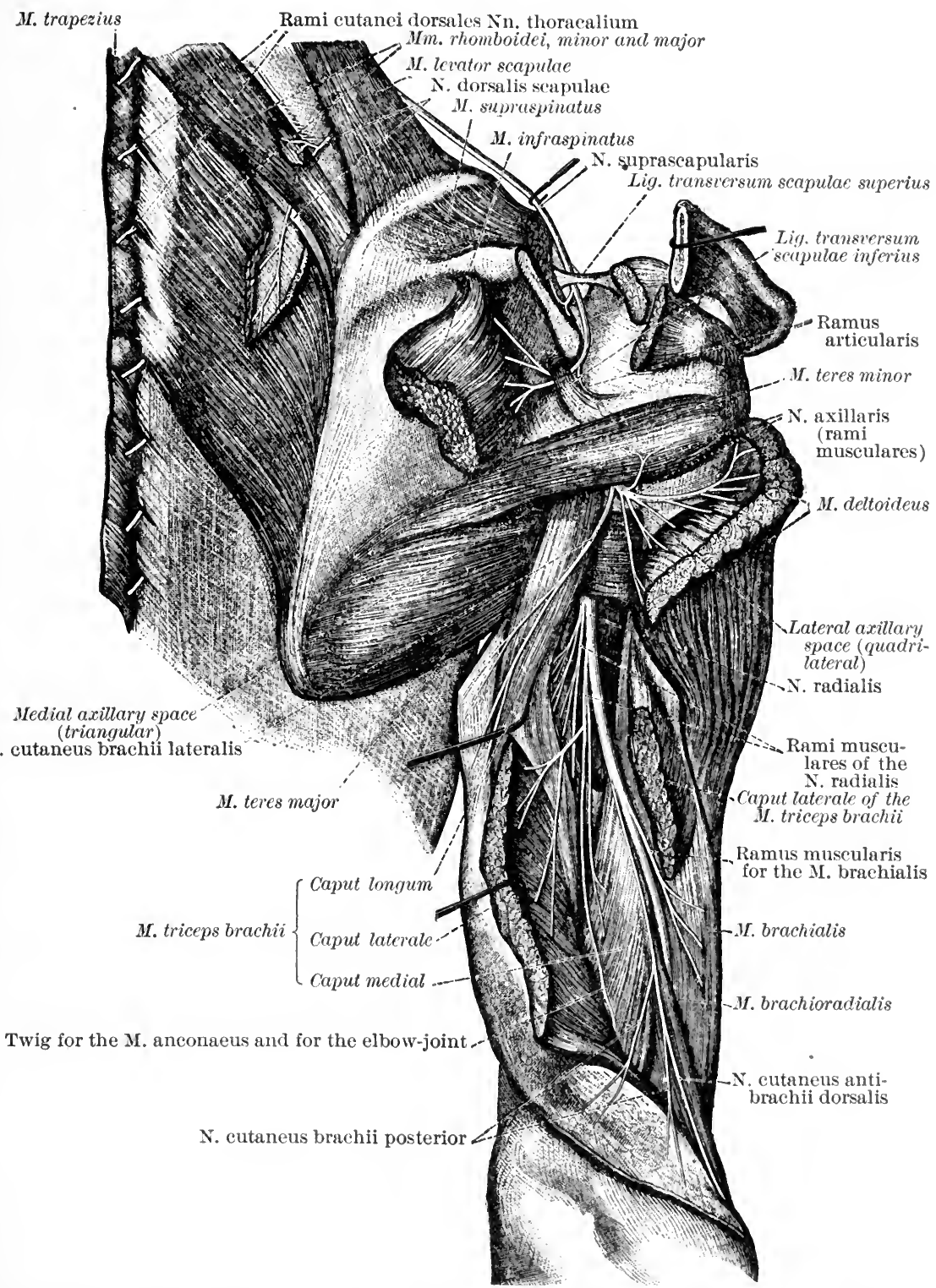

The nerves of the museles about the shoulder-joint and of the M. triceps, together with the eutaneous branches of the $\mathrm{Nn}$. axillaris and radialis, and the distribution of the $\mathrm{N}$. dorsalis seapulat. The acromion has been sawed off and drawn lateralward and the $\mathrm{Mm}$. supraspinatus and infraspinatus cut through. The lateral head of the M. triceps brachii has been ent through obliquely and its two parts turned back. (After Toldt, Anat. Atlas, Wien, 1903, 3 Aufl., p. 824, Fig. 1256.) 
tions of the nerve closely in the different parts of the upper arm. Branches in upper arm:

(ba) Posterior cutaneous nerve of upper arm (N. cutaneus brachii posterior) (O. T. upper external cutaneous branch of musculospiral). (Already studied; vide supra.)

(bb) Muscular rami (rami musculares).

(bba) To three heads of M. triceps brachii.

(bbb) To M. anconaeus.

(bbc) To M. brachioradialis (already studied).

$(b b d)$ To M. extensor carpi radialis longus (already studied).

(bbe) To M. brachialis.

(bc) Posterior cutaneous nerve of forearm ( $N$. cutaneus antibrachii dorsalis) (O. T. lower external cutaneous branch of musculospiral. (See Fig. 28.)

(c) Deep artery of upper arm (A. profunda brachii) (O. T. superior profunda artery).

Branches :

Study its relations in all parts of its course.

(ca) Deltoid ramus (ramus deltoideus).

(cb) Middle collateral artery ( $A$. collateralis media). Note its contribution to the rete articulare cubiti.

(cc) Radial collateral artery (A. collateralis radialis) ( $\mathrm{O} . \mathrm{T}$. articular branch of superior profunda).

(cd) Nutrient arteries of the humerus (Aa. nutriciae humeri). These sometimes come from the brachial artery proper.

(d) Other structures in back of arm.

Dissect carefully above the back of the elbow-

(da) Ulnar nerve ( $N$. ulnaris), through the sulcus N. ulnaris.

(db) Superior ulnar collateral artery (A. ulnaris collateralis superior) (O. T. inferior profunda). Note relation to rete articulare cubiti.

(dc) Ramus muscularis to medial head of $\mathrm{M}$. triceps. This is sometimes called the " ulnar collateral nerve."

$(d d)$ Inferior ulnar collateral artery (A. collateralis ulnaris inferior) (O. T. anastomotica magna). Look for it beneath the tendon of the M. triceps, a little above the olecranon.

(de) Bursa subtendinea olecrani.

Look for a bursa subcutanea olecrani also and a bursa intratendinea olecrani.

\section{SHOULDER-JOINT (ARTICULATIO HUMERI, OR AR- TICULATIO SCAPULOHUMERALIS).}

This joint is an enarthrosis. (What is meant by this statement?) Review the muscles related to the joint and then remove them as follows:

Cut through the origins of the M. coracobrachialis and the caput breve of the M. biceps brachii; divide the M. teres major 
midway between its origin and its insertion and the caput longum of the M. biceps four centimetres below its origin. Reflect these muscles. Carefully dissect from the capsule of the joint the $\mathrm{Mm}$. supraspinatus, infraspinatus, teres minor, and subscapularis.

FIg. 26.

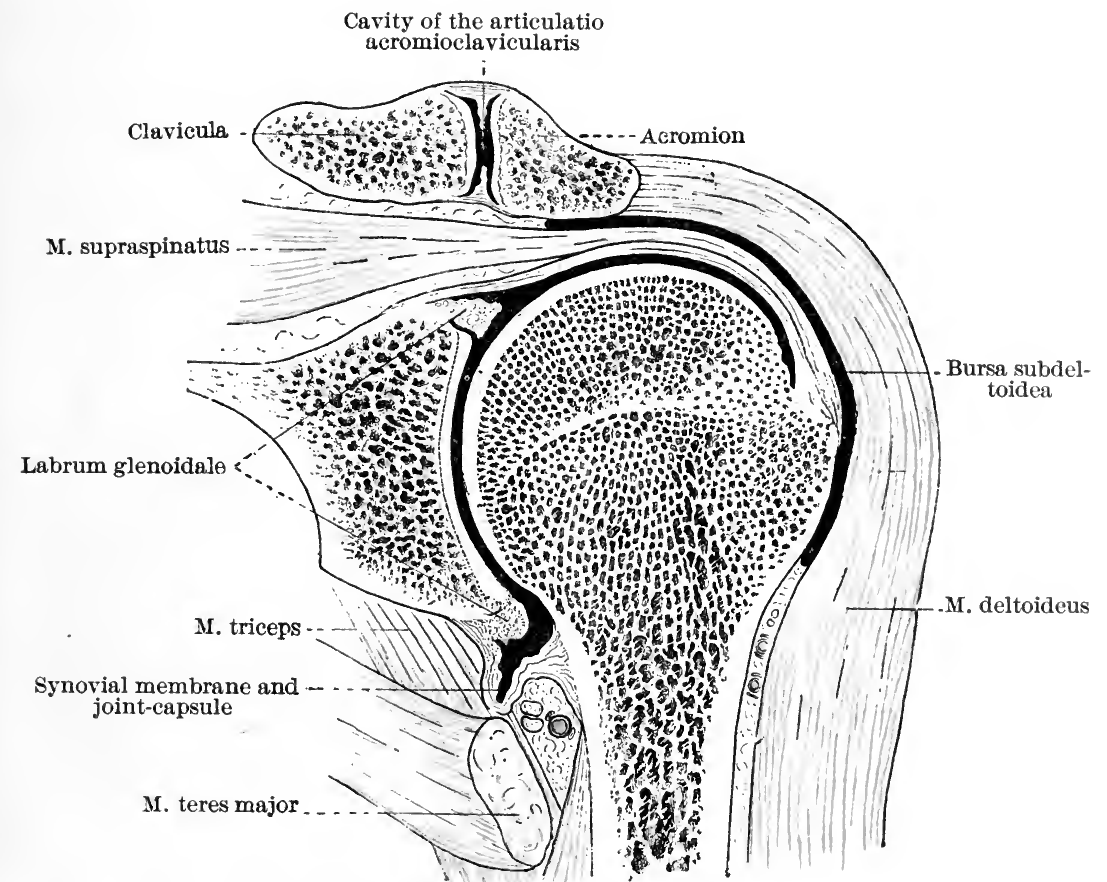

Articulatio humeri, frontal section passing through the tuberositas minor, the arm adducted. (From Poirier et Charpy, Traité d'Anat. hum., Paris, 1899, 2 ed., t. i. p. 624, Fig. 636.)

Clean the surfaces of the ligaments carefully. Study the following:

(a) Articular capsule (capsula articularis).

Note its cone shape, the summit attached to the labrum glenoidale, its base to the anatomical neck of the humerus. The attachment goes beyond the anatomical neck behind and below. Observe the variations in the thickness of the capsule in different parts.

(b) Coracohumeral ligament (ligamentum coracohumerale) (O. T. accessory ligament).

How is it attached medialward and lateralward? Note that it corresponds to the interval between the tendons of the M. supraspinatus and M. subseapularis, and so strengthens the capsule in a region otherwise feebly protected.

(c) Glenohumeral ligaments. (Cf. Fig. 27.)

Dissect off the posterior part of the eapsula articularis, pull the bones well apart, look into the joint from behind, 
and observe the following ligaments; they are very variable in their development.

(ca) Superior glenohumeral ligament (ligamentum glenohumerale superius) (O. T. coracobrachial ligament of Schlemm, supraglenosuprahumeral ligament of Farabœuf). Note that it with the coracohumeral ligament forms a gutter in which the long head of the M. biceps runs.

FIG. 27.

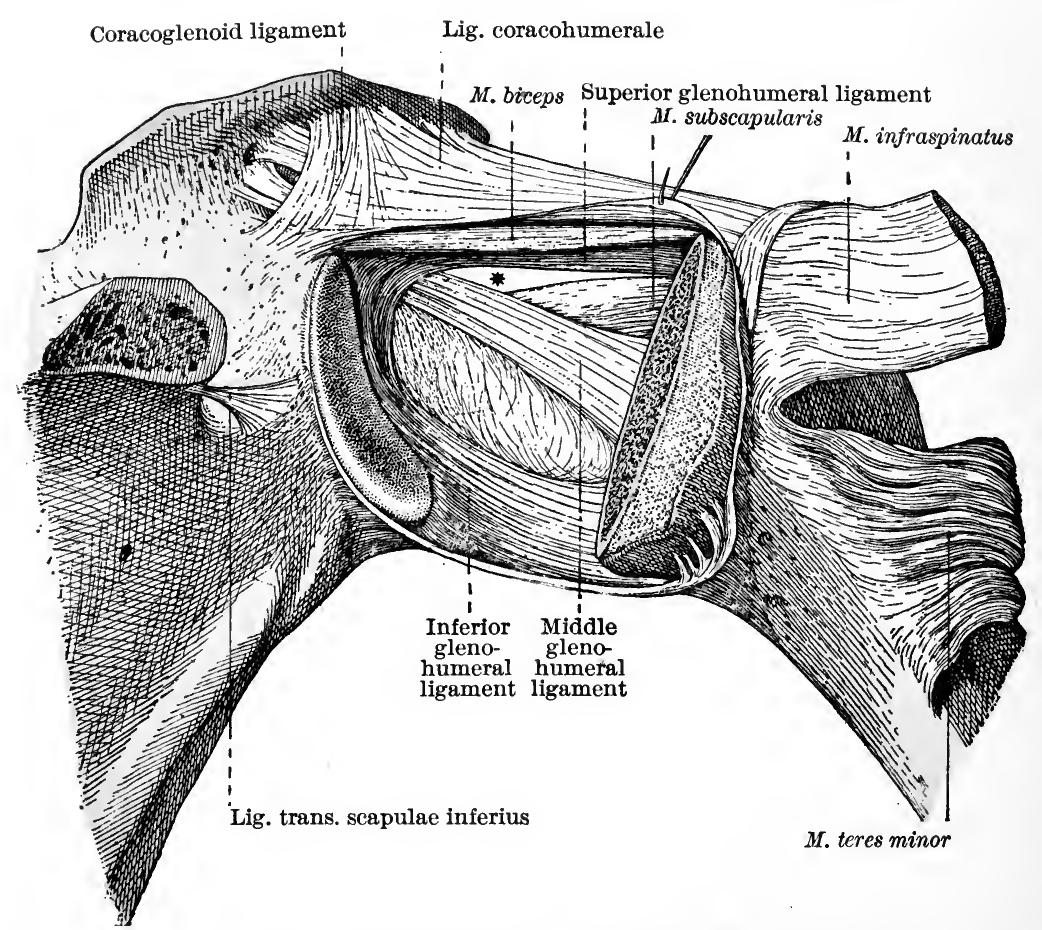

Articulatio humeri, posterior view (the posterior part of the capsule and the head of the humerus have been cut away to show the articular surface of the anterior part of the capsule). (From Poirier et Charpy, Traité d'Anat. hum., Paris, 1899, 2 ed., t. i. p. 628, Fig. 639.)

(cb) Middle glenohumeral ligament (ligamentum glenohumerale medium) ( $O$. T. Lig. glenoideobrachiale internum of Schlemm or supraglenoprehumeral ligament of Farabœuf).

Between $(c a)$ and $(c b)$ is the interstice known as the "foramen ovale" of Weitbrecht.

(cc) Inferior glenohumeral ligament (ligamentum glenohumerale inferius) (O. T. Lig. glenoideobrachiale inferius of Schlemm, pregleno-infrahumeral ligament of Farabœuf).

(d) Glenoid lip (labrum glenoidale) (O. T. glenoid ligament).

Divide the capsula articularis and the glenohumeral ligaments in front and pull the head of the humerus away from the glenoid cavity. Examine the labrum glenoidale. Note the relation to it of 
the long head of the M. biceps brachii above and of the long head of the M. triceps below.

(e) Long head of biceps muscle (caput longum M. bicipitis brachii).

Note its relations to the joint. Why is it of especial importance? Note its exact mode of origin. While in the joint, surrounded by the synovial membrane, it lies beneath a sort of inverted gutter formed by the superior glenohumeral ligament and by the posterior border of the coracohumeral ligament.

[In the horse the tendon is inserted into the external surface of the capsula articularis; in sheep it becomes invaginated in the joint-capsule, is immediately related to the synovial membrane, being swung by a meso of the latter; in higher animals and man it is free in the joint. All these phylogenetic stages are repeated in the ontogeny of the human embryo. (Cf. Welcker, H., Die Einwanderung der Bicepssehne in das Schultergelenk, Arch. $f$. Anat. u. Physiol., Anat. Abth., Leipzig, 1871, p. 20.)]

(f) Synovial membrane and its evaginations.

Note exact extent of synovial membrane. Observe the following constant evaginations:

( $f a$ ) Subscapular bursa, in the adult usually communicating with the M. subscapularis (already studied).

$(f b)$ Vagina mucosa intertubercularis (for tendon of long head of biceps muscle).

( $g$ ) Movements of the joint.

What muscles are concerned in the following movements?

1. Flexion.

2. Extension.

3. Abduction.

4. Adduction.

5. Circumduction.

6. Rotation.

What structures check excessive movements in the directions mentioned?

What agencies contribute to keeping the joint surfaces in contact,-i.e., to maintaining the integrity of the joint?

For the details of the anatomy of this and other joints, consult R. Fick, Handbuch der Anatomie und Mechanik der Gelenke (in Bardeleben's Handbuch), Jena, 1904.

\section{FOREARM AND HAND (ANTIBRACHIUM ET $M A N U S)$.}

\section{Superficial Fascia of Dorsum of Hand.}

Make incisions through the skin along the margo ulnaris and the margo radialis of the hand. Dissect off the skin of the back of the hand (dorsum manus), detaching the flap at the roots of the fingers. Make an incision along the middle line of the dorsum of each digit and reflect the skin radialward and ulnarward in each instance. Take no fat with the skin. 
Fig. 28.

N. cutaneus antibrachii dorsalis

Ramus ulnaris of the N. cutaneus antibrachii medialis

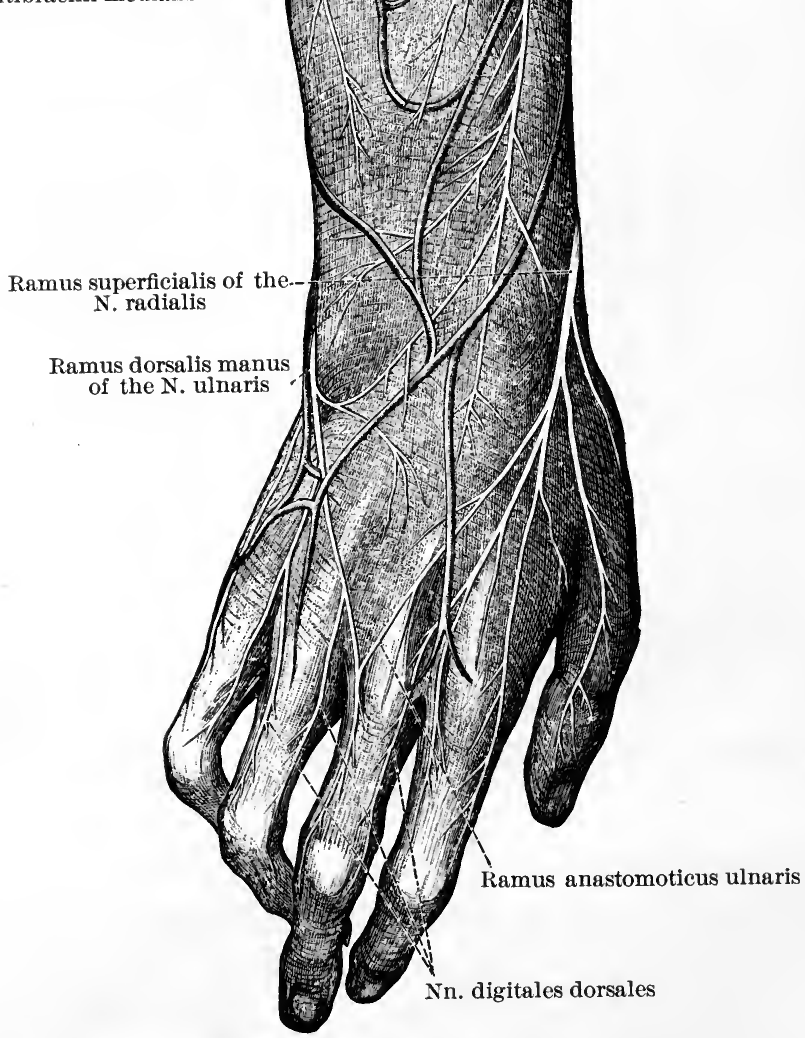

The cutaneous nerves of the dorsal surface of the forearm and hand. (After Toldt, Anat. Atlas, Wien, 1903, 3 Aufl., p. 831, Fig. 1265.) 


\section{In the superficial fascia find the following:}

Nerves. (Fig. 28.)

(a) Superficial ramus of radial nerve (ramus superficialis $N$. radialis)

(O. T. radial branch of musculospiral).

(aa) Ulnar anastomotic ramus (ramus anastomoticus ulnaris).

(ab) Dorsal digital nerves ( $N n$. digitales dorsales).

(b) Dorsal ramus of hand from N. ulnaris (ramus dorsalis manus $N$. ulnaris) (O. T. dorsal branch of ulnar nerve).

(ba) Dorsal digital nerves (Nn. digitales dorsales).

Study exact distribution.

FIG. 29.

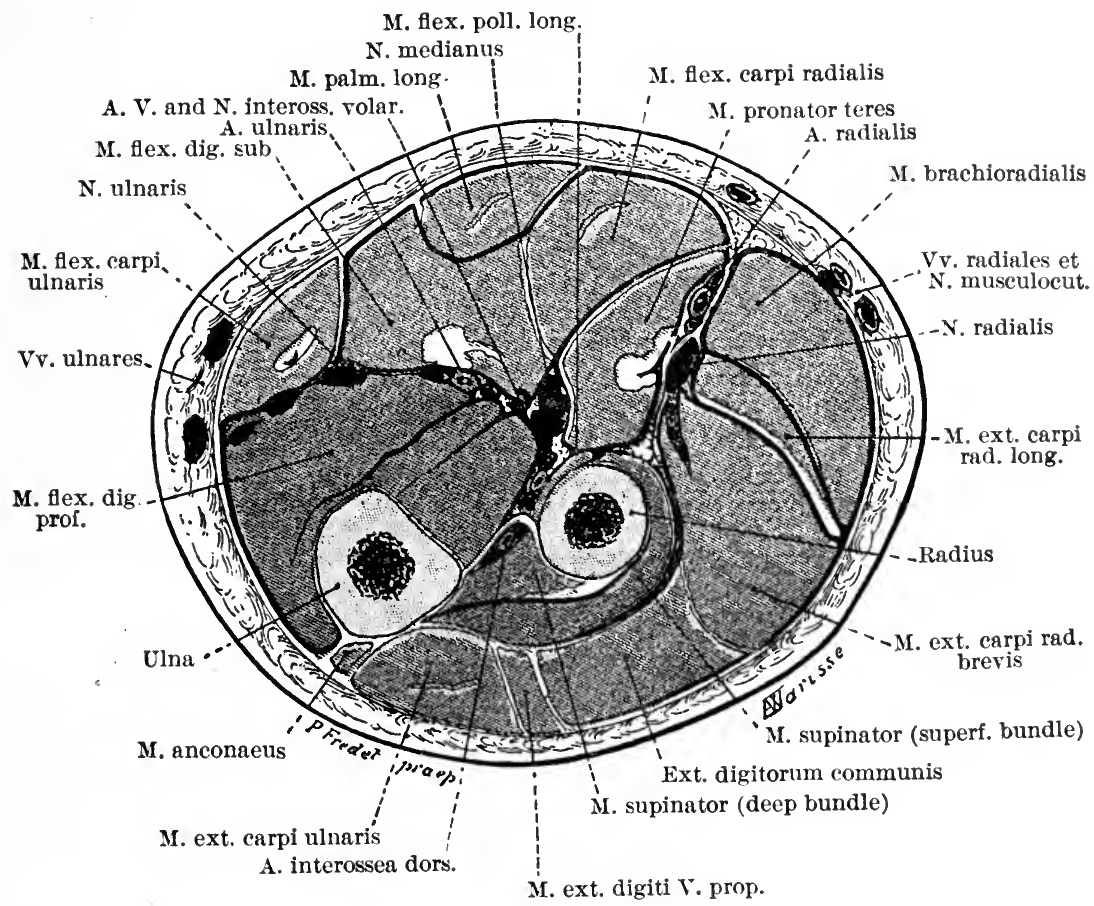

Section passing below the upper third of the forearm. Frozen subject; right forearm; segment distal to the cut. (P. Fredet.) (From Poirier et Charpy, Traité d'Anat. hum., Paris, 1901, 2 ed., t. ii. p. 166, Fig. 129.)

\section{Veins.}

(a) Venous network of back of hand (rete venosum dorsale manus). (aa) Dorsal metacarpal veins ( $V v$. metacarpeae dorsales).

(aaa) Digital venous arches (arcus venosi digitales). $(a a a a)$ Dorsal digital veins proper ( $V v$. digitales dorsales propriae).

Note the relation of these veins to the V. basilica and V. cephal- 
ica. Observe the communication of the deep veins with these superficial veins.

Arteries.

(a) Dorsal metacarpal and cutaneous rami of dorsal digital arteries (Aa. digitales dorsales, Aa. metacarpeae dorsales).

\section{Deep Fascia.}

Remove all remaining fat of superficial fascia, so as to clean carefully the deep fascia of the forearm and back of the hand.

(a) Deep fascia of forearm (fascia antibrachii).

How is it related to the fascia brachii? What becomes of it below? Note the relation of the lacertus fibrosus to this fascia. Look closely at the fibres composing the fascia and note their direction. What nerves and blood-vessels perforate the fascia on the volar and dorsal surfaces of the forearm? Besides those already mentioned find (1) ramus cutaneus palmaris $N$. ulnaris, (2) ramus palmaris N. mediani, and (3) ramus superficialis $N$. radialis. (See Fig. 24.)

(b) Deep fascia of back of hand (fascia dorsalis manus).

Is it as thick as the fascia antibrachii? Note its attachment proximalward to the ligamentum carpi dorsale and distalward to the fibrous sheaths of the extensor tendons. The deeper connections of the fascia may be studied later.

(ba) Dorsal ligament of the wrist (Lig. carpi dorsale) (O. T. posterior annular ligament). What are its relations proximalward and distalward? Ascertain exactly its attachments to the radius. What becomes of the ligament ulnarward?

\section{Radial Artery and its Relations in the Forearm (A. radialis).}

Turn aside the ramus volaris of the $N$. cutaneus antibrachii medialis, the N. cutaneus antibrachii lateralis, and the superficial veins. Dissect off the fascia antibrachii, but take care not to disturb the nerves going to the palm. Where muscle-fibres arise from its deep surface, do not remove the fascia. Note carefully the attachments of the fascia antibrachii in the depth. Dissect out the radial artery and its branches in the forearm, cleaning the adjacent muscles, but disturbing the relations as little as possible. Where does the artery begin? Note exact relations to various muscles in different parts of its course. Between what tendons does that portion of the artery usually palpated in taking the pulse lie? Note the relations of the radial artery to the radial veins ( $V v$. radiales) and to that part of the superficial ramus of the radial nerve beneath the fascia antibrachii. Study the origin and distribution of the following branches: 
FIG. 30.

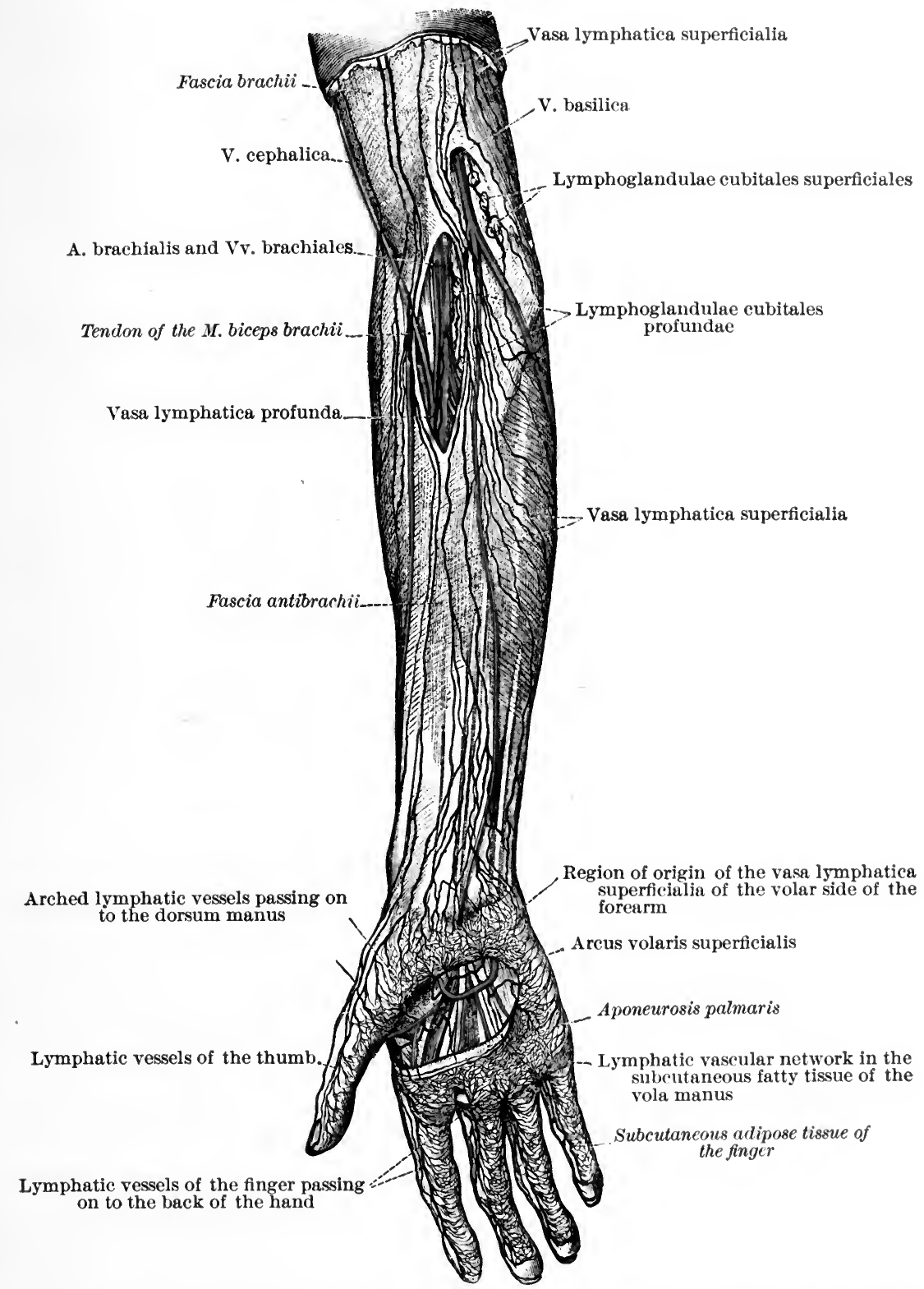

Lymphatic vessels of the volar surface of the forearm and hand. In front of the elbow the fascia has been divided to expose the deep lymphatic vessels and glands. (After Toldt, Anat. Atlas, Wien, 1900, 2 Aufl., p. 705, Fig. 1087.) 
(a) Radial recurrent artery (A. recurrens radialis).

(b) Superficial volar ramus (ramus volaris superficialis).

(c) Muscular rami (rami musculares).

(d) Volar carpal ramus (ramus carpeus volaris) (O. T. anterior radial earpal).

The other branches of the A. radialis are studied subsequently.

\section{Radial Nerve (N. radialis) (O. T. Musculospiral) in the Forearm.}

(a) Deep ramus (ramus profundus).

(aa) Museular rami (rami musculares).

Fig. 31.

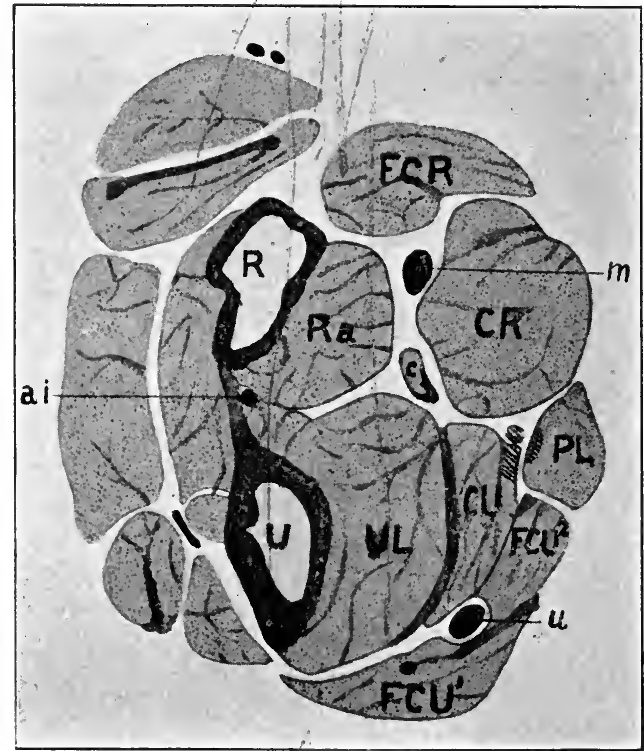

Transverse section through the forearm of the opossum.

$a i$, anterior interosseous nerve; $C, \mathrm{M}$. centralis; $C R, \mathrm{M}$. condyloradialis : $C U, \mathrm{M}$. condylo-ulnaris; $F C R$, M. flexor carpi radialis; $F C U^{1}, F C U^{2}$ lateral and medial portions of the $\mathrm{M}$. flexor carpi ulnaris; $m$, median nerve; $P L$, M. palmaris longus; $R$, radius; $R a$, M. radialis ; $U$, ulna ; $u$, ulnar nerve ; $U L$, M. ulnaris. The shaded areas represent the M. flexor sublimis digitorum. (After McMurrich, Amer. Jour. of Anat., Baltimore, 1903, vol. ii., No. 2, p. 196, Fig. 5.)

(b) Superficial ramus (ramus superficialis) (O. T. radial branch of musculospiral).

The branches of $(a)$ and $(b)$ are studied later.

\section{Superficial Muscles of Volar Surface and Ulnar Margin of Forearm.}

All the superficial muscles of the forearm should now be dissected out and cleaned. Avoid injury to the sheath of the flexor tendons in the lower part of the forearm (vagina tendinum $M m$. flexorum communium). Note that the tendon of the M. 
palmaris longus runs in front of the ligamentum carpi transversum (O. T. anterior annular ligament). The ulnar artery and nerve also run superficial to the ligamentum carpi transversum, though under cover of the more superficial ligamentum carpi volare. (Cf. Spalteholz, Figs. 362 and 363.) Study carefully the form, position, origin, and innervation of each of the following muscles:

(a) Brachioradial muscle (M. brachioradialis) (O. T. supinator longus). This will be studied more particularly later, when the back and radial margin of the forearm are dissected.

FIG. 32.

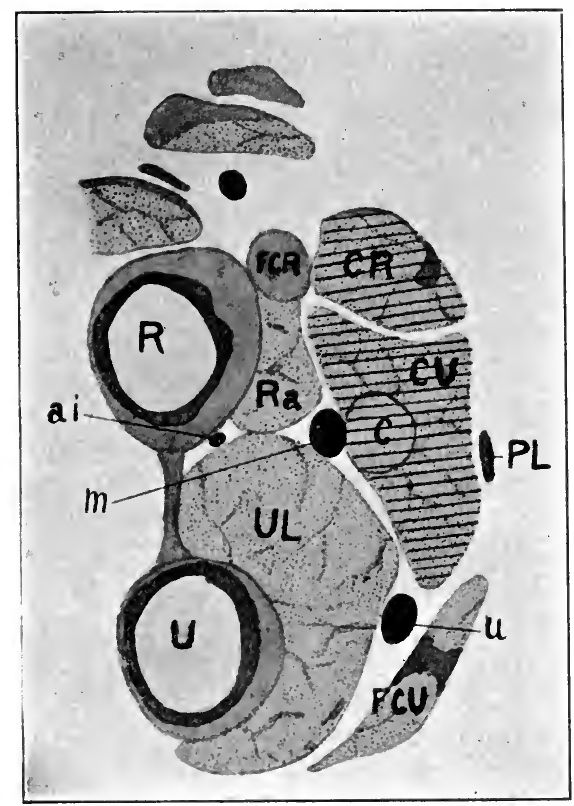

Transverse section through the forearm of a human embryo of $4.5 \mathrm{em}$.

$a i$, anterior interosseous nerve; $C, \mathbf{M}$. centralis; $C R, \mathbf{M}$. condyloradialis ; $C U$, M. condylo-ulnaris : $F C R$, M. flexor earpi radialis; $F C U$, M. flexor earpi ulnaris; $m$, median nerve; $P L$, M. palmaris longus ; $R$, radius; $R a$, M. radialis: $U$, ulna ; $u$, ulnar nerve; $U L$, M. ulnaris. The shaded areas represent the M. flexor sublimis digitorum. (After McMurrich, Amer. Jour. of Anat., Baltimore, 1903, vol. ii., No. 2, p. 200, Fig. 8.)

(b) Long palmar musele (M. palmaris longus).

(c) Round pronator muscle (M. pronator teres) (O. T. pronator radii teres).

(ca) Humeral head (caput humerale).

(cb) Ulnar head (caput ulnare) (O. T. coronoid head).

Note relation of ulnar head to median nerve and ulnar artery.

(d) Radial flexor muscle of wrist (M. flexor carpi radialis). 
(e) Ulnar flexor muscle of wrist (M. flexor carpi ulnaris).

(ea) Humeral head (caput humerale).

(eb) Ulnar head (caput ulnare).

What large nerve runs between these two heads?

(f) Superficial flexor muscle of the fingers (M. flexor digitorum sublimis).

(fa) Humeral head (caput humerale).

( $f b)$ Radial head (caput radiale).

Note space between these two heads for the passage of A. ulnaris and N. medianus.

(See study of these flexor muscles by Professor MeMurrich, of Ann Arbor, in the Amer. Jour. of Anat., Baltimore, vol. ii., No. 2.)

\section{Ulnar Blood-vessels and Ulnar and Median Nerves.}

These structures and their branches should next be carefully studied. Better to expose the ulnar artery, cut through the intermuscular septum between the M. flexor digitorum sublimis and the M. carpi ulnaris.

(a) Ulnar artery (A. ulnaris).

Study its origin, course, relations, and the following branches:

(aa) Recurrent ulnar arteries (Aa. recurrentes ulnares).

(ab) Museular rami (rami musculares).

(ac) Volar earpal ramus (ramus carpeus volaris) (O. T. anterior ulnar earpal).

(ad) Dorsal carpal ramus (ramus carpeus dorsalis) (O. T. posterior ulnar earpal).

(ae) Common interosseous artery (A. interossea communis).

(aea) Volar interosseous artery $(A$. interossea volaris $)(0$. T. anterior interosseous) (vide infra).

(aeb) Dorsal interosseous (A. interossea dorsalis) (O. T. posterior interosseous), to be studied later.

(b) Ulnar veins ( $V v$. ulnares).

(c) Ulnar nerve ( $N$. ulnaris).

(ca) Palmar cutaneous ramus (ramus cutaneus palmaris).

$(c b)$ Dorsal ramus of hand (ramus dorsalis manus) (O. T. dorsal cutaneous).

( $c b a)$ Dorsal digital nerves (Nn. digitales dorsales), already studied (vide supra).

(cc) Volar ramus of hand (ramus volaris manus) (branches in palm to be studied later).

(cd) Rami museulares to M. flexor earpi ulnaris and part of MI. flexor digitorum profundus.

(d) Median nerve ( $N$. medianus).

Reflect the humeral head of the M. pronator teres and the radial head of the M. flexor digitorum sublimis, and study the median nerve throughout its whole course in the forearm. Note its relation to the A. mediana. Examine-

(da) Muscular rami (rami musculares) to $\mathrm{Mm}$. pronator teres, flexor carpi radialis, palmaris longus, flexor sublimis digitorum,-i.e., to all muscles of superficial group except M. flexor carpi ulnaris. 
FIG. 33.

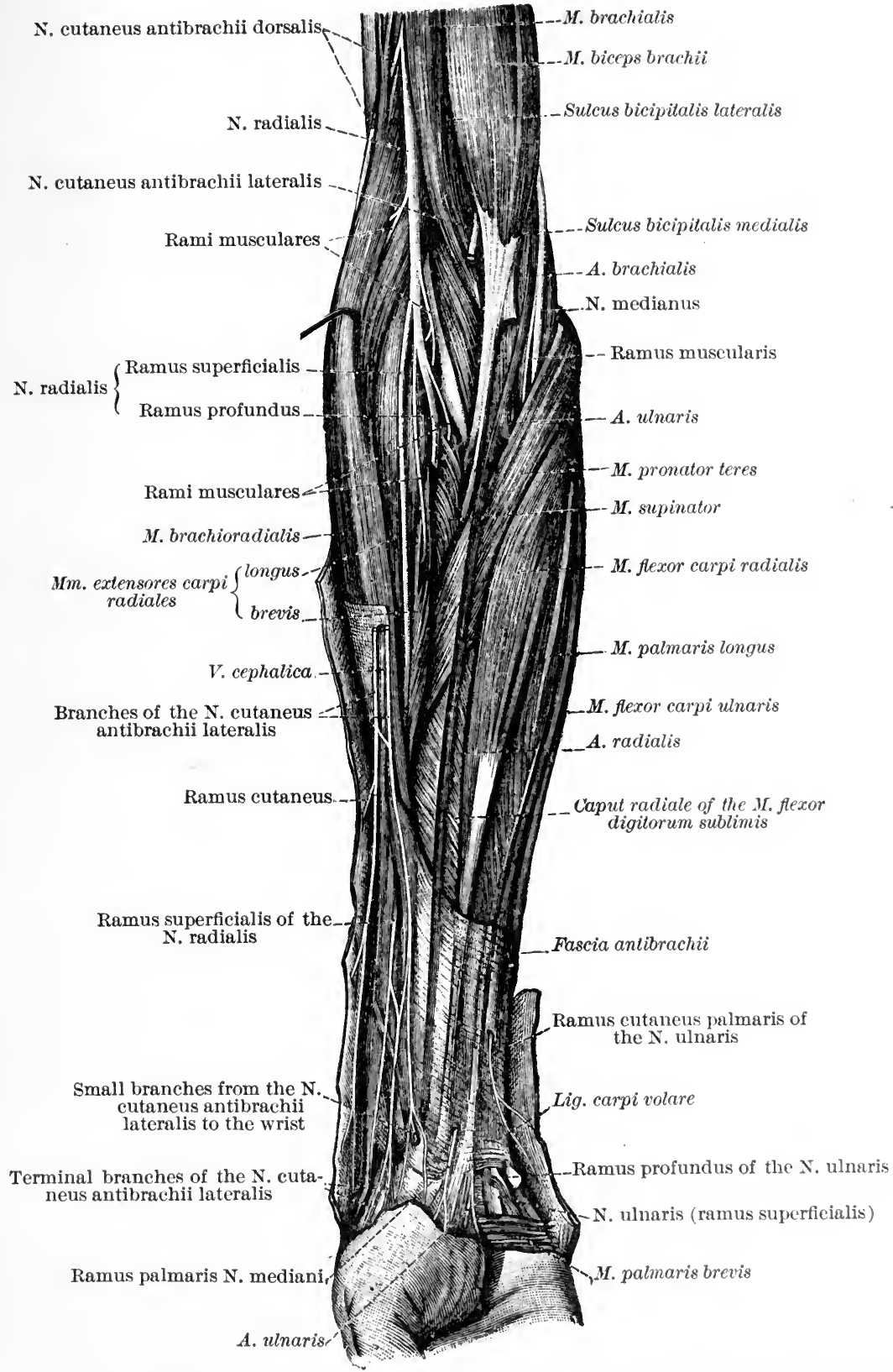

The deep nerves of the volar side of the forearm. (After Toldt, Anat. Atlas, Wien, 1903, 3 Aufl., p. 826 , Fig. 1258.) 
(db) Volar interosseous nerve of forearm ( $N$. interosseus antibrachii volaris) (O. T. anterior interosseous).

(dba) Rami musculares, to be studied a little further on.

(dc) Palmar ramus (ramus palmaris N. mediani) (O. T. median palmar cutaneous), already examined (vide supra).

\section{Deep Structures on Volar Surface of Forearm.}

The remaining structures on the volar surface are now to be dissected out and carefully cleaned.

Muscles.

(a) Deep flexor muscle of fingers (M. flexor digitorum profundus).

(b) Long flexor muscle of thumb (M. flexor pollicis longus).

(c) Quadrate (or square) pronator muscle (M. pronator quadratus).

Blood-vessels.

(a) Volar interosseous artery (A. interossea volaris) (O. T. anterior interosseous).

(aa) Median artery (A. mediana).

(ab) Muscular rami (rami musculares).

(b) Volar interosseous veins (Vv. interosseae volares). (Cf. Toldt, Fig. 1063.)

Volar interosseous nerve (N. interosseus [antibrachii] volaris) (O. T. anterior interosseous).

(a) Muscular rami (rami musculares) to the M. flexor digitorum profundus (lateral or radial part) and M. pronator quadratus.

\section{WRIST AND PALM (CARPUS ET VOLA MANUS $[P A L M A])$.}

Study the surface anatomy first, noting the thenar and hypothenar eminences. Palpate the bony prominences in the front of the wrist. To what is each due? Observe the three prominent furrows in the thick skin of the palm. Note also the transverse sulci on the palmar surfaces of the digits. In what relation do these stand to the metacarpophalangeal articulations and the finger-joints?

To reflect the skin make (1) a vertical incision along the middle line of the vola manus and (2) a transverse incision near the metacarpophalangeal junction from the margo radialis to the margo ulnaris of the hand. Reflect the flaps of skin ulnarward and radialward respectively, proceeding with caution. Do not reflect the ulnar flap quite to the ulnar margin of the hand until the insertion of the M. palmaris brevis into it has been made out. 


\section{Superficial Fascia of Palm.}

Note the subdivision of the fat into lobules. Study-

(a) The short palmar muscle ( $M$. palmaris brevis). After examination cut through its origin and reflect it ulnarward, avoiding injury to ulnar artery and nerve.

(b) Cutaneous nerves of palm.

(ba) Ramus palmaris N. mediani.

(bb) Ramus cutaneus palmaris $N$. ulnaris.

(bc) Ramus superficialis $N$. radialis.

FIG. 34.

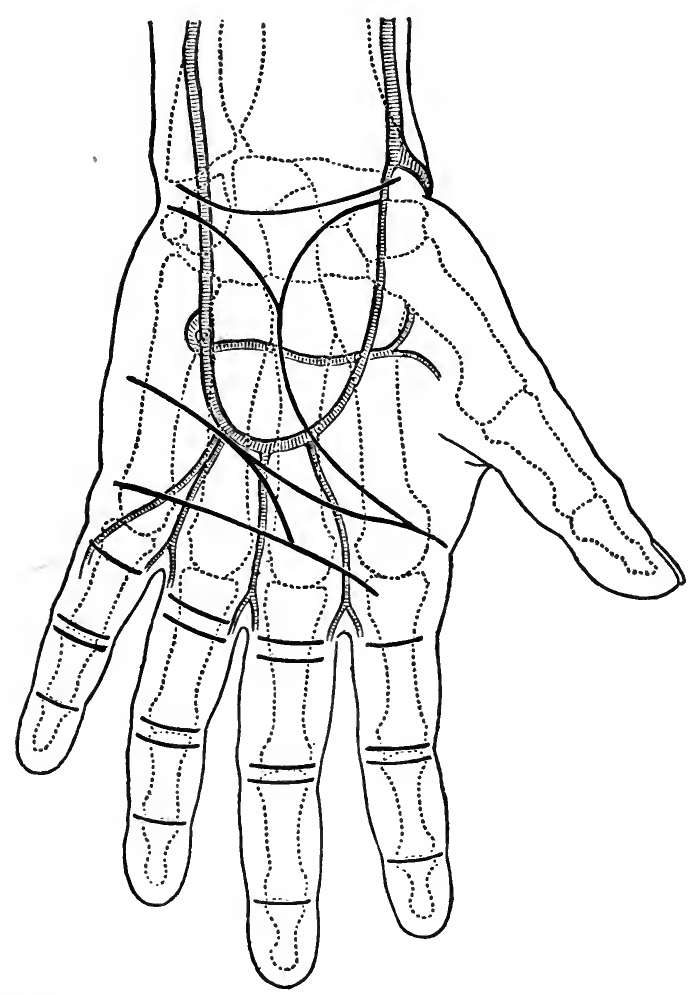

Surface markings of the palm of the hand. The thick black lines represent the chief creases in the skin. (After Treves, Surg. Ap. Anat., London, 1889, 4th ed., p. 260, Fig. 26.)

\section{Deep Fascia of Palm (Aponeurosis palmaris).}

Clean the surface of this and note its three parts.

(a) Radial or thenar aponeurosis.

(b) Middle or principal palmar aponeurosis.

(c) Ulnar or hypothenar aponeurosis.

$(a)$ and $(c)$ are relatively thin; $(b)$ is very thick. What is the shape of $(b)$ ? To what does the base of the triangle corre- 
spond? Note the distribution of the longitudinal and transverse fibres which constitute it. Examine carefully-

(d) Fasciculi transversi (O. T. transverse superficial ligament).

Between each two digital slips of the aponeurosis palmaris, note the vessels and nerves. Cut through the fasciculi transversi in order to expose them better. Observe the septa passing into the depth from the margins of $(b)$. What three compartments are thus formed and what do they contain?

\section{Superficial Volar Arch (Arcus volaris superficialis) (O. T. Superficial Palmar Arch) and Ulnar Artery.}

Cut through the narrow proximal part of the aponeurosis palmaris, reflect it distalward, and then remove it entirely. Remove the ligamentum carpi volare and dissect out the ulnar vessels and ramus volaris manus N. ulnaris in the palm. Dissect out also the N. medianus and its branches; avoid injury to the muscular rami near the distal margin of the ligamentum carpi transversum. To follow out the digital vessels and nerves, make an incision along the middle line of the volar surface of each digit and reflect the skin ulnarward and radialward.

How is the superficial volar arch formed? Study the ulnar artery and the superficial volar ramus of the radial artery. Where does the ulnar artery give off its deep volar ramus? On the skin of your own palm draw a line with a colored pencil corresponding to the position of the arcus volaris superficialis.

Branches :

(a) Common volar digital arteries (Aa. digitales volares communes) (O. T. palmar digital arteries).

(aa) Volar digital arteries proper (Aa. digitales volares propriae) (O. T. collateral digital arteries).

Note the point where the Aa. digitales volares communes receive the Aa. metacarpeae volares II.-IV. from the deep volar arch. Observe the relations of the digital arteries to the accompanying nerves; the relations of the proper digital differ from those of the common digital arteries.

\section{Median Nerve in Palm (N. medianus). (Fig. 35.)}

How does this nerve enter the palm? Note its relations to the ligamentum carpi transversum and to the mucous sheath of the flexor tendons. Study the following branches:

(a) Muscular rami (rami musculares) to M. abductor pollicis brevis, M. flexor pollicis brevis (caput superficialis), and M. opponens pollicis. 
(b) Common volar digital nerves ( $N n$. digitales volares communes).

Three from N. medianus.

The second supplies M. lumbricalis II.

The third gives off the ramus anastomoticus cum N. ulnari.

(ba) Proper volar digital nerves (Nn. digitales volares propriae)

(O. T. collateral digital nerves). Seven from N. medianus,-

Fig. 35.

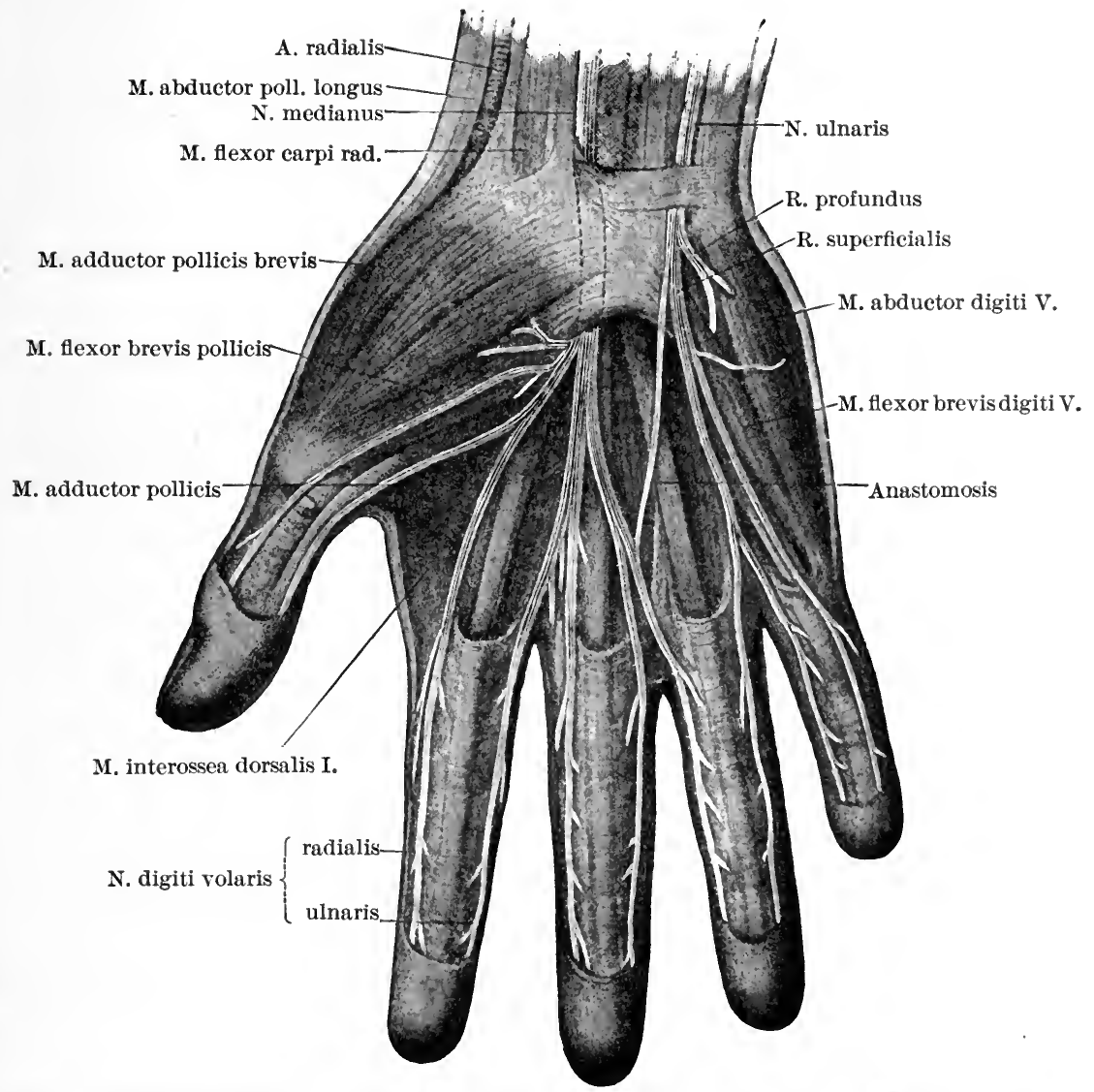

Nerves of the volar surface of the hand. (After Gegenbaur, Lehrb. der Anat. des Mensch., Leipzig, 1899, 7 Aufl., Bd. ii. S. 499, Fig. 660.)

I. and II. to skin of thumb;

III. to skin of radial side of index and to M. lumbricalis I.;

IV. to skin of ulnar side of index;

V. to skin of radial side of digitus medius;

VI. to skin of ulnar side of digitus medius;

VII. to skin of radial side of digitus annularis.

Work one of these out to its two terminals,--(1) volar, 
to the pulp, (2) dorsal, to the bed of the nail. On the terminals find the form of terminal corpuscle krown as the Pacinian corpuscle (corpuscula lamellosa [Vateri, Pacini]).

\section{Volar Ramus of Ulnar Nerve in Hand (Ramus volaris manus $\mathbf{N}$. ulnaris). (Fig. 35.)}

Note position of volar ramus of ulnar nerve in wrist (between ligamentum carpi volare and ligamentum carpi transversum). Observe branching as follows:

(a) Deep ramus (ramus profundus).

Note its disappearance between M. abductor digiti quinti and M. flexor digiti quinti brevis. It will be studied later.

(b) Superficial ramus (ramus superficialis).

(ba) Ramus muscularis to M. palmaris brevis.

(bb) Common volar digital nerve ( $N$. digitalis volaris communis).

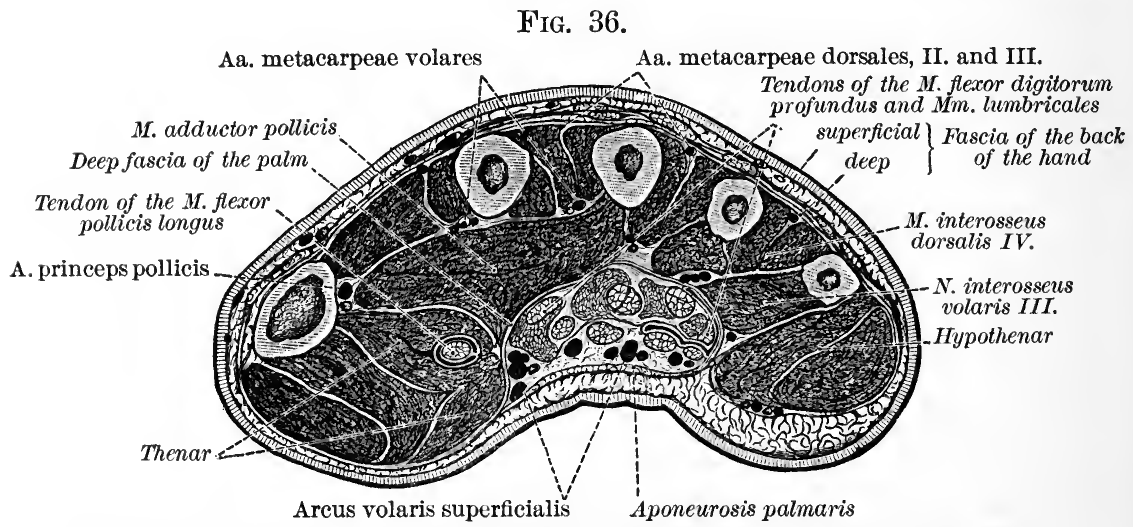

Cross-section of the palm of the hand through the bases of the metacarpal bones. Frozen section. Hand pronated. (After Toldt, Anat. Atlas, Wien, 1900, 2 Aufl., p. 621, Fig. 1000.)

1. Ramus anastomoticus cum N. mediani.

2. Rami cutanei to skin of palm.

3. Proper volar digital nerves $(N n$. digitales volares proprii),-

VIII. to ulnar side of ring-finger;

IX. to radial side of little finger.

(bc) Proper volar digital nerve ( $N$. digitalis volaris proprius),-

$\mathrm{X}$. to ulnar side of little finger.

Find the muscular rami given off by this nerve to the muscles of the hypothenar eminence.

Transverse Carpal Ligament (Ligamentum carpi transversum) ( $O$. T. Anterior Annular Ligament). (Cf. Spalteholz, Figs. $3^{63-}$ 365.)

Contrary to statements in many books, this ligament is not directly continuous either with the fascia antibrachii or with the 
aponeurosis palmaris. Note its attachments ulnarwayd and'o medialward. Study the relations of its deep and superficial surfaces. What is the canal beneath it called and what structures pass through this canal?

Flexor Tendons and their Mucous Sheaths. (Cf. Spalteholz, Fig. 366.)

(a) Mucous sheath of common flexor tendons (vagina [mucosa] tendinum $M m$. flexorum communium) (O. T. synovial sheath).

If uninjured by dissection thus far, insert a blow-pipe into sheath proximal to ligamentum carpi transversum and inflate. Study the exact relations to the flexor tendons in your cadaver and compare with text-book deseriptions. (Cf. Fig. 37.)

FIG. 37.

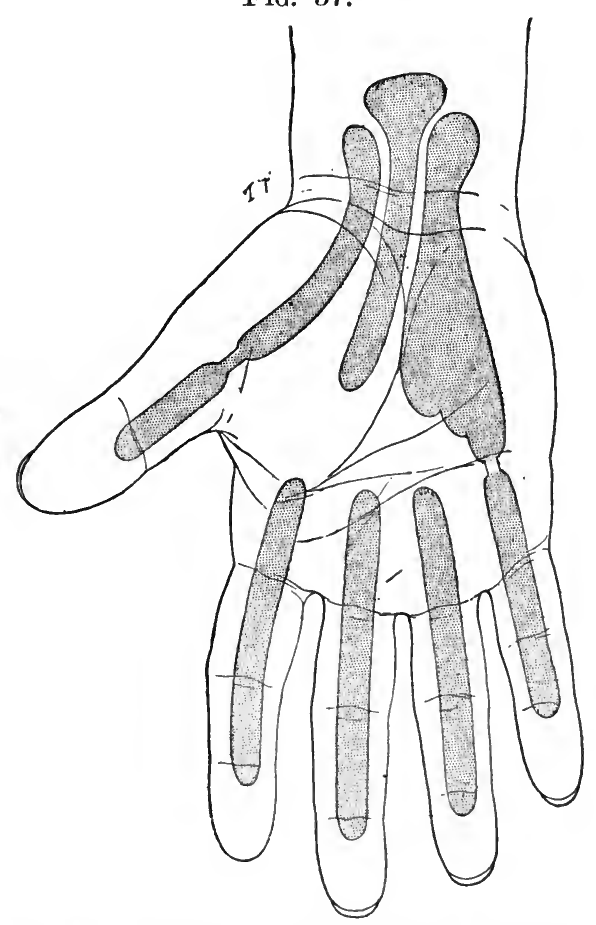

Palmar synovial sheaths (vaginae tendinum), normal adult type. (From Poirier et Charpy, Traité d'Anat. hum., Paris, 1901, 2 ed., t. ii. p. 190, Fig. 147.)

(b) Mucous sheath of tendon of long flexor of thumb (vagina tendinis $M$. flexoris pollicis longi).

(c) Digital sheaths of the tendons (vaginae tendinum digitales).

Note also the pairs of small folds extending between the first and second phalanges and the digital sheaths; these are the socalled "bands of union of the tendons" (vincula tendinum). They carry the blood-vessels to the tendons. 
Study the ligaments of the sheaths of the fingers (ligamenta vaginalia digitorum manus) and the accessory ligaments. Of the latter, examine (1) the ring-ligaments (ligamenta annularia digitorum manus) and (2) the cruciate ligaments (ligamenta cruciata digitorum manus). (Spalteholz, Fig. 363.)

Cut through these ligaments and open up the digital sheaths of the tendons. The vincula tendinum are well seen on raising the tendons from the phalanges.

(d) Flexor tendons and their insertions. (Fig. 38.)

Cut through the ligamentum carpi transversum by a vertical incision at its middle, thus opening the carpal canal (canalis carpi). Examine the arrangement of the flexor tendons and dissect each free from the vagina tendinum. Compare the arrangement found with the description in your systematic text-book. Note the relation of these tendons to the central compartment of the palm.

Examine carefully the insertions of the tendons of the various flexor muscles. Where and how are the tendons of the superficial flexor perforated by those of the deep flexor? What is meant by the chiasm of the tendons (chiasma tendinum)?

Fig. 38.

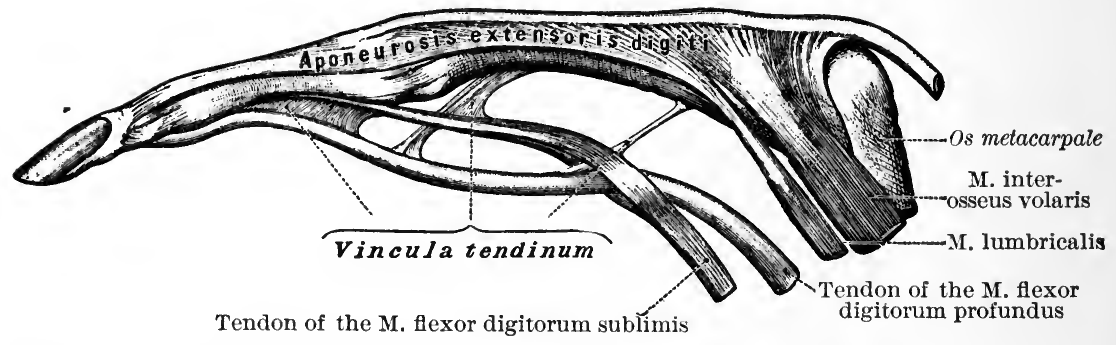

The terminal parts of the flexor and extensor tendons of the right middle finger, seen from the radial side. (After Toldt, Anat. Atlas, Wien, 1900, 2 Aufl., p. 323, Fig. 565.)

Follow the tendon of the M. flexor pollicis longus. Open the vagina tendinis and see how and where the tendon is inserted. What is its relation to the muscles of the thenar eminence?

\section{Lumbrical Muscles (Mm. lumbricales).}

Cut through the arcus volaris superficialis on the ulnar side distal from the deep volar ramus of the ulnar artery, and on the radial side at its junction with the superficial volar ramus of the radial artery. Reflect the arch distalward. Cut through the $\mathrm{N}$. medianus at the level of the wrist and reflect it radialward and distalward, noting again its branches to the muscles of the thenar eminence and the two (radial) Mm. lumbricales. Cut through the M. flexor digitorum sublimis at middle of forearm and reflect it and its tendons distalward as far as possible. Examine thoroughly the tendons of the M. flexor digitorum profundus and the 
Mm. lumbricales. Study origin, insertion, form, position, action, and innervation of each of the four lumbrical muscles. Divide the M. flexor digitorum profundus in its muscular part in the forearm and reflect it far distalward, along with the Mm. lumbricales. Take this opportunity to secure the branches of the ramus profundus of the ramus volaris manus of the N. ulnaris supplying the two (ulnar) lumbrical muscles.

\section{Short Muscles of Thumb and Little Finger.}

Study carefully the form, position, origin, insertion, action, and innervation of each of the following muscles :

(a) Short abductor muscle of thumb (M. abductor pollicis brevis) (O. T. abductor pollicis).

(b) Abductor muscle of little finger (M. abductor digiti quinti).

(c) Short flexor muscle of little finger ( $M$. flexor digiti quinti brevis).

(d) Opposing muscle of thumb (M. opponens pollicis).

(e) Short flexor muscle of thumb (M. flexor pollicis brevis).

(f) Adductor muscle of thumb (M. adductor pollicis).

(g) Opposing muscle of little finger ( $M$. opponens digiti quinti).

Deep Ramus of Volar Ramus of the Hand from the Ulnar Nerve ( $R$. profundus $R$. volaris manus N. ulnaris).

Study-

(a) Muscular rami (rami musculares) to all muscles of palm lying ulnarward from tendon of $M$. flexor longus pollicis (except two radial Mm. lumbricales).

Deep Volar Arch (Arcus volaris profundus) (O. T. Deep Palmar Arch). (See Fig. 34.)

How is it formed? (Cf. Spalteholz, Fig. 460.) Compare its convexity with that of the superficial volar arch. With a colored pencil indicate its position on your own palm. Study carefully its relation to neighboring muscles and nerves. Cut through the M. adductor pollicis at its origin and reflect it. Follow out carefully each of the four following branches:

(a) Volar metacarpal arteries (Aa. metacarpeae volares).

(aa) A. metacarpeae volaris $I$.

(aaa) Branch to thumb (O. T. arteria princeps pollicis).

$(a a b)$ Branch to radial side of index-finger (O. T. arteria radialis indicis).

(ab) A. metacarpea volaris II. (O. T. first palmar interosseous), forward between second and third phalanges to join $\mathrm{A}$. digitalis communis. 
(ac) A. metacarpea volaris III. (O. T. second palmar interosseous), forward between third and fourth phalanges to join $\mathrm{A}$. digitalis communis.

(ad) A. metacarpea volaris IV. (O. T. third palmar interosseous), forward between fourth and fifth phalanges to join $\mathrm{A}$. digitalis communis.

Note rami perforantes given off by each volar metacarpal artery.

Study the formation of the volar carpal network (rete carpi volare) (O. T. anterior carpal rete).

\section{DORSAL SURFACE AND RADIAL MARGIN OF FORE- ARM (FACIES DORSALIS ET MARGO RADIALIS ANTIBRACHII).}

The skin and superficial fascia with their nerves and bloodvessels have already been studied. The fat of the superficial fascia should now be completely removed and the deep fascia studied.

\section{Dorsal Portion of Deep Fascia of Forearm (Fascia antibrachii).}

How is it attached above and below? Note the relation of its deep surface to the extensor muscles. The dorsal ligament of the carpus (ligamentum carpi dorsale) (O. T. posterior annular ligament) is really a part of it.

\section{Superficial Muscles of Dorsum of Forearm.}

Dissect away the deep fascia on the dorsum of the forearm, with the exception of that portion of it near the elbow overlying and giving origin to muscles underneath. The ligamentum carpi dorsale should also be preserved. Isolate as far as possible and clean the surfaces of the following muscles:

(a) Brachioradial muscle ( $M$. brachioradialis) (O. T. supinator longus).

(b) Long radial extensor muscle of carpus ( $M$. extensor carpi radialis longus).

(c) Short radial extensor muscle of carpus ( $M$. extensor carpi radialis brevis).

(ca) Bursa M. extensoris carpi radialis brevis (Spalteholz, Fig. $360)$.

(d) Common extensor muscle of fingers ( $M$. extensor digitorum communis).

Study juncturae tendinum later.

(e) Proper extensor muscle of fifth finger ( $M$. extensor digiti quinti proprius) (O. T. extensor minimi digiti). 
(f) Elbow musele (M. anconaeus).

(g) Ulnar extensor muscle of earpus (M. extensor carpi ulnaris).

\section{Vessels and Nerves on Dorsum of Forearm.}

Divide the M. extensor digitorum communis and M. extensor digiti quinti proprius at about the middle of their fleshy bellies and reflect proximalward and distalward. Find the muscular rami from the ramus profundus $\mathrm{N}$. radialis innervating these muscles. Dissect out carefully-

(a) Dorsal interosseous artery (A. interossea dorsalis) (O. T. posterior interosseous artery). How does it arise? Follow its course and distribution.

(aa) Recurrent interosseous artery (A. interossea recurrens) ( $O$. $\mathrm{T}$. posterior interosseous recurrent).

(b) Arterial network about the elbow-joint (rete articulare cubiti).

Find the following tributaries: Above.

(ba) A. collateralis ulnaris superior.

(bb) A. collateralis ulnaris inferior.

(bc) A. collateralis media.

$(b d)$ A. collateralis radialis.

Below.

(be) A. recurrens ulnaris posterior.

(bf) A. interossea recurrens.

$(b g)$ A. recurrens radialis.

Ulnarward.

(bh) Transverse branch fed by A. collateralis ulnaris inferior.

(c) Terminal branch of volar interosseous artery (A. interossea volaris)

(O. T. anterior interosseous).

(d) Deep ramus of radial nerve (ramus profundus $N$. radialis).

(da) Museular branches (rami musculares).

$(d b)$ Dorsal interosseous nerve $(N$. interosseus [antibrachii] dorsalis) (O. T. posterior interosseous).

\section{Deep Muscles of Dorsum of Forearm.}

Clean and study carefully-

(a) Supinator muscle ( $M$. supinator) (O. T. supinator brevis).

(b) Long abductor muscle of thumb (M. abductor pollicis longus) (O. T. extensor ossis metacarpi pollicis).

(c) Short extensor muscle of thumb (M. extensor pollicis brevis) (O. T. extensor primi internodii pollicis).

(d) Long extensor muscle of thumb (M. extensor pollicis longus) (O. T. extensor secundi internodii pollicis).

(e) Proper extensor muscle of index-finger (M. extensor indicis proprius) (O. T. extensor indicis).

Whence does each get its nerve-supply? 


\section{DORSUM OF WRIST AND HAND.}

\section{Radial Artery and Veins.}

\section{Study-}

(a) Radial artery (A. radialis).

(aa) Dorsal earpal ramus (ramus carpeus dorsalis) (O. T. posterior radial carpal).

(ab) First dorsal metacarpal artery (A. metacarpea dorsalis I.). (aba) Aa. digitales dorsales.

(1) A. dorsalis pollicis ulnaris.

(2) A. dorsalis indicis.

(ac) Dorsal digital artery to radial side of thumb ( $A$. dorsalis pollicis radialis).

\section{Fig. 39.}

Tendon of the M. flexor digitorum sublimis $\quad$ Lig. carpi volare

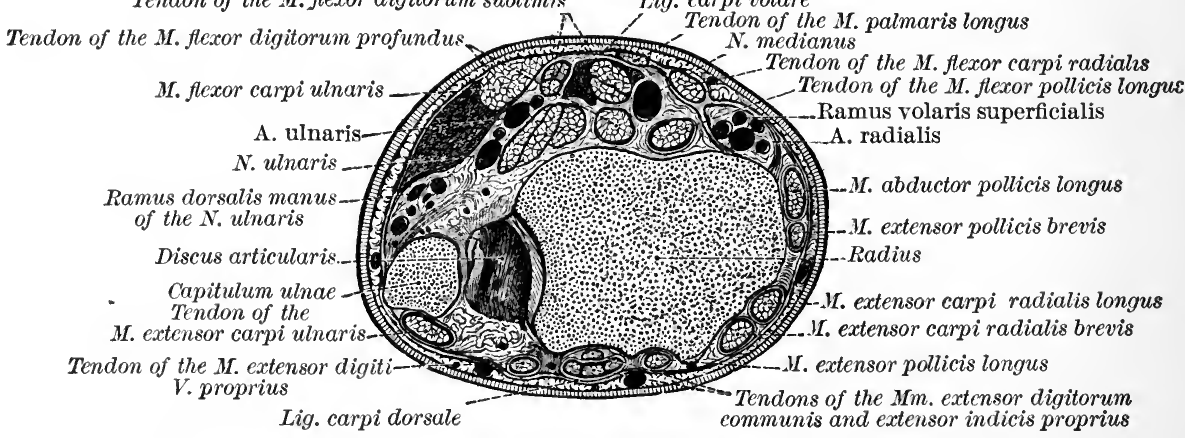

Cross-section of the distal extremity of the right forearm. Surface distal to the cut.

(After Toldt, Anat. Atlas, Wien, 1900, 2 Aufl., p. 621, Fig. 999.)

(ad) Dorsal carpal rete (rete carpi dorsale) (O. T. posterior carpal rete).

$(a d a)$ Dorsal metacarpal arteries (Aa. metacarpeae dorsales II., III., IV.) (O. T. dorsal interosseous arteries).

Note how each of these divides into two dorsal digital arteries (Aa. digitales dorsales) for adjacent margins of the second to the fifth finger.

Find the artery passing directly from the ulnar side of the rete carpi dorsale towards the ulnar margin of the little finger.

(b) Radial veins (Vv. radiales).

Dorsal Ligament of Wrist (Ligamentum carpi dorsale) (O. T. Posterior Annular Ligament).

How does it become attached radialward and ulnarward? How is the space beneath it subdivided? To what are the various septa attached? Open up the following six compartments and study their contents. 
First Compartment.

Tendons of M. abductor pollicis longus and M. extensor pollicis brevis.

Vagina tendinum Mm. abductoris longi et extensoris brevis pollicis.

Second Compartment.

Tendons of Mm. extensor carpi radialis longus et brevis.

Vagina tendinum Mm. extensorum carpi radialium.

Third Compartment.

Tendon of M. extensor pollicis longus.

Vagina tendinis M. extensoris pollicis longi.

Fourth Compartment.

Tendons of M. extensor digitorum communis and M. extensor indicis proprius.

Vagina tendinum $\mathrm{Mm}$. extensoris digitorum communis et extensoris indicis.

Fifth Compartment.

Tendon of M. extensor digiti quinti proprius.

Vagina tendinis M. extensoris digiti V.

Sixth Compartment.

Tendon of $\mathrm{M}$. extensor carpi ulnaris.

Vagina tendinis M. extensoris earpi ulnaris.

Note the variable extent of the vaginae tendinum into the dorsum manus. (Spalteholz, Fig. 369.)

Tendons of Extensor Muscles of Fingers. (Spalteholz, Fig. 36r; Poirier et Charpy, t. ii., Figs. Iog, Iro.)

Note the divergence of the tendons after leaving the osteofibrous canals at dorsum of wrist. At level of head of each metacarpal bone find fibrous expansion going from deep surface of tendon, becoming adherent to capsula articularis, and inserted into base of first phalanx. At this level note union of process of deep palmar fascia with lateral sides of tendon. Note aponeurotic expansion at sides of each tendon over first phalanx and find insertions of $\mathrm{Mm}$. interossei and $\mathrm{Mm}$. lumbricales into these. Observe division of tendon (reinforced by "dorsal expansion") into three tongues of fibrous tissue, the middle one becoming directly inserted into base of phalanx II.; the lateral tongues, stronger, go farther, become united over the second phalanx, and are inserted into the base of phalanx III. (ungual phalanx). Study the aponeurotic bridges (juncturae tendinum) uniting one tendon with its neighbor. Why is the independent movement of the ring-finger backward so limited?

In the thumb, the tendons of the two extensors are placed side by side at the level of the metacarpophalangeal joint and receive the same expansions of the palmar fascia and also interosseous muscles (represented here by the M. abductor pollicis brevis and the M. interosseus I.). 
Note the exact behavior of the tendons of the M. extensor indicis proprius and the M. extensor digiti quinti proprius at and near their terminations.

Dorsal Interosseous Nerve (N. Interosseus dorsalis) (O. T. Posterior Interosseous).

Find the terminal filament of this nerve. To what structures is it related? What joints are innervated by it?

Fig. 40.

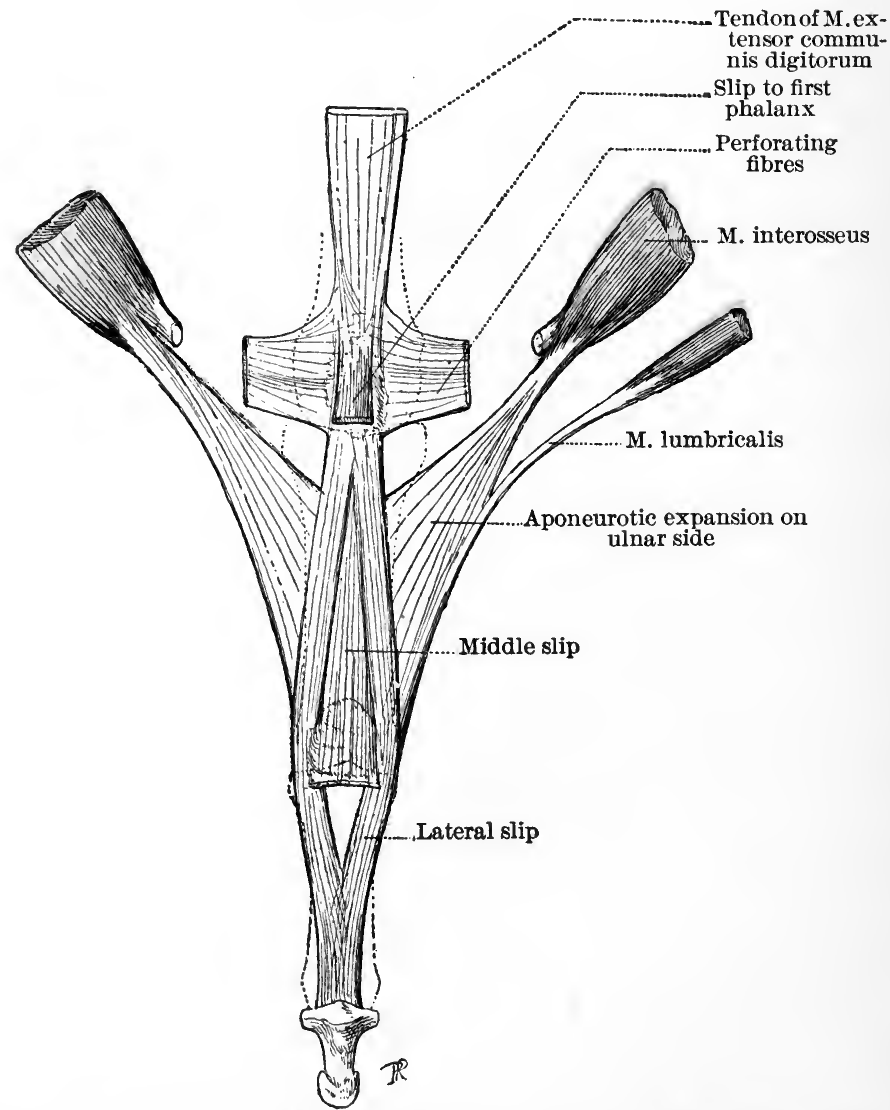

Tendons of the M. extensor communis digitorum: connections (deep surface). (From Poirier et Charpy, Traité d'Anat. hum., Paris, 1901, 2 ed., t. ii. p. 130, Fig. 109.)

Transverse Ligament of the Heads of the Metacarpal Bones and the Interosseous Muscles.

Place the volar surface of the hand upward and examine the transverse ligament of the heads of the metacarpal bones (liga- 
mentum capitulorum [ossium metacarpalium] transversum). Note that the Mm. lumbricales run volar and the Mm. interossei dorsal to this ligament.

Study the form, position, origin, insertion, action, and innervation of-

(a) Volar interosseous muscles (Mm. interossei volares I., II., III.).

(b) Dorsal interosseous muscles ( $\mathrm{Mm}$. interossei dorsales $\mathrm{I} \cdot-\mathrm{IV}$.).

Reflect the radial head of the M. interosseus dorsalis I. A good dorsal view of the deep head of the M. flexor pollicis brevis

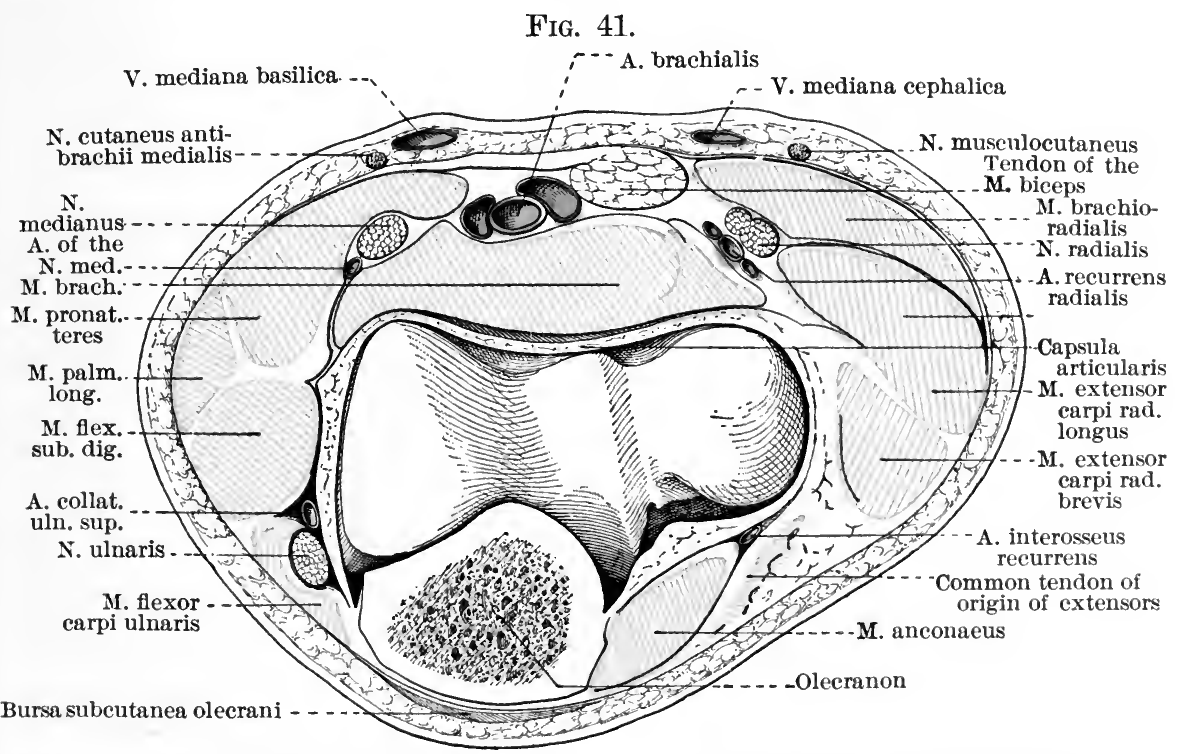

Transverse section of the left elbow (after Farabœuf). The distal end of the humerus is seen intact.

(From Poirier et Charpy, Traité d'Anat. hum., Paris, 1899, 2 ed., t. i. p. 615, Fig. 655.)

can now be obtained. How does this head arise? Where is it inserted? This part of the M. flexor pollicis brevis was designated by Henle the M. interosseus volaris primus.

The course of the tendon of the M. flexor radialis should now be followed and its exact insertion established.

\section{JOINTS.}

\section{Elbow-joint (Articulatio cubiti). (Figs. 4I, 42, and 43.)}

The muscles about the elbow may now be completely removed. Leave the M. supinator in position after removal of the others, in order that its exact origin, insertion, innervation, and mode 
of action may be made out. It may then be cut away also. What bones are concerned in the formation of the elbow-joint? Note that the articulatio cubiti really includes three articulations:

(a) Humero-ulnar articulation (articulatio humero-ulnaris);

(b) Humeroradial articulation (articulatio humeroradialis);

(c) Proximal radio-ulnar articulation (articulatio radio-ulnaris proximalis).

Joints $(a)$ and $(b)$ are studied at this stage; joint $(c)$, a little later.

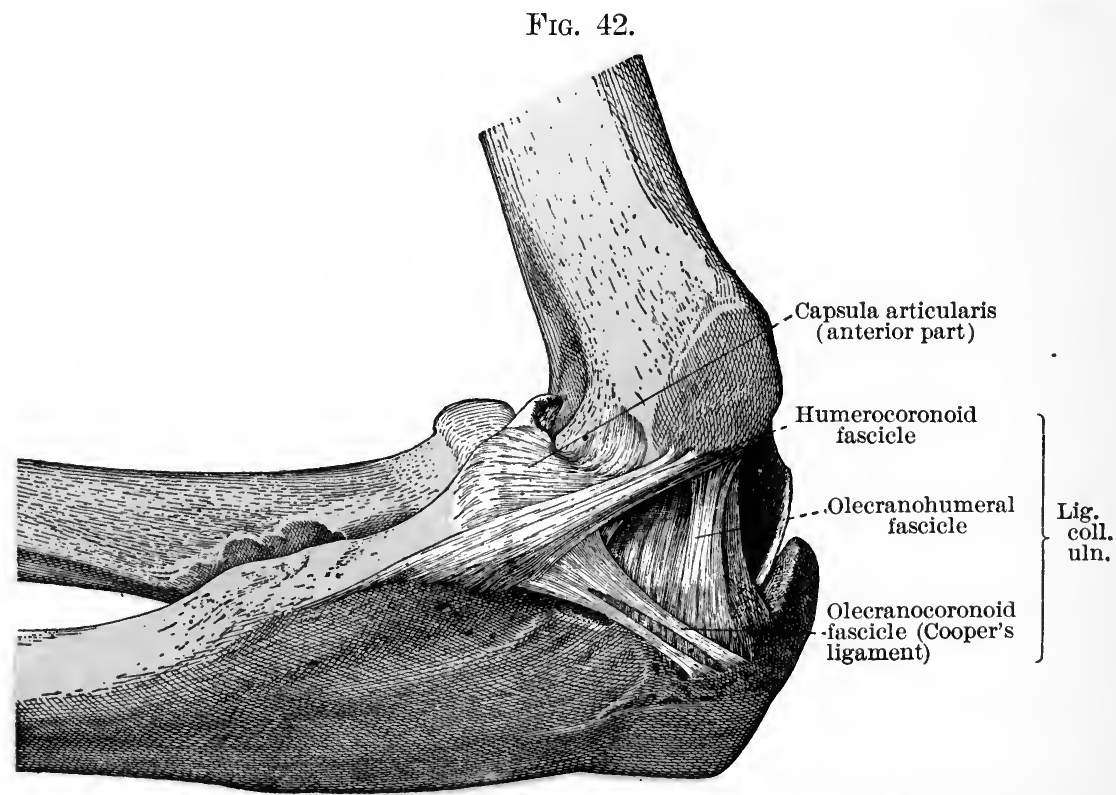

Articulatio cubiti. (From Poirier et Charpy, Traite d'Anat. hum., Paris, 1899, 2 ed., t. i. p. 641, Fig. 650.)

Study carefully the form, position, and attachments of each of the following ligaments :

(a) Joint-capsule (capsula articularis).

(aa) Ulnar collateral ligament (ligamentum collaterale ulnare) ( 0. $\mathrm{T}$. internal lateral ligament).

(ab) Radial collateral ligament (ligamentum collaterale radiale) (O. T. external lateral ligament).

Make a transverse cut across the anterior surface of the capsula articularis so as to open the joint. Examine the extent of the synovial membrane. Note the continuity of the cavities of the humero-ulnar and humeroradial articulations with that of the proximal radio-ulnar articulation, all forming one jointcavity, that of the articulatio cubiti. (See Fig. 43.) 
Study the possible movements of the humeroradial and humero-ulnar articulations.

\section{Joint of the Hand (Articulatio manus).}

Dissect away the whole of the ligamentum carpi transversum and the ligamentum carpi dorsale. Reflect the flexor and extensor tendons distalward, so as to leave the region of the wrist bare. Cut away the short muscles of the thenar and hypothenar eminences.

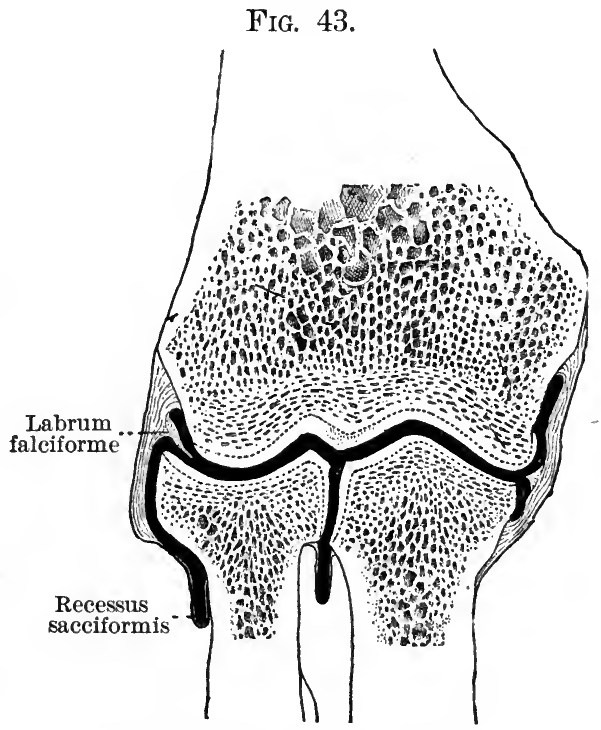

Articulatio cubiti, frontal section. (From Poirier et Charpy, Traité d'Anat. hum., Paris, 1899, 2 ed., t. 1. p. 644, Fig. 653.)

Note that the joint of the hand is divisible into two chief parts :

(a) Radiocarpal articulation (articulatio radiocarpea) (O. T. wristjoint).

(b) Intercarpal articulation (articulatio intercarpea) (O. T. carpal joints).

ad (a) What bony articular surfaces are concerned in the radiocarpal articulation? Study carefully the following:

(aa) Joint-capsule (capsula articularis).

$(a b)$ Strengthening ligaments:

(aba) Dorsal radiocarpal ligament (ligamentum radiocarpeum dorsale) (O. T. posterior ligament).

$(a b b)$ Volar radiocarpal ligament (ligamentum radiocarpeum volase $)$ (O. T. anterior ligament).

$(a b c)$ Ulnar collateral ligament of carpus (ligamentum 
collaterale carpi ulnare) ( $\mathrm{O} . \mathrm{T}$. internal lateral ligament).

$(a b d)$ Radial collateral ligament of carpus (ligamentum collaterale carpi radiale) ( $\mathrm{O} . \mathrm{T}$. external lateral ligament).

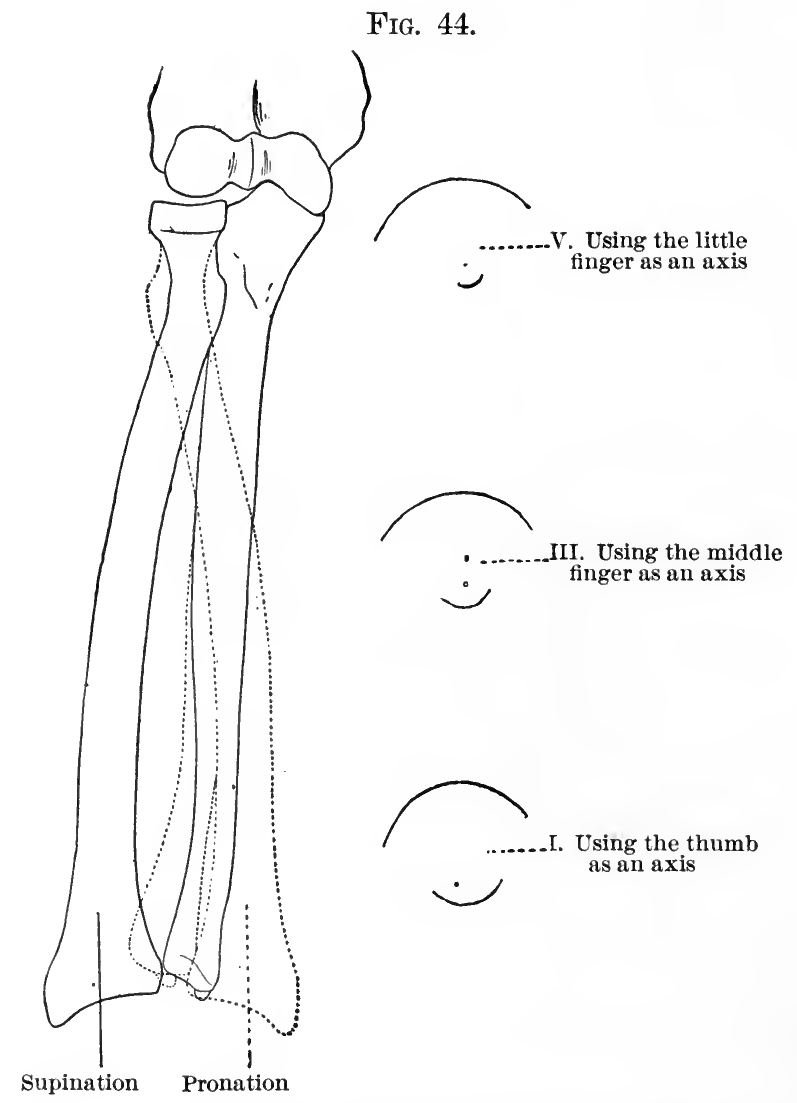

Schema of pronation and of supination.

On the right side of the figure are reproduced three tracings by the radius and the ulna in the movements of pronation and supination, taking as an axis the thumb $\left(I_{\text {. }}\right)$, the middle finger $(I I I$.$) , and$ the little finger $\left(V_{*}\right)$. (The larger curve belongs to the radius, the smaller to the ulna.) (From Poirier et Charpy, Traité d'Anat. hum., Paris, 1899, 2 ed., t. i. p. 658, Fig. 665.)

(b) What bony articular surfaces are concerned in the intercarpal articulation? Study carefully the following:

(ba) Joint-capsules (capsulae articulares).

(bb) Strengthening ligaments :

(bba) Radiate ligament of carpus (ligamentum carpi radiatum).

(bbb) Dorsal intercarpal ligaments (Ligg. intercarpea dorsalia). 
(bbc) Volar interearpal ligaments (Ligg. intercarpea volaria) (O. T. palmar intercarpal).

$(b b d)$ Interosseous intercarpal ligaments (Ligg. intercarpea interossea).

To examine these ligaments carefully the ulnar and radial collateral ligaments of the carpus and the dorsal intercarpal ligaments should be cut through.

In this connection study also the articulation of the pisiform bone (articulatio ossis pisiformis), examining the following:

FIG. 45.

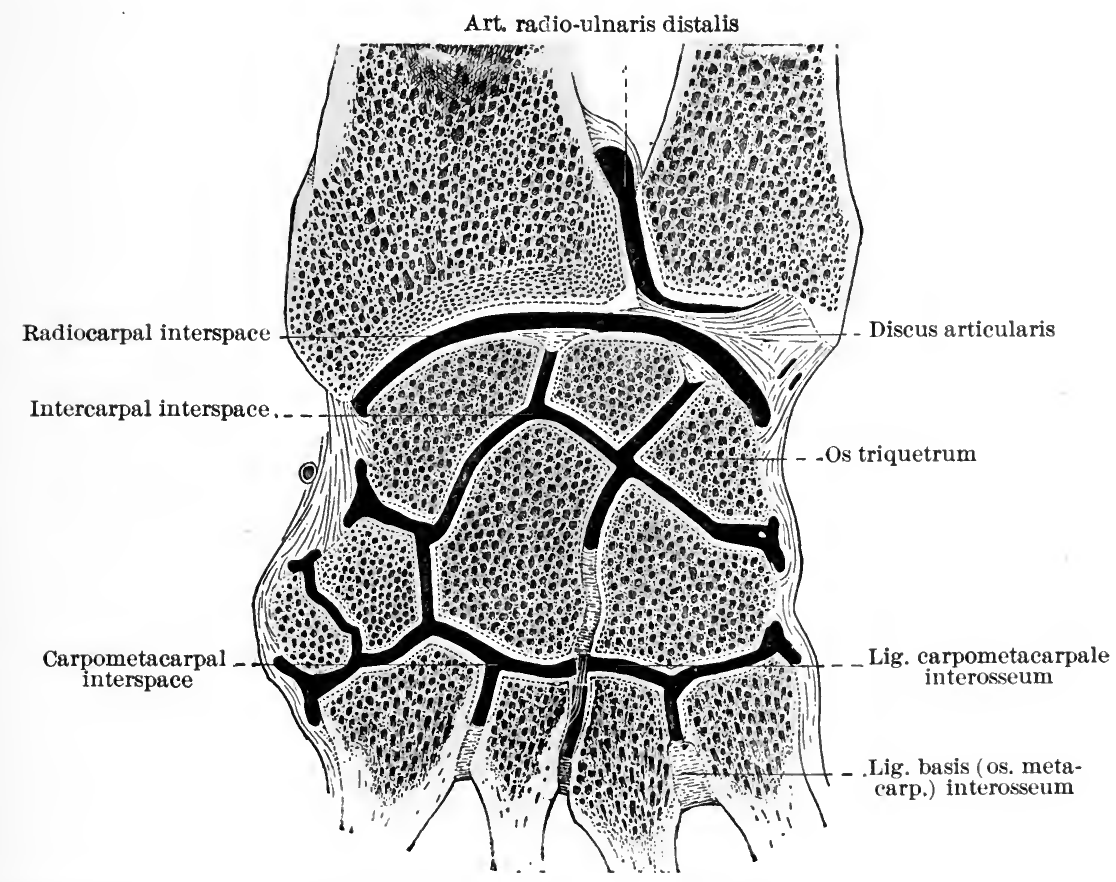

Frontal section of the radiocarpal, carpal, and carpometacarpal joints. (From Poirier et Charpy,

Traité d'Anat. hum., Paris, 1899, 2 ed., t. i. p. 660, Fig. 667.)

(a) Joint-capsule (capsula articularis).

(b) Strengthening ligaments:

(ba) Ligamentum pisohamatum.

(bb) Ligamentum pisometacarpeum.

How are these related to the tendon of the M. flexor carpi ulnaris, and of what importance are they?

Of what movements is the hand capable at the radiocarpal articulation? In how far are these movements favored or increased by means of the intercarpal articulation? What muscles 
are concerned in flexion, extension, abduction, and adduction of the hand? Of what especial advantage are the multiple bones and joints of the carpus?

Radio-ulnar Joints. (See Figs. 44, 45, and 46.)

These joints consist of-

(a) Proximal radio-ulnar articulation (articulatio radio-ulnaris proximalis) (0. T. superior radio-ulnar).

FIG. 46.

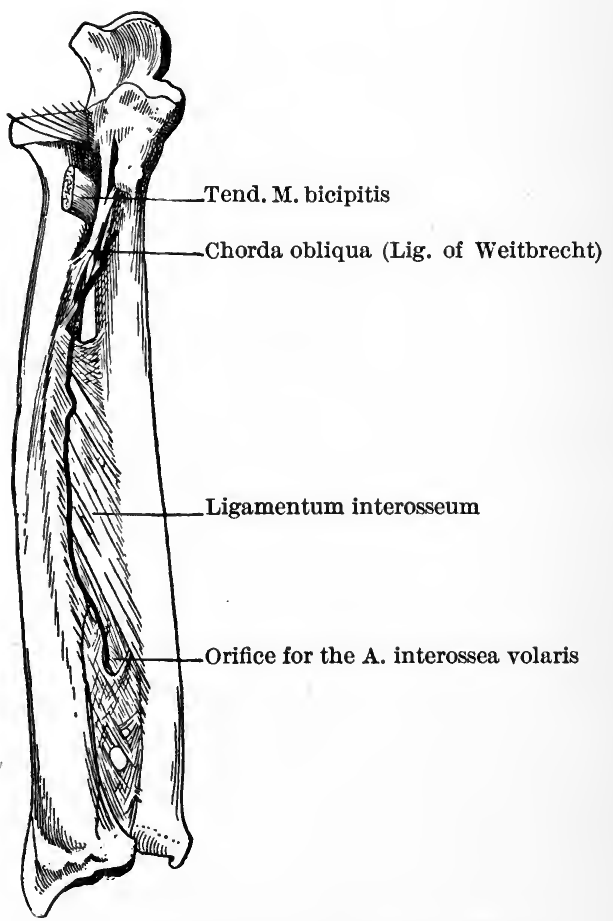

The interosseous membrane of the forearm, volar surface. (After Poirier et Charpy, Traité d'Anat. hum., Paris, 1899, t. i. p. 655, Fig. 664.)

(b) Distal radio-ulnar articulation (articulatio radio-ulnaris distalis) ( 0. $\mathrm{T}$. inferior radio-ulnar).

(c) Interosseous membrane and oblique cord (membrana interossea antibrachii et chorda obliqua).

To expose the ligaments, the muscles must be completely removed from the dorsal and volar surfaces of the forearm.

(a) Proximal radio-ulnar joint.

What bony surfaces are concerned? Study carefully the annular 
ligament of the radius (ligamentum annulare radii) (O. T. orbicu-

lar ligament) and the sacciform recess (recessus sacciformis).

(b) Distal radio-ulnar joint.

What bony surfaces are concerned? Study carefully-

(ba) Joint-capsule (capsula articularis).

(bb) Articular disk (discus articularis) (O. T. triangular fibrocartilage).

(bc) Saccular recess (recessus sacciformis).

(c) Interosseous membrane and oblique cord.

Study exact form and attachments. What vessels and nerves are related to the interosseous membrane?

In what movements are the radio-ulnar articulations concerned? Study carefully the movements of pronation and supination $(a)$ when the forearm is extended, $(b)$ when it is flexed. What muscles are concerned in these movements?

Saw through the ulna at the junction of its distal and middle thirds; cut through the membrana interossea. Draw the distal fragment of the ulna medialward; open the recessus sacciformis. The proximal surface of the discus articularis can be seen and its attachments carefully examined.

\section{Carpometacarpal Joints (Articulationes carpometacarpeae).}

How many of these are there? What bony surfaces are concerned in their formation? In order to examine these joints thoroughly, remove the $\mathrm{Mm}$. interossei from the metacarpal bones and detach the tendons of the flexor muscles and the Mm. lumbricales from the fingers. Do not disturb the extensor tendons at present. Clean the carpometacarpal and the intermetacarpal ligaments carefully. Study in the four ulnar carpometacarpal articulations the following:

(a) Joint-capsules (capsulae articulares).

(b) Strengthening ligaments:

(ba) Dorsal carpometacarpal ligaments (ligamenta carpometacarpea dorsalia).

(bb) Volar carpometacarpal ligaments (ligamenta carpometacarpea volaria).

Note the special carpometacarpal joint for the thumb (articulatio carpometacarpea pollicis). Study its capsula articularis.

Intermetacarpal Joints (Articulationes intermetacarpeae).

These joints are amphiarthroses. What is an amphiarthrosis? Study-

(a) Joint-capsules (capsulae articulares).

(b) Strengthening ligaments: 
(ba) Dorsal ligaments of basal extremities of metacarpal bones (Ligg. basium [ossium metacarpalium] dorsalia).

$(b b)$ Volar ligaments of basal extremities of metacarpal bones (Ligg. basium [oss. metacarp.] volaria).

(bc) Interosseous ligaments of basal extremities of metacarpal bones (Ligg. basium [oss. metacarp.] interossea). These fill up the spatia interossea metacarpi and can be studied to better advantage later, when the bases of the metacarpal bones are separated from one another.

Before leaving the joints of the carpus and metacarpus, the synovial membranes and articular surfaces of the joint-cavities should be carefully reviewed. (See Fig. 45.) Which joint-cavities are independent? Which communicate? Detach the metacarpus from the carpus and examine the articular surfaces. Then separate the various bones of the carpus and metacarpus from one another in order to see the extent of the various interosseous ligaments and articular surfaces.

Study the possible movements in the various joints under examination. What muscles are especially concerned in the various movements?

\section{Metacarpophalangeal Joints (Articulationes metacarpophalangeae).}

These five joints are arthrodiae. What is an arthrodia? Study-

(a) Joint-capsules (capsulae articulares).

(b) Strengthening ligaments:

(ba) Collateral ligaments (ligamenta collateralia), one radial and one ulnar for each joint.

(bb) Volar accessory ligaments (ligamenta accessoria volaria) ( 0. T. palmar ligaments).

(c) Transverse ligaments of the heads of the metacarpal bones (Ligg. capitulorum [oss. metacarpalium] transversa). These have already been examined (vide supra).

Raise the extensor tendon from the dorsal aspect of each joint and note the absence of dorsal ligaments, their place being taken by the extensor tendon.

Of what movements is each metacarpophalangeal joint capable? What are the muscles concerned in each case for the different joints?

\section{Joints of the Fingers (Articulationes digitorum manus).}

Note that the thumb has one, the other digits two ginglymi. What is a ginglymus? Study- 
(a) Joint-capsule (capsula articularis).

(b) Strengthening ligaments (ligamenta collateralia), one ulnar, one radial $(O . T$. lateral ligaments).

What muscles are concerned in flexion and in extension of each of these joints?

Review the bones of the upper extremity. 



\section{Part II}

DISSECTION OF THE LOWER EXTREMITY 



\section{LOWER EXTREMITY}

\section{Introductory.}

On the surfaces of the lower extremity mark out the regions (regiones extremitatis inferioris). Make four drawings indicating these, one anterior, one posterior, one medial, and one lateral. Make use of Figs. 1 and 2 (pp. 43 and 44).

In the thigh (femur) observe the general cylindrical shape, tapering distalward, the prominence in the middle line of the thigh in front due to the M. rectus femoris, and medial from this another due to the M. vastus medialis, extending distalward to the knee-cap. Note the direction of the streams of hairs (flumina pilorum).

At the knee (genu) observe the prominent knee-cap (patella). In the leg (crus) note the general shape and the lateral and medial prominences near the ankle (malleoli lateralis et medialis).

In the foot (pes) observe the heel (calx) behind, constituting part of the ankle (tarsus); anteriorly, the toes (digiti); and between the ankle and the toes, the metatarsus. Among the digits distinguish-

(a) First or great toe (digitus primus, or hallux).

(b) Second toe (digitus secundus).

(c) Third toe (digitus tertius).

(d) Fourth toe (digitus quartus).

(e) Fifth or little toe (digitus quintus).

How many phalanges has each digit? Examine the nails (ungues), and distinguish (a) root of nail (radix unguis), (b) free margin (margo liber), (c) covered margin (margo occultus), (d) semilunar white area (lunula).

On the lateral surface of the lower extremity (facies lateralis) observe the hip (coxa) and thigh (femur), with the iliac crest (crista iliaca) above. Note the general configuration; observe the depression over the trochanter major at the upper extremity of the thigh-bone. On the lateral surface of the knee observe the prominent fold of skin covering the tendon of the biceps muscle, and anterior to this the fold covering the iliotibial band of the fascia. At the ankle the lateral malleolus (malleolus lateralis) is prominent, with a depression (fossa retromalleo- 
laris) behind it. In the foot note that the lateral margin (margo lateralis) rests on the floor when the body is erect.

Examine the posterior surface (facies posterior) of the lower extremity. Observe the prominence of the buttocks (clunes or nates), separated from each other by the crena ani and from the thigh by the gluteal fold (sulcus glutaeus); behind the knee is the popliteal fossa (fossa poplitea). Examine its boundaries and extent. In the leg note the prominent calf (sura), and lower down the prominence due to the tendon of the calf muscles. In the sole of the foot (planta) observe the grooves in the skin (sulci cutis) and the ridges (cristae cutis) between. Observe the arch of the foot: it is higher on the medial than on the lateral margin. Note the ball of the great toe and the eminence of the little toe.

On the medial surface (facies medialis) of the lower extremity, observe the prominence due to the M. vastus medialis in the thigh, that due to the malleolus medialis at the ankle, and the curved medial margin of the foot (margo medialis pedis).

After inspection the student should resort to palpation of these parts. In addition to what has been mentioned above, note the groove at the groin (sulcus inguinalis) and the depression in the subinguinal region corresponding to a deeper triangle (fossa Scarpae major, trigonum femorale) (O. T. Scarpa's triangle). Can you feel the superficial lymph-glands (lymphoglandulae subinguinales superficiales)? Note the mobility of the skin over the patella, due to a bursa (bursa praepatellaris subcutanea). Feel the ligament of the patella (ligamentum patellae) and note the mobility of the skin over it (bursa infrapatellaris subcutanea). Flex and extend the knee-joint. What are the limits of movement? Feel the line of the joint during the movement. Palpate the condyles of the femur and of the tibia. Locate the tuberosity of the tibia and note the mobility of the skin over it (bursa subcutanea tuberositatis tibiae). Feel the anterior crest of the tibia. How much of it is immediately subcutaneous? Feel the medial surface of the tibia and the tendons of the extensor muscles of the foot. In the front of the foot find the rounded bony prominence of the anterior end of the heelbone (calcaneus), three or four centimetres anterior to the malleolus medialis. Just anterior to this note the depression indicating the position of Chopart's transverse joint of the ankle (articulatio tarsi transversa [Choparti]).

Run the finger along the iliac crest. Feel the sciatic tuberosity (tuber ischiadicum) and also the greater trochanter (trochanter major); note the mobility of the skin over the latter 
(bursa subcutanea trochanterica). Draw the shortest possible line on the surface from the tuber ischiadicum to the spina iliaca anterior superior and note the relation which the upper extremity of the trochanter major bears to this line. Palpate the structures forming the boundaries of the popliteal fossa. Note the mobility of the skin over the malleoli (bursa subcutanea malleoli lateralis et medialis). Feel the tendons in the retromalleolar fossa. Palpate the various bony prominences that can be felt about the ankle and foot, and identify each by comparison with the skeleton.

\section{GLUTEAL REGION (REGIO GLUTAEA).}

Make the following incisions (see Fig. 3) :

(1) From the spina iliaca posterior superior along the crista iliaca as far forward as possible.

(2) From the posterior extremity of this incision obliquely downward and medialward to the middle line of the sacral region, then vertically downward to the tip of the coccyx.

(3) From the tip of the coccyx downward and lateralward over the back of the thigh, intersecting the sulcus glutaeus at its middle point, and terminating a little below the upper third of the thigh.

The flap of skin thus formed should now be reflected lateralward, leaving intact the fat immediately beneath. There is now exposed to view-

\section{Superficial Fascia.}

Note the abundance of fat. How does the amount of fat contained compare in the male and female? Observe its tough, fibrous character over the tuber ischiadicum. This fascia should now be removed by blunt dissection. Find the following:

Nerves. (Fig. 47.)

(a) Middle nerves of buttock ( $N n$ : clunium medii). These pierce the glutaeus maximus and the deep fascia in a line passing from the spina iliaca posterior superior to the tip of the coccyx.

(b) Superior nerves of buttock ( $N n$. clunium superiores), crossing the crista iliaca at a point corresponding to the lateral limit of the attachment of the musculus sacrospinalis to the os ilium.

(c) Lateral cutaneous ramus of iliohypogastric nerve (ramus cutaneus lateralis nervi iliohypogastrici). It generally crosses the iliac crest opposite a tubercle projecting from its outer lip, about two and one-half inches dorsal to the spina iliaca anterior superior.

(d) Lateral cutaneous ramus of last thoracic nerve (ramus cutaneus lateralis $N$. thoracalis $I$.) (O. T. last dorsal nerve). This nerve crosses the iliac crest a short distance anterior to the preceding. 


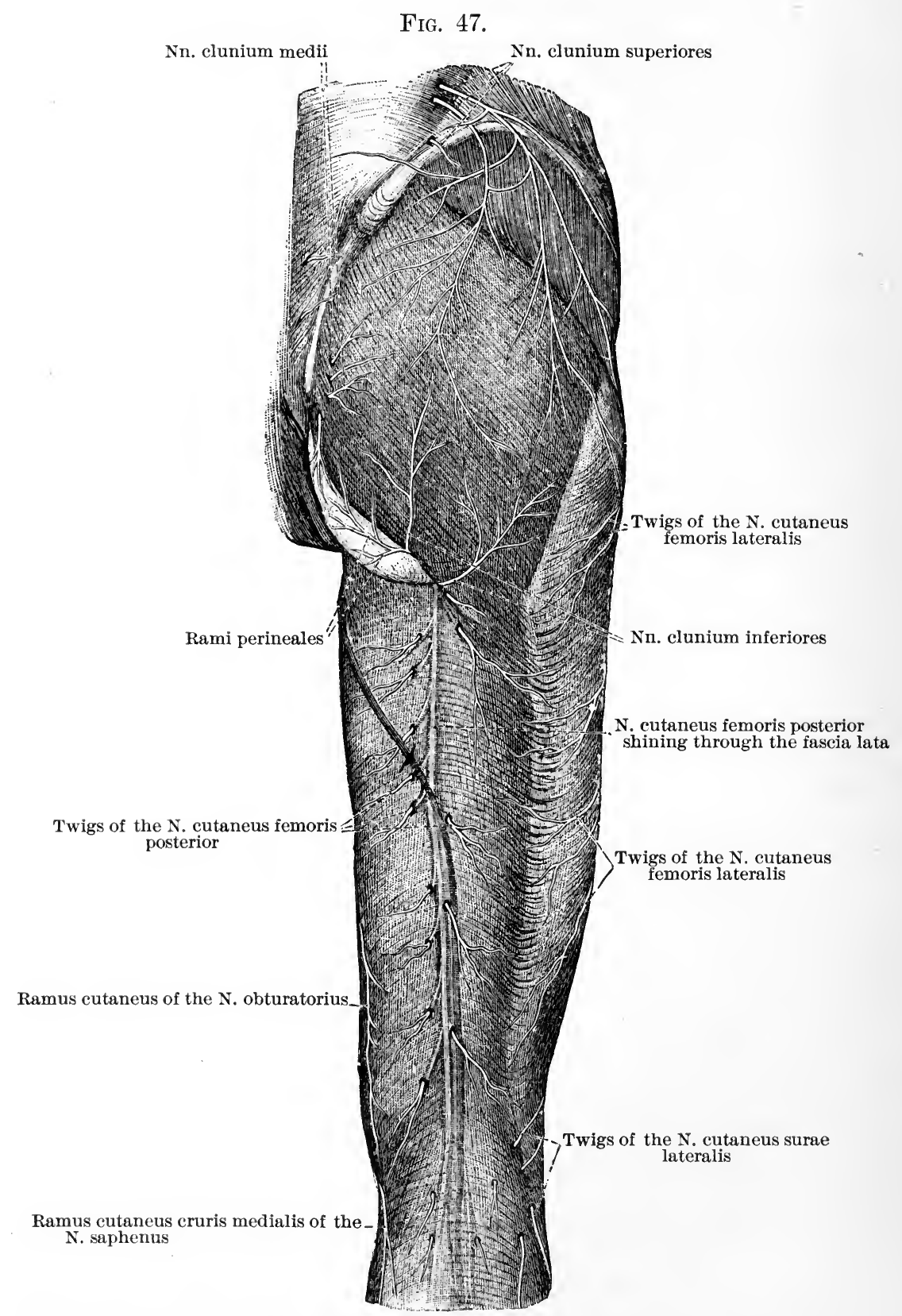

The cutaneous nerves of the gluteal region and on the posterior side of the thigh. (After Toldt, Anat. Atlas, Wien, 1903, 3 Aufl., p. 849, Fig. 1286.) 
(e) Cutaneous twigs from the posterior cutaneous nerve of the thigh ( $N$. cutaneus femoris posterior) (O. T. cutaneous branches of small sciatic), passing around the lower border of the glutaeus maximus.

(ea) Inferior nerves of buttock ( $N n$. clunium inferiores).

(eb) Perineal branches (rami perineales).

( $f$ ) Posterior branches of lateral cutaneous nerve of thigh (N. cutaneus femoris lateralis) ( $\mathrm{O}$. T. external cutaneous).

These nerves should be traced as far as possible and their distribution carefully noted.

\section{Deep Fascia.}

Remove the remaining portion of the superficial fascia, preserving the nerves, and expose the deep fascia.

Notice the general character of this fascia anteriorly, where it covers the M. glutaeus medius, and compare it in general characteristics with that portion of the fascia covering the M. glutaeus maximus. After having made a careful study of this fascia, remove it in a continuous layer from above downward, after having rendered the muscle tense beneath by rotating the thigh medialward. The borders of the muscle should be carefully defined; note that the thin fascia covering the latter becomes continuous with the dense pearly aponeurosis covering the muscle immediately anterior to it. In dissecting away this fascia, cut always in the direction of the muscle fibres.

\section{Glutaeus Maximus Muscle (M. glutaeus maximus).}

Note carefully the form, position, origin, insertion, action, and innervation of this muscle. What is the relation of the muscle to the dense fibrous band (tractus iliotibialis) passing from the ilium to the tibia? How does the size of this muscle in man compare with that in other animals? After having carefully examined and drawn this muscle, the student may reflect it in the following manner: The two borders of the muscle should be freed and the hand passed under the muscular mass. Detach the muscle from its attachment to the ilium. Proceed cautiously as the upper margin of the greater sacrosciatic foramen (foramen ischiadicum majus) is approached and avoid the gluteal vessels as they pass through this opening. Secure these and detach the muscle from the side of the sacrum, exposing the piriform muscle ( $\boldsymbol{M}$. piriformis) as it emerges from the pelvis. Separate the muscular fibres from the ligamentum sacrotuberosum (O. T. great sacrosciatic ligament) and the side of the coccyx, avoiding 
the Nn. clunium inferiores in order that they may later be traced to their source. The few coccygeal arteries passing through the ligamentum sacrotuberosum may be sacrificed. The muscle can now be partially reflected, but is still attached by blood-vessels and nerves entering its under surface. These should now be carefully cleaned. Having studied these last structures, they may be cut away, leaving a small piece of muscle connected to each. The whole muscle may then be reflected and its insertion examined. Clean and study the following:

Bursae. (Vide Spalteholz, Figs. 385, 386.)

(a) Bursa trochanterica $M$. glutaei maximi.

(b) Bursa ischiadica M. glutaei maximi.

(c) Bursae glutaeofemorales.

Muscles.

(a) Gluteus medius muscle (M. glutaeus medius).

(b) Piriform muscle ( $M$. piriformis). Note the relation it bears to the foramen ischiadicum majus.

(c) Tendon of obturator internus muscle ( $M$. obturator internus), passing through the foramen ischiadicum minus and having the $\mathrm{Mm}$. gemelli superior and inferior above and below it.

(d) Quadratus femoris muscle ( $M$. quadratus femoris).

(e) Smallest adductor muscle (M. adductor minimus) (O. T. upper adductor magnus).

( $f$ ) Tendon of the obturator externus muscle ( $M$. obturator externus).

Blood-vessels. (Fig. 48.)

(a) Above the M. piriformis:

(aa) Superior gluteal artery (A. glutaea superior). (aaa) Upper ramus (ramus superior).

(aab) Lower ramus (ramus inferior).

$(a b)$ Superior gluteal veins (Vv. glutaeae superiores).

(b) Below the M. piriformis:

(ba) Inferior gluteal artery (A. glutaea inferior) (O. T. sciatic artery).

(baa) Coceygeal branch, passing inward between the greater and lesser sacrosciatic ligaments to reach the integument and fascia in the region of the coccyx. Some twigs derived from this have already been cut in reflecting the muscle.

$(b a b)$ Companion artery of the great sciatic nerve ( $A$. comitans $N$. ischiadici).

(bac) Artery to the quadratus femoris. This artery will serve as a guide for the nerve to this muscle in the further dissection.

(bb) Inferior gluteal veins ( $V v$. glutaeae inferiores).

(bc) Internal pudendal artery ( $A$. pudenda interna). The pudic vessels can be seen emerging from the pelvis through the foramen ischiadicum majus and immediately re-entering through the foramen ischiadicum minus. No 
branches of these vessels are found at this stage of the dissection. What is the canal of Alcock?

(bd) Terminal branches of the medial circumflex artery (A. circumflexa femoris medialis). What is the "crucial anastomosis"?

FIG. 48.

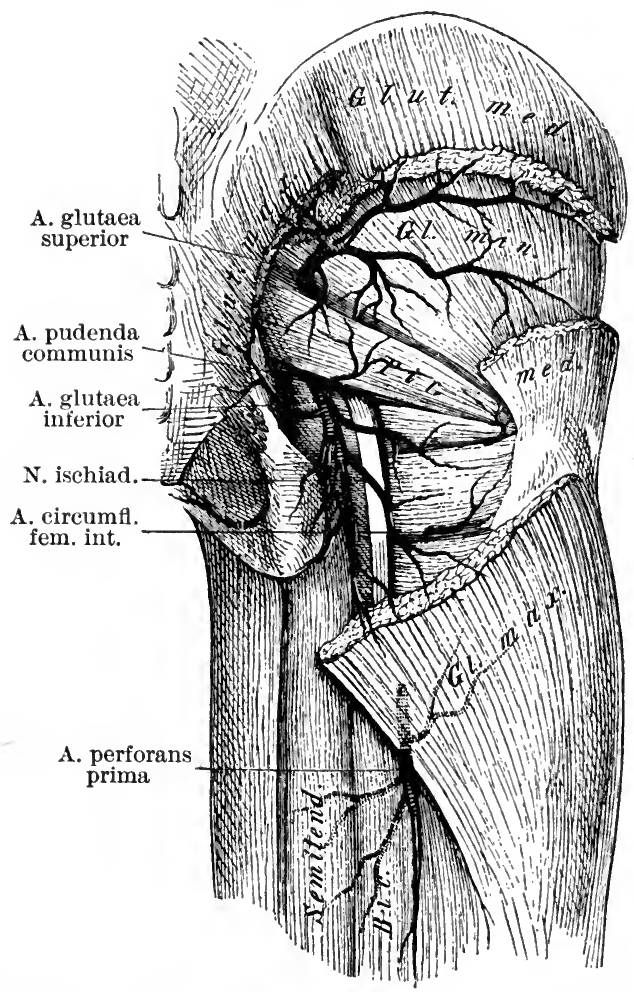

Arteries of the gluteal region. The Mm. glutaeus max. and med. are, in great part, removed. (After Gegenbaur, Lehrb. der Anat. des Mensch., Leipzig, 1899, 7 Aufl., Bd. il. p. 285, Fig. 545.)

Nerves. (Fig. 49.)

(a) Above the M. piriformis:

(aa) Superior gluteal nerve (N. glutaeus superior).

(b) Below the M. piriformis:

(ba) Great sciatic nerve ( $N$. ischiadicus). This nerve often divides into two branches (N. peronaeus communis and $\mathrm{N}$. tibialis) over the piriformis muscle. The nerve supplying the M. obturator internus and M. gemellus superior will be found lying just lateral to the pudic vessels. It rests upon the base of the spina ischiadica. To expose the nerve to the quadratus femoris and gemellus inferior, reflect the gemelli muscles under which it runs. When traced as far as the gemellus inferior exercise great care and avoid injury to the twig passing into 
this muscle. The quadratus femoris may now be reflected by detaching it from the femur and turning it towards the ischial tuberosity. The nerves supplying these muscles are derived from the tibial nerve ( $N$. tibialis), a branch of the $\mathrm{N}$. ischiadicus.

FIG. 49.

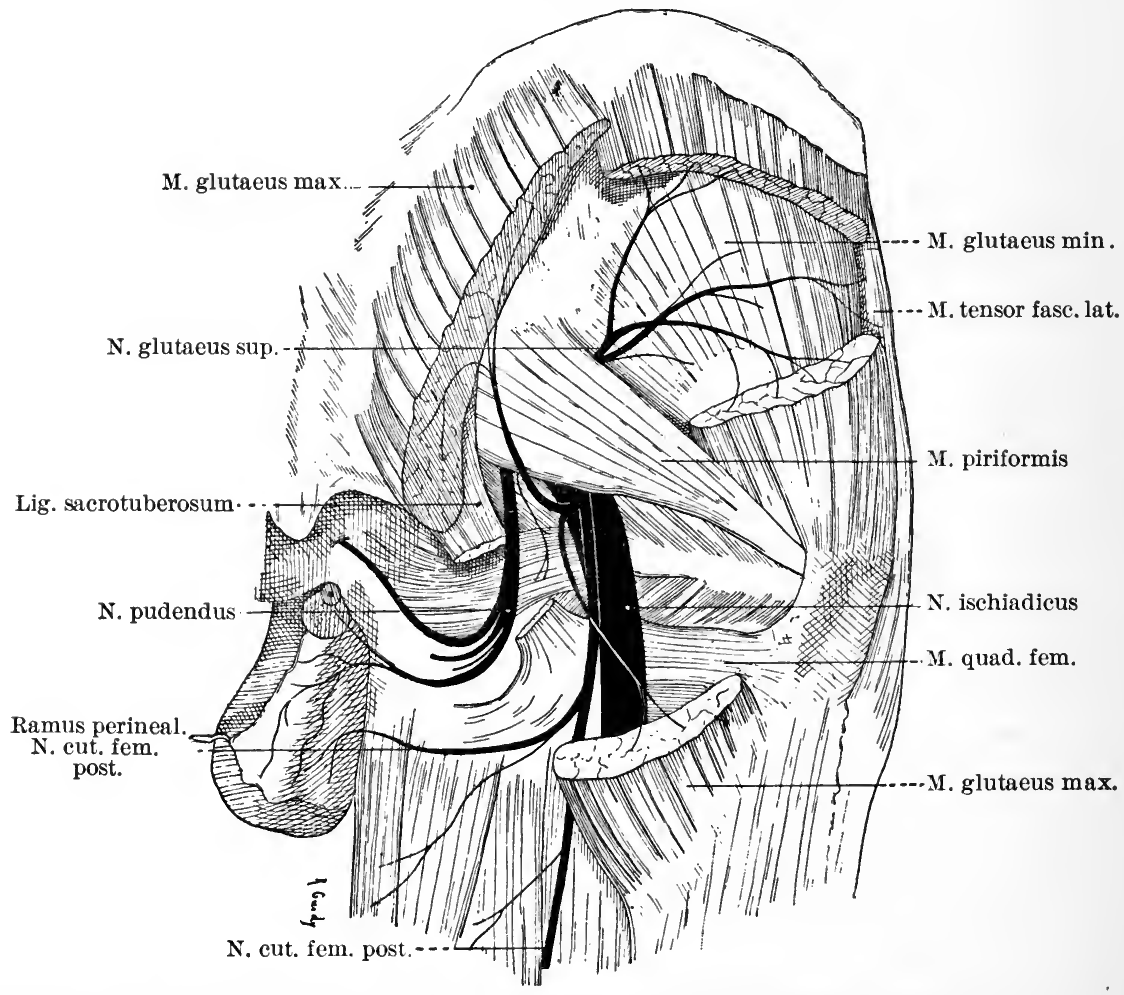

Nerves of the gluteal region (after Hirschfeld, simplified). (From Poirier et Charpy, Traité d'Anat. hum., Paris, 1899, t. iii. p. 1111, Fig. 580.)

(bb) Posterior cutaneous nerve of thigh ( $N$. cutaneus femoris posterior) (O. T. small sciatic).

$(b b a)$ Inferior nerves of buttock (Nn. clunium inferiores).

$(b b b)$ Perineal branches (rami perineales).

(bc) Pudendal nerve (N. pudendus) (O. T. internal pudic nerve).

(bd) Inferior gluteal nerve ( $N$. glutaéus inferior).

\section{Mm. Glutaei Medius et Minimus and Adjacent Structures.}

The M. glutaeus medius should be reflected after a careful study has been made of the preceding structures. Rotate the 
thigh lateralward. Pull the M. glutaeus maximus well outward and divide its aponeurosis of insertion downward for a distance of five to seven centimetres. Grasping the cut edge of the fascia lata just separated from the M. glutaeus maximus, pull it outward and dissect in the interval between it and the M. glutaeus medius. By traction upon this band the deep surface of the tensor muscle of the fascia lata (M. tensor fasciae latae) (O. T. tensor vaginae femoris) may be exposed. It is covered by a layer of fascia. The student should now find-

(a) Terminal branch of superior gluteal nerve, piereing the anterior border of the M. glutaeus minimus and entering the M. tensor fasciae latae.

(b) Ascending branch of lateral circumflex artery (ramus ascendens $A$. circumflexae femoris lateralis).

Separate the M. glutaeus medius from the M. glutaeus minimus by passing the fingers between their posterior border's. When completely isolated, divide the M. glutaeus medius five centimetres above the trochanter major and reflect the two parts upward and downward respectively. Carefully preserve the vessels and nerves between the two muscles and clean them thoroughly. What vessels and nerves are exposed? Trace each to its termination. Can you find-

(a) Bursa trochanterica M. glutaei medii posterior?

(b) Bursa trochanterica M. glutaei medii anterior?

Where is the bursa $M$. piriformis?

Detach the M. glutaeus minimus from its origin and reflect it downward. Observe the capsular ligament of the hip-joint (capsula articularis). Where is the bursa trochanterica M. glutaei minimi? Find the reflected head of the M. rectus femoris. What is the action of the muscles supplied by the superior gluteal nerve?

\section{POPLITEAL SPACE (FOSSA POPLITEA).}

Before beginning the dissection, review the surface anatomy of this space, noting-

(a) Lateral hamstring, formed by the biceps muscle.

(b) Medial hamstring, formed by the tendons of the semitendinosus and semimembranosus muscles.

(c) Tendon of adductor magnus muscle. Can you trace it as far downward as the adductor tubercle? In what position of the leg can this tendon be outlined most easily? 
(d) Epicondyles of femur. Which is the more prominent?

(e) Line of the knee-joint. Flex and extend the leg to render this study easier.

( $f$ ) Head of fibula (capitulum fibulae). Determine its relation to the tibia and femur.

(g) Position of common peroneal nerve ( $N$. peronaeus communis). What relation does it bear to the lateral hamstring muscle?

\section{Skin and Superficial Fascia.}

Make an incision in the middle line of the posterior surface of the thigh through the popliteal fossa from a point ten or twelve centimetres above the bend of the knee to a point ten centimetres below it. Two transverse incisions should next be made, one at each end of the vertical incision, and the flaps formed reflected medialward and lateralward respectively. (See Figs. 3 and 52.) Make the parts tense by means of a large block placed under the knee.

Note the general characteristics of the superficial fascia. Remove this fascia piecemeal, examining the following:

(a) Twigs of posterior cutaneous nerve of thigh ( N. cutaneus femoris posterior) (O. T. small sciatic). In what part of this space does the terminal branch of this nerve pierce the deep fascia?

(b) Small saphenous vein ( $V$. saphena parva). Preserve intact this vein and any of its radicles that you may find. What is the femoropopliteal vein (V. femoropoplitea)? (Vide Spalteholz, Fig. 509.)

(c) Superficial lymphatic vessels (vasa lymphatica superficialia). (Vide Fig. 50.)

\section{Boundaries of Fossa Poplitea.}

The fossa poplitea is diamond-shaped. Its roof is formed by the deep fascia (fascia cruris), sometimes called the " popliteal fascia." Note carefully the density of this fascia. After the fascia has been studied, incise it in the median line and reflect each way. Care should be exercised to avoid injuring the communicating fibular nerve ( $N$. communicans peronaeus) and a bursa situated between the M. semimembranosus and the medial head of the M. gastrocnemius.

Next study the lateral boundaries:

(a) Biceps musele (M. biceps femoris).

(b) Semitendinosus muscle (M. semitendinosus).

(c) Semimembranosus muscle (M. semimembranosus).

(d) Gastrocnemius muscle (M. gastrocnemius). (da) Lateral head (caput laterale).

(db) Medial head (caput mediale).

(e) Plantaris muscle (M. plantaris). 
Fig. 50.

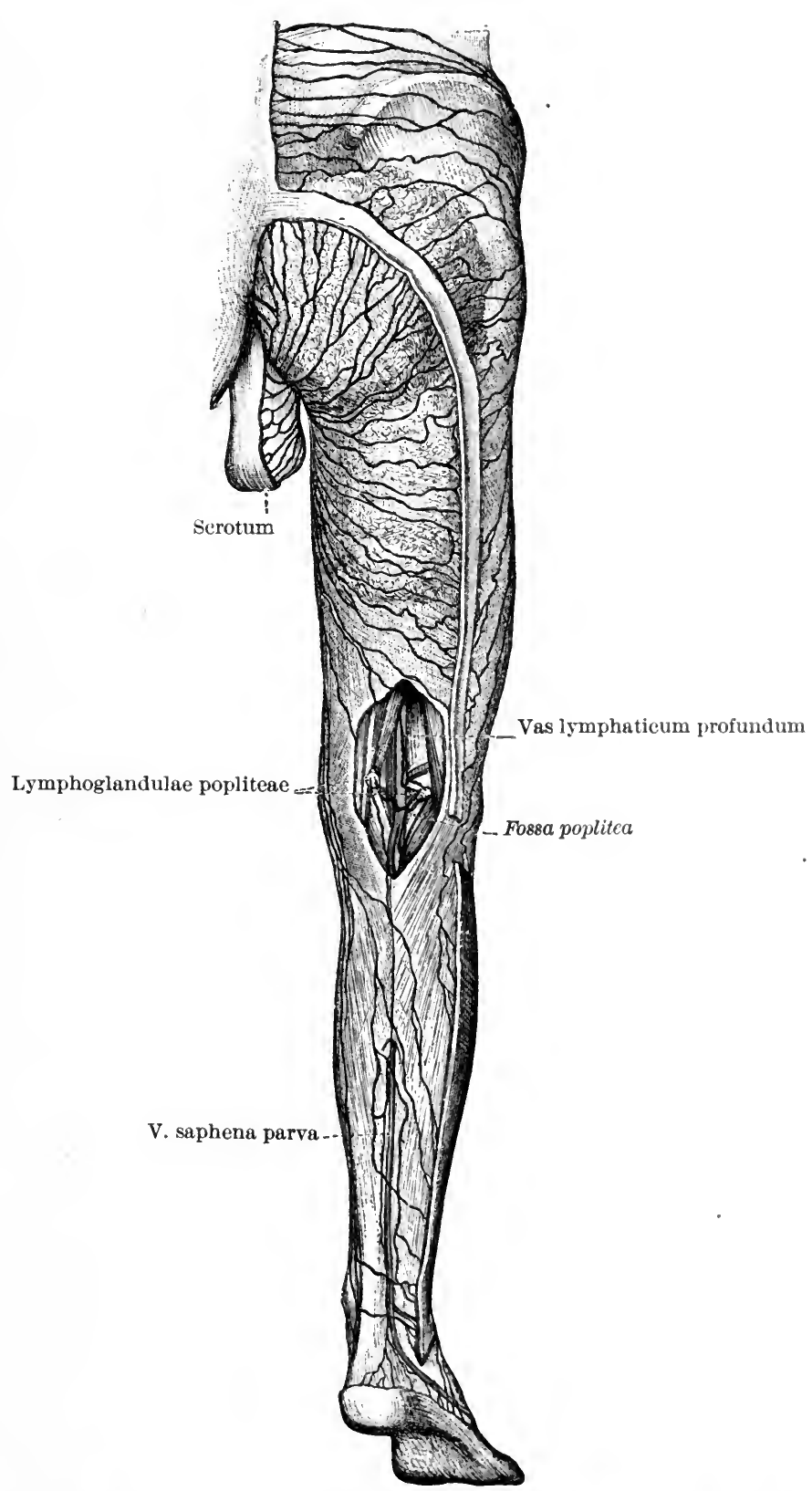

The superficial lymphatic vessels of the posterior side of the lower extremity and the deep lymphatics of the popliteal space. (After Toldt, Anat. Atlas, Wien, 1900, 2 Aufl., p. 709, Fig. 1091.) 
The exposed parts of each of these muscles should be carefully cleaned.

Remove the fat of the fossa, carefully dissecting out its contents (vide infra).

In cleaning the structures in the fossa, avoid disturbing their relations.

FIG. 51.

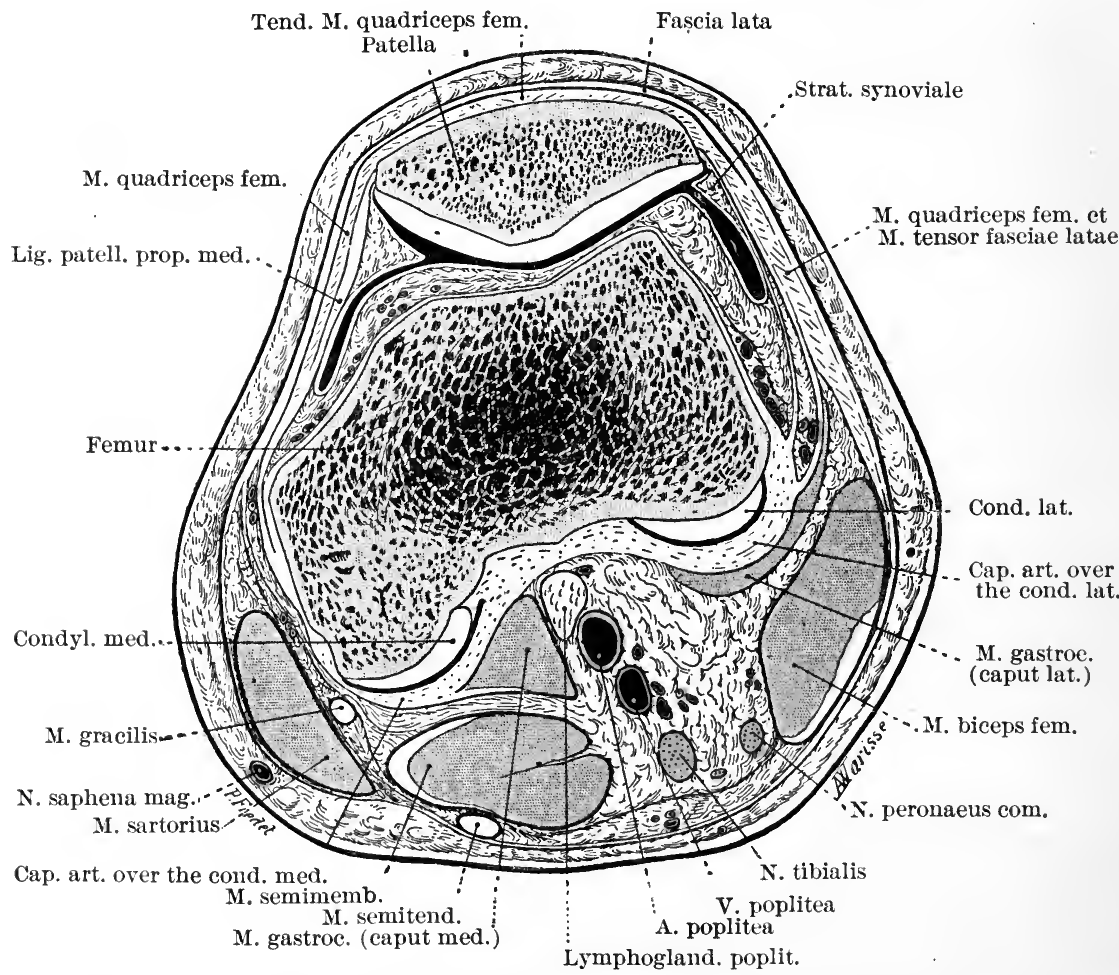

Cross-section of the artieulatio genu, passing through the middle of the patella. Right side, segment distal to the line of seetion (P. Fredet). (From Poirier et Charpy, Traité d'Anat. hum., Paris, 1901, 2 ed., t. ii. p. 242, Fig. 180.)

\section{Contents of Fossa Poplitea. (Figs. 5I-53.)}

Nerves. (Figs. 51 and 52.)

(a) Tibial nerve (N. tibialis) (O. T. internal popliteal nerve). What is its position in the fossa?

(aa) Muscular branches (rami musculares). Dissect each muscular branch out carefully and trace into the muscle.

(ab) Articular branch (ramus articularis).

(ac) Medial cutaneous nerve of the calf ( $N$. cutaneus surae medialis) ( 0. T. nervus communicans tibialis).

(ad) Interosseous nerve of the leg ( $N$. interosseus cruris). 
(b) Conmon peroneal nerve (N. peronaeus communis) (O. T. external popliteal).

(ba) Muscular branches (rami musculares).

$(b b)$ Lateral cutaneous nerre of calf ( $N$. eutaneus surae lateralis).

(bc) Peroneal anastomotic branch (ramus anastomoticus peronaeus) (O. T. nervus communicans fibularis).

(c) Posterior cutaneous nerve of thigh ( $N$. cutaneus femoris posterior)

(O. T. small sciatic nerve). This nerve is found directly underneath the fascia. Its branches have already been referred to.

(d) A branch of the obturator nerve ( $N$. obturatorius) descends into the space in close relation to the artery.

FIG. 52.

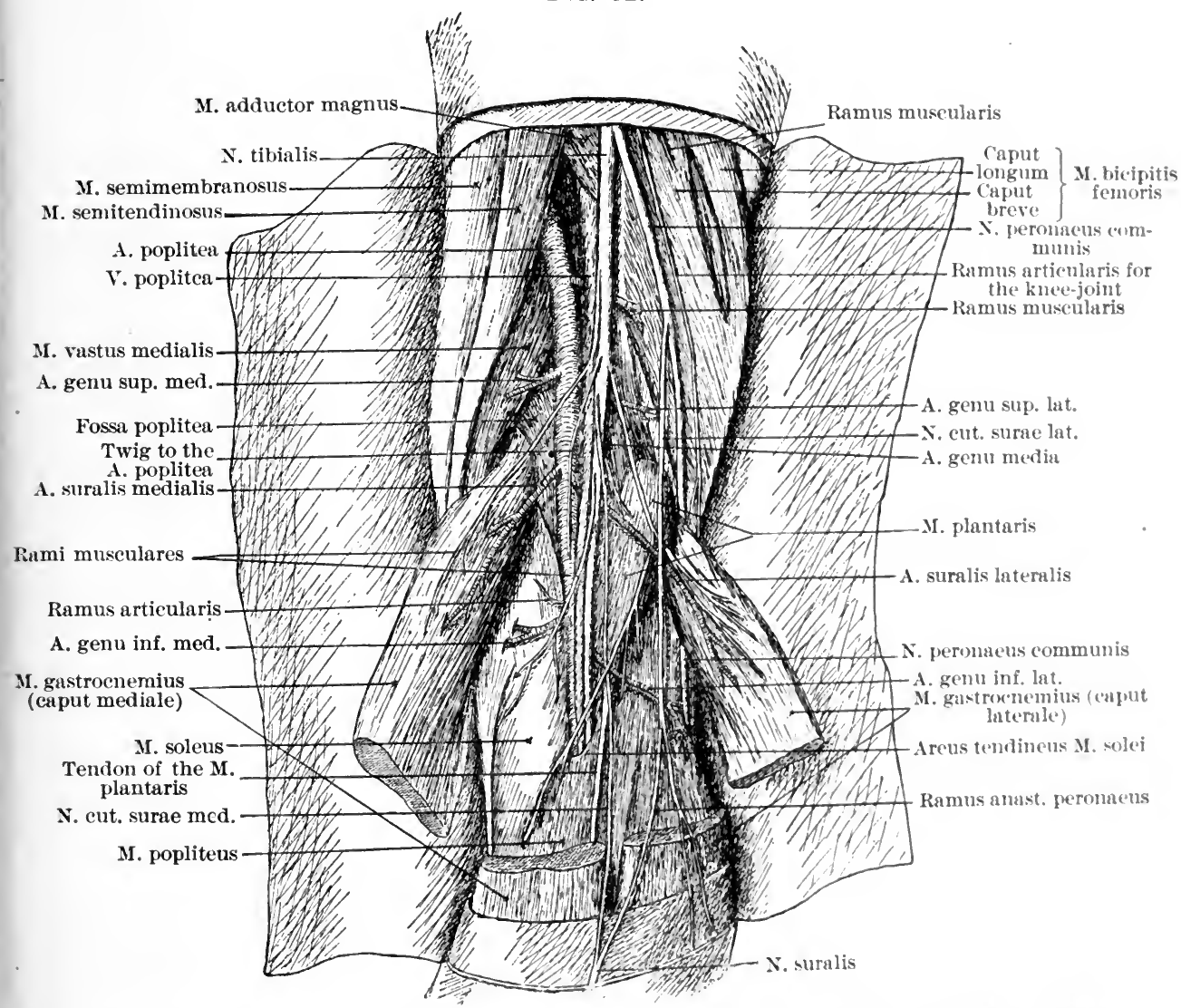

The popliteal space. (After Toldt, Anat. Atlas, Wien, 1900, 2 Aut., p. 631, Fig. 1010, and (3 Anfl.) p. 842,

Fig. 1278, and Cunningham, Manual of Prae. Anat., Phila., 1903, 3d ed., vol. i. p. 174, Fig. 54.)

Arteries. (Figs. 52 and 53.)

(a) Popliteal artery (A. poplitea). Note especially the relation that this artery bears to the structures contained in the space 
and dissect out its branches. Determine accurately the vessels which enter into the anastomosis about the knee.

(aa) Lateral superior artery of knee (A. genu superior lateralis) (O. T. superior external articular artery).

(ab) Medial superior artery of knee (A. genu superior medialis) (O. T. superior internal articular artery).

(ac) Middle artery of knee (A. genu media) (O. T. azygos articular artery).

(ad) Lateral inferior artery of knee (A. genu inferior lateralis) (O. T. inferior external articular artery).

(ae) Medial inferior artery of knee (A. genu inferior medialis) (O. T. inferior internal articular artery).

(af) Sural arteries (Aa. surales).

What is the rete articulare genu? What is the rete patellae?

FIG. 53.

A. genu sup. med.

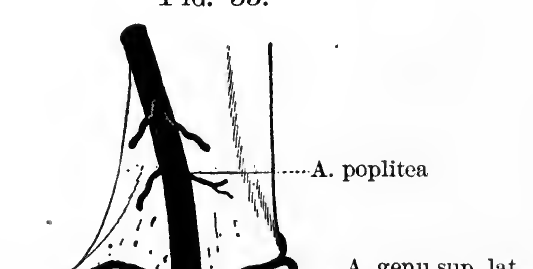

A. genu inf. med.

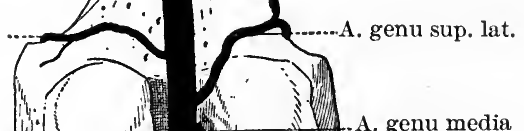

A. genu media

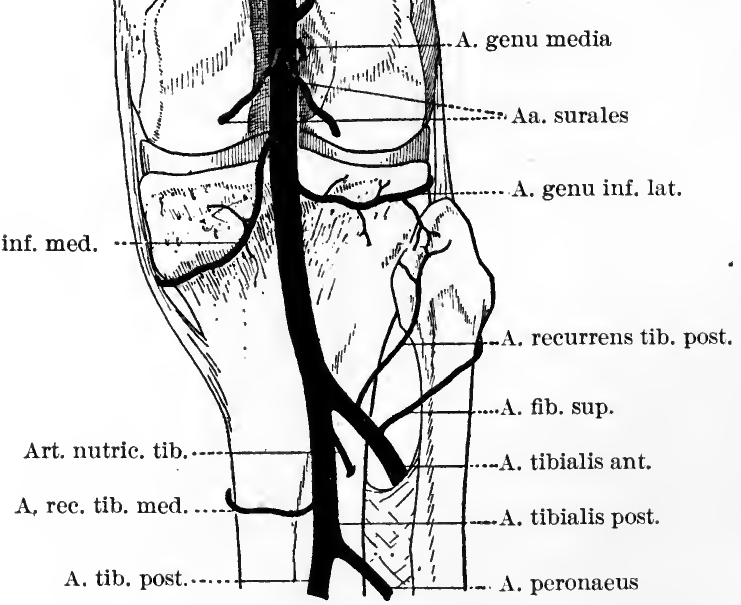

Schema of the A. poplitea. (From Poirier et Charpy, Traité d'Anat. hum., Paris, 1901, 2 ed., t. ii. p. 830 , Fig. 448.)

Veins.

(a) Popliteal veins (Vv. popliteae).

(aa) Small saphenous vein ( $V$. saphena parva).

( $a b)$ Articular veins of knee (Vv. articulares genu).

(ac) Lateral companion vein ( $V$. comitans lateralis).

(ad) Medial companion vein ( $V$. comitans medialis). 
Note especially the relation of the vein to the artery in the upper and lower parts of the fossa. Incise the vein longitudinally and seareh for valves. Are any present?

Lymphatics. (Vide Fig. 50.)

(a) Popliteal lymph-nodes (lymphoglandulae popliteae).

How many do you find?

(b) Deep lymphatic vessels (vasa lymphatica profunda).

Study in the floor of the fossa-

(a) Popliteal surface (planum popliteum) of the femur.

(b) Posterior ligament (ligamentum popliteum obliquum) of the kneejoint.

(c) Strong faseia covering popliteal musele. From what musele is this fascia derived?

A careful drawing of the boundaries and contents of this fossa should now be made. Each structure should be shown in its proper relation and carefully labelled. To complete the study make use of cross-sections. Make a drawing of a section through the middle of the space, labelling each structure found and giving correct relations.

\section{POSTERIOR SURFACE OF THIGH (FACIES FEMORIS POSTERIOR).}

Make a vertical incision through the belt of skin remaining in the middle of the thigh. Reflect the flaps thus formed lateralward and medialward respectively.

\section{Superficial Fascia and Cutaneous Nerves.}

Note carefully branches of arteries and radicles of veins. Preserve these as carefully as possible and trace back the larger vessels. Study the cutaneous nerves in the superficial fascia. Consult Fig. 47, p. 124.

(a) Branches of the posterior cutaneous nerve of the thigh ( $N$. cutaneus femoris posterior) (O. T. small sciatic).

(b) On the lateral surface of the thigh, passing backward, branches of the lateral cutaneous nerve of the thigh $(N$. cutaneus femoris lateralis) ( $0 . T$. external entaneous).

(c) On the inner aspect of the thigh, near the knee, the cutaneous branch of the obturator nerve (ramus cutaneus nervi obturatoris); also twigs from the rami cutanei anteriores nervi femoralis.

\section{Deep Fascia (Fascia lata). (“Broad Fascia.”)}

Clean away carefully and completely the superficial fascia and expose the deep layer of fascia. Note especially its thick- 
ness at different levels. The deep fascia may next be removed. Follow the rule already given for removal of fascia. Avoid the nervus cutaneus femoris posterior in the median line.

\section{Muscles of Back of Thigh.}

Note carefully the form, position, origin, insertion, action, and nerve supply of each of the following:

(a) Biceps muscle of thigh ( $M$. biceps femoris).

(aa) Long head (caput longum).

(ab) Short head (caput breve).

(b) Semitendinosus muscle (M. semitendinosus).

(c) Semimembranosus musele (M. semimembranosus).

What do you understand by the hamstring muscles?

\section{Nerves of Back of Thigh.}

(a) Posterior cutaneous nerve of thigh ( $N$. cutaneus femoris posterior) (O. T. small sciatic). This nerve will be found directly underneath the deep fascia in the median line of the thigh.

(b) Great sciatic nerve (N. ischiadicus). Note the level at which this nerve divides into the $\mathrm{N}$. tibialis and the $\mathrm{N}$. peronaeus communis. What are the muscular branches (rami musculares) of the nerve and to what muscles are they distributed? What is the relation of the $\mathrm{N}$. peronaeus communis to the biceps muscle? Is there a triangle formed by the hamstring muscles in which you can readily find the N. ischiadicus?

\section{Blood-vessels and Lymphatics of Back of Thigh.}

The following arteries and accompanying veins will be seen piercing the large muscle inserted into the linea aspera. What muscle is this?

Arteries.

(a) First perforating artery (A. perforans prima).

(b) Second perforating artery (A. perforans secunda).

(c) Third perforating artery (A. perforans tertia).

Determine from which of these arteries the superior nutrient artery of the femur (A. nutricia femoris superior) and the inferior nutrient artery of the femur (A. nutricia femoris inferior) are derived. Clean each artery and trace it back to the muscle which it pierces. Dissect out the fibrous passage-way through which each artery passes. Note that these openings are in the same line with and exactly analogous to the one through which the terminal portion of the deep femoral artery ( $A$. profunda femoris) passes to the back of the thigh. The terminal part of this artery is sometimes called the "fourth perforating artery." What is the purpose of these fibrous arches? 


\section{Veins.}

(a) First perforating vein ( $V$. perforans prima).

(b) Second perforating vein ( $V$. perforans secunda).

(c) Femoropopliteal vein ( $V$. femoropoplitea). Into what larger vein does each empty?

FIG. 54.

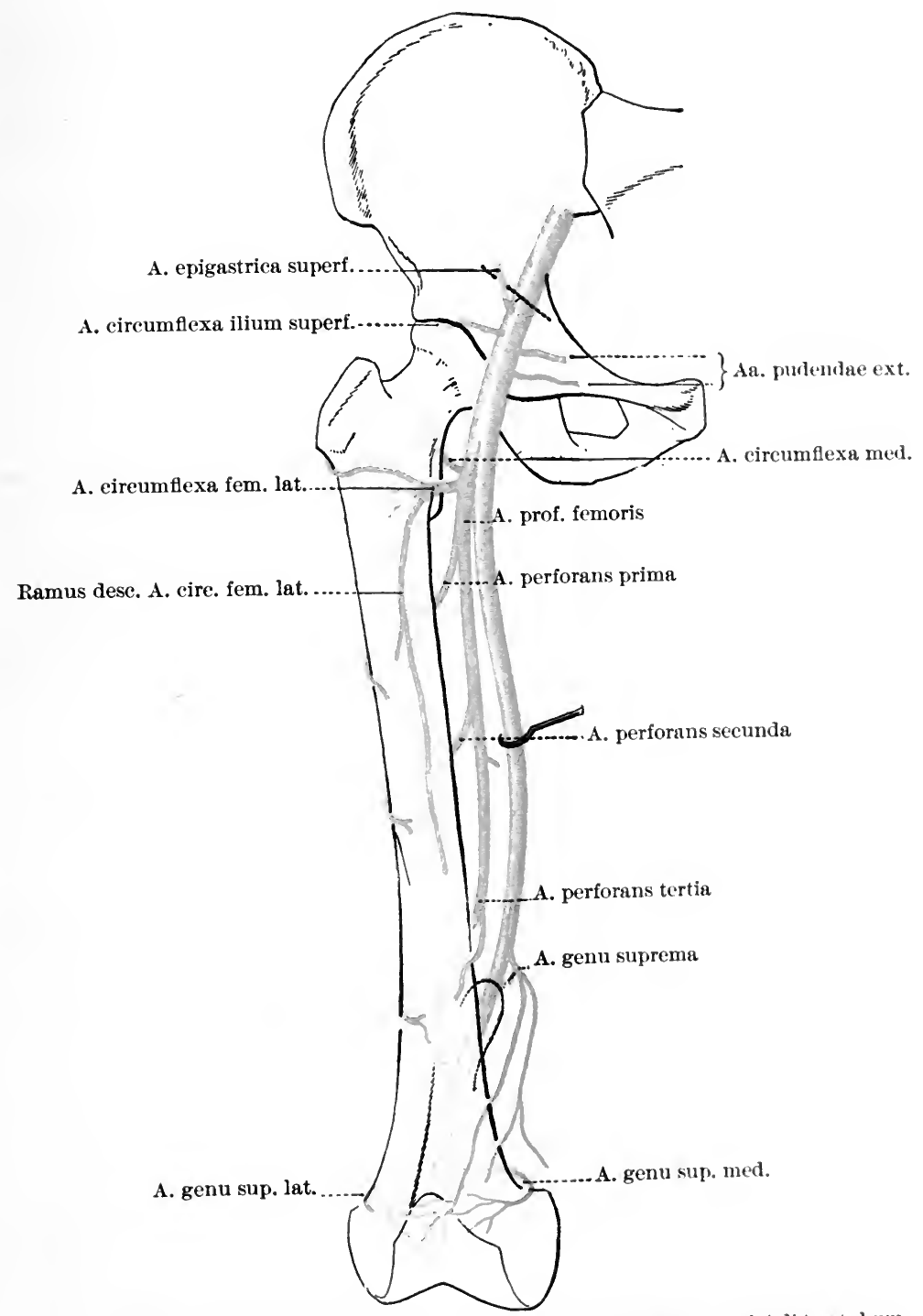

Schema of the arteria femoralis. (From Poiricr et Charpy, Traité d'Anat. hum., Paris, 1901, 2 ed., t. ii. p. 818 , Fig. 471.)

Determine accurately the course of each of the preceding arteries and veins. What is their relation to the M. vastus lateralis? 
Lymphatics. (Fig. 50.)

Do you find any lymphatic vessels? If so, determine their course and distribution. Are any lymph-nodes (lymphoglandulae) to be found in this region?

\section{Great Adductor Muscle (M. adductor magnus).}

After having completed the dissection of the popliteal surface, divide the conjoined tendon of the M. semitendinosus and M. biceps, exposing the origin of the M. semimembranosus. After studying the origin of this muscle and charting the length and breadth of its origin, reflect it downward.

Determine accurately the relation of the M. adductor magnus to the linea aspera. What is the adductor tubercle? The form, position, origin, insertion, action, and innervation of the great adductor muscle can best be studied later.

Anastomosis upon the Back of the Thigh. (Fig. 54.)

What vessels enter into the formation of this anastomosis? How would the circulation to the leg be carried on if the common femoral artery were ligated? Where may the femoral artery be ligated most advantageously, and with the greatest possibility of the establishment of a collateral circulation?

\section{ANTERIOR SURFACE OF THIGH (FACIES FEMORIS ANTERIOR).}

Skin and Superficial Fascia of Upper Part of Anterior Surface of Thigh.

Make the following incisions:

(a) From the spina anterior superior along the line of the inguinal ligament of Poupart to the symphysis pubis.

(b) From the medial extremity of $(a)$ downward, just lateral from the scrotum and along the medial surface of the thigh for a distance of ten centimetres.

(c) From the lower extremity of $(b)$ transversely lateralward over the anterior surface of the thigh to its lateral aspect.

Raise the quadrilateral flap thus outlined, taking no fat of the superficial fascia with it.

In the superficial fascia of the lower part of the anterior abdominal wall two layers are demonstrable,-(1) more superficial (Camper's fascia), fatty, continuous with the fatty superficial fascia of the thigh; and (2) a deeper layer (Scarpa's fascia), devoid of fat, inserted into the fascia lata a little below Poupart's ligament. Study this fascia in conjunction with the 
FIG. 55.

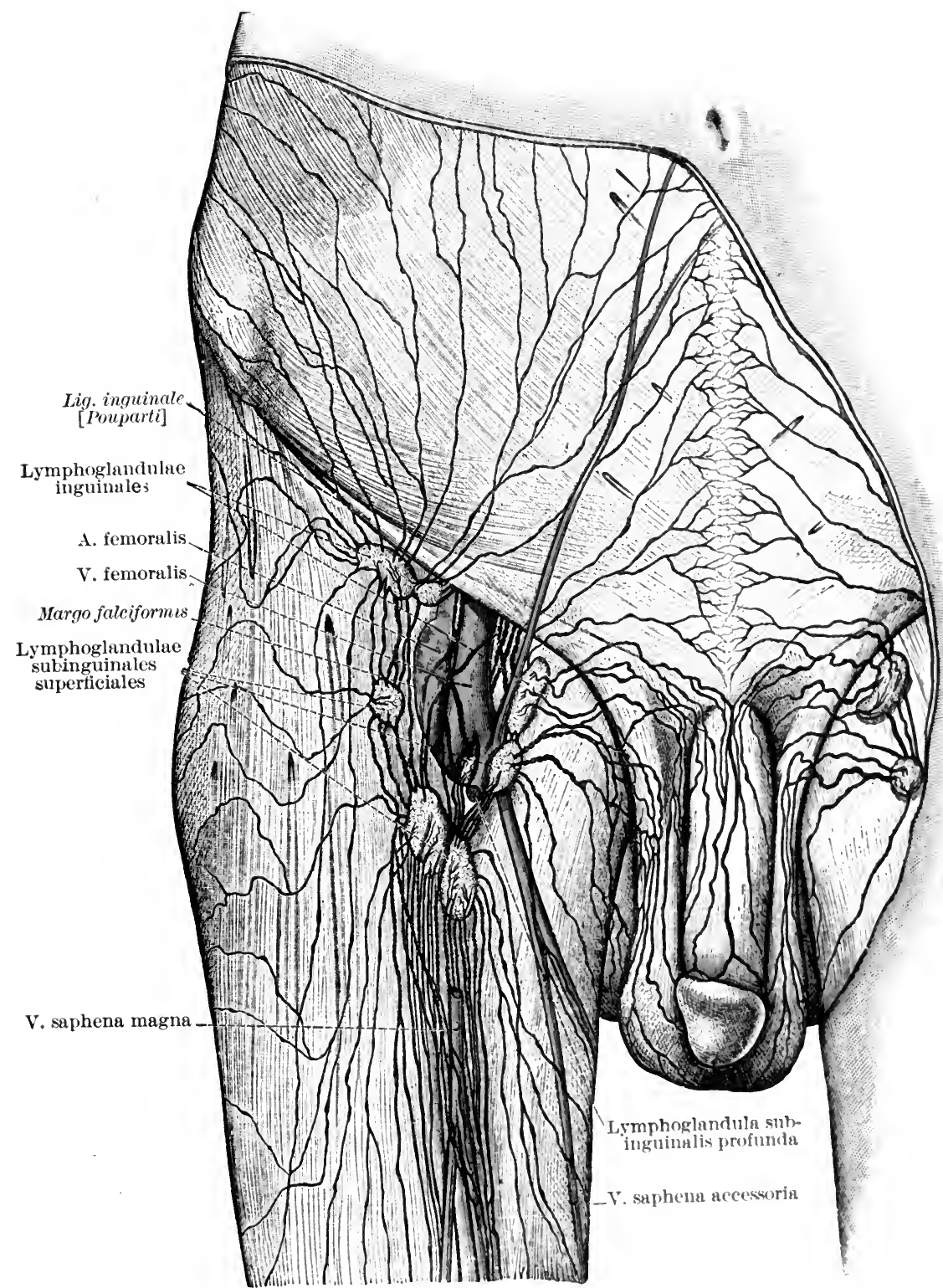

The superfieial y.mplaties of the inguinal and aljacent regions. (After Toldt, Anat. Atlas, Wien, 1900, 2 Aufl., p. 706, Fig. 1uss.) 
dissector of the abdomen. What bearing have the attachments of Scarpa's fascia upon the distribution of extravasated urine?

Dissect out and study carefully in the superficial fascia the following structures :

Veins.

(a) Large saphenous vein ( $V$. saphena magna) (O. T. internal saphenous).

(aa) Accessory saphenous vein ( $V$. saphena accessoria), inconstant.

(b) Superficial epigastric vein ( $V$. epigastrica superficialis).

(c) Superficial circumflex iliac vein ( $V$. circumflexa ilium superficialis).

(d) External pudendal veins ( $V v$. pudendae externae).

Arteries.

(a) Superficial epigastric artery (A. epigastrica superficialis).

(b) Superficial cireumflex iliac artery (A. circumflexa ilium superficialis).

(c) External pudendal arteries (Aa. pudendae externae) (O. T. superficial and deep external pudic arteries).

(d) Inguinal rami of femoral artery (rami inguinales A. femoralis).

Lymphatics. (Fig. 55.)

(a) Inguinal lymph-glands (lymphoglandulae inguinales).

Whence do they draw lymph? Whither does the lymph go from them?

(b) Superficial subinguinal lymph-glands (lymphoglandulae subinguinales superficiales).

Whence do they draw lymph? Whither does lymph go from them?

Nerves. (See Fig. 56, p. 143.)

Avoid injury to the N. ilio-inguinalis, N. spermaticus externus, N. lumbo-inguinalis, and $\mathrm{N}$. cutaneus femoris lateralis. These nerves are to be studied thoroughly a little later, when the rest of the skin on the anterior surface of the thigh is removed.

\section{Oval Fossa of Thigh (Fossa ovalis) (O. T. Saphenous Opening).}

The structures to be studied at and about the oval opening include-

(a) Falciform margin (margo falciformis).

(aa) Superior cornu (cornu superius).

(ab) Inferior cornu (cornu inferius).

(b) Covering of cribriform fascia (fascia cribrosa).

(c) Junction of V. saphena magna with V. femoralis.

(d) Lymph-glands.

Remove with great care the superficial fascia of the region, beginning over the upper part of the M. adductor longus and M. pectineus, cleaning the deep fascia (fascia lata) lying beneath. Note that the latter is attached above to the posterior margin of 
Poupart's ligament. Following this portion of the fascia, fascia pectinea (O. T. pubic portion of fascia lata), lateralward, it will be seen to pass beneath the femoral vessels so as to line the fossa iliopectinea, becoming continuous lateralward with the fascia iliopectinea. The inferior cornu of the falciform margin is now visible. It belongs to that part of the fascia lata which lies lateral from the fossa ovalis,-viz., the superficial layer of the fascia lata (O. T. iliac portion of fascia lata),- - and is inserted medialward into the fascia pectinea or deep layer. Observe the cribriform fascia (fascia cribrosa) closing the aperture of the fossa ovalis. The superficial subinguinal lymph-glands must be removed to display it properly. What vessels pass through this fascia cribrosa?

Remove the fascia cribrosa carefully, so that the edge of the margo falciformis and its cornu superius may be clearly defined. The upper horn (cornu superius) (O. T. femoral ligament or Hey's ligament or ligament of Allan Burns) consists of two layers, an anterior and a posterior.

The anterior layer of the cornu superius is a broad lamina, strengthened by Scarpa's fascia; it bounds the fossa ovalis above and lies rertically between it and Poupart's ligament, to which it is attached. The posterior, thicker layer of the cornu superius extends further upward than the anterior, passes superficial to the femoral vessels medialward and upward, assumes a horizontal position, filling up the acute angle between the medial end of Poupart's ligament and the pecten ossis pubis, and really forming (by fusion with the process of Poupart's ligament to the pecten) the anterior or inferior part of the ligamentum lacunare [Gimbernati].

The femoral canal will be studied farther on. Beneath the fascia cribrosa within the fossa ovalis observe the deep subinguinal lymph-glands (lymphoglandulae subinguinales profundae).

\section{Skin and Superficial Fascia of Anterior Surface of Thigh and Knee.}

Continue the vertical incision on the medial aspect of the thigh distalward as far as the medial condyle of the tibia. Make another incision from the medial condyle of the tibia lateralward across the front of the leg as far as the lateral condyle of the tibia. Reflect the large flap lateralward, taking no fat with the skin and avoiding injury to nerves and blood-vessels and to the subcutaneous praepatellar bursa.

In the fat of the superficial fascia dissect out carefully the following structures: 
Veins.

(a) Large saphenous vein ( $V$. saphena magna) ( $O$. T. internal saphenous vein) and its tributaries.

Nerves.

(a) Ilio-inguinal nerve ( $N$. ilio-inguinalis).

(b) Lumbo-inguinal branch of genitofemoral nerve (N. lumbo-inguinalis $N$. genitofemoralis) (O. T. crural branch of genitocrural).

(c) Lateral cutaneous nerve of thigh ( $N$. cutaneus femoris lateralis) (O. T. external cutaneous).

Follow this nerve into the sheath formed for it by the fascia lata. People whose occupations require excessive standing or walking (fascia lata tense) sometimes suffer from a severe neuralgia of this nerve,so-called "meralgia paraesthetica," or "Bernhardt's disturbance of sensibility in the thigh."

(d) Branches of femoral nerve ( $N$. femoralis) (O. T. anterior crural). (da) Anterior eutaneous rami (rami cutanei anteriores) (O. T. middle and internal cutaneous).

(db) Infrapatellar ramus of saphenous nerve (ramus infrapatellaris $N$. sapheni) (O. T. patellar branch of long saphenous). Look for it near the cutaneous branch of the A. genu suprema.

(dc) Cutaneous ramus of obturator nerve (ramus cutaneus $N$. obturatorii).

Bursa.

Subcutaneous praepatellar bursa (bursa praepatellaris subcutanea).

Pinch up a bit of tissue in front of the patella with forceps and make a transverse cut into the bursa. Explore with the finger.

\section{Deep Fascia of Thigh (Fascia lata).}

Clean the surface of the fascia lata carefully and examine its attachments above and below. Compare with the description in your systematic text-book. Pay especial attention to the following points :

(a) Superficial layer of fascia lata and margo falciformis of fossa ovalis (O. T. iliac portion of fascia lata).

(b) Deep layer of fascia lata or fascia pectinea (O. T. pubic portion of fascia lata).

(c) Cribriform fascia (fascia cribrosa). This is really a thin continuation of $(a)$.

(d) Iliotibial band (tractus iliotibialis [Maissiati]). What is its significance?

(e) White lines corresponding to intermuscular septa in depth.

(ea) Lateral intermuscular septum of thigh (septum intermusculare [femoris] laterale) (O. T. external intermuscular septum).

(eb) Medial intermuscular septum of thigh (septum intermusculare [femoris] mediale) (O. T. internal intermuscular septum). 
FIG. 56.

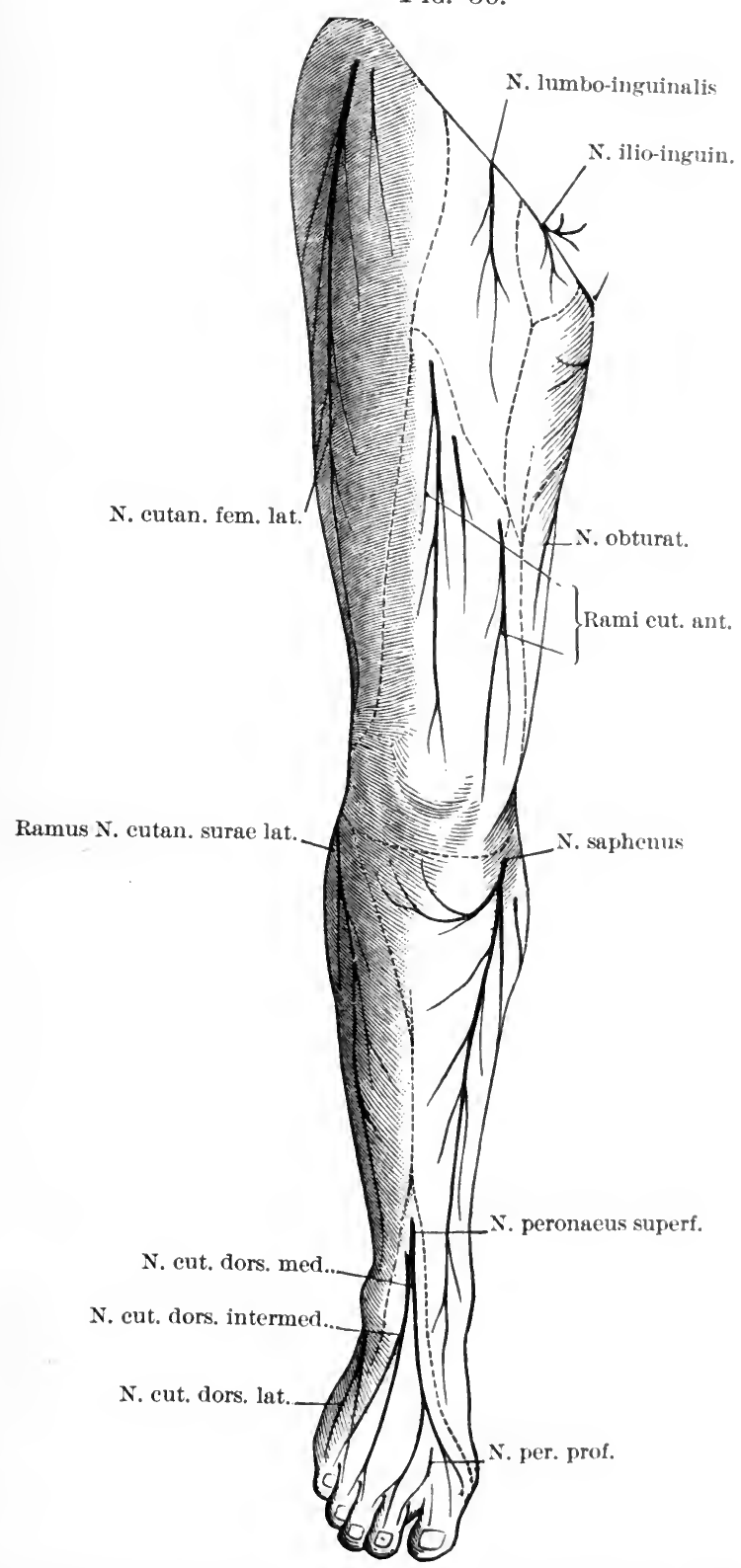

Cutaneous nerves of the flexor side of the lower extremity (schematic). (From Gegenbaur, Lehrb. der Anat. des Menseh., Leipzig, 1899, 7 A ufl., Bd. ii. p. 516, Fig. 6ti9.) 
Note that these two septa divide the thigh into two great osteofascial compartments, one in front, the other behind. The latter is further subdivided by a layer of fascia, sometimes called the " posterior intermuscular septum." The further dissection will reveal the following contents of these osteofascial compartments. (Fig. 57.)

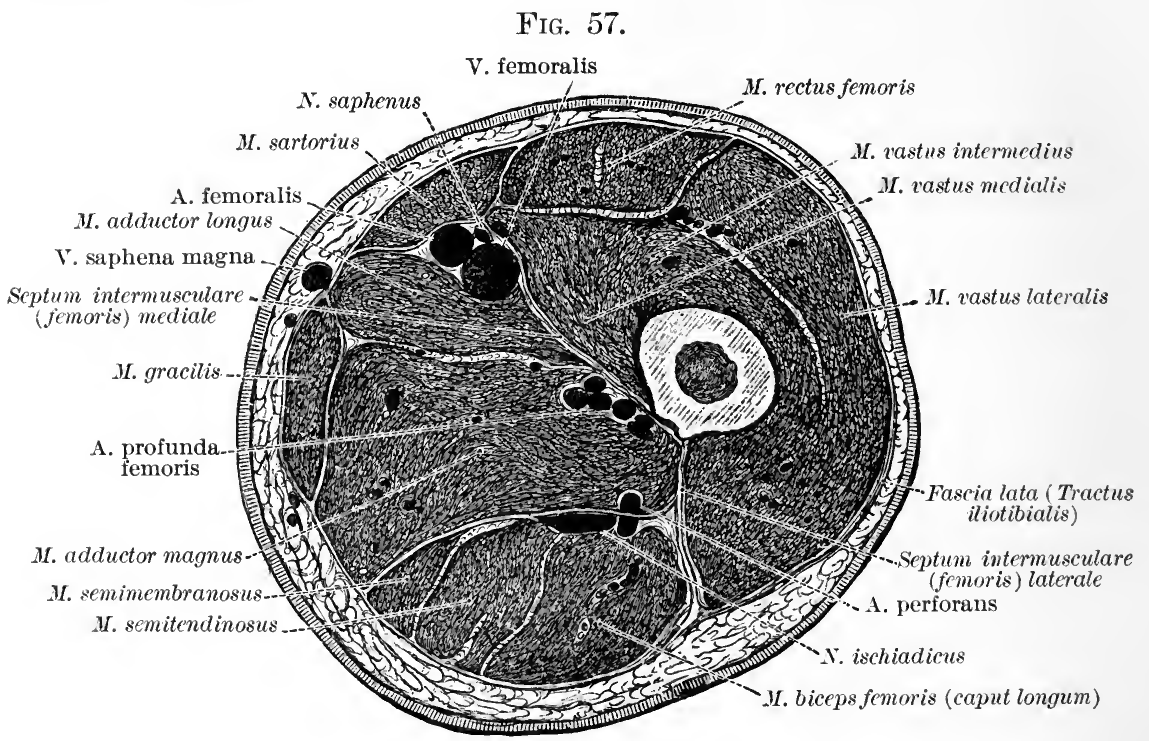

Cross-section of right thigh, a little above its middle. Surface distal to the cut. (After Toldt, Anat. Atlas, Wien, 1900, 2 Aufl., p. 640, Fig. 1020.)

(1) In the anterior osteofascial compartment (in front, between the medial and lateral septa) the extensor muscles and the femoral nerve.

(2) In the posterior osteofascial compartment (behind, between the medial and lateral septa), -

(a) Between the lateral and posterior septa, the flexor museles and the great sciatic nerve.

(b) Between the medial and posterior septa, the adductor muscles and the obturator nerve.

\section{Sheath for Femoral Vessels and Femoral Canal.}

Before proceeding to this dissection, the student should study thoroughly, on a dry preparation of the pelvis with its ligaments, the following:

(a) Inguinal ligament of Poupart (Lig. inguinale [Pouparti]).

(b) Lacunar. ligament of Gimbernat (Lig. lacunare [Gimbernati]).

After this, divide the cornu superius of the margo falciformis of the fossa ovalis and carry the knife lateralward just 
below Poupart's ligament to within two or two and a half centimetres of the spina iliaca anterior superior, so as to sever the attachment of this portion of the fascia lata from the ligament. Reflect the fascia and margo falciformis downward and lateralward. Carefully pick out the fat and deep subinguinal lymphglands immediately subjacent, so as to expose the connective tissue sheath of the femoral vessels. With the handle of the scalpel gently separate the sheath from Poupart's ligament in front and from Gimbernat's ligament medialward from it.

What is the shape of the femoral sheath? Note the points where it is perforated by-

(a) Lumbo-inguinal branch of genitofemoral nerve.

(b) Large saphenous vein.

(c) Lymph-vessels.

By a study of the cadaver, the use of models and a systematic text-book of anatomy, ascertain how it is that the anterior wall of the femoral sheath represents the continuation into the thigh of the fascia transversalis of the abdomen, the posterior wall of the sheath the continuation of the fascia iliaca of the abdomen.

Next open the femoral sheath by making three parallel and vertical incisions, the first over the femoral artery, the second over the femoral vein, and the third one centimetre medial from the second. The incisions all begin above at the Lig. inguinale [Pouparti], the medial one being one centimetre long, each of the other two from three to four centimetres long. Note the septa, dividing the sheath into three compartments (Fig. $58)$ -

(a) Lateral compartment.

It contains the $A$. femoralis and the $\mathrm{N}$. lumbo-inguinalis.

(b) Middle compartment.

It contains the V. femoralis.

(c) Medial compartment (femoral canal or canalis femoralis) (O. T. erural canal).

It contains a lymph-gland (Rosenmüller's lymph-gland), some lymphatic vessels, and loose areolar tissue.

Observe that the femoral sheath and its contents completely fill up the lacuna vasorum. How is the lacuna vasorum bounded? How is the lacuna musculorum bounded? What structures pass through the lacuna musculorum? This latter region will be dissected later.

Introduce the little finger into the canalis femoralis. How long is it? How is it bounded? Locate exact position of superior 
aperture,-the so-called "femoral ring" (annulus femoralis) (O. T. crural ring). What is directly medial and what directly lateral from this ring? How is it closed above? Can you find the femoral septum of Cloquet (septum femorale [Cloqueti])?

What is meant by femoral hernia? Why should it occur where it does and not elsewhere? Is it more common in males or in females, and why? What must have been the course of a hernia presenting over the fossa ovalis? In cutting down upon

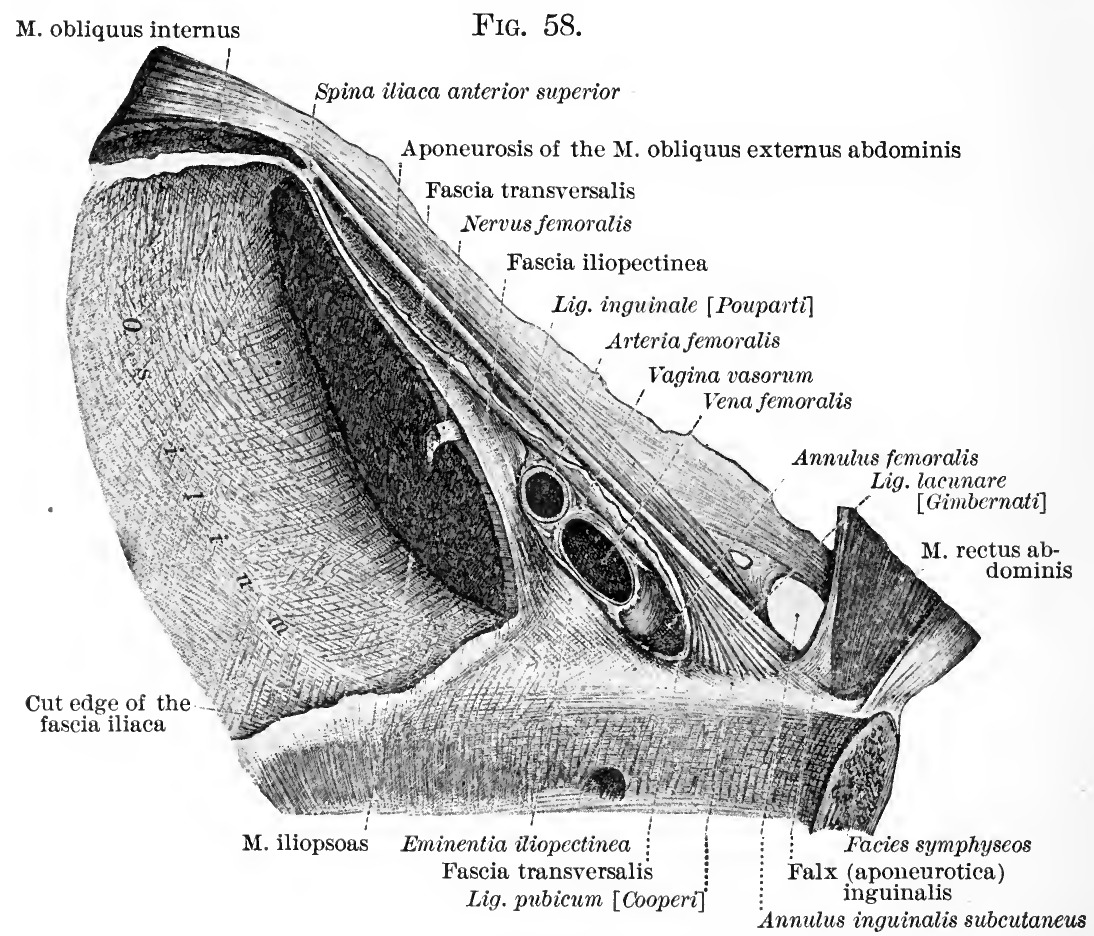

The lacuna musculorum and the lacuna vasorum of the left side, seen from the internal, medial side. (After Toldt, Anat. Atlas, Wien, 1900, 2 Aufl, p. 374, Fig. 616.)

it, what are the various coverings met with? Where is such a hernial sac most subject to constriction? How could such constriction be relieved by the knife? In cutting Gimbernat's ligament, what is to be remembered about the origin of the obturator artery?

Femoral Triangle, or Larger Fossa of Scarpa (Trigonum femorale [Fossa Scarpae major]) (O. T. Scarpa's Triangle).

Remove the fascia lata from the anterior aspect of the proximal third of the thigh. Do not disturb the fascia lata farther 


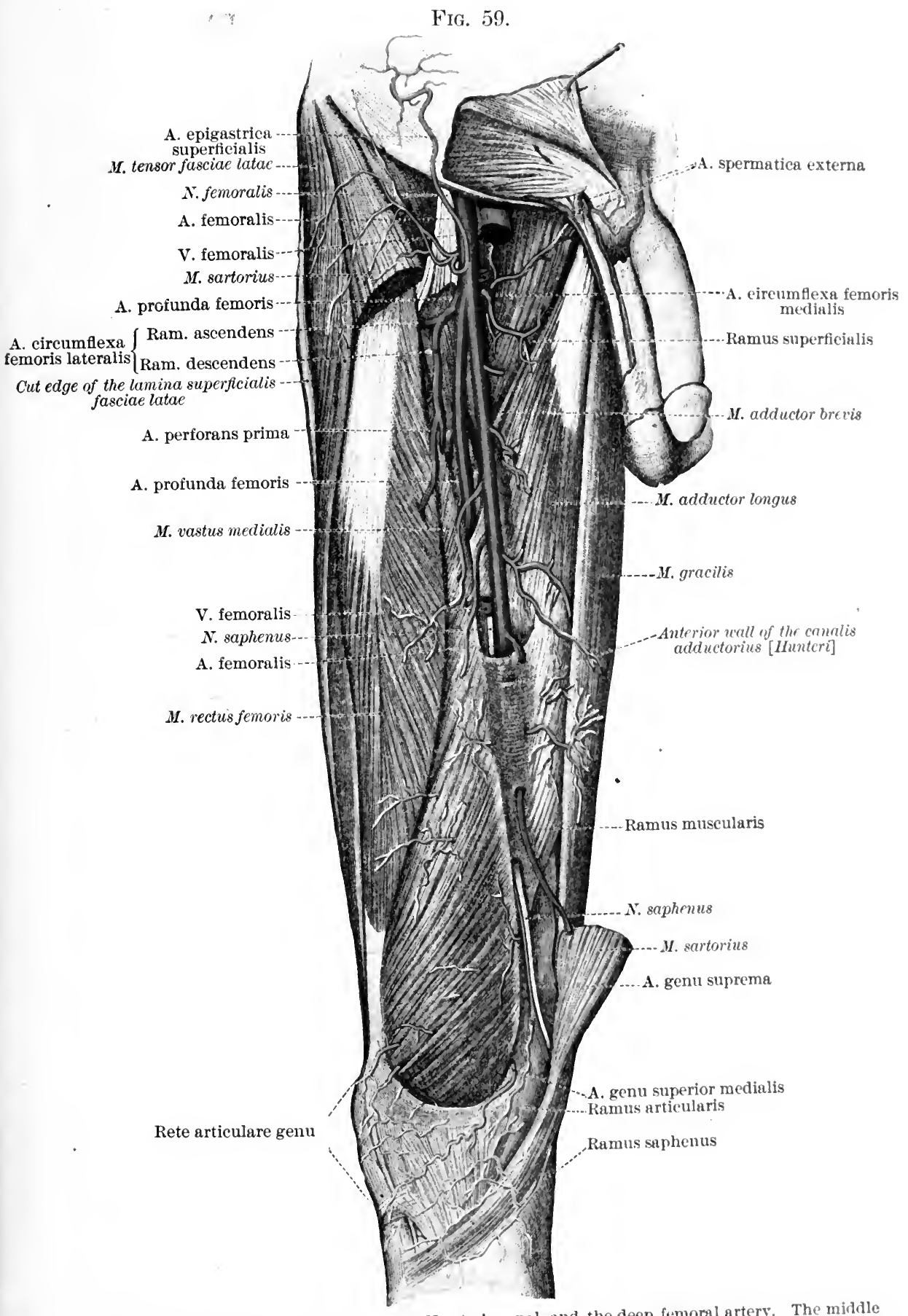

The femoral artery to its entrance into Hunter's canal, and the deep femoral artery. The middle segment of the M. sartorius has been taken away. (After Toldt, Anat. Atlas, Wien, 1900, 2 Aufl., p. 624, Fig. 1003.) 
distalward. The boundaries of the triangle may now be defined and its contents dissected out. Remove the femoral sheath and clean the vessels, nerves, and muscles related to the triangle.

How is the femoral triangle bounded above, lateralward, and medialward? How is its floor formed?

Among the contents find and study the following, comparing your findings with the descriptions in a systematic text-book.

Arteries. (Fig. 59.)

(a) Femoral artery (A. femoralis). Study carefully the relations of this artery in different parts of Scarpa's triangle.

(aа) Superficial epigastric artery (A. epigastrica superficialis).

$(a b)$ Superficial circumflex iliae artery (A. circumflexa ilium superficialis).

(ac) External pudendal arteries (Aa. pudendae externae) (0. T. superficial and deep external pudic arteries).

(ad) Inguinal rami (rami inguinales).

(ae) Muscular rami (rami musculares).

(af) Deep artery of thigh (A. profunda femoris).

(afa) Medial circumflex artery of thigh (A. circumflexa femoris medialis) (0. T. internal circumflex). (afb) Lateral circumflex artery of thigh (A. circumflexa femoris lateralis) (0. T. external circumflex). (afc) Superficial ramus (ramus superficialis).

Veins.

(a) Femoral vein ( $V$. femoralis).

(aa) Superficial epigastric vein ( $V$. epigastrica superficialis).

$(a b)$ Superficial circumflex iliac vein ( $V$. circumflexa ilium superficialis).

( $a c)$ Thoraco-epigastric vein ( $V$. thoraco-epigastrica).

(ad) External pudendal veins ( $V v$. pudendae externae) (O. T. external pudic veins).

(ae) Large saphenous vein (V. saphena magna) (O. T. internal saphenous).

(af) Deep vein of thigh ( $V$. profunda femoris).

(afa) Medial circumflex veins of thigh ( $V v$. circumflexae femoris mediales).

( $a f b)$ Lateral circumflex veins of thigh ( $V v$. circumflexae femoris laterales).

Nerves.

(a) Lumbo-inguinal nerve ( $N$. lumbo-inguinalis) (O. T. crural branch of genitocrural).

(b) Lateral cutaneous nerve of thigh ( $N$. cutaneus femoris lateralis) (O. T. external cutaneous).

How much of this nerve is in the triangle?

(c) Femoral nerve ( $N$. femoralis) (O. T. anterior erural).

What is the relation of the branches of this nerve, given off in this fossa, to the A. circumflexa femoris lateralis? 
Sartorius Muscle and Hunter's Adductor Canal (M. sartorius et Canalis adductorius [Hunteri]). (Figs. 59 and 60.)

Remove the fascia lata from the distal two-thirds of the thigh, leaving, however, the iliotibial band on the lateral surface. Clean the M. sartorius carefully, watching for the nerves which are near it or pass through it. Study its form, position, origin, insertion, action, and innervation. Whence has it received its name?

What is meant by "Hunter's canal" or the "adductor" canal" (canalis adductorius [Hunteri])? Note that it is the continuation distalward of the lacuna vasorum. How is the

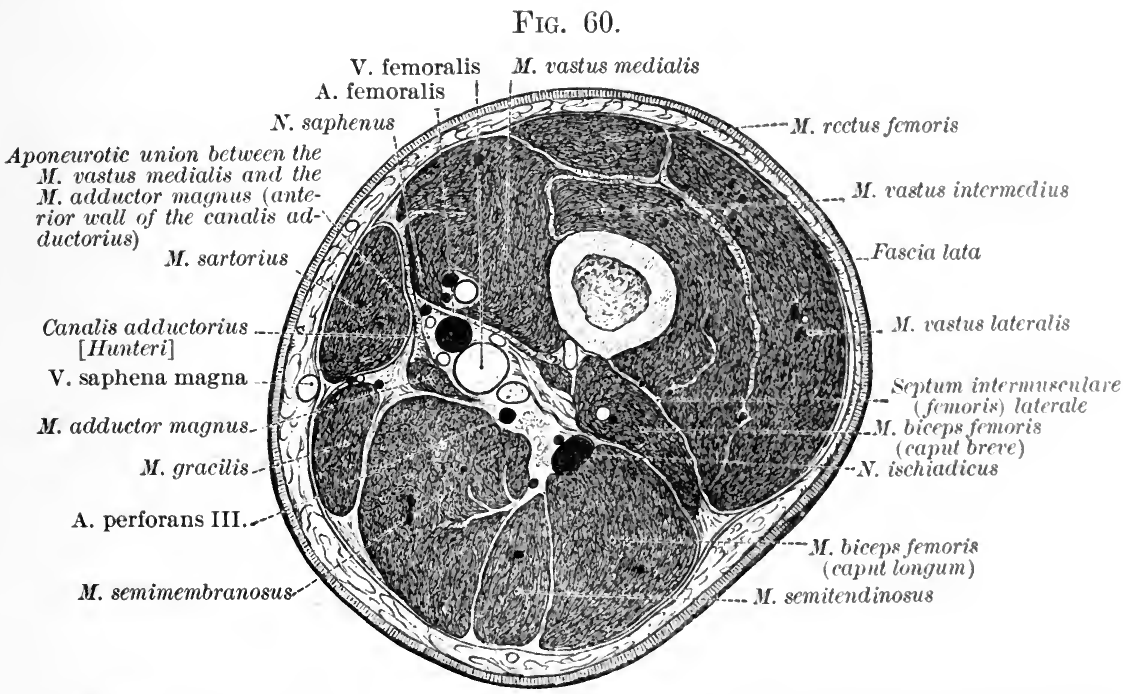

Cross-section of the right thigh through Hunter's canal, a little above the opening in the adductor magnus musele. Surface distal to the section. (After Toldt, Anat. Atlas, Wien, 1900, 2 Aufl., p. 6il, Fig. 1021.)

canal or tunnel formed? Study the constituents of the walls of the canal. Cut through the fibrous expansion forming the anterior wall of the adductor canal and study the contents of the canal. How do the femoral vessels leave the canal to enter the popliteal space? Describe the opening in the M. adductor magnus (hiatus tendineus adductorius). At what point do the $\mathrm{N}$. saphenus and the A. genu suprema leave the canal? Study the lower part of the A. femoralis and its relations. Examine the following branches:

(a) Muscular rami (rami musculares). What museles are supplied by these? 
(b) Highest artery of knee (A. genu suprema) (O. T. anastomotica magna).

(ba) Saphenous ramus (ramus saphenus).

(bb) Muscular ramus (ramus muscularis).

(bc) Articular rami (rami articulares).

Review now the femoral vein throughout its whole course, examining its various tributaries. How does its relative position as regards the femoral artery change as it proceeds distalward towards the hiatus tendineus adductorius? With scissors slit the vein open along its whole length and look for valves. The femoral vein is often thrombosed in the course of typhoid fever, or in pelvic infections, especially during the puerperal period (phlegmasia alba dolens).

\section{Femoral Nerve and Muscles of Front of Thigh. (Fig. 6r.)}

Clean the muscles of the front of the thigh carefully and follow the nerve branch to each.

Study thoroughly the femoral nerve (N. femoralis) (O. T. anterior crural) and all its branches, cutaneous and muscular. What are these and what do they supply? Does the femoral nerve innervate joints?

Study the form, position, origin, insertion, action, and innervation of each of the following muscles:

(a) Tensor muscle of fascia lata (M. tensor fasciae latae) (O. T. tensor vaginae femoris). Study exact relation to-

(aa) Iliotibial band (tractus iliotibialis [Maissiati]).

Cut through the iliotibial band below the tensor muscle, deflect it forcibly lateralward, and displace the M. vastus lateralis medialward so as to expose the lateral intermuscular septum (septum intermusculare [femoris] laterale). How is it attached at its two edges? What important structures perforate it?

Examine also the medial intermuscular septum (septum intermusculare [femoris] mediale). Which is the stronger, the medial or the lateral septum?

(b) Quadriceps ("four-headed") muscle of thigh (M. quadriceps femoris).

(ba) Rectus ("straight") muscle of thigh (M. rectus femoris). (baa) Bursa $M$. recti femoris.

(bb) Vastus lateralis muscle ("lateral great muscle") (M. vastus lateralis) ( $\mathrm{O} . \mathrm{T}$. vastus externus).

(bc) Vastus medialis muscle ("medial great muscle") (M. vastus medialis) (O. T. vastus internus).

(bd) Vastus intermedius muscle ("intermediate great muscle") (M. vastus intermedius) (O. T. erureus).

Cut through the $M$. rectus femoris at its middle and reflect distal end forcibly. Find the groove between the M. 
FIG. 61.

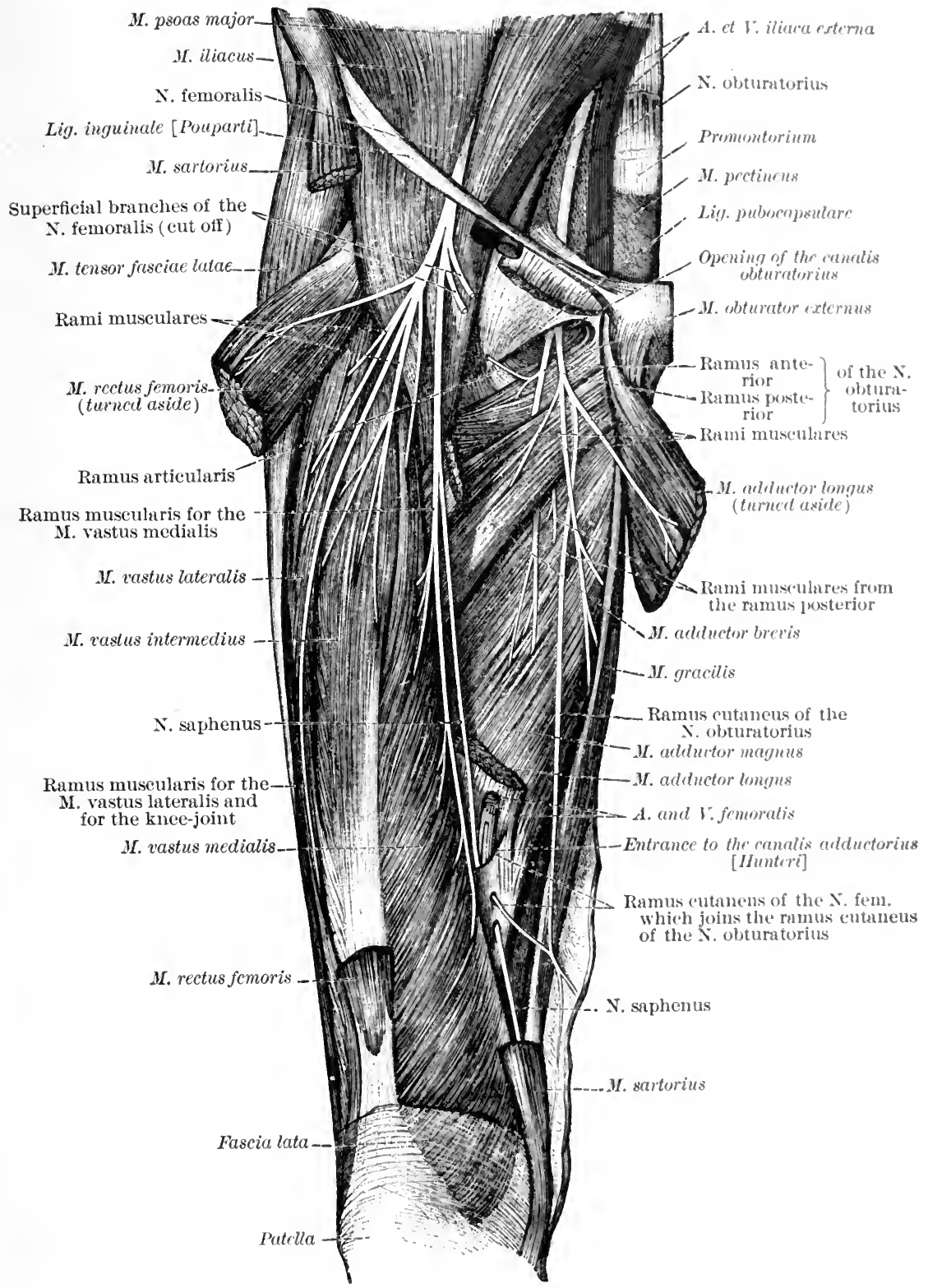

The branching of the femoral and obturator nerves seen from in front, after partinl removal of the $\mathrm{Im}$. sartorius, rectus femoris, adductor longus, and pectineus. (After Toldt, Alat. Atlas, Wien, 1903, 3 Aufl., p. 839, Fig. 1275.) 
vastus medialis and the M. vastus intermedius and follow it proximalward. Observe the nerve to the M. articularis genu ruming along the medial margin of the M. vastus intermedius. Cut through the body of the M. vastus medialis transversely five centimetres above the patella and reflect it medialward. Examine carefully the origin of the M. vastus medialis.

(be) Articular musele of knee (M. articularis genu) (O. T. subcrureus).

Make a vertical incision through the M. vastus intermedius so as to expose the M. articularis genu. What is the relation of the tendon of insertion of the latter muscle to the capsule of the knee-joint?

Study the relations of the various constituents of the M. quadriceps femoris to the patella and the ligamentum patellae. Examine the retinacula patellae mediale et laterale. When the patellar tendon is struck during life, what constituents of the M. quadriceps femoris contract most? Try it on yourself at home with the thigh exposed.

\section{MEDIAL SURFACE OF THIGH (FACIES MEDIALIS FEMORIS).}

\section{Long Adductor Muscle (M. adductor longus).}

Study its form, position, origin, insertion, action, and innervation. Then divide it near its origin and reflect it lateralward. Avoid injury to the ramus anterior of the N. obturatorius.

\section{Deep Artery of Thigh (A. profunda femoris) and M. Pectineus.}

The beginning of this artery and some of its branches have already been studied in the femoral triangle of Scarpa. Separate the aponeurotic tendon of the $\mathrm{M}$. adductor longus from the M. vastus medialis in front and the M. adductor magnus behind, and study the deeper portions of the deep artery and vein of the thigh ( $A$. and $V$. profunda femoris). The perforating branches of the artery deserve especial attention (cf. Fig. 54, p. 137):

(a) First perforating artery (A. perforans prima).

(aa) Superior nutrient artery of femur (A. nutricia femoris superior).

(b) Second perforating artery (A. perforans secunda).

(c) Third perforating artery (A. perforans tertia).

(ca) Inferior nutrient artery of femur ( $A$. nutricia femoris inferior). 
The pectineus muscle ( $M$. pectincus) should now be carefully studied. Ascertain its exact form, position, origin, insertion, action, and innervation. Note that it is covered by the fascia pectinea (O. T. pubic portion of fascia lata). Observe the bursa M. pectinei.

\section{Short Adductor Muscle (M. adductor brevis), Medial Circumflex Artery, and Obturator Nerve.}

Cut through the M. pectineus at its origin and reflect it distalward and lateralward. Avoid injury to the ramus anterior of the N. obturatorius. Is an accessory obturator nerve present? Dissect out the branches of the medial circumflex artery (A. circumflexa femoris medialis) in this region and review the artery as a whole. Follow especially-

(a) Superficial ramus (ramus superficialis).

(b) Deep ramus (ramus profundus).

(c) Acetabular ramus (ramus acetabuli).

Clean the short adductor muscle (M. adductor brevis) and study its form, position, origin, insertion, action, and innervation. Then cut through it at its origin and reflect it distalward and lateralward. Now dissect out the posterior ramus of the obturator nerve $(N$. obturatorius) and study the nerve as a whole. How does it get into the thigh? What muscles receive their motor innervation from it? How do the anterior and posterior rami differ in their relations? Which sends a branch to the hipjoint? Which to the knee-joint? How is the obturator innervation of the hip and knee of interest in hip-joint disease?

\section{Other Muscles on Medial Side of Thigh.}

Study the form, position, origin, insertion, action, and innervation of the following:

(a) Gracilis ("slender") muscle ( $M$. gracilis).

(aa) Bursa M. sartorii propria.

(ab) Bursa anserina.

(b) Smallest adductor muscle (M. adductor minimus) (O. T. upper portion of adductor magnus).

(c) Great adductor muscle ( $M$. adductor magnus). (ca) Hiatus tendineus adductorius (already studied).

Observe its double nerve-supply.

Detach $(b)$ and $(c)$ at their origins and reflect them.

(d) External obturator muscle (M. obturator externus).

(e) Psoas major ("larger lumbar") muscle (M. psoas major).

( $f$ ) Iliac muscle (M. iliacus).

The conjoined tendon of $(e)$ and $(f)$ may now be studied. 
Obturator Artery (A. obturatoria). (Cf. Spalteholz, Fig. 469.)

Reflect the M. obturator externus and in it and beneath it find the following branches of the obturator artery:

(a) Anterior ramus (ramus anterior).

(b) Posterior ramus (ramus posterior).

(ba) Artery of acetabulum (A. acetabuli).

\section{HIP-JOINT (ARTICULATIO COXAE).}

Cut through the A. femoralis, V. femoralis, and N. femoralis two or three centimetres below Poupart's ligament; tie the dis-

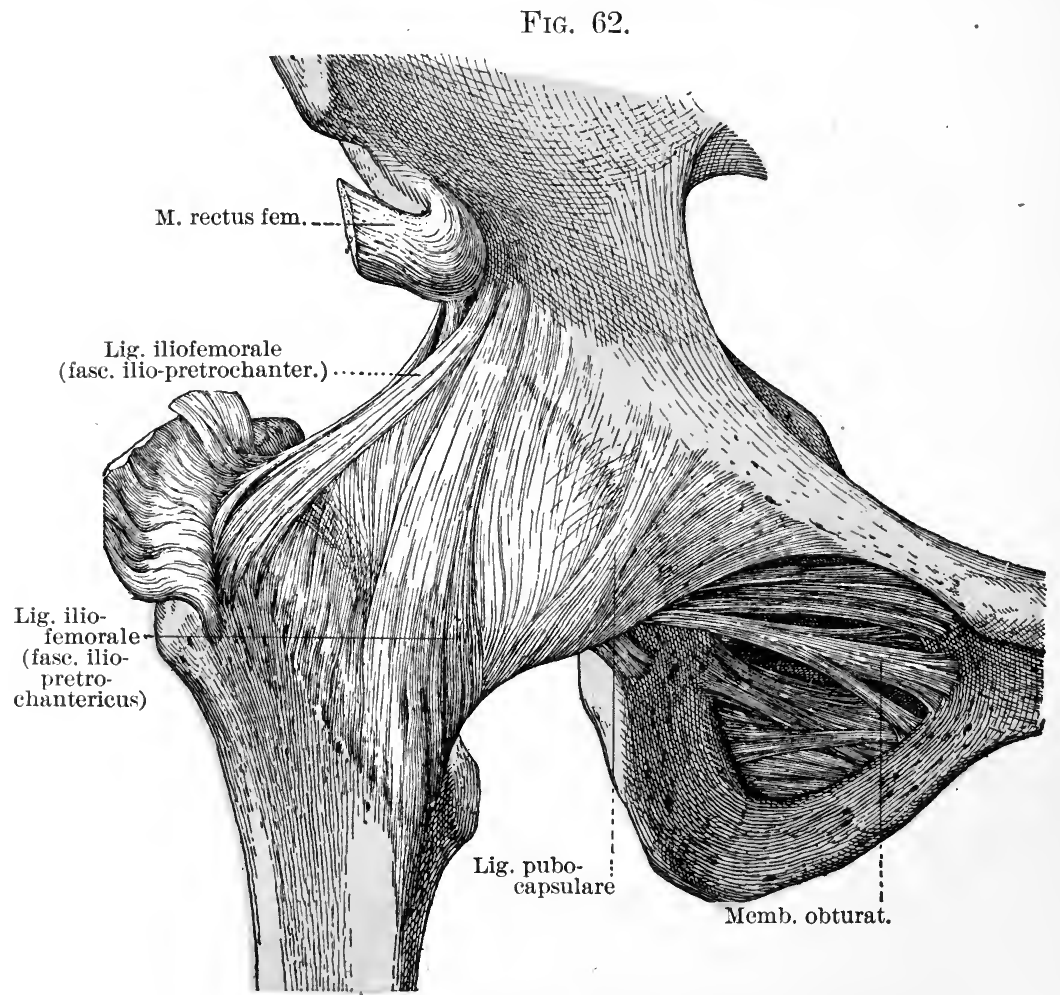

Articulatio coxae, anterior view. (From Poirier et Charpy, Traité d'Anat. hum., Paris, 1899, 2 ed., t. i. p. 716, Fig. 721.)

tal stumps together and reflect them distalward. Then cut through the M. sartorius and M. rectus femoris five centimetres from their origin and reflect them. Cut away the conjoined tendon of the M. iliacus and M. psoas at its insertion and turn it proximalward. Examine the bursa iliopectinea, the bursa $M$. 
recti femoris, and the bursa iliaca subtendinea. Reflect the M. tensor fasciae latae. Clean the outer surface of the hip-joint.

The joint is an enarthrosis. What is meant by this? What bony surfaces are concerned?

Study the following ligaments (Figs. 62 and 63):

(a) Joint-capsule (capsula articularis).

(b) Strengthening ligaments.

(ba) Iliofemoral ligament (ligamentum iliofemorale) (O. T. Yshaped ligament of Bigelow).

(baa) Superior fasciculus to tubercle in front of great trochanter (Lig. iliofemorale superius).

FIG. 63.

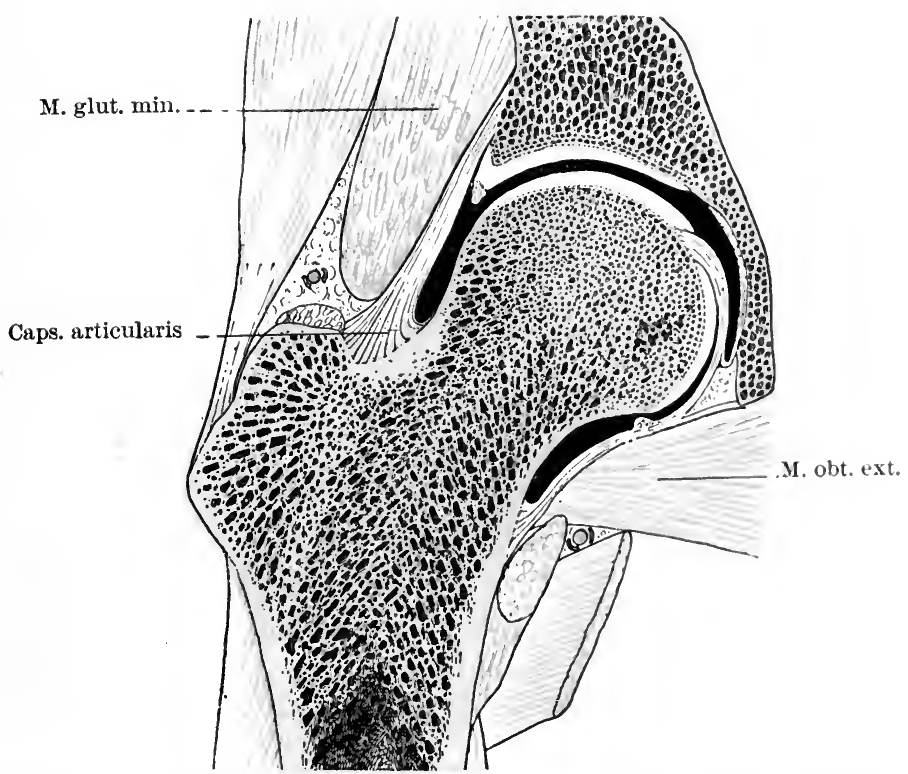

Frontal section of the articulatio coxae, passing through the fovea capitis femoris. (From Poirier et Charpy, Traité d'Anat. hum., Paris, 1899, 2 ed., t. i. p. 725, Fig. 729.)

(bab) Anterior or inferior fasciculus to tubercle in front of small trochanter (Lig. iliofemorale anterius).

This is perhaps the strongest ligament in the body. It will resist a strain of from two hundred and fifty to seven hundred and fifty pounds.

(bb) Ischiocapsular ligament (ligamentum ischiocapsulare) (O. T. ischiocapsular band).

(bc) Pubocapsular ligament (ligamentum mubocapsulare) (O. T. pubocapsular band or pubofemoral ligament).

(bd) Orbicular zone (zona orbicularis) (O. T. zonular band or ring ligament). 
Make the maximal movements in the joint in the direction of (1) extension, (2) flexion, (3) adduction, (4) abduction, (5) rotation medialward, (6) rotation lateralward, (7) circumduction. How are the ligaments affected in each instance? What muscles are concerned in each movement in the living body?

Open the articulation. Remove the whole capsule first, with the exception of the ligamentum iliofemorale. Test the strength of this and then remove it.

Study each of the following ligaments:

(a) Glenoid lip (labrum glenoidale) (O. T. cotyloid ligament).

(b) Transverse ligament of acetabulum (ligamentum transversum acetabuli).

(c) Round ligament of the femur (ligamentum teres femoris).

This would be better designated "triangular ligament," or, better still, Lig. intrarticulare coxae, a name suggested by Fick. It is probable that it is a vestige of a pubofemoral muscle the tendon of which has become invaginated into the joint.

Study the blood supply and nerve supply of the joint. (Cf. Poirier et Charpy, t. i., Figs. 731 and 732.) Follow the reflections of the synovial membrane.

Cut through the ligamentum teres, remove the lower extremity from the trunk, and continue the dissection at a side-table.

\section{LEG AND FOOT.}

Make the following incisions: (1) an incision distalward along the middle line of the leg and dorsum of the foot, in front, extending as far as the base of the middle toe; (2) transverse incisions extending $(a)$ across the ankle and $(b)$ across the bases of the toes. Reflect the flaps thus formed, taking no fat with the skin.

\section{Superficial Fascia.}

Note the characteristics of this fascia and determine with what fasciae it is continuous above and below. Dissect out the following:

Veins. (Vide Spalteholz, Figs. 507-509.)

(a) Dorsal digital veins of foot ( $V v$. digitales dorsales pedis).

(b) Intercapitular veins ( $V v$. intercapitulares).

(c) Common digital veins of foot ( $V v$. digitales communes pedis).

(d) Dorsal venous arch of foot (arcus venosus dorsalis pedis (cutaneus)).

(e) Dorsal cutaneous venous network of foot (rete venosum dorsale pedis cutaneum). 
( $f$ ) Lateral marginal vein ( $V$. marginalis lateralis).

(g) Medial marginal vein ( $V$. marginalis medialis).

Trace these veins proximalward and determine their relation to the large saphenous vein ( $V$. saphena magna) and the small saphenous vein (V. saphena parva).

Nerves. (See Fig. 56, p. 143.)

Refer frequently to charts in the study of cutaneous nerves and determine the exact area of skin supplied by each.

(a) Saphenous nerve (N. saphenus).

(aa) Medial cutaneous branches of leg (rami cutanei cruris medialis).

(b) Branches of lateral cutaneous nerve of calf ( $N$. cutaneus surae).

(c) Superficial peroneal nerve ( $N$. peronaeus superficialis).

(d) Terminal twigs of deep peroneal nerve ( $N$. peronaeus profundus).

$(e)$ Dorsal digital nerves ( Nn. digitales dorsales).

( $f$ ) Lateral dorsal cutaneous nerve ( $N$. cutaneus dorsalis lateralis), from the $\mathrm{N}$. tibialis.

Carefully remove the superficial fascia, preserving the structures which you have dissected, and expose the deep fascia.

\section{Deep Fascia of the Leg (Fascia cruris). (Figs. 64 and 67.)}

Observe that this fascia is continuous with the fascia lata; note also its shiny, aponeurotic character just below the knee, where it gives origin to muscles. Trace the fascia downward, noting that it tends to become thinner distalward, but is strengthened just above the ankle to form the transverse ligament of the leg (ligamentum transversum cruris) ( $\mathrm{O}$. T. upper or broad part of anterior annular ligament). Note the exact points of attachment of the ligament and observe that it sends a septum to the tibia, thus forming two compartments, one medial for the M. tibialis anterior, one lateral for the M. extensor longus hallucis and M. extensor longus digitorum. The fascia cruris becomes continuous below with the dorsal fascia of the foot (fascia dorsalis pedis), the latter being continuous at the sides of the foot with the plantar aponeurosis (aponeurosis plantaris). In the fascia dorsalis pedis examine the Lig. cruciatum cruris ( $O$. T. lower part of anterior annular ligament). Determine the exact attachments of the two limbs of the $\mathrm{Y}$ of this ligament, and their relations to the tendons of the muscles over which they pass. A good description is given in Spalteholz's Atlas, 1). 356. Observe the band passing from the lateral malleolus to the posterior prominence of the os calcis; this is the retinaculum $\mathrm{Im}$. peronaeorum superius (O. T. external annular ligament). (Fig. 67.) 
Incise now the deep fascia longitudinally, midway between the tibia and fibula, but do not cut through the Lig. transversum cruris or the Lig. cruciatum cruris. Note that the fascia becomes continuous medialward with the periosteum of the tibia, and that lateralward it sends a septum into the crista anterior of the fibula, forming the anterior [fibular] intermuscular septum (septum intermusculare anterius [fibulare]) (O. T. anterior peroneal septum). This latter septum separates the contents of the " anterior tibiofibular compartment" of the leg from the contents of the "peroneal compartment." The latter is bounded posteriorly by another septum going into the crista lateralis of

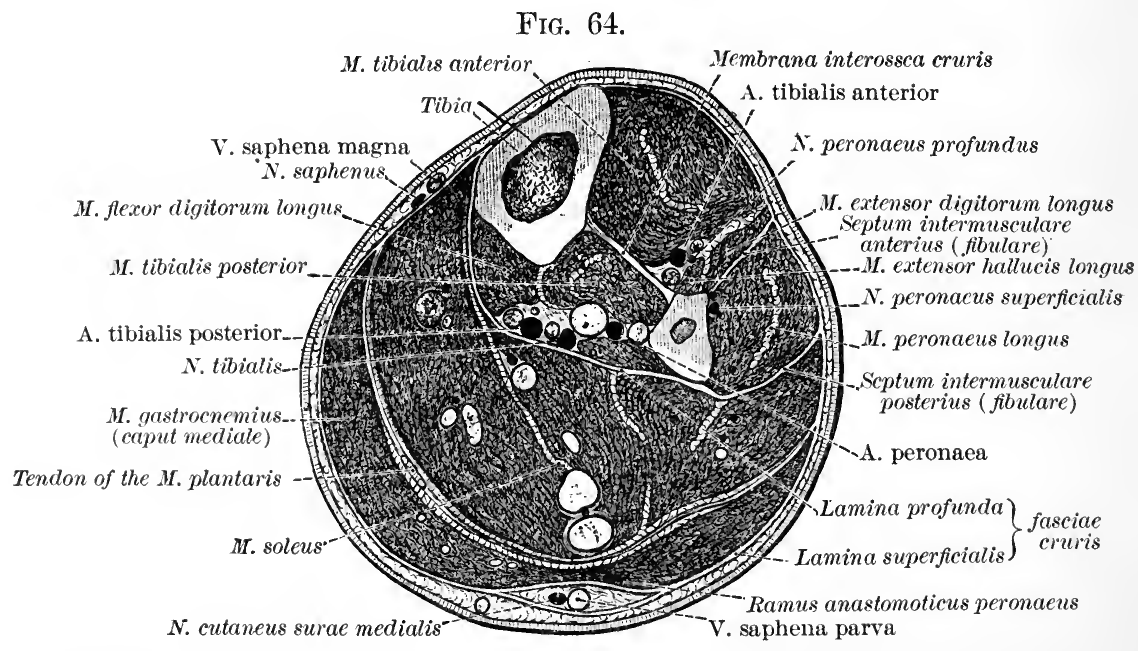

Cross-section of the right leg a little above its middle. Surface distal to the cut. (After Toldt, Anat. Atlas, Wien, 1900, 2 Aufl., p. 642, Fig. 1023.)

the fibula and known as the posterior intermuscular septum (septum intermusculare posterius) (O. T. posterior peroneal septum). It will be studied later. Examine Fig. 64 carefully.

\section{Muscles of Anterior Tibiofibular Region.}

Use cross-sections to complete this study. Study the contents of the anterior tibiofibular osteofascial compartment. Clean carefully each muscle and determine its form, position, origin, insertion, action, and nerve supply. What are flexion and extension of the foot? Study-

(a) Anterior tibial muscle ( $M$. tibialis anterior) (O. T. tibialis anticus).

(b) Long extensor of digits (M. extensor digitorum longus). 
Fig. 65.

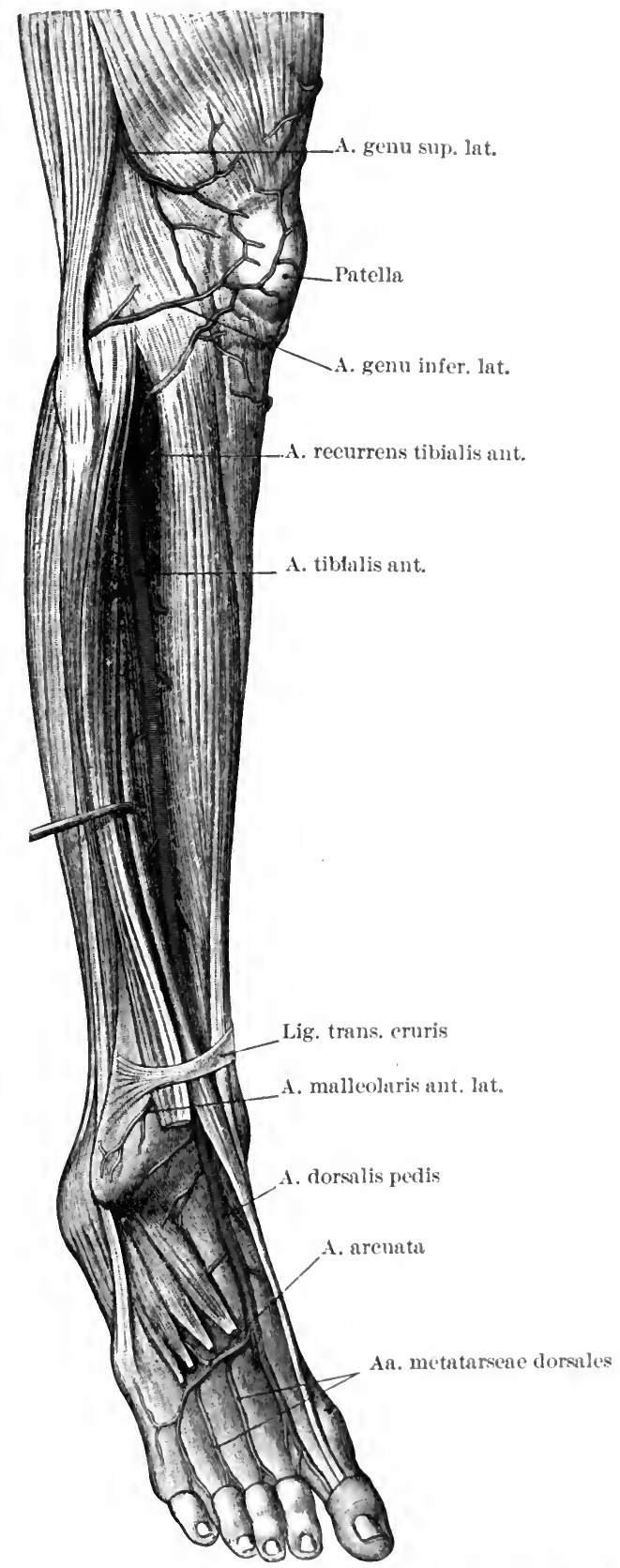

Arteries of the anterior part of the leg. The $\mathrm{Im}$. ext. hallucis long. and ext. dig. long. are drawn aside and the M. ext. dig. brevis is divided. (From Gegenbaur, Lehrb. der Anat, des Mensch., Leipzig, 1899, 7 Aufl., Bd. ii. p. 294, Fig. 547.) 
(c) Long extensor of great toe (M. extensor hallucis longus).

(d) Third peroneal muscle ( $M$. peronaeus tertius).

Examine the vagina tendinis M. tibialis anterioris, the vagina tendinis M. extensoris hallucis longi, and the vagina tendinum M. extensoris digitorum longi.

\section{Arteries of Anterior Tibiofibular Region and Dorsum of Foot.}

Separate the M. extensor digitorum longus from the M. tibialis anterior and expose the following; at the same time dissect out the structures on the dorsum of the foot.

(a) Anterior tibial artery (A. tibialis anterior). (Vide Fig. 65, and Spalteholz, Fig. 479.) How does this artery pass to the anterior compartment of the leg, and what is its relation to the membrana interossea cruris?

(aa) Posterior recurrent tibial artery (A. recurrens tibialis posterior).

(ab) Anterior recurrent tibial artery (A. recurrens tibialis anterior).

(ac) Lateral anterior malleolar artery (A. malleolaris anterior lateralis) ( $\mathrm{O} . \mathrm{T}$. external malleolar).

(ad) Medial anterior malleolar artery (A. malleolaris anterior medialis) ( $\mathrm{O} . \mathrm{T}$. internal malleolar).

(ae) Medial malleolar network (rete malleolare mediale).

(af) Lateral malleolar network (rete malleolare laterale).

The continuation of the anterior tibial artery from in front of the ankle-joint is known as the-

(b) Dorsal artery of the foot (A. dorsalis pedis).

(ba) Lateral tarsal artery (A. tarsea lateralis).

(bb) Medial tarsal arteries (Aa. tarseae mediales).

(bc) Arcuate artery (A. arcuata).

(bd) Dorsal network of foot (rete dorsale pedis).

(be) Dorsal metatarsal arteries (Aa. metatarseae dorsales).

(bf) Deep plantar branch (R. plantaris profundus).

\section{Nerves of Anterior Region of Leg and Dorsum of Foot.}

(a) Deep peroneal nerve ( $N$. peronaeus profundus) (O. T. anterior tibial).

(aa) Muscular branches (rami musculares).

(ab) Dorsal digital nerves to lateral surface of hallux and to medial surface of digit II. (Nn. digitales dorsales hallucis lateralis et digiti secundi medialis).

Determine how the deep peroneal reaches the anterior surface of the leg. Does it accompany the artery? What area of the skin does it supply? What muscles are supplied by this nerve?

(b) Superficial peroneal nerve (N. peronaeus superficialis) (O. T. musculocutaneous).

(ba) Muscular branches (rami musculares).

(bb) Medial dorsal cutaneous nerve ( $N$. cutaneus dorsalis medialis). 
(bc) Intermediate dorsal eutaneous nerve (N. cutaneus dorsalis intermedius).

$(b d)$ Dorsal digital nerves of the foot $(N n$. digitales dorsales pedis).

The proximal part of the N. peronaeus superficialis will be studied later.

FIG. 66.

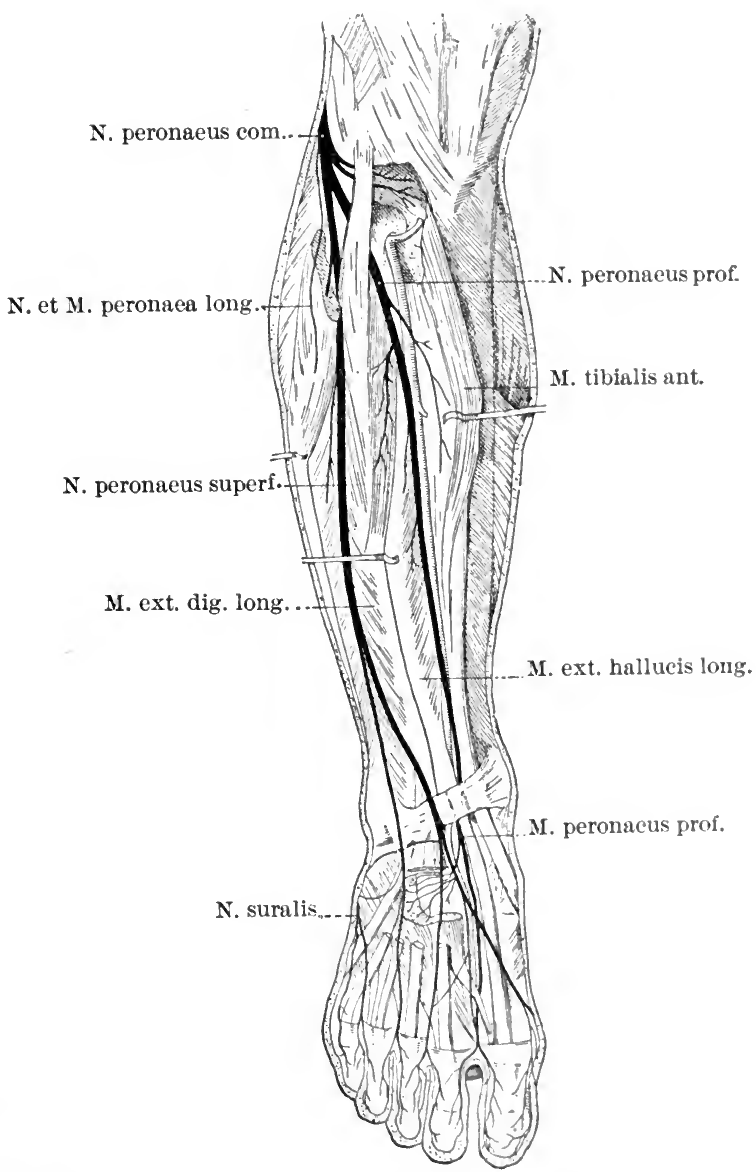

Nn. peronaei superficialis et profundus (after Hirschfeld). (From I'oirier et Charpy,

Traité d'Anat. hum., Paris, 1s99, t. iii. p. 112., Fig. 5xt.)

\section{Muscles of Dorsum of Foot.}

Dissect these out carefully, studying the form, position, origin, insertion, action, and innervation of each. (Vide Fig. 67 and Spalteholz, Fig. 402.) 
Fig. 67.

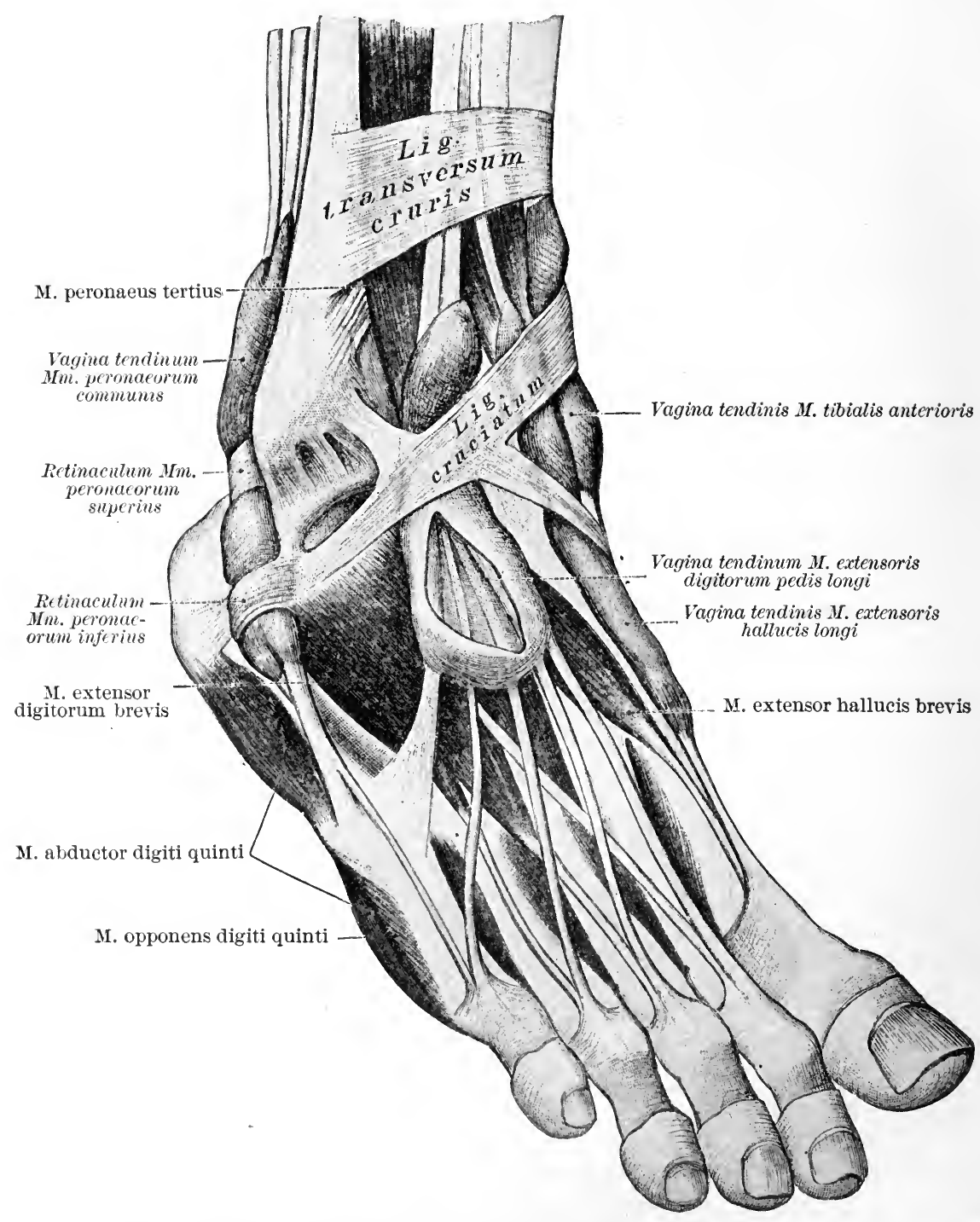

The museles on the dorsum and lateral side of the foot. The sheaths of the tendons on the dorsum pedis and in the lateral retromalleolar region have been injected with strong alcohol. (After Toldt, Anat. Atlas, Wien, 1900, 2 Aufl., p. 356, Fig. 597.) 
(a) Short extensor of great toe (M. extensor hallucis brevis).

(b) Short extensor of digits ( $M$. extensor digitorum brevis).

Determine the relation of these muscles to the tendons of the long extensor of the digits. What is their action? Have they homologues in the hand?

(c) Dorsal interosseous muscles (Mm. interossei dorsales).

Compare these with similar muscles found in the hand, as regards origin, insertion, action, etc.

Where are the bursae intermetatarsophalangeae situated?

\section{LATERAL OR PERONEAL REGION OF LEG (REGIO CRURIS LATERALIS).}

The superficial fascia of this region has already been studied. An anterior intermuscular septum (septum intermusculare anterius [fibulare]) has been found passing from the deep fascia into the crista anterior fibulae. Incise the deep fascia longitudinally in this region; reflect it posteriorly and note the corresponding posterior [fibular] intermuscular septum (septum intermusculare posterius [fibulare]) going to the crista lateralis fibulae. A lateral or peroneal osteofascial compartment is thus formed. Dissect its contents and supplement the dissection by the use of cross-sections made at different levels; note carefully the alterations in the relations of the different contents of the compartment at different levels. Study the following:

\section{Peroneal Muscles and Retinacula.}

Clean each muscle carefully, defining well its borders and noting the form, position, origin, insertion, action, and innervation of each.

(a) Long peroneal muscle (M. peronaeus longus). What action does this muscle have as regards the arch of the foot?

(b) Short peroneal muscle (M. peronaeus brevis).

Trace the tendons of both muscles distalward into the foot. What are their relative positions in the fossa retromalleolaris lateralis? (Cf. Fig. 68.) What is the trochlear process (processus trochlearis) of the os calcis? Fxamine the bony foot and determine the position of the peroneal sulcus (sulcus M. peronaei [longi]).

Define next the retaining bands of the peroneal muscles (retinacula). (Cf. Spalteholz's Atlas, Fig. 409.) 
(a) Retinaculum Mm. peronaeorum superius (O. T. external annular ligament).

(b) Retinaçulum Mm. peronaeorum inferius.

What is the relation of the latter to the ligamentum cruciatum cruris? (See Fig. 67.)

Dissect out carefully the common synovial sheath of the peroneal muscles (vagina tendinum Mm. peronaeorum communis).

Fig. 68.

M. tibialis ant. A. et V. tibial. ant. and N. peronaeus prof.

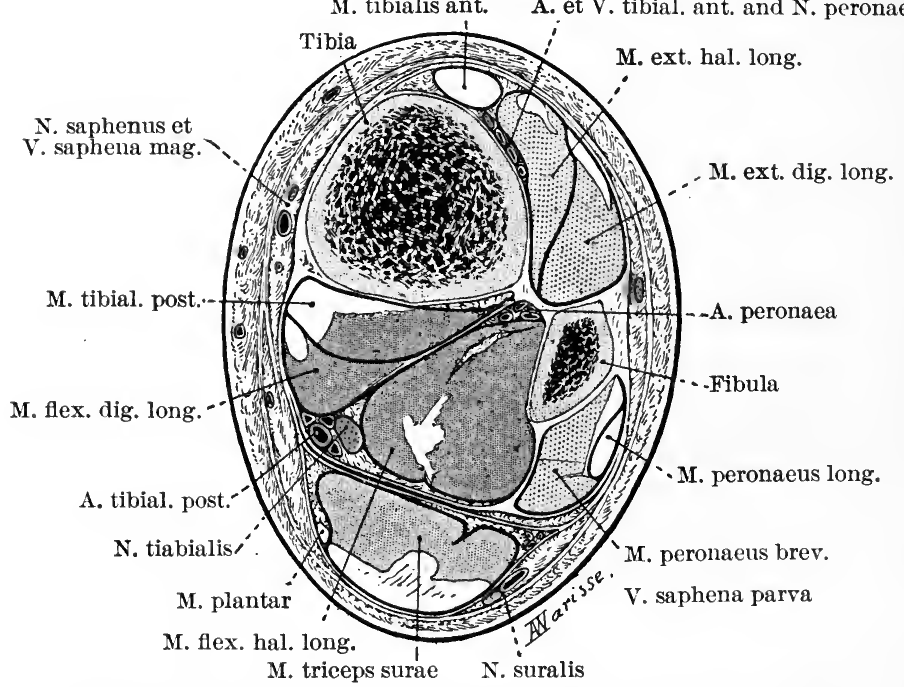

Cross-section passing through the inferior part of the leg. Right side, segment distal to the line of section.

Anterolateral region (lighter shade).--The two groups, anterior and lateral, are plainly separated. The M. tiabialis ant. has become tendinous and accompanies the A. tibialis ant. The fleshy fibres of the M. peronaeus long. have disappeared. The M. peronaeus brev. is attached to the corresponding surface of the fibula. The N. peronaeus superf. has emerged from between the two muscles, but runs beneath the fascia cruris, which it is about to perforate.

Posterior region (darker shade).-There is to be noted a displacement of the muscles. The M. tibialis post. has left the space between the flexors and, gaining the medial aspect of the leg, passes beneath the tendon of the M. flex. dig. long.

Superficial laver (intermediate shade).-The tendo calcaneus [Achillis] receives the lowest fibres of the M. soleus. (From Poirier et Charpy, Traité d'Anat. hum., Paris, 1899, 2 ed., t. ii. p. 268, Fig. 196.)

What is its extent proximalward and distalward? This sheath may be seen to better advantage if injected. Note here also the subcutaneous bursa over the lateral malleolus (bursa subcutanea malleoli lateralis).

\section{Peroneal Nerves.}

Trace the course of the N. peronaeus communis and dissect out all the branches: 
(a) Common peroneal nerve ( $N$. peronaeus communis) (O. T. external popliteal nerve).

(aa) Superficial peroneal nerve (N. peronaeus superficialis) (O. T. musculocutaneous nerve).

(ab) Deep peroneal nerve ( $N$. peronaeus profundus) (O. T. anterior tibial nerve).

What muscles are supplied by the superficial peroneal nerve? What cutaneous area? Review at this stage of the dissection the distribution of the deep peroneal nerve. What are the homologues of these nerves in the forearm?

\section{MEDIAL REGION OF LEG (REGIO CRURIS ME- DIALIS) (O. T. TIBIAL REGION).}

The student has but little to examine here, as the tibia is subcutaneous. Study-

(a) Tendons of insertion of Mm. sartorius, gracilis, and semitendinosus.

(b) Great saphenous vein ( $V$. saphena magna) (O. T. internal saphenous vein).

(c) Branches of saphenous nerve (N. saphenus) (O. T. internal saphenous nerve).

(ca) Infrapatellar branch (ramus infrapatellaris).

(cb) Medial cutaneous rami of leg (rami cutanei cruris mediales).

(d) Medial inferior artery of knee (A. genu inferior medialis) (O. T. inferior internal articular artery).

(e) Tibial collateral ligament of knee-joint (Lig. collaterale tibiale) (O. $\mathrm{T}$. internal lateral ligament).

\section{POSTERIOR REGION OF LEG AND HEEL (REGIO CRURIS POSTERIOR ET REGIO CALCANEA) (O. T. POSTERIOR TIBIOFIBULAR REGION).}

Note how the leg tapers from above downward. The large, convex, fleshy upper half is known as the calf (sura). The lower half rapidly diminishes in size, so that the tibia and fibula may be felt as they pass into their respective malleoli. Behind each malleolus will be found a fossa. Flex the foot and extend it and note the play of tendons in each fossa. These fossae become obliterated in pathological processes involving the synovial membrane of the ankle-joint. Outline the stronger tendon (tendo calcaneus [Achillis]) passing down to the os calcaneum.

\section{Superficial.Fascia of Leg.}

Make the muscles of the calf tense and continue the medial 
incision already made in the dissection of the fossa poplitea down to the heel; at the distal extremity of this make a transverse incision extending for five centimetres along the margo pedis lateralis and margo pedis medialis. Reflect the flaps.

Note the general characteristics of this fascia as regards the amount of fat contained, the general direction of its fibres, and the strength of the same. In it, dissect out carefully the following:

Veins. (Vide Spalteholz, Fig. 509.)

(a) Small saphenous vein (vena saphena parva) (O. T. external saphenous vein). What is its relation to the malleolus lateralis? Note that it is the continuation of the lateral marginal vein of the foot (vena marginalis lateralis) into the leg. In the upper half of the leg this vein is ensheathed by a duplicature of the fascia eruris. Dissect out the two branches into which it bifureates above and trace these out into the vena poplitea and the vena femoris profunda. What is the relation of the vena femoropoplitea to the latter? Trace one of the perforating veins through the fascia eruris. Incise the vein longitudinally and determine whether it has valves or not.

(b) Large saphenous vein (V. saphena magna) (O. T. internal saphenous). Determine its relation to the malleolus medialis. It is the continuation of the medial marginal vein (vena marginalis medialis) of the foot into the leg. Do you find a collateral vein accompanying it? When present, it will be found just posterior to the large vein. Incise the vein longitudinally and determine the characteristies of its valves.

Nerves. (Fig. 69.)

(a) Nerve of the calf ( $N$. suralis) (O. T. short saphenous nerve). This nerve is formed by the union of the following:

(aa) Medial cutaneous nerve of the calf ( $N$. cutaneus surae medialis). In the greater part of its course this nerve will be found beneath the fascia cruris. At the middle of the leg it pierces this fascia to become subcutaneous.

(ab) Anastomotic peroneal branch of the N. cutaneus surae lateralis (ramus anastomoticus peronaeus).

(b) Lateral cutaneous nerve of calf ( $N$. cutaneus surae lateralis) (O. T. nervus communicans fibularis).

(c) Posterior cutaneous nerve of thigh (N. cutaneus femoris posterior) (O. T. small sciatic). This nerve will be found beneath the fascia cruris. It sends twigs through the fascia to end in the skin.

(d) Medial cutaneous branches (rami cutanei cruris mediales) to the leg from the N. saphenus. Determine the area of skin supplied by each nerve. fascia.

Remove the superficial fascia of the leg, and study the deep 
Fig. 69.

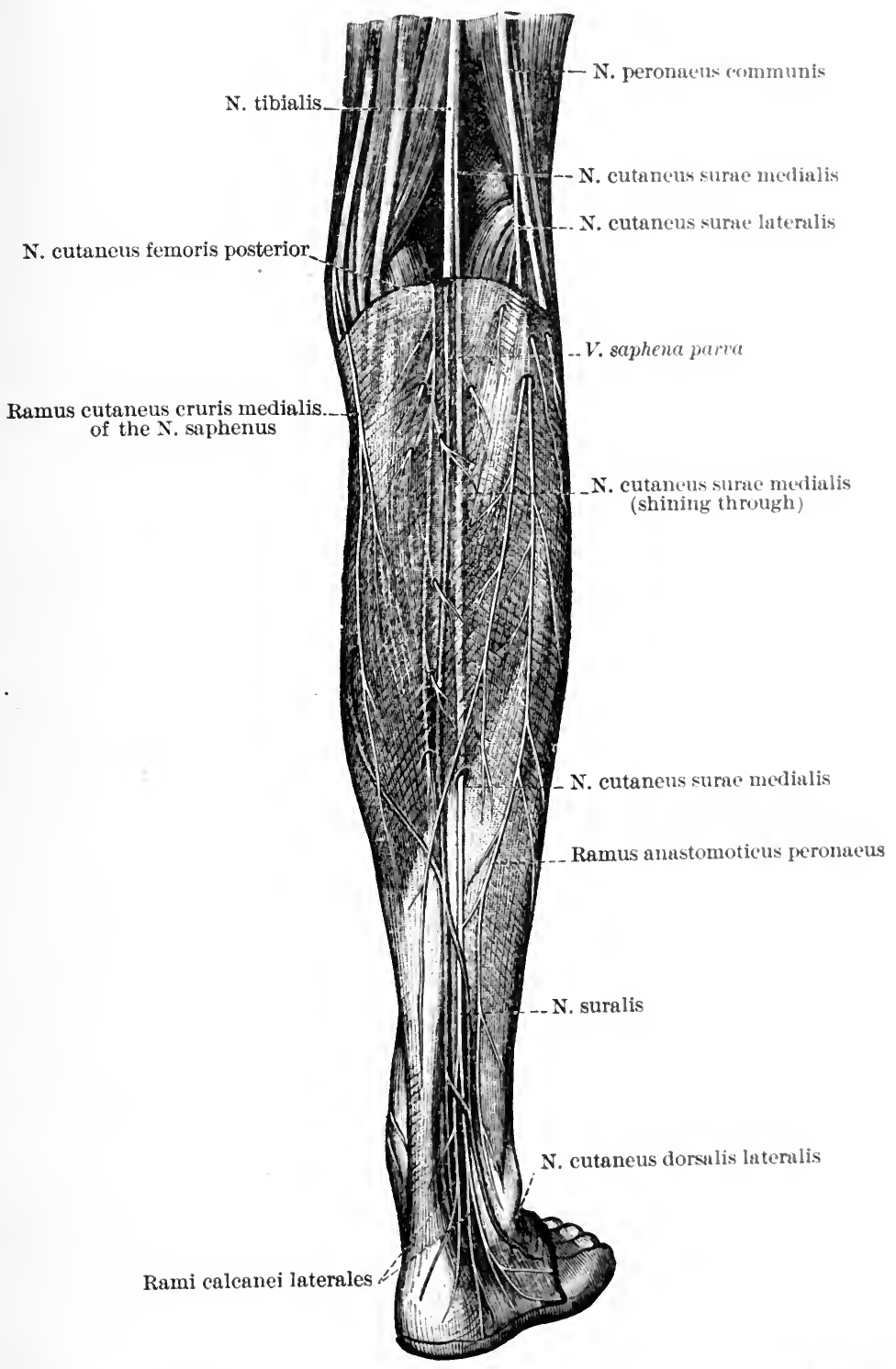

The cutaneus nerves of the posterior side of the leg. (After Toldt, Anat. Atlas, Wien, 1903, 3 Aufl., p. 849, Fig. 1257.) 


\section{Deep Fascia of Leg (Fascia cruris).}

This fascia extends from the knee to the malleoli. It represents almost a complete cone, being interrupted only at the facies medialis of the tibia. What is the relation of its superior circumference to the capitulum fibulae, the condyles of the tibia, and the fascia lata?

Its inferior circumference is attached to the malleoli and the tuber calcaneum. Between these three bony projections it becomes continuous with the annular ligaments about the ankle.

Examine cross-sections of the leg and note the different relations of the fascial reflections and the osteofascial compartments formed by septa passing from this fascia into the tibia and fibula. They have already been referred to above. The septum intermusculare anterius [fibulare] and the septum intermusculare posterius [fibulare] divide the subfascial space into three compartments. (See Figs. 64 and 68.)

(a) Anterior compartment, deep, prismatic in form. It lodges the M. tibialis anterior, the M. extensor digitorum longus, the M. extensor hallucis longus, the M. peronaeus tertius, and the anterior tibial vessels and nerves.

(b) Lateral compartment: this lodges the peroneal muscles and the superior part of the nervus peronaeus superficialis.

(c) Posterior compartment: this is divided into two secondary compartments by the deep transverse fascia of the leg passing from the margo medialis of the tibia to the crista lateralis of the fibula. Posterior to this fascia will be found the M. triceps surae and the M. plantaris; anterior to it will be found the M. tibialis posterior, M. flexor digitorum longus, M. flexor hallucis longus, the posterior tibial vessels and nerves, and the peroneal vessels.

The fascia cruris varies much in thickness in different regions. It is composed of transverse longitudinal and oblique fibres. The transverse fibres arise from the crista anterior of the tibia and after encircling the leg are inserted upon the margo medialis of the same bone. The oblique and longitudinal fibres arise from the bony projections into which the superior circumference of this fascia is attached. What muscles send off processes which reinforce the longitudinal and oblique fibres?

Incise the fascia in the median line from the fossa poplitea to the os calcaneum and reflect the flaps.

\section{Muscles of the Superficial Posterior Osteofascial Compartment.}

These may now be dissected out.

The muscles of the leg find their homologues in the muscles of the forearm. Any differences found are to be explained by the adaptation of the 
leg muscles to locomotion. The relative atrophy of the motor system of the toes is due to the loss of its femoral attachment and to a displacement of the flexors and extensors, deprived of their tibial and fibular attachments, downward into the intrinsic musculature of the fort. The pronator and supinator systems are almost absent, being represented by the popliteus alone, and this through skeletal modifications has lost its primitive rôle. The muscles of the leg, like those of the forearm, are delimited into an anterior, a posterior, and a lateral group by the bones of the leg and aponeurotic septa.

Study carefully the origin, insertion, form, action, and innervation of the following muscles:

(a) Triceps muscle of the calf ( $M$. triceps surae). (Vide Spalteholz, Figs. 393-94.)

(aa) Gastrocnemius muscle (M. gastrocnemius).

(aaa) Lateral head (caput laterale). Look for a small sesamoid cartilage or bone in this head. Observe the lateral bursa of the gastrocnemius (bursa $M$. gastrocnemii lateralis) and the bursa bicipitogastrocnemialis.

(aab) Medial head (caput mediale). Observe the medial bursa of the gastrocnemius (bursa M. gastrocnemii medialis) and the bursa M. semimembranosi. Dissect this bursa out carefully and determine whether or not it communicates with the joint-cavity.

Divide the gastrocnemius transversely near its attachment to the tendo Achillis and reflect it upward, preserving vessels and nerves passing into each head, and study the muscle beneath the M. gastrocnemius.

(ab) Soleus muscle (M. soleus). (Vide Spalteholz, Fig. 394.) Determine its exact origin from the fibula and tibia. Note that the tendon of this muscle fuses with the tendon of the gastrocnemius to form the-

(aba) Tendo calcaneus [Achillis].

Divide the soleus transversely at the level at which it joins the gastrocnemius. Incise the belly of the soleus in the middle line and expose the tendinous arch (arcus tendineus M. solei) and the blood-ressels and nerves entering the muscle. The plantaris muscle may be divided to permit of the turning of the tendo calcaneus [Achillis] downward. Determine its exact attachment to the os calcaneum and study the following bursae:

(1) Bursa subcutanea calcanea. (Vide Spalteholz, Fig. 393.)

(2) Bursa tendinis calcanea. (Vide Spaltelolz, Fig. 395.)

(b) Plantaris muscle (M. plantaris). (Vide Spalteholz, Fig. 394.) The tendon of this small muscle has already been divided to permit of 
the turning of the tendo calcaneus [Achillis] downward. Determine its insertion. It is the homologue of the palmaris longus in the forearm. In monkeys, whose feet are prehensile, it is the proper tensor muscle of the plantar fascia. It is well developed in all plantigrade animals.

\section{Deep Transverse Fascia of Leg and Structures beneath it.}

Note its attachment medially to the tibia and laterally to the fibula. Trace the fascia downward to the ankle, where it becomes thickened to form the ligamentum laciniatum (O. T. internal annular ligament). (Vide Spalteholz, Fig. 395.) Incise this fascia longitudinally, leaving the Lig. laciniatum intact, and proceed to the study of the structures beneath it.

Muscles. Note carefully the form, position, origin, insertion, action, and innervation of each. (Vide Spalteholz, Fig. 395.)

(a) Popliteus musele ( $M$. popliteus). Determine the relation of its tendon to the capsule of the knee-joint and the ligamentum popliteum areuatum. Note the dense aponeurosis covering this muscle. It receives a strengthening band from the tendon of the semimembranosus muscle. Examine the bursa of the popliteus muscle (bursa M. poplitei). (Vide Spalteholz, Fig. 396.) Does this bursa communicate with the knee-joint?

(b) Long flexor of the digits (M. flexor digitorum longus). (Vide Spalteholz, Fig. 395.) Examine the sheath of the long flexor tendons of the toes (vagina tendinum $M$. flexoris digitorum pedis longi). This sheath surrounds the tendon as it passes behind the malleolus medialis and is continued well down into the foot. Does it communicate with a sheath which surrounds the tendons of the tibialis posterior and the flexor longus hallucis?

(c) Posterior tibial muscle ( $M$. tibialis posterior) ( $O$. T. tibialis posticus). This muscle may be more or less fused with the long flexors, which is only an exaggeration of the normal disposition in man and a reproduction of the normal disposition in the cat. (Strauss-Dürckheim.)

Examine the sheath of the tendon of the M. tibialis posterior (vagina tendinis $M$. tibialis posterioris).

(d) Long flexor of the great toe (M. flexor hallucis longus). Examine the sheath of the tendon of the long flexor of the great toe (vagina tendinis $M$. flexoris hallucis longi).

At this stage in the dissection examine the bony leg and foot. Determine the exact position of the sulci through which these tendons pass. Note the exact relation of the tendons beneath the ligamentum laciniatum. (See Fig. 71.)

Varieties in the flexor system of the foot are frequent. The tendency to differentiate may be accentuated, but more frequently there is a return to the primitive undivided mass arising 
FIG. 70.

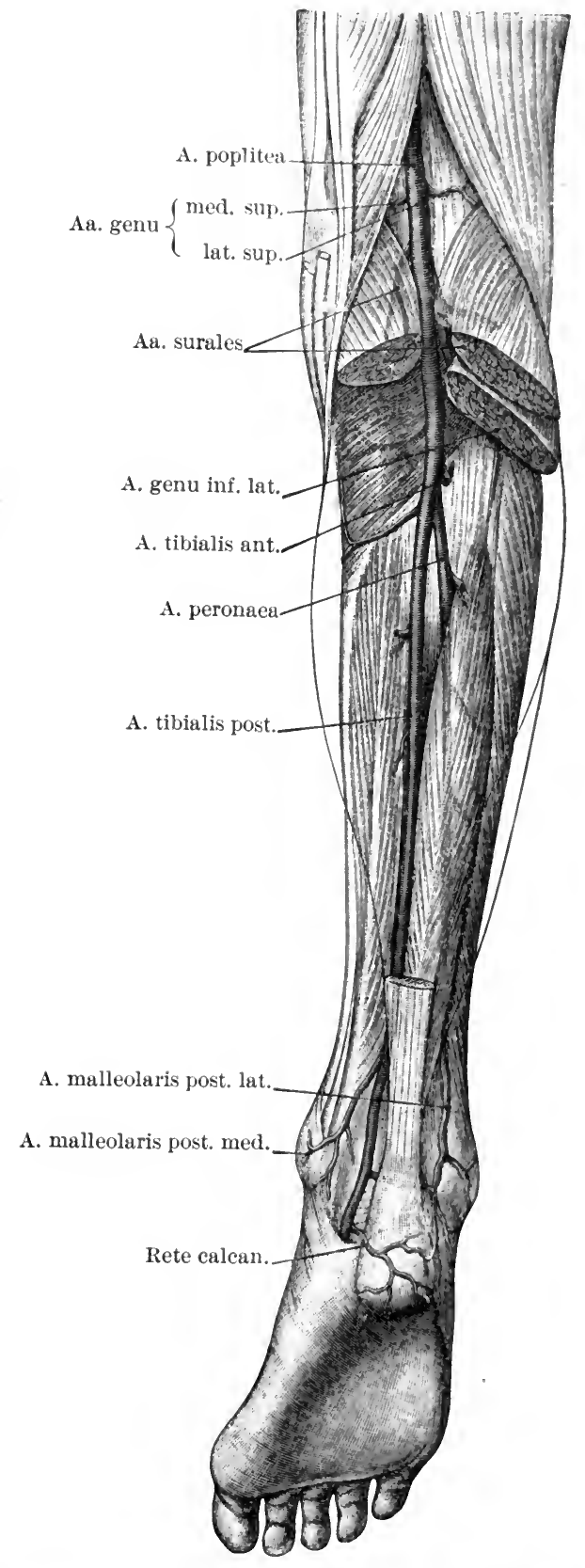

Arteries of the posterior part of the leg.

The museles of the calf have been removed. The A. peronaea is represented as showing through the muscle lying over it. (From Gegenbaur. Lehrb, der Anat. des Mensch., Leipzig, 1899, 7 Aufl., Bd. ii. p. 295, Fig. 548.) 
from the tibia and fibula. In the lower forms, such as the rabbit and kangaroo, this primitive undivided mass of flexors is the rule.

Arteries. (Vide Fig. 70, and also Spalteholz, Fig. 478.)

Trace the popliteal artery downward, and note that it bifurcates just anterior to the areus tendineus M. solei into the anterior and posterior tibial arteries. The anterior tibial has already been dissected. Trace out the course of the posterior tibial artery. Determine the course and position of its linear guide. Where would you take the pulse in the leg and foot? Study-

(a) Posterior tibial artery (A. tibialis posterior).

(aa) Fibular branch (ramus fibularis).

(ab) Peroneal artery (A. peronaea).

(1) Nutrient artery of the fibula (A. nutricia fibulae).

(2) Perforating branch (ramus perforans).

(3) Communicating branch (ramus communicans).

(4) Lateral posterior malleolar artery (A. malleolaris posterior lateralis).

(5) Lateral caleanean branches (rami calcanei laterales).

(ac) Nutrient artery of the tibia (A. nutricia tibiae).

How are the nutrient arteries of the lower extremities directed?

(ad) Medial posterior malleolar artery (A. malleolaris posterior medialis) (O. T. internal malleolar).

(ae) Medial calcanean branches (rami calcanei mediales).

(af) Network of heel (rete calcaneum).

The terminal branches of the A. tibialis posterior will be found when the dissection of the foot is made.

Trace out the veins accompanying this artery and determine their anastomoses and the large veins into which they empty.

Nerves. (Spalteholz, Fig. 823.)

(a) Tibial nerve (N. tibialis).

(aa) Muscular branches (rami musculares).

$(a b)$ Interosseous nerve of the leg ( $N$. interosseus cruris).

(ac) Medial cutaneous nerve of the calf ( $N$. cutaneus surae medialis). This nerve has already been dissected. It unites with the ramus anastomoticus peronaeus to form the-

(ad) Nerve of the calf (N. suralis).

(ada) Lateral ealeanean branches (rami calcanei laterales).

$(a d b)$ Lateral dorsal eutaneous nerve of the foot $(N$. cutaneus dorsalis lateralis pedis). This nerve is the continuation of the $\mathrm{N}$. suralis into the foot. Review at this stage of the dissection the cutaneous nerve supply of the dorsum of the foot.

(ae) Medial calcanean branches (rami calcanei mediales).

Dissect out the branch passing into the syndesmosis tibiofibularis. Do you find any branches accompanying the A. tibialis posterior? 
Make a drawing showing the structures exposed and their exact relations at different levels. Pay especial attention to the relations of the structures in the fossa retromalleolaris medialis.

Laciniate ("Fringed") Ligament (Ligamentum laciniatum) (O. T. Internal Annular Ligament). (Fig. 7I.)

Determine the different points of attachment of this fibrous triangular lamina and the relation that they bear to the malleolus medialis, the medial surface of the tuber calcanei, the medial

FIG. 71.

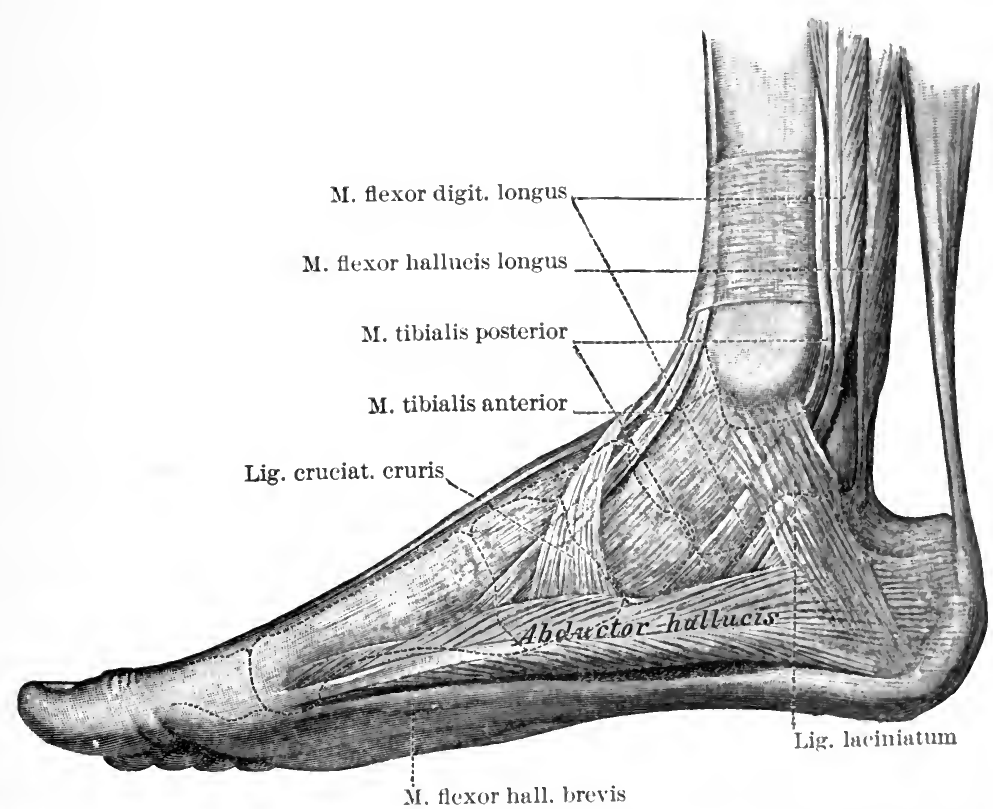

Margo pedis medialis, showing the tendons of the muscles of the leg. (From Gegenbaur, Lehrb. der Anat. des Mensch., Leipzig, 1899, 7 Aufl, Bd. i. p. 46s, Fig. 340.)

plantar aponeurosis, the abductor hallucis muscle, and the fascia cruris. It covers the tendons of the tibialis posterior, the flexor digitorum longus, and the flexor hallucis longus muscles, and the posterior tibial vessels and nerves. Each of these structures runs within a special sheath. The tibialis posterior and the flexor digitorum longus are separated from each other by a fibrous septum which passes off from the deep surface of the ligament. The fibres constituting the septum pass beneath the tendons; one process, ascending, is attached to the malleolus 
medialis and the ligamentum deltoideum; the other, descending, is attached to the processus medialis tuberis calcanei.

The tendon of the long flexor of the great toe is contained within a special sheath, altogether independent of the ligamentum laciniatum. The fibres of this sheath arise from the medial tip of the sulcus M. flexoris hallucis longi and from the summit of the sustentaculum tali. They terminate upon the medial tip of the same sulcus and the medial face of the os calcaneum about one centimetre below the sustentaculum tali. The vessels run above this sheath covered by the ligament, but generally the medial and plantar arteries are separated from each other by a septum.

Detach the ligament from the malleolus medialis and dissect it towards the os calcaneum, determining its exact attachment and the position of the septa above mentioned. Make a drawing of these structures at this stage of the dissection.

\section{PLANTAR REGION OF THE FOOT (REGIO PLAN- TARIS PEDIS).}

Before beginning this dissection, review the anatomy of the bones entering into the formation of the foot. Note the number and the exact position of each. (Vide Spalteholz, Figs. 198-99.) Study in the bony foot and upon your subject the following:

Arches.

(a) Longitudinal arch. This arch extends from the heel to the metatarsophalangeal joints. It is capped by the talus and may be divided into-

(aa) A posterior $\operatorname{limb}$, formed by the os calcaneum and the posterior part of the talus. Note the thickness and the strength of this posterior limb, which serves as a point of attachment for the tendo calcaneus [Achillis]. Study the skeletons of the white, the negro, and the monkey and compare the relative lengths of this posterior limb.

(ab) An anterior limb. This limb is formed by the remaining bones of the tarsus and metatarsus. Examine a model of a foot in which the joint-cavities are represented, and note the amount of synovial membrane found in the joints of the anterior limb. These membranes produce an elastic resistance to any force applied to the anterior limb of the longitudinal arch. Shocks transmitted through it to the leg, pelvis, and trunk are much lessened by this arrangement. The anterior limb is subdivided into a medial pillar, formed by the talus, the navicular, the three cuneiform, and the three medial metatarsal 
bones, and a lateral pillar, formed by the calcaneum, the cuboid, and the two lateral metatarsal bones.

(b) Transverse arch. Where is this arch to be found and what function does it subserve?

(c) Lateral arch. This arch is convex laterally and extends from the heel to the base of the little toe. Determine in what directions pressure exerted from above is radiated by this arrangement of the arches. What factors assist in the maintenance of the arch of the foot? Study, in this connection, especially the tendon of the M. peronaeus. What is flat-foot? Where is the spring of the foot? In flat-foot the line of tenderness generally corresponds to the line of Chopart's joint.

Examine the medial margin of the foot and determine the position of the following:

(a) Sustentaculum tali.

(b) Tuberositas ossis navicularis.

(c) Line of calcaneo-astragaloid joint (articulatio talocalcanea).

(d) Line of tarsometatarsal joints (articulationes tarsometatarseae).

(e) Lines of metatarsophalangeal joints (articulationes metatarsophalangeae).

Examine upon the lateral margin of the foot-

(a) Trochlear process (processus trochlearis), not always present.

(b) Line of calcaneocuboid joint (articulatio calcaneocuboidea). In conneetion with Chopart's amputation it is important to know the structures which serve as guides in finding the mid-tarsal joint.

(c) Tuberositas ossis metatarsalis. What is its relation to the articulationes tarsometatarseae? What is Lisfrane's ligament? (Vide Cunningham's Systematic Anatomy, p. 1218.)

Examine now the skin covering the sole of the foot. Note its general characteristics and compare it with the skin of the palm of the hand. How does it compare with the skin over the dorsum of the foot? Which is the more mobile, and which is the thinner? Note the callosities that form about the heel and the ball of the toes. In standing which parts of the sole come in contact with the floor?

The foot should be firmly fixed and the toes extended, thus rendering the plantar aponeurosis tense. Make the following incisions :

(1) A perpendicular incision extending down the middle of the sole.

(2) A transverse incision across the sole at the clefts of the toes.

(3) A medial incision extending the length of each toe.

Reflect the flaps of skin and study the fascia beneath. 


\section{Superficial Fascia of Sole.}

This fascia consists of but one layer, and resembles the fasciae of the palm of the hand and of the scalp. Note the peculiar character of the fat and its disposition. Thick fibrous processes will be found which connect this fascia to the skin. Dissect away this fascia carefully and study the following:

\section{Bursae.}

(a) Subcutaneous calcanean bursa (bursa subcutanea calcanea). (Vide Spalteholz, p. 348.)

(b) Bursa in the ball of the great and of the little toe. Occasionally a small artery and nerve may be found passing into these bursae. When inflamed they are very painful. How may the formation of these subcutaneous bursae be accounted for?

Veins.

(a) Digital plantar veins ( $V v$. digitales plantares).

(b) Plantar venous arch (arcus plantaris venosus).

(ba) Intercapitular veins ( $V v$. intercapitulares), single or double, passing between each pair of toes to join the Vv. digitales dorsales.

(c) Plantar venous network (rete venosum plantare).

Determine the exact course of the venous blood coming from the sole of the foot.

Nerves.

(a) Medial calcanean branches of the tibial nerve (rami calcanei $N$. tibialis).

(b) Cutaneous branches of the plantar nerves.

Lymphatics.

Determine the exact course of the lymphatics of the sole of the foot.

(Vide Toldt, Fig. 1090.)

\section{Plantar Aponeurosis (Aponeurosis plantaris.)}

Remove now the superficial fascia and study the aponeurosis plantaris. (Vide Spalteholz, Fig. 397.)

Note the density of this aponeurosis. It is attached posteriorly to the os calcaneum and passes forward to cover the whole of the musculature of the foot. It is attached to the medial and lateral margins of the foot, and passes without interruption into the fascia of the dorsum of the foot.

This aponeurosis, like the palmar aponeurosis, may be divided into a middle, a medial, and a lateral part. How does the strength of the middle part compare with that of the medial and lateral parts? Trace this medial part forward, after having determined its posterior attachment; dissect out the five processes into which it divides. Determine their relations to the Ligg. vaginalia and the phalanges. Dissect out carefully the transverse bundles (fasciculi transversi). 
The medial part is the weakest. It is continuous posteriorly with the ligamentum laciniatum, and is attached along the medial margin of the foot to the tarsal and first metatarsal bones. What muscles does this part cover?

The lateral part is stronger than the medial. It is attached behind to the lateral tubercle of the os calcaneum and in front to the tuberosity and the lateral border of the fifth metatarsal. Posteriorly all three divisions give origin to muscles.

From the deep surface of the plantar aponeurosis a medial and a lateral process are given off, which pass downward to fuse with the fascia interossea plantaris and to become attached to the tarsal bones. Three separate muscular compartments are thus formed. These septa may be seen if the plantar aponeurosis be incised in the median line from heel to toe and reflected each way.

Contraction of the plantar fascia gives rise to the condition known as pes cavum. The division of the aponeurosis is most easily accomplished one inch in front of its attachments to the os calcaneum, where it is narrowest. Pus may collect beneath the fascia, and it is apt to burrow, because the aponeurosis is so dense that pus cannot perforate it. Occasionally pus may pass through one of the small foramina formed by the passage of small arteries and nerves through the aponeurosis. In this case two abscess-cavities will be formed. Such a condition is known as the abcès en bissac.

Remove the aponeurosis plantaris and study the structures beneath.

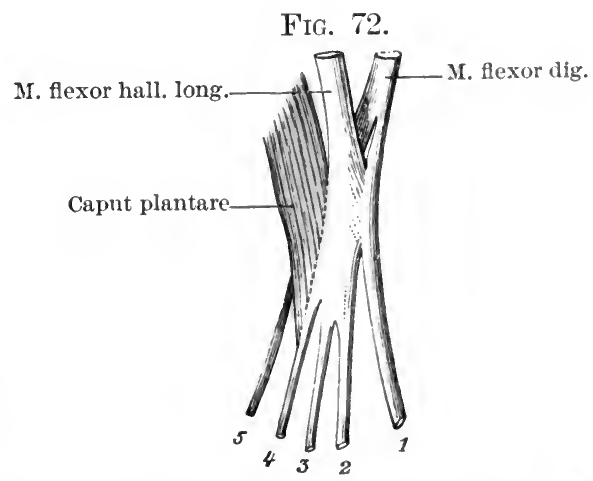

Relation of the tendons of the M. flexor digitorum longus and of the M. flexor hallucis longus, seren from the upper side. (From Gegenbaur, Lehrb. der Anat. des Mensch., Leipzig, 1N99, 7 Aufl., p. 4ti7, Fig. 339.)

Superficial Layer of Muscles of the Sole. (Vide Spalteholz, Fig. 398.)

Note the form, position, origin, insertion, action, and innervation of each. 
(a) Short flexor muscle of the toes (M. flexor digitorum brevis). Examine its ligamentous sheath (ligamentum vaginale), annular ligament (ligamentum annulare), and the cruciate ligament (ligamentum cruciatum).

After having made a careful study of this ligamentous sheath, incise it in the medial line and reflect each way, preserving carefully the vessels and nerves. Note the synovial sheath (vaginae tendinum digitales pedis) surrounding the flexor tendons. (Vide Spalteholz, Fig. 407.) What relations exist between the tendons of the long and of the short flexors of the toes? Compare these relations with those found in the hand.

(b) Abductor muscle of great toe (M. abductor hallucis).

(c) Abductor muscle of little toe ( $M$. abductor minimi digiti).

Divide these muscles at their origin and reflect them forward. Exercise great care and avoid injury to yessels and nerves immediately beneath.

\section{Plantar Arteries. (Vide Spalteholz, Fig. 48I.)}

Determine the relations of these different arteries. What is the linear guide for each artery? All the branches are mentioned here, although many will not be seen at this stage of the dissection.

(a) Medial plantar artery (A. plantaris medialis) (O. T. internal plantar).

(aa) Superficial branch (ramus superficialis).

(ab) Deep branch (ramus profundus).

(b) Lateral plantar artery (A. plantaris lateralis) (O. T. external plantar).

(ba) Plantar arch (arcus plantaris).

(baa) Plantar metatarsal arteries (Aa. metatarseae plantares).

(bab) Perforating branches (rami perforantes).

(bac) Digital plantar arteries (Aa. digitales plantares).

With what arteries do the above anastomose? Dissect out the veins accompanying the arteries and trace them into larger stems.

\section{Plantar Nerves. (Vide Spalteholz, Figs. 824 and 825.)}

Determine what muscle is supplied by each nerve, also the exact cutaneous supply. What is muscle sense?

(a) Medial plantar nerve ( $N$. plantaris medialis) (O. T. internal plantar).

(aa) Common digital plantar nerves $(N n$. digitales plantares communes).

(ab) Proper digital plantar nerves (Nn. digitales plantares proprii).

(b) Lateral plantar nerve ( $N$. plantaris lateralis) (O. T. external plantar).

(ba) Superficial branch (ramus superficialis).

Common digital plantar nerves $(N n$. digitales plantares communes). 
Proper digital plantar nerves $(\mathrm{Nn}$. digitales plantares proprii).

(bb) Deep branch (ramus profundus).

\section{Second Layer of Muscles and Tendons of the Sole.}

(a) Tendons of the M. flexor digitorum longus.

(aa) Quadrate muscle of the sole ( $M$. quadratus plantae) (O. T. accessorius muscle). (Fig. 72.)

Two planes of fibres will be found in this muscle. Dissect both out carefully and determine their relations to the different tendons.

(ab) Lumbrical muscles ( $\mathrm{Mm}$. lumbricales). Determine the exact modes of origin from the tendons of the long flexor and trace them forward to the tendons of the long extensor. These little muscles act chiefly through the above tendons and produce, when active, a flexion of the first phalanx and extension of the second and third. Dissect out the bursae Mm. lumbricalium pedis.

(b) Tendon of the long flexor of the great toe. Determine the relative position of this tendon and dissect out the tendinous process passing over to the tendon of the long flexor of the digits.

Divide the tendons of the M. flexor longus digitorum, M. flexor longus hallucis, M. quadratus plantae, and the plantar vessels and nerves near the os calcaneum and reflect them downward.

\section{Third Layer of Muscles of the Sole. (Vide Spalteholz, Fig. 400.)}

(a) Short flexor muscle of great toe ( $M$. flexor hallucis brevis).

(b) Adductor muscle of great toe (M. adductor hallucis).

(ba) Oblique head (caput obliquum).

(bb) Transverse head (caput transversum).

(c) Short flexor musele of fifth toe (M. flexor digiti quinti brevis).

(d) Opposing muscle of little toe (M. opponens digiti quinti).

Sever the M. flexor digitorum brevis and the caput obliquum of the M. adductor hallucis at their origins and reflect them downward. Complete now the study of the arcus plantaris. Determine where the deep branch of the dorsal artery of the foot passes into the planta. Note the deep branch of the lateral plantar nerve close by the arch.

Displace the digital vessels and nerves forward and sever the short flexor of the little toe and the transverse head of the adductor hallucis at their origins and reflect. Note the strong bands of fibrous tissue (ligamentum capitulorum transversum) passing between the heads of the metacarpal bones. 
Fourth Layer of Muscles of the Sole.

(a) Plantar interosseous muscles ( $M m$. interossei plantares).

(b) Dorsal interosseous muscles (Mm. interossei dorsales).

Determine the form, position, origin, insertion, action, and innervation of each, and compare them with their homologues in the hand. Note that they are covered by a thin layer of interosseous fascia (fascia interossea).

The points of insertion of the M. tibialis posterior will now be exposed. Dissect out the different reflections of its tendons and determine to what bones each is attached.

Dissect out the tendon of the M. peronaeus longus. Note that it runs in a sulcus covered over by the ligamentum longum plantae. Dissect down to the tendon and trace it to its insertion, noting the plantar synovial sheath (vagina tendinis $M$. peronaei longi plantaris). (Vide Spalteholz, Fig. 407.) What role does it play in maintaining the arch of the foot?

\section{JOINTS OF THE LOWER EXTREMITY.}

The hip-joint has been studied already. The other joints should now be carefully dissected.

Arterial Anastomosis about the Knee-joint.

Before dissecting the knee-joint, review the various bloodvessels about the knee and study the most important anastomoses. (Fig. 73.)

\section{Knee-joint (Articulatio genu). (Figs. 73-77.)}

Remove the popliteal vessels and nerves. The tendons of the muscles surrounding the joint should be left in place, so that their relations to the different ligaments may be studied. Divide the M. quadriceps extensor about eight centimetres above the patella and allow the lower part to remain in situ. Trace each of the articular arteries to its termination. Study the following:

(a) Articular capsule (capsula articularis).

(b) Fibular collateral ligament (Lig. collaterale fibulare) (O. T. long external ligament).

(c) Tibial collateral ligament (Lig. collaterale tibiale) (O. T. internal lateral ligament).

(d) Oblique popliteal ligament (Lig. popliteum obliquum) (O. T. posterior ligament).

(e) Arcuate popliteal ligament (Lig. popliteum arcuatum).

Observe the retinaculum Lig. arcuati. 
(f) Ligament of the patella (Lig. patellae).

(fa) Retinaculum patellae mediale.

(fb) Retinaculum patellae laterale.

FIg. 73.

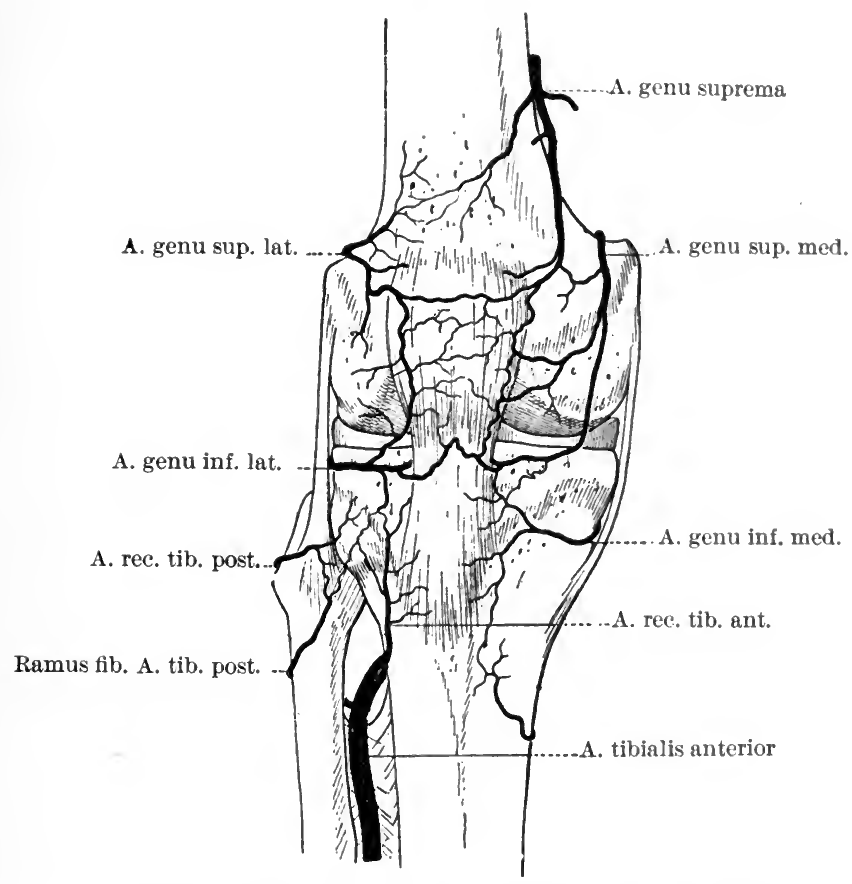

Arterial anastomosis about the knee. (After Poirier et Charpy, Traité d'Anat hum., Paris, 1901, 2 ed., t. ii. p. 831, Fig. 449.)

Make a vertical incision into the joint on either side of the patella and ligamentum patellae. The common extensor tendon and the patella should now be thrown downward and the following internal structures studied:

(g) Lateral meniscus (meniscus lateralis) (O. T. external semilunar fibroeartilage).

(h) Medial meniscus (meniscus medialis) (O. T. internal semilunar fibrocartilage).

(i) Transverse ligament of the knee (Lig. transversum genu).

(j) Crucial ligaments of the knee (Ligg. cruciata genu). (ja) Anterior crucial ligament (Lig. cruciatum anterius).

(jb) Posterior crucial ligament (Lig. cruciatum posterius).

(k) Patellar synovial fold (plica synovialis patellaris) (O. T. ligamentum mucosum).

What is the significance of this fold?

(l) Alar folds (plicae alares) (O. T. ligamenta alaria). 
Study with care the reflections of the synovial membrane. It is the most extensive of any in the body, and is of great practical importance because of its frequent involvement in disease.

Look for "loose bodies" in the cavity of the knee-joint; occasionally one is found. Remember that in a Röntgen-ray

FIG. 74.

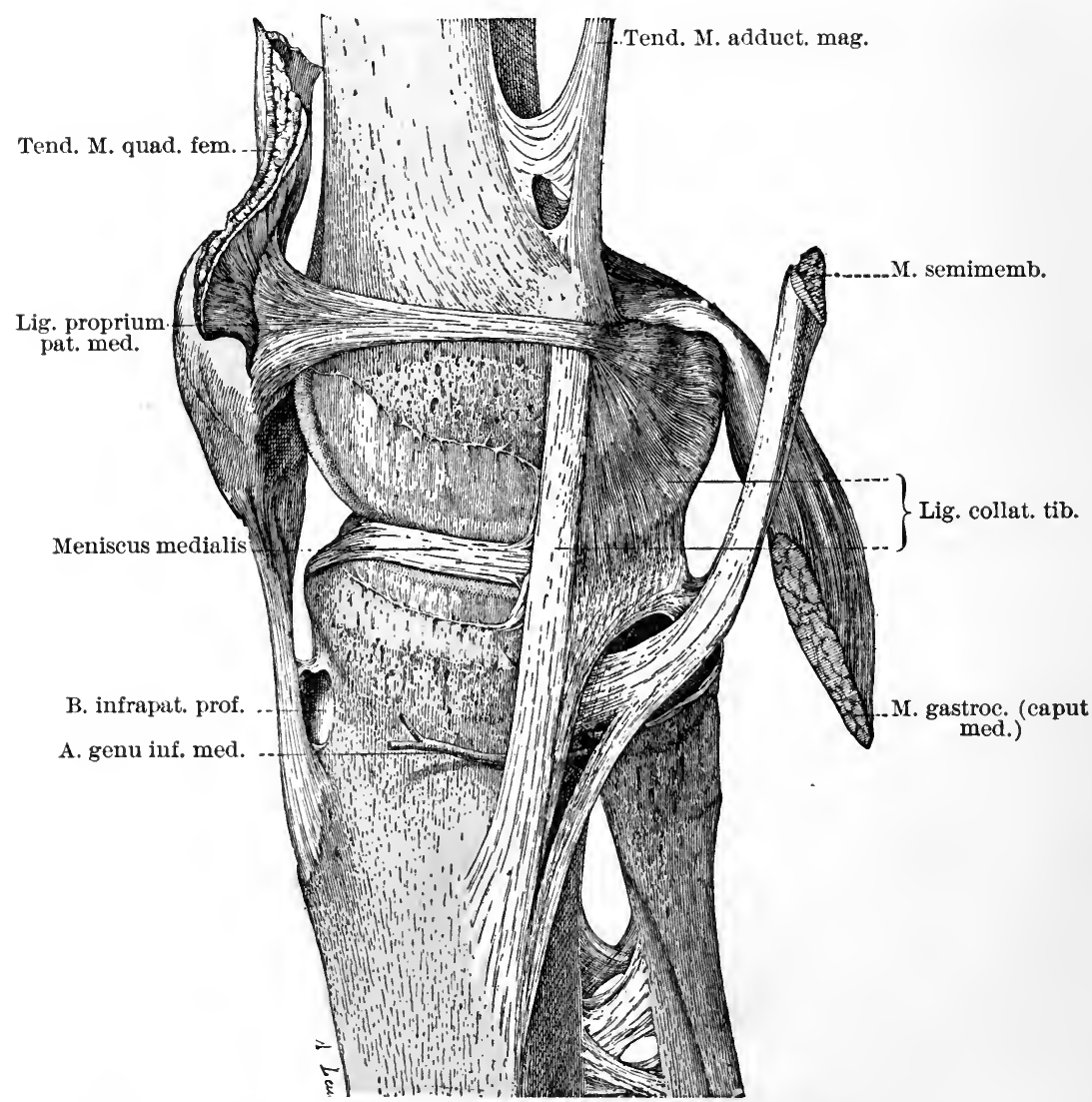

Articulatio genu, medial view. The capsula articularis is removed to demonstrate its lines of attachment to the femur and tibia. (From Poirier et Charpy, Traité d'Anat. hum., Paris, 1899, 2 ed., t. i. p. 737, Fig. 739.)

picture the sesamoid bone in the origin of the M. gastrocnemius has before now been mistaken for such a "loose body."

Having charted the length and breadth of the muscular attachments on the tibia and fibula, remove the muscles and study the articulation between the bones. 
FIG. 75.

Lig. transversum

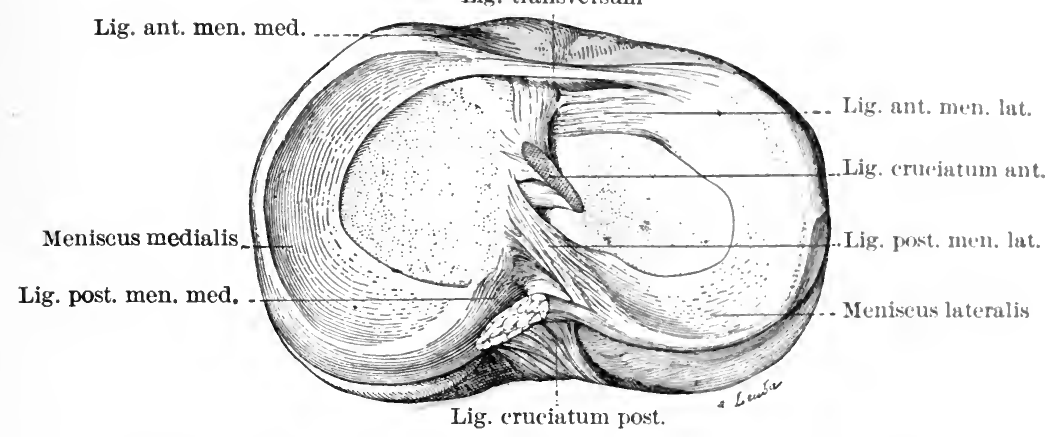

Condylus medialis ct lateralis with menisci. (From Poirier et Charpy, Tratié

d'Anat. hum., Paris, 1899, 2 ed., t. i. p. 733, Fig. 737.)

FIG. 76.

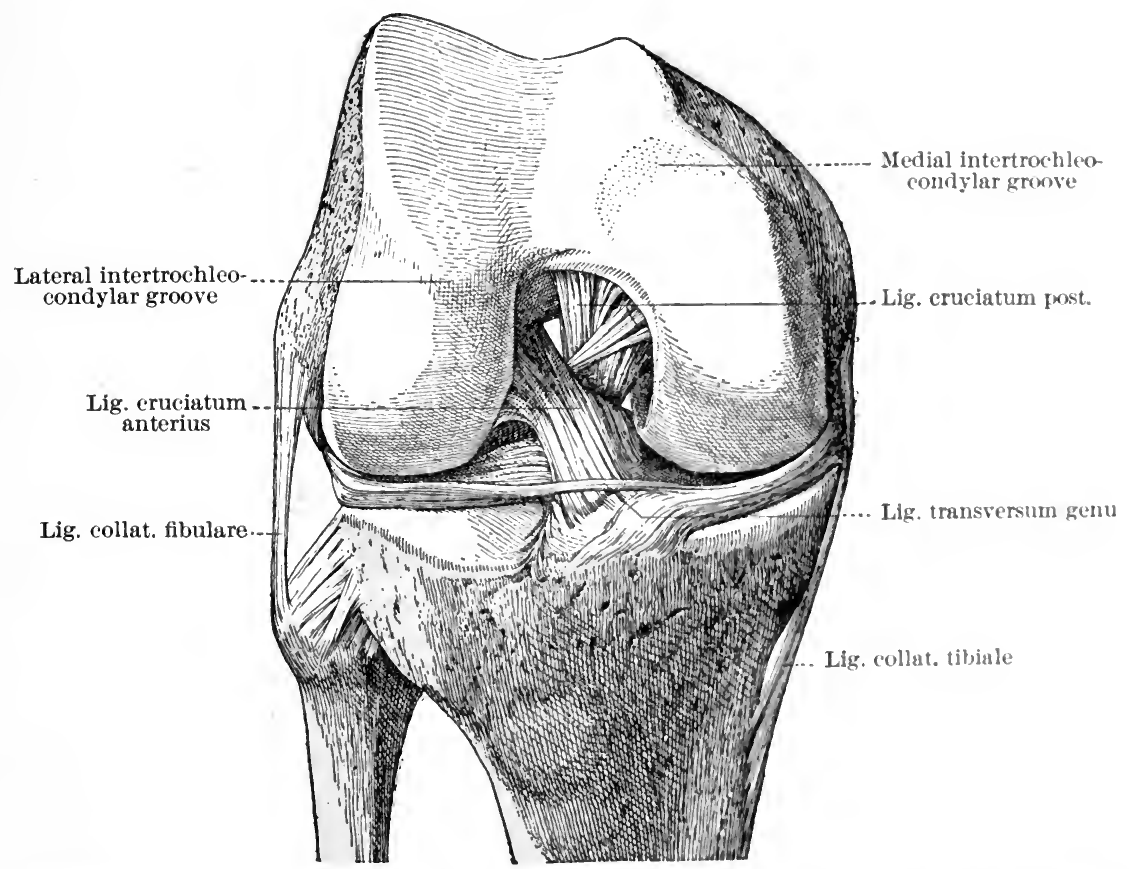

Crucial ligaments of the knee (Ligg. cruciata genu); anterior view. The femur is flexed at right angles to the tibia. (From Poirier et Charpy, Traité d'Anat. hum., Paris, 1899, t. i. p. 740 , Fig. 741.) 


\section{Tibiofibular Articulation (Articulatio tibiofibularis) (O. T. Superior Tibiofibular Articulation). (Fig. 78.)}

(a) Articular capsule (capsula articularis).

(b) Ligaments of the head of the fibula (Ligg. capituli fibulae) (O. T. anterior and posterior superior tibiofibular ligaments).

(c) Interosseous membrane of leg (membrana interossea cruris) (O. T. middle tibiofibular ligament).

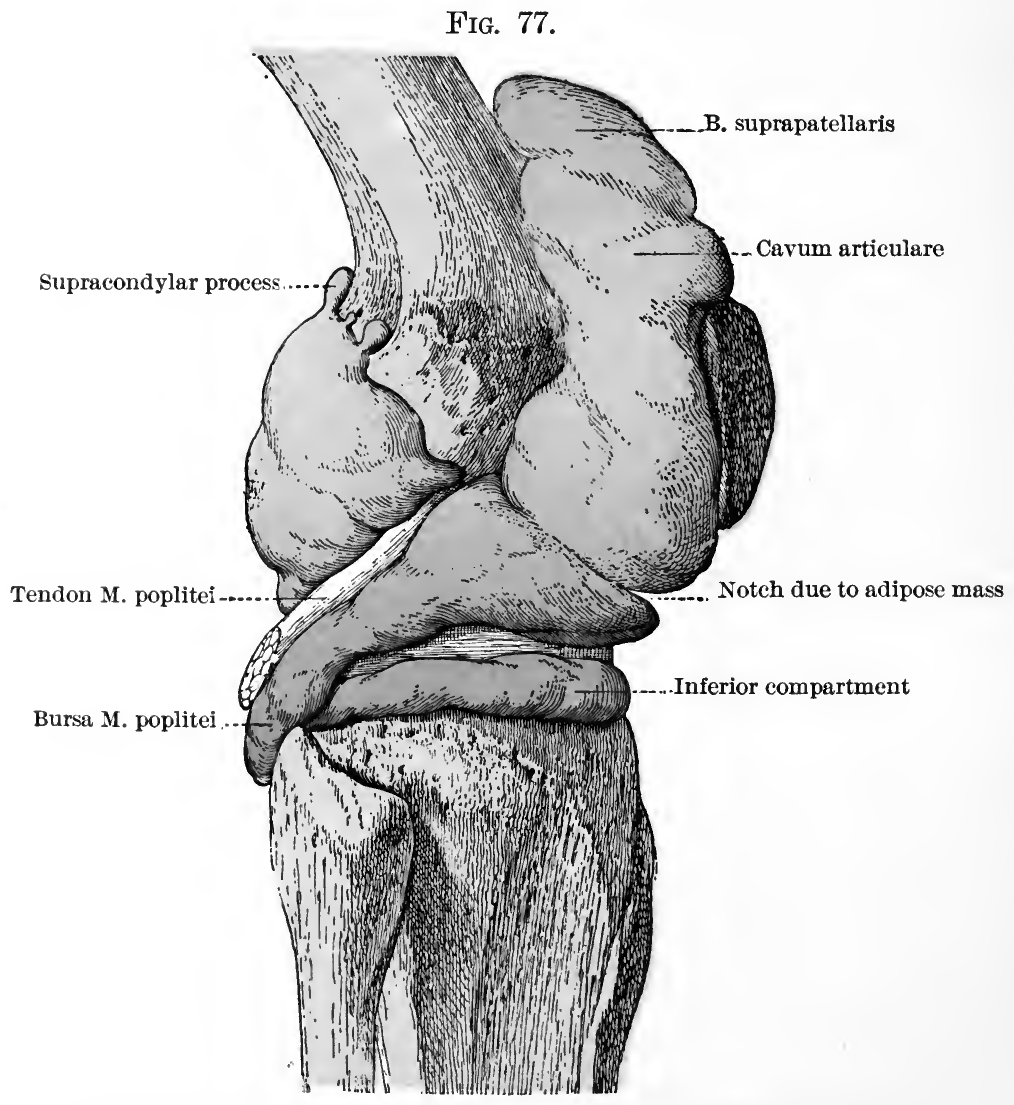

Synovial spaces of the articulatio genu, lateral view. (From Poirier et Charpy, Traité d'Anat. hum., Paris, 1899, 2 ed., t. i. p. 744, Fig. 745.)

\section{Tibiofibular Syndesmosis (Syndesmosis tibiofibularis) (O. T. Inferior Tibiofibular Articulation).}

Study-

(a) Anterior ligament of lateral malleolus (Lig. malleoli lateralis anterius).

(b) Posterior ligament of lateral malleolus (Lig. malleoli lateralis posterius). 
After the ankle-joint has been studied, the tibia and fibula should be sawn through about five centimetres above their lower articular surfaces. Make a coronal section of the bones and study the ligament passing between their lower extremities. Establish the continuity of the synovial membrane of this joint with that of the ankle.

Remove the crucial ligament of the leg and the transverse ligament of the foot, also the tendons passing over the ankle, and dissect out the joints of the foot.

FIG. 78.

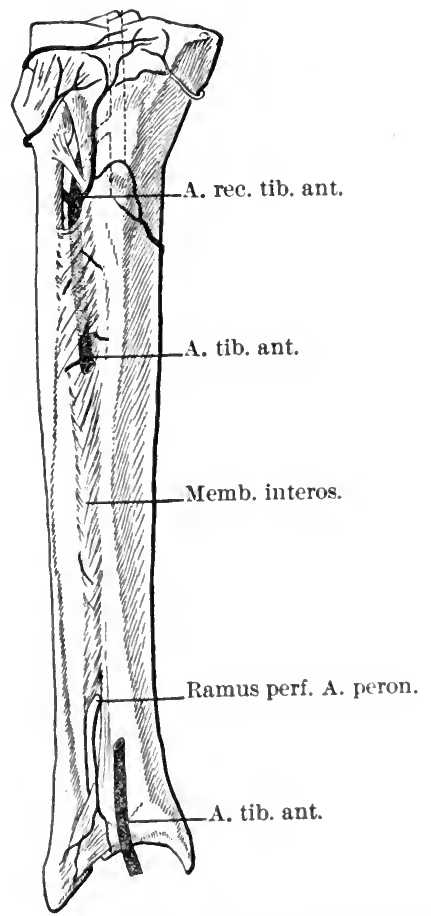

Membrana interossea cruris. (After Poirier et Charpy, Traité d'Anat. hum., Paris, 1599, 2 ed., t. i. p. 756, Fig. 755.)

Articulations of the Foot (Articulationes Pedis). (Figs. 79-82.)

Talocrural Articulation (Articulatio talocruralis) (Ankle-joint).

In removing the soft parts in order to expose the joint, take great care not to injure the very delicate anterior part of the articular capsule. 
(a) Articular capsule (capsula articularis).

(b) Deltoid ligament (Lig. deltoideum) (O. T. internal lateral ligament and anterior and posterior tibiotarsal ligaments).

(ba) Tibionavicular ligament (Lig. tibionaviculare).

(bb) Caleaneotibial ligament (Lig. calcaneotibiale).

(bc) Anterior talotibial ligament (Lig. talotibiale anterius).

(bd) Posterior talotibial ligament (Lig. talotibiale posterius).

(c) Anterior talofibular ligament (Lig. talofibulare anterius) (O. T. anterior fasciculus of external lateral ligament).

(d) Posterior talofibular ligament (Lig. talofibulare posterius) (O. T. posterior fasciculus of external lateral ligament).

(e) Calcaneofibular ligament (Lig. calcaneofibulare) (O. T. middle fasciculus of external lateral ligament).

FIG. 79.

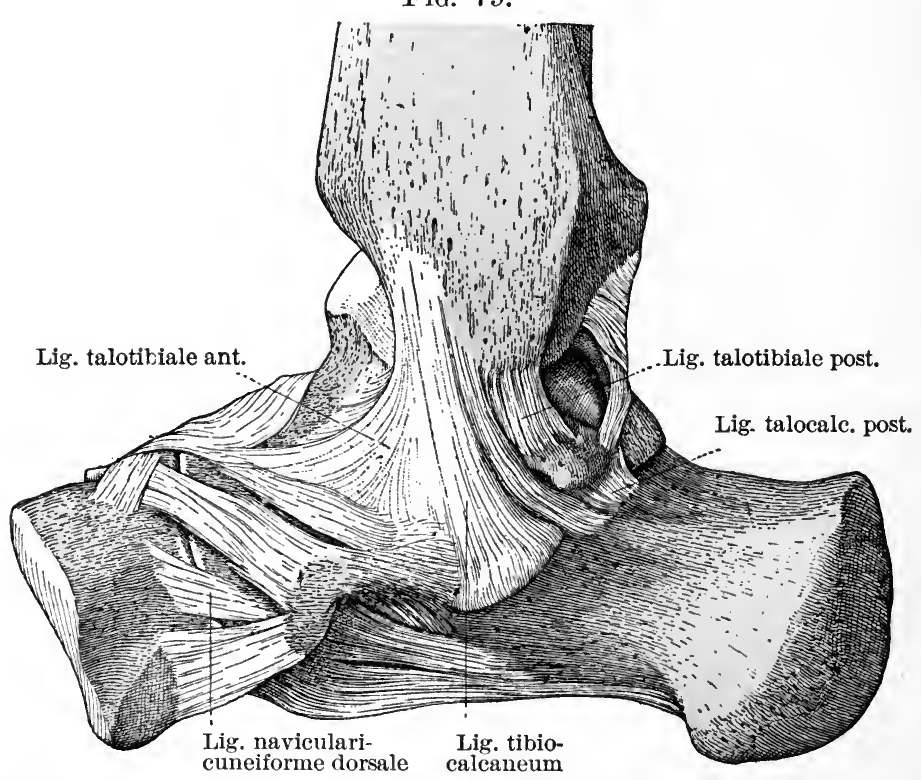

Articulationes talocruralis et intertarseae. Medial view. (From Poirier et Charpy, Traité d'Anat. hum., Paris, 1899, 2 ed., t. i. p. 761, Fig. 759.)

The articular capsule should be opened anteriorly and posteriorly after it has been cleaned. When opened, a good view of the interior of the joint may be had and the remaining ligaments more easily defined.

Study sagittal and frontal sections through this joint on preparations in the anatomical museum; examine closely the relations of the ligaments and the folds of the capsule to the joint-cavity.

Clean away all the muscles and tendons upon the foot, after 
the length and breadth of their attachments have been charted, and study the following joints.

\section{Intertarsal Articulations (Articulationes intertarseae).}

Talocalcaneonavicular Articulation (Articulatio talocalcaneonavicularis).

This is the more anterior of the two separate joint-cavities which exist between the talus on the one hand and the navicular bone and the calcaneus on the other. The posterior of the two joint-cavities is called the articulatio calcanea. (See Spalteholz, Fig. 271.)

FIG. 80 .

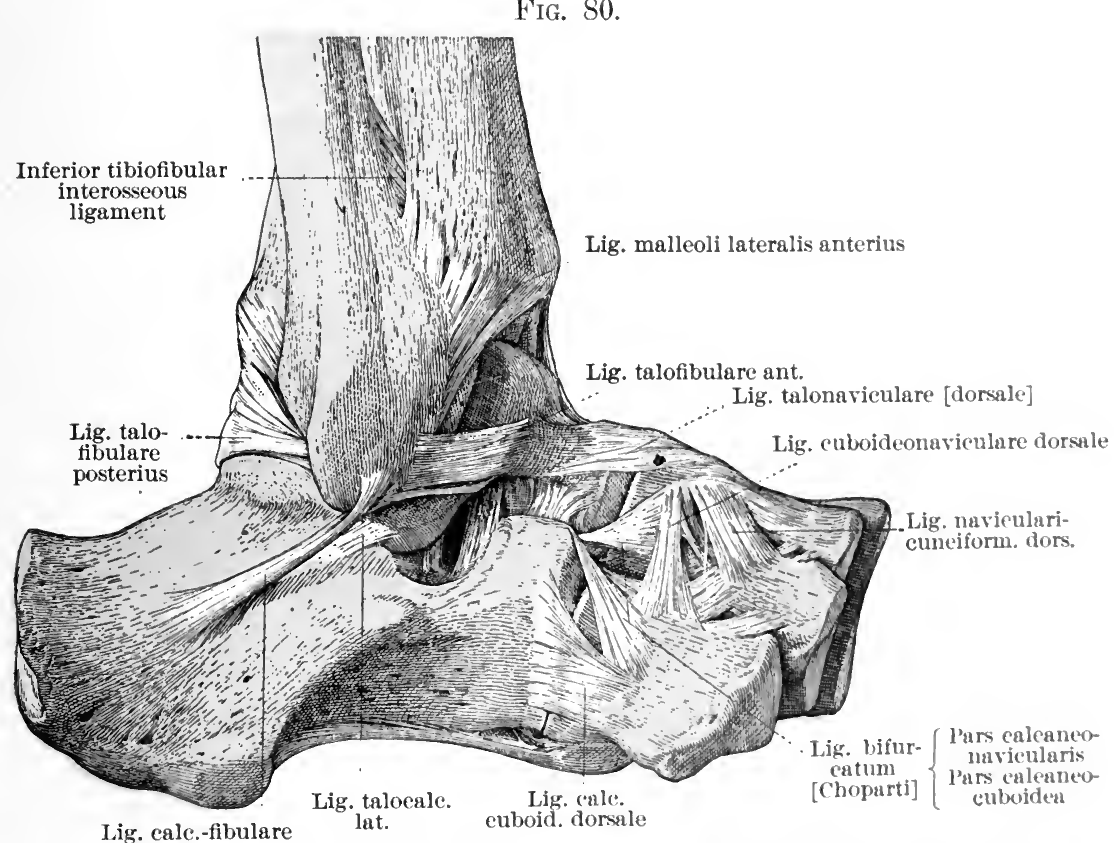

Articulationes talocruralis et intertarseac. Lateral view. (From Poirier et Charpy, Traité d'Anat. hum., Paris, 1899, 2 ed., t. i. p. 760, Fig. 758.)

\section{Study now :}

(a) Talocalcanean articulation (articulatio talocalcanea).

(aa) Articular capsule (capsula articularis).

(ab) Lateral talocalcanean ligament (Lig. talocalcaneum laterale) ( 0 . T. external calcaneo-astragaloid ligament).

(ac) Medial talocaleanean ligament (Lig. talocalcaneum mediale) (O. T. internal calcaneo-astragaloid ligament).

(ad) Anterior talocalcanean ligament (Lig. talocalcaneum anterius).

(ae) Posterior talocalcanean ligament (Lig. talocalcancum posterius).

(b) Chopart's transverse articulation of the tarsus (articulatio tarsi transversa [Choparti]). 
This demands particular attention because it is of great importance to the surgeon. Chopart's amputation of the foot is made through this joint. Besides, the pain of flat-foot is localized in this joint.

(ba) Talonavicular articulation (articulatio talonavicularis).

Observe that this is really only a part of the articulatio talocalcaneonavicularis.

(baa) Articular capsule (capsula articularis).

(bb) Calcaneocuboid articulation (articulatio calcaneocuboidea). (bba) Artieular capsule (capsula articularis).

(c) Cuneonavicular articulation (arsiculatio cuneonavicularis).

(d) Interosseous ligaments of tarsus (Ligg. tarsi interossea).

(da) Interosseous talocaleanean ligament ( $\mathrm{Lig}$. talocalcaneum interosseum).

(db) Interosseous euneocuboid ligament (Lig. cuneocuboideum interosseum).

(dc) Interosseous intercuneiform ligaments (Ligg. intercuneiformia interossea).

(e) Dorsal ligaments of tarsus (Ligg. tarsi dorsalia).

(ea) [Dorsal] talonavicular ligament (Lig. talonaviculare [dorsale]) ( $\mathrm{O}$. T. superior astragalonavieular ligament).

(eb) Dorsal cuneocuboid ligament (Lig. cuneocuboideum dorsale).

(ec) Dorsal cuboideonavicular ligament (Lig. cuboideonaviculare dorsale).

(ed) Bifureate ligament (Lig. bifurcatum). (eda) Calcaneonavicular part (pars calcaneonavicularis)

(O. T. superior or external calcaneonavicular ligament).

(edb) Caleaneocuboidal part (pars calcaneocuboidea) (O. T. internal calcaneocuboid ligament).

(ee) Dorsal calcaneonavicular ligament (Lig. calcaneonaviculare dorsale).

(ef) Dorsal navicular cuneiform ligaments (Ligg. navicularicuneiformia dorsalia).

(f) Plantar ligaments of tarsus (Ligg. tarsi plantaria).

( $f a)$ Long plantar ligament (Lig. plantare longum) (O. T. long calcaneocuboid ligament).

(fb) Deep ligaments of tarsus (Ligg. tarsi profunda).

(fba) Plantar caleaneocuboid ligament (Lig. calcaneocuboideum plantare).

( $f b b)$ Plantar calcaneonavieular ligament (Lig. calcaneonaviculare plantare) (O. T. inferior calcaneonavicular ligament).

$(f b c)$ Navicular fibrocartilage (fibrocartilago navicularis).

$(f b d)$ Plantar navicular euneiform ligaments (Ligg. navicularicuneiformia plantaria).

(fbe) Plantar cuboideonavicular ligament (Lig. cuboideonaviculare plantare). 


\section{DISSECTION OF THE LOWER EXTREMITY}

( $f c$ ) Plantar interemeiform ligaments (Ligg. intercuneiformia plantaria).

(fd) Plantar cuneocuboid ligament (Lig. cuneocuboideum plantare).

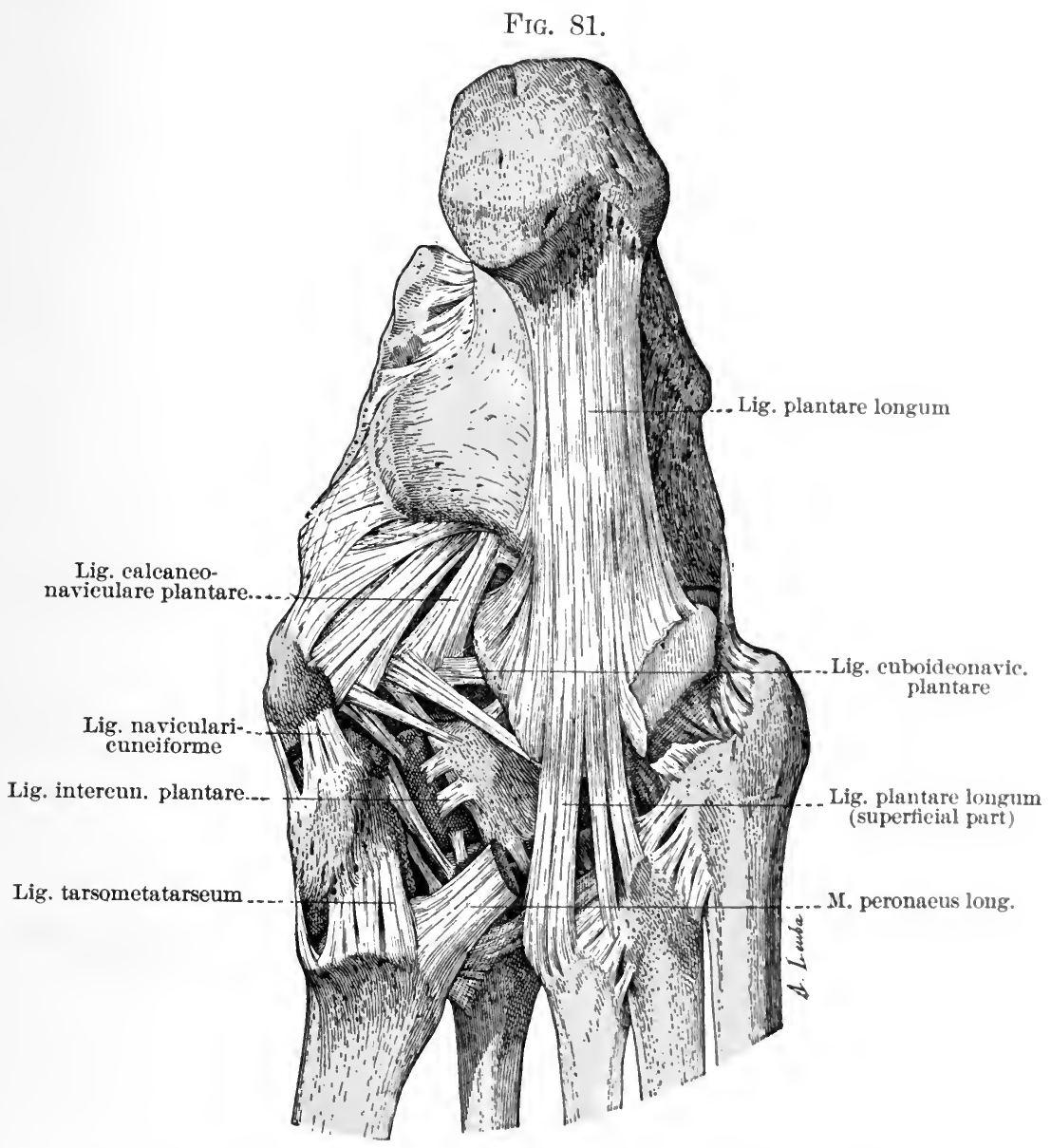

Articulationes intertarscae and tarsometatarseae. Plantar view. (From Poirier et Charpy, Traité d'Anat. hum., Paris, 1899, 2 ed., t. i. p. 773, Fig. 768.)

\section{Tarsometatarsal Articulations (Articulationes tarsometatarseae).}

(a) Articular capsules (capsulae articulares).

(b) Dorsal tarsometatarsal ligaments (Ligg. tarsometatarsea dorsalia).

(c) Plantar tarsometatarsal ligaments (Ligg. tarsometatarsea plantaria).

(d) Interosseous cuneometatarsal ligaments (Ligg. cuneometatarsea interossea). 


\section{Intermetatarsal Articulations (Articulationes intermetatarseae).}

(a) Articular capsules (capsulae articulares).

(b) Interosseous ligaments of the bases of the metatarsal bones (Ligg. basium [oss. metatars.] interossea).

(c) Dorsal ligaments of the bases of the metatarsal bones (Ligg. basium [oss. metatars.] dorsalia).

(d) Plantar ligaments of the bases of the metatarsal bones (Ligg. basium [oss. metatars.] plantaria).

(e) Interosseous spaces of metatarsus (spatia interossea metatarsi).

\section{Metatarsophalangeal Articulations (Articulationes metatarsopha- langeae).}

(a) Articular capsules (capsulae articulares).

(b) Collateral ligaments (Ligg. collateralia).

(c) Plantar accessory ligaments (Ligg. accessoria plantaria).

(d) Transverse ligaments of the heads of the metatarsal bones (Ligg. capitulorum [oss. metatars.] transversa).

FIG. 82.

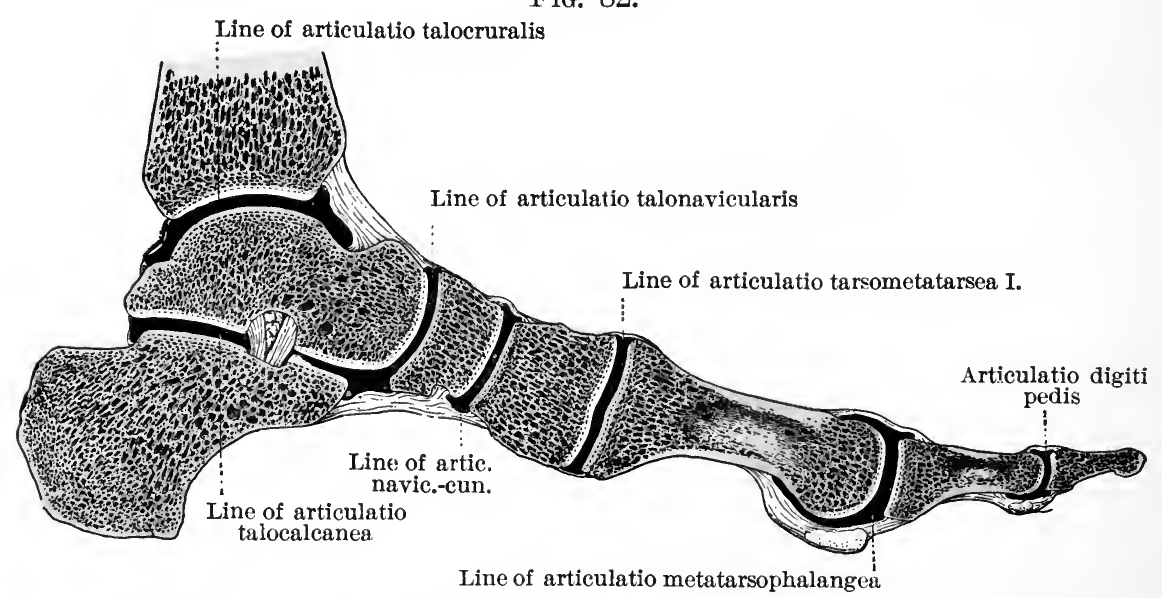

Sagittal section of the foot, passing through the great toe. (From Poirier et Charpy, Traité d'Anat. hum., Paris, 1899, 2 ed., t. i. p. 787, Fig. 781.)

\section{Articulations of the Toes (Articulationes digitorum pedis).}

(a) Articular capsules (capsulae articulares).

(b) Collateral ligaments (Ligg. collateralia).

As each ligament is dissected out it may be divided and the joint-cavity opened. Especial attention should be paid to the synovial cavities of the foot. Consult your systematic anatomy for full descriptions.

Review the bones of the lower extremity. 


\section{Part III}

DISSECTION OF THE HEAD, NECK, AND DORSUM OF THE TRUNK, INCLUDING DISSECTION

OF THE CENTRAL NERVOUS SYSTEM, EYE, AND EAR 


\section{HEAD AND NECK}

\section{Introductory.}

Make three drawings of the head (caput) and neck (collum) : (a) anterior view, (b) posterior view, (c) lateral view. Distinguish the portion corresponding to the skull proper (cranium) from the face (facies). Indicate the highest point of the head (vertex), separating the anterior part of the head (sinciput) from the posterior part (occiput). Note that the occiput is covered with hair; the sinciput also with the exception of its anterior inferior portion, the forehead (frons). Laterally, the temples (tempora) are naked in front but covered with hair. behind. The external ears (auriculae) are attached to the cranium.

In the drawings of the face (ef. Spalteholz, Fig. 513) label the nose (nasus) with its tip (apex nasi), back (dorsum nasi), and wings (alae nasi). Note the openings into the nose (nares) and the partition between (septum nasi). In the region of the mouth (regio oralis), draw the upper and lower lips (labia superius et inferius) which bound the mouth-slit (rima oris). Indicate exactly the furrow (sulcus nasolabialis) running downward from each ala nasi and separating the region of the mouth from that of the cheek (bucca); also, the curved transverse furrow (sulcus mentolabialis) separating the region of the mouth from the chin (mentum). Fill in with care the details in the eyeregions (regiones orbitales), bounded above at the junction with the frons by the eyebrow (supercilium) and below at the junction with the bucca by the infrapalpebral furrow (sulcus infrapalpebralis). Label the upper and lower eyelids (palpebrae superior et inferior) and the slit between them (rima palpebrarum), through which the anterior part of the eyeball (bulbus oculi) is visible.

Beneath the cheek, on each side, notice the region below the lower jaw (regio submaxillaris) and beneath the chin another area (regio submentalis). Note the deep depression (fossa retromandibularis) below the ear and behind the lower jaw. Examine the four cavities of the face,--the two eye-carities (orbitae), the nasal cavity (cavum nasi) divided by the septum, 
and the mouth-cavity (cavum oris) containing the tongue (lingua) and the fauces.

In the neck (collum), note in front the laryngeal prominence (prominentia laryngea) (O. T. Adam's apple, pomum Adami); beneath it, a depression (fossa jugularis); above the clavicles, the supraclavicular fossae (fossae supraclaviculares majores); running obliquely upward and backward from the junction of the clavicle with the sternum, an elevated area (regio sternocleidomastoidea).

Make three outline sketches of the head and neck of your subject, corresponding to the positions indicated in Figs. 281, 282, 283 of Spalteholz's Atlas. Outline the boundaries of all the regions and print in neatly the names of the regions. Be sure that you understand the reason of the name for each region.

What are meant by the following terms: nasion, glabella, inion, ophryon, maximum occipital point, bregma, lambda, asterion, stephanion, gonion? How is the cephalic index determined? Is the head of your subject mesaticephalic or does it show any tendency towards dolichocephaly or brachycephaly? (Cf. Cunningham's Systematic Anatomy, p. 173.)

Examine closely the skin (cutis) and hairs (pili) of the head and neck, first with the naked eye and afterwards through a lens. Is the skin equally movable in all parts? What are capilli, supercilia, cilia, barba, tragi, and vibrissae? Make a sketch of the front and of the back of the head and neck, illustrating the hair streams (flumina pilorum) and vortices (vortices pilorum). Demonstrate the orifices of sweat-glands (pori sudoriferi).

\section{THE SCALP AND SUPERFICIAL STRUCTURES IN THE TEMPORAL REGION.}

Place a block under the head. Shave the scalp carefully. Make an incision from the root of the nose (nasion), along the middle line over the top of the head, to a point a little beyond the external occipital protuberance (inion); at right angles to this make a second incision from the tip of the mastoid process (processus mastoideus) of one side, over the top of the head, to a corresponding point on the opposite side; then, from a point on the latter incision just above the ear, make a cut downward and a little forward to the root of the zygoma. The incision should extend through the skin only, not through the superficial fascia. Reflect carefully the four flaps of skin, carrying the knife close to the corium. The dissection is difficult, owing (1) to the firm 
attachment of the fascia to the skin, (2) to the fact that the roots of the hairs penetrate the superficial fascia, and (3) to the fact that the scalp is often dried out if the cadaver has been kept for some time.

Superficial Blood-vessels and Nerves. (Vide Fig. 105, p. 253.)

In the superficial fascia dissect out the following, cleaning vessels and nerves thoroughly.

In the Frontal Region.

(a) Frontal artery (A. frontalis) and supraorbital artery (A. supraorbitalis).

(b) Angular vein ( $V$. angularis).

(c) Supratrochlear nerve (N. supratrochlearis) and supraorbital nerve (N. supraorbitalis).

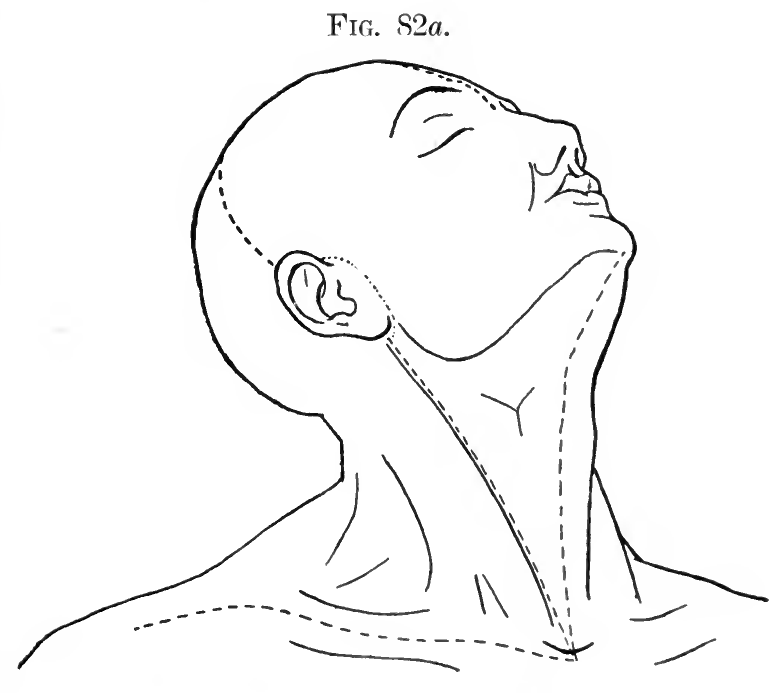

Skin incisions for head and neck.

In the Temporal Region.

(a) Superficial temporal artery (A. temporalis superficialis).

(aa) Frontal branch (ramus frontalis).

(ab) Parietal branch (ramus parietalis).

(b) Superficial temporal vein (V. temporalis superficialis).

(c) Temporal branches of facial nerve (rami temporales $N$. facialis).

(d) Zygomaticotemporal branch of zygomatic nerve (ramus zygomaticotemporalis $N$. zygomatici) (O. T. temporal branch of malar nerve).

(e) Auriculotemporal nerve ( $N$. auriculotemporalis).

In the Mastoid and Occipital Regions.

(a) Posterior auricular artery (A. auricularis posterior). 
(b) Occipital artery (A. occipitalis).

(c) Posterior auricular vein (V. auricularis posterior).

(d) Occipital vein ( $V$. occipitalis).

(e) Posterior auricular nerve ( $N$. auricularis posterior). (ea) Occipital ramus (ramus occipitalis).

(f) Posterior ramus of great auricular nerve (ramus posterior N. auricularis magni).

(g) Lesser occipital nerve ( $N$. occipitalis minor).

(h) Great occipital nerve ( $N$. occipitalis major). (See Fig. 4, p. 47.)

\section{Muscles of the Scalp.}

Having isolated the blood-vessels and nerves, remove the rest of the superficial fascia bit by bit, exposing the muscles of the scalp; these and the aponeuroses are to be carefully cleaned. Take care not to injure the muscles which move the ear; expose each muscle by putting it on the stretch. Write a description of the course and distribution of the nerves and blood-vessels exposed. Read the description given in your text-book of Systematic Anatomy of each vessel and nerve. How do the structures in your cadaver differ, if at all, from those described in the text-book? Note the extreme vascularity of the scalp. It is significant for the mobility and vitality of large flaps of detached scalp.

Study the exact origin and insertion and the innervation of each of the following muscles:

(a) Epicranius muscle (M. epicranius) (O. T. occipitofrontalis).

(aa) Frontal muscle ( $M$. frontalis).

(ab) Occipital muscle (M. occipitalis).

( $a b a)$ Procerus ("prolonged") muscle (M. procerus).

Study the "aponeurotic helmet" (galea aponeurotica) (O. T. epicranial aponeurosis).

(b) Anterior auricular muscle ( $M$. auricularis anterior) (O. T. attrahens auriculam).

(c) Superior auricular muscle (M. auricularis superior) (O. T. attolens auriculam).

(d) Posterior auricular muscle (M. auricularis posterior) (O. T. retrahens auriculam).

Approximate the origin and insertion of each muscle so as to understand clearly its action.

\section{Dangerous Area of the Scalp.}

Make an incision from four to eight centimetres long in the galea aponeurotica in the middle line at the vertex; at right angles to this make a short transverse incision. Lift the corners of the flaps with forceps and note the loose areolar tissue, free 
from fat, between it and the pericranium. The presence of this layer (1) permits of freedom of movement of scalp, (2) accounts for extensive effusion of blood which may follow scalp injury, and (3) explains wide distribution of pus or other inflammatory products in infections involving the tissue beneath the M. epicranius (hence the term " dangerous area of the scalp").

\section{Lymphatics of the Scalp. (Cf. Toldt, Fig. I085; also Poirier et Charpy, t. ii., fasc. iv., p. I282, Fig. 630.)}

Try to find the-

(a) Occipital lymph-glands (lymphoglandulae occipitales). These are of significance in the diagnosis of syphilis, pedieulosis, and other conditions.

(b) Posterior auricular lymph-glands (lymphoglandulae auriculares posteriores).

(c) Anterior auricular lymph-glands (lymphoglandulae auriculares anteriores).

\section{OPENING THE CAVITY OF THE SKULL.}

Nowadays the brain is usually removed immediately after the cadaver is turned over to the department of anatomy, in order that it may be better prepared in formalin for subsequent study. Occasionally, however, a cadaver with the brain still in situ arrives in the dissecting-room; and in any case the student should know how to remove a brain.

Raise the head of the cadaver by means of a wooden block. Make a coronal or frontal section, with a cartilage-knife, from the region behind the left ear, transversely over the vertex, to the same region behind the right ear. The cut should go through the galea aponeurotica down to the pericranium. 'Turn the front flap downward over the face and the hind flap downward over the neck. The temporal fascia and temporal muscle should be separated from the bone and reflected downward. Now make a horizontal cut with the saw along the largest horizontal circumference of the skull; the saw passes through the glabella in front and a little above the protuberantia occipitalis externa behind. Saw through the outer table of the skull only. With hammer and chisel split the inner table along the line of the cut. Insert a hook into the cut in front and forcibly wrench off the skullcap. The outer surface of the dura mater is now exposed. 
Dura Mater of Brain (Dura mater encephali). (Vide Figs. 83, 84.) Wipe the surface clean with a moist sponge. Study-

(a) Middle meningeal artery (A. meningea media).

(b) Arachnoideal granulations (granulationes arachnoideales [Pacchioni]) (O. T. Pacchionian granulations).

Place the head nearly upright and support it by blocks. Make two sagittal incisions through the dura mater, one on each side of the superior sagittal sinus, along its whole length. From

Fig. 83.

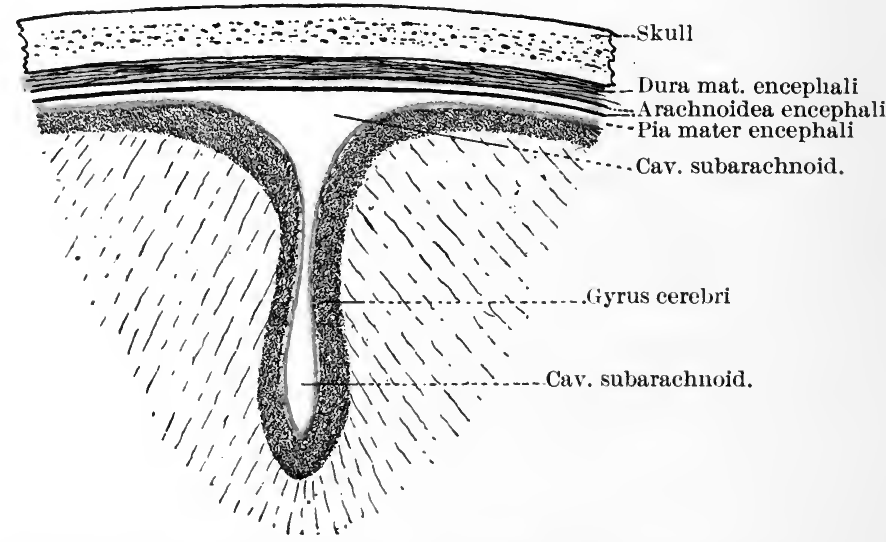

The meninges. Schematic section passing through a sulcus cerebri. The pia mater encephali in red. (From Poirier et Charpy, Traité d'Anat. hum., Paris, 1899, t. iii., 1, p. 96, Fig. 69.)

the middle of each incision make a cut lateralward through the dura as far down as the cut margin of the skull; reflect the flaps of dura forward and lateralward and backward and lateralward respectively. The subdural space (cavum subdurale) has now been opened up. Compare the inner surface of the dura mater with the outer.

The arachnoid of the brain (arachnoidea encephali) is nonvascular and transparent. Through it the vessels of the pia mater of the brain (pia mater encephali) are visible.

\section{Superior Sagittal Sinus}

The veins on the surface of the brain can be seen running towards the superior sagittal sinus (sinus sagittalis superior) (O. T. superior longitudinal sinus). This sinus should be opened throughout its whole length with a sharp knife or scissors. Observe in it the network-like bands (O. T. chordae Willisi).

Cut through the superior veins of the cerebrum which empty 
into the superior sagittal sinus and pull the hewisphere gently lateralward. The falx cerebri may now be examined.

Falx Cerebri.

Examine its extent. How is it formed? To what is it attached? Note that it contains three of the sinuses of the dura mater (sinus durae matris), as follows:

(a) Superior sagittal sinus (sinus sagittalis superior) (vide supra).

(b) Inferior sagittal sinus (sinus sagittalis inferior) (O. T. inferior longitudinal sinus).

(c) Straight sinus (sinus rectus).

FIG. 84.

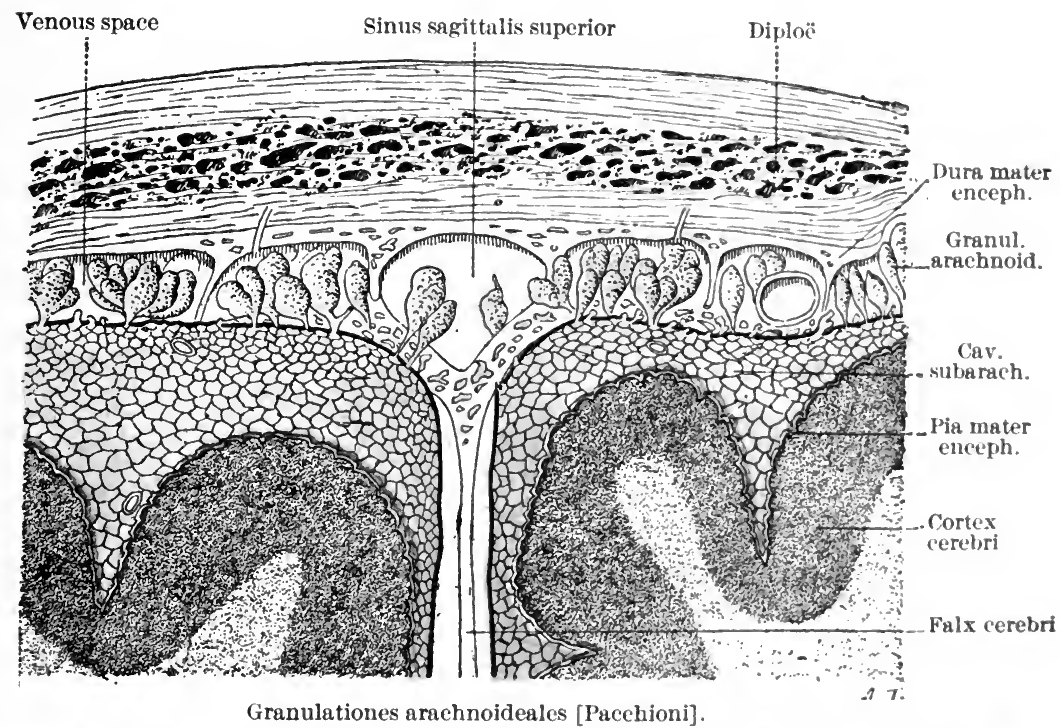

Transverse section (magnified) passing through the falx cerebri, and showing the skull, meninges, and cortex cerebri. (From Poirier et Charpy, Traité d'Anat. hum., Paris, 1899, t. iii., 1, p. 143, Fig. 98.)

\section{Removal of Brain.}

Cut through the attachment of the falx cerebri to the basis cranii interna in front and pull it backward. Let the head hang over the end of the table; support the occipital regions of the brain in the left hand. The frontal lobes will fall away from the floor of the skull; if the bulbi olfactorii remain adherent, separate them from the lamina cribrosa of the ethmoid bone with the handle of a scalpel; this easily tears across the Nn. olfactorii, which, coming through the lamina cribrosa from the nose, here plunge into the olfactory bulb to terminate there. Next cut 
through each optic nerve ( $N$. opticus) as it enters the skull near the foramen opticum. The internal carotid artery ( $A$. carotis interna) on each side should next be divided, and, behind and between the arteries of the two sides, the infundibulum. Behind and lateralward from each $\mathrm{A}$. carotis interna, cut through the oculomotor nerve ( $N$. oculomotorius). In cutting these nerves,

FIG. 85.

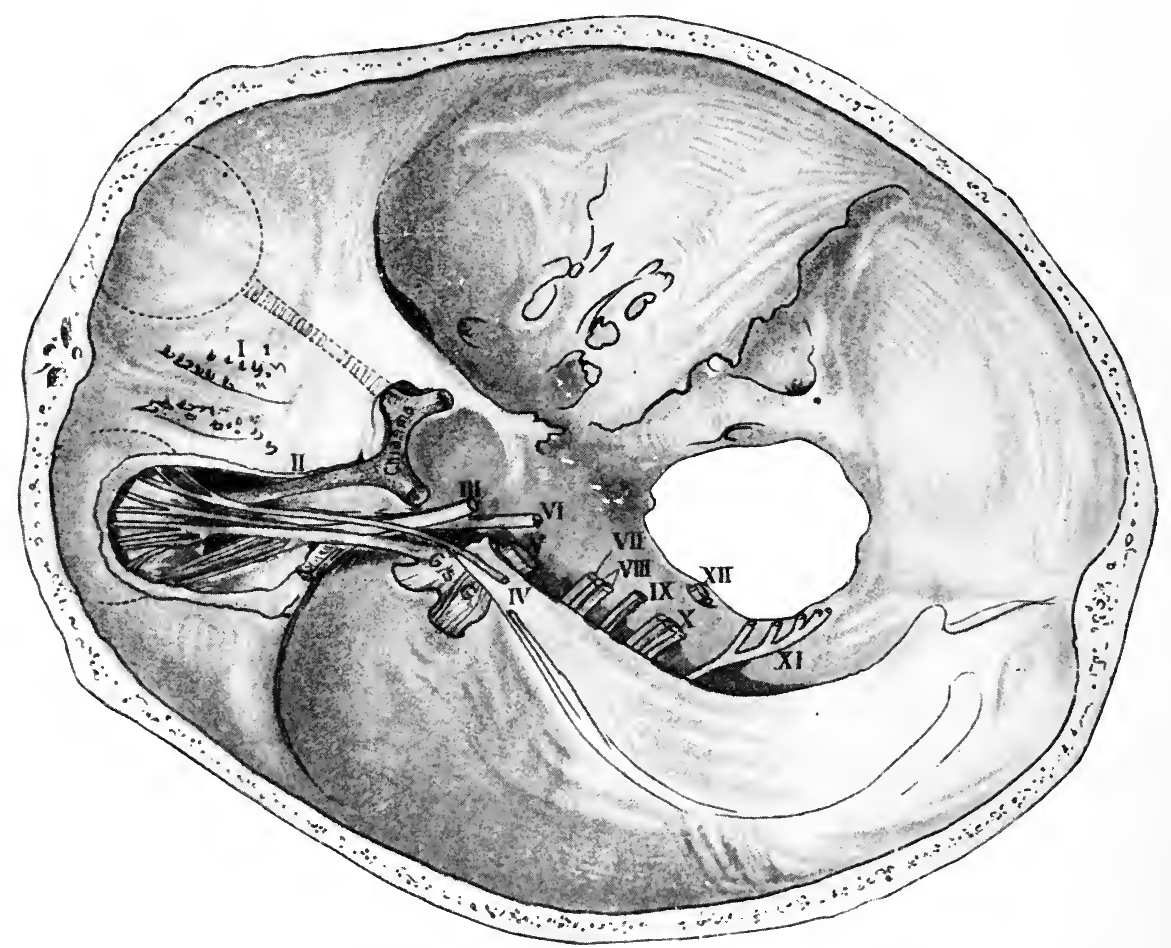

Exit and entrance of nervi cerebrales at the base of the skull.

$I$, Nn. olfactorii ; $I I, \mathrm{~N}$. opticus; $I I I, \mathrm{~N}$. oculomotorius ; $I I$, N. troehlearis; $I, \mathrm{~N}$. trigeminus; $I I$, $\mathrm{N}$. abducens; $I I I, \mathrm{~N}$. facialis; $I I I I, \mathrm{Nn}$. cochleae et vestibuli ; $I X, \mathrm{~N}$. glossopharyngeus; $\mathrm{X}, \mathrm{N}$. vagus ; $X I, N$. aceessorius; $X I I, N$. hypoglossus; 1 , peripheral olfactory neurones; G. $s . G$, garglion semilunare (Gasseri). (From Barker, The Nerv. Syst., New York, 19ง1, Plate I., Fig. 2.)

as well as the cerebral nerves farther back, the student is advised to cut close to the point where they pierce the dura mater on the right side and close to the brain on the left side. (Cf. Spalteholz, Fig. 705.) The anterior extremity of the medial free margin of the tentorium cerebelli is now visible at its attachment to the processus clinoideus anterior of the ala parva of the sphenoid bone. Displace it slightly lateralward and find the trochlear nerve (N. trochlearis); cut through it on each side. 
Turn the head of the cadaver forcibly to the left; raise the posterior part of the right cerebral hemisphere and expose the broad tentorium cerebelli. Cut through the tentorium along its attachment to the superior angle of the temporal bone (angulus superior pyramidis) and, with the point of the knife, shove the tentorium backward out of the way. Now turn the head forcibly to the right and divide the tentorium on the left side in a similar manner.

Next divide successively, from before backward-

(a) Trigeminal nerve (N.trigeminus).

(aa) Larger (sensory) root (portio major).

(ab) Smaller (motor) root (portio minor).

(b) Abducent nerve (N. abducens).

(c) Facial nerve ( $N$. facialis).

(d) Intermediate nerve $(N$. intermedius) ( $O$. T. pars intermedia of Wrisberg).

(e) Acoustic nerve (N. acusticus) (O. T. auditory nerve).

(ea) Vestibular root (radix vestibularis).

(eb) Cochlear root (radix cochlearis).

(f) Glossopharyngeal nerve ( $N$. glossopharyngeus).

(g) Vagus nerve (N. vagus).

(h) Accessory nerve (N. accessorius) (O. T. spinal accessory).

(i) Hypoglossal nerve (N. hypoglossus).

Now thrust a long, narrow, straight-bladed knife into the vertebral canal, cut the A. vertebralis on each side and the roots of the first cervical nerve on each side; just below this level sever the spinal cord, as nearly transversely as possible, with the knife or, better, with Pick's myelotome.

The brain may now be easily removed from the skull, usually by gravity. Tie a thread around the basilar artery (A. basilaris) and suspend the brain in a jar of ten per cent. formalin solution; in a few days it will be hardened sufficiently for study. The brain may be kept for a long time in formalin without injuring it for gross anatomical study.

\section{Dura Mater Encephali.}

The study of this membrane should now be resumed. Examine its relations to the cerebral nerves and to the bones of the base of the skull. At the partitions formed by processes of the dura mater the falx cerebri has already been studied. The student should now examine thoroughly-

(a) Tentorium cerebelli.

Note its form, position, and attachments. In it run the important 
(b) Falx cerebelli.

transverse sinus (sinus transversus) (O. T. lateral sinus) and the superior petrosal sinus (sinus petrosus superior).

Note the relation of the occipital sinus to this membrane.

(c) Diaphragma sellae.

In connection with the dura mater at the base of the skull, the entrance and exit of the various cerebral nerves ( $N n$. cerebrales) (O. T. cranial nerves) should be carefully studied.

\section{Sinuses of the Dura Mater. (Vide Spalteholz, Figs. 483 and 484.)}

These venous sinuses (sinus durae matris) should now be systematically examined. Open each throughout its length with a knife having a sharp point. Study them in the following order :

(a) Transverse sinus (sinus transversus) (O. T. lateral sinus).

Examine its course exactly and note its relations to the exterior of the skull. It is of very great practical importance to the physician and surgeon, not infrequently becoming infected as a sequel of inflammation of the cellulae mastoideae.

(aa) Confluence of the sinuses (confluens sinuum) (O. T. torcular Herophili).

$(a b)$ Internal auditory veins ( $V v$. auditivae internae).

(b) Occipital sinus (sinus occipitalis).

(c) Superior sagittal sinus (sinus sagittalis superior) (O. T. superior longitudinal sinus).

(d) Inferior sagittal sinus (sinus sagittalis inferior) (O. T. inferior longitudinal sinus).

(e) Straight sinus (sinus rectus).

Note that it receives the V. cerebri magna [Galeni].

( $f$ ) Inferior petrosal sinus (sinus petrosus inferior).

How does it leave the skull? Into what does it empty?

(g) Superior petrosal sinus (sinus petrosus superior).

(h) Cavernous sinus (sinus cavernosus).

Leave this unopened at present; it will be thoroughly studied subsequently.

It receives the $\mathrm{Vv}$. ophthalmicae superior et inferior and the sinus sphenoparietalis.

(i) Anterior and posterior intercavernous sinuses (sinus cavernosus anterior et posterior).

These together with the two cavernous sinuses constitute the circular sinus (sinus circularis).

(j) Sphenoparietal sinus (sinus sphenoparietalis) (O. T. sinus alae parvae).

Note that it receives one $\mathrm{V}$. meningea media, the $\mathrm{Vr}$. cerebri inferiores, the $\mathrm{V}$. ophthalmomeningea, and frequently the V. diploica temporalis anterior.

Certain veins should be studied at this juncture. 
(a) Basilar plexus (plexus basilaris) (O. T. basilar sinus).

(b) Diploic veins (venae diploicae). (See Spalteholz, Fig. 455.)

(ba) Frontal diploie vein ( $V$. diploica frontalis).

(bb) Anterior temporal diploic vein ( $V$. diploica temporalis anterior).

(bc) Posterior temporal diploic rein ( $V$. diploica temporalis posterior).

(bd) Occipital diploie rein ( $V$. diploica occipitalis).

(c) Emissary reins.

(ca) Parietal emissary (emissarium parietale).

(cb) Mastoid emissary (emissarium mastoideum).

(cc) Condyloid emissary (emissarium condyloideum).

(cd) Occipital emissary (emissarium occipitale).

\section{Entrance of Arteries into the Cavity of the Skull.}

The student should next examine the exact position and relations of the various arteries entering the cavum cranii.

(a) Internal carotid artery (A. carotis interna).

(b) Vertebral artery (A. vertebralis).

(c) Meningeal arteries (Aa. meningeae). (See Spalteholz, Fig. 447.)

(ca) Middle meningeal artery (A. meningea media), from the $A$. maxillaris interna.

( $c b)$ Anterior meningeal artery (A. meningea anterior), from the A. ethmoidalis anterior of the A. ophthalmica.

(cc) Accessory meningeal branch (ramus meningeus accessorius) (O. T. small meningeal artery), usually from the A. meningea media. It will be studied later.

(cd) Posterior meningeal artery (A. meningea posterior), from the A. pharyngea ascendens.

(ce) Meningeal branch (ramus meningeus), from the $\mathrm{A}$. oceipitalis. It is inconstant.

(cf) Meningeal branch (ramus meningeus), from the $\Lambda$. vertebralis.

\section{Hypophysis.}

Cut through the diaphragma sellae and extricate the hypophysis (O. T. pituitary body) from the sella tureica. Observe its shape and consistency and its division into an anterior lobe (lobus anterior) and a posterior lobe (lobus posterior). Make a sagittal section through it and observe the relations of the two lobes to each other. With which is the infundibulum continuous? Read up on the embryological origin of these two lobes. The cavity of the skull should now be packed with tow soaked in a solution of carbolic acid or in alcohol. Replace the skullcap and stitch the scalp flaps in position over it. This is very important for the further dissection. 


\section{SIDE OF NECK.}

Examination of Surface.

Review the names of the regions of the neck. In the middle line feel the hyoid bone; run the finger along its body and make out the greater cornua. A finger-breadth lower the thyreoid cartilage is reached. Examine it in detail. Lower still, palpate the cricoid cartilage, the cricothyreoid space, and the trachea. Can you make out the thyreoid gland? Ascertain the vertebral levels of the hyoid bone, the cricoid cartilage, and the upper border of the manubrium.

Make deep pressure in upper part of supraclavicular fossa and feel transverse process of seventh cervical vertebra. Is there a cervical rib present? Make also deep pressure over the line of the carotid vessels at the level of the cricoid cartilage and feel the prominent anterior tubercle of the transverse process of the sixth cervical vertebra. This is the so-called " carotid tuber. cle" (Chassaignac's), an important landmark to the surgeon, since the A. carotis lies directly anterior to it.

Palpate through the skin the anterior margin of the M. trapezius, the clavicle, the upper border of the manubrium, the lower margin of the mandible, and the mastoid process. Note the exact position of the M. sternocleidomastoideus, separating the anterior from the posterior triangle of the neck. Above the manubrium note the jugular fossa (fossa jugularis), sometimes called the "suprasternal fossa" or "fonticulus gutturis"; it is especially marked in marasmus.

\section{Skin, Superficial Fascia, and Platysma.}

The skin is thin and rather loose. Place a large block beneath the shoulders and put the parts upon the stretch. Make three incisions (see Fig. 82a, p. 195): (1) in the linea mediana anterior from the chin to the manubrium; (2) from the middle of the upper margin of the manubrium lateralward along the clavicle to the acromion; (3) from the processus mastoideus obliquely downward and medialward along the anterior margin of the M. sternocleidomastoideus to the angle formed by incisions (1) and (2). Reflect the anterior triangular flap upward and the posterior one backward.

In the superficial fascia, compare the amount of fat present in your subject with that in the cadaver of the opposite sex. Note the relative abundance of fat between the chin and the hyoid bone. To what is " double chin" due? Take care to avoid 
FIG. 86.

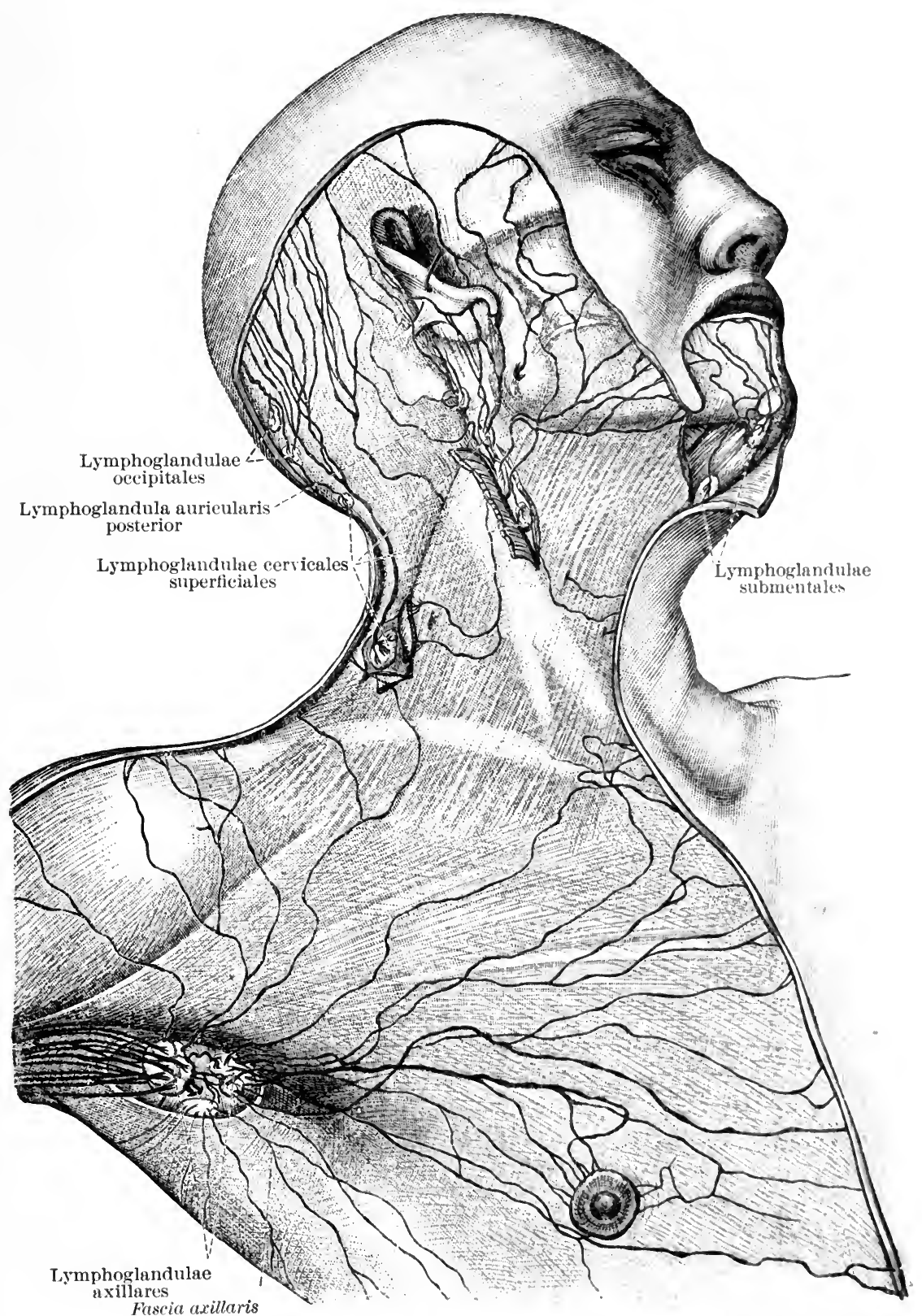

The superficial lymphatic vessels and lymph-glands of the head, neck, upper thoracic and shoulder regions. (After Toldt, Anat. Atlas, Wien, 1900, 2 Aut., p. 702, Fig. 10s4.) 
FIG. 87.

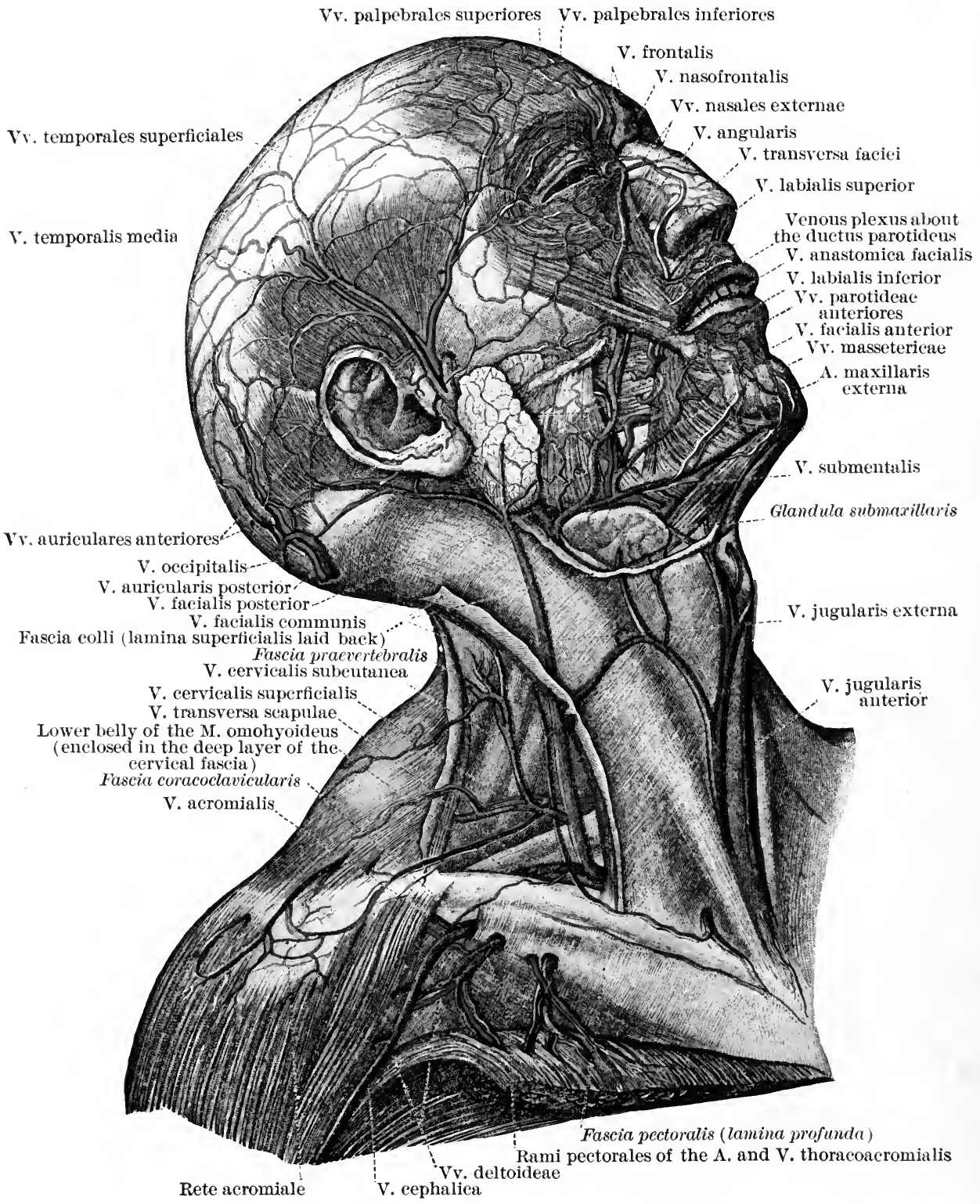

The superficial veins of the head and neck. (After Toldt, Anat. Atlas, Wien, 1900, 2 Aufl., p. 664, Fig. 1046.) 
injury to the platysma, which lies in the superficial fascia. Observe the superior rami of the cutaneous nerve of the front of the neck (rami superiores $N$. cutanei colli), superficial to the platysma. The superficial lymphatic channels can be well seen only in especially injected specimens. (Vide Fig. 86.)

Study the form, position, origin, insertion, innervation, and action of the platysma. Is the muscle in general placed superficial or deep as regards the panniculus adiposus? Is the fat of double chin premuscular, retromuscular, or both? Note that the platysma always moves with the skin of the neck. The loose areolar tissue between the platysma and the superficial layer of the fascia colli permits of free movement. What is the relation of the anterior jugular and of the external vein to the platysma? Why is the platysma called the muscle of fright? (Cf. Duchenne, Mécanisme de la physionomie humaine, Figs. 61, 62, and 63.) Why is it easier to pinch up a fold of skin vertically than transversely in the neck? What effect would the platysma have on the edges of a wound passing through the skin and muscles at right angles to the course of its fibres?

Structures between the Platysma and the Surface of the Fascia Colli.

Reflect the platysma from below upward, taking care not to injure the subjacent nerves and veins.

Veins.

External jugular ( $V$. jugularis externa).

(a) Posterior auricular ( $V$. auricularis posterior).

(b) Anterior jugular ( $V$. jugularis anterior).

How would you draw a line on the surface of the neck corresponding to the position of the external jugular vein? Observe the superficial lymphatic glands (lymphoglandulae cervicales superficiales) along the external jugular vein. How many are there? Whence do they draw lymph? (Vide Figs. 86 and 93.)

Nerves.

Branches of the cervical plexus.

(a) Lesser occipital nerve ( $N$. occipitalis minor).

(b) Great auricular nerve ( $N$. auricularis magnus).

(c) Cutaneous nerve of the front of the neck ( N. cutaneus colli) ( $\mathrm{O}$. T. superficial cervical).

(ca) Upper rami (rami superiores).

$(c b)$ Lower rami (rami inferiores).

Is a cutaneous nerve of the back of the neck ( $N$. cutaneus cervicis) present?

(d) Supraclavicular nerves (Nn. supraclaviculares).

(da) Anterior (Nn. supraclaviculares anteriores) (O. T. suprasternal). 
Fig. $87 a$.

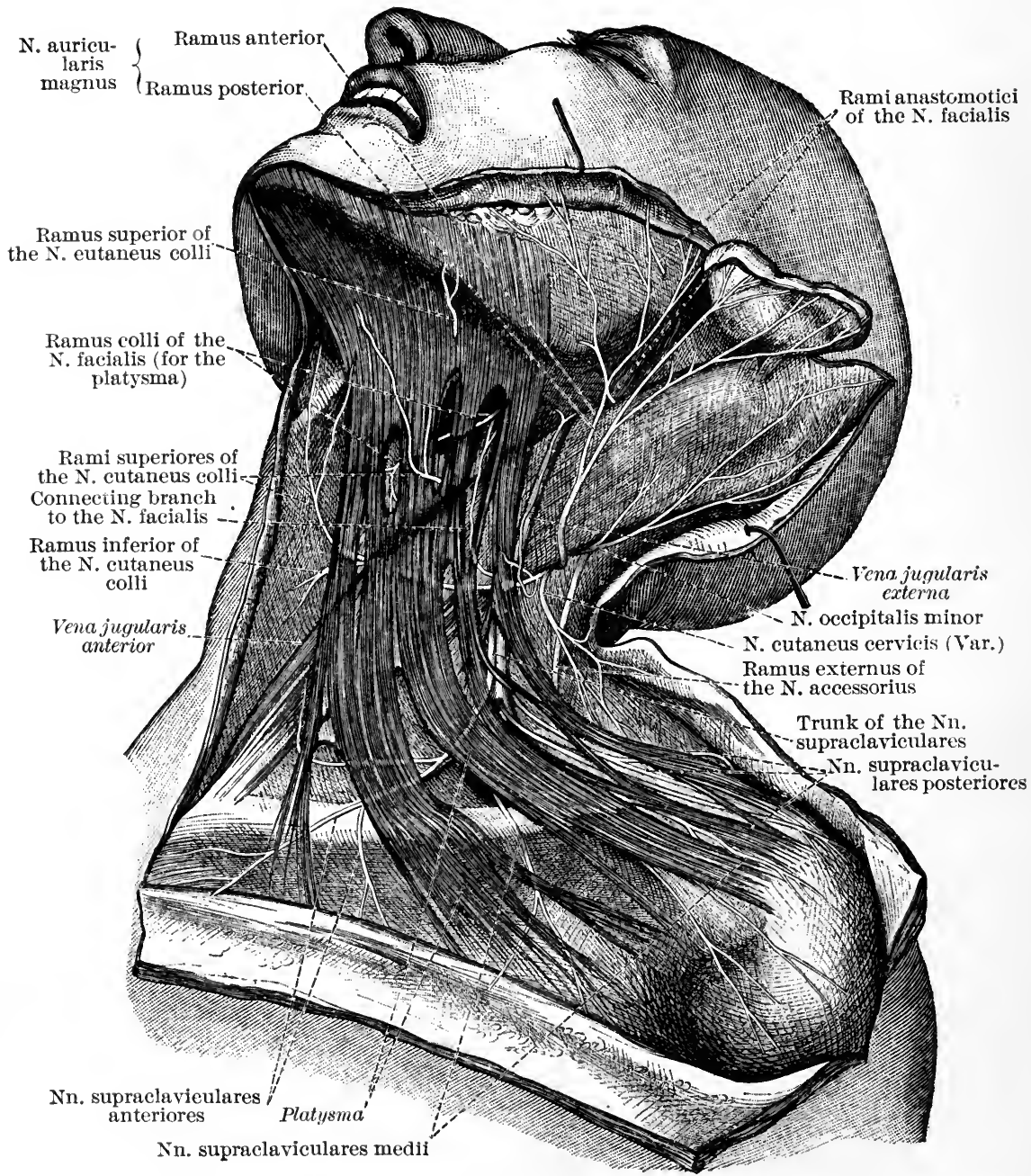

The cutaneous branches from the cervical plexus to the head and neck and their relation to the platysma. The bundles of the latter have been pulled apart in places to show partially the nerves under it. (After Toldt, Anat. Atlas, Wien, 1903, p. 819, Fig. 1251.) 
(db) Middle (Nn. supraclaviculares medii) (O. T. supraciavicular).

Note that the direction of the six principal branches corresponds to six lines radiating from a point at the middle of the posterior border of the M. sternocleidomastoideus.

Branches of facial nerve to neck.

(a) Branch to front of neck (ramus colli $N$. facialis).

Note its anastomosis with a branch of the $\mathrm{N}$. cutaneus colli.

\section{Deep Cervical Fascia and Aponeuroses. (Figs. 87, 88, and 89.)}

At this stage, before proceeding further with the dissection, the student will do well to read a good description of the deep cervical fascia. One of the best descriptions is that in Poirier et Charpy, t. ii. pp. 409-430. If the student does not read French easily, he will find a brief description in Cunningham's TextBook of Anatomy, 1902, pp. 373, 374, 1178, 1179. In the description, pay attention especially to the following points and verify each as the dissection goes on:

General subdivision of the deep fascia and aponeuroses of the front of the neck into-

(a) A superficial part (fascia colli, superficial layer), corresponding to the sternocleidomastoid muscles.

(b) A middle part (fascia colli, deep layer) (O. T. pretracheal fascia), corresponding to the subhyoid muscles.

(c) A deep part (fascia praevertebralis), corresponding to the prevertebral muscles.

Between $(b)$ and $(c)$ are a large central compartment containing viscera and a small lateral compartment on each side containing the great vessels, each compartment having a sheath.

The Superficial Part (Fascia colli, superficial layer).

Attachments.

Above, to prominentia occipitalis externa, linea nuchae superior, processus mastoideus, and to the fascia parotideomasseterica and the inferior border of the mandibula.

Below, to the spina scapulae, anterior border of clavicle, upper border of sternum.

Behind, to the ligamentum nuchae.

In front, to same layer of opposite side.

Note splitting of layer to enclose sternocleidomastoid muscle (M. sternocleidomastoideus) and again to enclose the trapezius muscle (M. trapezius); it does not split above the sternum to make suprasternal compartments, as ordinarily described.

Observe firm transverse fixation of superficial layer of fascia colli to anterior surface of hyoid bone. This is the true line of demarcation between the head and the neck; the suprahyoid muscles are all innervated by cerebral nerves. The intermuscu- 
lar septa separating the muscles of the front of the neck (collum) from those of the back of the neck (cervix) are attached to the deep surface of the layer under description; they pass medialward and a little forward, and become attached to the lateral portion of the fascia praevertebralis which encloses the scalene

FIG. 88 .

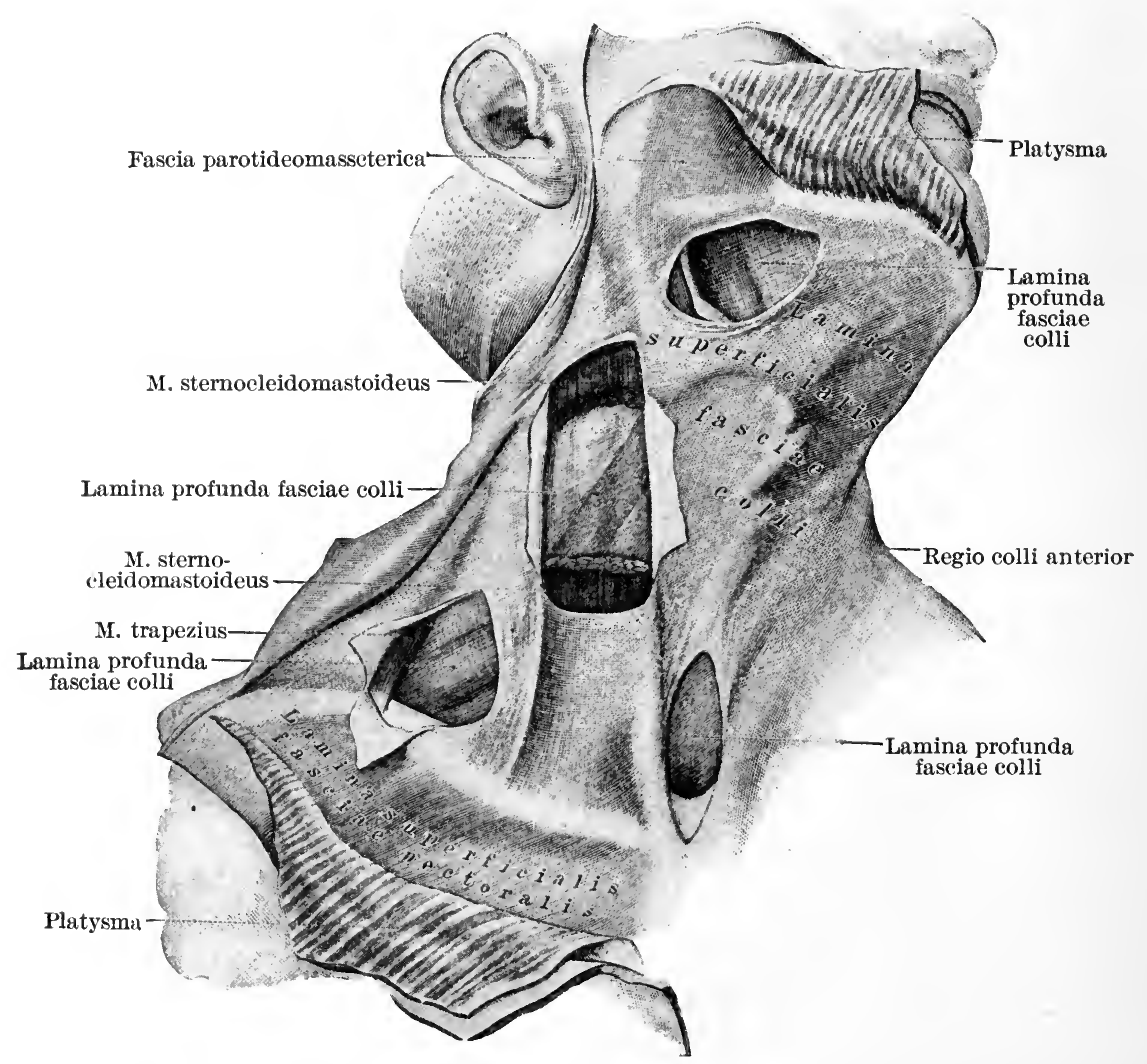

The fascia colli on the right side after the removal of the platysma. The superficial lamina is partly taken away in places where it is sharply separated from the deep lamina. The middle portion of the sternocleidomastoid muscle has been removed to show the deep layer of the fascia colli, under which is seen the upper belly of the omohyoid muscle shining through. (After Toldt, Anat. Atlas, Wien, 1900, 2 Aufl., p. 287, Fig. 525.)

muscles (Mm. scaleni) and extends between the anterior and posterior tubercles of the transverse processes of the cervical vertebrae.

Note that where the superficial layer of the fascia colli is covered by the platysma it is thinner than elsewhere. Examine closely various parts of the fascia under description: 
1. Sternomastoid portion.

2. Parotid portion.

3. Submaxillary portion.

4. Median suprahyoid portion.

5. Median infrahyoid portion.

6. Supraclavicular portion.

The Middle Part (Fascia colli, deep layer) ("omoelavicular aponeurosis" of Richet).

Extent.

Vertically, from os hyoideum to thorax.

Laterally, from one M. omohyoideus to the other.

FIG. 89.

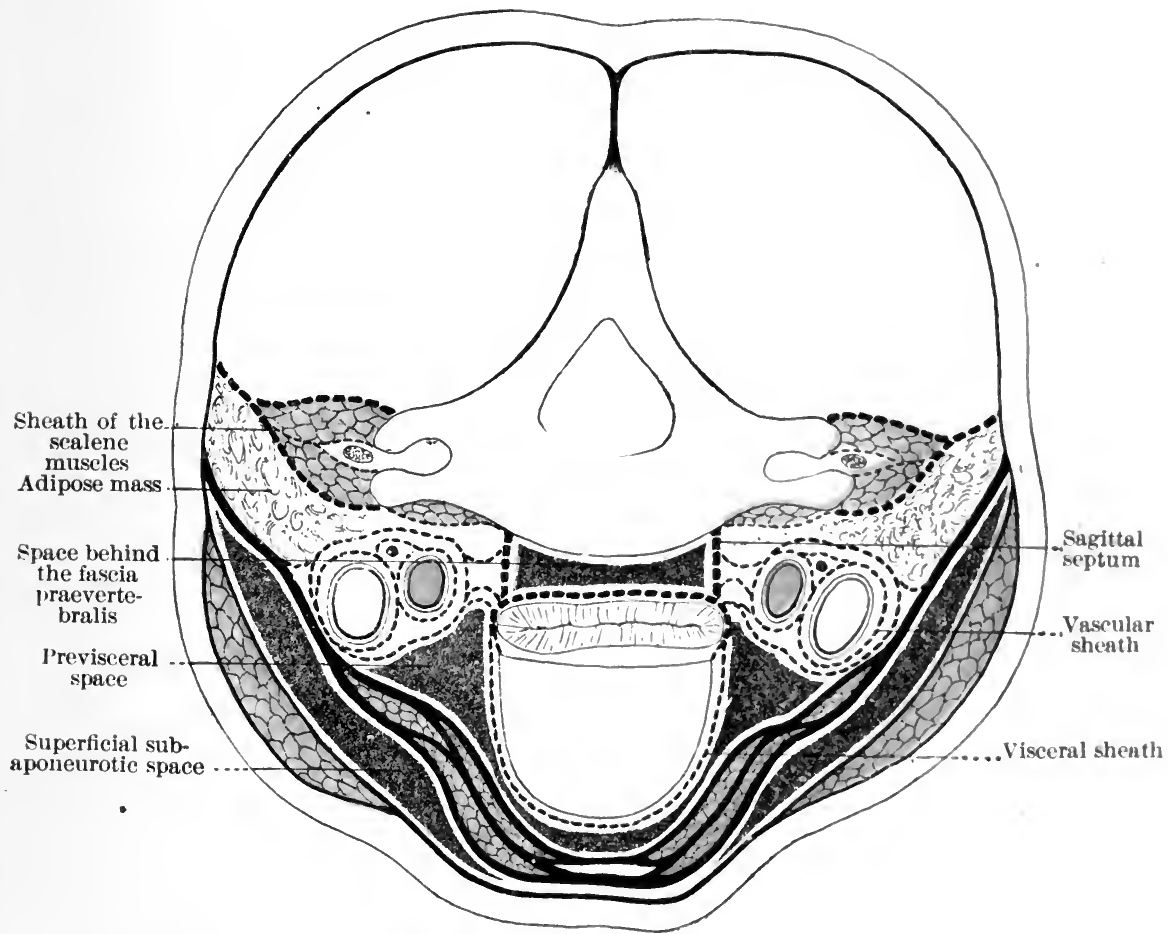

The interaponeurotic spaces, seen in section und supposed to be injected. The visceral and vascular sheaths, the sagittal septa, and the faseia praevertebralis are represented by dotted lines, the middle aponeurosis by a heary black line. The section passes immediately below the larynx. (From Polrier et Charpy, Traité d'Anat. hum., Paris, 1901, 2 ed., t. ii. p. 426, Fig. 269.)

Embedded in it are the omohyoid, sternohyoid, cleidohyoid, and thyreohyoid muscles. In lower forms this fascia is represented by a single broad cleidohyoid muscle; in man it has become differentiated into several muscles with connective tissue between. (Gegenbaur.) 
Distinguish in it-

(1) A deep or intermuscular layer.

(2) A superficial or premuscular layer.

The suprasternal space (sometimes called Burns' space) is situated between the superficial layer of the fascia colli and the premuscular layer of the middle layer of the fascia colli. (Charpy.) Examine its contents. The interaponeurotic veins near the base of the neck are aspirated during inspiration. Hence this is the most dangerous area for the entrance of air into the veins during surgical operations.

\section{The Deep Part (Fascia praevertebralis).}

Note its two parts:

(1) That extending from the anterior longitudinal ligament of the spine to the anterior tubercles of the transverse processes; it covers the prevertebral muscles proper ( $\mathrm{Mm}$. longi colli et capitis). Observe the relation of the $\mathrm{N}$. sympathicus to this part.

(2) That extending from the anterior to the posterior tubercles around the $\mathrm{Mm}$. scaleni. Observe the relation of the $\mathrm{N}$. phrenicus and of the Nn. cervicales to this part.

The fascia praevertebralis is part of the great common sheath of the body-cavity, being for the neck what the endothoracic fascia, the transversalis fascia, and the pelvic fascia are for the other visceral cavities. (Fawcett.)

\section{The Visceral Sheath.}

Contents. - Thyreoid gland, trachea, oesophagus, and pharynx.

Shape.-In cross-section, semicylindrical.

Attachments.

Behind, by lateral angles to the vertebral column; between these two attachments is left a retrovisceral or prevertebral space. This is of especial interest in the retropharyngeal region (postpharyngeal abscess, etc.).

Above, to base of skull.

Below, extends into mediastinum.

The Vascular Sheath.

(a) Common sheath. (Note relation of ramus descendens N. hypoglossi.) Observe attachment lateralward to deep layer of fascia colli and medialward to visceral sheath. The common sheath encloses $b, c$, and $d$.

(b) Sheath for A. earotis (loose, to permit of pulsation).

(c) Sheath for V. jugularis interna (dense, especially between artery and vein, septum vasorum).

(d) Sheath for N. vagus (always satellite of artery, even in embryonic life, when vein is distant). 
Some Enclosed Spaces of the Neck.

(a) Subcutaneous space-between fascia superficialis and superticial layer of fascia colli.

(b) Superficial subaponeurotic space-between superficial and deep layers of fascia colli.

(c) Suprasternal space.

(d) Previsceral space-between the deep layer of the fascia colli in front and the visceral and rascular sheaths behind. It contains fat and deep lymphatic glands.

(e) Retrovisceral or prevertebral space-bounded in front by visceral sheath, behind by spine, and laterally by attachments of visceral sheath to spine.

\section{Surgical Anatomical Points.}

(a) Abscesses within the visceral sheath either are secondary to infections involving the organs contained within it or are due to primary pyogenic infection of the areolar tissue inside it.

(b) Abscesses secondary to disease of the retropharyngeal lymph-glands lie in front of the fascia praevertebralis in the space between it and the visceral sheath.

(c) Abscesses secondary to disease of the cervical vertebrae lie behind the fascia praevertebralis and spread lateralward behind the rascular sheath; they usually point behind the M. sternocleidomastoideus, and may be opened by an incision at the posterior border of this muscle.

(d) The earotid ehain of lymph-glands (lymphoglandulae cerricales profundae) lies inside the common part of the vasenlar sheath. Abscess resulting from disease of these glands usually points upon the surface, owing to the formation of adhesions: first bet ween the gland and the vascular sheath, then between the latter and the superficial layer of the fascia colli, and finally between the latter and the skin.

\section{Sternocleidomastoid Muscle (M. sternocleidomastoideus). (Vide} Fig. 91, p. 219.)

Note that it divides the neck into an anterior and a posterior triangle. Remove from its surface the covering derived from the superficial layer of the fascia colli. Ascertain exactly its origin, insertion, innervation, and action. Why does Kiause designate it " the quadrigeminal muscle of the head"? Which of the four parts (sternomastoid, sterno-occipital, cleidomastoid, and cleido-occipital) are present in your cadaver?

Posterior Triangle of the Neck. (Vide Fig. 9I, p. 219.)

Remove, over the posterior triangle, the superficial and deep layers of the fascia colli; note that the former covers the whole triangle, the latter only its inferior part as far as the upper 
border of the inferior belly (venter inferior) of the M. omohyoideus. Beneath the tendinous part of the M. omohyoideus, where it passes below the sternomastoid, secure the small nervetwig from the descending ramus of the hypoglossal nerve $(R$. descendens $N$. hypoglossi) which goes to it. How is the posterior triangle bounded in front, behind, and below? How is its apex formed? The inferior belly of the omohyoid muscle divides it into $(a)$ an upper, larger part, the occipital triangle, and (b) a lower, very much smaller part, the supraclavicular triangle. Notice the chain of lymph-glands at the posterior border of the M. sternocleidomastoideus. They are often inflamed in diseases of the scalp (impetigo pedicularis).

\section{The Occipital Triangle.}

What are its boundaries? How is its floor formed from above downward? Observe among its contents the following:

Arteries. (Vide Fig. 97, p. 236.)

(a) Occipital artery (A. occipitalis) occasionally.

(b) Transverse artery of neck (A. transversa colli). Veins.

(a) Occipital vein ( $V$. occipitalis).

(b) Transverse vein of neck ( $V$. transversa colli). Nerves.

(a) Supraclavicular branches of the cervical plexus (Nn. supraclaviculares).

(b) Accessory nerve ( $N$. accessorius) (O. T. spinal accessory).

(c) Branches of eervical nerves to M. trapezius and to M. levator seapulae (rami musculares Nn. cervicalium).

Many of these structures will be studied more particularly when the body is turned on its face and the back of the neck is dissected.

\section{The Supraclavicular Triangle (O. T. Subclavian Triangle).}

The fascia praevertebralis may be removed where it covers the vessels and nerves. Note its continuity with the axillary sheath. What are the boundaries of the supraclavicular triangle? Find and study the following structures, and compare each as found in the cadaver with the description in your textbook of systematic anatomy.

Arteries. (Vide Fig. 16, p. 63.)

(a) Third portion of subclavian artery (A. subclavia).

(b) Transverse artery of neck (A. transversa colli).

(c) Transverse artery of scapula (A. transversa scapulae) (O. T. suprascapular). 
Veins. (Vide Fig. 87, p. 206.)

(a) External jugular vein ( $V$. jugularis externa).

(b) Transverse vein of scapula (V.transversa scapulae) (O. T. suprascapular).

(c) Transverse vein of neck ( $V$. transversa colli).

(d) Subclavian vein ( $V$. subclavia).

FIG. 90.

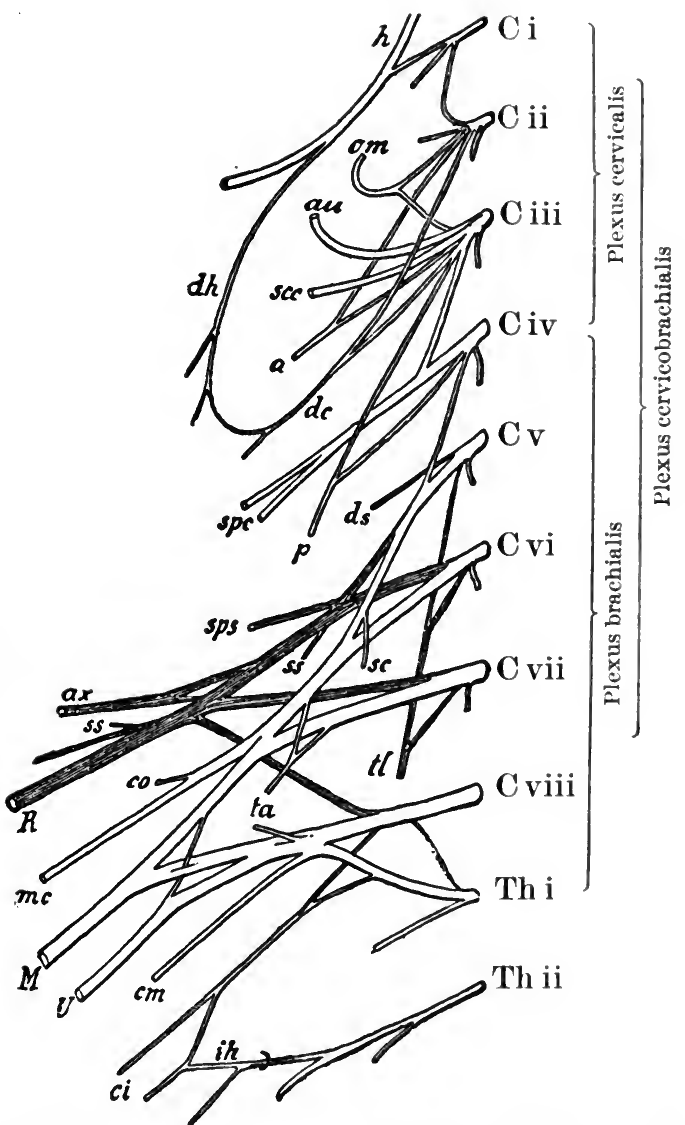

Plexus cervicobrachialis. (After P. Eisler, from Rauber's Text-Book.) Ventral view.

$h$, N. hypoglossus; $d h$, ramus descendens N. hypoglossi, which, along with $d c$, the ramus descendens cervicalis, forms the ansa hypoglossi; om, N. occipitalis minor; $a u, N$. auricularis magnus; $8 c c$, N. cutaneus colli; $a$, to N. accessorius; $s p c, \mathrm{Nn}$. supraclaviculares; $p, \mathrm{~N}$. phrenicus ; $d s, \mathrm{~N}$. dorsalis scapulae; $s p s, \mathrm{~N}$. suprascapularis; $s s, \mathrm{Nn}$. subscapulares; $s c, \mathrm{~N}$. subclavius; $a x, \mathrm{~N}$. axillaris; $c o, \mathrm{~N}$. to $\mathrm{M}$. coracobrachialis; $R, \mathrm{~N}$. radialis ; $m c, \mathrm{~N}$. musculocutaneus; $M, N$. medianus; $t a$, Nn. thoracales anteriores ; $t l, \mathrm{~N}$. thoracalis longus ; $U, \mathrm{~N}$. ulnaris ; $\mathrm{cm}$, N. cutaneus antibrachii medialis ; $c i, \mathrm{~N}$. cutaneus brachii medialis; ih, N. intercostobrachialis. (From Barker, The Nervous System, New York, 1899, p. 324, Fig. 186.)

Nerves. (To be studied in conjunction with the dissector of the upper extremity.)

(a) Brachial plexus (plexus brachialis). (Vide Fig. 90.) 
(aa) Supraclavicular part (pars supraclavicularis).

(aaa) Posterior thoracic nerves (Nn. thoracales posteriores).

(1) Dorsal nerve of scapula ( $N$. dorsalis scapulae) (O. T. nerve to the rhomboids).

(2) Long thoracic nerve ( $N$. thoracalis longus)

(O. T. external respiratory nerve of Bell, or posterior thoracic).

(aab) Anterior thoracic nerves (Nn. thoracales anteriores).

(aac) Subclavius nerve (N. subclavius).

(aad) Suprascapular nerve (N. suprascapularis).

(aae) Subscapular nerves (Nn. subscapulares).

(aaf) Thoracodorsal nerve ( $N$. thoracodorsalis) (O. T. long subseapular nerve).

(aag) Axillary nerve (N. axillaris) (O. T. circumflex nerve).

(1) Muscular branches (rami musculares).

(2) Lateral cutaneous nerve of arm (N. cutaneus brachii lateralis).

\section{Surgical Anatomical Points.}

Note that the supraclavicular triangle is the "seat of election" for ligature of the subclavian artery; its relations to neighboring structures are, therefore, exceedingly important. Examine these carefully, using your text-book as a guide. Note especially the relation of the artery to the external jugular vein and its tributaries. Run the finger down the lateral margin of the M. scalenus anterior (just beneath the posterior margin of the M. sternocleidomastoideus) until the scalene tubercle (tuberculum scaleni) on the first rib is reached. This is the guiding-point for the surgeon in the operation of ligation. If the finger-nail rest on the tuberculum scaleni, the artery will be felt, in the living subject, to pulsate against the finger-pulp. The proximity of the lowest trunk of the plexus brachialis and of the cupula pleurae to the artery are important points to the surgeon. If a cervical rib be present, the artery may either be in front of it or arch above it.

The V. jugularis externa is often opened in the posterior triangle to relieve the right heart in asphyxia; its position corresponds to a line drawn from the angle of the jaw to the middle of the clavicle. It should not be opened in the lower part of its course, where it is held open by the fascia, owing to the danger of suction of air into the vessel during inspiration.

\section{How is the floor of the supraclavicular triangle formed?}

\section{Anterior Triangle of the Neck. (Vide Figs. 87 and 9r.)}

Turn the N. cutaneus colli and the ramus colli N. facialis backward and remove the superficial and deep layers of the fascia colli. Note that the deep layer does not extend above the omohyoid muscle or the hyoid bone. How is the anterior 
triangle of the neck bounded? Locate $(a)$ superior belly of omohyoid muscle (venter superior $M$. omohyoidei), $(b)$ digastric muscle (M. digastricus), and (c) stylohyoid muscle ( $M$. stylohyoideus). Clean their surfaces, but avoid injury to (1) the branches of the ramus descendens $N$. hypoglossi entering the upper border of the venter superior M. omohyoidei, (2) the anterior and posterior facial veins ( $V v$. faciales anterior et posterior) where they unite to form the common facial vein ( $V$. facialis communis) on the posterior belly of the M. digastricus, and (3) the mylohyoid nerve (N. mylohyoideus) entering the upper border of the venter anterior of the M. digastricus. When these muscles have been thoroughly eleaned, it will be noticed that they divide the anterior triangle of the neck into three subsidiary triangles from above downward:

(a) Submaxillary or digastric triangle.

(b) Carotid triangle (sometimes called "superior earotid" triangle).

(c) Muscular triangle (sometimes ealled "inferior carotid" triangle).

Review the boundaries of these three triangles. Note especially that the muscular triangle is covered by both the superficial and deep layers of the fascia colli, the digastric and carotid triangles by the superficial layer only.

The anterior triangle should be dissected as a whole, rather than the subsidiary triangles one by one; the structures passing through more than one triangle are then studied in their continuity. The areolar tissue binding arteries, veins, nerves, and muscles together is to be removed bit by bit. Atlases should be open before the student constantly; as soon as a structure is exposed, it should be identified by reference to the plates in the atlases and the name repeatedly associated with it as it is further handled. From time to time, a description of all structures exposed should be read in the systematic text-book. It is very helpful to write down in full each new term met with in order to learn it more quickly and surely. It will be found that the majority of the structures can be exposed without cutting through any muscle or larger vessel or nerve.

Submaxillary or Digastric Triangle. (Vide Fig. I13, p. 266.)

It is arbitrarily subdivided into an anterior and a posterior portion by the stylomandibular ligament (ligamentum stylomandibulare) (O. T. stylomaxillary ligament) (ef. Spalteholz, vol. i., Fig. 206) and a line drawn downward from it.

In the anterior portion find- 
(a) Lymph-glands.

(aa) Submaxillary lymph-glands (lymphoglandulae submaxillares $)$. There are three,-anterior, middle, and posterior.

( $a b$ ) Submental lymph-glands (between the anterior bellies of $\mathrm{Mm}$. digastrici of the two sides).

In eancer of the lip which glands are likely to be the seat of metastatic growth?

(b) Submaxillary salivary gland (glandula submaxillaris).

Study also the following:

Arteries.

(a) External maxillary artery (A. maxillaris externa) (O. T. facial artery).

(aa) Ascending palatine (A. palatina ascendens).

(ab) Tonsillar ramus (ramus tonsillaris).

(ac) Submental (A. submentalis).

(ad) Glandular rami (rami glandulares).

(b) Mylohyoid ramus of internal maxillary artery (ramus mylohyoideus A. maxillaris internae).

(c) Lingual artery (A. lingualis). This is really not in the triangle, being beneath its floor, as it is completely covered by the $M$. hyoglossus. It is very important to study it, however, in this situation, as it is the point where it is ligated. Notice that it lies in a minute triangle (Lesser's triangle), formed by the margin of the M. mylohyoideus, the venter posterior M. digastrici, and the N. hypoglossus. If the M. hyoglossus be exposed within this little triangle and the muscle split vertically, the lingual artery is exposed. (Fig. 113.)

Veins. (Vide Fig. 87, p. 206.)

(a) Anterior facial vein ( $V$. facialis anterior) and tributaries.

(aa) Submental vein ( $V$. submentalis).

(ab) Palatine vein ( $V$. palatina).

(b) Tributaries of internal jugular vein ( $V$. jugularis interna).

(ba) Lingual vein ( $V$. lingualis).

(bb) Vein accompanying hypoglossal nerve ( $V$. comitans $N$. hypoglossi).

Nerves.

(a) Hypoglossal nerve (N. hypoglossus).

(b) Mylohyoid nerve ( $N$. mylohyoideus)-from the third division of the fifth cerebral nerve.

\section{In the posterior portion of the triangle find-}

(a) Lower part of parotid gland (glandula parotis).

(b) External carotid artery (A. carotis externa).

$(b a)$ Posterior auricular artery (A. auricularis posterior).

Carotid Triangle. (Vide Fig. 9r.)

Why is it so called? Find in it and study the following structures : 
FIG. 91.

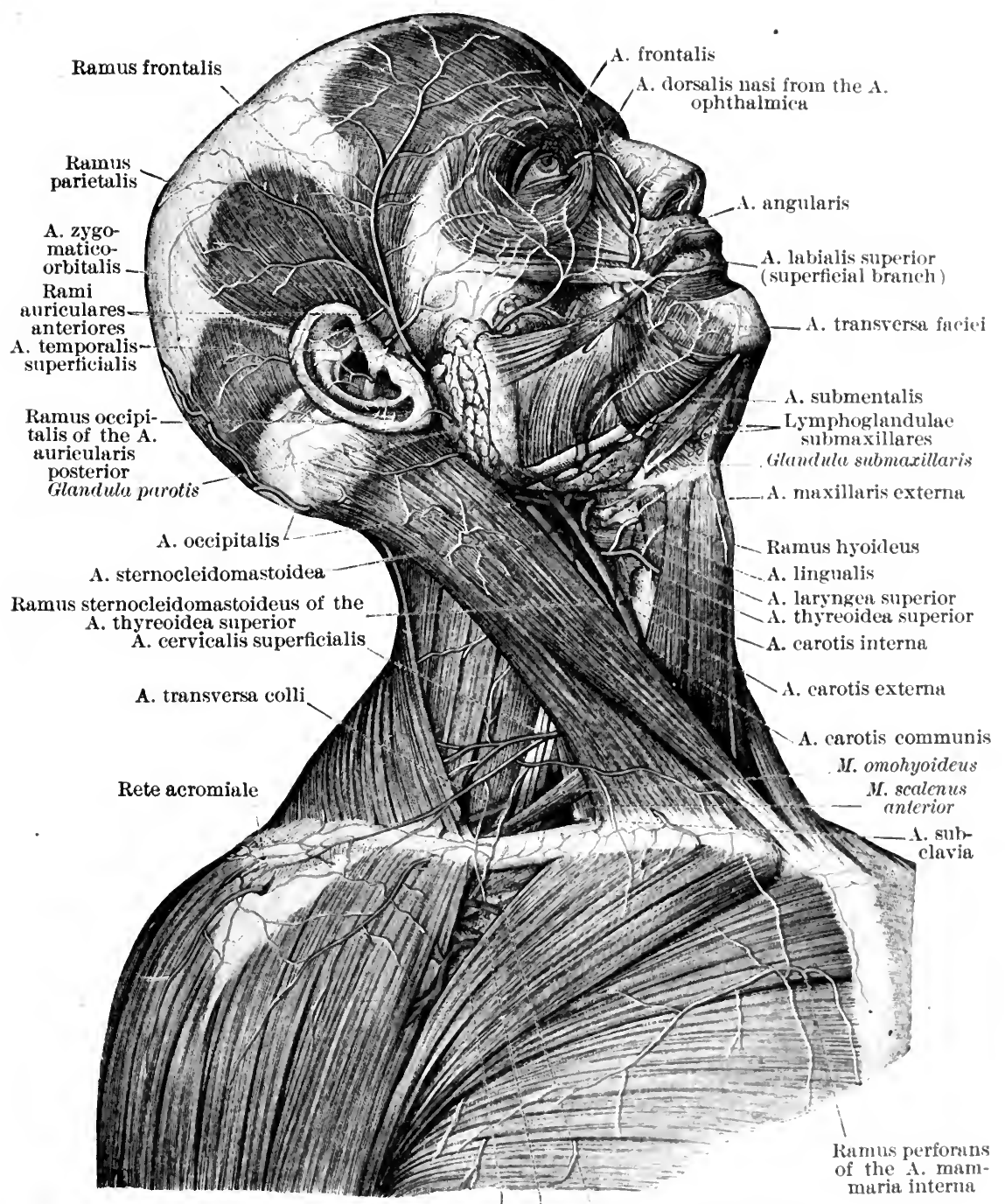

A. thoracoacromialis $\{$ Ramus deltoideus $\quad$ Fascia coracoclavicularis of the $A$. mam
maria interna

The superficial arteries of the head, neck, upper mammary and shoulder regions, seen from the right side. The fascia parotideomasseterica has been retained over the anterior part of the parotid gland. The lower end of the M. quadratus labii superioris has been removed. (After Toldt, Anat. Atlas, Wien, 1900, 2 Aufl., p. 592, Fig. 967.) 
Arteries.

(a) Common carotid artery (A. carotis communis).

(aa) External carotid artery (A. carotis externa).

(aaa) Superior thyreoid (A. thyreoidea superior).

(1) Hyoid ramus (ramus hyoideus).

(2) Sternomastoid ramus (ramus sternomastoideus).

(3) Superior laryngeal artery (A. laryngea superior).

(aab) Lingual artery (A. lingualis).

(1) Hyoid ramus (ramus hyoideus).

(aac) External maxillary artery (A. maxillaris externa) (O. T. facial).

(aad) Sternocleidomastoid artery (A. sternocleidomastoidea).

(aae) Occipital artery (A. occipitalis).

(aaf) Ascending pharyngeal artery (A. pharyngea ascendens).

Veins.

(ab) Internal carotid artery (A. carotis interna).

(a) Internal jugular vein (V. jugularis interna).

$(a a)$ Lingual vein ( $V$. lingualis).

( $a b)$ Superior thyreoid veins ( $V v$. thyreoideae superiores). $(a b a)$ Sternocleidomastoid vein ( $V$. sternocleidomastoidea).

$(a b b)$ Superior laryngeal vein ( $V$. laryngea superior).

(ac) Common facial vein ( $V$. facialis communis).

(aca) Anterior facial vein ( $V$. facialis anterior).

$(a c b)$ Posterior facial vein ( $V$. facialis posterior).

Nerves. Many of these will be much better seen later; at present they should simply be located in the triangle.

(a) Hypoglossal nerve (N. hypoglossus).

(aa) Descending ramus (ramus descendens).

(ab) Loop of the hypoglossus (ansa hypoglossi).

(ac) Thyreohyoid ramus (ramus thyreohyoideus).

(b) Accessory nerve ( $N$. accessorius) (O. T. spinal accessory).

(ba) Internal ramus (ramus internus).

(bb) External ramus (ramus externus).

(c) Vagus nerve (N. vagus) (O. T. pneumogastrie nerve).

(ca) Superior laryngeal nerve ( $N$. laryngeus superior).

(caa) External ramus (ramus externus) (O. T. external laryngeal nerve).

(cab) Internal ramus (ramus internus) (O. T. internal laryngeal nerve).

(d) Glossopharyngeal nerve ( $N$. glossopharyngeus).

(e) Sympathetic trunk (truncus sympathicus [pars cervicalis]).

(ea) Superior cervical ganglion (ganglion cervicale superius).

(eb) External carotid nerves ( Nn. carotici externi).

(ec) External carotid plexus (plexus caroticus externus).

(ed) Superior thyreoid plexus (plexus thyreoideus superior).

(ee) Lingual plexus (plexus lingualis).

(ef) External maxillary plexus (plexus maxillaris externus). 
FIG. 92.

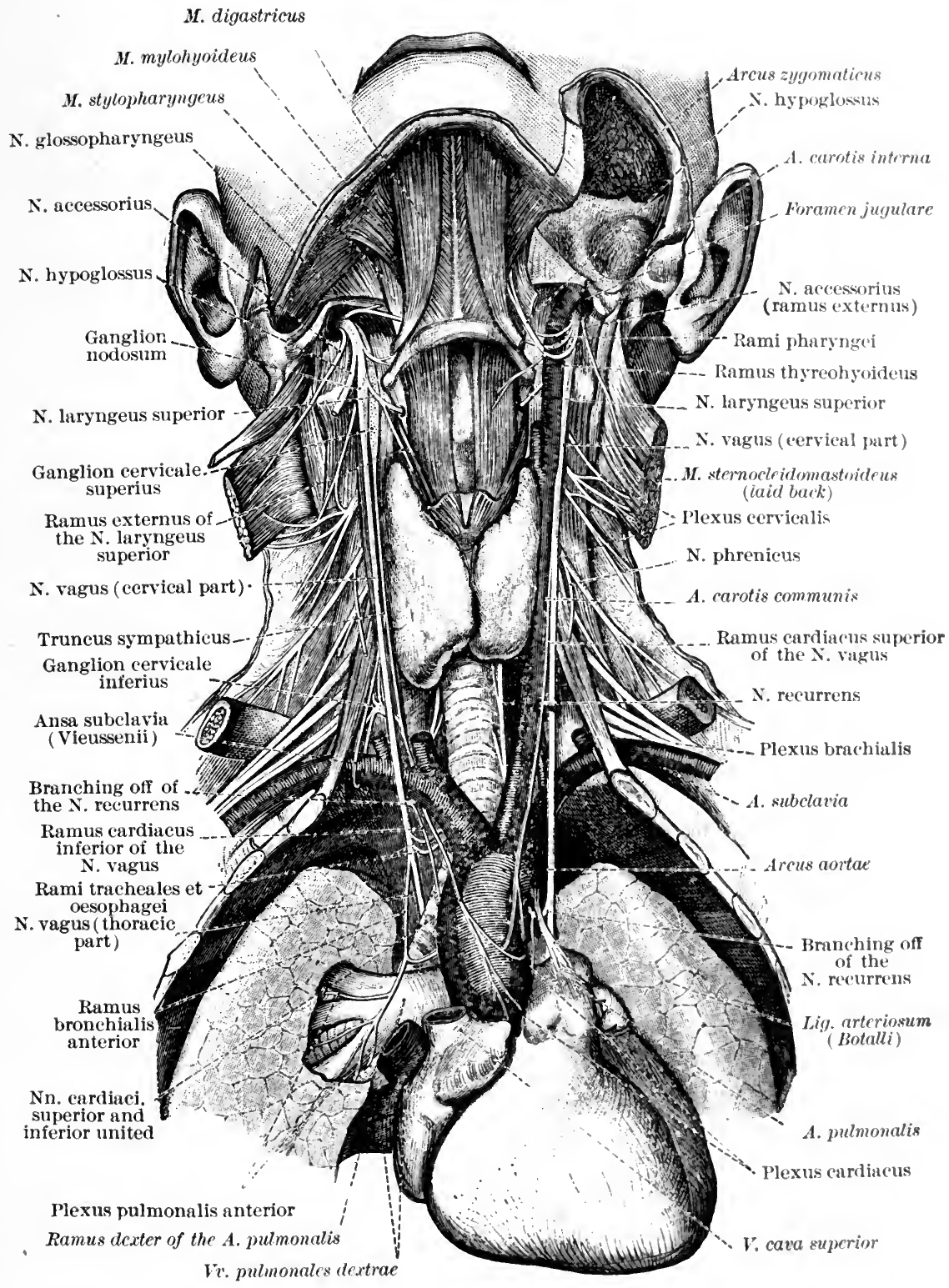

The anterior region of the neck. The head is strongly pulled back. (After Toldt, Anat. Atlas, Wien, 1903, 3 Autl, p. 878 , Fig. 1321.) 
Lymph-glands. (Vide Fig. 93.)

(a) Superior deep cervical lymph-glands (lymphoglandulae cervicales profundae superiores).

Viscera.

(a) Larynx.

(b) Pharynx.

(c) Carotid skein (glomus caroticum) (O. T. intercarotid gland).

Observe that some of the structures above enumerated become visible only after the parts have been loosened by dissection, that the greater cornu of the os hyoideum is in this space, and that, while the N. hypoglossus runs transversely in the spaces, the $\mathrm{N}$. vagus and truncus sympathicus run vertically and the other large nerves obliquely.

\section{Muscular Triangle.}

Why is it so called? Find and study the following structures : Muscles.

(a) Sternohyoid (M. sternohyoideus).

(b) Sternothyreoid (M. sternothyreoideus).

Arteries.

(a) Superior thyreoid artery (A. thyreoidea superior).

Nerves.

(a) Muscular rami to M. sternohyoideus and M. sternothyreoideus (from the ramus descendens $N$. hypoglossi).

(b) External ramus of superior laryngeal nerve (ramus externus $N$. laryng. sup.).

(c) Recurrent laryngeal nerve ( $N$. recurrens $N$. vagi).

Viscera.

(a) Larynx.

(b) Trachea.

(c) Thyreoid gland (glandula thyreoidea).

(d) Oesophagus (on left side).

Structures in the Anterior Median Line of the Neck and near it. (See Fig. 92, p. 22r.)

These should now be successively examined and studied.

Suprahyoid Region.

(a) Platysma.

(b) Fascia superficialis. (Note double chin.)

(c) Space between anterior bellies of Mm. digastrici of two sides.

(d) Mylohyoid muscles (Mm. mylohyoidei) and raphe between them.

Infrahyoid Region.

(a) Hyothyreoid membrane (membrana hyothyreoidea) (O. T. thyrohyoid membrane).

(b) Thyreoid cartilage (cartilago thyreoidea). 
FIG. 93.

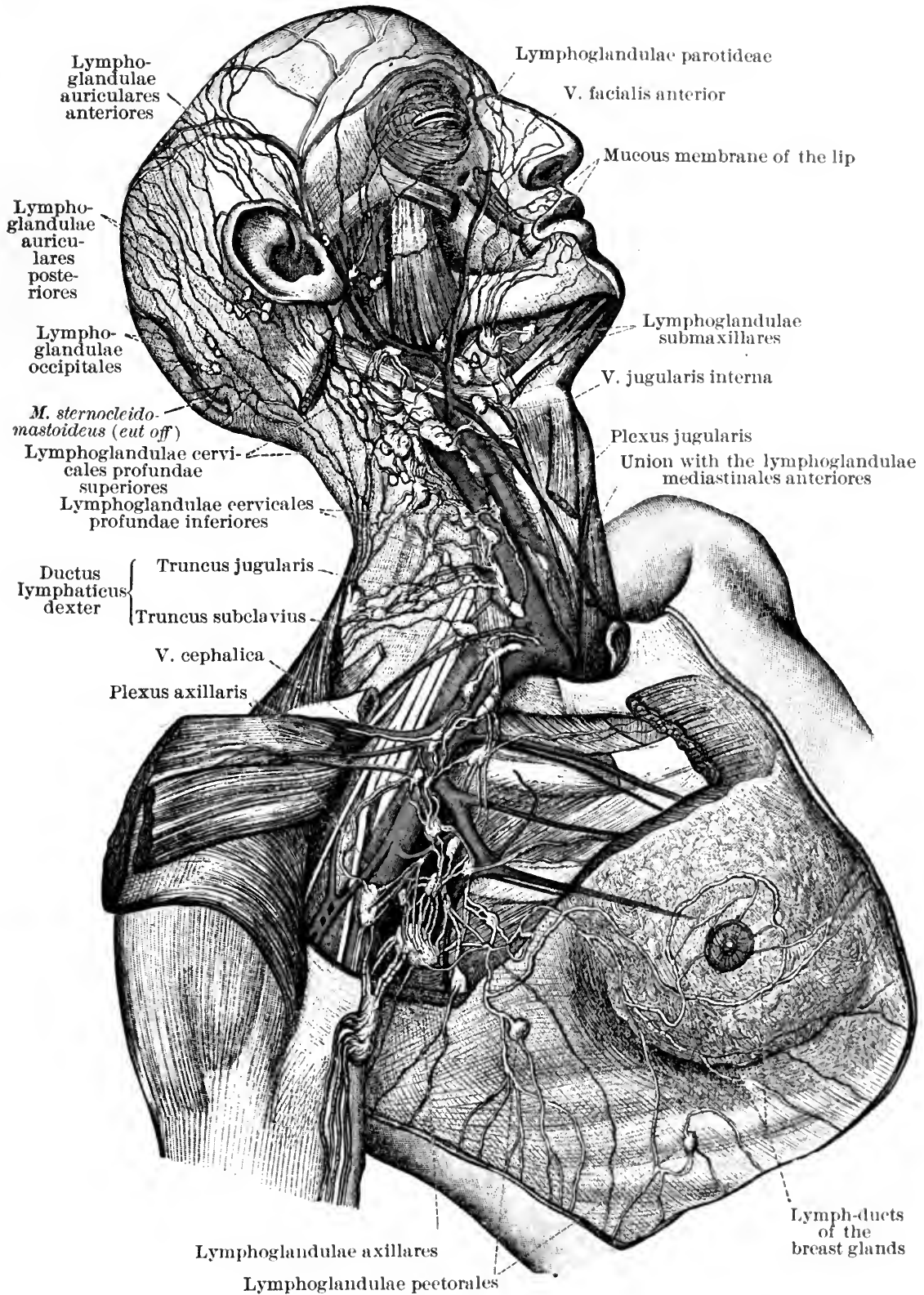

The lymphatic ressels of the head, the deep lymphatic vessels and lymph-glands of the nerk and axillary space, and the lymphatie vessels of the female breast. (After Toldt, Anat. Atlas, Wien, 1900, 2 Aufl., p. 703, Fig. 1085.) 
(c) Elastic cone and middle cricothyreoid ligament (conus elasticus, ligamentum cricothyreoideum [medium $]$ ) (O. T. cricothyroid membrane). Upon it lies the ramus cricothyreoideus of the $A$. thyreoidea superior.

(d) Cricoid eartilage (cartilago cricoidea).

(e) Cricothyreoid muscles (Mm. cricothyreoideae).

(f) Trachea.

FIG. 94.

M. sternocleidomastoideus
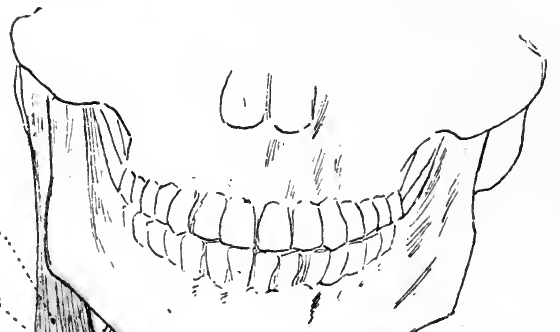

M. omohyoideus

M. sternohyoideus

M. scalenus medius

M. trapezoideus
M. scalenus posterior

M. omchyoideus

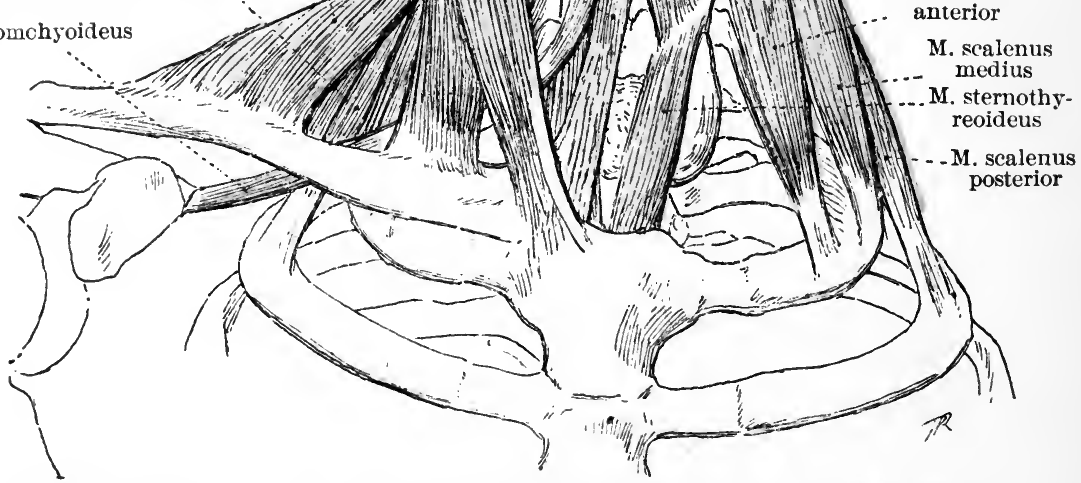

Muscles of the neck. (From Poirier et Charpy, Traité d'Anat. hum., Paris, 1901, 2 ed., t. ii. p. 395, Fig. 249.)

(g) Isthmus of thyreoid gland (isthmus glandulae thyreoideae).

(h) Pyramidal lobe of thyreoid gland (lobus pyramidalis [Gl. thyreoideae]).

(i) Inferior thyreoid veins ( $V v$. thyreoideae inferiores).

$(j)$ Innominate artery ( $A$. anonyma).

( $j a)$ Lowest thyreoid artery (A. thyreoidea ima).

(k) Left innominate vein ( $V$. anonyma sinistra).

(l) Thymus.

\section{Surgical Anatomical Points.}

In the operation of laryngotomy the ligamentum cricothyreoideum is cut through transversely in its lower part, inferior to the ramus cricothyreoideus of the $A$. thyreoideus superior. 
In the operation of tracheotomy the incision into the trachea is preferably made between the cricoid cartilage and the isthmus of the thyreoid gland (so-called high operation).

\section{Muscles of the Neck.}

Study the form, position, insertion, and innervation of the following muscles :

Those of the Second Layer.

(a) Digastric muscle (M. digastricus).

(b) Stylohyoid muscle (M. stylohyoideus).

These are supplied by $\mathrm{Nn}$. cerebrales.

Those of the Third Layer.

(a) Omohyoid muscle (M. omohyoideus).

(b) Sternohyoid muscle ( $M$. sternohyoideus).

(c) Sternothyreoid muscle (M. sternothyreoideus).

(d) Thyreohyoid muscle ( $M$. thyreohyoideus).

These are supplied by branches of the $\mathrm{Nn}$. cervicales running in the descending ramus of the $\mathrm{N}$. hypoglossus.

The Sternoclavicular Joint and the Ligaments about the Clavicle, Sternum, and First Rib.

The dissector of the upper extremity has removed the M. pectoralis major from the clavicle and sternum, and the sternoclavicular joint (articulatio sternoclavicularis) may now be studied. (Cf. Spalteholz, Fig. 227.) What bony surfaces are here concerned? Note the following:

(a) Sternoclavicular ligament (Lig. sternoclaviculare).

(b) Interclavicular ligament (Lig. interclaviculare).

(c) Costoclavicular ligament (Lig. costoclaviculare) (O. T. rhomboid ligament).

Cut through these ligaments and pull the clavicle lateralward so as forcibly to open the sternoclavicular joint. Observe that the articular disk of fibrocartilage (discus articularis) separates two joint-cavities, each with a special capsule (capsula articularis).

\section{The Root of the Neck.}

Remove with great care the areolar tissue at the root of the neck, avoiding injury to the finer rami of the sympathetic system. Study the structures on both sides of the body.

Muscles.

(a) Anterior scalene (M. scalenus anterior).

(b) Middle scalene (M. scalenus medius).

(c) Posterior scalene (M. scalenus posterior). 
(d) Smallest scalene (M. scalenus minimus) (inconstant).

Study the relation of these to surrounding structures and note especially the very important structures related to the most anterior of the three muscles.

Arteries.

(a) Subclavian artery (A. subclavia). (Vide Fig. 16.)

Branches :

Medialward from the M. scalenus anterior.

(aa) Vertebral (A. vertebralis).

( $a b)$ Internal mammary (A. mammaria interna).

(ac) Thyreocervical trunk (truncus thyreocervicalis).

(aca) Inferior thyreoid (A. thyreoidea inferior).

(acb) Ascending cervical (A. cervicalis ascendens).

(acc) Superficial cervical (A. cervicalis superficialis).

Behind the M. scalenus anterior.

(acd) Transverse scapular (A. transversa scapulae).

(ad) Costocervical trunk (truncus costocervicalis) (O. T. superior intercostal).

(ada) Highest intercostal artery (A. intercostalis suprema) (O. T. superior intercostal proper).

( $a d b)$ Deep cervical artery (A. cervicalis profunda).

Between the Mm. scaleni.

(ae) Transverse artery of neck (A. transversa colli).

Veins.

(a) Right and left innominate veins ( $V v$. anonymae dextra et sinistra).

(aa) Inferior thyreoid veins ( $V v$. thyreoideae inferiores).

$(a b)$ Inferior laryngeal vein ( $V$. laryngea inferior).

(ac) Lowest thyreoid vein ( $V$. thyreoidea ima).

(ad) Unpaired thyreoid plexus (plexus thyreoideus impar).

(ae) Vertebral vein ( $V$. vertebralis).

(aea) Deep cervical vein (V. cervicalis profunda).

$(b)$ Lower part of internal jugular vein $(V$. jugularis interna).

$(b a)$ An inferior thyreoid vein ( $V$. thyreoidea inferior).

Note the bulbus venae jugularis inferior.

(c) Subclavian vein ( $V$. subclavia).

(ca) Thoraco-acromial vein ( $V$. thoraco-acromialis).

$(c b)$ Transverse vein of neck ( $V$. transversa colli).

Lymphatics.

(a) Thoracic duct (ductus thoracicus). (Vide Fig. 93.)

(b) Right lymphatic duct (ductus lymphaticus dexter).

These may easily be mistaken for veins when first met with by the student.

Nerves. (Vide Fig. 92.)

(a) Phrenic nerve (N. phrenicus). Why should this nerve, arising in the neck, supply a muscle situated at the lower opening of the thorax?

(b) Vagus nerve (N. vagus). Note its relation to the M. scalenus anterior, the trachea, and the A. carotis communis.

(c) Lower part of cervical part of sympathetic trunk (truncus sympathicus). 
- (ca) Middle cervical ganglion (ganglion cervicale medium).

Does it rest on the A. thyreoidea inferior?

( $c b)$ Inferior cervical ganglion (ganglion cervicale inferius). Look for it in the depression between the transverse process of the seventh cervical vertebra and the neck of the first rib.

(cc) Superior cardiac nerve ( $N$. cardiacus superior).

(cd) Middle cardiac nerve ( $N$. cardiacus medius).

(ce) Inferior cardiac nerve ( $N$. cardiacus inferior).

(cf) Subclavian loop (ansa subclavia [Vieussenii]).

(cg) Subclavian plexus (plexus subclavius).

FIG. 95.

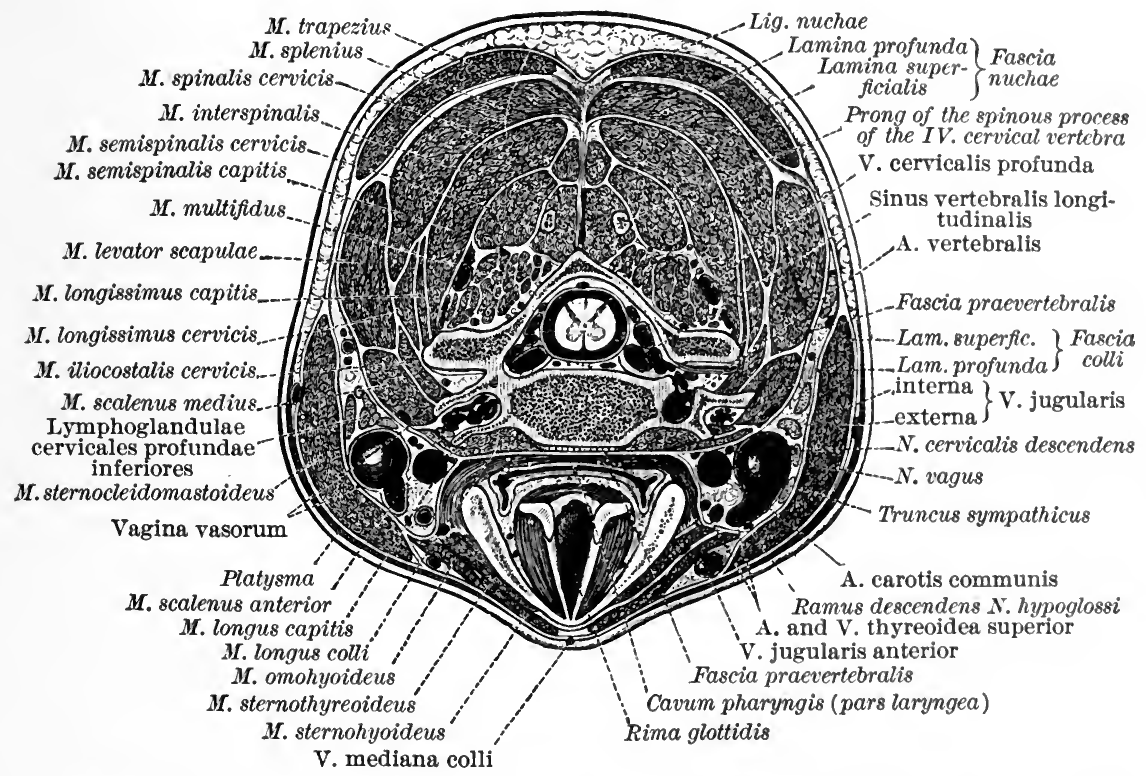

Cross-section through the neck at the level of the rima glottidis and through the body of the fifth cervical vertebra. Cut surface below the plane of section. (After Toldt, Anat. Atlas, Wien, 1900, 2 Aufl., p. 608, Fig. 984.)

\section{Viscera.}

(a) Summit of pleura (cupula pleurae).

How far above the level of the sternal extremity of the clavicle does it extend into the neck? Which is higher, the left or the right? Is there a $M$. scalenus minimus or tensor of the cupula pleurae present? What is Sibson's fascia? What structures come into contact with the cervical pleura on the right side? What on the left?

Note the fibrous expansions helping to support and fix the cupula (cf. Poirier et Charpy, t. iv., Figs. 250-252) :

(1) From the apex of the dome to the fourth to seventh eervical vertebrae. 
(2) From the lower half of the dome to the visceral sheath of the deep cervical fascia (pretracheal aponeurosis).

(3) From the sixth and seventh eervical vertebrae to the first rib, often replacing the M. scalenus minimus. This is the ligamentum costopleurovertebrale.

(4) From the anterior surface of neck of first rib to anterior surface of cupula. This is the ligamentum costopleurale.

The two latter ligaments may be absent altogether.

(b) Apex of lung (apex pulmonis).

(c) Trachea.

(d) Oesophagus.

Cervical Plexus. (Vide Figs. 90 and 122.)

The cervical plexus (plexus cervicalis) should now be studied. How is it formed? What is its relation to the brachial plexus? What are its exact situation and relation to surrounding parts? What muscles receive their innervation from it? Review the communications formed by the cervical plexus with the cerebral nerves ( $N$. hypoglossus, $N$. vagus, $N$. accessorius). How is the sympathetic nervous system related to the cervical plexus? Make drawings or diagrams to show the cervical plexus and its relations as they exist in your cadaver.

General Review of Blood-vessels of Side of Neck.

The dissector should now make a systematic review of all the bloodvessels of the side of the neck, reading on the structures from his textbook and making a sketch of the large arteries and their branches and the large veins and their tributaries.

Further Study of Viscera of Neck.

(a) Thyreoid gland (glandula thyreoidea).

(aa) Isthmus of thyreoid (isthmus Gl. thyreoideae).

(ab) Pyramidal lobe (lobus pyramidalis) (inconstant).

(ac) Right and left lobes (lobi dexter et sinister).

(ad) Lobules of gland (lobuli Gl. thyreoideae).

(ae) Stroma of gland (stroma Gl. thyreoideae).

(b) Trachea. Are any accessory thyreoid glands present?

(ba) Tracheal cartilages (cartilagines tracheales).

(bb) Annular ligaments (Ligg. annulares trachealia).

(bc) Membranous wall (paries membranacea).

(c) Cervical part of gullet (oesophagus, pars cervicalis).

\section{BACK OF HEAD, NECK, AND TRUNK.}

The dissectors of the head and neck work in conjunction with those of the upper extremity.

Make an incision in the middle line from the inion to the spine of the vertebra prominens, a second from the spine lateralward to the medial border of the acromion, and a third from the inion transversely lateralward (vide Fig. 3). Reflect the skin, 
taking no fat with it; the superficial fascia (fascia superficialis) is thus exposed. Why should the pain accompanying inflammation at the nape of the neck-as, for example, in carbuncle-be particularly severe?

\section{Superficial Vessels and Nerves. (Vide Fig. 4, p. 47.)}

Remove the superficial fascia piecemeal. The $\mathrm{A}$. and $\mathrm{V}$. occipitalis have already been met with in the dissection of the scalp, as have the terminals of the $\mathrm{N}$. occipitalis major and M. occipitalis minor. The trunk of the N. occipitalis major pierces the M. trapezius; note exact spot, usually about two and onehalf centimetres below the inion and one centimetre from the middle line. Is a $\mathrm{N}$. occipitalis tertius present? If so, where does it pierce the M. trapezius and how is it related in distribution to the $\mathrm{N}$. occipitalis major? Find the dorsal cutaneous branches of the fourth, fifth, sixth, seventh, and eighth cervical nerves (rami cutanei dorsales $N n$. cervicalium IV., V., VI., VII., VIII.). Ascertain as closely as possible the area of skin innervated by each.

Isolate the great auricular nerve ( $N$. auricularis magnus) and follow its posterior branch (ramus posterior); its anterior branch (ramus anterior) will be studied later.

Dissect out the cutaneous branches of the superficial cervical artery (A. cervicalis superficialis) and of the transverse artery of the scapula ( $A$.transversa scapulae). Note the corresponding veins. Into what lymph-glands do the lymphatics from this region empty? Make a drawing of the dissection at this stage.

\section{Upper Part of the Posterior Triangle of the Neck.}

Clean the surface of the trapezius muscle (M.trapezius) and the posterior border of the sternocleidomastoid muscle ( $M$. sternocleidomastoideus), which form the posterior and anterior boundaries of the triangle. Do not raise the M. sternocleidomastoideus and take care not to disturb the nerves which lie beneath it at this stage of the dissection. Clean the floor of the triangle. Note the direction of the fibres of the splenius muscle of the head (M. splenius capitis) and of the elevator muscle of the scapula (M. levator scapulae) (O. T. levator anguli scapulae). Avoid injury to the rami musculares of the plexus cervicalis supplying the latter muscle; they lie usually upon its surface in close apposition with it. How is the apex of the triangle formed? Is the semispinalis muscle ( $M$. semispinalis capitis) (O. T. complexus) visible? If so, in what direction do its fibres run? Is 
the occipital artery $(A$. occipitalis $)$ to be seen in the triangle? Dissect out the portion of the accessory nerve ( $N$. accessorius) belonging to the triangle. Note its exact point of emergence from the substance of the M. sternocleidomastoideus. Find the rami musculares of the plexus cervicalis which run across the triangle to disappear beneath the anterior margin of the $\mathrm{M}$. trapezius. How are they related in position to the N. accessorius? Do you find any large cutaneous nerve of the cervix (N. cutaneus cervicis)?

\section{Broad Muscles of the Back (First Layer). (Vide Fig. 5, p. 49.)}

(a) Trapezius muscle (M. trapezius). After its surface has been thoroughly cleaned, in conjunction with the dissector of the upper extremity, the exact origin and insertion of the M. trapezius are to be established. Test the effect of contraction of its various portions, fixing alternately the head and the shoulder girdle. With the dissector of the upper limb, reflect the muscle and dissect the structures on its deep surface. Divide the attachment to the linea nuchae superior and protuberantia oceipitalis externa and cut through it about one centimetre from the cervical vertebral spines and five centimetres from the thoracic spines, taking care that the knife does not injure the underlying structures. Turn the muscle lateralward and allow the dissector of the upper limb to work out the distribution of the $\mathrm{N}$. accessorius and the nerves from the cervical plexus going to the M. trapezius. Determine the origin and distribution of the A. cervicalis superficialis. In your cadaver does it come from the truncus thyreocervicalis or from the A. transversa colli?

(b) Broadest muscle of back (M. latissimus dorsi). The dissector of the arm has worked this muscle out and the dissector of the head should review his knowledge of it.

(c) Elevator muscle of scapula (M. levator scapulae) (O. T. levator anguli scapulae) and neighboring vessels and nerves. Examine the M. levator scapulae and the nerves on its surface (from the plexus cervicalis). Lift the muscle carefully and find beneath it -

(ca) Descending branch (ramus descendens) of the A. transversa colli, often ealled the "posterior seapular artery."

$(c b)$ Dorsal nerve of scapula ( $N$. dorsalis scapulae) (O. T. nerve to the rhomboids). Note the twigs from this nerve to the M. levator scapulae.

Establish the exact origin and insertion of the muscle and test its action.

Expose the scapular portion of the inferior belly (venter inferior) of the M. omohyoideus, and note the position of the A. transversa scapulae (O. T. suprascapular, or transversalis humeri) and the suprascapular nerve (N. suprascapularis) and 
the relation of each to the ligamentum transversum scapulae superius. Make a drawing of the part of the posterior triangle dissected and read in your systematic text-book descriptions of the various structures.

Broad Muscles of the Back (Second Layer). (Fig. 96.)

The larger and smaller rhomboid muscles (M. rhomboideus major, M. rhomboideus minor) have been worked out by the dissector of the arm. and may be conveniently reviewed.

\section{Broad Muscles of the Back (Third Layer). (Fig. 96.)}

The dissector of the head and neck now proceeds with the dissection of the muscles of the back, and permits the dissector of the upper extremity to study the parts. Observe the fascia nuchae.

(a) Superior posterior serratus muscle (M. serratus posterior superior).

Note its form and position. Establish its exact origin and insertion. Test its action. Is it concerned in inspiration or expiration? Find the nerves supplying it. What are they ealled? Is it a monomeric or a polymeric muscle? Divide it close to its attachment to the vertebral spines and reflect it lateralward.

(b) Inferior posterior serratus muscle (M. serratus posterior inferior).

(c) Splenius ("bandage") muscle of head (M. splenius capitis).

(d) Splenius muscle of neck (M. splenius cervicis) (O. T. splenius eolli).

Lumbodorsal Fascia (Fascia lumbodorsalis). (Vide Figs. 5 and 6.)

The posterior layer of this fascia is now visible. That portion which corresponds to the thoracic spine is often spoken of as the "vertebral aponeurosis"; that portion corresponding to the small of the back is often called the "lumbar fascia."

Examine the posterior layer of the fascia lumbodorsalis. What are its medial and inferior attachments? How is it attached lateralward? What becomes of it above? These questions may be answered (1) by making an incision through it in the middle of the thoracic region and passing the handle of the scalpel medialward and lateralward, and (2) by dividing the lumbar portion by a vertical cut made two or three centimetres from the median line and raising it from the subjacent M. sacrospinalis. Then displace the M. sacrospinalis medialward and expose the anterior layer of the fascia lumbodorsalis. How is the anterior layer attached medialward? Note the fusion of the anterior and posterior layers lateralward. Cut through the anterior layer close to its medial attachment and lift it from the 
FIG. 96.

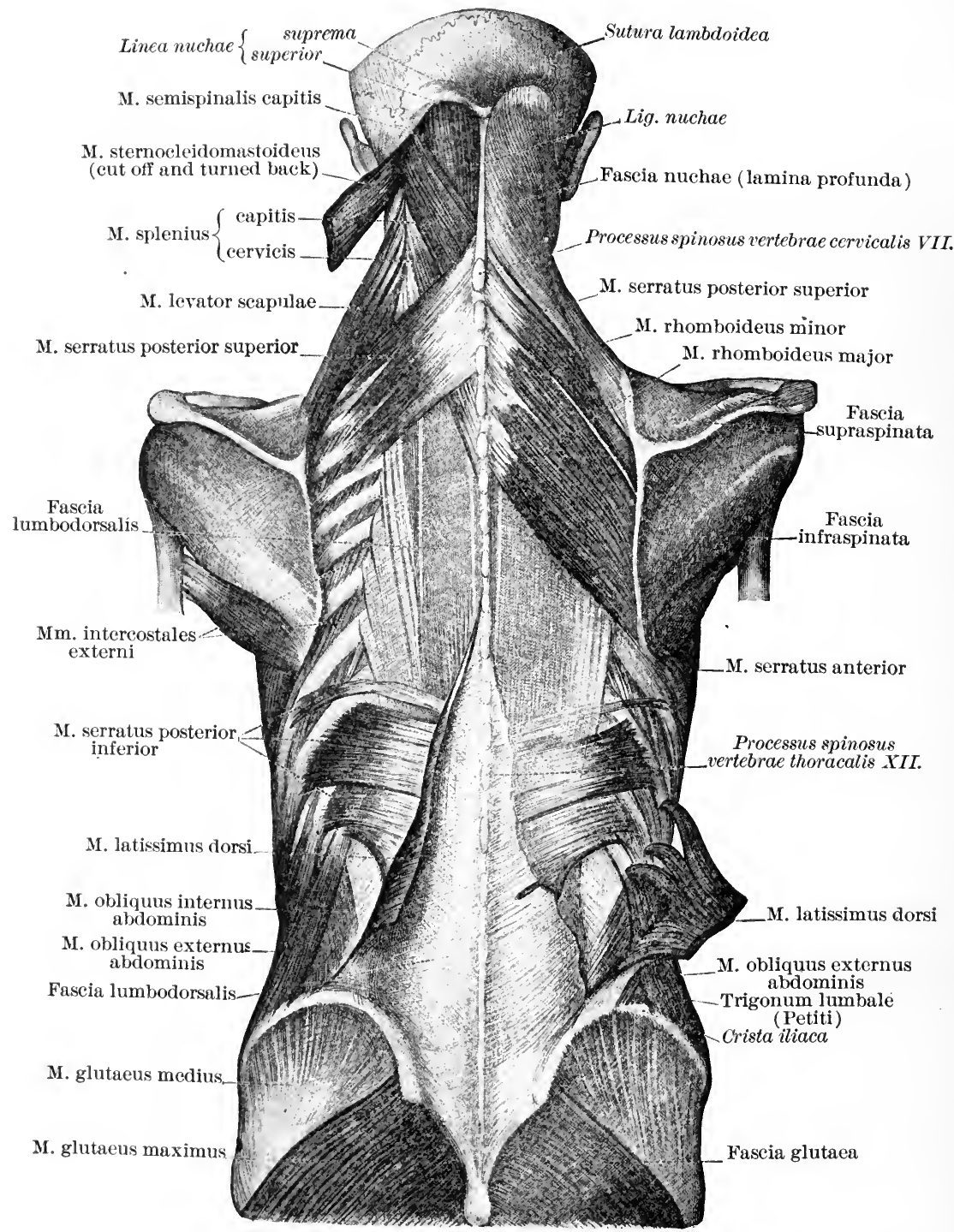

The second layer of the broad muscles of the back, shown after removal of the latissimus dorsi and trapezius muscles; on the left side the rhomboid muscles also have been taken away. (After Toldt, Anat. Atlas, Wien, 1900, 2 Aufl., p. 267, Fig. 505.) 
surface of the M. quadratus lumborum. Displace the latter medialward and the fascia covering the anterior surface of the latter will be brought into view. (This fascia was formerly called the anterior layer of the lumbar fascia.) Make a drawing of the structures in your cadaver corresponding to Spalteholz, Fig. 334 .

Long Muscles of the Back (First and Second Layers) (O. T. Third Layer of the Back). (Vide Fig. 4, p. 47.)

Cut through the attachments of the M. splenius capitis and M. splenius cervicis, close to the spines of the vertebrae, and reflect the muscles upward and lateralward. Turn the whole of the posterior layer of the fascia lumbodorsalis with the M. serratus posterior inferior lateralward.

(a) Sacrospinal muscle (M. sacrospinalis) (O. T. erector spinae). What are the names given to the three main divisions of this muscle? Which is the largest of the three, and which the smallest? Pull the M. iliocostalis lateralward on the right side; reflect it on the left. Blood-vessels and nerves emerge between the M. iliocostalis and the M. longissimus dorsi and are the guide in dissection. (Vide Spalteholz, Fig. 336.)

(aa) Lateral subdivision: iliocostal muscle (M. iliocostalis).

(aaa) Iliocostal muscle of loins (M. iliocostalis lumborum)

(O. T. sacrolumbalis).

(aab) Iliocostal muscle of back (M. iliocostalis dorsi) (O. T. musculus accessorius).

(aac) Iliocostal muscle of neck (M. iliocostalis cervicis) (O. T. cervicalis ascendens).

Establish the exact origin and insertion of each. Test action. Find innervation.

(ab) Middle subdivision: longest muscle (M. longissimus). $(a b a)$ Longest muscle of back (M. longissimus dorsi).

$(a b b)$ Longest muscle of neck ( $M$. longissimus cervicis) (O. T. transversalis cervicis).

(abc) Longest muscle of head (M. longissimus capitis) ( $O$. T. trachelomastoid).

Establish exact origin and insertion of each. Test action. Find innervation.

(ac) Medial subdivision: spinal muscle (M. spinalis).

(aca) Spinal muscle of back (M. spinalis dorsi).

(acb) Spinal muscle of neck (M. spinalis cervicis) (O. T. spinalis colli).

(acc) Spinal muscle of head (M. spinalis capitis).

Establish exact origin and insertion of each. Test action.

Find innervation. 


\section{Short Muscles of the Back (First, Second, and Third Layers). (Vide Spalteholz, Figs. 338, 339, and 340.)}

Cut away the attachments of the M. spinalis dorsi and reflect the M. longissimus dorsi lateralward. Reflect the M. longissimus capitis lateralward and upward. The M. semispinalis is now exposed. Clean it thoroughly. While cleaning the M. semispinalis capitis avoid injuring the posterior cutaneous rami of the cervical nerves (rami cutanei posteriores $\mathrm{Nn}$. cervicalium) emerging from the substance of the muscle near the middle line.

(a) Semispinal muscle (M. semispinalis). (Cf. Spalteholz, Fig. 338.)

(aa) Semispinal musele of back (M. semispinalis dorsi).

(ab) Semispinal musele of neck (M. semispinalis cervicis) (O. T. semispinalis colli).

(ac) Semispinal muscle of head (M. semispinalis capitis) (O. T. complexus).

Establish exact form, position, origin, insertion, action, and innervation of each.

Cut away the attachments of the M. semispinalis from the vertebral spines and from the transverse processes, and study-

(b) Multifidus ("much-divided") muscle (M. multifidus).

What is its origin? Establish insertion, action, and innervation.

Cut away the M. multifidus and expose the third layer of short muscles. Observe in the sacral region the posterior rami of the sacral and coceygeal nerves (rami posteriores Nn. sacralium et N. coccygei).

(c) Rotator muscles (Mm. rotatores).

(ca) Long rotator muscles ( $M m$. rotatores longi).

$(c b)$ Short rotator muscles ( $\mathrm{Mm}$. rotatores breves).

(d) Elevators of the ribs ( $\mathrm{Mm}$. levatores costarum).

(da) Short elevators of the ribs (Mm. levatores costarum breves).

(db) Long elevators of the ribs (Mm. levatores costarum longi).

(e) Interspinal museles ( $\mathrm{Mm}$. interspinales).

( $f$ ) Intertransverse muscles (Mm. intertransversarii).

( $f a)$ Cervical.

( $f a a)$ Anterior (Mm. intertransversarii anteriores).

( $f a b)$ Posterior (Mm. intertransversarii posteriores).

( $f b)$ Lumbar.

( $f b a)$ Medial (Mm. intertransversarii mediales).

$(f b b)$ Lateral (Mm. intertransversarii laterales).

\section{Short Muscles of the Neck. (Cf. Spalteholz, Fig. 34r).}

Carefully clean the surface of the following muscles:

(a) Larger posterior straight muscle of head (M. rectus capitis posterior major).

(b) Lesser posterior straight muscle of head (M. rectus capitis posterior minor).

(c) Superior oblique muscle of head (M. obliquus capitis superior).

(d) Inferior oblique muscle of head (M. obliquus capitis inferior). 
Find the posterior ramus of the suboceipital nerve (ramus posterior $N$. suboccipitalis), the branches of which supply all four. Establish the origin, insertion, and action of each muscle. What are the boundaries of the "suboccipital space, or triangle"? (Fig. 97.) Note in the space-

(a) Posterior ramus of the first cervical nerve (N. suboccipitalis).

(b) Portion of the vertebral artery (A. vertebralis).

Examine the floor of the space and distinguish-

(a) Posterior arch of the atlas (arcus posterior atlantis).

(b) Posterior atlanto-occipital membrane (membrana atlanto-occipitalis posterior).

\section{Blood-vessels and Nerves of Neck and Back.}

The more superficial vessels of the neck have already been studied. The student should carefully work out the following:

Arteries. (Fig. 97.)

(a) Occipital artery (A. occipitalis). How is it related to neighboring structures?

(aa) Muscular rami (rami musculares).

To what muscles are these distributed? Note especially the descending ramus (ramus descendens) ( $\mathrm{O} . \mathrm{T}$. arteria princeps cervicis).

(ab) Mastoid branch (ramus mastoideus).

Into what foramen does this enter?

(ac) Auricular branch (ramus auricularis).

(ad) Occipital branches (rami occipitales).

(b) Ascending cervical artery (A. cervicalis ascendens).

(ba) Muscular branches (rami musculares).

$(b b)$ Deep ramus (ramus profundus).

(c) Deep cervical artery (A. cervicalis profunda).

With what vessels does this artery anastomose?

(d) Posterior ramus of highest intercostal artery (ramus posterior $A$. intercostalis supremae).

(e) Posterior rami of intereostal arteries (rami posteriores Aa. intercostalium).

(ea) Muscular branches (rami musculares).

(eb) Medial cutaneous rami (rami cutanei mediales).

(ec) Lateral cutaneous rami (rami cutanei laterales).

Veins.

(a) Occipital vein ( $V$. occipitalis).

(aa) Mastoid emissary vein (emissarium mastoideum).

Why are the emissary veins of special importance?

Why is it a common practice to apply leeches and blisters behind the ear in certain intracranial affections?

(b) Deep cervical vein (V. cervicalis profunda).

(c) Posterior rami of intercostal veins (rami posteriores Vv. intercostalium). 
(d) Posterior rami of lumbar veins (rami posteriores Vv. lumbalium). Nerves.

In addition to those already studied note the following:

(a) Posterior rami of cervical nerves (rami posteriores $\mathrm{Nn}$. cervicalium).

(aa) Medial rami (rami mediales).

(ab) Lateral rami (rami laterales).

FIG. 97.

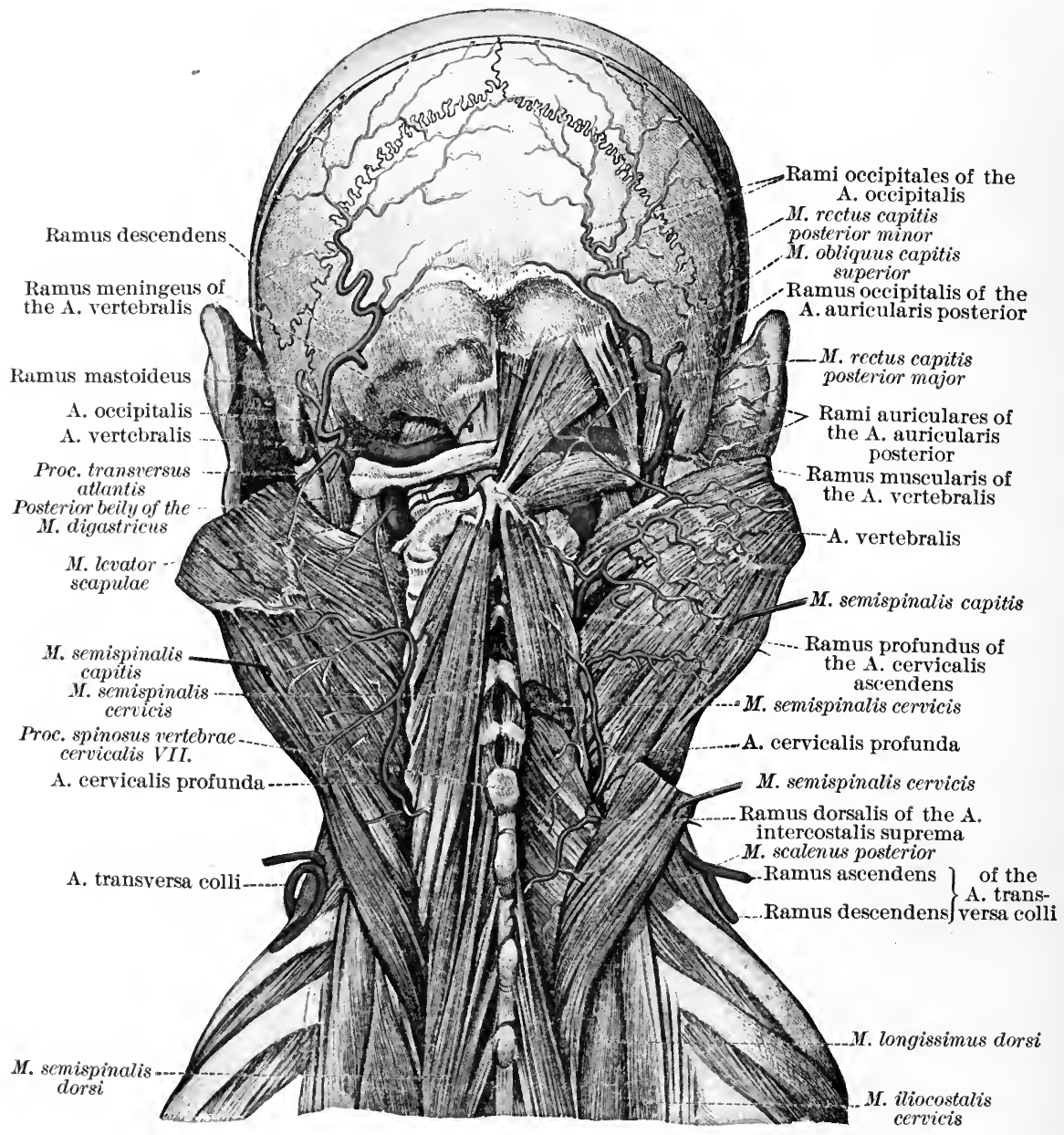

The arteries of the posterior region of the head and the deep arteries of the neck.

(After Toldt, Anat. Atlas, Wien, 1900, 2 Aufl., p. 597, Fig. 972.)

(b) Posterior rami of thoracic nerves (rami posteriores Nn. thoracalium).

(ba) Medial rami (rami mediales).

(bb) Lateral rami (rami laterales). 
(c) Posterior rami of lumbar nerves (rami posteriores Nn. lumbalium).

(ca) Medial rami (rami mediales).

$(c b)$ Lateral rami (rami laterales).

(d) Posterior rami of sacral and coccygeal nerves (rami posteriores Nn. sacralium et coccygei).

\section{Opening the Vertebral Canal.}

Carefully remove all muscles and tendons from the spinous processes and arches of the vertebrae from the oceipital bone to the tip of the coccyx, avoiding injury, however, to the posterior rami of the spinal nerves (rami posteriores $N n$. spinalium). Place high blocks under the thorax and let the head hang over the end of the table.

The vertebral canal (canalis vertebralis) may be opened from behind in any one of four different ways: $(a)$ with the double rhachiotome, $(b)$ with a saw, $(c)$ with mallet and chisel, $(d)$ with bone-forceps.

(a) With the Double Rhachiotome.-Place the saws of Luer's double rhachiotome on the cervical portion of the vertebral column; seize the handle with the right hand, pressing down from above upon the rhachiotome with the left hand; saw (in the direction in which the teeth are set) with quick, rapidly repeated strokes through the vertebral arches. As soon as the saw has gone through, the same process is repeated a little lower down, and so on until the whole posterior wall of the vertebral eolumn has been eut through as far as the last lumbar vertebra. Between the last lumbar vertebra and the sacrum cut through the ligamenta flava; introduce the vertebral forceps below the posterior areh of the last lumbar vertebra, seize hold of it and, grasping the forceps with both hands, tear away in one piece the whole posterior wall of the vertebral canal from the lumbar region to the occipital bone. If the cervical portion be not quite sawn through, apply the rhachiotome again and cut in a reverse direction.

(b) With a Saw.-One may use a simple saw, eutting through first one side and then the other. The saw should pass elose behind the articular processes and should be directed slightly obliquely, so that the cut passes a little medialward in the depth. It may be necessary to use the hammer and chisel also. Neelsen reeommends the use of the "fox-tail saw."

(c) With Mallet and Chisel.- The vertebral arches may be chiselled instead of sawn through, if desired, but the tyro is apt to injure the cord. Special chisels (one for each side) are manufactured for the purpose, but a good heavy plain chisel works well.

(d) With Bone-forceps. - Cut through the root of each vertebral arch (radix arcus vertebrae) (O. T. pedicle) on each side, just ventral to the superior articular processes, and thus remove the whole posterior wall of the vertebral canal. This method has the advantage that it opens up all intervertebral foramina and permits of the removal of the spinal ganglia and the proximal portion of each spinal nerve along with the spinal cord. 
It will be well also to remove the posterior wall of the spinal canal in the sacrum and coceyx.

The cord lying in its external covering, the dura mater, or pachymeninx spinalis, is now exposed. Notice the interspace between the third and fourth lumbar vertebrae. This is the site of Quincke's " lumbar puncture." Here a needle can be introduced into the cavum subarachnoideale during life without danger.

Read carefully the description of the veins of the spinal column and study Spalteholz, Figs. 493 and 494.

Examine carefully-

(a) Spinal ramus of posterior ramus of each intercostal artery (ramus spinalis rami post. A. intercostalis), passing through an intervertebral foramen. (Cf. Spalteholz, Fig. 462.)

(b) Spinal ramus of posterior ramus of each lumbar artery (ramus spinalis rami post. A. lumbalis).

(c) Spinal rami of vertebral artery (rami spinales $A$. vertebralis) and the spinal rami of the ascending cervical artery (rami spinales $A$. cervicalis ascendentis).

\section{Spinal Meninges. (Fig. 98.)}

Clean the external surface of the dura mater, removing fat and areolar tissue. Lift the dura gently with fine forceps, and, with fine, sharp scissors, cut through the dura mater spinalis in the middle line throughout its whole length. Take great care not to injure the spinal arachnoid (arachnoidea spinalis). Trace the terminal thread of the dura mater (filum durae matris spinalis) to its bony attachment. Note that the dura mater spinalis corresponds to the inner layer of the dura mater encephali, the outer layer having fused with the periosteum of the vertebral canal. The space (cavum epidurale) between the lining of the vertebral canal and the dura mater spinalis contains the plexus venosi, which in turn correspond to the cerebral sinuses. Note disproportion between the size of the cord and the size of the dura mater, especially in the regions where the mobility of the vertebral column is great. Observe the fibrous filaments from the anterior middle line of the dura which run obliquely downward ventralward to be inserted into the ligamentum longitudinale posterius; note their excessive development and fusion from the fourth lumbar vertebra downward; this is the ligamentum sacrodurale anterius (ligament sacré antérieur of Trolard). (Cf. Poirier et Charpy, iii., Fig. 77.) What is its function? Note the dural sheaths of the nerve roots. 
Examine the membrane (arachnoidea spinalis) which bounds the cavum subdurale internally. Note that this is what many anatomists call the "visceral layer" of the arachnoid; below, at the summit of the conus terminalis (second sacral), it is reflected upon the dura mater to form the "parietal layer" of the arachnoid; the "arachnoid cavity" of various writers is sit-

FIG. 98.

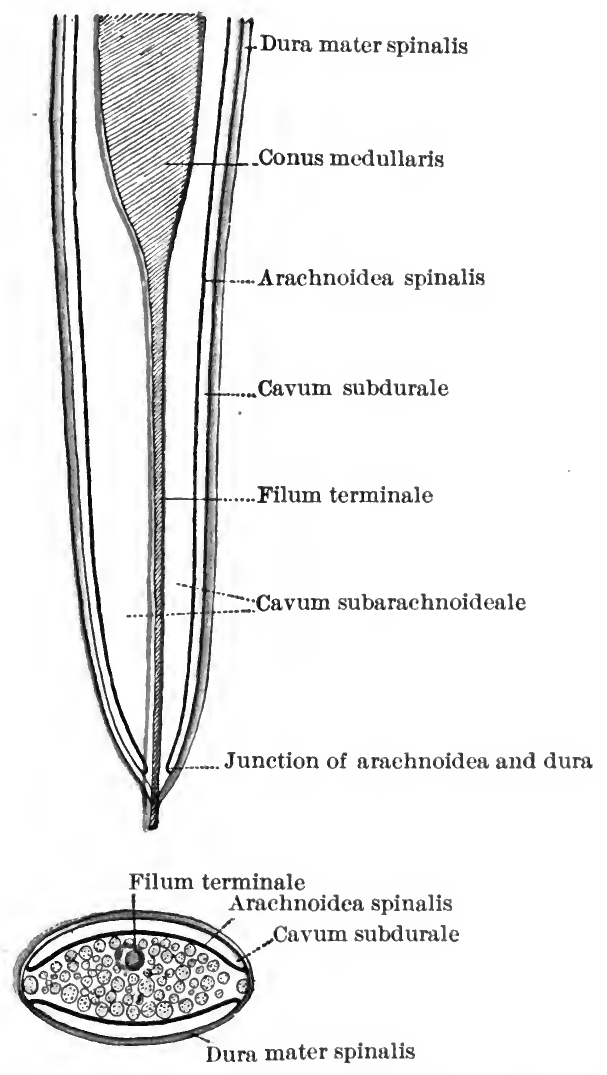

Disposition of the arachnoidea spinalis at the inferior extremity of the medulla spinalis. The sections are schematic (longitudinal and transverse). The pia mater is in red. The transverse section passes through the cauda equina. (From Poirier et Charpy, Traité d'Anat. hum., Paris, 1899, t. lii., 1, p. 118, Fig. 83.)

uated between the parietal layer and the visceral layer, being the cavum subdurale of the present nomenclature. It is a serous cavity like the pleura. Can you separate the "parietal layer" from the dura? Do you see any blood-vessels in the "visceral layer"? Are there any calcified plates in the latter? Note the 
prolongation of the arachnoid over the nerve roots and over the summits of the teeth of the ligamentum denticulatum.

Lift the visceral layer of the arachnoid, cut through it with fine scissors, insert grooved director, and cut it open longitudinally a little to one side of median line. Examine the space (cavum subarachnoideale) beneath the visceral layer of the arachnoid and the pia mater. Note the expanse of the space especially in the region of the cauda equina, which it contains.

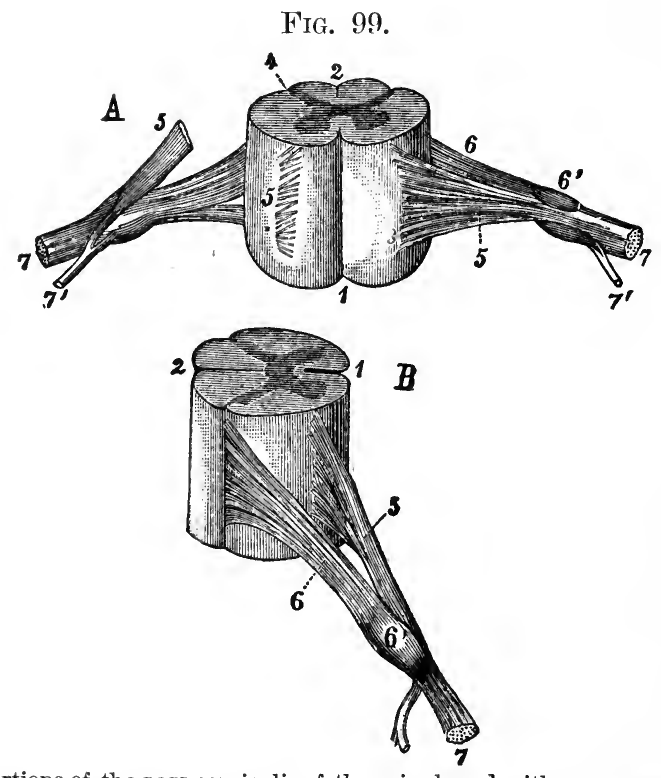

Portions of the pars cervicalis of the spinal cord with nerve-roots.

$A$, spinal cord seen from the ventral surface. On the right side the ventral fila radicularia have been cut through. $B$, spinal cord seen from the lateral surface. -1 , fissura mediana anterior; 2 , sulcus medianus posterior; 3 , sulcus lateralis ventralis, whence the ventral fila radicularia emerge; 4 , sulci laterales dorsales through which the dorsal root fibres enter the spinal cord; 5 , radix ventralis going past spinal ganglion cut through on the right side in Fig. $A ; 6$, radix dorsalis emerging from the ganglion spinale $\left(6^{\prime}\right) ; 7, \mathrm{~N}$. spinalis immediately after its formation through the union of the radix ventralis and dorsalis dividing into 7 , a ramus ventralis, and $7^{\prime}$, a ramus dorsalis. The ramus communicans and the ramus meningeus are not shown in this figure. (After Allen Thompson, from A. Rauber, Lehrbuch der Anatomie des Menschen, V. Aufl., Leipz., 1898, Bd. ii. S. 283, Fig. 248.)

Where would the cerebrospinal fluid accumulate in greatest amount? Note precise spot where cavum subarachnoideale is tapped in Quincke's lumbar puncture. There is no communication between the cavum subdurale and the cavum arachnoideale. Notice the areolar tissue everywhere present between the arachnoid and the pia. Make out the septum subarachnoideale posterius.

Examine the spinal pia mater (pia mater spinalis). How 
closely does this membrane invest the cord? Note its extreme vascularity. What is meant by the term "leptomeninx", ?

Study the ligamentum denticulatum. Where is the medial border of the ligament inserted? On the lateral border note the teeth and the free arcades intermediate between the teeth. Into what are the summits of the teeth inserted? What is the relation of the ligamentum denticulatum to the anterior and posterior roots? The number of teeth varies from eighteen to twenty-three. How many are there in your subject? What is the function of the ligament?

Study the relations of the spinal nerve roots to the pia, the arachnoid, and the dura. (Cf. Poirier et Charpy, iii., Fig. 103.)

\section{The Spinal Nerves (Nervi spinales). (Figs. 99 and 100.)}

Count these on each side. Distinguish the cervical nerves $(N n$. cervicales, I.-VIII.), the thoracic nerves (Nn. thoracales, I.-XII.), the lumbar nerves (Nn.lumbales, I. $-V$.), the sacral

\section{Spinal cord in connection above with the medulla} oblongata and pons.

$V$, nervus trigeminus; $X I I$, nervus hypoglossus; $C_{1}$, first ccrvical nerve ; $C 2-8$, second to eighth cervical nerve; $T 1-12$, first to twelfth thoracic nerve; $L 1-5$, first to fifth lumbar nerve; $S 1-5$, first to fifth sacral nerve; 6 , nervus coccygeus ; $x, x$, filum terminale of the spinal cord. From the root marked $L_{1}$ to $x$, cauda equina; $R r$, plexus brachialis; $C r$, nervus femoralis; $S c$, nervus ischiadicus; $O$, nervus obturatorius; the enlargements opposite $L 3,4$, and 5 represent the spinal ganglia on the dorsal roots. On the left side of the figure the sympathetic trunk is shown. $a$ to $8 s$ are ganglia; $a$, ganglion cervicale superius; $b$ and $c$, ganglion ecrvieale medium et inferius; $d$, first thoracic ganglion; $d$, last thoracic ganglion; $l$, first lumbar ganglion ; $s s$, first sacral ganglion. (After Allen Thompson, from A. Rauber, Lehrbuch der Anatomie des Mensehen, V. Aufl., Leipz., 1898, Bd. ii. S. 504, Fig. 485. )
Fig. 100.

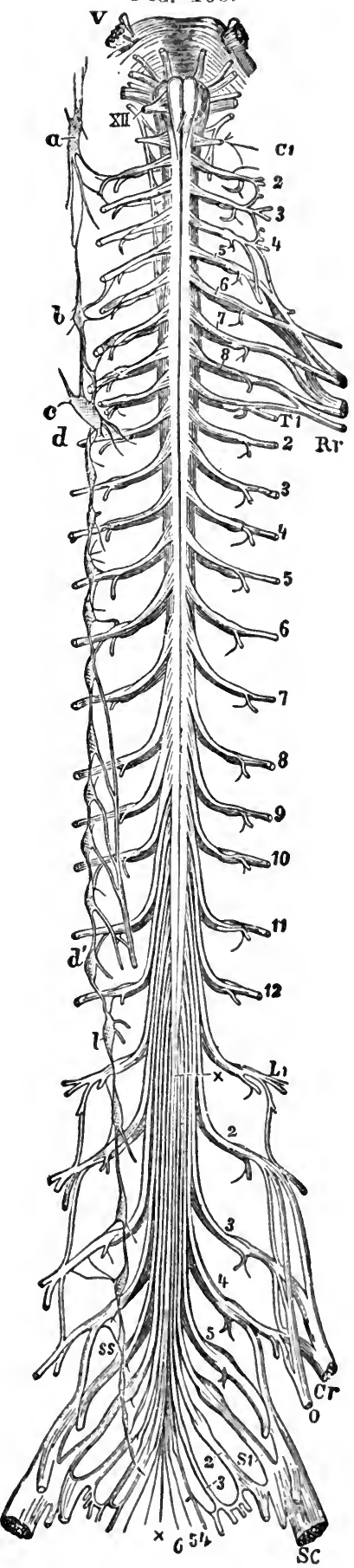


nerves (Nn. sacrales, I.- V.), and the coccygeal nerve ( $N$. coccygeus). Note the subdivision of each spinal nerve trunk into an anterior and a posterior ramus. Observe the roots of the spinal nerves (radices nervorum spinalium). Where does the ventral root (radix anterior) fuse with the dorsal root (radix posterior)? Note the ganglion (ganglion spinale) on the latter. What are the fila radicularia? Observe the relative lengths of the various nerve roots. How do you account for this? Which are the largest and which the smallest nerve roots? Examine the cauda equina carefully. How do the nerves leave and enter the vertebral canal? Look for the ramus meningeus.

\section{Removal of Spinal Cord.}

Divide each spinal nerve trunk midway between the point of . fusion of its two roots and the point of division into an anterior and a posterior ramus.

Cut through the cord at the level of the first cervical nerve. Lift the cord and its membranes gently from the vertebral canal and transfer to formalin for subsequent study.

\section{THE SPINAL CORD.}

\section{Blood-vessels of the Spinal Cord.}

As a rule, special injections are necessary to demonstrate the blood-vessels of the cord well, but a good deal can be made out in ordinary cadavers. Study the following:

Arteries.

(a) Branches of vertebral artery (A. vertebralis).

(aa) Spinal rami (rami spinales).

(ab) Posterior spinal artery (A. spinalis posterior).

(ac) Anterior spinal artery (A. spinalis anterior).

(b) Branches of ascending cervical artery ( $A$. cervicalis ascendens).

(ba) Spinal rami (rami spinales).

(c) Branches of highest intercostal artery (A. intercostalis suprema). (ca) Spinal rami (rami spinales).

(d) Branches of intercostal arteries (Aa. intercostales).

(da) Spinal ramus of posterior ramus (ramus spinalis rami posterioris).

(e) Branches of lumbar arteries (Aa. lumbales).

(ea) Spinal ramus (ramus spinalis).

(f) Branch of iliolumbar artery (A. iliolumbalis). (fa) Spinal ramus (ramus spinalis).

(g) Branches of lateral sacral artery (A. sacralis lateralis). (ga) Spinal rami (rami spinales). 
(For a description of the course of the vessels in the meninges and in the cord itself, see article "Spinal Cord" in last edition of Wood's Reference Hand-book of the Medical Sciences.)

Veins.

(a) Internal spinal veins ( $V v$. spinales internae).

(b) Posterior external spinal veins ( $V v$. spinales externae posteriores).

(c) Anterior external spinal veins ( $V v$. spinales externae anteriores).

(d) Intervertebral veins ( $V v$. intervertebrales).

\section{External Morphology of Spinal Cord.}

What is the shape of the spinal cord? How is it curved? Note the position and extent of each of the following:

(a) Cervical portion (pars cervicalis). (aa) Cervical enlargement (intumescentia cervicalis).

(b) Thoracic portion (pars thoracalis) (O. T. dorsal portion).

(c) Lumbar portion (pars lumbalis). (ca) Lumbar enlargement (intumescentia lumbalis).

(d) Medullary cone (conus medullaris). ( $d a)$ Swelling due to terminal ventricle (ventriculus terminalis).

(e) Terminal thread (filum terminale).

On the surface of the cord observe the following grooves:

(a) Anterior median fissure (fissura mediana anterior).

(b) Posterior median sulcus (sulcus medianus posterior).

(c) Anterior lateral sulcus (sulcus lateralis anterior).

(d) Posterior lateral sulcus (sulcus lateralis posterior).

(e) Posterior intermediate sulcus (sulcus intermedius posterior) (O. T. paramedian furrow).

( $f$ ) Anterior intermediate sulcus (sulcus intermedius anterior) (inconstant).

Between these grooves the funiculi of the spinal cord (funiculi medullae spinalis).

(a) Anterior funiculus (funiculus anterior) (O. T. anterior column).

(b) Lateral funiculus (funiculus lateralis) (O. T. lateral column).

(c) Posterior funiculus (funiculus posterior) (O. T. posterior column).

\section{Transverse Sections of Spinal Cord. (Fig. ror.)}

Sections of the spinal cord (sectiones medullae spinalis) should next be studied. With a sharp, thin knife, cut through the cord transversely at the following levels: (1) just below roots of fourth pair of cervical nerves, (2) through middle of cervical enlargement, (3) through middle of thoracic cord, (4) through middle of lumbar enlargement, and (5) through conus terminalis. In the sections observe the differentiation into white matter (substantia alba) and gray matter (substantia grisea). 


\section{The Gray Matter.}

What is its form in cross-section? How does this vary at different levels? Localize the central canal (canalis centralis)

Fig. 101.
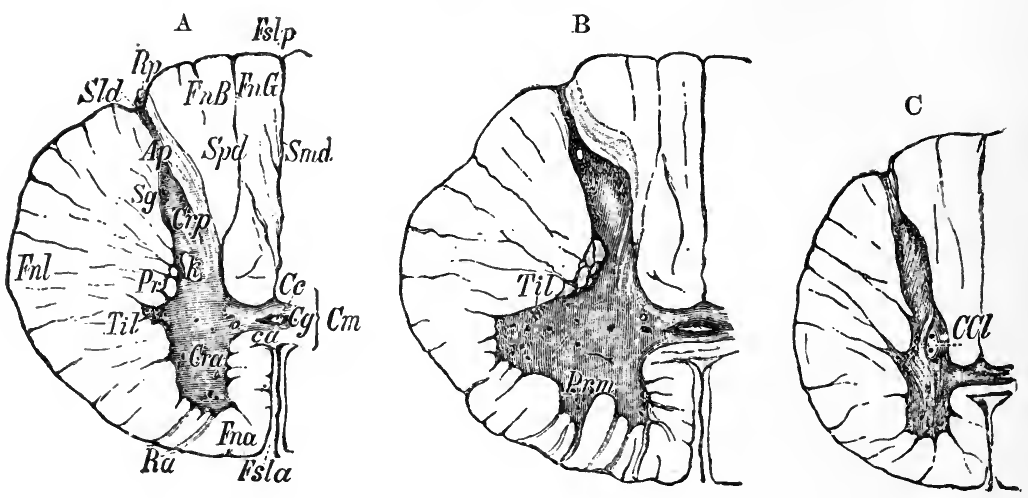

$\mathbf{E}$
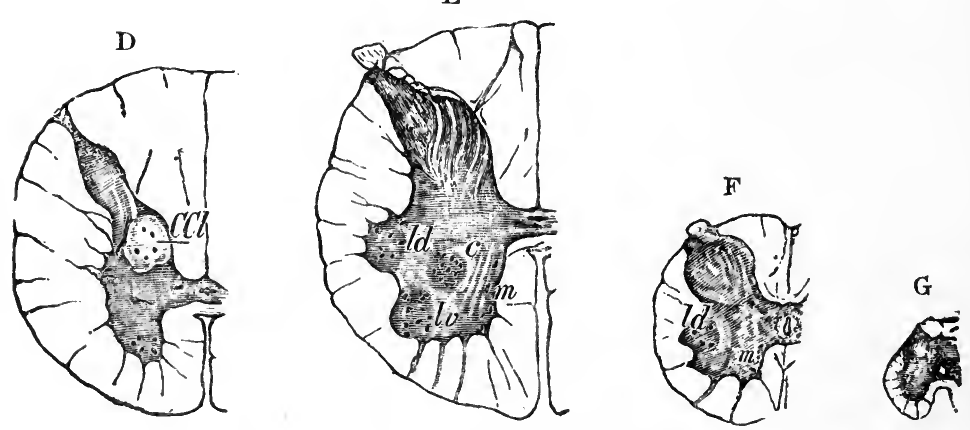

Cross-sections through the human spinal cord, stained with carmine.

A, transverse section at the level of $\mathrm{C}$ iii : $C g$, commissura grisea ; $A p$, apex columnæ dorsalis ; $C a$, commissura ventralis alba ; $C c$, canalis centralis; $C \mathrm{~m}$, commissura medullæ spinalis ; $\mathrm{Cra}$, cornu ventrale; Crp, cornu dorsale; Fna, funiculus ventralis; $F n B$, fasciculus cuneatus Burdachi; $F n G$, fasciculus gracilis Golli; $F n l$, funiculus lateralis; $F s l a$, fissura mediana ventralis; $F s l p$, sulcus medianus dorsalis; $k$, tractus solitarius; $P r$, formatio reticularis ; $R a$, radix ventralis ; $R p$, radix dorsalis ; $S g$, substantia gelatinosa Rolandi; Sld, sulcus lateralis dorsalis; Smd, septum medianum dorsale; Spd, septum intermedium dorsale; Til, tractus intermedio-lateralis. $\mathrm{B}$, transverse section at the level of C vi : Prm, processus cervicalis medius cornu ventralis; Til, columna intermedio-lateralis. C, transverse section at the level of $\mathrm{T}$ iii : $\mathrm{CCl}$, nucleus dorsalis Clarkii. D, transverse section at the level of T xii : $C C l$, nucleus dorsalis. $\mathrm{E}$, transverse section at the level of $\mathrm{L} \mathrm{v}: m$, medial cell group of the cornu ventrale; $l v$, lateral ventral, $l d$, lateral dorsal, and $c$, central cell group. $\mathrm{F}$, transverse section at the level of S iii : $m$, medial, $l d$, lateral-dorsal cell group. G, transverse section through the lower part of the conus medullaris at the level of origin of the N. coccygeus. (After H. Obersteiner, Anleitung beim Studium des Baues der nervösen Centralorgane im gesunden und kranken Zustande, III. Aufl., Leipz. u. Wien, 1896, S. 227, Figs. 96-102.)

and the gray matter close to it, the central gray substance (substantia grisea centralis). In front of it is the anterior gray com- 
missure (commissura grisea anterior) and behind it the posterior gray commissure (commissura grisea posterior). Study in crosssection each of the following gray columns (columnae griseae) :

(a) Anterior column of gray matter (columna anterior) (O. T. anterior horn).

(b) Lateral column of gray matter (columna lateralis) (O. T. lateral horn).

(ba) Reticular formation (formatio reticularis).

Fig. 102.

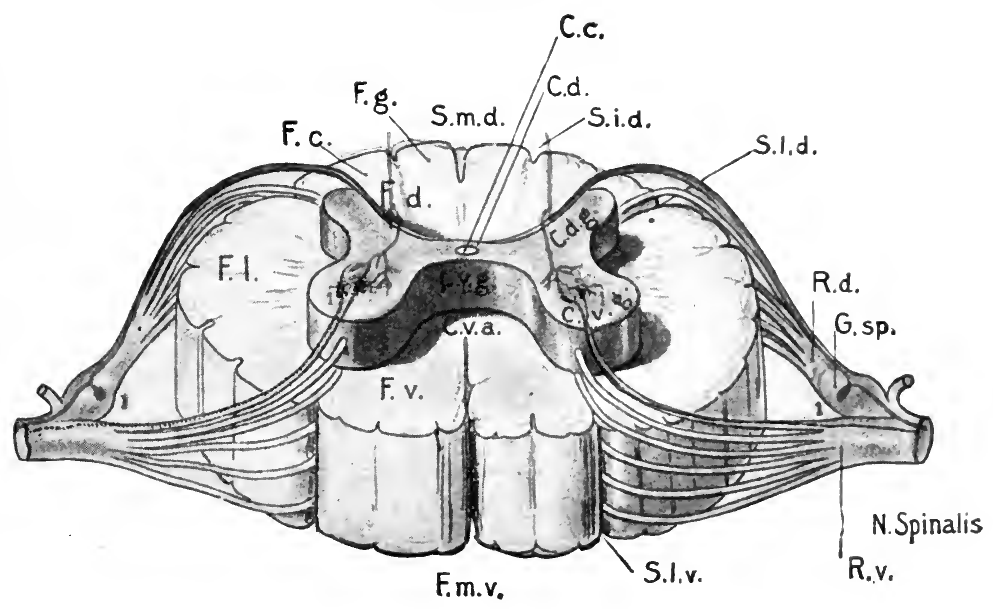

Schematic representation of the portion of a spinal cord corresponding to the attrehment of the ventral and dorsal roots of one pair of spinal nerves. (From Barker, The Nerv. Syst., New York, 1901, Pl. II. Fig. 2.)

Black.-C.c., canalis centralis; C.d., commissura posterior (O.Y., commissura dorsalis); C.d.g., columna posterior (O.Y., cornu dorsale griseum) ; C.v.g., columna anterior (O.Y., cornu ventrale griseum); Cm.v.a., commissura anterior alba (O.Y., com. ventralis alba); Cm.v.g., commissura anterior grisea (O.Y. eom. ventralis grisea); $F c$. funiculus euneatus; $F g$., funieulus graeilis; $F d$., funiculus posterior (O.Y. funieulus dorsalis); $F l$., funieulus lateralis; $F$. , funiculus anterior (O.Y., fun. ventralis) ; $F . m . v$., fissura mediana anterior (O.Y., fis. med. ventralis); G.sp., ganglion spinale; R.d., radix posterior nervi spinalis (O.Y., rad. dorsalis); R.v., radix anterior (O.Y., ventralis) nervi spinalis (O.Y., rad. ventralis) ; S.l d., suleus lateralis posterior (O.Y., sule. lat. dorsalis); S.l.v., suleus lateralis anterior (O.Y., sule. lat. ventralis); S.i.d., sulcus intermedius posterior (O.Y., sule. intermed. dorsalis); S.m.d., suleus medianus posterior (O.Y., sulc. med. dorsalis.)

Rcd.-Peripheral sensory neurones. The eell-bodies, 1 , are situated in the spinal ganglion. The peripheral proeesses enter the peripheral nerves, the central axones pass by way of the radix posterior into the funieulus cuneatus of each side. There each axone bifureates into an ascending and a deseending limb, giving off also collaterals to the gray substanee.

Blue.-Lower motor neurones. The cell-bodies, 1, are situated in the columnae anteriores. They send their axones by way of the anterior roots into the peripheral nerves. figure.

The neural meehanism believed to form the basis of the simplest reflex are is illustrated in the

(c) Posterior column of gray matter (columna posterior) (O. T. posterior horn).

(ca) Neck of posterior column (cervix columnae posterioris).

(cb) Apex of posterior column (apex columnae posterioris). 
(cc) Gelatinous substance of Rolando (substantia gelatinosa [Rolandi]).

(cd) Dorsal nucleus (nucleus dorsalis [Clarkii, Stillingi]) (O. T. Clarke's column).

Fig. 103.

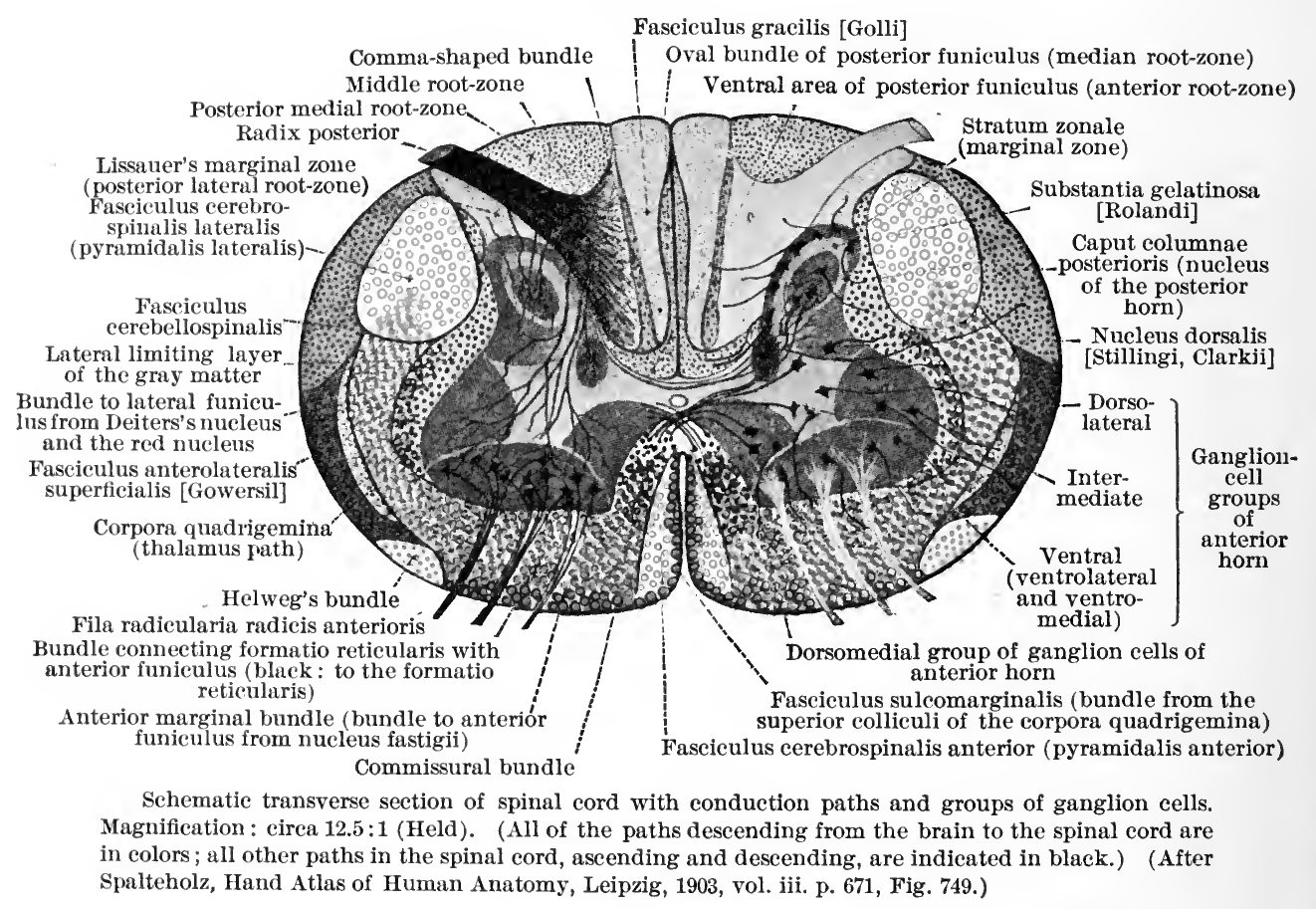

The White Matter.

In the sections recognize in the white matter the position of each of the following:

(a) In the anterior funiculus (funiculus anterior):

(aa) Anterior cerebrospinal or pyramidal fasciculus (fasciculus cerebrospinalis anterior [pyramidalis anterior]) ( $0 . \mathrm{T}$. direct pyramidal tract).

(ab) Proper anterior fasciculus (fasciculus anterior proprius [Flechsigi $]$ ) (O. T. anterior ground-bundle).

(b) In the lateral funiculus (funiculus lateralis):

(ba) Lateral cerebrospinal or pyramidal fasciculus (fasciculus cerebrospinalis lateralis [pyramidalis lateralis]) (O. T. crossed pyramidal tract).

(bb) Cerebellospinal fasciculus (fasciculus cerebellospinalis) ( 0 . T. direct cerebellar tract of Flechsig). ${ }^{1}$

${ }^{1}$ This would be better designated fasciculus spinocerebellaris dorsolateralis. 
(bc) Superficial anterolateral fasciculus (fasciculus anterolateralis superficialis [Gowersi]) (O. T. Gower's's tract).

(bd) Proper lateral fasciculus (fasciculus lateralis proprius [Flechsigi]) (O. T. lateral ground-bundle).

(c) In the posterior funiculus (funiculus posterior):

(ca) Slender fasciculus (fasciculus gracilis [Golli]) (O. T. column or tract of Goll).

(cb) Wedge-shaped fasciculus (fasciculus cuneatus [Burdachi]) (O. T. column or tract of Burdach).

(d) Anterior white commissure (commissura anterior alba).

\section{FACE.}

\section{Surface Anatomy.}

The general features of the surface have already been examined. The student will do well at this time to make especial drawings of the external appearance of the eye and mouthopening.

\section{The Eye.}

Indicate the eyebrow (supercilium), with the hairs (supercilia). Examine the lids (palpebrae), closed and open. After noting the appearance of the anterior surface (facies anterior palpebrarum), evert each lid and view the posterior surface (facies posterior palpebrarum). The slit between is called the palpebral fissure (rima palpebrarum). At the junction of the two lids are situated the medial and lateral palpebral commissures (commissurae palpebrarum lateralis et medialis) (O. T. external and internal canthi). These are at the lateral and medial angles of the eye (anguli oculi lateralis et medialis). Observe that the edge of each lid has two margins (limbus palpebralis anterior,-posterior). What is the relation of the eyelashes (cilia) and of the openings of the Meibomian follicles (glandulae tarsales [Meibomi]) to these margins? Trace the continuity of the palpebral conjunctiva (tunica conjunctiva palpebrarum) with the bulbar conjunctiva (tunica conjunctiva bulbi). Note the recesses in the region where the palpebral conjunctiva is reflected upon the eyeball; these are the superior and inferior fornices (fornix conjunctivae superior,-inferior). Observe the triangular space (lacus lacrimalis) at the medial extremity of the rima palpebrarum; in its centre is a reddish flesh-like mass, the lacrimal caruncle (caruncula lacrimalis). Observe the small vertical semilunar fold of conjunctiva (plica semilunaris conjunctivae) just lateral from the caruncle. It cor- 
responds to the third eyelid, or membrana nictitans, of lower animals. The stream (rivus lacrimalis) of tears (lacrimae) running over the conjunctiva passes to the medial angle of the eye. At the point on each lid where the eyelashes cease is a small eminence, the lacrimal papilla (papilla lacrimalis). These eminences are perforated, the openings looking like two minute black dots (puncta lacrimalia). The openings lead into small canals, lacrimal ducts (ductus lacrimales). Pass a fine bristle into each. The lacrimal apparatus will be further studied later.

\section{The Mouth-opening.}

Note that it is bounded by the two lips (labia oris), one superior (labium superius), one inferior (labium inferius). At the junction of the lips at the two angles of the mouth (anguli oris) are the commissures of the lips (commissurae labiorum). The slit between the lips is called the rima oris. Note the curious transition between skin and mucous membrane in the lip.

\section{Skin and Superficial Fascia.}

Observe the thinness and fineness of the skin of the face. Compare its mobility with that of other parts. Which portions are most firmly attached to subjacent structures? Are any comedones visible? Note the distribution of the hairs of the beard $(b a r b a)$. Observe the long, rather stiff hairs at the nose openings (vibrissae). Through the skin feel the external maxillary artery where it passes over the margin of the mandible. Distend the cheeks and lips with moist cotton and stitch the margins of the lips together with a fine needle.

Make an incision through the anterior median line and remove the skin of the face, taking no fat with it. Avoid injury to the upper part of the platysma, the M. risorius, and the structures of the eyelids. After the platysma has been carefully studied, it may be removed.

\section{Cutaneous Muscles of Face. (Vide Fig. 104.)}

Note that the majority of the muscles of the face and scalp have attachments to the skin; indeed, these muscles have been derived by progressive differentiation of a primitive cervicofacial cutaneous muscle; all of them are innervated by branches of the N. facialis, which is the nerve of the hyoid arch. Observe the tendency to a grouping around various orifices, the muscles acting as dilators or constrictors of these orifices. What part do these muscles play in facial expression? 
Platysma.

Muscles of the external ear.

(a) M. aurieularis anterior (already studied).

(b) M. auricularis posterior (already studied).

(c) M. auricularis superior (already studied).

Muscles of the eyelids.

Dilators.

(a) M. epicranius (already studied).

Note that the prineipal dilator, the M. levator palpebrae superioris, is not a skin-muscle.

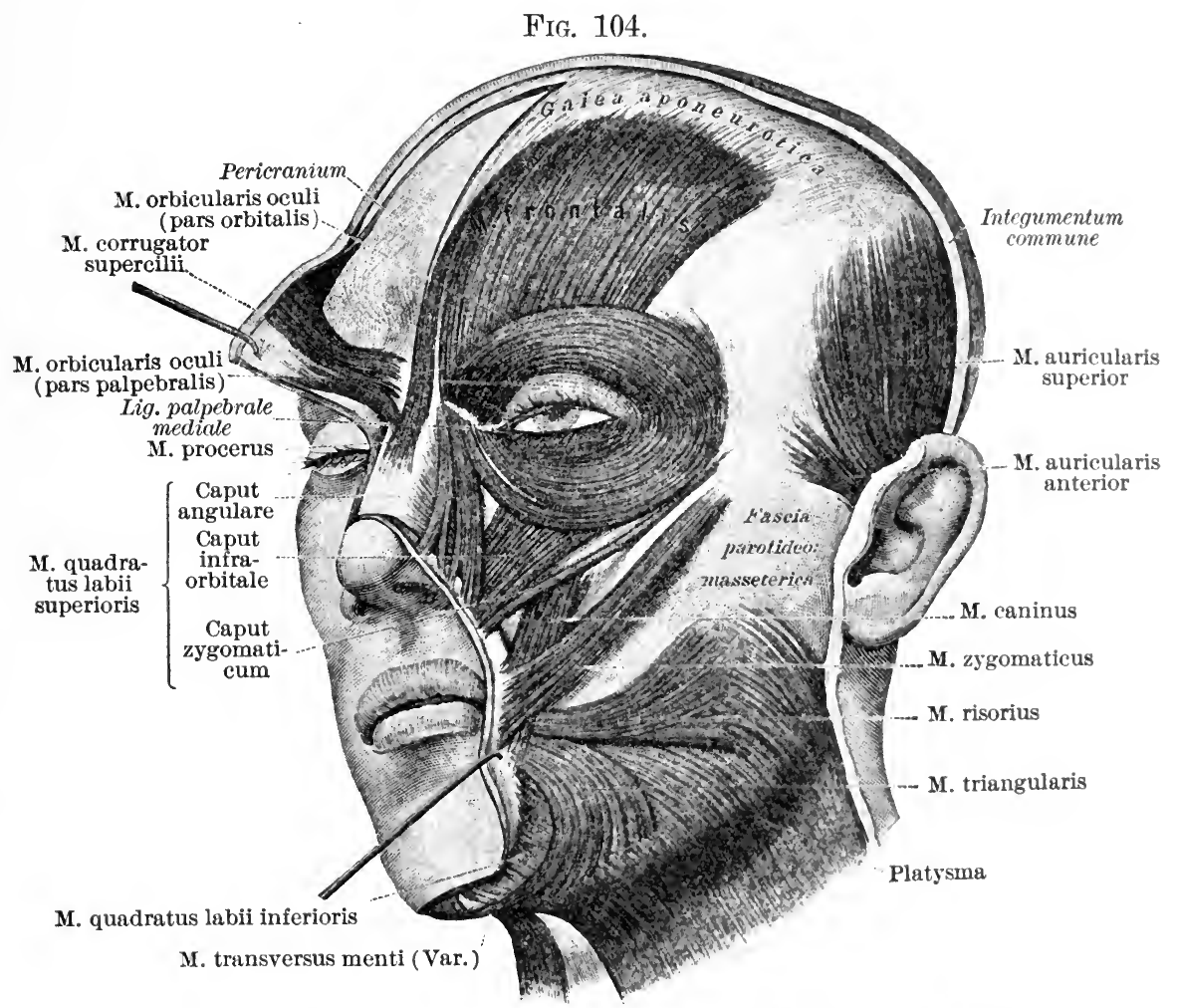

The muscles of the face. (After Toldt, Anat. Atlas, Wien, 1900, 2 Aufl., p. 296, Fig. 537.)

\section{Constrictors.}

(a) Procerus ("prolonged") muscle (M. procerus) (O. T. pyramidalis nasi).

This muscle is wrongly classed as a part of the M. frontalis. Comparative anatomy has shown it to have been derived from the muscle which lifts the lip. Note that the M. procerus is an antagonist of the M. frontalis.

(b) Orbicular muscle of eye (M. orbicularis oculi) (O. T. orbicularis palpebrarum).

(ba) Palpebral part (pars palpebralis). 
(baa) Medial palpebral ligament (ligamentum palpebrale mediale).

(bab) Lateral palpebral raphe (raphe palpebralis lateralis).

(bb) Orbital part (pars orbitalis).

(bc) Lacrimal part (pars lacrimalis [Horneri]).

(c) Corrugator muscle ( $M$. corrugator).

Note the relation of the muscle to physical or mental effort.

Muscles of the nose.

Dilators.

(a) Transverse part of nasal muscle (pars transversa M. nasalis).

Note the origin on the back of the nose and insertion into skin of nasolabial groove-not jugulum alveolare of upper canine tooth. It is called the "muscle of sensuality."

(b) Dilator muscle of nose ( $M$. dilator naris).

(c) Angular head of quadrate muscle of upper lip (caput angulare M. quadrati labii superioris) (O. T. superficial elevator of lip).

(d) Infraorbital head of quadrate muscle of upper lip (caput infraorbit. M. quadrat. labii superioris) (O. T. deep elevator of upper lip).

Constrictors.

(a) Alar part of nasal muscle (pars alaris $M$. nasalis) (O. T. depressor alae nasi).

(b) Depressor muscle of septum (M. depressor septi).

(c) Triangular muscle of nose ( $M$. triangularis nasi).

Muscles of the lips.

Dilators of mouth.

Group 1.

(a) Muscle of laughing (M. risorius).

(b) Zygomatic muscle (M. zygomaticus) (muscle of grimace).

Group 2.

(c) Quadrate muscle of upper lip (M. quadratus labii superioris).

(ca) Zygomatic head (caput zygomaticum) (O. T. zygomaticus minor).

( $c b$ ) Infraorbital head (caput infraorbitale) (O. T. levator labii superioris).

(cc) Angular head (caput angulare) (O. T. superficial eleGroup 3. vator of lip, or levator labii superioris alaeque nasi).

(d) Canine muscle (M. caninus) (muscle of menacing hate) ( $O$. T. levator anguli oris).

(e) Triangular muscle of mouth (M. triangularis oris) (muscle of Group 4. sadness) (O. T. depressor anguli oris).

( $f$ ) Quadrate muscle of lower lip (M. quadratus labii inferioris) (O. T. depressor labii inferioris).

(g) Chin muscle ( $M$. mentalis) (musele of hesitation, doubt, disGroup 5. dain, disgust).

(h) Cheek muscle (M. buccinator) (muscle of suckling; trumpetblower's or glass-blower's muscle). 
(ha) Buccopharyngeal fascia (fascia buccopharyngea).

In cleaning this muscle avoid injury to the motor branch from the $\mathrm{N}$. facialis and the sensory buccinator nerve from the $\mathrm{N}$. trigeminus.

Constrictors of mouth.

(a) Orbicular muscle of mouth (M. orbicularis oris).

As a muscle of vegetable life it closes the mouth to hinder exit of saliva and food, prevents entrance of foreign bodies, is prehensile in taking food and liquid, and is active in suction and mastication; as a muscle of relational life it closes lips partly or completely in physical effort, in articulation of words, whistling, playing musical instruments, kissing, etc.

Note the division into a pars externa and a pars interna. The latter is unaffected in cerebral hemiplegia, and the patient can still close the mouth, whistle, and speak. Both are affected in glosso-labio-laryngeal paralysis, and the patient can no longer whistle, retain saliva, or pronounce the letters $o$ and $u$.

(b) Compressor muscle of lips ( $M$. compressor labiorum [Kleinii]) (O. T. rectus labii of Aeby).

(c) Incisive muscle of upper lip (M. incisivus labii superioris).

(d) Incisive muscle of lower lip (M. incisivus labii inferioris).

The incisive muscles act along with the M. orbicularis oris in pointing the mouth: they are sometimes called the "muscles of pouting."

\section{References.}

1. On the origin of the facial muscles:

Gegenbaur, C., Lehrbuch der Anatomie des Menschen.

Ruge, Untersuchungen über die Gesichtsmuskulatur der Primaten, Leipzig, 1887.

2. On the muscles as agents of expression:

Bell, C., Anatomy and Physiology of Expression, 1844.

Duchenne (of Boulogne), Mécanisme de la physionomie humaine, 1852.

Darwin, C., Expression of Emotions in Man and Animals.

\section{Region of Parotid Gland.}

In the study of the deep cervical fascia the parotideomasseteric fascia (fascia parotideomasseterica) has been observed passing upward to become attached to the zygoma. On its surface the ramus anterior of the $\mathrm{N}$. auricularis magnus was noted. The fascia is to be very carefully removed and the parotid gland defined.

Observe the exact position and relations of the parotid gland (glandula parotis). Find its excretory duct (ductus parotideus [Stenonis]) and follow it throughout its course. Cut into it and pass a probe through into the mouth. Note the position of the oral termination of the duct. In relation to the upper border of the duct, note the accessory parotid gland (Gl. parotis accesso- 
ria) (O. T. socia parotidis). Follow the retromandibular process of the gland (processus retromandibularis) in behind the mandible. How is the pocket in which the parotid gland is contained separated from that in which the submaxillarr gland is situated? Pick awar the parotid gland bit by bit, in order to studr the blood-ressels and nerres of the region, many of which pass directly through the gland-substance.

Superficial Blood-vessels and Nerves of Face. (Vide Figs. 87, 87a, and I05.)

Arteries.

(a) External carotid (A. carotis externa).

(aa) Superficial temporal (A. temporalis superficialis).

(aaa) Parotid rami (rami parotidei).

(aab) Transverse artery of face (A. transversa faciei).

(aac) Anterior auricular rami (rami auriculares anteriores).

(aad) Middle temporal artery (A. temporalis media).

(aae) Zygomatico-orbital artery (A. zygomatico-orbitalis).

The terminal rami have already been studied in the dissection of the scalp.

(ab) Internal maxillary artery (A. maxillaris interna).

Its branches cannot ret be seen.

(ac) External maxillary artery (A. maxillaris externa) (O. T. facial arterc).

(aca) Inferior labial (A. labialis inferior).

(acb) Superior labial (A. labialis superior).

(acc) Angular artery (A. angularis).

Teins. (Cf. Spalteholz, Figs. 457. 455.)

(a) Posterior facial rein ( $\Gamma$. facialis posterior).

(aa) Superficial temporal rein ( $V$. temporalis superficialis).

$(a b)$ Middle temporal rein ( $\Gamma$. temporalis media).

(ac) Anterior auricular reins ( $\Gamma x$. auriculares anteriores).

(ad) Posterior parotid reins ( $V t$. parotideae posteriores).

(ae) Transterse rein of face ( $T$. transversa faciei).

(b) Anterior facial rein ( $\Gamma$. facialis anterior).

(ba) Angular rein ( $\Gamma$. angularis).

(baa) Frontal rein ( $V$. frontalis).

(bab) Supraorbital rein ( $\Gamma$. supraorbitalis).

(bac) Nasofrontal rein ( $\Gamma$. nasof rontalis).

(bad) External nasal reins ( $\Gamma v$. nasales externae).

(bae) Teins of the lips ( $\Gamma x$. labiales superior et inferior).

(baf) Anterior parotid reins ( $\nabla v$. parotideae anteriores).

(bag) Masseteric veins ( $\nabla v$. massetericae).

Nerces. Cut away free projecting part of processus mastoideus, with saw 
FIG. 105.

Rami temporales superficiales of the $\mathrm{X}$. auriculo temporalis

Nin. auriculares anteriores

Rami temporales of the I. facialis

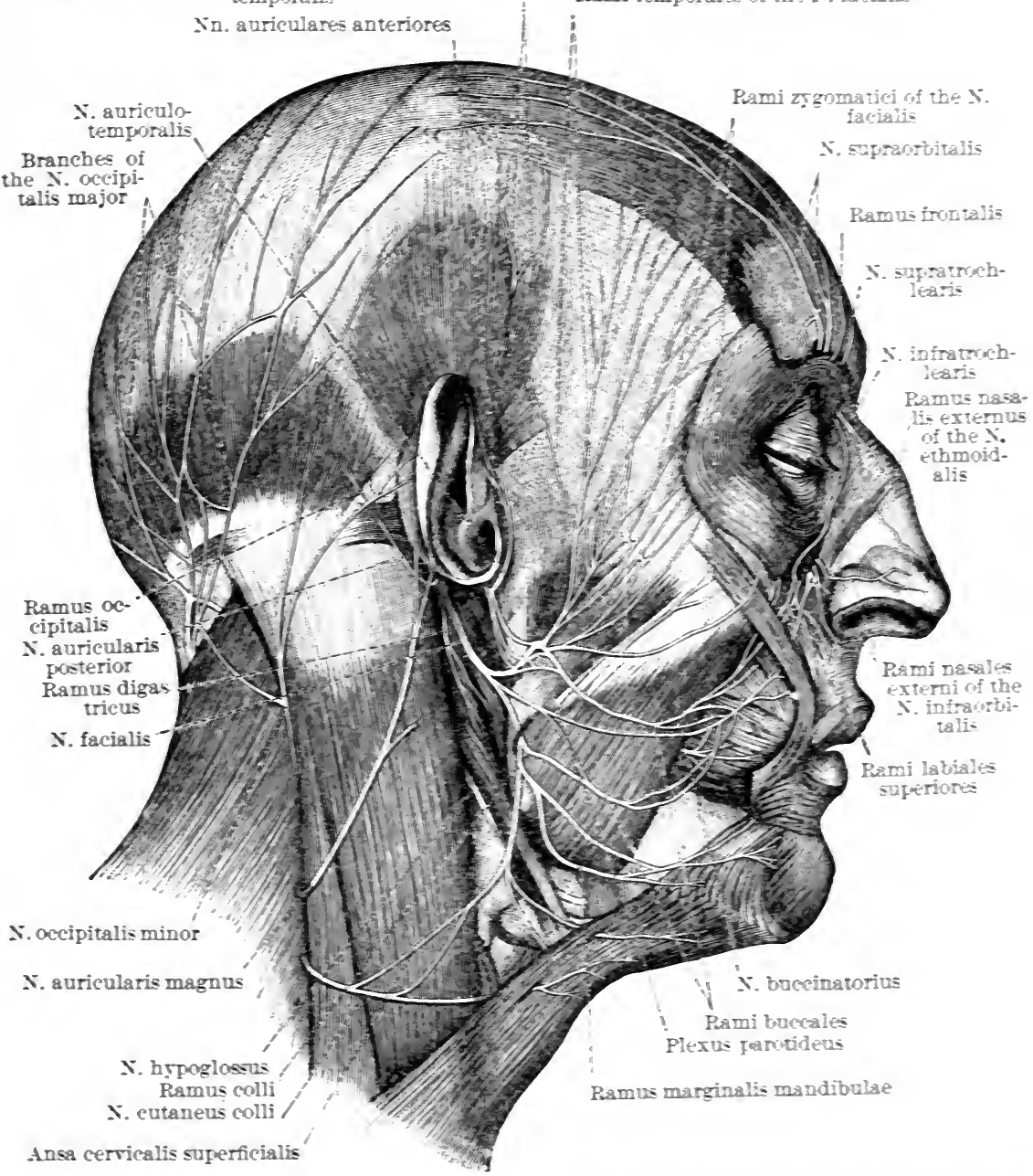

The distribution of the facial, aurieulotempral. great amricular. greater and smallet mecipital nerves and their communications. The perotid gland has been remored and also parts of the orbiculas

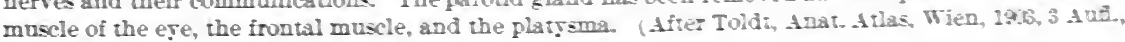
p. s.1, Fig. 131\%.) 
first and chisel afterwards. Do not injure N. auricularis posterior.

(a) Anterior ramus of $\mathrm{N}$. auricularis magnus (ramus anterior $N$. auricularis magni).

(b) Branches of facial nerve ( $N$. facialis). (Vide Fig. 105.)

(ba) Parotid plexus (plexus parotideus).

(bb) Temporal rami (rami temporales).

(bc) Zygomatic rami (rami zygomatici).

(bd) Buccal rami (rami buccales).

(be) Ramus of margin of jaw (ramus marginalis mandibulae).

Examine also-

(bf) Digastrie ramus ( $R$. digastricus).

(bfa) Stylohyoid ramus ( $R$. stylohyoideus).

(bfb) Ramus anastomosing with glossopharyngeal nerve

(ramus anastomoticus cum N. glossopharyngeo).

(c) Branches of trigeminal nerve ( $N$. trigeminus).

(ca) Branches of ophthalmic nerve (N. ophthalmicus).

(caa) Branches of frontal nerve ( $N$. frontalis).

(caaa) Supraorbital nerve ( $N$. supraorbitalis).

(caab) Frontal ramus (ramus frontalis).

(caac) Supratrochlear nerve (N. supratrochlearis).

(cab) Branches of nasociliary nerve (N. nasociliaris).

$(c a b a)$ One of anterior nasal rami (rami nasales anteriores).

(cabaa) External nasal ramus (ramus nasalis externus).

(cac) Branches of infratrochlear nerve ( $N$. infratrochlearis).

(caca) Superior palpebral ramus (ramus palpebralis superior $)$.

(cacb) Inferior palpebral ramus (ramus palpebralis inferior).

( $c b)$ Branches of the maxillary nerve (N. maxillaris) (O. T. superior maxillary).

( $c b a)$ Branches of zygomatic nerve (N. zygomaticus).

(cbaa) Zygomaticotemporal ramus (ramus $z y$ gomaticotem poralis).

$(c b a b)$ Zygomaticofacial ramus (ramus zygomaticofacialis ).

$(c b b)$ Branches of infraorbital nerve (N. infraorbitalis).

$(c b b a)$ Inferior palpebral rami (rami palpebrales, inferiores).

$(c b b b)$ External nasal rami (rami nasales externi).

$(c b b c)$ Superior labial rami (rami labiales superiores).

(cc) Branches of the mandibular nerve (N. mandibularis) (O. T. inferior maxillary).

(cca) Branch of masticatory nerve (N. masticatorius). 
(ccaa) Buccinator nerve (N. buccinatorius) ( $\mathrm{O}$. T. long buccal).

( $c c b)$ Auriculotemporal nerve (N. auriculotemporalis).

(ccba) Parotid rami (rami parotidei).

$(c c b b)$ Anastomotic branch of facial nerve (ramus anastomoticus cum N. facialis).

$(c c b c)$ Anterior auricular nerves (Nn. auriculares anteriores).

$(c c b d)$ Superficial temporal rami (rami temporales superficiales).

(ccc) Mental nerve ( $N$. mentalis).

(ccca) Mental rami (rami mentales).

$(c c c b)$ Inferior labial rami (rami labiales inferiores).

Eyelids. (Figs. 106 and 107.)

Dissect up the M. orbicularis palpebrarum in its entirety and reflect it medialward, avoiding injury to the underlying vessels and nerves. Study-

(a) Orbital septum (septum orbitale) (O. T. palpebral ligaments). Note

(b) Tarsi. its attachments.

(ba) Upper tarsus (tarsus superior).

$(b b)$ Lower tarsus (tarsus inferior).

How do these differ?

(c) Tarsal glands (glandulae tarsales [Meibomi]) (O. T. Meibomian glands).

Compare those of the upper with those of the lower lid. Examine the orifices with a hand lens along the limbi palpebrales posteriores. Squeeze the lid between the thumb and finger, so as to express some of the greasy secretion (sebum palpebrale).

(d) Lateral palpebral raphe (raphe palpebralis lateralis) (O. T. external tarsal ligament).

Examine its attachments to the os zygomaticum and to the tarsi.

(e) Medial palpebral ligament (ligamentum palpebrale mediale) ( $\mathrm{O} . \mathrm{T}$. internal tarsal ligament).

Examine its attachments to the maxilla and to the tarsi.

(f) Nerves and blood-vessels of the lids.

These have been enumerated above in connection with the arteries, veins, and nerves of the face.

( $g$ ) Tendon of insertion of the M. levator palpebrae superioris. This can be exposed by cutting transversely through the upper part of the septum orbitale close to the bone and reflecting it downward towards the tarsus superior. .

\section{Lacrimal Apparatus (Apparatus lacrimalis).}

Dissect out the upper and lower lacrimal glands (glandula lacrimalis superior,-inferior). Are any accessory lacrimal 
FIG. 106.

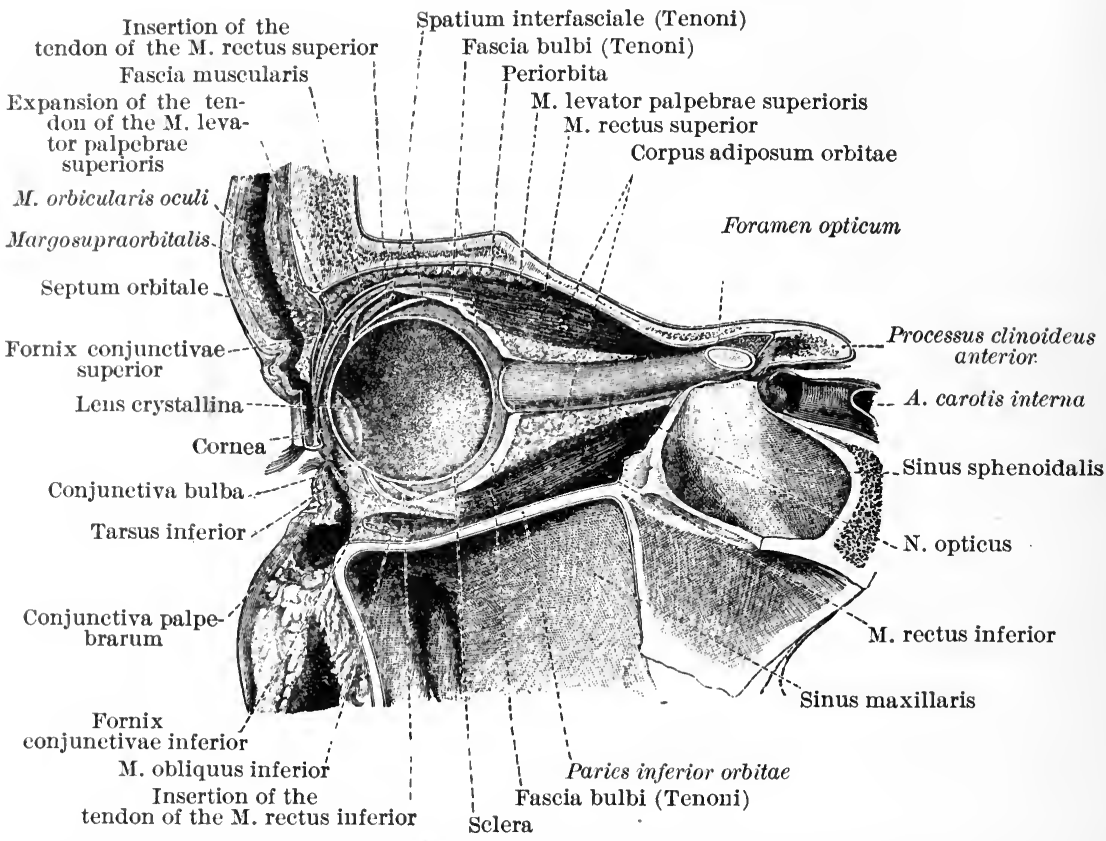

The capsule of Tenon (fascia bulbi) and its relation to the tendons of the superior and inferior straight muscles (Mm. rectus superior and rectus inferior). (After Toldt, Anat. Atlas, Wien, 1903, 3 Aufl., p. 907, Fig. 1378.)

FIr. 107.

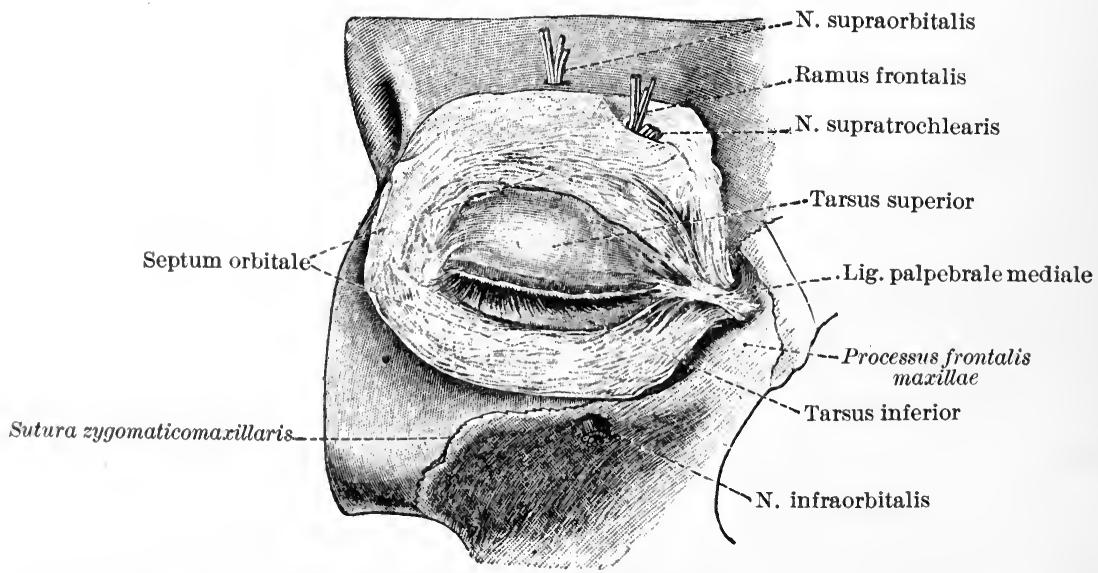

The orbital septum (septum orbitale) and the tarsal cartilages. The skin and orbicular muscle of the eye have been removed. (After Toldt, Anat. Atlas, Wien, 1903, 3 Aufl., p. 909, Fig. 1385.) 
glands (Gl. lacrimales accessoriae) present? Underneath the gland find the minute excretory ducts (ductuli excretorii Gl. lacrimalis). About how many are there? Where do they open?

Reflect the ligamentum palpebrale mediale and expose the ampulla ductus lacrimalis and the lacrimal sac (saccus lacrimalis). Note its summit (fornix sacci lacrimalis) and the duct leading from it down into the nose (ductus nasolacrimalis). (Fig. 108.)

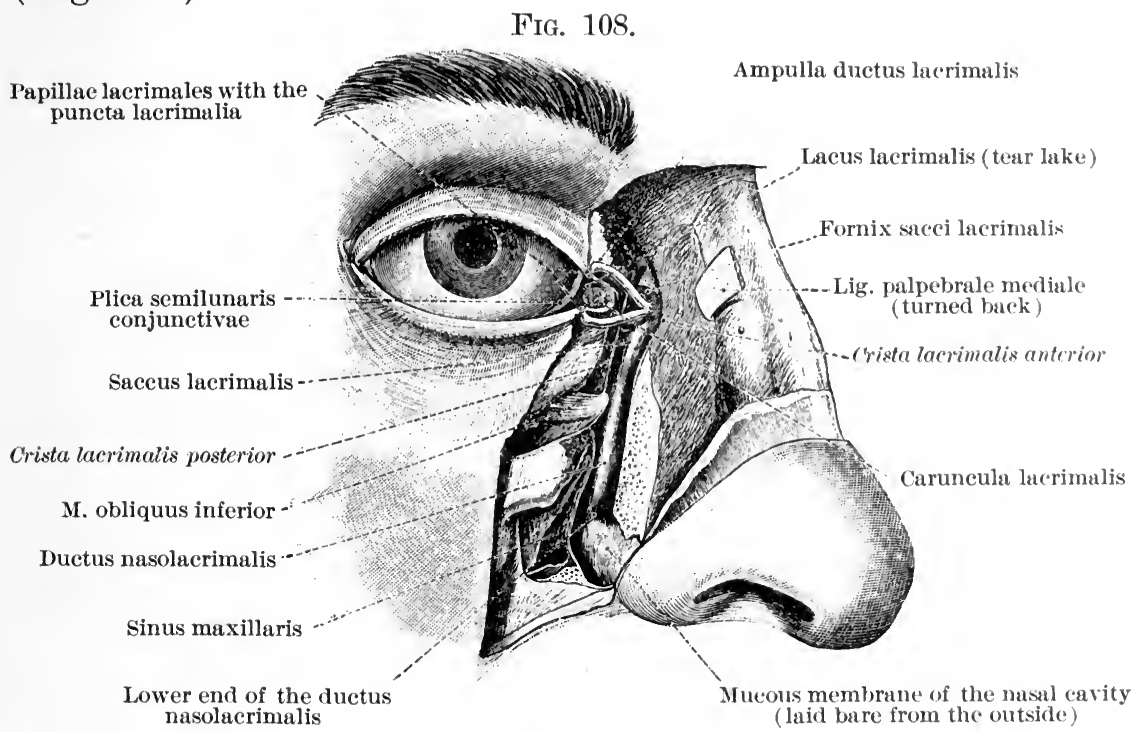

The tear sac (saccus lacrimalis), the lacrimal canals (ductus lacrimales), and the nasolacrimal duct (ductus nasolacrimalis). Part of the maxilla has bcen removed. (After Toldt, Anat. Atlas, Wien, 1903, 3 Aufl., p. 912, Fig. 1391.)

Divide the lids vertically through the middle and turn the medial halves of the lids upward and downward respectively. Dissect away the conjunctiva at the medial angle of the eye and expose the superior tarsal muscle (M.tarsalis superior) and the inferior tarsal muscle (M.tarsalis inferior) (O. T. tensor tarsi). Note the relation of the saccus lacrimalis to the tarsal muscles on one hand and to the medial palpebral ligament on the other. What is the action of the tarsal muscles?

\section{Nose.}

At this stage the nasal bones (ossa nasalia) and cartilages (cartilagines nasi) may be conveniently examined. Dissect out and study the following:

(a) Lateral cartilage of nose (cartilago nasi lateralis).

(b) Cartilage of septum of nose (cartilago septi nasi) (anterior margin). 
(c) Larger alar eartilage (cartilago alaris major).

(ca) Lateral crus (crus laterale).

(cb) Medial crus (crus mediale).

(d) Lesser alar cartilages (cartilagines alares minores).

Are any sesamoid cartilages present?

External Ear, or Auricle. (Fig. Iog.)

Though the auricle (auricula) (O. T. pinna) belongs to the cranium rather than to the face, it can be most conveniently dissected at this stage.

FIG. 109.

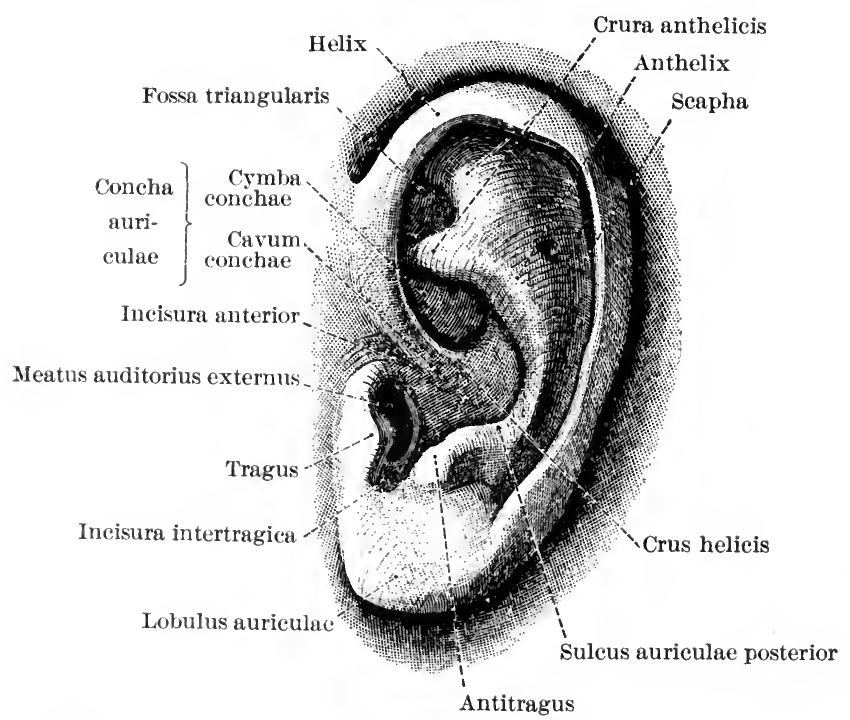

The external ear (auricula) of a young woman. (After Toldt, Anat. Atlas, Wien, 1903, 3 Aufl., p. 920, Fig. 1406.)

Make a careful drawing first of the lateral surface, then of the medial surface. Indicate-

On the lateral surface-

(a) Lobule of auricle (lobulus auriculae).

(b) Tragus (tragus).

(ba) Hairs of tragus (tragi).

(c) Cartilage of antitragus (antitragus).

(d) Intertragic notch (incisura intertragica).

(e) Anterior notch (incisura anterior).

(f) Concha of auricle (concha auriculae).

( $f a)$ Cymba of concha (cymba conchae) ("boat of the concha").

(g) Coil (helix).

( $f b)$ Cavity of concha (cavum conchae)

(ga) Crus of coil (crus helicis). 
(gb) Spine of coil (spina helicis).

(gc) Tail of coil (cauda helicis).

(h) Anthelix (anthelix).

(ha) Crura of anthelix (crura anthelicis).

(i) Triangular fossa (fossa triangularis).

(j) Tubercle of auricle (tuberculum auriculae [Darwini]), if present. On the medial surface-

(a) Fossa of anthelix (fossa anthelicis).

(b) Eminence of concha (eminentia conchae).

(c) Eminence of scapha (eminentia scaphae).

(d) Eminence of triangular fossa (eminentia fossae triangularis).

Remove the skin from the whole auricle, taking care not to injure the minute muscles and ligaments beneath. Isolate the latter.

Fig. 110.

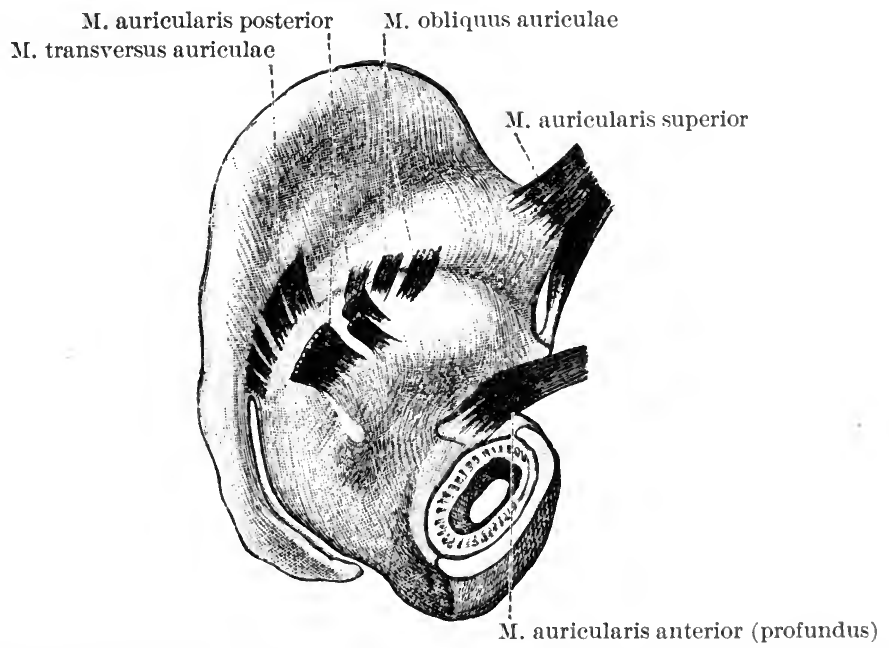

The muscles on the medial surface of the cartilage of the ear. Left car. (After Toldt, Anat. Atlas, Wien, 1903, 3 Aufl., p. 921, Fig. 1413.)

Muscles.

(a) Larger muscle of helix (M. helicis major).

(b) Smaller muscle of helix (M. helicis minor).

(c) Muscle of tragus (M. tragicus).

(d) Pyramidal muscle of ear (M. pyramidalis auriculae [Jungi]).

(e) Muscle of antitragus ( $M$. antitragicus).

(f) Transverse muscle of ear (M. transversus auriculae).

(g) Oblique muscle of auricle (M. obliquns auriculae).

(h) Muscle of noteh of helix (M. incisurae helicis [Santorini]).

Ligaments.

(a) Auricular ligaments (ligamenta auricularia [Valsalvae]). (aa) Anterior auricular (Lig. auriculare anterius). (ab) Superior auricular (Lig. auriculare superius). (ac) Posterior auricular (Lig. auriculare posterius). 
Cut the auricle off close to the skull; macerate the cartilages and scrape clean.

Study the following:

(a) Isthmus of cartilage of ear (isthmus cartilaginis auris).

(b) Terminal notch of ear (incisura terminalis auris).

(c) Antitragohelicine fissure (fissura antitragohelicina).

(d) Transverse anthelicine groove (sulcus anthelicis transversus).

(e) Groove of sulcus of crus (sulcus cruris helicis).

\section{TEMPORAL REGION AND RETROMANDIBULAR FOSSA.}

Clean away the superficial fascia and expose the deep fascia above the zygomatic process. Preserve the arteries, veins, and nerves on its surface, and watch especially for blood-vessels and nerves perforating the fascia.

\section{Temporal Fascia.}

The temporal fascia (fascia temporalis) should now be studied. What are its attachments? Demonstrate the two layers below. What structures perforate the fascia? Trace the peripheral distribution of the following:

(a) Zygomaticotemporal ramus of facial nerve (ramus zygomaticotemporalis $N$. facialis) (O. T. temporal branch of temporomalar).

(b) Temporal rami of facial nerve (rami temporales $N$. facialis).

(c) Zygomaticofacial ramus of zygomatie nerve (ramus zygomaticofacialis N. zygomatici).

Follow the middle temporal artery (A. temporalis media) from its origin from the A. temporalis superficialis to the point where it pierces the fascia.

\section{Masseter Muscle.}

The masseter muscle ( $M$. masseter) may now be dissected out. Divide the N. facialis below the ear and throw it forward. If the other structures superficial to the M. masseter impede the dissection, they may also be reflected. Study the form, position, origin, and insertion of the muscle. Cut through the fascia temporalis just above the processus zygomaticus and reflect it upward, saving the vessels and nerves which pass through it. Cut through the bony arch of the zygoma in front of and behind the origin of the M. masseter. The anterior saw-cut must be obliquely made. Throw the bony arch with the M. masseter 
lateralward and downward and find the artery (A. masseterica) and nerve (N. massetericus, V. 3) passing through the incisura mandibulae (O. T. sigmoid notch) to it. Cut through these after they have been cleaned and studied, but do not detach the M. masseter from its insertion.

\section{Temporal Muscle.}

The temporal muscle (M. temporalis) may now be examined. How are the fibres arranged? Saw nearly through the coronoid process of the mandible, making the cut pass obliquely from the middle of the incisura mandibulae downward and forward to the junction of the ramus with the basis mandibulae. Complete the division with fine forceps. Avoid injury to the N. buccinatorius. Reflect the processus coronoideus with the attached M. temporalis upward and separate the muscle from the bone. Study the anterior and posterior deep temporal nerves ( $N$. temporalis profundus anterior-posterior) and arteries ( $A$. temporalis profunda anterior-posterior); follow the distribution of the A. temporalis media; find the point of exit of the ramus zygomaticotemporalis $\mathrm{N}$. zygomatici (O. T. temporal branch of temporomalar).

\section{Pterygoid Region.}

Make a cut through the neck of the condyloid process of the mandible and another transversely through the ramus mandibulae just above the level of the mandibular foramen (O. T. inferior dental foramen). Begin the cuts with the saw and complete with bone-forceps. Remove the incised portion of the ramus and dissect away the fat and areolar tissue, so as completely to expose the pterygoid muscles and the pterygomandibular raphe (raphe pterygomandibularis) of the fascia buccopharyngea.

Examine carefully the following structures:

Muscles. (Spalteholz, Figs. 294 and 295.)

(a) External pterygoid (M. pterygoideus externus).

(b) Internal pterygoid (M. pterygoideus internus).

(c) Buccinator musele (M. buccinator).

(ca) Buccopharyngeal fascia (fascia buccopharyngea) with its raphe pterygomandibularis.

Arteries. (Vide Spalteholz, Fig. 439.)

(a) Internal maxillary (A. maxillaris interna).

First portion.

(a) Deep auricular (A. auricularis profunda).

(ab) Anterior tympanic (A. tympanica anterior). 
(ac) Inferior alveolar (A. alveolaris inferior) (O. T. inferior dental).

Second portion.

(aca) Mylohyoid ramus (ramus mylohyoideus).

(ad) Middle meningeal (A. meningea media).

(ada) Accessory meningeal ramus (ramus meningeus accessorius) ( $0 . \mathrm{T}$. small meningeal).

(ae) Masseteric (A. masseterica).

(af) Posterior and anterior deep temporal (Aa. temporales posterior et anterior profundae).

(ag) Pterygoid rami (rami pterygoidei).

(ah) Buccinator (A. buccinatoria) (O. T. buccal).

(ai) Posterior superior alveolar (A. alveolaris superior posterior) (O. T. posterior dental).

Third portion.

It cannot be studied at this stage of the dissection.

Veins.

These are seldom well enough preserved to be studied in the dissection satisfactorily. Atlases illustrating the part and, if possible, museum preparations of special venous injections, should be consulted.

Tributaries of the posterior facial vein ( $V$. facialis posterior).

(a) Articular mandibular veins ( $V v$. articulares mandibulares).

(b) Stylomastoid vein ( $V$. stylomastoidea).

(c) Pterygoid plexus (plexus pterygoideus).

(ca) Middle meningeal veins ( $V v$. meningeae mediae).

$(c b)$ Deep temporal veins ( $V v$. temporales profundae).

(cc) Masseteric veins ( $V v$. massetericae).

$(c d)$ Inferior alveolar vein ( $V$. alveolaris inferior) (O. T. inferior dental).

(d) Transverse vein of face ( $V$. transversa faciei).

Joint of Jaw.

The jaw-joint (articulatio mandibularis) (O. T. temporomaxillary articulation) should now be studied. What bony surfaces enter into its formation?

Examine-

(a) Joint-capsule (capsula articularis) (O. T. capsular ligament).

(b) Joint-disk (discus articularis) (O. T. interarticular fibrocartilage).

(c) Temporomandibular ligament (ligamentum temporomandibulare) (O. T. external lateral ligament).

(d) Sphenomandibular ligament (ligamentum sphenomandibulare) (O. T. internal lateral ligament).

(e) Stylomandibular ligament (ligamentum stylomandibulare) (0. T. stylomaxillary ligament). 


\section{Nerves of Pterygoid Region.}

To see these well the joint of the jaw should be opened and the condyloid process together with the M. pterygoideus externus turned forward. Avoid the N. auriculotemporalis.

(a) Mandibular nerve ( $N$. mandibularis) (O. T. inferior maxillary division of the trigeminal nerve).

(aa) Spinosus nerve (N. spinosus) (O. T. recurrent nerve).

Fig. 111.

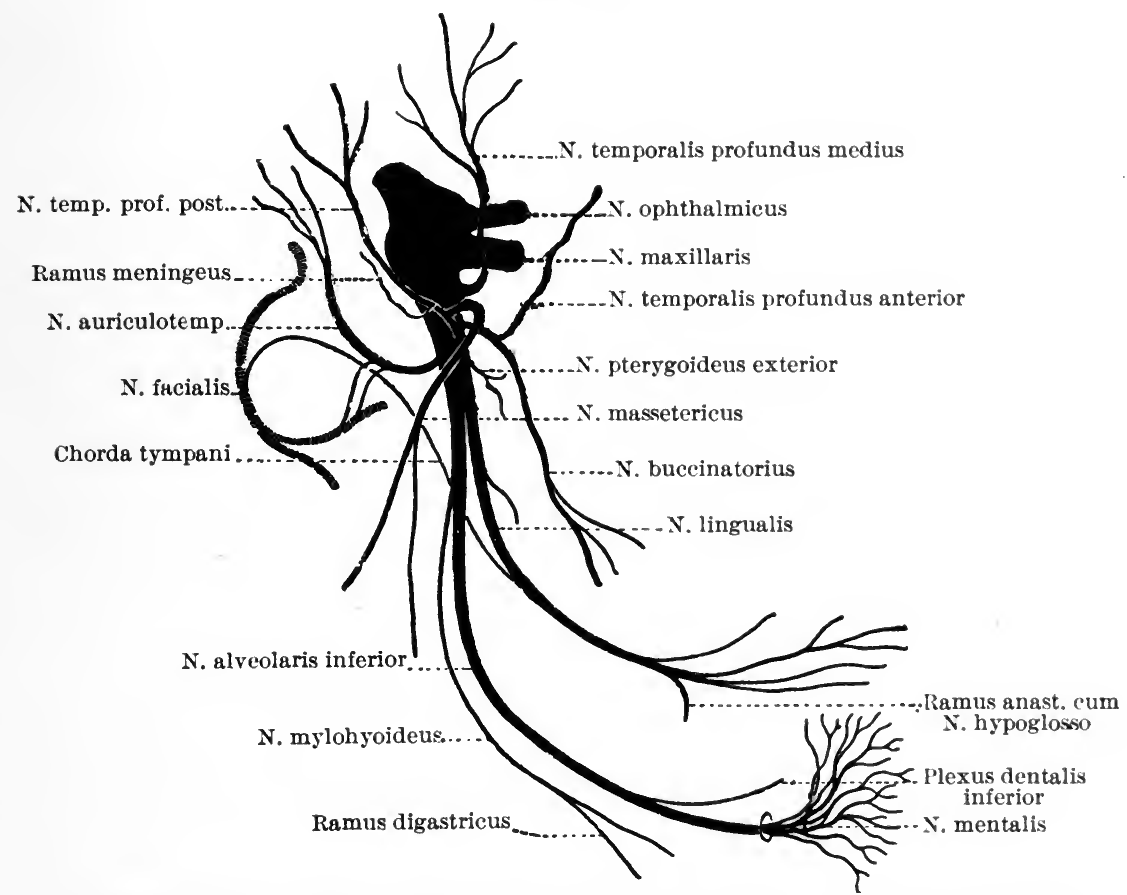

Diagram of the N. mandibularis. (From Poirier et Charpy, Traitê d'Anat. hum., Paris, 1899, t. iii., 3, p. 824 , Fig. 463 .)

(ab) Masticator nerve (N. masticatorius).

( $a b a)$ Masseteric nerve (N. massetericus).

$(a b b)$ Anterior and posterior deep temporal nerves ( $N n$. temporales profundi anterior et posterior).

$(a b c)$ Buccinator nerve (N. buccinatorius). (abd) External pterygoid (N. pterygoideus externus). (abe) Internal pterygoid (N. pterygoideus internus).

(ac) Auriculotemporal nerve ( $N$. auriculotemporalis).

(aca) Nerve of external auditory meatus (N. meatus auditorii externi).

(acaa) Branch to tympanic membrane (ramus membranae tympani). 
The other branches have been already studied, viz.(acb) Parotid rami (rami parotidei).

(acc) Anastomotic with the facial (rami anastomotici cum $N$. faciali).

(acd) Anterior auricular (Nn. auriculares anteriores).

(ace) Superficial temporal rami (rami temporales superficiales).

(ad) Lingual nerve ( $N$. lingualis) (branches studied later).

(ae) Inferior alveolar nerve ( $N$. alveolaris inferior) (O. T. inferior dental).

(aea) Mylohyoid nerve (N. mylohyoideus).

Other branches studied later.

(b) Chorda tympani of N. intermedius.

(c) Otic ganglion (ganglion oticum). This can be exposed now, but can be better studied later.

\section{Mandibular Canal.}

The mandibular canal (canalis mandibularis) (O. T. inferior dental canal) is somewhat difficult to open.

With Hey's saw, mallet and chisel, and bone-forceps remove the outer compact layer of the mandible so as to expose the contents of the canal. Study the following:

(a) Inferior alveolar artery (A. alveolaris inferior) (O. T. inferior dental).

(aa) Mylohyoid ramus (ramus mylohyoideus) (not in the canal).

(ab) Mental artery (A. mentalis).

(b) Inferior alveolar nerve ( $N$. alveolaris inferior) (O. T. inferior dental).

(ba) Inferior dental plexus (plexus dentalis inferior). (baa) Inferior dental rami (rami dentales inferiores).

(bab) Inferior gingival rami (rami gingivales inferiores).

(bb) Mylohyoid nerve (N. mylohyoideus) (not in the canal).

(bc) Mental nerve ( $N$. mentalis). (bca) Rami to chin (rami mentales).

(bcb) Rami to lower lip (rami labiales inferiores).

\section{SUBMAXILLARY REGION.}

Draw the tongue forcibly forward and stitch the tip of it to the end of the nose. Throw the head backward to the full extent and turn slightly to the opposite side.

\section{Mylohyoid Muscle (M. mylohyoideus) and Subjacent Structures.}

The structures which have been dissected, cut superficial to this muscle, may now be reflected. Clean the muscle. Study its form, position, origin, insertion, action, and innervation. Cut 
Fig. 112.

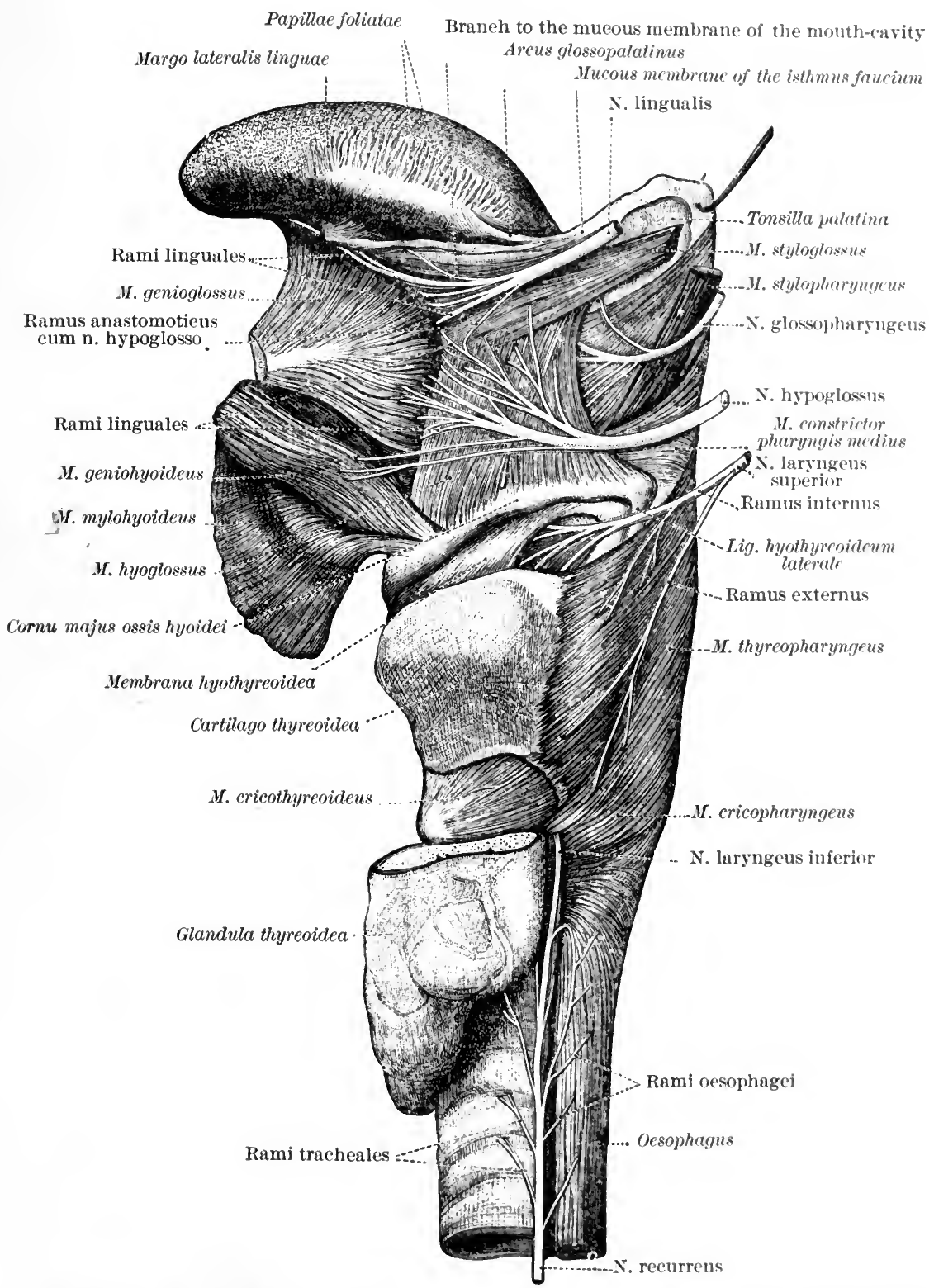

The distribution of the Nn. lingualis, glossopharyngets, and hypoglossus, and also of the Nn. larrn. geus superior and reeurrens. The head and neck viseera are isolated. The upper part of the thyrecisi gland has been removed. (After Toldt, Anat. Atlas, Wien, 1903, 3 Aufl., p. 880, Fig. 1323.) 
through the muscle close to the linea obliqua mandibulae and along the median raphe and turn it downward over the hyoid bone. Saw through the corpus mandibulae a little lateral from the median line. Lift the edge of bone and fasten it by a suture to structures above. Avoid injury to the mucous membrane in the floor of the mouth.

Beneath the mylohyoid muscle study carefully the following: Muscles.

(a) Hyoglossus muscle (M. hyoglossus).

(b) Styloglossus muscle (M. styloglossus).

(c) Genioglossus musele (M. genioglossus).

(d) Geniohyoid muscle (M. geniohyoideus).

\section{FIG. 113.}

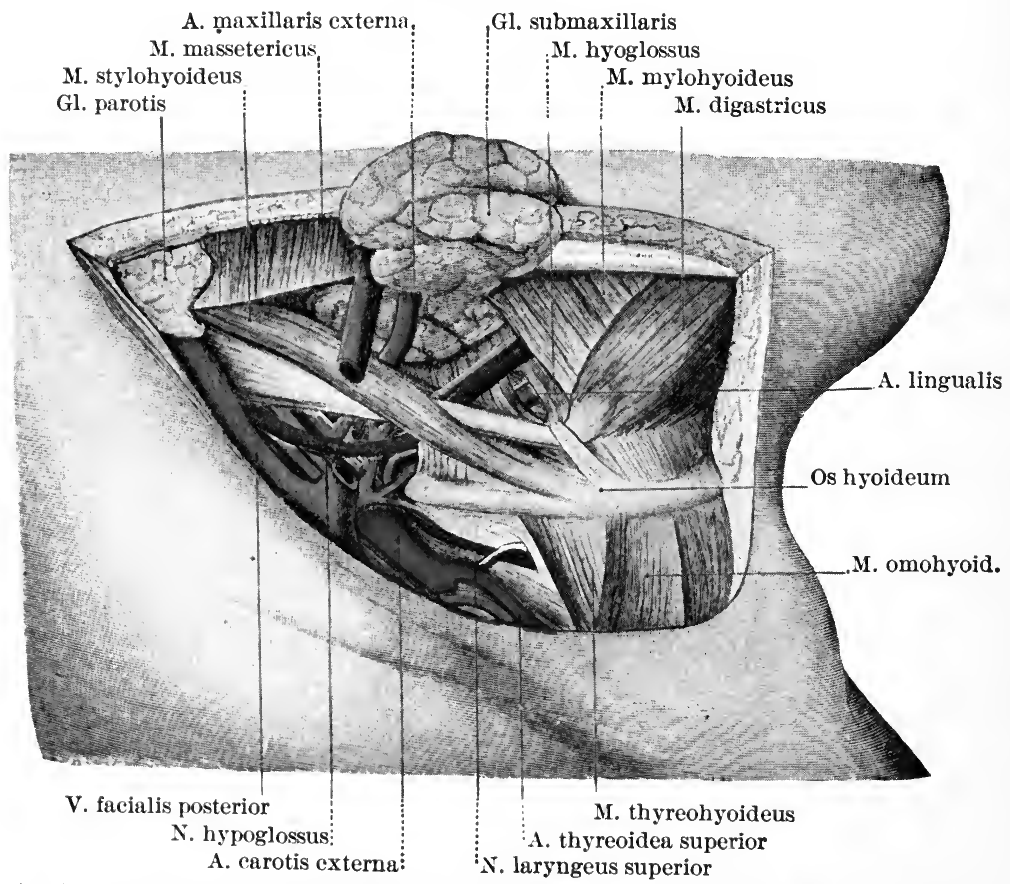

The structures in the submaxillary region. (After Haeckel in Atlas des Topograph. Anat. des Mensch.-v. Bardeleben, Haeckel, and Frohse-Jena, 1901, Fig. 49.)

Nerves. (Fig. 112.)

(a) Lingual nerve ( $N$. lingualis). (aa) Sublingual nerve ( $N$. sublingualis).

(ab) Lingual rami (rami linguales).

(b) Hypoglossal nerve (N. hypoglossus).

(ba) Lingual rami (rami linguales).

What muscles are innervated by the N. hypoglossus?

(c) Submaxillary ganglion (ganglion submaxillare). 
To find this dissect in the interval between the N. lingualis and the deep part of the glandula submaxillaris. Find-

(ca) Branches communicating with the lingual nerve (rami communicantes cum N. linguali).

(cb) Submaxillary rami (rami submaxillares).

(d) Glossopharyngeal nerve ( $N$. glossopharyngeus).

Arteries.

How does it behave as regards the M. hyoglossus?

(a) Lingual artery (A. lingualis). (Fig. 113.)

Veins.

(a) Lingual vein ( $V$. lingualis).

(aa) Sublingual vein ( $V$. sublingualis).

$(a b)$ Companion veins to hypoglossal nerve ( $V v$. comites $N$. hypoglossi).

Salivary Glands.

(a) Deep part of submaxillary gland (glandula submaxillaris).

(aa) Submaxillary duct (ductus submaxillaris [Whartoni]).

(b) Sublingual gland (glandula sublingualis).

(ba) Larger sublingual duct (ductus sublingualis major) (O. T. duct of Bartholin).

(bb) Smaller sublingual ducts (ductus sublinguales minores) ( $O$. T. ducts of Rivini).

\section{Hyoglossal Muscle (M. hyoglossus).}

Study its form, position, origin, insertion, action, and innervation. Then detach it from the hyoid bone and reflect it upward. Study the structures beneath.

Arteries.

(a) Lingual (A. lingualis) in its second and third parts.

(aa) Dorsal rami of tongue (rami dorsales linguae).

$(a b)$ Sublingual artery ( $A$. sublingualis).

(ac) Deep artery of tongue (A. profunda linguae) (O. T. ranine

Veins. artery).

(a) Tributaries of lingual vein.

(aa) Companion veins of lingual artery ( $V v$. comites A. lingualis).

\section{DEEP DISSECTION OF NECK.}

Stylopharyngeus Muscle (M. stylopharyngeus). (Vide Spalteholz, Fig. 53I.)

Remove the calvarium; sponge the floor of the skull-cavity with alcohol. Cut through the venter posterior M. digastrici near its origin from the incisura mastoidea of the temporal bone, and turn it forward and downward. At this point observe the 
anastomotic ramus connecting the facial with the glossopharyngeal nerve (ramus anastomoticus cum N. glossopharyngeo). From which branch of the $\mathrm{N}$. facialis does it come? Cut through the A. carotis externa at a point just inferior to the terminal bifurcation into the A. temporalis superficialis and the A. maxillaris interna; cut through also the $A$. auricularis posterior and the A. occipitalis at their origins and turn the A. carotis externa forward out of the way. Clean the M. stylopharyngeus, avoiding injury to the N. glossopharyngeus. Study the form, position, origin, action, and innervation of the muscle. The insertion can be seen best at a later stage of the dissection.

\section{Internal Carotid Artery (A. carotis interna).}

With bone-forceps cut through the base of the processus styloideus and reflect it, with the muscles attached to it, downward and forward. The vessels and nerves more medially situated may now be carefully dissected out of the tough fascia in which they lie and traced up to the base of the skull. Secure the pharyngeal rami of the $\mathrm{N}$. vagus early; they will be found on the lateral surface of the A. carotis interna. Study with especial care the interval between the $\mathrm{V}$. jugularis interna and the $\mathrm{A}$. carotis interna just beneath the base of the skull.

What is the level of origin of the A. carotis interna? How does it pass into the cranial cavity? Study carefully the complicated relations of its cervical portion. Why is its proximity to the pharynx and palatine tonsil emphasized in the text-books?

\section{Smaller Arteries deep in the Neck. (Vide Spalteholz, Figs. 44I and 442.)}

(a) Ascending pharyngeal artery (A. pharyngea ascendens).

Note its relations to the A. carotis interna and to the pharynx. Study the following branches:

(aa) Pharyngeal rami (rami pharyngei).

(ab) Posterior meningeal (A. meningea posterior).

(ac) Inferior tympanic (A.tympanica inferior).

(b) Ascending palatine branch of external maxillary (A. palatina ascendens) (O. T. inferior palatine).

(c) Tonsillar ramus of external maxillary (ramus tonsillaris) (O. T. tonsillitic artery).

\section{Internal Jugular Vein (V. jugularis interna) and its Tributaries.}

With what cerebral sinus is the internal jugular vein continuous? What are the relations of the vein in the jugular foramen? Where is the superior bulb of the jugular vein (bulbus 
venae jugularis superior) situated? What is the relation of this vein to the other structures contained within the "vascular sheath" of the deep cervical fascia? Observe the entrance of the inferior petrosal sinus (simus petrosus inferior) into the superior bulb of the vein. Pass a probe through the sinus from the skull cavity into the bulb. Find the pharyngeal plexus (plexus pharyngeus) and tributary veins (Vv. pharyngeae). 'The following tributaries of the internal jugular have been studied already, but they may now be reviewed with advantage:

(a) Lingual vein ( $V$. lingualis).

(b) Superior thyreoid veins ( $V v$. thyreoideae superiores).

(c) Common facial vein ( $V$. facialis communis).

Slit open the internal jugular vein and observe the valve. In what part of the vein is it situated? How many flaps are to be seen?

\section{Hypoglossal Nerve (N. hypoglossus). (Vide Figs. 92 and II2.)}

Divide the V. jugularis interna five centimetres below the base of the skull and turn the upper part upward so as to expose the $\mathrm{N}$. hypoglossus better as it emerges from the canalis hypoglossi (O. T. anterior condyloid foramen). Note the close connection of the nerve with the ganglion nodosum of the N. vagus. At what point does the N. hypoglossus enter the anterior triangle of the neck? Note its relation to the A. occipitalis. Demonstrate, if possible, the branches of communication (1) with the superior cervical ganglion of the sympathetic, (2) with the ganglion nodosum of the N. vagus, and (3) especially with the Nn. cervicales I. and II. What is the significance of the fibres to the N. hypoglossus from the cervical nerves? Make a list of (1) the muscles supplied by the N. hypoglossus proper and (2) the muscles supplied by fibres of cervical nerves running in the $\mathrm{N}$. hypoglossus. Review the branches of the N. hypoglossus already studied,-viz., (1) ramus descendens, (2) ansa hypoglossi, (3) ramus thyreohyoideus, and (4) rami linguales.

Accessory Nerve (N. accessorius) (O. T. Spinal Accessory). (Vide Fig. 92.)

What is its relation to the $\mathrm{N}$. vagus and $\mathrm{N}$. glossopharyngeus in the jugular foramen? Study-

(a) Internal ramus (ramus internus) (O. T. aceessory portion). Follow it to where it fuses with the $\mathrm{N}$. vagus. 
(b) External ramus (ramus externus) (O. T. spinal portion). Follow it to the sternocleidomastoid muscle. Its peripheral distribution has been studied in the neck.

Vagus Nerve (N. vagus) (O. T. Pneumogastric Nerve). (Vide Fig. 92.)

Study the two ganglia from the cells of which its constituent sensory fibres arise:

(a) Upper or jugular ganglion (ganglion jugulare) (O. T. ganglion of the root).

(b) Lower or knotty ganglion (ganglion nodosum) (O. T. ganglion of the trunk).

In special dissections from the museum, with the aid of models and atlases, study the following:

(a) Meningeal ramus (ramus meningeus) (O. T. recurrent branch).

(b) Auricular ramus (ramus auricularis) (O. T. Arnold's nerve).

(c) Anastomotic ramus with glossopharyngeal nerve (ramus anastomoticus cum N. glossopharyngeo).

The following branches, already examined in the dissection of the side of the neck, may be conveniently reviewed:

(a) Pharyngeal rami (rami pharyngei).

(b) Superior laryngeal nerve ( $N$. laryngeus superior).

(ba) External ramus (ramus externus).

(bb) Internal ramus (ramus internus).

(bc) Ramus anastomosing with.inferior laryngeal nerve (ramus anastomoticus cum $N$. laryngeo inferiori).

(c) Superior eardiac rami (rami cardiaci superiores).

(d) Depressor nerve (N. depressor).

(e) Recurrent nerve ( $N$. recurrens) (O. T. recurrent laryngeal).

The other branches are studied when the thorax and abdomen are dissected.

Glossopharyngeal Nerve (N. glossopharyngeus). (Vide Fig. II2.)

Examine its relations in the jugular foramen. Find the two ganglia which give origin to those of its fibres that are sensory:

(a) Upper ganglion (ganglion superius) (O. T. jugular ganglion).

(b) Lower or petrous ganglion (ganglion petrosum) (O. T. ganglion of Anderseh).

Find as many of the branches of the N. glossopharyngeus in your own dissection as you can and supplement the study of the 
cadaver by that of museum preparations, models, and atlases; include the following:

(a) Tympanie nerve (N. tympanicus) (O. T. Jacobson's nerve). (Vide Spalteholz, Fig. 773.)

(aa) Tympanic plexus (plexus tympanicus [Jacobsoni]).

(aaa) Superior earoticotympanic nerve (N. caroticotympanicus superior).

$(a a b)$ Inferior caroticotympanic nerve $(N$. caroticotympanicus inferior).

(aac) Tubal ramus (ramus tubae).

(b) Ramus anastomosing with the auricular branch of the vagus (ramus anastomoticus cum ramo auriculari $N$. vagi).

(c) Pharyngeal rami (rami pharyngei).

(d) Stylopharyngeal branch (ramus stylopharyngeus).

(e) Tonsillar rami (rami tonsillares) (O. T. tonsillitic branches).

( $f$ ) Lingual rami (rami linguales) (O. T. terminal branches).

Cervical Part of Sympathetic System (Pars cervicalis S. sympathici). Note that the sympathetic trunk (truncus sympathicus) in the neck has only three ganglia upon it. (Fig. 92.)

(a) Superior cervical ganglion (ganglion cervicale superius).

What evidence is there that it represents four segmental sympathetic ganglia?

(b) Middle cervical ganglion (ganglion cervicale medium).

Why does it probably correspond to two primitive ganglia?

(c) Inferior cervieal ganglion (ganglion cervicale inferius).

Note that it represents at least two segmental ganglia.

Study the exact level and relations of each of these ganglia. Find the rami communicantes connecting them with the $\mathrm{Nn}$. cervicales.

How much have you made out in your dissection of the following :

(a) Jugular nerve ( $N$. jugularis).

(b) Internal carotid nerve ( $N$. caroticus internus).

(c) Internal carotid plexus (plexus caroticus internus).

(d) External carotid nerves ( $N$. carotici externi).

(e) External earotid plexus (plexus caroticus externus).

( $f$ ) Superior thyreoid plexus (plexus thyreoideus superior).

(g) Lingual plexus (plexus lingualis).

(h) External maxillary plexus (plexus maxillaris externus).

(i) Sympathetic root of submaxillary ganglion (radix sympathica ganglii submaxillaris, .

(j) Occipital plexus (plexus occipitalis).

(k) Posterior auricular plexus (plexus auricularis posterior).

(l) Superficial temporal plexus (plexus temporalis superficialis).

( $m$ ) Internal maxillary plexus (plexus maxillaris internus). 
(n) Common carotid plexus (plexus caroticus communis).

(o) Laryngopharyngeal rami (rami laryngopharyngei).

(p) Ascending pharyngeal plexus (plexus pharyngeus ascendens).

(q) Superior cardiac nerve (N. cardiacus superior).

(r) Middle cardiac nerve ( $N$. cardiacus medius).

(s) Subclavian loop (ansa subclavia [Vieussenii]).

$(t)$ Inferior cardiac nerve ( $N$. cardiacus inferior).

(u) Subclavian plexus (plexus subclavius).

(v) Internal mammary plexus (plexus mammarius internus).

(w) Inferior thyreoid plexus (plexus thyreoideus inferior).

$(x)$ Vertebral plexus (plexus vertebralis).

Note that all the above, except $(a),(i),(o),(q),(r),(s),(t)$, are plexuses about the larger arteries.

\section{Lateral Straight Muscle of the Head (M. rectus capitis lateralis).} (Vide Spalteholz, Fig. 304.)

Clean the muscle; find its origin and its insertion. What is its action? What nerve supplies it? Find the ramus anterior of the first cervical nerve. How is it related to the M. rectus capitis lateralis? How is the first loop of the plexus cervicalis formed? Divide the attachment of the M. rectus capitis lateralis close to the transverse process of the atlas and turn the muscle upward. Sever the origin of the M. obliquus capitis superior. Find the nerve to the $M$. rectus capitis lateralis and follow it to its origin from the first cervical nerve ( $N$. suboccipitalis).

\section{STRUCTURES IN FOSSA CRANII MEDIA.}

\section{Removal of Dura Mater.}

(a) Cut through the dura mater just lateral from the openings through which the III., IV., and V. nerves pass, from the processus clinoideus anterior to the apex pyramidis (O. T. tip of petrous bone).

(b) Cut through the dura mater from the apex pyramidis backward and lateralward along the line of the sinus petrosus superior as far as the sulcus sigmoideus.

(c) Cut through the dura from the processus clinoideus anterior lateralward and forward along the posterior margin of the ala parva of the sphenoid bone to its lateral extremity.

Lift the dura carefully, keeping the edge of the knife close to the membrane, and thus avoid injury to the nerves attached to it beneath. 


\section{Cavernous Sinus (Sinus cavernosus). (Vide Fig. Ir4.)}

Where is it situated? What are its boundaries? What important structures pass through it? Note the following tributaries :

(a) Superior ophthalmic vein ( $V$. ophthalmica superior).

(b) Inferior ophthalmic vein ( $V$. ophthalmica inferior).

(c) Sphenoparietal sinus (sinus sphenoparietalis).

\section{Arteries of the Region.}

(a) Internal carotid artery (A. carotis interna). How does it enter the eranial cavity? Study its course.

(aa) Ophthalmic artery (A. ophthalmica). (Vide Fig. 117.)

Fig. 114.

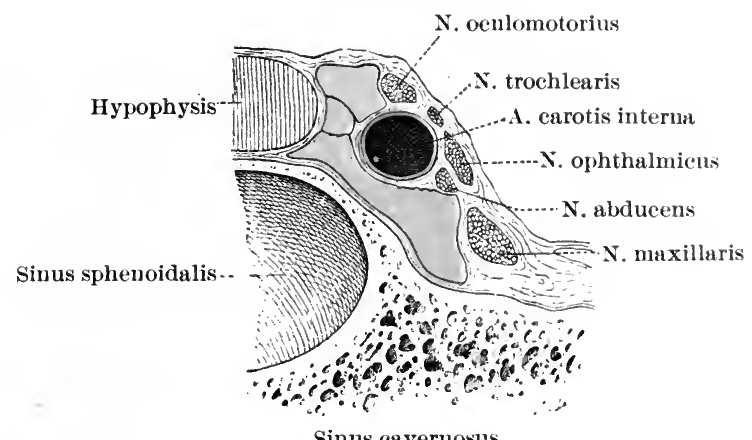

Sinus cavernosus

Histological section passing in a frontal plane through the hypophysis, adult (after Langer). (From Poirier et Charpy, Traité d'Anat. hum., Paris, 1901, 2 ed., t. ii. p. 767, Fig. 497. )

(b) Middle meningeal artery (A. meningea media). Through what opening does it enter the middle cranial fossa? In what portion of your dissection did you meet with the origin of this artery? Find the following branches:

(ba) Superficial petrosal ramus (ramus petrosus superficialis).

(bb) Superior tympanic artery (A. tympanica superior).

(c) Accessory meningeal ramus (ramus meningeus accessorius) of $\mathrm{A}$. meningea media. Look for it entering the skull through the foramen ovale.

\section{Nerves of the Region.}

(a) Trigeminal nerve (N. trigeminus) (O. T. N. cerebralis V.).

Observe the exact position of the larger portion (portio major) (sensory) and of the smaller portion (portio minor) (motor) and their relations to the semilunar ganglion (ganglion semilunare [Gasseri]) (O. T. Gasserian ganglion), the cells of which give origin to the sensory fibres of the nerve (vide Fig. 115). Read carefully a description of the semilunar ganglion in your systematic text- 
book and compare this with the findings in the cadaver. Study the mode of formation of the cavum Meckelii. Examine the three great trunks:

(aa) Ophthalmic nerve ( $N$. ophthalmicus).

(ab) Maxillary nerve (N. maxillaris) (O. T. superior maxillary).

FIG. 115.

$\mathrm{N}$. lacrimalis $\mathrm{N}$. nasociliaris

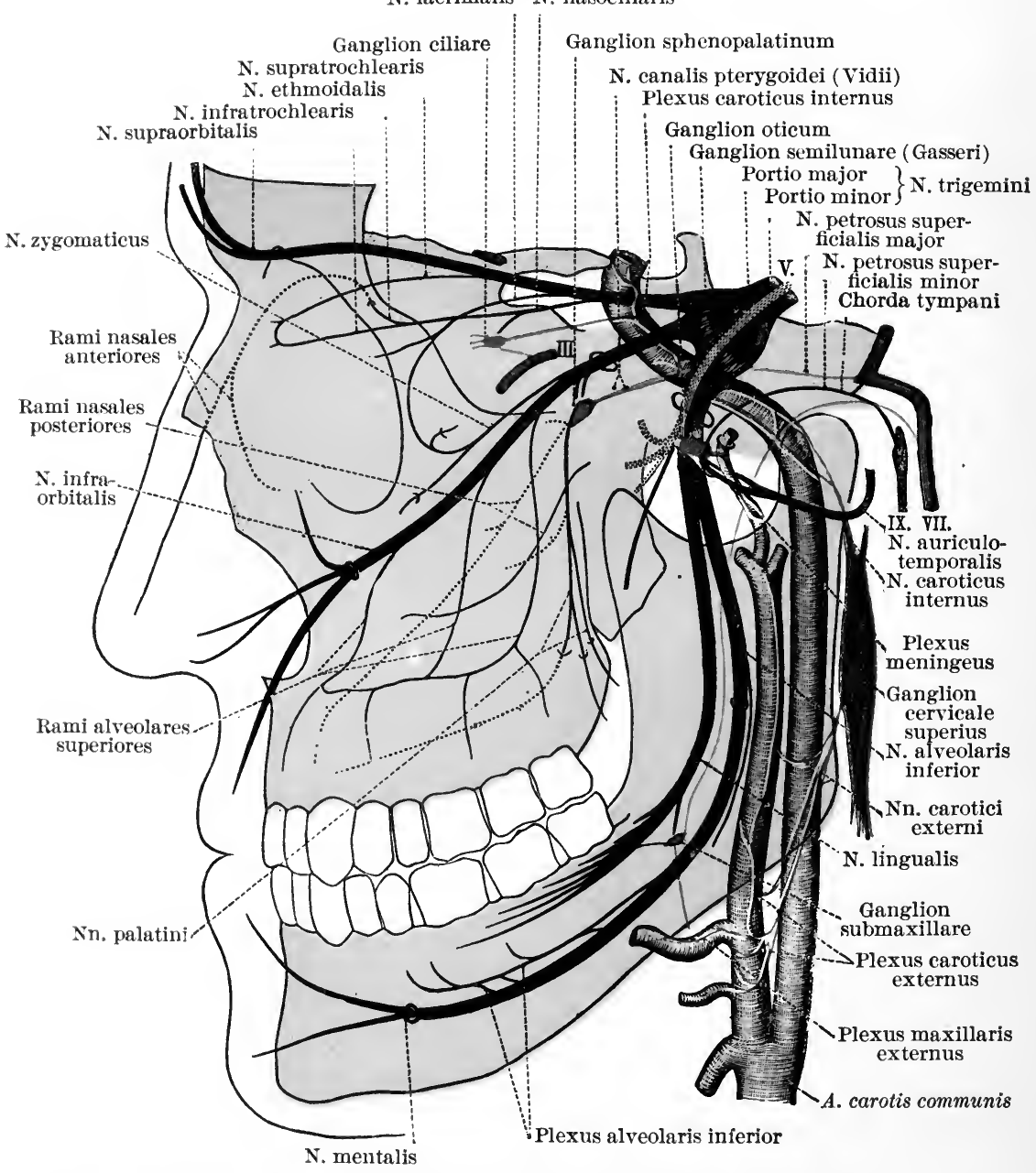

Schematic representation of the $\mathrm{N}$. trigeminus and its connections. (After Toldt, Anat. Atlas, Wien, 1903, 3 Aufl., p. 859, Fig. 1298.)

(ac) Mandibular nerve (N. mandibularis) (O. T. inferior maxillary).

Do these trunks arise from the convexity or from the concavity of the ganglion? With which is the motor root (portio minor) combined? Find the following branches of the N. ophthalmicus: 
(aca) Frontal nerve (N. frontalis).

(acb) Lacrimal nerve (N. lacrimalis).

(acc) Nasociliary nerve ( $N$. nasociliaris).

Through what openings does the N. maxillaris leave the skull? Find the meningeal branch $(N$. meningeus (medius)). Through what openings does the N. mandibularis leave the skull? Observe its relation to the ramus meningeus accessorius of the A. meningea media.

(b) Oculomotor nerve (N. oculomotorius) (O. T. N. cerebralis III.).

(ba) Superior branch (ramus superior).

$(b b)$ Inferior branch (ramus inferior).

(c) Trochlear nerve (N. trochlearis) (O. T. nervus patheticus; N. cerebralis IV.).

(d) Abducent nerve (N. abducens) (O. T. N. cerebralis VI.).

(e) Cavernous plexus of sympathetic (plexus cavernosus).

(f) Larger superficial petrosal nerve (N. petrosus superficialis major).

(g) Smaller superficial petrosal nerve ( $N$. petrosus superficialis minor).

\section{ORBIT.}

\section{Removal of Roof of Orbit (Facies superior orbitae).}

Chisel away the thick bone forming the cranial wall above the aditus orbitae, but leave unbroken the supraorbital margin (margo supraorbitalis). Remove carefully the whole of the thin roof of the orbit (paries superior). With bone-forceps cut away that part of the ala parva of the sphenoid bone which forms the superior orbital fissure (fissura orbitalis superior) (O. T. sphenoidal fissure). Cut away the processus clinoideus anterior. The periosteum of the paries superior is now exposed; note its continuity with the dura mater through the superior orbital fissure. Cut through it sagittally midway between the lateral and medial walls of the orbit and also transversely near the anterior margin of the paries superior. Reflect the two halves medialward and lateralward respectively. Grasp the front of the bulbus oculi with forceps, pull it forward, and fasten to the nose by means of needle and thread passed through the tunica conjunctiva bulbi. Avoid perforation of the eyeball proper.

\section{Frontal Nerve (N. frontalis).}

Find it lying upon the upper surface of the M. levator palpebrae superioris. Clean it and the upper surface of the muscle, avoiding, in front, the A. supraorbitalis. Study the following branches of the N. frontalis : 
(a) Supraorbital nerve (N. supraorbitalis).

(b) Frontal ramus (ramus frontalis).

(c) Supratrochlear nerve (N. supratrochlearis).

The peripheral distribution of these nerves has already been studied in the dissection of the face.

\section{Trochlear Nerve (N. trochlearis) (O. T. Patheticus, or Fourth Cran- ial Nerve).}

Carefully remove the fat along the medial wall of the orbit until the M. obliquus is found. On its hinder part find the trochlear nerve. Notice the abundance of fat in the orbit (corpus adiposum orbitae).

\section{Lacrimal Nerve (N. lacrimalis).}

Look for it on the lateral wall of the orbit along with the A. lacrimalis above the superior margin of the M. rectus lateralis. Besides the branches to the lacrimal gland, secure the anastomotic ramus to the zygomatic nerve (ramus anastomoticus cum N. zygomatico). The student will recall having studied the terminals of the $\mathrm{N}$. lacrimalis (rami palpebrales) in the dissection of the upper eyelid.

Elevator Muscle of Upper Eyelid (M. levator palpebrae superioris).

Study this muscle carefully. On lifting it, the minute branch of the N. oculomotorius innervating it may be seen approaching it from the upper surface of the M. rectus superior.

\section{Lacrimal Glands.}

(a) Superior lacrimal gland (glandula lacrimalis superior).

(b) Inferior lacrimal gland (glandula lacrimalis inferior).

(c) Accessory lacrimal glands [inconstant] (glandulae lacrimales accessoriae).

(d) Excretory ductules (ductuli excretorii [Gl. lacrimalis]).

Fascia of Eyeball (Fascia bulbi [Tenoni]) (O. T. Capsule of Tenon).

Divide the N. frontalis in the middle of the orbit and reflect the stumps. Cut through the M. levator superioris in the middle and reflect. With the point of a sharp narrow-bladed knife make an oblique valvular opening into the eyeball at the junction of cornea and sclera. Introduce the tip of a blow-pipe and inflate the eyeball with air. On withdrawal, the valve-like character of the opening prevents the escape of the air. Pick up with 
forceps the upper part of the loose bursa-like tissue (fascia bulbi [Tenoni]) (O. T. capsule of Tenon) at the back of the eyeball and remove a bit with scissors. Through the opening thus made introduce the handle of the knife, and explore the interfascial space (spatium interfasciale [Tenoni]). Determine the extent of the space. What is the relation of the tendons of the eyemuscles to the fascia bulbi?

Superior Straight and Superior Oblique Muscles of Eye (M. rectus superior; $M$. obliquus superior).

Establish the form, position, origin, insertion, innervation, and action of each. Examine carefully the pulley (trochlea) through which the tendon of the superior oblique muscle runs.

FIG. 116.

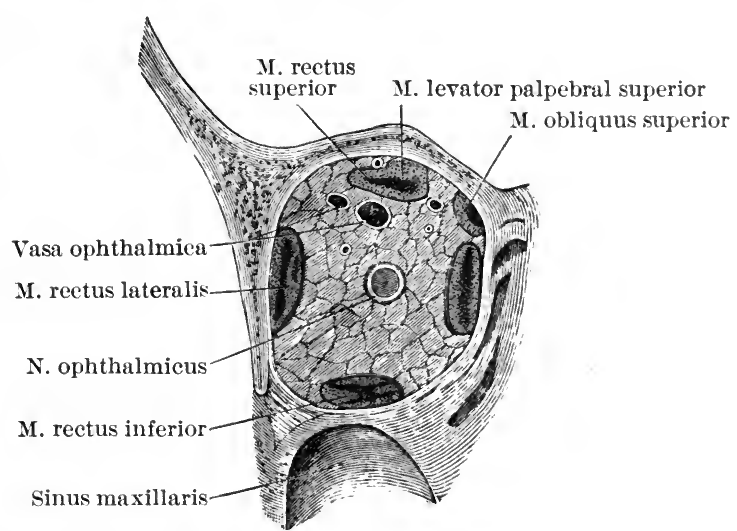

Frontal section through the right orbit behind the bulbus oculi. Posterior surface. (From Gegenbaur, Lehrb. der Anat. des Menseh., Leipzig, 1899, 7 Aufl., Bd. ii. p. 589, Fig. 715.)

\section{Optic Nerve and Neighborhood. (Vide Fig. II6.)}

Cut through the M. rectus superior and reflect the ends. Beneath its posterior part find the ramus superior of the $\mathrm{N}$. oculomotorius. Remove the fat over the optic nerve and study the following structures:

(a) Optic nerve (N. opticus).

(b) Nasociliary nerve ( $N$. nasociliaris) (O. T. nasal nerve).

(ba) Long root of ciliary ganglion (radix longa ganglii ciliaris).

(bb) Long ciliary nerves ( $N n$. ciliares longi).

(bc) Posterior ethmoidal nerve ( $N$. èthmoidalis posterior).

$(b d)$ Anterior ethmoidal nerve ( $N$. ethmoidalis anterior).

(be) Infratrochlear nerve ( $N$. infratrochlearis).

(bea) Superior palpebral branch (ramus palpebralis superior). 
(beb) Inferior palpebral branch (ramus palpebralis inferior) (already studied in dissection of the face).

(bf) Anterior nasal rami (rami nasales anteriores) (to be studied further when the nasal cavity is dissected).

(c) Ciliary ganglion (ganglion ciliare) (O. T. lenticular or ophthalmic ganglion).

(ca) Short ciliary nerves (Nn. ciliares breves).

Try to find also the sympathetic roots of the ciliary ganglion (radices sympathicae ganglii ciliaris).

\section{Blood-vessels of Orbit.}

Arteries.

(a) Ophthalmic artery (A. ophthalmica).

(aa) Central artery of retina (A. centralis retinae).

(ab) Lacrimal artery (A. lacrimalis).

$(a b a)$ Lateral palpebral arteries (Aa. palpebrales laterales).

(ac) Muscular rami (rami musculares).

FIG. 117.

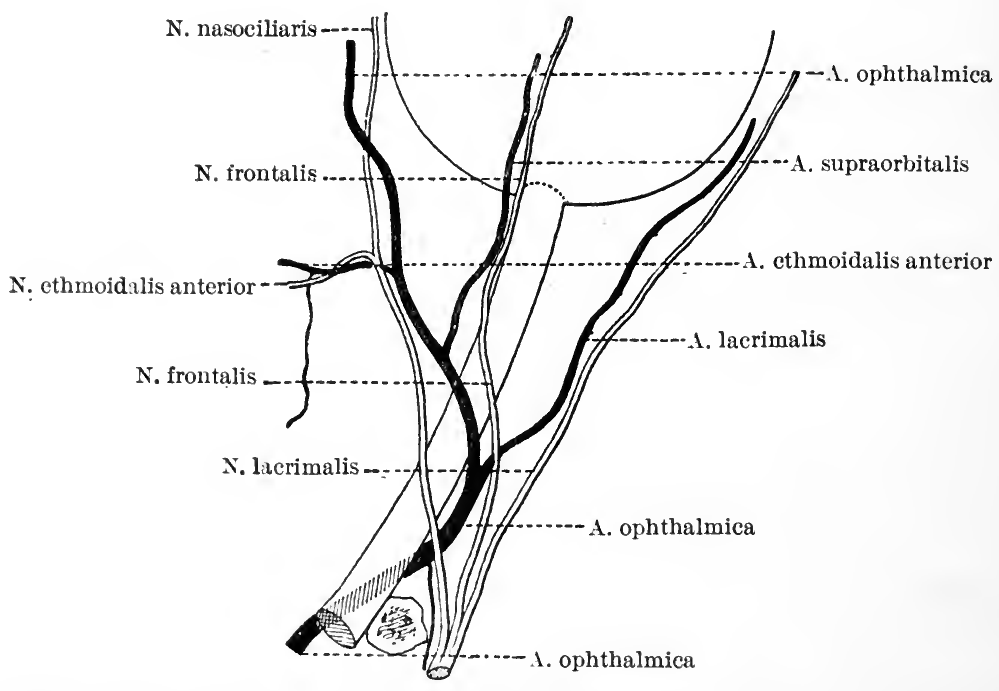

Relation of the branches of the ophthalmic artery to the nerves. (After Poirier et Charpy, Traite d'Anat. hum., Paris, 1901, 2 ed., t. ii. p. 698, Fig. 103.)

(ad) Short posterior ciliary arteries (Aa. ciliares posteriores breves).

(ae) Long posterior ciliary arteries (Aa. ciliares posteriores longae).

(af) Anterior ciliary arteries (Aa. ciliares anteriores). (afa) Episcleral arteries (Aa. episclerales).

$(a f b)$ Anterior conjunctival arteries (Aa. conjunctivales anteriores). 
(ag) Supraorbital artery (A. supraorbitalis).

(ah) Posterior ethmoidal artery (A. ethmoidalis posterior).

(ai) Anterior ethmoidal artery (A. ethmoidalis anterior).

(aj) Medial palpebral arteries (Aa. palpebrales mediales). Note especially-

(aja) Superior tarsal arch (arcus tarseus superior).

(ajb) Inferior tarsal arch (arcus tarseus inferior).

(ajc) Posterior conjunctival arteries (Aa. conjuncti-. vales posteriores).

(ak) Dorsal artery of nose (A. dorsalis nasi) (O. T. nasal branch).

(al) Frontal artery (A. frontalis).

Veins. (Tributaries of the sinus cavernosus.)

(a) Superior ophthalmic vein ( $V$. ophthalmica superior).

(aa) Nasofrontal vein ( $V$. nasofrontalis).

$(a b)$ Anterior and posterior ethmoidal veins ( $V v$. ethmoidales anterior et posterior).

(ac) Lacrimal vein ( $V$. lacrimalis).

(ad) Muscular veins ( $V v$. musculares).

(b) Inferior ophthalmic vein (V. ophthalmica inferior).

(c) Central vein of retina ( $V$. centralis retinae).

The following veins of the bulbus oculi and of the eyelids are drained partly by the V. ophthalmica superior, partly by the V. ophthalmica inferior:

(d) Vortex veins ( $V v$. vorticosae).

(e) Posterior ciliary veins ( $V v$. ciliares posteriores).

(f) Anterior ciliary veins ( $V v$. ciliares anteriores).

(g) Episcleral veins ( $V v$. episclerales).

(h) Palpebral veins ( $V v$. palpebrales).

(i) Anterior and posterior conjunctival veins ( $V v$. conjunctivales posteriores et anteriores).

\section{Other Straight Muscles of Eyeball.}

The M. rectus superior has already been studied and reflected. Examine the origin, insertion, position, action, and innervation of each of the following muscles:

(a) Medial straight muscle ( $M$. rectus medialis) (O. T. rectus internus).

(b) Inferior straight muscle ( $M$. rectus inferior).

(c) Lateral straight muscle ( $M$. rectus lateralis) (O. T. external rectus). (ca) Lacertus of lateral straight muscle (lacertus musculi recti lateralis).

Divide the N. opticus close to the foramen opticum and turn the eyeball forward. Examine the attachment of the eye-muscles behind. What is meant by the common tendinous ring of Zinn (annulus tendineus communis [Zinni]) (O. T. ligament of Zinn) ? 
How are the muscles of the eye (musculi oculi) inserted into the eyeball (bulbus oculi)?

Oculomotor and Abducent Nerves (Nn. oculomotorius et abducens). Study these two nerves through their course.

(a) Oculomotor nerve (N. oculomotorius) (O. T. third nerve).

(a) Superior ramus (ramus superior).

(ab) Inferior ramus (ramus inferior).

(aba) Short root of ciliary ganglion (radix brevis ganglii ciliaris) (O. T. motor root of lenticular ganglion).

(b) Abducent nerve (N. abducens) (O. T. sixth nerve). What muscle does it innervate?

FIG. 118.

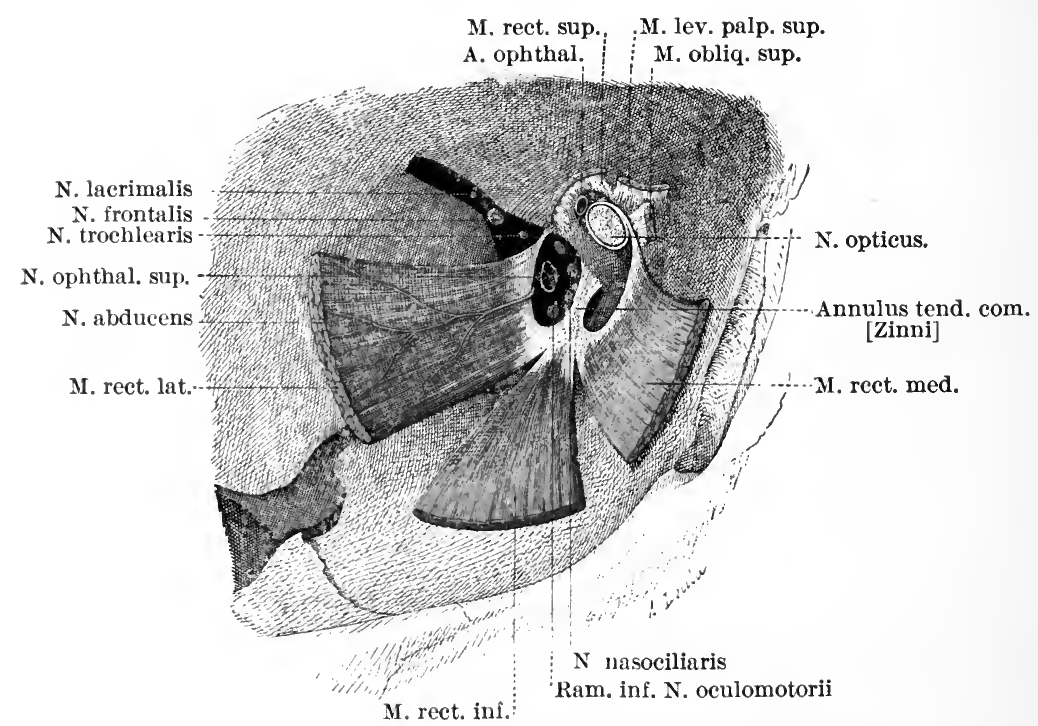

Schema of the annulus tendineus communis [Zinni] and its relations to the nerves of the orbit. (From Poirier et Charpy, Traité d'Anat. hum., Paris, 1899, t. iii., 3, p. 794, Fig. 440.)

Compare the arrangement of the various nerves in the superior orbital fissure (fissura orbitalis superior) (O. T. sphenoidal fissure) with that of the nerves in the sinus cavernosus. What are the principal differences?

\section{Inferior Oblique Muscle of Eye (M. obliquus inferior).}

Replace the eyeball in its natural position. Evert lower eyelid, and dissect off conjunctiva in the region of the fornix conjunctivae inferior. Find the inferior oblique muscle and clean it. Study its origin, insertion, action, and innervation. 
Fig. 119.

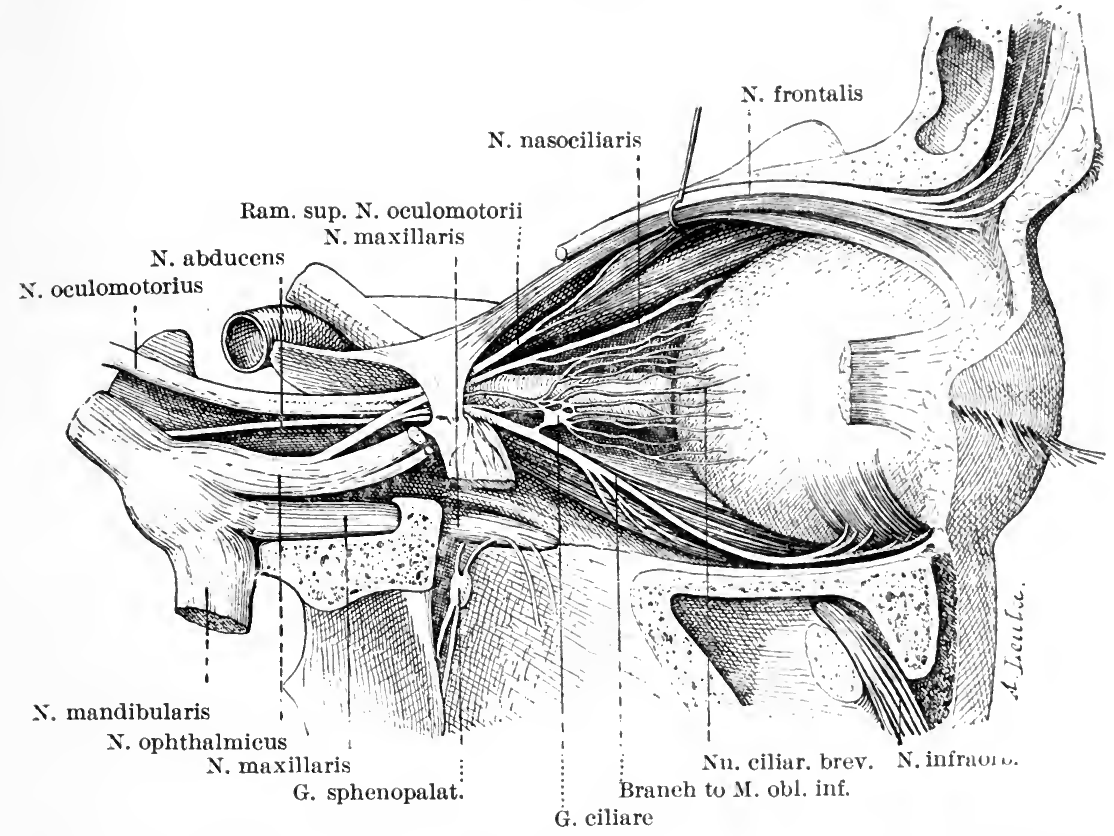

Distribution of the N. oculomotorius (after Hirschfeld). (From Poirier et Charpy, Traité d'Anat. hum., Paris, 1899, t. iii., 3, p. 796, Fig. 441.)

Fig. 120.

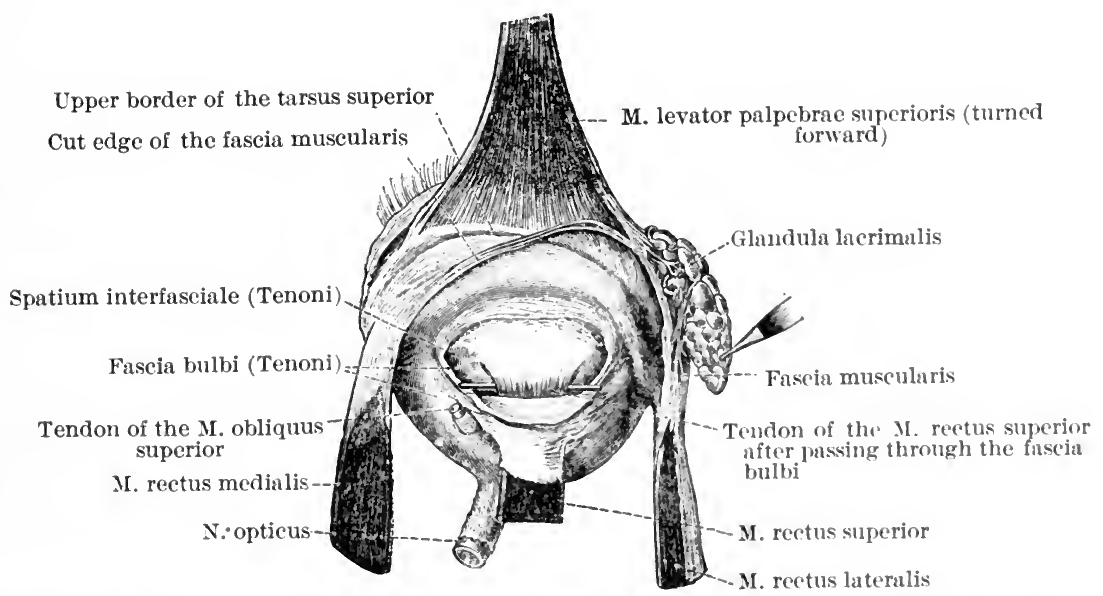

The capsule of Tenon (fascia bulbi) and its relation to the superior rectus muscle of the right eye. Seen from above. (After Toldt, Anat. Atlas, Wien, 1903, 3 Aufl., p. 907, Fig. 1379.) 


\section{Fascia of Bulb (Fascia bulbi [Tenoni]) (O. T. Capsule of Teron).}

Compare the fascia in the eye before you with a description of it in some good systematic text-book. Open the interfascial space (spatium interfasciale [Tenoni]). (Compare Lockwood, J. Anat. and Physiol., Lond., vol. xx., 1885.) What is meant by the "fat body of the orbit" (corpus adiposum orbitae)?

\section{Zygomatic Nerve (N. zygomaticus) (O. T. Temporomalar Nerve).}

Find this nerve entering the orbit through the inferior orbital fissure (fissura orbitalis inferior) (O. T. sphenomaxillary fissure). Follow its two branches:

(a) Zygomaticotemporal ramus (ramus zygomaticotemporalis) (O. T. temporal branch of temporomalar).

(b) Zygomaticofacial ramus (ramus zygomaticofacialis) (O. T. malar branch of temporomalar).

Recall where the terminals of these two rami were previously met with in the dissection.

\section{REGION IN FRONT OF CERVICAL SPINE (PREVER- TEBRAL REGION).}

Place the dissection upside down, the cut margin of the cranium resting on the table. Cut through the A. carotis communis, $\mathrm{V}$. jugularis interna, $\mathrm{N}$. vagus and truncus sympathicus on each side at level of neck of first rib. Displace trachea, oesophagus, large vessels and nerves forward, separating them from the front of the spine. Complete the separation to the base of the skull, but be careful not to injure adjacent structures. Make a transverse cut through the thick periosteum on the pars basilaris of the occipital bone, the knife passing between the pharynx in front and the prevertebral muscles behind. Rest the inside of the base of the skull (basis cranii interna) upon a wooden block, apply a chisel in the line of the cut through the periosteum of the basis cranii externa (between pharynx and prevertebral muscles), and with a wooden mallet cut through the pars basilaris of occipital bone.

Turn the head first on one side and then on the other, in each instance making a saw-cut through the side of the cranium from a point one centimetre behind the processus mastoideus, obliquely forward and medialward, to a point just behind the jugular foramen. Next place the dissection in such a position 
that the basis cranii interna looks upward; on each side complete the division of the base of the skull by chiselling through the solid interval still left,-i.e., from the lateral extremity of the chisel-cut through the pars basilaris backward upon the medial side of the jugular foramen to the medial end of the saw-cut.

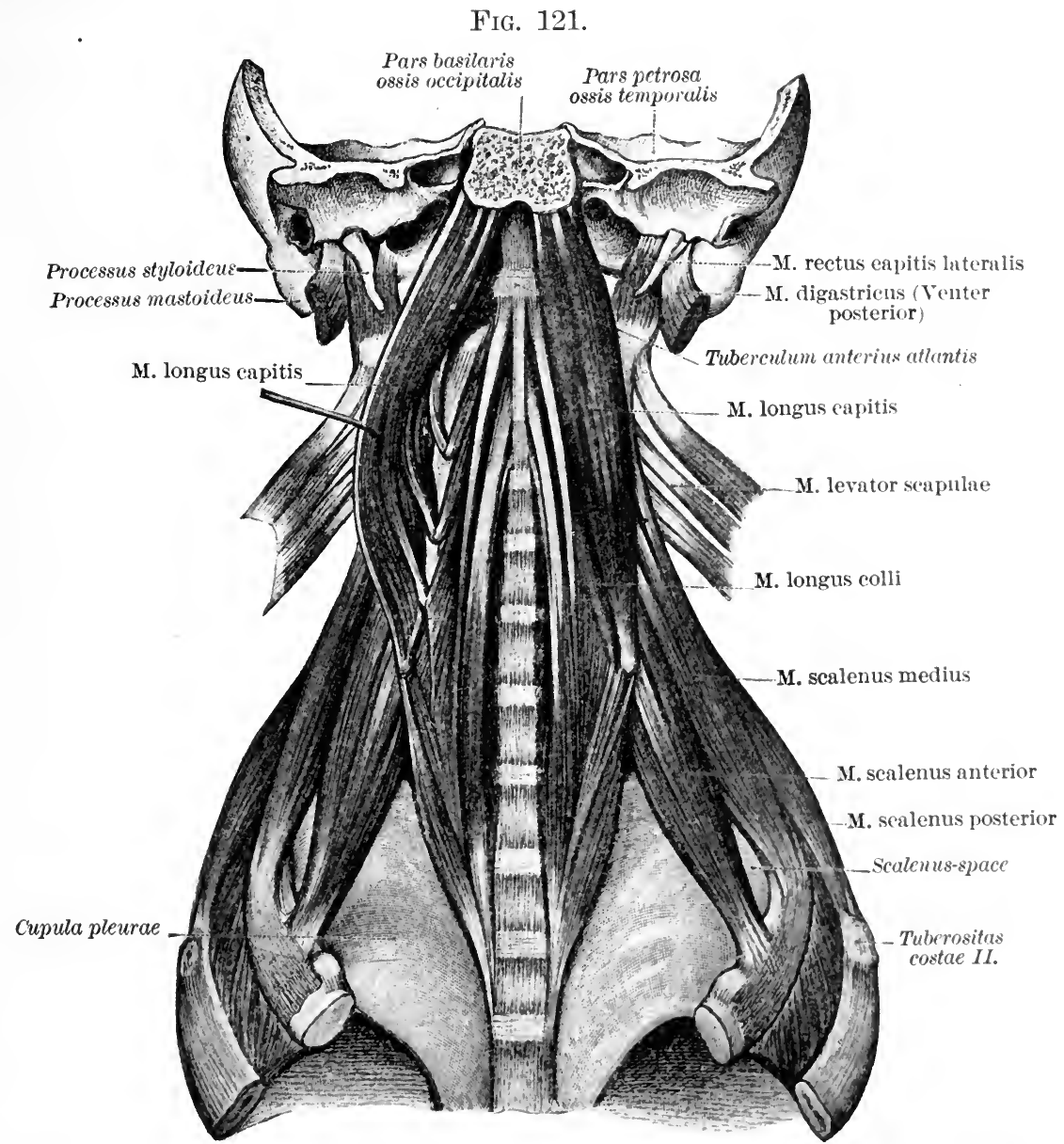

The decp muscles of the front of the neck. (After Toldt, Anat. Atlas, Wien, 1900, 3 Aufl., p. 294, Fig. 535.)

The forepart of the skull (with the pharynx and great vessels and nerves) is now to be separated from the hind part (with the cervical spine). The N. hypoglossus is cut through close to the base of the skull, but should not be separated from the ganglion of the N. vagus. Wrap the pharynx and forepart of the 
skull in moist carbolized cloths and oil-cloth and lay aside for subsequent dissection.

In the posterior part of the dissection work out the following:

Muscles.

(a) Lateral straight muscle of head ( $M$. rectus capitis lateralis).

This has been studied before in the deep dissection of the neck.

(b) Anterior straight muscle of head (M. rectus capitis anterior) ( $O$. T. rectus capitis anticus minor).

(c) Long muscle of head (M. longus capitis) (O. T. rectus capitis anticus major).

(d) Long muscle of neck ( $M$. longus colli).

Determine the form, position, origin, insertion, action, and innervation of each of the above muscles. Review the attachments of the $\mathrm{Mm}$. scaleni. Then cut away entirely the prevertebral and scalene muscles and study from in front-

(e) Intertransverse muscles ( $M m$. intertransversarii).

(ea) Anterior (Mm. intertransversarii anteriores).

(eb) Posterior (Mm. intertransversarii posteriores).

Nerves.

Eight cervical nerves (Nn. cervicales I.-VIII.).

(a) First cervical nerve ( $N$. cervicalis $I$.).

(aa) Anterior ramus (ramus anterior) (O. T. anterior primary division).

(ab) Posterior ramus (ramus posterior, or N. suboccipitalis) (O. T. posterior primary division).

(b) Second cervical nerve (N. cervicalis II.).

(ba) Anterior ramus (ramus anterior) (O. T. anterior primary division).

(bb) Posterior ramus (ramus posterior) (O. T. posterior primary division).

(bba) Medial ramus (ramus medialis, or $N$. occipitalis major).

$(b b b)$ Lateral ramus (ramus lateralis).

(c) Third to eighth cervical nerves (Nn. cervicales III.-VIII.).

(ca) Anterior rami (rami anteriores) (O. T. anterior primary divisions).

(cb) Posterior rami (rami posteriores) (O. T. posterior primary divisions).

Blood-vessels.

Remove the intertransverse muscles ( $\mathrm{Mm}$. intertransversarii), M. rectus capitis lateralis, M. obliquus eapitis superior, and M. obliquus capitis inferior. With bone-forceps cut away the anterior tubercles and costal processes of the transverse processes of the third, fourth, fifth, and sixth cervical vertebrae. Study-

(a) Vertebral artery (A. vertebralis).

(aa) Spinal rami (rami spinales).

Find the plexus vertebralis of the sympathetic around the artery.

(b) Vertebral vein (V.vertebralis). 
Fig. 122.

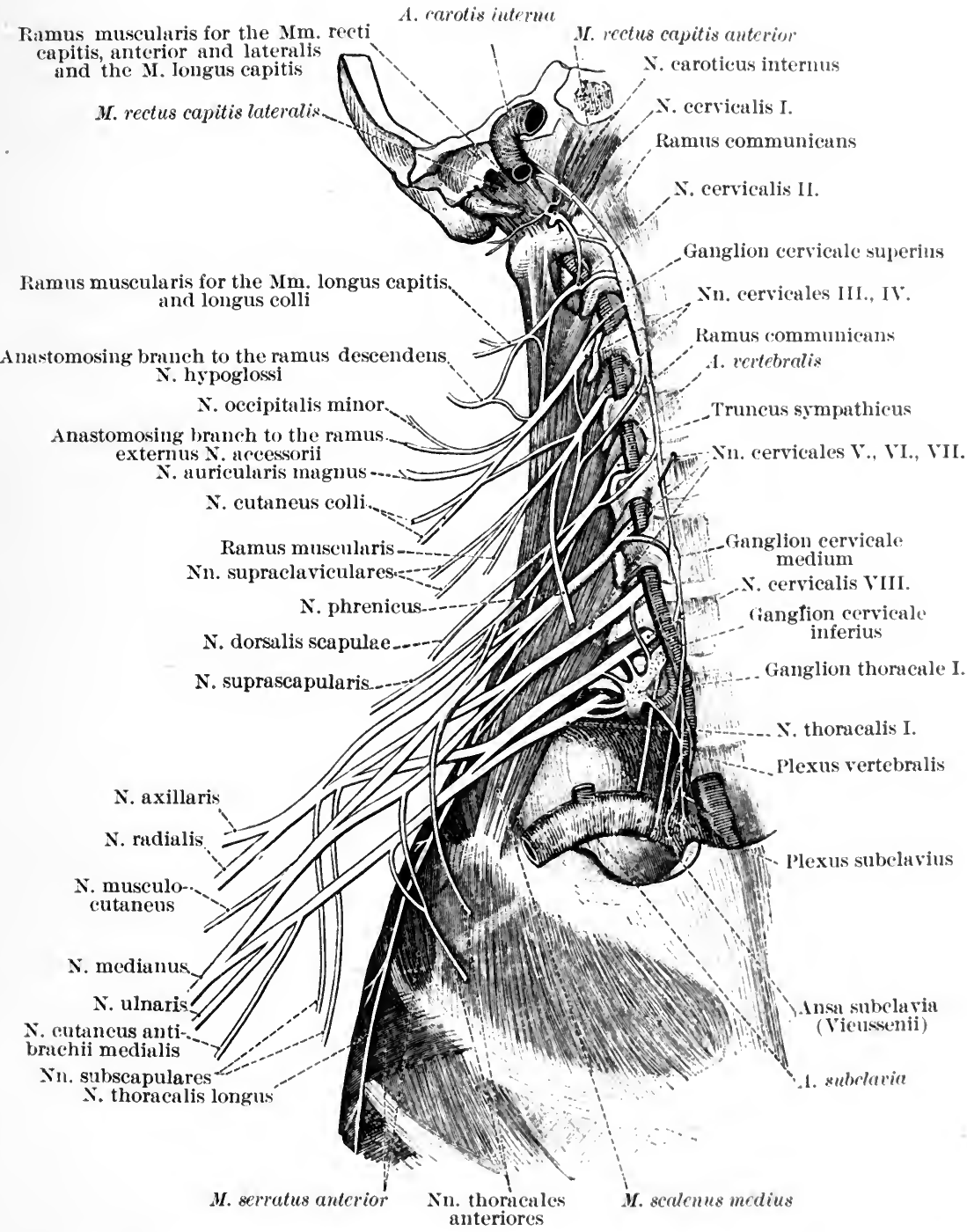

The cervical and brachial plexuses. (After Toldt, Anat. Atlas, Wien, 1903, 3 Aufl., p. 816, Fig. 1248.) 


\section{LIGAMENTS OF VERTEBRAL COLUMN AND SKULL (LIGAMENTA COLUMNAE VERTEBRALIS ET CRANII).}

Remove all muscles from the spinal column and posterior part of the bones of the skull and study the following:

(a) Joints of the lower five cervical vertebrae.

(aa) Intervertebral fibrocartilages (fibrocartilagines intervertebrales).

(aaa) Fibrous ring (annulus fibrosus).

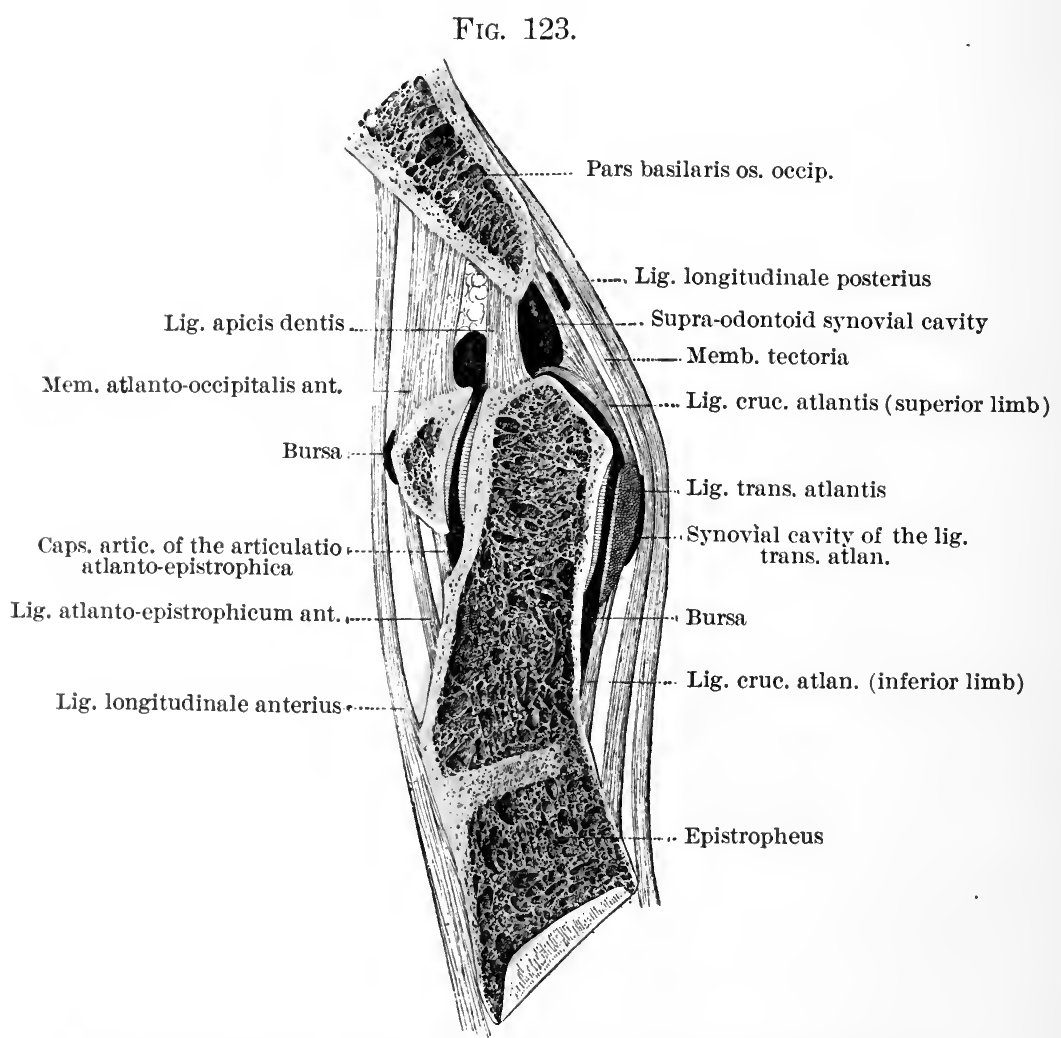

Sagittal section through the articulationes atlanto-occipitalis and atlanto-epistrophica.

(From Poirier et Charpy, Traité d'Anat. hum., Paris, 1899, 2 ed., t. i. p. 814, Fig. 799.)

(aab) Pulp-like nucleus (nucleus pulposus). What is the relation of this to the chorda dorsalis of the embryo?

( $a b)$ Yellow ligaments (ligamenta flava) (O. T. ligamenta subflava). 
(ac) Joint-capsules (capsulae articulares).

(ad) Intertransverse ligaments (ligamenta intertransversaria).

(ae) Interspinous ligaments (ligamenta interspinalia).

(af) Supraspinous ligament (ligamentum supraspinale). Here called the ligamentum nuchae.

(ag) Anterior longitudinal ligament (Lig. longitudinale anterius) (O. T. anterior common ligament).

(ah) Posterior longitudinal ligament (Lig. longitudinale posterius) (O. T. posterior common ligament).

(b) Joint between atlas and occipital bone (articulatio atlanto-occipitalis).

What kind of joint is it? Study-

(ba) Joint-capsules (capsulae articulares).

(bb) Anterior atlanto-oceipital membrane (membrana atlantooccipitalis anterior).

(bc) Posterior atlanto-occipital membrane (membrana atlantooccipitalis posterior).

(c) Joint between atlas and epistropheus or axis (articulatio atlantoepistrophica).

(ca) Joint-capsules (capsulae articulares).

Fig. 124.

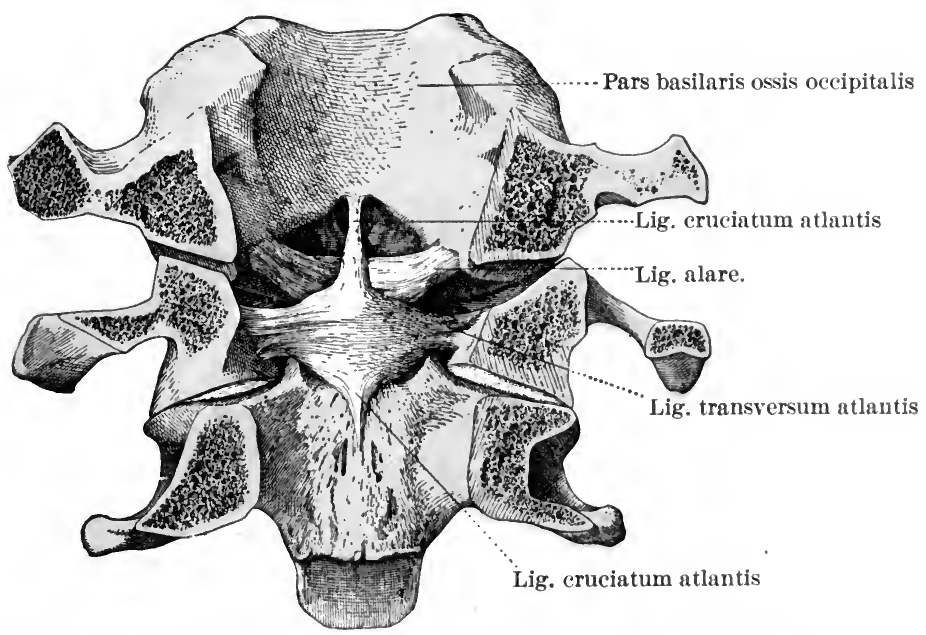

Lig. cruciatum atlantis-posterior view. (From Poirier et Charpy, Traité d'Anat. hum., Paris, 1899, 2 ed., t. i. p. 818 , Fig. 802 .)

Before studying further, eut away the posterior arches of the atlas and epistropheus with heavy bone-foreeps. With a saw, eut through the occipital bone on each side from a point behind the processus jugularis and the condylus occipitalis into the foramen magnum, so that the whole squama occipitalis may be removed. Remove the dura mater and study the following:

$(c b)$ Tectorial membrane ("roof-membrane") (membrana tectoria) (O. T. posterior occipito-axial ligament). 
Detach this from the epistropheus and reflect it upward. Study the following:

(cc) Cruciform ligament of atlas (ligamentum cruciatum atlantis). Observe its two parts (ef. Spalteholz, vol. i., Fig. 218) :

(cca) Transverse ligament (ligamentum transversum atlantis).

$(c c b)$ Vertical part.

Detach the vertical part, reflect it downward, and study-

(cd) Alar ligaments (ligamenta alaria) (O. T. odontoid or check ligaments).

(ce) Ligament of apex of tooth (ligamentum apicis dentis) (O. T. suspensory ligament). How is this ligament related to the chorda dorsalis?

Name from before backward the ligaments of the articulatio atlanto-occipitalis and articulatio atlanto-epistrophica. (Cf. Spalteholz, p. 169.)

What movements can occur at these joints? What muscles are concerned in such movements? How is excessive movement in any direction checked?

\section{MOUTH AND FAUCES.}

Returning to the forepart of the skull and neck, the dissector now studies the following, comparing his findings in the cadaver with illustrations in atlases and descriptions in his systematic text-book.

\section{Cavity of Mouth (Cavum oris).}

\section{General.}

(a) Cheek (bucca). (aa) Fat body of cheek (corpus adiposum buccae).

(b) Entrance to mouth (vestibulum oris). This is bounded in front and laterally by lips and cheeks, behind by teeth and gums.

(c) Mouth eavity proper (cavum eris proprium). This is internal to the teeth.

(d) Mouth slit or oral fissure (rima oris).

(e) Lips of mouth (labia oris). (ea) Upper lip (labium superius).

(eb) Lower lip (labium inferius).

( $f$ ) Junction of lips (commissura labiorum).

(g) Angles of mouth (angulus oris).

(h) Palate (palatum).

(ha) Hard palate (palatum durum).

$(h b)$ Soft palate (palatum molle).

(hc) Middle ridge of palate (raphe palati). 
FiG. 125.

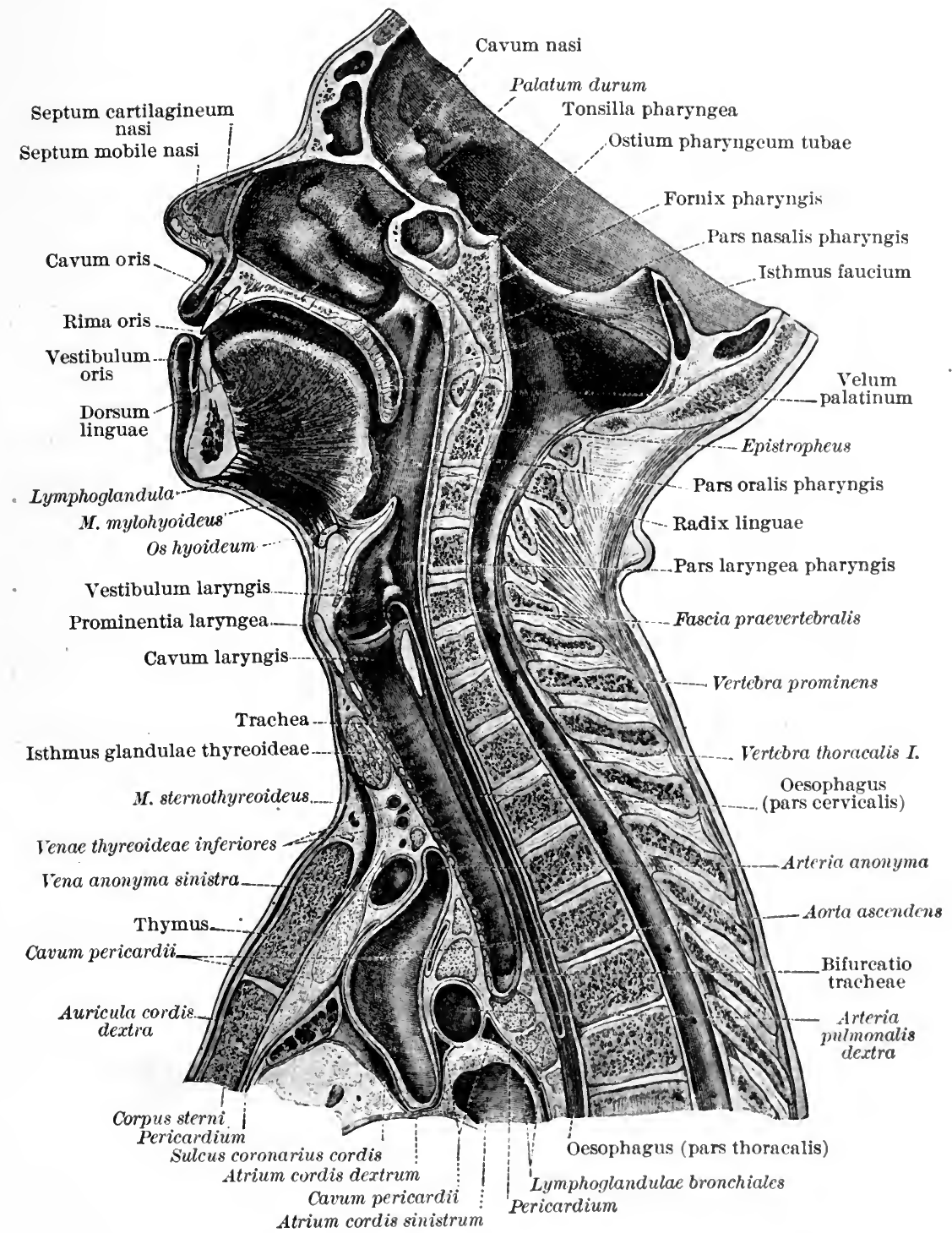

The viscera of the head and neck and their connections in the thorax. (After Toldt, Anat. Atlas, Wien, 1900, 2 Aufl., p. 395, Fig. 636.) 
Mucous Membrane of Mouth (Tunica mucosa oris).

(a) Cheek-cord of upper lip (frenulum labii superioris).

(b) Check-cord of lower lip (frenulum labii inferioris).

(c) Gum (gingiva).

(d) Sublingual caruncle (caruncula sublingualis).

(e) Sublingual fold (plica sublingualis).

(f) Transverse palatine folds (plicae palatinae transversae).

(g) Incisor papilla ( papilla incisiva).

Glands of Mouth (Glandulae oris).

(a) Labial glands (glandulae labiales).

(b) Buceal glands (glandulae buccales).

(c) Molar glands (glandulae molares).

(d) Palatine glands (glandulae palatinae).

(e) Lingual glands (glandulae linguales).

(f) Anterior lingual gland (glandula lingualis anterior [Blandini, Nuhni]) (O. T. gland of Nuhn).

(g) Sublingual gland (glandula sublingualis).

(ga) Larger sublingual duct (ductus sublingualis major).

(gb) Smaller sublingual ducts (ductus sublinguales minores).

(h) Submaxillary gland (glandula submaxillaris).

(ha) Submaxillary duct (ductus submaxillaris [Whartoni]) (O. T. Wharton's duct).

(i) Parotid gland (glandula parotis).

(ia) Retromandibular process (processus retromandibularis) ( 0. T. socia parotidis).

(ib) Accessory parotid gland (glandula parotis accessoria).

(ic) Parotid duct (ductus parotideus [Stenonis]) (O. T. Steno's duct).

(j) Mixed mouth secretions (saliva).

Teeth (Dentes).

(a) Superior dental arch (arcus dentalis superior).

(b) Inferior dental arch (arcus dentalis inferior).

How many teeth does each arch contain?

(c) Incisor teeth (dentes incisivi).

(d) Canine teeth (dentes canini).

(e) Premolar teeth (dentes praemolares) (O. T. bicuspids).

(f) Molar teeth (dentes molares).

( $f a$ ) Late tooth (dens serotinus) (O. T. wisdom tooth).

How many of these teeth are present in your cadaver? What is meant by deciduous teeth (dentes decidui) and permanent teeth (dentes permanentes)?

Tongue. (Vide Figs. 125 and 137.)

(a) Dorsum of tongue (dorsum linguae).

What is a "coated tongue"?

(b) Root of tongue (radix linguae).

(c) Inferior surface (facies inferior [linguae]).

(d) Lateral margin (margo lateralis [linguae]).

(e) Apex or tip of tongue (apex linguae).

(f) Mucous membrane (tunica mucosa linguae).

The muscles of the tongue and the details of the mucous membrane will be studied later. 
Fauces.

(a) Isthmus of fauces (isthmus faucium).

(b) Palatine curtain (velum palatinum).

(c) Uvula (uvula palatinum). This little body is often cut off by the surgeon if it becomes so relaxed and lengthened that it causes continued tickling of the throat.

(d) Palatine arches (arcus palatini).

( $d a$ ) Glossopalatine arch (arcus glossopalatinus) (O. T. anterior pillar of fauces).

(db) Pharyngopalatine arch (arcus pharyngopalatinus) (O. T. posterior pillar of fauces).

(e) Tubopalatine or salpingopalatine fold (plica salpingopalatina). This eannot be seen well until later, when the pharynx is opened from behind.

(f) Palatine tonsil (tonsilla palatina) (O. T. ordinary tonsil). This will be studied later.

The muscles of the palate and fauces will be studied with those of the pharynx.

\section{PHARYNX.}

Place the chin of the cadaver on a block with the pharynx hanging downward, its posterior surface turned towards dissector. Distend the cavity of the pharynx with cotton or tow.

\section{Buccopharyngeal Fascia (Fascia buccopharyngea).}

(a) Pterygomandibular raphe (raphe pterygomandibularis) (O. T. pterygomaxillary ligament). This will be seen as a dense eord between the hamulus pterygoideus and the posterior end of the crista buecinatoria [mandibulae]).

Observe that the part of the fascia in front of $(a)$ covers the M. buccinatorius, that behind $(a)$ covers the muscles of the pharynx.

\section{Muscular Tunic of Pharynx (Tunica muscularis pharyngis).}

Study the form, position, origin, insertion, action, and innervation of each of the following muscles:

(a) Inferior constrictor muscle of pharynx (M. constrictor pharyngis inferior).

(aa) Thyreopharyngeal muscle (M. thyreopharyngeus).

(ab) Cricopharyngeal muscle (M. cricopharyngeus).

(b) Middle constrictor muscle of pharynx ( $M$. constrictor pharyngis medius).

(ba) Chondropharyngeal muscle ( $M$. chondropharyngeus) (from cornu minus os. hyoid.).

(bb) Ceratopharyngeal muscle (M. ceratopharyngeus) (from cornu majus os. hyoid.). 
Between $(a)$ and $(b)$ observe the A. laryngea inferior and the ramus internus of the $\mathrm{N}$. laryngeus superior perforating the membrana hyothyreoidea.

Fig. 126.

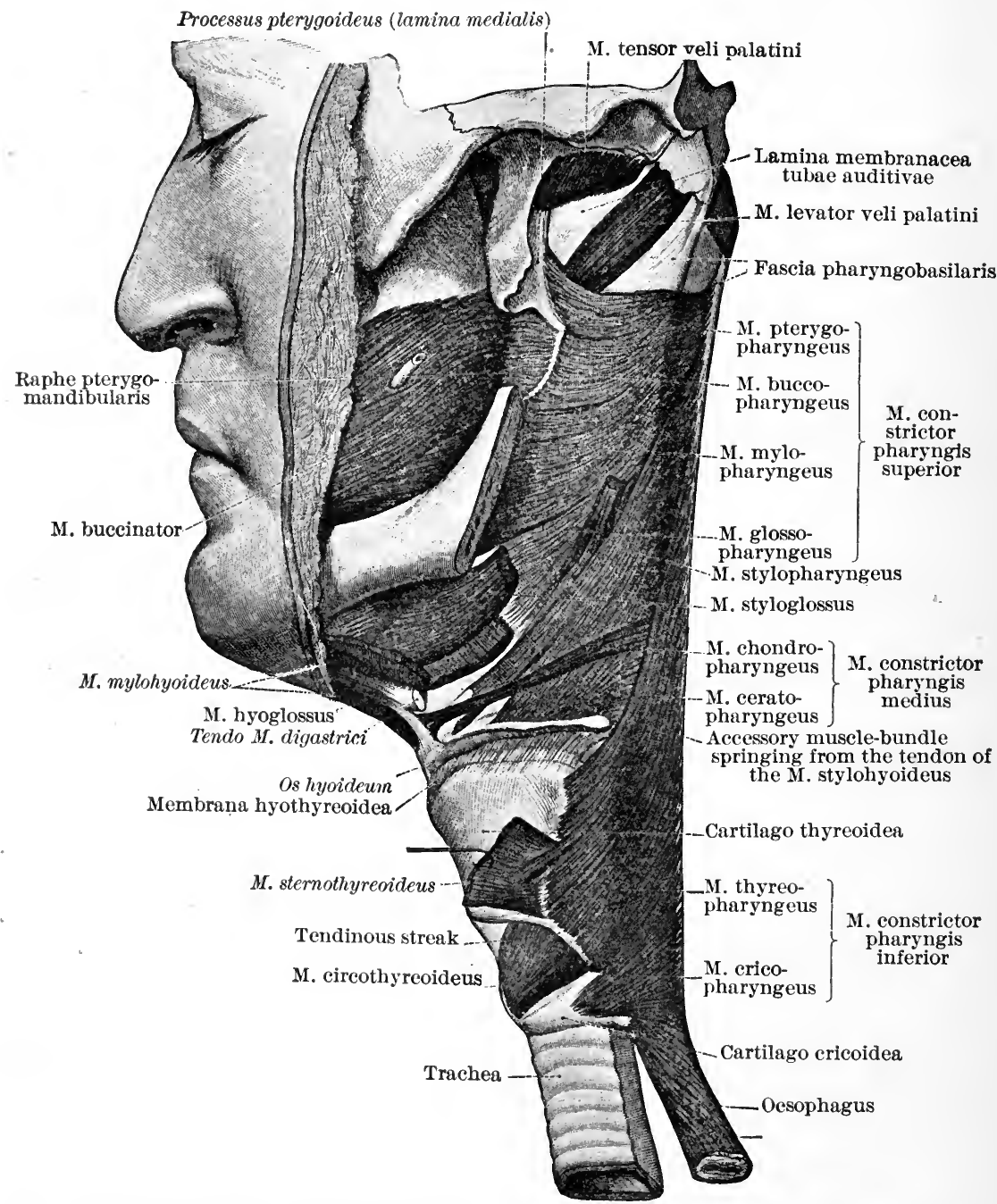

The muscles of the pharynx. The ramus of the mandible and the styloid process of the temporal bone bave been removed. (After Toldt, Anat. Atlas, Wien, 1900, 2 Aufl., p. 417, Fig. 682.)

Next cut through the M. pterygoideus internus at its middle and reflect the two ends; avoid injury to M. tensor veli palatini just medial to it.

(c) Superior constrictor muscle of pharynx ( $M$. constrictor pharyngis superior). 
(ca) Pterygopharyngeal muscle (M. pterygopharyngeus).

( $c b)$ Buccopharyngeal muscle (M. buccopharyngeus).

(cc) Mylopharyngeal muscle (M. mylopharyngeus).

(cd) Glossopharyngeal muscle (M. glossopharyngeus).

Between $(b)$ and $(c)$ observe the M. stylopharyngeus and the N. glossopharyngeus. Observe the interval between the uppermost constrictor muscle and the base of the skull; here a thickened portion of the tela submucosa pharyngis ( $\mathrm{O}$. T. pharyngeal aponeurosis), called the pharyngobasilar fascia (fascia pharyngobasilaris), is visible. The semilunar space where the muscle is absent is sometimes called the "sinus of Morgagni." In the lateral part of this on each side the M. levator veli palatini, the M. tensor veli palatini, and the cartilage of the Eustachian tube (cartilago tubae auditivae) can be seen; these structures will be dissected out earefully later.

\section{Cavity of Pharynx (Cavum pharyngis).}

Open the pharynx from behind by a vertical incision in the median line of its posterior wall along its whole length. Divide the fascia pharyngobasilaris close to the basis cranii externa on each side, carrying the knife from the median line lateralward as far as the cartilage of the Eustachian tube. Remove the stuffing of tow or cotton. Sponge the tunica mucosa clean and study the cavum pharyngis. With what cavities or tubes does the cavity of the pharynx communicate? Explore the "vault of the pharynx" (fornix pharyngis). How is the cavity of the pharynx bounded? What very important structures are related to its lateral walls? Where does the pharynx end and the oesophagus begin? Observe the three parts of the cavum pharyngis (see Fig. 125) :

(a) Nasal part (pars nasalis) (O. T. nasopharynx).

(b) Oral part (pars oralis) (O. T. oral pharynx).

(c) Laryngeal part (pars laryngea) (O. T. laryngopharynx).

Find-

(d) Pharyngeal opening of Eustachian tube (ostium pharyngeum tubae auditivae).

(da) Anterior lip (labium anterius).

( $d b)$ Posterior lip (labium posterius). This is sometimes called the tubal projection (torus tubarius), owing to the rounded eminence it forms.

(dc) Salpingopharyngeal fold (plica salpingopharyngea).

It extends downward from the lower end of the labium posterius.

(e) Pharyngeal recess of Rosenmüller (recessus pharyngeus [Rosenmülleri]) (O. T. recessus infundibuliformis).

(f) Pharyngeal tonsil (tonsilla pharyngea) (O. T. Luschka's tonsil). It is enlargement of this tonsil that constitutes the " postadenoids" so often met with in children.

(fa) Tonsillar crypts (fossulae tonsillares). 
(g) Pharyngeal bursa (bursa pharyngea).

Look into the nasal cavity behind and observe the septum of the nose (septum nasi) separating the two choanae. The following are visible:

(1) Nasopharyngeal meatus (meatus nasopharyngeus).

(2) Inferior meatus of nose (meatus nasi inferior).

(3) Middle meatus of nose (meatus nasi medius).

(4) Inferior turbinated bone (concha nasalis inferior).

(5) Middle turbinated bone (concha nasalis media).

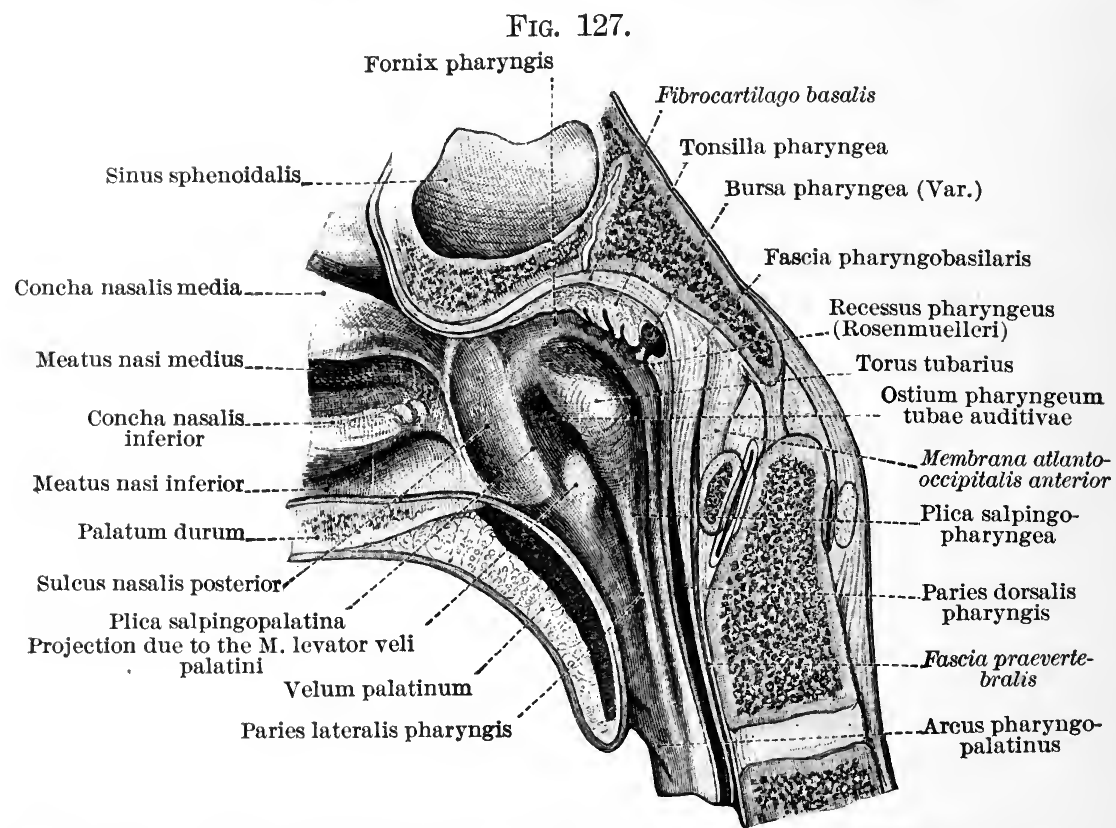

Medial aspect of right nasopharynx. (After Toldt, Anat. Atlas, Wien, 1900, 2 Aufl., p. 420, Fig. 686.)

The posterior surface of the velum palatinum, the uvula, and the arcus pharyngopalatinus are to be examined; they have already been seen in front. Find the following:

(h) Pharyngo-epiglottic fold (plica pharyngo-epiglottica).

(i) Aryepiglottic fold (plica aryepiglottica).

(j) Opening into larynx (aditus laryngis) (vide Fig. 95).

Observe the epiglottis guarding the opening.

(k) Piriform recess (recessus piriformis) (O. T. sinus pyriformis). Foreign bodies often become lodged in this pocket.

(ka) Fold of laryngeal nerve (plica nervi laryngei). Look for it in anterior part of recessus piriformis.

\section{Soft Palate (Palatum molle). (Vide Figs. I27 and I30.)}

The mucous membrane of this structure has already been viewed from the mouth; it is now to be studied also from the pharyngeal side. 
Make the velum palatinum tense by means of a hook and dissect the tunica mucosa off both its surfaces (oral and pharyngeal) and also off the arcus glossopalatinus and the areus pharyngopalatinus. Observe the distribution of the glandulae palatinae.

Study the form, position, origin, insertion, action, and innervation of each of the following:

Muscles of the Palate and Fauces (Mm. palati et faucium). (Cf. Spalteholz, Figs. 551 and 552.)

(a) Glossopalatine muscle (M. glossopalatinus) (O. T. palatoglossus).

(b) Pharyngopalatine muscle ( $M$. pharyngopalatinus) (O. T. palatopharyngeus).

(Observe here the salpingopharyngeal muscle (M. salpingopharyngeus); it is really a part of the tunica muscularis pharyngis.).

(c) Muscle of uvula (M. uvulae) (O. T. azygos uvulae).

Now remove the wall of the pharynx between the Eustachian tube (tuba auditiva) above and the upper border of the M. constrictor pharyngis superior below. Study-

(d) Levator muscle of palatine curtain (M. levator veli palatini) ( $O$. T. levator palati).

(e) Tensor muscle of palatine curtain ( $M$. tensor veli palatini) ( $O$. T. tensor palati). Note especially the nerve supply of this muscle; also the bursa $M$. tensoris veli palatini. What is meant by the "palatal aponeurosis"?

\section{Study also-}

Arteries.

(a) Ascending palatine artery (A. palatina ascendens) (O. T. inferior palatine). Of what is it a branch? Where have you met it before?

(b) Pharyngeal rami of ascending pharyngeal artery (rami pharyngei A. pharyngeae ascendentis) ( $\mathrm{O}$. T. palatine branch of ascending pharyngeal).

(c) Descending palatine artery from internal maxillary artery ( $A$. palatina descendens).

Veins.

(a) Pharyngeal veins ( $V v$. pharyngeae), from pharyngeal plexus (plexus pharyngeus) to V. jugularis interna.

(b) Palatine vein ( $V$. palatina), corresponding to A. palatina ascendens and emptying into $\mathrm{V}$. facialis anterior.

Nerves. (Figs. 128 and 132.)

(a) Branches of $\mathrm{N}$. accessorius (through the rami pharyngei N. vagi) to the M. levator palati, M. uvulae, M. pharyngopalatinus, and M. glossopalatinus.

(b) Nerve of the palatine tensor ( $N$. tensoris palatini). from motor part of $\mathrm{N}$. trigeminus (through ganglion oticum) to the M. tensor veli palatini. 
(c) Palatine nerves $(N n$. palatini), from the N. trigeminus through the ganglion sphenopalatinum.

(ca) Middle palatine nerve (N. palatinus medius) (O. T. external palatine).

$(c b)$ Posterior palatine nerve (N. palatinus posterior).

Palatine Tonsils (Tonsillae palatinae). (Vide Spalteholz, Figs. 545548.)

Observe the exact situation of the palatine tonsil on each side. How is it related to neighboring structures? What especial danger might be run in lancing a peritonsillar abscess?

(a) Tonsillar crypts or fossulae (fossulae tonsillares). Are any of them filled with yellowish-white plugs?

(b) Tonsillar sinus (sinus tonsillaris). Is it completely filled by the tonsil or is there a fossa supratonsillaris? Note the plica triangularis.

How is the tonsil supplied with blood? How is the tonsil related to the cervical lymph-glands?

\section{Auditory or Eustachian Tube (Tuba auditiva [Eustachii]).}

Of its two parts, one is lateral and bony (pars ossea tubae auditivae), the other medial and cartilaginous (pars cartilaginea tubae auditivae). The cartilaginous part is now studied. Its pharyngeal opening (ostium pharyngeum tubae auditivae) has already been examined. Pass a bristle or probe into it and ascertain the direction of the tuba auditiva. What is the position of the tuba as regards the M. levator veli palatini and the M. tensor veli palatini?

Dissect the mucous membrane cautiously away around the pharyngeal opening of the tube and examine the constitution of the cartilaginous portion.

The cartilage of the Eustachian tube (cartilago tubae auditivae) consists of two laminae, one medial (lamina cartilaginis medialis) and one lateral (lamina cartilaginis lateralis). ( $\mathrm{Cf}$. Spalteholz, Fig. 552.) Where cartilage is lacking, a strong membranous layer (lamina membranacea) takes its place. Open the tuba auditiva and examine the mucous membrane (tunica mucosa). Are there any glands (glandulae mucosae) or solitary lymph-nodules (noduli lymphatici tubarii) visible? Observe the trumpet-shape of the canal.

From which of the inner branchial pockets of the embryo do the Eustachian tube and cavity of the middle ear arise? 


\section{CAROTID AND INFRAORBITAL CANALS (CANALIS CAROTICUS ET CANALIS INFRAORBITALIS).}

\section{Carotid Canal (Canalis caroticus).}

Remove the inferior wall of the carotid canal with strong forceps. Do not disturb the position of the Eustachian tube. Inside the carotid canal study the following:

(a) Internal carotid artery (A. carotis interna). (Cf. Spalteholz, Fig. 442.)

(aa) Caroticotympanic ramus (ramus caroticotympanicus) (O. T. tympanic branch).

(b) Venous plexus of internal carotid (plexus venosus caroticus internus).

(c) Internal carotid nerve of sympathetic system ( $N$. caroticus internus). (Cf. Spalteholz, Figs. 773 and 779.)

(ca) Internal carotid plexus (plexus caroticus internus). (caa) Deep petrosal nerve (N. petrosus profundus) (O. T. large, deep petrosal nerve), which runs to the pterygoid canal (O. T. Vidian canal) and unites with the $\mathrm{N}$. petrosus superficialis major to form the nerve of the pterygoid canal ( $N$. canalis pterygoidei [Vidii]) (O. T. Vidian nerve).

\section{Infraorbital Canal (Canalis infraorbitalis).}

Remove the M. temporalis and the upper head of the M. pterygoideus externus; saw through the squama temporalis and the ala magna oss. sphenoidalis, beginning on the cut margin of the skull just above the meatus acusticus externus and passing obliquely downward and forward towards the medial end of the fissura orbitalis superior (O. T. sphenoidal fissure). The saw-cut should enter the fissura orbitalis superjor just lateral from the foramen rotundum. Make a second saw-cut from the eut margin of the cranial wall, just above the anterior margin of the ala magna oss. sphenoidalis, downward into the fissura orbitalis superior, to meet the first saw-cut. Remove the bone thus freed and with bone-forceps remove what remains of the ala magna lateral from the foramen rotundum, keeping intact, however, the entire bony margin of this aperture. Next open the canalis infraorbitalis with bone-forceps (or chisel if necessary). Study the following:

(a) Maxillary nerve ( $N$. maxillaris) (O. T. superior maxillary). (Fig. 115, p. 274.)

Trace it from the ganglion semilunare [Gasseri] to its termination. 
(aa) Middle meningeal nerve ( $N$. meningeus [medius]) (O. T. recurrent).

This has been studied already within the cranium.

(ab) Zygomatic nerve (N. zygomaticus) (O. T. orbital or temporomalar).

Already studied, with its two rami, in the orbit.

(ac) Sphenopalatine nerves (Nn. sphenopalatini), going to the ganglion sphenopalatinum.

(ad) Superior alveolar nerves (Nn. alveolares superiores).

(ada) Posterior superior alveolar rami (rami alveolares superiores posteriores) (O. T. posterior superior dental).

(ae) Infraorbital nerve ( $N$. infraorbitalis).

(aea [also $a d b]$ ) Middle superior alveolar ramus (ramus alveolaris superior medius) (O. T. middle superior dental).

(aeb [also $a d c]$ ) Anterior superior alveolar rami (rami alveolan ss superiores anteriores) ( 0 . $\mathrm{T}$. anterior superior dental).

How is the superior dental plexus (plexus dendentalis superior) formed? Find-

(1) Superior dental rami (rami dentales superiores).

(2) Superior gingival rami (rami gingivales superiores).

The other terminal rami of the infraorbital nerve have been studied in the dissection of the face.

(b) Infraorbital artery (A. infraorbitalis).

Its origin has already been studied. Examine its course and-

(ba) Anterior superior alveolar arteries (Aa. alveolares superiores anteriores) (O. T. anterior superior dental).

\section{SAGITTAL SECTION OF FOREPART OF HEAD NEAR MEDIAN PLANE.}

Remove the mandible, with the tongue and larynx, by making a cut backward from the angle of the mouth through the cheek, the raphe pterygomandibularis, and the lateral wall of the pharynx. Cut through also the vessels and nerves still connecting the pharynx with the skull. Wrap the larynx and tongue in moist cloth for subsequent dissection.

Make a sagittal saw-cut through the forepart of the skull just lateral from the septum nasi; if the septum is not vertical, but deviates somewhat to one side, make the section close to the concave side of the septum. Before sawing, cut through the cartilaginous part of the nose as far as the nasal bone with a 
knife and cut through the soft palate in the same plane. Then saw through the bone from behind forward; the saw passes through the hard palate and the roof of the nose.

FIG. 128.

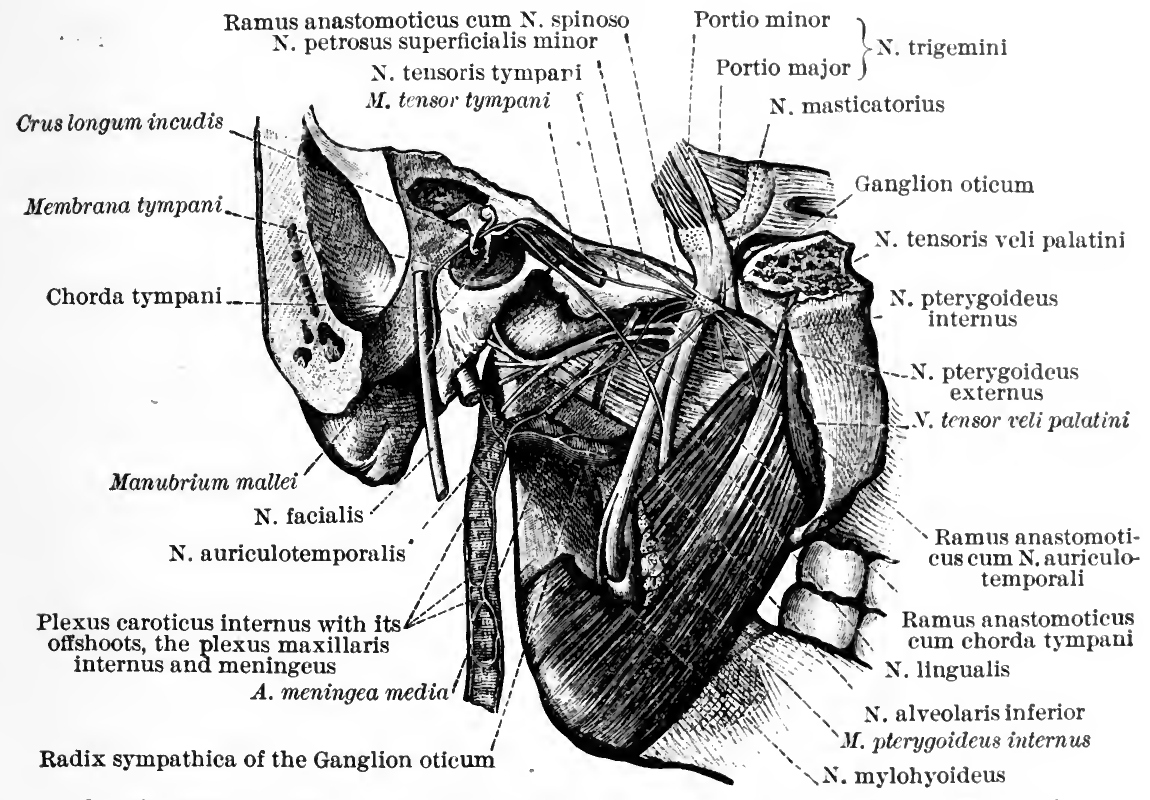

The otic ganglion and its connections seen from the medial side. (After Toldt, Anat. Atlas, Wien, 1903, 3 Aufl., p. 867, Fig. 1307.)

Otic Ganglion (Ganglion oticum) (O. T. Ganglion of Arnold). (Fig. I28.)

Place the sagittal cut surface upward; detach the M. levator veli palatini at its origin and reflect it downward. Remove very cautiously the pars cartilaginea tubae auditivae; the otic ganglion lies just beneath, between it and the N. mandibularis. A useful guide is the nerve to the M. pterygoideus internus; follow it upward and backward to the otic ganglion. Find the following " roots":

(a) Short root: from the N. mandibularis.

(b) Long root: lesser superficial petrosal nerve ( $N$. petrosus superficialis minor); probably contains motor fibres from $\mathrm{N}$. facialis and sensory fibres from N. glossopharyngeus.

(c) Ganglionic or sympathetic root (radix sympathica): from plexus in middle meningeal artery.

Branches of Distribution.

(a) Nerve of tensor muscle of palatine curtain $(N$. tensoris veli palatini). 
(b) Nerve of tensor muscle of tympanum (N. tensoris tympani). Connecting Branches.

(a) With spinous nerve of mandibular nerve (ramus anastomoticus cum N. spinoso).

(b) With auriculotemporal nerve of mandibular nerve (ramus anastomoticus cum N. auriculotemporali).

(c) With chorda tympani (ramus anastomoticus cum chorda tympani).

\section{Cavity of Nose (Cavum nasi). (Vide Figs. I30 and I3r.)} Study-

(a) Septum of nose (septum nasi).

Remove the mucous membrane and examine-

(aa) Cartilaginous septum (septum cartilagineum).

(ab) Membranous septum (septum membranaceum).

Remove the cartilage and bones of septum piecemeal, avoiding injury to the mucous membrane of the opposite side of the septum. Find-

Fig. 129.

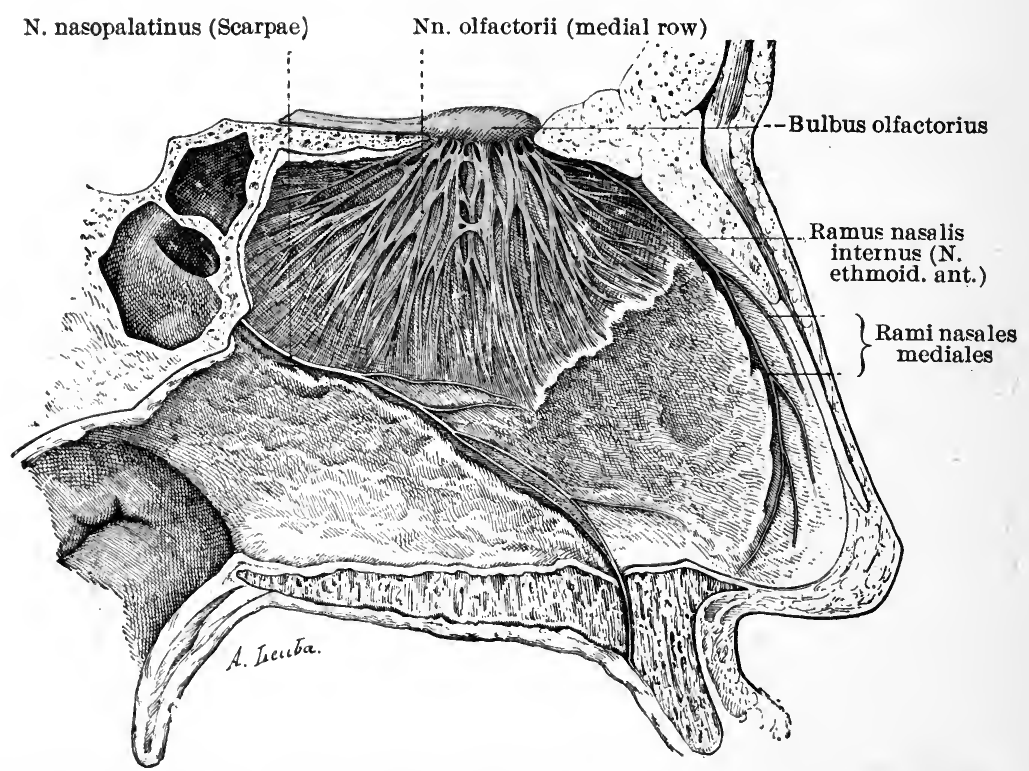

Medial branches of the olfactory nerve (after Hirschfeld). (From Poirier et Charpy, Traité d'Anat. hum., Paris, 1897, t. iii., 3, p. 775, Fig. 418.)

(ac) Nerves of the septum nasi.

(aca) Olfactory nerves (Nn. olfactorii).

(acb) Medial superior posterior nasal rami (rami nasales posteriores superiores mediales).

(acba) Nasopalatine nerve (N. nasopalatinus [Scarpae]) (O. T. nerve of Cotun- 
nius), from the sphenopalatine ganglion.

(acc) Medial nasal rami of internal nasal rami of anterior nasal rami of $\mathrm{N}$. nasociliaris (rami nasales mediales) (O. T. septal branch of nasal nerve).

(ad) Arteries going to the septum.

Of what are they branches?

Now eut through the tunica mucosa septi, with scissors, along the roof of the nasal cavity and reflect it; the nasopalatine nerve and medial nasal rami are to be preserved for further examination.

FIG. 130.

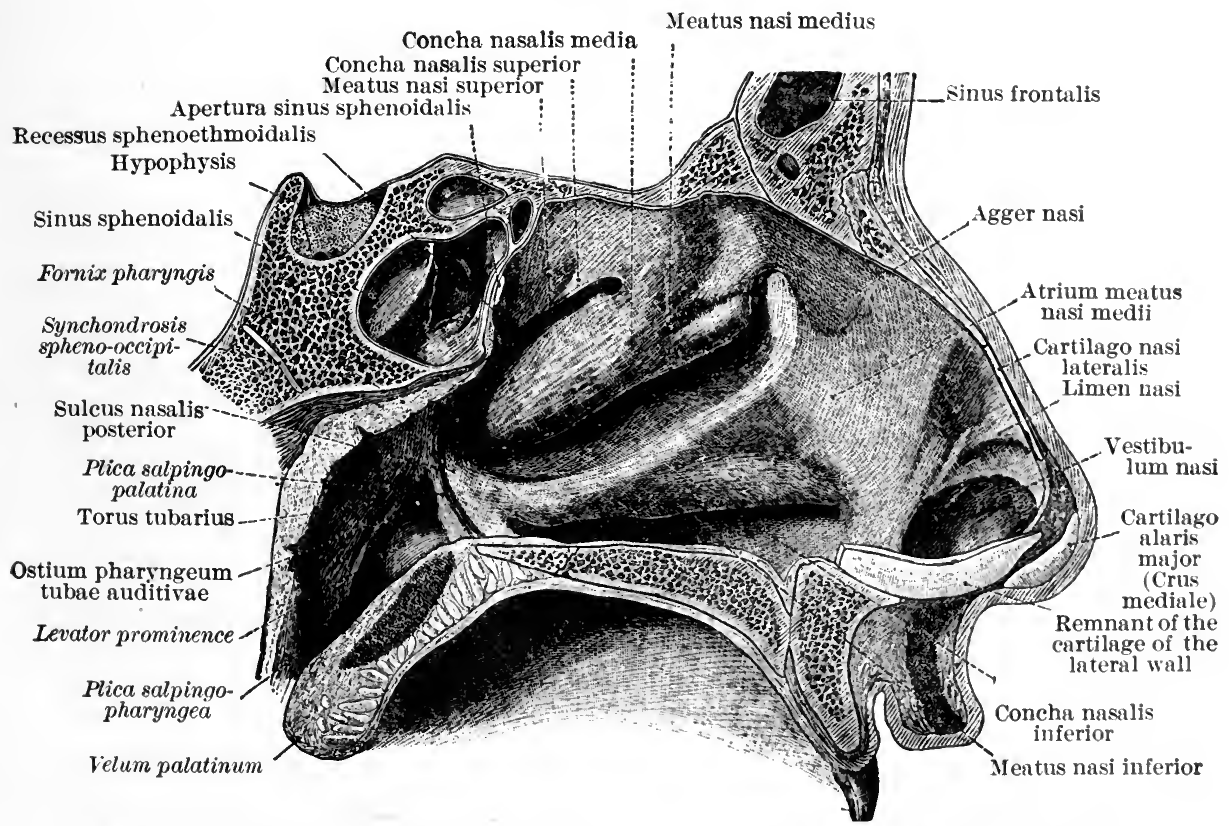

Medial aspect of the left wall of the nasal cavity (After Toldt, Anat. Atlas, Wien, 1903, 3 Aufl., p 944, Fig 1473.)

(b) Anterior apertures (nares).

(c) Posterior apertures (choanae).

(d) Vestibule of nose (vestibulum nasi).

(da) Hairs in vestibule (vibrissae).

(e) Threshold of nose (limen nasi).

( $f$ ) Olfactory sulcus (sulcus olfactorius).

(g) Conchae (O. T. turbinated bones).

(ga) Superior nasal concha (concha nasalis superior) (O. T. superior turbinated bone).

( $g b$ ) Middle nasal concha (concha nasalis media) (O. T. middle turbinated bone). 
(gc) Inferior nasal concha (concha nasalis inferior) (O. T. inferior turbinated bone).

Is a highest nasal concha (concha nasalis suprema [Santorini]) present? Observe the mucous membrane of the nose (membrana mucosa nasi). Over the concha there is much erectile tissue (plexus cavernosi concharum). What is the spheno-ethmoidal recess (recessus spheno-ethmoidalis)?

(h) The agger ("elevation") of the nose (agger nasi) (O. T. rudiment of anterior turbinal).

Fig. 131.

Infundibulum ethmoidale with the apertura sinus maxillaris Opening of the labyrinthus ethmoidalis Recessus sphenoethmoidalis

Apertura sinus sphenoidalis Concha nasalis superior

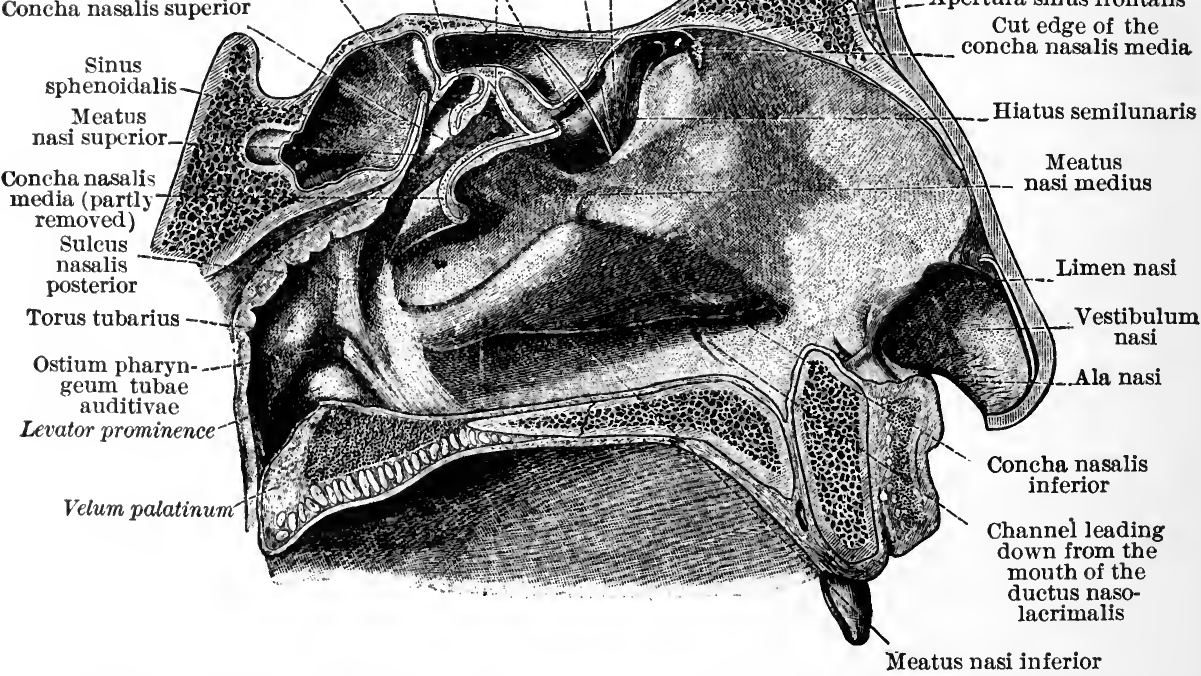

Medial aspect of the left wall of the nasal cavity, after removal of the greater part of the middle and the anterior part of the superior turbinated bones (concha nasalis media and concha nasalis superior). (After Toldt, Anat. Atlas, Wien, 1903, 3 Aufl., p. 944, Fig. 1474.)

(i) Meatuses of the nose (meatus nasi).

(ia) Superior meatus (meatus nasi superior).

(ib) Middle meatus (meatus nasi medius).

(iba) "Entrance-hall" of middle meatus (atrium meatus medii).

(ic) Inferior meatus (meatus nasi inferior).

(id) Common meatus (meatus nasi communis).

(ie) Nasopharyngeal meatus (meatus nasopharyngeus).

(j) Regions of the nose.

(ja) Respiratory region (regio respiratoria).

(jb) Olfactory region (regio olfactoria).

What is the extent of the latter?

(k) Glands of nose. 
( $k a)$ Olfactory glands ( $G l$. olfactoriae).

(kb) Nasal glands (Gl. nasales).

Force the concha media upward and backward and examine-

(l) Ethmoidal infundibulum (infundibulum ethmoidale).

(la) Semilunar opening (hiatus semilunaris).

( $m$ ) Ethmoidal "bubble" (bulla ethmoidalis).

Where are the ethmoidal cells (cellulae ethmoidales) and how do they communicate with the nose?

(n) Sinuses near nose (sinus paranasales).

(na) Maxillary sinus (sinus maxillaris [Highmori]) (O. T. antrum of Highmore).

Open this by removing its lateral wall. How does it communicate with the nose?

$(n b)$ Sphenoidal sinus (sinus sphenoidalis).

(nc) Frontal sinus (sinus frontalis).

(o) Nerves of lateral wall of nose. (Fig. 132.)

(oa) Olfactory nerves ( $N n$. olfactorii).

$(o b)$ Anterior nasal rami of ethmoidal nerve (rami nasales anteriores).

(oba) Internal nasal rami (rami nasales interni).

(obaa) Lateral nasal rami (rami nasales laterales).

(obb) External nasal ramus (ramus nasalis externus).

(oc) From ganglion sphenopalatinum.

(oca) Lateral superior posterior nasal rami (rami nasales posteriores superiores laterales).

$(o c b)$ [Lateral] inferior posterior nasal rami (rami nasales posteriores inferiores [laterales]).

(od) Internal nasal rami (rami nasales interni), from anterior superior alveolar rami of $\mathrm{N}$. infraorbitalis).

( $p$ ) Arteries of lateral wall of nose.

What is the arterial supply here? Work out the large branches.

\section{SPHENOPALATINE GANGLION (GANGLION SPHENO- PALATINUM) (O. T. MECKEL'S GANGLION).}

The lateral posterior nasal rami (superior and inferior) have already been found; follow them back to the pterygopalatine canal (canalis pterygopalatinus) (O. T. posterior palatine canal); open this canal thoroughout its length and follow the palatine nerves $(\mathrm{Nn}$. palatini) upward to the sphenopalatine ganglion which lies in the pterygopalatine fossa (fossa pterygopalatina) (O. T. sphenomaxillary fossa). In connection with the ganglion sphenopalatinum study the following:

(a) Orbital rami (rami orbitales).

(b) Nerve of the pterygoid canal, or Vidian nerve ( $N$. canalis pterygoidei

[Vidii]). 
(ba) Larger superficial petrosal nerve (N. petrosus superficialis major).

(bb) Deep petrosal nerve (N. petrosus profundus) (O. T. great deep petrosal branch of carotid plexus).

To display $(b)$, eut away with bone-forceps the sphenoidal process (processus sphenoidalis) of the palate bone and carefully open the pterygoid or Vidian canal, which passes through the root of the pterygoid process of the sphenoid bone.

FIG. 132.

Rami lat. Nn. olf.

N. nasopalatinus

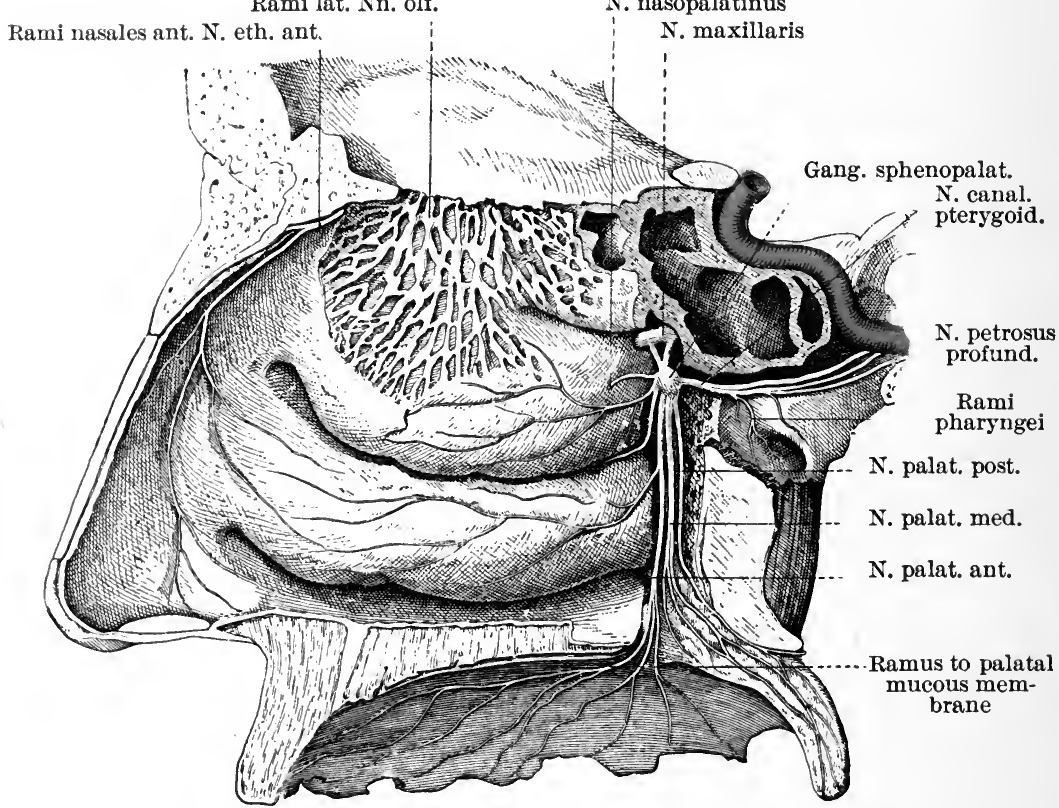

Lateral branches of the olfactory nerves (after Hirschfeld). (From Poirier et Charpy, Traité d'Anat. hum., Paris. 1899, t. iii., 3, p. 774, Fig. 417.)

(c) Nasal rami (rami nasales). (Vide Fig. 132.)

(ca) Lateral superior posterior nasal rami (rami nasales posteriores superiores laterales).

(cb) Medial superior posterior nasal rami (rami nasales posteriores superiores mediales).

$(c b a)$ Nasopalatine nerve of Scarpa (N. nasopalatinus [Scarpae $]$ ).

(cc) [Lateral] inferior posterior nasal rami (rami nasales posteriores inferiores [laterales]).

These have been previously examined in their peripheral distribution.

(d) Palatine nerves (Nn. palatini).

$(d a)$ Anterior palatine nerve ( $N$. palatinus anterior).

Trace it to its terminals in the tunica mucosa of the hard palate. 
(db) Middle palatine nerve ( $N$. palatinus medius) (O. T. external palatine).

(dc) Posterior palatine nerve (N. palatinus posterior).

Near this note the tendinous expansion of the M. tensor veli palatini.

Trace the N. nasopalatinus [Scarpae] also back to its origin. A better view still of the pterygopalatine fossa will be obtained if the orbital process (processus orbitalis) of the palate bone and a portion of the body of the sphenoid bone be cut away with bone-forceps.

The terminal branches of the internal maxillary artery ( $A$. maxillaris interna) should now be finally reviewed.

\section{INTRAOSSEOUS COURSE OF N. FACIALIS, N. INTER- MEDIUS, AND N. ACUSTICUS.}

The N. facialis and N. acusticus should now be followed from the internal acoustic meatus (meatus acusticus internus) in their course through the interior of the petrous portion of the temporal bone.

Remove the temporal bone from the skull, fasten it firmly in a vice in its natural position, and remove the squama temporalis by sawing horizontally through it just above the level of the pars petrosa.

Make a second horizontal cut through the pars petrosa just above the roof of the internal acoustic meatus; the vestibule (vestibulum), cavity of the tympanum (cavum tympani), and mastoid cells (cellulae mastoideae) will thus be opened. Upon the mastoid wall (paries mastoidea) will be seen a prominent anteroposterior ridge (prominentia canalis facialis), due to the canal in which the facial nerve runs, the facial canal of Fallopius (canalis facialis [Fallopii]) (O. T. aqueduct of Fallopius). Notice that with this is continuous a part of the canal upon the labyrinthine wall of the cavum tympani, above and behind the stapes and the fenestra vestibuli (O. T. foramen ovale). Gain space by removing more of the roof of the tympanum (tegmen tympani) with bone-forceps; open up the facial canal on the labyrinthine wall (paries labyrinthica) with a chisel and expose corresponding part of the N. facialis. Next expose the nerve in the proximal portion of the facial canal by chiselling away the roof of the internal acoustic meatus and following the nerve along forward and lateralward. When the geniculate ganglion 
of the $\mathrm{N}$. intermedius is reached, it will be recognizable as a swelling at the point where the $\mathrm{N}$. facialis bends backward to enter the tympanic portion of the facial canal of Fallopius. Avoid injury to the delicate nerve threads connected with the ganglion geniculi.

Push a fine pin upward through the stylomastoid foramen (foramen stylomastoideum) to ascertain the course of the distal portion of the canalis facialis [Fallopii]. This can be opened by first removing the mastoid process by a coronal saw-cut in a plane just behind the foramen stylomastoideum. When this cut has gone as deep as the foramen, make a second cut at right angles to it,- - that is, a sagittal saw-cut,- to meet the extremity

FIG. 133.

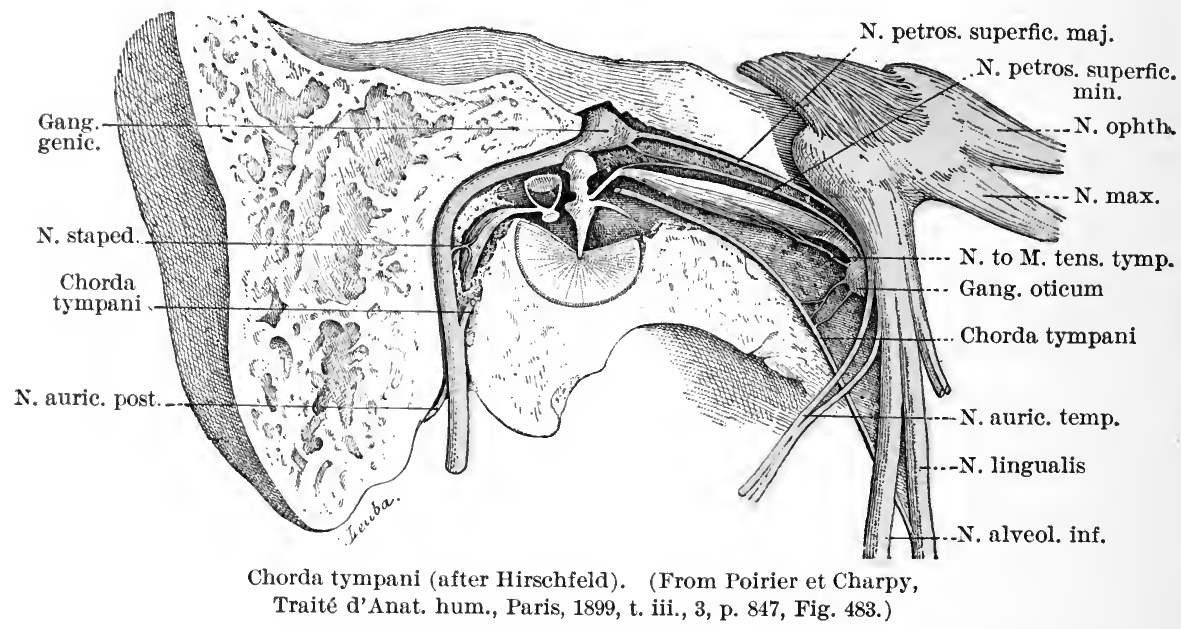

of the first cut. Remove the bone thus excised and open the rest of the facial canal with a chisel.

Study the direction followed by the N. facialis in each of its four stages. Examine the following:

Facial Nerve (N. facialis). (Vide Fig. I33.)

Note-

(a) Knee of the facial nerve (geniculum $N$. facialis) and the ganglion at the knee (ganglion geniculi), which really belongs to the N. intermedius.

(b) Nerve to stapedius muscle (N. stapedius).

(c) Ramus anastomosing with the tympanic plexus (ramus anastomoticus cum plexu tympanico) (O. T. tympanic branch). This helps to form the lesser superficial petrosal nerve $(N$. petrosus superficialis minor). 
Observe also (1) the communication of Arnold's nerve (ramus auricularis $N$. vagi) with the trunk of the N. facialis, a little above the foramen stylomastoideum; (2) a little branch, inconstant, which sometimes runs to join the sympathetic plexus upon the A. meningea media; it is called the external superficial petrosal nerve.

\section{Intermediate Nerve of Wrisberg (N. intermedius) (O. T. Sensory Part of Facial Nerve).}

To this the geniculate ganglion belongs. Study-

(a) Larger superficial petrosal nerve ( $N$. petrosus superficialis major).

This carries sensory fibres from the N. intermedius by way of the Vidian nerve to the sphenopalatine ganglion.

(b) Cord of tympanum (chorda tympani). (Fig. 133.)

This carries secretory fibres (sympathetic?) to the Gl. submaxillaris and Gl. sublingualis and taste fibres from the tongue to the ganglion geniculi of the N. intermedius. Read carefully the complicated course of the chorda tympani and follow it as well as possible in the specimen and in models.

\section{Acoustic Nerve (N. acusticus) (O. T. Auditory Nerve).}

Note its position as regards the $\mathrm{N}$. facialis in the meatus acusticus internus. Observe that it is formed by the fusion of two roots,-one from the vestibule (radix vestibularis), the other from the cochlea (radix cochlearis). The former root carries sensory fibres from the vestibule and semicircular canals and is concerned with the maintenance of equilibrium of the head and eyes; the latter root carries the impulses concerned in the sense of hearing and in auditory reflexes.

\section{LARYNX.}

Examine the position and relations of the larynx. Note that to its presence in the neck is due the laryngeal prominence, or "Adam's apple" (prominentia laryngea, pomum Adami). With a model of the larynx and the specimen before you, read a general description of its construction before beginning the dissection.

Examine the relation of the larynx to the tongue. Study the following:

(a) Median glosso-epiglottic fold (plica glosso-epiglottica mediana) ( $O$. T. middle glosso-epiglottidean fold, or fraenum of the epiglottis). 
(b) Lateral glosso-epiglottic fold (plica glosso-epiglottica lateralis) ( 0 . T. lateral glosso-epiglottidean folds).

(c) Epiglottic vallecula (vallecula epiglottica).

Cavity of Larynx (Cavum laryngis). (Vide Figs. I25, I34, and 135.)

Look into it from above, the epiglottis being pulled well forward, and observe its subdivision by the true and false vocal cords into three portions,- the " upper subdivision," or vestibule of the larynx, the " middle subdivision," corresponding to the ventricles of the larynx, and the "inferior subdivision," below the true vocal cords or folds.

FIG. 134.

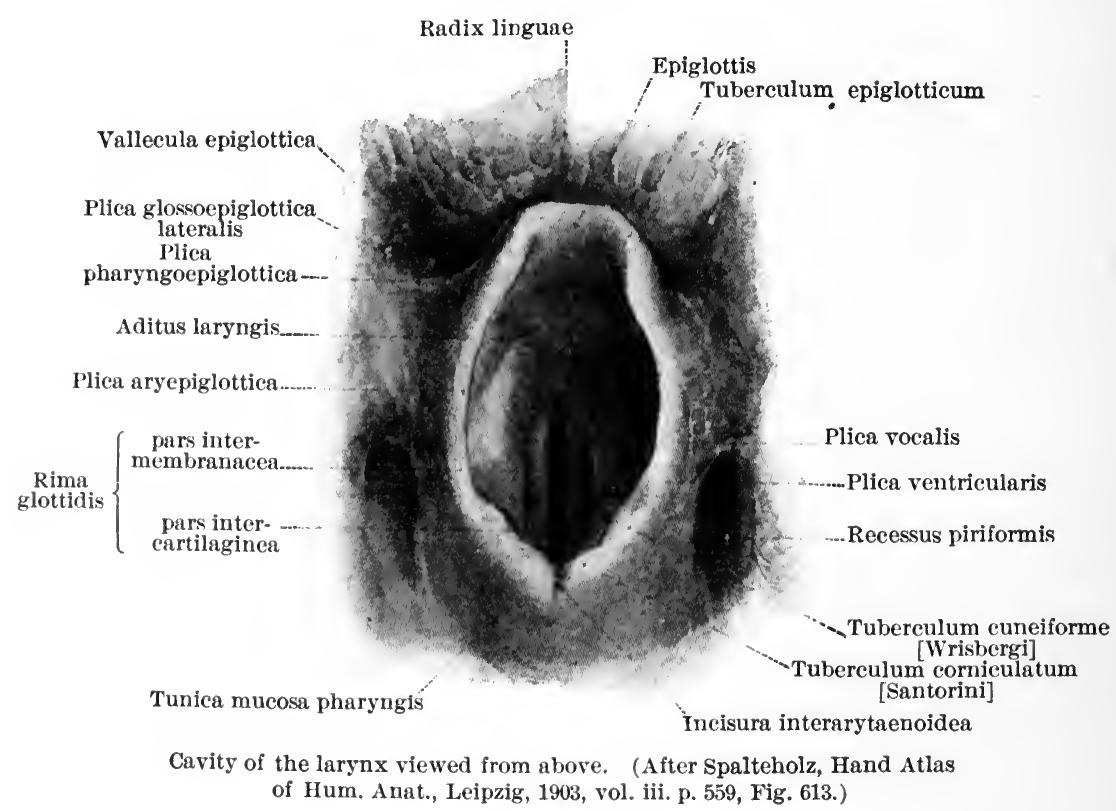

The entrance to the larynx, or superior aperture (aditus laryngis), may now be observed. What is its shape? How is it bounded? The aryepiglottic fold (plica aryepiglottica) (O. T. arytaeno-epiglottidean fold) extends, on each side, from the lateral margin of the epiglottis in front to the tip of the arytaenoid cartilage behind. Note that it is formed by the junction of the tunica mucosa pharyngis with the tunica mucosa laryngis. In it near its posterior extremity are two tubercles, the cuneiform tubercle (tuberculum cuneiforme [Wrisbergi]) and the corniculate tubercle (tuberculum corniculatum [Santorini]), on each 
side, due to the cuneiform and the corniculate cartilage respectively; between the tubereula corniculata of the two sides,- that is, in the median plane behind,- - note the interarytaenoid notch (incisura interarytaenoidea). The cavity between the aditus and the ventricular folds (plicae ventriculares) (O. T. false vocal cords) is called the vestibule of the larynx (vestibulum laryngis). This cavity communicates, by means of the slit (rima vestibuli) (O. T. false glottis) between the ventricular folds (plicae ventriculares) (O. T. false vocal cords), with the superior entrance to the glottis (aditus glottidis superior).

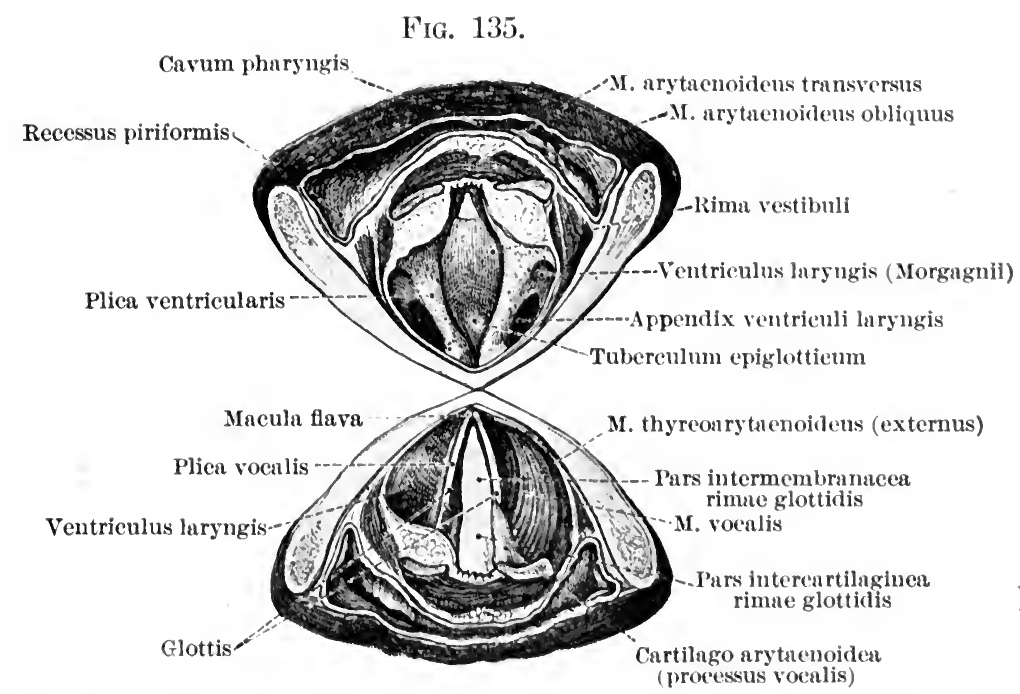

Both sections of a larynx ent transversely across in the region of the ventricle. The mucous membrane has been removed from the right side of the lower section. (After Toldt, Anut. Atlas, Wien, 1900, 2 Aufl., p. 445, Fig. 757.)

On each side of the aditus glottidis superior is situated the ventricle of the larynx (ventriculus laryngis [Morgagnii]) ( $O$. T. laryngeal sinus), with its blind sac-like appendage, appendix ventriculi laryngis (O. T. laryngeal pouch or sac).

The true vocal cord, or vocal fold (plica vocalis) on each side, is the free margin of the three-sided prismatic projection known as the vocal lip (labium vocale). Note that the plica vocalis is situated farther medialward than the plica ventricularis. Observe near the anterior extremity of the plica vocalis the "yellow spot"' (macula flava). 'To what is this due? 


\section{Glottis.}

The two plicae vocales together form the glottis (O. T. glottis vera), the slit between them being known as the rima glottidis. The rima glottidis is divisible into-

(a) Intermembranous part (pars intermembranacea) (O. T. glottis vocalis), the anterior longer part, between the plicae vocales.

(b) Intercartilaginous part (pars intercartilaginea) (O. T. glottis respiratoria), the posterior shorter part, between the medial surfaces of the arytaenoid cartilages.

The cavity below the rima glottidis-i.e., the inferior cavity of the larynx-is called the aditus glottidis inferior. It leads below into the trachea.

\section{Mucous Membrane of the Larynx (Tunica mucosa laryngis). Observe-}

(a) Mucous laryngeal glands (Gl. laryngeae).

(aa) Anterior ( $G l$. laryngeae anteriores), in front of and behind the epiglottis.

(ab) Middle (Gl. laryngeae mediae), on the plicae ventriculares, in the ventricles, and around the cuneiform cartilages.

(ac) Posterior (Gl. laryngeae posteriores), in front of, in, and behind the M. arytaenoideus transversus.

(b) Lymph-nodules of larynx (noduli lymphatici solitarii), especially on the posterior surface of the epiglottis and in the ventricles.

\section{Hyothyreoid Membrane and Laryngeal Muscles.}

With a probe stuff the ventricle of the larynx and its appendix full of moist cotton. Fasten the larynx with pins on a wooden block, the anterior surface upward.

Find the ramus externus and ramus internus of the N. laryngeus superior, the N. recurrens, the superior and inferior laryngeal vessels, and avoid injury to them in the subsequent dissection. Remove the Gl. thyreoidea and the Mm. omohyoideus, sternohyoideus, sternothyreoideus, thyreohyoideus, and constrictor pharyngis inferior. Clean and study the following structures:

(a) Hyothyreoid membrane (membrana hyothyreoidea) (0. T. thyrohyoid membrane). Observe how it is perforated by the $\mathrm{A}$. and $V$. laryngea superior and by the ramus internus $\mathrm{N}$. laryngei superioris.

(aa) Middle hyothyreoid ligament (ligamentum hyothyreoideum medium).

$(a b)$ Lateral hyothyreoid ligament (ligamentum hyothyreoideum laterale). 
(aba) Wheat-like or triticeous cartilage (cartilago triticea).

(b) Cricothyreoid muscle ( $M$. cricothyreoideus).

(ba) Straight part (pars recta) (O. T. anterior or oblique part).

(bb) Oblique part (pars obliqua) (O. T. posterior or horizontal part).

Study its origin, insertion, action, and innervation.

(c) Middle cricothyreoid ligament (ligamentum cricothyreoideum [medium ]) (O. T. middle portion of cricothyreoid membrane).

This is really a part of the conus elasticus (vide infra).

Reverse position of larynx on wooden block, so that posterior surface looks upward. Slit open oesophagus along middle line behind. Dissect the tunica mucosa cautiously off the posterior aspect of the cricoid and arytaenoid cartilages, avoiding injury to the A. laryngea inferior and N. recurrens (between thyreoid and cricoid cartilages).

Clean carefully the following muscles and ascertain their form, position, origin, insertion, action, and innervation :

(a) Posterior cricoarytaenoid muscle (M. cricoarytaenoideus posterior).

(b) Oblique arytaenoid muscle (M. arytaenoideus obliquus).

(ba) Aryepiglottic muscle ( $M$. aryepiglotticus) (O. T. arytenoepiglottidean muscle).

(c) Transverse arytaenoid muscle (M. arytaenoideus transversus).

From this point on, the dissection is conducted differently on the two sides, that on the right being made especially for the muscles, that on the left for the vessels and nerves.

Fasten larynx by its left side to the wooden block. Remove the M. cricothyreoideus of the right side. Cut through the right ligamentum hyothyreoideum laterale; disarticulate the right inferior cornu of thyreoid cartilage from the facies articularis thyreoidea on the side of the cricoid cartilage. Cut vertically through the lamina dextra of the thyreoid cartilage a little lateral from the anterior median line and remove the detached right lamina.

Clean carefully and study the form, position, origin, insertion, action, and innervation of the following muscles:

(a) Lateral erico-arytaenoid muscle (M. cricoarytaenoideus lateralis).

(b) Thyreo-arytaenoid muscle ( $M$. thyreoarytaenoideus [externus]).

(c) Thyreo-epiglottic muscle (M. thyreoepiglotticus) (O. T. thyro-epiglottidean muscle).

(d) Vocal muscle ( $M$. vocalis) (O. T. internal thyro-arytenoid muscle). (This muscle is best seen in a cross-section through the whole larynx at the level of the true vocal cord. It lies medial from the 
M. thyreoarytaenoideus [externus], the two being scarcely separable.)

(e) Ventricular muscle (M. ventricularis).

This is a minute muscle in the plica ventricularis, scarcely to be made out except with the aid of the microscope.

Remove cautiously the M. cricoarytaenoideus lateralis and try to separate the M. thyreoarytaenoideus [externus] from the more medially situated M. vocalis. Ascertain the relation of the latter to the labium vocale and then remove it. Study the parts now exposed.

FIG. 136.

Bundle of the M. stylopharyngeus

M. ceratocricoideus (Var.)

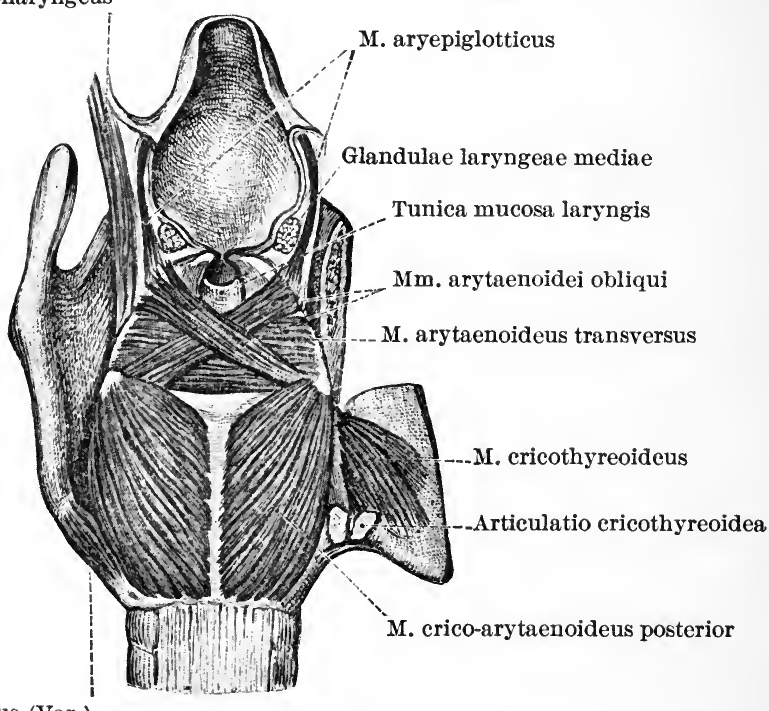

Muscles of larynx seen from behind. Part of the right lamina of the thyreoid cartilage has been removed. (After Toldt, Anat. Atlas, Wien, 1900, 2 Aufl., p. 444, Fig. 751.)

\section{Elastic Membrane of Larynx (Membrana elastica laryngis).}

(a) Elastic cone (conus elasticus) (O. T. cricothyroid membrane).

(aa) Vocal ligament (ligamentum vocale) (O. T. inferior thyroarytenoid ligament).

Observe that this is really the upper free thickened border of the conus elasticus. Note especially its anterior and posterior attachments. Between the two ligamenta vocalia in front observe-

(aaa) Sesamoid cartilage (cartilago sesamoidea).

(b) Quadrangular membrane (membrana quadrangularis).

(ba) Ventricular ligament (ligamentum ventriculare) (O. T. superior thyro-arytenoid ligament). 
Observe that this ligament is simply the thickened lower margin of the quadrangular membrane.

Next dissect away on the right side the remains of the plica aryepiglottica, membrana quadrangularis, plica ventricularis, plica vocalis, and conus elasticus, but leave the arytaenoid and corniculate cartilages undisturbed. Remove the cuneiform cartilage from the right aryepiglottic fold and preserve it for subsequent study. The left wall of the cavity of the larynx may now be thoroughly studied, and any points not well made out in the preliminary study of the cavity before it was opened should be cleared up before going further.

\section{Vessels and Nerves of Larynx.}

The dissection of the vessels and nerves of the interior of the larynx should now be undertaken.

(a) Internal ramus of superior laryngeal nerve (ramus internus 'N. laryngei superioris).

(aa) Ramus anastomosing with inferior laryngeal nerve (ramus anastomoticus cum $N$. laryngeo inferiore) (O. T. Galen's loop).

Make traction on this nerve outside the larynx where it pierces the membrana hyothyreoidea and eut through the tunica mucosa laryngis on the inner surface of this membrane and find the nerve and artery. Gradually dissect off the tunica mucosa and expose their branches. What is the function of the internal ramus of the N. laryngeus superior?

(b) Inferior laryngeal nerve from the recurrent nerve (N. laryngeus inferior $N$. recurrentis) ( $\mathrm{O} . \mathrm{T}$. recurrent laryngeal nerve).

To see it well, draw the thyreoid cartilage well lateralward.

(ba) Anterior ramus (ramus anterior).

What muscles are innervated by it?

(bb) Posterior ramus (ramus posterior).

What muscles does it supply?

(c) Superior laryngeal artery (A. laryngea superior).

(d) Inferior laryngeal artery (A. laryngea inferior).

\section{Cartilages and Ligaments of Larynx.}

Some of these have already been examined.

Remove the rest of the mucous membrane and the muscles from the cartilages, taking care not to injure the ligaments or the smaller cartilages. Study-

Epiglottis and its Ligaments.

(a) Epiglottis.

(aa) Stem of epiglottis (petiolus epiglottidis). 
(ab) Epiglottic tubercle (tuberculum epiglotticum) (O. T. cushion of epiglottis).

(ac) Epiglottic cartilage (cartilago epiglottica).

(b) Thyreo-epiglottic ligament (ligamentum thyreoepiglotticum) (0. T. thyro-epiglottidean ligament).

(c) Hyo-epiglottic ligament (ligamentum hyoepiglotticum) (O. T. hyoepiglottidean ligament).

Thyreoid Cartilage (Cartilago thyreoidea).

(a) Right and left plates (laminae [dextra et sinistra]) (O. T. ala).

(b) Superior thyreoid notch (incisura thyreoidea superior).

(c) Inferior thyreoid notch (incisura thyreoidea inferior).

(d) Superior thyreoid tubercle (tuberculum thyreoideum superius).

(e) Inferior thyreoid tubercle (tuberculum thyreoideum inferius).

(f) Oblique line (linea obliqua).

(g) Superior horn (cornu superius).

(h) Inferior horn (cornu inferius).

(i) Thyreoid foramen (foramen thyreoideum).

Cricothyreoid Joint (Articulatio cricothyreoidea).

(a) Capsule of joint (capsula articularis cricothyreoidea).

(b) Lateral ceratocricoid ligaments (Ligg. ceratocricoidea lateralia).

(c) Anterior ceratocricoid ligament (Lig. ceratocricoideum anterius).

(d) Posterior ceratocricoid ligaments (Ligg. ceratocricoidea posteriora).

Divide the structures connecting the thyreoid cartilage to the cricoid and remove the former.

Cricoid Cartilage (Cartilago cricoidea).

- (a) Arch (arcus [cartilaginis cricoideae]).

(b) Lamina (lamina [cartilaginis cricoideae]).

(c) Arytaenoid articular surface (facies articularis arytaenoidea).

(d) Thyreoid articular surface (facies articularis thyreoidea).

Observe also the cricotracheal ligament (ligamentum cricotracheale).

Corniculate Cartilage (Cartilago corniculata [Santorini]) (O. T. cartilage of Santorini).

(a) Arycorniculate joint (synchondrosis arycorniculata).

(b) Corniculopharyngeal ligament (Lig. corniculopharyngeum) (O. T. Lig. jugale).

(ba) Cricopharyngeal ligament (Lig. cricopharyngeum).

Arytaenoid Cartilage (Cartilago arytaenoidea).

Remove one in order to study the cartilage itself; leave the other in situ for the study of the ligaments.

(a) Tip or apex (apex [cartilaginis arytaenoideae]).

(b) Base (basis [cartilaginis arytaenoideae]).

(ba) Articular surface (facies articularis).

(c) Posterior surface (facies posterior).

(d) Medial surface (facies medialis) (O. T. internal surface).

(e) Lateral surface (facies lateralis) (O. T. antero-external surface).

(ea) Nodule or hillock (colliculus).

(eb) Arched ridge (crista arcuata).

(ec) Triangular depression (fovea triangularis).

(ed) Oblong depression (fovea oblonga). 
(f) Vocal process (processus vocalis).

(g) Muscular process (processus muscularis).

Cuneiform Cartilage (Cartilago cuneiformis [Wrisbergi]).

Was this present on each side in the subject under dissection?

Crico-arytaenoid Joint (Articulatio cricoarytaenoidea).

(a) Joint-capsule (capsula articularis cricoarytaenoidea).

(b) Posterior crico-arytaenoid ligament (Lig. cricoarytaenoideum posterius).

The student is advised to read at this juncture on the physiology of voice-production and to study the action of the various laryngeal muscles. Why does food not enter the larynx during deglutition?

\section{TONGUE (LINGUA).}

Examine the following:

(a) Back of tongue (dorsum linguae). (Vide Figs. 125, 137.)

(b) Root of tongue (radix linguae).

To what is it attached?

FIG. 137.

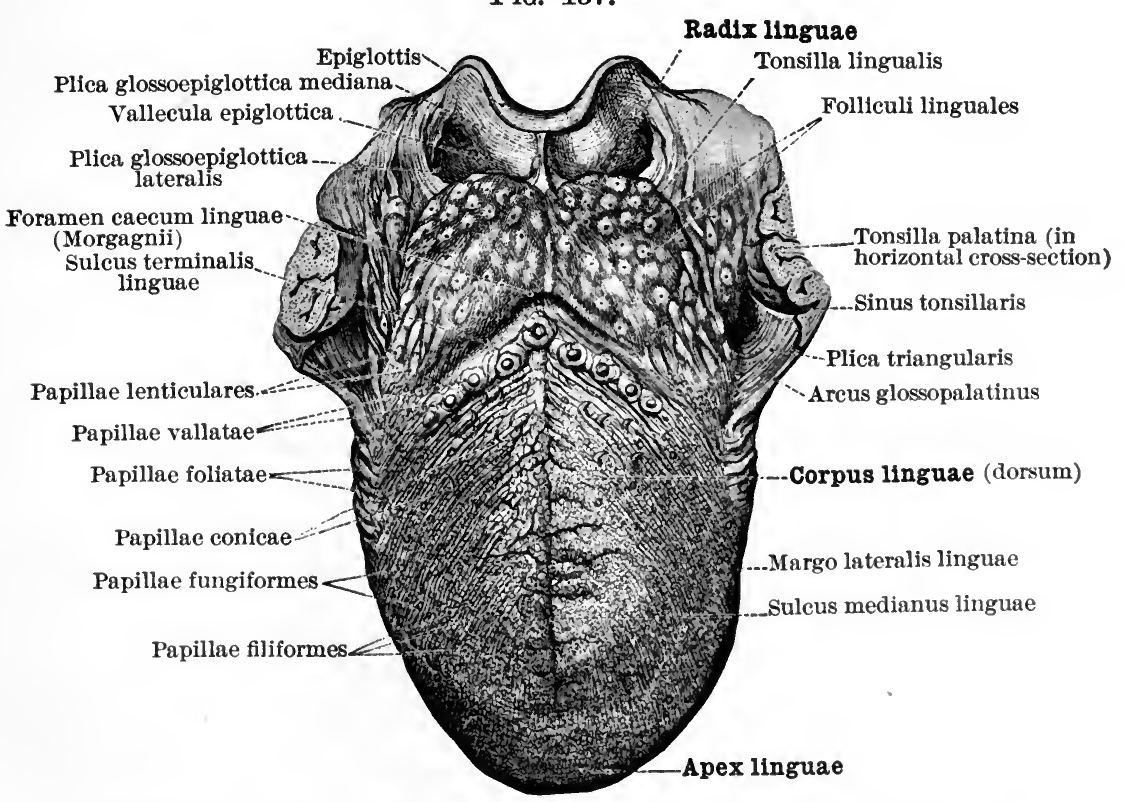

Dorsal surface of tongue. (After Toldt; Anat. Atlas, Wien, 1900, 2 Aufl., p. 403, Fig. 649.)

(c) Body of tongue (corpus linguae).

(d) Inferior surface (facies inferior [linguae]).

(da) Fimbriated fold (plica fimbriata). 
(e) Tip of tongue (apex linguae).

( $f$ ) Mucous membrame of tongue (tunica mucosa linguae).

This has already been studied in connection with the mouth eavity, but should now be reviewed.

( $f a$ ) Frenulum ("bridle" or " check-rein") of tongue (frenulum linguae).

$(f b)$ Lingual papillae (papillae linguales).

$(f b a)$ Filiform papillae (papillae filiformes).

( $f b b)$ Conical papillae (papillae conicae).

$(f b c)$ Fungiform papillae (papillae fungiformes).

$(f b d)$ Lenticular papillae (papillae lenticulares).

(fbe) Vallate papillae (papillae vallatae) (O. T. circumvallate).

( fbf) Foliate papillae (papillae foliatae).

(g) Median sulcus of tongue (sulcus medianus linguae).

(h) Terminal sulcus (sulcus terminalis).

FiG. 138.

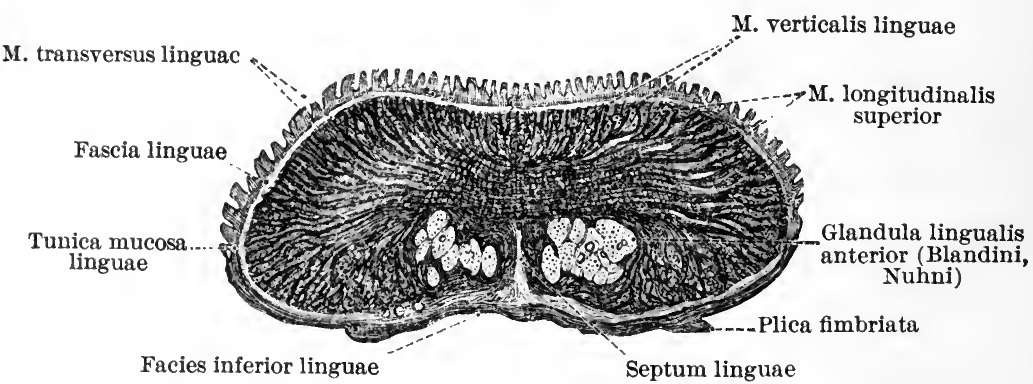

Transverse section through the tongue near the tip. (After Toldt, Anat. Atlas, Wien, 1900, 2 Aufl., p. 405, Fig. 652.

(i) Blind foramen of tongue (foramen caecum linguae [Morgagnii]).

(ia) Lingual duet (ductus lingualis).

(iaa) Thyreoglossal duct (ductus thyreoglossus).

(j) Lingual tonsil (tonsilla lingualis).

(ja) Lingual folliculi (folliculi linguales).

(k) Muscles of tongue.

Remove the tunica mucosa linguae from the right side of the tongue; isolate the individual muscles and review their form, position, origin, insertion, action, and innervation.

Extrinsic museles (vide Fig. 112) :

(ka) Genioglossus muscle (M. genioglossus).

(kb) Hyoglossus muscle (M. hyoglossus).

(kc) Chondroglossus muscle (M. chondroglossus).

(kd) Styloglossus muscle (M. styloglossus).

Intrinsic muscles (vide Fig. 138) :

(ke) Superior longitudinal muscle of tongne ( $M$. longitudinalis superior linguae) (O. T. superficial lingual).

$(k f)$ Inferior longitudinal muscle of tongue (M. longitudinalis inferior linguae) (O. T. inferior lingual). 
( $k g$ ) Transverse muscle of tongue (M. transversus linguae).

( $k h)$ Vertical muscle of tongue (M. verticalis linguae).

(l) Septum of tongue (septum linguae).

( $m$ ) Nerves of tongue (to be studied on left side of tongue).

( $m a$ ) Glossopharyngeal nerve ( $N$. glossopharyngeus).

(maa) Lingual rami (rami linguales).

$(m b)$ Lingual nerve ( $N$. lingualis).

(mba) Sublingual nerve (N. sublingualis).

(mbb) Lingual rami (rami linguales).

Read on the relations of the N. trigeminus and the $\mathrm{N}$. intermedius, respectively, to the $\mathrm{N}$. lingualis.

(mc) Hypoglossal nerve (N. hypoglossus).

(mca) Lingual rami (rami linguales).

What do they innervate?

(n) Lingual artery (A. lingualis).

(na) Dorsal rami of tongue (rami dorsales linguae).

( $n b)$ Deep artery of tongue (A. profunda linguae) (O. T. ranine artery).

\section{BRAIN (ENCEPHALON).}

The student should now undertake the systematic study of the brain.

\section{Membranes of Brain (Meninges encephali).}

(a) Dura mater of the brain (dura mater encephali). This has been examined before (p. 198).

(b) Arachnoid of brain (arachnoidea encephali).

(ba) Subarachnoid cavity (cavum subarachnoideale).

(bb) Subarachnoid cisterns (cisternae subarachnoideales).

Divide the arachnoid in the middle line over the anterior surface of the medulla oblongata and pons, and turn the two halves lateralward.

(bba) Cerebellomedullary eistern (cisterna cerebellomedullaris) (O. T. eisterna magna).

$(b b b)$ Cistern of the lateral fossa of the cerebrum (cisterna fossae lateralis cerebri [Sylvii]). Note relation to $A$. cerebri media.

$(b b c)$ Cistern of the chiasm (cisterna chiasmatis).

(bbd) Interpeduncular cistern (cisterna interpeduncularis) (O. T. eisterna basalis).

(bbe) Cistern of the large veins of the cerebrum (cisterna venae magnae cerebri).

(bc) Arachnoideal granulations (granulationes arachnoideales

[Pacchioni]) (O. T. Pacchionian bodies). (Vide Fig. S4.)

(c) Pia mater of brain (pia mater encephali). Study this in its relation to the surface of the brain. The portions of the pia in the interior of the brain will be studied later on. 
FIg. 139.

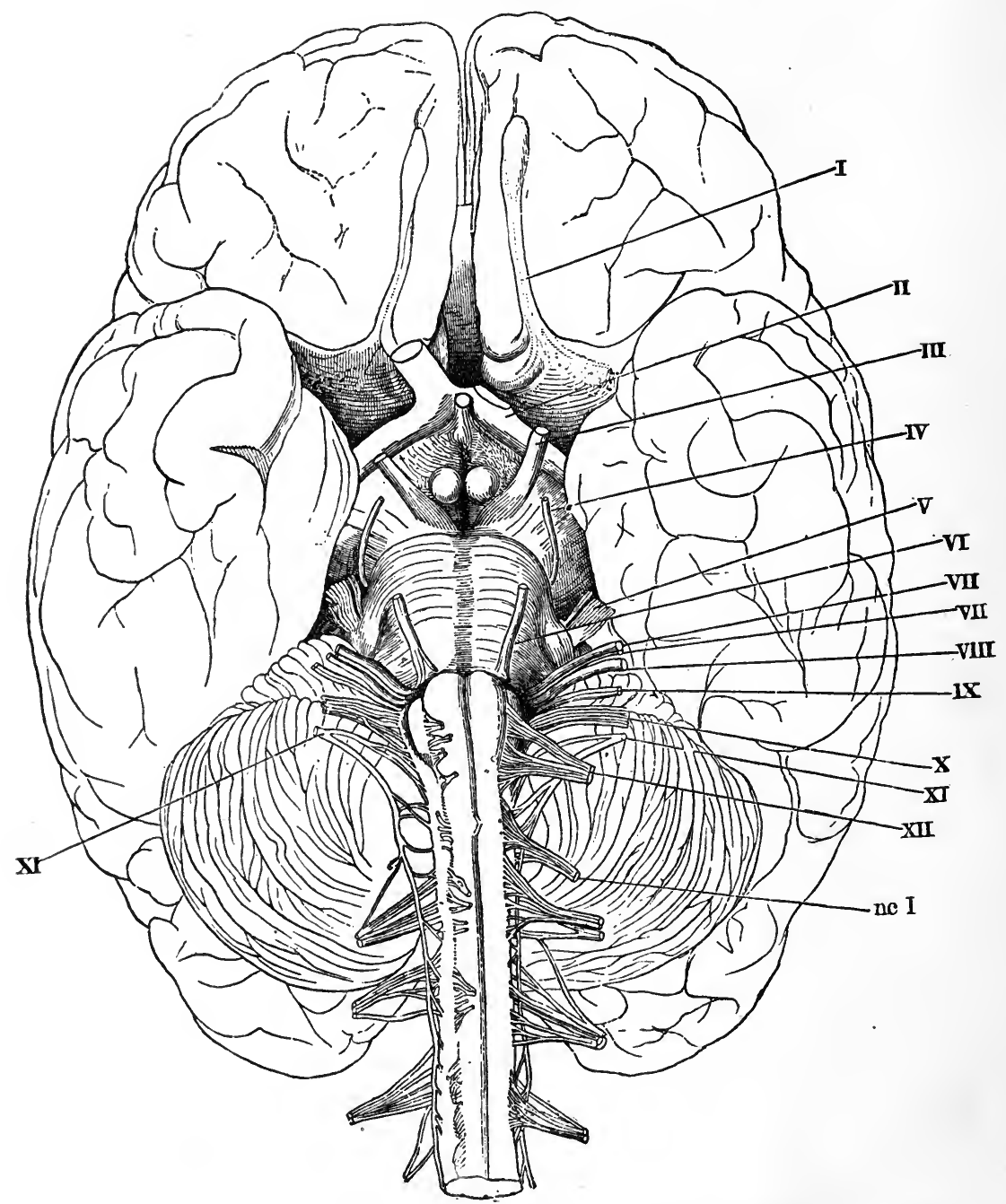

Cerebrum, with a portion of the spinal cord, viewed from the ventral surface. On the right-hand side the ventral roots are cut off short and turned medialward.

$I$, tractus olfactorius; $I I$, tractus opticus ; $I I I, \mathrm{~N}$. oculomotorius ; $I V, \mathrm{~N}$. trochlearis; $V, \mathrm{~N}$. trigeminus, portio major et portio minor; VI, N. abducens; $I I I, \mathrm{~N}$. facialis; $V I T, \mathrm{~N}$. intermedius; VIII, $\mathrm{N}$. acusticus; $I X, \mathrm{~N}$. glossopharyngeus; $X, \mathrm{~N}$. vagus ; $X I, \mathrm{~N}$. accessorius ; $X I I, \mathrm{~N}$. hypoglossus ; $n c I$, N. cervicalis primus. (After Rüdinger and Henle, from A. Rauber's text-book.)

\section{Blood-vessels of Brain. (Vide Fig. I40.)}

Remove the arachnoidea encephali from the base of the cerebrum and from the anterior surface of the rhombencephalon. With scissors and forceps follow the blood-vessels, beginning at 
the large trunks and passing out to the finer subdivisions. Do not injure the brain substance.

FIG. 140.

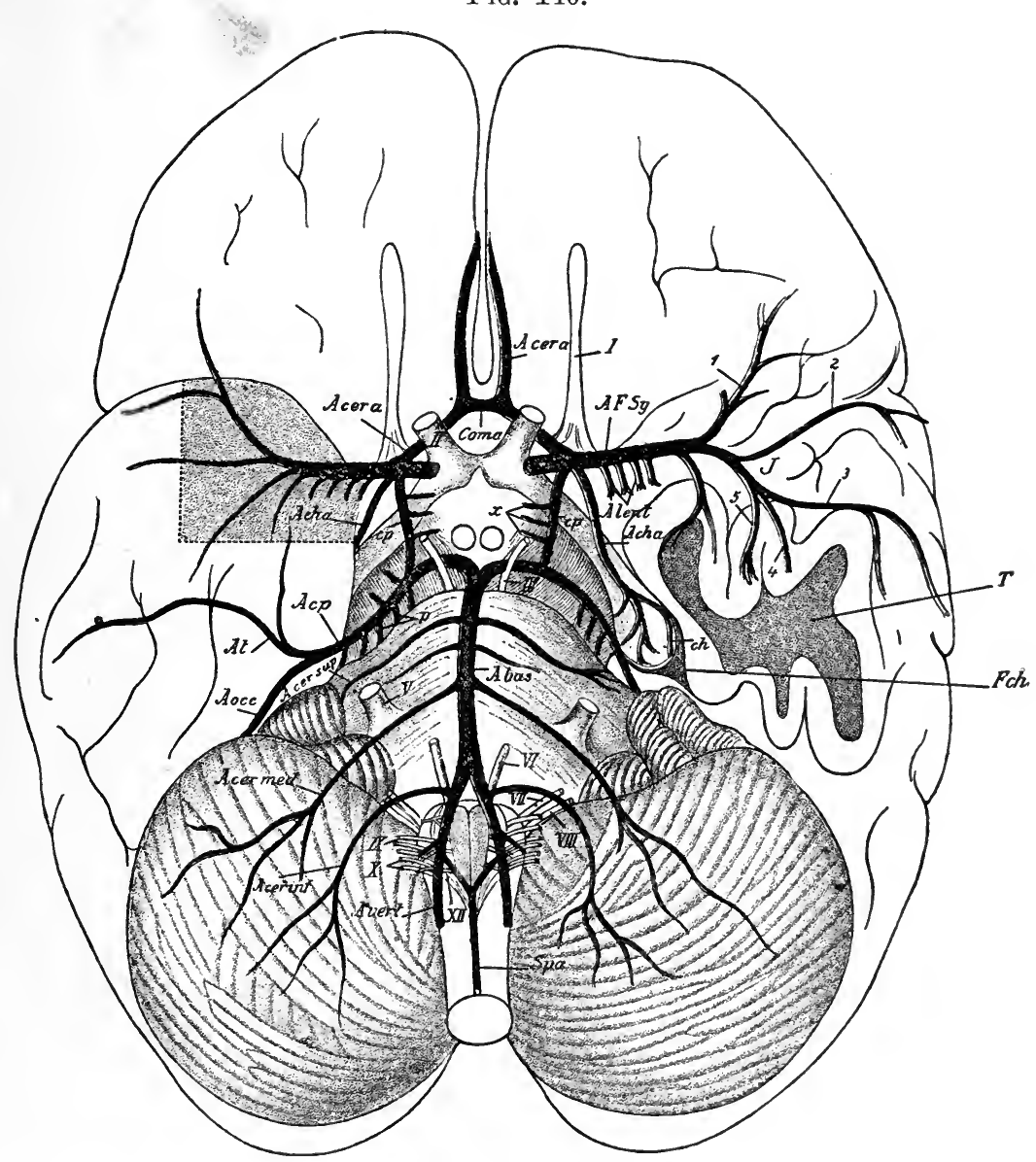

The arteries of the base of the brain.

$T$, lobus temporalis (cut off) ; $I-X I I$, Nn. cerebrales ; A ecr a, art. cercbri anterior ; Com a, art. communieans anterior; A F Sy, art. cerebri media; A lent, art. lenticularis; 1-5, the cortical chief branches of the $A$ cer. med. ; $c p$, art. communicans posterior; $A$ cha, art. chorioidea; $c h$, a small branch of the preceding which goes into the plexus chorioidei; $x$, lateral branches of the A. com. post. ; $A c p$, art. cerebri posterior; $p$, short branches of the preceding; $A b a s$, art. basilaris; $A t$, art. temporalis (Duret) ; $A$ occ, art. oceipitalis (Duret) ; A ccr sup, art. cerebelli superior; A ccr $m c d$, art. cerebelli inferior anterior ; A ccrinf, art. cerebelli inferior posterior ; A vert, art. vertebralis ; Sp a, art. spinalis anterior. (After Monakow, Gehirnpathologie, Wien, 1897, p. 668, Fig. 151.)

\section{Arteries.}

(a) Vertebral artery (A. vertebralis).

(aa) Posterior spinal artery (A. spinalis posterior).

(ab) Anterior spinal artery (A. spinalis anterior). 
(ac) Meningeal ramus (ramus meningeus) (O. T. posterior meningeal branch).

(ad) Posterior inferior cerebellar artery (A. cerebelli inferior posterior).

(b) Basilar artery (A. basilaris).

(ba) Anterior inferior cerebellar artery (A. cerebelli inferior anterior).

(bb) Internal auditory artery (A. auditiva interna) (O. T. auditory artery).

(bc) Rami to pons (rami ad pontem) (O. T. transverse arteries).

$(b d)$ Superior cerebellar artery (A. cerebelli superior).

(be) Posterior cerebral artery (A. cerebri posterior).

(bf) Arterial circle of Willis (circulus arteriosus [Willisi]).

Fig. 141.

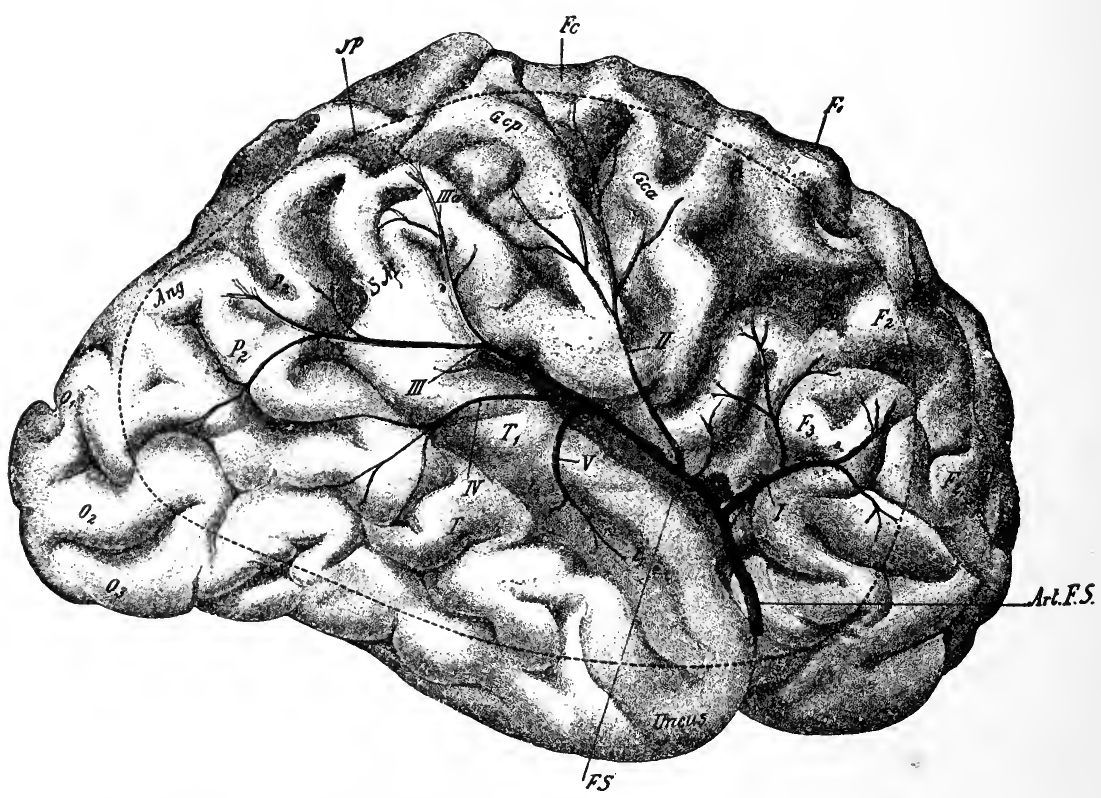

Lateral view of the right hemisphere with the distribution of the A. cerebri media.

Art. FS, art. eer. med.; $I, I I, I I I, I V, V$, the five ehief branches of the preceding; IIIa, lateral twigs of the third branch of the A. cer. med.; Fc, sulcus centralis; FS, fissura cerebri lateralis [Sylvii]; $J P$, sulcus interparietalis; $t$, suleus temporalis superior; $F_{1}-F_{3}$, superior, middle and inferior frontal gyri ; SM, gyrus supramarginalis; $A$ g g, gyrus angularis; $O_{1}-O_{3}$, gyri occipitales laterales. The dotted line indicates the extent of the distribution of the A. cer. med. (After v. Monakow, Gehirnpath., Wien, 1897, p. 673, Fig. 154.)

(c) Internal carotid artery (A. carotis interna).

(ca) Posterior communicating artery (A. communicans posterior).

( $c b)$ Chorioid artery (A. chorioidea) (O. T. anterior choroidal).

(cc) Anterior cerebral artery (A. cerebri anterior).

(cca) Anterior communicating artery (A. communicans anterior). 
(cd) Middle cerebral artery (A. cerebri media) (O. T. arteria fossae Sylvii). (Vide Figs. 140-142.)

Distinguish between basal and central branches and cortical branches of the cerebral arteries. Why should emboli pass more

FIG. 142.

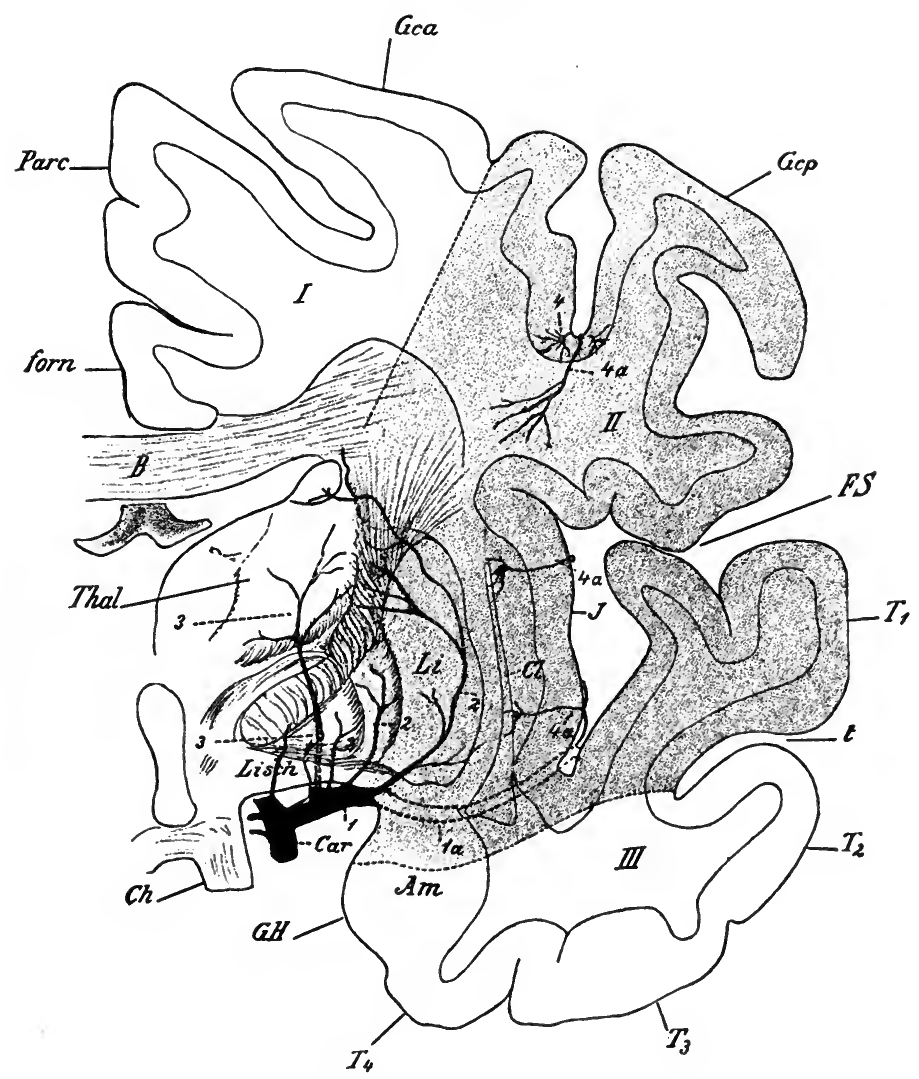

Frontal section through the right cerebral hemisphere (plane of the gyrus centralis and ehiasma opticum). Origin and course of the $\mathrm{A}$. lenticularis as well as of the eortical branches of the $\mathrm{A}$. cerebri media, schematically showu.

Gca, anterior, Gcp, posterior central gyrus; Parc, lobulus paracentralis; $T_{1}-T_{4}$, first to fourth temporal gyrus; $B$, eorpus callosum; $A m$, nucleus amygdalae; $J$, island; $F S$, fissura cerebri lateralis [Sylvii]; $L i$, nucleus lentieularis; Thal, thalamus; CH, gyrus hippocampi ; I, field of the A. cer. ant.; $I I$, of the A. cer. med.; III, of the A. cer. post. The dotted lines show the boundaries between these. Ch, chiasma opticum ; Car, A. eer. inf. ; 1 , stem of the A. cer. med. ; ta, course to the island is shown by dotted outline; 2 , lentieulo-striate arteries; 3, lentieulo-optic arteries; 4, cortical branches of the A. eer. med, ; $4 a$, medullary branehes of the eortical twigs of the A. eer. med. (After v. Monakow, Gehirnpath., Wien, 1897, p. 674, Fig. 155.)

frequently into the middle cerebral than into the anterior cerebral artery? The "artery of cerebral hemorrhage" (Charcot), 
so called on account of the frequency of its involvement, as compared with the other cerebral arteries, is one of the lenticular branches of the A. cerebri media. For a full description of the cerebral arteries and their relation to cerebral hemorrhages, see v. Monakow, Gehirnpathologie, pp. 667-792.

Veins (Venae cerebri).

Superficial.

(a) Superior cerebral veins ( $V v$. cerebri superiores).

(b) Middle cerebral vein ( $V$. cerebri media).

(c) Inferior cerebral veins ( $V v$. cerebri inferiores).

(d) Superior cerebellar veins (Vv. cerebelli superiores).

(e) Inferior cerebellar veins ( $V v$. cerebelli inferiores).

( $f$ ) Basal vein ( $V$. basalis [Rosenthali]).

Deep.-These may be studied later.

\section{Removal of Meninges and Superficial Blood-vessels.}

Place the brain on the table, with the base upward. With forceps and scissors, cautiously remove the membranes and. blood-vessels from the surface. The pia over the medulla oblongata and lower part of pons may be left until after the cerebral nerves have been identified and studied. The base of the brain is now exposed, including a part of the basis cerebri and the inferior (anterior) surfaces of the pons, medulla oblongata, and cerebellum. Observe-

(a) Longitudinal fissure of cerebrum (fissura longitudinalis cerebri).

(b) Lateral fissure of cerebrum (fissura cerebri lateralis [Sylvii]) (O. T. fissure of Sylvius).

(c) Temporal pole (polus temporalis).

(d) Optic chiasm (chiasma opticum) with optic nerves (Nn. optici) and optic tracts (tractus optici).

(e) Hypophysis (hypophysis) (O. T. pituitary body). This may have been left in the sella turcica.

( $f$ ) Infundibulum (infundibulum) ("funnel").

(g) Ash-like tuber (tuber cinereum).

(h) Mammillary bodies (corpora mammillaria).

(i) Cerebral peduncles (pedunculi cerebri) (O. T. crura cerebri).

(j) Interpeduncular fossa (fossa interpeduncularis [Tarini]).

( $j a)$ Anterior recess (recessus anterior).

( $j b)$ Posterior recess (recessus posterior).

(k) Posterior perforated substance (substantia perforata posterior).

(l) Pons (pons [Varolii]) ("bridge").

( $m$ ) Medulla oblongata.

\section{Cerebral Nerves (Nn. cerebrales). (Figs. I39 and r43.)}

The "superficial origins" of the cerebral nerves-i.e., their regions of exit from or entrance into the brain-may now be examined. 
I. Olfactory nerves ( $N n$. olfactorii). Do not confuse these with the olfactory tracts. Try to find the fine threads of the Nn. olfactorii penetrating the ventral surface of each bulbus olfactorius. They come from the nasal mucous membrane, through the lamina cribrosa of the ethmoid bone.

II. Optic nerve ( $N$. opticus). It runs from the back of the eyeball to the optic chiasm. The student's later studies will show him that the optic nerve is really not a peripheral nerve, like the other cerebral nerves, but rather a part of the central nervous system.

FIg. 143.

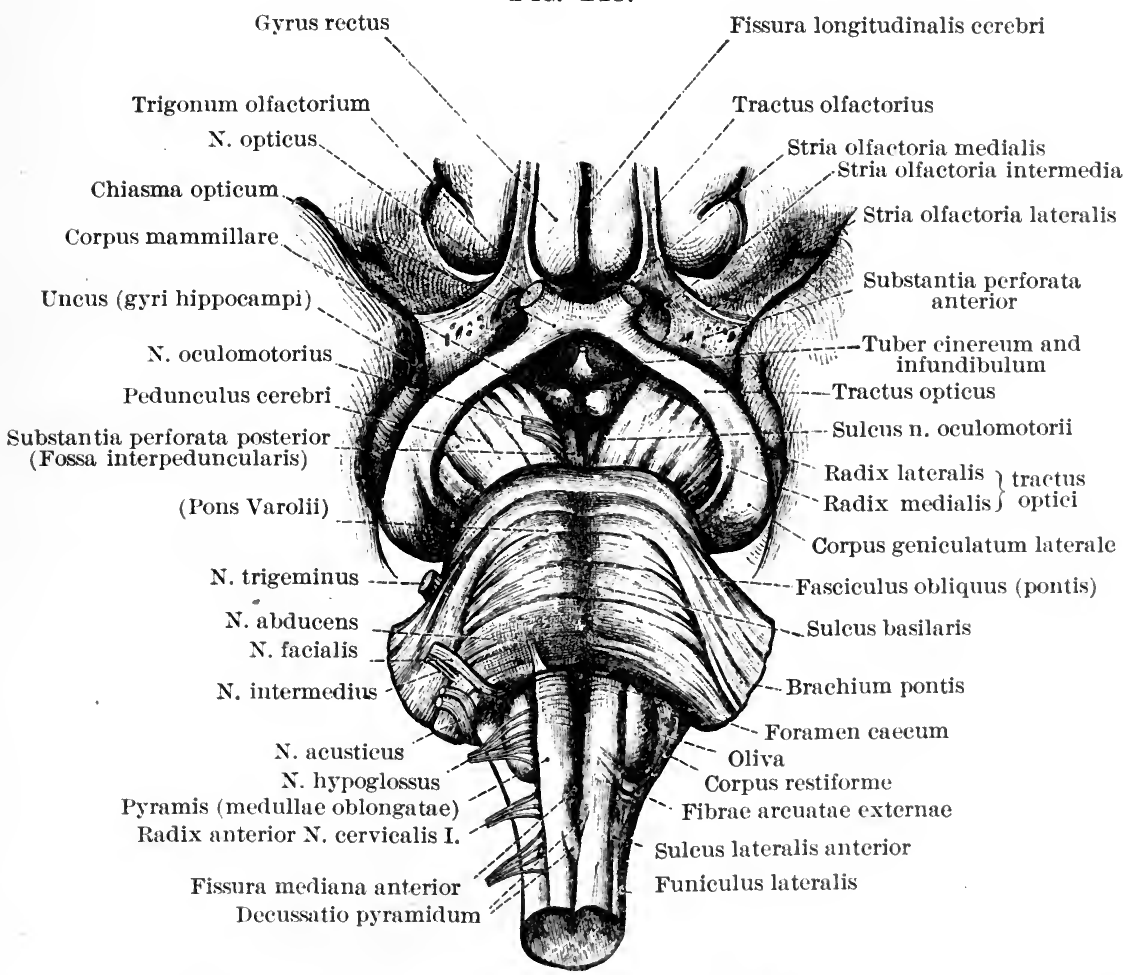

The medulla oblongata, pons (Varolii), cerebral peduncles, and part of the floor of the midbrain. Scen from the basal surface. (After Toldt, Anat. Atlas, Wien, 1903, 3 Aufl., p. 765, Fig. 1174.)

III. Oculomotor nerve (N. oculomotorius). Look for it in the fossa interpeduncularis. It belongs to the midbrain.

IV. Trochlear nerve ( $N$. trochlearis). While all the other cerebral nerves emerge from the ventral aspect of the brain-stem, this nerve emerges on the dorsal aspect. Its exact origin will be seen later. Note that it is on the lateral aspect of the pedunculus cerebri, while the oculomotor nerve is on the medial aspect of that structure.

V. Trigeminal nerve (N. trigeminus).

(a) Larger portion (portio major), sensory.

(b) Smaller portion (portio minor), motor. 
VI. Abducent nerve (N. abducens). Emerges on each side at the junction of the pons with the medulla oblongata.

VII. Facial nerve ( $N$. facialis).

(a) Intermediate nerve ( $N$. intermedius) (O. T. pars intermedia, or nerve of Wrisberg).

VIII. Acoustic nerve (N. acusticus) (O. T. auditory nerve).

(a) Vestibular root (radix vestibularis). This runs medial to the restiform body.

(b) Cochlear root (radix cochlearis). This runs lateral from the restiform body.

IX. Glossopharyngeal nerve ( $N$. glossopharyngeus). It may be difficult to decide exactly how many fila radicularia belong to this nerve and how many to $\mathrm{X}$. and $\mathrm{XI}$.

$\mathrm{X}$. Vagus nerve ( $N$. vagus) (O. T. pneumogastric nerve).

XI. Accessory nerve ( $N$. accessorius) (O. T. spinal accessory).

XII. Hypoglossal nerve (N. hypoglossus). Emerges between the oliva and the pyramis, by several fasciculi (fila radicularia).

\section{Larger Subdivisions of the Brain.}

Note the subdivision into rhomboid brain (rhombencephalon) and cerebrum, the junction being that between pons and midbrain.

The rhombencephalon includes-

(a) After-brain (myelencephalon) ("medullary brain").

(b) Hind-brain (metencephalon), subdivisible into-

(ba) Cerebellum.

(bb) Pons [Varolii].

The cerebrum includes-

(a) Midbrain (mesencephalon).

(b) Forebrain (prosencephalon), consisting of-

(ba) Interbrain (diencephalon) and

(bb) End-brain (telencephalon).

Examine carefully the longitudinal fissure of the cerebrum (fissura longitudinalis cerebri) (O. T. great longitudinal fissure).

The following table shows the derivatives of the three cerebral vesicles. 

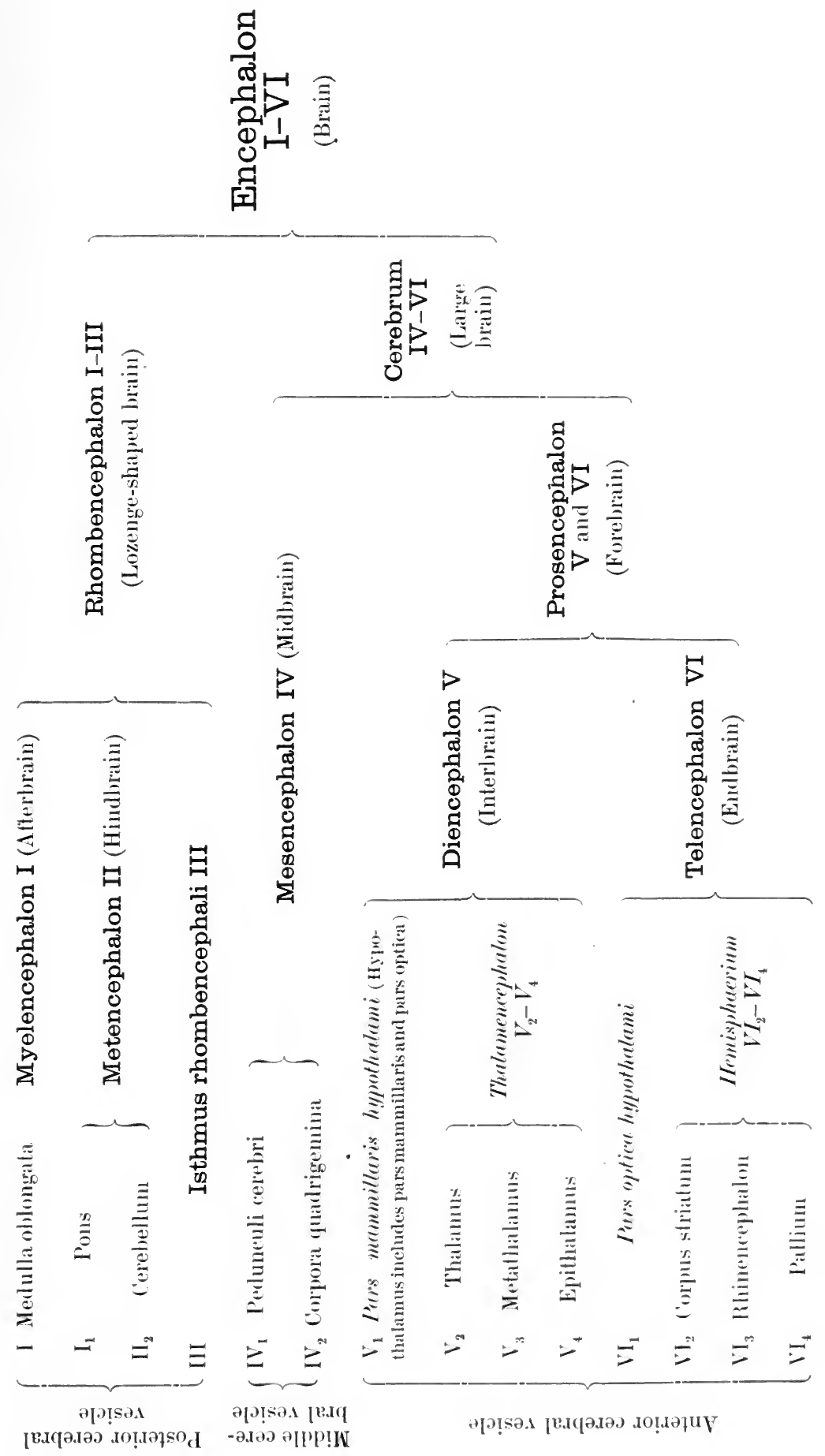
The student may now remove one cerebral hemisphere (hemisphaerium cerebri). With a very sharp, thin knife, cut through the right cerebral peduncle just behind the right corpus mammillare. Turn the brain over; press the two hemispheres apart by widening the longitudinal fissure of the cerebrum; expose the corpus callosum and cut through it and the tissues beneath, as near the middle line as possible, or a very little to the left of it, so as to leave the septum pellucidum on the right hemisphere. Carry the incision backward to meet the transverse incision already made through the cerebral peduncle. This right hemisphere may be kept in the preserving fluid while the rest of the brain is being studied.

\section{External Morphology of Rhomboid Brain and Midbrain (Rhombencephalon and Mesencephalon).}

Lift the cerebellum gently from behind and observe the relation of its inferior surface to the medulla oblongata and ventriculus quartus. With a sharp, thin brain-knife, cut through the cerebellum in the middle line of the worm (vermis). Do no injury to the floor of the fourth ventricle, but permit the incision to pass forward through the anterior medullary velum as far as the inferior colliculi of the midbrain. Cut through the three cerebellar peduncles on the right side,-the superior peduncle (brachium conjunctivum), the middle peduncle (brachium pontis), and the inferior peduncle (corpus restiforme). Remove the right half of the cerebellum and preserve it for further study.

Make three drawings showing anterior, lateral, and posterior views of rhombencephalon, and illustrating the following structures:

\section{Medulla Oblongata.}

(a) Anterior median fissure (fissura mediana anterior).

(b) Posterior median fissure (fissura mediana posterior).

(c) Blind foramen (foramen caecum).

(d) Pyramid (pyramis [medullae oblongatae]).

(e) Decussation of pyramids (decussatio pyramidum).

( $f$ ) Anterior lateral sulcus (sulcus lateralis anterior).

(g) Posterior lateral sulcus (sulcus lateralis posterior).

(h) Olive (oliva) (O. T. olivary eminence).

(i) Restiform body (corpus restiforme) (O. T. inferior cerebellar peduncle).

(j) Lateral funiculus (funiculus lateralis).

( $k$ ) Cuneate funiculus (funiculus cuneatus) (O. T. column of Burdach). 
(l) Gray or ashen tubercle (tuberculum cinereum) (O. T. tuberele of Rolando).

( $m$ ) Slender funiculus (funiculus gracilis) (O. T. column of Goll). ( ma) Club (clava).

(n) External arcuate fibres (fibrae arcuatae externae) (O. T. superficial arcuate fibres).

\section{Pons [Varolii].}

(a) Basilar groove (sulcus basilaris).

(b) Oblique bundle of pons (fasciculus obliquus [pontis]).

(c) Brachium ("arm") of pons (brachium pontis) (O. T. middle cerebellar peduncle).

FIG. 144.

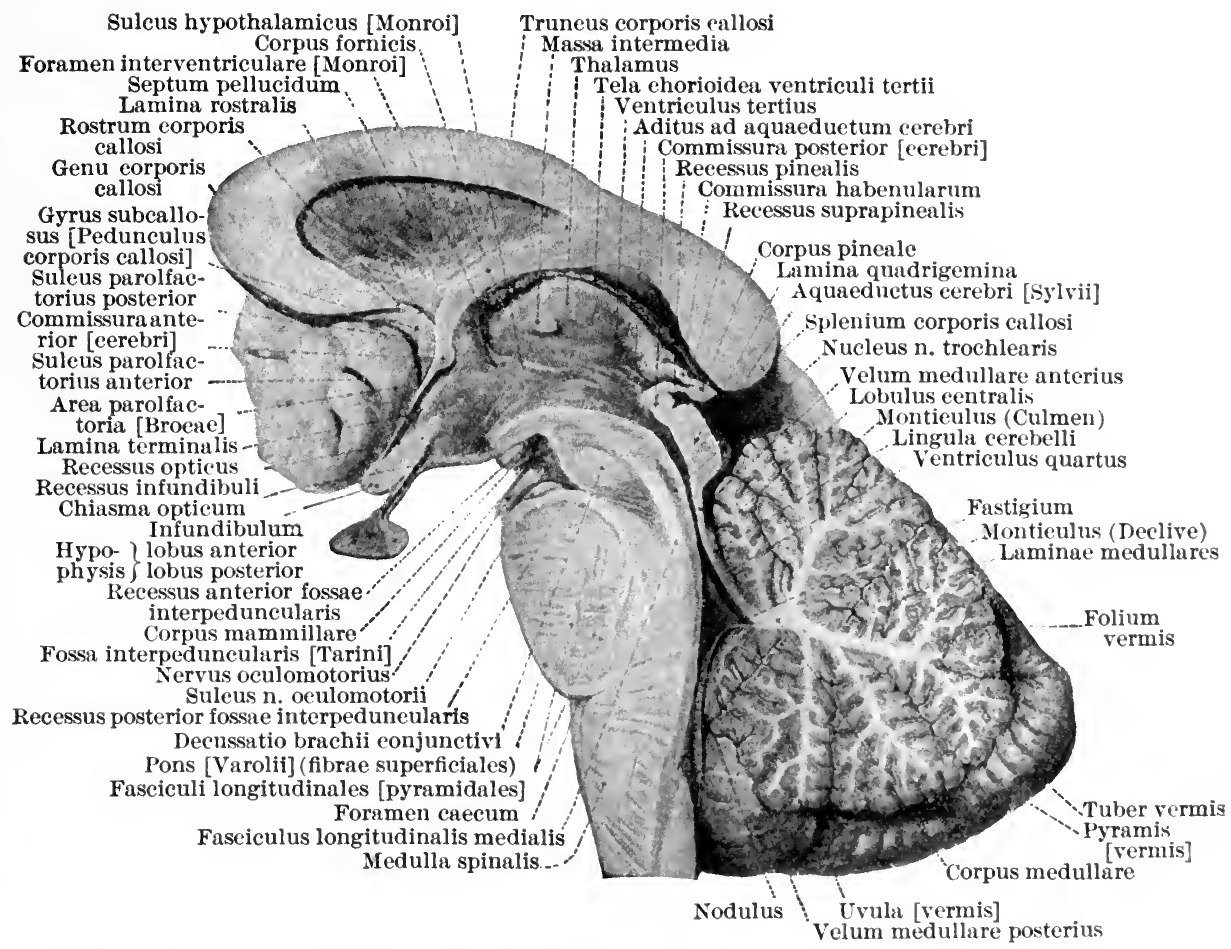

Median seetion of the brain-stem. Right half seen from the left. (Only a small part of the pallium is shown.) (After Spalteholz, Hand Atlas of Hum. Anat., Leipzig, 1903, p. 636, Fig. 695.)

\section{Isthmus of Rhombencephalon (Isthmus rhombencephali).}

(a) Brachium conjunctivum ("connecting arm") of cerebellum (brachium conjunctivum [cerebelli]) (O. T. superior cerebellar peduncle).

(b) Fillet or ribbon (lemniscus).

(ba) Lateral fillet (lemniscus lateralis).

(bb) Medial fillet (lemniscus medialis) (O. T. ribbon of Reil).

(bc) Trigone of fillet (trigonum lemnisci). 
(c) Anterior medullary velum (velum medullare anterius) (O. T. valve of Vieussens).

(ca) Frenulum ("rein" or "check-rein") of anterior medullary velum (frenulum veli medullaris anterioris).

\section{Midbrain (Mesencephalon).}

(a) Peduncle of cerebrum (pedunculus cerebri) (O. T. crus cerebri).

(aa) Aqueduct of cerebrum (aquaeductus cerebri [Sylvii]) (O. T. iter e tertio ad quartum ventriculum).

$(a b)$ Lateral sulcus (sulcus lateralis).

(ac) Sulcus of oculomotor nerve (sulcus $N$. oculomotorii).

(b) Quadrigeminal bodies (corpora quadrigemina) (O. T. optic lobes).

(ba) Quadrigeminal layer (lamina quadrigemina).

(bb) Superior hillock (colliculus superior) (O. T. anterior body or nates).

(bc) Inferior hillock (colliculus inferior) (O. T. posterior body or testis).

(bd) Superior quadrigeminal brachium (brachium quadrigeminum superius).

(be) Inferior quadrigeminal brachium (brachium quadrigeminum inferius).

FIG. 145.

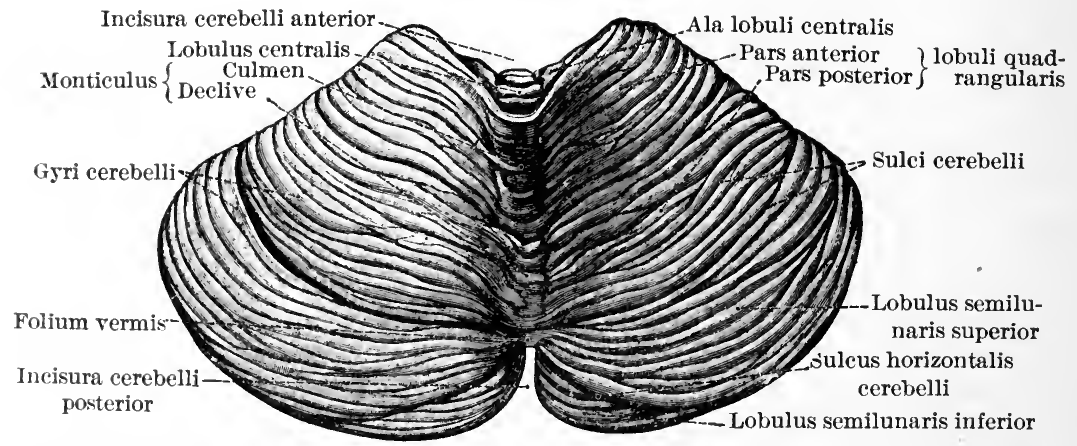

The upper surface of the cerebellum. (After Toldt, Anat. Atlas, Wien. 1903, 3 Aufl., p. 770,

Cerebellum. Fig. 1182.)

(a) Convolutions of cerebellum (gyri cerebelli).

(b) Sulci of cerebellum (sulci cerebelli).

(c) Cerebellar vallecula ("little valley") (vallecula cerebelli).

(d) Anterior notch of cerebellum (incisura cerebelli anterior) (O. T. semilunar notch).

(e) Posterior notch of cerebellum (incisura cerebelli posterior) ( $\mathrm{O}$. T. marsupial notch).

( $f$ ) Horizontal sulcus of cerebellum (sulcus horizontalis cerebelli) (O. T. great horizontal fissure).

(g) Transverse fissure of cerebellum (fissura transversa cerebelli).

(h) Vermis ("worm") (vermis).

(ha) Lingua ("tongue") of cerebellum (lingua cerebelli). 
(haa) Vineulum of the lingua ("tongue-band") (vinculum linguae cerebelli).

( $h b$ ) Central lobule (lobulus centralis) (O. T. lobus centralis).

(hc) Monticulus (monticulus) ("little mountain").

(hca) Culmen (culmen) ("summit").

(hcb) Declive (declive) ("slope or descent").

\section{FIG. 146.}

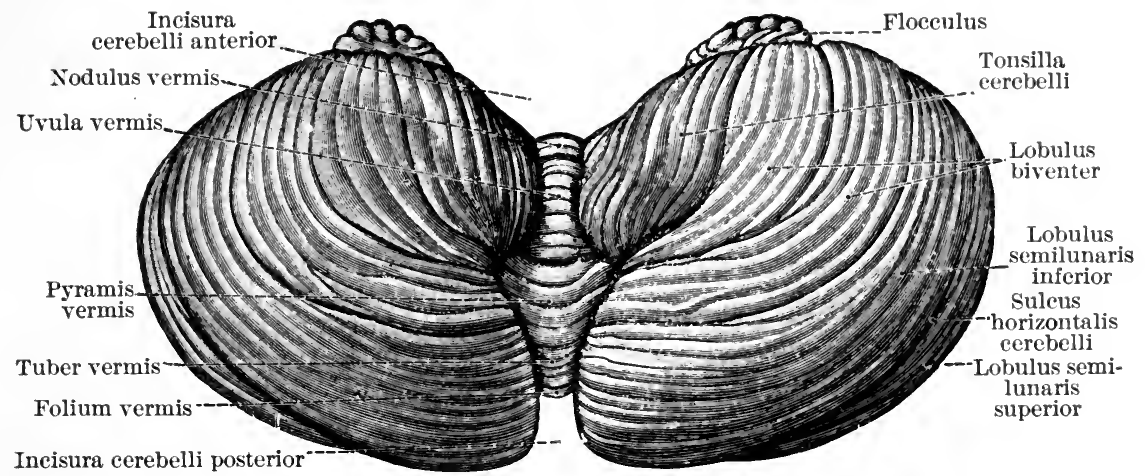

The lower surface of the cerebellum. (After Toldt, Anat. Atlas, Wien, 1903, 3 Aufl., p. 770, Fig. 1183.)

(hd) Folium vermis (folium vermis) ("leaflet of worm") (O. T. folium cacuminis).

(he) Tuber of vermis (tuber vermis) (O. T. tuber valvulus).

(hf) Pyramid of vermis (pyramis $[$ vermis]).

FIG. 147.

Vermis supcrior

Vellum medullare anterius $\quad$ Culmen

Ala lobuli centralis. Lingula cerebelli

Vinculum lingulae cerebelli. Brachium conjunetivum

$\begin{aligned} & \text { Hemisphaerium eerebelli } \\ & \text { (faeies superior) }\end{aligned}$
Flocculi secundarii

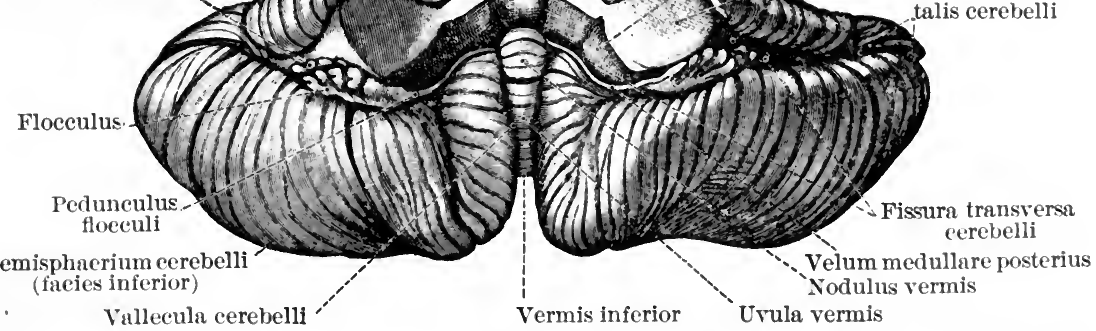

The anterior surface of the cerebellum. (After Toldt, Anat. Atlas, Wien, 1903, 3 Auf., p. 771, Fig. 1184.)

( $h g$ ) Uvula of vermis (uvula [vermis]).

(hh) Nodule (nodulus).

(i) Hemisphere of cerebellum (hemisphaerium cerebelli).

(ia) Superior surface (facies superior). 
(iaa) Wing of central lobule (ala lobuli centralis).

- (iab) Quadrangular lobule (lobulus quadrangularis) (O.

T. quadrate lobule).

(1) Anterior part (pars anterior),

(2) Posterior part (pars posterior),

(O. T. two anterior erescentic lobules).

(iac) Superior semilunar lobule (lobulus semilunaris superior) (O. T. posterior crescentic lobule).

(ib) Inferior surface (facies inferior).

(iba) Inferior semilunar lobule (lobulus semilunaris inferior) (O. T. postero-inferior lobule).

(ibb) Slender lobule (lobulus gracilis).

(ibc) Biventral lobule (lobulus biventer).

(ibd) Tonsil of cerebellum (tonsilla cerebelli).

(ibe) Floceulus (flocculus) ("wool-tuft-like body").

(1) Secondary flocculi (flocculi secundarii), inconstant.

(2) Peduncle of flocculus (pedunculus flocculi). (ibf) Nidus avis (nidus avis) ("bird's nest").

FIG. 148.

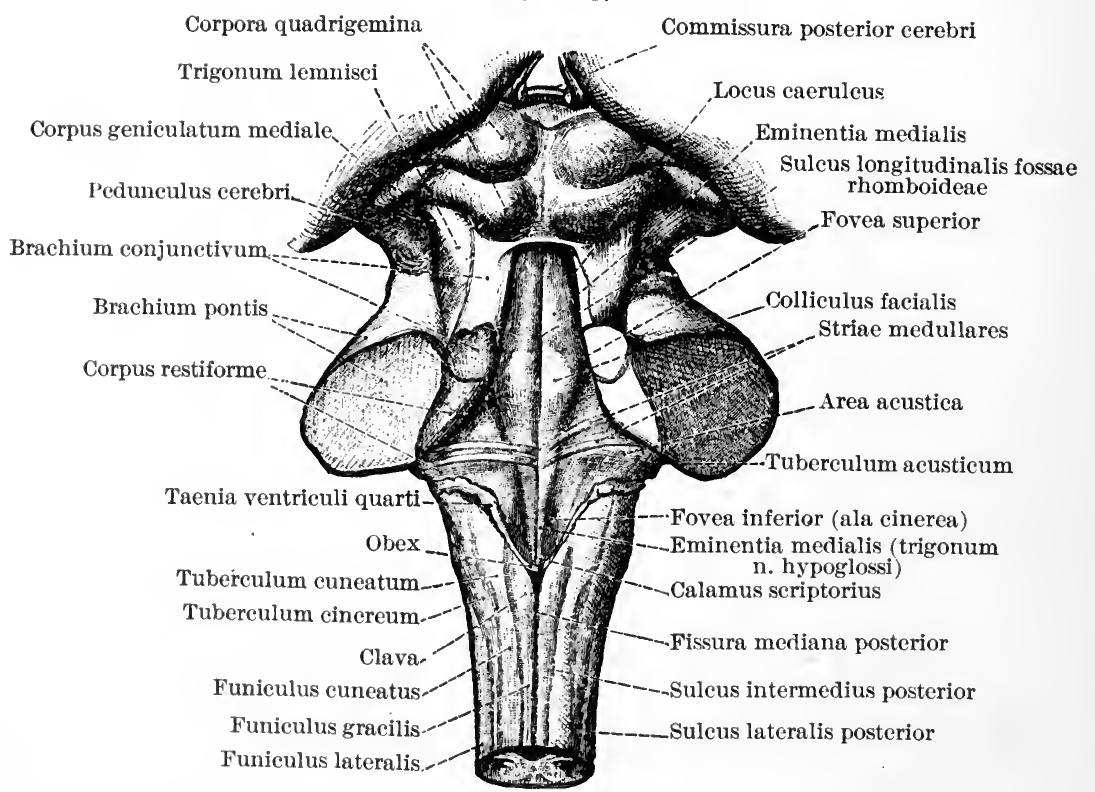

The dorsal surface of the medulla oblongata and midbrain and the floor of the fourth ventricle. The cerebellum has been removed. (After Toldt, Anat. Atlas, Wien, 1903, 3 Aufl, p. 768, Fig. 1178.)

\section{Fourth Ventricle (Ventriculus quartus).}

(a) Rhomboid fossa (fossa rhomboidea).

(aa) Inferior part (pars inferior fossae rhomboideae [calamus scriptorius]). 
(ab) Intermediate part (pars intermedia fossae rhomboideae). $(a b a)$ Lateral recess (recessus lateralis fossae rhomboideae).

(ac) Superior part (pars superior fossae rhomboideae).

(ad) Limiting groove (sulcus limitans [fossae rhomboideae]). (ada) Inferior pit (fovea inferior). ( $a d b)$ Superior pit (fovea superior).

(b) Trigone of hypoglossal nerve (trigonum N. hypoglossi).

(c) Medullary striae (striae medullares) (O. T. striae acusticae).

(d) Medial eminence (eminentia medialis) (O. T. eminentia teres).

(e) Facial hillock (colliculus facialis).

(f) Ash-like wing (ala cinerea) (O. T. trigonum vagi).

(g) Acoustic area (area acustica) (O. T. trigonum acustici).

(h) Locus caeruleus (locus caeruleus) ("blue place").

(i) Roof of fourth ventricle (tegmen ventriculi quarti).

(ia) Posterior medullary velum (velum medullare posterius).

(ib) Junction of epithelial part of roof with compact nerve substance (taenia ventriculi quarti). (iba) Obex (obex) ("bar").

(ic) Epithelial chorioid layer (lamina chorioidea epithelialis).

(ica) Median aperture (apertura mediana ventriculi quarti [foramen Magendii]).

(icb) Lateral aperture (apertura lateralis ventriculi quarti).

(id) Fastigium (fastigium) ("summit of roof").

\section{External Morphology of Forebrain (Prosencephalon).}

Cut cautiously through the remaining cerebral peduncle and place the rhombencephalon and the mesencephalon in preserving fluid for further study later. In the prosencephalon distinguish the following parts:

Forebrain (prosencephalon).

Interbrain (diencephalon).

Thalamic brain (thalamencephalon).

Mammillary part of hypothalamus (pars mammillaris hypothalami).

End-brain (telencephalon).

Hemisphere (hemisphaerium).

Optic part of hypothalamus (pars optica hypothalami).

Make three drawings of the half of the prosencephalon before you-

(a) Of medial surface (facies medialis cerebri). (Fig. 151.)

(b) Of convex surface (facies convexa cerebri). (Fig. 149.)

(c) Of base (basis cerebri). (Fig. 150.)

Take up systematically the study of the external morphology as follows: 


\section{End-brain (Telencephalon).}

Note that it consists of the optic part of the hypothalamus (pars optica hypothalami) and the hemisphere (hemisphaerium).

Hemisphere (Hemisphaerium).

This includes the brain-mantle (pallium), the olfactory brain (rhinencephalon), and the striate body (corpus striatum). The fissura longitudinalis cerebri was examined before the hemispheres were separated. Locate now the transverse fissure of the cerebrum (fissura transversa cerebri).

FIG. 149.

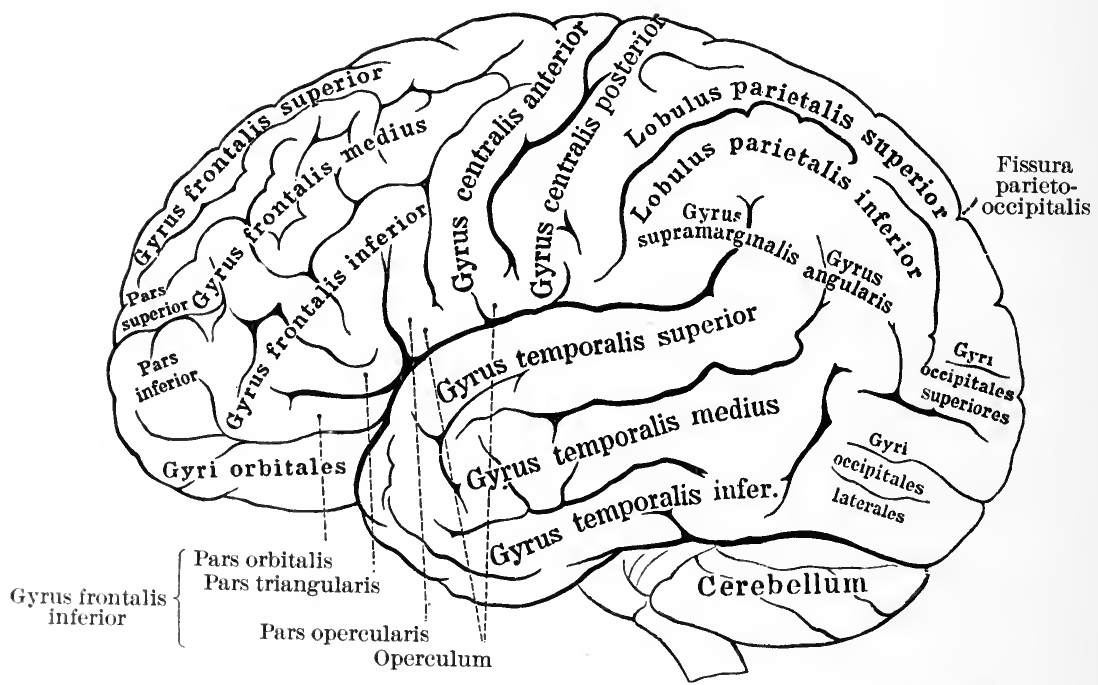

The left cerebral hemisphere. Lateral aspect. (After Toldt, Anat. Atlas, Wien, 1903, 3 Aufl., p. 777, Fig. 1194.)

\section{Brain-mantle (Pallium).}

Note its subdivision by fissures (fissurae cerebri) and grooves (sulci cerebri) into convolutions (gyri cerebri). What is the difference arbitrarily made between a "fissure" and a "sulcus"? Besides the main gyri, there are others in the depth (gyri profundi) and small gyri connecting adjacent gyri, the so-called gyri transitivi (O. T. annectent gyri). On the basis cerebri note the petrosal impression (impressio petrosa) due to the petrous portion of the temporal bone.

The student should begin his study of the topography of the pallium with a careful examination of the lateral fissure of the 
cerebrum (fissura cerebri lateralis [Sylvii]), ordinarily known as the "fissure of Sylvius." Besides its main trunk (truncus), observe-

(a) Posterior ramus (ramus posterior).

(b) Ascending anterior ramus (ramus anterior ascendens).

(c) Horizontal anterior ramus (ramus anterior horizontalis).

FIG. 150.

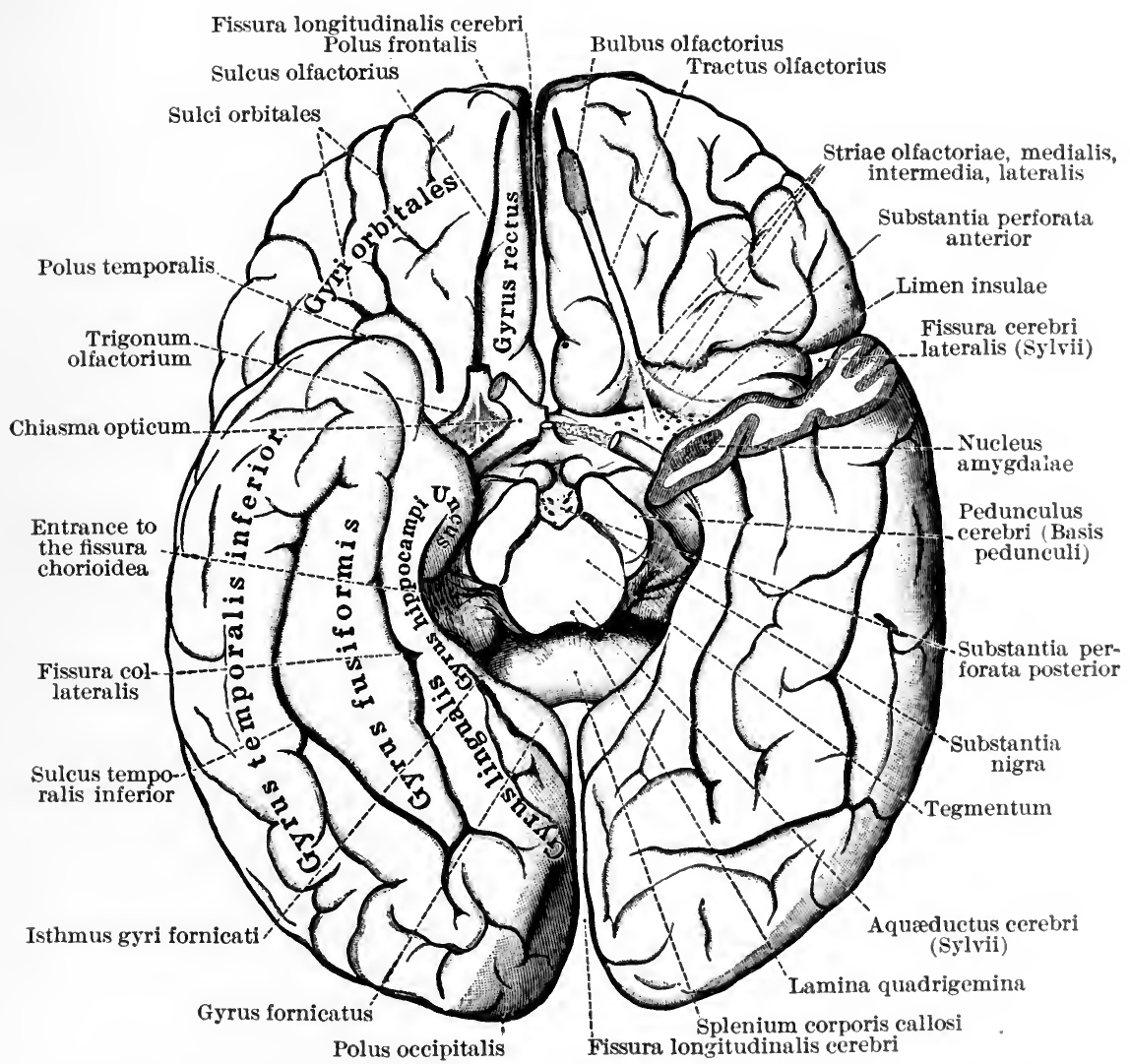

Basal surface of the cerebrum. (After Toldt, Anat. Atlas, Wien, 1903, 3 Aufl., p. 775, Fig. 1191.)

\section{Lobes of Cerebrum (Lobi cerebri) and their Boundaries.}

The pallium has been subdivided arbitrarily into five lobes:

(1) Frontal lobe (lobus frontalis).

(2) Parietal lobe (lobus parietalis).

(3) Temporal lobe (lobus temporalis).

(4) Occipital lobe (lobus occipitalis).

(5) Island of Reil (insula). 
These five lobes are bounded by six fissures:

(1) Fissure of Sylvius (fissura cerebri lateralis [Sylvii]).

(2) Central sulcus of Rolando (sulcus centralis [Rolandi]) (O. T. fissure of Rolando).

(3) Sulcus of cingulum (sulcus cinguli) (O. T. callosomarginal sulcus).

(4) Parieto-occipital fissure (fissura parietooccipitalis).

(5) Collateral fissure (fissura collateralis).

(6) Circular suleus of Reil (sulcus circularis [Reili]) (O. T. limiting sulcus of Reil).

\section{Frontal Lobe (Lobus frontalis).}

Find its posterior boundary on the convex surface by locating the sulcus centralis [Rolandi]; the latter is always between the two parallel, obliquely placed, central gyri. What is the inferior boundary of the frontal lobe on the convex surface? How is it bounded on the medial surface of the hemisphere? In the frontal lobe study the following:

(a) Frontal pole (polus frontalis).

(b) Anterior central gyrus (gyrus centralis anterior).

(c) Precentral sulcus (sulcus praecentralis).

(d) Superior frontal gyrus (gyrus frontalis superior).

(e) Superior frontal sulcus (sulcus frontalis superior).

( $f$ ) Middle frontal gyrus (gyrus frontalis medius).

(fa) Superior part (pars superior).

$(f b)$ Inferior part (pars inferior).

(g) Inferior frontal sulcus (sulcus frontalis inferior).

(h) Inferior frontal gyrus (gyrus frontalis inferior).

(ha) Opercular part (pars opercularis) (on the left side this constitutes the celebrated Broca's convolution, concerned in the function of speech).

(hb) Triangular part (pars triangularis).

(hc) Orbital part (pars orbitalis).

(i) Straight gyrus (gyrus rectus).

(j) Olfactory sulcus (sulcus olfactorius).

(k) Orbital gyri (gyri orbitales).

(l) Orbital sulci (sulci orbitales).

\section{Parietal Lobe (Lobus parietalis).}

How is it bounded-

(a) On the convex surface?

(b) On the medial surface?

Examine on the convex surface-

(a) Posterior central gyrus (gyrus centralis posterior) (O. T. ascending parietal convolution). 
(b) Interparietal sulcus (sulcus interparietalis) (O. T. intraparietal sulcus of Turner). Note the sulcus paroccipitalis of Wilder.

(c) Superior parietal lobule (lobulus parietalis superior).

(d) Inferior parietal lobule (lobulus parietalis inferior).

(da) Supramarginal gyrus (gyrus supramarginalis) around end of fissure of Sylvius.

(db) Angular gyrus (gyrus angularis), around end of sulcus temporalis superior.

The portion of the parietal lobe on the medial surface of the hemisphere will be studied later.

\section{Occipital Lobe (Lobus occipitalis).}

How is it bounded-

(a) On the convex surface?

(b) On the medial surface?

On the convex surface, examine-

(a) Transverse occipital sulcus (sulcus occipitalis transversus).

(b) Superior occipital gyri (gyri occipitales superiores).

(c) Superior occipital sulci (sulci occipitales superiores).

(d) Lateral occipital gyri (gyri occipitales laterales).

(e) Lateral occipital sulci (sulci occipitales laterales).

Locate the occipital pole (polus occipitalis).

The medial surface of the occipital lobe will be studied later.

\section{Temporal Lobe (Lobus temporalis).}

What are its boundaries? Observe the temporal pole (polus temporalis). Examine the following:

(a) Transverse temporal sulci (sulci temporales transversi).

(b) Transverse temporal gyri (gyri temporales transversi).

(c) Superior temporal gyrus (gyrus temporalis superior) (O. T. first temporal gyrus).

(d) Superior temporal sulcus (sulcus temporalis superior) (O. T. parallel sulcus, or first temporal sulcus).

(e) Middle temporal gyrus (gyrus temporalis medius) (O. T. second temporal gyrus).

( $f$ ) Middle temporal sulcus (sulcus temporalis medius) (O. T. second temporal sulcus).

(g) Inferior temporal gyrus (gyrus temporalis inferior) ( $O$. T. third temporal gyrus).

(h) Inferior temporal sulcus (sulcus temporalis inferior) (O. T. oceipitotemporal suleus).

(i) Fusiform gyrus (gyrus fusiformis) (O. T. occipitotemporal convolution).

(j) Lingual gyrus (gyrus lingualis). In some English books this is regarded as a part of the occipital rather than of the temporal lobe. 


\section{Medial Surface of Hemisphere (Facies medialis hemisphaerii).}

Here study-

(a) Sulcus of corpus callosum (sulcus corporis callosi) (O. T. callosal sulcus).

(b) Fissure of hippocampus (fissura hippocampi) (O. T. dentate fissure, or fissura dentata) (between gyrus hippocampi and fascia dentata).

(c) Fornicate gyrus (gyrus fornicatus) (O. T. limbic or falciform lobe). (ca) Gyrus of eingulum (gyrus cinguli) (O. T. callosal convolution, or gyrus fornicatus).

(cb) Gyrus of hippocampus (gyrus hippocampi) (O. T. hippocampal convolution ).

(cba) Hook of gyrus hippocampi (uncus gyri hippocampi) (O. T. uncinate gyrus).

$(c b b)$ White reticular substance of Arnold (substantia reticularis alba [Arnoldi]).

(cc) Isthmus of fornicate gyrus (isthmus gyri fornicati).

FIG. 151.

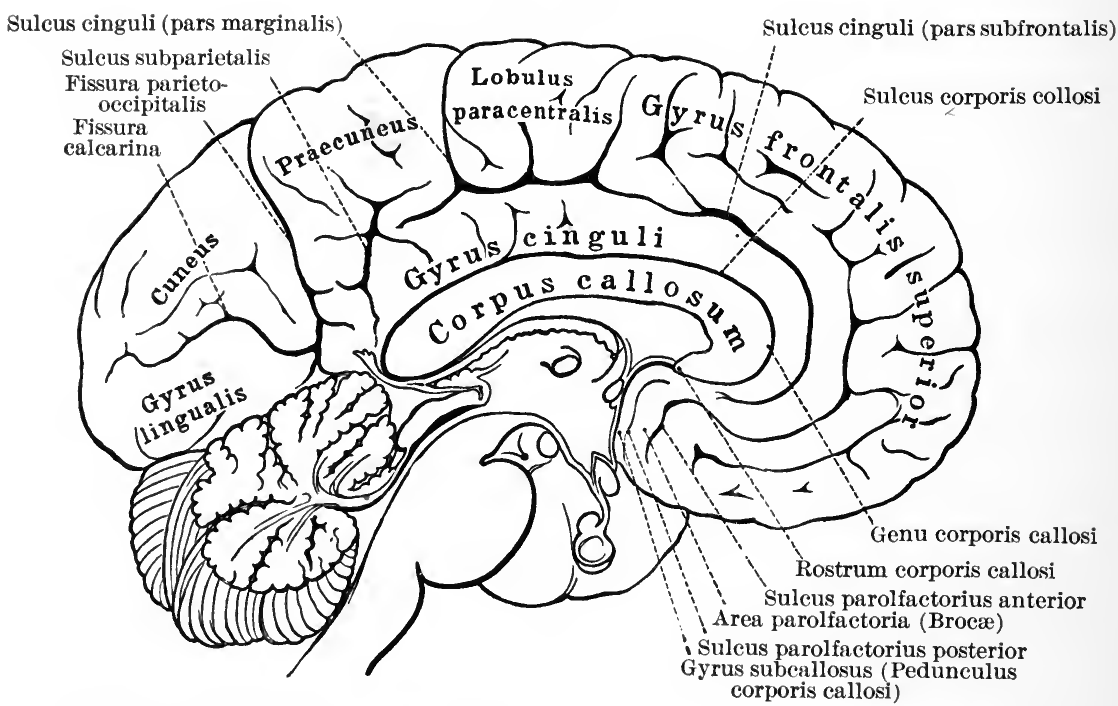

The left cerebral hemisphere. Medial aspect. (After Toldt, Anat. Atlas, Wien, 1903, 3 Aufl., p. 777, Fig. 1195)

(d) Sulcus of cingulum (sulcus cinguli) (O. T. callosomarginal fissure).

(da) Subfrontal part (pars subfrontalis).

(db) Marginal part (pars marginalis).

(e) Subparietal sulcus (sulcus subparietalis).

(f) Paracentral lobule (lobulus paracentralis). This belongs to the frontal lobe.

(g) Precuneus (praecuneus). This belongs to the parietal lobe. 
(h) Parieto-occipital fissure (fissura parietooccipitalis).

(i) Cuneus (cuneus) ("wedge"). This belongs to the occipital lobe.

(j) Calcarine fissure (fissura calcarina).

\section{Island (Insula) (O. T. Island of Reil, or Central Lobe).}

Pull the lips of the Sylvian fissure apart and examine the island. If possible, study the island on a fresh brain or on one prepared especially to show the island. How is it bounded? Examine carefully the overhanging operculum, and note that it is subdivisible into a frontal part (pars frontalis), a parietal part (pars parietalis), and a temporal part (pars temporalis). In the island study-

(a) Sulci of island (sulci insulae).

(b) Gyri of island (gyri insulae).

(ba) Long gyrus of island (gyrus longus insulae).

(bb) Short gyri of island (gyri breves insulae).

\section{Olfactory Brain (Rhinencephalon). (Vide Fig. I5I.) \\ Examine-}

(a) Anterior parolfactory sulcus (sulcus parolfactorius anterior).

(b) Anterior part of rhinencephalon (pars anterior [rhinencephali]).

(ba) Olfactory lobe (lobus olfactorius).

(baa) Olfactory bulb (bulbus olfactorius).

(bab) Olfactory tract (tractus olfactorius) (incorrectly sometimes designated "olfactory nerve").

(bac) Olfactory trigone (trigonum olfactorium).

(bad) Medial stria (stria medialis).

(bae) Intermediate stria (stria intermedia).

(bb) Parolfactory area of Broca (area parolfactoria [Brocae]).

(c) Posterior parolfactory sulcus (sulcus parolfactorius posterior).

(d) Posterior part of rhinencephalon (pars posterior [rhinencephali]).

(da) Subcallosal gyrus (gyrus subcallosus [pedunculus corporis callosi]).

(daa) Anterior perforated substance (substantia perforata anterior).

(db) Lateral olfactory stria (stria olfactoria lateralis).

(dc) Threshold of island (limen insulae).

Note that, in the broader sense, the gyrus fornicatus, fimbria and fascia dentata hippocampi, fornix, and corpora mammillaria are often classed as belonging to the rhinencephalon. In the fissura hippocampi note the fimbria hippocampi and the fascia dentata hippocampi (O. T. gyrus dentatus), lying side by side. The handle of the scalpel shoved deeply into the brain just above the fimbria would enter the inferior cornu of the lateral ventricle. 


\section{Hypothalamus (O. T. Subthalamic Region).}

Note that it is subdivisible into-

(a) Mammillary part of hypothalamus (pars mammillaris hypothalami).

This belongs to the diencephalon and includes-

(aa) Mammillary body (corpus mammillare) on each side.

(b) Optic part of hypothalamus (pars optica hypothalami). This belongs

to the telencephalon and includes-

(ba) Tuber cinereum (tuber cinereum) ("ash-like tuber").

(bb) Infundibulum (infundibulum) ("funnel").

(bc) Hypophysis (O. T. pituitary body). Cut through this in the median sagittal plane and note-

(bca) Anterior lobe (lobus anterior), from the hypoblast.

(bcb) Posterior lobe (lobus posterior), from the epiblast.

(bd) Optic tract (tractus opticus).

$(b d a)$ Medial root (radix medialis).

. (bdb) Lateral root (radix lateralis).

(be) Optic chiasm (chiasma opticum).

(bf) Terminal lamina (lamina terminalis).

\section{Corpus Callosum (Great Transverse Commissure of Cerebrum).}

With a sharp, thin, broad brain-knife, the upper part of the right hemisphere should be sliced off at the level of the sulcus cinguli. The cortical substance (substantia corticalis) and semioval centre (centrum semiovale) of the hemisphere are thus beautifully displayed. Cut transversely through the middle of the gyrus cinguli; insinuate the fingers cautiously beneath it and tear it away lateralward, observing how the fibres of the corpus callosum enter the hemisphere. These fibres constitute the radiation of the corpus callosum (radiatio corporis callosi). This radiation is subdivisible into a frontal part (pars frontalis) (O. T. forceps minor), a parietal part (pars parietalis), a temporal part (pars temporalis), an occipital part (pars occipitalis) (O. T. forceps major), and the "tapestry" (tapetum), the layer of fibres coming from the truncus corporis callosi and curving lateralward and downward over the inferior and posterior horns of the lateral ventricle. In the gyrus cinguli, just torn away, note on the deep surface a very definite bundle of sagittally directed arcuate fibres, easily lifted out of the bed in which it lies. This is the cingulum or "girdle," an association bundle pertaining to the rhinencephalon. (See full description in Barker's Nervous System, p. 1061.) Examine-

(a) Splenium ("bandage") of corpus callosum (splenium corporis callosi).

(b) Trunk of corpus callosum (truncus corporis callosi) (O. T. body).

(c) Knee of corpus callosum (genu corporis callosi). 
(d) Beak of corpus callosum (rostrum corporis callosi).

(da) Rostral lamina (lamina rostralis).

(e) Transverse striae (striae transversae).

(f) Medial longitudinal stria (stria longitudinalis medialis).

(g) Lateral longitudinal stria (stria longitudinalis lateralis).

(h) Fasciola cinerea (fasciola cinerea) (" ash-like little bandage").

Lateral Ventricle (Ventriculus lateralis).

Make a sagittal incision through corpus callosum one centimetre from sagittal median plane; the part of the corpus callosum lateral from the incision is to be reflected lateralward and removed; leave the part of the corpus callosum medial from the incision in place. Note the relation of the splenium corporis

FIG. 152.

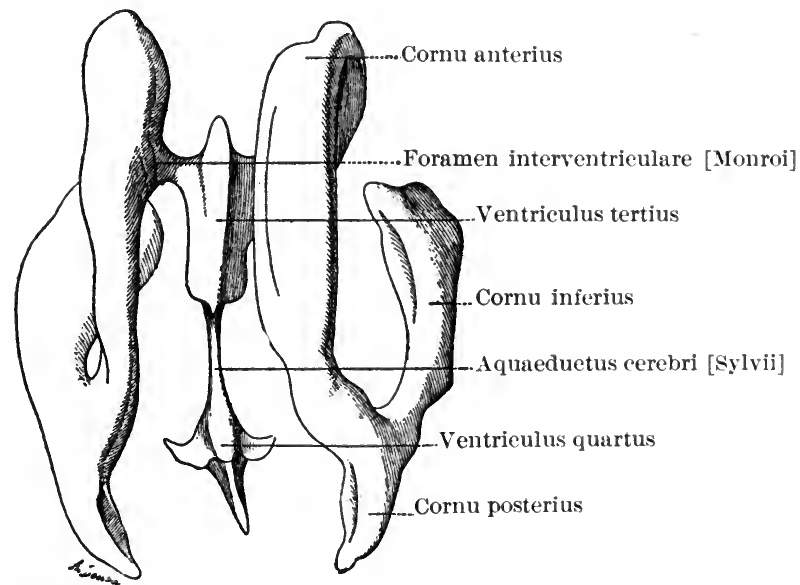

Cast of the ventricles. (After Welcker.) (From Poirier et Charpy, Traité d'Anat. hum., Paris, 1901, 2 ed., t. iii., 1, p. 365, Fig. 260.)

callosi to the pars occipitalis of the radiatio corporis callosi, or forceps major; leave the latter in place.

The central part and anterior cornu of the lateral ventricle are now exposed. Make a cut backward and lateralward through the white matter which forms the roof of the posterior horn and excise enough of this roof to permit of thorough inspection of the cavity. To open the inferior horn, place the tip of the knife in the central part of the ventricle at the entrance to the inferior horn and make a cut downward and forward through the outer part of the temporal lobe towards the polus temporalis, following the course of the inferior horn, which runs nearly parallel to the sulcus temporalis superior. Now remove the part of the 
temporal lobe above this incision, including the pars temporalis of the operculum; take care not to injure the island.

Observe the general form, and study the walls, of the various subdivisions of the lateral ventricle; compare your observations with atlases, models, and text-book descriptions.

FIG. 153.

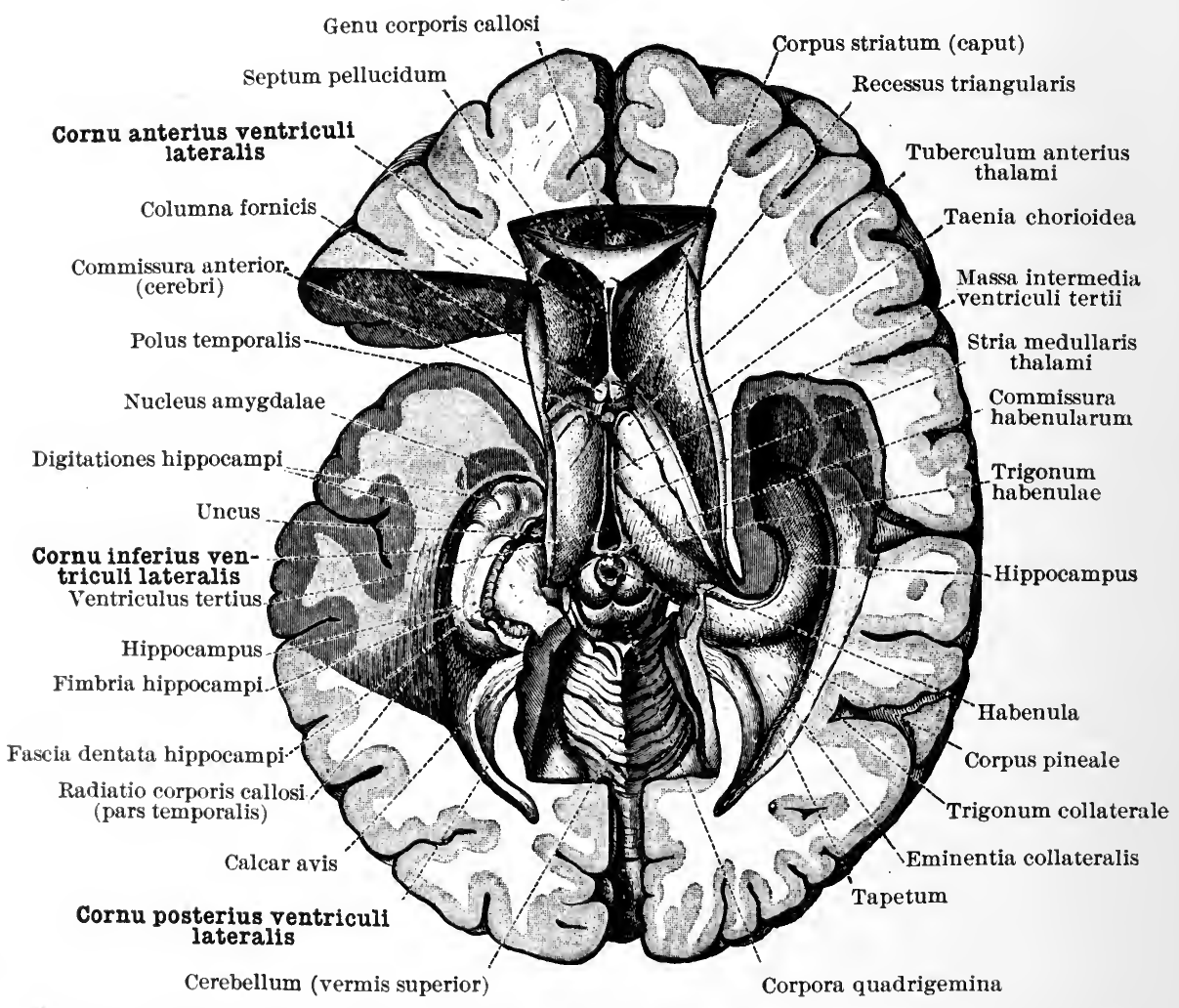

The ventricles of the brain shown completely opened from above. The upper part of the cerebral hemispheres, the corpus callosum, the fornix, and the tela chorioidea have been removed, and the corpora quadrigemina, corpus pineale, and vermis superior laid bare. (After Toldt, Anat. Atlas, Wien, 1903, 3 Aufl., p. 782, Fig. 1200.)

(a) Head of caudate nuclens (caput nuclei caudati).

(b) Anterior horn (cornu anterius).

(c) Posterior horn (cornu posterius).

(d) Inferior horn (cornu inferius) (O. T. descending horn).

In the anterior horn study-

(a) Head of candate nucleus (caput nuclei caudati).

In the central part of the ventricle study- 
(a) Caudate nucleus (nucleus caudatus).

(b) Terminal stria (stria terminalis) (O. T. taenia semicircularis).

(c) Terminal vein (vena terminalis) ( $O$. T. vein of the corpus striatum).

(d) Lamina affixa (lamina affixa) ("fastened layer").

('The embryology of the part must be studied, in order to understand the origin of this structure.)

(e) Chorioid plexus of lateral ventricle (plexus chorioideus ventriculi lateralis).

( $f$ ) Epithelial chorioid layer (lamina chorioidea epithelialis).

(g) Chorioid taenia (taenia chorioidea).

(h) Thalamus (thalamus) (showing through).

(i) Taenia of fornix (taenia fornicis) (O. T. sharp edge of fornix).

Fig. 154.

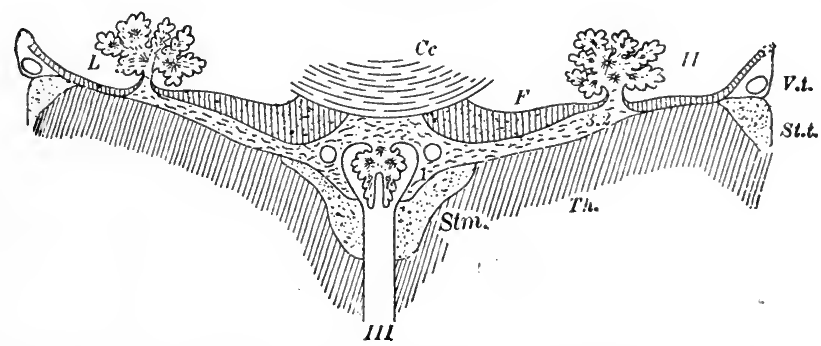

Transverse section through the tela chorioidea, ventriculi tertii, and adjacent parts.

$I I$, lateral ventricle; $I I I$, third ventricle; $C c$, corpus callosum; $F$, fornix ; $T h$, thalamus; St. $m$, stria medullaris; St. $t$, stria terminalis; $V . t$, vena terminalis; $L$, lamina affixa; 1 , taenia thalami ; 2 , taenia chorioidea; $s$, taenia fornicis. The figure shows the continuity of the taeniae and the epithelial layer of the plexus chorioidea. (After His, Die Anat. Nomenclatur, Leipzig, 1895, p. 166, Fig. 21.)

\section{In the posterior horn of the ventricle study-}

(a) Roof and lateral wall formed by tapetum.

(b) Two ridges on medial wall.

(ba) Upper ridge $=$ bulb of posterior horn (bulbus cornu posterioris), due to pars occipitalis of radiatio corporis callosi, the so-called "forceps major," hooking around the fissura parieto-occipitalis.

( $b b)$ Lower ridge $=$ calcar avis (calcar aris) ("cock's spur") $(0$. T. hippocampus minor), due to fissura calcarina.

Before studying the inferior horn of the ventricle in detail, insert the fingers cautiously beneath the pars frontalis and pars parietalis of the operculum of the island, and tear away the cortex. This exposes the island thoroughly, and its relations to the lateral ventricle can be examined.

In the inferior horn of the ventricle study-

(a) Its narrow inferior wall.

(aa) Collateral eminence (eminentia collateralis), corresponding to the fissura collateralis; it is sometimes absent. 
(aaa) Collateral trigone (trigonum collaterale) ( $0 . \mathrm{T}$. trigonum ventriculi).

(b) Its lateral and superior walls.

(ba) Tapetum.

(bb) Lowermost end of striate body (corpus striatum).

(bc) Swelling due to amygdaloid nucleus (nucleus amygdalae).

(bd) Terminal stria (stria terminalis).

(be) Tail of caudate nucleus (cauda nuclei caudati).

Fig. 155.

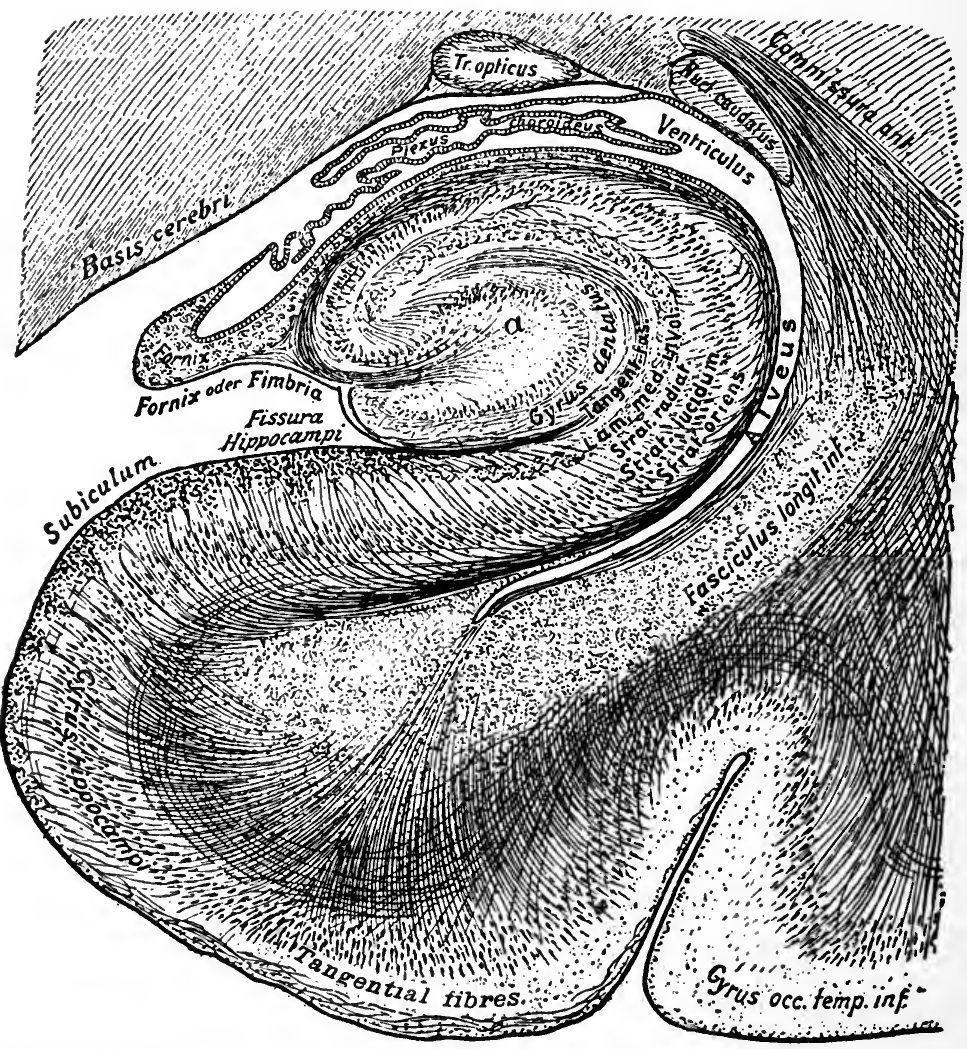

Section through the base of the brain and the hippocampus lying beneath it. Plexus chorioideus made simpler than the actual. . (After L. Edinger, Nervöse Centralorgane, V. Aufl., Leipzig, 1896, S. 225. Fig. 154.)

(c) Its medial wall.

(ca) Hippocampus (hippocampus) ("sea-horse") (O. T. hippocampus major, cornu Ammonis, or Ammon's horn). It corresponds to the fissura hippocampi of the facies medialis hemisphaerii.

(caa) Digitations of the hippocampus (digitationes hippocampi) (O. T. pes hippocampi). 
(cb) Chorioid plexus (plexus chorioideus ventriculi lateralis). The fimbria hippocampi and fascia dentata hippocampi have already been examined. The edge of the fimbria after tearing away the chorioid plexus is called the taenia fimbriae.

The student should next remove the remains of the right temporal and occipital lobes; cut through $(a)$ the fimbria hippocampi, where it passes into the crus fornicis, and $(b)$ the occipital part of the radiation of the corpus callosum ( $O$. T . forceps major). Next make an incision from the anterior end of the cornu inferius forward, above the level of the uncus, through the polus temporalis. Now separate the lobus temporalis, with the gyrus hippocampi medial to it, from the rest of the hemisphere, the separation taking place along the line of the inferior part of the fissura transversa cerebri. Cut away enough of the lateral edge of the remaining medial part of the corpus callosum to permit of a good view of the septum pellucidum and the fornix, lying beneath it. Having noted their relations, cut across the remains of corpus callosum behind the genu, gently raise the truncus corporis callosi, and dissect backward, freeing it from the septum pellucidum and, further back, from the fornix.

Septum pellucidum.

(a) Layer of septum pellucidum (lamina septi pellucidi).

(b) Cavity of septum pellucidum (cavum septi pellucidi) (O. T. fifth ventricle).

Fornix.

(a) Body of fornix (corpus fornicis).

(aa) Taenia of fornix (taenia fornicis).

(b) Crus of fornix (crus fornicis) (O. T. posterior pillar of fornix).

(c) Column of fornix (columna fornicis) ( 0 . T. anterior pillar of fornix).

(ca) Free part (pars libera columnae fornicis).

(cb) Covered part (pars tecta columnae fornicis).

Next cut transversely through the corpus fornicis at its middle and gently reflect the two ends forward and backward respectively. Running medialward from the crus fornicis, inferior to the corpus callosum, towards the crus fornicis of the opposite side, observe the commissure of the hippocampus (commissura hippocampi) (O. T. lyra or lyre of David). The space between the commissura hippocampi and the inferior surface of the corpus callosum has been called "Verga's ventricle." One-half of the so-called chorioid tela of the third ventricle (tela chorioidea ventriculi tertii) (O. T. velum interpositum) is now 


\section{exposed; note how it is formed by the pia mater encephali} coming in through the fissura transversa cerebri. In connection with it, study-

(a) Upper lamella, next to inferior surface of corpus callosum and fornix.

(b) Lower lamella, over the surface of the thalanus.

$(c)$ Between $(a)$ and $(b)$ some loose connective tissue, a continuation of the subarachnoideal connective tissue of the cisterna venae cerebri magnae. In this study-

Fig. 156.

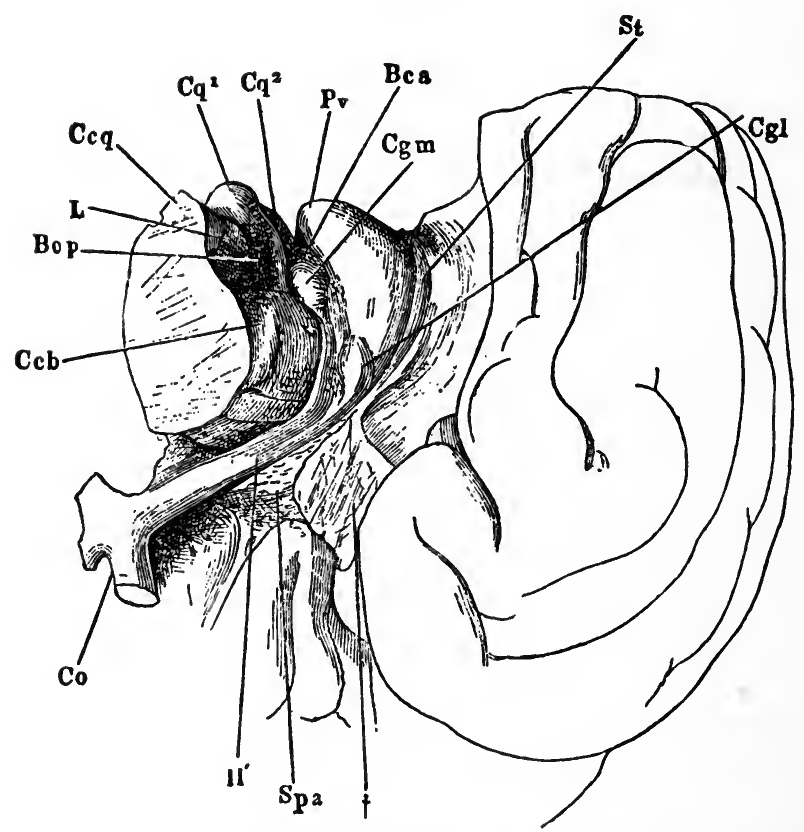

A portion of the right cerebral hemisphere resting on the polus frontalis, to illustrate the basis cerebri.

$B c a$, braehium quadrigeminum superius ; $B c p$, brachium quadrigeminum inferius ; $C c b$, pedunculus cerebri ; $C g l$, corpus geniculatum laterale; $C g m$, eorpus geniculatum mediale ; $C c q$, brachium conjunctivum; $C o$, chiasma opticum; $C q^{1}$, colliculus inferior; $C q^{2}$, colliculus superior; $L$, lemniscus; $P v$, pulvinar of thalamus; Spa, substantia perforata anterior; $S t$, stria terminalis; $I I^{\prime}$, tractus opticus. The radix medialis and the radix lateralis are well illustrated; $\dagger$, eut surface of tip of temporal lobe which has been removed. (After J. Henle, Handbuch der Nervenlehre des Menschen, II. Aufl., Braunsch., 1879, S. 155, Fig. 80.)

(ca) The two internal veins of the cerebrum (venae cerebri internae) (O. T. veins of Galen), and their tributaries-

(caa) Vein of septum pellucidum (V. septi pellucidi).

(cab) Terminal vein (V. terminalis) (O. T. vein of the corpus striatum).

$(c a c)$ Chorioid vein ( $V$. chorioidea).

The venae cerebri internae unite to form the 
large vein of the cerebrum (vena magna cerebri [Galeni]), which in turn opens into the straight sinus (sinus rectus).

(d) Chorioid plexus of lateral ventricle (plexus chorioideus ventriculi lateralis).

Fig. 157.

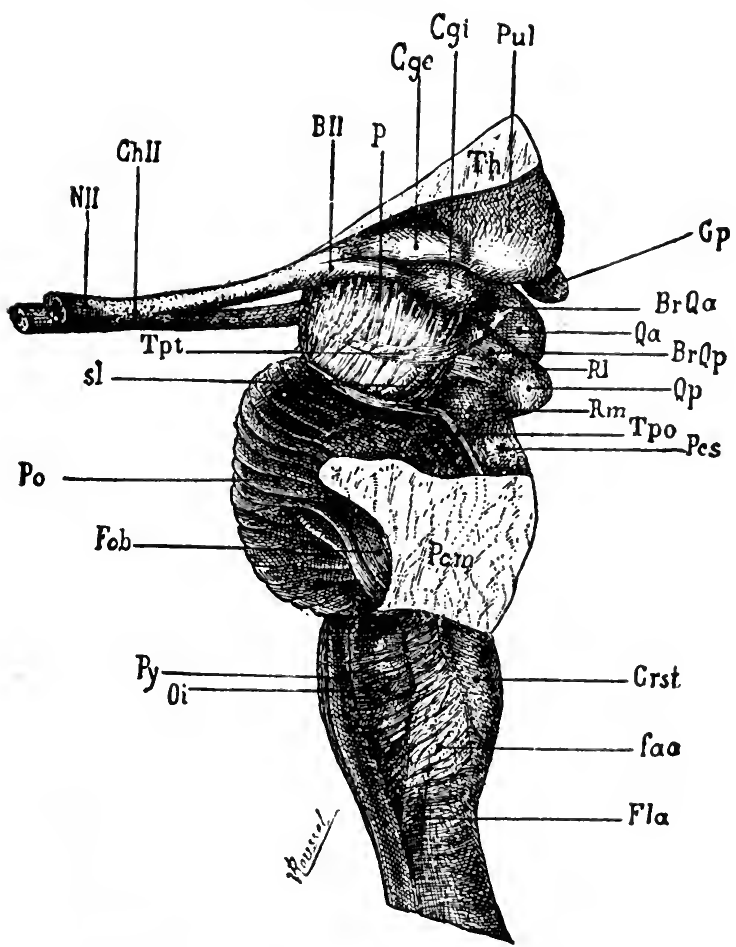

Isthmus rhombencephali seen in profile.

$B I I$, tractus opticus; $B r Q q$, brachium quadrigeminum superius; $B r Q p$, brachium quadrigeminum inferius ; $C g e$, corpus geniculatum laterale ; $C g i$, corpus geniculatum mediale ; $C h I I$, chiasma opticum; Crst, corpus restiforme; $F l a$, funiculus lateralis of medulla oblongata; faa, fibrac arcuatae externae; $F o b$, fibrae superficiales pontis ; $C p$, corpus pineale ; $N I I$, N. opticus; $O i$, oliva ; $P$, pedunculus cerebri ; $P c m$, brachium pontis ; $P c s$, brachium conjunctivum ; Po, pons Varoli ; Pul, pulvinar; $P y$, pyramis ; $Q a$, colliculus superior; $Q p$, colliculus inferior; $R l$, trigonum lemnisei; $R m$, lemniscus medialis; $s l$, sulcus lateralis; $T h$, thalamus; Tpo, taenia pontis; $T p t$, tractus peduncularis transversus. (After J. Déjerine, Anatomie des centres nerveux, t. 1., Paris, 1895, p. 328, Fig. 192.)

(da) Chorioid skein (glomus chorioideum).

Now eut through the $\mathrm{V}$. terminalis at its junction with the V. cerebri interna, seize the apex of the right half of the tela chorioidea ventriculi tertii, and pull it with the chorioid plexuses backward, but be careful not to tear away the corpus pineale beneath it behind. Examine-

(e) Chorioid plexus of third ventricle (plexus chorioideus ventriculi tertii). 


\section{Thalamencephalon.}

This part of the diencephalon includes (1) the thalamus, (2) the metathalamus, and (3) the epithalamus.

\section{Thalamus (O. T. Optic Thalamus).}

The upper and medial surfaces of the thalamus may now be examined. Observe-

(a) Pulvinar (pulvinar) ("cushion").

(b) Anterior tubercle of the thalamus (tuberculum anterius thalami).

(c) Medullary stria (stria medullaris) (O. T. stria fornicis or stria pinealis).

(d) Epithelial chorioid layer (lamina chorioidea epithelialis).

This covers the thalamus where it appears to be, but is not, in the lateral ventricle.

\section{Metathalamus.}

Behind and beneath the thalamus observe-

(a) Medial geniculate body (corpus geniculatum mediale) (O. T. internal geniculate body). This has to do chiefly with the auditory conduction path.

(b) Lateral geniculate body (corpus geniculatum laterale) (O. T. external geniculate body). This has to do with the visual conduction path.

\section{Epithalamus.}

Here the student should study-

(a) Pineal body (corpus pineale) (O. T. conarium, or epiphysis cerebri).

(b) Pineal recess (recessus pinealis).

(c) Suprapineal recess (recessus suprapinealis).

(d) Habenula (O. T. peduncle of the pineal body).

(e) Commissure of the habenulae (commissura habenularum).

(f) Trigone of the habenula (trigonum habenulae).

\section{Third Ventricle (Ventriculus tertius).}

This has been cut in two in the median plane, and its roof, tela chorioidea ventriculi tertii, with the lamina chorioidea epithelialis, has been removed. The student should now study the following structures and openings :

(a) Entrance to the aqueduct of the cerebrum (aditus ad aquaeductum cerebri).

(b) Posterior commissure of the cerebrum (commissura posterior [cerebri]) (above the aditus ad aquaeductus cerebri).

(c) Interventricular foramen of Monro (foramen interventriculare [Monroi]). One on each side, connecting ventriculus tertius with ventriculus lateralis. 
(d) Hypothalamic suleus of Monro (sulcus hypothalamicus [Monroi]). Note the importance of this in connection with the embryology of the brain. Compare with the suleus limitans in the floor of the fourth ventricle.

(e) Intermediate mass (massa intermedia) (O. T. middle or soft or gray commissure).

(f) Optic recess (recessus opticus). (Cf. W. His's models of developing brain.)

(g) Recess of infundibulum (recessus infundibuli).

(h) Anterior commissure of cerebrum (commissura anterior [cerebri]).

(i) Triangular recess (recessus triangularis).

\section{SECTIONS THROUGH BRAIN (SECTIONES ENCEPHALI).}

\section{Sections of Medulla Oblongata (Sectiones medullae oblongatae).}

Make three transverse sections through the medulla oblongata: (1) at the level of the decussation of the pyramids; (2) between the decussation of the pyramids and the olives; (3)

FIG. 158.

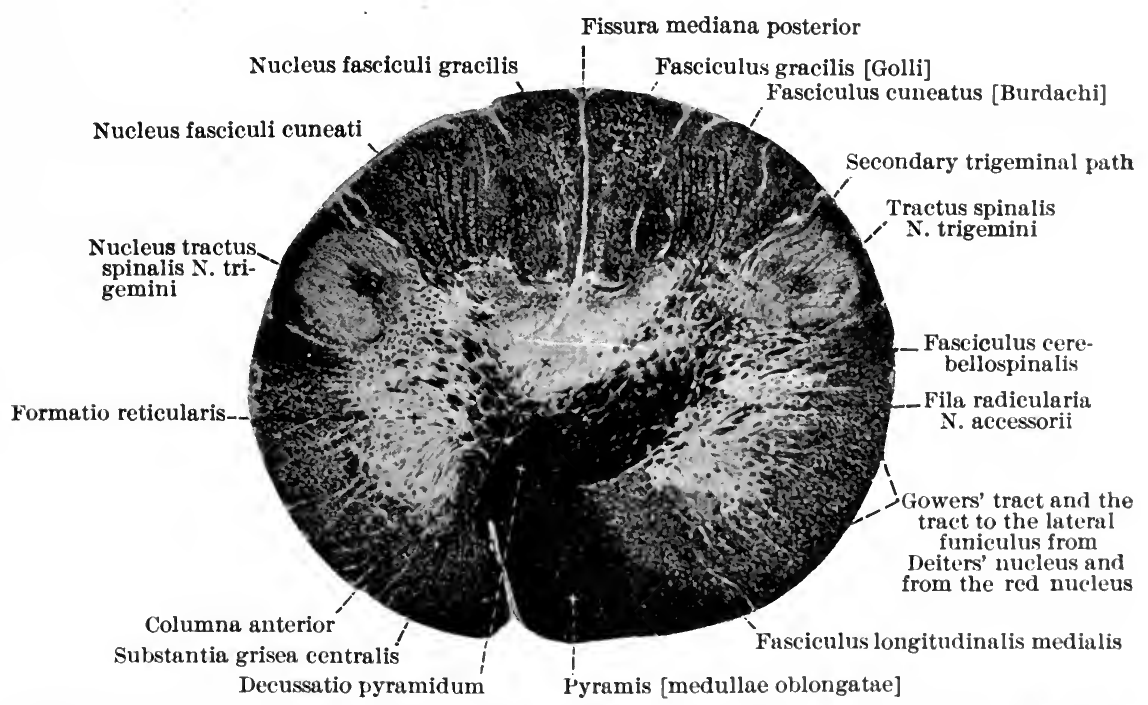

Transverse section of the brain-stem of an adult, through the decussation of the pyramids. Magnificaation : 6:1. (After Spalteholz, Hand Atlas of Hum. Anat., Leipzig, 1903, vol. iii., p. 656, Fig. 725.)

through the middle of the olives. (Cf. Spalteholz, Fig. 723.) Examine first the unstained cross-sections and subsequently sections, from the same levels, stained by Weigert's method. ${ }^{1}$ Use

${ }^{1}$ The study is much simplified if sections through the brain-stem of a newborn babe are used; see Figs. 162-169. 
a hand-lens or dissecting microscope. Make drawings indicating-

(1) Median raphe (raphe).

(2) Nuclear layer (stratum nucleare).

(3) Nucleus of hypoglossal nerve (nucleus $N$. hypoglossi).

(4) Ambiguous nucleus (nucleus ambiguus).

(5) Nucleus of ala cinerea (nucleus alae cinereae).

(6) Solitary tract (tractus solitarius) (O. T. respiratory bundle).

(a) Nucleus of solitary tract (nucleus tractus solitarii).

Fig. 159.

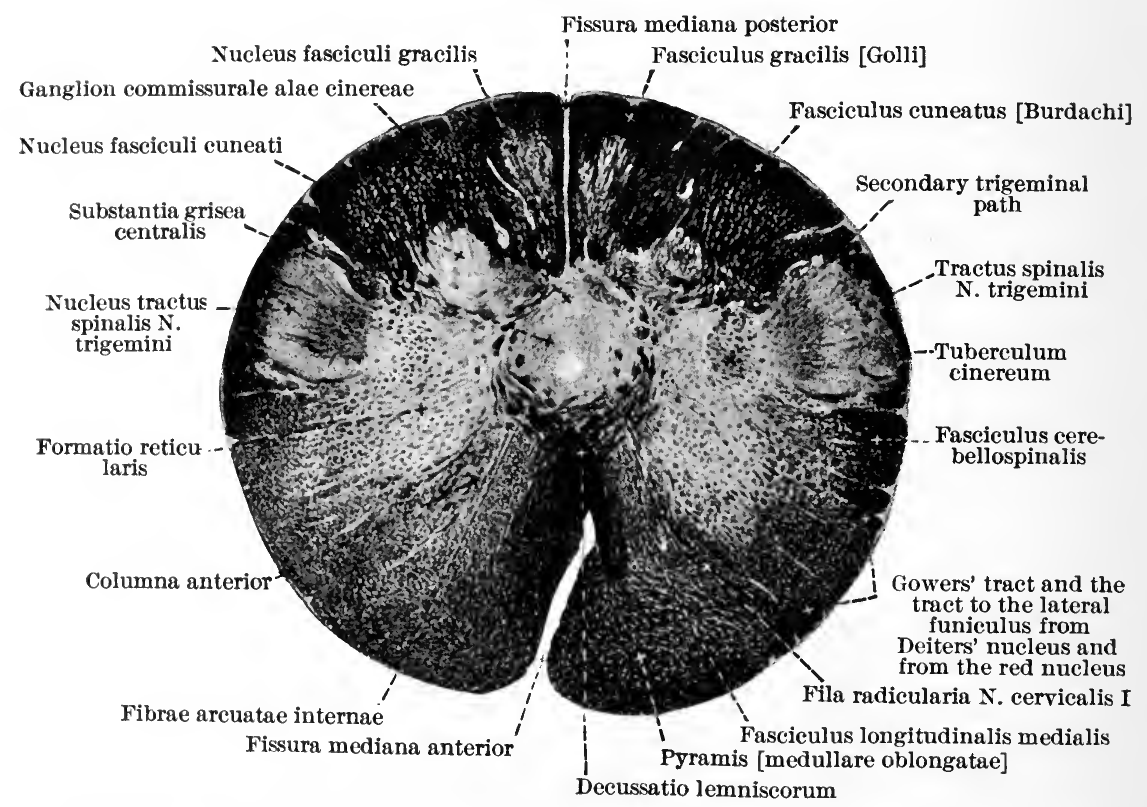

Transverse section through the brain-stem of an adult, between the decussation of the pyramids and the olives. Magnification: 6:1. (Held.) (After Spaltcholz, Hand Atlas of Hum. Anat., Leipzig, 1903, vol. iii., p. 656, Fig. 726.)

(7) Spinal tract of trigeminal nerve (tractus spinalis N. trigemini) (O. $T$. ascending root of trigeminal nerve - a very bad name, for the fibres descend).

(a) Nucleus of spinal tract of trigeminal nerve (nucleus tractus spinalis $N$. trigemini).

(8) Nucleus of slender funiculus (nucleus funiculi gracilis) (O. T. nucleus of Goll's column).

(9) Nucleus of wedge-shaped funiculus (nucleus funiculi cuneati) (O. T. nueleus of Burdach's column).

(10) Lateral nuelei (nuclei laterales).

(11) Inferior olivary nucleus (nucleus olivaris inferior).

(a) Hilus of olivary nucleus (hilus nuclei olivaris).

(b) Medial accessory olivary nucleus (nucleus olivaris accessorius medialis). 
FIG. 160.

Area acustica Taenia ventriculi quarti

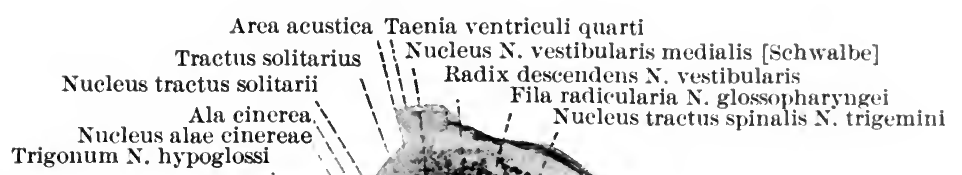
Nucleus alae einere
Trigonum $\mathrm{N}$. hypoglossi

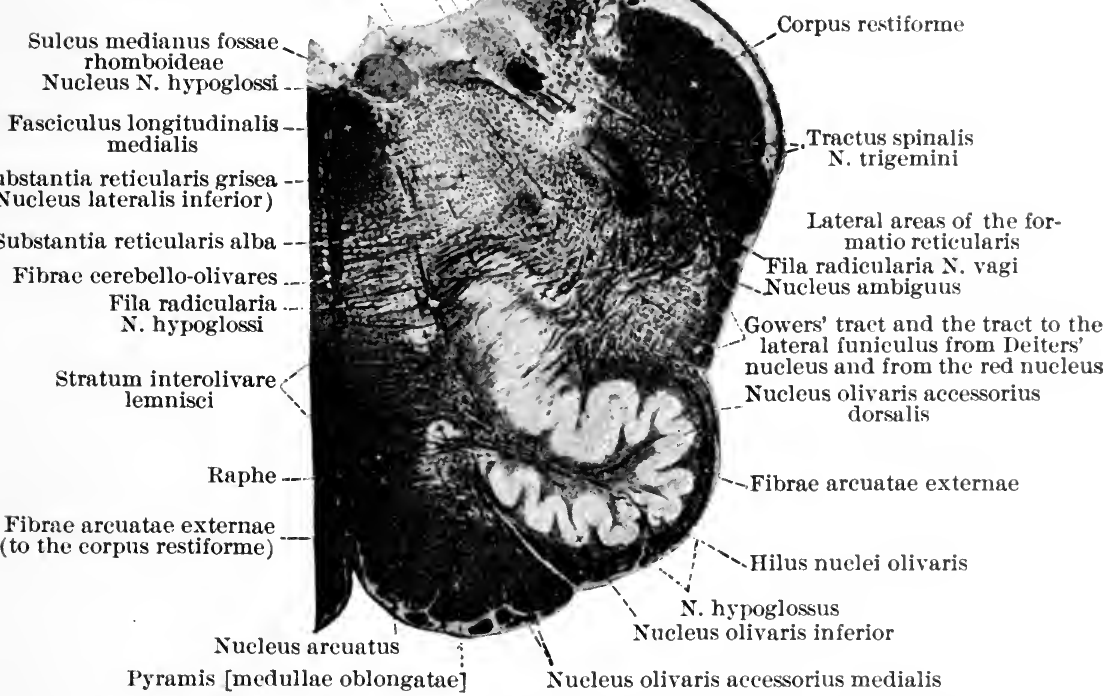

Transverse section of the brain-stem of an adult through the middle of the olive. Magnification : 4.5:1

(Held.) (After Spalteholz, Hand Atlas of Hum. Anat., Leipzig, 1903, vol. iii., p. 65i, Fig. 727.)

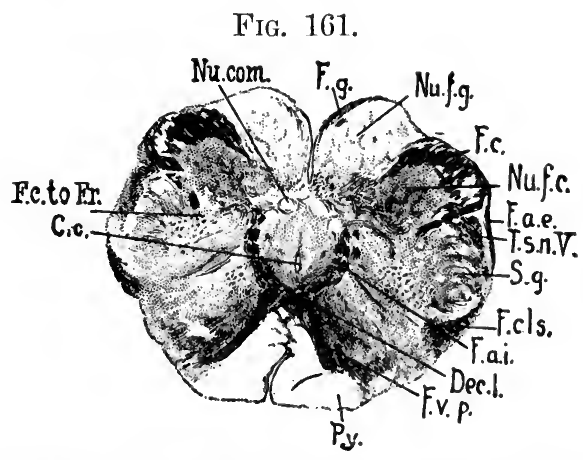

Transverse section throngh medulla oblongata of new-born child at level of decussatio lemniscorum. (Series ii. section No. 50.) ${ }^{1}$

C.c., canalis centralis; Dec.l., decussatio lemniscorum; F.a.i., fibrae areuatae internae ; F.a.c., fibrae arcuatae externae; F.c., fasciculus cuneatus Burdachi ; F.c. to F.r., bundles from faseiculus cuneatus to formatio reticularis ; F.cls., fasciculus cerebellospinalis or direct cerebellar traet ; $F . g$., fasciculus gracilis Golli; F.v.p., fasciculus ventralis proprius; Nu.com., nucleus commissuralis; Nuf.c., nucleus funieuli cuneati et gracilis; $\nu u, f . g .$, nueleus funiculi graeilis; Py., pyramis; T.s.n. I., tractus spinalis N. trigemini ; S.g., substantia gelatinosa [Rolandi]. (From Barker, The Nerv. Syst., New York, 1899, p. 479, Fig. 308.)

\footnotetext{
${ }^{1}$ Figs. 161-169 are from Weigert-Pal preparations by Dr. John Hewetson.
} 
Fig. 162.

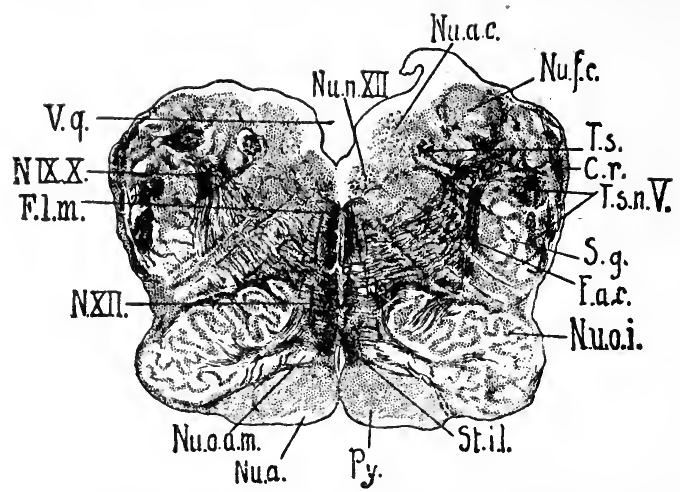

Transverse section of medulla oblongata of new-born child passing through the nucleus olivaris inferior. (Series ii. section No. 102.)

C.r., corpus restiforme; F.a.c., fibrae arcuatae internae from the anterior half of the nucleus funiculi euncati ; F.l.m., fasciculus longitudinalis medialis; N.IX.X., N. glossopharyngeus et vagus; N.XII., N. hypoglossus; $N u . a$. , nucleus areuatus; Nu.a.c., nucleus alae cinereae ; Nu.f.c., nucleus funiculi cuneati ; Nu.o.a.m., nucleus olivaris accessorius medialis; Nu.o.i., nucleus olivaris inferior; Nu.n.XII., nucleus N. hrpoglossi; Py., pyramis (non-medullated); S.g., substantia gelatinosa Rolandi; St.i.l., stratum

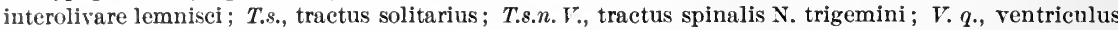
quartus. (Weigert-Pal preparation by Dr. John Hewetson.) (From Barker, The Nerv. Syst., New York, 1899, p. 479, Fig. 309.)

FIG. 163.

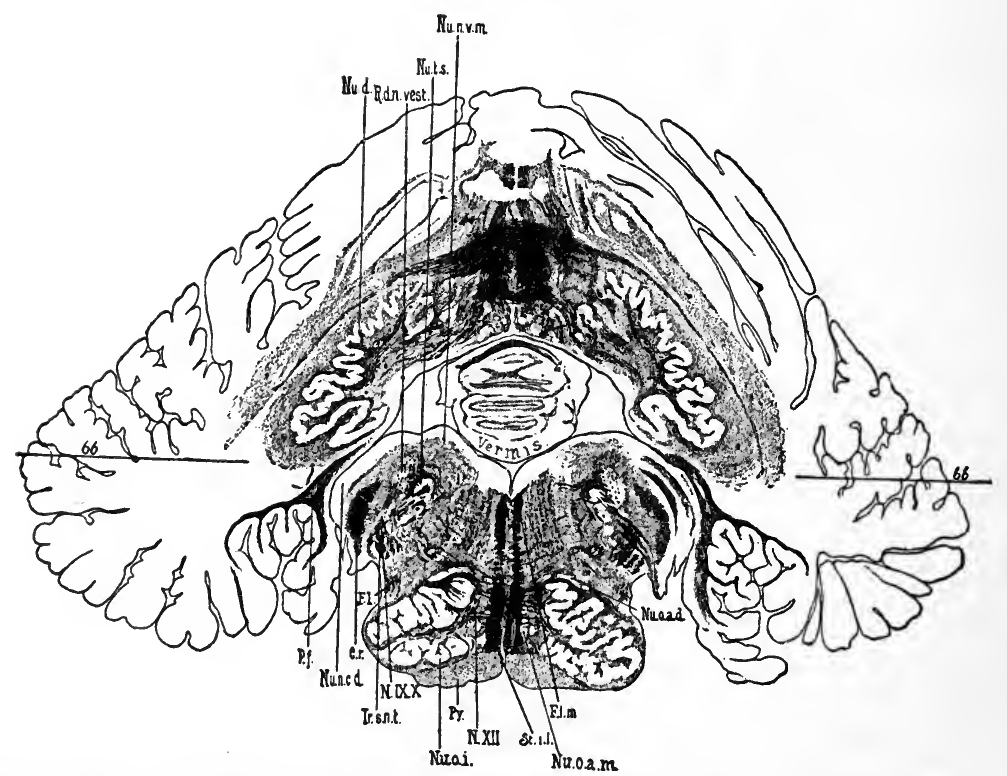

Transverse section of medulla oblongata and cerebellum of new-born child. (Series ii. section No.146.)

C.r., corpus restiforme (the part medullated corresponds in the main to the direct cerebellar tract) ; F.l., bundle continuous with the funiculus lateralis of the cord; F.l.m., fasciculus longitudinalis medialis; N.IX.X., N. glossopharyngeus et vagus; N.XII., N. hypoglossus; Nu.d., nucleus dentatus; Nu.n.c.d., nucleus N. cochleae dorsalis; Nu.n.v.m., nucleus $\mathrm{N}$. vestibuli medialis; Nu.o.a.d., nucleus olivaris accessorius dorsalis; Nu.o.a.m., nucleus olivaris accessorius medialis; Nu.o.i., nucleus olivaris inferior; Nu.t.s., nucleus tractus solitarii ; $P f$., pedunculus floceuli ; $P y$., pyramis; R.d.n.vest., radix descendens N. vestibuli; St.i.l., stratum interolivare lemnisci; 66 , plane of longitudinal section No. 66 . [NOTE.-This figure has been disproportionately reduced in the reproduction.] (From Barker, The Nerv. Syst., New York, 1899, p. 480, Fig. 310.) 
FIG. 164.

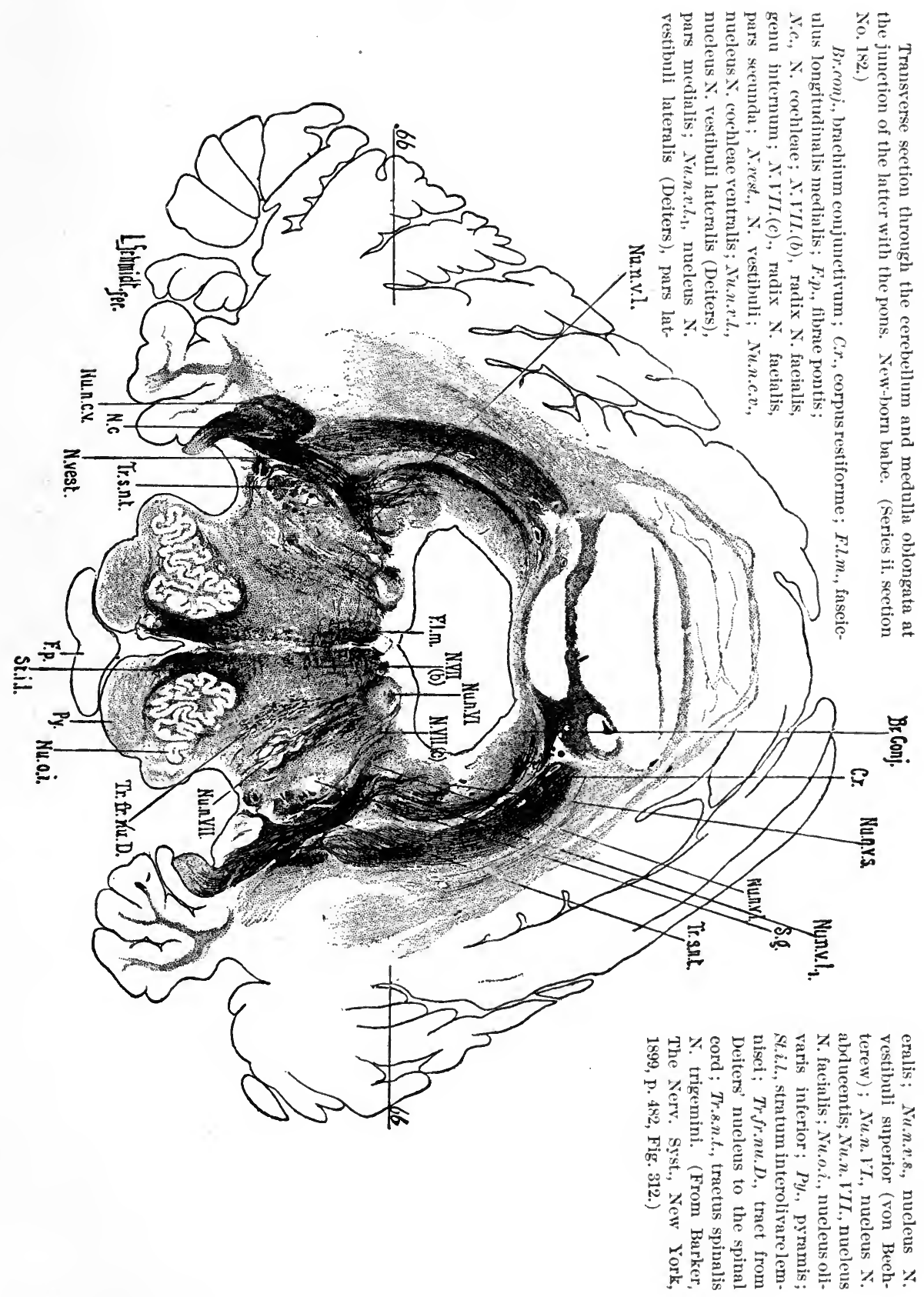


(c) Dorsal accessory olivary nucleus (nucleus olivaris accessorius dorsalis).

(12) Arcuate nuclei (nuclei arcuati).

(13) Internal arcuate fibres (fibrae arcuatae internae).

(14) Gray reticular substance (substantia reticularis grisea).

(15) White reticular substance (substantia reticularis alba).

FIG. 165.

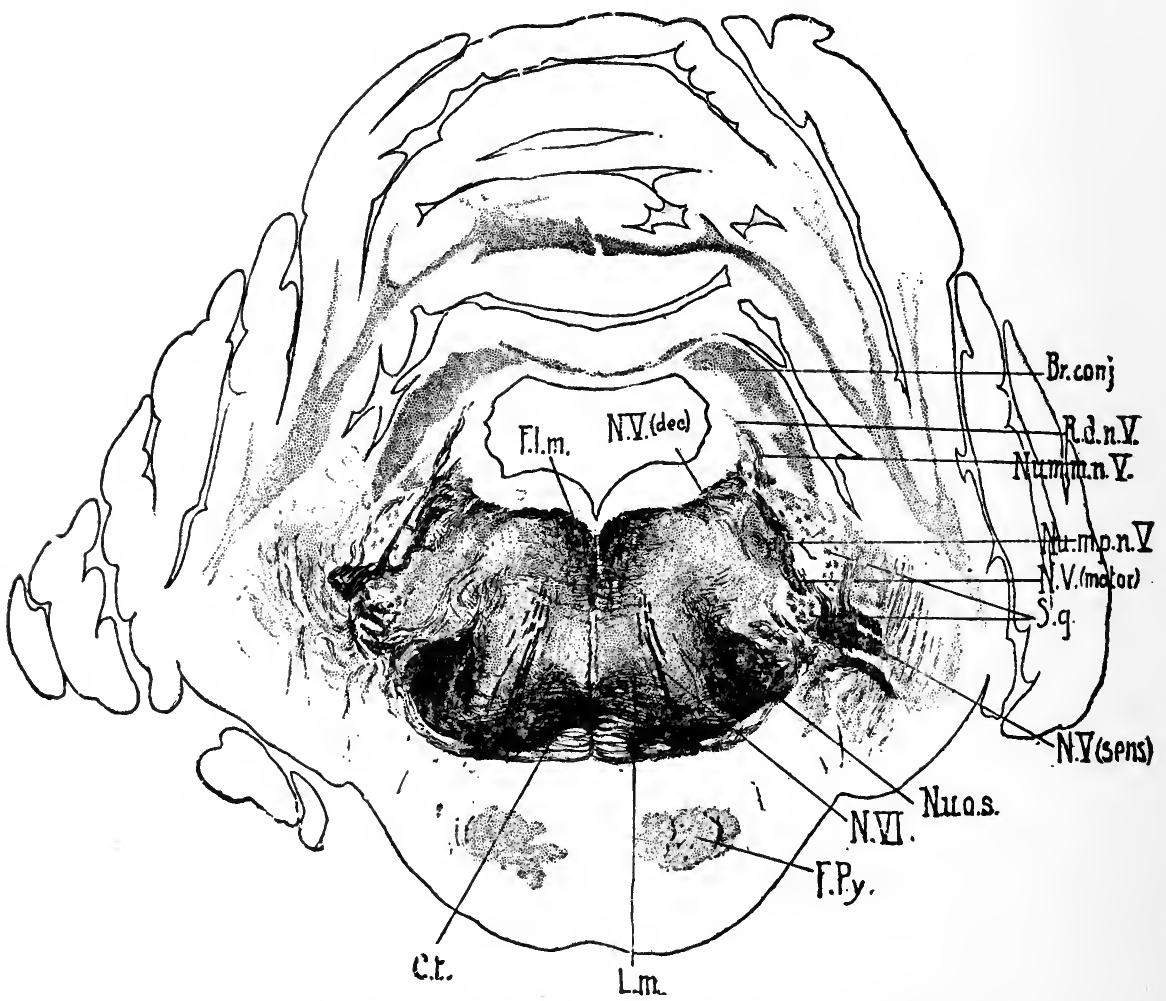

Transverse section through the pons and cerebellum at the level of the principal motor nucleus of the trigeminal nerve. New-born babe. (Weigert-Pal, series ii. section No. 212.)

Br.conj., brachium conjunctivum; C.t., corpus trapezoideum; F.l.m., fasciculus longitudinalis medialis ; F.Py., faseiculi pyramidales in the pars basilaris pontis; L.m., lemniscus medialis ; N.V.(dec.), decussating part of root of $\mathrm{N}$. trigeminus; $N . \mathrm{V}$.(motor), root of $\mathrm{N}$. trigeminus; $N . V .($ sens $)$, sensory root of N. trigeminus; N.V.I., root fibres of N. abducens; Nu.m.m.n.V., nuclei motorii minores N. trigemini ; Nu.m.p.n.V., nueleus motorius princeps $\mathrm{X}$. trigemini; Nu.o.s., nucleus olivaris superior and superior olivary complex; R.d.n.V., radix descendens [mesencephalica] N. trigemini ; S.g., substantia gelatinosa. (From Barker, The Nerv. Syst., New York, 1899, p. 483, Fig. 313.)

(16) Medial longitudinal fasciculus (fasciculus longitudinalis medialis) (O. T. posterior longitudinal bundle).

(17) Interolivary layer of lemniscus (stratum interolivare lemnisci).

(18) Decussation of lemniscus or fillet (decussatio lemniscorum) (O. T. sensory decussation of medulla oblongata). 
(19) Restiform body (corpus restiforme) (O. T. inferior cerebellar peduncle).

(a) Fasciculi of restiform body (fasciculi corporis restiformis).

(20) Cerebello-olivary fibres (fibrae cerebelloolivares).

(21) Pyramidal fasciculi (fasciculi pyramidales).

(22) External arcuate fibres (fibrae arcuatae externae).

\section{Sections of Pons (Sectiones pontis).} 723.)

Make four sections through the pons. (See Spalteholz, Fig.

FIG. 166.

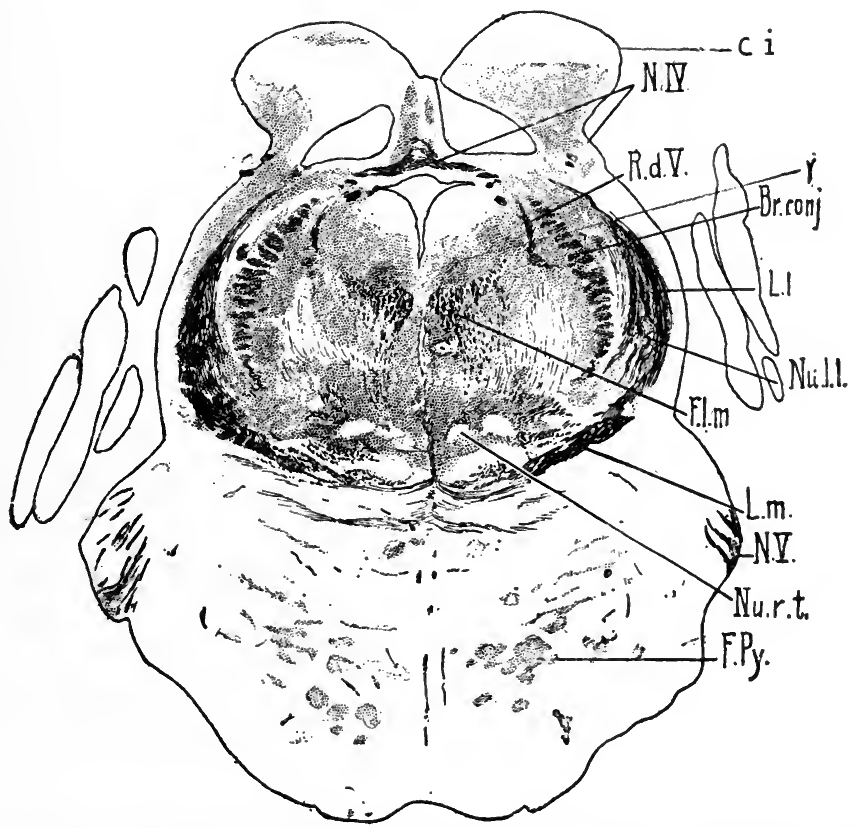

Transverse section through isthmus rhombencephali of new-born babe. (Weigert-Pal, series ii. section No. 268.)

Br.conj., brachium conjunctivum ; C.i., colliculus inferior; $\gamma$, nucleus described by Westphal as probably eoncerned in the origin of the N. trochlearis; F.l.m., fasciculus longitudinalis medialis ; F.Py., fasciculi longitudinales [pyramidales]; L.l., lemniscus lateralis; L.m., lemniscus medialis; N.IV., decussatio nervorum trochlearium; N.V., N. trigeminus; Nu.l.l., nucleus lemnisei lateralis; Nu.r.t. nucleus reticularis tegmenti pontis; $R . d$. I., radix descendens [mesencephalica] nervi trigemini. (From Barker, The Nerv. Syst., New York, 1899, p. 484, Fig. 314.)

(1) About at the junction of the pons and the medulla, passing through the level of entrance of $\mathrm{N}$. acusticus.

(2) At the level of the roots of $\mathrm{N}$. facialis and N. abducens.

(3) At the level of the roots of $N$. trigeminus.

(4) Through the velum medullare anterius and the middle of the pons.

Examine first the unstained cross-sections and subsequently sections, from the same levels, stained by Weigert's method. 
Use a hand-lens or dissecting microscope. Make drawings indicating-

In dorsal part of pons (pars dorsalis pontis).

(1) Median raphe (raphe).

(2) Nucleus of N. abducens (nucleus N. abducentis) (O. T. nucleus of sixth nerve).

(3) Motor nuclei of trigeminal nerve (nuclei motorii N. trigemini).

(4) Descending or mesencephalic root of trigeminal nerve (radix mesencephalica $N$. trigemini).

(5) Spinal tract of trigeminal nerve (tractus spinalis N. trigemini).

(a) Nucleus of spinal tract of trigeminal nerve (nucleus tractus spinalis $N$. trigemini).

(6) Nucleus of facial nerve (nucleus $N$ : facialis).

(7) Root of facial nerve (radix $N$. facialis).

(a) First part (pars prima).

(b) [Internal] knee (genu [internum]).

(c) Second part (pars secunda).

(8) Nuclei of acoustic nerve (nuclei N. acustici) (O. T. auditory nucleus).

(a) Nuclei of cochlear nerve (nuclei $N$. cochlearis).

(aa) Ventral (nucleus $N$. cochlearis ventralis).

(ab) Dorsal (nucleus N. cochlearis dorsalis [tuberculum acusticum]).

(b) Nuclei of vestibular nerve (nuclei $N$. vestibularis).

(ba) Medial nucleus (nucleus $N$. vestibularis medialis).

(bb) Lateral nucleus (nucleus $N$. vestibularis lateralis [Deitersi] $)$.

(bc) Superior nucleus (nucleus $N$. vestibularis superior [Bechterewi]).

$(b d)$ Nucleus of descending root (nucleus N. vestibularis radicis descendentis).

(9) Superior olivary nucleus (nucleus olivaris superior).

(10) Nucleus of lateral lemniscus (nucleus lemnisci lateralis).

(11) Medial longitudinal fasciculus (fasciculus longitudinalis medialis)

(O. T. posterior longitudinal bundle).

(12) Reticular formation (formatio reticularis).

(13) Trapezoid body (corpus trapezoideum).

(14) Fillet or lemniscus (lemniscus).

(a) Medial (sensory) fillet (lemniscus medialis [sensitivus]).

(b) Lateral (acoustic) fillet (lemniscus lateralis [acusticus]).

In basilar part of pons (pars basilaris pontis).

(1) Deep fibres of pons (fibrae profundae pontis).

(2) Longitudinal pyramidal fasciculi (fasciculi longitudinales [pyramidales]).

(3) Nuclei of pons (nuclei pontis).

(4) Superficial fibres of pons (fibrae pontis superficiales).

\section{Sections of Cerebellum (Sectiones cerebelli).}

Make a sagittal median section through the vermis. (Vide Fig. 144.) 
Study-

(1) Medullary body (corpus medullare).

(2) Medullary laminae (laminae medullares).

(3) Arbor vitae (arbor vitae) ("tree of life").

(4) Cortical substance (substantia corticalis).

(a) Inner, yellowish or reddish-brown, "granular" layer (stratum granulosum).

(b) Outer gray layer (stratum cinereum).

(c) Layer of Purkinje's cells between $(a)$ and (b) (stratum gangliosum).

Fig. 167.

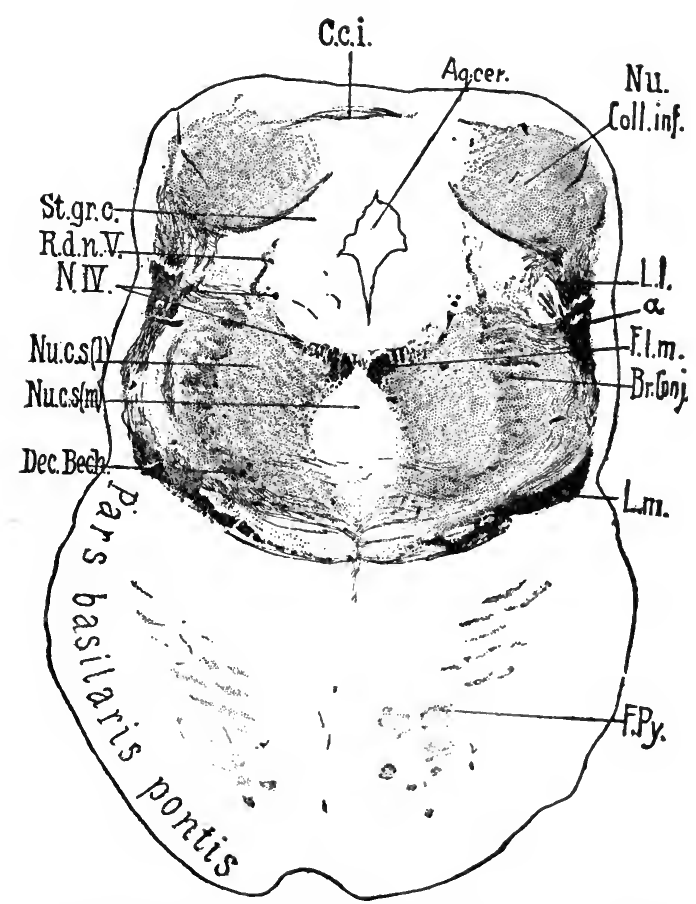

Transverse section through brain of new-born babe. Level of colliculi inferiores of corpora quadrigemina. (Weigert-Pal, series ii. section No. 290.)

Aq.cer., aquacductus cerebri; a, fibres running from lateral lemniseus toward dorsal border of brachium conjunctivum; Br.Conj., brachium eonjunctivum; C.c.i., commissure between the colliculi inferiores; $D c c . B c c h$., ventral portion of brachium conjunctivum, which in reality forms a eommissure between the superior nuclei of the vestibular nerves of the two sides; F.l.m., fasciculus longitudinalis medialis; F.Py., fasciculi longitudinales pontis (pyramidales); L.l., lemniscus lateralis in large part terminating in the nucleus of the colliculus inferior; $L . m$., lemniseus medialis; N.IV., N. troehlearis ; Nu.Coll.inf., nueleus colliculi inferioris; Nu.c.s. (l), nucleus centralis superior, pars lateralis ; Nu.c.s. $(m)$, nucleus centralis superior, pars medialis; R.d.n.V., radix descendens [mesencephalica] N. trigemini; St.gr.c., stratum griseum centrale. (From Barker, The Nerv. Syst., New York, 1899, p. 485, Fig. 315. )

Make a frontal section through the left half of the cerebellum at about its middle (cf. Spalteholz, Fig. 743), and a horizontal section through the right half of the cerebellum in the general 
direction of the brachium conjunctivum (cf. Spalteholz, Fig. 744). In these, study the following:

(1) Dentate nucleus (nucleus dentatus).

(a) Hilus of dentate nucleus (hilus nuclei dentati).

(b) Capsule of nucleus dentatus (capsula nuclei dentati).

(2) Nucleus of fastigium ("roof") (nucleus fastigii).

(3) Nucleus globosus (nucleus globosus) ("spherical nucleus").

(4) Emboliform nucleus (nucleus emboliformis) (O. T. cork or plug).

FIG. 168.

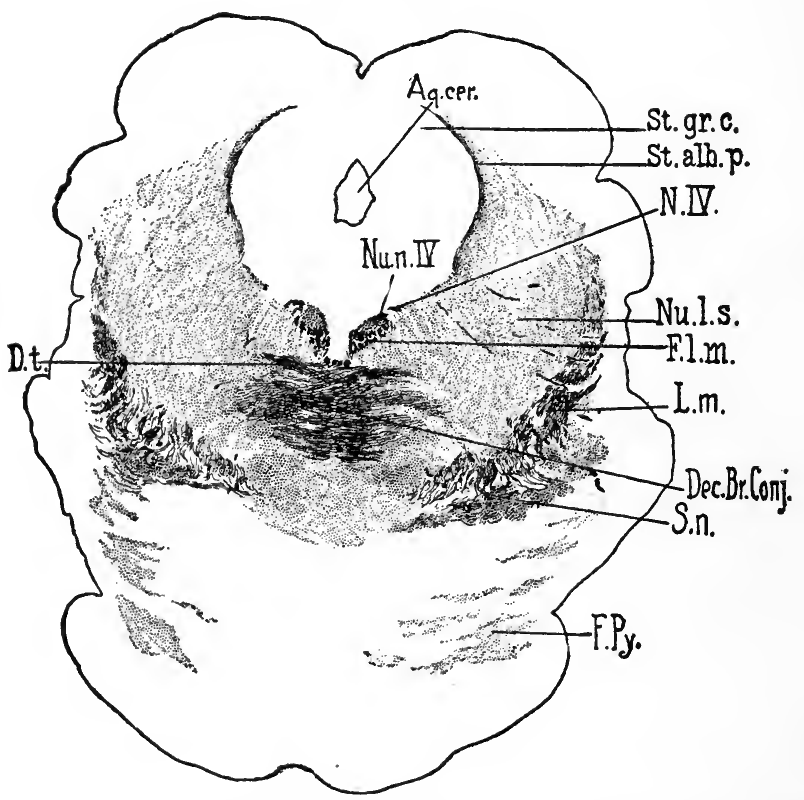

Transverse section through mesencephalon of new-born babe. Level of colliculi superiores of corpora quadrigemina. (Weigert-Pal, series ii. section No. 338.)

Aq.cer., aquaeductus cerebri ; Dec.Br.Conj., decussatio brachii conjunctivi ; D.t., decussatio tegmenti ventralis (ventrale Haubenkreuzung of Forel); F.l.m., fasciculus longitudinalis medialis; F.Py., fasciculi pyramidales in the pars basilaris pontis; L.m., lemniscus medialis; N.IV., N. trochlearis; Nu.l.s., nucleus lateralis superior of Flechsig; Nu.n.IV., nucleus N. trochlearis; St.alb.p., stratum album profundum; St.gr.c., stratum griseum centrale; S.n., substantia nigra. (From Barker, The Nerv. Syst., New York, 1899, p. 486, Fig. 316.)

\section{Sections of Rhombencephalic Isthmus, Corpora Quadrigemina, and} Cerebral Peduncle (Sectiones isthmi, corporum quadrigeminorum, et pedunculi cerebri).

Make two sections: (1) through the inferior colliculus at the level of the nucleus of the N. trochlearis; (2) through the level of the roots of the $\mathrm{N}$. oculomotorius, nucleus ruber, superior colliculus, and lateral geniculate body. (Cf. Spalteholz, Fig. 723.) 


\section{Study-}

In the isthmus rhombencephali.

(1) Interpeduncular ganglion (ganglion interpedunculare).

(2) Nucleus of trochlear nerve (nucleus N. trochlearis).

In the pedunculus cerebri.

(A) Tegmentum.

(1) Central gray layer (stratum griseum centrale).

(2) Reticular formation (formatio reticularis).

(3) Medial longitudinal fasciculus (fasciculus longitudinalis medialis) (O. T. posterior longitudinal bundle).

FIG. 169.

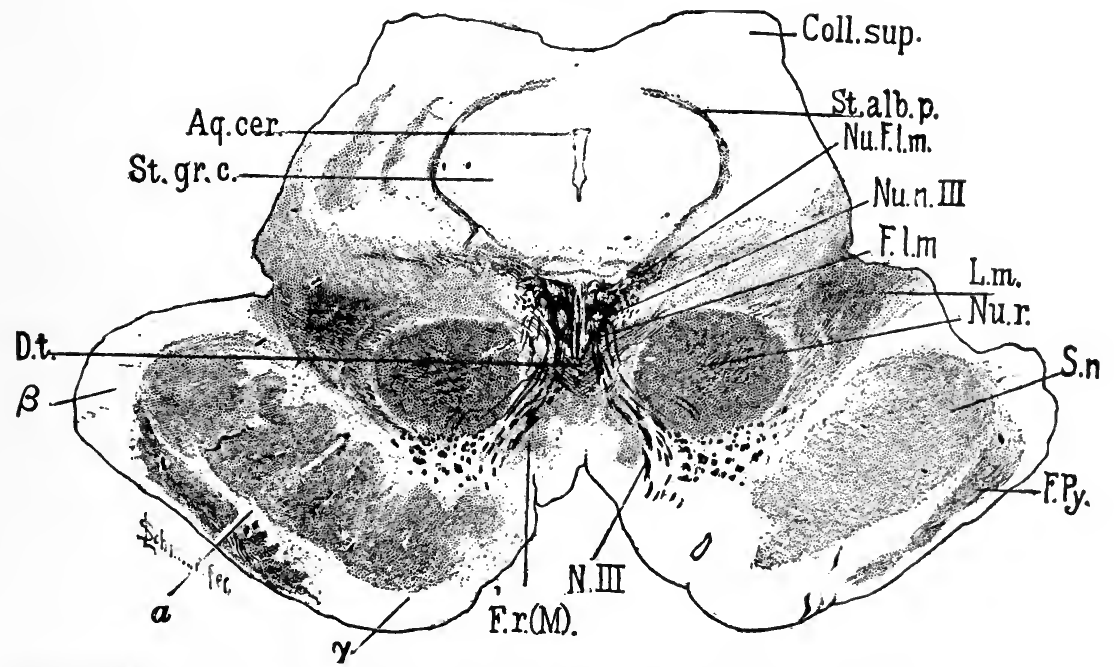

Transverse section through mesencephalon, colliculi superiores of corpora quadrigemina and cerebral peduncle of new-born babe. (Weigert-Pal, series ii. section No. 384.)

Aq.cer., aquaeductus cerebri ; Coll.sup., colliculus superior; D.t., decussatio tegmenti dorsalis (fontaineartige Haubenkrcuzung of Meynert); F.l.m., fasciculus longitudinalis medialis; F.Py., fasciculi pyramidales in the basis pedunculi ; F.r. (M), fasciculus retroflexus Meynerti ; L.m., lemniscus medialis; Nu.F.l.m., nucleus fasciculi longitudinalis medialis or nucleus commissurae posterioris (oberer Oculomotoriuskern of Darkschewitsch); Nu.n.III, nucleus N. oculomotorii ; Nu.r., nucleus ruber; N.III, N. oculomotorius; St.alb.p., stratum album profundum; St.gr.c., stratum griseum centrale ; S.n., substantia nigra ; a, region of Flechsig's Fussschleife; $\beta$, temporo-occipital tract to pons; $\gamma$, frontal tract from pallium to pons. (From Barker, The Nerv. Syst., New York, 1899, p. 487, Fig. 317.)

(4) Descending root of trigeminal nerve (radix descendens $N$. trigemini).

(5) Nucleus of descending root of trigeminal nerve (nucleus radicis descendentis $N$. trigemini $=$ nuclei motorii $\mathrm{mi}^{-}$ nores $N$. trigemini).

(6) Nucleus of oculomotor nerve (nucleus N. oculomotorii) (O. T. nucleus of III. nerve).

(7) Nuclei of tegmentum (nuclei tegmenti).

(a) Red nucleus (nucleus ruber).

(8) Decussations of tegmenta (decussationes tegmentorum). 
(a) Decussation of brachium eonjunctivum (decussatio brachii conjunctivi).

(b) Dorsal decussation of tegmentum (decussatio tegmenti dorsalis) (German O. T. fontaineartige Haubenkreuzung).

(c) Ventral decussation of tegmentum (decussatio tegmenti ventralis).

(9) Lateral fillet (lemniscus lateralis).

(10) Medial fillet (lemniscus medialis).

(B) Base of peduncle (basis pedunculi) (O. T. "foot" of cerebral peduncle, or pes pedunculi).

(1) Pyramidal path (fasciculi cerebrospinales).

(2) Temporal pontile path (fasciculus temporopontilis) (O. T. Türk's bundle).

(3) Frontal pontile path (fasciculus frontopontilis) (O. T. Arnold's bundle).

Between $(A)$ and $(B)$, study the substantia nigra (substantia nigra) ("black substance").

In the corpora quadrigemina.

(1) Zonal layer (stratum zonale).

(2) Gray layer of superior colliculus (stratum griseum colliculi superioris).

(3) Nucleus of inferior colliculus (nucleus colliculi inferioris).

(4) Deep white layer (stratum album profundum).

\section{Sections through Prosencephalon (Sectiones diencephali et telen- cephali).}

Take the portion still remaining of the right cerebral hemisphere; with a broad, thin, sharp brain-knife, make horizontal slices, one-half or one centimetre thick, down as far as the level of the commissura anterior cerebri. Make a drawing of the surface of each slice. (Vide Fig. 177.)

The left half of the brain, thus far preserved intact, should now be divided, at the choice of the instructor, either into vertical frontal or into obliquely frontal sections, the latter according to the method of Pitres. ${ }^{1}$ All the oblique sections pass in planes parallel to the course of the sulcus centralis Rolandi. Place the four fingers of the left hand in the sulcus centralis [Rolandi], the hemisphere lying upon its medial surface with the polus occipitalis directed towards you. With the sharp, broad, thin brain-knife, make six sections from before backward, as indicated in the accompanying diagram. (Cf. Fig. 170.)

\footnotetext{
${ }^{1}$ Pitres, Recherches sur les lésions du centre oval des hémisphères cérébraux, étudieés au point de vue des localisations cérébrales. Paris, 1877.
} 
(1) Prefrontal section, passing through the front of the lobus frontalis about five centimetres in front of the sulcus centralis and parallel to it.

(2) Operculofrontal or pediculofrontal section, through the pars opereularis of the gyrus frontalis inferior and the corresponding portions of the gyrus frontalis medius and gyrus frontalis superior; in other words, through the "feet" of the frontal gyri.

(3) Frontal section, through the middle of the gyrus centralis anterior.

(4) First parietal section, through the middle of the gyrus centralis posterior.

(5) Second parietal or pediculoparietal section, through the anterior extremities ("feet") of the lobulus parietalis superior and lobulus parietalis inferior.

(6) Occipital section, about one centimetre in front of the fissura parietooccipitalis.

FIG. 170.

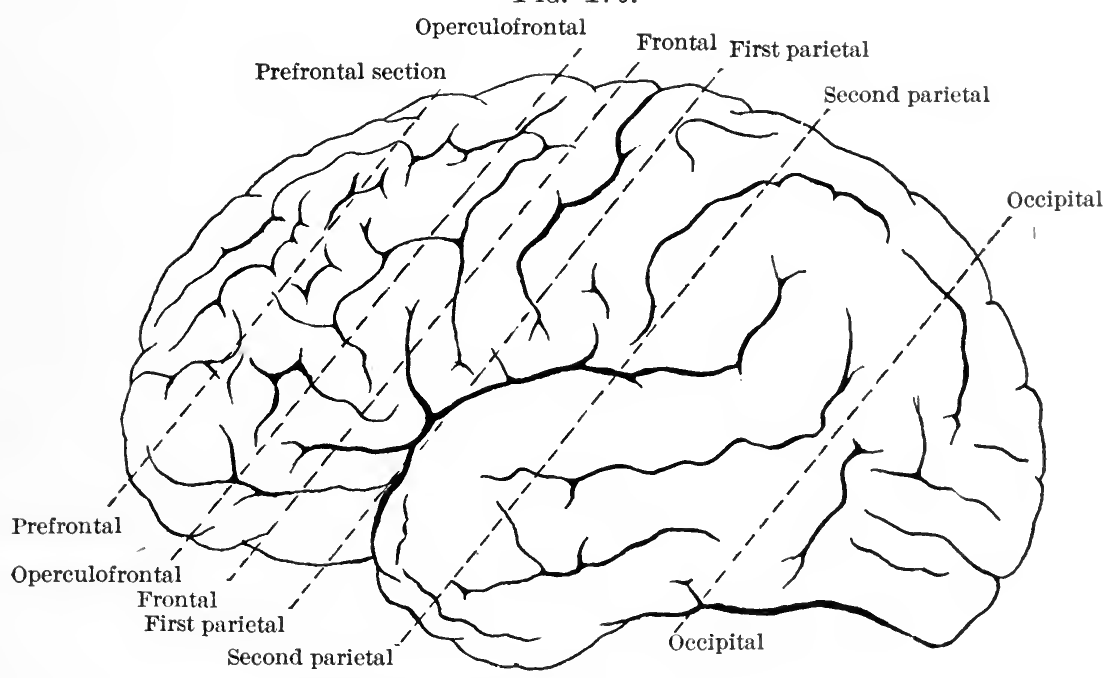

Pitres' method of sectioning the cerebral hemisphere. ${ }^{1}$

It will be advantageous to the student while studying anatomy to become familiar with these obliquely frontal sections, as well as with the vertical frontal, for it is the former that are more often employed at the autopsy table of the pathological laboratories.

The prefrontal section shows the cortex and white matter of the three frontal gyri, the orbital gyri, and the medial surface of the frontal lobe.

The pediculofrontal section passes through the three frontal gyri, anterior end of island, orbital gyrus, corpus callosum, caput nuclei caudati, anterior part of nucleus lentiformis, and lenticulostriate part of eapsula interna.

${ }^{1}$ The lines indicating the planes of the sections slope too much in this figure. 
The frontal section passes through the gyrus centralis anterior, island, temporal gyri, corpus callosum, cauda nuclei caudati, thalamus, middle piece of nucleus lentiformis, anterior part of lenticulo-optic part of capsula interna, capsula externa, and claustrum.

The first parietal section passes through the gyrus centralis posterior, island, temporal gyri, corpus callosum, cauda nuclei caudati, posterior end of thalamus and of nucleus lentiformis, posterior end of lenticulooptic part of capsula interna, capsula interna, capsula externa, and claustrum.

The pediculoparietal section passes through the lobuli parietalis superior et inferior, temporal gyri, corpus callosum, most posterior part of thalamus, and cauda nuclei caudati.

The occipital section passes through the cortex and white matter of the lobus occipitalis.

In addition to the horizontal sections and frontal sections above mentioned, the student should study, if possible (perhaps from the anatomical museum), two other sections :

(1) Section through the brain in a plane passing parallel to the course of the brachia conjunctiva and through them. (Cf. Spalteholz, Fig. 744.)

(2) Section through the brain in a plane parallel to the course of the cerebral peduncles and through them. (Cf. Spalteholz, Fig. 745.)

Fig. 171.

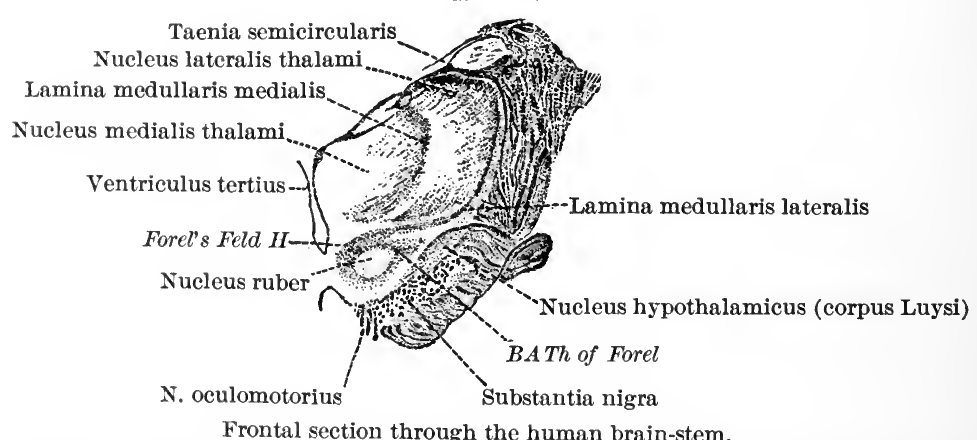

$B A T h$, large bundle of fibres which, coming from the nucleus ruber and its capsule, runs upward, lateralward, and dorsalward to the ventral part of the thalamus to the reticular layer, to the lamina medullaris lateralis, etc., where the fibres become so interwoven with others that they cannot be followed farther; Forel's Feld H, dorsal white matter of regio subthalamica. (After A. Forel, Arch. f. Psychiat., Berl., Bd. vii., 1877, Taf. vii., Fig. 10.)

Drawings should be made of all these sections, and the following structures designated when met:

\section{Sections of Hypothalamus (Sectiones hypothalami).}

(1) Hypothalamic nucleus, or Luys's body (nucleus hypothalamicus [corpus Luysi]) (O. T. subthalamic nucleus).

(2) Gray part of hypothalamus (pars grisea hypothalami). 
(3) Superior commissure of Meỳnert (commissura superior [Meynerti]).

(4) Inferior commissure of v. Gudden (commissura inferior [Guddeni]).

(5) Nuclei of mammillary body (nuclei corporis mammillaris).

FIG. 172.

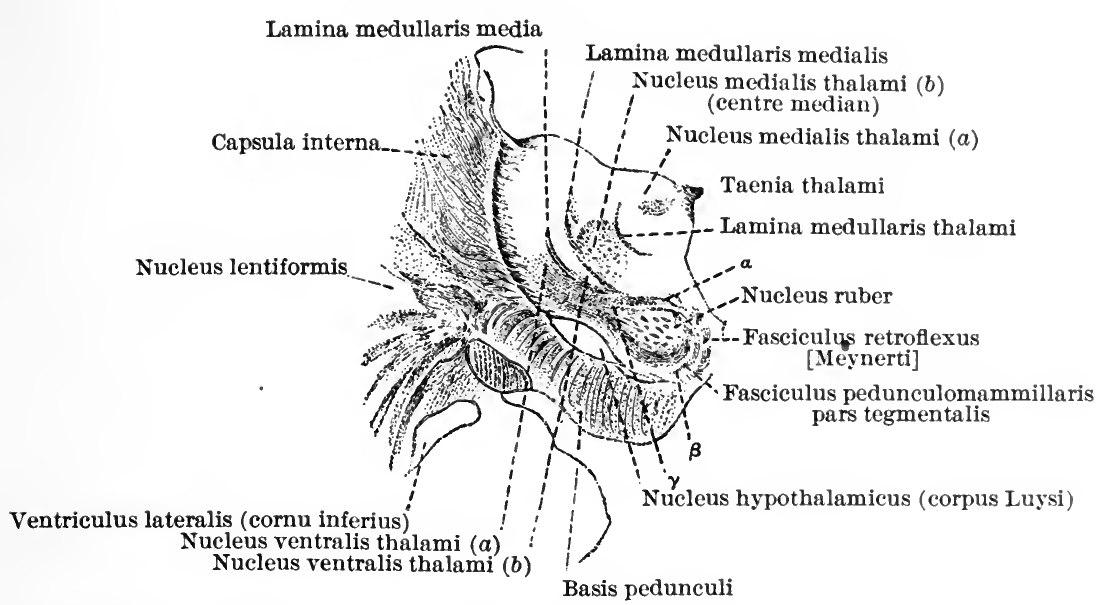

Frontal section through a normal human brain at the level of the lower end of the nucleus hypothalamicus.

$\alpha$, dorsal white matter of nucleus ruber; $\beta$, ventral white matter of nucleus ruber ; $\gamma$, lateral white matter of nucleus ruber. (After C. von Monakow, Arch. f. Psychiat., Berl., Bd. xxvii., 1895, Taf. iii., Fig. 20.)

(6) Thalamomammillary fasciculus (fasciculus thalamomammillaris [Vicq d'Azyri]) (O. T. bundle of Vicq d'Azyr).

FIG. 173.

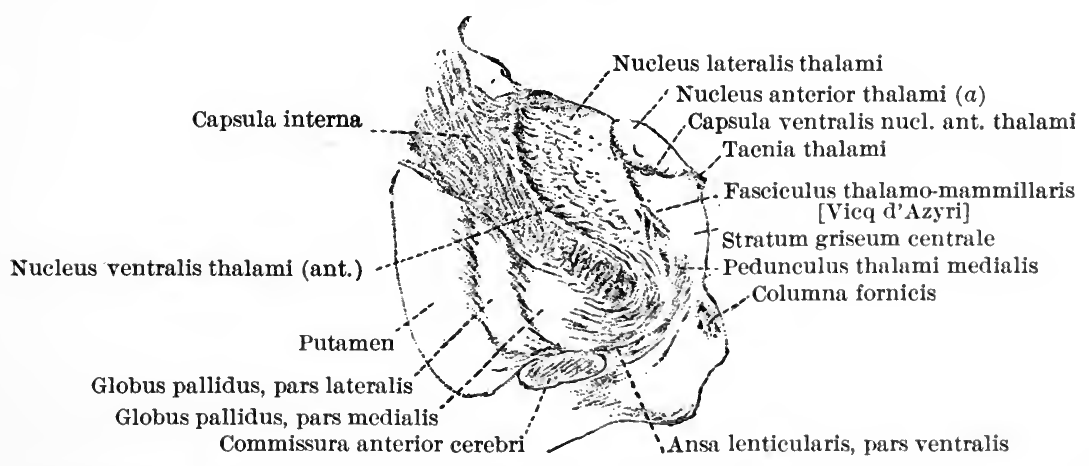

Frontal section through a normal human brain at level of anterior part of thalamus. (After C. von Monakow, Arch. f. Psychiat., Berl., Bd. xxvii., 1895, Taf. iv., Fig. 33.)

(7) Pedunculomammillary fasciculi (fasciculi pedunculomammillares).

(a) Tegmental part (pars tegmentalis).

(b) Basilar part (pars basilaris). 
(8) Peduncular loop (ansa peduncularis).

(a) Lenticular loop (ansa lenticularis).

(b) Inferior peduncle of thalamus (pedunculus thalami inferior).

\section{Sections of Thalamencephalon (Sectiones thalamencephali).}

(1) Zonal layer (stratum zonale).

(2) Anterior nucleus of thalamus (nucleus anterior thalami).

(3) Medial nucleus of thalamus (nucleus medialis thalami).

(4) Lateral nucleus of thalamus (nucleus lateralis thalami).

FIG. 174.

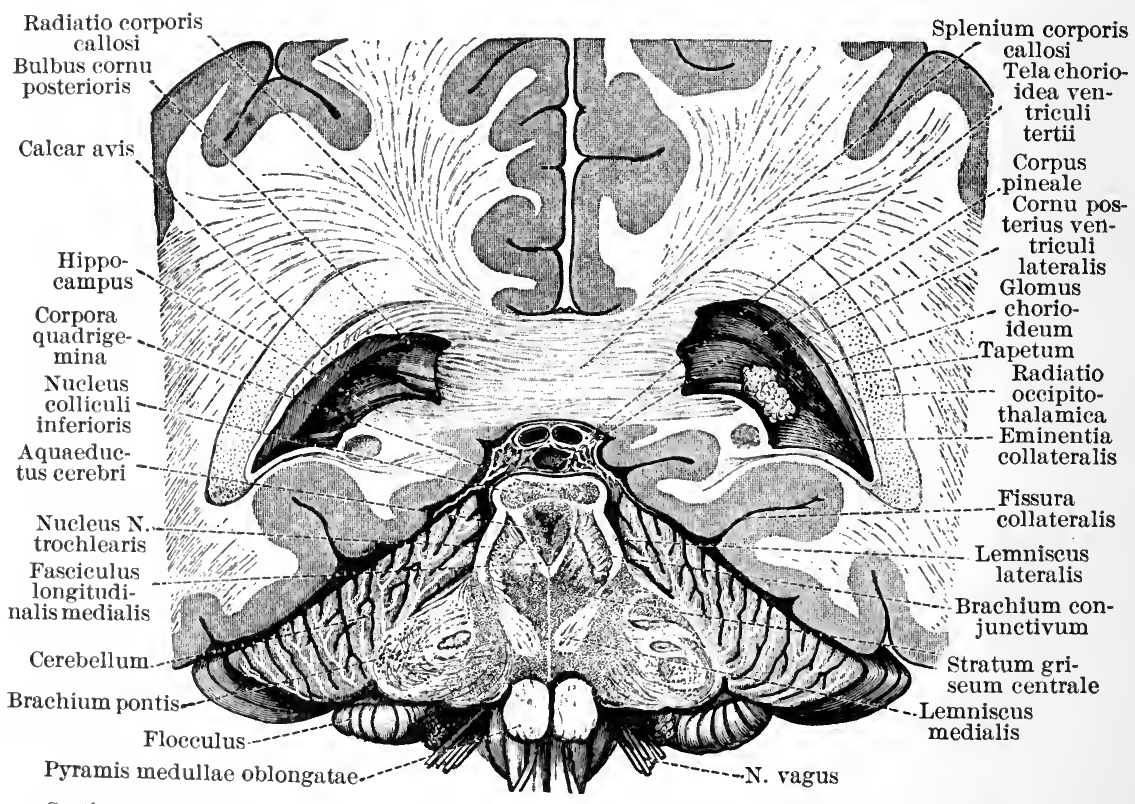

Section through the upper ends of the pyramids of the medulla oblongata, through the splenium corporis callosi, and through the posterior parts of the lateral ventricles. (After Toldt, Anat. Atlas, Wien, 1903, 3 Aufl., p. 791, Fig. 1217.)

(5) Medullary layers of thalamus (laminae medullares thalami).

(6) Nucleus of medial geniculate body (nucleus corporis geniculati medialis). (7) Nucleus of lateral geniculate body (nucleus corporis geniculati lat-
eralis).

(8) Nucleus of habenula (nucleus habenulae).

(9) Retroflex fasciculus (fasciculus retroflexus [Meynerti]).

\section{Sections of Telencephalon (Sectiones telencephali).}

(1) Cortical substance (substantia corticatis).

Observe the difference in macroscopic appearance in different areas in the cortex.

(2) Semioval centre (centrum semiovale). 
Fig. 175.

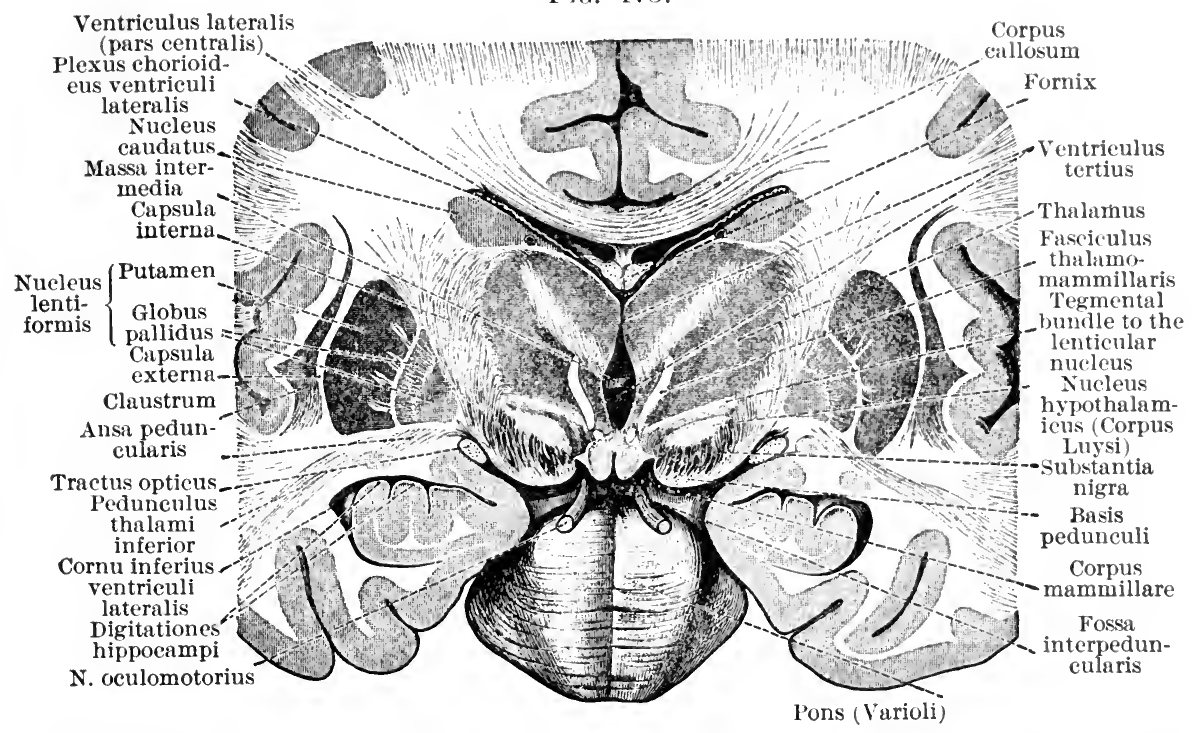

Section through the third ventricle, cerebral peduncles, and corpora mammillaria. (After Toldt, Anat. Atlas, Wien, 1903, 3 Aufl., p. 792, Fig. 1219.)

FIG. 176.

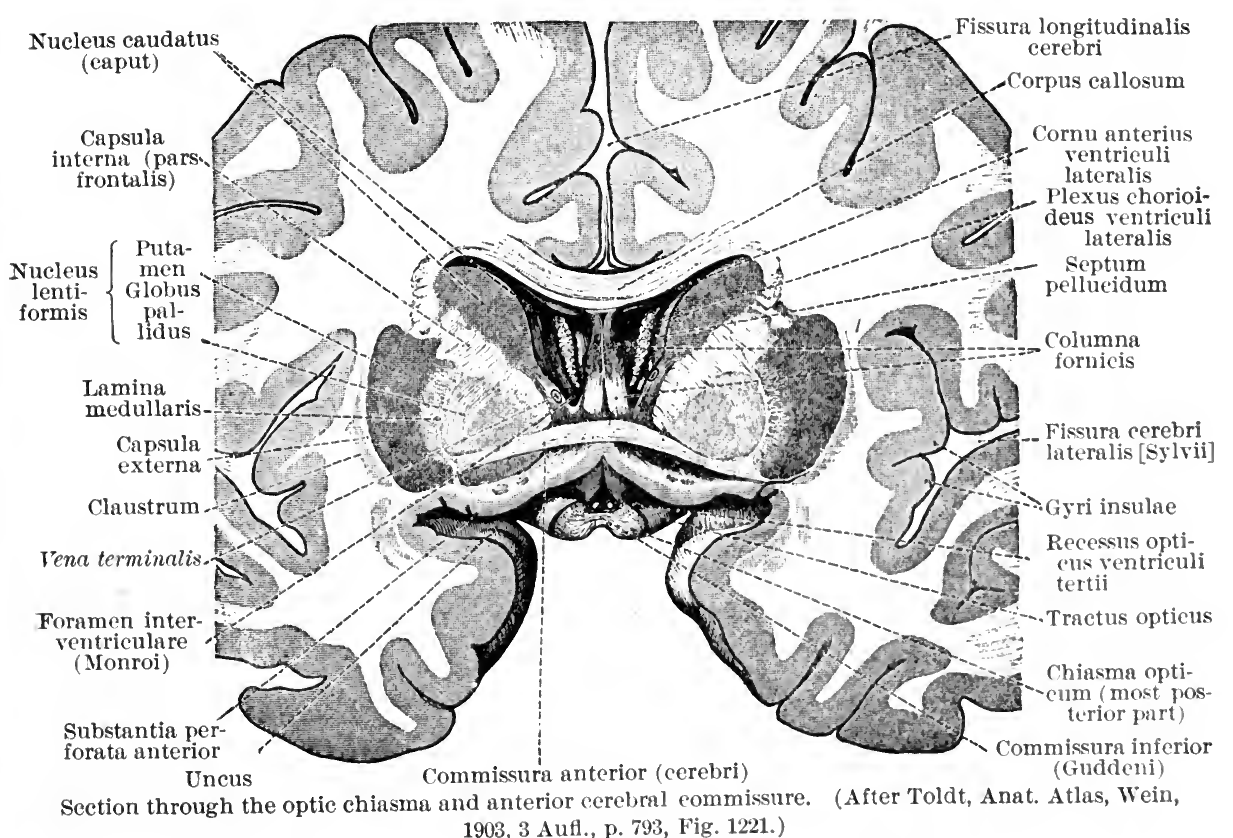


(3) Decursus ("running down") of cerebral fibres (decursus fibrarum cerebralium).

(4) Areuate fibres of cerebrum (fibrae arcuatae cerebri).

(a) Cingulum (cingulum) ("girdle").

(b) Superior longitudinal fasciculus (fasciculus longitudinalis superior).

(c) Inferior longitudinal fascieulus (fasciculus longitudinalis inferior).

(d) Uncinate ("hooked") fasciculus (fasciculus uncinatus).

(e) Radiation of corpus callosum (radiatio corporis callosi). (ea) Frontal part (pars frontalis) (O. T. forceps minor). (eb) Parietal part (pars parietalis).

(ec) Temporal part (pars temporalis).

(ed) Occipital part (pars occipitalis) (O. T. forceps major).

(f) Tapetum (tapetum) ("carpet" or "tapestry").

(5) Lentiform nucleus (nucleus lentiformis) (O. T. lenticular nucleus).

(a) Putamen (putamen) ("shell" or "paring").

(b) Globus pallidus (globus pallidus) ("pale sphere").

(6) Claustrum (claustrum) ("bulwark" or "barrier").

(7) External capsule (capsula externa).

(8) Internal capsule (capsula interna).

(a) Knee of internal capsule (genu capsulae internae).

(b) Frontal part of internal capsule (pars frontalis capsulae internae) (O. T. anterior limb).

(c) Occipital part of internal capsule (pars occipitalis capsulae internae) (O. T. posterior limb).

(9) Amygdaloid nucleus (nucleus amygdalae) ("almond" nucleus).

(10) Corona radiata.

(a) Frontal part (pars frontatis).

(b) Parietal part (pars parietalis).

(c) Temporal part (pars temporalis).

(d) Occipital part (pars occipitalis).

(11) Radiation of corpus striatum (radiatio corporis striati).

(12) Occipitothalamic radiation (radiatio occipitothalamica [Gratioleti])

(O. T. optic radiation).

(13) Anterior commissure of cerebrum (commissura anterior cerebri).

(a) Anterior part (pars anterior).

(b) Posterior part (pars posterior).

\section{CONDUCTION PATHS OF THE NERVOUS SYSTEM.}

The student should now take up systematically the study of the various motor, sensory, and associative conduction paths of the nervous system. For frequent consultation, Section VI. of "The Nervous System and its Constituent Neurones"' (D. Appleton and Co., New York) is recommended. A study of sections of various spinal cords in which secondary degenerations have 
taken place is desirable for a thorough understanding of the complex relations which exist.

\section{Descending Fibre Systems in Spinal Cord.}

(1) Pyramidal tracts (anterior and lateral), from cortex of central gyri of same and opposite side.

(2) Anterior marginal bundle from nucleus fastigii.

(3) Sulcomarginal fasciculus $=$ path from superior colliculus of corpora quadrigemina.

(4) Descending paths in fasciculus lateralis proprius.

(a) Tract from Deiters's nucleus to lateral funiculus.

(b) Tract from red nucleus of opposite side to lateral funiculus.

(c) Tract from nuclei laterales superior, medius, inferior, to lateral funiculus.

(5) Descending paths in posterior funiculus.

(a) Descending limbs of bifureation of posterior root-fibres.

(b) Endogenous fibres of posterior funiculi (comma bundle, ventral area, oval centre, median triangle).

(6) Other descending paths in lateral and anterior funiculi.

(a) Endogenous fibres (tautomeric and heteromeric).

\section{Ascending Fibre Systems in Spinal Cord. (Vide Fig. 103.)}

(1) Direct continuation of ascending limbs of bifurcation of posterior root-fibres to nucleus funiculi gracilis, nucleus funiculi cuneati, and partly to cerebellum.

Secondary path: from nuclei of dorsal funiculi, through decussatio lemniscorum to stratum interolivare lemnisci of opposite side, and then through lemniscus medialis to [ventro-]lateral nucleus of thalamus.

Tertiary path: from ventrolateral nucleus of thalamus to cortex of central gyri (somaesthetic area).

(2) Fibre systems leading from the end-stations of posterior root-fibres in the spinal cord to higher centres.

(a) Cerebellospinal fasciculus (fasciculus cerebellospinalis) (O. T. direct cerebellar tract), from nucleus dorsalis of Clarke to cerebellum.

(b) Gowers's tract (fasciculus anterolateralis superficialis [Gowersi]), from base of anterior horn to cerebellum.

(c) From posterior horn upward in lateral limiting layer of gray matter, and in opposite anterior funiculus.

(3) Fibre systems from some of cells in anterior liorns upward in fasciculus anterior proprius and fasciculus lateralis proprius to formatio reticularis.

\section{Fibre Systems of Cerebral Nerves.}

Like the motor spinal nerves, the motor cerebral nerves arise from nuclei of origin (nuclei originis) in the brain-stem, the fibres leaving the central system to terminate in the muscles. 
Like the sensory spinal nerves, the sensory cerebral nerves arise from ganglia outside the central nervous system and their axones terminate in certain end-nuclei (nuclei terminales) inside the central system; in these end-nuclei are cells which give off the axones of secondary fibre systems.

\section{Olfactory Nerves (NN. olfactorit).}

Nuclei originis: bipolar cells of regio olfactoria of nasal mucous membrane.

Nuclei terminales: in olfactory glomeruli of bulbus olfactorius.

Secondary path: from mitral cells of bulbus olfactorius through tractus olfactorius to-

(a) Gray matter of trigonum olfactorium.

(b) Through stria olfactoria lateralis to uncus and thence to hippocampus and gyrus cinguli.

(c) Through stria olfactoria intermedia to substantia perforata anterior.

(d) Through stria olfactoria medialis to gyrus subcallosus.

Bipolar Cells of Retina (analogous, for visual path, to sensory fibres of posterior roots of spinal nerves).

Nuclei terminales: ganglion cell layer of retina.

Secondary path: from ganglion cell layer of retina, through (badly named) N. opticus, chiasma opticum, and tractus optici, to-

(a) Corpus geniculatum laterale.

(b) Pulvinar thalami.

(c) Superficial and middle gray strata of superior colliculus of corpora quadrigemina.

Tertiary paths:

(a) From corpus geniculatum laterale and pulvinar thalami, through radiato occipitothalamica [Gratioleti], to cortex of occipital lobe about the fissura calcarina.

(b) From middle gray layer of colliculus superior, through decussatio tegmenti dorsalis of Meynert and fasciculus longitudinalis medialis of opposite side, to eye-muscle nuclei and spinal cord; this is the so-called "optic-acoustic reflex path" (Held).

\section{OCUlomotor NeRve (N. OCULOMOTORIUS).}

Nuclei originis: nucleus of oculomotor nerve (nucleus N. oculomotorii) in tegmentum of cerebral peduncle.

Cortical control:

(a) From cortex of central gyri through capsula interna (?).

(b) From cortex about fissura calcarina to superior colliculus and then through optic-acoustic reflex path.

\section{Trochlear Nerve (N. trochlearis).}

Nucleus originis: nucleus of trochlear nerve (nucleus $N$. trochlearis) in isthmus rhombencephali on opposite side, through decussatio nervorum trochlearium.

Cortical control: probably same as for N. oculomotorius. 
Trigeminal Nerve (N. Trigeminus).

(A) Motor part (portio minor).

Nuclei originis :

(a) Principal motor nucleus (nucleus motorius princeps N. trigemini) in pars dorsalis pontis.

(b) Lesser motor nuclei (nuclei motorii minores N. trigemini).

(ba) Cells of locus eaeruleus.

(bb) Cells along descending or mesencephalic root (nucleus radicis descendentis).

Cortical control: from lower third of gyrus centralis anterior and feet of frontal gyri, through capsula interna and partly through basis pedunculi (pyramidal tract), partly through area of lemniscus medialis (accessory lemniscus of v. Bechterew).

(B) Sensory part (portio major).

Nucleus originis: semilunar ganglion (ganglion semilunare [Gasseri]). Nuclei terminales :

(a) Main sensory nucleus (nucleus sensibilis N. trigemini).

(b) Nucleus of spinal tract (nucleus tractus spinalis $N$. trigemini).

Secondary paths:

(a) To opposite medial lemniscus and through it to [ventro-] lateral nucleus of thalamus.

(b) To substantia reticularis alba of same and of opposite side, and through it to [ventro-] lateral nucleus of thalamus.

Tertiary path: from [ventro-]lateral nucleus of thalamus to central gyri (somaesthetic area).

Abducent Nerve (N. abducens).

Nucleus originis: nucleus of abducent nerve (nucleus $N$. abducentis) in pars dorsalis pontis.

Cortical control:

(a) Pyramidal tract (?).

(b) "Accessory lemniscus" (?).

(c) Indirectly through optic-acoustic reflex path.

Facial Nerve (N. facialis).

Nucleus originis: nucleus of facial nerve (nucleus $N$. facialis) in ventral lateral part of pars dorsalis pontis close to medulla oblongata.

Cortical control:

(a) Through pyramidal tract of the same and of the opposite side.

(b) Through the "accessory descending lemniscus" of the same and of the opposite side (Hoche).

INTERMEdiate Nerve (N. intermedius).

Nucleus originis: geniculate ganglion (ganglion geniculi).

Nucleus terminalis: probably the same as for $\mathrm{N}$. vagus and $\mathrm{N}$. glossopharyngeus.

Secondary path: uncertain.

Acoustic Nerve (N. acusticus).

(A) Vestibular nerve ( $N$. vestibularis).

Nucleus originis: vestibular ganglion (ganglion vestibulare).

Nuclei terminales:

(a) Lateral nucleus (nucleus N. vestibularis lateralis [Deitersi]). 
(b) Medial nucleus (nucleus $N$. vestibularis medialis).

(c) Superior nucleus (nucleus $N$. vestibularis superior).

(d) Nucleus of descending root (nucleus radicis descendentis). Secondary paths:

(a) From lateral nucleus of Deiters to lateral funiculus of cord.

(b) From medial and superior nuclei to fasciculi longitudinales mediales of 'two sides for eye-muscle nuclei, ete.

(c) From lateral and superior nuclei to nucleus fastigii, nucleus dentatus, and cortex vermis.

(d) From all the terminal nuclei, through the substantia reticularis alba to the [ventro-] lateral nuclei of the thalamus (?).

Tertiary path: from [ventro-]lateral nuclei of thalamus to central gyri (somaesthetic area).

(B) Cochlear nerve ( $N$. cochlearis).

Nucleus originis: spiral ganglion (ganglion spirale).

Nuclei terminales:

(a) Ventral nucleus (nucleus $N$. cochlearis ventralis).

(b) Dorsal nucleus (nucleus N. cochlearis dorsalis [nucleus tuberculi acustici]).

Secondary paths:

(a) Fibres from ventral nucleus through corpus trapezoideum to upper olives, and through lateral lemniscus to nucleus of lateral lemniscus, inferior colliculus, middle gray layer of superior colliculus, and through brachium quadrigeminum inferius to medial geniculate body, possibly some even to temporal cortex.

(b) Fibres from dorsal nucleus through stria medullares to trapezoid body.

Tertiary paths: from end-stations of secondary path (a), especially from nucleus colliculi inferioris and nucleus corporis geniculati medialis, through capsula interna and pars temporalis of corona radiata, to gyrus temporalis superior (junction of third and fourth fifths) and adjacent gyri temporales transversi. [When this cortical area is diseased, the patient has "word-deafness."]

Glossopharyngeal and Vagal Nerves (N. glossopharyngeus et N. vagus).

(A) Motor part.

Nuclei originis :

(a) Dorsal nucleus (nucleus dorsalis Nn. glossopharyngei et vagi) in medulla oblongata just medial from ala cinerea.

(b) Ventral nucleus (nucleus ambiguus) in the formatio reticularis of medulla oblongata just dorsal from nucleus olivaris accessorius dorsalis.

Cortical control:

(a) Pyramidal tract from central gyri.

(b) Accessory lemniscus (?).

(B) Sensory part.

Nuclei originis:

(a) For N. glossopharyngeus.

(aa) Superior ganglion (ganglion superius).

(ab) Petrosal ganglion (ganglion petrosum).

(b) For N. vagus. 
(ba) Jugular ganglion (ganglion jugulare).

(bb) Ganglion nodosum (ganglion nodosum) ("knotty" ganglion).

Nuclei terminales:

(a) Nucleus of ala cinerea (nucleus alae cinereae).

(b) Nucleus of tractus solitarius (nucleus tractus solitarii).

(c) Conmissural nucleus (nucleus commissuralis) (O. T. ganglion commissurale).

Secondary paths:

(a) From nuclei terminales through fibrae arcuatae internae to opposite stratum interolivare lemnisci and thence through lemniscus medialis to [ventro-]lateral nucleus of thalamus.

(b) From nuclei terminales through lateral bundles of substantia reticularis alba of same and of opposite side to [ventro-] lateral nuclei of thalamus.

(c) From nuclei terminales through medial portion of corpus restiforme to nucleus fastigii and cortex vermis.

Tertiary path: probably from [ventro-]lateral nucleus of thalamus through capsula interna and corona radiata to central gyri (somaesthetic area).

\section{Conduction Paths of Cerebellar Peduncles.}

Paths of Corpus Restiforme (O. T. Inferior Peduncle).

(a) Paths of corpus restiforme proper.

(aa) Cerebellospinal fasciculus (fasciculus cerebellospinalis, or, better, fasciculus spinocerebellaris dorsolateralis) ( $\mathrm{O} . \mathrm{T}$. direct cerebellar tract).

(ab) External arcuate fibres (fibrae arcuatae externae) $=$ crossed and uncrossed medullated axones from nuclei funiculi cuneati et gracilis.

(ac) Cerebello-olivary fibres (fibrae cerebelloolivares), partly ascending, partly descending.

(ad) Medullated axones from nuclei laterales to cerebellum. These paths ( $a a, a b, a c$, and $a d)$ run into the cerebellum to end in the cortex of the vermis; they give off collaterals and some terminals to the nucleus fastigii, nucleus dentatus, nucleus emboliformis, and nucleus globosus as these are passed.

(b) Paths of medial part of corpus restiforme.

(ba) Ascending paths, corresponding to secondary paths from the nuclei terminales of the Nn. vagus, glossopharyngeus, vestibularis, et trigeminus to the nucleus fastigii and cortex vermis.

$(b b)$ Descending paths from nucleus fastigii to opposite nucleus $\mathrm{N}$. vestibularis [Deitersi]. This connects at Deiters' nucleus with the path leading to the lateral funiculus of the spinal cord.

Paths of Brachium Pontis (O. T. Middle Cerebellar Peduncle).

(a) Cerebellopontal paths, from cortex of hemisphaerium cerebelli to nuclei pontis of same and of opposite side. 


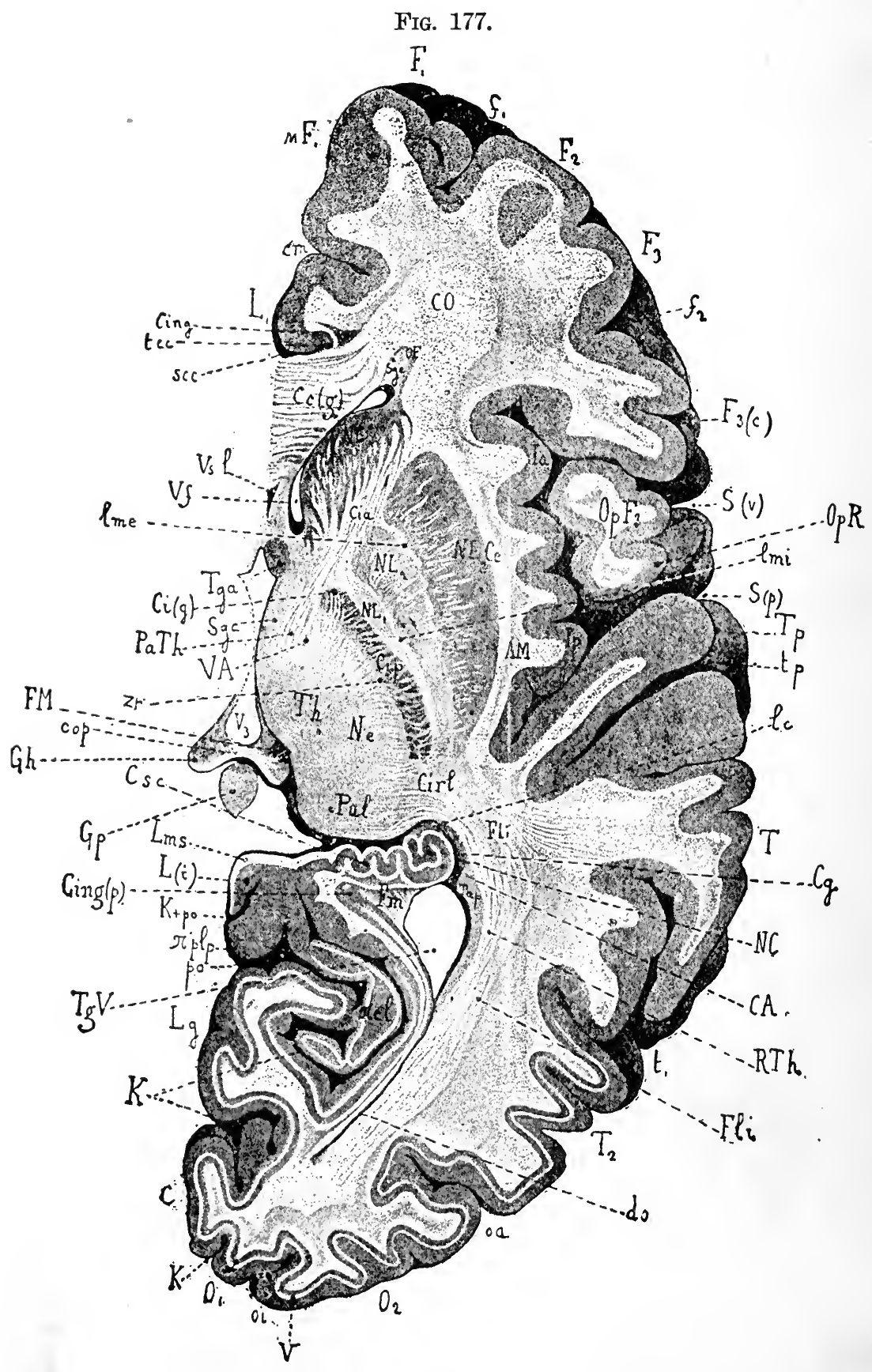


Horizontal section through the right cerebral hemisphere cut at a distance of $61 \mathrm{~mm}$. below its superior border; natural size.

$A M$, claustrum ; $C$, cuneus; $C A$, hippocampus (cornu ammonis) ; $C c(g)$, genu corporis eallosi ; $C e$, capsula externa ; $C g$, gyrus dentatus ; $C i a$, pars frontalis capsulae internae; $C i(g)$, genu capsulae internae ; Cing, horizontal bundle of the cingulum; $\operatorname{Cing}(p)$, posterior bundle of the eingulum ; Cip, pars occipitalis capsulae internae ; $\mathrm{Cirl}$, retrolenticular portion of internal capsule ; $\mathrm{cm}$, sulcus cinguli ; $\mathrm{CO}$, centrum semiovale; cop, commissura posterior cerebri ; Csc, gyrus subeallosus ; do, cornu posterius ventrieuli lateralis ; $F_{1}$, gyrus frontalis superior ; $F_{2}$, gyrus frontalis medius ; $F_{3}$, gyrus frontalis inferior; $f_{1}$, sulcus rontalis superior; $f_{2}$, sulcus frontalis inferior; $F_{3}(c)$, pars triangularis gyri frontalis inferioris; $F l i$, fasciculus longitudinalis inferior; FM, fasciculus retroflexus Meynerti; $\mathrm{Fm}^{\prime}$, faseiculus inferior or minor of the fornix ; $G h$, nucleus habenulae ; $G p$, corpus pineale ; $I a$, insula (pars anterior) ; $I p$, insula (pars posterior) ; $K$, fissura calcarina; $K_{+} p o$, union of the fissura calcarina with the fissura parictooccipitalis; $L_{1}$, gyrus cinguli ; $L(i)$, isthmus gyri fornicati ; $l c$, lamina cornea and fibres of the taenia semicircularis; $L g$, gyrus lingualis; $l m e$, lamina medullaris lateralis nuclei lentiformis; $l m i$, lamina medullaris medialis nuclei lentiformis; $l n s$, lamina medullaris superficialis ; $m F_{1}$, facies medialis gyri frontalis superioris; $N C$, caput nuclei caudati; $N C^{\prime}$, cauda nuclei caudati; $N e$, nucleus lateralis thalami; $N L_{1}, N L_{2}$, globus pallidus (of nucleus lentiformis); $N L_{3}$, putamen (of nueleus lentiformis); $O_{1}, O_{2}$, gyri occipitales; $o a$, suleus occipitalis anterior of Wernicke; $O F$, fasciculus occipito-frontalis; $o i$, sulcus interoccipitalis; $O p, F_{3}$, pars opercularis gyrus frontalis inferioris; $O p R$, Rolandic operculum; $\mathrm{PaTh}$, pedunculus anterior thalami; $p o$, fissura parieto-occipitalis; $P u l$, pulvinar; $\pi c l$, cuneo-limbic fold; $\pi p l p$, posterior parieto-limbic fold; $R T h$, radiatio oceipito-thalamica [Gratioleti] ; $S(p)$, ramus posterior fissurae eerebri lateralis Sylvii ; $S(v)$, ramus ascendens; $s c c$, sinus corporis eallosi ; $S g c$, substantia grisea centralis; Sge, subependymal gray matter; $T_{1}$, gyrus temporalis superior; $T_{2}$, gyrus temporalis medius; $t_{1}$, sulcus temporalis superior; Tap, tapetum; tec, taenia tecta; Tga, anterior pillar of the fornix; $T g V$, ventriculus lateralis; $T h$, thalamus; $T p$, gyrus temporalis transversus ; $t p$, sulcus temporalis transversus; $V_{3}$, ventriculus tertius; $V$, stripe of Vieq d'Azyr; $V A$, fasciculus thalamomammillaris Vicq d'Azyri; $V f$, cornu anterius ventriculi lateralis; $V s l$, cavum septi pellucidi; $Z r$, zona reticularis. (After J. Déjerine, Anatomie des Centres Nerveux, Paris, 1895, p. 408, Fig. 226.) 
(b) Pontocerebellar paths, from nuclei pontis to cortex of hemisphaerium cerebelli of same and of opposite side.

Paths of Brachium Conjunctivum (O. T. Superior Cerebellar Peduncle).

(a) Paths from nucleus dentatus cerebelli through brachium conjunctivum to tegmentum of cerebral peduncle, there to decussate in large part in the decussatio brachii conjunctivi, then to give off large numbers of collaterals to the nucleus ruber, and finally, joining the fibres of the lemniscus medialis, to end in the [ventro-]lateral nucleus of the thalamus. There it connects with the thalamocortical path to the central gyri (somaesthetic area).

This path influences the spinal cord in at least two ways:

(1) By collateral fibres which leave main axones soon after exit from the cerebellum and pass down to the spinal cord (ramus descendens of Ramón y Cajal).

(2) Through the red nucleus which sends a path to the lateral funiculus.

(b) Path from spinal cord to eerebellum running in Gowers' tract upward to pons and corpora quadrigemina, then hooking around N. trigeminus and running, in velum medullare anterius, close to brachium conjunctivum, to cerebellar vermis.

\section{Conduction Paths of Diencephalon and Telencephalon.}

\section{Projection Fibre Systems.}

These are the fibre systems which connect the gray matter of the telencephalon (pallium, nucleus caudatus, nucleus lentiformis, rhinencephalon) with lower parts of the nervous system (thalamus, brainstem, spinal cord).

(A) Ascending projection systems (corticopetal paths).

(1) Uppermost neurone systems of general sensory path. The medullated axones pass from the cells in the [ventro-]lateral nucleus of the thalamus, chiefly through the posterior third of the capsula interna and corona radiata, to the cortex of the central gyri (somaesthetic area); a few fibres go through or around the nucleus lentiformis, and by way of the capsula externa to the cortex.

(2) Upper part of visual conduction path. The medullated axones pass from the cells in the nucleus corporis geniculati laterale and pulvinar thalami, through the radiatio occipitothalamica, to the cortex about the fissura calcarina.

(3) Upper part of auditory conduction path. The medullated axones pass from the cells in the nucleus corporis genieulati medialis and nucleus colliculi inferior, by way of the posterior part of the capsula interna, lateralward to the gyrus temporalis superior (junction of third and fourth fifths), and adjacent gyri temporales transversi.

(B) Descending projection systems (corticofugal paths).

(1) Pyramidal tract (fasciculi pyramidales), from large pyramidal cells of central gyri and lobulus paracentralis, through corona radiata and through knee and anterior two-thirds of oceipital limb of capsula interna (head-movement fibres in front, arm 
fibres in middle, and leg fibres behind), into basis pedunculi, to motor nuclei of cerebral nerves and to anterior horns of spinal cord.

(2) "Accessory lemniscus" of v. Bechterew, from cortex to motor nuclei of cerebral nerves.

(3) Frontal corticopontile path (fasciculus corticopontilis frontalis), from posterior part of frontal lobe, through frontal limb of eapsula interna and through medial part of basis pedunculi (Arnold's bundle), to nuclei pontis.

(4) Temporal corticopontile path (fasciculus corticopontilis temporalis).

(5) Occipitomesencephalic path (fasciculus occipitomesencephalica) (O. T. secondary optic radiation of Flechsig), fibres from visual sense area about fissura calcarina, downward through occipitothalamic radiation to superior colliculus.

(6) Projection fibre systems of central olfactory paths.

(a) Hippocampo-mammillary fibre system, going from cells in hippocampus, through fornix and columna fornicis, to nuclei corporis mammillaris of same and of opposite side; there connecting with-

(aa) Principal mammillary fasciculus (fasciculus mammillaris princeps), which divides into-

(aaa) Thalamomammillary fasciculus (fasciculus thalamomammillaris [Vicq d'Azyri]), going to nucleus anterior thalami, and

(aab) Tegmental part of pedunculomammillary fasciculus (fasciculus pedunculomammillaris, pars tegmentalis), to the tegmentum of the cerebral peduncle.

(ab) Basilar part of peduneulomammillary fasciculus (fasciculus pedunculomammillaris, pars basilaris) (O. T. peduncle of mammillary body).

\section{Associative Fibre Systems.}

(A) Connecting different parts of same hemisphere.

(a) Those with short axones, including the fibrae propriae of the gyri cerebri.

(b) Those with long axones.

(ba) Cingulum (cingulum) ("girdle"), in the gyrus cinguli and gyrus hippocampi.

(bb) Superior longitudinal fasciculus (fasciculus longitudinalis superior), extending between lobus frontalis and lobus occipitalis.

(bc) Inferior longitudinal fasciculus (fasciculus longitudinalis inferior), between lobus occipitalis and more anterior parts of brain.

(bd) Uncinate fasciculus (fasciculus uncinatus), between uncus and base of lobus frontalis.

(B) Connecting the two hemispheres with each other (commissural fibre systems).

(a) Corpus callosum (corpus callosum) ("callous" or "lard" body).

(b) Anterior commissure of cerebrum (commissura anterior [cerebri]).

(c) Commissure of hippocampus (commissura hippocampi). 
Fig. 178.

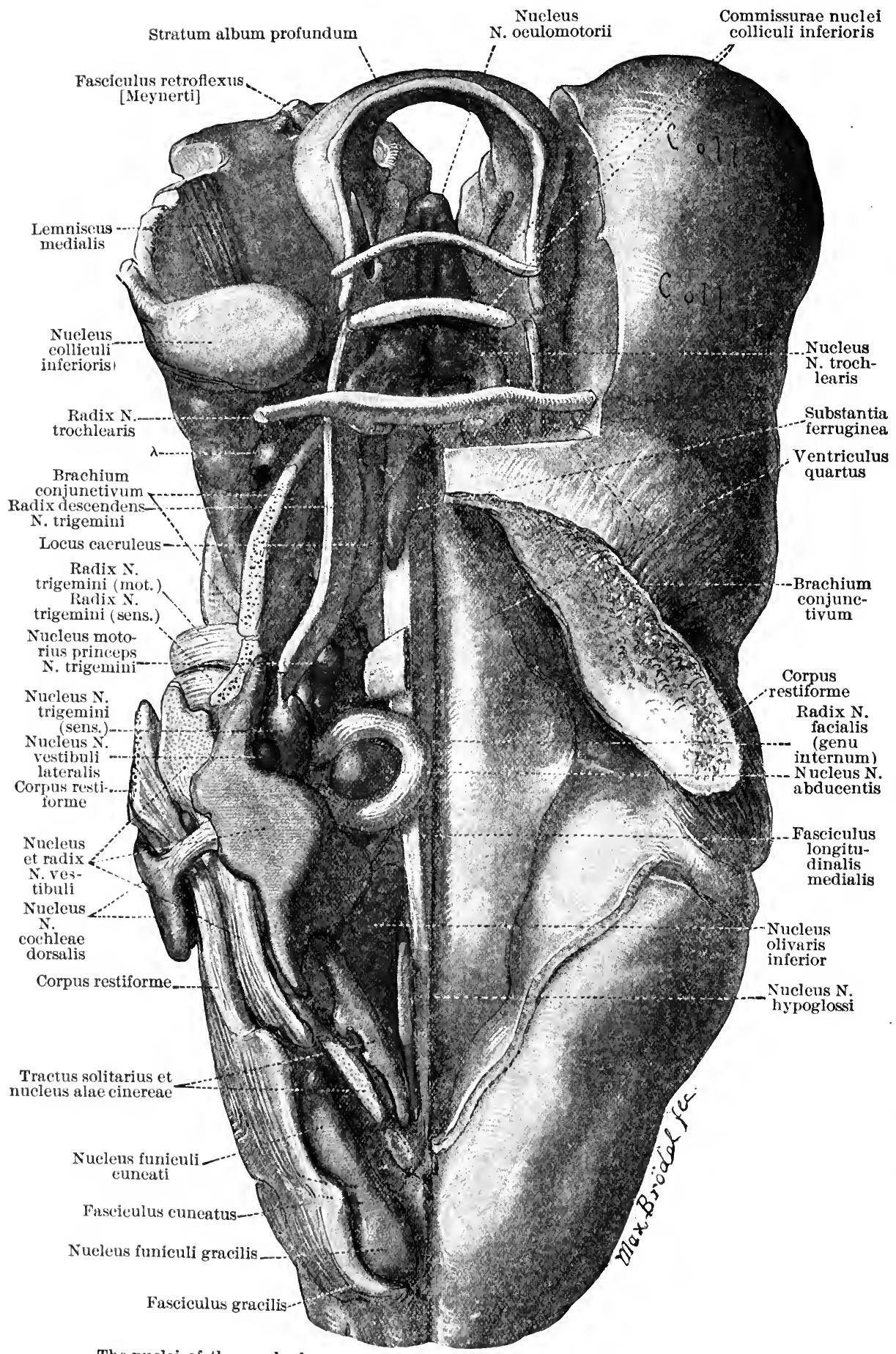

The nuclei of the cerebral nerves. Dorsal surface of model. (From Sabin, An Atlas of the Medulla and Midbrain, Baltimore, 1901, Plate iii.) 


\section{ORGAN OF VISION (ORGANON VISUS).}

This includes the eyeball (bulbus oculi), the optic nerve $(N$. opticus), and the accessory organs of the eyeball (organa oculi accessoria). The latter, including the extrinsic muscles of the eye, orbital fasciae, eyelids and eyebrows, conjunctiva, and lacrimal apparatus, as well as the optic nerve, have already been studied. The eyeball itself should now be examined.

The student should provide himself with several eyeballs from the ox and pig; some of these may be dissected fresh,

FIG. 179.

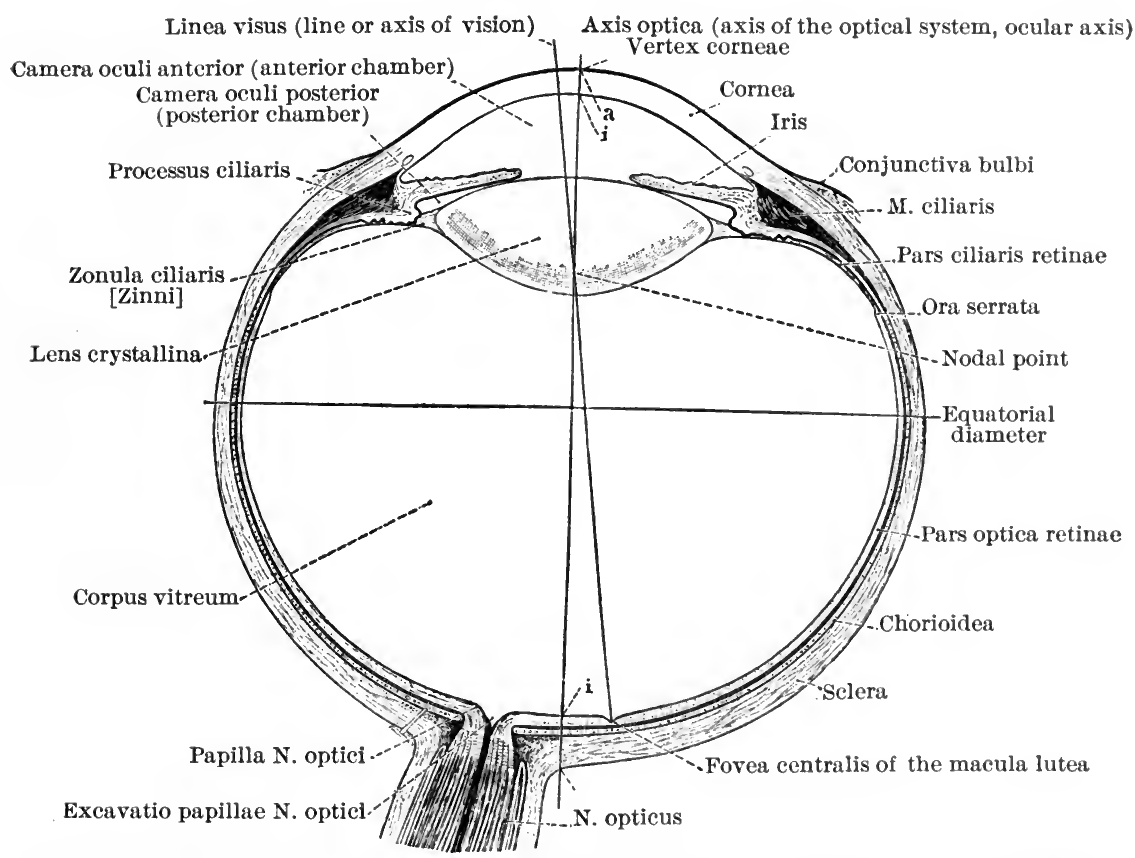

Schematic section in the horizontal meridian of the right eyeball. (After Toldt, Anat. Atlas, Wien, 1903, 3 Aufl., p. 892, Fig. 1334.)

others after hardening in formalin. Sections in different planes should be made of eyeballs hardened in formalin. These may easily be permanently preserved in formalin gelatin. The eyeballs should first be cleaned. Remove the conjunctiva, eyemuscles, fat, and capsule of Tenon. This is best done by grasping the conjunctiva and fascia bulbi with the point of the forceps close to the limbus corneae and snipping through them with fine 
sharp scissors, going right around the cornea in this way. All the soft parts may now be stripped off the sclera back as far as the N. opticus. Take two eyes, hardened in formalin, and divide one by a sagittal section into a medial and a lateral half, and the other by a coronal section through the equator into an anterior and a posterior half. With a sharp razor this can be easily accomplished in formalinized eyes without freezing.

Ascertain the exact meaning of the following terms :

(a) Anterior pole (polus anterior).

(b) Posterior pole (polus posterior).

(c) Equator (aequator).

(d) Meridians (meridiani).

(e) External axis of eyeball (axis oculi externa).

$(f)$ Internal axis of eyeball (axis oculi interna).

(g) Optic axis (axis optica).

(h) Line of vision (linea visus).

And of the two embryological terms:

(i) Ophthalmic vesicle (vesicula ophthalmica).

(j) Ophthalmic eup (caliculus ophthalmicus).

Note that the eyeball possesses the following:

(a) Fibrous tunic (tunica fibrosa oculi).

(aa) Cornea.

(ab) Sclera.

(b) Vascular tunic (tunica vasculosa oculi).

(ba) Chorioid coat (chorioidea).

(bb) Ciliary body (corpus ciliare).

(bc) Iris (iris).

(c) Pigment layer (stratum pigmenti).

(d) True nervous portion of eye (retina).

(e) Anterior and posterior chambers (camera oculi anterior, posterior).

(f) Vitreous body (corpus vitreum).

(g) Crystalline lens (lens crystallina).

(h) Ciliary zonule (zonula ciliaris [Zinni]).

\section{Fibrous Coat of Eye (Tunica fibrosa oculi).}

Make an incision with a very sharp knife through the sclera of an eyeball at the equator; let the knife cut only as far as the black chorioidea, then lay it aside. Catch the edge of the sclera in forceps, and with sharp fine scissors eut through the sclera all the way around the eyeball, along the line of the equator. Raise the anterior and posterior segments of the sclera from the subjacent parts. In turning the anterior half forward, overcome the resistance met near the margin of the cornea, due to the attachment of the M. ciliaris to the deep surface of the sclera, 
with the probe or the blunt point of the closed forceps; on breaking through this resistance the so-called " aqueous humor" escapes. To separate completely the posterior segment of the sclera from the rest of the eyeball, cut through the fibres of the

Fig. 180.

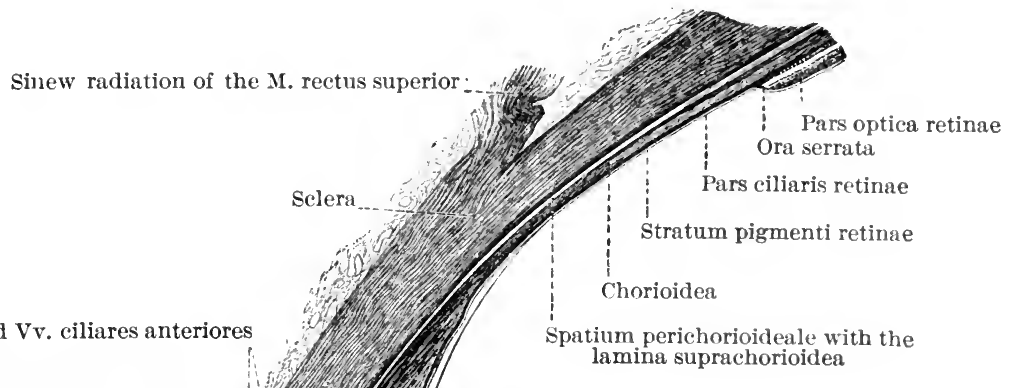

Aa. and Vv. ciliares anteriores

Circulus arteriosus major

Angulus iridis

Sinus venosus sclerae

(canalis Schlemmi)

Episcleral connective

tissue with Aa. and Vv.

Rima cornealis

for the cornea) Limbus corneae
(border of the
cornea) Iris (facie anterior)

\section{Camera oculi} anterior Cornea (substantia propria)

Facies posterior corneae Epithelium corneae

Lamina elastica anterior [Bowmani]

Lamina elastica posterior [Descemeti] with the M. endothelium camerae anterioris
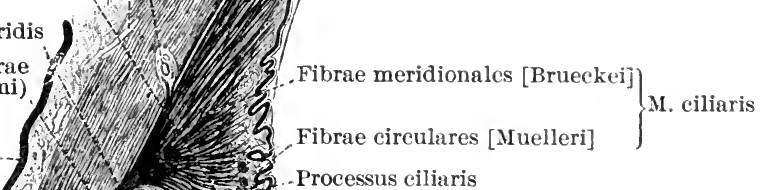

The upper half of a section in the vertical meridian of the anterior part of the eyeball.

(After Toldt, Anat. Atlas, Wien, 1903, 3 Aufl., p. 893, Fig. 1337.)

$\mathrm{N}$. opticus in a plane corresponding to the inner surface of the sclera. Place the denuded eyeball in a dish of water and study the sclera and cornea. 
In the scLERA (O. T. sclerotic coat, or white of the eye) examine the following:

(a) Sulcus of the sclera (sulcus sclerae).

Make a meridional incision through the sclerocorneal junction and note-

(b) Cleft for the cornea (rima cornealis).

(c) Venous sinus of the sclera, or eanal of Schlemm (sinus venosus sclerae [canalis Schlemmi, Lauthi]).

(d) Brown layer (lamina fusca).

To what is this due?

(e) Perforated layer of sclera (lamina cribrosa sclerae).

In the CORNEA study the following:

(a) Ring of conjunctiva (annulus conjunctivae).

(b) Vertex of cornea (vertex corneae).

(c) Border of cornea (limbus corneae).

(d) Anterior surface (facies anterior).

(e) Posterior surface (facies posterior).

Make a razor section or a frozen section through the cornea, and under the dissecting microscope observe-

FIG. 181.

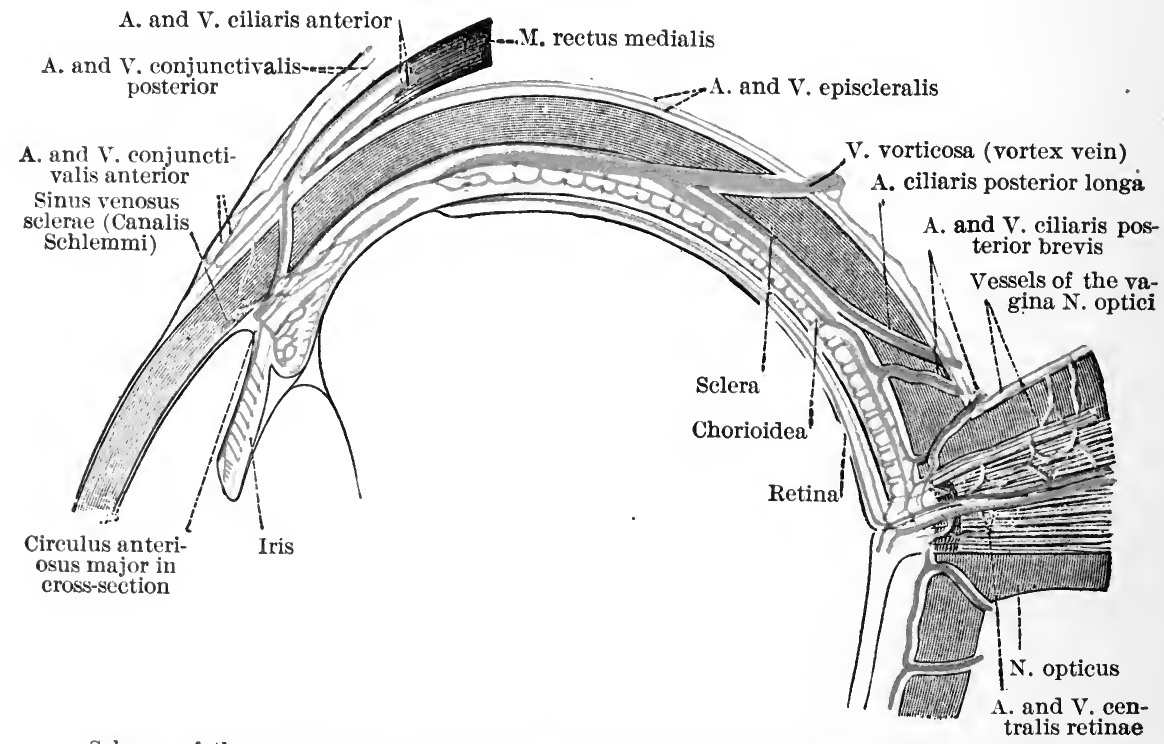

Scheme of the arrangement of blood-vessels in the cyeball. Horizontal section. (After

Th. Leber.) (From Toldt, Anat. Atlas, Wien, 1903, 3 Aufl., p. 897, Fig. 1351.)

(f) Epithelium of cornea (epithelium corneae).

(g) Anterior elastic layer (lamina elastica anterior [Bowmani]) (O. T.

Bowman's membrane).

(h) Proper substance (substantia propria). 
(i) Posterior elastic layer (lamina elastica posterior [Demoursi, Descemeti]) (O. T. Descemet's membrane).

(j) Endothelium of anterior chamber (endothelium camerae anterioris).

\section{Vascular Coat of Eye (Tunica vasculosa oculi).}

In the CHORIOID COAT (chorioidea) study the following:

(a) Suprachorioid layer (lamina suprachorioidea).

(b) Perichorioideal space (spatium perichorioideale).

(c) Vascular layer (lamina vasculosa).

Observe the Vv. vorticosae. If the pigment be washed out with a camel's-hair brush, these will become very evident.

(d) Choriocapillary layer (lamina choriocapillaris) (O. T. tunica Ruysehiana).

Between $(c)$ and $(d)$ observe the Aa. ciliares posteriores breves.

In the CILIARY BODY (corpus ciliare) the ciliary wreath (corona ciliaris) and the ciliary disk (orbiculus ciliaris) are to be studied. To expose the ciliary wreath make a coronal section through an eyeball a little in front of the equator, and remove cautiously the vitreous humor from the anterior segment of the eyeball. Examine-

Fig. 182.

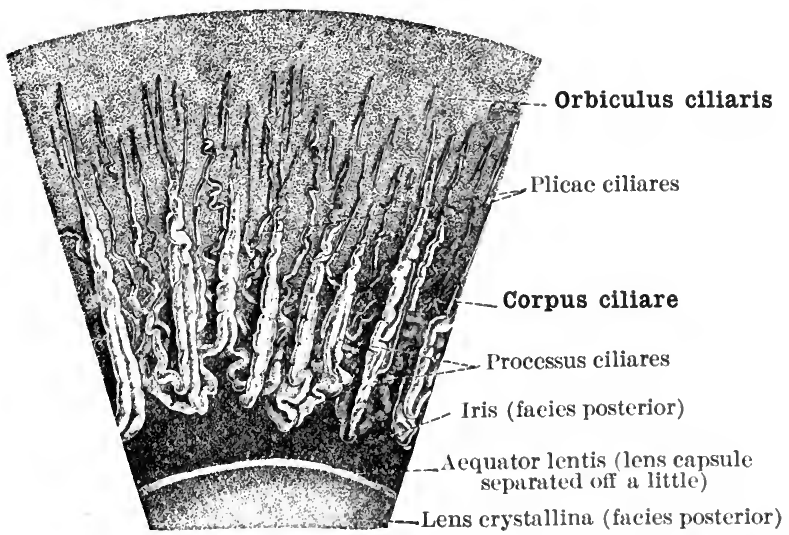

A part of the ciliary processes and ciliary folds, enlarged. (After Toldt, Anat. Atlas, Wien, 1903. 3 Aufl., p. 895, Fig. 1342.)

(a) Ciliary processes (processus ciliares).

(b) Ciliary folds (plicae ciliares).

With a camel's-hair brush wash out the pigment and note the exact disposition of the processes and folds.

The ciliary wreath may be exposed from in front in another eyeball as follows: remove cornea with scissors at sclerocorneal junction. Make four meridional cuts through sclera and reflect 
its parts backward, separating each from the M. ciliaris; pin these down to the bottom of a cork-lined tray. Examine the iris and then cautiously remove it.

In the ciliary disk (orbiculus ciliaris) examine-

(a) Ciliary muscle (M. ciliaris).

(aa) Meridional fibres (fibrae meridionales [Brueckei]).

(ab) Circular fibres (fibrae circulares [Mülleri]).

These are best distinguished in a thin meridional section through the corpus ciliare under the microseope.

(b) Ganglionie eiliary plexus (plexus gangliosus ciliaris).

Look for it at the junction of the chorioidea with the corpus ciliare.

In the DIAPHRAGM of THE Eye (iris) study-

(a) Pupillary margin (margo pupillaris).

(b) Ciliary margin (margo ciliaris).

(c) Anterior surface (facies anterior).

(d) Posterior surface (facies posterior).

FIG. 183.

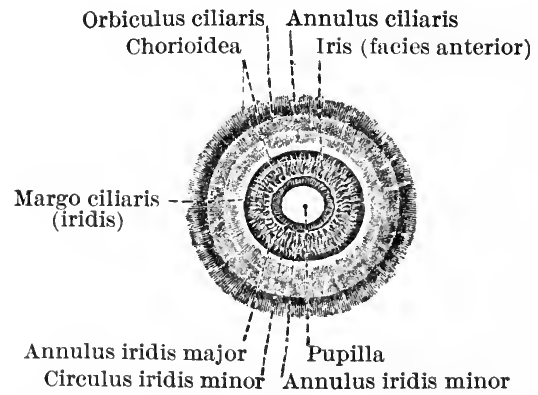

The iris ("rainbow") of a clear gray eye, with the adjacent part of the sclerotic coat. Seen from in front. (After Toldt, Anat. Atlas, Wien, 1903, 3 Aufl., p. 896, Fig. 1347.)

(e) Greater ring of iris (annulus iridis major), between iris and corpus ciliare.

( $f$ ) Lesser ring of iris (annulus iridis minor), between ciliary zone (zona ciliaris) and pupillary zone (zona pupillaris) of iris.

(g) Folds of iris (plicae iridis).

(h) Pupil (pupilla).

(i) Sphincter muscle of pupil (M. sphincter pupillae).

(j) Stroma of iris (stroma iridis).

(k) Dilator muscle of pupil (M. dilator pupillae).

(l) Pectinate ("comb-like") ligament of iris (Lig. pectinatum iridis) (O. T. pillars of the iris). Observe the relation of the fibres of this ligament to those of Descemet's lamina.

$(m)$ Spaces of Fontana at the angle of the iris (spatia anguli iridis [Fontanae]). 
(n) Greater arterial circle (circulus arteriosus major).

(o) Lesser arterial circle (circulus arteriosus minor). life?

What is the pupillary membrane (membrana pupillaris) of foetal

The ciliary nerves and ciliary arteries and the Vv. vorticosae should now be reviewed. (See pp. 277-281.) Examine especially, at the back part of the eye, between the chorioid and the sclera, the passage of the $\mathrm{Vv}$. vorticosae into the sclera; cut through these and observe the Aa. ciliares posteriores breves coming from the sclera to the chorioid.

\section{Layer of Pigment (Stratum pigmenti).}

In an eyeball which has had its sclera and cornea removed and is under water, remove the chorioidea piecemeal, so as to expose the stratum pigmenti and the retina. Note that the stratum pigmenti is divisible into three parts:

(a) Pigment layer of retina (stratum pigmenti retinae).

(b) Pigment layer of ciliary body (stratum pigmenti corporis ciliaris).

(c) Pigment layer of iris (stratum pigmenti iridis).

\section{Retina.}

In this study the following:

(a) Optic part of retina (pars optica retinae). (aa) Serrated edge (ora serrata).

(b) Ciliary part of retina (pars ciliaris retinae).

(c) Papilla of optic nerve (papilla N. optici).

(d) Excavation of papilla of optic nerve (excavatio papillae N. optici).

(e) Yellow spot (macula lutea).

Present in man, but not in the ox or sheep.

(f) Central fovea (fovea centralis).

(g) Blood-vessels of the retina (vasa sanguinea retinae). (Fig. 184.)

The blood-vessels of the retina may now be studied. The student should sometime control this study by an examination of the eye-ground of a living person through the ophthalmoscope. Note-

(a) Superior arteriole [venule] of temporal retina (arteriola [venula] temporalis retinae superior).

(b) Inferior arteriole [venule] of temporal retina (arteriola [venula] temporalis retinae inferior).

(c) Superior arteriole [venule] of nasal retina (arteriola [venula] nasalis retinae superior).

(d) Inferior arteriole [venule] of nasal retina (arteriola [venula] nasalis retinae inferior). 
(e) Superior macular arteriole [venule] (arteriola [venula] macularis superior).

Fig. 184.

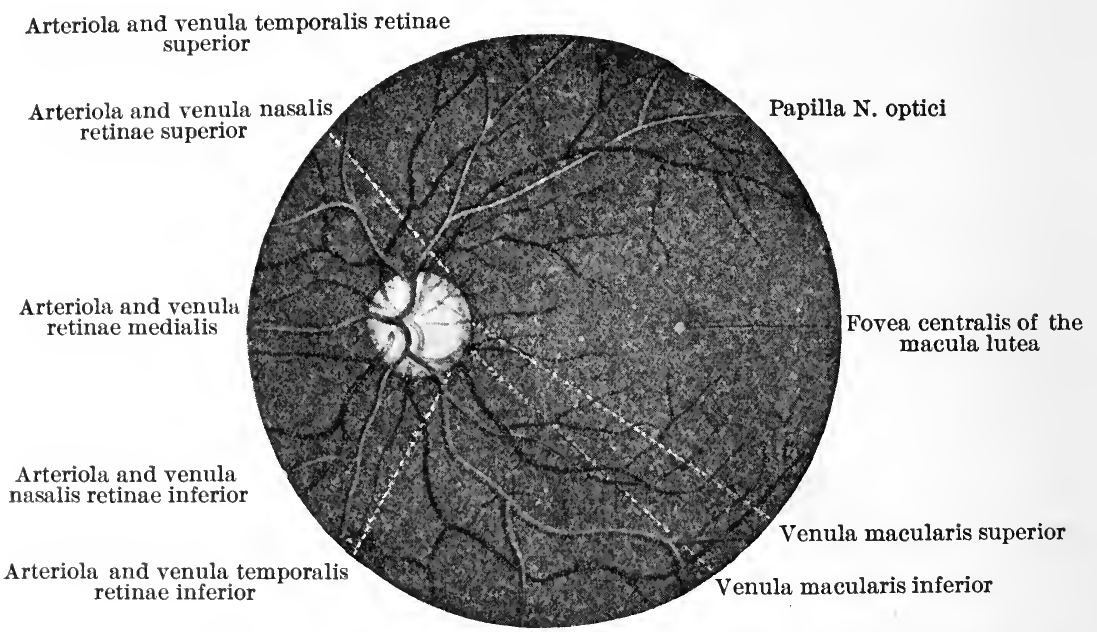

The fundus of the eye, with the retinal vessels; in the left normal eye of a dark-haired young man, as seen with the ophthalmoscope. Erect image. (After E. v. Jäger, in Toldt, Anat. Atlas, Wien, 1903, 3 Aufl., p. 898, Fig. 1355.)

(f) Inferior macular arteriole [venule] (arteriola [venula] macularis inferior).

(g) Medial arteriole [venule] of retina (arteriola [venula] retinae medialis).

\section{Vitreous Body (Corpus vitreum).}

In an eyeball no longer fresh; one that has been kept for from one to four days, divide the tunicae of the eye at the equator, reflect the cut edges backward and forward, and, as Anderson Stuart has suggested, allow the " eye-kernel" (corpus vitreum + lens crystallina) to slip out into a vessel filled with clean water. Transfer it for a few minutes to a strong solution of picrocarmin, then wash in water. The membrana hyaloidea, capsula lentis, and zonula ciliaris [Zinni] will be stained red, and their connections are easily visible.

In the vitreous body study-

(a) Hyaloid canal (canalis hyaloideus) (O. T. canal of Stilling).

(b) Hyaloid fossa (fossa hyaloidea) (O. T. fossa patellaris).

(c) Hyaloid membrane (membrana hyaloidea).

(d) Vitreous stroma (stroma vitreum).

(e) Vitreous humor (humor vitreus). 
Where does the hyaloid artery (A. hyaloidea) run in the embryo?

Ciliary Zonule (Zonula ciliaris [Zinni]). (See Figs. $\mathrm{x} 79$ and $\mathrm{1} 80$. .)

How is this formed? What is its relation to the corpus vitreum and to the lens? What part of it is known as the "suspensory ligament of the lens"? Study-

(a) Zonular fibres (fibrae zonulares).

(b) Zonular spaces (spatia zonularia) (O. T. canal of Petit).

The well-known saccular appearance of these spaces may be easily produced if the point of a fine blow-pipe be inserted into them and they be inflated with air.

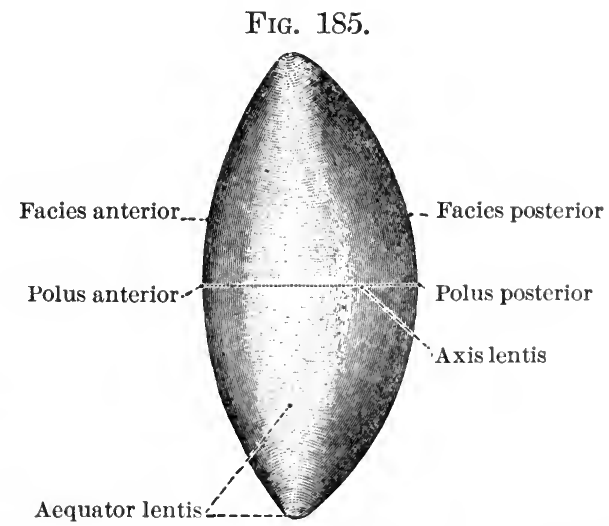

The terms used for the orientation of the lens. (After Toldt, Anat. Atlas, Wien, 1903, 3 Aufl, p. 900 , Fig. 1363.)

\section{Crystalline Lens (Lens crystallina).}

Cut through the fibrae zonulares of the zonula ciliaris [Zinni] and remove the lens. Examine the following:

(a) Capsule of lens (capsula lentis).

(b) Anterior pole of lens (polus anterior lentis).

(c) Posterior pole of lens (polus posterior lentis).

(d) Anterior surface of lens (facies anterior lentis).

(e) Posterior surface of lens (facies posterior lentis).

(f) Axis of lens (axis lentis).

(g) Equator of lens (aequator lentis) (O. T. circumference).

(h) Radii of lens (radii lentis).

Now divide the anterior part of the capsula lentis with a sharp knife and press the lens out through the opening. Study the stained capsule as it floats in water. Press the substance of the lens (substantia lentis) between the finger and thumb, and 
note that the cortical substance (substantia corticalis) is soft while the central part or nucleus of the lens (nucleus lentis) is firmer. Place a bit of the lens in thirty-three and one-third per cent. alcohol for twenty-four hours, then tease it apart and under the microscope examine-

(a) Epithelium of lens (epithelium lentis).

(b) Fibres of lens (fibrae lentis).

\section{Chambers of Eyeball (Camerae oculi).}

These are two in number:

(a) Anterior chamber of eyeball (camera oculi anterior).

(aa) Angle of iris (angulus iridis) (O. T. iridocorneal angle).

Why is this of great importance?

(b) Posterior chamber of eyeball (camera oculi posterior).

Study the form, position, and boundaries of each. Both contain the so-called aqueous humor.

\section{ORGAN OF HEARING (ORGANON AUDITUS).}

This consists of several parts, viz. :

(a) Internal ear (auris interna).

(aa) Membranous labyrinth (labyrinthus membranaceus).

(ab) Osseous labyrinth (labyrinthus osseus).

(b) Middle ear.

(ba) Cavity of tympanum (cavum tympani).

(bb) Eustachian tube (tuba auditiva [Eustachii]).

(bc) Mastoid cells (cellulae mastoideae).

(c) External ear (auricula) (O. T. pinna).

The dry temporal bone should be thoroughly reviewed in connection with the study of the organ of hearing. The external ear has been studied already. (Cf. p. 258.)

Take a temporal bone, preferably one with the auricula still attached to it; saw off the squama temporalis by making a horizontal cut at the level of the root of the zygomatic process; remove roof and anterior wall of the external acoustic meatus piecemeal, with the aid of bone-forceps and chisel, until the membrane of the tympanum is exposed. 
External Acoustic Meatus (Meatus acusticus externus).

Examine the external acoustic meatus (meatus acusticus externus) thus opened, and compare it with a dry temporal bone, and if possible with a macerated specimen showing the cartilaginous part well. Study-

(a) External acoustic porus (porus acusticus externus).

(b) Tympanic incisure (incisura tympanica [Rivini]).

(c) Cartilaginous external acoustic meatus (meatus acusticus externus cartilagineus).

(d) Cartilage of acoustic meatus (cartilago meatus acustici).

$(d a)$ Notches in cartilage of external meatus (incisurae cartilaginis meatus acustici externi [Santorini]).

(db) Layer of tragus (lamina tragi).

FIG. 186.

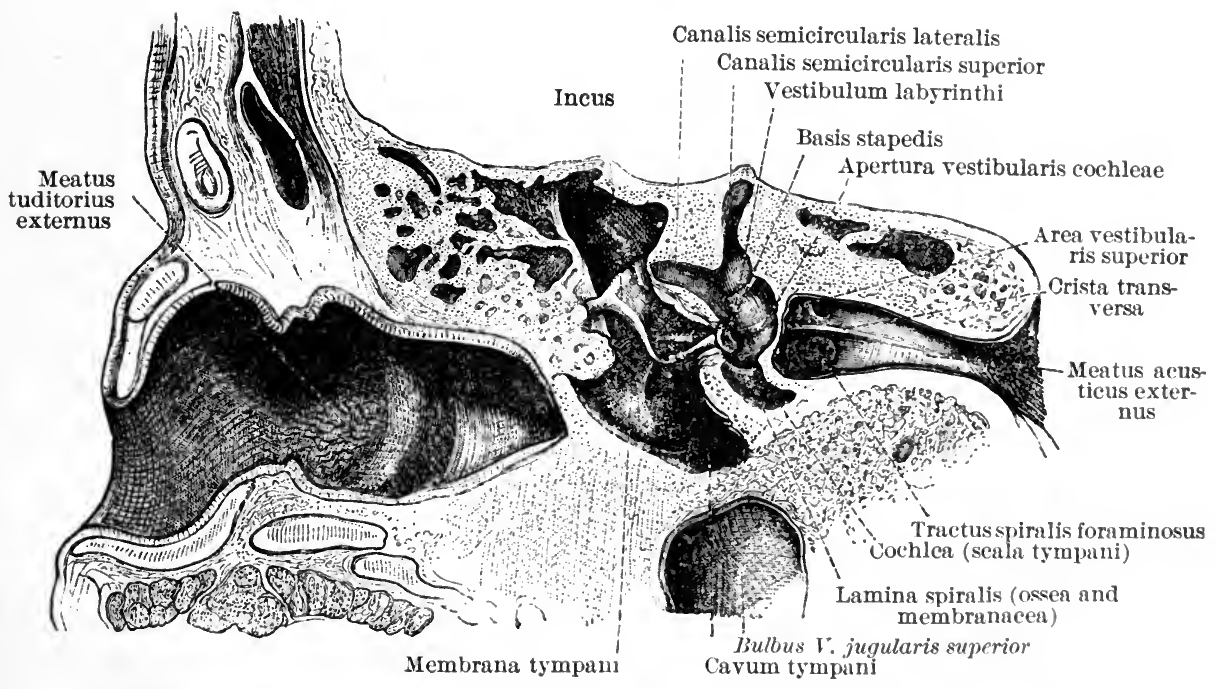

Nearly frontal section through the outer, middle, and inner ear. (After Toldt, Anat. Atlas,

Wien, 1903, 3 Aufl., p. 932, Fig. 1447.)

\section{Cavity of Tympanum (Cavum tympani).}

This cavity (cavum tympani) should next be studied. Make an opening through the roof of the tympanum (tegmen tympani) just lateral from the eminentia arcuata (due to the superior semicircular canal) and about one centimetre in front of the angulus superior pyramidis. The opening leads into the tympanic antrum of the mastoid wall of the cavity of the tympanum. Enlarge the opening carefully with bone-forceps, gradually removing piece by piece the whole roof or tegmental wall (paries 
tegmentalis). Note that the epitympanic recess (recessus epitympanicus) is opened into; observe its cupolar portion (pars cupularis). The general shape of the cavity and walls of the tympanum may easily be remembered by thinking of a miniature cigar-box obliquely placed inside the temporal bone. Study the following:

(a) Jugular wall (paries jugularis) (O. T. floor).

$(a b)$ Styloid prominence (prominentia styloidea).

(b) Mastoid wall (paries mastoidea) (O. T. posterior wall).

(ba) Tympanic antrum (antrum tympanicum) (O. T. mastoidal antrum).

$(b b)$ Prominence of lateral semicircular canal (prominentia canalis semicircularis lateralis).

(bc) Prominence of facial canal (prominentia canalis facialis).

(bd) Pyramidal eminence (eminentia pyramidalis).

(be) Fossa of incus (fossa incudis).

(bf) Posterior sinus (sinus posterior).

(bg) Tympanic aperture of canaliculus of chorda (apertura tympanica canaliculi chordae).

(c) Carotid wall (paries carotica) (O. T. anterior wall).

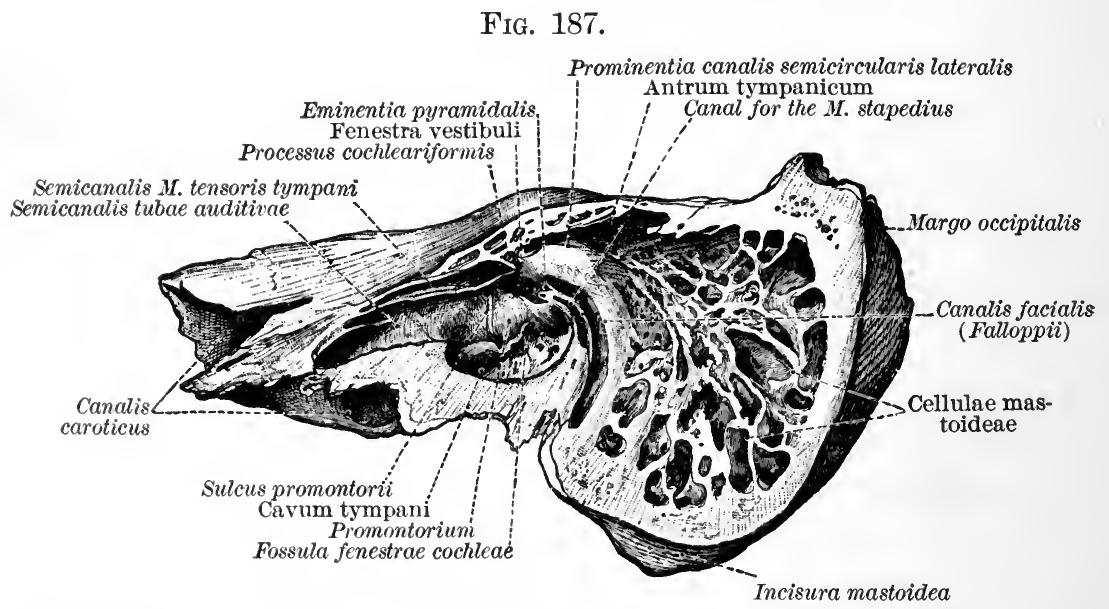

The medial wall, paries labyrinthicus, of the left bony tympanic cavity, and the mastoid cells, cellulae mastoidcae, shown in a section through the mastoid process and the anterior part of the petrous part of the temporal bone. Seen from the anterior and lateral side. (After Toldt, Anat. Atlas, Wien, 1903, 3 Aufl., p. 927, Fig. 1432.)

(d) Labyrinthic wall (paries labyrinthicus) (O. T. inner wall). (Fig. 187.)

(da) Fenestra ("window") of vestibule (fenestra vestibuli) (O. T. fenestra ovalis).

(daa) Fossula of fenestra of vestibule (fossula fenestrae vestibuli).

(db) Promontory (promontorium). 
( $d b a)$ Suleus of promontory (sulcus promontorii).

( $d b b$ ) Subiculum ("support") of promontory (subiculum promontorii).

(dc) Sinus of tympanum (sinus tympani).

(dd) Fenestra ("wiridow") of cochlea (fenestra cochleae) (O. T. fenestra rotunda).

$(d d a)$ Fossula of fenestra of cochlea (fossula fenestrae cochleae).

$(d d b)$ Crest of fenestra of cochlea (crista fenestrae cochleae).

(de) Cochleariform process (processus cochleariformis).

Observe the mastoid cells (cellulae mastoideae) and the tympanic cells (cellulae tympanicae).

(e) Membranous wall (paries membranaceus) (O. T. outer wall).

This consists largely of the membrane of the tympanum, which should now be studied.

Fig. 188.

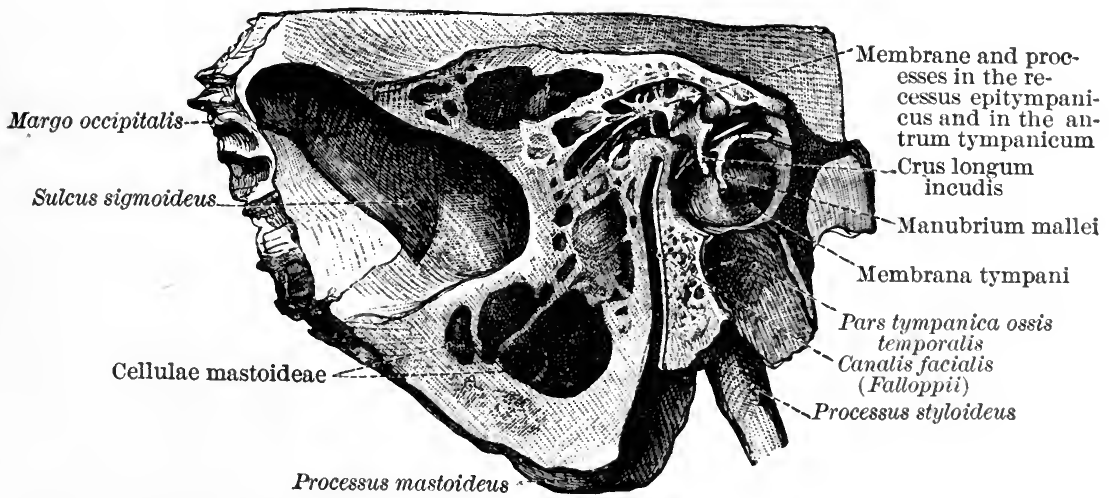

The lateral wall, paries membranaceus, of the left tympanic cavity, and the entrance to the mastoid cells. The antrum tympanicum contains branching connective-tissue strands. Seen from the medial side. (After Toldt, Anat. Atlas, Wien, 1903, 3 Aufl., p. 927, Fig. 1433.)

\section{Membrane of the Tympanum (Membrana tympani) (O. T. Drum- head). (Figs. 186, 188, and I9I-I93.)} Examine-

(a) Flaccid part (pars flaccida) (O. T. Shrapnell's membrane).

(b) Tense part (pars tensa).

(c) Border of membrane of tympanum (limbus membranae tympani).

(d) Anterior malleolar fold (plica malleolaris anterior).

(e) Posterior malleolar fold (plica malleolaris posterior).

These two folds are sometimes called "Prussak's striae."

(f) Malleolar prominence (prominentia malleolaris).

(g) Malleolar stria (stria malleolaris).

(h) Umbo ("prominent part") of tympanic membrane (umbo membranae tympani).

(i) Cutaneous layer (stratum cutaneum). 
(j) Fibrocartilaginous ring (annulus fibrocartilagineus).

(k) Radiate layer (stratum radiatum).

(l) Circular layer (stratum circulare).

( $m$ ) Mucous layer (stratum mucosum).

\section{Tympanic Mucous Membrane.}

This membrane (tunica mucosa tympanica) presents a number of folds and recesses. Examine-

Fig. 189.

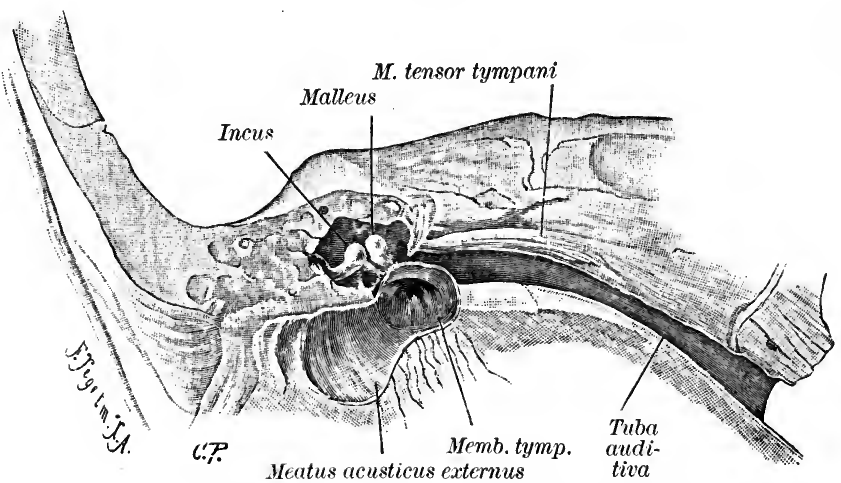

The cavum tympani and membrana tympani. A part of the meatus acusticus externus and the tuba auditiva are seen from in front, and somewhat from above and the side. (From Gegenbaur, Lehrb. der Anat. des Mensch., Leipzig, 1899, 7 Aufl., Bd. II. p. 619, Fig. 730.)

(a) Posterior malleolar fold (plica malleolaris posterior).

(b) Anterior malleolar fold (plica malleolaris anterior).

(c) Anterior recess of membrane of tympanum (recessus membranae tympani anterior).

(d) Superior recess of membrane of tympanum (recessus membranae tympani superior).

(e) Posterior recess of membrane of tympanum (recessus membranae tympani posterior).

( $f$ ) Fold of anvil (plica incudis).

(g) Fold of stirrup (plica stapedis).

(h) Secondary membrane of tympanum (membrana tympani secundaria).

Are any tympanic glands (Gl. tympanicae) visible?

\section{Auditory Ossicles.}

These small bones (ossicula auditus) are three in number,stirrup (stapes), anvil (incus), and hammer (malleus).

In the stapes study-

(a) Head of stirrup (capitulum stapedis).

(b) Anterior limb (crus anterius). 
(c) Posterior limb (crus posterius).

(d) Base of stirrup (basis stapedis) (O. T. foot-piece).

\section{Fig. 190.}

Incisura tympanica (Rivini)

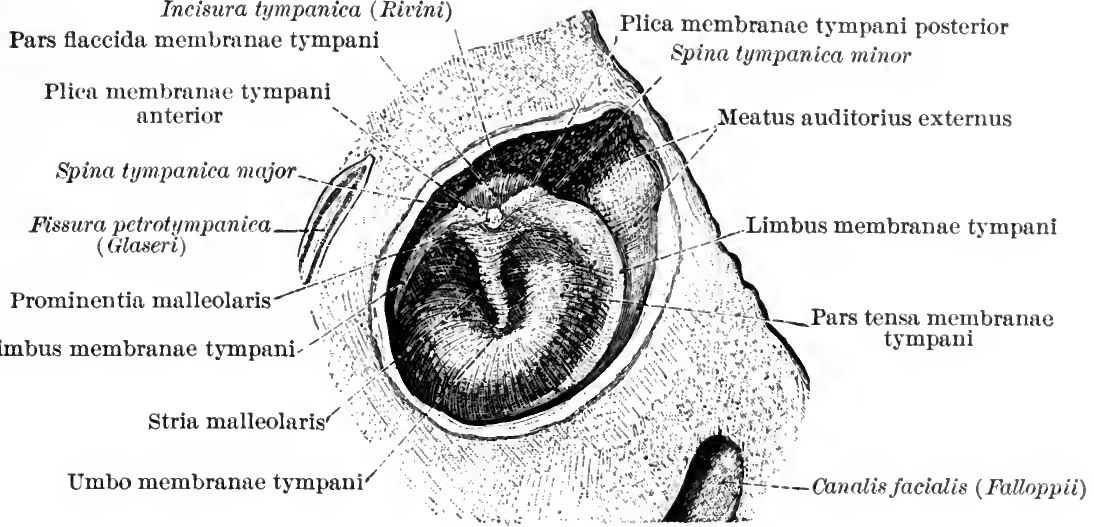

The lateral surface of the left tympanic membrane (suface towards the meatus acusticus externus; the latter has been removed by a saw-cut parallel and close to the tympanie membrane). (After Toldt, Anat. Atlas, Wien, 1903, 3 Aufl., p. 924, Fig. 1420.)

\section{In the incus study-}

(a) Body (corpus incudis).

(b) Long limb (crus longum). (ba) Lenticular process (processus lenticularis).

(c) Short limb (crus breve).

FIG. 191.

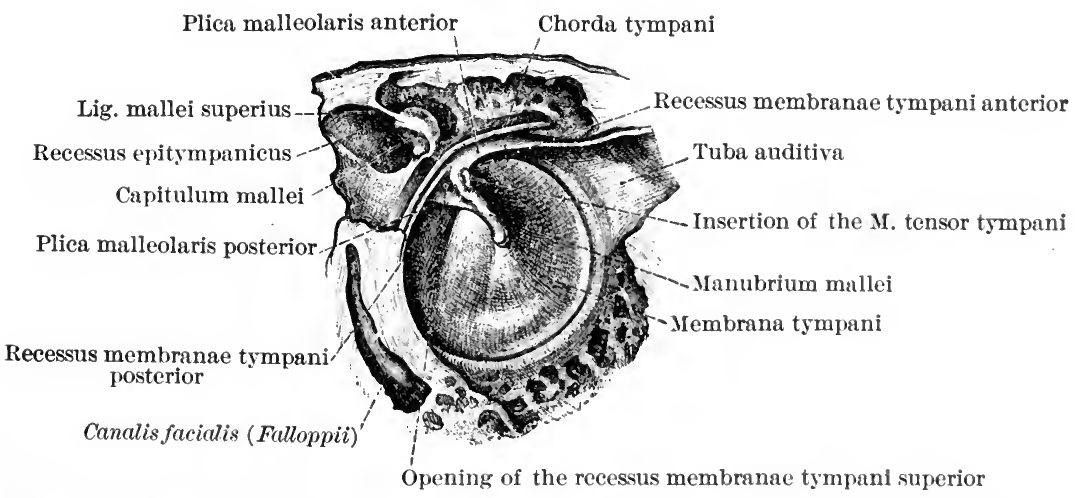

The medial surface and the anterior and posterior recesses of the tympanic membrane, brought into view by a saw-cut through the tympanum elose to and parallel to the membrane and by removal of the anvil. (After Toldt, Anat. Atlas, Wien, 1903, 3 Aufl., p. 924, Fig. 1421.)

\section{In the malleus study-}

(a) Handle of hammer (manubrium mallei).

(b) Head of hammer (capitulum mallei). 
(c) Neck of hammer (collum mallei).

(d) Lateral process (processus lateralis) (O. T. processus brevis).

(e) Anterior process (processus anterior [Folii]) (O. T. processus gracilis).

\section{Joints and Ligaments of the Auditory Ossicles.}

\section{Study the following:}

Joints (Articulationes ossiculorum auditus).

(a) Joint between anvil and hammer (articulatio incudomalleolaris).

(b) Joint between anvil and stirrup (articulatio incudostapedia).

(c) Junction of stirrup and tympanum (syndesmosis tympanostapedia).

FIG. 192.

Posterior wall of the apex

of the recess (fold of mucous membrane)

$$
\text { Apex of the recess }
$$

Lig. mallei superius

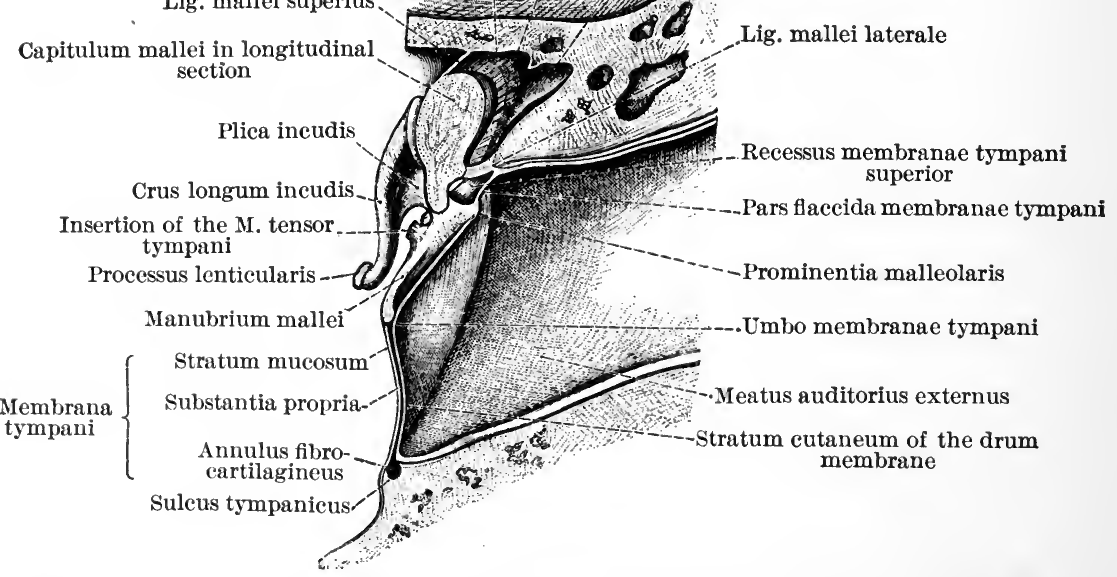

Frontal section of the left tympanic membrane and of the adjacent part of the external auditory passage, meatus acusticus externus, made after hardening in chromic acid and alcohol and decalcification with hydrochloric acid. The section passes through the head of the malleus and in front of the manubrium mallei. Seen from in front. (After Toldt, Anat. Atlas, Wien, 1903, 3 Aufl., p. 925, Fig. 1423.)

\section{Ligaments (Ligg. ossiculorum auditus).}

(a) Anterior ligament of hammer (Lig. mallei anterius).

(b) Superior ligament of hammer (Lig. mallei superius).

(c) Lateral ligament of hammer (Lig. mallei laterale) (O. T. external ligament).

(d) Superior ligament of anvil (Lig. incudis superius).

(e) Posterior ligament of anvil (Lig. incudis posterius).

( $f$ ) Obturator membrane of stirrup (membrana obturatoria [stapedis]).

(g) Annular ligament of base of stirrup (Lig. annulare baseos stapedis). 
(h) Fixing muscle of the base of the stapes (M. fixator baseos stapedis).

\section{Muscles of Auditory Bones.}

These muscles (musculi ossiculorum auditus) are two in number :

(a) Tensor muscle of tympanum (M. tensor tympani).

(b) Stapedius muscle (M. stapedius).

Study form, position, origin, insertion, action, and innervation of each.

Fig. 193.

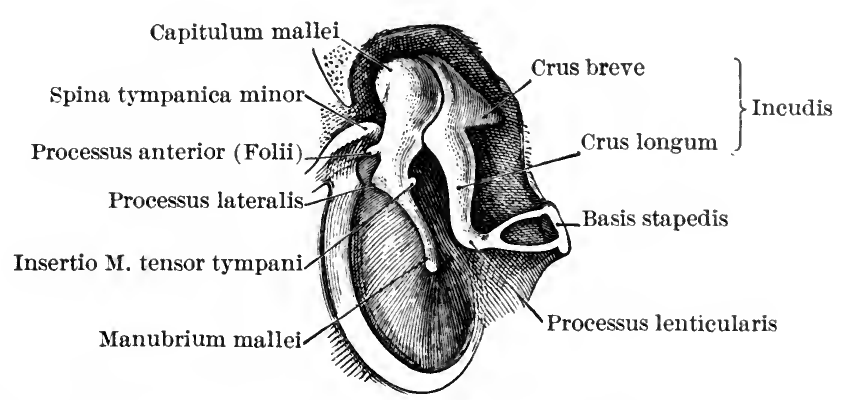

The carum tympani with the membrana tympani and the ossicula auditus. Only that part of the cavum tympani supporting the membrana tympani is represented. (From Gegenbaur, Lehrb. der Anat. des Mensch., Leipzig, 1899, 7 Aufl., Bd. ii. p. 615, Fig. 728.)

The student should next review certain structures previously studied,-viz, the chorda tympani (see p. 264 and Figs. 111 and 115), the plexus tympanicus and the N. tympanicus (see p. 271), and the tuba auditiva [Eustachii] (see pp. 293 and 296).

The study of the internal ear is very difficult. Much can be learned by fastening a temporal bone in a vice and chiselling cautiously until the osseous labyrinth is exposed. The temporal bone of a new-born babe or of a younger foetus will be found very helpful for study. The labvrinth of a guinea-pig and an embryo pig will also well repay study. In the museum, the exquisite preparations of isolated labyrinths, Wood's metal corrosions, and models are available for study. The student should use these and should study his atlases and good descriptive texts in connection with them.

\section{Osseous Labyrinth (Labyrinthus osseus).}

Here the student should study successively the vestibule (vestibulum), the snail-like body (cochlea), and the internal acoustic meatus (meatus acusticus internus). 


\section{In the vestibulum examine the following:}

(a) Spherical recess (recessus sphaericus) (O. T. fovea hemisphaerica).

(b) Elliptical recess (recessus ellipticus) (O. T. fovea hemielliptica).

(c) Crest of vestibule (crista vestibuli).

(d) Pyramid of vestibule (pyramis vestibuli).

(e) Cochlear recess (recessus cochlearis).

(f) Perforated spots (maculae cribrosae).

(fa) Superior (macula cribrosa superior).

( $f b)$ Middle (macula cribrosa media).

( $f c)$ Inferior (macula cribrosa inferior).

(g) Osseous semicireular canals (canales semicirculares ossei).

(ga) Superior (canalis semicircularis superior).

(gb) Posterior (canalis semicircularis posterior).

(gc) Lateral (canalis semicircularis lateralis) (O. T. external).

Fig. 194.

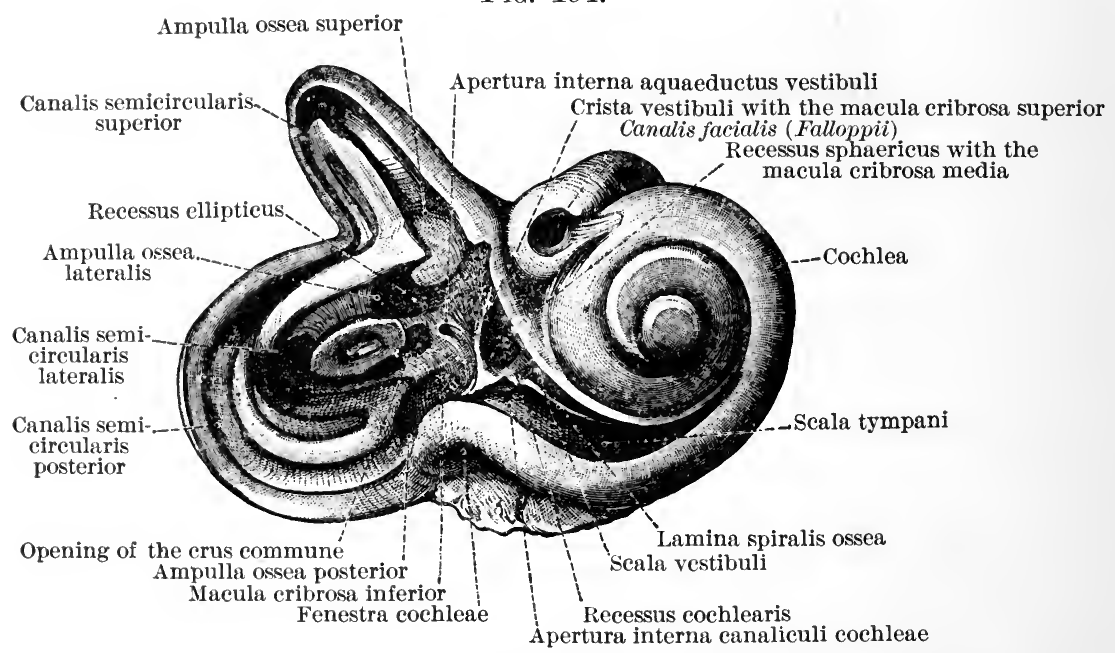

The bony labyrinth of the right ear, seen from in front. The lateral wall of the vestibule has been removed and the semicireular canals opened up in their entire length. (After Toldt, Anat. Atlas, Wien 1903, 3 Aufl., p. 930, Fig. 1442.)

(h) Osseous ampullae (ampullae osseae).

(ha) Superior (ampulla ossea superior).

(hb) Posterior (ampulla ossea posterior).

(hc) Lateral (ampulla ossea lateralis).

(i) Ampullary limbs (crura ampullaria).

(ia) Common limb (crus commune).

(ib) Simple limb (crus simplex).

In the cochlea study-

(a) Cupola (cupula cochleae).

(b) Base (basis cochleae).

(c) Spiral canal of cochlea (canalis spiralis cochleae). 
(d) Modiolus.

(da) Base of modiolus (basis modioli).

$(d b)$ Shelf of modiolus (lamina modioli).

(e) Osseous spiral shelf (lamina spiralis ossea).

(ea) Hooklet of spiral shelf (hamulus laminae spiralis).

$(f)$ "Stairease" of vestibule (scala vestibuli).

(g) "Staircase" of tympanum (scali tympani).

(h) Helicotrema (helicotrema) ("pore of the helix").

(i) Secondary spiral shelf (lamina spiralis secundaria).

(j) Spiral canal of modiolus (canalis spiralis modioli).

(k) Longitudinal canals of modiolus (canales longitudinales modioli).

FIG. 195.

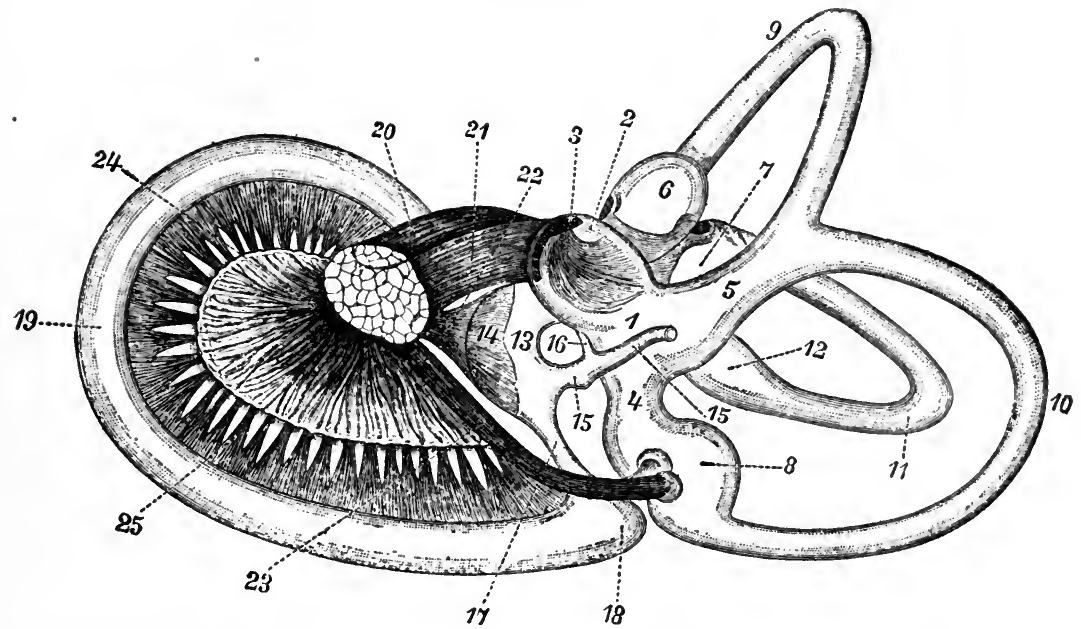

The labyrinthus membranaceus of the right internal ear of a human embryo at the fifth month, secn from the medial side.

1-5, utriculus ; 2 , recessus utriculi ; 3 , macula acustica utriculi ; 4 , sinus posterior ; 5 , sinus superior ; 6 , ampulla membranacea superior; 7 , ampulla membranacea lateralis; 8 , ampulla membranacea posterior; 9 , ductus semicircularis superior; 10 , ductus semicircularis posterior; 11 , ductus semicircularis lateralis; 12 , widened mouth of crus simplex of the lateral scmicircular canal opening into the utriculus; 13 , sacculus; 14 , macula acustica sacculi ; 15 , ductus endolymphaticus; 16 , ductus utriculosaccularis; 17 , ductus reuniens; 18 , caecum vestibulare of ductus cochlearis; 19 , ductus cochlearis ; 20 , $\mathrm{N}$. facialis ; $21-24, \mathrm{~N}$. acusticus; $21, \mathrm{~N}$. vestibuli ; $22, \mathrm{~N}$. saccularis ; $23, \mathrm{~N}$. ampullaris inferior; $24, \mathrm{~N}$. cochleae ; 25 , distribution of $\mathrm{N}$. cochleae within the lamina spiralis ossca. (After G. Retzius, as slightly modified by A. Rauber.)

\section{In the meatus acusticus internus study-}

(a) Internal acoustic opening (porus acusticus internus).

(b) Bottom of internal acoustic meatus (fundus meatus acustici interni).

(ba) Transverse crest (crista transversa).

(bb) Area of facial nerve (area $N$. facialis).

(bc) Area of cochlea (area cochleae).

(bca) Foraminous spiral tract (tractus spiralis foraminosus).

(bd) Superior vestibular area (area vestibularis superior). 
(be) Inferior vestibular area (area vestibularis inferior).

(bf) Isolated foramen (foramen singulare).

\section{Membranous Labyrinth (Labyrinthus membranaceus). (Vide Fig. I95.) ${ }^{1}$}

In the membranous labyrinth the following structures are to be studied: to make out some of them, sections should be examined under a hand-lens or the dissecting microscope. The whole internal ear is simple if approached from the embryological side. (Cf. Kollmann, Minot, McMurrich, et al.)

(a) Endolymphatic duct (ductus endolymphaticus).

(b) Endolymphatic sac (saccus endolymphaticus).

(c) Utriculosaceular duct (ductus utriculosaccularis).

(d) Utricle (utriculus).

(e) Semicircular ducts (ductus semicirculares). (ea) Superior (ductus semicircularis superior).

(eb) Posterior (ductus semicircularis posterior).

(ec) Lateral (ductus semicircularis lateralis) (O. T. external).

(f) Membranous ampullae (ampullae membranaceae).

(1) Ampullary sulcus (sulcus ampullaris).

(2) Ampullary erest (crista ampullaris).

( $f a)$ Superior (ampulla membranacea superior).

(fb) Posterior (ampulla membranacea posterior).

(fc) Lateral (ampulla membranacea lateralis).

(g) Saccule (sacculus).

(h) Uniting duct (ductus reuniens [Henseni]) (O. T. canalis reuniens).

(i) Acoustic spots (maculae acusticae).

(ia) In utricle (macula acustica utriculi).

(ib) In saccule (macula acustica sacculi).

(j) Ear-stones (otoconia).

(k) Endolymph (endolympha).

(l) Perilymph (perilympha).

( $m$ ) Perilymphatic space (spatium perilymphaticum).

(n) Perilymphatic ducts (ductus perilymphatici).

(o) Cochlear duct (ductus cochlearis) (O. T. membranous cochlea, or scala media).

(oa) Cupular blind sac (caecum cupulare).

(ob) Vestibular blind sac (caecum vestibulare).

(oc) Basilar layer (lamina basilaris).

(od) Vestibular membrane of Reissner (membrana vestibularis [Reissneri]).

(oe) Spiral ligament of cochlea (Lig. spirale cochleae).

(of) Spiral prominence (prominentia spiralis).

(og) Vascular stripe (stria vascularis).

${ }^{1}$ For an account of the blood supply of the inner ear, consult " The Distribution of the Blood-vessels in the Labyrinth of the Ear of Sus scrofa domesticus," by G. E. Shambaugh, in Decennial Publications, vol. x., of the University of Chicago, Univ. of Chic. Press, 1903. 
(oh) Spiral suleus (sulcus spiralis).

(oi) Tympanic lip (labium tympanicum).

(oia) Openings for nerves (foramina nervosa).

(oj) Vestibular lip (labium vestibulare).

(ok) Spiral ganglion of cochlea (ganglion spirale cochleae).

(ol) Spiral organ of Corti (organon spirale [Cortii]). (Fig. 197.)

( $p$ ) Vessels of internal ear (vasa auris internae). (Cf. articles by Siebenmann and by Shambaugh.)

FIg. 196.

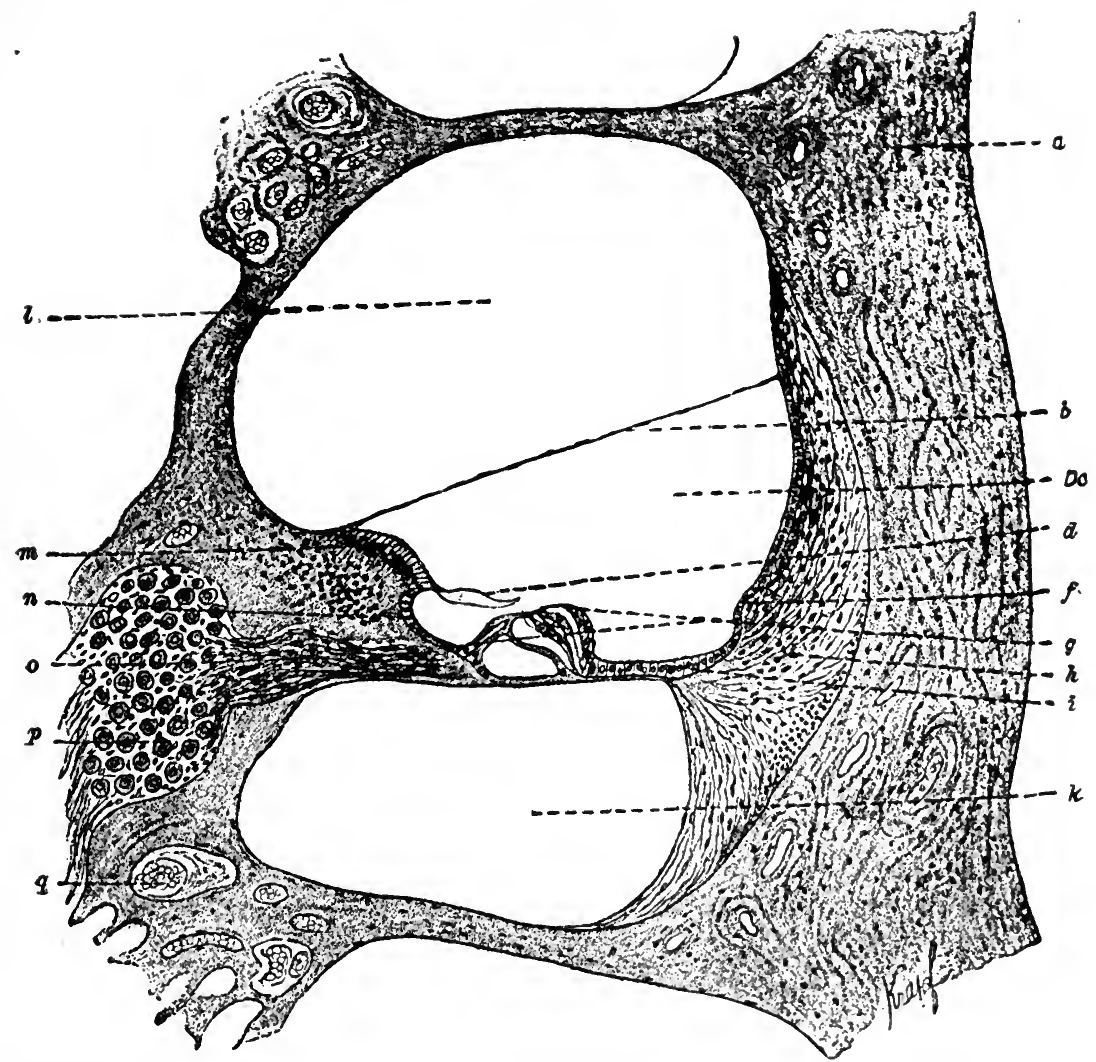

Seetion through the cochlear region of the labyrinthus osseus et membranaceus of a guinea-pig.

$l$, seala vestibuli ; $m$, labium vestibulare of the limbus; $n$, sulcus spiralis ; $o$, medullated peripheral fibres arising from cells in the ganglion spirale and being distributed to the organon spirale (Cortii); $p$, perikaryons in the ganglion spirale; $q$, blood-ressel ; $a$, bone; $b$, membrana vestibularis (Reissneri); $D c$, ductus cochlearis; $d$, Corti's membrane; $f$, prominentia spiralis; $h$, ligamentum spirale coehleae; $i$, lamina basilaris; $k$, scala tympani. (After A. A. Boehm and M. von Davidoff, Lehrbueh der Histologie des Menschen, etc., Wiesb., 1895, S. 362, Fig. 243.)

Arteries.

( $p a)$ Internal auditory artery (A. auditiva interna).

Of what is it a branch?

(paa) Vestibular rami (rami vestibulares). 
( $p a b)$ Cochlear ramus (ramus cochleae).

( $p a c)$ Arterial glomeruli of cochlea (glomeruli arteriosi cochleae).

Veins.

$(p b)$ Internal auditory veins ( $V v$. auditivae internae). Of what are they tributaries?

( $p b a)$ Spiral vein of modiolus ( $V$. spiralis modioli). ( $p b a a)$ Prominent vessel (vas prominens).

$(p b b)$ Vestibular veins ( $V v$. vestibulares).

$(p b c)$ Vein of aqueduct of vestibule ( $V$. aquaeductus vestibuli).

$(p b d)$ Vein of canaliculus of cochlea ( $V$. canaliculus cochleae).

Fig. 197.

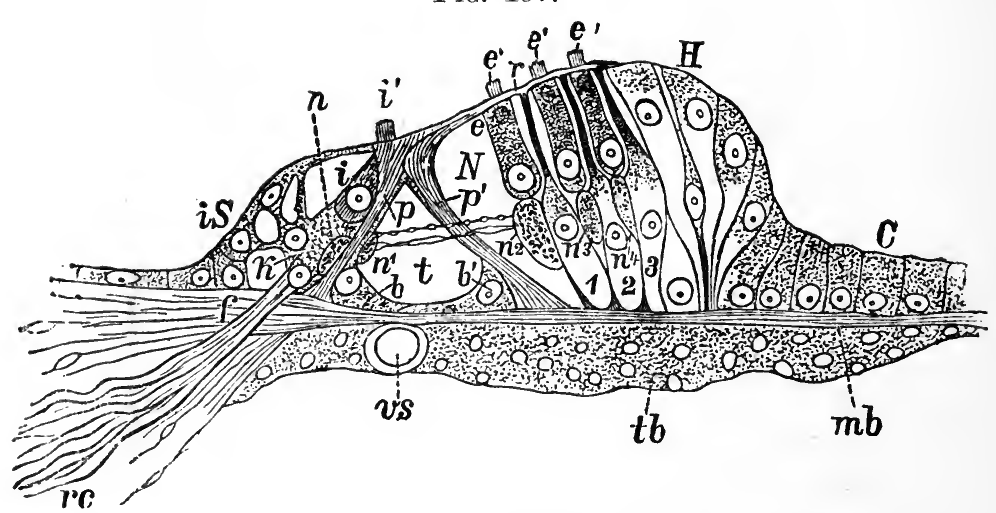

Spiral organ of Corti of the ductus cochlearis in transverse or radial section.

$r c$, medullated distal processes of bipolar nerve cells in ganglion spirale; $f$, foramen nerrosum in labium tympanicum giving passage to a bundle of the cochlear nerve fibres; $t b$, tympanal covering of lamina basilaris; $v s$, vas spirale; iS, internal supporting cells which on the left side are continuous with the epithelium of the sulcus spiralis ; $p$, internal pillar with an inner basal cell $(b)$ next to it ; $p^{\prime}$, external pillar with its external basal cell, $b^{\prime} ; 1,2,3$, Deiters' supporting cells with phalangeal processes arriving at the surface of Corti's organ, there attached to the lamina reticularis, $r$; $H$, Hensen's supporting cells which diminish in height toward the right side of the figure and are continuous with $C$, the cells of Claudius; $k$, epithelial cells of the so-called "layer of granules"; $i$, internal hair cell, the upper end of which is hidden by the "head" of the internal pillar; $i$, hairs of internal hair cell; $e$, external hair cell; $e^{\prime}, e^{\prime}, e^{\prime}$, hairs of three external hair cells; $n, n^{1}$ to $n^{4}$, various cross-sections of the spiral cord of nerve distribution; the "tunnel cord" extends from $n^{1}$ to $n^{2}$ as a radial bundle; $t$, tunnel space; $\boldsymbol{N}$, Nuel's space. (After G. Retzius. from A. Rauber's text-book, 1898, S. 818, Fig. 743.)

The student should now make careful drawings of sections made in various directions through macerated temporal bones, labelling correctly the different structures. 


$$
\begin{gathered}
\text { Part IV } \\
\text { DisSECTION OF THE THORAX }
\end{gathered}
$$




\section{DISSECTION OF THE THORAX}

\section{WALLS AND VISCERA OF THORAX.}

BEFore beginning the dissection of the thorax, the student should review the thorax in the articulated skeleton. Examine-

(a) Cavity of thorax (cavum thoracis).

(b) Superior aperture of thorax (apertura thoracis superior) (O. T. inlet of thorax).

(c) Inferior aperture of thorax (apertura thoracis inferior) (O. T. outlet or base of thorax).

(d) Costal arches (arcus costarum).

(e) Intereostal spaces (spatia intercostalia).

(f) Infrasternal angle (angulus infrasternalis).

(g) Pulmonary groove (sulcus pulmonalis).

\section{Thoracic Walls.}

The dissector of the upper extremity and the dissector of the abdomen will have left certain structures still attached to the walls of the thorax. Of these should be noticed the $\mathrm{Mm}$. pectoralis major, pectoralis minor, serratus anterior, rectus abdominis, obliquus externus abdominis, latissimus dorsi, subclavius, scalenus posterior; remove all these except the M. scalenus posterior, and so expose completely the arcus costarum and the $\mathrm{Mm}$. intercostales. Preserve, however, the rami cutanei laterales and rami cutanei anteriores of the $\mathrm{Nn}$. intercostales, as well as the perforating rami of the A. mammaria interna.

\section{Intercostal Muscles ( $\mathrm{Mm}$. intercostales).}

Clean carefully and study the form, position, origin, insertion, action, and innervation of the-

(a) External intercostal muscles (Mm. intercostales externi).

(Observe anteriorly the external intercostal ligaments (ligamenta intercostalia externa) (O. T. anterior intercostal membrane).)

(b) Internal intercostal muscles ( $\mathrm{Mm}$. intercostales interni).

Bring them into view by dividing in all the spaces the MIm. intercostales externi and Ligg. intercostalia externa along their inferior margins and reflecting them upward. Avoid injury to the Aa. intercostales. Observe the internal intercostal ligaments (ligamenta intercostalia interna) ( $\mathrm{O}$. T. posterior intercostal membranes). 


\section{Intercostal Nerves (Nn. intercostales). (Vide Fig. I98.)}

The intercostal nerves should now be studied. Each nerve runs on the internal surface of a rib near the lower margin in the sulcus costae, along with the $\mathrm{V}$. and $\mathrm{A}$. intercostalis. The nerve may be brought into view by pulling downward upon the ramus cutaneus lateralis. Follow two or three of the nerves from their origin to their termination. Note that the Nn. inter-

\section{FIG. 198.}

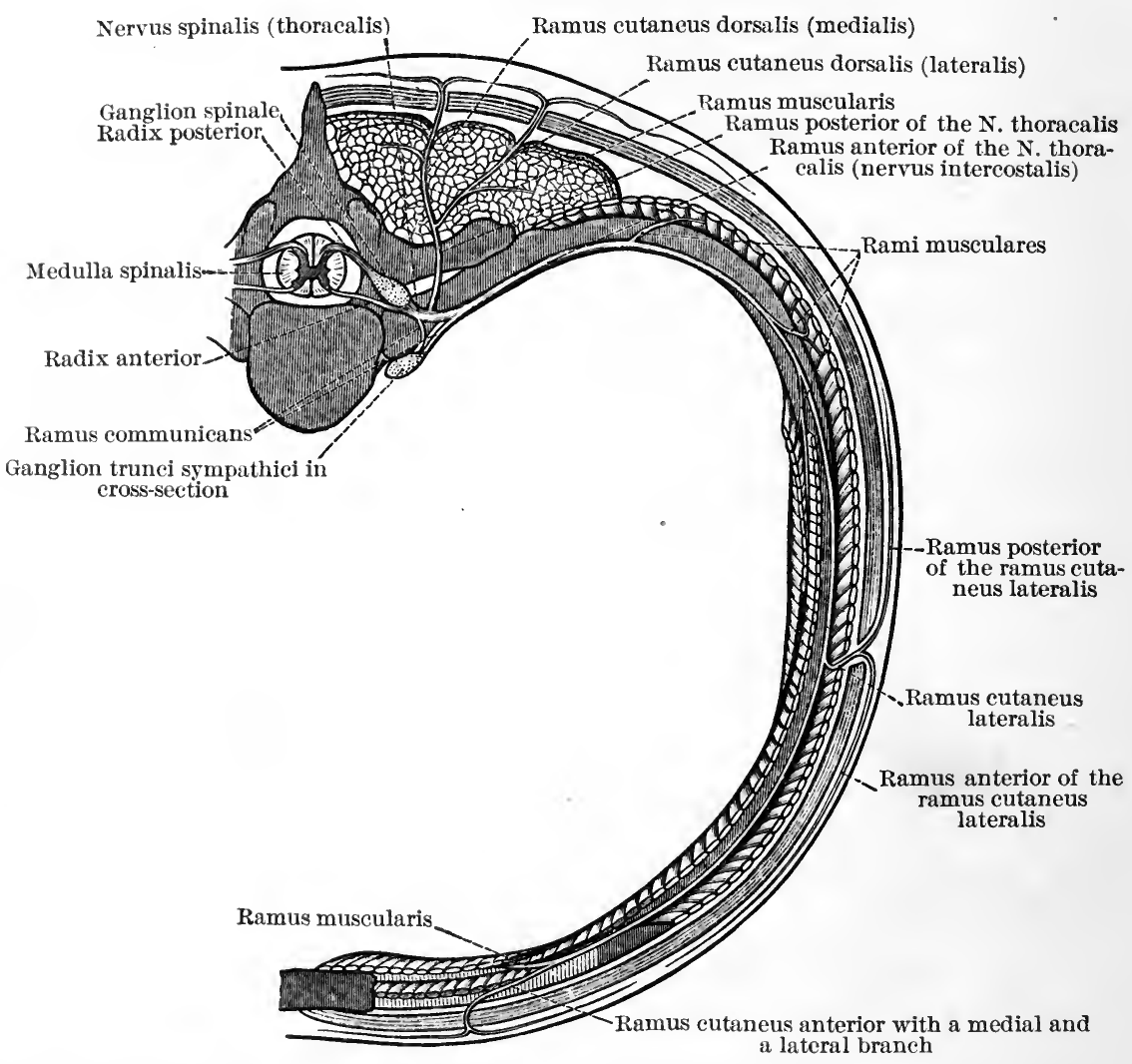

The course and branching of a thoracic nerve, nervus thoracicus, in a segment of the body. Schematic. (After Toldt, Anat. Atlas, Wien, 1903, 3 Aufl, p. 810, Fig. 1240.)

costales are really the anterior rami (rami anteriores) of the thoracic nerves (Nn.thoracales). Study the following branches:

(a) Muscular rami (rami musculares).

(b) Lateral cutaneous ramus (ramus cutaneus lateralis [pectoralis et abdominalis]).

(c) Anterior cutaneous ramus (ramus cutaneus anterior [pectoralis et abdominalis]). 


\section{Intercostal Vessels.}

Study-

(a) Highest intercostal artery (A. intercostalis suprema), from the truncus costocervicalis of the A. subclavia.

(aa) Dorsal rami (rami dorsales).

(ab) Spinal rami (rami spinales).

(b) Intercostal arteries (Aa. intercostales) (vide Fig. 198), from the aorta thoracalis, and hence often called "aortic intercostal arteries." The posterior relations and posterior branches may be better studied later.

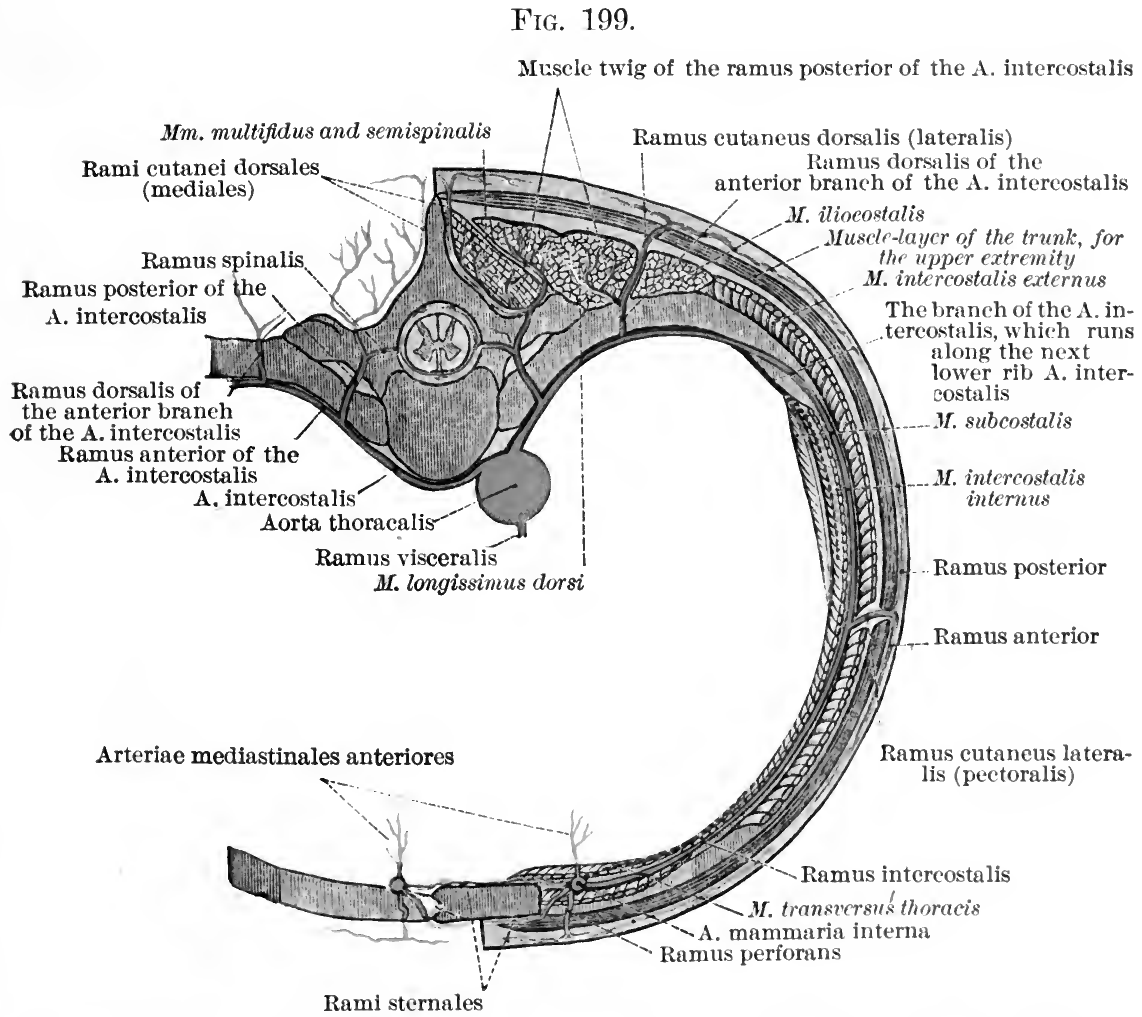

The branching of the arteries of the body-wall. Schematically represented in a segment of the thorax. (After Toldt, Anat. Atlas, Wien, 1900, 2 Aufl., p. 571, Fig. 947.)

(ba) Posterior rami (rami posteriores).

(baa) Spinal ramus (ramus spinalis).

(bab) Muscular rami (rami musculares).

(bac) Medial cutaneous ramus (ramus cutaneus medialis).

(bad) Lateral cutaneous ramus (ramus cutaneus lateralis).

(bb) Anterior rami (rami anteriores).

(bba) Muscular rami (rami musculares). 
$(b b b)$ Lateral cutaneous rami (rami cutanei laterales [pectorales et abdominales]).

(bbba) Posterior ramus (ramus posterior).

$(b b b b)$ Anterior ramus (ramus anterior).

$(b b b c)$ Lateral mammary rami (rami mammarii laterales).

$(b b c)$ Anterior cutaneous rami (rami cutanei anteriores

[pectorales et abdominales]).

(bc) Medial mammary rami (rami mammarii mediales).

(c) Intercostal veins ( $V v$. intercostales).

Note that they run above the arteries in the sulci costarum. Their posterior relations will be studied later.

\section{Internal Mammary Artery (A. mammaria interna). (Vide Fig. 9.)}

Remove the intercostal muscles, but work most cautiously, and be sure to avoid injury to the subjacent pleura, which must be preserved intact and left undisturbed in contact with the internal surface of the ribs. Clean the A. mammaria interna and the outer surface of the M. transversus thoracis. Observe how far down the pleura passes in the recess between the diaphragm and the costal arches. Below the pleura do not mistake the diaphragm for unremoved intercostal muscles.

Examine the following branches of the A. mammaria interna:

(a) Sternal rami (rami sternales).

(b) Perforating rami (rami perforantes).

(ba) Mammary rami (rami mammarii).

(bb) Muscular rami (rami musculares).

(bc) Cutaneous rami (rami cutanei).

(c) Intercostal rami (rami intercostales) (O. T. anterior intercostals).

(d) Musculophrenic artery (A. musculophrenica).

(e) Superior epigastric artery (A. epigastrica superior).

Note that $(d)$ and $(e)$ are terminal branches, the bifurcation usually occurring in the sixth intercostal space; the sixth costal cartilage may be excised in order to display this better. The anterior mediastinal, thymic, bronchial, and pericardiacophrenic branches of the internal mammary artery cannot be studied until later.

Transverse Muscle of Thorax (M. transversus thoracis) (O. T. Triangularis Sterni).

Study this muscle as far as is possible at the present stage of the dissection. It will be better exposed later." 


\section{THORACIC CAVITY (CAVUM THORACIS).}

It is well to precede the dissection of this region by the study of a frozen or formalin section ${ }^{1}$ through the thorax, a little below its middle, in order that the general relations of the thoracic viscera to one another may be understood. ${ }^{2}$ (Vide Fig. 200.)

FiG. 200.

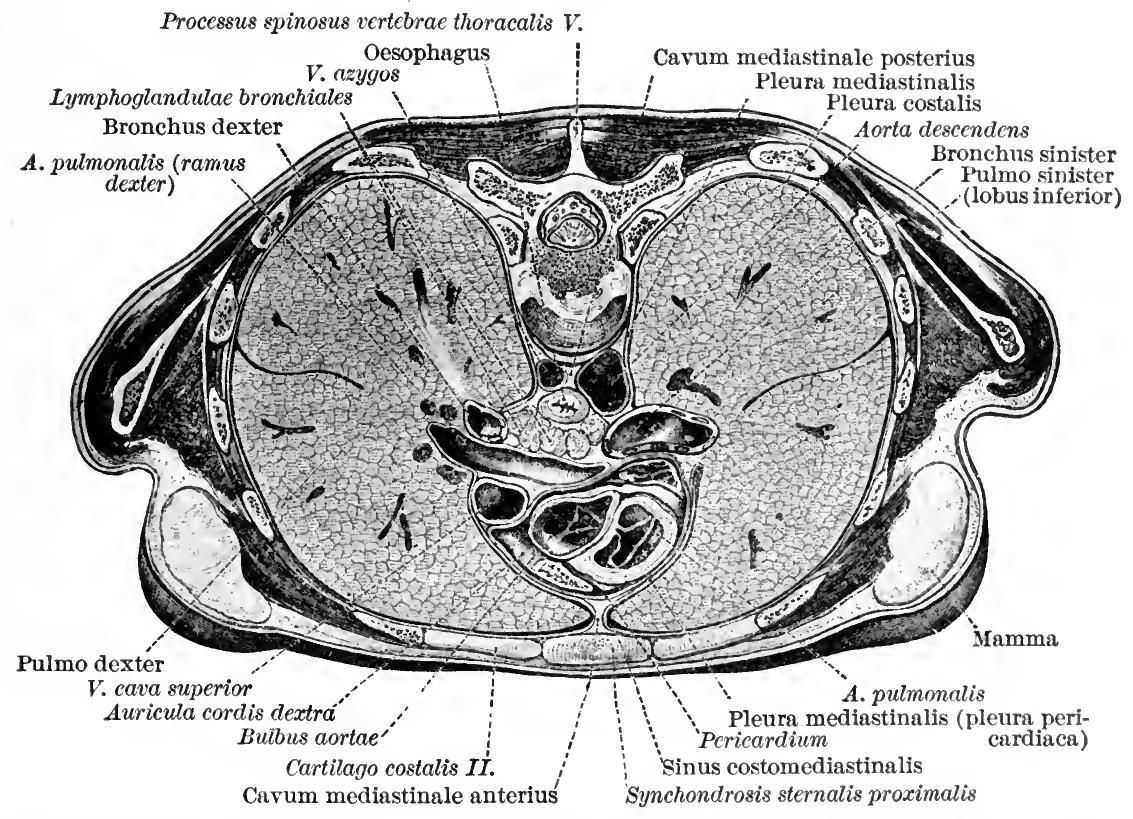

Horizontal section of the body between the bodies of the fifth and sixth thoracic vertebrae. (After Toldt, Anat. Atlas, Wien, 1900, 2 Aufl., p. 455, Fig. 773.)

\section{Pleura.}

The student has to study-

(a) Pulmonary pleura (pleura pulmonalis).

(b) Parietal pleura (pleura parietalis).

Between $(a)$ and $(b)$ is the cavity of the pleura (cavum pleurae).

${ }^{1}$ See foot-note on making sections, p. 474.

${ }^{2}$ The student is strongly advised to study a series of cross-sections through the trunk of a human being; in this work he will find the plates and descriptions by Dr. Potter, of St. Louis, of great help; these are published under the title "Topographical Anatomy of the Viscera of the Thorax and Abdomen," in the "University Studies of the University of Missouri, 1904." 
(ba) Costal pleura (pleura costalis).

(bb) Mediastinal pleura (pleura mediastinalis).

(bc) Diaphragmatic pleura (pleura diaphragmatica).

The external surface of the pleura costalis has been exposed already by the dissection. The loose areolar tissue between the pleura costalis and the Mm. intercostales interni and internal

Fig. 201.

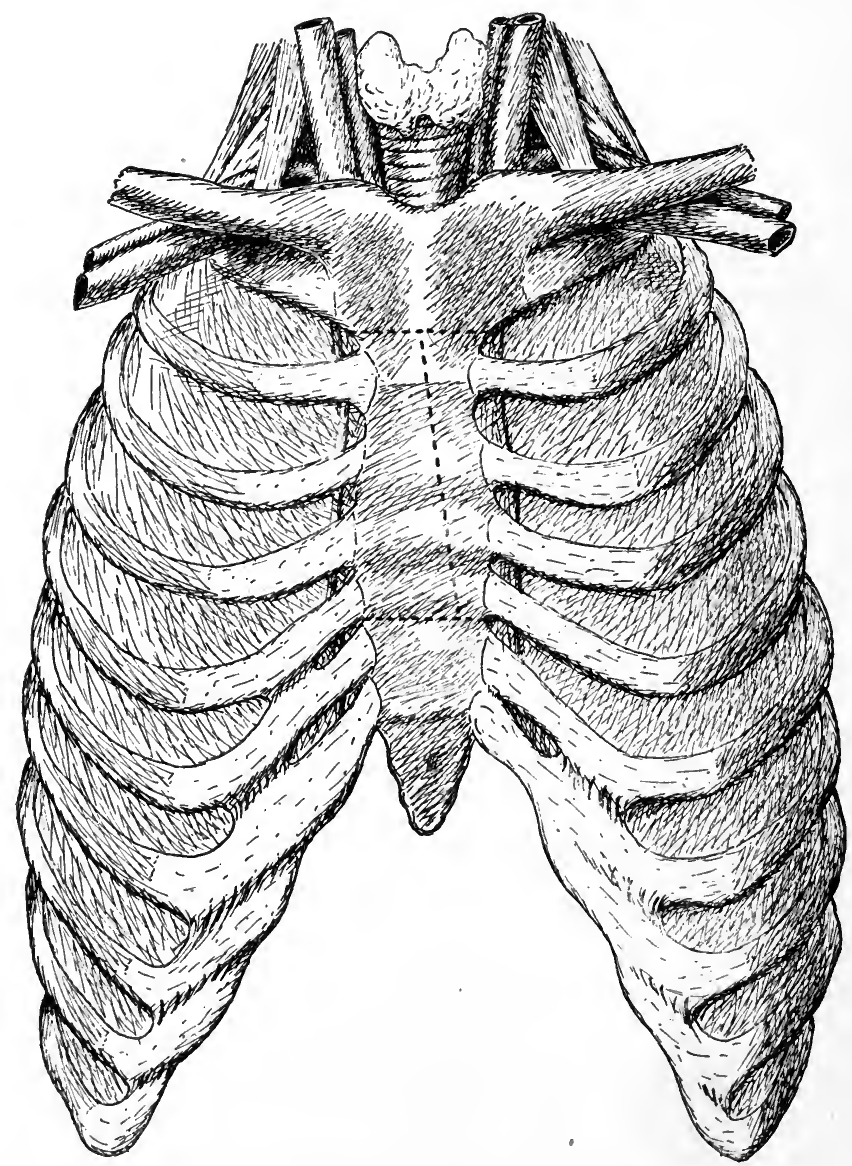

Saw-cuts in sternum in Cunningham's method of opening the anterior mediastinum.

surface of the costal arches is a part of the endothoracic fascia (fascia endothoracica).

The mode of reflection backward of the pleura on each side, from the posterior aspect of the sternum, may now be investi- 
gated by the method devised by Sir William Turner. (See Fig. 201.)

Saw through the sternum as follows:

(1) Transversely through manubrium sterni on a line connecting the lower margins of the incisurae costales I.,-i.e., parallel to the lower margins of the first pair of costal cartilages.

(2) Transversely through the lower part of the corpus sterni, midway between incisura costalis V. and incisura costalis VI.

(3) Obliquely, almost vertically, from the inferior transverse cut near the left margin of the sternum, upward to the middle of the superior transverse cut.

The saw should not be permitted to pass through the periosteum on the back of the sternum; this is to be cautiously divided with a scalpel.

Gently separate the two lateral portions of the central piece of the sternum; observe the parietal pleura on each side passing backward towards the pericardium. Insert the finger between the right and the left pleura, and pass it upward and downward through the loose areolar tissue of the anterior mediastinal cavity (cavum mediastinale anterius) ( $\mathrm{O}$. T. anterior mediastinum), between them, until the pericardium is exposed.

Next separate the pleura costalis from the ribs, as far forward as the cartilages, by carefully passing the index-finger between the pleura and each rib and running it backward and forward; be sure not to detach the pleura from the costal cartilages. Excise, with bone-forceps, the second, third, fourth, fifth, and sixth ribs, cutting through them in front at their junctions with the costal cartilages, and behind as far back as is possible. Leave the sternum and costal cartilages, with adherent pleura, for the present, in position.

Cut vertically through the pleura costalis, midway between spine and sternum, from level of costa II. to level of costa VII. Make two transverse incisions medialward for a distance of five or seven centimetres, one from each end of this vertical incision, and reflect the large flap of costal pleura medialward. The cavity of the pleura (cavum pleurae) has now been opened.

\section{Exploration of Pleural Cavity.}

Pass the hand into the cavum pleurae and trace the membrane (1) in a transversal direction, (2) in the vertical direction. Control your exploration by a study of the figures in atlases and by a description in a good systematic text-book. Trace the parietal 
pleura in its costal, diaphragmatic, and mediastinal parts. (Vide Fig. 202.) Study-

(a) Pulmonary ligament (ligamentum pulmonale) (triangular duplicature extending from hilus of lung to mediastinal pleura).

(b) Dome or cupola of pleura (cupula pleurae).

Examine its relations.

(c) Mediastinal septum (septum mediastinale).

( ca) Anterior mediastinal eavity (cavum mediastinale anterius).

(cb) Posterior mediastinal eavity (cavum mediastinale posterius).

Fig. 202.

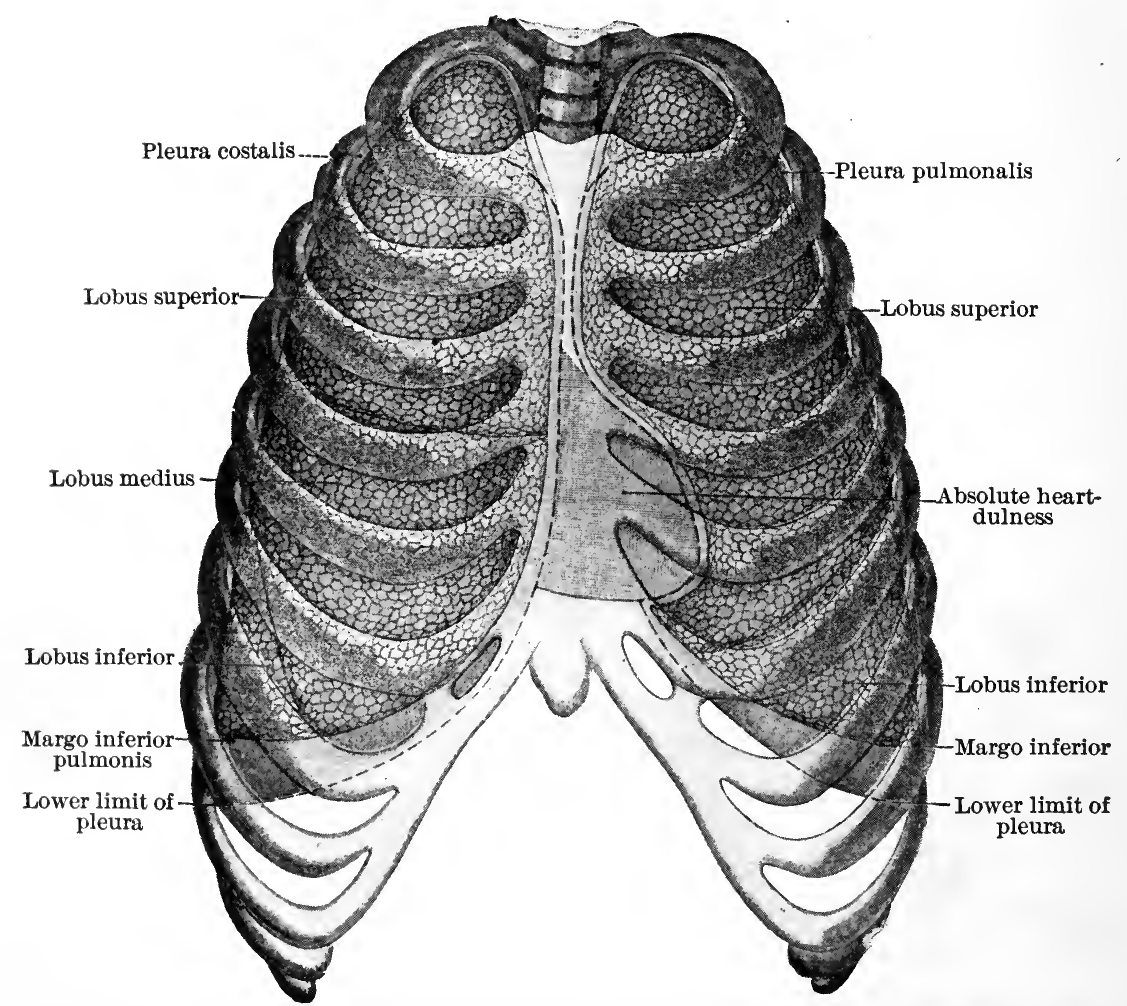

Boundaries of the lungs and pleura, from in front. Absolute heart-dulness. (After Joessel, from Bardeleben and Haeckel, Atlas der Topog. Anat. des Menschen, Jena, 1901, Fig. 98.)

(d) Two parts of mediastinal pleura.

(da) Pericardiac pleura (pleura pericardiaca).

(db) Mediastinal layers (laminae mediastinales).

(e) Sinuses of the pleura (sinus pleurae).

(ea) Phrenicocostal sinus (sinus phrenicocostalis).

(eb) Costomediastinal sinus (sinus costomediastinalis).

( $f$ ) Adipose folds (plicae adiposae).

( $f a)$ Pleural villi (villi pleurales). 
Look for them in the sinus phrenicocostalis and at the junction of the pleura mediastinalis with the pleura diaphragmatica.

(g) Differences between lines of pleural reflection on right and left sides.

\section{Removal of Central Portion of Sternum.}

Carefully remove the portion of the sternum between the superior and inferior transverse cuts, with costal cartilages attached, but save the piece for the study of the sternocostal articulations later. Separate the pleura pericardiaca (of the pleura mediastinalis) from the side of the pericardium. The following structures become visible:

(a) Phrenic nerve (N. phrenicus).

(b) Branches of A. mammaria interna.

(ba) Pericardiacophrenic artery (A. pericardiacophrenica) (O. T. arteria comes nervi phrenici).

(bb) Anterior mediastinal arteries (Aa. mediastinales anteriores).

(bc) Thymic arteries (Aa. thymicae).

(bd) Bronchial rami (rami bronchiales).

Fig. 203.

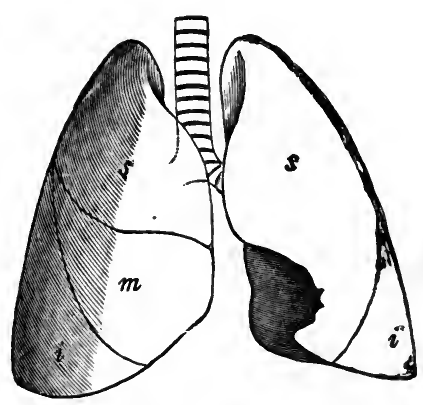

The lungs seen from in front. $s$, lobus superior; $m$, lobus medius; $i$, lobus inferior. (From Gegenbaur, Lerhb. der Anat. des Mensch., Leipzig, 1899, 7 Aufl., Bd. ii. p. 111, Fig. 432.)

\section{Lungs (Pulmones). (Figs. 202 and 203.)}

The right lung (pulmo dexter) and the left lung ( $p u l m o$ sinister) may next be studied. With the consent of the dissector of the head and neck.inflate the lungs with air. Do this by inserting the nozzle of the bellows in the cervical portion of the trachea. After inflation ligate the trachea. Now study the external form of each lung, comparing the findings in the cadaver with atlas illustrations, text-book descriptions, and the His-Steger models. In each lung study the following: 
(a) Apex of lung (apex pulmonis).

(b) Base of lung (basis pulmonis).

(c) Costal surface (facies costalis) (O. T. outer surface of lung).

(d) Mediastinal surface (facies mediastinalis) (O. T. inner surface of lung).

(e) Diaphragmatic surface (facies diaphragmatica).

( $f$ ) Anterior border or margin (margo anterior).

(g) Inferior border or margin (margo inferior).

(h) Hilus of lung (hilus pulmonis).

(i) Root of lung (radix pulmonis).

(j) Cardiac impression (impressio cardiaca).

(k) Subclavian groove (sulcus subclavius).

(l) Interlobar incisure (incisura interlobaris).

The student should next pay attention to the differences between the right lung and the left. Which is the larger? Which is the shorter and wider? Why should it be so?

In the right lung observe the form, position, and boundaries of-

(a) Superior lobe (lobus superior).

(b) Middle lobe (lobus medius).

(c) Inferior lobe (lobus inferior).

In the left lung study-

(a) Superior lobe (lobus superior).

(b) Inferior lobe (lobus inferior).

(c) Cardiac notch (incisura cardiaca).

(d) Lingula of lung (lingula pulmonis).

\section{Root of Lung (Radix pulmonis).}

Strip the pleura off the root of the lung; study the relations of neighboring structures to the root. Observe-

On both sides.

In front of the root:

(a) Anterior pulmonary plexus of N. vagus (plexus pulmonalis anterior).

(b) Phrenic nerve (N. phrenicus).

(c) Pericardiacophrenic artery (A. pericardiacophrenica).

Behind the root:

(a) Vagus nerve (N. vagus) (O. T. pneumogastric).

(b) Posterior pulmonary plexus of $\mathrm{N}$. vagus (plexus pulmonalis posterior).

Below the root:

(a) Broad ligament of lung (ligamentum latum pulmonis). On right side.

(a) Azygos vein ( $V$. azygos) (O. T. V. azygos major).

(b) Superior vena cava ( $V$. cava superior). 
On left side.

(a) Arch of aorta (arcus aortae).

(b) Descending aorta (aorta descendens).

The student should next undertake the dissection of the structures in the root of each lung. Isolate them in the following order :

(a) Anterior pulmonary plexus (plexus pulmonalis anterior).

(b) Posterior pulmonary plexus (plexus pulmonalis posterior).

To expose this, throw the lung medialward over the pericardium and remove the pleura from the back part of the radix pulmonis. Secure the N. vagus and follow it into the plexus.

(c) Bronchial arteries ( $A a$. bronchiales), from the aorta thoracalis.

(d) Pulmonary artery (A. pulmonalis). (da) Right ramus (ramus dexter). (db) Left ramus (ramus sinister).

(e) Pulmonary veins (venae pulmonales). (ea) Right pulmonary veins ( $V v$. pulmonales dextrae). (eb) Left pulmonary veins ( $V v$. pulmonales sinistrae).

( $f$ ) Bronchus, right and left (bronchus [dexter et sinister]). (fa) Bronchial rami (rami bronchiales). (Vide Fig. 216.)

(faa) Eparterial ramus (ramus bronchialis eparterialis).

(fab) Hyparterial rami (rami bronchiales hyparteriales).

(g) Bronchial lymph-glands (lymphoglandulae bronchiales).

Isolate these parts thoroughly and clean each structure; it may be necessary to remove the bronchial lymph-glands in order to expose the vessels and bronchi adequately. Fix in the memory the relations of bronchus, artery, and vein, from before backward and from above downward, on each side.

\section{Phrenic Nerve and Cardiac Plexus (N. phrenicus and plexus car- diacus).}

The phrenic nerve should be followed upward and downward on each side, and the differences in relations on the two sides studied. Isolate the following branches of the N. phrenicus:

(a) Pericardiac ramus (ramus pericardiacus).

(b) Phrenico-abdominal rami (rami phrenicoabdominales).

In dissecting out the cardiac plexus of nerves (plexus cardiacus) in the concavity of the aortic arch, follow down-

(a) Superior cardiac nerve ( $N$. cardiacus superior), from the cervical sympathetic.

(b) Inferior cardiae ramus (ramus cardiacus inferior), from the left $\mathbf{N}$. vagus. 
These will be found upon the arcus aortae between the $\mathrm{N}$. vagus and the $\mathrm{N}$. phrenicus. On dissection of the plexus, look for the cardiac ganglion of Wrisberg (ganglion cardiacum [Wrisbergi]).

\section{Pericardium.}

Clean the external surface of the pericardium thoroughly; observe the ligaments which connect the pericardium to the sternum (ligamenta sternopericardiaca). Examine the exact relations of the pericardial sac to the diaphragm. Compare the general relations of the pericardium in the cadaver with the description in a good text-book.

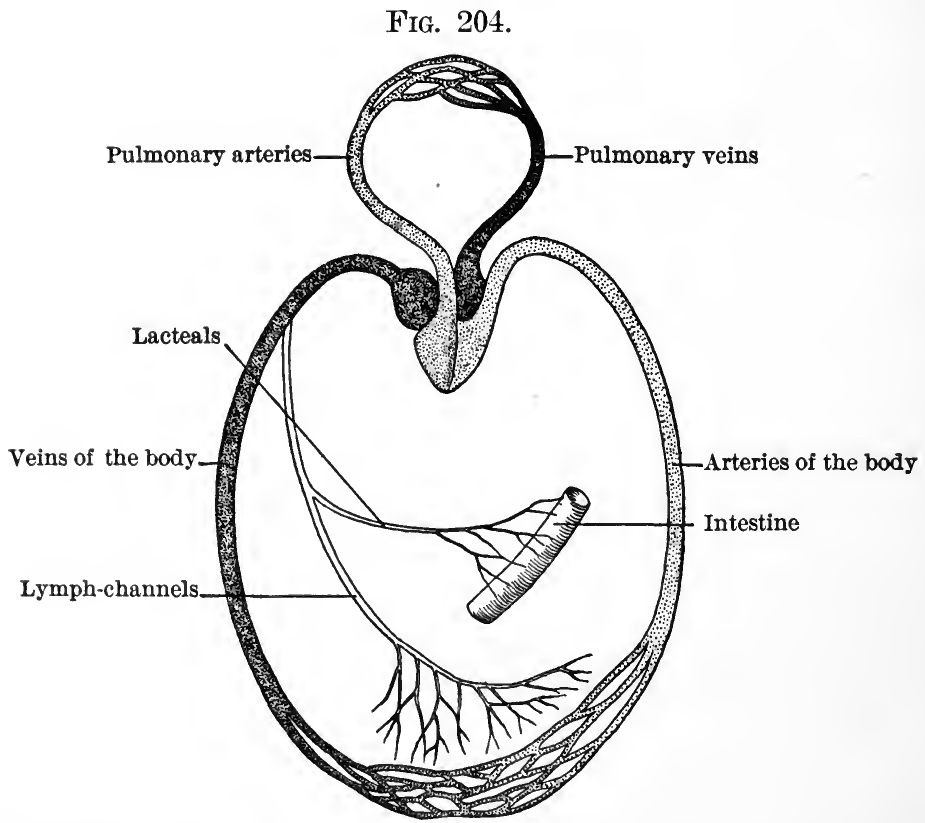

Schematic representation of the vascular system with the lymph- and chyle-vessels. (After Gegenbaur, Lehrb. der Anat. des Mensch., Leipzig, 1899, 7 Aufl., Bd. ii. p. 203, Fig. 498.)

Open the pericardium by a crucial incision, making a vertical cut through it from the aorta to the diaphragm and a transverse cut in a line from the middle of the root of one lung to that of the other. Examine-

(a) Parietal pericardium (pericardium).

(b) Visceral pericardium (epicardium).

(c) Pericardial fluid (liquor pericardii).

(d) Transverse sinus of pericardium (sinus transversus pericardii). 
Ascertain exactly the distribution of the pericardium at the base of the heart and about the great vessels.

\section{Thymus.}

The appearances will vary according to the age of the individual. If the cadaver be that of a child, study the following:

(a) Right and left lobes (lobi dexter et sinister).

(b) Central tract (tractus centralis).

(c) Lobules of thymus (lobuli thymi).

FIG. 205.

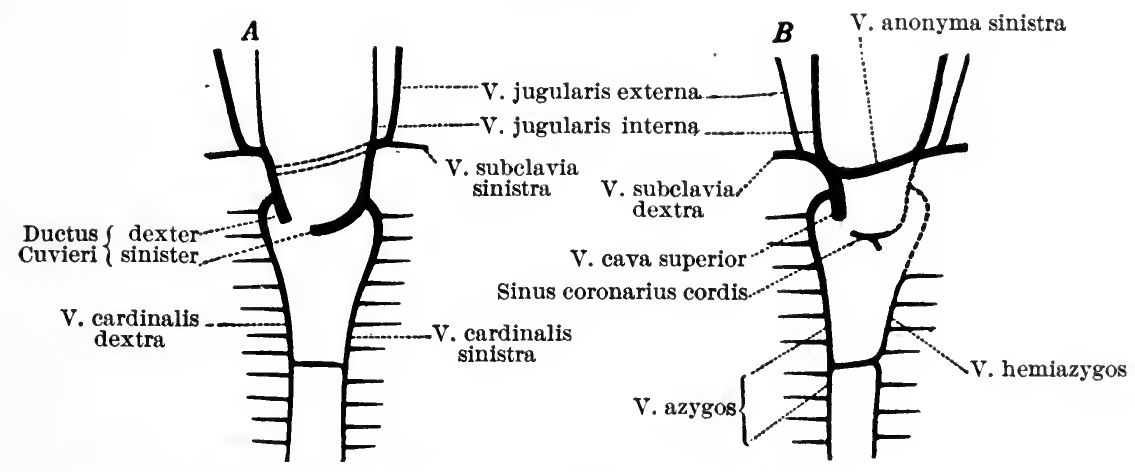

Diagrams of $A$, the primitive anlage of the venous system of the body and $B$, the formation of the system of the v. cava superior. (Rathke's schema with slight alterations by F. Hochstetter.) (After Toldt, Anat. Atlas, Wien, 1900, 2 Aufl., p. 563, Fig. 939.)

Great Veins of Thorax and their Tributaries. (Vide Fig. 205.)

Remove the thymus or the fat-body representing it; dissect out the great veins of the thorax. Clean and study-

(a) Superior vena cava ( $V$. cava superior).

(aa) Right and left innominate veins ( $V v$. anonymae dextra et sinistra).

(aaa) Inferior thyreoid veins ( $V v$. thyreoideae inferiores).

(aab) Lowest thyreoid vein (V. thyreoideae ima).

(aac) Thymic veins ( $V v$. thymicae).

(aad) Pericardiac veins ( $V v$. pericardiacae).

(aae) Superior phrenic veins ( $V v$. phrenicae superiores).

(aaf) Anterior mediastinal veins ( $V v$. mediastinales anteriores).

(aag) Anterior bronchial veins ( $V v$. bronchiales anteriores).

(aah) Vertebral vein (V. vertebralis).

(1) Deep cervical vein (V. cervicalis profunda).

(aai) Internal mammary vein (V. mammaria interna). 
(1) Subcutaneous veins of abdomen ( $V v$. subcutaneae abdominis).

(2) Superior epigastric vein ( $V$. epigastrica superior).

(aaj) Highest intercostal vein ( $V$. intercostalis suprema)

(O. T. left superior intercostal vein).

( $a b)$ Azygos vein ( $V$. azygos) (O. T. vena azygos major).

Its tributaries ean be studied better later.

(b) Inferior vena cava ( $V$. cava inferior).

\section{FIG. 206.}

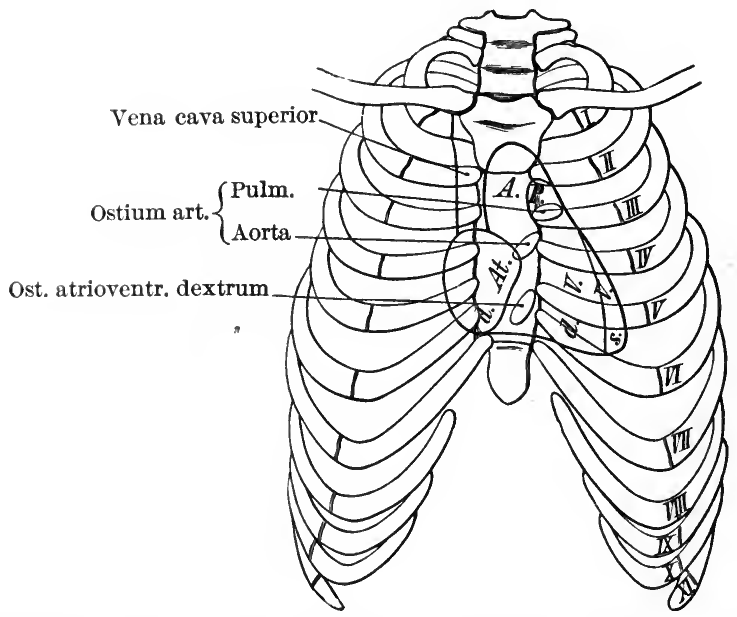

Thorax from in front with the position of the heart. $d$. At., right atrium; $d . V$. , right ventricle; s. V., left ventricle; $A$., aorta; $P$., A. pulmonalis; $I$-XII, the ribs. (From Gegenbaur, Lehrb. der Anat. des Mensch., Leipzig, 1899, 7 Aufl., Bd. ii. p. 221, Fig. 514.)

\section{Heart (Cor). (Vide Fig. 207.)}

Study thoroughly its form, position, and general relations. Examine the following:

(a) Base of heart (basis cordis).

(b) Apex of heart (apex cordis).

(c) Right arterial orifice (ostium arteriosum dextrum) (O. T. pulmonary orifice).

(d) Left arterial orifice (ostium arteriosum sinistrum) (O. T. aortic orifice).

(e) Sternocostal surface (facies sternocostalis) (O. T. anterior surface).

(f) Diaphragmatic surface (facies diaphragmatica) (O. T. posterior surface).

(g) Surfaces of chambers of heart.

(ga) Forechambers (atria cordis) (O. T. auricles).

(gaa) Right atrium (atrium dextrum) (O. T. right auricle).

(gaaa) Right auricle (auricula dextra) (O. T. right auricular appendix). 
(gab) Left atrium (atrium sinistrum) (O. T. left auricle). (gaba) Left auricle (auricula sinistra) (O. T. left auricular appendix).

(gb) Ventricles (ventriculi cordis).

( $g b a)$ Right ventricle (ventriculus dexter).

$(g b b)$ Left ventricle (ventriculus sinister).

(h) Coronary sulcus (sulcus coronarius) (O. T. auriculoventricular groove).

(i) Anterior longitudinal sulcus (sulcus longitudinalis anterior) (O. T. anterior interventricular groove).

(j) Posterior longitudinal sulcus (sulcus longitudinalis posterior) (O. T. posterior interventricular groove).

(k) Notch at apex of heart (incisura [apicis] cordis).

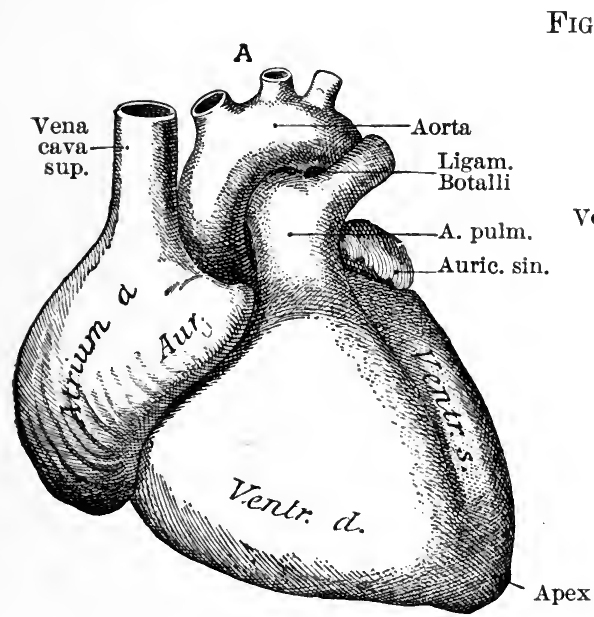

FIG. 207.

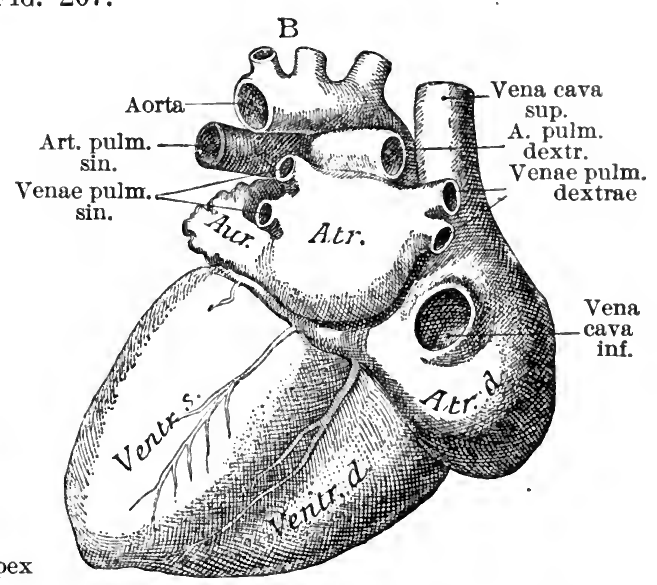

The heart: $A$, from in front and somewhat above and to the right; $B$, from behind and somewhat below and to the left. $\times 1 / 3$. (From Gegenbaur, Lehrb. der Anat. des Mensch., Leipzig, 1899, 7 Aufl., Bd. ii. p. 208, Fig. 502.)

\section{Blood-vessels and Nerves of the Heart.}

In the sulci on the surface of the heart, the larger trunks of the blood-vessels will be found. Remove the epicardium over these and clean the vessels of adherent fat. The nerves are extremely delicate and difficult to isolate. Study the arteries:

(a) Right coronary artery of heart (A. coronaria [cordis] dextra). (aa) Posterior descending ramus (ramus descendens posterior).

(b) Left coronary artery of heart (A. coronaria [cordis] sinistra).

(ba) Circumflex ramus (ramus circumflexus).

(bb) Anterior descending ramus (ramus descendens anterior).

The veins of the heart ( $V v$. cordis) should next be examined. Pull the apex forward so as to bring the diaphragmatic surface into view. Examine- 
(a) Coronary sinus (sinus coronarius).

Open it with scissors throughout its entire length.

(aa) Large vein of heart ( $V$. cordis magna) (O. T. great cardiac vein).

$(a b)$ Posterior vein of left ventricle ( $V$. posterior ventriculi sinistri).

(ac) Oblique vein of left atrium (V. obliqua atrii sinistri [Marshalli]).

Note the embryological significance of this vein. Can you find the ligamentum $\mathrm{V}$. cavae sinistrae in the fold of epicardium known as the "vestigial fold of Marshall"?

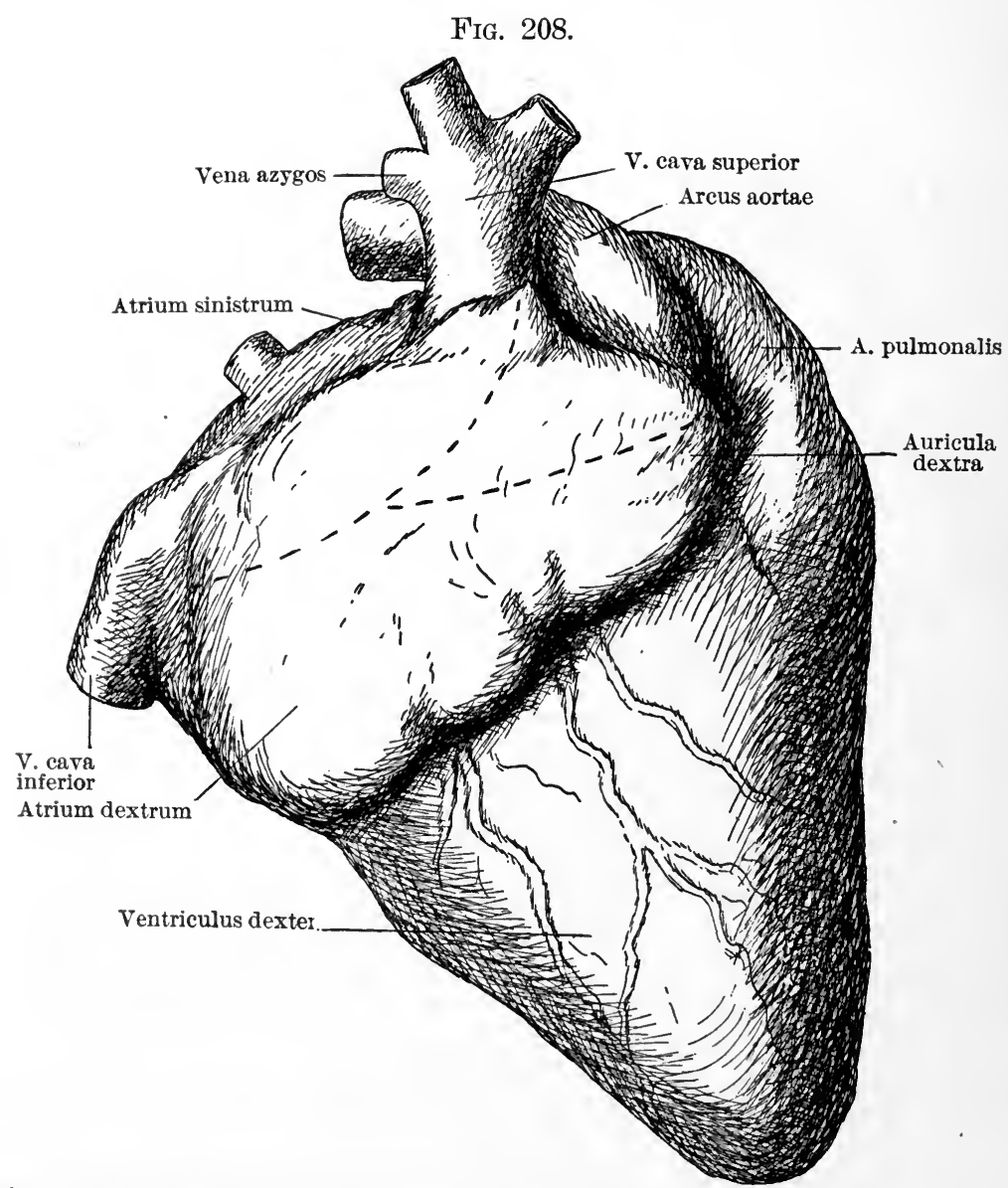

Incisions to open the right atrium of the heart. (After Cunningham, Man. of Pract. Anat., Edinburgh, 1896, 2d ed., vol. ii. p. 47, Fig. 182.)

(ad) Middle vein of heart ( $V$. cordis media).

$(a e)$ Small vein of heart ( $V$. cordis parva).

(b) Anterior veins of heart ( $V v$. cordis anteriores), to right atrium.

(c) Smallest veins of heart ( $V v$. cordis minimae) (O. T. Vv. Thebesii). 
The nerves of the heart may next be studied, as far as they can be made out.

(a) Anterior coronary plexus (plexus coronarius cordis anterior).

(b) Posterior coronary plexus (plexus coronarius cordis posterior).

\section{Cavities of Heart.}

Pull the heart over to the left side so that the right atrium will be fully in view; open the right atrium by two incisions,-

\section{FIG. 209.}

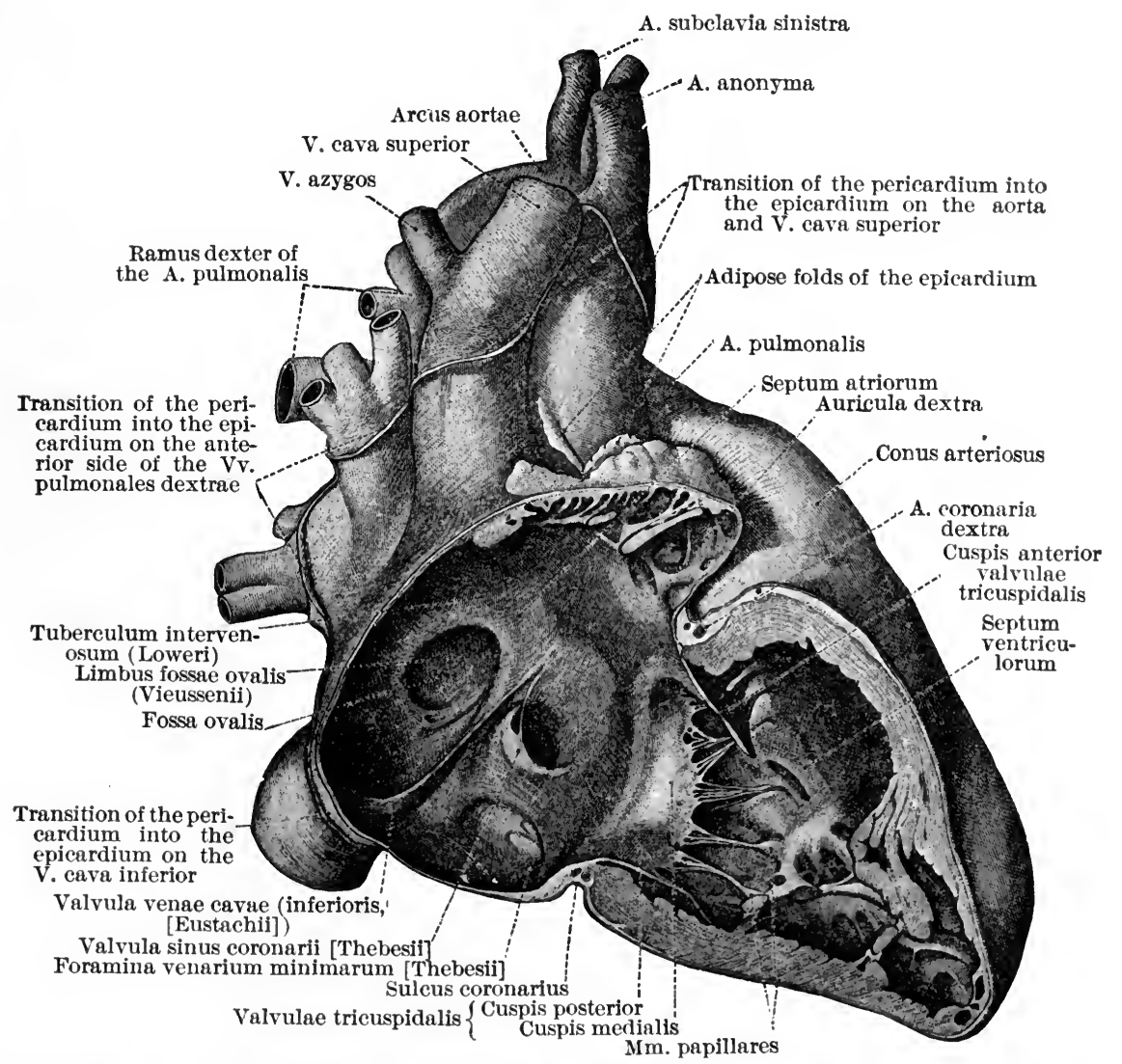

The heart seen from the right side. After hardening in formalin in distention, the right side of the right atrium and ventricle were removed. (After Toldt, Anat. Atlas, Wien, 1900, 2 Aufl., p. 549, Fig. 918.)

(1) an almost vertical one from in front of the $V$. cava superior downward and a little backward to the V. cava inferior, and (2) an oblique one from the middle of the vertical incision to the tip of the auricula dextra cordis (O. T. right auricular appendix). 
(Vide Fig. 208.) Clean out the cavity of the right atrium with a wet sponge.

\section{Right Atrium (Atrium dextrum) (O. T. Right Auricle).}

Examine the endocardium and the myocardium of its wall, the openings of the $\mathrm{V}$. cava superior, $\mathrm{V}$. cava inferior, and sinus coronarius. Note the subdivision of the right atrium into two parts by the terminal sulcus of the right atrium (sulcus terminalis atrii dextri), which corresponds to the terminal crest (crista terminalis) in the interior; the posterior, medial part of the atrium is called the venous sinus (sinus venarum [cavarum]). Observe the exact situation of the right auricle (auricula dextra) (O. T. right auricular appendix). In the right atrium, examine also-

(a) Intervenous tuberele of Lower (tuberculum intervenosum [Loweri]).

(b) Septum of the atria (septum atriorum) (O. T. interauricular septum).

(ba) Membranous part of septum of atria (pars membranacea septi atriorum).

(bb) Oval fossa (fossa ovalis).

(bba) Edge of oval fossa (limbus fossae ovalis [Vieusseni]) (O. T. annulus ovalis).

(c) Valve of inferior vena cava (valvula venae cavae [inferioris Eustachii]) (O. T. Eustachian valve).

(d) Valve of coronary sinus (valvula sinus coronarii [Thebesii]) (O. T. coronary valve, or valve of Thebesius).

(e) Venous ostium of right ventricle (ostium venosum) (O. T. right auriculoventricular orifice).

(f) Foramina of the smallest veins (foramina venarum minimarum [Thebesii]) (O. T. foramina Thebesii).

(g) Pectinate ("comb-like") museles (Mm. pectinati).

\section{Right Ventricle (Ventriculus dexter) and Pulmonary Artery (A. pul- monalis).}

Cut into the right ventricle by a vertical incision half a centimetre to the right of the sulcus longitudinalis anterior, extending all the way from the conus arteriosus to the facies diaphragmatica. Make carefully a second incision from the upper end of the first one, transversely to the right, parallel to the sulcus coronarius and one centimetre below it. (Vide Fig. 210.) Avoid injury to the tricuspid valve; the incision may be controlled by inserting the index-finger of the left hand from the right atrium into the ventricle through the ostium venosum. Reflect the $\mathrm{V}$-shaped flap of the ventricular wall to the right and clean the cavity of the ventricle with a wet sponge. 
Study the shape and general relations of the cavity and with the aid of Fig. 209 examine the following:

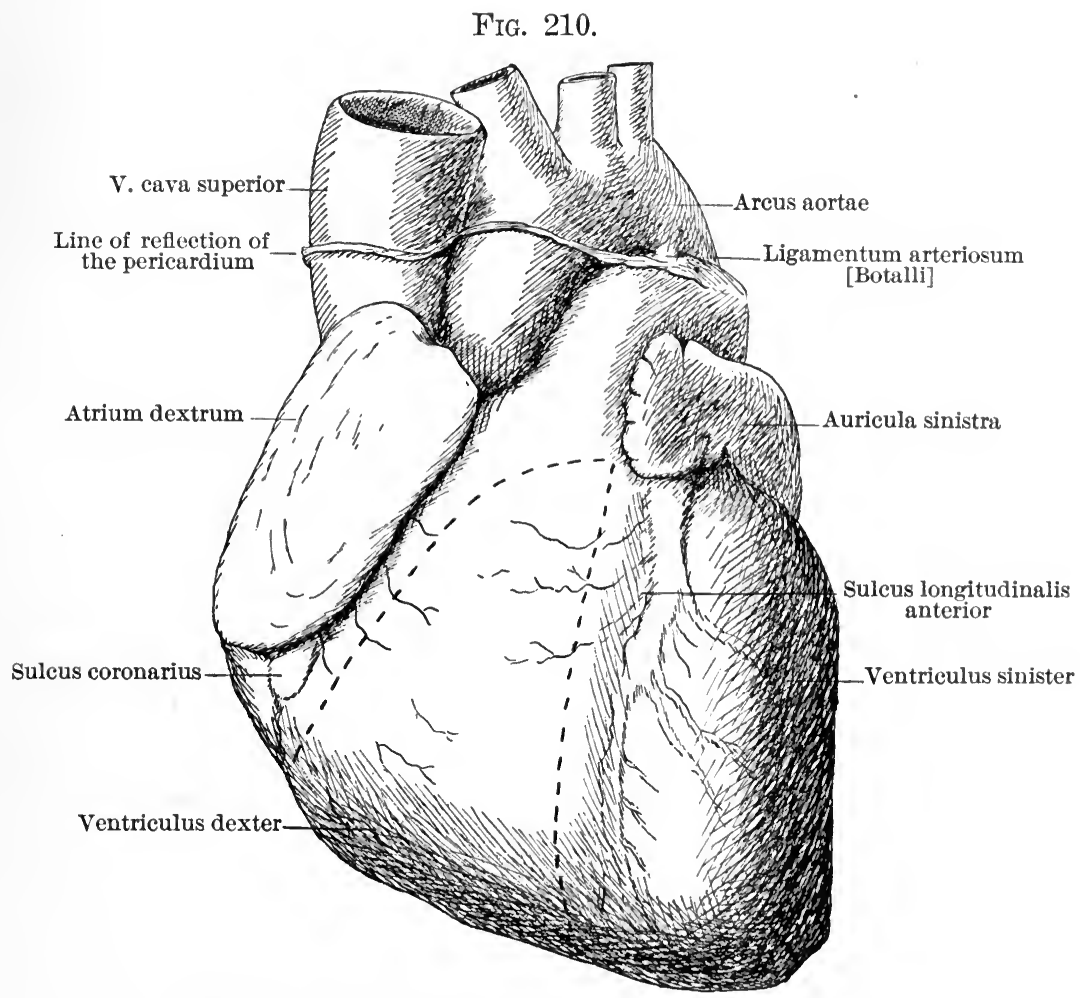

The heart, to show lines of incisions to open the right ventricle.

(a) Arterial cone (conus arteriosus).

(b) Supraventricular crest (crista supraventricularis).

(c) Venous orifice (ostium venosum).

(ca) Tricuspid valve (valvula tricuspidalis) (O. T. right auriculoventricular valve).

(caa) Anterior cusp (cuspis anterior) (O. T. infundibular cusp).

(cab) Posterior cusp (cuspis posterior) (O. T. marginal cusp).

(cac) Medial cusp (cuspis medialis) (O. T. septal cusp).

(d) Papillary muscles ( $\mathrm{Mm}$. papillares).

(da) Tendinous cords (chordae tendineae).

Is there a "moderator band" ?

(e) Pulmonary artery (A. pulmonalis).

(ea) Right ramus (ramus dexter).

(eb) Left ramus (ramus sinister).

(ec) Ligamentum arteriosum (ligamentum arteriosum). 
What is the relation of this to the ductus arteriosus [Botalli] of foetal life? (Fig. 211.)

(f) Arterial orifice of right ventricle (ostium arteriosum ventriculi dextri)

(O. T. pulmonary orifice).

( $f a)$ Semilunar valves of pulmonary artery (valvulae semilunares A. pulmonalis ).

(faa) Anterior semilunar valve (valvula semilunaris anterior).

( $f a b)$ Right semilunar valve (valvula semilunaris dextra).

(fac) Left semilunar valve (valvula semilunaris sinistra).

Fig. 211.

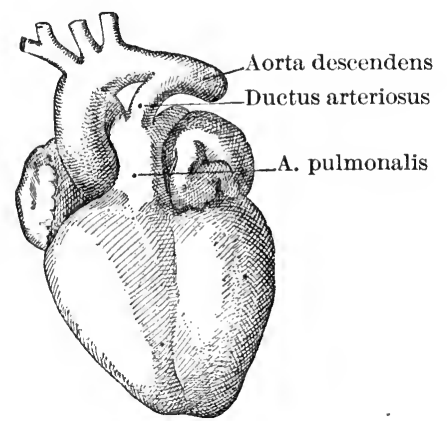

Heart of a foetus of 7 months. ${ }_{1}^{1}$. (From Gegenbaur, Lehrb. der Anat. des Menseh., Leipzig, 1899, 7 Aufl., Bd. ii. p. 232, Fig. 521.)

Slit open the pulmonary artery between the anterior and right semilunar valves. Examine the nodules of the semilunar valves (noduli valvularum semilunarium) and the so-called " sails" of the valves (lunulae valvularum semilunarium).

\section{Pulmonary Veins and Left Atrium.}

The pulmonary veins ( $V v$. pulmonales) may again be examined. Note the differences in the relations of the right pulmonary veins ( $V v$. pulmonales dextrae) and the left pulmonary veins ( $V v$. pulmonales sinistrae).

Cut through the vena cava inferior and turn the heart upward. Observe the entrance of the venae pulmonales into the left atrium.

The left atrium (atrium sinistrum) (O. T. left auricle) may next be examined. Note the size, shape, and position of its auricle (auricula sinistra) (O. T. left auricular appendix).

Turn the heart well to the right, with the apex forward. Make an oblique cut, from the middle of the posterior margin of the atrium behind, forward to the tip of the auricula sinistra. 
Clean the cavity. Observe the openings of the pulmonary veins and the opening into the left ventricle. Examine the valve of the oval foramen (valvula foraminis ovalis).

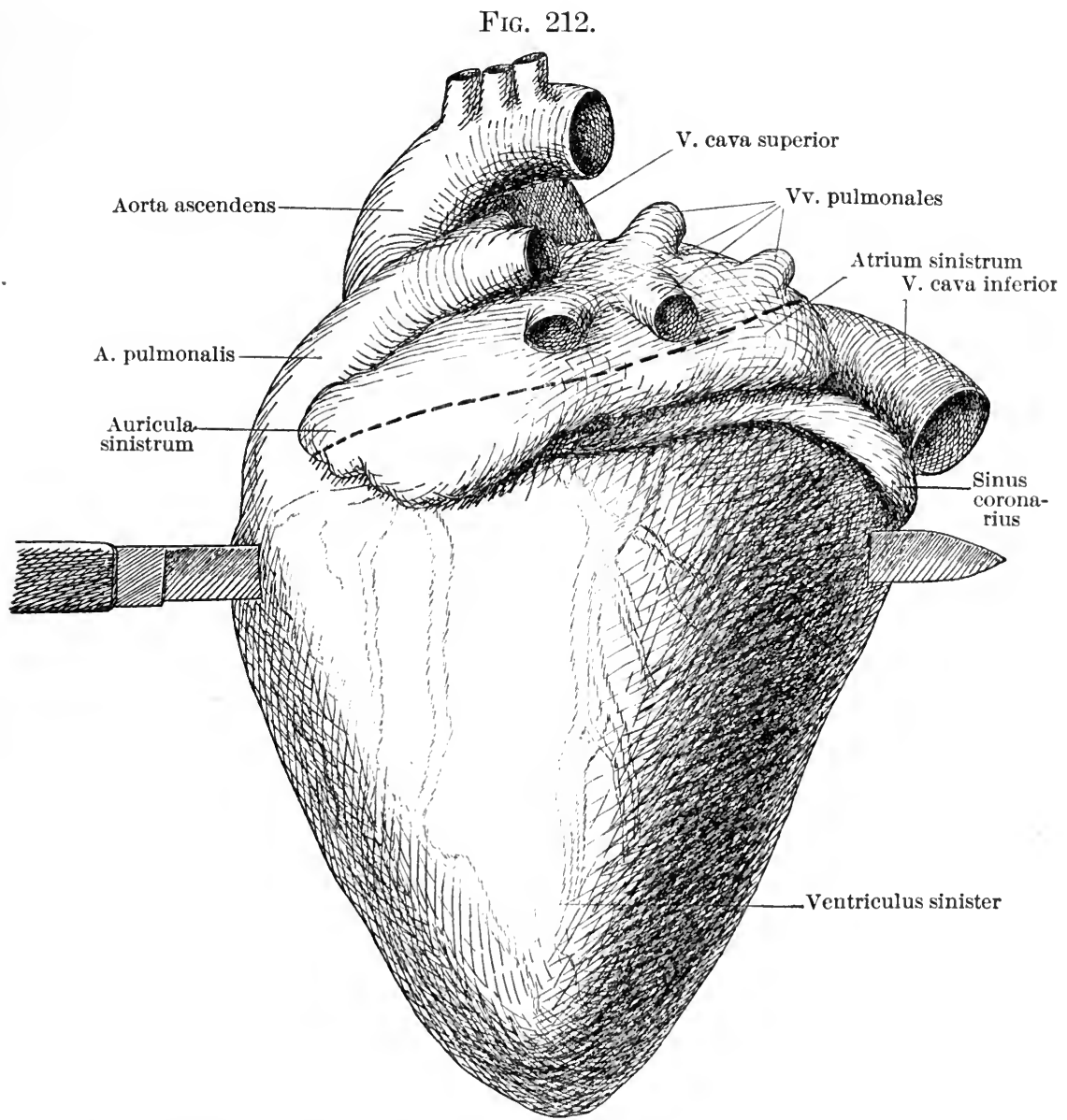

Incisions to open the left atrium and ventriele by Cunningham's method. (After Cunningham, Man. of Praet. Anat., Edinburgh, 1896, 2d ed., vol. ii. p. 58, Fig. 189.)

\section{Left Ventricle (Ventriculus sinister).}

Note its shape, size, and normal position. Observe that it forms the apex cordis.

Stand upon the right side of the cadaver, grasp the heart with the left hand, the index-finger upon the upper part of the sulcus longitudinalis posterior, the thumb upon the upper part of the sulcus longitudinalis anterior. Plunge the long knife through the ventricle from a point below the thumb one centi- 
metre to the left of the sulcus longitudinalis anterior to a point below the index-finger one centimetre to the left of the sulcus longitudinalis posterior. Cut through to the apex, the incisions

FIG. 213.

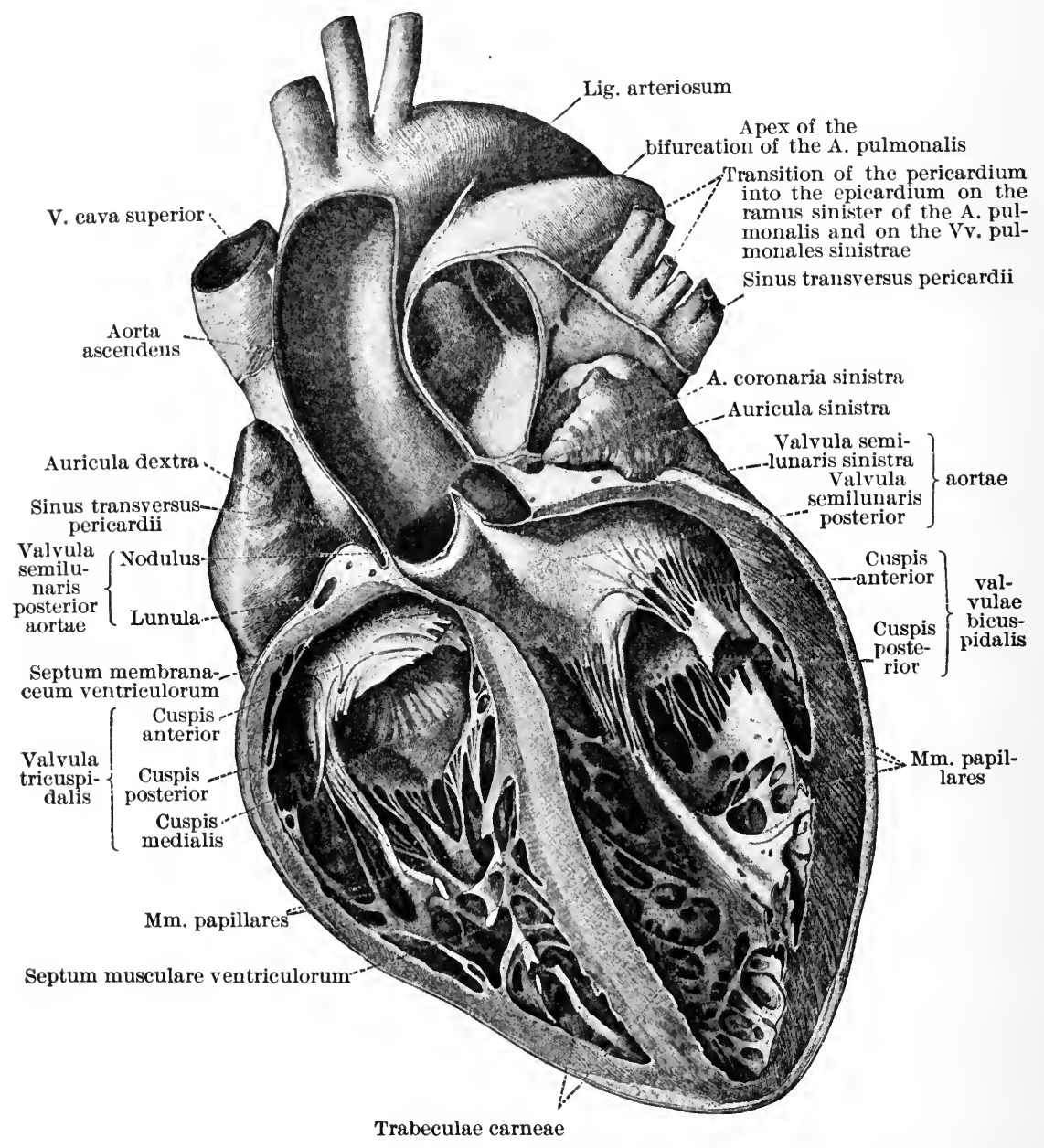

The heart seen from in front. After complete hardening distended in formalin, the anterior part of the ventricles, of the ascending aorta, and of the pulmonary artery have been taken away by a frontal cut. (After Toldt, Anat. Atlas, Wien, 1900, 2 Aufl., p. 552, Fig. 921.)

running along lines parallel to the anterior and posterior sulci and about one centimetre distant from them. (Vide Fig. 212.)

Clean out the blood and injection mass and examine the cavity and walls of the left ventricle; compare them with those of the right. 
Observe the fleshy ridges and cords (trabeculae carneae) (O. T. columnae carneae), the papillary muscles ( $\mathrm{Mm}$. papillares), and tendinous cords (chordae tendineae). Here again are two ostia, one venous (ostium venosum) and one arterial (ostium arteriosum); note their relative positions. The ostium venosum (O. T. left auriculoventricular opening) is guarded by the bicuspid or mitral valve (valvula bicuspidalis [mitralis]). In it study-

(a) Anterior cusp (cuspis anterior).

(b) Posterior cusp (cuspis posterior).

FIG. 214.

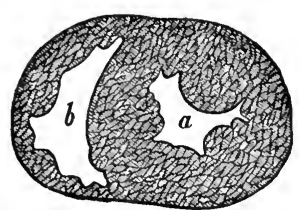

Cross-section of the ventricles. (After H. v. Meyer.) $a$, cavity of the left ventricle; $b$, cavity of the right ventricle. (From Gegenbaur, Lehrb. der Anat. des Mensch., Leipzig, 1899, 7 Aufl., Bd. ii. p. 217, Fig. 511.)

The ostium arteriosum (O. T. aortic opening) is guarded by the semilunar valves of the aorta (valvulae semilunares aortae), to be studied later.

Septum of the Ventricles (Septum ventriculorum).

Study its anterior and posterior insertions and examine its thickness in different parts. Note-

(a) Muscular septum of ventricles (septum musculare ventriculorum).

(b) Membranous septum of ventricles (septum membranaceum ventriculorum).

What is the embryological explanation? Make a note of the importance of the septum ventriculorum in connection with congenital heart disease.

\section{Aorta and its Branches.}

Clean that part of the aorta lying within the thorax, as well as the structures related to it. Study the position, shape, and relations of the-

(a) Ascending aorta (aorta ascendens).

(b) Arch of aorta (arcus aortae).

(c) Thoracic portion of descending aorta (aorta descendens, pars thoracalis). 
In the ascending aorta, study from the outside-

(a) Bulb of aorta (bulbus aortae).

(b) Sinuses of aorta (sinus aortae [Valsalvae]).

(c) Coronary arteries of heart (Aa. coronariae [cordis]).

The distribution of these arteries has already been studied.

FIG. 215.
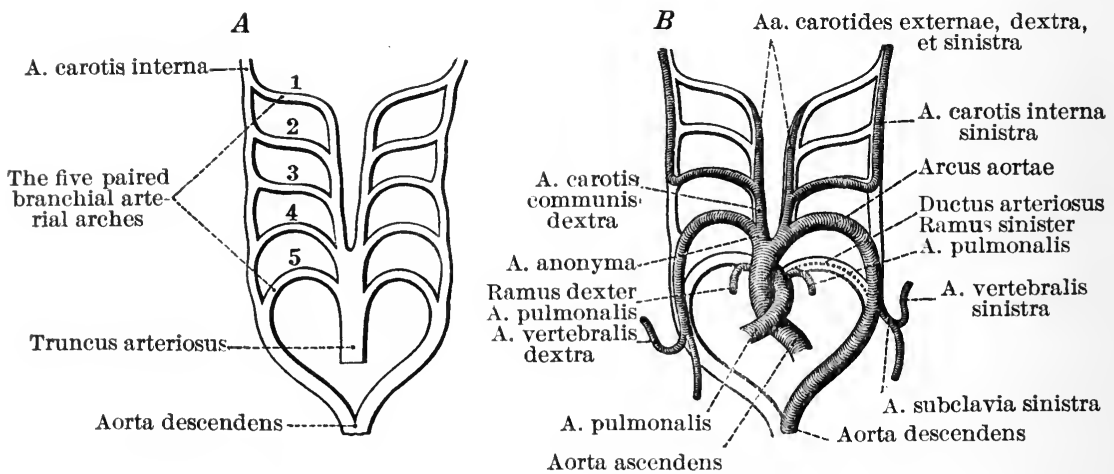

Diagrams of the transformation in the arteries of the branchial arches: $A$, original, $B$, persistent disposition of the arterial stems (Rathke's schema with a slight alteration according to F. Hochstetter.) (After Toldt, Anat. Atlas, Wien, 1901, 2 Aufl., p. 563, Fig. 938.)

In connection with the arcus aortae, study,its exact relations, note the isthmus aortae at its junction with the aorta descendens, and dissect out and study thoroughly the following great arterial trunks :

(a) Innominate artery (A. anonyma).

(aa) Lowest thyreoid artery (A. thyreoidea ima), inconstant.

(b) Left common carotid artery (A. carotis communis sinistra).

(c) Left subclavian artery (A. subclavia sinistra).

The thoracic part of the aorta descendens will be studied later on.

\section{Cardiac Plexus (Plexus cardiacus).}

The superficial portion of this plexus has already been studied. Its deeper portion should next be examined. Place two ligatures about the arcus aortae at its junction with the aorta descendens and divide the aorta between them; similarly ligate twice and divide between at the junction of the arcus aortae with the aorta descendens. Cut through the V. cava superior, just below the entrance into it of the V. azygos. Divide the liga- 
mentum arteriosum. The arcus aortae should now be drawn aside and the trachea and Nn. cardiaci exposed. Find the following nerves, which help to form the deep cardiac plexus:

(a) From the sympathetic system:

(aa) Middle cardiac nerve ( $N$. cardiacus medius).

(ab) Inferior cardiac nerve ( $N$. cardiacus inferior).

(b) From the $N$. vagus:

(ba) Superior cardiac rami (O. T. cervical cardiac branches of pneumogastric).

Is an isolated "N. depressor" present?

(bb) Inferior cardiac rami from the N. recurrens (rami cardiaci inferiores nervi recurrentis) ( $\mathrm{O}$. T. cardiac branches of recurrent laryngeal).

Notice the subdivision of the plexus cardiacus into a right and a left portion.

How is the plexus cardiacus related to the anterior and posterior coronary plexuses?

\section{Removal of Heart.}

Divide the A. pulmonalis and the Vv. pulmonales and remove the heart from the body. Continue the incision in the anterior wall of the left ventricle through the ascending aorta, but make sure that it passes between two of the semilunar valves.

\section{Valves of Aorta.}

The semilunar valves of the aorta (valvulae semilunares aortae) are now to be studied.

(a) Posterior semilunar valve (valvula semilunaris posterior).

(b) Right semilunar valve (valvula semilunaris dextra).

(c) Left semilunar valve (valvula semilunaris sinistra).

Examine the nodules (noduli valvularum semilunarium [Arantii]) (O. T. corpora Arantii) and "sails" or " lunulae", (lunulae valvularum semilunarium) of the valves. Examine the sinus aortae [Valsalvae] from the inside and note exact origin, on the inside, of the Aa. coronaria.

\section{Myocardium.}

Its relations to the epicardium and endocardium should be reviewed. The student should study a sheep's heart or a calf's heart that has been macerated in fifty per cent. $\mathrm{HCl}$ or in equal parts of glycerin, alcohol, and nitric acid. Or a sheep's heart filled with a paste of flour and water may be boiled for fifteen 
minutes. then placed in cold water and dissected. Other fresh hearts should be distended under pressure. fired and hardened in five or ten per cent. formalin for sereral dars; windows may then be cut out in rarious wars in order to displar internal structures.

Follow the course of the muscle bundles $(a)$ in the atria, (b) in the rentricles. Studr the mode of formation of the rortex of the heart (cortex cordis). Look up the articles br Ludwig, J. B. MacCallum, and Retzger on the distribution of the heart-muscle fibres.

Fibrous Rings of Heart (Annuli fibrosi).

Cut away the atria with scissors. Examine the relative positions of the orifices at the base of the ventricular portion of the heart. Situdi-

(a) Pight flbrons ring (annuluz fbrosus derter). around ostium renosum dertrum

(b) Lefti fbrots ring (annwhis fibroske sivister), around ostium renosum sinistrum

(ba) Fibrous trigumes (trigana fibrasa).

\section{Trachea and Bronchi.}

Separate the ramus dexter from the ramus sinister of the A. pulnonalis and reffect each lateralward. Expose the trachea and bronchi thoroughly by drawing the arcus aortae aside and remoring the bronchial Irmph-glands (lymphoglandulae bron(hiales) in the angle of bifurcation of the trachea. Examine carefulls the relations of the trachea. Note the level of the bifurcation (bifurcatio tracheae) into the right and left bronchus (bronchus [dester et sinister]). Look for the broncho-oesophageal muscle (M. broncho-oesophageus).

\section{Posterior Mediastinal Cavity and its Contents.}

To open the posterior mediastinal cavity (cavum mediastinale posterius) (O.T. posterior mediastinum), make a vertical cut through the pericardium along the vesophagus and reflect the pericardium lateralward. Look for the pleuro-oesophageal muscle ( $M$. pleuro-oesophageus), extending across the aorta descendens from the oesophagus to the left pleura. In the cavum mediastinale posterius dissect out and study thoroughly the relations of the following: 
(a) Tagus nerves (Ain. ragi) (O. T. pnewmogasic nerves).

(aa) Reeurrent nerve ( $N$. remrrevs) on lefi side.

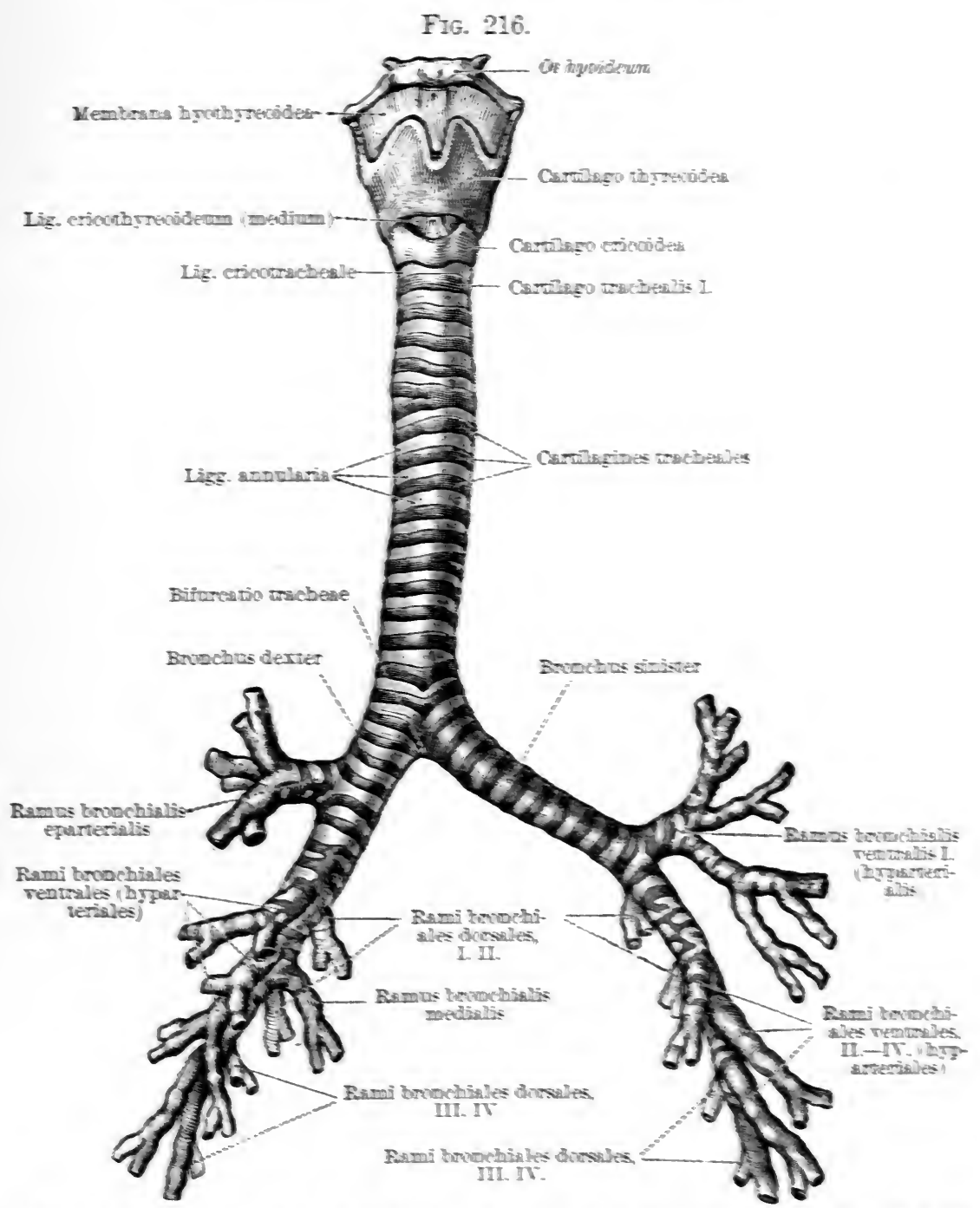

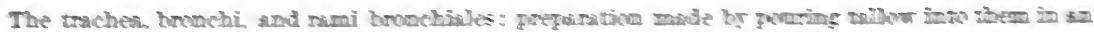

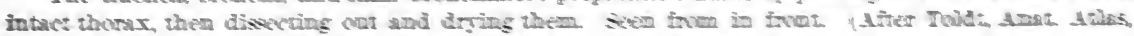

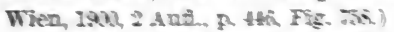

(ab) Anterior and pusterior bnonchial rami ( nawi browolniales andurions at pasterions?.

(aba) Anterior and pusterior palmonary plexuses (plcaws

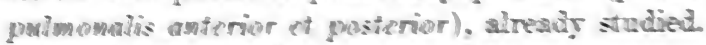

(ac) Oesophagesl rami (nami moghagri). 
(aca) Anterior and posterior oesophageal plexuses (plexus oesophageus anterior et posterior) (O. T. plexus gulae).

(b) Thoracic part of oesophagus (oesophagus, pars thoracalis).

(c) Thoracie part of aorta descendens (aorta thoracalis).

Examine the aortic spindle. Study-

(ca) Visceral rami (rami viscerales).

(caa) Bronchial arteries (Aa. bronchiales).

(cab) Oesophageal arteries (Aa. oesophageae).

(cac) Pericardiac rami (rami pericardiaci).

(cb) Parietal rami (rami parietales).

( $c b a)$ Mediastinal rami (rami mediastinales).

$(c b b)$ Superior phrenic arteries (Aa. phrenicae superiores).

$(c b c)$ Intereostal arteries (Aa. intercostales).

Fig. 217.

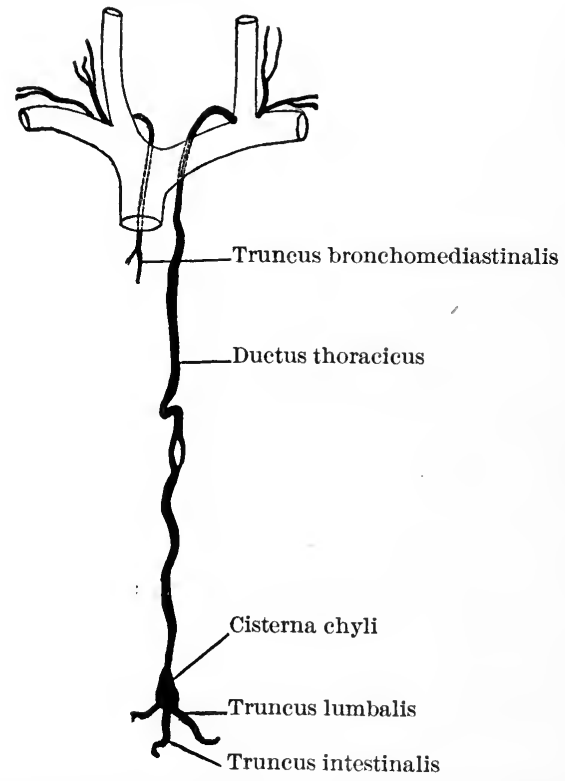

The large lymph-vessels opening into the venous system. Schematic. (After Gegenbaur, Lehrb. der Anat. des Mensch., Leipzig, 1899, 7 Aufl., Bd. ii. p. 339, Fig. 566.)

(d) Thoracic duct (ductus thoracicus). (Fig. 217.)

(e) Posterior mediastinal lymph-glands (lymphoglandulae mediastinales posteriores).

\section{Interior of the Lungs.}

Cut through the trachea three centimetres above its bifurcation. Remove the lungs from the body. With a medium-sized scissors with one blunt-ended blade, cut open the bronchus and 
its subdivisions, also the branches of the pulmonary artery and pulmonary veins in each lung. Compare the intrapulmonary bronchi of the two lungs. Dissect out the wall of a larger bronchus; examine its muscular coat (tunica muscularis), the submucous layer (tela submucosa), and the mucous coat (tunica mucosa); in the latter note the bronchial glands (Gl. bronchiales) and the bronchial lymph-nodules (noduli lymphatici bronchiales). How far down in a bronchus does the cartilage extend?

Study a corrosion-preparation of the bronchi and their subdivisions. With the help of atlases, sections, and the model (Fig. 218) by W. S. Miller, of the University of Wisconsin, study the size, shape, and relations of the following:

FIG. 218.

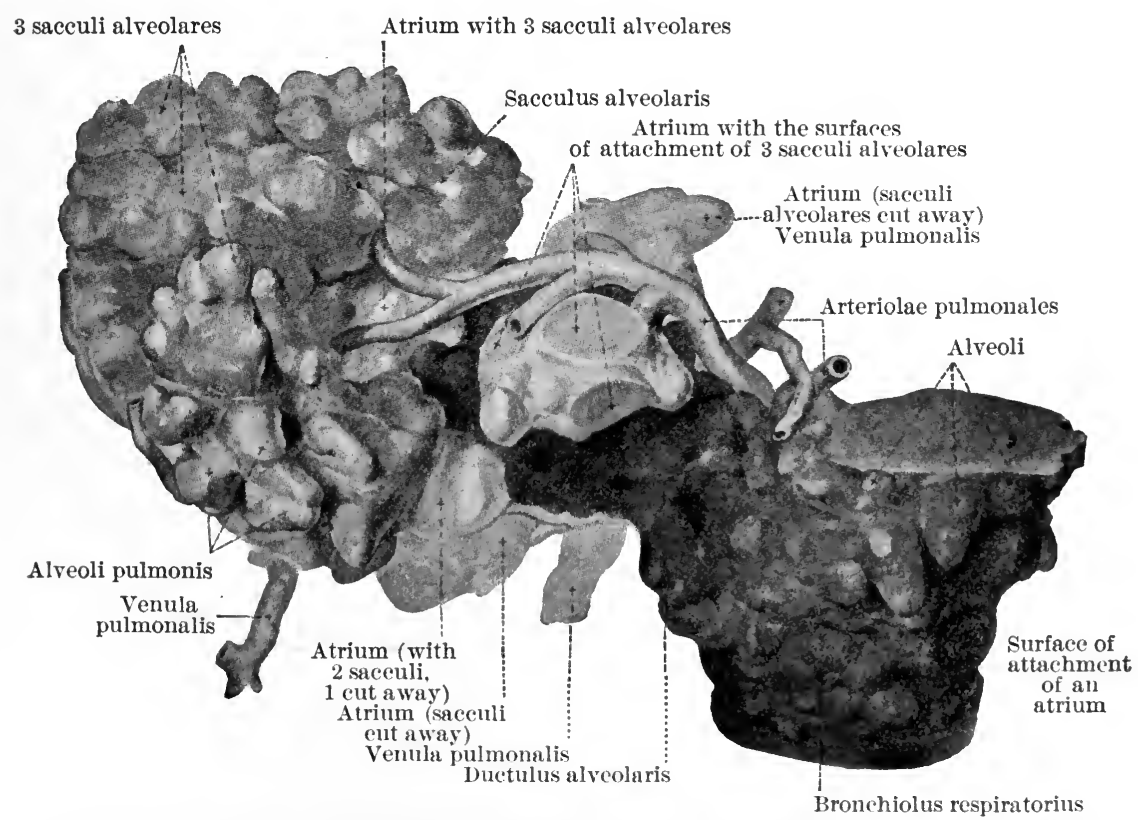

Alveolar duct, with branches and blood-ressels, from the dog. (After Spalteholz, Hand Atlas of Human Anatomy, Leipzig, 1903, vol. iii. p. 567, Fig. 624.)

(a) Bronchioles (bronchioli).

(b) Respiratory bronchioles (bronchioli respiratorii).

(c) Terminal bronchi, or alveolar ductules (ductuli alveolares).

These open through the vestibules (vestibuli) into the-

(d) Atria.

The latter communicate through the air-sac passages with-

(e) Air-sacs (sacculi alveolares) (O. T. infundibula), which are studded

- with many-

(f) Air-cells or pulmonary alveoli (alveoli pulmonis). 
Each ductulus alveolaris with all its branches makes up a lobule of the lung (lobulus pulmonis). The term "lobule" as used by the pathologists includes several of these anatomical lobuli. Note the central position of the terminal branch of the A. pulmonis and the peripheral distribution of the roots of the pulmonary veins in each lobulus pulmonis.

\section{Thoracic Portion of Sympathetic Nervous System.}

Remove the pleura parietalis from the sides of the vertebral column and from the inner surface of the ribs. Dissect out the thoracic portion of the sympathetic nervous system (pars thoracalis S. sympathici). Study-

(a) Thoracie ganglia (ganglia thoracalia).

How many are there? How are they located?

(b) Great splanchnic nerve (N. splanchnicus major).

How is it formed? Where does it leave the thorax? Find-

(ba) Splanchnic ganglion (ganglion splanchnicum).

(c) Small splanchnie nerve (N. splanchnicus minor).

How is it formed? How does it leave the thorax? In the abdomen it gives off a renal ramus (ramus renalis).

(d) Lowermost splanchnic nerve (N. splanchnicus imus).

The plexus aorticus thoracalis, plexus cardiacus, plexus coronarii, rami pulmonales, and plexus pulmonalis have been studied already.

\section{Wall of Thorax from within.}

Examine the Mm. intercostales interni and the ligamenta intercostalia interna from within. Clean, isolate, and study-

(a) Subcostal muscles (Mm. subcostales).

(b) External intercostal muscles ( $M$ m. intercostales externi).

These ean be exposed from within by removing the ligamenta intercostalia interna in one or two intercostal spaces.

(c) Intercostal arteries from the aorta (Aa. intercostales).

The rami posteriores can now be examined at their origins.

(d) Highest intercostal artery from the subclavian (A. intercostalis suprema).

(e) Intercostal nerves $(N n$. intercostales $)=$ anterior rami of thoracic nerves (rami anteriores $\mathrm{Nn}$. thoracalium).

Examine their proximal portions.

$(f)$ Intercostal veins ( $V v$. intercostales).

Study carefully the differences in termination on the two sides.

(g) Azygos vein (vena azygos) (O. T. vena azygos major). (Cf. Fig. 205.) 
Study its tributaries-

(ga) Hemiazygos vein (V. hemiazygos) (O. T. V. azygos minor inferior).

(gb) Aecessory hemiazygos vein ( $V$. hemiazygos accessoria) (O. T. V. azygos minor superior).

Make out the exact relations of the intercostal veins ( $V v$. intercostales), oesophageal veins ( $V v$. oesophageae), and posterior bronchial veins ( $V v$. bronchiales posteriores) to $(g),(g a)$, and $(g b)$. What is the relation of the venous system of the vertebral column to the V. azygos?

\section{Joints of the Thorax. (Vide Spalteholz, Vol. i., pp. I56-I6I and I70-} I75.)

These are divisible into three sets-

(a) Sternocostal joints (articulationes sternocostales).

(b) Costovertebral joints (articulationes costovertebrales).

(c) Joints of vertebral column.

These should now be dissected out. Study-

(aa) Joint-capsules (capsulae articulares).

(ab) Interarticular sternocostal ligament (Lig. sternocostale interarticulare) ( $\mathrm{O} . \mathrm{T}$. interarticular chondrosternal ligament).

(ac) Radiate sternocostal ligaments (Ligg. sternocostalia radiata) (O. T. anterior and posterior chondrosternal ligaments).

(ad) Membrane of sternum (membrana sterni).

(ae) Costoxiphoid ligaments (Ligg. costoxiphoidea) (O. T. chondroxiphoid ligaments).

(af) Interchondral joints (articulationes interchondrales).

$(a g)$ Intercostal ligaments (Ligg. intercostalia), already studied.

In the costovertebral joints study-

(ba) Capitular joints (articulationes capitulorum) (articulations between the heads of the ribs and the vertebrae).

(baa) Joint-capsules (capsulae articulares).

(bab) Radiate ligament of head of rib (Lig. capituli costae radiatum $)(\mathrm{O} . \mathrm{T}$. anterior costovertebral or stellate ligament).

(bac) Interarticular ligament of head of rib (Lig. capituli costae interarticulare).

This is best displayed by removing the radiate ligament from the front of the joint.

(bb) Costotransverse joints (articulationes costotransversariae).

$(b b a)$ Joint-capsules (capsulae articulares).

(bbb) Ligament of tubercle of rib (Lig. tuberculi costae).

$(b b c)$ Ligament of neck of rib (Lig. colli costae).

$(b b d)$ Anterior costotransverse ligament (Lig. costotransversarium anterius).

(bbe) Posterior costotransverse ligament (Lig. costotransversarium posterius).

(bbf) Lumbocostal ligament (Lig. lumbocostale). 
(bbg) Costotransverse foramen (foramen costotransversarium ).

In the vertebral column study-

(ca) Anterior longitudinal ligament (Lig. longitudinale anterius)

(O. T. anterior common ligament).

(cb) Posterior longitudinal ligament (Lig. longitudinale posterius)

(O. T. posterior common ligament).

Make vertical and transverse sections through three intervertebral disks and study-

(cc) Intervertebral fibrocartilages (fibrocartilagines intervertebrales).

(cca) Fibrous ring (annulus fibrosus).

(ccb) Pulpy nucleus (nucleus pulposus) (O. T. nucleus polyposus).

The joint-capsules (capsulae articulares) and intertransverse ligaments (Ligg. intertransversaria) should also be examined. The other ligaments of the spine have been injured or removed in the study of the vertebral canal. 


\section{Part V}

DISSECTION OF THE ABDOMEN AND PELVIS 


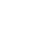




\section{ABDOMEN AND PELVIS}

Mark out on the abdomen, perineum, and small of the back the various regions. Make three drawings of these regions, labelling them accurately, one of the anterior surface, one of the posterior surface, and one of the perineum when the body is in the lithotomy position. (Vide Figs. 1, 2, 219, and 220.)

Inspect the surface of the abdomen. Note the lower margin of the thorax, counting the ribs (costae) and marking each with its number. Locate the heart fossa (scrobiculus cordis) and the navel (umbilicus). What is its position with reference to $(a)$ the lumbar vertebrae, $(b)$ the thorax and pubis? How does its position differ in the two sexes?

Observe the furrow in the median line (linea mediana anterior) and note the difference in this furrow above and below the umbilicus. Examine the pubic eminence (mons pubis) covered with hair (pubes). Note the prominences due to the Mm. recti abdominis and the furrows bounding these lateralward. Examine the direction of the hair streams (flumina pilorum). Note the distribution of pigment in the skin; in female subjects look for white, depressed streaks in the skin over the side and front of the abdomen (striae albicantes).

In the depression of the groin (inguen) observe the linear elevation due to Poupart's ligament (ligamentum inguinale [Pouparti]). Follow it out to the spina iliaca anterior superior.

On the lateral surface of the abdomen define the limits of the loin (latus). On the posterior surface of the abdomen examine the small of the back (lumbus). Follow the furrow in the middle line (linea mediana posterior) downward to the cleft between the buttocks (crena ani). Note the hair streams (fumina pilorum) here and vortices pilorum. Observe the prominence on each side due to the M. sacrospinalis. Note that the surface of the lumbus passes upward over the twelfth rib into the back and lateralward to the loin (latus) without any perceptible delimitation; below it is separated from the surface of the hip (coxa) by the iliac crest. Observe the foveola coccygea.

After thorough inspection, palpate the anterior and posterior surfaces. Feel the cartilage of the xiphoid process (processus 
xiphoideus), the anterior ends of the seventh, eighth, ninth, tenth, eleventh, and twelfth ribs, the spina iliaca anterior superior and inferior, the ramus superior oss. pubis, the symphysis pubis, the pubic tubercle (tuberculum pubicum), the subcutaneous inguinal ring (annulus inguinalis subcutaneus) (O. T. external abdominal ring), and the spermatic cord (funiculus spermaticus) in the male, the round ligament in the female, the tuber ischiadicum and the ramus inferior ossis ischii.

In the small of the back palpate the spinous processes, the crista media sacralis, the tip of the coccyx, the spina iliaca posterior superior, and the crista iliaca.

\section{PERINEAL REGION (REGIO PERINEALIS).}

Draw the body to the end of the table; place a block beneath the buttocks and place the legs in the leg-rests. (See Fig. 221.) Review the surface anatomy of this region, noting the symphysis

FiG. 219.

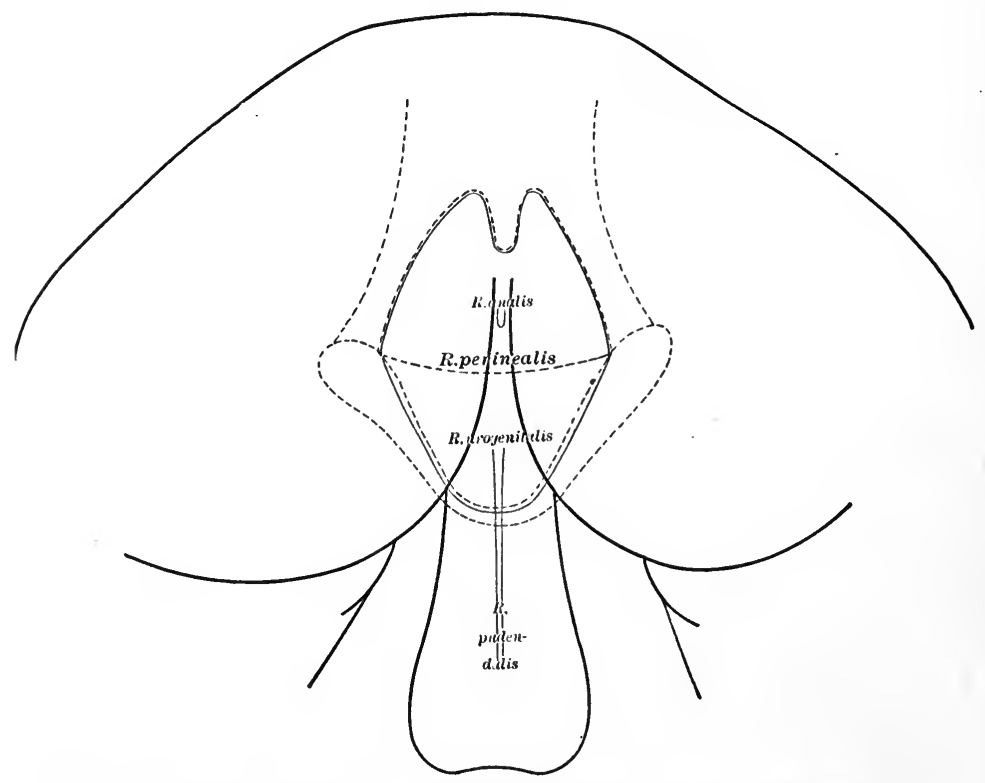

The perineal region in the male. (After His, Die Anat. Nom., Leipzig, 1895, p. 104.)

pubis, the tip of the coccyx, the tuber ischiadicum of each side, and the rami of the ischium and pubis. Note the subdivisions of this region into- 
(1) Anal region (regio analis).

(2) Urogenital region (regio urogenitalis).

(Vide Figs. 219 and 220.)

The region anterior to the latter, comprising the external organs of generation, is known as the pudendal region (regio pudendalis). Determine exactly the boundaries of these different regions.

FIG. 220.

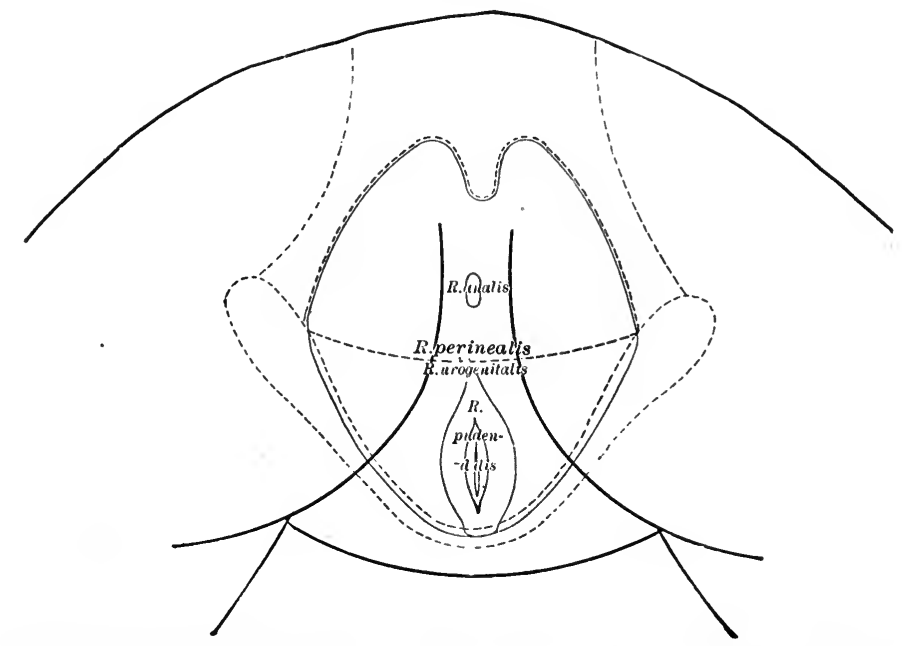

The perineal region in the female. (After His, Die Anat. Nom., Leipzig, 1895, p. 105.)

Examine the external genitalia, comparing the parts of the male and the female, noting differences and homologies.

\section{External Genital Parts in Male (Partes genitales externae).}

(a) Body of penis (corpus penis).

(b) Dorsum of penis (dorsum penis).

(c) Urethral surface (facies urethralis).

(d) Glans penis.

(da) Corona glandis.

(db) Septum of glans (septum glandis).

(dc) Neck of glans (collum glandis).

(e) Prepuce (praeputium).

(ea) Frenulum of prepuce (frenulum praeputii).

(eb) Preputial glands (Gl. praeputiales).

(eba) Secretion (smegma praeputii).

(f) External urethral orifice (orificium urethrae externum).

(g) Scrotum.

(ga) Raphe of scrotum (raphe scroti), continuous with the raphe penis. 
Note the amount of pigmentation about the external genitalia and its distribution.

What is the perineal raphe (raphe perinei)?

\section{MALE PERINEUM.}

Pass a sound into the bladder, fill the rectum with cotton or strips of cheese-cloth, and close the anal orifice by sutures. The sound should rest upon the anterior abdominal wall, and the scrotum may be stitched to the prepuce, thus rendering the field of dissection larger: Make the following incisions (Fig. 221) :

(1) A transverse incision from the anterior extremity of the tuber ischiadicum of one side to that of the other.

(2) An incision passing in the median raphe from the serotum in front to the tip of the coceyx behind.

Refleet earefully the four flaps marked out by these incisions.

FIG. 221.

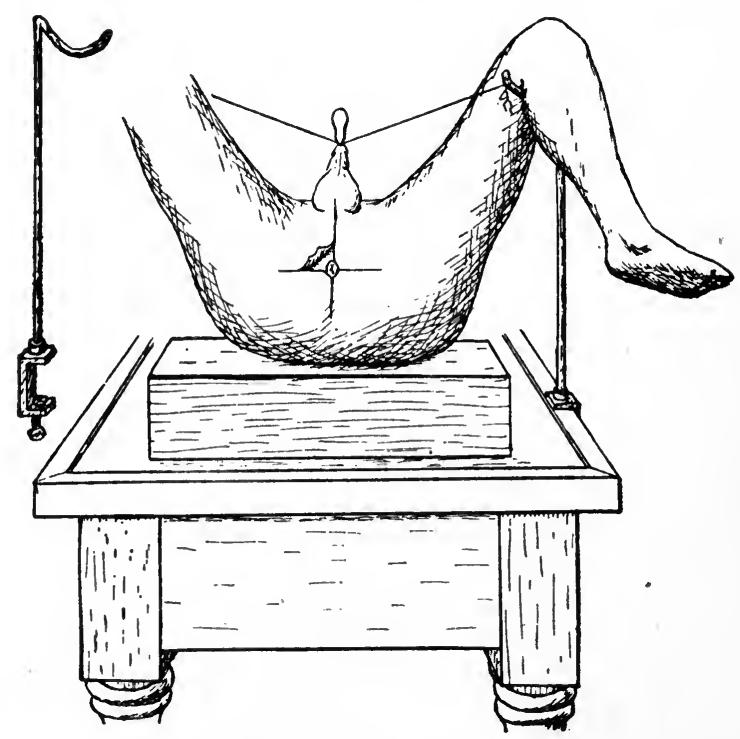

Skin incision, and the lithotomy position for the dissection of the perineum. At the left is shown a convenient iron leg-support which clamps on to the edge of the table. The table-top is covered with zinc.

Superficial Perineal Fascia (Fascia superficialis perinei). (Figs. 222, 227-229.)

Note the general characteristics of this fascia in the regio analis. In the regio urogenitalis it will be found to consist of 
two distinct layers. The fascia in this region is spoken of occasionally as the "inferior perineal aponeurosis." The inferior layer is continuous with all the neighboring superficial fascia, while the superior layer is dense and membranous. It is called Colles' fascia; it gains smooth muscular fibres anteriorly to form the tunica dartos of the scrotum. It is the continuation of Scarpa's fascia of the abdomen into the perineum.

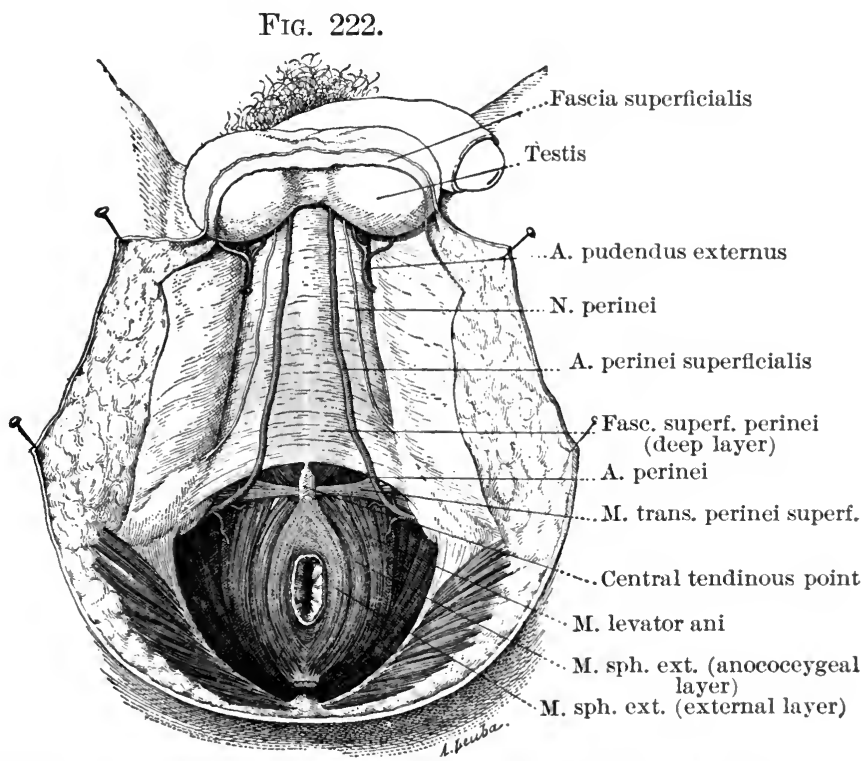

Fascia superficialis perinei. (From Poirier et Charpy, Traité d'Anat. hum., Paris, 1901, t. ii. p. 215, Fig. 143.)

To demonstrate the attachments of this deep layer proceed as follows: Enter the knife in the median line at the base of the scrotum and carry it dorsalward and lateralward to the tuber ischiadicum on each side, exercising caution to avoid injury to important vessels and nerves just below this fascia. Carefully dissect back the central and the two lateral flaps, noting the attachment of the fascia. How is it related to the muscles immediately beneath?

After studying this fascia fully, proceed to the study of the contents of the "inferior perineal compartment."

\section{Structures in "Inferior Compartment" of Perineum between the Fas- cia Superficialis Perinei and the Diaphragma Urogenitale.}

Muscles of the Perineum (Musculi perinei). (See Figs. 223 and 224.)

These museles are all attached to the organs of copulation and 
they. are all derived phylogenetically from the sphineter of the cloaca. (Vide Holl, Die Muskelen im Beckenausgange des Menschen; Merkel-Bonnet, Ergebnisse der Anatomie, xi., 1901.) Study the form, position, origin, insertion, action, and innervation of each.

(a) Superficial transverse muscle of perineum ( $M$. transversus perinei superficialis).

In the embryo and child this muscle is well developed and constant in its relations. In the adult the muscle varies in size, strength, and position; in the foetus muscle bands are found connecting it to the M. sphincter ani externus and the M. ischiocavernosus, and it may be regarded as a connecting link between anal musculature and the musculature of the urogenital region. In the adult it seems to be underging retrogressive changes.

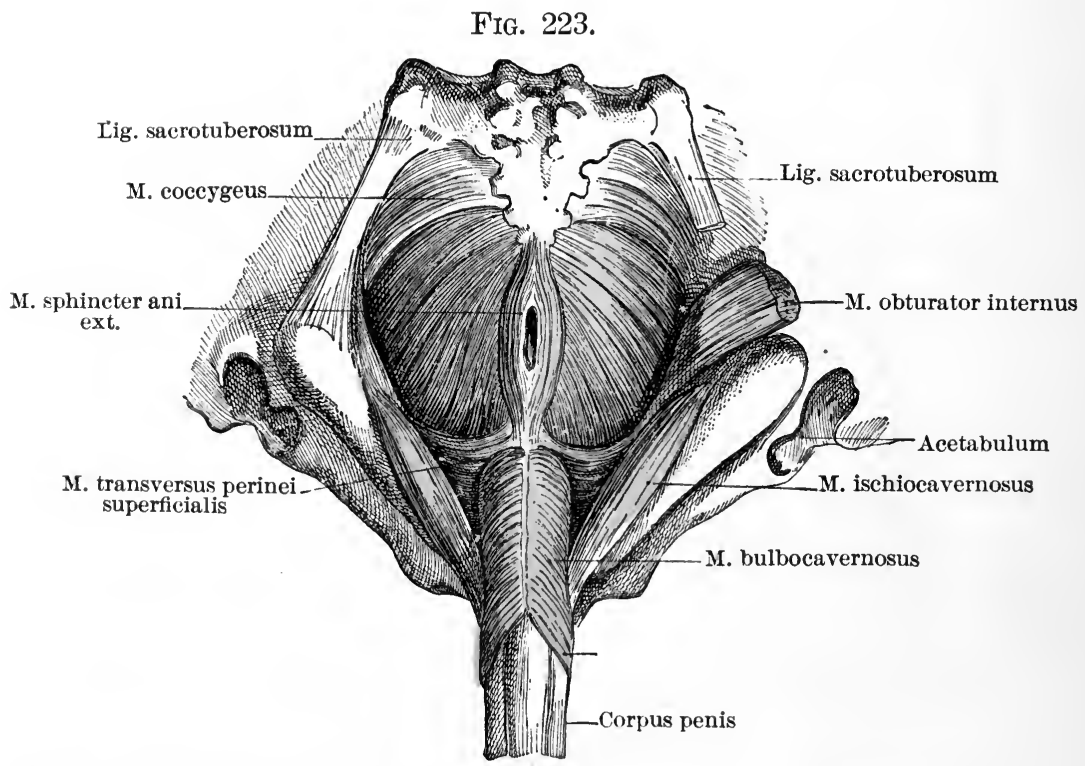

Perineal muscles of man. On the right side the anterior part of the lig. sacrotuberosum is removed, the M. obturator internus is divided, the M. levator ani is shown, but not labelled. (From Gegenbaur, Lehrb. der Anat. des Mensch., Leipzig, 1899, 7 Aufl., Bd. ii. p. 197, Fig. 496.)

(b) Ischiocavernous muscle (M. ischiocavernosus) (O. T. erector penis muscle).

According to Merkel, the tendon of this muscle fuses, as a rule, with the lower and lateral surfaces of the fibrous tunic of the corpora eavernosa. At times a tendinous slip is given off, which fuses with a corresponding process from the opposite side, on the dorsum of the penis, and thus forms a.loop about the dorsal vein of the penis. These processes may be muscular. If so, they are called the "compressor muscle of the dorsal vein of the penis" (M. compressor venae dorsalis penis). 
(c) Bulbocavernous muscle (M. bulbocavernosus) (O. T. ejaculator seminis or accelerator urinae). Dissect out the following parts of it:

(ca) Proper compressor of the bulb ( $M$. compressor bulbi proprius).

( $c b)$ Constrictor of root of penis ( $M$. constrictor radicis penis).

(cc) Compressor of the bulbar hemispheres ( $M$. compressor hemisphaerium bulbi [Kobelti]).

Is a M. ischiobulbosus present? Note the median tendinous raphe of this muscle and the relation of the muscular fibres to it.

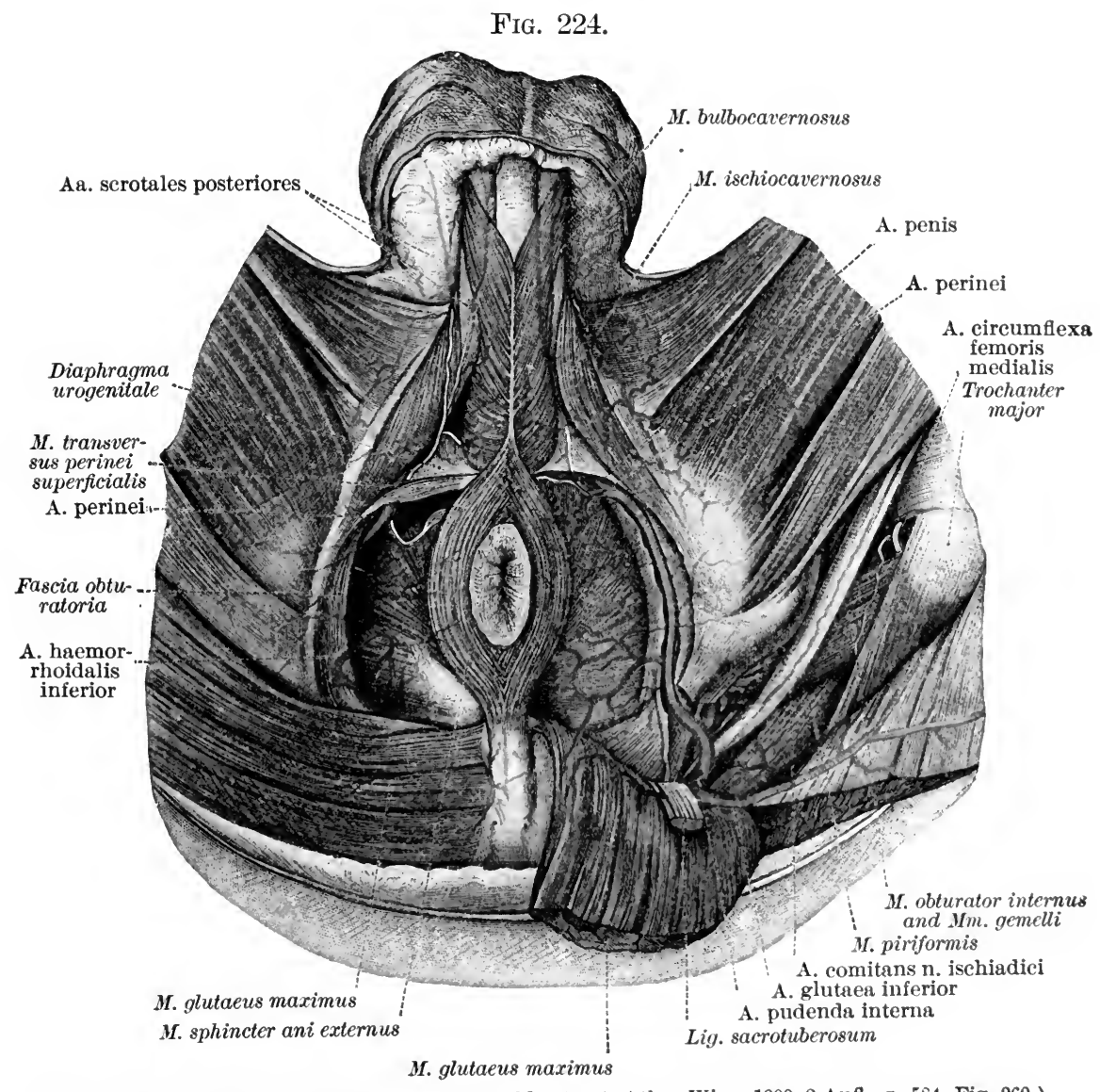

The arteries of the male perineum. (After Toldt, Anat. Atlas, Wien, 1900, 2 Aufl., p. 584, Fig. 960.)

Arteries. (Vide Fig. 224 and Spalteholz, Fig. 473.)

(a) Artery of perineum (A. perinei). Trace this artery forward to the scrotum and find-

(aa) Posterior scrotal arteries (Aa. scrotales posteriores).

Note the course and anastomoses of the veins accompanying the arteries. 
Nerves. (Fig. 225.)

(a) Nerve of the perineum ( $N$. perinei).

(aa) Posterior serotal nerves (Nn. scrotales posteriores).

(b) Perineal branches of posterior eutaneous nerve of thigh (O. T. branches of small sciatic).

Lymphatics. (Vide Toldt, Fig. 1092.)

What is the course of the lymphaties in this region? Are there any lymph-nodes?

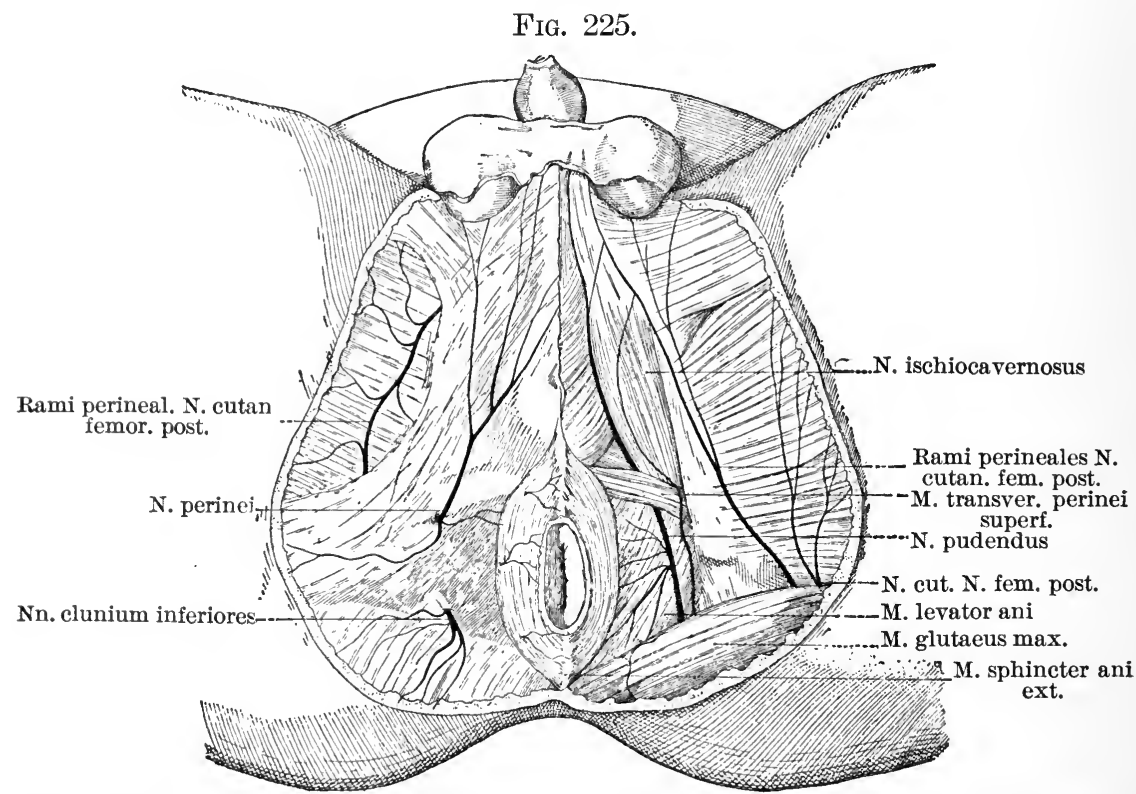

Perineal nerves in the male (after Paulet). (From Poirier et Charpy, Traité d'Anat. hum., Paris, 1899, t. iii., 3, p. 1154, Fig. 593.)

Dissect away carefully the skin covering the penis, avoiding vessels and nerves. Observe-

(a) Root of penis (radix. penis).

(b) Crura of penis (crura penis).

Use models in this study. Note the divergence of the crura behind, also their exact relations to the ischiadic and pubic rami. What is their relation to the urogenital diaphragm?

(c) Cavernous bodies of penis (corpora cavernosa penis).

(d) Cavernous body of urethra (corpus cavernosum urethrae).

This body may be exposed if the M. bulbocavernosus is incised in the median line and carefully reflected. Note how it expands posteriorly to form the-

(e) Bulb of urethra (bulbus urethrae).

(ea) Hemispheres of bulb of urethra (hemisphaeria bulbi urethrae).

(eb) Septum of bulb of. urethra (septum bulbi urethrae). 
Urogenital Diaphragm (Diaphragma urogenitale) and " Middle Perineal Compartment." (Figs. 226-229, 273, and 274.)

The two dissectors should now work together. On one side remove the M. ischiocavernosus and detach the crus penis from

FIG. 226.

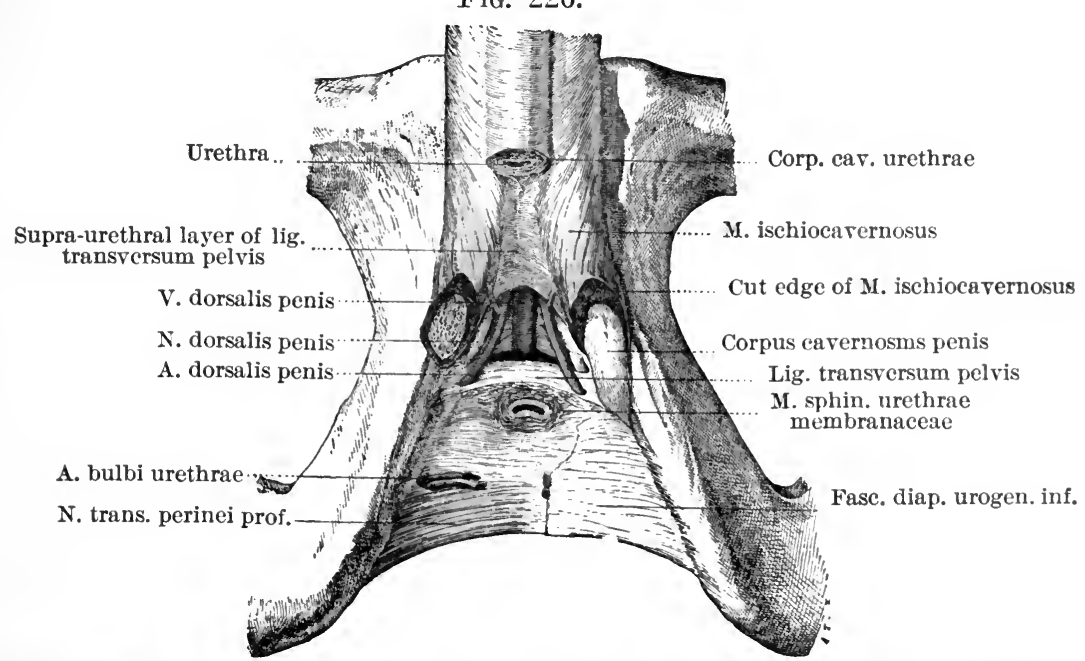

Inferior surface of the urogenital diaphragm. (From Poirier et Charpy, Traité d'Anat. hum., Paris, 1901, t. v. p. 216, Fig. 145.)

the pubic and ischiadic rami. Turn the crus aside, avoiding injury to the dense fascia immediately beneath. Avoid injury

FIG. 227.

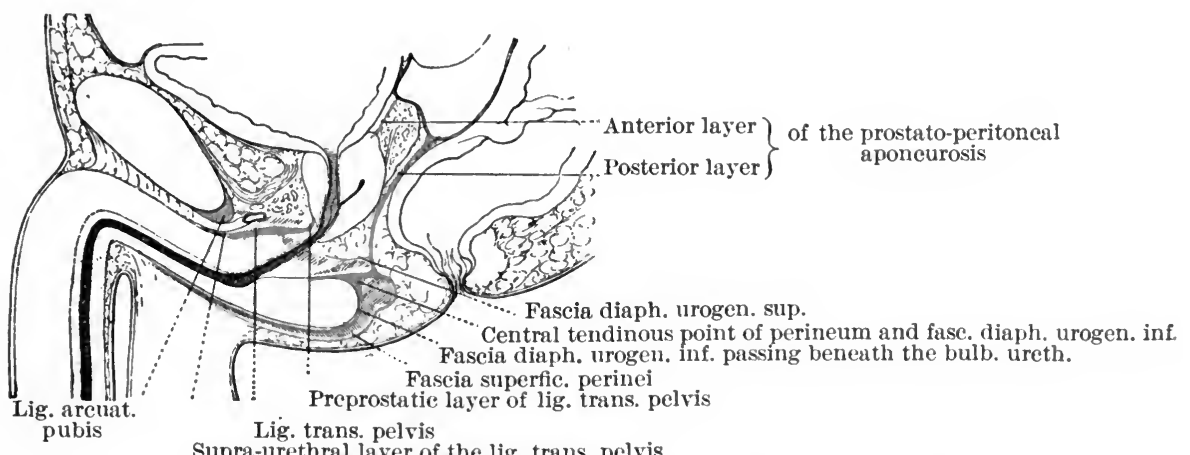

Supra-urethral layer of the lig. trans. pelvis

Pelvic and perineal fasciae; sagittal section. (From Poirier et Charpy, Traité d'Anat. hum., Paris, 1901, t. v. p. 216, Fig. 144.)

to the pudic artery and the dorsal nerve of the penis. On the other side the dissection may be made without the removal of the muscles. Use models to complete the study. Examine the 
urogenital diaphragm (diaphragma urogenitale) (O. T. triangular ligament). This is also known as the "middle perineal aponeurosis.'” (Vide Spalteholz, Figs. 657, 658, 673.)

FIG. 228.

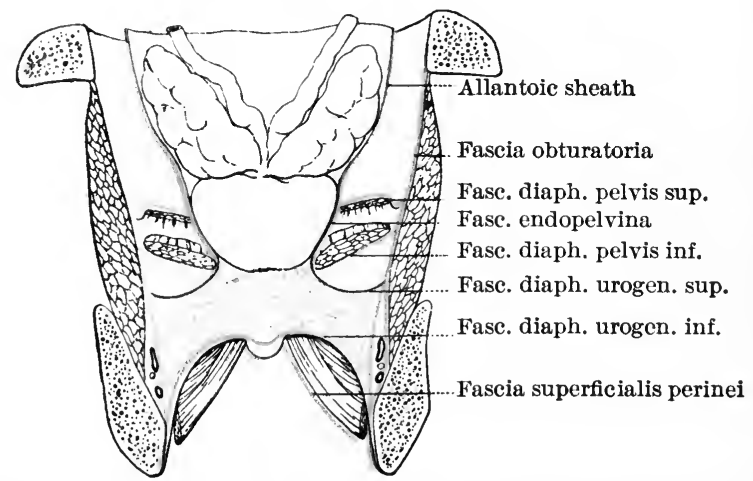

The perineal aponeuroses; frontal section. (From Poirier et Charpy, Traité d'Anat. hum., Paris, 1901, t. v. p. 217, Fig. 146.)

Note carefully the attachments of the following layers of fascia and their relations to neighboring parts.

(a) Inferior fascia of urogenital diaphragm (fascia diaphragmatis urogenitalis inferior) (O. T. superficial layer of triangular ligament).

(b) Superior fascia of urogenital diaphragm (fascia diaphragmatis urogenitalis superior) (O. T. deep layer of triangular ligament).

What is the relation posteriorly of these two layers of fascia to the fascia of Colles?

The superior fascia is composed of three lamellae. In order from the pubic arch backward note-

(ba) Areuate ligament of pubis (Lig. arcuatum pubis). Note the relation of the dorsal vein of the penis ( $V$. dorsalis penis) and the dorsal nerve of the penis ( $N$. dorsalis penis) to this ligament. (Vide Spalteholz, Fig. 673.)

(bb) Transverse ligament of pelvis (Lig. transversum pelvis) ( $O$. T. median puboprostatic ligament of Krause). It is a fibrous band extending between the ischiopubic rami immediately behind the dorsal vein of the penis and in front of the urethra. Note the following aponeurotic divisions.

(1) The anterior division passes anteriorly beneath the dorsal vein and is attached to the fibrous tunic of the corpora cavernosa, forming a union between these two bodies. It is called the "supra-urethral part of the middle aponeurosis."

(2) The posterior division passes in front of the urethra and the prostate, fusing with the proper capsule of the latter. It is known as the "preprostatic layer." 
(bc) The third lamella covers the deep transverse muscle of the perineum superiorly. It extends from the urethra in the median line to the line extending between the tuber ischiadicum of each side posteriorly. The anterior border is intimately adherent to the urethra, while the posterior gives off processes which are united to the inferior aponeurosis of the levator ani muscle. It is also intimately adherent to the central tendinous point of the perineum.

Fig. 229.

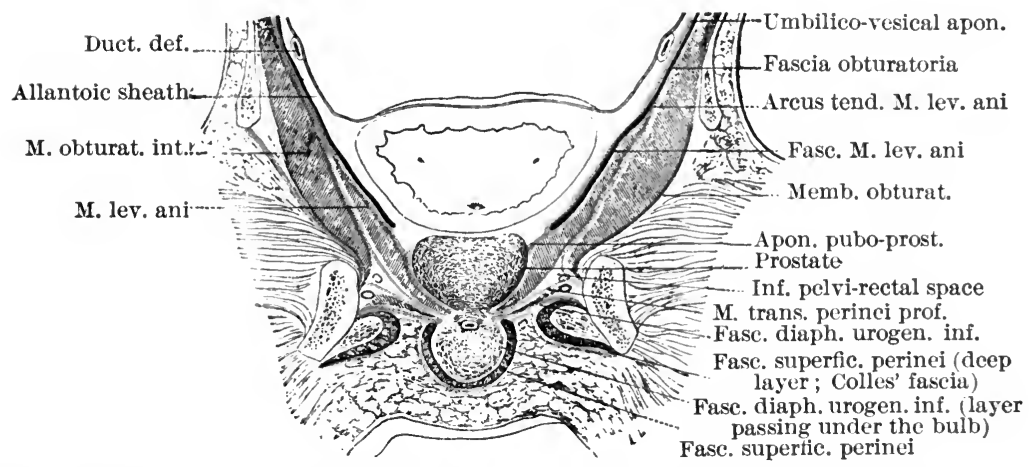

The fasciae of the pelvic floor and of the perineum. Frontal section through the bulbus urethrae.

(From Poirier et Charpy, Traité d'Anat. hum., Paris, 1901, t. v., fasc. 1, p. 218, Fig. 147.)

Remove the inferior fascia of the urogenital diaphragm (fascia diaphragmatis urogenitalis inferior) on one side and note the contents of the " middle perineal compartment" (O. T. space between the two layers of triangular ligament).

Muscles. (Vide Figs. 226 and 229, and Spalteholz, Fig. 673.)

(a) Deep transverse muscle of perineum (M. transversus perinei profundus). What is the general direction of the fibres composing this muscle? What is the relation to the perineal body? (Vide Poirier et Charpy, t. v. p. 202.)

(b) Sphincter muscle of the membranous urethra (M. sphincter urethrae membranaceae). It is also known as the "striated sphineter of the urethra." Consult Poirier et Charpy, t. v. p. 204, concerning the disposition of the fibres forming this muscle.

Arteries. (Vide Fig. 224, and Spalteholz, Fig. 473.)

(a) Artery of the penis (A. penis).

(aa) Artery of bulb of urethra (A. bulbi urethrae).

(ab) Urethral artery (A. urethralis).

(ac) Deep artery of penis (A. profunda penis).

(ad) Dorsal artery of penis (A. dorsalis penis).

Determine the exact relation of each artery. Dissect out the veins accompanying each, carefully noting relations and anastomoses. What is the course of the dorsal vein of the penis ( $V$. dorsalis penis), and into what does it empty? 
Nerves.

(a) Dorsal nerve of penis (N. dorsalis penis). (Vide Toldt, Fig. 1291.) Do any branches of this nerve pass to the muscles of the middle perineal compartment?

Examine also-

(a) Bulbo-urethral glands (glandulae bulbo-urethrales [Cowperi]).

(aa) Body of gland (corpus glandulae bulbo-urethralis).

(ab) Excretory duct (ductus excretorius).

(b) Membranous part of urethra (pars membranaceae urethrae).

Observe that this is the most fixed portion of the urethra.

Note that the rectum passes posteriorly and the urethra anteriorly. A triangle is thus formed, the base of which is the skin. This is known as the " recto-urethral triangle."

For a description of this triangle and its contents see Poirier et Charpy, t. v. p. 140.

The dissection may now be completed on the other side. Leave the muscles intact, preserving relations.

\section{UROGENITAL REGION (REGIO UROGENITALIS) IN THE FEMALE.}

Before proceeding to the dissection, review the embryology of the external genital parts. (Vide Kollmann, Lehrbuch der Entwickelungsgeschichte des Menschen, pp. 434, 435.) What are the genital ridge, the genital folds, the genital eminence, and the urogenital sinus? Observe the following:

\section{External Genital Parts in Female (Partes genitales externae), (Fig.} 230.)

(a) Pubic eminence (mons pubis).

(b) Vulva (pudendum muliebre).

(c) Labium majus of vulva (labium majus pudendae).

(d) Anterior labial commissure (commissura labiorum anterior).

(e) Posterior labial commissure (commissura labiorum posterior).

(f) Frenulum of pudendal labia (frenulum labiorum pudendi).

(g) Pudendal slit (rima pudendi).

(h) Navicular fossa (fossa navicularis [vestibuli vaginae]).

(i) Labium minus of vulva (labium minus pudendi).

(j) Vestibule of vagina (vestibulum vaginae).

(k) Orifice of vagina (orificium vaginae).

(l) Clitoris (clitoris).

(la) Body of clitoris (corpus clitoridis).

(lb) Glans of clitoris (glans clitoridis).

(lc) Prepuce of clitoris (praeputium clitoridis).

(ld) Frenulum of clitoris (frenulum clitoridis). 
(m) External orifice of urethra (orificium urethrae externum).

(n) Hymen (hymen [femininus]).

(o) Hymenal caruncles (carunculae hymenales).

Notice the characteristics of the external genitalia in different subjects. How do the nulliparous differ from the multiparous? Note the homologies existing between the female and the male genitals and tabulate these in your drawing-books.

FIG. 230.

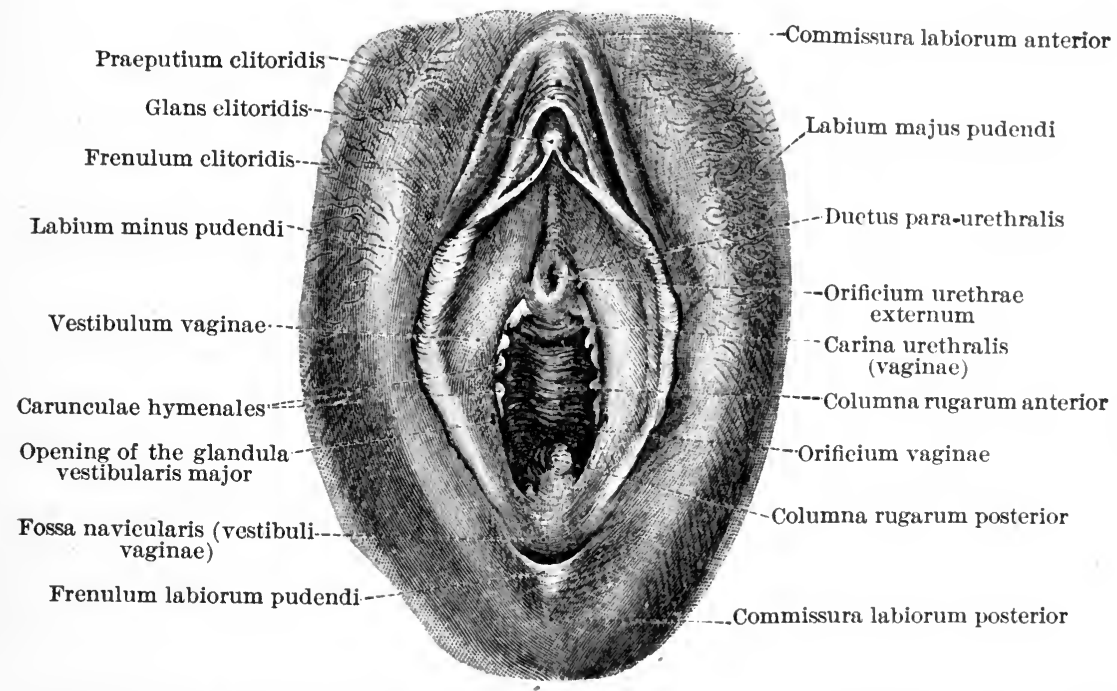

The female external genitalia. The labia majora have been drawn apart and the pudendal cleft thereby opened wide. (After Toldt, Anat. Atlas, Wien, 1900, 2 Aufl., p. 498, Fig. 855.)

Distend the rectum with cotton and close the anal and vulvar orifices by sutures. Make the following incisions (cf. Fig. 221) :

(1) A transverse incision passing from the tuber ischiadicum of one side to that of the other.

(2) An incision in the median line encireling the labii majora and the anal orifice, passing from the mons pubis in front to the tip of the coceyx behind.

Reflect the four flaps.

\section{Superficial Perineal Fascia (Fascia perinei superficialis) and Con- tents of "Superficial Perineal Compartment."}

Note the general characteristics of this fascia and compare it with the fascia in the male. Note the two layers, superficial and deep. Determine the exact disposition of each. What is the 
attachment of the deep layer? Does it acquire smooth muscle fibres in the labium majus to form a tunica dartos? Study-

Arteries.

(a) Artery of perineum (A. perinei). Trace this artery forward to the labia majora and dissect out-

(aa) Posterior labial arteries (Aa. labiales posteriores).

Look for a small artery passing into the superficial transverse muscle of the perineum, the "transverse perineal artery."

Nerves. (Fig. 232.)

Fig. 231.

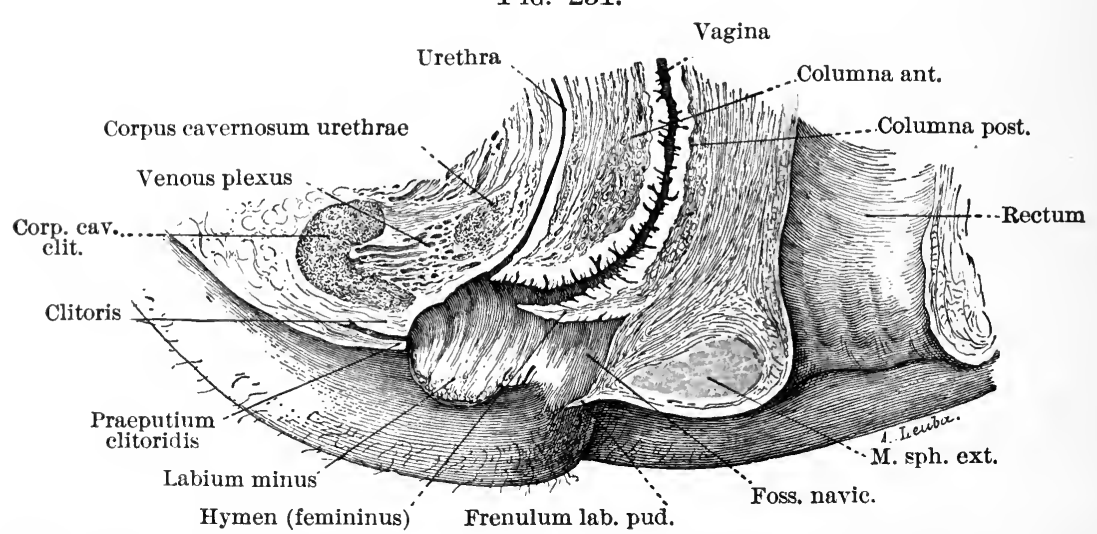

Sagittal section through the vulva and vagina to show the lateral walls of the vestibule. (From Poirier et Charpy, Traité d'Anat. hum., Paris, 1901, t. v. p. 558, Fig. 382.)

(a) Nerve of perineum (N. perinei).

(aa) Posterior labial nerves (Nn. labiales posteriores).

(b) Perineal branches of posterior cutaneous nerve of thigh ( $0 . \mathrm{T}$. Lymphatics. small sciatic).

Determine the course of the lymphaties found in this region, also the lymph-nodes with which they communicate. (Vide Toldt, Fig. 1093.)

Remove this fascia and expose to view the contents of the " superficial perineal compartment"' (between fascia perinealis and diaphragma urogenitale). (Cf. Figs. 227, 229.)

Clean off the fascia from the muscles, determine the form, position, origin, insertion, action, and nerve-supply of the following :

Muscles. (Vide Spalteholz, Fig. 669.)

(a) Superficial transverse muscle of perineum ( $M$. transversus perinei superficialis). This muscle is not constant. It is found in the female in a little more than thirty per cent. of the eases. In the male it is more frequent. 
(b) Bulbocavernous muscle (M. bulbocavernosus) (O. T. sphincter vaginae). This muscle extends from the perineal septum behind to the clitoris in front. Note its homologue in the male.

(c) Ischiocavernous muscle (M. ischiocavernosus) (O. T. erector elitoridis).

FIG. 232.

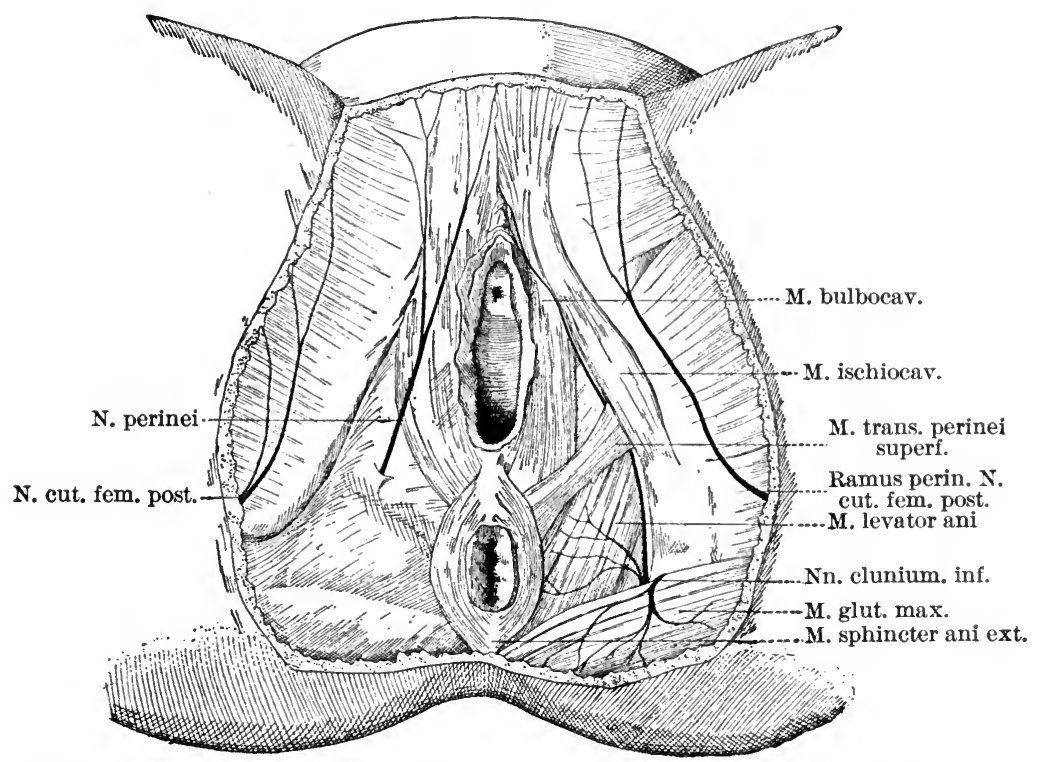

Nerves of the perineum in the female (after Paulet.) (From Poirier et Charpy, Traité d'Anat. hum., Paris, 1899, t. iii., 3, p. 1156, Fig. 594.)

\section{What is the perineal body?}

Detach the M. bulbocavernosus and reflect it forward. The M. transversus perinei superficialis and M. ischiocavernosus may also be detached. Study also-

(a) Bulb of the vestibule (bulbus vestibuli).

The bulb will be found just beneath the M. bulbocavernosus. Note its relation to the labia minora and urethra. Trace these anteriorly and note that the two oblong bodies forming the bulb are united below the elitoris by an intermediate venous plexus (pars intermedia of Kobelt).

(b) Larger vestibular gland of Bartholin (glandula vestibularis major [Bartholini]). (Vide Spalteholz, Fig. 668.)

Note the relation of this gland to the bulbous vestibule and the M. bulbocavernosus. Dissect out its excretory duct and determine where it opens. 


\section{Clitoris.}

The sutures inserted to close the vulvar cleft may now be removed. Remove the mucous membrane from the clitoris, leaving intact any vessels and nerves found. Study-

(a) Crura of clitoris (crura clitoridis). Determine exactly their attachments.

(b) Body of clitoris (corpus clitoridis).

(ba) Cavernous bodies of clitoris (corpora cavernosa clitoridis).

(bb) Septum of cavernous bodies (septum corporum cavernosorum).

(bc) Fascia of clitoris (fascia clitoridis).

(c) Glans of clitoris (glans clitoridis). Note that the glans is not a part of the corpora cavernosa, but that it is quite independent of these bodies.

Urogenital Diaphragm or Trigone (Diaphragma [s. Trigonum] urogenitale).

Remove the crura clitoridis from their bony attachments, avoiding injury to the vessels and nerves in relation to them, and study the "urogenital diaphragm." (Vide Spalteholz, Figs. 673-675.) Make use of models to complete the study. Examine-

(a) Inferior fascia of urogenital diaphragm (fascia diaphragmatis urogenitalis inferior) ( O. T. anterior layer of triangular ligament).

Cautiously remove this inferior layer of fascia and study the contents of the " middle perineal compartment," as follows :

Muscles.

(a) Deep transverse muscle of perineum ( $M$. transversus perinei profundus).

(b) Sphincter muscle of membranous urethra (M. sphincter urethrae membranaceae).

Arteries. (Vide Spalteholz, Fig. 474.)

(a) Artery of clitoris (A. clitoridis).

(aa) Artery of bulb of vestibule (A. bulbi vestibuli [vaginae]).

(ab) Urethral artery (A. urethralis).

(ac) Deep artery of clitoris (A. profunda clitoridis).

(ad) Dorsal artery of clitoris (A. dorsalis clitoridis).

Note that the two latter are given off after the A. elitoridis has pierced the inferior fascia of the urogenital diaphragm. Find the internal pudendal veins (Vv. pudendae internae).

\footnotetext{
${ }^{1}$ Sometimes called the "fascia trigoni urogenitalis inferior."
} 
Nerves.

(a) Dorsal nerve of clitoris ( $N$. dorsalis clitoridis).

Determine the exact course of this nerve. What relation does it bear to the dorsal artery of the clitoris and to the dorsal vein? What relation does the latter vein bear to the ligamentum arcuatum pubis and the ligamentum transversum pelvis? Demonstrate the presence of a part of the glandulae vestibulares majores in Urethra. the " middle compartment of the perineum."

Note the general direction and extent of the urethra in the female. What is its relation to the anterior vaginal wall? (Vide Figs. 231, 291, and 293.)

The superior boundary of the diaphragma urogenitale is formed by the superior fascia of the urogenital diaphragm (fascia diaphragmatis urogenitalis superior) (O. T. deep or posterior layer of triangular ligament).

Note that these (the superior and inferior) layers fuse posteriorly and become continuous with the deep layer of the superficial fascia of the perineum, or "Colles' fascia." Anteriorly they also fuse to form the transverse ligament of the pelvis (ligamentum transversum pelvis). The dorsal vein of the clitoris separates the latter from a ligament just below the pubis, which is known as the arcuate ligament of the pubis (ligamentum arcuatum pubis).

\section{ANAL REGION (REGIO ANALIS) AND DIAPHRAGM OF PELVIS (DIAPHRAGMA PELVIS) IN BOTH SEXES.}

Review at this stage of the dissection the general characteristics and disposition of the fascia in this region. After having reviewed this, remove it by blunt dissection.

\section{External Sphincter Muscle of Anus (M. sphincter ani externus).}

(Figs. 222-224, 231, 273, 289.)

Clean this muscle, noting carefully its form, position, origin, insertion, action, and innervation, and dissect out the following layers, proceeding from without inward:

(a) External layer ("superficial sphineter" of Cruveilhier, the "skin muscle" of Luschka). It is inserted into the skin about the anus.

(b) Anococeygeal layer, formed by the fibres arising from the coccyx and the ligamentum anococeygeum, encireling the anus and inserted 
anteriorly into the central tendinous point of the diaphragm, becoming continuous with some of the fibres of the M. bulbocavernosus.

(c) Internal circular layer, made up of annular fibres surrounding the terminal part of the rectum.

Note that the external sphincter is made up of two symmetrical halves.

Clean out the fat of the ischiorectal fossa, carefully preserving the structures found therein. Proceed to the study of it, noting exactly the position of its boundaries.

\section{Ischiorectal Fossa (Fossa ischiorectalis).}

(Figs. 224, 232, 274, 278.)

(a) Levator ani muscle ( $M$. levator ani).

(b) Obturator internus muscle (M. obturator internus).

(c) Inferior fascia of pelvic diaphragm (fascia diaphragmatis pelvis inferior) (O. T. anal fascia).

(d) Obturator fascia (fascia obturatoria).

At the junction of $(c)$ and $(d)$ as seen from below, in the angle between them is seen the tendinous arch of the levator ani muscle (arcus tendineus $M$. levatoris ani) (O. T. white line of pelvic fascia).

(e) Posterior border of urogenital diaphragm (diaphragma urogenitale).

( $f$ ) Gluteus maximus muscle (M. glutaeus maximus).

(g) Sacrotuberous ligament (ligamentum sacrotuberosum) (O. T. great sacrosciatic ligament).

After having located accurately the boundaries of this fossa, proceed to the study of its contents :

(a) Inferior hemorrhoidal artery (A. haemorrhoidalis inferior). (Vide Spalteholz, Fig. 473.)

Determine the course of the inferior hemorrhoidal veins $(V v$. haemorrhoidales inferiores). What is the plexus haemorrhoidalis? With what vessels do the above anastomose?

(b) Inferior hemorrhoidal nerve ( $N$. haemorrhoidalis inferior).

(c) Anococeygeal nerves ( $N n$. anococcygei) (O. T. perineal branches of fourth sacral nerve).

Trace the arteria haemorrhoidalis inferior back to the internal pudendal artery ( $A$. pudenda interna), its parent stem. Note that it is accompanied by the $V$. pudenda interna and the $N$. pudendus. These three structures run in the "canal of Alcock," which is formed by the fusion of the fascia obturatoria with the falciform process of the ligamentum sacrotuberosum. 


\section{ANTERIOR ABDOMINAL WALL.}

Inflate the abdominal cavity (cavum abdominis) by means of an aspirating needle and bicycle pump, and make the following incisions through the skin only (vide Fig. 7 ) :

(1) Along the anterior median line from the level of the tip of the xiphoid process to the mons pubis; carry the knife around the umbilicus, leaving it intact.

(2) From the upper limit of the first, transversely around the body, as far back as possible.

(3) From the lower limit of the first to the tuberculum pubicum, thence over the ligamentum inguinale to the spina iliaca anterior superior and along the iliac crest as far back as possible.

Reflect the flaps so formed, taking no fat with the skin, but leaving intact the layer beneath (fascia superficialis) and the vessels and nerves which ramify in it.

\section{Superficial Fascia (Fascia superficialis).}

Observe the distribution of fat, especially in the hypogastric region; the dense white band of tissue (linea alba) in the anterior median line; the arrangement of the superficial fascia in two layers, especially in the hypogastric region; superficially the fascia of Camper, and more deeply the fascia of Scarpa. Dissect out the structures in the superficial fascia, preserving them carefully:

Arteries. (Vide Fig. 9.)

(a) Superficial circumflex artery of ilium (A. circumflexa ilium superficialis) (O. T. superficial circumflex iliac artery).

(b) Superficial epigastric artery (A. epigastrica superficialis).

(c) External pudendal arteries (Aa. pudendae externae) (O. T. external pudic arteries).

Veins.

(a) Parumbilical vein (V. parumbilicalis).

(b) Thoraco-epigastric vein ( $V$. thoraco-epigastrica).

(c) Superficial epigastric vein ( $V$. epigastrica superficialis).

(d) External pudendal veins ( $V v$. pudendae externae).

(e) Superficial circumflex vein of ilium (V. circumflexa ilium superficialis).

Nerves. ${ }^{1} \quad$ (Vide Fig. 8.)

(a) Anterior cutaneous rami of intercostal nerves (rami cutanei anteriores).

${ }^{1}$ Regarding the variations in the nerves of the abdominal wall, see the paper by Professor Bardeen in the American Journal of Anatomy, vol. i., No. 2, p. 203. 
(b) Anterior cutaneous ramus of iliohypogastric nerve (ramus cutaneus anterior $N$. iliohypogastrici) (O. T. hypogastric branch).

(c) Ilio-inguinal nerve ( $N$. ilio-inguinalis).

(d) Lateral cutaneous nerves (rami cutanei laterales of $\mathrm{Nn}$. intercostales).

(e) Lateral cutaneous ramus of twelfth thoracic nerve (ramus cutaneus lateralis $N$. thoracalis $\mathrm{XII}$.) (O. T. iliac branch of last dorsal nerve).

(f) Lateral cutaneous ramus of iliohypogastric nerve (ramus cutaneus lateralis $N$. iliohypogastrici) (O. T. iliac branch of iliohypogastric).

\section{Deep Fascia (Fascia profunda).}

Remove the superficial and expose the deep fascia. Compare the latter with the superficial. Does it contain fat? Does it contain blood-vessels and nerves?

\section{Muscles of Abdomen (Musculi abdominis). (Cf. Fig. 6.)}

Remove the deep fascia, keeping the muscles tense and using a belly-bladed scalpel in the dissection of the fibres. Expose the underlying muscles. Make drawings of each muscle as exposed. Study its form, position, origin, insertion, action, and nervesupply.

(a) External oblique muscle of abdomen ( $M$. obliquus abdominis externus).

Find the lumbar triangular space (trigonum lumbale [Petiti]). What is the relation of the muscle to the inguinal ligament of Poupart (ligamentum inguinale [Pouparti])? The reflection of this ligament should be carefully studied. Study especially Gimbernat's ligament (ligamentum lacunare [Gimbernati]), the reflex ligament of Colles (ligamentum reflexum [Collesi]) (O. T. triangular fascia). Both these structures will be frequently referred to. Note the opening just lateral to the tuberculum pubicum, the "subcutaneous inguinal" or " external abdominal" ring (annulus inguinalis subcutaneus). What structures pass through it in the male? In the female? (See Spalteholz, vol. ii., Fig. 317.)

In connection with the annulus inguinalis subcutaneus, study-

(aa) Superior pillar (crus superius).

$(a b)$ Inferior pillar (crus inferius).

Dissect out carefully the fibres passing between the two crura (fibrae intercrurales); expose the fascia descending 
upon the spermatic cord from this muscle; it is the intercolumnar or external spermatic fascia (fascia spermatica externa).

FIG. 233.

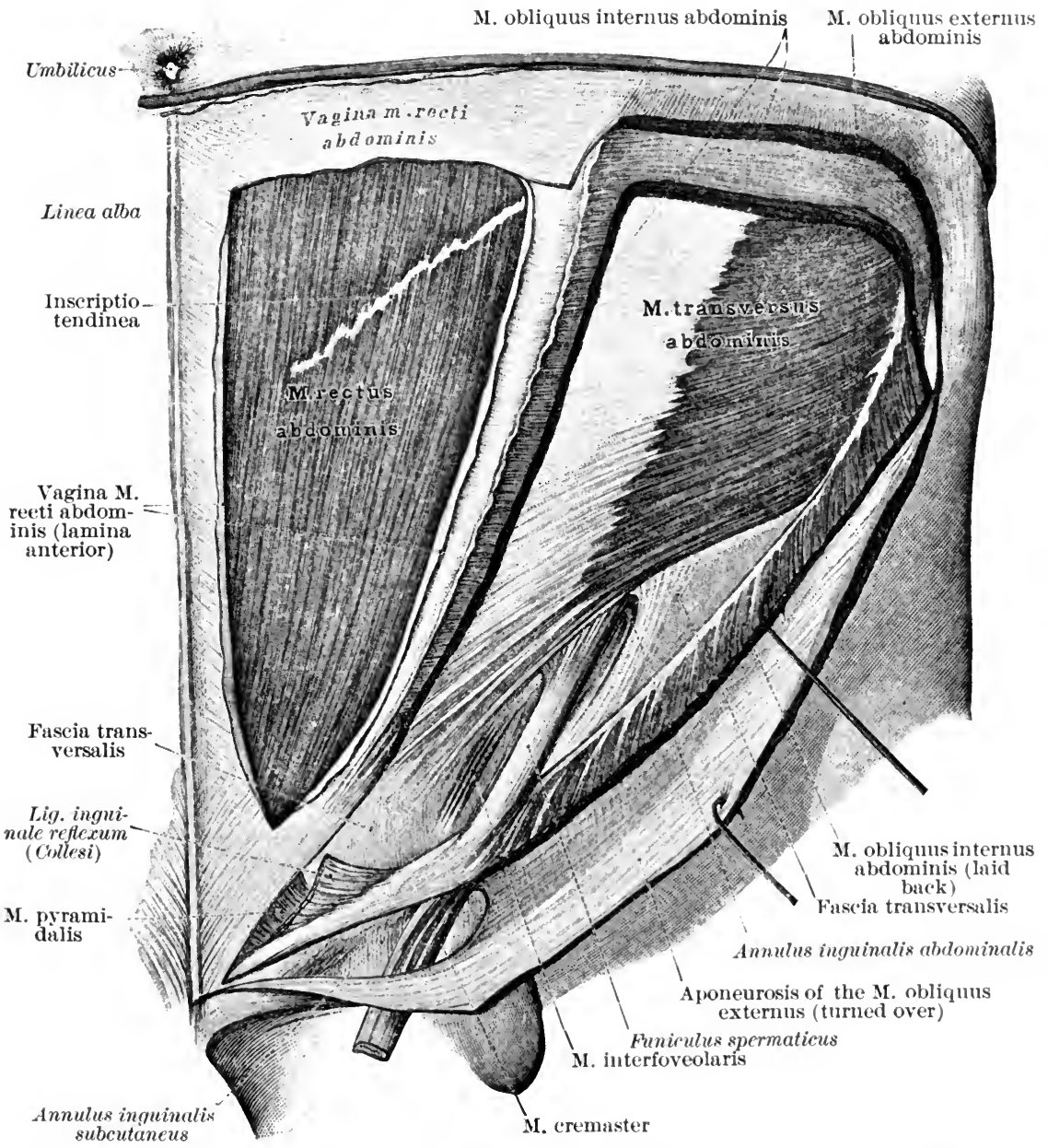

The lateral part of the inguinal eanal, from the abdominal inguinal ring to the passage of the spermatic cord through the II. obliquus abdominis, shown by mrtial removal of the M. obliquus abdominis. The II. interfoveolaris lies on the anterior surface of the Lig. interfoveolare [Hesselbachi]. (After Toldt, Anat. Atlas, Wien, 1900, 2 Aufl., p. 369, Fig. 610.)

Divide the attachment of the M. obliquus externus abdominis along a line one centimetre from the crista iliaca. From the spina iliaca anterior superior carry the incision medialward, then upward in the line along which the muscle-fibres pass into the aponeurosis. Reflect the muscle dorsalward and cranial- 
ward, noting its digitations and the muscles to which it is related. (Cf. Spalteholz, Fig. 317.) Examine the course of the N. ilioinguinalis.

(b) Internal oblique muscle of abdomen (M. obliquus internus $a b$ dominis).

What is meant by the inguinal aponeurotic falx (falx aponeurotica inguinalis) (O. T. conjoined tendon)?

On the right side only, divide this muscle where the fibres pass over into the aponeurosis and also along a line five millimetres above the inguinal ligament and crista iliaca. Reflect the muscle dorsalward and cranialward, noting carefully the vessels and nerves on its deep surface. What are they? (Cf. Spalteholz, Figs. 319 and 324.) Note where the lateral cutaneous rami of the intercostal nerves perforate this muscle. What is the relation of the M. cremaster to the M. obliquus internus abdominis?

(c) Transverse muscle of abdomen (M. transversus abdominis).

Note carefully the relation of the falx inguinalis to the annulus inguinalis subcutaneus. Note where the fibres go over into the aponeurosis, the semilunar line (linea semilunaris [Spigeli]).

On each side make an incision through the aponeuroses parallel to the linea alba and distant three centimetres from it, extending throughout the entire length of the M. rectus abdominis. Reflect the flaps of the aponeuroses lateralward and medialward respectively. Difficulty in reflecting is experienced along the transverse line, owing to attachment to the subjacent muscle. These transverse lines are the inscriptiones tendineae. How many of them are there? Where are they situated? What is their morphological significance?

(d) Rectus ("straight") muscle of abdomen (M. rectus abdominis).

Study its sheath (vagina recti abdominis). Note the disposition in its upper two-thirds and in the lower third. What theories have been advanced to explain this arrangement? How is the semicircular line of Douglas (linea semicircularis [Douglasi]) formed?

Note the vessels and nerves which pass through the posterior layer of the sheath; also the artery passing from below upward (A. epigastrica inferior) and the one passing from above downward (A. epigastrica superior). Do these anastomose in the muscle?

(e) Pyramidal muscle ( $M$. pyramidalis).

What is its relation to the M. rectus abdominis and its sheath? What is the phylogenetic significance of this muscle?

This muscle is frequently absent, and also varies greatly in length, sometimes extending as high as the umbilicus. 
White Line (Linea alba).

What aponeuroses enter into the formation of the linea alba? Between what points does it extend? Note that it is broad in the upper two-thirds and rapidly diminishes in breadth in the lower third. Do you find foramina in the linea alba above the umbilicus?

Make a drawing of the dissection at this stage.

Transversal Fascia (Fascia transversalis) (O. T. Transverse Fascia).

On the right side cut through the M. transversus along the linea semilunaris and carefully reflect this muscle lateralward. Note under it two layers: externally, the fascia transversalis; internally, the peritoneum. These are separated by "extraperitoneal fatty tissue.' Trace the fascia inferiorly. Note its

FIG. 234.

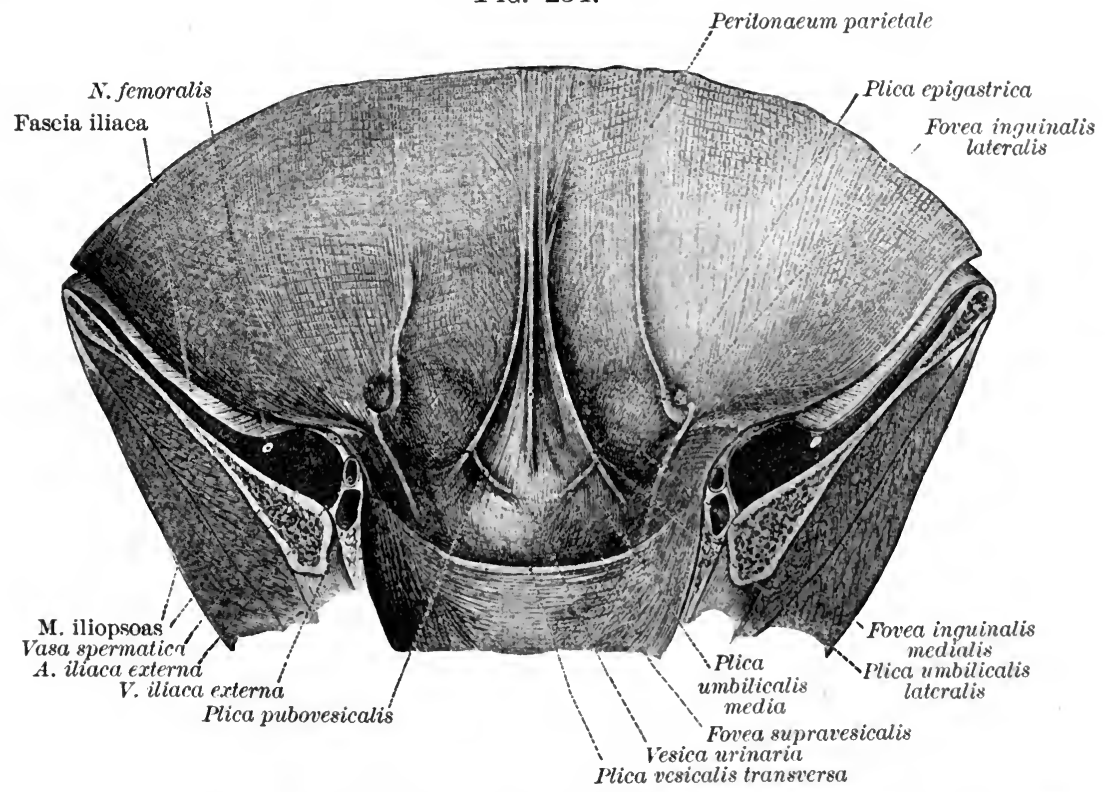

Frontal section of the lower part of the trunk to show the lower part of the parietal peritoneum and its folds. Seen from behind. (After Toldt, Anat. Atlas, Wien, 1900, 2 Aufl., p. 370, Fig. 611.)

relation to the ligamentum inguinale, to the crista iliaca, and medialward to the linea alba.

On the left side, from a point immediately above the umbilicus, make two incisions, one passing to the spina iliaca interior superior, the other to the right of the umbilicus and two centimetres to the right of the linea alba, down to the ramus supe- 
rior ossis pubis. Reflect this flap laterally and downward. Note the fan-like attachment of the cord-like linea alba to the ossa pubis (this is the adminiculum lineae albae).

\section{Internal Surface of Anterior Abdominal Wall. (Fig. 234.)} Note-

(a) A fibrous eord (urachus), passing from the umbilicus downward to the urinary bladder (vesica urinaria).

(aa) Middle umbilical fold of peritoneum, over this (plica umbilicalis media).

(b) A second cord, passing from the umbilicus to the lateral margin of the obliterated umbilical artery. (Spalteholz, vol. ii., Fig. 511.)

(ba) Lateral umbilical fold of peritoneum, over this (plica umbilicalis lateralis).

(c) Band extending from linea semicireularis [Douglasi] to a point opposite the middle of the inguinal ligament. This is the A. epigastrica inferior and accompanying vein.

(ca) Epigastric fold (plica epigastrica).

(d) The three foveae thus formed:

(da) Medial supravesical fovea (fovea supravesicalis). By which plicae is this fovea limited?

(db) Medial inguinal fovea (fovea inguinalis medialis).

(dc) Lateral inguinal fovea (fovea inguinalis lateralis). Note especially its relation to the plica epigastrica. The deepest part of this fovea is a depression in the peritoneum indieating the position of the abdominal inguinal ring (annulus inguinalis abdominis) ( $\mathrm{O} . \mathrm{T}$. internal abdominal ring), the beginning of the inguinal canal. In what structure is this annulus formed?

What is Hesselbach's triangle? Note carefully its boundaries.

\section{Inguinal Canal (Canalis inguinalis). (Vide Fig. 233.)}

Dissect the peritoneum away carefully from the anterior wall of the abdomen. Examine-

Posterior wall of inguinal canal.

Note the following structures:

(a) Thickened fold of fascia forming the lower and medial boundary of the annulus inguinalis abdominis (ligamentum interfoveolare) (O. T. Hesselbach's ligament).

(b) Thin layer of fascia transversalis.

(c) Interfoveolar muscle (M. interfoveolaris) (O. T. Luschka's muscle).

(d) Medialward the inguinal aponeurotic falx (falx aponeurotica inguinalis) (O. T. conjoined tendon). What muscles enter into the formation of it? Note earefully its insertion. Pay particular attention to its relation to the annulus inguinalis subeutaneus. 
(e) Reflex ligament of Colles (ligamentum reflexum [Collesi]) (O. T. triangular fascia of the abdomen). From which musele is it derived? This ligament is often referred to as the crus posterius. Roof of inguinal canal.

What structures enter into its formation?

Floor of inguinal canal.

How is it formed? What are the contents of the canal in the male? In the female? (Vide Fig. 235.)

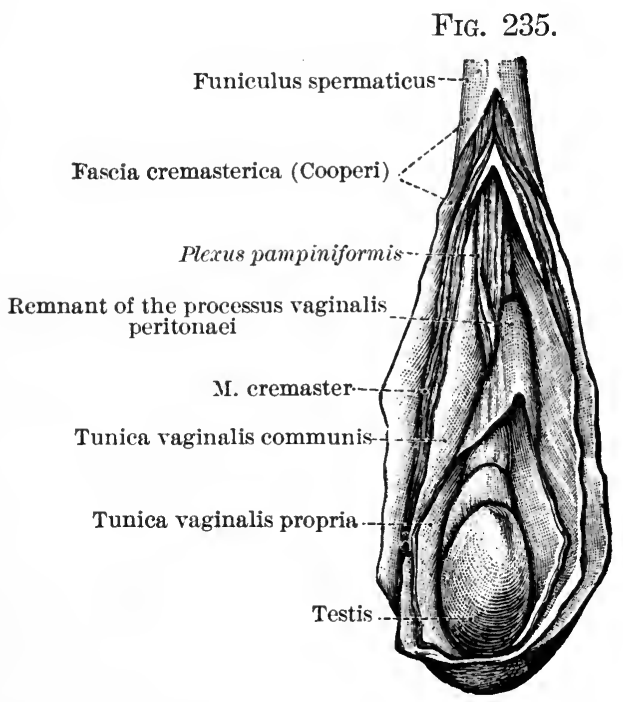

The spermatic cord of a boy two months old, with the coverings of the cord opened up and showing the remnant of the vaginal process of the peritoneum. (After Toldt, Anat. Atlas, Wien, 1900, 2 Aufl., p. 507, Fig. 878.)

\section{Spermatic Cord (Funiculus spermaticus).}

Each covering should be carefully dissected out and traced back to the muscle or fascia from which it is derived.

(1) External spermatic fascia (fascia spermatica externa).

(2) Cremasteric fascia (fascia cremasterica [Cooperi]).

(3) Cremasteric muscle (M. cremaster).

Dissect out carefully these muscular fibres and determine accurately their origin and insertion. Consult Poirier et Charpy, t. ii. p. 451 , concerning the different views held regarding the origin and significance of the M. cremaster.

What is its nerve supply and action? What part does it play in the cremasteric reflex?

(4) Vaginal process of fascia transversalis (processus vaginalis fasciae transversalis) (O. T. infundibuliform fascia).

(5) What is the fate of the peritoneal process that descended into the scrotum (processus vaginalis peritonaei)? What is the tunica vaginalis communis [testis et funiculi spermatici])? 
Examine the excretory duct of the testicle (ductus deferens). Note its consistence. Is it resistant to the touch? Determine its length. What is its course and what are its relations to the important structures with which it is associated?

Arteries.

(1) Internal spermatic artery (A. spermatica interna).

(2) External spermatic artery (A. spermatica externa) (O. T. cremasteric artery).

(3) Deferential artery (A. deferentialis) (O. T. artery of the vas deferens).

Determine from what vessel each is derived, the relation that they bear to each other and to the component parts of the funiculus spermaticus. Cross-sections should be used to determine these relations. (See Poirier et Charpy, t. v., Fig. 236.) Do these arteries anastomose with one another?

Veins.

Observe an anterior and a posterior group. (Cf. Poirier et Charpy, t. v., Fig. 238.)

What is the pampiniform plexus (plexus pampiniformis)? What is the general course of the spermatic veins ( $V v$. spermaticae)? Into what veins do they empty? Is there any difference on the right and left sides?

Nerves.

(a) External spermatic nerve (N. spermaticus externus) (O. T. genital branch of genito-crural).

(b) Deferential plexus (plexus deferentialis). Note that the ilio-inguinal nerve ( $N$. ilio-inguinalis) is found in the canal, but that it is not a constituent part of the spermatic cord.

Lymphatics.

Whence do they come and to what lymph-glands do they go?

\section{Round Ligament of Uterus [in Female] (Lig. teres uteri). (Vide Fig. 236.)}

Coverings.

The coverings should be compared with those of the funiculus spermaticus in the male. Note differences. What is the canal of Nuck?

Arteries.

(a) External spermatic artery (A. spermatica externa), a branch of the A. epigastrica inferior. [Later the course of the $A$. ovarica, which corresponds to the arteria spermatica interna, will be studied.] Dissect out the terminal branches of this artery in the mons pubis and the labium majus.

(b) Artery of round ligament, a small artery occasionally found within the cellular tissue of the round ligament, which anastomoses with Veins. a branch of the A. uterina.

Many veins accompanying the round ligament appear mostly on the surface. They communicate with the uterine veins, the veins of the labia majora and clitoris. 
Nerves.

(a) External spermatic nerve (N. spermaticus externus), as in male.

(b) Sympathetic nerves from plexus uterinus.

Lymphatics:

FIG. 236.

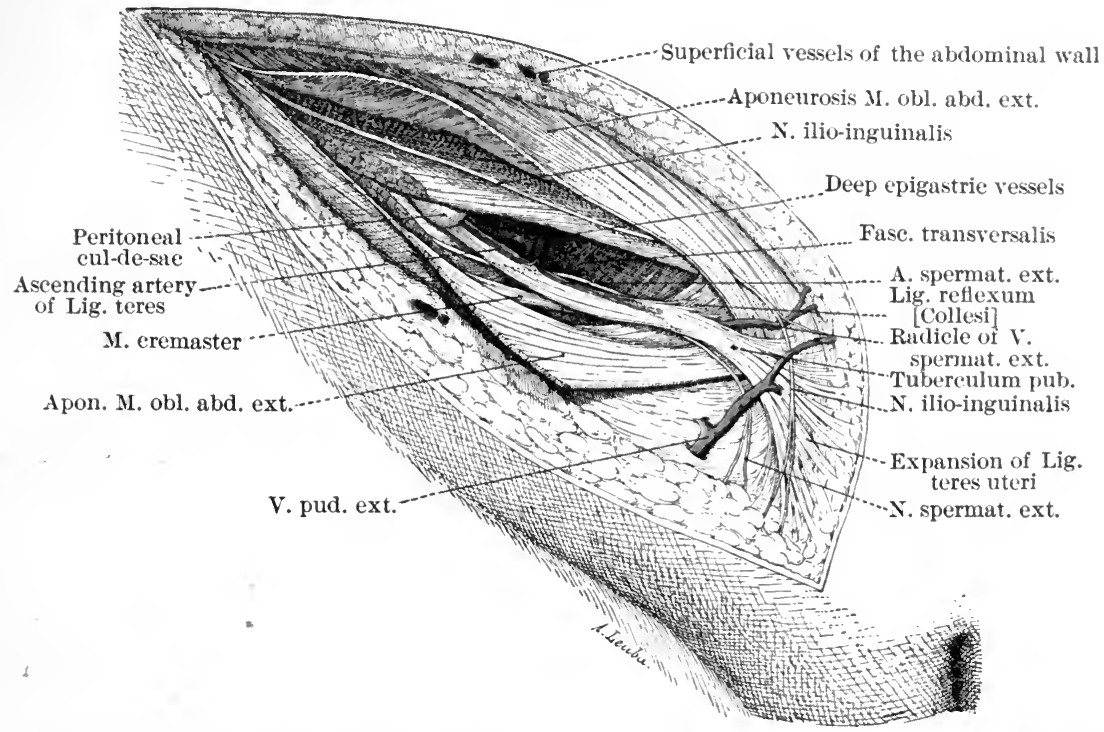

The round ligament in its course through the eanalis inguinalis. The ligament is drawn slightly downward. (In greater part after Waldeyer.) (From Poirier et Charpy, Traité d'Anat. hum., Paris, 1901, t. ii. p. 436, Fig. 315.)

\section{Testis (Testicle). (Vide Figs. 237, 239, 240.)}

Note its position in the scrotum and the relation to it of the proper vaginal tunic (tunica vaginalis propria testis). Distinguish a parietal layer (lamina parietalis) and a visceral layer (lamina visceralis). Where does the testis originate and how does it reach its position in the scrotum? Where may it be arrested in its descent and what is the resultant condition?

Cut through the lamina parietalis along its anterior aspect. Examine-

(a) Superior extremity of testicle (extremitas superior).

(b) Inferior extremity (extremitas inferior).

(c) Lateral surface (facies lateralis).

(d) Medial surface (facies medialis).

(e) Anterior margin (margo anterior).

(f) Posterior margin (margo posterior).

In the epididymis study- 
(a) Head (caput epididymidis) (O. T. globus major).

(b) Body (corpus epididymidis).

(c) Tail (cauda epididymidis) (O. T. globus minor).

What is the relation of the ductus deferens to the epididymis? Dissect the testicle under water. (Vide Fig. 239.) Study-

(a) Tunica albuginea.

(b) Mediastinum of testis (mediastinum testis [corpus Highmori]).

(c) Septules of testicle (septula testis).

(d) Lobules (lobuti testis).

(e) Parenchyma (parenchyma testis).

FIG. 237.

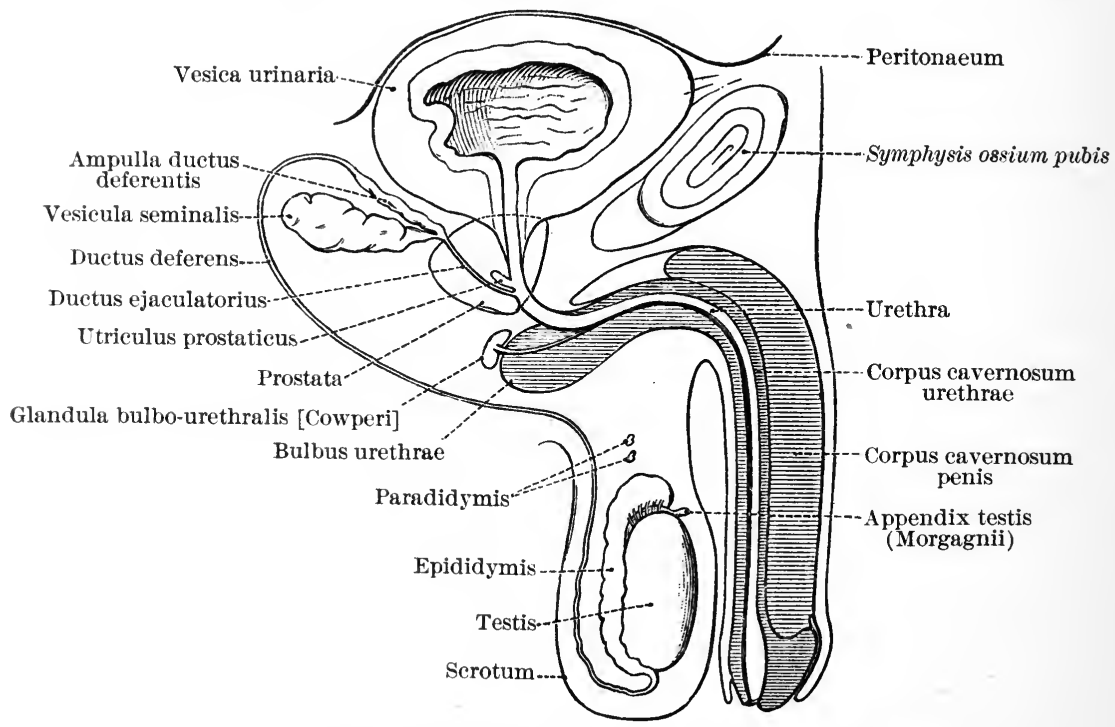

Diagram of the male genital organs and their relation to the urinary bladder and urethra. Lateral view. (After Toldt, Anat. Atlas, Wien, 1900, 2 Aufl., p. 484, Fig. 816.)

( $f$ ) Convoluted seminiferous tubules (tubuli seminiferi contorti).

(g) Straight seminiferous tubules (tubuli seminiferi recti). (ga) Proper coat (tunica propria).

(h) Network (rete testis [Halleri]).

(i) Efferent ductules (ductuli efferentes testis).

(j) Sperm or semen (sperma [semen]).

In the epididymis study-

(a) Lobules (lobuli epididymidis).

(b) Ducts (ductus epididymidis).

(c) Aberrant ducts (ductuli aberrantes).

(ca) Superior (ductulus aberrans superior). 
FIG. 238.

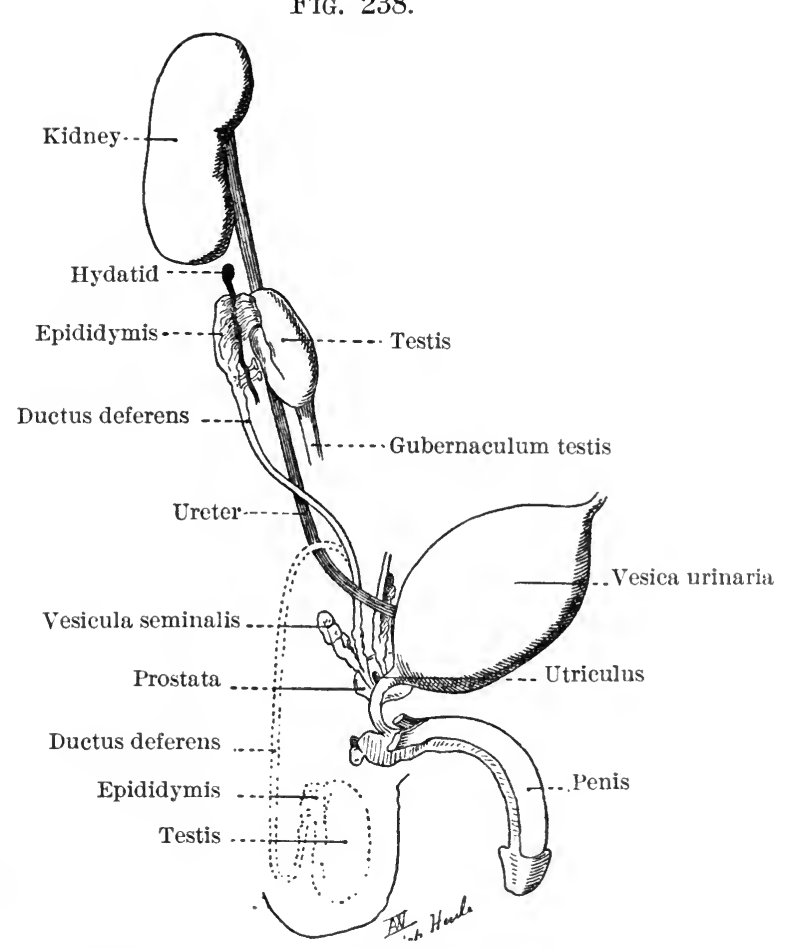

Development of the urogenital system in man. The dotted line shows the final position of the organs after the descent of the testicle into the scrotum. (From Poirier et Charpy, Traité d'Anat. hum., Paris 1901, t. v. p. 302, Fig. 234.)

FIG. 239.

Transition of the lamina parietalis tunicae vaginalis propriae into the lamina visceralis

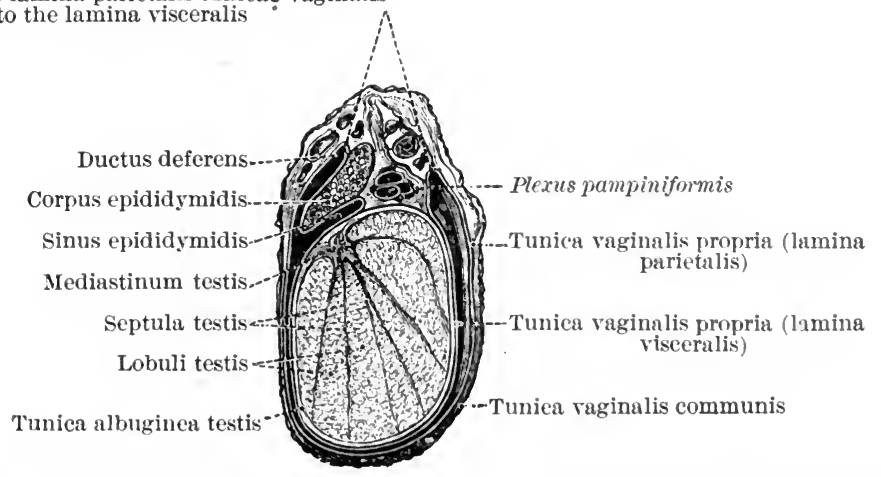

Horizontal section of the right testis, epididymis, and tunica raginalis (both the parietal and the risceral layer.) (After Toldt, Anat. Atlas, Wien, 1900, 2 . Aufl., p. 487, Fig. 824.) 


\section{Examine also-}

(a) Appendages of testis (appendices testis).

(aa) Appendix testis [Morgagnii]).

(b) Paradidymis (O. T. organ of Giraldés).

FIG. 240.

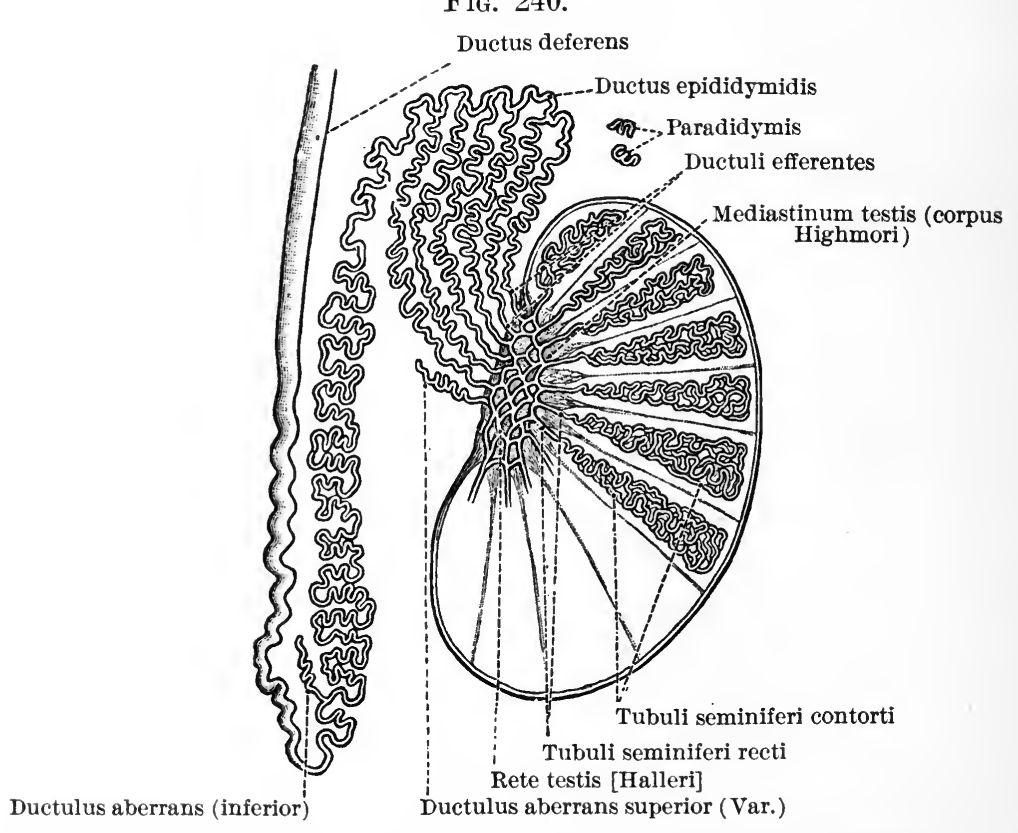

The course and constituents of the canal-system of the testis and epididymis, schematically shown. (After Toldt, Anat. Atlas, Wien, 1900, 2 Aufl., p. 487, Fig. 827.)

Penis.

This organ, already studied to a certain extent, should now be further examined.

Dissect out the suspensory ligament (Lig. suspensorium penis) and the dorsal vessels and nerves.

\section{PERITONEUM AND VISCERA.}

\section{Developmental Relations. (Cf. Figs. 24I-244.)}

Before beginning the study of the peritoneum, the development of the coelomic cavities and the transitional stages in the mesenterium commune should be carefully studied. Frequent reference should be made to Cunningham, pp. 1054-1058; Poirier et Charpy, pp. 870-898. What are the foregut, midgut, hindgut, and vitelline duct, and what relation does this latter bear to the diverticulum occasionally found upon the ileum? In what percentage of the cases is this diverticulum present, and how far distant is it from the iliocaecal junction? 
The following folds of peritoneum, connecting the intestinal tract with the anterior and posterior abdominal walls, should be noted (cf. Fig. 241) :

(a) Ventral mesentery (mesenterium anterius). What is its extent?

(b) Dorsal mesentery (mesenterium posterius). Note its extent and divisions.

Note that, while these structures are found in the embryo, they persist in part in different forms and relations in the adult.

(c) Duodenal loop (ansa duodenalis).

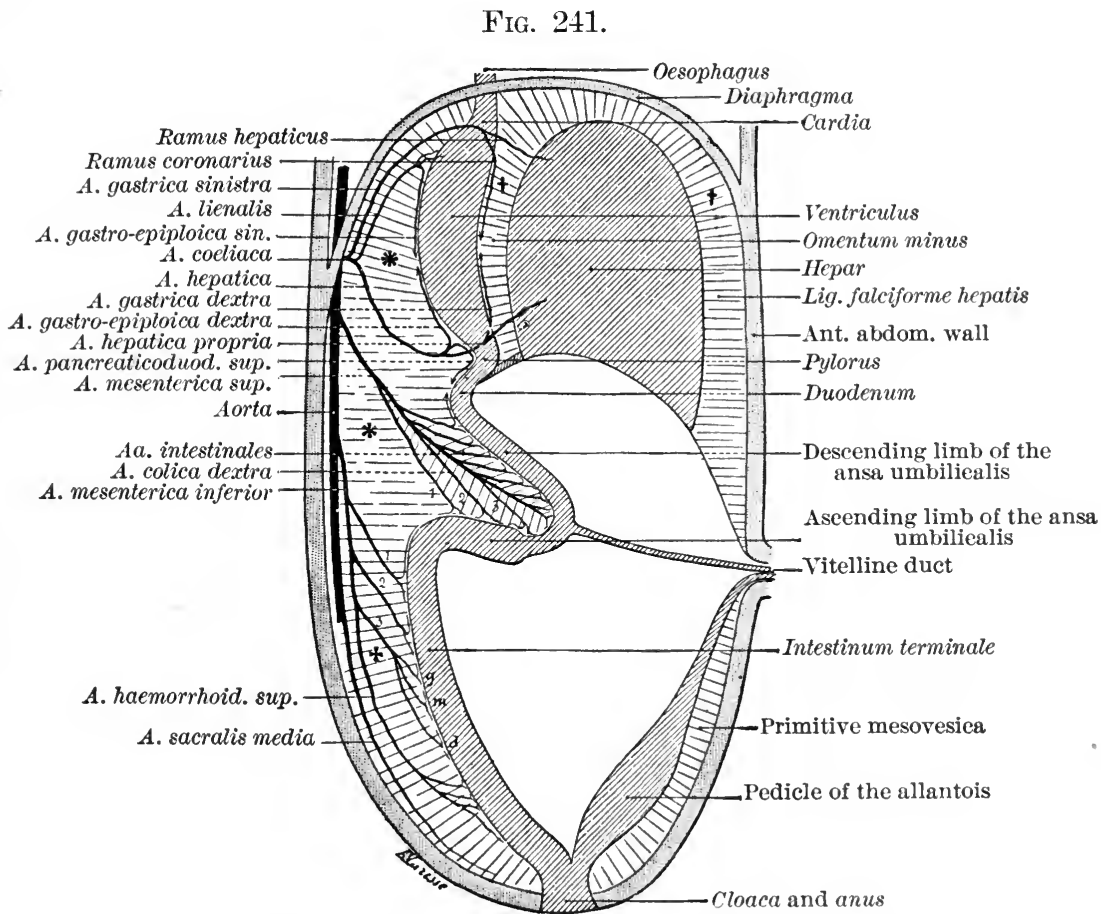

Schematic section of the abdomen of an embryo in which the segments of the alimentary canal have been differentiated. The seetion is made in the sagittal plane and is to the right of the median plane. The right face of the mesenterium commune dorsale and of the mesenterium ventrale is shown. The liver is represented isolated from the anterior abdominal wall and the diaphragm. Star with 8 points, territory of the A. coeliaea; star with 6 points, territory of the A. mes. sup. ; star with 4 points, territory of the A. mes. inf.; $1,2,3$, Aa. colicae dextra, media et sinistra ; $g, m, d$, Aa. sigmoideae; cross, mesenterium ventrale. (From Fredet in Poirier et Charpy, Traité d'Anat. hum., Paris, 1901, 2 ed., t. iv. p. 881 , Fig. 455.)

(d) Intestinal loop (ansa intestinalis or umbilicalis). The summit of this ansa is in communication with the vitelline duct and this determines its convexity anteriorly. This ansa is divided into two segments:

(da) Superior descending or proximate segment, forming an obtuse angle with the ansa duodenalis (flexura duodenojejunalis of the embryo).

(db) Inferior or ascending segment. This is prolonged to the 


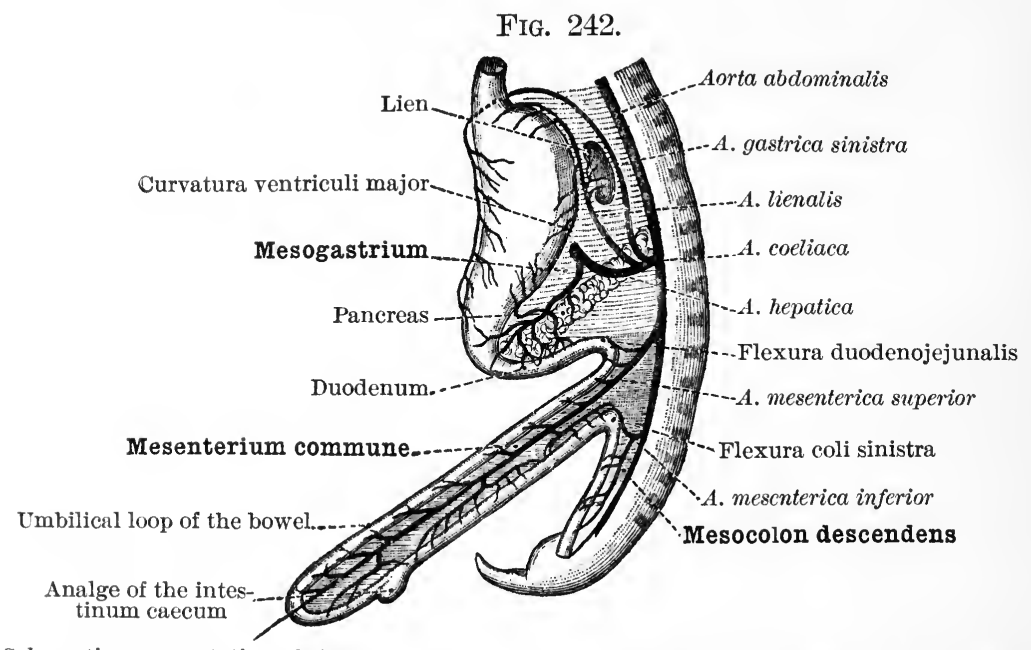

Schematic representation of the form of the intestinal canal and of the mesentery in the sixth week of fetal life. (After Toldt, Anat. Atlas, Wien, 1900, 2 Aufl., p. 435, Fig. 726.)

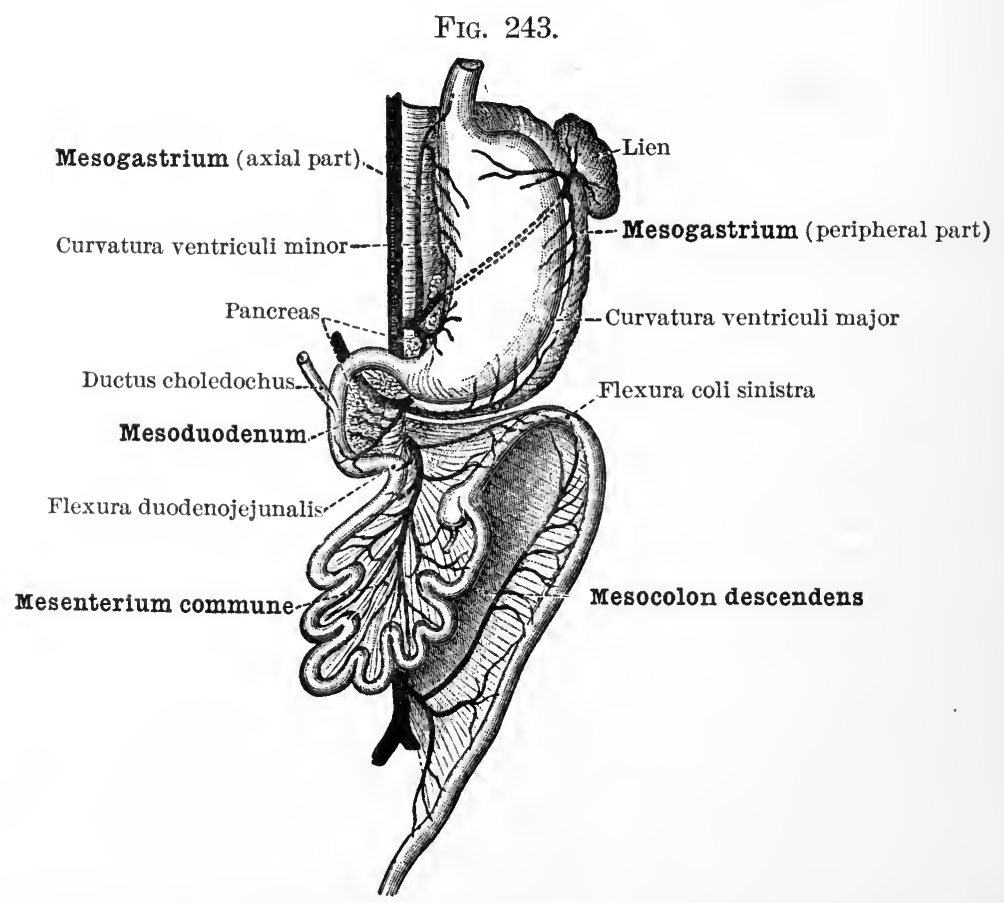

The form of the human alimentary intestinal canal and mesentery in the eighth week of fetal life. Schematic. (After Toldt, Anat. Atlas, Wien, 1900, 2 Aufl, p. 435, Fig. 727.) 
following segment, forming an acute angle (flexura colico-lienalis of the embryo).

(dc) Derivatives.

(1) From the descending segment: intestinum tenue.

(2) From the ascending segment:

a) Terminal part of the intestinum tenue.

b) Caecum.

c) Colon ascendens.

d) Colon transversum.

The remainder of the primitive intestinal tube, from the flexura colicolienalis on, is called the intestinum terminale. What are the derivatives of this? Special attention should be paid to the vascular relation of the different segments.

FIg. 244.

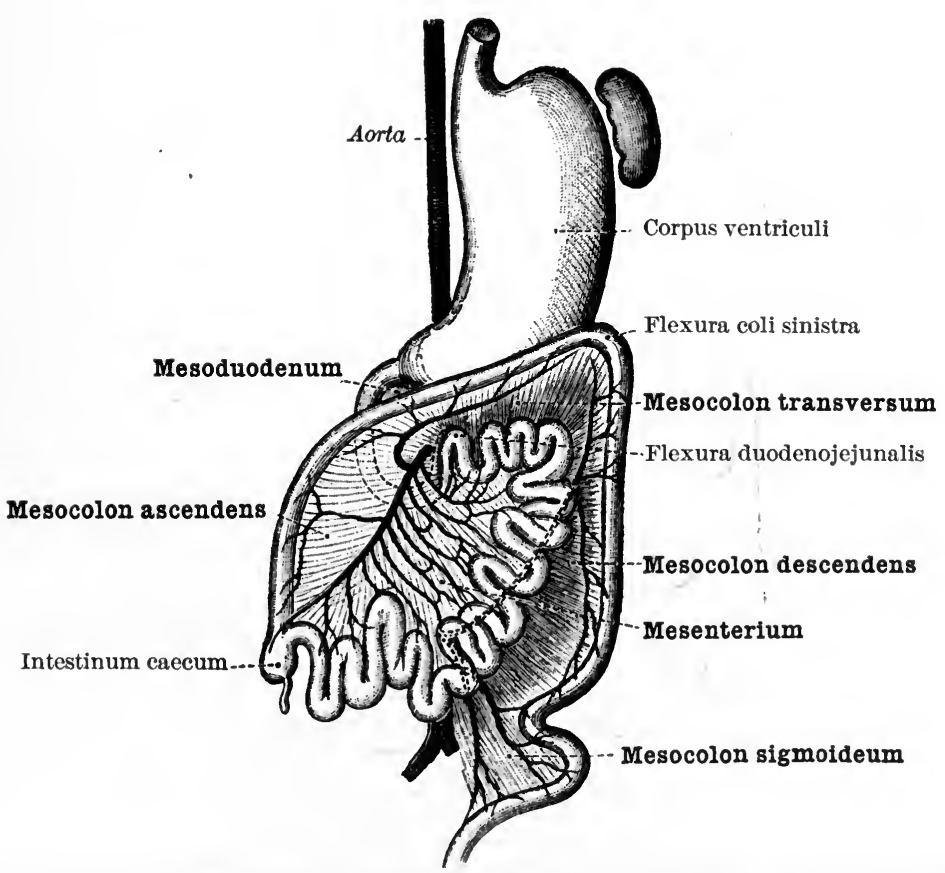

The form of the human intestinal canal and mesentery in the middle of the fourth month of foetal life. (After Toldt, Anat. Atlas, Wien, 1900, 2 Aufl., p. 435, Fig. 728.)

Make a drawing showing the viscera in position, and name each viscus visible. Make a preliminary survey of the organs in the abdominal cavity, examining exact position and general characters of the stomach (ventriculus), small intestine (intestinum tenue), large intestine (intestinum crassum), rectum (intestinum rectum), pancreas (pancreas), liver (hepar), and spleen (lien).

Note that the peritoneum (tunica serosa) lines the walls of 


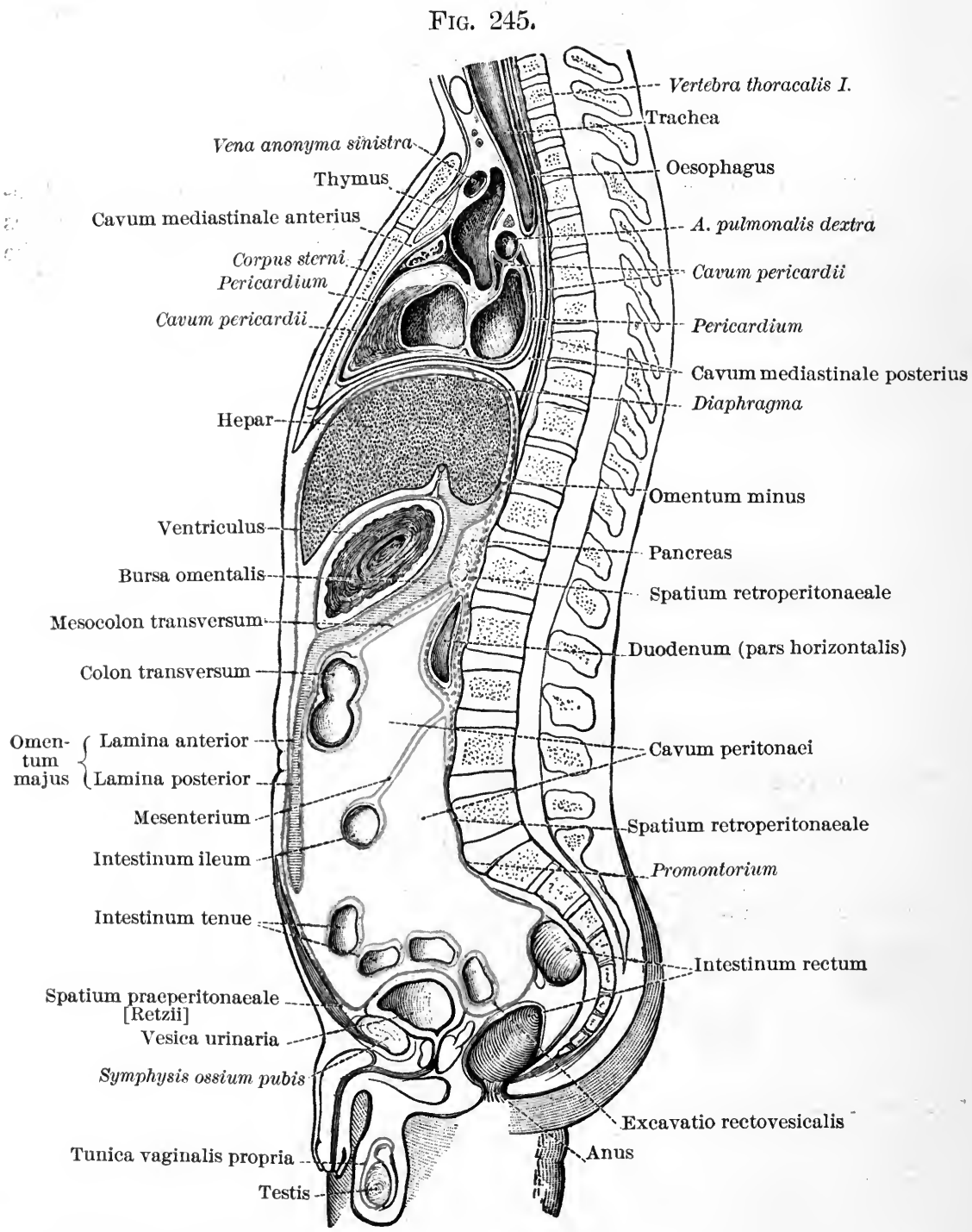

Schematic representation of the course and arrangement of the peritoneum, mesentery, and omental bursa in normal condition. The blue line indicates the primary parietal peritoneum; the red line, the mesogastrium; and the yellow line, the visceral peritoneum and peritoneal covering of the mesentery. The continuous lines show the free surface of the peritoneum; the dotted lines show those parts of the peritoneum of which the free surfaces have been lost by fusion. (After Toldt, Anat. Atlas, Wien, 1900, 2 Aufl., p. 440, Fig. 737.) 
the abdominal cavity as the parietal peritoneum (peritonaeum parietale) and covers the viscera as the visceral peritoneum (peritonaeum viscerale). The space between these two layers, which exists only upon dissection, is the peritoneal cavity (cavum peritonaei). In the male this is completely closed, but in the female it communicates with the exterior through the abdominal openings of the Fallopian tubes.

\section{Great Omentum (Omentum majus). (Figs. 245, 258.)}

What is its position and form and of what tissue is it composed?

What is its relation to that part of the large intestine passing transversely across the abdomen (colon transversum)? Is its relation in the adult the same as in the embryo? How is it re-

FIG: 246.

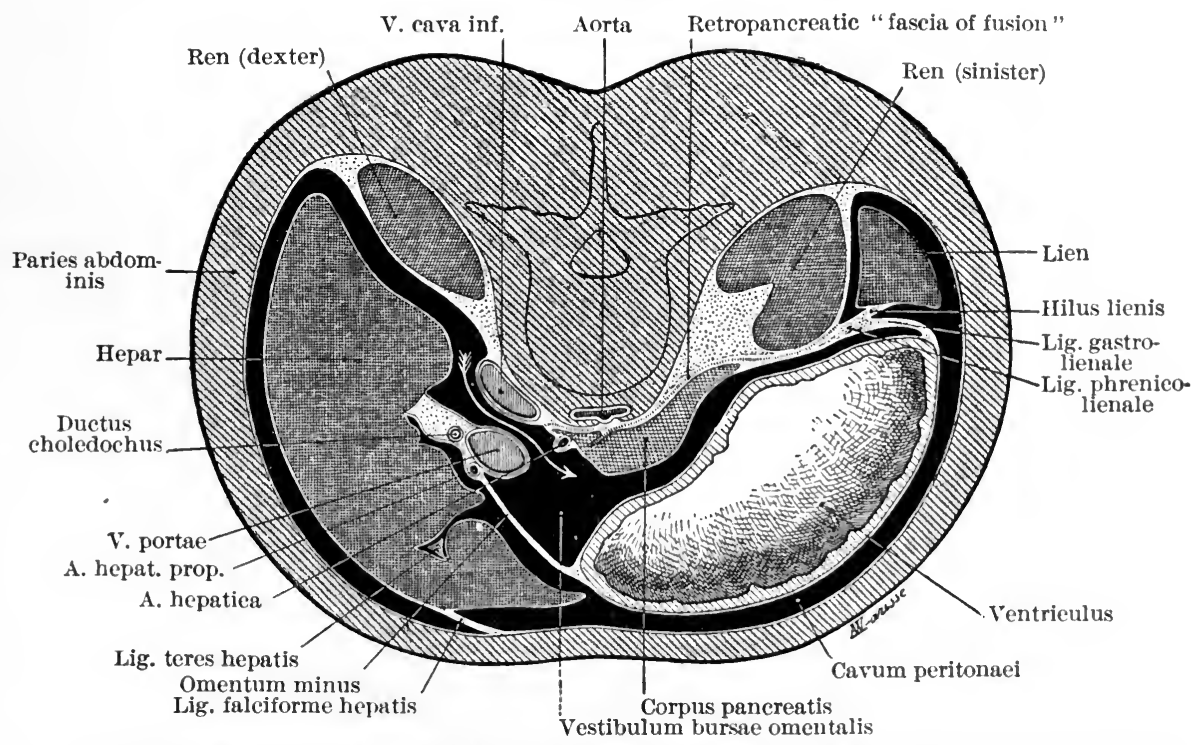

Transverse section passing through the bursa omentalis at the level of the foramen epiploicum, that is, below the hepatocaval meso. Surface below the plane of section. Schematic. The arrow indieates the opening between the $\mathrm{V}$. cava inferior behind and the $\mathrm{V}$. portae, ductus choledochus, and $\mathrm{A}$. hepatica propria in front. The great extent of the bursa omentalis is due to its lodging, at this level, the projecting lobus caudatus [Spigelii]. (From Fredet in Poirier et Charpy, Traité d'Anat. hum., Paris, 1900, 2 ed., t. iv. p. 1011, Fig. 576.)

lated to the stomach? How many layers of peritoneum form it? Does it enclose any cavity?

Trace the anterior two layers to the lower margin of the stomach. These two layers separate to enclose the stomach, 
meeting at its superior border. The double layer of peritoneum thus formed, passing between the stomach and the transverse fissure of the liver, is called the lesser or gastrohepatic omentum (omentum minus).

\section{Lesser Omentum (Omentum minus). (Figs. 24r, 245, 246.)}

Examine the thickened right border, the hepatoduodenal ligament (ligamentum hepatoduodenale); the remaining portion is sometimes referred to as the hepatogastric ligament (ligamentum hepatogastricum).

How many layers of peritoneum help to form this lesser omentum? What relation do these layers bear to the different peritoneal cavities? From which primitive mesentery is the omentum minus derived?

\section{Mesentery (Mesenterium). (Fig. 252.)}

What is the length of attachment to the posterior abdominal wall? This attachment is called the root of the mesentery (radix mesenterii). What is the length of the attachment to the bowel? What is the greatest length between its two attachments?

Note carefully between which vertebrae the mesentery extends; also its general direction. It divides the abdominal cavity (cavum abdominis) into an upper right and a lower left compartment. (Cf. Waldeyer's Kolon nischen.)

Determine the relation of the radix mesenterii to the pancreas and to the terminal portion of the duodenum. (Vide Poirier et Charpy, t. iv., Fig. 494; Spalteholz, Fig. 563.)

\section{Mesocolon (Mesocolon).}

(a) Ascending mesocolon (mesocolon ascendens). It is found in only twenty-six per cent. of the cases in the adult. (Treves.) How do you explain its absence in the remaining seventy-four per cent., while it is constantly present in the embryo? For a discussion concerning the factors leading up to the disappearance of these different peritoneal folds and the fixation of organs previously mobile, see Poirier et Charpy, 2 ed., t. iv., Fig. 498. What is Toldt's theory? What are the "fasciae of fusion"? Read about the relation of this fascia to the head of the pancreas, the right kidney, and the right ureter. What is the fascia of Treitz? From what is it derived and what relation does it bear to the head of the pancreas?

(b) Transverse mesocolon (mesocolon transversum). Note the general direction of this mesocolon. How many layers of peritoneum form this fold, and to which peritoneal sac does each belong? Is the arrangement in the adult the same as in the embryo? Determine 
its relation to the duodenum, the head of the pancreas, and the duodenojejunal angle. What is the length of this mesocolon? Does it readily permit of displacement of the colon? Note that the peritoneal relations of the first part of the transverse mesocolon depend somewhat upon the position and size of the liver. (Vide Cunningham, p. 1033.)

(c) Descending mesocolon (mesocolon descendens). The descending colon is fixed in eighty-five per cent. of the cases (Jonnesco and Charpy); in sixty-four per cent. (Treves). The disappearance of the primitive mesocolon descendens is accounted for by Toldt's theory. Determine the relation of the fascia remaining to the left kidney and ureter. Determine with accuracy the relation of the left kidney to the descending colon. Do you find any paracolic fossae (recessus paracolici)?

(d) Sigmoid mesocolon (mesocolon sigmoideum). Note its general directions and relations. (Vide Cunningham, p. 1035.) The length of this mesocolon bears a relation to the frequent torsion of the bowel (volvulus) occurring here. Does the length of this part of the intestine differ in carnivora, herbivora, and omnivora? Where does the mesocolon sigmoideum end?

\section{Mesorectum.}

Where does the mesorectum begin? How is it related to the sacral vertebrae? How far is it attached to the rectum?

After having made a careful study of these different peritoneal folds, the student should turn the omentum and transverse colon up on to the thoracic wall and proceed to the study of-

\section{Peritoneal Fossae about the Duodenojejunal Angle. (Vide Fig. 247;} Cunningham, p. Ior8; Poirier et Charpy, t. iv. pp. 265-267.)

(a) Inferior duodenal fossa (fossa duodenalis inferior). How frequently is this fossa present? Determine its relation to the terminal portion of the duodenum and to the vertebrae. Is this fossa vaseular? What is the inferior duodenal plica (plica duodenalis inferior)? Determine depth and width of this fossa, comparing it with fossae found in other subjects.

(b) Superior duodenal fossa (fossa duodenalis superior). This fossa is found in about fifty per cent. of the cases, occurring less constantly than the preceding. What relation does it bear, if present, to the second lumbar vertebra and the body of the pancreas? What is the superior duodenal plica (plica duodenalis superior)? Is this fossa vascular? If so, determine what vessels course in the plica. The two fossae frequently occur together. If both are present in your subject, compare as to depth, width, vascular relations, etc.

(c) Duodenojejunal fossa (fossa duodenojejunalis of Jonnesco). This fossa occurs in about twenty per cent. of the cases and is never coincident with the other fossae found at this flexura. Determine the relation that this fossa bears to the aorta, the left kidney, and the left renal vein. What are the plica duodenojejunalis dextra and sinistra? 
(d) Paraduodenal fossa (fossa paraduodenalis) (fossa of Landzert).

This fossa is rarely found in the adult, occurring in a little less than three per cent. of the cases. It is more frequently found in the new-born. It is situated some distance to the left of the terminal portion of the duodenum. If present, note especially its relation to the inferior mesenteric vein and the ascending branch of the left colic artery. The peritoneal folds raised by these vessels produce the fossa. What is the plica mesentericomesocolica?

\section{FIG. 247.}

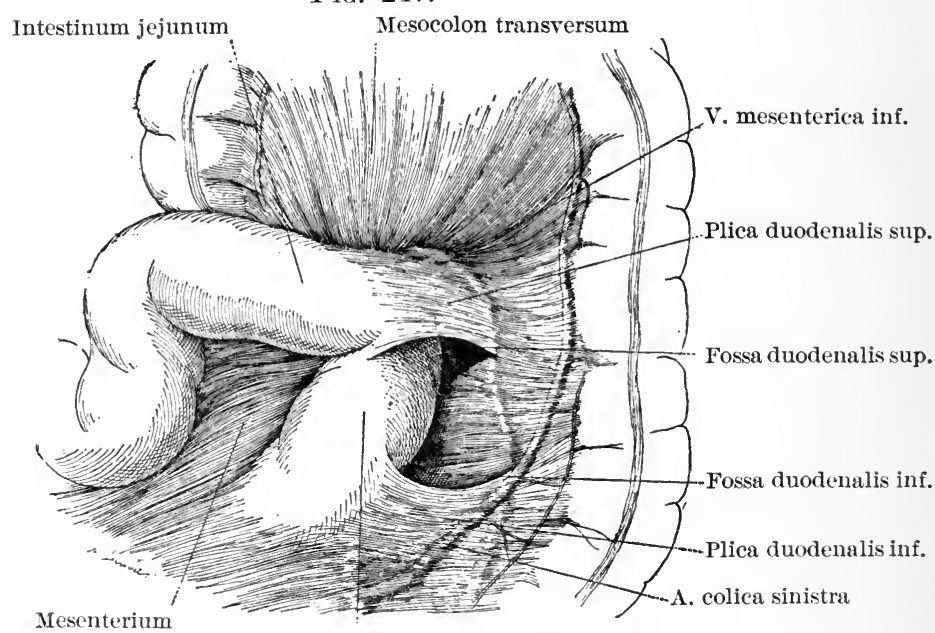

Pars aseendens duodeni

Fossae duodenales supcrior et inferior (after Jonnesco). (From Poirier et Charpy, Traité d'Anat. hum., Paris, 1901, 2 ed., t. iv. p. 907, Fig. 483.)

(e) Retroduodenal fossa (fossa retroduodenalis).

Infrequent in occurrence. Upon what does the formation of this fossa depend? In this connection reference should again be made to the fascia of Treitz.

Many anatomists recognize the existence of but one fossa at this angle. It is called the duodenojejunal fossa of Treitz. The student should refer to Poirier et Charpy, t. iv. p. 269.

\section{Peritoneal Plicae and Fossae about the Caecum.}

Before proceeding to a study of these plicae and fossae, some idea should be had concerning the disposition of the folds of peritoneum forming the mesentery at the iliocaecal junction. Arriving at this junction, the right leaf of the mesentery passes from the anterior surface of the ileum upon the caecum, and the left leaf takes a similar course posteriorly. They then rejoin upon the external border and inferior extremity of the caecum and the base of the appendix. In passing from the ileum to the caecum and appendix, the folds of the mesentery meet the short 
anterior caecal and the appendicular and recurring iliac arteries, and also a band of muscle passing from the base of the appendix to the ileum. There are thus formed three

Folds (plicae).

(a) Mesentericocaecal fold (plica mesentericocaecalis), which contains the short anterior caecal artery.

(b) Meso-appendix (mesentericum processus vermiformis), in which the appendicular artery runs.

(c) Ileocaecal or ileo-appendicular fold (plica ileocaecalis or plica ileoappendicularis), containing the muscular band passing from the base of the appendix to the ileum and the recurring ileal branch of the appendicular artery. This plica is often spoken of as the "bloodless fold of Treves."

These three plicae form two fossae.

Fossae. (See Cunningham, pp. 1030, 1031; Poirier et Charpy, pp. 338340.)

(a) Superior ileocaecal recess (recessus ileocaecalis superior), situated behind the plica mesentericocaecalis. This fossa and fold correspond to the ileocolic of Cunningham. It, is well developed in the new-born, but is less distinct in the adult. Increase in the diameters of the eaecum and deposition of fat within the plica account for the diminution in size.

(b) Inferior ileocaecal recess or ileo-appendicular fossa (recessus ileocaecalis inferior). This is the ileocaecal fossa of Cumningham. If the appendix is drawn downward and the finger passed along the lower border of the terminal part of the ileum towards the caecum, it will generally enter a fossa situated in the angle between the ileum and the caecum. The fold bounding this fossa in front is the "bloodless fold of Treves," or plica ileocaecalis; that behind, the meso-appendix, or mesenteriolum processus vermiformis.

Turn the caecum and adjacent parts of the ileum upward and expose the following:

Folds (plicae).

(a) Parietocaecal fold (plica parietocaecalis), extending from the iliac fossa or lumbar region to the external wall of the eaecum or colon ascendens. Note the general shape and attachments of this fold. Does it contain vessels? Between what vessels do they establish an anastomosis? (Vide Poirier et Charpy, t. iv., 340.)

(b) Mesentericoparietal fold (plica mesentericoparietalis). This fold represents not only the attachment of the mesentery to the iliac fossa, but also its prolongation below along the posterior abdominal wall into the pelvic eavity. Is this fold ever prolonged as far downward as the openings of the femoral and inguinal canals?

Fossae or recesses.

(a) Retrocaecal fossa (recessus retrocaecalis).

Determine accurately the boundaries, depth, breadth, etc., of 
this recess. It frequently lodges the appendix, a condition which is said to favor the development of appendicitis.

(b) Iliaco-subfascial fossa (fossa iliaco-subfascialis [Biesiadecki]).

This is a depression or pouch lined by peritoneum in the middle of the iliac fossa.

(c) Paracolic recess (recessus paracolici).

(d) Intersigmoid recess (recessus intersigmoideus).

Raise the sigmoid colon and its peritoneal fold upward and a small opening will be found, corresponding to the apex of the $\mathrm{V}$-shaped attachment of its root to the posterior abdominal wall.

This fossa is due to the imperfect fusion of the mesentery of the descending colon of the foetus with the parietal peritoneum. (See Cunningham, p. 1036; Poirier et Charpy, t. iv., Fig. 191.)

(e) Phrenicohepatic recesses (recessus phrenicohepatici).

\section{Omental Bursa, or Lesser Peritoneal Cavity (Bursa omentalis).} (Fig. 246.)

Pass the finger beneath the right free border of the omentum minus, through the opening there into the omental bursa.

Examine-

(a) Vestibule of bursa (vestibulum bursae omentalis).

(b) Extension upward behind liver, superior omental recess (recessus superior omentalis), downward behind the stomach, inferior omental recess (recessus inferior omentalis), to the left as far as the spleen, splenic recess (recessus lienalis).

(c) Gastropancreatic fold (plica gastropancreatica).

What is the method of formation of this pouch in the embryo? (Vide Cunningham, p. 1073; Poirier et Charpy, t. iv., Figs. 517520.)

Epiploic Foramen (Foramen epiploicum [Winslowi]) (O. T. Foramen of Winslow). (Fig. 246.)

How is it formed? Examine its boundaries: Anteriorly, the Lig. hepatoduodenale. Feel it. Special attention should be paid to this ligament, because of the important vessels and ducts contained in it. Superiorly, the liver. What part of the liver forms this boundary? Posteriorly, the posterior abdominal wall and inferior vena cava. Inferiorly, a fold of peritoneum, covering the hepatic artery as it passes upward to reach the ligamentum hepatoduodenale. The dissection of this ligament will be made later.

\section{Peritoneal Ligaments.}

Locate accurately the following peritoneal folds:

(1) Phrenicocolic ligament (Lig. phrenicocolicum) (O. T. costocolic ligament). From what is it developed? (Vide Cunningham, p. 1033.) 
What is its relation to the spleen? (Vide Spalteholz, Figs. 592, 593.) Why has it been called the sustentaculum lienis?

(2) Phrenicosplenic ligament (Lig. phrenicolienale). Is it present?

(3) Ligaments of liver.

(a) Coronary ligament of liver (Lig. coronarium hepatis). (Vide Cumningham, p. 1066.)

(b) Falciform ligament of liver (Lig. falciforme hepatis).

(c) Right and left triangular ligaments (Lig. triangulare dextrum et sinistrum).

(4) Ligaments of omentum minus.

(a) Hepatoduodenal ligament (Lig. hepatoduodenale).

(b) Hepatogastric ligament (Lig. hepatogastricum).

(5) Hepatorenal ligament (Lig. hepatorenale). (Cf. Spalteholz, Fig. 594.)

(6) Duodenorenal ligament (Lig. duodenorenale). (Cf. Spalteholz, Fig. 595.)

(7) Gastrolienal ligament (Lig. gastrolienale) (O. T. gastrosplenic omentum).

(8) Gastrocolic ligament (Lig. gastrocolicum).

In the pelvic cavity study the general disposition of the peritoneum. To what extent does it cover the rectum, uterus, and bladder?

Note especially the following:

In the male.

(a) Anterior surface of the bladder. Is it covered by peritoneum? What is the prevesical space of Retzius (cavum Retzii)? (Vide Cunningham, p. 410.)

(b) Pouch between bladder and rectum. This is known as the excavatio rectovesicalis.

(c) Pubovesical fold (plica pubovesicalis), also the transverse vesical fold (plica vesicalis transversa).

In the female.

(a) Broad ligament of uterus (Lig. latum uteri). What are the mesometrium, the mesovarium, and the mesosalpinx? (Vide Figs. 290 and 293; Spalteholz, Fig. 662; Toldt, Fig. 845.)

(b) Ovarian bursa (bursa ovarica).

(c) Suspensory ligament of ovary (Lig. suspensorium ovarii) (Toldt, Fig. 900). Why is this fold often referred to as the infundibulopelvic ligament? What artery runs in this fold?

(d) Recto-uterine fold (plica recto-uterina).

(e) Recto-uterine excavation, or cul-de-sac of Douglas (excavatio rectouterina [Douglasi]).

What viscera are said to be retroperitoneal?

Determine what viscera have a complete peritoneal covering. What viscera are only partially covered by this membrane?

How can you expose the third portion of the duodenum (pars inferior duodeni)? What is its relation to the parietal perito- 
neum? Demonstrate three methods by which the pancreas can be exposed anteriorly.

How can the kidney be reached from the anterior abdominal wall? What structures may be taken as guides to it? Note especially the relation of the kidneys to the ascending colon and descending colon, duodenum, and liver.

Locate accurately the position of the appendix. Do the longitudinal bands passing out on to the caecum ever serve as a guide? What are these longitudinal bands called? The tip of the appendix is found sometimes directly beneath the liver. How do you explain this?

After having gained an accurate knowledge of these different folds, fossae, etc., the student should trace the peritoneum longitudinally and transversely, demonstrating that the layers forming the greater and lesser sacs are continuous. Transverse tracings should be made $(a)$ at the level of the foramen epiploicum [Winslowi] and $(b)$ at a level below the transverse colon. (See Figs. 245 and 246.) Use transverse sections of a body hardened in formalin in this study. ${ }^{1}$

\section{Superior Mesenteric Artery (A. mesenterica superior) and its \\ Branches. (Fig. 248.)}

Turn the great omentum and transverse colon upward upon the thorax. Remove the anterior leaf of the mesentery by blunt dissection from the flexura duodenojejunalis downward as far as the terminal portion of the ileum; remove the peritoneum passing from the posterior abdominal wall upon the intestinum caecum and colon ascendens and also the inferior layer of the mesocolon transversum.

Study the following branches of the superior mesenteric artery. (Vide Fig. 248, and Spalteholz, Fig. 466.)

(a) Inferior pancreaticoduodenal artery (A. pancreaticoduodenalis inferior).

(b) Intestinal arteries (Aa. intestinales).

(ba) Jejunal arteries (Aa. jejunales).

(bb) Ileal arteries (Aa. ileae) (O. T. rami intestini tenuis).

(c) Ileocolic artery (A. ileocolica).

(ca) Appendicular artery (A. appendicularis).

(d) Right colic artery (A. colica dextra).

(e) Middle colic artery $(A$. colica media).

${ }^{1}$ See foot-note, p. 403. For a method of making cross-sections consult Professor C. M. Jackson's paper in the Jour. Am. Med. Assoc. 
Determine the number of arcades formed by the superior mesenteric artery before the ultimate intestinal branches are given off. Do these ultimate arteries anastomose with each other? What are "terminal arteries"?

The blood supply of the caecum and appendix is derived from the arteria ileocolica. It runs in the terminal part of the

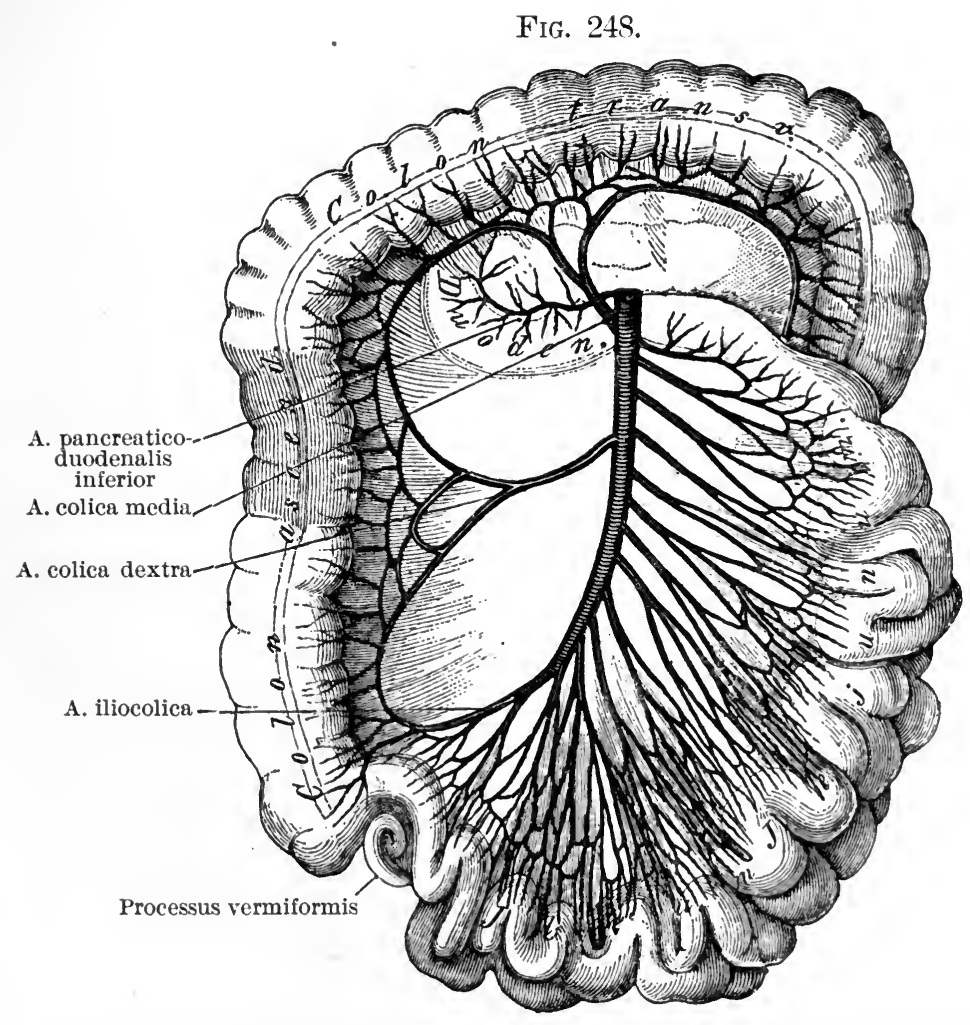

The superior mesenteric artery. The small intestine has been laid over to the left and the transverse colon turned upward. (After Gegenbaur, Lehrb. der Anat. des Mcusch., Leipzig, 1899, 7 Aufl, Bd. ii. p. 277, Fig. 540.)

mesentery and, as it approaches the ileocaecal junction, gives off four small twigs. Two of these supply the anterior and posterior surfaces of the caecum and are known respectively as the anterior and the posterior caecal artery. The anterior caecal artery gives rise to a fold in the peritoneum, which we already know as the plica mesentericocaecalis. The artery to the appendix is directed below and to the left, passing behind the terminal portion of the ileum to enter the mesenteriolum processus vermi- 
formis. It may pass in front of the ileum, then giving a special form and disposition to the meso-appendix. The fourth branch passes to the left along the ileum.

The following arteries may occasionally be derived from the superior mesenteric:

(1) Hepatic artery (A. hepatica), or right branch of the proper hepatic (ramus dexter arteriae hepaticae propriae). The importance of the anomalous origin of these arteries will be discussed when the dissection of the hepatoduodenal ligament is made.

(2) Cystic artery (A. cystica).

(3) Coronary left gastric artery, or artery of stomach (A. gastrica sinistra).

(4) Left colie artery (A. colica sinistra).

(5) Superior hemorrhoidal (A. haemorrhoidalis superior).

What relation does the superior mesenteric artery bear to the omphalomesenteric artery in the embryo? Does Meckel's diverticulum, when present, receive any branches from this artery?

Superior Mesenteric Vein (V. mesenterica superior) and its Tributaries. (Vide Spalteholz, Fig. 497.)

This vein accompanies the artery. Follow its course until it joins the splenic vein (V. splenica).

The following tributaries may be found:

(a) Intestinal veins ( $V v$. intestinales).

(b) Right gastro-epiploic vein ( $V$. gastro-epiploica dextra).

(c) Pancreatic veins ( $V v$. pancreaticae).

$(d)$ Ileocolic vein ( $V$. ileocolica).

(e) Right colic veins ( $V v$. colicae dextrae).

$(f)$ Middle colic vein ( $V$. colica media).

(g) Panereaticoduodenal veins ( $V v$. pancreaticoduodenales).

(h) Duodenal veins ( $V v$. duodenales).

Where the intestine is applied directly to the posterior abdominal wall without the interposition of peritoneum, an anastomosis is established between the systemic and portal eirculations. These anastomoses have been well established in pathological cases. What do you understand by the veins of Retzius?

Superior Mesenteric Plexus (Plexus mesentericus superior). (Vide Toldt, Fig. 1332.)

A dense plexus of sympathetic nerves surrounds the superior mesenteric artery, and filaments from it accompany the different branches of the artery to the gut. Dissect out carefully these filaments and determine whether they join in the spaces between 
FIG. 249.

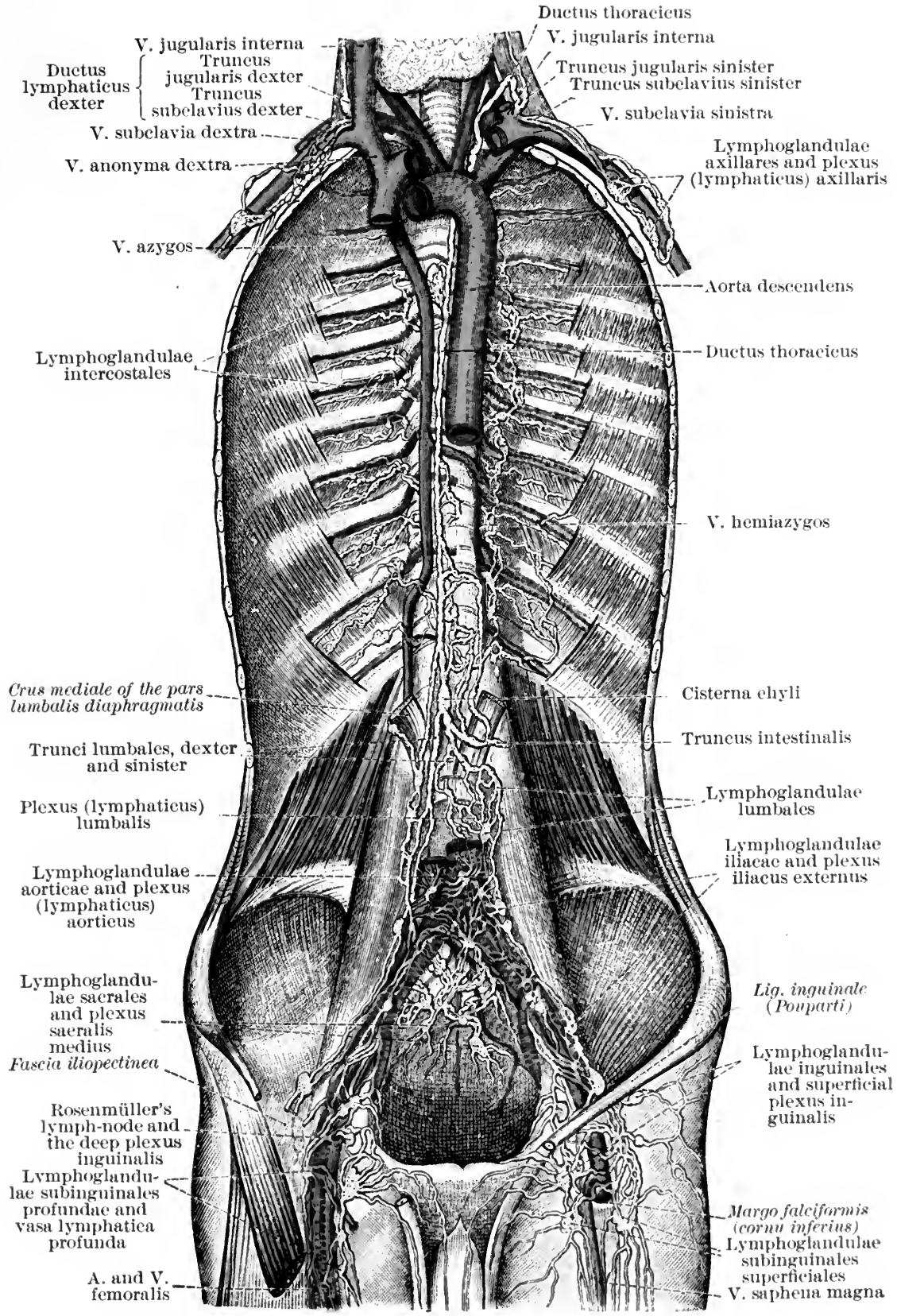

The thoracic duct and its tributaries. (After Toldt, Anat. Atlas, Wien, 1900, 2 Aufl., p. 700, Fig. 1082.) 
the arteries. From what larger sympathetic plexus are the nerves of this plexus derived?

Mesenteric Lymph-glands (Lymphoglandulae mesentericae). (Vide Fig. 249, and Toldt, Fig. 1083.)

How large are these glands? Where are they most numerous? Do you find any along the intestinal margin of the mesentery? Examine the lymph-nodes in the mesocolon (lymphoglandulae mesocolicae). What is the course and destination of the lacteals?

\section{Inferior Mesenteric Vessels and Nerves.}

Turn the loops of the small intestine to the right side of the body cavity. Remove by blunt dissection the peritoneum of the posterior abdominal wall and that passing from the posterior abdominal wall upon the colon descendens and colon sigmoideum. Study-

(a) Inferior mesenteric artery (A. mesenterica inferior). (Vide Fig. 250, and Spalteholz, Fig. 465.)

(aa) Left colic artery (A. colica sinistra). Determine with what vessels this artery anastomoses above and below. Does it form arcades before the intestinal branches are given off?

(ab) Sigmoid arteries (Aa. sigmoideae).

(ac) Superior hemorrhoidal artery (A. haemorrhoidalis superior).

This artery descends into the pelvis between the layers of the pelvic mesocolon, and is found upon the posterior surface of the rectum. Its distribution will be studied in a later dissection. When it reaches the rectal ampulla it divides into two branches which descend upon the sides of the rectum as far as the anus.

(b) Inferior mesenteric vein (V. mesenterica inferior). (Vide Spalteholz, Fig. 497.)

The beginning of this vein will be found later when the dissection of the rectal wall is made. Two large lateral tributaries will be found which run upon each side of the rectum. They unite to form the-

(ba) Superior hemorrhoidal vein ( $V$. haemorrhoidalis superior). An anastomosis is established in the rectal wall between the systemic and portal circulation. Hemorrhoids often develop as a result of disease of the liver or occlusion of the portal vein. Why?

(bb) Sigmoid veins (Vv. sigmoideae).

(bc) Left colic vein ( $V$. colica sinistra).

What relation does the $\mathrm{V}$. mesenterica inferior bear to the artery? Into what large vein does it empty above? Use models to study the relation of this vein to the pancreas and the surrounding viscera. 
(c) Inferior mesenteric plexus (plexus mesentericus inferior).

Dissect out earefully the filaments of the sympathetic nerves surrounding the branches of the artery and trace them to the intestinal wall. What relation do they bear to the aortic plexus (plexus aorticus abdominalis)? Study the latter now also.

FIG. 250.

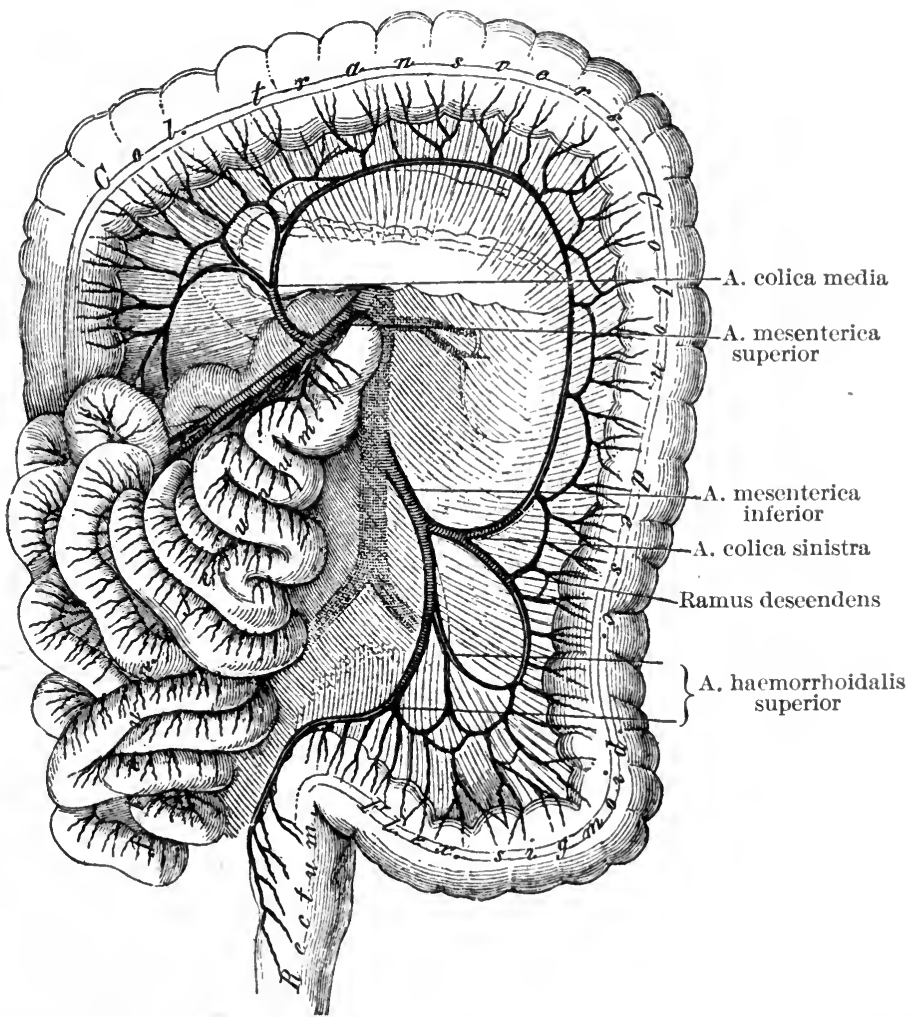

The inferior mesenteric artery. The small intestine has been laid over to the right. The transverse colon has been turned upward and the sigmoid flexure spread out. (After Gegenbaur, Lehrb. der Anat. des Mensch., Leipzig, 1899, 7 Aufl., Bd. ii. p. 279, Fig. 512.)

(d) Left mesocolic lymphaties.

Do you find any lymph-nodes? What is the course and destination of the lymphaties draining the colon descendens and colon sigmoideum?

\section{Mesenterial Small Intestine (Intestinum tenue mesenteriale).}

That portion of the small intestine which in the adult is attached to the posterior abdominal wall by a mesentery is known as the intestinum tenue mesenteriale. (Vide Figs. 251-3 and Spalteholz, Figs. 565, 566, 568, 569.)

It is arbitrarily divided into an upper one-third (two-fifths, 
Winslow; three-fifths, Hyrtl, Merkel), which is known as the empty intestine, or jejunum (intestinum jejunum), and a lower two-thirds, known as the twisted intestine, or ileum (intestinum

FIG. 251.

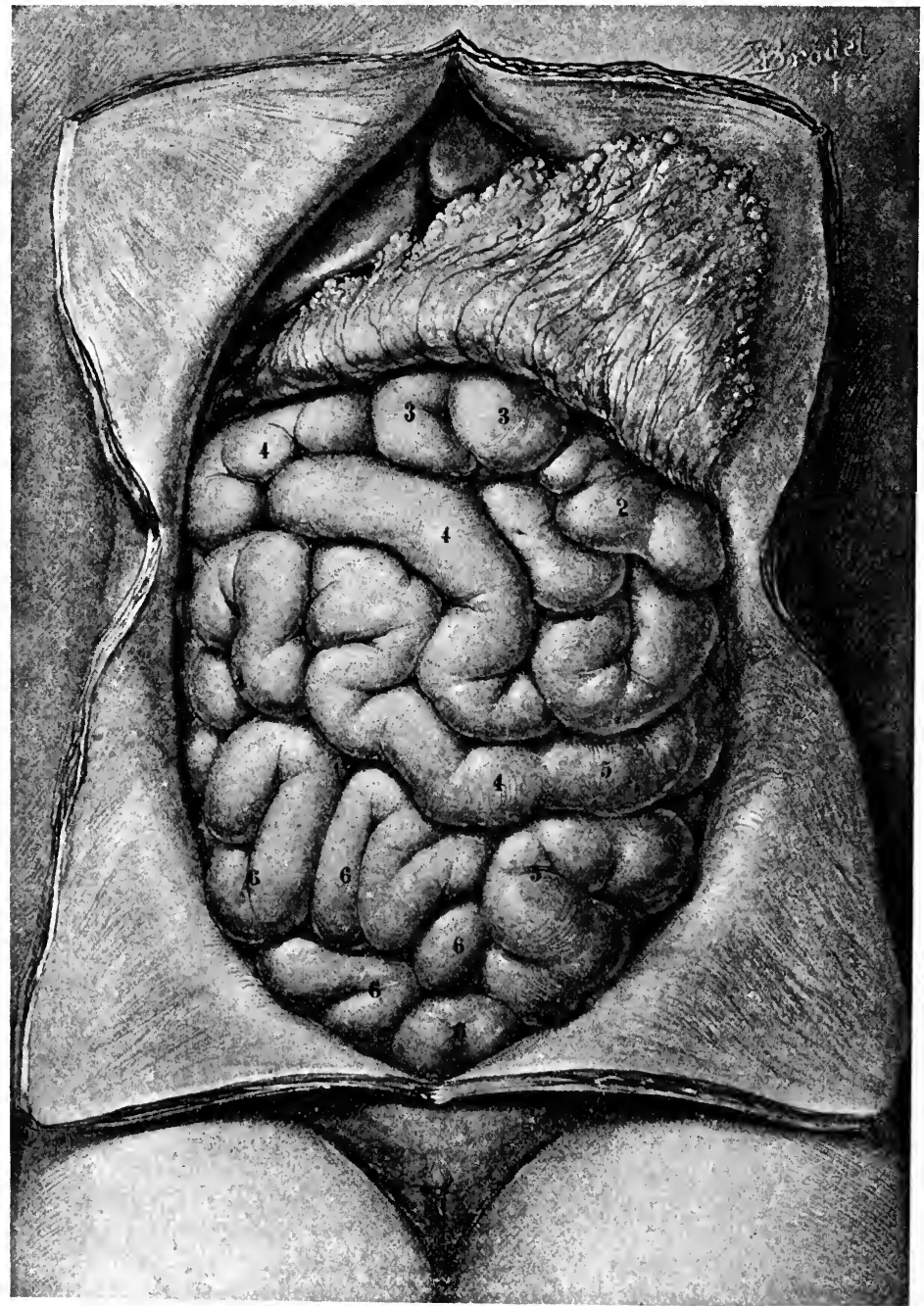

Usual position of the intestines in the abdominal cavity. Although this is an actual specimen, it represents the condition in twenty-one of forty-one cadavers. The numbers in the figure mark the parts which are homologous with the loops correspondingly numbered in Figs. 252 and 253 . (After Mall, Devel. of the Hum. Int. and its Position in the Adult, Bull. J. H. Hosp., Baltimore, 1898, vol. ix.. Nos. 90 and 91 , Plate iv., Fig. 16.)

ileum). The duodenum in the adult has no mesentery, and accordingly does not belong to the "mesenterial small intestine." 
Use models for the study of the position and relations. The viscera soon lose their characteristic shapes and relations in the cadaver, unless they have been hardened in situ by the injection of some hardening fluid, such as formalin.

Are the loops of the intestinum tenue mesenteriale arranged

FIG. 252.

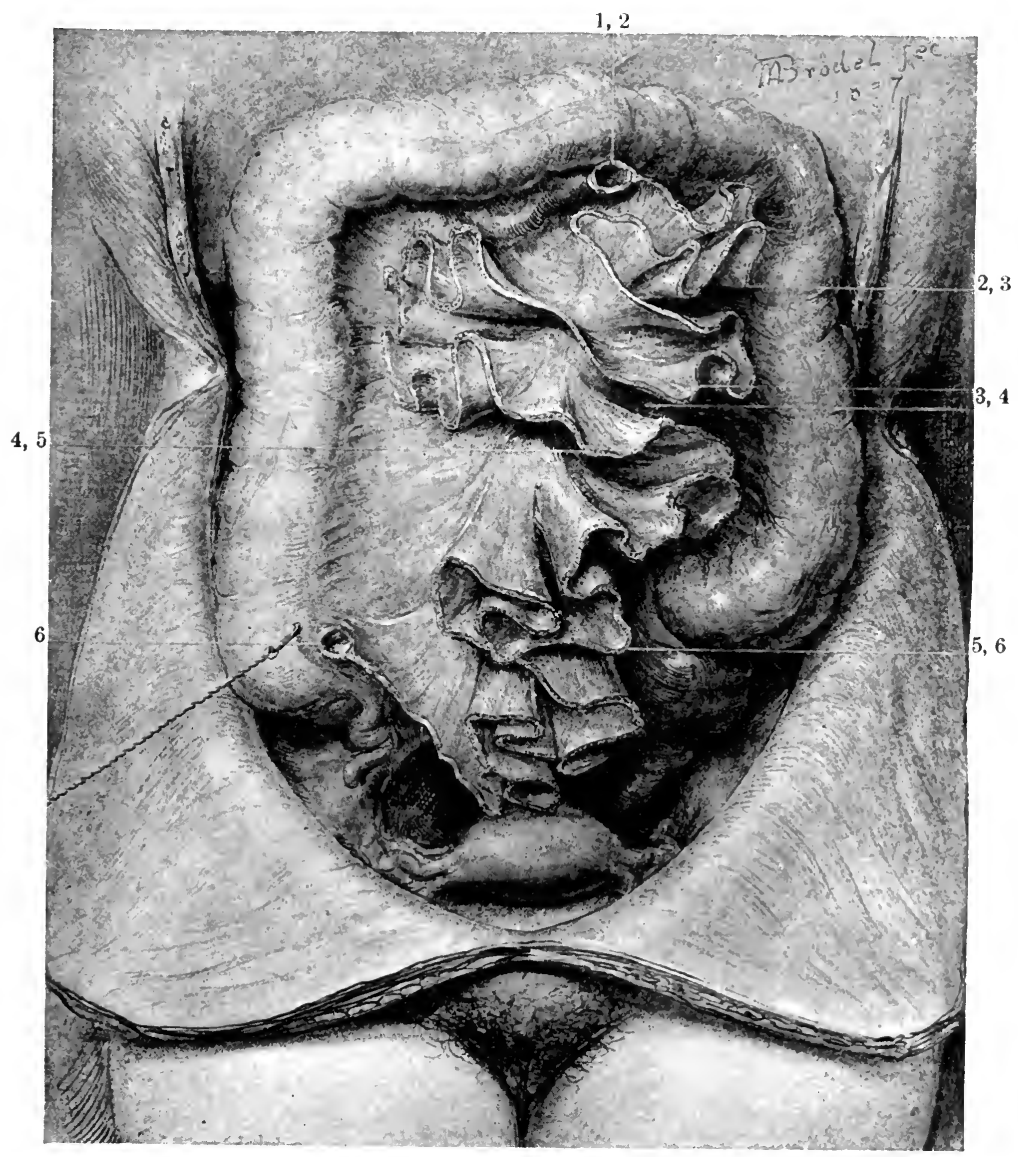

Usual position of the mesentery. Cf. Fig. 251. (After Mall, ibid., Plate v., Fig. 17.)

in any definite order? Can you find in the model a superior group directly underneath the colon and passing transversely across the abdominal cavity, two vertical groups situated on either side of the vertebral column, and an inferior group within the small pelvis and passing transversely across it? Note that 
the convolutions placed between the preceding four groups have no definite orientation. ${ }^{1}$

Forty-one per cent. of the total length of the intestinum tenue mesenteriale is situated in the abdomen to the left of the median

FIG. 253.

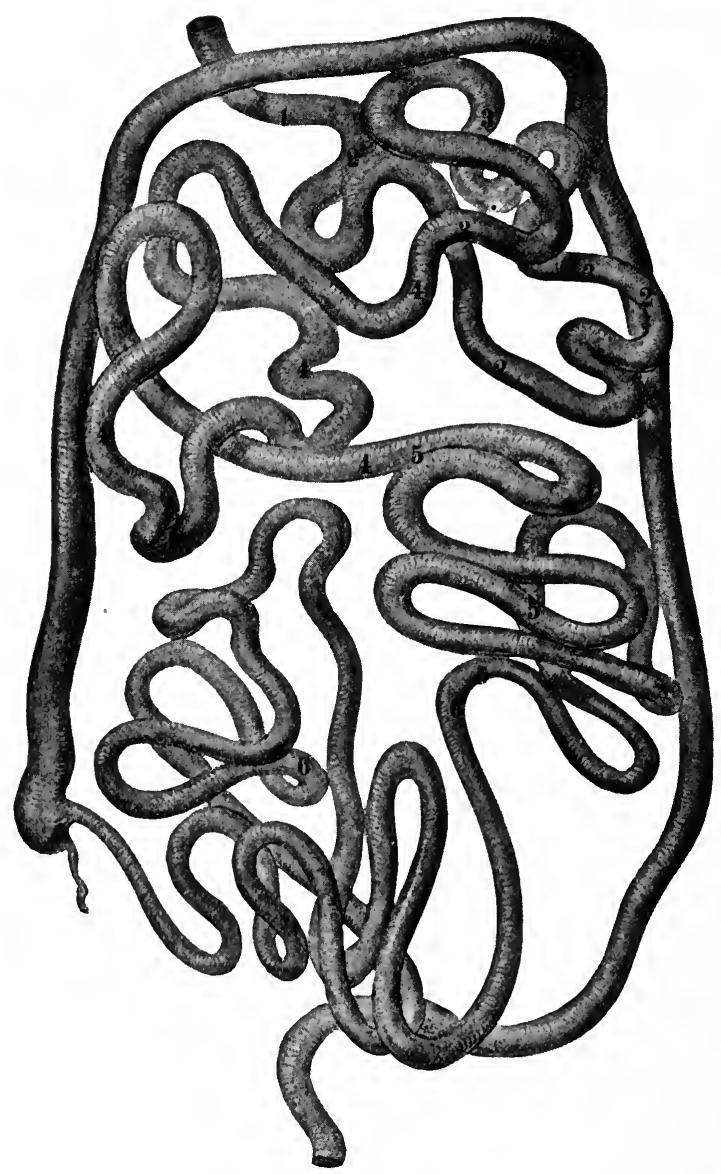

Course of the intestine. This figure is taken from a model made from the same cadaver from which Figs. 251 and 252 were drawn. (After Mall, ibid., Plate v., Fig. 18.)

sagittal plane, eighteen per cent. to the right of it, and forty-one per cent. within the pelvis.

${ }^{1}$ For a full discussion concerning the arrangement of the intestinal loops, consult Mall, F. P., The Development of the Human Intestine, Bulletin of Johns Hopkins Hospital, 1898; Archiv f. Anat., 1897; Sernoff, Zur Kenntniss d. Dünndarms, Intern. Monatschr. f. Anat., 1894. 
The loops of the jejunum will be found in the superior half of the regio mesogastrica and on each side of the vertebral column; the convolutions of the ileum are situated in the lower half of the same region, in the regio hypogastrica, and in the pelvis. The convolutions occupying the space between the two psoas muscles are the most mobile. They are the most frequent content in inguinal and femoral herniae. Determine the relations of the small intestine to the colon transversum and its mesocolon above; to the intestinum caecum and colon ascendens on the right and colon descendens and colon sigmoideum on the left; to the annulus inguinalis abdominalis and annulus femoralis below and lateralward, and to the urinary bladder, uterus, and rectum in the median plane of the body.

What factors are concerned in maintaining the loops of the small intestine in their normal position? What is enteroptosis?

\section{Removal of the Intestines.}

Apply two ligatures around the upper end of the jejunum about two inches below the flexura duodenojejunalis. Cut between these. Cut through the blood-vessels and the remaining fold of peritoneum of the mesentery along the line of their attachment to the intestinal wall as far down as four inches above the ileocaecal junction. Apply two more ligatures there and cut between them. The intestine should now be taken to the sink, the ligatures removed, and all contents of the bowel thoroughly washed away.

Remove a small piece of equal length from each end of the intestine and weigh. Which is the heavier? Which has the thicker walls? Feel them between the fingers. To what is the greater thickness of the one due? Which is the more vascular? Which has the larger lumen, the jejunum or the ileum?

Remove about six inches from the upper part of the jejunum, open it along its mesenteric border, and thoroughly clean the mucous membrane. Spread it out carefully and pin it down upon a cork dissecting tray, with the serous surface upward, and study its coats.

\section{Coats of Small Intestine.}

(a) Serous coat (tunica serosa).

(b) Muscular coat (tunica muscularis).

(ba) Longitudinal layer (stratum longitudinale).

(bb) Circular layer (stratum circulare).

(c) Submucous coat (tela submucosa). 
(d) Mucous coat (tunica mucosa). This will be studied in continuity. Can you make out the lamina muscularis mucosae?

Open the remainder of the small intestine along its mesenteric border, using an enterotome, and proceed to the study of the-

(a) Circular folds (plicae circulares [Kerkringi]) (O. T. valvulae conniventes). (Fig. 254.) What happens to these folds when the intestinal wall is put upon the stretch? How far above the ileocaecal valve (valvula coli) do they disappear? These folds are occasionally found extending as far as the ileocaecal junction in diseases such as tuberculosis of the peritoneum, where the total length of the small intestine is shortened. Cruveilhier and Kazzander have

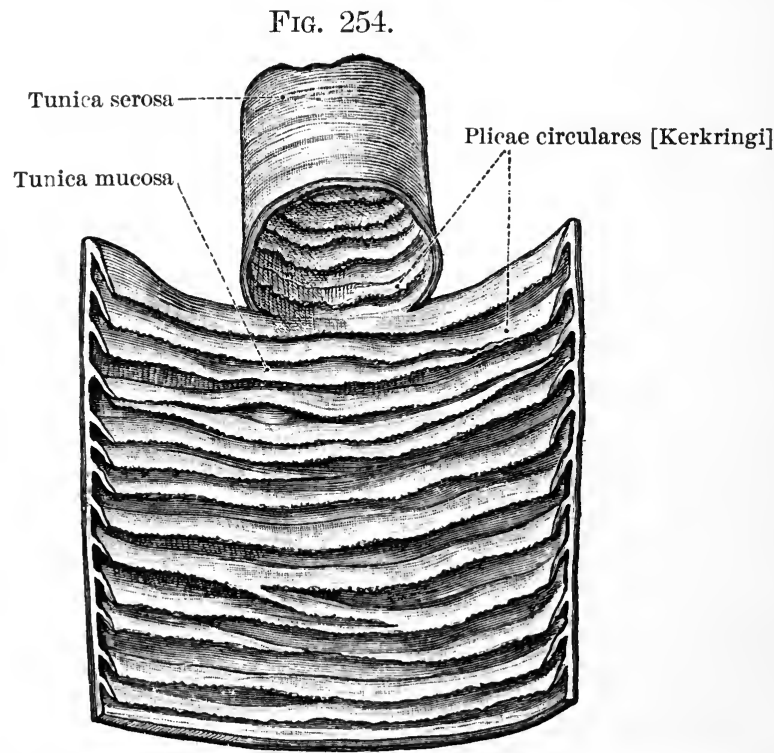

A piece of the intestinum jejunum in part cut open. (After Toldt, Anat. Atlas, Wien, 1900, 2 Aufl., p. 426, Fig. 698.)

seen them extending thus far in cadavers in which no disease of the peritoneum or intestine could be detected. Three forms are to be found, according to Kazzander. Most of the folds extend only part way about the wall of the gut; others surround the wall completely; while the third set has a spiral arrangement. Can you find one of each variety? They number 678 in the male and 644 in the female (Kazzander). What coats of the intestine enter into the formation of these folds? What effect does this arrangement have? How do you explain their formation? Folds which are parallel to the long axis of the bowel are occasionally found. Upon what factors does their formation depend? Do you find any in your subject?

(b) Intestinal villi (villi intestinales). Wash the mucous membrane clean 
and examine it with a pocket lens. About twelve villi are found to the square millimetre (Sappey). Their total number is about ten millions. Where are they most numerous?

(c) Intestinal glands (Gl. intestinales [Lieberkuehni]) (O. T. erypts of Lieberkuehn). Look for their orifices with a lens.

(d) Solitary lymph-nodules (noduli lymphatici solitarii). They are enlarged in many of the acute infectious diseases so as to be easily visible.

(e) Aggregated lymph-nodules of Peyer, or Peyer's patches (noduli lymphatici aggregati [Peyeri]). These should be studied carefully and a correct idea of normal appearance be gained. They are the seat of pathological changes in typhoid fever and often also in tuberculosis. Notice that they are situated opposite the mesenteric attachment. They are most easily found if the bowel is held to the light. They number from thirty-five to forty (Sappey; fifteen to fifty, Frey). What is their general form? Measure an elliptical patch and note in drawing-book the measurements. What is the general appearance of a patch? How is its long axis directed? (Vide Fig. 255.)

FIg. 255.

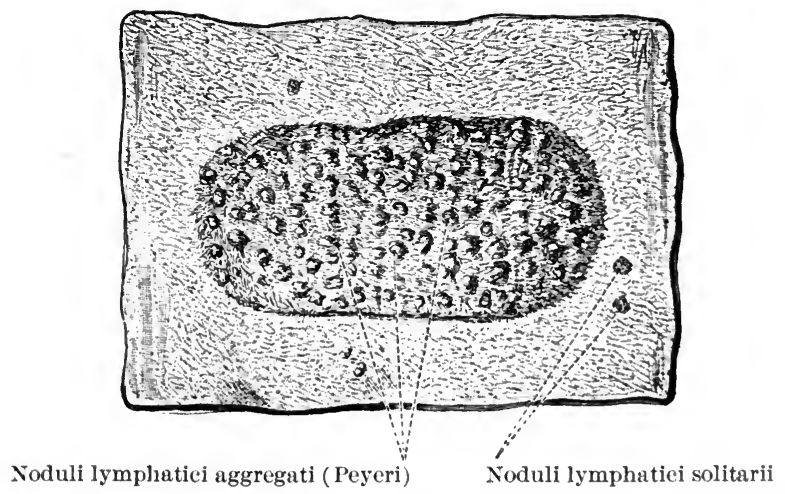

Noduli lymphatiei aggregati [Peyeri], "Peyer's pateh" of the intestinum ileum. Natural size. (After Toldt, Anat. Atlas, Wien, 1900, 2 Aufl., p. 426, Fig. 701.)

Tabulate in your note-book the differences you have observed between the intestinum jejunum and the intestinum ileum. What are chyme (chymus), chyle (chylus), and intestinal juice (succus entericus)?

Large or Thick Intestine (Intestinum crassum). (Vide Fig. 256 and Spalteholz, pp. 522-524.)

Make use of models and determine accurately the course of the intestinum crassum. Study its different parts :

(a) Blind intestine (intestinum caecum). What are the foetal, infantile, 
and adult types of caeca? How does the caecum differ in carnivora and herbivora? (Vide Poirier et Charpy, t. iv. p. 323.)

(b) Vermiform process or appendix (processus vermiformis). What is the average length of the appendix? What is its relation to the caecum? What positions may the process occupy?

(c) Colon.

(ca) Ascending colon (colon ascendens).

( $c b)$ Right colic flexure (flexura coli dextra).

(cc) Transverse colon (colon transversum).

(cd) Left colic flexure (flexura coli sinistra).

(ce) Descending colon (colon descendens).

(cf) Sigmoid colon (colon sigmoideum) (O. T. sigmoid flexure).

Note carefully the relation of each of these parts to the neighboring viscera. Note especially the relation to the kidneys, duodenum, pancreas, stomach, and spleen.

Free the remaining portions of the intestine, as far as the left sacro-iliac synchondrosis. Apply two ligatures there and cut between. Take the intestines to the sink, remove the ligatures, and wash out the contents. Then proceed to the study of the following external features (cf. Fig. 256) :

(a) Bands of the colon (taeniae coli).

(aa) Mesocolic band (taenia mesocolica).

(ab) Omental band (taenia omentalis).

(ac) Free band (taenia libera).

Note the relation of the taeniae to the base of the appendix and the caecum. Compare specimen with models and determine the number of taeniae to be found upon the rectum.

(b) Sacculations of colon (haustra coli).

Compare the size of the sacculations upon the caecum, colon ascendens, and colon sigmoideum. Which are the largest? How is their formation explained?

(c) Epiploic [fatty] appendages (appendices epiploicae).

How many series do. you find upon the colon ascendens and colon descendens? Along which taeniae are they arranged? How many series do you find on the colon transversum?

Remove a section of the colon ascendens, open it, and clean its mucous surface; pin down upon a cork tray and study its coats.

(a) Serous coat (tunica serosa). Has the colon ascendens a complete serous coat? What relation do the appendices epiploicae bear to this coat?

(b) Muscular coat (tunica muscularis).

(ba) Longitudinal layer (stratum longitudinale).

Note that the longitudinal muscular fibres are collected together, forming the taeniae. A complete, but thin, continuous longitudinal layer of muscle will also be found.

(bb) Circular layer (stratum circulare). 
(c) Submucous coat (tela submucosa).

(d) Mucous coat (tunica mucosa).

(da) Muscular layer of mucosa (lamina muscularis mucosae).

(db) Solitary lymph-nodules (noduli lymphatici solitarii).

$(d c)$ Intestinal glands of Lieberkuehn (Gl. intestinales [Lieberkuehni]).

What are the semilunar folds (plicae semilunares coli)?

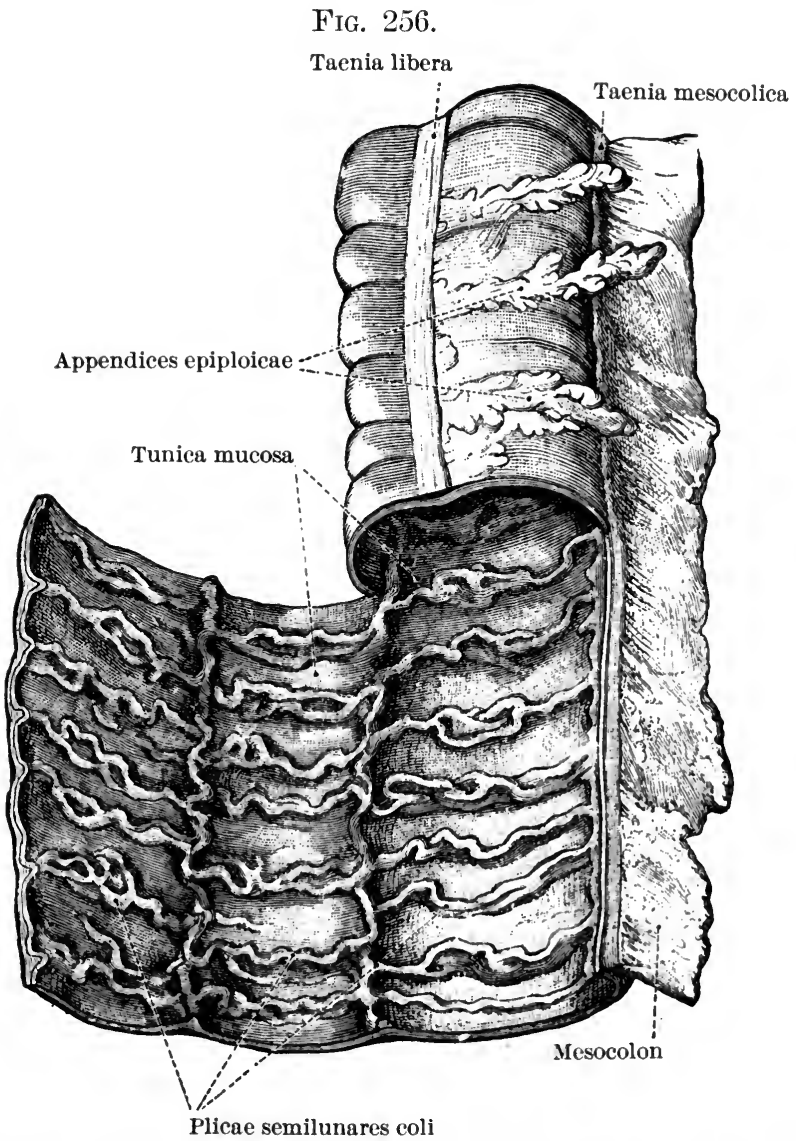

A piece of the large intestine, intestinum crassum, partly opened along the line of attachment of the mesocolon. The bowel is in a state of contraction. (After Toldt, Anat. Atlas, Wien, 1900, 2 Aufl., p. 427, Fig. 704.)

Note carefully the differences between the mucous membrane of the large and small intestines and tabulate these in your drawing-book.

Open the anterior and right wall of the caecum and study the valve of the colon (valvula coli) (O. T. ileocaecal valve). (See 
Fig. 257.) What is the general direction of the valve? What coats of the intestine enter into its formation?

Note-

(a) Inferior lip (labium inferius).

(b) Superior lip (labium superius).

(c) Frenula ("bridles" or "reins") of valve of colon (frenula valvulae coli).

What is the function of the valve?

Note the location of the opening of the vermiform process into the caecum. Is it guarded by a valve (valvula processus vermiformis)? This valve was first fully described by Gerlach

FIG. 257.

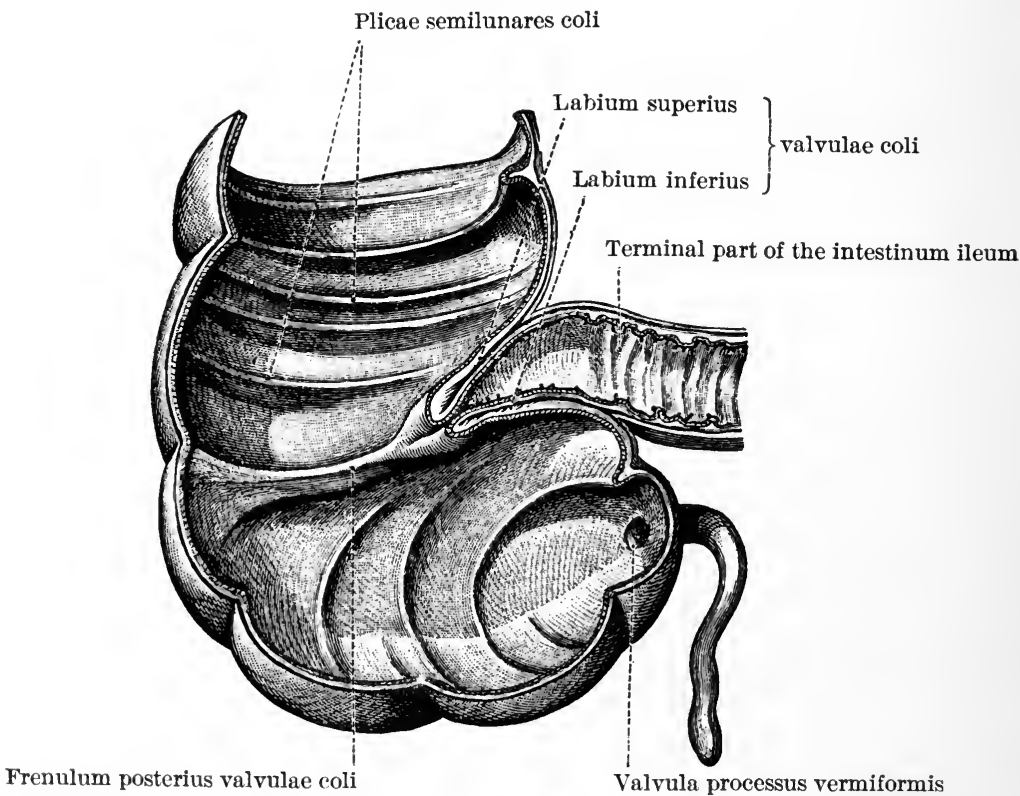

The intestinum caecum hardened in formalin and cut open in a plane passing through the entrance of the intestinum ileum (valvula coli). The latter is closed and is eut in a frontal plane. (After Toldt, Anat. Atlas, Wien, 1900, 2 Aufl., p. 428, Fig. 709.)

in 1847. According to him, it is found in the embryo, but only vestiges of it occur in the adult. Laffargue thinks that the formation of this valve is pathological and is due to mechanical pressure exerted by enteroliths in the lumen of the appendix. The lumen of the appendix tends to become obliterated in the adult. (Vide Fitz, Am. J. Med. Sc., 1886.)

Open the vermiform process along its mesenteriolic border 
and study its internal structure. Examine the aggregated nodules of the vermiform process (noduli lymphatici aggregati processus vermiformis). Good specimens for study can be made by blowing up the caecum and lower part of the colon ascendens; when dry, windows can be cut out to expose the interior.

\section{Structures in the Omentum Minus. (See Fig. 258.)}

Elevate the anterior margin of the liver and stitch it to the lower margin of the thorax. Then remove, by blunt dissection, the anterior layer of the hepatoduodenal ligament (ligamentum hepatoduodenale) and study-

(a) Common bile-duct (ductus choledochus).

Make frequent use of models to complete this study. Dissect out carefully the duct, as far as its entrance into the wall of the duodenum. What is its general direction? Does the direction of the eanal depend upon the form of the duodenum? To what vertebra is it related above and below?

Notice that the duct may be divided for descriptive purposes into a duodenal, a pancreatic, and a parietal portion. What is the course of the pancreatic division? Determine accurately the relations of the duct to the rena cava inferior, the fascia of Treitz, the termination of the V. mesenterica superior, the ductus accessorius and ductus pancreaticus.

Now clean this duct towards the liver and examine-

(aa) Hepatic duct (ductus hepaticus). Notice the two biliary ducts passing from the porta hepatis and uniting to form the hepatic duct. Determine their relation to other structures found here. Are any aberrant vessels of the liver (vasa aberrantia hepatis) present?

(ab) Cystic duct (ductus cysticus). Determine its relation to the gall-bladder (vesica fellea).

The study of this duct will be completed when the dissection of the liver is made.

(b) Portal vein (vena portae). (Vide Spalteholz, Fig. 498.)

Exereise great eare in eleaning this vein. Free the pancreas from the posterior abdominal wall; disturb its relations as little as possible. Determine the relation of the portal vein to the head of the panereas and the first or superior portion of the duodenum (pars superior duodeni). Supplement dissection by use of models. Examine the following tributaries:

(ba) Coronary vein of stomach (V. coronaria ventriculi).

$(b b)$ Superior mesenteric vein ( $V$. mesenterica superior).

(bc) Inferior mesenteric vein ( $V$. mesenterica inferior).

The tributaries of the last two veins have been studied in the earlier dissection. 
$(b d)$ Lienal or splenic vein ( $V$. lienalis).

( $b d a)$ Short gastric veins ( $V v$. gastricae breves).

(bdb) Left gastro-epiploic vein (V. gastro-epiploica sinistra).

(bdc) Cystic vein (V. cystica).

(be) Parumbilical veins (Vv. parumbilicales [Sappeyi]).

What are the following anastomoses in the domain of the $\mathrm{V}$. portae?

(1) Oesophageal anastomosis.

(2) Rectal anastomosis.

(3) Peritoneal anastomosis (veins or system of Retzius).

(4) Umbilical anastomosis (veins of Sappey). What is the "caput medusae," sometimes met with when the V. portae is obstructed in the liver?

Are any of the accessory portal veins present?

(1) Deep cystic veins, passing from the fossa vesicae felleae and the non-peritoneal surface of the gall-bladder directly into the liver.

(2) Epiploic veins, passing from the omentum minus and the hepatocolic and hepatorenal ligaments.

(3) Veins of the hilus.

(4) Veins from the diaphragm, which pass through the coronary ligament of the liver.

(5) Veins of the ligamentum falciforme hepatis.

(c) Hepatic artery (A. hepatica). (Vide Spalteholz, Fig. 464.)

(ca) Right gastric artery (A. gastrica dextra).

(cb) Proper hepatic artery (A. hepatica propria).

(cba) Right branch (ramus dexter).

$(c b b)$ Cystic artery (A. cystica).

$(c b c)$ Left branch (ramus sinister).

(cc) Gastroduodenal artery (A. gastroduodenalis).

(cca) Superior pancreaticoduodenal artery (A. pancreaticoduodenalis superior).

(1) Pancreatic branches (rami pancreatici).

(2) Duodenal branches (rami duodenales).

(ccb) Right gastro-epiploic artery (A. gastro-epiploica dextra).

(1) Epiploic branches (rami epiploici).

Later this artery (right gastro-epiploic) will be traced back to its origin, the coeliac artery ( $A$. coeliaca). Pay especial attention to the relations of the hepatic artery to the ductus choledochus and vena portae. What anomalies of the hepatic artery frequently occur?

(d) Hepatic plexus of sympathetic nerves (plexus hepaticus).

The filaments of this plexus will be found accompanying the arteria hepatica propria and each of its branches. Dissect these filaments out and study their distribution.

(e) Lymph-glands.

What is the exact position of the cystic lymph-glands (lymphoglandulae cysticae) and the lymph-gland of the portal vein 
(lymphoglandula venae portae)? (Vide Bardeleben, Haeckel u. Frohse, Atlas der Topographischen Anatomie des Menschen, 2 Aufl., Fig. 111.)

Look for hepatic lymph-glands (lymphoglandulae hepaticae).

FIG. 258.

A. phrenica inferior sinistra Rami oesophagei

A. gastrica sinistra :

A. coeliaca

A. phrenica inferior dextra

A. hepatica
inferior

A. hepatica $\{$ Ramus sinister propria $\{$ Ramus dexter

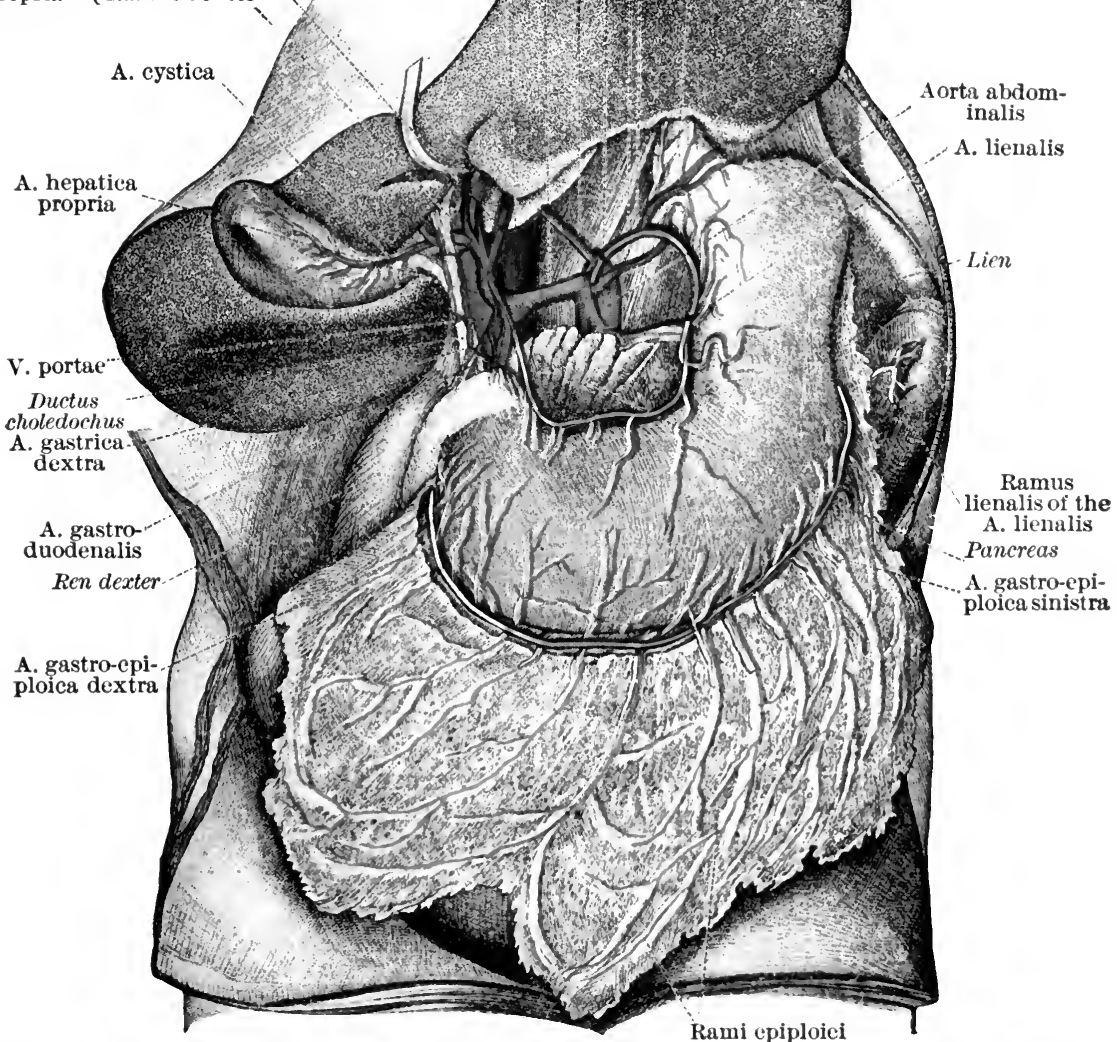

The tri-partition of the coeliac artery, Tripus coeliacus (Halleri), shown from in front after removal of the lesser omentum. (After Toldt, Anat. Atlas, Wien, 1900, 2 Aufl., p. 576, Fig. 952.)

Coeliac Artery (Arteria coeliaca) and its Branches. (Fig. 258.)

Trace the hepatic artery, the main branches of which have already been dissected out, back to its parent stem, the coeliac artery (A. coeliaca) (O. T. coeliac axis).

Note its exact relations to the vertebrae, pancreas, etc. Dis- 
sect out carefully the sympathetic nerves (plexus coeliacus) accompanying this artery and its branches. Preserve these as far as possible. Examine the origins of the following branches:

(a) Left gastric artery (A. gastrica sinistra). (aa) Oesophageal branches (rami oesophagi).

(b) Hepatic artery (A. hepatica). The branches of this artery have already been traced to their destinations.

(c) Lienal or splenic artery (A. lienalis). This artery can be seen to the best advantage if the stomach is turned upward towards the thorax. Note carefully its relations to the panereas and the pancreaticolienal and lienorenal ligaments.

(ca) Pancreatic branches (rami pancreatici).

( $c b)$ Left gastro-epiploic artery (A. gastro-epiploica sinistra).

(cc) Short gastric arteries (Aa. gastricae breves).

(cd) Splenic branches (rami lienales).

\section{Duodenum. (Vide Figs. 259, 261, 262, and Spalteholz, Fig. 563.)}

Study this part of the small intestine in your cadaver and compare it with the His models.

Divisions and angles.

(a) Superior part (pars superior), or first portion.

(b) Descending part (pars descendens), or second portion.

(c) Inferior part (pars inferior).

(ca) Horizontal part (pars horizontalis [inferior]), or third part.

$(c b)$ Ascending part (pars ascendens), or fourth part.

(d) Superior duodenal flexure (flexura duodeni superior).

(e) Inferior duodenal flexure (flexura duodeni inferior).

( $f$ ) Duodenojejunal flexure (flexura duodenojejunalis).

Three types of duodena are described.

(1) The annular type is met with in the second half of foetal life (Toldt). It is found almost always in the infant and is known as the infantile type of Jonnesco. Occasionally it persists to adult life.

(2) The U-shaped. The pars inferior is very long.

(3) The V-shaped. The pars descendens passes down almost vertically. The ascending portion passes upward and to the left, making an angle of $25^{\circ}$ to $40^{\circ}$ with the descending part. The pars horizontalis is lacking.

In what subdivisions of the abdomen is the duodenum situated? What is its relation to the upper and lower abdominal compartments and to the right and left colon pockets (separated by the radix mesenterii)?

How is it related to the liver, the pancreas, the ductus choledochus, the vertebrae, the right and left kidneys, the superior mesenteric artery and vein, the right spermatic artery, etc.? 
What are its peritoneal relations? Examine-

(a) Duodenorenal ligament (ligamentum duodenorenale).

(b) Duodenocolic ligament (ligamentum duodenocolicum). (Inconstant.)

The latter has been dissected away. Examine model and other cadavers and determine its location, if present.

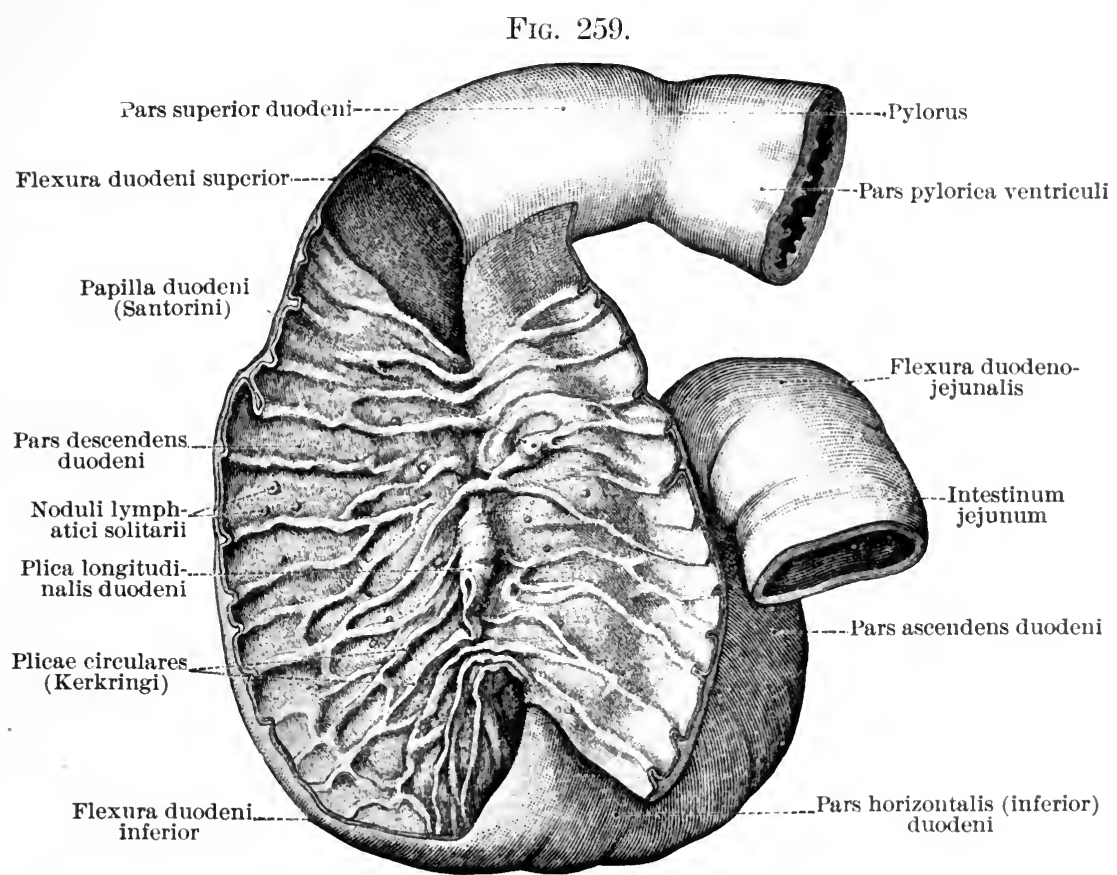

The duodenum seen from in front. The descending part has been cut open and its anterior wall turned back to the left. (After Toldt, Anat. Atlas, Wien, 1900, 2 Aufl., p. 424, Fig. 694.)

The duodenum is never displaced en masse, for the flexura duodenojejunalis is attached to the vertebral column. The first two portions are often displaced laterally or prolapsed secondary to the downward displacement of the stomach and liver. Determine by what means the duodenum is held in position.

Dissect out the suspensory muscle of the duodenum (M. suspensorius duodeni).

This muscle was described by Treitz in 1853 . It arises from the superior border of the flexura duodenojejumalis and the superior half of the pars ascendens. Passing upward behind the head of the pancreas and in front of the aorta, it is attached to the left pillar of the diaphragm by a tendon composed of elastic and fibrous tissue. It is composed of smooth muscle-fibres. According to Braune, it is derived from both coats of the intestine. 
Incise the ductus choledochus, the ductus pancreaticus [Wirsungi], and the ductus pancreaticus accessorius [Santorini], pass probes (or broom-straws) through these into the duodenum; just below the pylorus, apply two ligatures about the duodenum, cut between, and remove the duodenum from the abdomen. Remove ligatures, wash the lumen out carefully, incise along the convex margin, and study in the duodenum-

(a) Circular folds (plicae circulares [Kerkringi]).

(b) Intestinal villi (villi intestinales).

(c) Solitary lymph-nodes (noduli lymphatici solitarii).

(d) Duodenal papilla (papilla duodeni [Santorini]).

(e) Longitudinal fold of duodenum (plica longitudinalis duodeni).

Note that this fold is formed by the ductus choledochus and leads up to the common opening of the ductus choledochus and ductus pancreaticus.

Spread the duodenum out upon a cork dissecting tray, pin it down, and study its coats.

(a) Serous coat (tunica serosa).

(b) Muscular coat (tunica muscularis).

(ba) Longitudinal layer (stratum longitudinale).

(bb) Circular layer (stratum circulare).

(c) Submucous coat (tela submucosa).

(d) Mucous coat (tunica mucosa).

(da) Muscular layer of the mucosa (lamina muscularis mucosae). (db) Proper mucous coat (tunica propria mucosa).

Fig. 260.

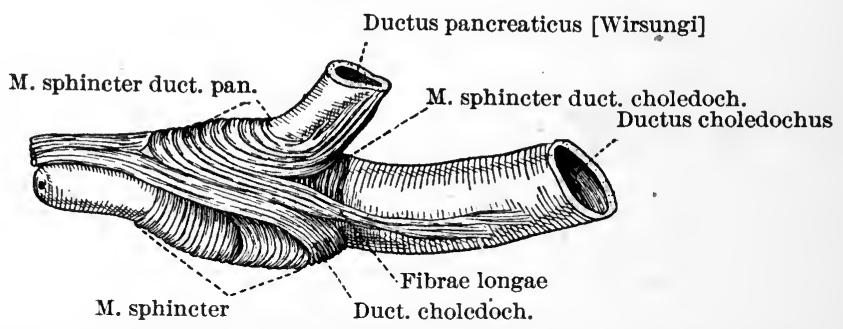

Sphincter muscle of the ductus choledochus and of the ductus pancreaticus in man (after Hendrickson). (From Poirier et Charpy, Traité d'Anat. hum., Paris, 1901, 2 ed., t. iv. p. 796, Fig. 411.)

Use a lens and look for the duodenal glands of Brunner ( $G l$. duodenales [Brunneri]) in the tela submucosa.

Throw the lower end of the ductus choledochus and a small adjacent portion of the duodenum into a dish containing equal parts of nitric acid, alcohol, and glycerin for a few hours. Then transfer to water, and with delicate instruments isolate the 
sphincter muscle of the bile-duct. (Cf. Fig. 260, and Hendrickson's description.)

\section{Pancreas. (Vide Figs. 26I-3, and Spalteholz, Figs. 584, 585.)}

Review the embryology of the pancreas. What are the ventral and dorsal anlages? (Vide Kollmann, Entwickelungsgeschichte des Menschen, p. 370.)

Study the pancreas in your cadaver and make frequent use of models and atlases. The secretion of the gland is called the pancreatic juice (succus pancreaticus).

FIG. 261.

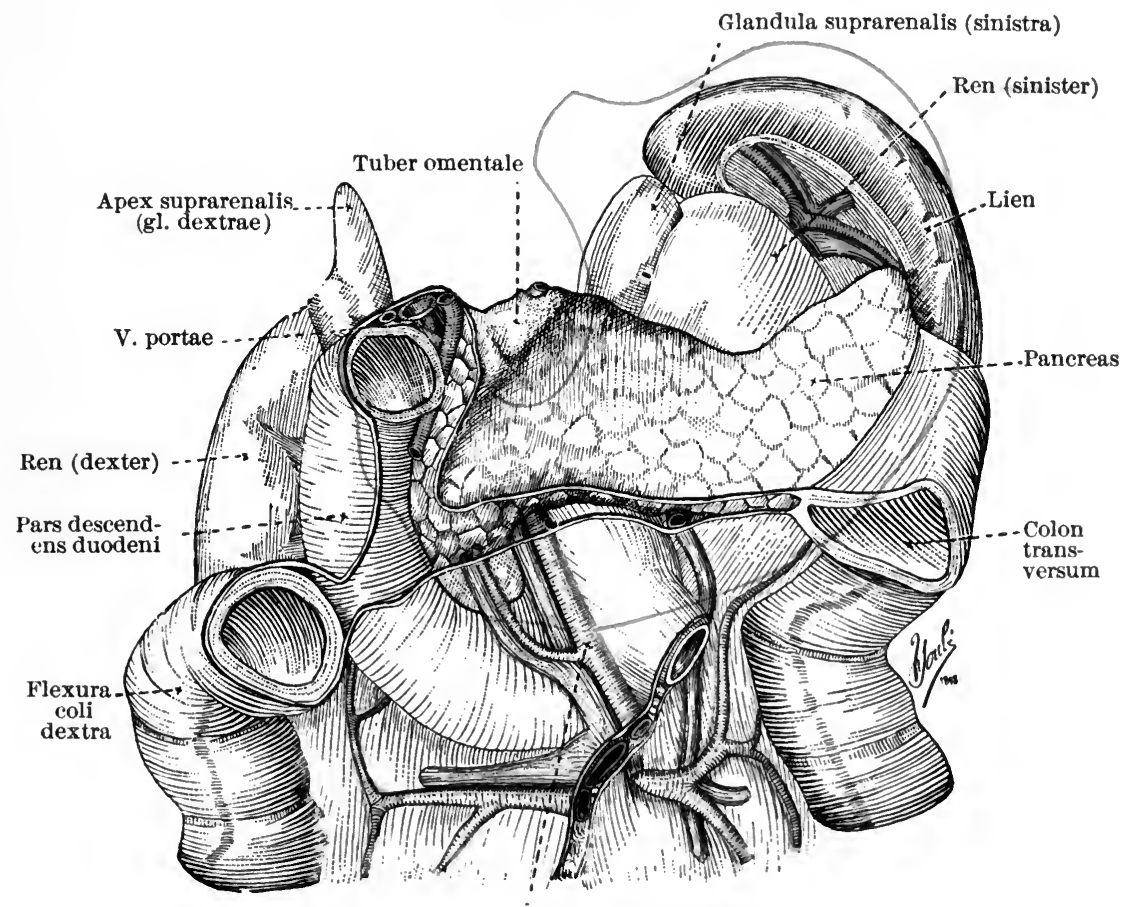

A. mesenterica superior

Relations of the pancreas. After models by His. The contour of the stomach is projected in red.

(From Poirier et Charpy, Traité d'Anat. hum., Paris, 1901, 2 ed., t. iv. p. 806, Fig. 415.)

Divisions.

(a) Head of pancreas (caput pancreatis).

(b) Uncinate process (processus uncinatus [pancreas Winslowi]).

(c) Notch of the pancreas (incisura pancreatis).

(d) Body of pancreas (corpus pancreatis). (da) Anterior surface (facies anterior).

(db) Posterior surface (facies posterior). 
(dc) Inferior surface (facies inferior).

(dd) Superior margin (margo superior).

(de) Anterior margin (margo anterior).

$(d f)$ Posterior margin (margo posterior).

(e) Omental tuber (tuber omentale).

(f) Tail of pancreas (cauda pancreatis).

Relations.

Note carefully the relations of the pancreas to the lumbar vertebrae, the stomach, the left kidney, the spleen, the superior mesenteric vessels, the splenic vessels, the inferior mesenteric vein, the ligamentum pancreaticolienale, and the duodenum. What are "fasciae of fusion," and what relation has the pancreas to such? (Vide Figs. 263, $A$ and $B$.) Dissect away some of the parenchyma of the gland and determine, if possible, the source and relations of the pancreatic ducts: ${ }^{1}$

FIG. 262.

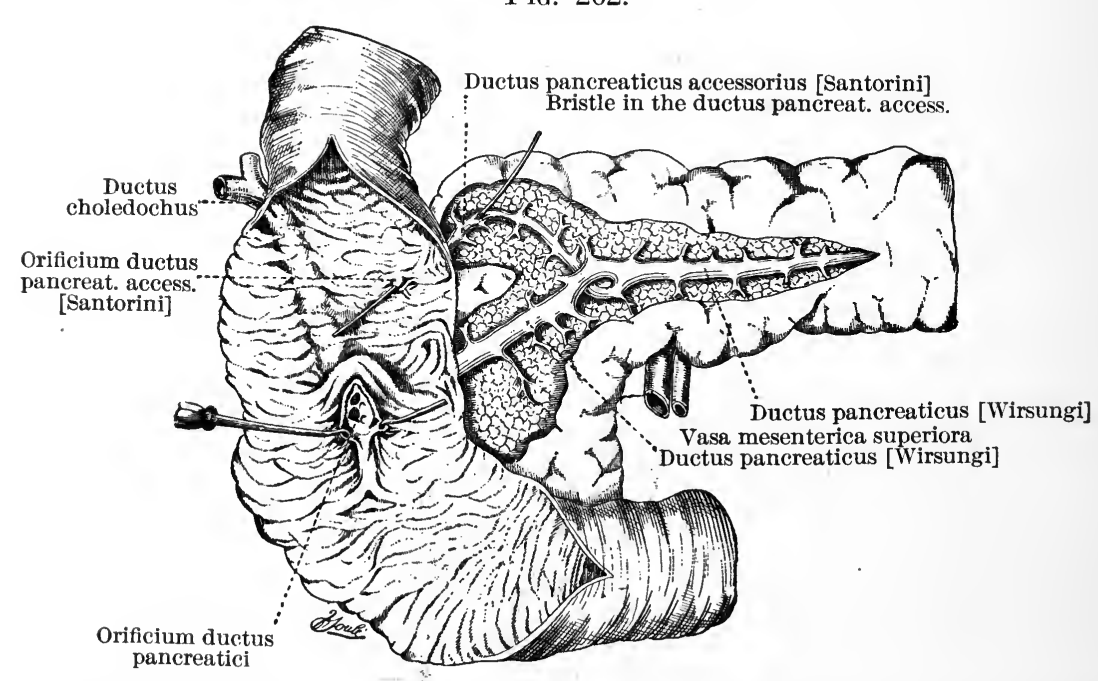

Duodenal openings of the ductus pancreaticus [Wirsungi] and ductus pancreaticus accessorius [Santorini]. (After Schiomer.) A bristle has been passed into the accessory duct. (From Poirier et Charpy, Traité d'Anat. hum., Paris, 1901, 2 ed., t. iv. p. 811, Fig. 417.)

(a) Pancreatic duct of Wirsung (ductus pancreaticus [Wirsungi]).

(b) Accessory pancreatic duct of Santorini (ductus pancreaticus accessorius [Santorini]).

\section{Is an "accessory pancreas" (pancreas.accessorium) pres- ent?}

${ }^{1}$ For a method of studying the pancreatic ducts; see "Pancreatic Ducts in the Dog," by D. G. Revell, in Amer. Jour. Anat., vol. i. p. 443. 


\section{Vagus Nerves (O. T. Pneumogastric Nerves). (Vide Toldt, Fig.} I322.)

Depress the stomach and bring well into view the abdominal part (pars abdominalis) of the oesophagus. Dissect out carefully the nerves descending upon the anterior and posterior sur-

Fig. 263.

A

Omentum minus A. gastrica sinistra

A. hepatica propria Ductus choledochus

Paries abdom. post. Peritonaeum parietale

Ren (dexter)

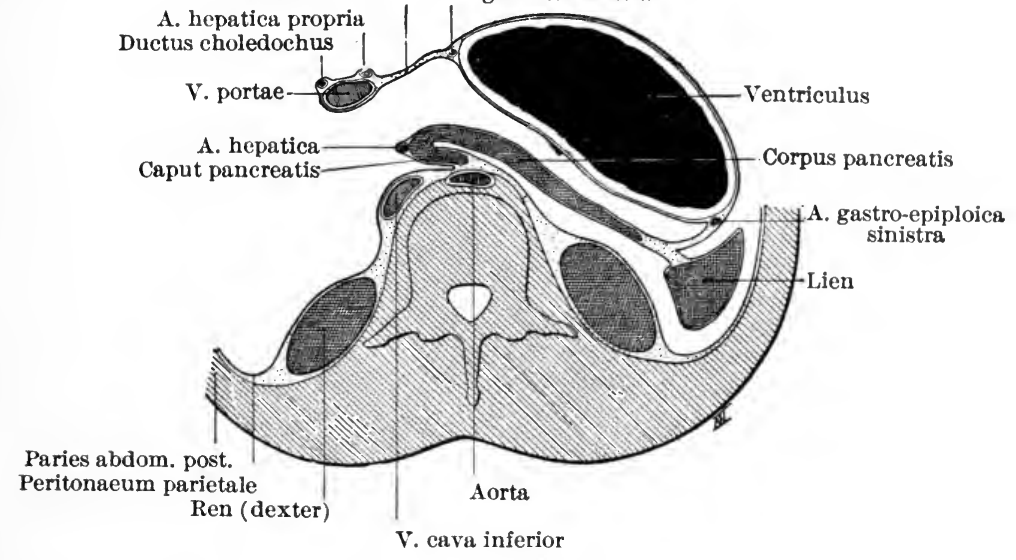

$B$

Omentum minus

A. hepatica propria Ductus choledochus

A. gastrica sinistra

V. portae

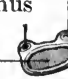

A. hepatica

Fusion of the caput pancreatis to the paries abdominis (fascia of Treitz)

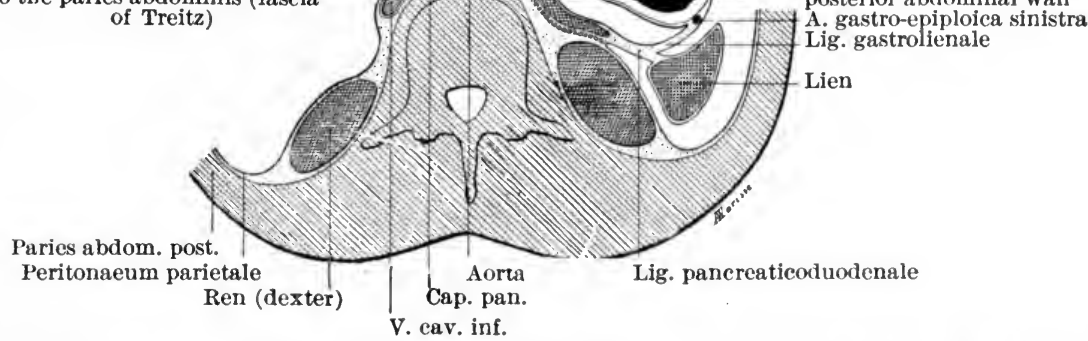

Scction passing through the hepatic arch, above the pylorus and through the body and head of the pancreas, to show the situation and origin of the "fasciae of fusion." The pancreatic plica is to be seen above and to the left of the hepatic. In $A$ the fusions have been destroyed and the mesos thereby restored to their primitive independence. In $B$ the adult type is shown. The caput pancreatis and the mesoduodenum lodging it have been turned over to the right. The corpus et cauda pancreatis, contained within the omentum majus, lie in front of the caput pancreatis on the right side, and in front of the posterior abdominal wall on the left. In $B$ the retropancreatic "fasciac of fusion" may be seen (the fascia of Trietz behind the caput pancreatis, the fascia of Toldt behind the corpus pancreatis). (From Poirier et Charpy, Traité d'Anat. hum., Paris, 1901, 2 ed., t. iv. p. 976, Fig.548 $A$ and $B$.) 
faces and trace these upon the stomach. Some filaments will have been destroyed in the earlier dissections.

In connection with the abdominal part of the vagus nerve (N. vagus) study-

(a) Gastric branches (rami gastrici).

(b) Anterior gastric plexus (plexus gastricus anterior).

(c) Posterior gastric plexus (plexus gastricus posterior).

(d) Hepatic branches (rami hepatici).

(e) Coeliac branches (rami coeliaci).

(ea) Splenic branches (rami lienales).

(eb) Renal branches (rami renales).

Stomach (Ventriculus). (Vide Spalteholz, Figs. 557-560.)

Pass two ligatures about the abdominal portion of the oesophagus, cut between, and remove the stomach. Use models to determine its exact form, position, relations, etc. What is the direction of the axis of this organ? Note that the upper twothirds is vertical, while the lower one-third passes to the right, upward and slightly backward. These two parts make an angle with each other which varies between $60^{\circ}$ and $70^{\circ}$. For a full discussion concerning the exact position of these two parts, consult the systematic text-books.

Examine-

(a) Anterior wall (paries anterior).

(b) Posterior wall (paries posterior).

(c) Greater curvature of stomach (curvatura ventriculi major).

(d) Lesser curvature of stomach (curvatura ventriculi minor).

(e) Cardia (cardia).

(f) Fundus of stomach (fundus ventriculi).

(g) Body of stomach (corpus ventriculi).

(h) Pylorus (pylorus).

(i) Cardiac part (pars cardiaca).

(j) Pyloric part (pars pylorica).

(k) Cardiac antrum (antrum cardiacum). This is only occasionally present.

(l) Pyloric antrum (antrum pyloricum).

How much of the stomach is to the left of the median plane? How much to the right? What is the relation of the cardia to the sternal extremity of the seventh costal cartilage on the left side? What is the relation of the pylorus to the median plane and to the umbilicus? Draw a line uniting the medial extremities of the ninth costal cartilages. What is the position of the pylorus in relation to this line? What is the position of the greater curvature? Does it vary? The relation of the stomach to each 
neighboring viscus as shown in the models should be carefully studied.

What is the "semilunar space of Traube"? What is a bilocular or hour-glass stomach? Fill the stomach with water and determine its capacity. Does the axis of the stomach change when filled?

Fig. 264.

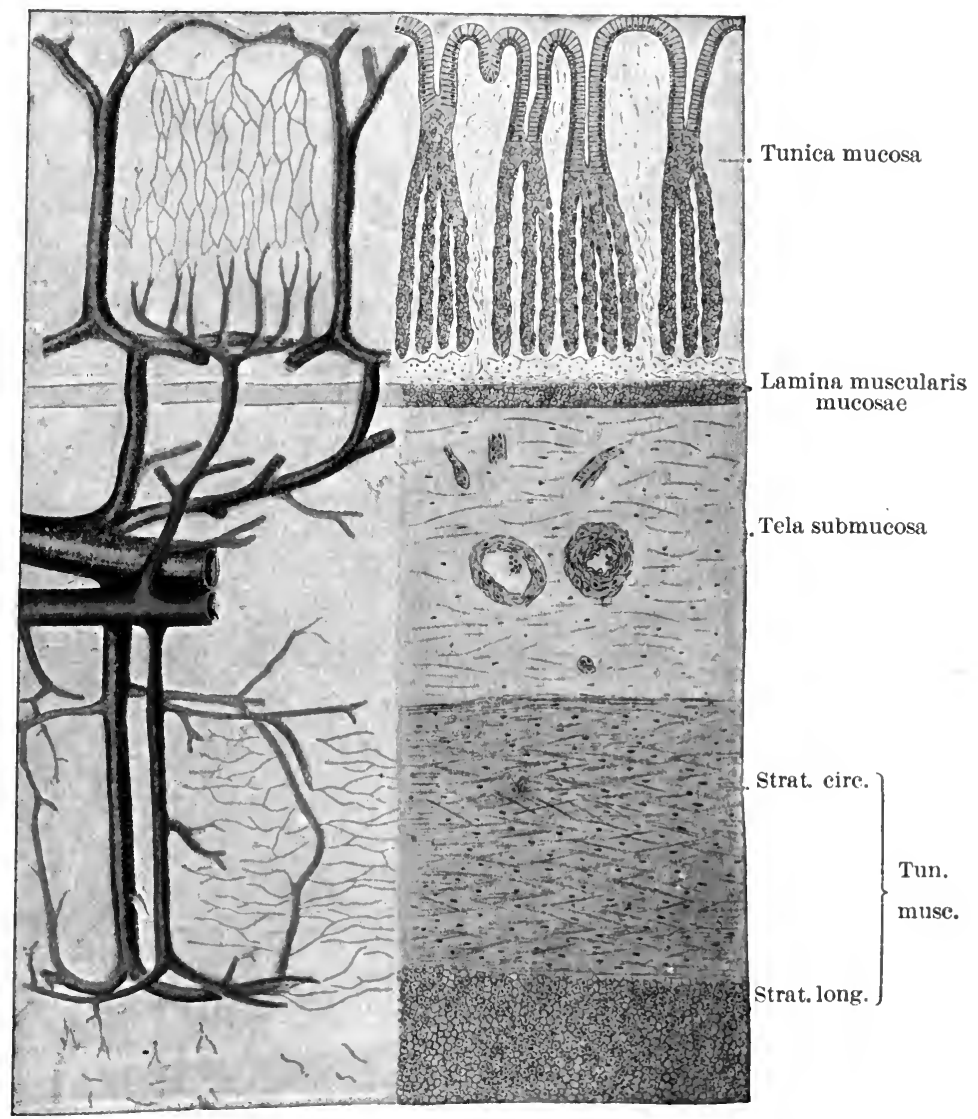

Transverse section of the wall of a dog's stomach. (After Mall.) On the right the tunics are superimposed, on the left the vessels and vascular plexuses are shown. (From Poirier et Charpy, Traite d'Anat. hum., Paris, 1901, 2 ed., t. iv. p. 229, Fig. 122.)

Open the stomach along its greater curvature, spread it out, pin it down upon a cork dissecting tray, and study its coats and the valve and muscle derived from them. (Cf. Fig. 264.) Also consult "The Vessels and Walls of the Dog's Stomach," by Professor Mall, in Johns Hopkins Hosp. Reports, vol. i. p. 1. 
(a) Serous coat (tunica serosa).

(b) Muscular coat (tunica muscularis).

(ba) Longitudinal layer (stratum longitudinale).

(bb) Pyloric ligaments (Ligg. pylori).

(bc) Circular layer (stratum circulare).

$(b d)$ Sphincter muscle of pylorus (M. sphincter pylori).

(be) Oblique fibres (fibrae obliquae).

(c) Pyloric valve (valvula pylori).

(d) Submucous layer (tela submucosa).

(e) Mucous coat (tunica mucosa). Can you make out the lamina muscularis mucosae?

(ea) Gastric areas (areae gastricae).

(eb) Mucous folds (plicae mucosae).

(ec) Gastric pits (foveolae gastricae).

(ed) Gastric lymph-nodules (noduli lymphatici gastrici).

With a loop (lens) look at the openings of the gastric glands.

(a) [Proper] gastric glands (glandulae gastricae [propriae]).

(b) Pyloric glands (glandulae pyloricae).

There are only a few true " cardiac" glands in the human stomach. (Cf. Bensley, R. R., "The Cardiac Glands of Mammals,"' Am. J. Anat., Balt., 1902, vol. ii. p. 105.)

The mixed secretion of the glands is called the gastric juice (succus gastricus).

\section{Abdominal Part of Sympathetic System (Pars abdominalis S. sym- pathici). (Vide Toldt, Fig. I332.)}

Dissect out carefully the sympathetic nerves and ganglia situated about the coeliac artery. Determine the course of the different plexuses which surround the arteries given off from the main trunk.

(a) Coeliac plexus (plexus coeliacus).

(b) Coeliac ganglia (ganglia coeliaca).

(c) Phrenic plexus (plexus phrenicus).

(ca) Phrenic ganglia (ganglia phrenica).

(d) Hepatic plexus (plexus hepaticus).

(e) Splenic plexus (plexus lienalis).

(f) Renal plexus (plexus renalis).

(g) Suprarenal plexus (plexus suprarenalis).

(h) Superior gastric plexus (plexus gastricus superior).

(i) Inferior gastric plexus (plexus gastricus inferior).

The two latter have been destroyed in the removal and dissection of the stomach. Examine models to determine their exact position and the arteries that they accompany.

Dissect carefully the abdominal aorta and study-

(j) Abdominal aortic plexus (plexus aorticus abdominalis). It has already been examined. 
What is the relation between the ganglia coeliaca and the $\mathrm{Nn}$. splanchnici majores? What are the lesser splanchnic nerve $(N$. splanchnicus minor) and its renal branch (ramus renalis)? These nerves are studied in the upper part of their course in the dissection of the thorax.

FIG. 265.

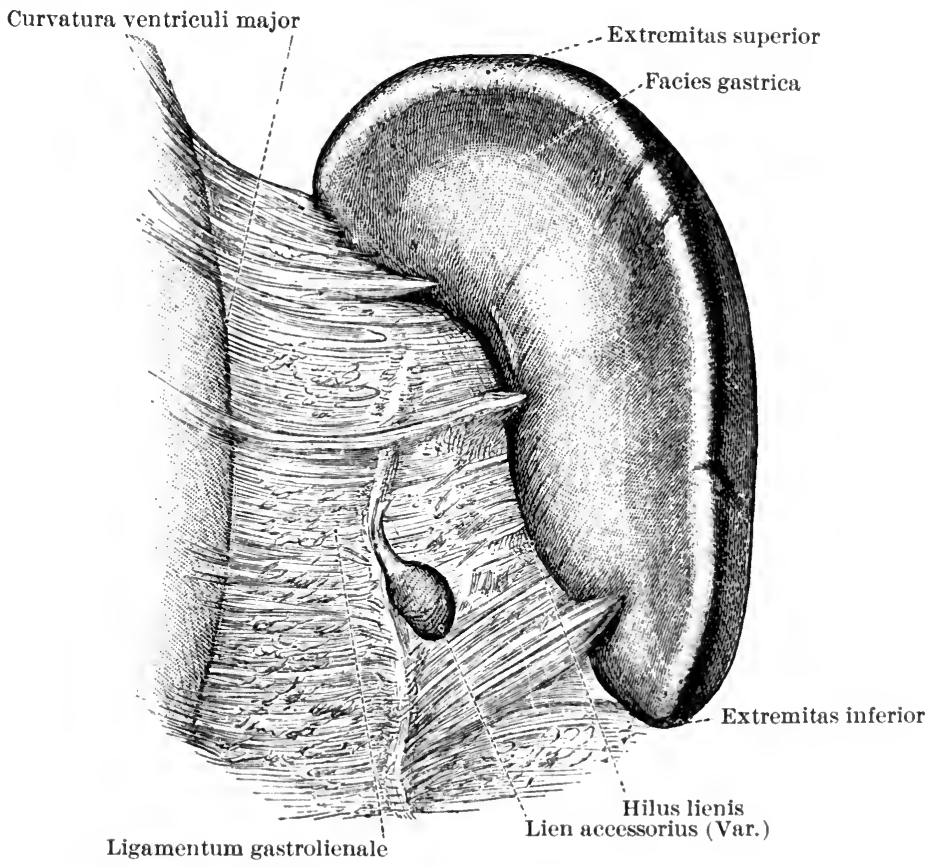

The spleen in conjunction with the ligamentum gastrolienale. Seen from in front. (After Toldt, Anat. Atlas, Wien, 1900, 2 Aufl., p. 434, Fig. 723.)

\section{Spleen (Lien). (Vide Figs. 261, 263, 265, and Spalteholz, Fig. 587.)}

Remove the spleen from your subject. It has already been examined in position in the cadaver. Examine models to determine the exact position of the organ in cadavers with the organs hardened in situ and its relation to vertebrae, ribs, and neighboring viscera. Note the following:

Surfaces.

(a) Diaphragmatic surface (facies diaphragmatica).

(b) Renal surface (facies renalis).

(c) Gastric surface (facies gastrica).

Extremities.

(a) Superior extremity (extremitas superior).

(b) Inferior extremity (extremitas inferior). 
Margins.

(a) Posterior margin (margo posterior).

(b) Anterior margin (margo anterior).

Note especially the crenations in this anterior border. It is often referred to as the "margo crenatus." These crenations are of importance, as they enable the clinician to differentiate between enlargement of the spleen and of the kidney. The rounded prominence separating the gastric and renal surfaces is sometimes described as the margo intermedius.

Examine the hilus of the spleen (hilus lienis).

Four layers of peritoneum will be found at the hilus. The two anterior layers are derived from the ligamentum gastrolienale, while the two posterior layers are derived from the ligamentum pancreaticolienale. Determine from which peritoneal sac each layer of this peritoneum is derived, noting that the relation is the same as found in the omentum majus. Between the two layers of the ligamentum pancreaticolienale will be found the tail of the pancreas and the splenic vessels.

Incise the spleen and examine-

(a) Serous coat (tunica serosa).

(b) Albugineous coat (tunica albuginea).

(c) Splenic pulp ( $p$ ulpa lienis).

(d) Splenic rami of splenic artery (rami lienales [arteriae lienalis]).

(e) Splenic lymph-nodules (noduli lymphatici lienales [Malpighii]) (O. T. Malpighian corpuscles).

Instructive pictures of the splenic framework can be obtained by allowing the organ to decompose partially and then washing out the pulp and blood and drying by inflation through one of the veins.

What is an accessory spleen (lien accessorius)? How many of these may be found, and where are they generally located?

Review the development of the spleen and its relation to the mesogastrium posterius.

Liver (Hepar). (Vide Figs. 266, 267, and Spalteholz, Figs. 579-58r.)

Review the development of the liver. Its position in the cadaver has already been studied. Use models to determine its exact relations, form, and position in a hardened bady. Remove the liver from the abdominal cavity. Cut through the ligamentum teres and ligamentum falciforme. Divide the triangular ligaments on each side and the upper layer of the coronary ligament. Separate the posterior surface of the liver from the dia- 
phragm by blunt dissection, noting the amount of areolar tissue. Next divide the vena cava inferior and the lower layer of the coronary ligament. The inferior vena cava must be divided a second time at the point where it first comes in contact with this viscus before the liver can be removed from the abdominal cavity.

Lobes.

(a) Right lobe of liver (lobus hepatis dexter).

(b) Quadrate lobe (lobus quadratus).

(c) Caudate lobe (lobus caudatus [Spigeli]).

(ca) Papillary process (processus papillaris).

( $c b)$ Caudate process (processus caudatus).

(d) Left lobe of liver (lobus hepatis sinister).

Fig. 266.

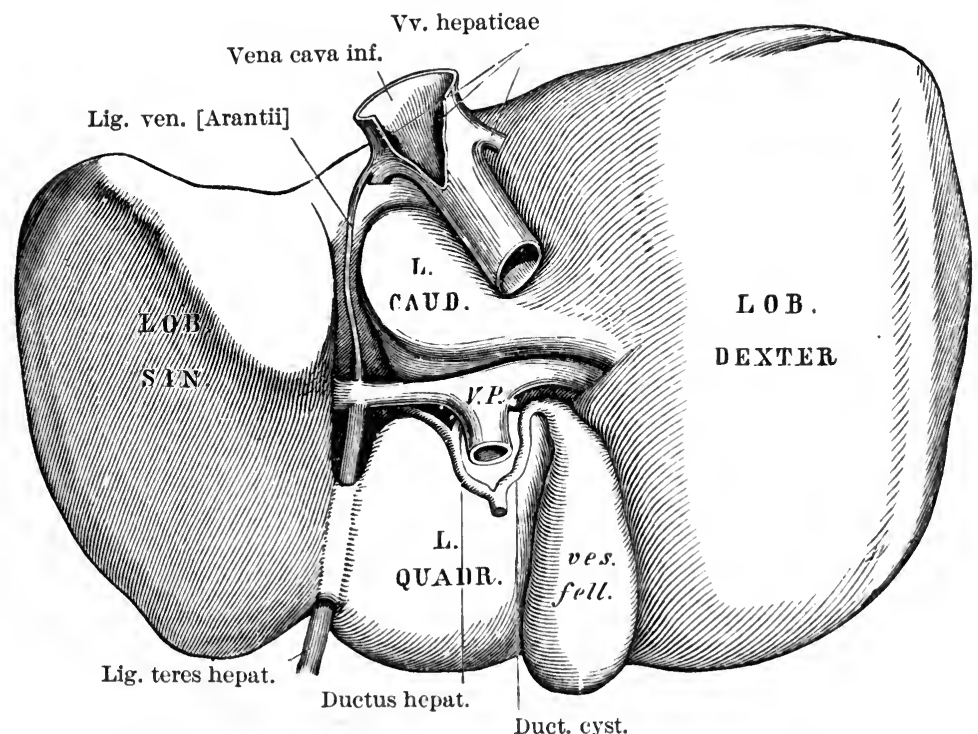

The liver, from behind and below. (From Gegenbaur, Lehrb. der Anat. des Mensch., Leipzig, 1899, 7 Aufl., Bd. ii. p. 75, Fig. 401.)

Surfaces.

(a) Superior surface (facies superior).

(b) Posterior surface (facies posterior).

(c) Inferior surface (facies inferior).

Ligaments.

(a) Round ligament of liver (ligamentum teres hepatis).

(b) Venous ligament (ligamentum venosum [Arantii]).

(c) Falciform ligament (ligamentum falciforme hepatis).

(d) Coronary ligament (ligamentum coronarium hepatis).

(e) Right triangular ligament (ligamentum triangulare dextrum).

(f) Left triangular ligament (ligamentum triangulare sinistrum). 
Impressions.

(a) Cardiac impression (impressio cardiaca).

(b) Oesophageal impression (impressio oesophagea).

(c) Gastric impression (impressio gastrica).

(d) Duodenal impression (impressio duodenalis).

(e) Colic impression (impressio colica).

(f) Renal impression (impressio renalis).

(g) Suprarenal impression (impressio suprarenalis).

Fig. 267.

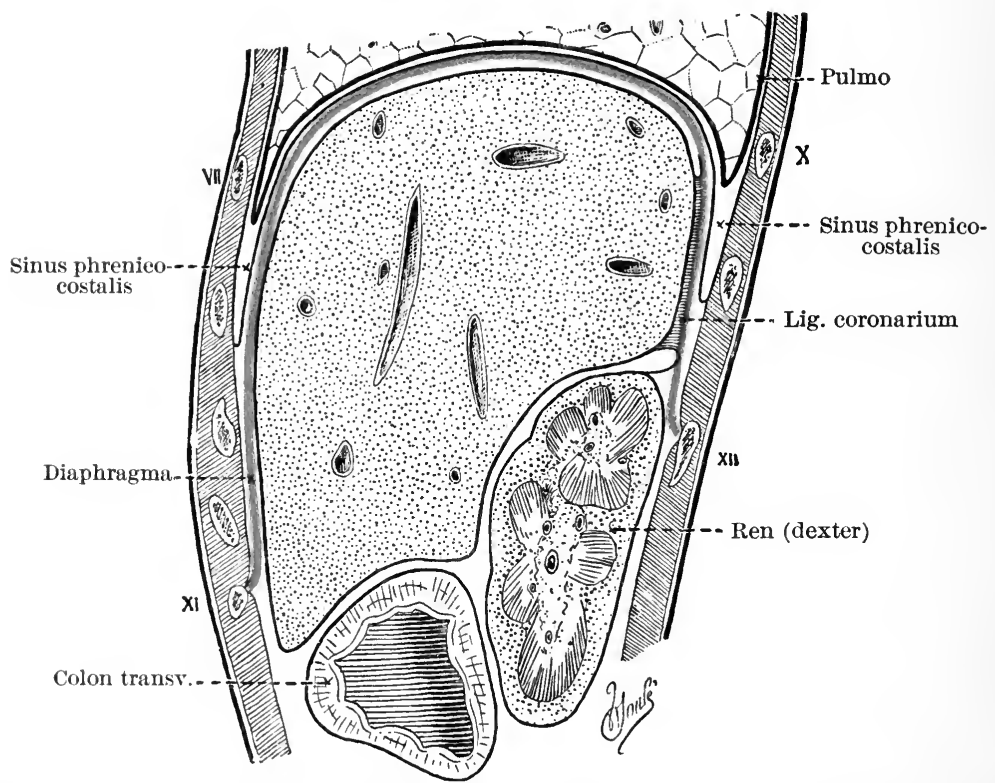

Sagittal section through the liver. The section passes through the regio hypochondriaca dextra.

(From Poirier et Charpy, Traité d'Anat, hum., Paris, 1901, 2 ed., t. iv. p. 711, Fig. 367.)

Fossae.

(a) Right sagittal fossa (fossa sagittalis dextra).

(aa) Fossa for gall-bladder (fossa vesicae felleae).

(ab) Fossa for vena cava (fossa venae cavae).

(b) Left sagittal fossa (fossa sagittalis sinistra).

(ba) Fossa for umbilical vein (fossa venae umbilicalis).

(bb) Fossa for venous duct (fossa ductus venosi).

Examine also-

(a) Anterior margin (margo anterior).

(b) Omental tuber (tuber omentale).

(c) Umbilical notch (incisura umbilicalis).

(d) Fibrous appendix of liver (appendix fibrosus hepatis). 
Make several incisions in the liver from right to left. Do not injure the gall-bladder. Examine first with naked eye and then with a loop-

(a) Lobules of liver (lobuli hepatis).

(b) Fibrous capsule of Glisson (capsula fibrosa [Glissoni]).

(c) Interlobular arteries (rami arteriosi interlobulares).

(d) Interlobular veins (venae interlobulares).

(e) Central veins (venae centrales). How can you differentiate $(d)$ and $(e)$ from each other?

( $f$ ) Bile-ducts (ductus biliferi) (O. T. bile canaliculi).

(g) Interlobular ducts (ductus interlobulares).

What are costal and diaphragmatic sulci in the liver? What is the general direction of these, when present? What is a " Riedel's lobe" in the liver?

Note the exact peritoneal relations, determining how much of the liver is not covered by peritoneum.

\section{Gall-bladder (Vesica fellea).}

Study-

(a) Fundus of gall-bladder (fundus vesicae felleae).

(b) Body of gall-bladder (corpus vesicae felleae).

(c) Neck of gall-bladder (collum vesicae felleae).

Is the gall-bladder completely covered by peritoneum?

Open the gall-bladder and dissect out the following coats:

(a) Serous coat (tunica serosa vesicae felleae).

(b) Muscular coat (tunica muscularis vesicae felleae).

(c) Mucous coat (tunica mucosa vesicae felleae).

(ca) Folds of mucous coat (plicae tunicae mucosa vesicae felleae).

Note the relation of the cystic duct to the gall-bladder. Open it and study the spiral valve of Heister (valvula spiralis [Heisteri]). How far does it extend in your subject? What coats take part in the formation of this valve? In the biliary mucous membrane look for glands (Gl. mucosae biliosae).

\section{UROPOËTIC ORGANS (ORGANA UROPOËTICA).}

These include the kidney (ren), ureter, and urinary bladder (vesica urinaria). Sometimes the suprarenal gland (Gl. suprarenalis) is classed here, but probably incorrectly. 


\section{Kidney (Ren). (Vide Fig. 27I, and Spalteholz, Figs. 634-636.)}

Study the exact relation of the kidneys to the vertebrae, the psoas and quadratus lumborum muscles, the twelfth rib, and the crest of the ilium. Which kidney is the lower? How do you account for this low position? Note the relation of the right kidney to the liver, the duodenum, and the flexura coli dextra, and the relation of the left kidney to the pancreas; the stomach, the spleen, and the flexura coli sinistra.

\section{Direction and Orientation.}

The kidney is not vertically placed. Its axis is directed obliquely downward and lateralward. The superior extremity approaches the median line. The anterior surface is directed lateralward and the posterior medialward.

Perirenal Fascia. (Figs. 268 and 269.) ${ }^{1}$

(a) Retrorenal layer.

This layer of fascia is found between the posterior surface of the kidney and the aponeurosis of the quadratus lumborum muscle. It covers the sheath of the psoas muscle, reinforeing it, and is attached to the vertebrae and intervertebral disks, medial to the attachment of the psoas. It does not become continuous with the corresponding fascia of the opposite side.

(b) Prerenal fascia.

This layer of fascia follows closely the reflections of the peritoneum about the kidneys. It passes in front of each kidney and its corresponding pedicle, the aorta and inferior vena cava, to become continuous with the corresponding fascia of the opposite side.

If these two fasciae are traced longitudinally, they will be found to fuse above and become attached to the diaphragm. Below, fusion does not occur; the anterior layer follows the reflections of the peritoneum, while the posterior layer divides into several lamellae which fuse with the cellular tissue of the iliac fossa.

\section{Vessels of the Kidney.}

In injected specimens study-

Arteries of Kidney (Arteriae renales).

(a) Interlobar arteries of kidney (Aa. interlobares renis).

(aa) Arciform arteries or renal arches (Aa. arciformes).

(ab) Interlobular arteries (Aa. interlobulares).

${ }^{1}$ For a description of the perineal fascia, consult "The Present Conception of the Perineal Fascia and its Rôle in Fixation of the Kidney," by D. D. Lewis, in Jour. Amer. Med. Assoc., vol. xlii. p. 701. 
Fig. 268.

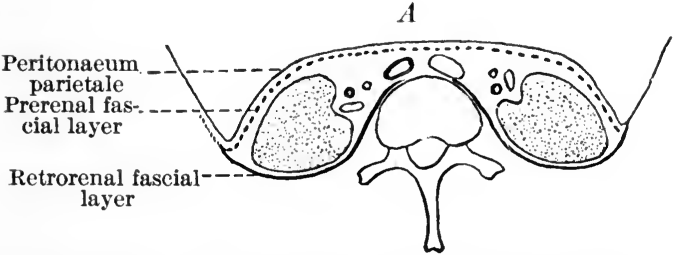

The perirenal fascia, comprising the two layers, prerenal and retrorenal. (After Poirier et Charpy, Traité d'Anat. hum., Paris, 1901, 2 ed., t. v. p. 10, Fig. 6.)

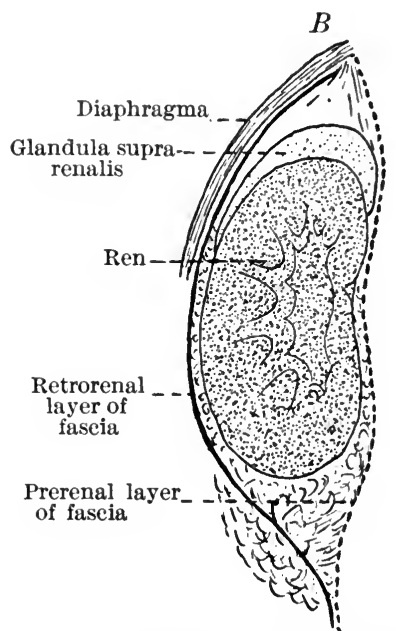

The perirenal fascia as seen in a longitudinal section of the kidney. Diagrammatic. (After Poirier et Charpy, Traité d'Anat. hum., Paris, 1901, 2 ed., t. v. p. 11, Fig. 7.)

FIG. 269.
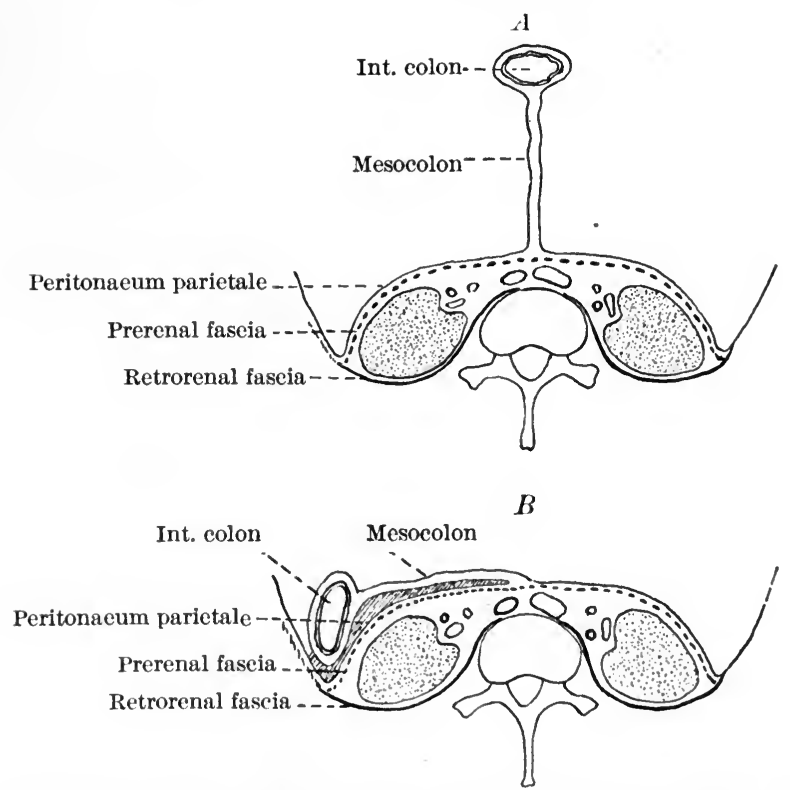

The formation of the "faseia of Toldt." The colon being pushed into the posterolateral part of the abdominal cavity, $(B)$ has its mesocolon brought into contact with the posterior abdominal wall with the peritoneum of which it fuses as indicated by the shading. (After Poirier et Charpy, Traite d'Anat. hum., Paris, 1901, t. v. p. 12, Figs. 8 and 9.) 
(aba) Afferent vessel (vas afferens). ( $a b b)$ Efferent vessel (vas efferens).

(ac) Capsular branches (rami capsulares).

(ad) Straight arterioles (arteriolae rectae).

(ae) Nutrient arteries of renal pelvis (Aa. nutricae pelvis renalis).

Veins of Kidney (Venae renales).

(a) Interlobar veins ( $V v \cdot$ interlobares).

(aa) Arciform veins ( $V v$. arciformes).

(ab) Interlobular veins ( $V v$. interlobulares).

(ac) Straight venules (venulae rectae).

(ad) Stellate veins (venae stellatae) (O. T. veins of Verheyn).

Examine corrosion preparations of the renal blood-vessels in the anatomical museum.

Examine the adipose capsule (capsula adiposa). Does it assist in maintaining the organ in position? What is the "pararenal adipose body" of Gerota?

Cut the blood-vessels and ureter some distance from the kidney and examine-

(a) Lateral margin (margo lateralis).

(b) Medial margin (margo medialis).

(ba) Renal hilus (hilus renalis).

(bb) Renal sinus (sinus renalis).

(c) Anterior surface (facies anterior).

(d) Posterior surface (facies posterior).

(e) Superior extremity (extremitas superior).

(f) Inferior extremity (extremitas inferior).

(g) Muscular impression (impressio muscularis).

(h) Hepatic impression (impressio hepatica).

(i) Gastric impression (impressio gastrica).

Incise the kidney along its lateral margin, cutting through its fibrous capsule (tunica fibrosa). Does it strip readily from the surface of the kidney? What is the muscular tunic (tunica muscularis)?

Divide the kidney into two symmetrical halves. (Cf. Spalteholz, Fig. 637.) Study-

(a) Cortical substance (substantia corticalis).

(b) Medullary substance (substantia medullaris).

(c) Renal lobes (lobi renales) (O. T. reniculi).

(d) Renal pyramids (pyramides renales [Malpighii]).

(e) Base of pyramid (basis pyramidis).

(f) Renal papillae (papillae renales).

(g) Papillary foramina (foramina papillaria).

(h) Renal columns (columnae renales [Bertini]).

(i) Cortical lobules (lobuli corticales). 
(ia) Radiate part (pars radiata [processus Ferreini]) (O. T. pyramid of Ferrein).

(ib) Convoluted part (pars convoluta) (O. T. labyrinth).

(j) Renal corpuseles (corpuscula renis [Malpighii]).

(ja) Glomeruli.

(jaa) Capsula glomeruli.

(k) Renal pelvis (pelvis renalis). (Fig. 270.)

(l) Renal calyces (calyces renales).

(la) Greater renal calyces (calyces renales majores).

(lb) Smaller renal calyces (calyces renales minores).

$(m)$ Glands of renal pelvis (Gl. pelvis renalis).

How can you determine to which side each kidney belongs?

Suprarenal Glands (Glandulae suprarenales). (Vide Fig. 27I, and Spalteholz, Fig. 644. $)^{1}$

Study-

(a) Hilus of suprarenal gland (hilus glandulae suprarenalis).

(b) Anterior surface (facies anterior).

(c) Posterior surface (facies posterior).

(d) Base of suprarenal gland (basis glandulae suprarenalis).

(e) Apex of the right suprarenal (apex suprarenalis [Gl. dextrae]).

(f) Superior margin (margo superior).

(g) Medial margin (margo medialis).

Cut through the suprarenal gland and study the general characteristics of-

(a) Cortical substance (substantia corticalis).

(b) Medullary substance (substantia medullaris).

Observe the central vein (vena centralis).

What are accessory suprarenal glands (Gl. suprarenales accessoriae), and where are they most frequently situated? Are portions of the suprarenal glands ever found in the kidney substance?

\section{Ureter.}

Trace the ureter downward into the pelvis. It is divisible into an abdominal part (pars abdominalis) and a pelvic part (pars pelvina). Study the exact relations of the abdominal part. The pelvic division will be studied later during the dissection of the pelvic viscera.

${ }^{1}$ Consult “ Blood-vessels, Angiogenesis, Organogenesis, Reticulum, and Histology of the Adrenal," by J. M. Flint, in Contributions to the Sci. of Med. by the pupils of Wm. H. Weleh, Baltimore, 1900. 
FIG. 270.
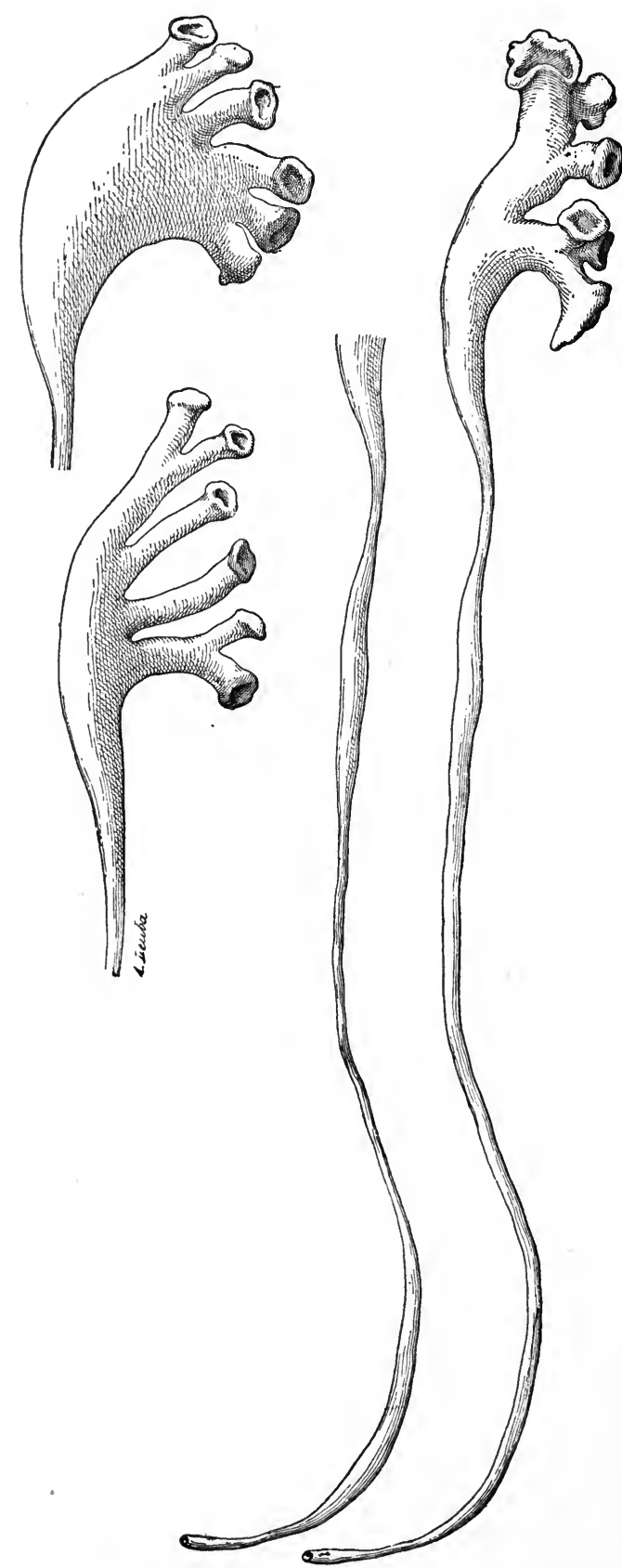

Casts of the calices renales, pelvis renalis, and ureter (Poirier). (From Poirier et Charpy, Traite d'Anat. hum., Paris, 1901, t. v. p. 57, Fig. 47.) 
Excise a portion of the abdominal part of the ureter. Make a dissection of its different coats, using a loop.

(a) Adventitious coat (tunica adventitia).

(b) Muscular coat (tunica muscularis).

(ba) External layer (stratum externum).

(bb) Middle layer (stratum medium).

(bc) Internal layer (stratum internum).

(c) Mucous coat (tunica mucosa).

(ca) Mucous glands of ureter ( $G l$. mucosae ureteris).

\section{MUSCLES, VESSELS, AND NERVES.}

\section{Diaphragm (Diaphragma).}

Strip the peritoneum from the under surface of the diaphragm, preserving carefully the vessels and nerves which ramify on its under surface. Review the development of the diaphragm. How do you explain a partial innervation by a cervical nerve? Make a careful study of the architecture of this muscle, noticing carefully the form, position, origin, insertion, and action of its different parts. (Vide Spalteholz, Fig. 314.)

(a) Lumbar part (pars lumbalis).

(aa) Medial crus (crus mediale).

(ab) Intermediate crus (crus intermedium).

(ac) Lateral crus (crus laterale).

(b) Costal part (pars costalis).

(c) Sternal part (pars sternalis).

(d) Aortic opening (hiatus aorticus).

(e) Oesophageal opening (hiatus oesophageus).

( $f$ ) Central tendon (centrum tendineum).

(g) Opening for vena cava (foramen venae cavae).

(h) Medial lumbocostal arch (arcus lumbocostalis medialis [Halleri]) (O. T. ligamentum areuatum internum ).

(i) Lateral lumbocostal arch (arcus lumbocostalis lateralis [Halleri]) (O. T. ligamentum arcuatum externum).

Examine the structures passing through the hiatus oesophageus, the hiatus aorticus, and the foramen venae cavae. What is the weak spot of the diaphragm? Where is Larrey's space?

\section{Abdominal Aorta and Inferior Vena Cava. (Vide Spalteholz, Figs.} 463,499 .)

Clean these two vessels, avoiding injury to the truncus sympathicus. The branches of the aorta will not be given in detail where they have already been met, with in earlier dissections. 
FIG. 271.

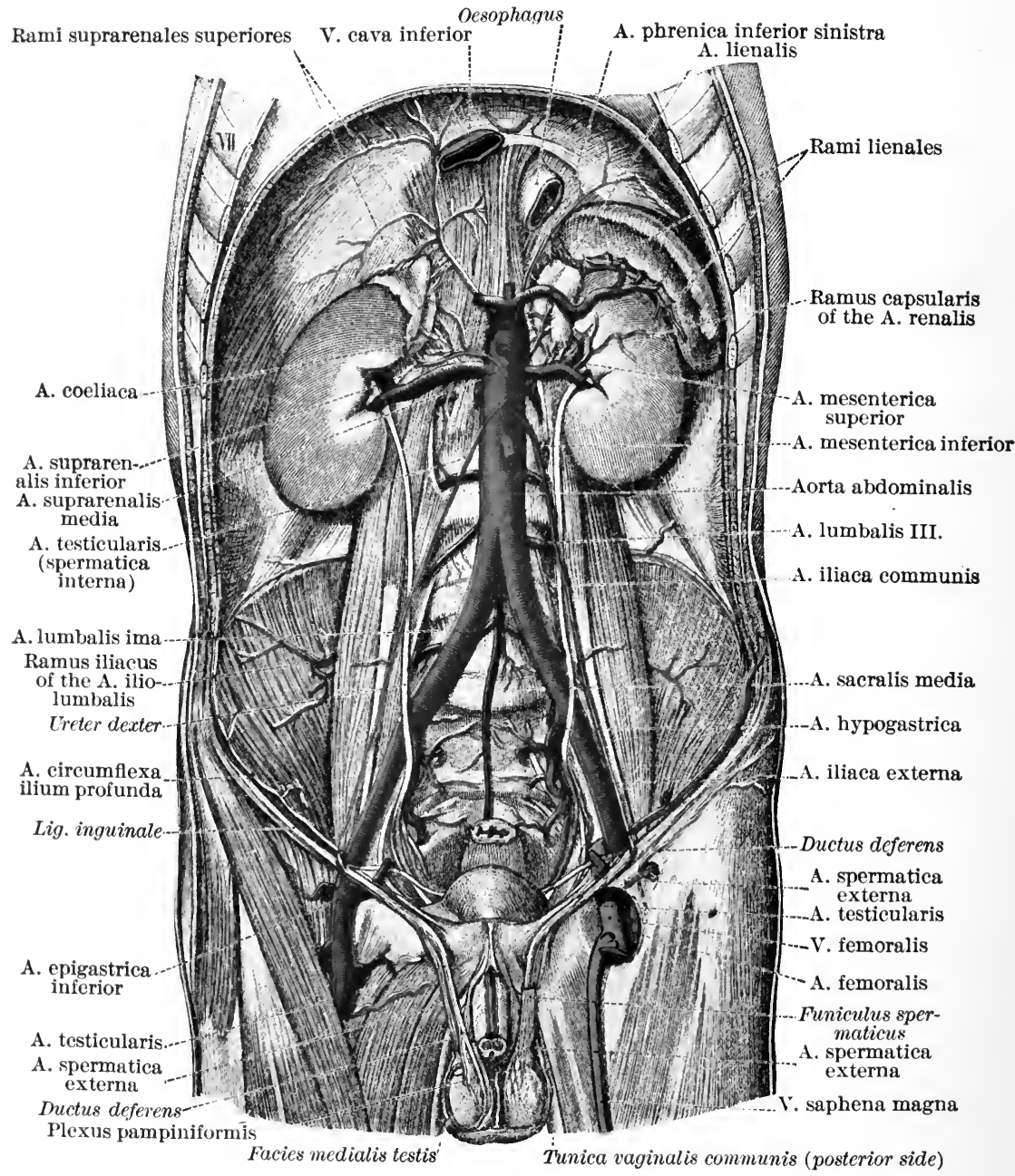

The parietal and the paired viseeral branches of the abdominal aorta. The abdominal viscera have been removed back to the spleen, kidney, and suprarenals. The medial surface of the right testis has been laid bare to show the entrance of the A. testicularis. The left testis has been turned medialward to show the terminal branching of the A. spermatica interna on the tunica vaginalis communis and between the bundles of the M. cremaster. (After Toldt, Anat. Atlas, Wien, 1900, 2 Aufl., p. 580,
Fig. 956.) 
Abdominal Aorta (Aorta abdominalis). (Fig. 271.)

(a) Parietal branches (rami parietales).

(aa) Inferior phrenic artery (A. phrenica inferior).

(aaa) Superior suprarenal branches (rami suprarenales superiores).

(ab) Lumbar arteries (Aa. lumbales). These run behind the truncus sympathicus.

(aba) Dorsal branch (ramus dorsalis).

$(a b b)$ Spinal branch (ramus spinalis).

(ac) Middle sacral artery (A. sacralis media).

(aca) Lowest lumbar artery (A. lumbalis ima).

(acb) Coceygeal skein (glomus coccygeum).

(b) Visceral branches (rami viscerales).

(ba) Coeliac artery (A. coeliaca).

(baa) Left gastric artery (A. gastrica sinistra).

(bab) Hepatic artery (A. hepatica).

(bac) Splenic artery (A. lienalis).

(bb) Superior mesenteric artery (A. mesenterica superior).

(bc) Inferior mesenteric artery (A. mesenterica inferior).

(bd) Middle suprarenal artery (A. suprarenalis media) (O. T. middle capsular artery).

(be) Renal artery (A. renalis).

(bea) Inferior suprarenal artery (A. suprarenalis inferior).

(bf) Internal spermatic artery (A. spermatica interna).

(1) Testicular artery (A. testicularis) (in the male).

(2) Ovarian artery ( $A$. ovarica) (in the female).

Notice the levels at which these arteries are given off and their relation to the vertebrae. Do you find any evidences of the segmental origin of the aorta?

Inferior Vena Cava (Vena cava inferior).

(a) Parietal roots (radices parietales).

(aa) Inferior phrenic vein ( $V$. phrenica inferior).

$(a b)$ Lumbar veins ( $V v$. lumbales).

(b) Visceral roots (radices viscerales).

(ba) Hepatic veins ( $V v$. hepaticae).

(bb) Renal veins ( $V v$, renales).

(bc) Suprarenal veins ( $V v$. suprarenales).

(bd) Spermatic vein (V. spermatica).

(be) Testicular vein (V.testicularis).

$(b f)$ Ovarian vein ( $V$. ovarica).

(bg) Pampiniform plexus (plexus pampiniformis).

Note the division of the abdominal aorta into the

Common Iliac Arteries (Aa. iliacae communes).

At what level does this bifurcation occur? Note the general direction of the artery and its length. What is the relation of each common iliac artery to the intestinum tenue, the sympathetic nerves connecting the plexus aorticus abdominalis, and 
the plexus hypogastricus and the ureter? What relation does the A. haemorrhoidalis superior bear to the artery of the left side? Note that the common iliac artery bifurcates below (vide Fig. 285) into-

(a) Hypogastric artery (A. hypogastrica) (O. T. internal iliac). The branches of this artery will be studied when the pelvis is dissected.

(b) External iliae artery (A. iliaca externa).

(ba) Inferior epigastric artery (A. epigastrica inferior) (O. T. deep epigastric).

(baa) Pubic branch (ramus pubicus).

(1) Obturator branch (ramus obturatorius).

(bab) External spermatic artery (A. spermatica externa) (O. T. cremasteric) (in the male).

(bac) Artery of round ligament (A. Lig. teretis uteri) (in the fenale).

(bb) Deep circumflex iliac artery (A. circumflexa ilium profunda).

\section{Common Iliac Veins (Vv. iliacae communes).}

(a) External iliac veins ( $V v$. iliaca externa).

(aa) Inferior epigastric vein ( $V$. epigastrica inferior).

$(a b)$ Deep circumflex iliac vein ( $V$. circumflexa ilium profunda).

(b) Hypogastric vein ( $V$. hypogastrica) (O. T. internal iliac vein).

Dissect out at this stage the-

(ba) Iliolumbar vein ( $V$. iliolumbalis).

The middle sacral vein ( $V$. sacralis media) will be found emptying into the left common iliac vein. Other branches of the hypogastric vein may be worked out later when the pelvis is dissected.

\section{Lymphatics.}

Dissect out the different lymph-glands; also the lymphtrunks (trunci lumbales, truncus intestinalis), which empty into the dilated beginning of the ductus thoracicus. This dilatation is known as the chyle-cistern (cisterna chyli) (O. T. receptaculum chyli). (Vide Fig. 249.) Look for it between the aorta abdominalis and the crus mediale of the diaphragm on the right side.

Examine the following:

Lymph-glands.

(a) Iliac (lymphoglandulae iliacae).

(b) Coeliac (lymphoglandulae coeliacae).

(c) Lumbar (lymphoglandulae lumbales).

Lymph-plexuses.

(a) External iliac (plexus [lymphaticus] iliacus externus). 
(b) Coeliae (plexus [lymphaticus] coeliacus).

(c) Lumbar (plexus [lymphaticus] lumbalis).

(d) Aortic (plexus [lymphaticus] aorticus).

Azygos and Hemiazygos Veins (V. azygos and V. hemiazygos).

Study the abdominal tributaries of these veins. (Cf. Spalteholz, Fig. 495.) Examine especially the ascending lumbar vein (V. lumbalis ascendens).

\section{NERVES IN THE ABDOMEN.}

Abdominal Part of Sympathetic Trunk (Truncus sympathicus, pars abdominalis). (Vide Toldt, Fig. 1327.)

Study the position of the truncus sympathicus in the abdomen. Determine its relation to the lumbar vessels and inferior vena cava. Examine the lumbar ganglia (ganglia lumbalia). Whither do the lateral and medial branches from these ganglia go? Review the general disposition of the systema nervorum sympathicum. What are the rami communicantes, and in what relation do they stand to the spinal nerves?

\section{Lumbar Plexus (Plexus lumbalis). (Figs. 272 and 273.)}

Remove by blunt dissection the M. psoas major and expose the lumbar plexus, preserving carefully the nerves derived from it. (Cf. Toldt, Fig. 1327.) Study-

(a) Muscular branches (rami musculares).

(b) Iliohypogastric nerve ( $N$. iliohypogastricus).

(ba) Muscular rami (rami musculares).

(bb) Lateral cutaneous ramus ( $R$. cutaneus lateralis).

(bc) Anterior cutaneous ramus ( $R$. cutaneus anterior).

(c) Ilio-inguinal nerve ( $N$. ilio-inguinalis).

(ca) Muscular branches (rami musculares).

( $c b)$ Anterior scrotal nerves (Nn. scrotales anteriores).

or Anterior labial nerves (Nn. labiales anteriores).

(d) Genitofemoral nerve ( $N$. genitofemoralis) (O. T. genitocrural).

(da) Lumbo-inguinal nerve ( $N$. lumbo-inguinalis) (O. T. crural branch).

(db) External spermatic nerve (N. spermaticus externus) (O. T. genital branch).

(e) Lateral cutaneous nerve of thigh ( $N$. cutaneus femoris lateralis) ( $\mathrm{O}$. T. external cutaneous).

( $f$ ) Obturator nerve ( $N$. obturatorius).

( $f a)$ Anterior branch (ramus anterior).

( $f b)$ Posterior branch (ramus posterior). 
FIG. 272.

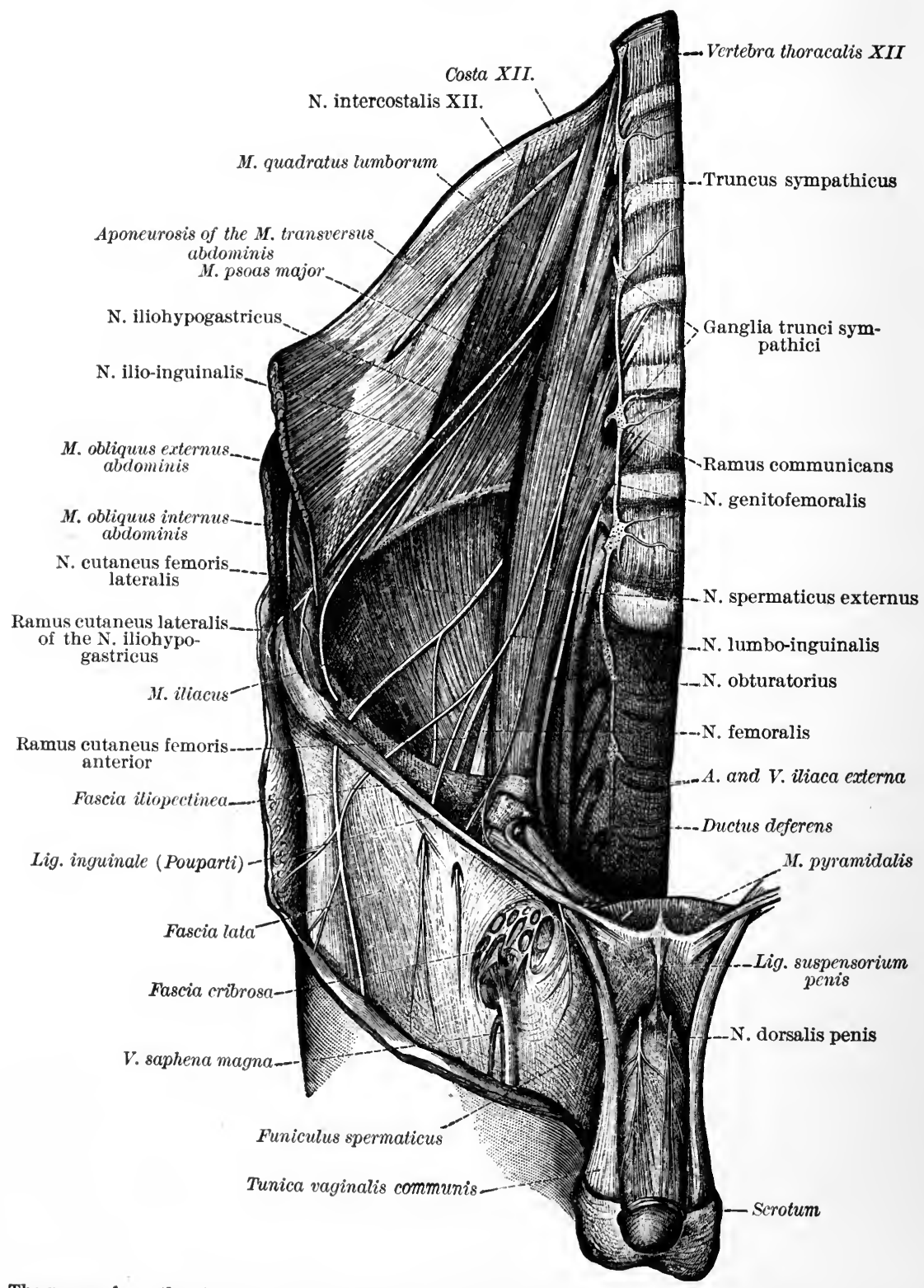

The nerves from the plexus lumbalis. (The N. ilio-inguinalis has been cut off just above the anterior superior iliac spine.) (After Toldt, Anat. Atlas, Wien, 1903, 3 Aufl., p. 837, Fig. 1273.) 
FiG. 273.

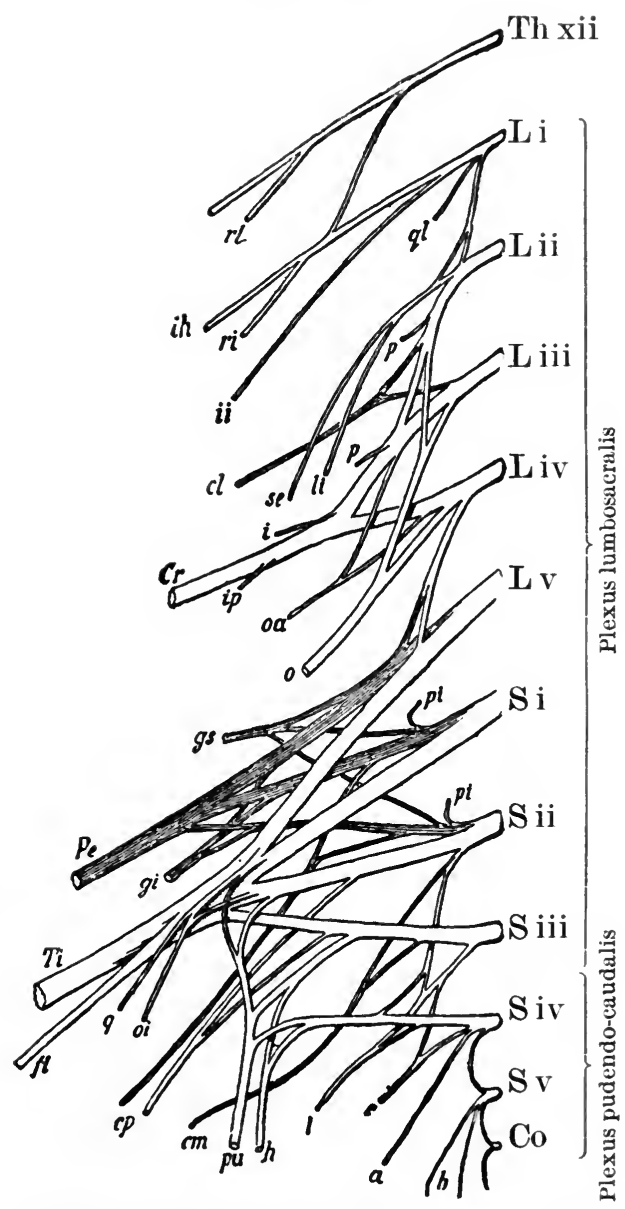

Plexus lumbosacralis, including the plexus lumbalis, the plexus sacralis, and the plexus pudendus. Ventral aspect.

$r l$, ramus cutaneus lateralis of the $\mathrm{N}$. subcostalis; $i h, \mathrm{~N}$. iliohypogastricus; $r i$, its ramus iliacus; $i i$, $\mathrm{N}$. ilio-inguinalis ; $q l$, nerve for quadratus lumborum; $s e, \mathrm{~N}$. spermaticus externus ; $l i, \mathrm{~N}$. lumbo-inguinalis ; $p$, ramus muscularis to M. psoas; $c l, \mathbf{N}$. cutaneus femoris lateralis; $i$, ramus muscularis to $\mathbf{M}$. iliacus; $i p$, ramus muscularis to $\mathrm{M}$. iliopsoas; $\mathrm{Cr}, \mathbf{N}$. femoralis; $o a, \mathbf{N}$. obturatorius accessorius; $o, \mathbf{N}$. obturatorius; $g s, \mathrm{~N}$. glutaeus superior ; $p i$, ramus muscularis to $\mathrm{M}$. piriformis ; $\mathrm{Pe}, \mathrm{N}$. peronaeus; $g i, \mathrm{~N}$. glutacus inferior; $T i, \mathrm{~N}$. tibialis ; $f$, rami musculares to $\mathrm{Mm}$. flexores cruris; $q$, ramus muscularis to $\mathrm{M}$. quadratus femoris and M. gemellus inferior; $o$, ramus muscularis to M. obturator internus and $\mathbf{M}$. gemellus superior; $c p, \mathbf{N}$. cutaneus femoris posterior ; $c m, \mathbf{N}$. cut. clun. inf. medialis (N. perforans lig. tuberoso-sacrum) ; $p u, \mathbf{N}$. pudendus ; $h, \mathbf{N}$. haemorrhoidalis ext. ; $l$, ramus muscularis to M. levator ani ; $c$, ramus muscularis to M. coccygeus; $a, b, \mathrm{Nn}$. anococcygei. (After P. Eisler, taken from Rauber's Lehrbuch der Anatomie des Menschen.)

(g) Femoral nerve (N. femoralis).

(ga) Anterior cutaneous branches (rami cutanei anteriores).

(gb) Muscular branches (rami musculares). 
(gc) Saphenous nerve (N. saphenus).

Its branches are studied in the dissection of the lower extremity.

What is the lumbosacral trunk (truncus lumbosacralis) (O. T. lumbosacral cord), and what nerves unite to form it: What rami anteriores of spinal nerves unite to form the lumbar plexus? ${ }^{1}$

\section{FASCIA AND MUSCLES.}

Cut through the aorta and vena cava inferior at the level of the bifurcation of the aorta. Separate the pelvis from the rest of the trunk at the level of the disk between the third and fourth lumbar vertebrae.

\section{Iliac Fascia (Fascia iliaca). (Vide Spalteholz, Fig. 370.)}

Note the relation of this fascia to the M. iliopsoas, the lumbar vertebrae, the crista iliaca, and medially to the pelvic fascia (fascia pelvis). Show that these two fasciae are continuous. Determine the line of attachment of the fascia to Poupart's ligament (ligamentum inguinale [Pouparti]) and trace it downward to the eminentia iliopectinea. What are the fascia iliopectinea, lacuna musculorum and lacuna vasorum? (Vide Fig. 58.) What is the relation of this fascia to the femoral vessels? Locate at this stage of your dissection the annulus femoralis, and, if possible, its septum (septum femorale [Cloqueti]). From what fascia is this septum derived? Dissect out the lymph-nodes of Rosenmüller. Determine their exact position and communications. Dissect out the fascia covering the M. quadratus lumborum, noting its exact attachments and general disposition.

\section{Muscles at the Back of the Abdomen.}

Clean the following muscles, noting the exact form, position, origin, insertion, action, and innervation of each. Avoid the sympathetic cord found medial from the iliopsoas muscle, also the nerves of the lumbar plexus, which may be readily seen, and take care not to destroy the iliac fascia as it passes into the fascia pelvis.

(a) Quadrate muscle of the loins (M. quadratus lumborum).

(b) Iliopsoas muscle (M. iliopsoas).

(ba) Greater psoas (M. psoas major).

(bb) Lesser psoas (M. psoas minor).

(bc) Iliac (M. iliacus).

${ }^{1}$ For a statistical study of the variations which occur in this region, see the paper by Bardeen and Elting, Anatomischer Anzeiger, 1901, vol. xix. p. 124. 


\section{DISSECTION OF THE MALE PELVIS.}

Before beginning the dissection of the pelvic viscera, review the anatomy of the bones entering into the formation of the pelvis. Compare the male and female pelves, noting the differences in the diameters, angles of inclination, size and general structure of the bones. Before disturbing any relations examine the position of each viscus. Compare the positions found in the adult with those in the child. Note the peritoneal relations of each viscus and study the peritoneal folds.

\section{Peritoneal Folds. (Vide Spalteholz, Fig. 667.)}

(a) Transverse vesical fold (plica vesicalis transversa). This fold disappears when the bladder is distended.

(b) Pubovesical fold (plica pubovesicalis).

(c) Rectovesical fold (plica rectovesicalis).

The rectovesical folds are raised by the obliterated umbilical arteries. They form the lateral boundaries of a deep excavation between the bladder and the rectum (excavatio rectovesicalis). In the folds will be found the rectovesical muscles ( $M m$. rectovesicales), which are composed of smooth muscle-fibres derived from the walls of the neighboring viscera.

(d) Mesorectum. A fold of peritoneum connecting the first part of the rectum to the posterior wall of the pelvis.

\section{Hypogastric Plexus (Plexus hypogastricus). (Vide Toldt, Figs. I33I and I333.)}

This sympathetic plexus rests upon the body of the fifth lumbar vertebra between the common iliac arteries. Establish its relation to the plexus aorticus and the ganglia lumbalia. Inferiorly it divides into two parts, which accompany the hypogastric arteries into the pelvis. Dissect out the plexus, trace it downward into the pelvis, and dissect out the following plexuses and nerves derived from it:

(a) Middle hemorrhoidal plexus (plexus haemorrhoidalis medius).

(b) Prostatic plexus (plexus prostaticus).

(c) Deferential plexus (plexus deferentialis).

(d) Vesical plexus (plexus vesicalis).

(da) Superior vesical nerves (Nn. vesicales superiores).

$(d b)$ Inferior vesical nerves ( $N n$. vesicales inferiores).

(e) Cavernous plexus of penis (plexus cavernosus penis).

(ea) Greater cavermous nerve of penis ( $N$. cavernosus penis major).

(eb) Lesser cavernous nerves of penis (Nn. cavernosi penis minores). 


\section{Pelvic Fascia (Fascia pelvis). (Vide Spalteholz, Fig. 677.)}

The lower aperture of the pelvis is closed by the pelvic diaphragm, which consists mainly of the M. levator ani with a layer of fascia above it and another beneath it.

The inferior fascia of the pelvic diaphragm (fascia diaphragmatis pelvis inferior) has already been studied in the dissection of the ischiorectal fossa, and its relation to the obturator fascia (fascia obturatoria) and the arcus tendineus M. levatoris

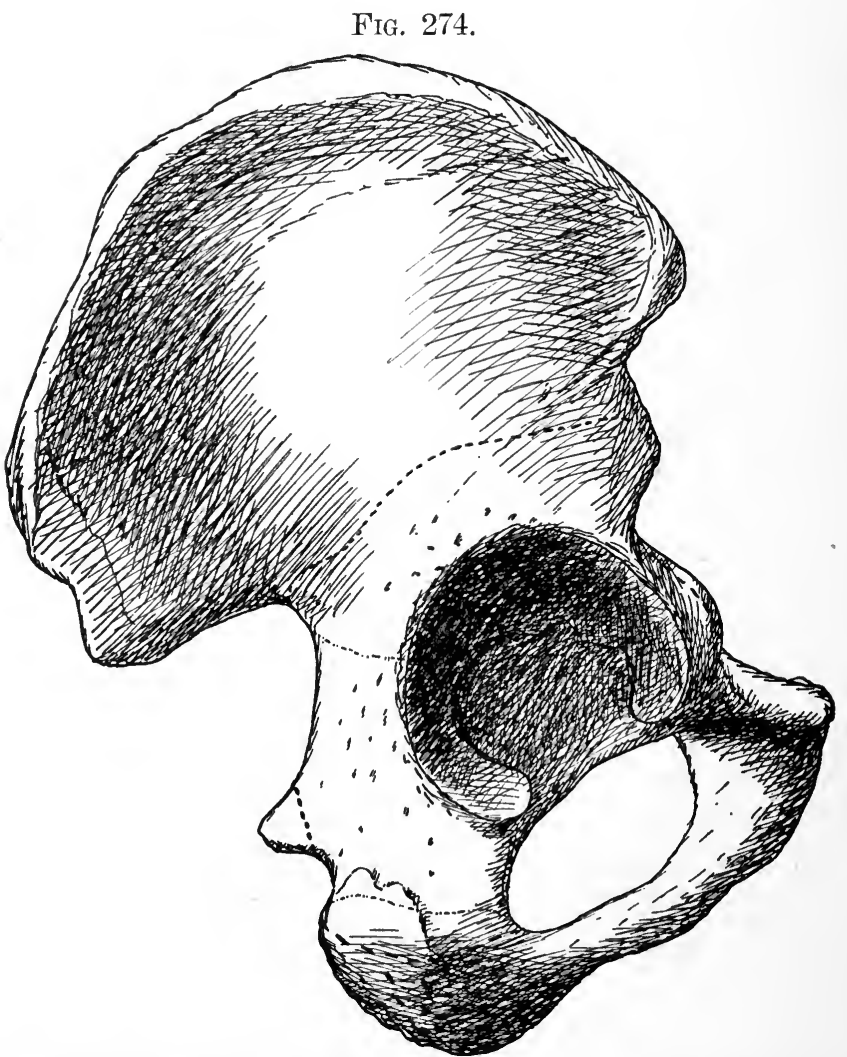

To show the lines of the saw-cuts to remove the part of the os coxae bearing the acetabulum to expose the external surface of the fascia pelvis by Cunningham's method.

ani (O. T. white line of the pelvis ${ }^{1}$ ) established. The white line of the pelvis may be exposed from the external side by removing the acetabular part of the os coxae by saw-cuts (Figs. 274, 275).

${ }^{1}$ The German anatomists have misapplied the term "white line of the pelvic fascia" to the arcus tendineus fasciae pelvis. 
To expose the superior fascia of the pelvic diaphragm (fascia diaphragmatis pelvis superior), remove the peritoneum from the sides of the pelvic wall and bladder by blunt dissection. Scrape away carefully the extraperitoneal fatty tissue with the handle of the scalpel. The bladder must be forcibly separated from the pubic bones and the intervening fatty tissue removed, to secure adequate exposure of the fascia anteriorly.

After the fascia has been exposed establish its continuity

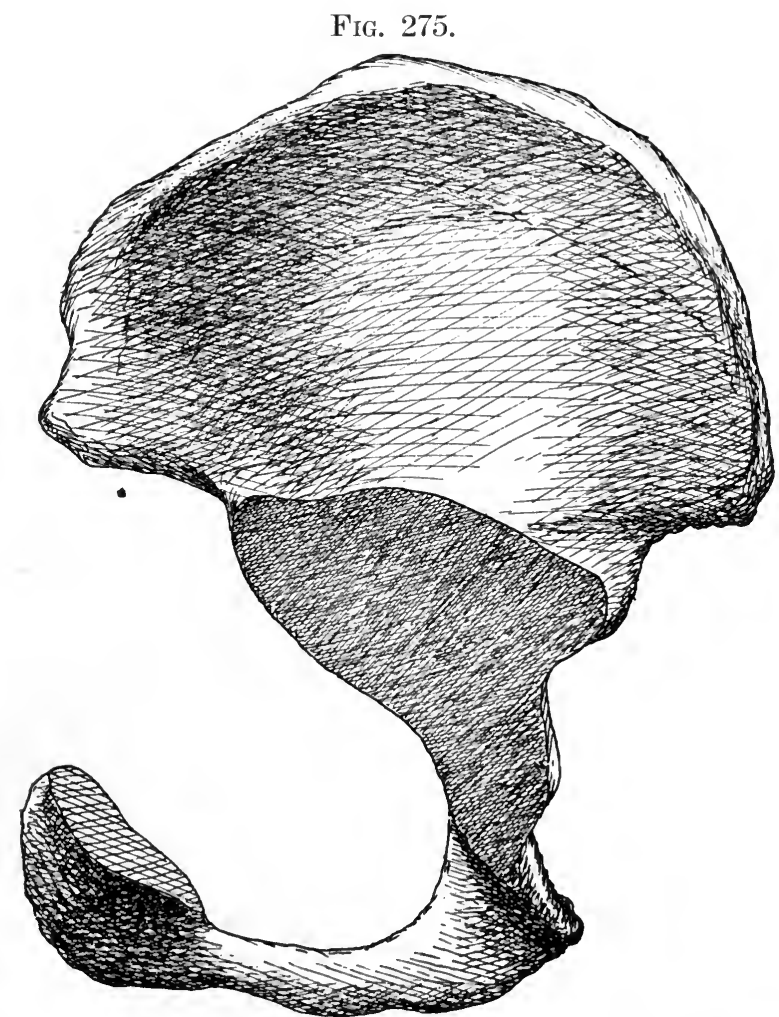

To show the cut surfaces of the os coxae after removal of the piece cut out to expose the external surface of the pelvic fascia.

with the fascia transversalis and the fascia iliaca at the terminal line (linea terminalis). Trace it downward and demonstrate that it covers the ilium and the upper part of the fascia obturatoria. It then becomes thickened and passes downward and medialward upon the upper surface of the pelvic diaphragm as far as the tendinous arch of the pelvic fascia (arcus tendineus fasciae pelvis); the tendinous arch passes in a curve, convexity directed downward and lateralward, from the symphysis ossium 
Fig. 276.

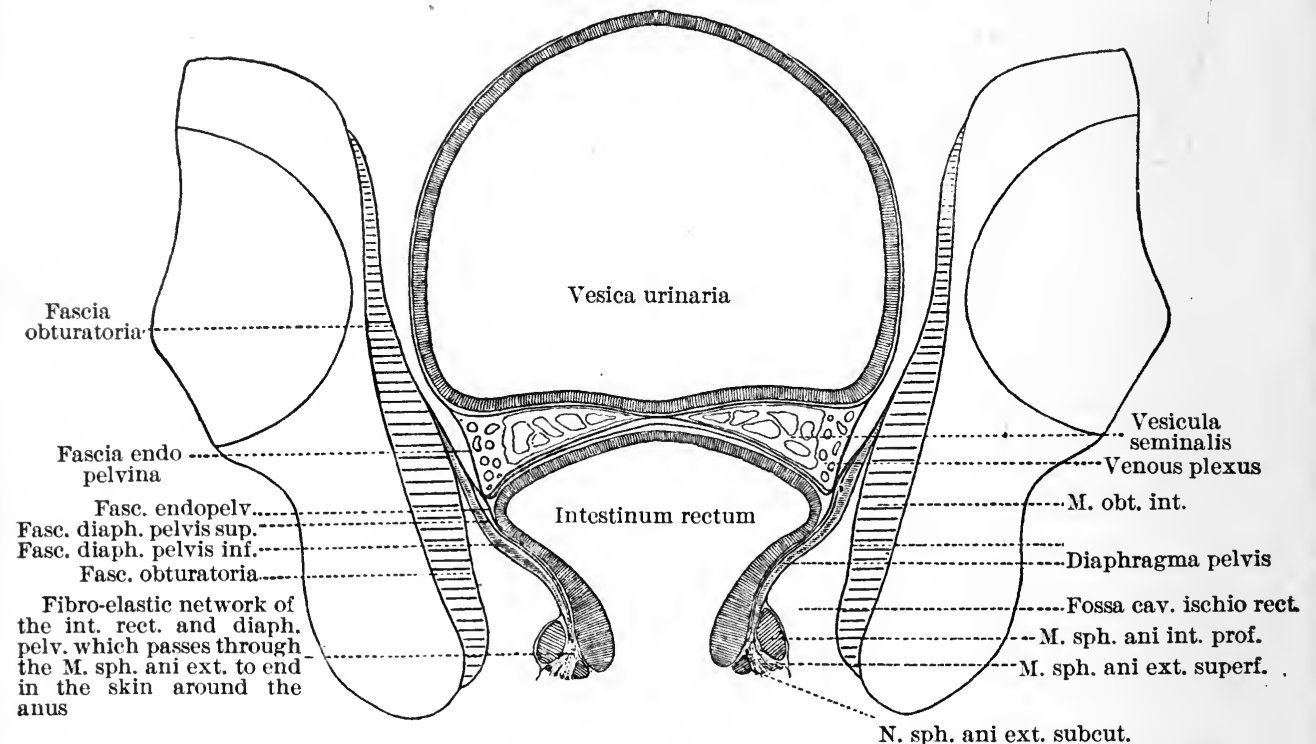

Diagram of the pelvic fasciae as seen in a frontal section of the pelvis passing through the rectum. (After Holl, in Handb. der Anat. des Mensch. von Bardeleben, Jena, 1896, Bd. vii., T. 2, Abt. 1, p. 162, Fig. 1.)

Fig. 277.

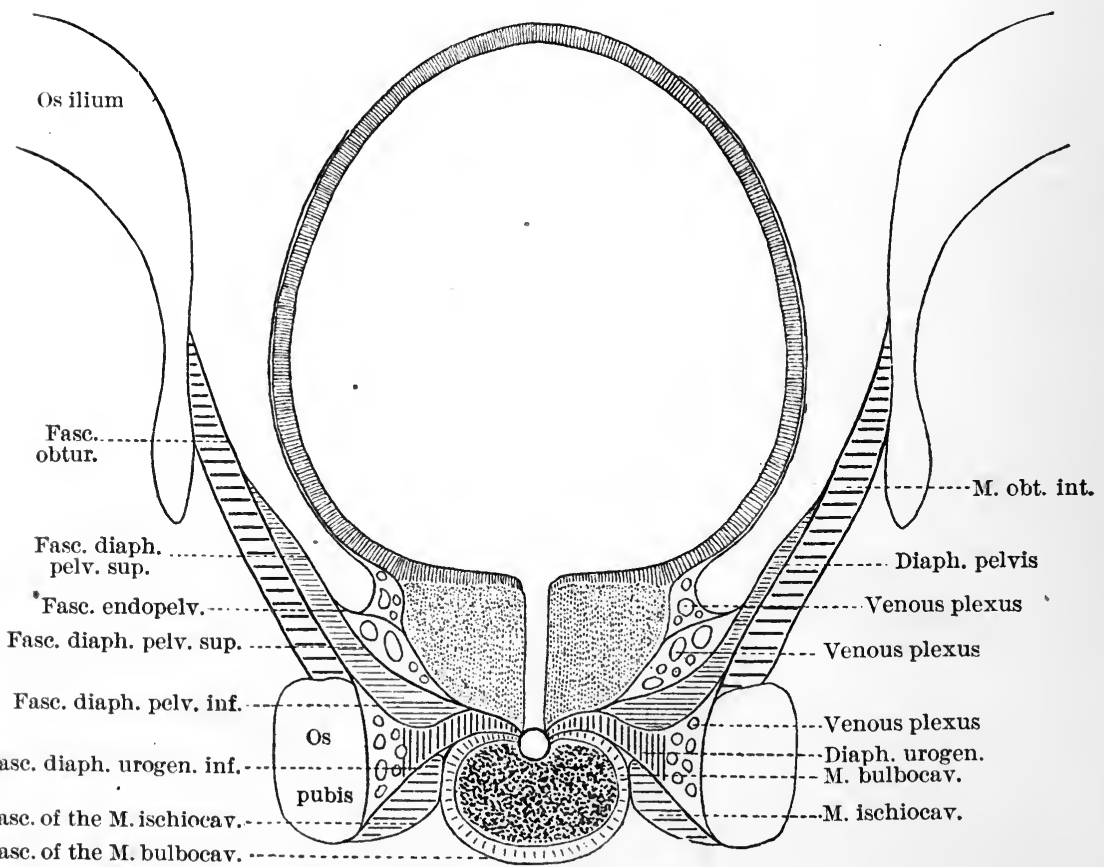

Diagram of the pelvic fasciae shown in a frontal section through the urogenital region. (After Holl, in Handb. der Anat. des Mensch. von Bardeleben, Jena, 1896, Bd. 7, T. 2, Abt. 1, p. 163, Fig. 2.) 
pubis to the spina ischiadica. Demonstrate that medialward from the arcus tendineus fasciae pelvis the greater part of the fascia is reflected upon the viscera (urinary bladder and rectum) and venous plexuses about them as the endopelvic fascia (fascia endopelvina), and that other strands of fascia follow the medial surface of the levator as far as the prostate, where they fuse with the capsule of the latter organ, sometimes called the fascia prostatae. English writers describe the fascia endopelvina as consisting of three layers,-(1) vesical, (2) rectovesical, and (3) rectal.

Fig. 278.

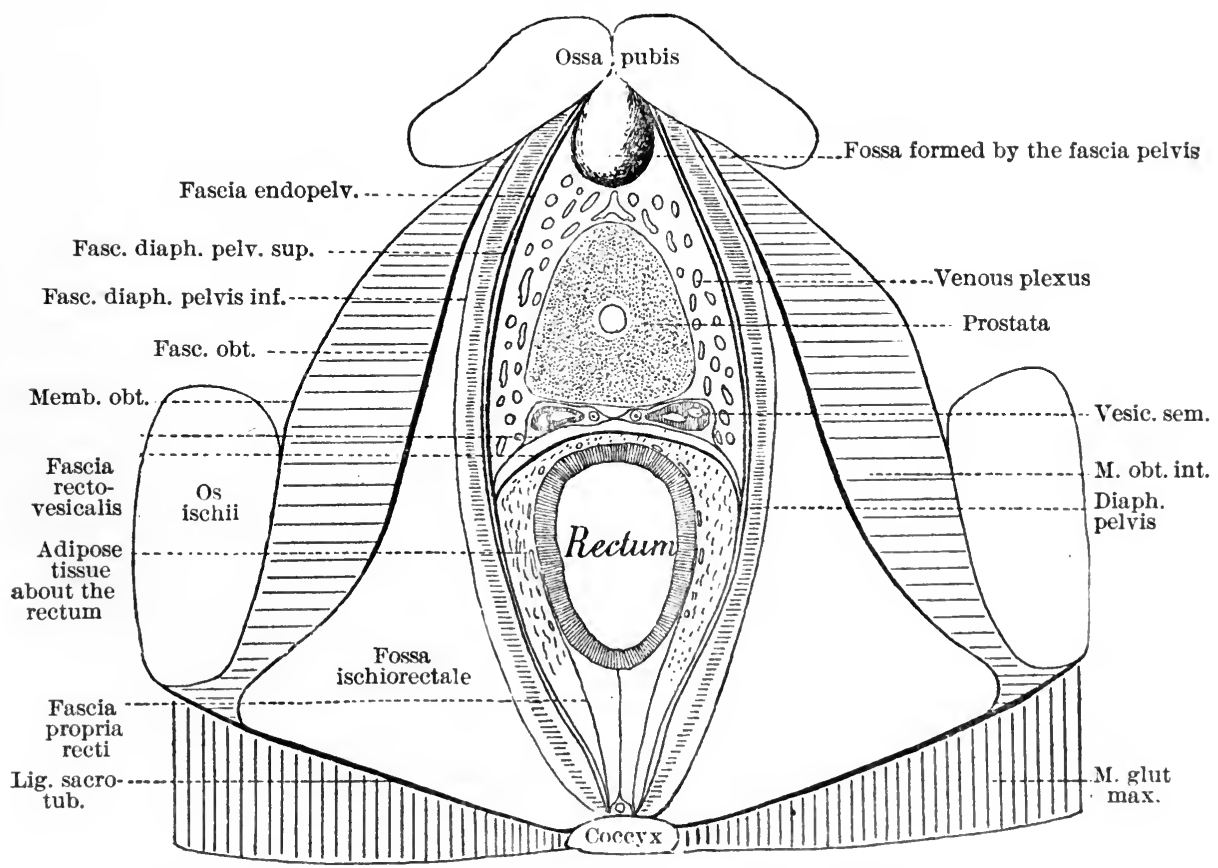

Diagram of the fasciac of the pelvis shown in a horizontal section of the pelvis. The fascia vesic. sem., faseia rectovesiea, and fascia recti prop. are parts of the fascia endopelvina. (After Holl, in Handb. der Anat. des Menseh. von Bardeleben, Jena, 1896, Bd. vii., T. 2, Abt. 2, p. 285, Fig. 31.)

Demonstrate that the fascia descends deeper between the anterior ends of the two arcus tendinei fasciae pelvis and forms a small fossa. (Cf. Fig. 279.) Isolate the prominent bands bounding this fossa laterally, the lateral puboprostatic ligaments (Ligg. puboprostatica lateralia) (O. T. lateral true ligaments of the bladder); in the female they are the Ligg. pubovesicalia lateralia (O. T. vesical layer of fascia endopelvina). Dissect in the 
depth of the fossa, and expose the middle puboprostatic ligament (Lig. puboprostaticum medium) (O. T. anterior true ligament, of bladder); in the female it is called the middle pubovesical ligament (Lig. pubovesicale medium).

Where are the pubovesical muscles ( $M m$. pubovesicales) to be found, and from what are they derived? What relations do the vessels and nerves of the pelvis bear to the pelvic fascia?

\section{Rectum (Intestinum rectum). (Vide Spalteholz, Fig. 575.)}

Determine the extent, general direction, and peritoneal relations of the rectum. Supplement the dissection by the use of

FIG. 279.

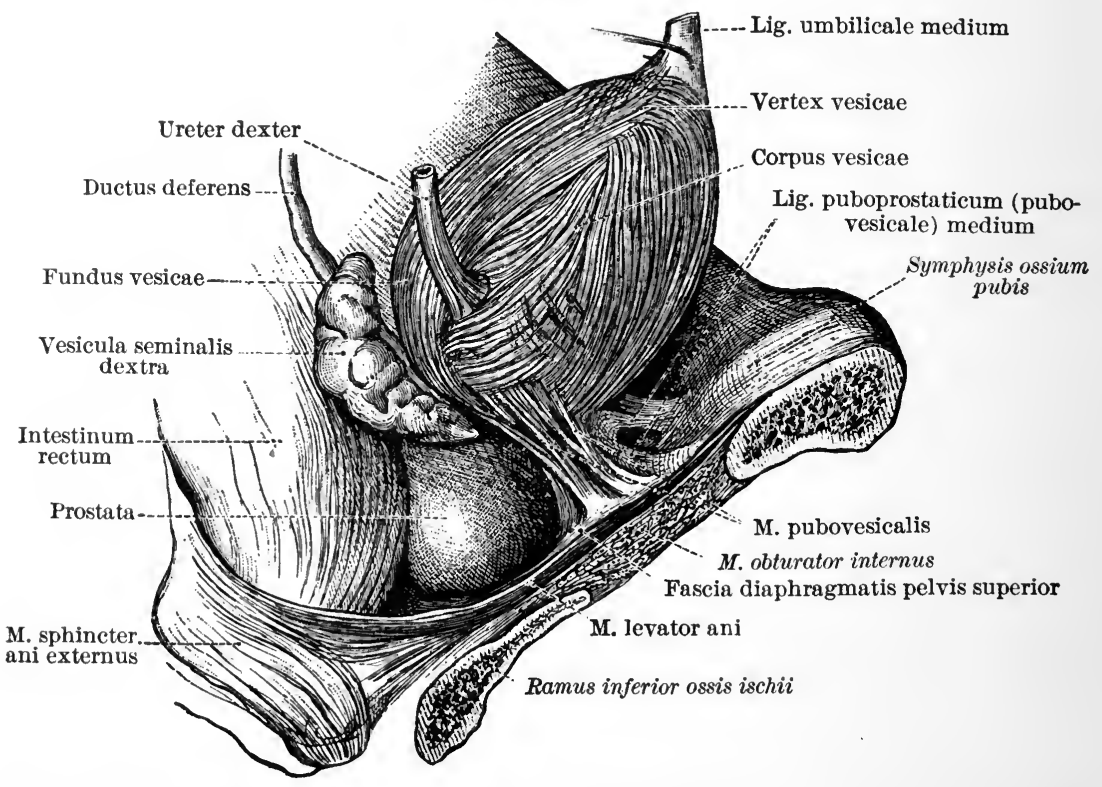

The stratum externum of the tunica muscularis of the urinary bladder, and the relations of the seminal vesicles and prostate to the bladder and rectum. Seen from the right side. (The prostate is abnormally enlarged.) (After Toldt, Anat. Atlas, Wien, 1900, 2 Aufl., p. 481, Fig. 812.)

models and cross-sections. Compare the external markings of the rectum with those of the colon, noting carefully any differences. Establish the position of the following:

(a) Flexures:

(aa) Sacral flexure (flexura sacralis).

$(a b)$ Perineal flexure (flexura perinealis).

(b) Ampulla of rectum (ampulla recti). 
This ampulla is the ovoid enlargement in the lower part of the rectum, and serves as a reservoir for faeces. It is sometimes spoken of as the "faecal bladder." Note the curves in the frontal direction, produced by lateral furrows, which correspond to the transverse folds of the rectum (plicae transversales recti). What is the rectococcygeal muscle ( $M$. rectococcygeus)? The rectum will be opened and its interior studied later.

Urinary Bladder (Vesica urinaria). (Vide Figs. 279, 28I, and Spalteholz, Figs. 641, 642.)

Examine the position and form (Fig. 280) of the bladder. To complete this study make use of models and cross-sections. How do the position and form of the bladder differ in the two sexes? How does the adult bladder differ in position and form from that of the child? From what does the bladder develop embryologically? (Cf. Kollmann, Lehrbuch der Entwickelungsgeschichte des Menschen, p. 348.) Study the following features:

(a) Apex of bladder (apex vesicae).

(b) Body of bladder (corpus vesicae).

(c) Fundus of bladder (fundus vesicae).

(d) Neck of bladder (collum vesicae).

What is the urachus?

Pelvic Part of Ureter (Pars pelvina ureteris). (Vide Spalteholz, Figs. 640, 64I).

Isolate the ureter as it enters the pelvis at the bifurcation of the common iliac artery and trace it downward to the bladder. Determine its relation to the plica rectovesicalis. What are the relative positions of the obliterated hypogastric artery and the ureter in this plica? As the ureter is traced forward, determine its relations to the ductus deferens and the vesicula seminalis. At what angle does it pierce the bladder wall?

Prostate (Prostata). (Vide Figs. 227, 279, and Spalteholz, Figs. 640, $643,653,655,656,677$.

Note the general size, shape, and position of this organ. Determine its relations to the neighboring structures. Review the anatomy of the capsule of the prostate. Avoiding injury to the venous plexus surrounding the organ, study the following:

Lobes.

(a) Right lobe (lobus dexter).

(b) Left lobe (lobus sinister). 
(c) Middle lobe (lobus medius).

The latter lobe is not always present. To bring it into view, if present, separate the seminal vesicles and the vasa deferentia

Fig. 280.

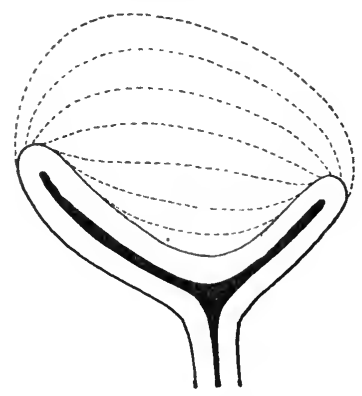

Schema showing the modifications in the form of the bladder during repletion. (From Poirier et Charpy, Traité d Anat. hum., Paris, 1901, t. v. p. 79, Fig. 63.)

from the base of the bladder and throw them backward. Many authorities maintain that this lobe is to be found only in pathological cases.

Fig. 281.

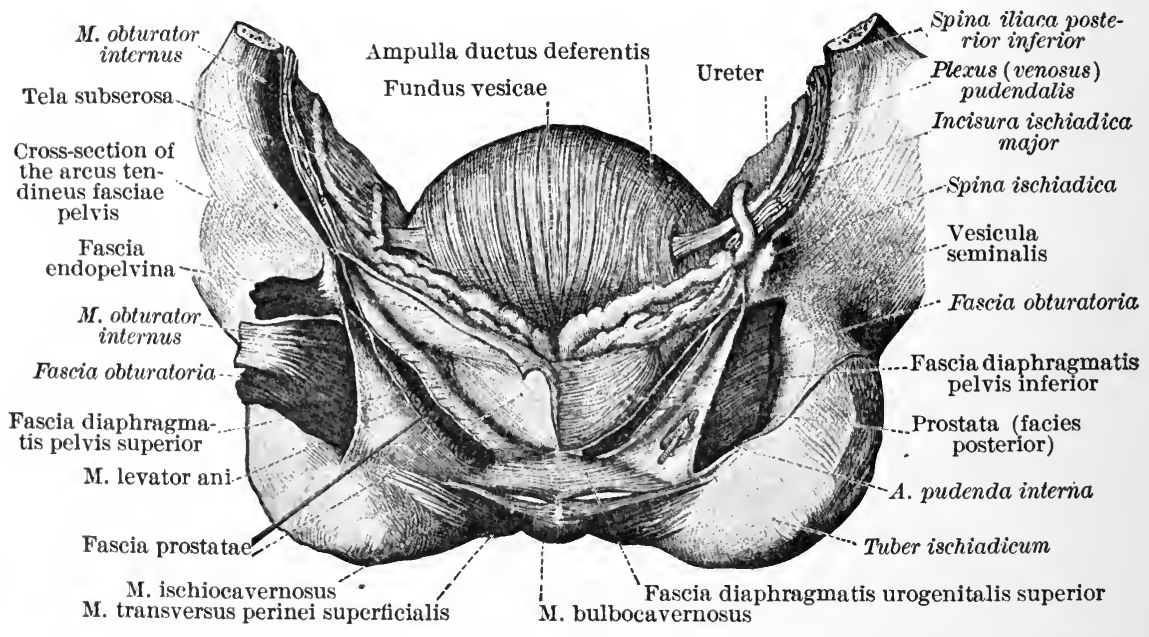

The position of the seminal vesicles and ampullae of the ductus deferentes in relation to the fundus of the bladder and to the prostate. The M. levator ani has been cut across in a frontal plane. The fascia prostatae has been removed on the right side: on the left side its upper part, passing on to the scminal vesicle, has been loosened and turned down. Seen from behind. (After Toldt, Anat. Atlas, Wien, 1900, 2 Aufl., p. 516, Fig. 895.)

\section{Surfaces.}

(a) Anterior surface (facies anterior).

(b) Posterior surface (facies posterior). 
Examine further-

(a) Base of prostate (basis prostatae).

(b) Apex of prostate (apex prostatae).

(c) Isthmus of prostate (isthmus prostatae).

Seminal Vesicles (Vesiculae seminales). (Vide Figs. 28I, 282, and Spalteholz, Figs. 599, 640, 653, 654.)

Note the general shape of the seminal vesicles; also the general direction of their long axes. Determine their relations to the prostate, the fundus and neck of the bladder, the ampulla of the deferential duct, and the ureter. Examine the body of the seminal vesicle (corpus vesiculae seminalis) and its excretory duct (ductus excretorius). With what structure does the ductus excretorius join below?

FIG. 282.

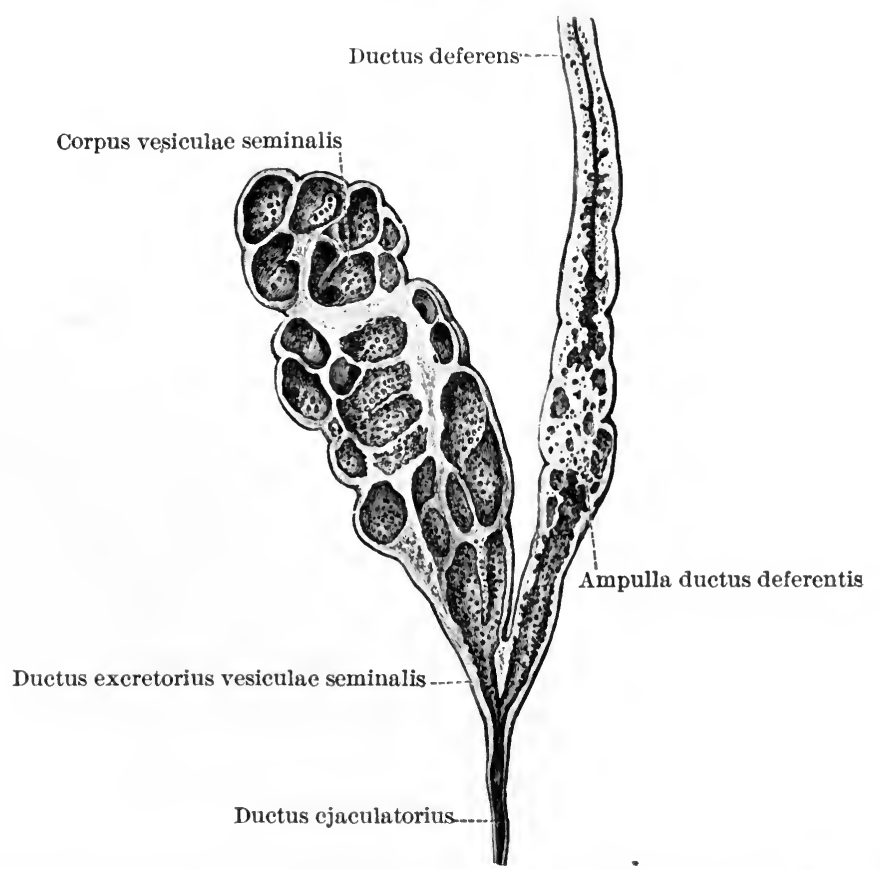

Longitudinal section through the right seminal vesicle. (After Toldt, Anat. Atlas, Wien, 1900, 2 Auf. p. 488, Fig. 829.)

Deferent Duct (Ductus deferens) (O. T. Vas deferens). (Vide Spalteholz, Figs. 599, 633, 640, 653, 654, 656.)

Trace the ductus deferens from the annulus inguinalis abdominalis downward into the pelvis. Determine again its relation to the A. et V. epigastrica, the A. et V. iliaca externa, and the 
vessels and nerves of the lateral pelvic wall. Note the spindleshaped dilatation or ampulla (ampulla ductus deferentis) behind the bladder. Observe the diverticula (diverticula ampullae). With what structure does the ampulla communicate? What duct is formed by this communication? Open the ampulla and dissect its wall. Study-

(a) Adventitious coat (tunica adventitia).

(b) Muscular coat (tunica muscularis).

(ba) External, middle, internal layer (stratum externum, medium, internum).

(c) Mucous coat (tunica mucosa).

\section{ARTERIES OF PELVIS.}

Carefully clean each vessel and its branches. Study its course and relations. Before beginning the dissection, remove

FIG. 283.

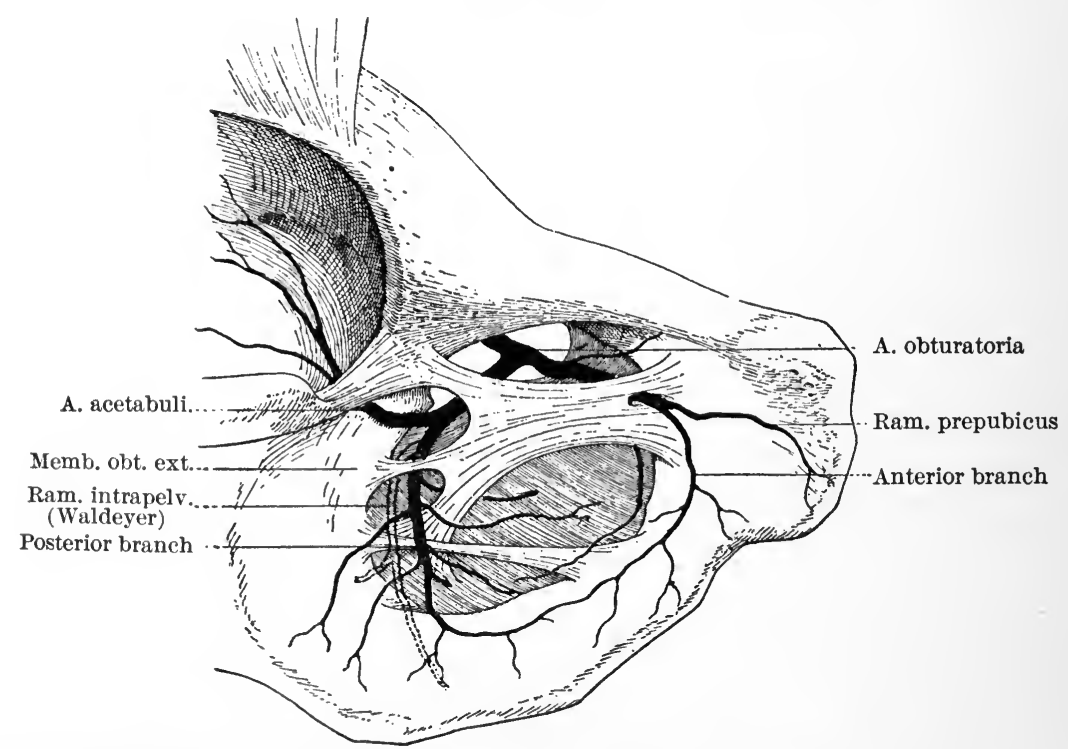

The obturator artery. (From Poirier et Charpy, Traité d'Anat. hum., Paris, 1901, 2 ed., t. ii. p. 802, Fig. 440.)

the cotton from the rectum, and if the bladder is still distended allow the air to escape. Preserve the sympathetic twigs following each artery. 
Hypogastric Artery (A. hypogastrica) (O. T. Internal Iliac Artery). (Vide Fig. 285, and Spalteholz, Figs. 470, 47 I.)

Read an account of the foetal circulation before dissecting out this artery. Note the differences between the adult and the foetus.

Parietal Branches (Rami parietales).

(a) Iliolumbar artery (A. iliolumbalis).

(aa) Lumbar branch (ramus lumbalis).

(ab) Spinal branch (ramus spinalis).

(ac) Iliac branch (ramus iliacus).

(b) Lateral saeral artery (A. sacralis lateralis).

(ba) Spinal branches (rami spinales).

(c) Obturator artery (A. obturatoria). (Figs. 283-285.)

(ca) Pubic branch (ramus pubicus).

(cb) Anterior branch (ramus anterior).

(cc) Posterior branch (ramus posterior).

(cd) Artery of the acetabulum (A. acetabuli).

(d) Superior gluteal artery (A. glutaea superior).

(da) Superior branch (ramus superior).

(db) Inferior branch (ramus inferior).

(e) Inferior gluteal artery (A. glutaea inferior).

(ea) Companion artery of sciatic nerve (A. comitans $N$. ischiadici).

Visceral Branches (Rami viscerales).

(a) Umbilical artery (A. umbilicalis).

(aa) Superior vesical arteries (Aa. vesicales superiores).

[Ligamentum umbilicale laterale].

(b) Inferior vesical artery (A. vesicalis inferior).

(c) Deferential artery (A. deferentialis).

(d) Uterine artery (A. uterina) (in the female).

(da) Vaginal artery (A. vaginalis).

$(d b)$ Ovarian branch (ramus ovarii).

(dc) Tubal branch (ramus tubarius).

(e) Middle hemorrhoidal artery (A. haemorrhoidalis media).

( $f$ ) Internal pudendal artery (A. pudenda interna).

The branches of this artery have already been met with in earlier disseetions, but are repeated here for review.

( $f a)$ Inferior hemorrhoidal artery (A. haemorrhoidalis inferior).

(fb) Artery of perineum (A. perinei).

( $f b a)$ Posterior scrotal arteries (Aa. scrotales posteriores) (in the male).

$(f b b)$ Posterior labial arteries (Aa. labiales posteriores) (in the female).

( $f c)$ Artery of penis (A. penis) (in the male).

(fca) Urethral artery (A. urethralis).

( $f c b)$ Artery of the bulb of urethra (A. bulbi urethrae).

( $f c c$ ) Deep artery of penis (A. profunda penis). 
FIG. 284.
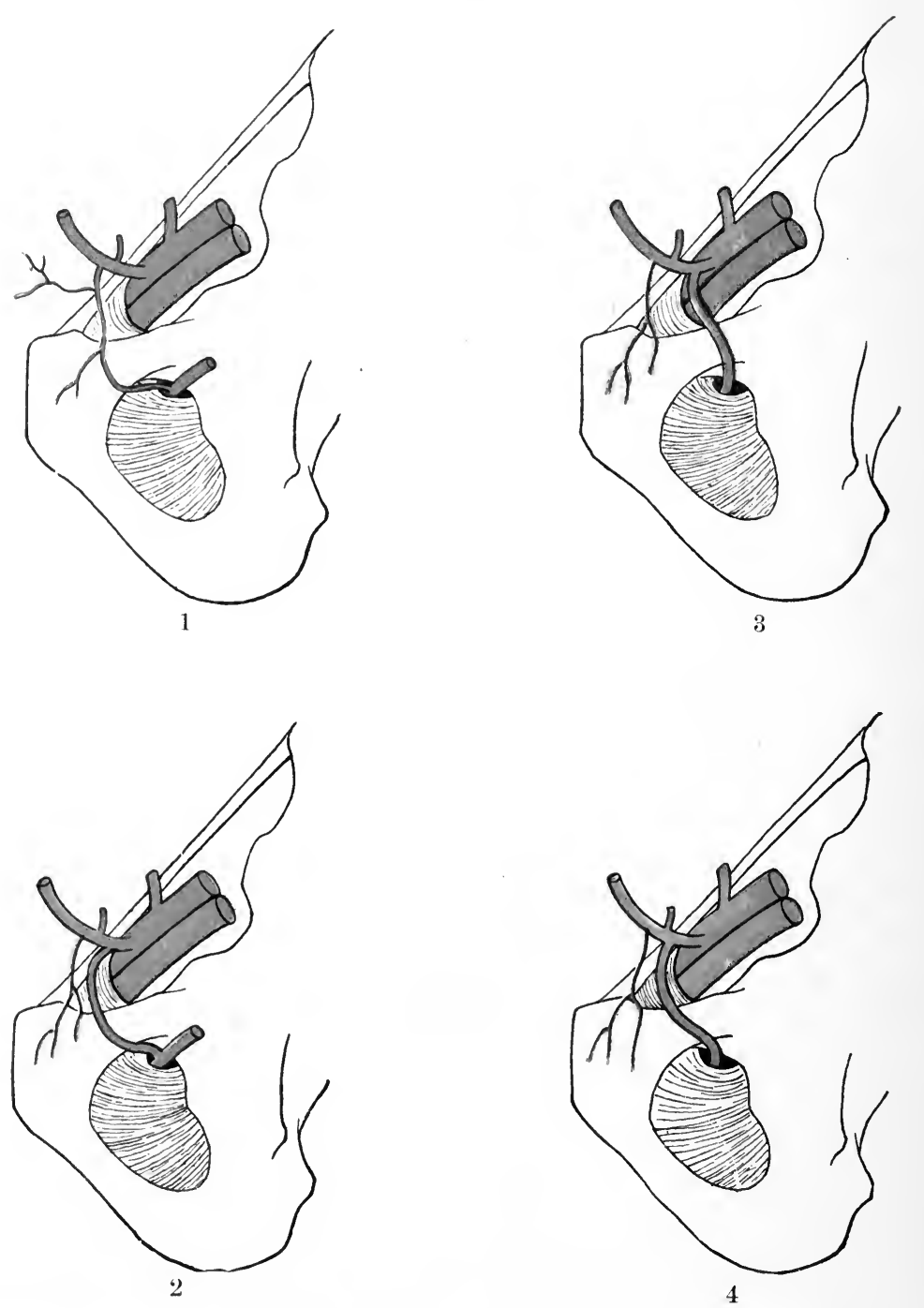

Schema showing the different ways in which the A. obturatoria may arisc. (From Poirier et Charpy, Traité d'Anat. hum., Paris, 1901, 2 ed., t. ii. p. 801, Fig. 441.) 
( $f c d)$ Dorsal artery of penis (A. dorsalis penis).

$(f d)$ Artery of elitoris (A. clitoridis) (in the female).

$(f d a)$ Artery of vestibular bulb of vagina (A. bulbi vestibuli [vaginae $])$.

FIG. 285.

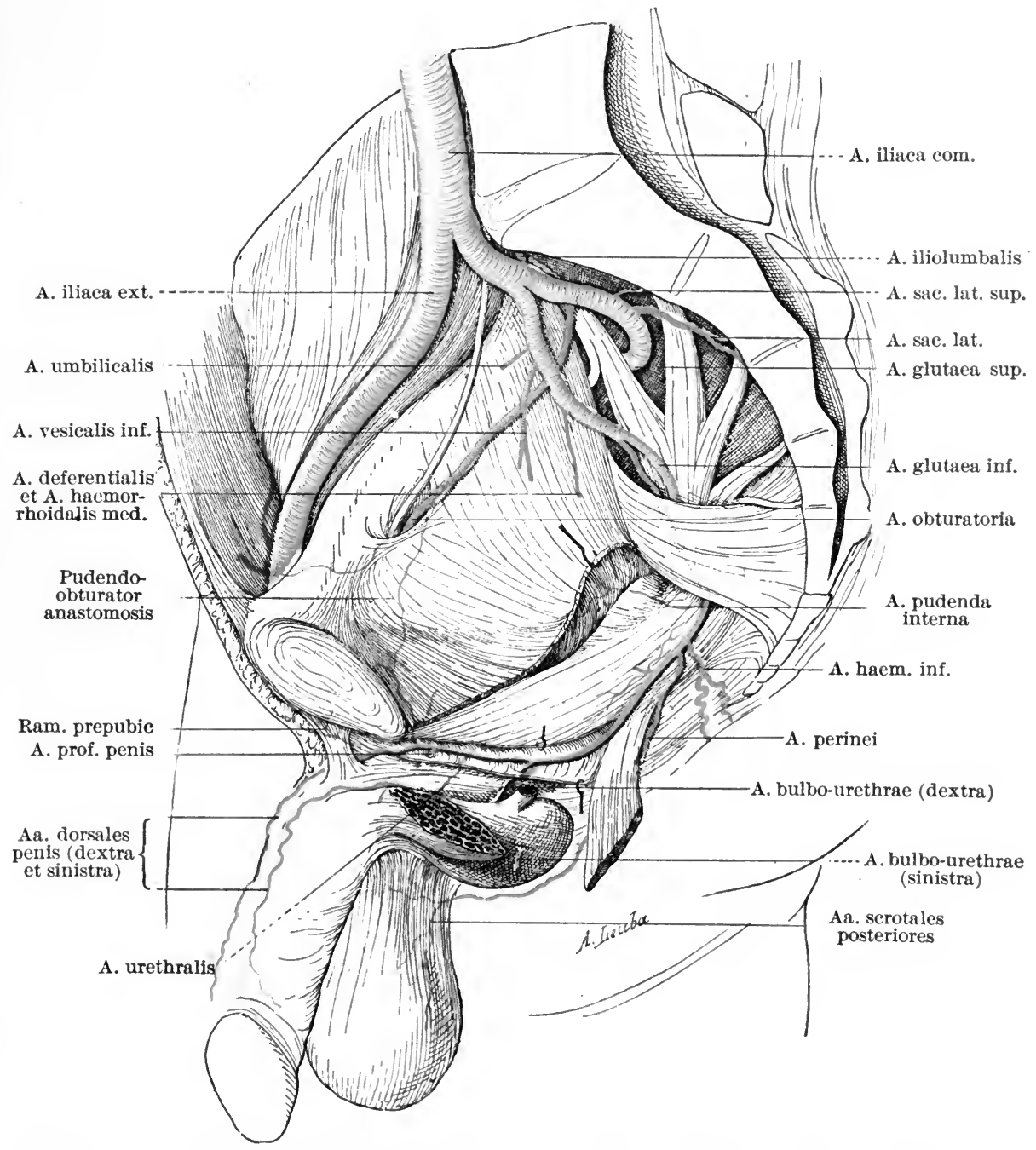

The A. pudenda interna. (From Poirier et Charpy, Traité d'Anat. hum., Paris, 1901, 2 ed., t. ii. p. 806, Fig. 442.)

(fdb) Urethral artery (A. urethralis).

$(f d c)$ Deep artery of clitoris (A. clitoridis).

( $f d d)$ Dorsal artery of clitoris (A. dorsalis clitoridis). 
Superior Hemorrhoidal Artery (A. haemorrhoidalis superior). (Vide

Fig. 286, and Spalteholz, Fig. 470.)

Trace this artery down into the mesorectum. What course does it pursue below, and how does it end in the rectal wall?

FIG. 286.

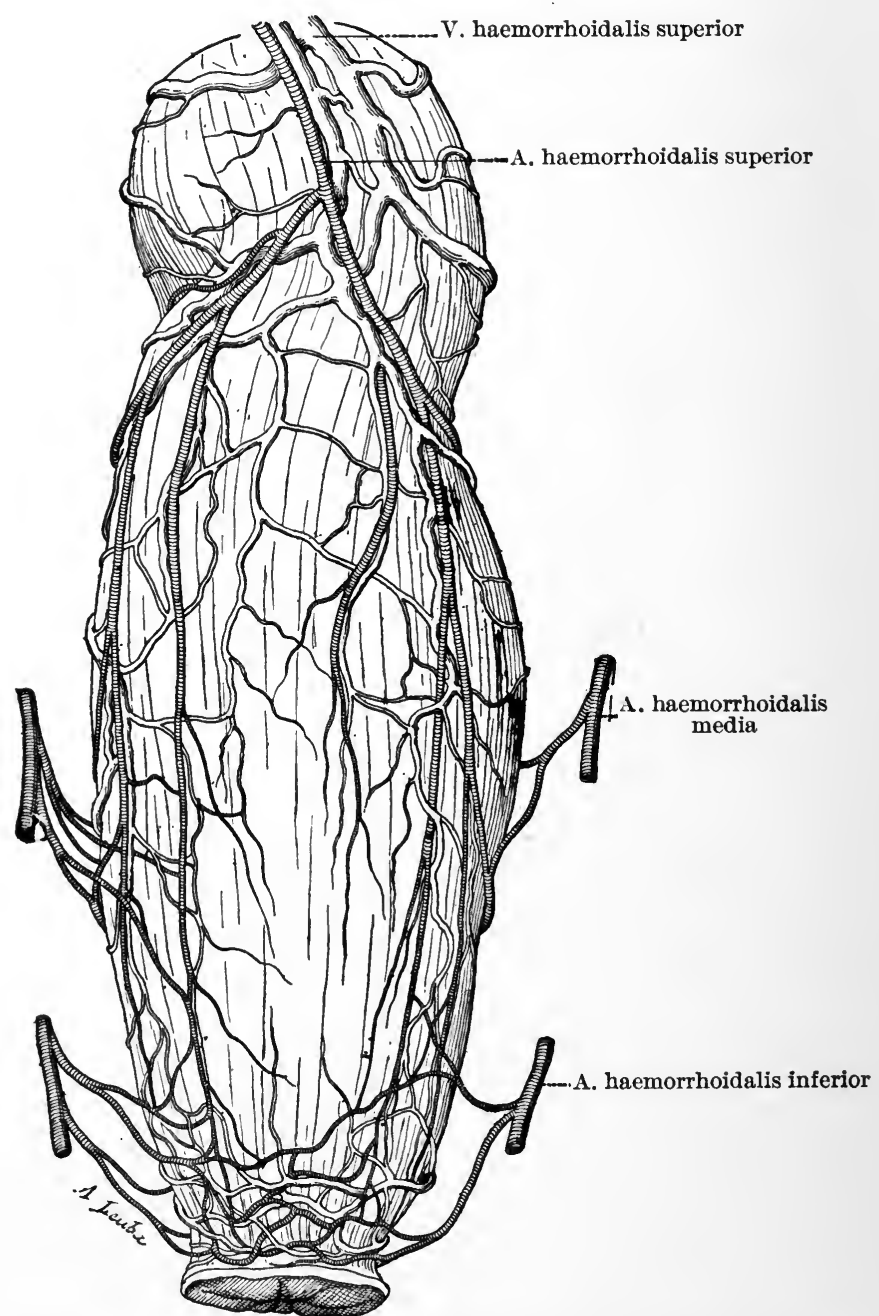

The arteries and veins of the rectum and anus. Their disposition upon the periphery of the posterior wall. (From Poirier et Charpy, Traité d'Anat. hum., Paris, 1901, 2 ed., t. iv. p. 389, Fig. 200.)

Middle Sacral Artery (A. sacralis media).

(a) Lowest lumbar artery (A. lumbalis ima).

(b) Coceygeal skein (glomus coccygeum). 


\section{VEINS OF THE PELVIS.}

Study the following:

Hypogastric Vein (V. hypogastrica). (Vide Spalteholz, Figs. 500, 50r.)

Many of the veins which are radicles of the vena hypogastrica have been met with in early dissections. They may be reviewed to advantage:

(a) Internal pudendal vein ( $V \cdot$ pudenda interna).

(aa) Deep veins of penis ( $V v$. profundae penis).

(ab) Urethral veins ( $V v$. urethrales).

(ac) Veins of bulb of urethra ( $V v$. bulbi urethrae).

$(a d)$ Posterior scrotal veins (Vv. scrotales posteriores).

In the female:

(ae) Inferior hemorrhoidal veins ( $V v$. haemorrhoidales inferiores).

(af) Deep veins of elitoris ( $V v$. profundae clitoridis).

$(a g)$ Veins of bulb of vestibule of vagina ( $V v$. bulbi vestibuli [vaginae]).

(ah) Posterior labial veins ( $V v$. labiales posteriores).

(b) Internal hemorrhoidal plexus (plexus haemorrhoidalis internus), which will be seen in the dissection of the rectal wall. The veins pierce the rectal wall and form the external hemorrhoidal plexus (plexus haemorrhoidalis externus). What veins arise from this latter plexus, and into what larger veins do they empty?

(c) Vesical plexus (plexus vesicalis). (Vide Spalteholz, Figs. 500, 503, 677.)

Determine the relation of this plexus to the bladder and to the prostate. By what veins is it drained?

(d) Pudendal plexus (plexus pudendalis).

What is the relation of the dorsal vein of the penis to this plexus? What course must this vein pursue to reach it?

(e) Uterovaginal plexus (plexus uterovaginalis).

Middle Sacral Vein (V. sacralis media).

Determine its relation to the Vv. sacrales laterales. What is the anterior sacral plexus (plexus sacralis anterior)?

\section{MUSCLES AND NERVES OF THE PELVIS.}

Muscles of the Pelvic Diaphragm. (Vide Fig. 287, and Spalteholz, Figs. 670, 67r.)

Clean away all that remains of the fascia diaphragmatis pelvis superior and study the muscles of the pelvic diaphragm. In cleaning the coccygeus muscles avoid injury to the fifth sacral 
and coccygeal nerves. Supplement the dissection by use of models and moist preparations. Compare the arrangement of the muscles in the two sexes. Study comparative preparations of the $\mathrm{dog}$ pelvis from the museum, and note how homologous muscles are related to the tail.

(a) Levator ani muscle ("elevator muscle of the anus") (M. levator ani). (aa) Pubococeygeus muscle (M. pubococcygeus).

$(a b)$ Iliococeygeus muscle (M. iliococcygeus).

(b) Coceygeus muscle (M. coccygeus).

Are the anterior and posterior sacrococcygeus muscles present in your subject?

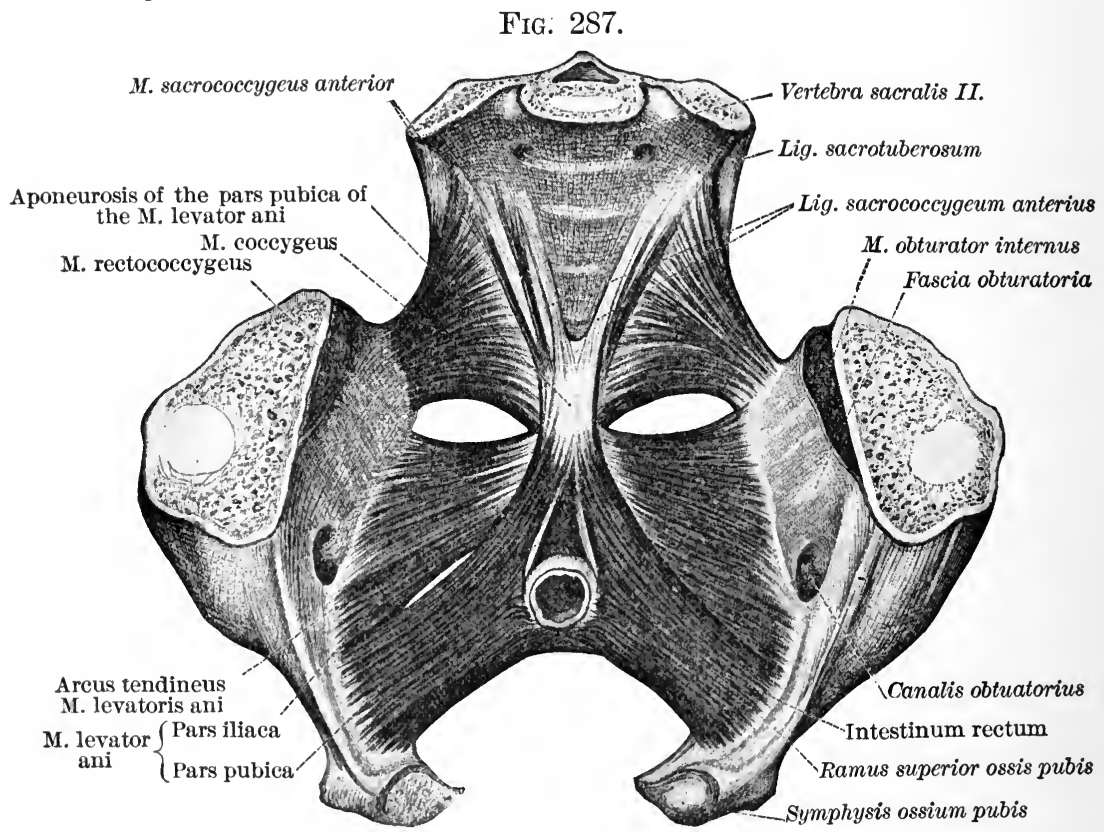

The levator ani and coccygeus muscles, seen from above. The hip-bones have been pulled apart after splitting the symphysis ossium pubis. (After Toldt, Anat. Atlas, Wien, 1900, 2 Aufl., p. 514, Fig. 891.)

\section{Pelvic Spinal Nerves. (Vide Toldt, Fig. 1295.)}

Determine the number of nerves entering into the formation of each of the following plexuses. Are the plexuses formed by the anterior or posterior primary divisions of spinal nerves? Trace each nerve as far as possible, noting course and distribution. Make a drawing of the plexuses and determine the general plan of their formation.

(a) Sacral plexus (plexus sacralis).

(aa) Superior gluteal nerve ( $N$. glutaeus superior).

(ab) Inferior gluteal nerve ( $N$. glutaeus inferior). 
(ac) Posterior cutaneous nerve of thigh (N. cutaneus femoris posterior).

(aca) Inferior nerves of buttocks ( $N n$. clunium inferiores).

(acb) Perineal branches (rami perineales).

(ad) Sciatic nerve ( $N$. ischiadicus).

(ada) Muscular branches (rami musculares).

( $a d b)$ Common peroneal nerve ( $N$. peronaeus communis). (adba) Museular branches (rami musculares).

(b) Pudendal plexus (plexus pudendus).

(ba) Middle hemorrhoidal nerves (Nn. haemorrhoidales medii).

(bb) Inferior vesical nerves ( $N n$. vesicales inferiores).

(bc) Vaginal nerves ( $N n$. vaginales) (in the female).

(bd) Pudendal nerve (N. pudendus).

$(b d a)$ Inferior hemorrhoidal nerves $(N n$. haemorrhoidales inferiores).

(bdb) Nerve of perineum (N. perinei).

$(b d c)$ Posterior scrotal or labial nerves $(N n$. scrotales vel labiales posteriores).

(bdd) Dorsal nerve of penis or of elitoris ( $N$. dorsalis penis vel clitoridis).

(be) Coceygeal nerve (N. coccygeus).

(bea) Anococygeal nerves ( $N n$. anococcygei).

Sacral Part of Sympathetic (Systema sympathicum, pars pelvina). (Vide Toldt, Fig. I327.)

Determine the number of sacral ganglia (ganglia sacralia) and their relation to the sacral foramina. What is the relation of the sacral division of the sympathetic trunk to the lumbar division? What is the unpaired ganglion (ganglion impar)? Determine the distribution of fibres given off from this part of the sacral trunk.

\section{Removal of the Pelvic Viscera.}

Divide the vessels and nerves which are connected with the viscera, also the M. levator ani and the puboprostatic ligaments. Free the membranous portion of the urethra from the pubic arch, avoiding any injury to the urethral walls. Dissect the urethra away from the coccyx and remove the viscera.

\section{Pelvic Muscles.}

Study now the form, position, origin, insertion, action, and innervation of the following muscles. (Vide Spalteholz, Fig. 373.)

(a) Internal obturator muscle ( $\boldsymbol{M}$. obturator internus).

(b) Piriform muscle (M. piriformis). 


\section{PELVIC VISCERA.}

Rectum (Intestinum rectum).

Dissect the rectum away from the bladder and the prostate. Open the rectum along its anterior wall, pin it down upon a cork dissecting-tray, and study its coats. (Cf. Fig. 288.)

(a) Mucous membrane (tunica mucosa). (Vide Spalteholz, Fig. 576.)

(aa) Transverse rectal folds (plicae transversales recti) (O. T. valves of Houston).

How many do you find? Where is each situated? What is the relation of the folds to the tela submucosa and the muscular wall of the rectum?

(ab) Lymphatic nodules (noduli lymphatici).

FIG. 288.

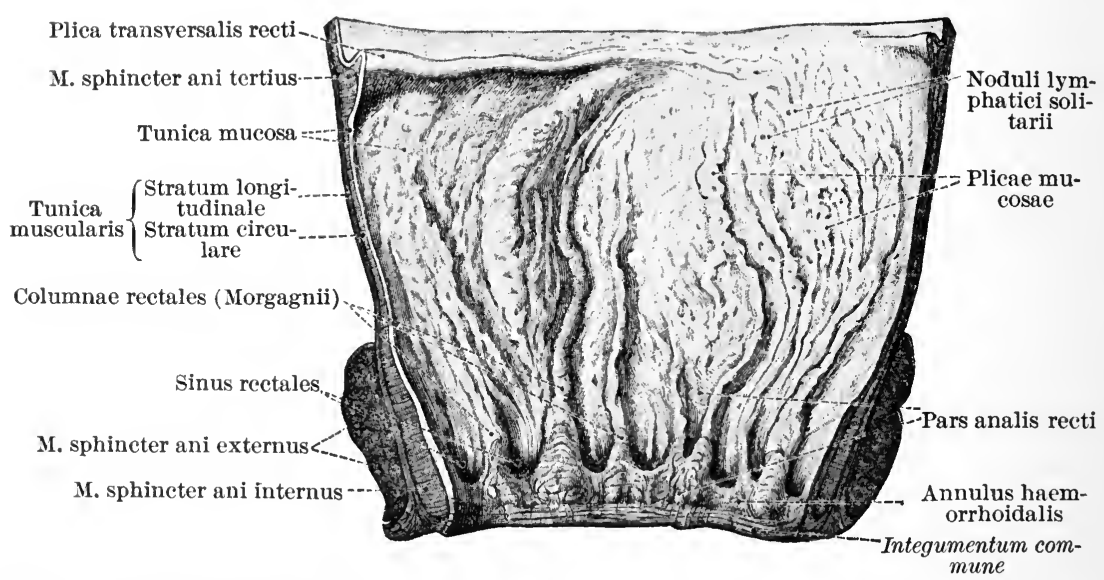

The tunica mucosa of the lower part of the intestinum rectum and its transition into the integumentum commune. (After Toldt, Anat. Atlas, Wien, 1900, 2 Aufl., p. 429, Fig. 712.)

In the anal part of the rectum (pars analis recti) study-

(ac) Rectal columns of Morgagni (columnae rectales [Morgagnii]).

(ad) Rectal sinuses (sinus rectales).

(ae) Hemorrhoidal ring (annulus haemorrhoidalis).

(b) Submucous tela (tela submucosa).

(c) Muscular coat (tunica muscularis).

(ca) Longitudinal layer (stratum longitudinale).

(caa) Rectococcygeus muscle (M. rectococcygeus).

(cb) Circular layer (stratum circulare).

Note that the fibres of the circular layer are increased in number in the base of each of the transverse rectal plicae.

$(c b a)$ Internal sphincter muscle of anus (M. sphincter ani internus). 
Compare the general structure of the rectum with that of the colon, noting any differences.

Urinary Bladder (Vesica urinaria). (Vide Spalteholz, Figs. 640642.)

Distend the bladder with air by a bicycle-pump before the dissection of its wall is made. Study the layers.

(a) Serous tunic (tunica serosa).

(aa) Transverse vesical fold (plica vesicalis transversa), when the bladder is empty.

(b) Muscular coat (tunica muscularis).

(ba) External layer (stratum externum).

(baa) Pubovesical muscle (M. pubovesicalis).

(bab) Rectovesical muscle (M. rectovesicalis).

(bb) Middle layer (stratum medium).

(bba) Sphineter musele of bladder (M. sphincter urethrae).

What is the relation of this latter to the prostatic muscle (M. prostaticus)?

(bc) Internal layer (stratum internum).

(c) Submucous tela (tela submucosa).

The mucous membrane of the bladder may be seen to the best advantage if the bladder is opened along the anterior surface from the apex to the neck. The incision should be made in the median line and the urethra opened up along its whole length, to the external urethral orifice. (See Fig. 289.)

(d) Mucous membrane (tunica mucosa).

Note that when the bladder is empty or only partially distended the mucous membrane is thrown into folds. Study the following:

(da) Vesical lymph-nodules (noduli lymphatici vesicales).

$(d b)$ Lieutaud's trigone of the bladder (trigonum vesicae [Lieutaudi]).

(dba) Vesical uvula (uvula vesicae).

$(d b b)$ Ureteral fold (plica ureterica).

$(d b c)$ Orifice of ureter (orificium ureteris).

Pass a probe through the ureter into the bladder and note the angle at which it pierces the bladder wall. Why does urine not pass from the bladder back through the ureter?

(dc) Internal orifice of urethra (orificium urethrae internum).

(dd) Urethral ring (annulus urethralis).

Raise carefully the mucous membrane covering the trigone and note the muscular tissue in the folds which bound it.

The penis should now be pinned down upon a block or a dissecting-tray and the mucous membrane of the different divisions of the urethra examined. 


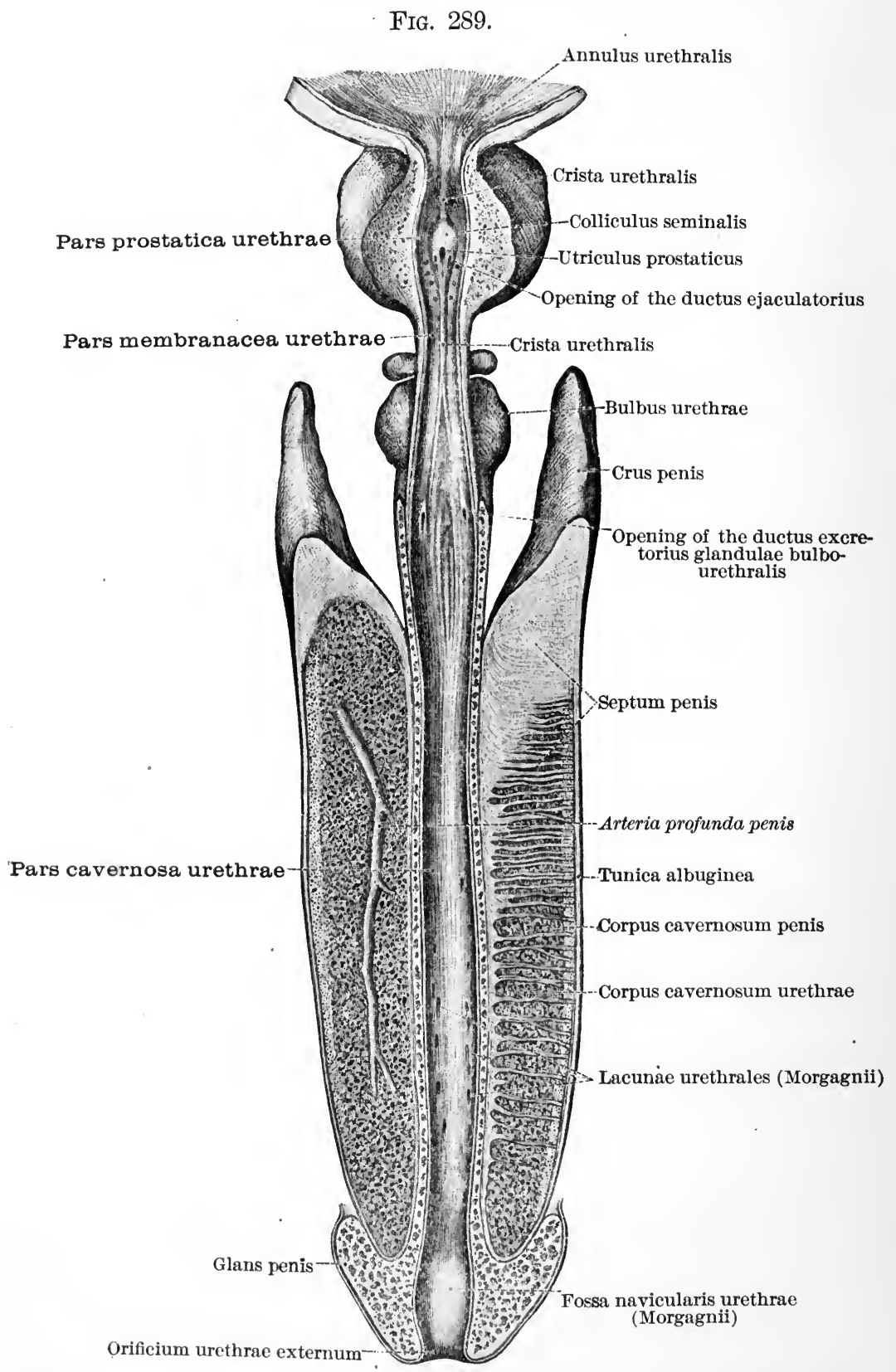

The male urethra, urethra virilis, laid open by a sagittal incision beside the septum penis. (After Toldt, Anat. Atlas, Wien, 1900, 2 Aufl, p. 490, Fig. 835.) 


\section{Male Urethra (Urethra virilis).}

Review at this stage of your dissection the general direction of the urethral canal, also the variations in the diameter of its lumen in different parts. Study the following (Fig. 289) :

(a) Prostatic part (pars prostatica). (Vide Spalteholz, Fig. 643.)

(aa) Urethral crest (crista urethralis).

( $a b)$ Seminal hillock (colliculus seminalis).

(ac) Prostatic utricle (utriculus prostaticus).

Pass a probe into the prostatic utricle and determine its length. What is its homologue in the female? It is of importance surgically, as it may render difficult the passing of a small sound or bougie and may harbor infection.

(ad) Mouth of ejaculatory ducts.

Pass a bristle into the duct and determine its general course and direction.

(ae) Prostatic ducts (ductus prostatici).

Squeeze the prostate. The fluid that exudes is the prostatic juice (succus prostaticus).

(af) Prostatic muscle (M. prostaticus).

(b) Membranous part (pars membranacea).

Note the fixity of this part, also the narrowness of the lumen. It is the portion most frequently ruptured. Being the narrowest part of the urethra, it is also the most frequently involved in stricture.

(c) Cavernous part (pars cavernosa).

(ca) Navicular fossa of urethra (fossa navicularis urethrae [Morgagnii]).

(cb) Valve of navicular fossa (valvula fossae navicularis).

(cc) External urethral orifice (orificium urethrae externum).

(cd) Urethral lacunae of Morgagni (lacunae urethrales [Morgagnii]).

(ce) Urethral glands of Littré (glandulae urethrales [Littrei]).

To complete this study the student should examine sections of the prostate and penis under the dissecting microscope.

\section{Wall of the Seminal Vesicle. (Vide Spalteholz, Fig. 653.)}

Straighten out the seminal vesicles and study the following coats :

(a) Adventitious coat (tunica adventitia).

(b) Muscular coat (tunica muscularis).

(c) Mucous coat (tunica mucosa). 


\section{DISSECTION OF THE FEMALE PELVIS.}

Before beginning this dissection review the anatomy of the bony pelvis. Compare the male and female pelvis, noting differences as regards pelvic diameters and the size and general structure of the bones. What is the meaning of the terms dolichopelvic, mesatipelvic, and platypelvic? What is the "pelvic index" and how is it estimated?

\section{Fig. 290.}

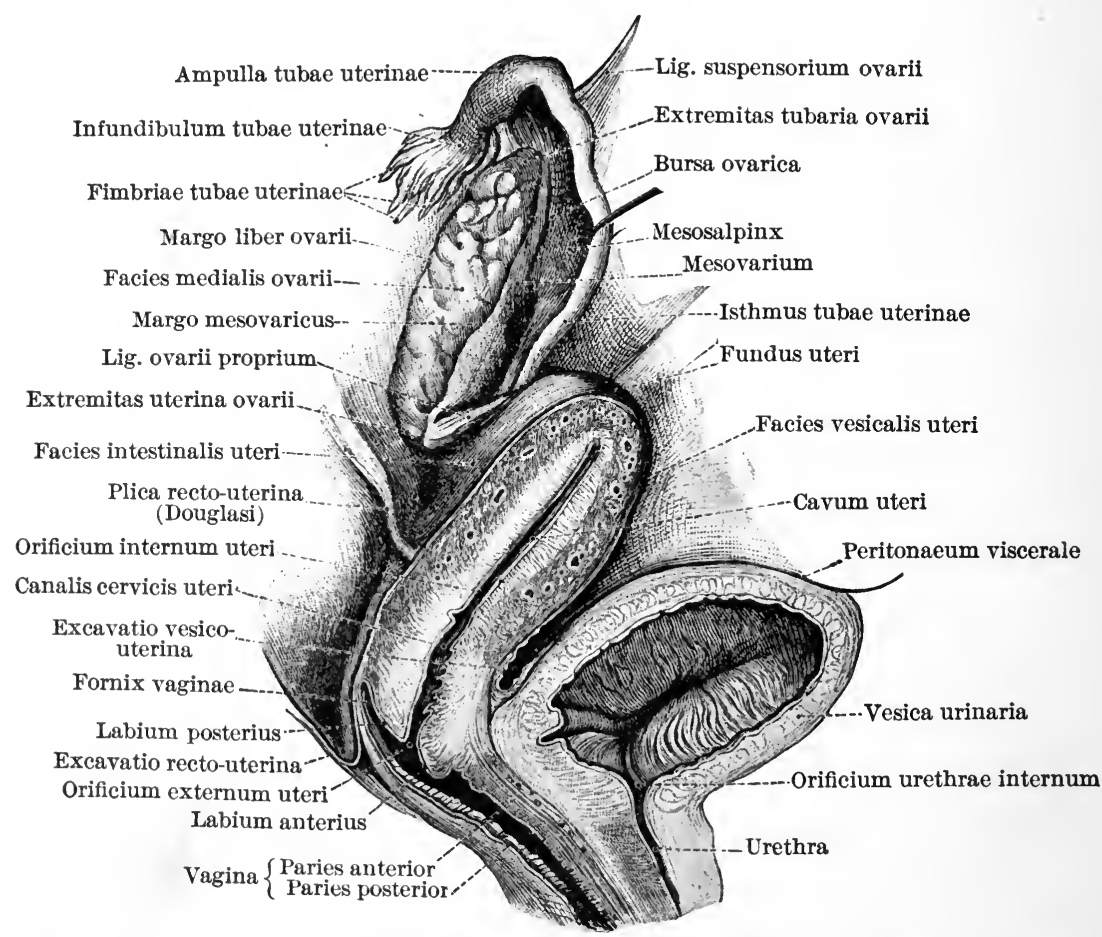

The genital organs of a girl ten years old, in median section. Left side. (After Toldt, Anat. Atlas, Wien, 1900, 2 Aufl., p. 495, Fig. 846.)

Before disturbing any relations establish exactly the position of each viscus. What is the relation of the body of the uterus to the bladder and to the rectum? Is the body of the uterus flexed upon the neck of the uterus? Make frequent use of models, cross-sections, and moist preparations in the further study of these parts. 
Peritoneum of Female Pelvis. (Vide Figs. 290, 292, and Spalteholz, Fig. 667.)

Trace the reflections of the peritoneum in the female pelvis. Establish the relations of the peritoneum to each viscus and the pelvic wall, noting-

(a) Vesico-uterine excavation (excavatio vesico-uterina).

(b) Recto-uterine excavation (excavatio recto-uterina).

(c) Recto-uterine folds of Douglas (plicae recto-uterinae [Douglasi]).

(d) Broad ligament of uterus (ligamentum latum uteri).

(da) Mesometrium (mesometrium).

(db) Mesosalpinx (mesosalpinx).

(dc) Mesovarium (mesovarium).

What structures are contained between the two layers of the broad ligaments?

(e) Suspensory ligament of ovary (Lig. suspensorium ovarii).

Feel this ligament. Do you feel any vessels in it? If so, what vessels are they?

Hypogastric Plexus (Plexus hypogastricus). (Vide Toldt, Fig. I333.)

This plexus is similar to the one in the male. Refer to the pages on the male pelvis for directions as to dissection. The following additional plexuses are found in the female:

(a) Uterovaginal plexus (plexus uterovaginalis).

(b) Cavernous plexus of clitoris (plexus cavernosus clitoridis).

(ba) Greater cavernous nerve of clitoris ( $N$. cavernosus clitoridis major).

(bb) Lesser cavernous nerves of clitoris ( $N n$. cavernosi clitoridis minores).

Pelvic Fascia (Fascia pelvis) in the Female. (Vide Spalteholz, Fig. 667.)

To expose the pelvic fascia from above, remove the peritoneum by blunt dissection from the viscera and the pelvic wall. This should be done on one side only. Avoid injury to the vessels and nerves. The anterior leaf of the broad ligament may also be left. Study now the following divisions of the fascia:

(a) Superior fascia of pelvic diaphragm (fascia diaphragmatis pelvis superior).

(b) Tendinous arch of pelvic fascia (arcus tendineus fasciae pelvis).

(c) Tendinous arch of levator ani muscle (arcus tendineus M. levatoris ani).

(d) Endopelvic fascia (fascia endopelvina). 
Determine the relation of this fascia to the uterine and vaginal walls.

(e) Middle pubovesical ligament (Lig. pubovesicale medium).

(f) Lateral pubovesical ligaments (Ligg. pubovesicalia lateralia).

(g) Inferior fascia of pelvic diaphragm (fascia diaphragmatis pelvis inferior).

The latter fascia has already been studied in the dissection of the fossa ischiorectalis. It should now be reviewed and its relation to the fascia obturatoria and the arcus tendineus $M$. levatoris ani established.

\section{Pelvic Vessels and Nerves.}

Dissect out the arteries and veins of the pelvis. Consult the pages on the male pelvis, noting the differences. Study the following:

Arteries.

(a) Uterine artery (arteria uterina). (Vide Spalteholz, Fig. 471.)

(aa) Vaginal artery (A. vaginalis).

(ab) Tubal branch (A. tubarius).

(ac) Branch to ovary (ramus ovarii).

Pay especial attention to the relation between the uterine artery and the ureter. At what distance from the cervix uteri does the artery eross the ureter?

(b) Ovarian artery (A. ovarica).

Through what ligament does the ovarian artery pass to reach the ovary? What is its relation to the ligamentum suspensorium ovarii?

Veins.

(a) Uterovaginal plexus (plexus uterovaginalis). (Vide Spalteholz, Fig. 502.)

Lymphatics.

Use special preparations for this study. (Consult Poirier et Charpy, i. 5, Figs. 338, 339.) Determine into what lymph-nodes the lymphaties of the cervix and the body of the uterus empty.

Pelvic Spinal Nerves.

Consult the directions in regard to the male pelvis for the dissection of these nerves.

Rectum (Intestinum rectum). (Vide Spalteholz, Figs. 575, 576, 59r, $655,656,665$.

The female rectum does not differ materially in structure from that of the male. Its relations should be carefully studied. Establish its relations to the posterior vaginal wall and the perineal body. Use models and cross-sections as supplementary to the cadaver for this purpose. 
Bladder (Vesica urinaria). (Vide Fig. 292, and Spalteholz, Figs. 633, 640.)

Compare the form of the bladder in the two sexes; also its position in the pelvis. Distend the bladder with air. How does the naturally distended bladder compare in form with the empty? Consult the pages on the male pelvis for the nomenclature of the bladder and the directions for dissection.

Pelvic Part of Ureter (Pars pelvina ureteris). (Vide Fig. 29I, and Spalteholz, Fig. 665.)

Isolate the ureter as it passes into the pelvis at the bifurcation of the common iliac arteries. Compare the lengths of the pelvic divisions in the male and female. Trace the ureter forward to the base of the broad ligament. Establish its relations to the uterine artery. What surgical significance has this anatomical relation?

$$
\text { Fig. } 291 .
$$

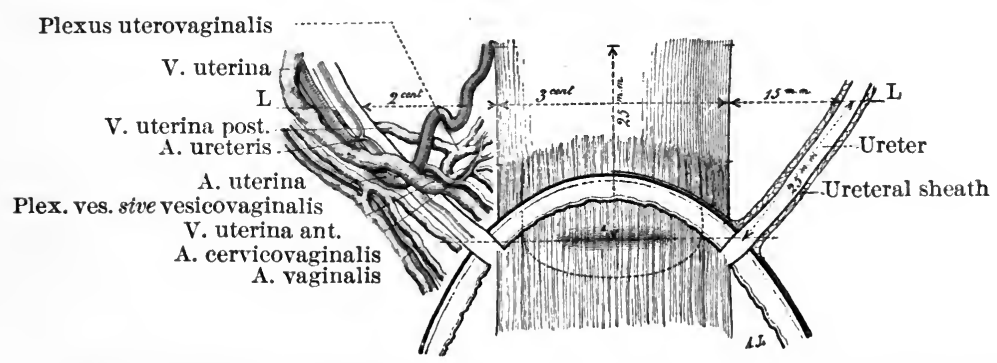

Diagram showing the relations of the ureter to the cervix [uteri] and adjacent vessels. The line LL passes a little below the vaginal isthmus of the uterus. The longitudinal striations indicate the vaginal wall, and the dotted line shows the contour of the cervix uteri. The vertical line, $25 \mathrm{~mm}$., indicates the length of the cervix uteri. (From Poirier et Charpy, Traité d'Anat. hum., Paris, 1901, t. v. p. 457 , Fig. 320.)

Trace the ureter forward to the bladder and establish its relation to the anterior vaginal wall. At what angle does the ureter enter the bladder?

Uterus (Uterus) (O. T. Womb). (Vide Spalteholz, Figs. 662, 664, $665,667$.

Use models and special preparations for this study. Establish the position and relations of the uterus. How does the infantile compare with the adult organ?

(a) Body of uterus (corpus uteri).

(aa) Fundus of uterus (fundus uteri).

(ab) Lateral margin (margo lateralis). 
(ac) Vesical surface (facies vesicalis).

(ad) Intestinal surface (facies intestinalis).

(ae) Cavity of uterus (cavum uteri).

(b) Neck of uterus (cervix uteri).

(ba) Supravaginal part of neck (portio supravaginalis [cervicis]).

(bb) Vaginal part of neck (portio vaginalis [cervicis]).

FIG. 292.

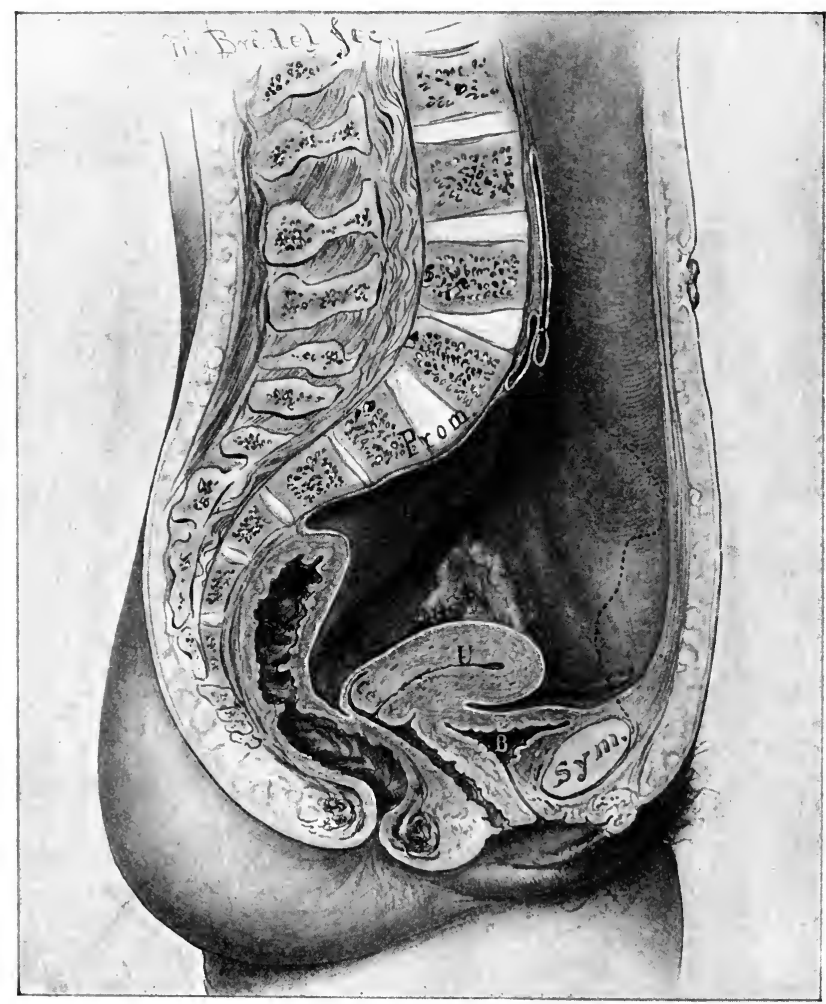

Median section of the body of a woman.

$U$, uterus; $B$, urinary bladder; Prom, promontory of the pelvis; Sym, symphysis ossium pubis; Cerv, cervix of the uterus. The dotted line shows the position of the upper boundary of the hip-bone. (After Cullen, Cancer of the Uterus, Baltimore, 1900, p. 7, Fig. 3.)

(bc) External orifice of uterus (orificium uteri externum) (O. T. external os).

(bca) Anterior lip (labium anterius).

(bcb) Posterior lip (labium posterius).

(bd) Canal of neck of uterus (canalis cervicis uteri).

(be) Internal orifice of uterus (orificium internum uteri) (O. T. internal os). 
Vagina. (Vide Figs. 290, 292, and Spalteholz, Figs. 664, 665.)

Note the length of its anterior and posterior walls. Palpate the cervix uteri. How does it differ in the parous and nulliparous? What is the relation of its long axis to the pelvic brim?

Fig. 293.

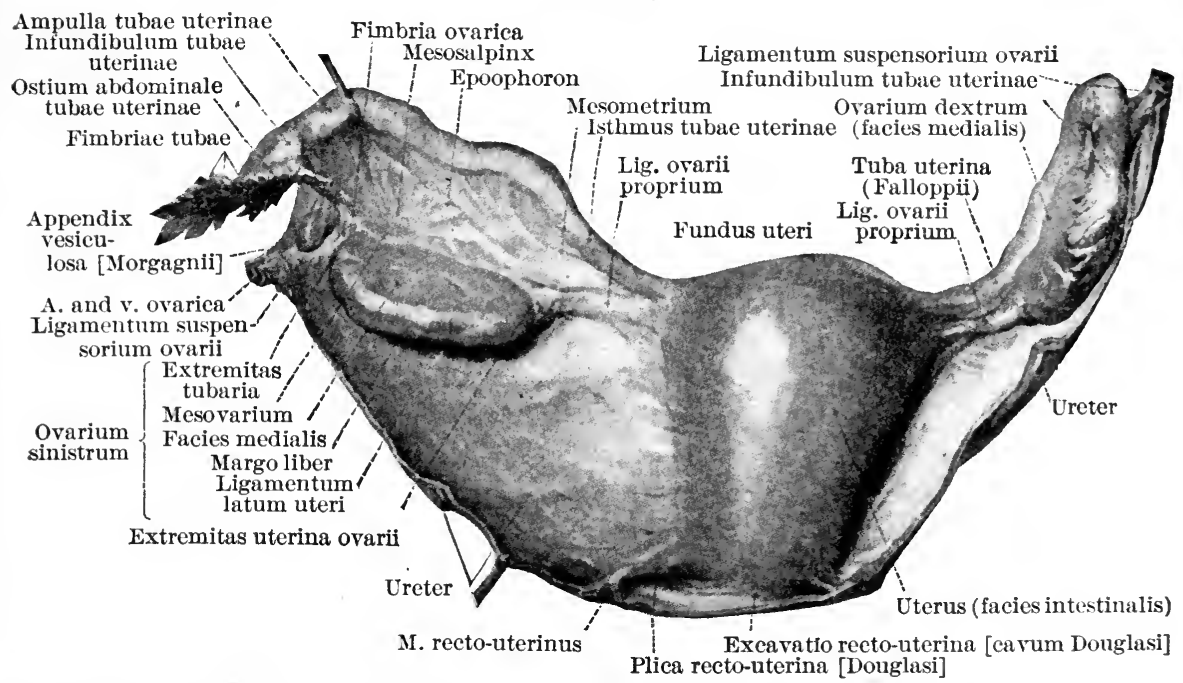

Uterus, Fallopian tubes, and ovaries, viewed from behind. (On the right the parts have been left in their natural position, on the left the ligamentum latum has been unfolded.) (After Spalteholz, Hand Atlas of Hum. Anat., Leipzig, 1903, vol. iii. p. 596, Fig 662.)

Determine its relations to the bladder and rectum. Pass a probe through the ureter from the pelvis and determine the relations of the ureter to the vaginal wall. What is the relation of the urethra? Study-

(a) Fornix of vagina (fornix vaginae).

(b) Anterior wall (paries anterior).

(c) Posterior wall (paries posterior).

(d) Hymen (hymen [femininus]).

(e) Hymeneal earuncles (carunculae hymenales).

Are the latter present? If so, what is their significance?

( $f$ ) Muscular coat (tunica muscularis).

(g) Mucous coat (tunica mucosa).

(ga) Vaginal lymph-nodules (noduli lymphatici raginales).

( $g b)$ Rugae (rugae vaginales).

(gc) Columns of the rugae (columnae rugarum).

(gca) Posterior (columna rugarum posterior).

( $g c b)$ Anterior (columna rugarum anterior).

(gcc) Carina urethralis [vaginae]. 
Ovary (Ovarium). ${ }^{1}$ (Vide Figs. 293, 295, and Spalteholz, Fig. 632.)

(a) Hilus of ovary (hilus ovarii).

(b) Medial surface (facies medialis).

(c) Lateral surface (facies lateralis).

(d) Free margin (margo liber).

(e) Mesovarian margin (margo mesovarica).

(f) Tubal extremity (extremitas tubaria).

(g) Uterine extremity (extremitas uterina).

(h) Proper ligament of ovary (ligamentum ovarii proprium).

(i) Corpus luteum (corpus luteum) ("yellow body").

(j) Corpus albicans (corpus albicans) ("white body").

Examine section of ovary under hand-lens.

(k) Stroma of ovary (stroma ovarii).

(l) Primary ovarian follicles (folliculi oophori primarii).

(m) Vesicular ovarian follicle (folliculus oophori vesiculosus [Graafi]).

(n) Theea of follicle (theca folliculi). (na) External tunic (tunica externa).

$(n b)$ Internal tunic (tunica interna).

(o) Liquor of follicle (liquor folliculi).

(p) Granular layer (stratum granulosum).

(q) Cumulus oophorus (O. T. discus proligerus). (qa) Ovule (ovulum).

What is the relation of the ovary to the posterior layer of the broad ligament? What is the "germinal epithelium"? Has the ovary a serous covering comparable to that of the testicle?

Examine the broad ligament with the naked eye and under a hand-lens and study the following structures. Determine what embryonic structures they are developed from, and what their homologues in the male are.

(a) Paroophoron (paroophoron).

(b) Epoophoron (epoophoron).

(ba) Longitudinal duet of epoophoron (ductus epoophori longitudinalis [Gärtneri]).

(bab) Transverse ductules (ductuli transversi).

(bb) Vesicular appendages (appendices vesiculosi [Morgagnii]).

Uterine Tube (Tuba uterina [Fallopii]) (O. T. Fallopian Tube). (Vide Spalteholz, Figs. 662-664, 667.)

Determine the positions and axes of the tubes, also their relations to the neighboring viscera. From what does the Fallopian tube develop? Note the following:

${ }^{1}$ For a detailed account of the blood-supply of the ovary, see "Origin, Development, and Degeneration of the Blood-vessels of the Human Ovary," by J. G. Clark, in Contributions to the Sci. of Med., Baltimore, 1900. 
(a) Abdominal mouth of uterine tube (ostium abdominale tubae uterinae).

This communicates with the peritoneal cavity.

(b) Infundibulum of uterine tube (infundibulum tubae uterinae).

(c) Fimbriae of tube (fimbriae tubae). (ca) Ovarian fimbria (fimbria ovarica).

(d) Ampulla of uterine tube (ampulla tubae uterinae).

(e) Isthmus of uterine tube (isthmus tubae uterinae).

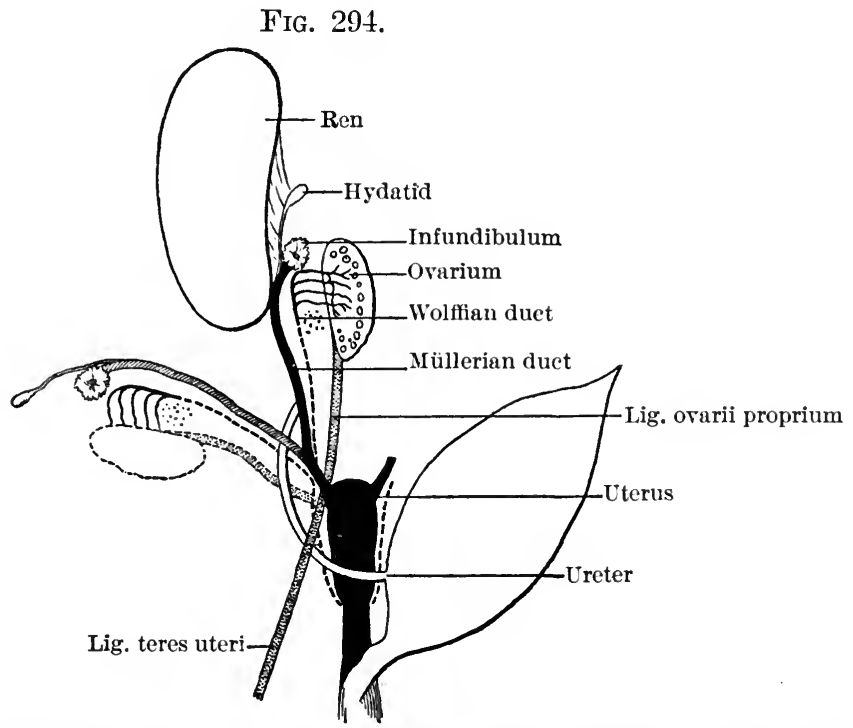

Scheme of the development of the internal female genital organs, showing their initial situation and their situation again after apparent descent. Organs which persist are in unbroken outline (except the ovary in the lower position), those that atrophy are in dotted outline (Hertwig). (After Poirier et Charpy, Traité d'Anat. hum., Paris, 1901, 2 ed., t. v. p. 399, Fig 290.)

\section{Round Ligament of Uterus (Ligamentum teres uteri). (Vide Spalte-} holz, Figs. 665, 667, 675.)

Dissect out the round ligament forward to the annulus inguinalis abdominalis. Do any lymphatics accompany the round ligament? If so, with what lymph-nodes do they communicate? From what is the round ligament developed, and what is its homologue in the male? What is its function? What is the vaginal process of the peritoneum (processus vaginalis peritonaei) (O. T. canal of Nuck)?

Diaphragm of the Pelvis (Diaphragma pelvis). (Vide Spalteholz, Figs. 670, 671.)

Dissect out the following muscles and determine their relations to the bladder, vagina, uterus, and rectum. Compare these muscles with those in the ma'e, noting carefully any differences. 
(a) Levator muscle of anus (M. levator ani).

(aa) Pubococeygeus muscle (M. pubococcygeus).

(ab) Iliococeygeus muscle (M. iliococcygeus).

(b) Coceygeus muscle ( $M$. coccygeus).

Remove the viscera from the pelvic cavity. Divide the vessels and nerves connected with the viscera, the levator ani muscle, and the pubovesical ligaments. Divide the structures holding the urethra and vagina to the pubic arch. Separate the rectum from the sacrum and coccyx. Now study-

\section{Other Pelvic Muscles and Fascia.}

(a) Obturator internus muscle (M. obturator internus). (Vide Spalteholz, Fig. 373.)

(b) Obturator fascia (fascia obturatoria).

(c) Piriform muscle (M. piriformis).

\section{Rectum (Intestinum rectum). (Vide Spalteholz, Fig. 576.)}

Dissect the rectum away from the vagina and uterus. Open the rectum along its anterior wall and study its-

(a) Mucous membrane (tunica mucosa).

(aa) Transverse rectal folds (plicae transversales recti).

(ab) Lymph-nodules (noduli lymphatici).

In the anal part of the rectum (pars analis recti) study-

(ac) Rectal columns of Morgagni (columnae rectales [Morgagnii]).

(ad) Hemorrhoidal ring (annulus haemorrhoidalis).

(b) Submucous tela (tela submucosa).

(c) Muscular tunic (tunica muscularis).

(ca). Longitudinal layer (stratum longitudinale). (caa) Rectococcygeus muscle (M. rectococcygeus).

( $c b)$ Circular layer (stratum circulare).

Note that the circular fibres are increased in number in the base of each transverse rectal fold.

$(c b a)$ Internal sphincter muscle (M. sphincter ani internus).

\section{Female Urethra.}

Open the bladder along its anterior surface. Carry the incision along the anterior wall of the urethra. Pin the urethra and bladder down upon a cork dissecting-tray and study the female urethra (urethra muliebris). (Vide Spalteholz, Fig. 642.) Compare it with the male urethra, noting differences.

(a) External urethral orifice (orificium urethrae externum).

(b) Spongy body of urethra (corpus spongiosum urethrae).

(c) Muscular tunic (tunica muscularis). 
(ca) Circular layer (stratum circulare).

( $c b)$ Longitudinal layer (stratum longitudinale).

(d) Submucous tunic (tunica submucosa).

(e) Mucous tunic (tunica mucosa).

(ea) Urethral glands ( $G l$. urethrales).

(eb) Urethral crest (crista urethralis).

Urinary Bladder (Vesica urinaria). (Vide Spalteholz, Fig. 642.)

Refer to the pages on the male pelvis. Compare the bladder of the female with that of the male as to form, size, and musculature.

\section{Interior of Vagina.}

Open the vagina along its anterior wall. Carry the incision into the uterus and lay open the Fallopian tube on each side. Study at this time also the relations of the ovary and its ligament to the uterus.

Pin down the structures upon a dissecting-block and study the following. (Vide Spalteholz, Fig. 664.)

(a) Muscular tunic (tunica muscularis).

(b) Mucous tunic (tunica mucosa).

(ba) Vaginal lymphatic nodules (noduli lymphatici vaginales).

(bb) Vaginal rugae (rugae vaginales).

(bc) Columns of rugae (columnae rugarum).

(bca) Posterior column of rugae (columna rugarum posterior).

(bcb) Anterior column of rugae (columna rugarum anterior).

(bcc) Urethral carina (carina urethralis [Morgagnii]).

Interior of Uterus. (Vide Fig. 295, and Spalteholz, Fig. 664.)

(a) Cavity of uterus (cavum uteri).

(b) Internal orifice of uterus (orificium uteri internum) (O. T. internal os).

(c) Neck of uterus (cervix uteri).

(ca) Supravaginal part of cervix (portio supravaginalis [cervicis]).

(cb) Vaginal part of cervix (portio vaginalis [cervicis]).

(cba) External orifice of uterus (orificium uteri externum)

(O. T. external os).

(1) Anterior lip (labium anterius).

(2) Posterior lip (labium posterius).

(d) Canal of neck of uterus (canalis cervicis uteri).

(da) Palmate folds (plicae palmatae).

( $d b)$ Cervical glands (Gl. cervicales).

(e) Parametrium (parametrium).

(f) Serous tunic (tunica serosa [perimetrium]). 
(g) Muscular tunic (tunica muscularis).

(h) Muscular tunic of neck (tunica muscularis [cervicis]).

(i) Mucous tunic (tunica mucosa).

(ia) Uterine glands (Gl. uterinae).

\section{Interior of Uterine Tube (Tuba uterina [Fallopii]) (O. T. Fallopian} Tube). (Vide Fig. 295, and Spalteholz, Fig. 664.)

(a) Uterine part (pars uterina).

(b) Uterine opening of tube (ostium uterinum tubae).

(c) Serous tunic (tunica serosa).

(d) Adventitious tunic (tunica adventitia).

Fig. 295.

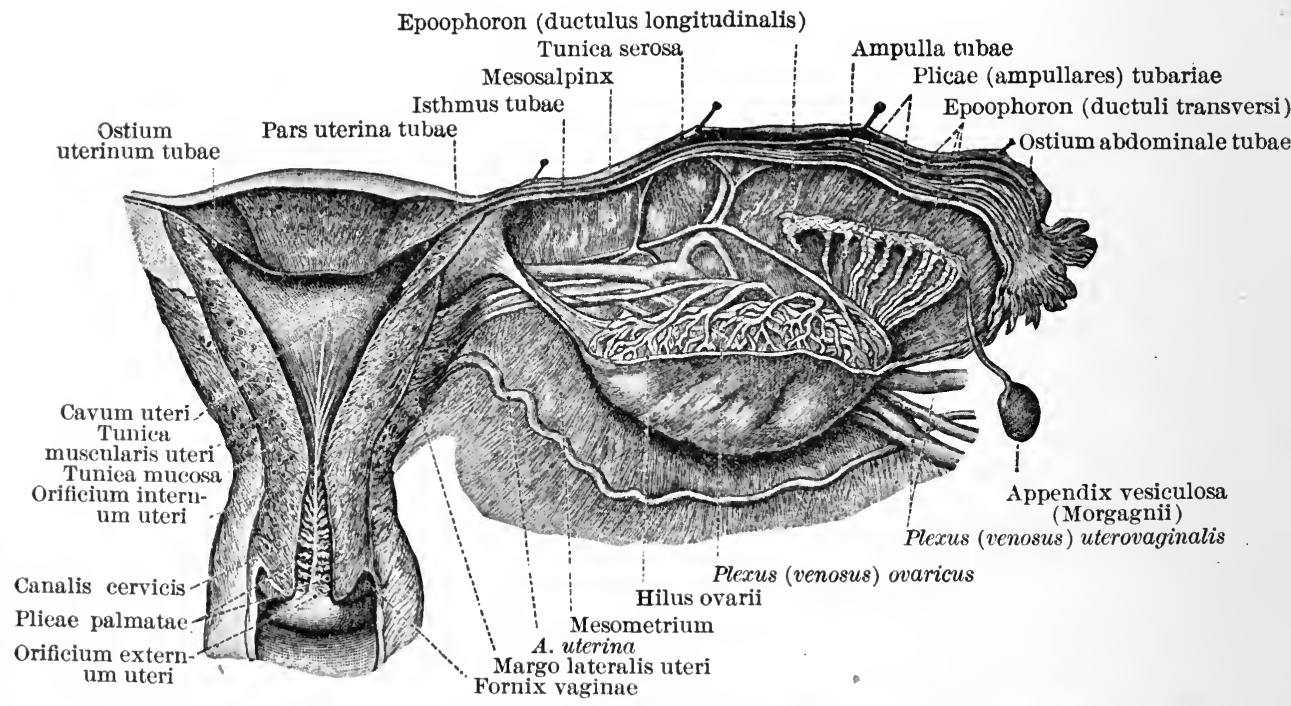

The uterus and right Fallopian tube, tuba uterina, opened up posteriorly. The peritoneum has been stripped off the posterior surface of the broad ligament. (After Toldt, Anat. Atlas, Wien, 1900, 2 Aufl., p. 496, Fig. 847.)

(e) Muscular tunic (tunica muscularis).

(ea) Longitudinal layer (stratum longitudinale).

(eb) Circular layer (stratum circulare).

( $f$ ) Submucous tela (tela submucosa).

(g) Mucous tunic (tunica mucosa).

(ga) Tubal folds (plicae tubariae).

(gaa) Ampullar folds (plicae ampullares).

(gab) Isthmian folds (plicae isthmicae). 


\section{JOINTS OF THE PELVIS.}

In studying the following joints determine the general characteristics of the bony surfaces entering into their formation. Study the movements which occur between the joint surfaces, the muscles which produce these movements, and the mechanism by which they are restricted. Pay particular attention to the

FIG. 296.

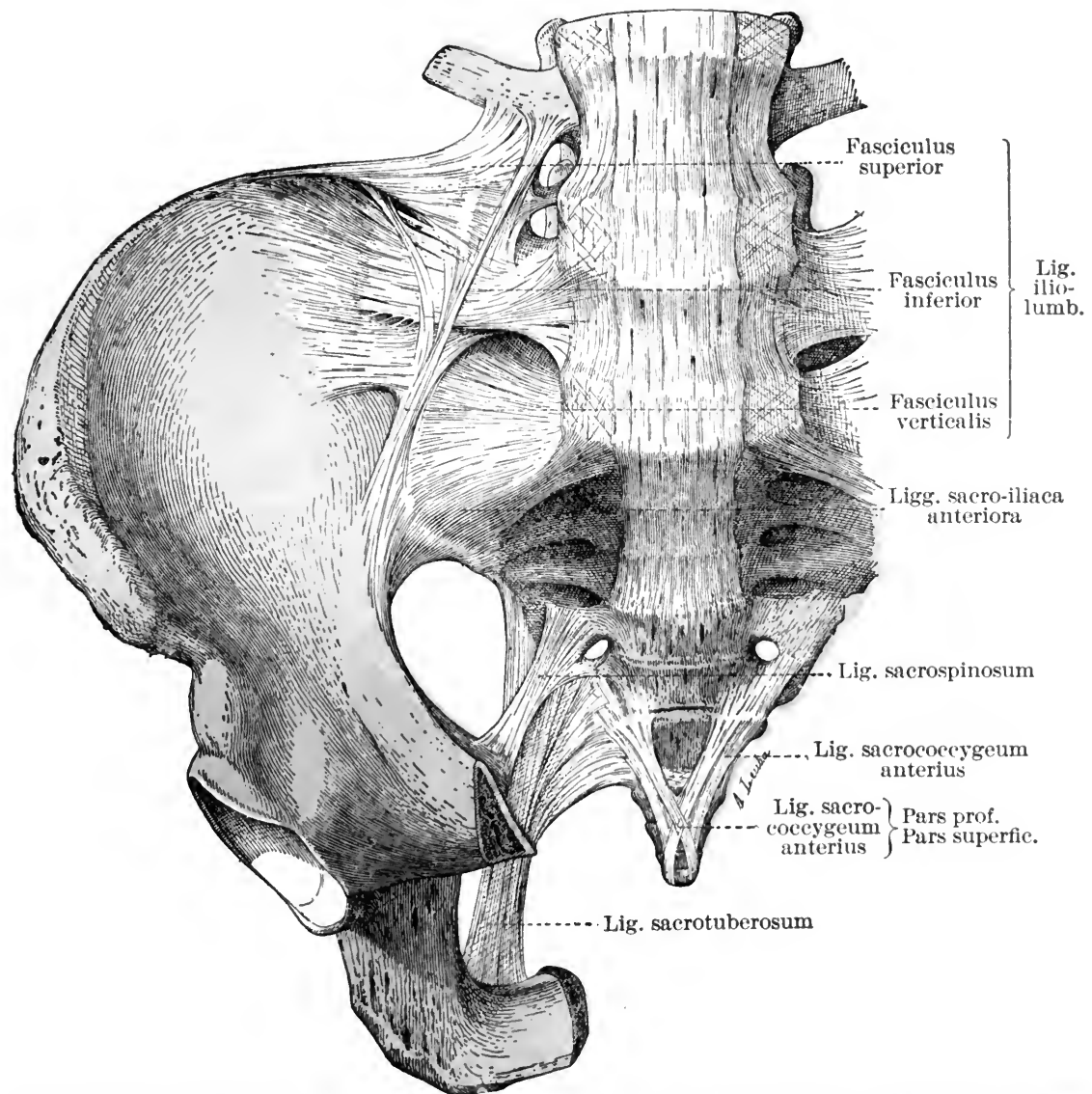

Articulations and ligaments of the pclvis. Anterior view. The os pubis and the os ischii have been partly eut away. (From Poirier et Charpy, Traité d'Anat. hum., Paris, t. i. p. 700, Fig. 706.)

synovial membranes and their relations to the joint-cavity. Review the bursae which you have found in your previous dissection, and establish their relations to the joints. If the ligaments have dried, their dissection may be rendered easier by soaking 
them in water. Remove the pelvic muscles and nerves. The origins and insertions of the muscles should be carefully measured and charted before they are removed.

FIG. 297.

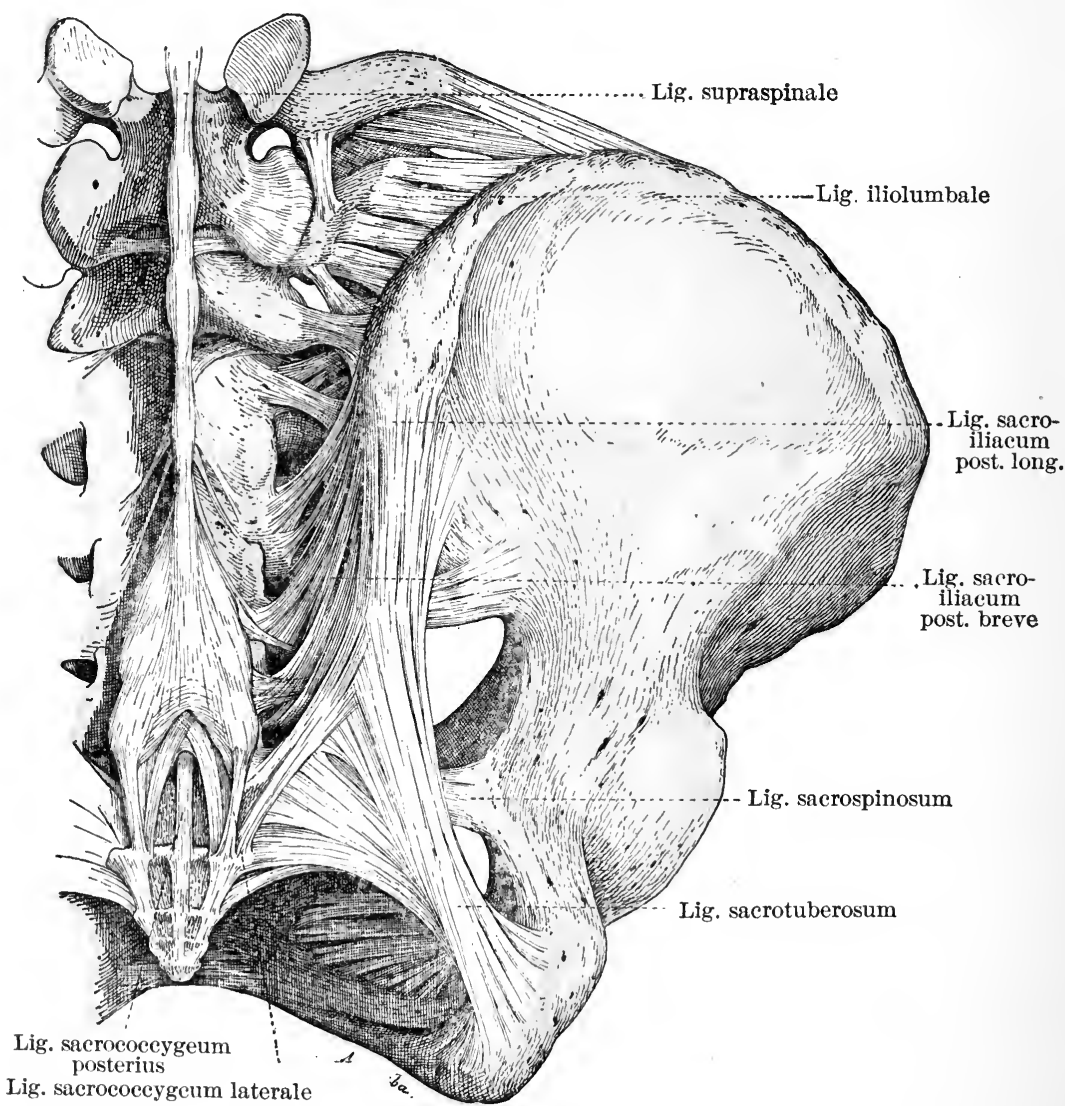

Articulations and ligaments of the pelvis. Posterior view. (From Poirier et Charpy, Traité d'Anat. hum., Paris, t. i. p. 701, Fig. 707.)

Ligaments of the Pelvic Girdle (Ligg. cinguli extremitatis inferioris). (Figs. 296 and 297.)

(a) Obturator membrane (membrana obturatoria). (aa) Obturator canal (canalis obturatorius).

(b) Iliolumbar ligament (ligamentum iliolumbale).

(c) Sacrotuberous ligament (ligamentum sacrotuberosum) (O. T. posterior or great sacrosciatic ligament).

(ca) Falciform process (processus falciformis) (O. T. falciform ligament). 
(d) Sacrospinous ligament (ligamentum sacrospinosum) (O. T. anterior or small sacrosciatic ligament).

(da) Greater sciatic foramen (foramen ischiadicum majus).

(db) Lesser sciatic foramen (foramen ischiadicum minus).

\section{Sacro-iliac Joint (Articulatio sacro-iliaca).}

(a) Anterior sacro-iliac ligaments (Ligg. sacroiliaca anteriora).

(b) Interosseous sacro-iliae ligaments (Ligg. sacroiliaca interossea).

(c) Short posterior sacro-iliae ligament (Lig. sacroiliacum posterius breve).

(d) Long posterior sacro-iliac ligament (Lig. sacroiliacum posterius longum).

Divide the ligaments and separate the bones. Study the synovial space.

FIG. 298.

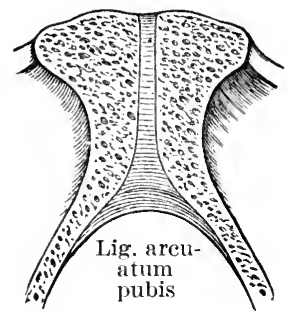

Frontal section through the symphysis ossium pubis. (From Gegenbaur, Lehrb. der Anat. des Mensch., Leipzig, 1899, 7 Aufl., Bd. i. p. 293, Fig. 231.)

Symphysis of Pubic Bones (Symphysis ossium pubis). (Fig. 298.)

(a) Superior pubic ligament (Lig. pubicum superius).

(b) Areuate ligament of pubis (Lig. arcuatum pubis).

(c) Interpubic fibrocartilaginous lamina (lamina fibrocartilaginea interpubica).

Use the chisel and remove a piece of the bone from the front of the joint. Study the interpubic fibrocartilaginous lamina and the synovial membrane of the joint-cavity.

\section{Symphysis of Sacrum and Coccyx (Symphysis sacrococcygea).}

(a) Superficial posterior sacrococygeal ligament (Lig. sacrococcygeum posterius superficiale).

(b) Deep posterior sacrococeygeal ligament (Lig. sacrococcygeum posterius profundum).

(c) Anterior sacrococeygeal ligament (Lig. sacrococcygeum anterius).

(d) Lateral sacrococeygeal ligament (Lig. sacrococcygeum laterale). 


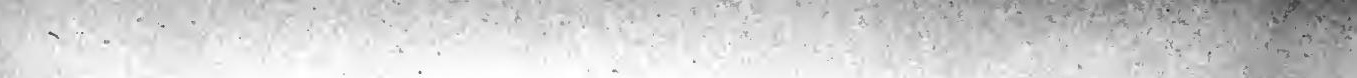




\section{CORRIGENDA}

Page 31.-Among "General Works" the books of Henle and Hyrtl should have been included.

Page 77.-At side of figure, instead of "M. brachiodorsalis" read "M. brachioradialis."

Page 99.-At side of figure, instead of "M. adductor pollicis brevis" read "M. abductor pollicis brevis."

Page 127.-At side of figure, instead of "A. pudenda communis" read "A. pudenda interna."

Page 132.-At side of figure, instead of " $\mathrm{N}$. saphena mag." read "V. saphena magna."

Page 161.-At side of figure, instead of "N. et M. peronaea long." read "Nerve to the M. peronaeus longus."

In same figure, instead of "M. peronaeus prof." read " $\mathrm{N}$. peronaeus profundus."

Page 164.-At side of figure, instead of " $\mathrm{N}$. tiabialis" read " $\mathrm{N}$. tibialis."

Page 186.-On figure, instead of "Lig. tibiocalcaneum" read "Ligamentum calcaneotibiale."

Page 198.-On figure, instead of "Gyrus cerebri" read "Cortex cerebri."

Page 224.-At side of figure, instead of " $M$. trapezoideus" read "M. trapezius."

Page 252, line 11.-Instead of "External carotid" read "Branches of external carotid."

Page 412.-At side of figure, instead of "Ost. atrioventr. dextrum" read "Ostium venosum dextrum."

Page 437.-At side of figure, instead of "A. pudendus externus" read "A. pudenda externa;" also, instead of "A. perinei superficialis" read " A. perinei."

Page 440.-At side of figure, instead of " $\mathrm{N}$. ischiocavernosus" read " $\mathrm{M}$. ischiocavernosus ;" also, instead of "N. pudendus" read "N. perinei ;" instead of "N. cut. N. fem. post." read "N. cutaneus femoris posterior."

Page 441.-At side of figure, instead of "N. trans. perinei prof." read "M. transversus perinei profundus."

Page 497.-At side of Fig. B, instead of "fusion to" read " fusion with." places.

Page 506.-In foot-note, instead of "perineal" read "perirenal" in two

Page 522.-At side of Fig. 276, instead of "Fibro-elastic network of the int. rect." read "Fibro-elastic network of the rectum;" also, instead of "N. sph. ani ext. subcut." read "M. sphincter ani subcutaneus."

Page 523.- In figure, instead of "Fossa ischiorectale" read "Fossa ischiorectalis."

Page 531.- In figure, instead of "A. bulbo-urethrae" read "A. bulbi urethrae."

Page 563 et seq.-Instead of "Index to New Terms" read "Index to Terms now used." 


\section{INDEX TO ILLUSTRATIONS}

Absolute heart dulness, 456

Annulus inguinalis abdominalis, 453

- - subcutaneus, 453

- tendineus communis, 280

- urethralis, 538

Aorta abdominalis and branches, 512

Appendix vesiculosa [Morgagnii], 550

Arcus tendineus M. levatoris ani, 534

Arteria axillaris, 63

- carotis, 274

- cerebri media, 320,321

- coeliacus, 491

- femoralis, 137, 147

- glutaea, 127

- intercostalis, 401

- lingualis, 267

- maxillaris externa, $26 \pi$

- meningea media, 299

- mesenterica infelior, 479, 495, 496

- - superior, 475

- obturatoria. 529, 530, 531

- ophthalmica, 278

- phrenica, 491, 512

- poplitea, 134

- profunda penis, 538

- pudenda interna. 530

- subclavius, 63, 221

- thyreoidea superior, 267

- uterina, 543, 550

- vertebralis, 285

Arterles of anterior body-wall, 56

- of base of brain, 319

- of body wall, in section, 401

- of head, 236, 319

- of knee. 181

- of leg, 159, 171. 185

- of neck, 219,236

- of palm, 97

- of pelvic wall and floor, 531

- of perineum, 13:

- of popliteal space, 133, 134

- of rectum, 532

- of retina, 382

- of shoulder. 219

transformation of branchial, 421

Articulations :

- acromioclavicular, 72,85

- ankle, 186. 187

- atlanto-epistrophic, 286, 287

- atlanto-occlpital, 286, 287, 294

- carpal, 113

- elbow, 110, 111

- foot 189.190

- hip. 154.155

- knee, 132, 181, 182, 183, 184

- pelric, 551, 5.52. 553

- radio-11lnar, 112, 113, 114

- shoulder, 85, 86

Axlllary arch, 59

- fascia, 59, 60

- fossa, 60

Base of skull, 200

Blood-vessels of eyeball, 378

Brachial arch, 5!

- plexus, 63, 75, 215

Brain, base, $318,319,333,344$

- lateral surface, 320,332

- median surface, 336

Bronchi, 425

Bulbus urethrae, 538

Capsule of Tenon, 256

Cartilages of larynx, 425
Cerebellum, 327, 328, 329

Cerebrum, 332, 335

Cervicobrachial plexus, 66, 215, 221, 285

Cervix uteri, 54:3

Chorda tympani, 274, 306

Ciliary body, 379

- processes, 379

Cisterna chyli, 478

Columnae rectales, 536

Conjunctiva bulbl, $256,375,377$

- palpebrarum, 2:6

Crista urethralis, 538

Development of female genitalia, 547

Ductus choledochus, 491, 494, 496

- deferens, 512,527

- ejaculatorius, 527, 538

- Iymphaticus dexter, 469

- thoracicus, 478

Duodenal fossae, 469

Epididymis, 462

Epoophoron, 550

Excavatio recto-uterina, 540

Exit of cerebral nerves, 200

Exposing pelvic fascia, 520, 521

Fascia, 76,77

- bulbi, 256

- colli, 206, 210, 211, 227

- coracobrachialis, 219

- cribrosa. 515

- of fusion, 497

- infraspinata, 232

- lata j15

- Iumbodorsalis. 232

- parotideus. 249

- of pelvis, 522, 523

- of perineum. $437,441,442,443$

- perirenal, 507

prerenal. 507

prevertebral, 289

retrorenal, 507

supraspinata, 232

of Toldt, 507

of Treitz, 497

Flexura coli dextra, 495

- duodeni inferior, 493

- duodenojejunalis, 493

- - superior, 493

Fundus oculi, 382

Ganglion, cervical sympathetic, 285

- ciliary, 281

- geniculate, 306

otic, 299. 306

- semilunar, 299, 306

- sphenopalatine, 281, 304

sympathetic, 515

Gland, lacrimal, 281

- parotid, 206, 219, 267

- submaxillary, 206, 267

suprarenal, 49.

Glans penis, 538

Granulationes arachnoideae, 199

Great omentum, 491

Heart, duIness, 406

- opening chambers of, 414, 416, 419

Inguinal canal, 453, 459

- ligament. $146.512,515$

- region, 146, 151

Intestine, caecum, 488 
Intestine, course of, 482

- duodenum, 493,496

- jejunum, 484,493

- large, 487

Iris, 380

Isthmus rlombencephalì, 345

Joints. See Articulations.

Kidney, 495, 497, 504, 512

Labyrinth, bony, 295, 392

- membranous, 393, 395

Lacrimal ducts, 257

Lacunae urethrales, 538

Larynx, 292, 308, 309

Leg-supports, 436

Lens, 383

Ligaments. See also Articulations.

Ligament(s), Ligamentum(i) :

- coronarium hepatis, 504

- gastrolienale. 501

- inguinal, 146, 512, 515

- of ankle, 16.2. 173

- of pelvis, 551. 552

- of scapula, 72

- puboprostatic. 524

- round, of uterus, 459

- suspensorium penis, 515

- teres hepatis, 503

- umbilicale medium, 524

- venosum (hepatis) [Arantii], 503

Lithotomy position, 436

Liver. 503, 504

Lungs, 403, 406, 407

Lymphatics of arm. 62

- of axilla, $62,205,223,478$

- of breast, $62,205,223$

- of forearm, 91

- of hand, 62.91

— of head, 205,223

- of iliac region, 478

- of inguinal region, 139,478

- of leg. 131

- of lower limb, 131

- of neck. 205. 223

- of penis. 139

- of posterior wall of trunk, 478

- of thigh, 131

- of thorax, 62, 205

Mamma, 57

Medulla oblongata, 323, 330, 345

IIesentery, 481

IIidbrain, 323,330

Niddle ear 209,306

Miller's model of lung, 427

Iouth, 289

Muscle, Muscles :

- coccygeus 53

- iliacus, 515

- levator ani, 534

- obliquus abdom. ext., 515

- of abdomen, 146, 453

- of back, 49,232

- of cheek 202

- of ear, 249,253

- of eye, $277,280.281$

- of face. 249253

- of fingers. 102,108

- of floor of mouth, 221

- of foot, $162,172,177$

- of forearm. 95

- of hand, 99

- ố larynx. 26.5. 312

- of leg. 161, 173, 174

- of neck. 210, 221,224,227,236,283

- of orbit .277, 280. 281

- of pectoral region, is

- of pelvic floor. 534

- of perineum, $437,438,440,441,443,446$

- of pharynx, 26.5, 292

- of platysma, 208,249

- of shovilder, 83

- of submaxiliary region, 267
Muscle, Muscles :

- of thigb, 147. 151

- of tongue, 265,316

- of wall of trunk. 50, 401

- psoas major, 515

- pubovesicalis, 529

- quadratus iumborum, 515

Nasolacrimal duct, 257

Nerves of anterior surface of trunk, 54 - of arm, 69, 75

- of face, 253

- of foot, 143, 161, 167

- of forearm, $81,88,95$

- of gluteal region, 124,128

- of hand, 88,89

- of leg. 143, 161, 167

- of lumbar plexus, 515

- of neck. 208,253

- of nose. 304

- of orbit, $278,280,281$

- of perineum. 440,446

- of scalp. 253

- of shoulder, 69, 75, 83

- of thigh, 124, 143, 151

Nervus, Nervi :

- abducens, $2 \pi 3$

- auricularis magnus, 253

- auriculotemporalis, 253

- cardiaci. 221

- cerebrales, 200. 318

- chorda tympani, 274,306

- dorsalis penis. 515

- facialis, 253,306

- glossopharyngeus, 265

- hypoglossus. 265

- lingualis, 265

- mandibularis and rami, 263

- maxillaris. 273

- nasopalatinus, 300

- occipitalis, 253

- oculomotorius, 273, 281

- olfactorii, 300,304

- ophthalmicus, 273

- palatinus (ant., post., med.), 304

- recurrens, 265

- spinalis, $47,240,241,245,400$

- trigeminus and rami, 274,281, 299, 306

- trochlearis. 273

- vagus, 221

Noduli lymphatici aggregati, 485

- solitarii, 485

Nose, 289

Nuclel of cerebral nerves, 374

Opening mediastinum, 404

Orbital septum, 256

Organ of Corti, 396

Ovary, 540, 545, 550

Pacchionian bodies, 199

Palmar sheaths. 101

Pancreas, 491, 495, 497

Papilla duodeni. 493

Pelvis renalis, 510

Penis, 538

Peritoneum, 455, 495, 497, 544

Pharynx. 289, 292

Pleura, 406

Plexus lumbalis, 517

- iumbosacralis, 517

- pudendus. 517

- sacralis, 517

Plica longitudinalis duodeni, 493

Plicae circulares, 493

Pons. 322, 327, 330, 345

Popliteal fossa, 133

Poupart's ligament, 146

Pronation. 112

Pylorus, 493

Reconstruction of lobule of lung, 427

Regions of anterior surfaces, 43

- of posterior surfaces, 43

- perineal, 434,435 
Regions urogenital, 434,435

Renal calices, 510

Rosenmüller's lymph-gland. 478

Schema of blood-supply of alimentary tract, 463,464

- of circulation, 410

- of embryonic alimentary tract, 463,464 , 465

- of male genital organs. 460

- of peritoneum, 466,467

of veins, 411

Sections of arm, 76,77

of atlanto-epistrophic articulation, 286

of atlanto-occipital articulation, 286

of axillary fossa, 60

of brain, $321,336,340,342$

of brain-stem, $327,360,361$

- of breast. 57

- of cerebellum, $327,350,351,352$

of cerebral hemisphere. 370

of corpora mammillaria, 363

- of corpora quadrigemina, $355,356,357$

- of corpus callosum, 327, 362, 363

- of ear, $385,386,387,388,389,390$

of elbow, iog 110

- of eyeball, $375,377,378$

- of falx cerebri, 199

- of female genitalia. 540, 544

of foot, 190

- of forearm, $89,92,93,106$

- of hand, 100, 113

- of hlp-joint, 155

- of hippocampus, 342

- of hypophysis. 302

- of isthmus rhombencephall, 353

- of knee, 132

of larynx, 309

- of leg, 158, 164

- of liver, 504

- of median plane of female body. 544

- of median plane of head, neck, thorax, 28 ?

- of medulla oblongata, $347,348,349$, 350 , 351. 362

- of meninges, 198, 199, 239

- of mesencephalon, 356,357

- of nasopharynx, 294

- of neck, $211,227,289$

- of nose, 289, 300, 301, 302

of orbit, 256,277

- of palate, $294,300,301,302,304$

- of pelvic and perineal fasciae, 441, 442, 443

of pelvis, $455,522,523$

- of pharynx, 227,294

- of pons. $352,353,355$

- of shoulder. 61,85

- of sinus cavernosus, 273

- of sinus sphenoidalis. 273

- of spinal cord, $240,244,245,246$

- of stomach wall, 499
Sections of symphysis pubis, 553

- of testis, 461

- of thigh, 144, 14!

- of third ventricle, 341

- of thorax, 400,401, 403

- of tongue, 316

- of trunk in median plane, 466

- of trunk, transverse, 467,497

- of uterine tube, 550

- of uterus, 550

- of vagina, 446

- of vesicula semInalis, 527

- of vulva, 446

Sinus cavernosus. 273

sphenoidalis, 273

Skin incisions of anterior surfaces, 53

- of head and neck, 195

- of perineum, 436

- of posterior surfaces, 46

Spermatic cord. 457

spinal cord, 323,330

Spleen, 491, 495, 497, 501

Stomach, 491, 497

Supination, 112

Surface of palm, 97

Sympathetic in neck, 221, 285

- in trunk, 241

Synovial spaces of knee, 184

Tarsal cartilages, 256

Tear-sac, 257

Tendons of fingers. 102, 108

Testis, 457, 461, 462

Thoracic dinct, 426

Tongue, 315,316

Trachea, 289, 425

Truncus intestinalis, 478

Tuba uterina, 545

Tympanum, 385, 386, 387, 388, 389, 390, 391

Ureter, 510, 512, 543

Urethra, 538, 544

Trogenital system, 461

Uterus, 540, 544, 545

Utriculus prostaticus, 538

Vagina, 540, 543

Valvula coli, 488

- processus vermiformis, 488

Ventricles of brain, $339,340,362,363$

Veins :

- azygos, 478

- hemiazygos, 478

- inferior caval, 503

- of head and neck, 206, 223

- portal, 495, 497, 503

- of rectum, 532

Veslca urinaria, 524, 526, 540

Vesicula seminalis, $524,526,527$ 


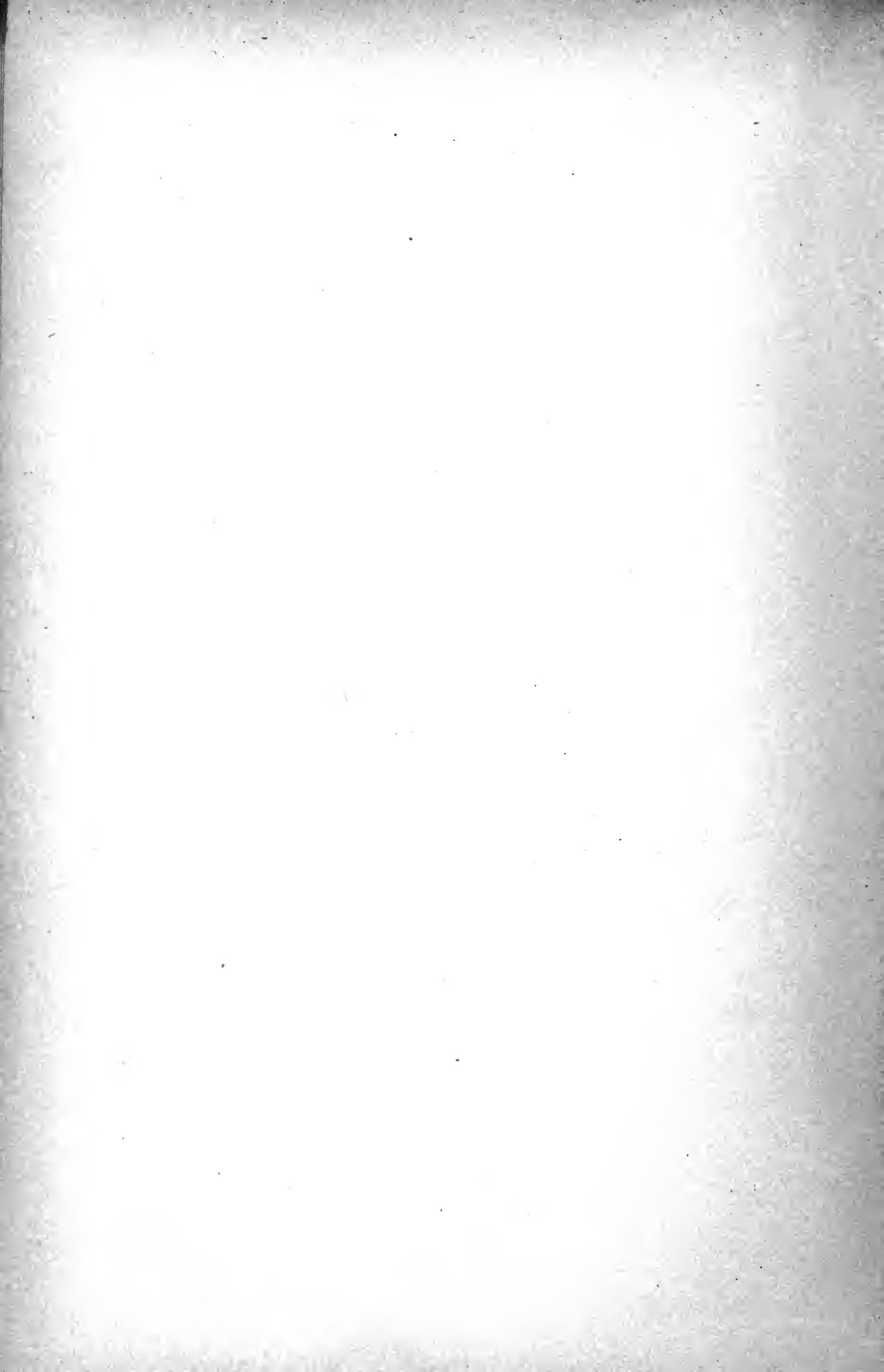




\section{INDEX TO OLDER TERMS}

Abdominal ring, external, 434

- - internal, 456

Adam's apple, 194

Alcock's canal, 450

Ammon's horn, 342

Anal fascia, 450

Angle of Louis, 52

Angulus Ludovici, 52

Anterior carpal rete, 104

Antrum of Highmore, 303

Aortic opening, 421

- orifice, 412

Aqueduct of Fallopius, 305

Arch, deep palmar, 103

- superficial palmar, 98

Arnold's bundle, 358

- nerve, 270

Arteria comes nervi phrenici, 407

- fossae Sylvil, 321

- princeps cervicis, 235

- - pollicis, 103

- radialis indicls, 103

Artery, Arteries :

- acromiothoracic or thoracic axis, 64

- anastomotica magna, 78 , 82,84

- - - of knee, 150

- anterior choroidal, 320

- - interosseous, 94, 196,105

- - radial carpal, 92

- - ulnar carpal, 94

- articular, of knee, 134

- auditory, 320

- azygos articular, 134

- buccal, 262

- collateral digital, 98

- cremasteric, 458

- deep epigastric, 514

- dorsal interosseous, 106

- dorsalis scapulae, 64, 69,73

external circumflex, of thigh, 148

- malleolar, 160

- - plantar, 178

- pudic, $140,148,451$

- facial, 218, 252

- first palmar interosseous, 103

- humeral thoracic 58

- inferior dental, 262, 264

- internal articular. of knee, 165

- - palatine, 268, 295

- - profunda, 78, 84

Internal circumflex, of thigh. 148

- iliac, 514, 528

- malleolar, 160, 172

- plantar. 178

- long thoracic. 64

- middle capsular. 513

- of the vas deferens. 458

- palatine branch of ascending pharyngeal, 295

palmar digital, 98

posterior dental, 262

- - interosseous, 105
Artery, Arteries :

posterior meningeal

branch of vertebral, 320

- - radial carpal, 106

- - scapular, 51

- ulnar carpal, 94

- rami intestini tenuis, 474

- ranine, 267. 317

- sciatic, 127

- second palmar interosseous, 104

- small meningeal, 203 , 262

superficial circumflex

iliac, 451

superior intercostal, 226

- profunda, 78, 84

- - thoracic, 63

- suprascapular, 51, 73,

214,230

tonsillitic, 268

- transverse, 320

- tympanic branch, of in-

ternal carotid, 297

Auditory nucleus, 354

Auricles of heart. 412

Auricular appendix, 412 , 413

Auriculoventricular groove, 413

Azygos uvulae, 295

Bicipital fascia, 82

Bicuspid teeth, 290

Bile canaliculi, 505

Bowman's membrane, 378

Brachial aponeurosis, 77

- plexus, Inner cord, 68

- outer cord, 63

Bundle of Vicq d'Azyr, 361

Callosomarginal fissure, 336

Canal, crural, 145

- inferior dental, 264

- of Petit, 38:

- of Stilling, 382

- posterior palatlne, 303

- Vidian. 297

Canalis reuniens, $\mathbf{3 9 4}$

Capsule of 'Tenon, 276 , 277,282

Carpal joints, 111

Cartilage of Santorini, 314

Cerebellar peduncle, inferior, 326,353

- - middle. 327

- superior, 327. 372

Chordae Willisí, 198

Circumvallate, papillae, 316

Cisterna basalis, 317

- magna, 317

Clarke's column, 246

Coellac axis, 491

Column of Burdach, 326

- of Goll, 327

- or tract of Burdach, 247

- - - of Goll, 247
Columnae carneae, 421

Conarium, $\mathbf{3 4 6}$

Conjoined tendon, 454, 456

Convolution, ascending parietal, 334

callosal, or gyrus fornicatus, 336

- hippocampal, 336

- occipitotemporal, 335

Corpora Arantil, 423

Cranial nerves, 202

Cricothyroid membrane, $224,311,312$

Crura cerebri, 322

Crural canal, 145

- ring. 146

Crus cerebri, 328

Crypts of Lieberkuehn, 485

Cushion of epiglottis, 314

Dentate fissure, or fissura dentata, 336

Descemet's membrane, 379

Direct cerebellar tract, 365

$3 \overline{69}$

Discus prollgerus, 546

Duct of Bartholin, 266 , 267

Ducts of Rivini, 266, 267

Ensiform process, 52

Epiphysis cerebri, 346

External intermuscular septum, 78

- - of thigh, 142

- os, 544, 549

Fallopian tube, 546, 550

Fallopius, aqueduct of, 305

False glottis, 309

- vocal cords 309

Fascia lata, iliac portion of, 141

pubic portion of

141,153

Fauces, anterior pillar of, 291

- posterior pillar of, 291

Fifth ventricle, 343

Fissure of Rolando, 334

- of Sylvius, 322

Fold, arytaeno-epiglottidean. 308

- middle glosso-epiglottidean, 307

Folds, lateral glosso-eplglottidean, $30 \mathrm{~s}$

"Foot" of cerebral peduncle. or pes pedunculi, $35 \mathrm{~S}$

Foramen, inferior dental, 261

— ovale, 305

- of Winslow, 472

Forceps major, $338, \quad 343$. 364

- minor, 338, 364

Fornix, anterior pillar of, 343

- posterior pillar of, 343 
Vossa patellaris, 382

Fovea hemielliptica, 392

- hemisphaerica, 392

Galen's loop, :313

Ganglion commissurale, 369

- of Andersch, 270

- of Arnold, 299

Gastrosplenic omentum, 473

Geniculate body, external, 346

- internal, 346

Gladiolus, 52

Glands of Nuhn, 290

Globus major, 460

- minor. 460

Glottis respiratoria, 310

- vera, 310

- vocalis, 310

Gower's tract, 247

Great horizontal fissure, 328

Ground-bundle, anterior. 246

- lateral, 247

Gylus dentatus, 337

- first temporal, 335

- second temporal, 335

- thild temporal, 335

- uncinate, 336

Hesselbach's ligament, 456

Hey's ligament, 141

Hippocampus major, 342

- minor, 341

Ioln of Ammon, 342

Ileocaecal valve, 487

Iliac portion of fascia lata, 142

Inferior peduncle, 369

Infundibula of lung, 427

Infundibuliform fascia, 457

Intercarotid gland, 222

Intercostal membranes, 399

Internal capsule, anterior limb, 364

- posterior limb, 364 78 intermuscular septum

— os, 544,549

Iridocorneal angle, 384

Ischiocapsular band, 155

Iter e tertio ad quartum ventriculum, 328

Jacobson's nerve, 271

Jolnt, inferior radio-ulnar, 114

- tibiofibular, 184 superior radio-ulnar 114

- - tibiofibular, 184

- temporomaxillary, 262

Joints, carpal, 111

Jugular ganglion, 270

Iabyrinth of kidney, 509

Laryngeal pouch or sac, 309

- sinus, 309

Laryngopharynx. 293

Lenticular ganglion, motor root of, 280

- nucleus, 364

- or ophthalmic ganglion, 278

I.esser's triangle, 218

Iigamenta alaria, 181

- subflara, 286
Ligament, Ligaments :

- anterior annular, 93 , 100

—- - of leg, 157

- common, of spine,

287,430

- costovertebral or stellate, 429

astragalonavicular, 189

calcaneocuboid, 189

calcaneonavicular, 189

chrondoxiphoid, 429

coracobrachial,

Schlemm, 86

costocolic, 472

cotyloid, $\mathbf{1 5 6}$

external annular, of leg. 157,164

- L lateral, of ankle. 186 - - of elbow, 110

- of jaw-joint, 262

- of wrist, 112

- tarsal, 2.55

falciform, 55

femoral, 141

glenoid, 86

glenoideobrachiale inferius of Scblemm, 86

- Internum

great sacrosciatic, 125 450,552

- Hesselbach's, 456

- IIey's, 141

- hyo-epiglottidean, 314

- internal annular, at ankle, 170,173

- lateral, of ankle, 186

- - of elbow, 110

- - - of jaw-joint, 262

- - of knee, 165, 180

- _ - of wrist, 112

- tarsal, 255

- long external, of knee, 180

median puboprostatic, of Krause, 442

- odontoid or check, 288 of Zinn, 279

- palmar intercarpal, 113 palpebral, 255

- posterior annular, 90 . 104,106

- common, of spine, 287,430

- occipito-axial, 287 - - of knee, 180 pregleno-infrahumeral, of Farabcuf, 86

- pterygomaxillary, 291

pubocapsular or pubo-

femoral, 155

- rhomboid, 225

- small sacrosciatic, $\mathbf{5 5 3}$

- stylomaxillary, 217, 262 supraglenoprehumeral, of Farabcuf, 86

- supraglenosuprahumeral, of Farabœuf, 86 suspensory, of axis, 88

thyro-arytaenoid, 312

thyro-epiglottidean. 314

tibiofibular, 184

transverse superficial 98

triangular, 442,448 , 449 true, of the bladder, 523,524

- Y-sliaped, of Bigelow 155

- zonular or ring, 155
Ligamentum jugale, 314

mucosum, 181

Limbic or falciform lobe 336

Lumbosacral cord, $\mathbf{5 1 8}$

Luschka's muscle, 456

- tonsil, 293

Lyra or lyle of David, 343

Marsupial notch, 328

Mediastinum, anterior, 405

posterior, 424

Meibomian glands, 255

Membranous cochlea, or scala media, 394

Iuscle, Muscles:

- abductor pollicis, 103

- accelerator urinae. 439

- adductor magnus, upper part, 127,153

aryteno-epiglottidean, 311

- attolens auriculam, 196

106

- azygos uvulae, 295

- brachialis anticus, 80 233

233

- complexus. 229, 234

- crureus, 150

- depressor alae nasi, 250

- - anguli oris, 250

- labii inferioris, 250

ejaculator seminis, 439 elevator of lip, superficial, 250

250

erector clitoridis, 447

- penis, 438

_ - spinae, 233

- extensor indicis, 105

- minimi digiti, 104

- ossis metacarpi pollicis, 105

- primi internodii pol-

licis, 105

- secundi internodi pollicis, 105

external rectus, 279

- inferior lingual, 316

- levator anguli oris, 250

- - - scapulae, 229, 230

- labii superioris, 250 250

- palati, 295

orbicularis palpebrarum, 249

- occipitofrontalis, 196

- palatoglossus, 295

- palatopharyngeus, 295

- pronator radii teres, 93

- pyramidalis nasi, 249

- rectus capitis anticus major, 284

- minor, 284

- internus, 279

_ labii, of Aeby, 251

retrabens auriculam 196

sacrolumbalis, 233

- semispinalis colli, 234

- serratus magnus, 68 sphincter vaginae, 447

spinalis colli, 233

splenius colli, 231

- subcrureus, 152

- superficial lingual, $\mathbf{3 1 6}$

- supinator brevis, 82 , 105 
Muscle, Muscles:

- tensor palati, 295

- - tarsi. 257

- vaginae femoris, 129,150

- thyro-arytenoid. 311

thyro-epiglottidean. 31

tibialis anticus, 158

- posticus, 170

- trachelomastoid, 233

- transversalis cervicis 233

- triangularis sterni. 402

vastus externus. 150 - internus, 150

- zygomaticus minor, 250

Musculospiral groove, 82

Nasopharynx, 293

Nates, 328

Nerve, Nerves

anterior crural, 142 , 148,150

- - interosseous, 96

- - superior dental, 298

- tibial, 160, 165

- Arnold's, 270

- auditory, 201, 307. 324

circumflex, 64, 67, 68, 71, 216

- collateral digital, 99

communicans fibularis

133,166

- tibialis, 132

crural branch of gen-

itocrural, 142, 148, 516

- dorsal branch of ulnar, 89

- cutaneous, 94

external cutaneous, 125,

$135,142,143,516$

- - laryngeal, 220

- palatine, 296,305

- plantar, 178

popliteal, 133, 165

- respiratory, of Bell, 67,216

facial, pars intermedia, 324

- genital branch of genitocrural, 458

- genitocrural, 516

iliac branch of iliohypogastric, 452 452 - of last dorsal,

inferior dental, 264

-7 maxillary, 254, 263, 274 74

ercostohumeral, 60

internal cutaneous, 64, $68,76,78$

- laryngeal. 220

- plantar, 17

- popliteal, 132 pudic, 128

- saphenous. 165

- last dorsal, 123

- lesser internal cutaneous, or nerve of Wrisberg. $64,68,76,78$

- long buccal, 255

- malar branch of temporomalar. 282

median palmar cutaneous. 96

- middle and internal cutaneous. 142 67

- superior dental, 298

musculocutaneous, 160, 165
Nerve, Nerves :

- musculospiral, 64, 68, 76. 82,92

nasal, 277

of Cotunnius, 301

orbital, 298

pars intermedia of

W'risberg, 201, 324

- patellar branch of Jong saphenous, 142

- perineal branches of fourth sacral, 4.j0)

- petrosal, large, deep. 297

- pneumogastric, 220 . $270,324,408,425,497$ posterior interosseous, 105,108

- superior dental, 298

radial branch of musculospiral, 89,92

- recurrent branch of inferior maxillary, 263

- - laryngeal, 270,313

- to the rhomboids, 51, $67,216,230$

- septal branch of nasal, 301

- short saphenous, 166

- sixth, 280

- small sciatic, 128, 130, 133, 135, 136, 166, 447 - - cutaneous branches of, 125

spinal accessory, 201, 214. 220,324

- superficial cervical, 207

superior maxillary, 254 , 274

supraclavicular. 209

- suprasternal, 207

- temporal branch of malar, 195

- of temporomalar. $260,261,282$

temporomalar, 282, 298

- third, 280

- tympanic branch of facial, 306

- Vidian, 297

Nervus cerebralis III., 275

- IV., 275

- - V., 273

- VI., 275

- patheticus, 275,276

Nucleus of Burdach"s column. 348

- of Goll's column, 348

- of sixth nerve, 354

- of III. nerve. 357

- polyposus, 430

Optic lobes, 328

radiation, 364

Oral pharynx. 293

Orbicular ligament, 115

Organ of Giraldes, 462

Pacchionian bodles. 317

- granulations, 198

Pes hippocampi. 342

l'illars of the iris, 380

I'inna, 258

I'itultary body, 203, 322, 338

I'lexus gulae, 426

l'omum Adami, 194

Posterior carpal rete, 106

- longitudinal bundle, $3 \% 4,357$

Pretracheal fascia. 20 9

I'ubic portion of fascia lata, 142

Pulmonary orlfice, 412

36
Pyramid of Ferrein, 509

I'yramidal tract, crossed, 241

- - direct, 246

Receptaculum chyli 514

Iiecessus infundibuliformis, 293

Reniculi, 508

Respiratory bundle, 348

Rete, anterior carpal, 104

Iibbon of Reil, 327

Saphenous opening. 140

Scarpa's triangle, 122, 146

Sclerotic coat. or white of the eye, 378

Semilunar fascia, 80

- fibrocartilages of knee, 181

- notch, 328

Sensory decussation of me dulla oblongata, 352

Septum, anterior peroneal, 158

posterior peroneal, 15s

Shoulder-joint, accessory ligament of, 85

Sigmoid flexure, 486

- notch, 261

Sinus alae parvae, 202

- basilar. $2(1) 3$

- inferior longitudinal, 199. 202

- lateral, 202

- pyriformis, 294

- superior longitudinal, $20 \%$

Socia parotidis, 252,290

Sphenoidal fissure, 275 . 280,297

Sphenomaxillary fissure, 282

- fossa, 303

Spinal cord. anterior column of, 243

- - - horn of, 245 - lateral column of 243

- horn of, 245 243

- - horn of, 245

Stria fornicis or stria pinealis, 346

Subclavian triangle, 214

Subthalamic nuclens, 360

- region, 338

Sulcus, callosal, 336

- callosomarginal, 334

- intraparietal, of 'Turner. 335

- limiting. of Reil. 334

- occipitotemporal 33.

- parallel, or first temporal, 335

— second temporal. 335

Superficial arcuate fibres, 327

Supra-acromial branches, 69

Suprasternal noteh, 52

Taenia semicircularis, $3+1$

Testis, 328

Thoracic axis, 6is

Thyrohyoid membrane. 222,310

Transverse fascia, 455

Triangular fascia of the abdomen, 4.7.

- fibrocartilage, 115

- ligament, 442,448, 449

Trigonum ventriculi. $3+2$ 
Torcular Herophili, 202

Tubercle of Rolando, 327

Tunica Ruyschiana. 379

Turbinated bones. 301

Turk's bundle, 358

Valve of Houston, 536

- of Vieussens, 328

Valvulae conniventes, 484

Vein, Veins :

azygos major, 408, 412, 428

- - minor superior, 429
Vein, Veins :

- external pudic, 148

- - saphenous, 166

- great cardiac. 414

- inferior dental, 262

- internal iliac, 514

- - saphenous, 140, 142 .

$148,165,166$

- of corpus striatum, 341 , 344

— of Galen, 344

- of Verheyn, 508

- suprascapular, 215
Velum interpositum, 343

Venae Thebesii, 414

Vesical layer of fascia endopelvina, 523

Vidian canai, 297

- nerve, 297

Wharton's duct, 290

White line of peivic fascia, 450,520

Wisdom tooth, 290

Womb, 543

Wrist-joint, 111 


\section{INDEX TO NEW TERMS}

Abdomen, 42

- contents of, 462

Accessory lemniscus, 367 , 373

Acromion, 41

Aditus laryngis, 308

Adminiculum lineae albae, 456

Aequator buibi, 376

- lentis, 383

Agger nasi, 302

Ala cinerea, 331

- lobuli centralis, 330

Alae nasl, 193

Alcock's canal, 450

Aiveoli pulmonis, 427

Amphiarthroses intercarpeae, 111

Ampulla ductus deferentis, 528

- - lacrimalis, 257

- recti, 524

- tubae uterinae [Fallopii], 547

Ampullae membranaceae, 394

- osseae, 392

Anal canal, 536-548

Anastomosis around ankle, 160

- - eibow, 80, 105

- - knee, 131

Angle of Louis, 52

Anguiar gyrus, 335

Angulus iridis, 384

- oculi (lateralis, medialis), 247

Annulus conjunctivae, 378

- femoralis, 146, 511

- fibrocartilagineus (membran. tympani), 388

- fibrosi (cordis), 424

- fibrosus (fibrocartilaginis intervertebralis, 286 , 430

haemorrhoidalis, 536 , 548

inguinalis abdominalis, 456

- subcutaneus, 436, 452 - iridis (major, minor), 384

- ovalis, 417

- tendineus communis (Zinni), 279

urethralis, 537

Ansa duodenalis. 463

- hypoglossi, 220, 269

- intestinalis, 463

- lentícularis, 36.

- peduncularis. 362

- subclavia [Vieussenii]. 227. 272

- umbilicalis, 463

Anterior commissure of vulva, 444

- fornix of vagina, 545

- intercostal arteries. 402

- interosseous nerve of forearm, 96

- marginal bundle, 365

- median line, 42

- mediastinum. 406

- pyramid, 326
Anterior tibiofibular compartment of leg, 168 - triangle of neck, 216

Anthelix, 259

Antibrachium, 41

Anticubital fossa, 80

Antitragus, 258

Antrum pyloricum, 448

- tympanicum, 386

Aorta, 409, 422, 424, 426, 512,513

abdominalis, 513

- ascendens, 422

- descendens, 409, 524

thoracalis, 422,426

- ramus visceralis, 426

Aortic opening in diaphragm, 511

valve, 421,423

Apertura lateralis ventriculi quarti, 331

- mediana ventriculi quarti, 331

- tympanica canalicuii chordae, 386

Apex cartilaginis arytaenoideae, $\mathbf{3 1 4}$

- columnae posterioris, 145

cordis, 412

- linguae. 316

nasi. 192

- prostatae, 527

- pulmonis, 408

suprarenalis (gl. dextrae), 509

Aponeurosis, brachial, 60. 77

of external oblique, 454 M. obliqui externi abdominis. 454

- palmaris, 97, 98

- plantaris, 157, 173, 176

- urogenitalis, 505

Appendices epiploicae, 486

Appendix fibrosa hepatis. 504

- testis [Morgagnii 1. 462

- ventriculi laryngis, 309

- vesiculosa [Morgagnii], 546

Aquaeductus cerebri [Sylvii 7.228

Arachnoidea encephali. 198. 317

- spinalis, 238, 239

Arachnoideal villi, 198 , 317

Arbor vitae cerebelii, 355

- - of uterus, 549

Arcuate fibres, $\mathbf{3 6 4}$

Arcus aortae, 409, 422

- (arteriosus) plantaris, 178,179

- - rami perforantes. 178

cartilaginis cricoideae, 314

- costarum, 399

- lumbocostalis lateralis

[Halleri]. 511

- medialls. 511

- M. solei, 16 ?

- tarseus, 279
Arcus tarseus inferior, 279 - superlor, 279

tendineus fasciae pel vis, 521,541

M. levatoris ani. 520,541

- venosi digitales, 89

venosus dorsalis pedis, 156

- - plantaris, 176

- volaris (arteriosus) profundus, 103

- - - superficialis, 102

Area acustica, 331

- cochleae, 393

- nervi facialis, 393

- parolfactoria [Brocae] 337

- vestibularis inferior, 394

- - superior, 393

Areola mammae, 4 :

Arnold's nerve, 270

Arteria, Arteriae:

- acetabuli, 528

- alveolares superiores anteriores, 298

- alveolaris inferior, 262, 264

- ramus mylohyoideus, 262,264

- superior posterior, 262

rami gingivaies

superiores, 262

- angularis, 252

- anonyma, 224, 442

- appendicularis 474

- arciformes renis, 506

- arcuata, 160

- auditiva interna, 320

- auricularis posterior, 195,218

- profunda, 261

- axillaris, $63,65,66.73$, 78

basilaris, 201, 320

- rami ad pontem. 320

- brachialis, $78,79,82$

- bronchiales, 409,426

- buccinatoria, 262

- bulbi urethrae, 443, 529

- vestibuli (vaginae), 448, 5.31

carotis communis, 220 , 282, 422

- externa, 218, 220, 252,268

- Interna, 200. 203

220, 268, 273, 297, 320

- ramus caroticotym panicus. 297

centralis retinae, 278

cerebelli inferior anterior, 320

- - posterior. 320

- superior, 320

cerebri anterior, 320

- media. 320

- posterior. 320

cervicalis ascendens,

226. 235. 242

235 rami musculares, 
Arteria, Arteriae : - cervicalis ramus profundus, 235 242

- profunda, 226, 235

- superficialis, 226 , 229,230

- chorioidea, 320

cillares anteriores, 278

- - posteriores. 27s

- circumflexa femoris lateralls, 148

- - ramus ascendens, 129 148, 153

- - - profundus, 153

- - - acetabuli, 153

- - - superficialis, 153 71 71 - posterior, 64, 69,

- ilium profunda, 514 - superficlalis, 140

148,451

-73 scapulae, 64,70 , 72 ,

- clitoridis, 448, 531

- coellaca, 490, 491, 513

- colica dextra, 474

- - media, 474

- - sinistra, 476, 477

- collateralis media, 84 105

- - radialis, 84,105

- ulnaris inferior, 68 ,

$82,84,105$ $105-$ superior, 68,84 ,

comitans ischiadici,

127,128

communicans anterior. 320

- - posterior. 320

- conjunctivales (ante-

riores, posteriores) 278

- coronaria (cordis)

dextra, 413, 422

- ramus descendens posterior. 413

- (cordis) sinistra,

413

413

- descendens an

terior, 413

- cystica, 476,490

- deferentialis, 458, 529

- digitales (manus) dor sales. 90,106

- - plantares. 178

- volares communes,

- - propriae, 98

dorsalis clltoridis, 448 531

- - indicis, 106

- nasi, 279

- pedis, 160

- ramus plantaris

profundus, 160

- penis. 443. 541

- epigastrica inferior.

$140,454,456.514,527$

-11 ramus obturatorius,

- superficialls, 148 ,

- - superior, 402, 454

- episclerales. 278

- ethmoídalis anterior, 278

- posterior, 279
Arteria, Arteriae :

- femoralis, $145,148,149$, 154

- rami inguinales, 140 , $1+8$

$1 \overline{49}$

- frontalis, 195, 279

- gastrica dextra, 490

- sinistra, 476, 492,

513 - rami oesophagei,

492

- gastricae breves, 492

- gastroduodenalis, 490

gastro-epiploica dextra and sinistra, 490,492

- rami epiploici, 490

genu inferior lateralis, 134

_ _ - medialis, 134, 165

- - media, 134

134

— - medialis, 134

- - suprema, 149, 150

- rami articulares, 150

150

- ramus saphenus, 150

glutaea inferior, 127 , 528

- - superior, 126, 528

- ramus inferior 126,528

$5 \overline{28}$

- haemorrhoidalis inferior, 450 , 52 ?

- media. 529

- super

- hepatica, 476, 490, 492 , 513

- - propria, 490

ramus dexter, 476

490

- - sinister, 490

- hypogastrica, 514, 528

- ileae, 474

- ileocolica, 474

- iliaca communis, 513

- - externa, 14.527

- iliolumbalis, 242, 528

- ramus iliacus, 528

- - lumbalis, 528

- - spinalis, 242. 528

- infraorbitalis, 298

- intercostales, 242, 399, $400,401,426,428$

- intercostalis suprema,

$226,242,401$

- interlobulares renis, 506

- Interossea communis, 94

- dorsalis. 94. 105

- recurrens. 105

- volares, 94 96, 105

- intestinales. 474

- jejunales. 474

- labiales posteriores,

447.529

- labialis inferior, 252

- - superior. 252

- laryngea inferior, 292 311,313 310, 313 superior, 220, 252,

- lienalls. 472, 513

- rami lienales, 492

- - pancreatici. 492

- lig. teretis uteri. 514

- lingualis, 218, 220, 267
Arteria, Arteriae

- lingualis rami dorsales

linguae, 267, 317

- - ramus hyoideus, 220

- iumbales, 242, 513

- - ramus dorsalis, 513

- - spinalis, 238, 242,

513

- lumbalis ima, 513, 532

- malleolaris anterior lateralis, 160

- _ - medialis. 160 $\overline{172}$ posterior laterails

- medialis, $\mathbf{1 7 2}$

mammaria interna, 226 , 402,407

402

399, $\overrightarrow{402}$ perforantes, 55 .

- - rami sternales, 402

- marginalis lateralis, $\mathbf{1 5 7}$

- - medialis, 157

- masseterica, 261, 262

- maxillaris externa, 218, 220,252

- rami glandulares. 218

- ramus tonsillaris,

maxillaris interna, 252 261. 268

- mediana, 96

- mediastinales anteriores, 407

- meningea anterior, 203 - media, 198, 203, 262. 273

- ramus meningeus accessorius, 203, 262. 273

- - petrosus superficialis, 273

- meningea posterior, 203 268

- mentalis, 264

- mesenterica inferior, 477,513

- - superior, 474, 513

- metacarpeae dorsales. 90,106

- - volares, 98, 103, 104

- metatarseae dorsales, 160

- plantares, 178

- musculophrenica, 402

- nutricia femoris inferior, 136

- - - superior, 136,152

- fibulae, 172

- nutriciae humeri. 84

- nutricia tibiae. 172

- obturatoria, 154,528

- ramus anterior, 154 528

$5 \overline{28}$

- posterior, 154,

- - pubicus, 528

- occipitalis. 196, 203 $214,220,230,235,268$, 269

- - ramus meningea, 203

- - musculares, 235

- - — descendens, 235

- mastoideus, 235

- oesophageae, 426

- ophthalmica, 203, 273. 278

- rami musculares, 278

- ovarica, 513, 542

palatina ascendens, 218 268,295

- descendens, 295

palpebrales lateraies, 
Arteria, Arteriae :

- mediales, 279

- pancreaticoduodenales. inferior et superior, 474 490

rami duodenales, 490 penis, 443, 529

perforans prima, 136 152

- - secunda, 136, 152

- - tertia, 136, 152 pericardiacophrenica, 407,408

- perinei, 439, 446, 529

- peronaea, 172

- rami calcanei laterales, 172

172

- ramus perforans, 172 pharyngea ascendens, $203,220,268$

- rami pharyngei, 268 295

phrenica inferior, 513 - rami suprarenales superiores, 513

phrenicae superiores, 426

- plantaris lateralis, 178

- medialis, 178

$1 \overline{78}$

- - superficialls, 178

- poplitea, 133

profunda brachii, 78 , $80,82,84$

84

- clitoridis, 448

152 femoris, 136, 148,

- linguae, 267, 317

- penis, 443, 529

pudenda interna, 126, 450,529

pudendae externae, 140 . 148,451

pulmonalis, 409, 417, 423,428

$4 \overline{17}$

- - sinister, 409,417 radialis, $74,82,90,106$ - ramus carpeus dorsalis, 106

_ _ _ volaris, 92

ficialis, 92

recurrens radialis, 92 , 105

- - tibialis anterior, 160

- ulnaris, 82, 94, 105 - renalis, 506. 513

- sacrales laterales, 242 , 528

- rami spinales, 242 ,

- sacralis media, 513, 532

- - posteriores. 439, 529

sigmoideae, 477

spermatica externa. 458,514

- - interna, 458, 513

- spinalis anterior, 242 , 319

- posterior, 242. 319

sternocleidomastoidea, 220

- subclavia. 63, 226, 422

- sublingualis, 267

- submentalis. 218

- subscapularis, 64, 73
Arteria, Arteriae :

- supraorbitalis, 195, 279

- suprarenales, inferior and media, 513

surales, lateralis and medialis, 134

- tarsea lateralis. 160

- tarseae mediales, 160

- temporales profundae, anterior and posterior, 261,262

temporalis media, 252, 260

- superficialis, 195 ,

$252,260,268$

- ramus frontalis, 195

- - parietalis, 195

- testicularis 513

- thoracalis lateralis, 55, $61,64,68$

- rami mammari externii, 55, 64

- - suprema, 63

- thoraco-acromialis, 64 65,70

- rami pectorales, 65

- ramus acromialis, $65,70,71$

- - - deltoideus, 65, 70

- thoracodorsalis, 64

- thymicae, 407

- thyreoidea ima (Var.) 224,422

- - inferior, 226

- - superior, 220, 222

- - ramus hyoideus, 220

- sternomastoideus, 220

- - - cricothyreoltibialis anterior 160

- - posterior, 172

- - rami calcanei mediales, 172

- - - fibularis, 172

transversa colli, 73,214 , 226

dens, 230

- - faciei, 252

- scapulae, 73, 226 , 229,230

- tympanica anterior, 261

- - inferior, 268

- - superior, 273

- ulnaris, 82, 92, 84

- ramus carpeus dorsalis, 94

- - - volaris, 94

- umbilicales, 529

urethalis, 443, 448, 529

521

- uterina, 529, 542

- - ramus ovarii. 542

- - tubarius, 542

- vaginales. 529 , $54^{2}$

- vertebralis, 201, 203 $226,235,242, \quad 284$ 319

- rami spinales, 238 . 242. 284

203

vesicales superiores, 529

- vesicalis inferior, 529

- zygomatico-orbitalis,

Arterial circle of Willis, 320

- crucial anastomosis, 127

Arteries, facial, 218,220 . 252

- obliterated hypogastric. 514. 528

Arteriolae retinae, 381,382
Articulatio, Articulationes: acromioclavicularis, 7 atlanto-epistrophica, 287

- atlanto-occipitalis, 237

calcaneocuboidea, 175 , 188

capitulorum (costa-

rum ), 429

('arpometacarpea pollicis, 115

carpometacarpeae, $\mathbf{1 1 5}$

- costotransversariae, 429

- costovertebrales, 429

- coxae, 156

- cricoarytaenoidea. $\mathbf{3 1 5}$

- cricothyreoidea, 314

- cubiti, 109

- cuneonavicularis, 188

- digitorum (manus),

116

- pedis, 190

et ligamenta capitis, 286

inferinguli extremi-

tatis inferioris, 552

$71,-\overline{2 \cdot 5}--$ superioris,

- genu, 180

- humeri, 8

- humeroradialis, 110

- humero-ulnaris, 110

- incudomalleolaris. 390

- incudostapedia. 390

- intercarpea. 115

- interchondrales, 429

- intermetatarseae, 190

- intertarseae. 187

- intervertebrales, 430

- mandibularis, 262

- manus, 111

- metacarpophalangeae. 116

- metatarsophalangeae,

175

ossiculorum auditus, 390

ossis pisiformis, 113

- pedis, 109

- radiocarpea, 111

radio-ulnaris distalis,

110,114

- - proximalis, 110, 114

- sacroiliaca, 553

- scapulohumeralis. 84

- sternoclavicularis. 225

- sternocostales, 420

- talocalcanea, 175,187

- talocalcaneonavicularis 187. 188

- talocruralis, 185

- talonavicularis, 18

- tarsi transversa (Choparti), 122, 187

tarsometatarseae. $\quad 175$

tibiofibularis, 184

Articulation, Articulations :

- of atlas, axis, and occipital bone, 287 capitular, 429

- costo-transverse, 429

- costo-vertebral. 429

- crico-arytaenoid. 315

- cricothvreoid. 314

- of the foot, 185

- interchondral. 429

- intervertebral 430

- manubrio-gladiolar. 429

- sternoclavicular. 225

- sternocostal 429

- temporomaxillary, 262

- thorax. 429

Arytaenoid cartilages, 314

Ascending fibre system, 365 
Association fibre systems, 373

Atrium coldis, 412

- dextrum, 41.

- sinistrum, 413, 418

Auditory apparatus, 384

- ossicles, 388

- ligaments of, 390

Auricle, left, 413,418

- ligaments of, 259

- right, $412,415,416$

Auricula cordis dextra, $412,415,416$

- - sinistra, 413, 418

Auriculae, 193

Auricular cartilage, 260

Auriculoventricular groove, 413

- orifice, 412

Auris externa, 258

- interna, 384

- media, 384, 385

Axilla, 41

Axis lentis, 383

- oculi (externa, interna), 376

- optica, 376

Bartholin, duct of, 447

Bartholin's glands, 447

Basal ganglia of cerebral hemispheres, $\mathbf{3 6 4}$

Basilar sinus, 203

Basis cartilaginis arytaenoideae, 314

- cochleae, 392

- cordis, 412

- encephali. 318

- modioli, 392

- pedunculi, 358

- prostatae, 527

- pulmonis. 408

- pyramidis (renis), 508

- stapedis, 389

Bell, nerve of, 67

Bertin, columns of, 508

Bifurcatio tracheae, 424

Bile ducts, 489

Bipolar cells of retina, 366

Bladder. urinary, 505, 525, $537,543,549$

- in distended condition, 527

- in empty condition, 527

- orifices of, 537

- trigone of, 537

IBody of clitoris, 444, 448

- of the penis, 435

- of testicle. 450

Brachial aponeurosis, 60 77

plexus, 66, 67, 215, 216,

Brachium, 41

- conjunctivum, 327

Brain, base of, 331

- preservation of, 201

- removal of, 201

Broca. convolution of, 334

Bronchioli, 427

- resplratorii, 427

Broncbus (dexter, sinis ter ), 424

Brunner's glands, 494

Bucca. 288

Buccai glands, 290

Buccopharyngeal fascia, $251,261,291$

Bulb of the penis, 440

- of the vestibule, 447

Bulbus aortae, 422

- cornu posterioris, 341

- ocull, 192, 280, 375

- olfactorius, 337

- pontis, 327
Rulbus quadrigeminum inferius, $3: 8$

- - superius, 328

- urethrae, 440

- V. jugularis inferior, $226^{\circ}$

venae jugularis superior, 269

- vestibuli (vaginae), 447

Bulla ethmoidalis, 303

Bursa. Rursae:

- anserina, 153

- bicipitogastrocnemialis, 169

- bicipitoradialis. 82

- cubitalis interossea, 82

- glutaeofemoralis, 126

- iliaca subtendinea, 155

— iliopectinea, 154

- - subcutanea, 122

- intermetatarsophalangeae, 163

- intratendinea olecrani, 84

- ischiadica M. glutaei maximi, 126

ligamenti coracoclavicularis, 72

- M. coracobrachialis, 72 , 80

- extensoris carpi radialis brevis, 104

- gastrocnemii lateralis, 169

$-\div-$ medialis, 169

- - infraspinati. 73

- - latissimi dorsi, 71

- pectinei, 153

- pectoralis majoris,

59

- piriformis, 129

- poplitei, 170

- recti femoris, 150 .

154

- sartorii propria, 153

- - semimembranosi, 169

- subscapularis. 72

- teretis majoris, 71

- musculorum lumbrica

lium pedis, 179

- omentalis, 472

- ovarica, 473

- pharyngea, 294

praepatellaris subcutanea, 122,142

- subacromialis, 71

- subcutanea acromialis, 70

- - calcanea, 169, 176

123

— - lateralis, 164

- olecrani, 84

- trochanterica, 123

tuberositatis tibiae

122

- subdeltoidea, 71

84

- suprapatellaris, 184

- tendinis calcanei

[Achillis], $\mathbf{1 6 9}$

- trochanterica M. glu-

taei maximi, 126

- medii anterior 129

$1 \overline{29}$

- - - minimi, 129

Caecum, 445

Calamus scriptorius, 330

Calcar avis, 341

Calices of ureter, 509
Caliculus ophthalmicus, 376

Callosal convolution, 336 , 338

- sulcus, 336

Calloso-marginal fissure, 336

Calyces renales, 509

- - majores, 509

- - minores, 509

Camera oculi anterior, 376 , 384

- posterior, 376,384

Camper, fascia of, 451

Canalis, Canales:

- adductorius [Hunteri], 149

- caroticus, 297

- carpi, 102, 113

- centralis (medullae

spinalis), 244

- cervicis uteri, 544, 549

- femoralis, 145

- hyaloideus, 382

- infraorbitalis, 297

_ inguinalis, 456

- longitudinales modioli, 393

- mandibularis, 264

- obturatorius, 552

- Schlemmi [Lauthi], 378

semicirculares ossei 392

spiralis cochleae, 392

- modioli, 393

vertebralis, 237

Capilli, 194

Capitulum mallei, 389

- stapedis, 388

Capsula, Capsulae :

- adiposa renis, 508

- articularis See Articulatio.

- cricothyreoidea, 314 cricoary taenoidea

- externa, 364

- fibrosa [Gilissoni], 505

- glomeruli. 509

- interna, 364

- lentis (crystallinae) 383

- nuclei dentati, 356

Capsule of the kidney, 505 , 508

- of lens. 383

- of Tenon, 276, 277, 282

Caput. 193

- epididymidis, 460

- nuclei caudati, 340

- pancreatis, 495

Cardia, 498

Carina urethralis (va-

ginae), 545,549

Carotid canal, 297

- sheath, 212

- triangle, 218

Cartilago, Cartilagines :

- alares minores. 258

- alaris major, 258

- arytaenoidea, 314

- auriculae, 260

- corniculata [Santorini] 314

- costales, 52

- cricoidea, 314

cuneiformis [Wrisbergi], 315

- epiglottica. 314

- laryngis, 313

- meatus acustici, 385

- nasi, 257

- lateralis, 257

- septi nasi, 257 
Cartilago, Cartilagines :

- sesamoidea, 31:

- thyreoldea, 31

- triticea, 311

Caruncula lacrimalis, 247

Cablingualis, 290

Carunculae hymenales, 445,545

Cauda epididymidis, 460

- equina, 242

- helicis, 259

- nuclei caudati. 342

- pancreatis, 496

Caudate nucleus, 341

Cavernous sinus, 20:2 273

Cavities of heart, 415

Cavum abdominis, 451,468

- conchae, 258

- epidurale, 238

- laryngis, 308

- Meckelii, 274

- mediastinale anterius. 405,406

- - posterius, 406, 424

- nasi, 300

- oris, 288

- peritonaei, 467

- pharyngis, 293

- pleurae, 403,405

- Retzii, 473

- septi pellucidi. 343

- subarachnoideale, 240 , 317

- subdurale, 198, 238, 240

- thoracis. 399,403

- tympani, 305, 384, 385

- uteri, 544. 549

Cellulae ethmoidales, 303

mastoideae, 305, 384 . 387

- tympanicae, 387

Centrum semiovale, 338 , 362

- tendineum diaphrag. matis. 511

Cerebellum, 324, 325, 328

Cerebral hemispheres, 325 . 331,332 - $\frac{1}{34}$ gyi and sulci of,

- lobes, 333

- nuclei, 366

Cerebrum, 324, 325

Cervical glands, deep, 222

- superficial, 207 svmpathetic, 220, 222, $27 i$

Cervix. 210

columnae posterioris 245

- uteri, 544, 549

Chambers of eye, 376, 384

Chiasma opticum, 322, 323 , 338,366

- tendinum, 102

Chorda obliqua, 715

- trmpani, 264, 300, 327

Chordae tendineae, 417,421

Chorloldea, 376, 379

Chorioid plexus, 343,345

Chyle. 485

Chyme. 485

Cilia. 194

Ciliary processes, 376. 379

Cingulum. 338, 364, 373

Circle of Willis. 320

Circular sinus. 202

Circulus arteriosus [Willisi] 320

- - iridis major. 381

- - - minor. 381

Cisterna cerebeilomedul

laris, 317

- chiasmatis, 317
Cisterna chyli. 514

- fissurae lateralis cere bri. 317

- interpeduncularis, 317

- venae cerebri magnae, $31 \pi$

(isternae subarachnoi-

deales, 317

Clanstrum, 364

Clava. $32 \pi$

Clavicle, 51

Clitoris, 444

Clunes, 12:2

Cochlea, 392

Collateral fissure, 334,341

Colles, fascia of, 437

Colliculus cartilaginis arytaenoideae, 314

- inferior, 328

- superior, 328

- facialis, 331

- seminalis, 539

Collum. 210

- mallei, 390

- vesicae felleae. 505

Colon, 465, 467, 486

- ascendens, 46.5. 486

- descendens, 486

- sigmoideum, 486

- transversum, 465, 467, 486

Columna, Columnae:

- fornicis. 343

- medullae spinalis, 245

- rectales [Morgagnii], 536. 548

- renales [Bertini]. 508

- rugarum ant.. post., 545,549

vertebralis, 42

Columns of Bertin, 508

Columns of Morgagni, 536, 548

Commissura (cerebri) anterlor. $347,364,373$

- - posterior, 346

- habenularum 346

- hippocampi, 343, 373

- inferior [Guddeni]. 361

- labiorum (oris), 248, 288

- - (pudendi), 444

- (medullae spinalis) anterior (alba grisea), $\mathbf{2 4 5}$, 247

- posterior. 245 palpebrarum (iateralis, medialis), 247

- superior [Meynerti]. 361

Commissures of spinal cord, 245, 247

Compartment (s) of femoral sheath, 145 - anterior, of arm, 78

- posterior, of arm, 82

- leg, 168

- of neck, 212

Concha auriculae. 258

- nasalis inferior, 294 302

- - media. 294. 301

- superior, 301

- suprema [Santorini],

302

Conduction paths, 366-373

- of cerebral nerves, 366-369

- - of cerebellar peduncles, 369

- of central nervous system, 364

Confluens sinuum. 202

Conlcal papllae. 316
Conjoined tendon, 454, 456 Connections of several parts of the brain, 372

Conus arteriosus, 417

- elasticus (laryngis). 31:

- medullaris, 239. 243

Convolutions of cerebrum. See Gyri.

Cor, +12

Cornea, 376, 37s

Cornu anterius ventriculi lateralis, 340

- inferius cartilaginis

thyreoideae. $: 314$

ventriculi lateralis,

$337,340,341$

- (marginis falciformis), 140

- posterius ventriculi

lateralis, 340,341

- superius cartilaginis

thyreoideae. 314

- superius, inferius (mar-

ginis falciformis), 140 , 141

Cornua of spinal cord. See

Columna.

Corona ciliaris, 379

- glandis penis, 435

- radiata, 364

Coronary sinus, 414, 416 . 417

- valve, 417

Corpora quadrigemina,

$325,328,358$

Corpus adiposum orbitae, 276. 282

- - buccae, 288

- albicans. 546

- callosum, 338. 373

- cavernosum clitoridis, 448

531 - artery of, 448 ,

- penis, 440

529

- wethrae, 440

ciliare. 376,379

clitoridis, 444,448

epldidymidis. 460

fornicis, 343

geniculatum laterale. 346

- mediale, 346

glandulae bulbo-urethralis. 444

Highmori. 460

incudis, 389

- linguae, 315

luteum. 546

- Lnysi, 360

ma

medullare cerebelli, 355

pancreatis, 495

penis, 435

pineale, 346

restiforme. $326, \quad 353$

spongiosum penls. 440

- urethrae (muliebris).

striatum. 325. 332, 342

- trapezoideum. 354

uteri, 543

ventriculi 498

vesicae felleae. 505

vesiculae seminalis, 527

vitreum, 376. 382

Cornuscula lamellosa [Va. terl. ['acini] 100

- renis [Malpighlil. 509

Cortical cont rol. $366-68$

Corticopetal paths, 372 
Corticojugal paths, 372

Costae, 42, 433

Costal arches, 52

Cowper"s glands, 444

- - duets of. 444

Coxa. 121, $4: 33$

Cranial blood sinuses, 199

Cranium, 193

"Crazy-bone," 74

Crena ani, 433

Crista ampullaris. 344

- supraventricularis, 417

- terminalis, 416

- transversa, 39:3

- urethralis (muliebris), 549

- - (virilis). 539

- vestibuli, 392

Crural canal. 145

- ring, 145

Crus, crura

- ampullaria, 392

- anthelieis, 259

- clitoridis, 448

- commune, 392

- fornicis, 343

- helicis, 258

- incudis, 389

- inferius annuli inguínalis, 4.52

— of diaphragm, 511

- penis. 440

- posterius, 457

- simplex, 392

- stapedis, 388

- superius annuli inguinalis, 4.52

Crystalline lens, 376, 383

Cubitus, 41

Culmen, 329

Cumulus oophorus, 546

Cuneus, 337

Cupula cochleae, 392

- pleurae. 406

Curvatura ventriculi major, 498

- minor, 498

Cutis, $19 t$

Cymba conchae, 258

Dartos, 437

Declive. 329

Decursus fibrarum cerebralium, 364

Decussatio brachii conjunctivi, 358

- lemniscorum, 352

- Nn. trochlearium, 366

- pyramidum, 326

Decussationes tegmentorum, 357, 358

Deep palmar arch, 103

Deiter"s nucleus, 369

Dentes. 290

- canini, 290

- decidul. 290

- Incisiví, 290

- molares, 290

- permanentes, 290

- praemolares, 290

Descendens hypoglossi, 220 , 269

- fibre systems, 36.

- paths. 365

Descensus testis, 459,461

Dianhragma 511

- pelvis, 449

- sellae, 202

urogenitale, 441, 442 , 448,450

Digitus, 121

Diencephalon, 324, 325, 327,331

Digastric: triangle, 217
Digitationes hippocampi, $3+2$

Discus articularis :

- articulationis acromioclavicularis, 71

- mandibularis, 262

- radio-ulnaris distalis, 115

- sternoclavicularis,

Dissection of -

- abdomen, 433

- abdominal cavity, 462

- nerves, 516

- - vessels, 511

- - viscera, 462

- wall, 451

- anal region, 449

- arm, back of, 82

- - front of. 73

- - and forearm, 73

- axillary space, 51, 59

- back, 45, 228

- - muscles of :

48,230

- - second layer,

51,231

231

$-\frac{1}{233}$ - long, first layer,

233

- short, 234

- brain, 317

- brain-mantle. 332

carotid canal. 297

cerebellum, 324, 325 , 328

cerebral hemispheres, 324

- diaphragm, 511

- — of pelvis, 449, 533

- urogenital, 441, 448

ear, external, 258

- internal, 384

- middle, 384

Eustachian tube, 296

eyeball, 375

eyelids, 255

- face. 248

- facial, intermediate and acoustic nerves, intraosseous course of, 305

- femoral triangle, 146

foot, dorsum of 156

- plantar region. 174

forearm and hand, 87

- dorsal surface and

radial margin, 104

fourth ventricle, 332

gluteal region, 123

head, neck, etc., 193

- heart, 412

- infraorbital canal. 297

inguinal canal, 456

isthmus, 327

joints, ankle, 185

finger. 11

foot, 185

hand 111

- bip. 154

- intermetacarpal, 115

intermetatarsal, 190

intertarsal, 187

jaw, 264

- knee 180

- metacarpophalan-

geal, 116

geal, 190

pelvic, 551

radio, 551
Dissection of -

- joints, sacro-iliac, 553

- - shoulder, 84

- - sternoclavicular, 225

- - symphysis pubis, $\mathbf{5 5 3}$

- - tarsometatarsal, 189

- - thorax, 429

- - tibiofibular, 184

- toes, 190

- - vertebral column,

- lacrimal apparatus, 255

- larynx, 307

leg, anterior surface, 156

- - lateral surface, 153

- medial surface, 165 - posterior surface,

16.5

- lower limb, 121

- lung. 407

- mandibular canal, 264

- Meckel's ganglion, 303

- median plane of head,

298

mediastinum, posterior,

424

- medulla oblongata, 326

- meninges, 322

- mesencephalon, 328

- mouth and fauces, 288

- neck, deep dissection, 267

- posterior aspect, 228

282

- - side of, 204

- triangle of:

- - - anterior, 216

- - muscular, 222

- - occipital, 214

- - posterior, 213,

214

- nose, 257,300

- opening of the skull,

197

- orbit, 275

- organ of hearing, 384

- of vision, 375

- otic ganglion, 299

- pelvic muscles and nerves, 533

- viscera, 536

pelvis, female, 540

- male, 519

perineal region, 434

perinenm, female, 444

- male, 436

peritoneum, 462

- peroneal region, 163

- pharynx, 291

plantar region of foot 174

- popliteal space, 129

- prevertebral region, 282

- removal of brain, 199 of intestines, 483 spinal cord, 242

- scalp. 194

- shoulder, 68

- spinal meninges, 238

submaxillary region, 264

- telencephalon, 332

- temporal region, 260

thigh, anterior surface of, 138

- medial surface, 152

135 posterior surface of,

- thoracic cavity, 403 
Dissection of -

- thorax, 399

51

- tongue. 315

- upper limb, 39

- urogenital region in female, 444

- uropoëtic organs, 505

- wrist and hand, dorsal aspect of, 106

- - and palm, 96

Dorsum, 42

- manus. 42

- nasi, 193

Ductuli alveolares, 427

- efferentes testis, 460

- epoophori transversi, $5+6$

- excretoris glandulae lacrimalis, 257,271

Ductulus aberrans, 460

Ductus arteriosus [Botalli], 418

- choledochus, 487

- cochlearis, 394

- cysticus, 489

- deferens, 458, 527

- endolymphaticus. 394

- ejaculatorius, 527

- epididymidis, 460

- epoophori longitudinalis [Gärtneri], 546 - excretorius, 527

- vesiculae seminalis. 527

- hepaticus, 489

- lacrimalis, 248

- lactiferi, 57

- lingualis, 316

- lymphaticus dexter, 226

- nasolacrimalis, 257

- pancreaticus [Wirsungi ], 496

- _ accessorius [Santorini ], 496

- parotideus [Stenonis] 255. 290

- perilymphaticus, 394

- prostatici, $\$ 39$

- reuniens [Henseni]. 394

- semicirculares, 394

- sublinguales minores, 267. 290 sublingualis major. 267. 290 submaxillaris [ Whartonil, 267, 290

- thoracicus, 226. 426

- utriculosaccularis. 39

- venosus, fissure of, 504

Duodenojejunal fossa, 470

Duodenum, 473, 492

Dura mater encephali, 201 272,317

- - spinalis, 238, 239

Far. extrinsic muscles of, 196,249

- internal. 384

- middle, 385

Eminentia collateralis, 341

- conchae. 259

- fossae triangularis. 259 medlalis (fossae rhomboideae). 331

- pyramidalis. 386

- scaphae 250

Emissarium condyloideum, 203

- mastoideum. 203, 235

- occipitale, 203

- parietale, 203

Encephalon, 325
Endolympha, 394

Ensiform process, 52

Epicranial aponeurosis, 196

Epididymis, 459,460

Epithalamus, 325,346

spithelium lentis, 384

Epoophoron, 546

Eustachian tubes, 296, 38

- valve, 417

Excavatio papillae N. optici, 381

- recto-uterina, 473,541

- rectovesicalis, 473

- vesico-uterina, 541

External auditory meatus, 35.5

- capsule, 364

Extremitas inferior, 121

- superior, 41

- tubaria ovaril, 546

- uterina ovarii, 546

Eyeball, 375

Eyelids, 247

\section{Facies, 193}

anterior, lateralis, etc. Cf. structure involved. diaphragmatica (cor-

dis) 412,417

lienis, 501

pulmonis, 408

- gastrica lienis, 501

- intestinalis uteri, 544

- mediastinalis pulmonis, 408

- renalis lienis, 501

- sternocostalis (cordis), 412

- urethralis penis. 435

- vesicalis uteri. 544

Falx (aponeurotica) inguinalis, 454,456

- cerebelli, 202

- cerebri. 201

Fasciae, anal, 449, 450, 520,542

- antibrachil, 90, 104

- axillaris. $59,60,77$

- bicipital, 77, 80, 90

- brachii 60,77

- buccopharyngeal, 251, 261. 291

- bulbi [Tenoni], 276 277,280

- colli, 209, 211

- coracoclavicularis, 57 , $60,64,65$

- cremasterica [Cooperi], 4.7

cribrosa, 140, 191, 192 cruris. 157, 168, 173

deltoldea, 70

- dentata ( hippocampi), 337

- diaphragmatis pelvis inferior, $449,450,520$ 542

—- - superior. 520. 541 - urogenitalis inferior, $442,443,448$

- - - superior. 442

- dorsalis manus. 90

- - pedis, $15 \%$

- endopelvina. 520,541

glutaea, 125

- iliaca, 145. 518

- lllac portion of fascla

lata, 135, 140, 142

illopectinea, 141,518

- lliotiblal band, 125, 142 , 150

- infrasninata, 70

- infundibuliform, 457
Fasciae, intercolumnar, 452

- intermuscular septa,

142,150

- lata, 13.5, 140, 14.

- lumbodorsalis, 231

- obturatoria, $5 \pm 20,548$

- of Camper, 451

- of Colles, 437

- of Scarpa. $45 \mathrm{I}$

- pectinea, 141, 142,153

- pectoralis. 57

- pelvis, 520, 541

- perirenal, 5oo

- pharyngobasilaris, 293

- praevertebralis, 209

212

- prerenal, 506

- pretracheal, 209

- prevertebral, 209, 212

- prostatae, 520

- pubic portion of fascia

lata, $135,140,142$

- rectovaginal, 520, 541

rectovesical, 442,443 ,

448

- retrorenal. 506

- Sibson's, 227

- spermatica externa, 453

- - interna, 452

- subscapularis, 70

- superficialis abdominis, 138

of back. 231

of female perineum,

$436,437,445$

of gluteal region, 125

- of male perineum

$436,437,445$

- - of pectoral region. 57 - of thigh, 135, 140, 142

$-\frac{1}{45}$ perinel, 436. 437,

supraspinata, 70

- temporalis. 260

- transversalis. 145, 455

- visceral pelvic. recto-

vesical layer, $520,5+1$

- - vesical layer, 442

Fascial compartments of palm, 97, 98

Fasciola cinerea, 339

Fastigium, 331

Femoral hernia, 146

- sheath, 144

Fenestra cochleae, 387

Fibrae arcuatae cerebri, 364

36

- internae. 352

cerebello-olivares, 353 , 36 !

- intercrurales, 452

- lentis. 384

pontis profundae. 3.54

- - superficiales. 354

- propriae gyri cerebrl, 373

- zonulares, 383

Fasciculus, Fasciculi :

- anterior proprius [Flechsig], 246 anterolateralis superficlalis, 247,365

cerebellospinalis, 246 . 365. 369 cerebrospinalis anterior, 246,38 :

- lateralis, 246

corporis restiformis, 353

corticopontilis frontalis, 373

- - temporalls, 373 
Fasciculus, Fasciculi : - cuneatus [Burdachi], 247

- frontopontilis, 358

- gracilis [Golli], 247

- lateralis proprius

[Flechsigi], 247

longitudinales (pyra-

midales) pontis, 354

- longitudinalis inferior, superior, 364,373

- medialis, 352, 354 357

- mammillaris princeps, 373

obliquus (pontis), 327

occipitomesencephalica, 373

pedunculomammillaris,

361,373

- plexus brachialis, 68

pyramidalis, 353, 372

retroflexus [Meynerti], 362

spinocerebellaris dorsolateralis, 369

- temporopontilis, 358

thalamomammillaris

[Vicq' d'Azyri], 361, 373

transversi aponeurosis palmaris, 98

- plantaris, 176

- uncinatus, 362, 373

Fibre systems in spinal cord, 365

- - associated, 373

of cerebral nerves, 365

— - projection, 372

Fibrocartilagines intervertebrales, 286, 430

Fifth ventricle, 343

Fila radicularia, 242

Fillet, 354

Filum terminale, 243

- durae matris spinalis, 238

Fimbria hippocampi, 337

- ovarica, 547

Fimbriae tubae, 547

Fissura antitragohelicina, 260

- calcarina, 337

- cerebri, 332

- cerebri lateralis [Sylvii], $322,333,334$

- collateralis, $334 \quad 341$

- hippocampi, 336,342

- longitudinalis cerebri, 322,324

- mediana anterior, 243 326

- - posterior, 326 parieto-occipitalis, 334 ,

transversa cerebelli, 328

- cerebri, 332

Fissure of Rolando, 334

of Sylvius, 322, 333 334

Flexor sheaths of fingers, 101

- tendons and mucous sheaths, 101

- of toes, 178

Flexura coli dextra, 486

- - sinistra, 486

- duodeni inferior, 492

- - superior, 492

- duodenojejunalís, 463 , 492

- perinealis (recti). 524

- sacralis (recti) 524

Flocculi secundarii, $\mathbf{3 3 0}$

Flocculus, 330
Flumina pilorum, 121

Fold of the nates, 433

Folds of the axilla, 52

Folium cacuminis, 327

Folliculi linguales, 316

- oophori primarli, 546 -

Fonticulus gutturis, 204

Foramen, Foramina :

- caecum linguae [Morgagnii ], 360, 366 - (medullae oblongatae), 326

costotransversarium, 430

epiplolcum [Vinslowi], 472

- interventriculare [Mon. roi] 346

ischiadicum majus, 553 - minus, 553

of Monro, 346

papillaria (renis), 508

singulare, 394

thyreoideum, 314

- venae cavae, 511

venarum minimarum

[Thebesii]. 417

Formatio reticularis, 245 , 354,357

Foruix (cerebri), 337, 343

- conjunctivae, 247

- pharyngis, 293

- sacci lacrimalis, 257

- vaginae, 545

Fossa anthelicis, 259

- axillaris, $41,60,64,65$

- cranii anterior, 272

cranii media, 272

- posterior, 272

cubitalis, 80

- ductus venosi, 504

duodenalis inferior, superior, 470

for gall-bladder, 504

- hyaloidea, 382

- iliopectinea, 141

- iliacosubfascialis, 472

- incudis, 386

- interpeduncularis [Ta-

rini] 322

- ischiorectalis, 450

- jugularis, 194, 204

- navicularis urethrae

[Morgagnii], 539

$-\overline{444}$ (vestibuli vaginae),

ovalis, 140

- (cordis), 417

paraduodenalis, 470

poplitea, 129, 130, 132

retroduodenalis, 470

retromalleolaris, 121

rhomboidea, 330

sagittalis dextra (hepatis), 504

- sinistra (hepatis),

- Scarpae major, 122

- supratonsillaris, 296

triangularis, 25

- umbilicalis, 504

- vesicae felleae, 504

Fossula fenestrae cochleae, 387

- vestibull, 386

Fossulae tonsillares, 293, 296

Fovea centralis, 381

- inferior, 331

- inguinalis lateralis, 456

- medialis, 456

- oblonga (cartilaginis

arytaenoideae), $\mathbf{3 1 4}$
Fovea superior, 331

- supravesicalis, 456

- triangularis (cartilagi

nis arytaenoideae), 314

Foveola coccygea, 433

Frenula valvulae coli, 488

Frenulum clitoridis, 444

- labii inferioris, 290

- - superioris, 290

- labiorum (pudendi), 444

- linguae, 316

- praeputii, 435

- veli medullaris anterioris, 328

Frons, 193

Fundus meatus acustici interni, 393

- uteri, 343

- ventriculi, 498

- vesicae felleae, 505

Funiculi medullae spinalis, $243,246,247,326$

Funiculus cuneatus, 327

- gracilis, 327

- spermaticus, 434

"Funny-bone," 74

Galea aponeurotica, 196

Galen, veins of, 202

Gall-bladder, 505

Ganglia of spinal nerve roots, 242

Ganglion(ia) cardiacum

[Wrisbergi], 410

cervicale inferius, 227 . 271

- medium, 227, 271

- superius, 269,271

ciliare, 278

- - radix brevis, 280

- - longa, 277

- - sympathica, 278

- geniculi, 306, 307, 367

interpedunculare isth.

rhomb. 357

- jugulare (N. vagi), 270 , 369

- lumbalia, 516

- nodosum, 269, 270, 369

- of Meckel, $301,303,307$

of Wrisberg, 410

oticum, 264, 295, 299

radix sympathica, 278

petrosum, 270, 368

phrenica, 500

radix sympathica, 271

sacralia, 535

semilunare [Gasseri], $273,274,297,367$

sphenopalatinum, 301

303,307

spinale, 242

spirale (cochleae), 368 , 395

splanchnicum, 428

submaxillare, 266

superius N. glossophar-

yngei, 270,368

thoracalla, 428

vestibulare, 367

Gasserian ganglion, 273 . $274,297,367$

Gaster, 465, 498

Gastrohepatic omentum, 468,489

Geniculum N. facialis, 306

Genu corporis callosi, 338

- internum (N. facialis), $\mathbf{3 5 4}$

Gimbernat's ligament, 141 , 452

Gíngiva, 290

Gladiolus, 52 
Glandula, Glandulae :

- areolares [Montgomerii], 52

- buccales, 290

- bulbourethralis [Cowperi], 444

- ciliares [Molli]. 278

- duodenales [isrunneri]. 494

- intestinales [Lieberkuehni]. 484, 487

- labiales. 290

- lacrimalis, 255,276

- laryngeae, 310

- linguales, 290

- lingualis anterior [Blandini, Nuhni], 290

- mucosae biliosae, 505

- palatinae. 290, 294 - parotis. $218,267,290$

- - a ccessoria, 251,290 - sublingualis, 267,290

- submaxillaris, 218, 267 . 290

- suprarenalis, 505, 509

- - accessoria, 505, 509

tarsales [Mcibomi]. 247,255

- thyreoidea, 222, 228

- urethrales (mullebres) 537

vestibularis major [Bartholini], 447

Glans clitoridis, 444,448

- penis, 435

Glisson's capsule, 505

Globus major. 460

- minor, 460

- pallidus, 364

Glomeruli arteriosi cochleae, 396

- (renis), 509

Glomus chorioideum, 345

- coccygeum, 513

Glottis, 310

Gluteal aponeurosis, 125

Granulationes arachnoideales [Pacchioni], 198, 317

Gray matter of spinal cord, 243

Great sciatic nerve, 127 , 136. 144,525

Greater sac of peritoneum, 467,473

Gyri and sulci, 328, 332 . 334-337

Gyrus, Gyri :

- angularis, 375

- breves insulae, 337

- centralis (anterior, posterior). 334

- cerebelli. 328

- cerebri, 332

- cinguli, 336, 338

- fornicatus. 336

- frontalis inferior, 334

- - medius, 334

- superior, 334

- fusiformis. 335

- hippocampi, 336

- insulae. 337

- lingualis, 335

- longus insulae, 337

- occipitales laterales. 335

- - superiores, 335

- orbitales, 334

- profundus 336

- rectus. 334

- subcallosus, 337

- supramarginalis, 335

- temporalis inferior, 335

- - medius, 335

- - superior, 335
Habenula, 346

Ilamulus laminae spiralis, 393

Haustra coli, 486

llead and neck, 193

lleart, 412

I Ielicotrema, 393

Ilelix, 258

Ilemisphaeria bulbi urethrae, $4+0$

IIemisphaerium cerebelli, 329

- cerebri, 325, 331, 33

Hepar, 465, 502

Hepatic lobules, 505

Ilesselbach's ligament, 456

- triangle, 456

Hiatus aorticus, 511

- oesophageus, 511

- semilunaris, 303

- tendineus adductorius, 149,153

IIilus nuclei dentati, 356

- olivaris, 348

IIippocampal convolution, 336

IIippocampi, 342

Humor vitreus, 382

II unter's canal, 149

Hymen (femininus), 445 , 545

Hypophysis, 203, 322, 338

Hypothalamus, 338

Ileocaecal valve, 487

Ileum, 480

Iliotibial band of fascia

lata, 125, 142, 150

Impressio colica (hepatis), 504

_ duodenalls (hepatis), 504

- gastrica (hepatis), 504

- hepatica (renis), 508

- muscularis (renis). 508

- oesophagea (hepatis). 504

_ petrosa (cerebri), 332

- renalis (hepatis), 504

- suprarenalis (hepatis), 504

Incisura (ae) anterior auriculae, 258

- (apicis) cordis, 413

- cartilaginis meatus auditori externi [Santorini], 385

- cerebelli anterior. 328

- posterior, 328

- Intertragica, 258

- jugularis. 52

- terminalis auris, 260

- thyreoidea (inferior, superior), 314

- tympanica [Rivini], 385

- umbilicalis (hepatis). 504

Incus, 389

Infundibulum, 203, 322

- ethmoidale, 303, $33 \mathrm{~s}$

- tubae uterinae. 547

Inguen, 433

Inguinal peritoneal fossae 456

Inion, 194

Inscriptiones tendineae $\mathrm{NI}$ recti abdominis. 458

Insula, $333,337,341$

Intermuscular septa of arm, 78,80

Internal abdominal ring. 456

- capsule, 364

- ear, 384
Interosseous membrane of forear'm, 114

- - of leg, 18.

Interventricular septum. 421

Intervertebral disks, 286 430

Intestinum caecum, 485

- crassum, 465, 485

- ileum, 480

- jejunum. 480

- rectum, 465, 536, 542, 548

- pars analis. 536, 548

- tenue, 465, 479

- mesenteriale, 479

- terminale, 465

Intumescentia cervicalis. $24: 3$

Iumbalis. 243

Iris, 376,380

Ischiorectal fossa, 450

Island of Reil, 333, 337.

341

Isthmus aortae, 422

- cartilaginis auris, 260

- faucium, 291

- gvri fornicati. 336

- of Fallopian tube, 547

- prostatae, 527

- rhombencephali, 325.

$327,356,357$

- tubae uterinae, 547

Jejunum, 480

Joints. See Dissection of.

See also Articulatio.

Symplysis, and Syndesmosis.

Juncturae tendinum, 107

Kidneys, 506, 565

Iabial glands, 290

Labia superius et inferius, 193

oris (inferius, supe-

rius), 288, 428

Labium anterius uteri, 544, 549

- majus pudenda. 444

- minus pudendi. 444

- inferius valvulae coll. 488

- tympanicum. 395

- vestibulare, 395

- vocale. 309

Labrum glenoidale, 86,156

Labyrinthus membranaceus, 384, 394

- osseus, 384, 391

Lacertus fibrosus, 7 . 80 , 90

- II, recti lateralis. 279

Lachrymal sac. 257

Lacuna musculorum. 146

- vasorum, 146. 51s

Lacunae urethrales [Morgagnii], 539

Lacus lacrimalis, 247

Lamina, Iaminae :

- affixa, 341

- basilaris, 394

- cartilaginis cricoideae. 314

- choriocapillaris. $\mathbf{3 7 9}$ chorioidea epithelialis, $331,341,346$

cribrosa (sclerae), 378 elastica anterior [Bowmani], 378 - posterior [Descemeti], 379

- fibrocartllaginea inter-

pubica, $\mathbf{5 5 3}$ 
Lamina, Laminae:

- fusca (sclerae), 378

- medullares cerebelli, 35.

- - thalami, 362

modioli, 393

muscularis mucosae,

$487,494,500$

quadrigemina, 328

rostralis, 339

- septi pellucidi, 343

- spiralis membranacea. 392

- - ossea, 392

- - secundaria, 392

- suprachorioidea, 379

- terminalis. 338

- tragi, 385

- vasculosa (chorioideae), 379

Larynx, 307

Lateral occipital sulcus, 209

Latus; 433

Left auricle, 413

- hepatic duct, 489

- lateral ligament of liver, 473, 503

Lemniscus lateralis (acusticus ), 327, 354, 358

medialis (sensitivus) $327,354,358$

Lens crystallina, 376,383

Lenticular nucleus, 364

Lesser sac of peritoneum, 468,489

Lieberkühn, crypts of, 484 , 487

Lien, 465, 501

Ligaments of joints. $\mathrm{Cf}$ the joint.

Ligamenta, Ligamentum :

- accessoria plantaria, 190

- - volaria, 116

- acromioclaviculare, 71

- alaria. 288

- annulare, 178

- baseos stapedis, 390

- - radii, 115 annularia digitorum manus, 102

- anococcrgeum, 449

- apicis dentis. 288

arcuatum pubis, 442 , 449,553

- arteriosum, 417

- articulationum meta carpophalangearum. 116 - metatarsophalan gearum, 190

$\overline{116}(--)$ interossea, auriculare anterius, 259

- - posterius, 259

- - superius, 259

- basium (ossium metacarpalium) dorsalia, 116 - - (- - ) volaria, 116

- metatarsalium ) dorsalia, 190

-100 (- - ) plantaria, 190

- bifurcatum, 189

- calcaneofibulare. 186 calcaneonavicuiare dorsale, 189

- plantare, 189

calcaneotibiale, 186

- capituli fibulae, 184

- capitulorum (ossium

metacarpalium) (ransversa, 116

transversa, 190

- carpi dorsale, 90
Ligamenta, Ligamentum : carpi radiatum, 90 ,

104,106

- - transversum, 93, 100

- volare, 93, 180

carpometacarpea dorsa-

lia, 115

— volaria, 115

ceratocricoideum anterius. 314

- - laterale, 314

- posterius, 314 collaterale carpi ra diale, 112

- - ulnare, 111

- fibulare. 180

- - radiale, 110

— - tibiale, 165, 180

- - vinare. 110

- collateralia articulationum digitorum (manus), 116

- - pedis, 190

colli costae, 429

- columnae vertebralis, 286

- conoideum. $7^{\circ}$

- coraco-acromiale, 71

- coracoclaviculare, 71

- coracohumerale, 85

- corniculopharyngeum, 314

coronarium (hepatis), 473,503

- costoclaviculare. 225

- costotransversarium anterius, 429

- - posterius, 429

- costoxiphoidea, 429

- cricoarytaenoideum posterius, 315

- cricopharyngeum. 314

- cricothyreoideum (me-

dium ), 311

- cricotracheale. 314

- cruciata (genu), 181

- cruciatum, 177

- cruciatum atlantis, 288

- cruris, 157

cuboideonaviculare dorsale, 189

- - plantare, 189

cuneocuboideum dorsale. 189

- - interosseum, 189

- plantare. 189

cuneometatarsea inter ossea, 189

- deltoideum, 186

- denticulatum 241

- duodenocolicum 493

- duodenorenale, 473, 493

- falciforme (hepatis), 473,503

- flava, 286

- gastrocolicum, 473

- gastrolienale, 473

- glenohumerale inferius,

- - medium, 86 superius. 86

- hepatoduodenale, 468. 473,489

- hepatogastricum, 468, 473

- hepatorenale, 473

- hyothyreoideum laterale, 310

- hyoepiglotticum, 319

- medium, 310

- illofemorale 155

- iliolumbale, 552

- incudis posterius, 390

- superius. 390

- inguinale [Pouparti],

$144,433,452,511$
Ligamenta, Ligamentum :

- inguinale reflexum [Collesi], 457

- - superius, 390

- interarticulare coxae, 156

- intercarpea dorsalia,

112

interossea, 113

volaria, 113

- interclaviculare, 225

- intercostalia externa, 399

- - interna. 399

- intercuneiformia interossea, 189

- interfoveolare [Hesselbachi ], 456

- interspinalia, 386

- intertransversaria, 386 , 430

- ischiocapsulare, 155

- laciniatum, 170, 173 , 177

lacunare [Gimbernati], $141,144,452$

- latum pulmonis, 408

- - (uteri), 473, 541

- longitudinale anterius, 286,430

- - posterius, 286, 430

- lumbocostale, 430

- mallei anterius, 390

- laterale, 390

- malleoli lateralis anterius, 184

- - - posterius, 184

- mucosum, 181

navicularicuneiformia dorsalia, 189

- - plantaria, 189

- nuchae, 239

- ovarii proprium, 546

- palpebrale laterale. 250

- 257 mediale, 250, 255,

- patellae, 122

- pectinatum iridis, 380

- phrenicocolicum, 473

- pisohamatum, 113

- pisometacarpeum, 113

- plantare longum, 180 popliteum arcuatum, 180

- obliquum, 135, 180

- pubicum superius. 553

- pubocapsulare. 155

- puboprostaticum (pubovesicale) laterale), 523 , 542

$-\frac{}{54}(-)$ medium, 523, 524,542

- pulmonale. 406

- pylori, 500

- radiocarpeum dorsale, 111

volare, 111

- reflexum [Collesii], 452

- sacrococcygeum. 553

- sacrodurale anterius, 238

sacroiliaca anteriora, 553

- - interossea, 553 sacroiliacum posterius breve, 553

- _ - longum, 553

- sacrospinosum, 553

- sacrotuberosum, 450 . 552

- sphenomandibulare. 262

- spirale cochleae, 394

- sternoclaviculare, 225

sternocostale interar-

ticulare, 429 
Ligamenta, Ligamentum : sternocostalia radiata. 429

sternopericardiaca inferius et superius, 410

- stylomandibulare, 217

subflava, 286

supraspinale, 286

- ovarii, 473,541

suspensorium penis, 461

talocalcaneum anterius. 187

- - interosseum, 188

- laterale, 187

- mediale, 187

- posterius, 187

- talofibulare anterius, 186

- - posterius, 186

- talonaviculare (dorsale), 189

talotibiale anterius, 186

- posterius, 186

tarsi dorsalia, 189

- interossea, 188

- plantaria, 189

tarsometatarsea dorsalia, 189

- plantaria, 189

temporomandibulare 262

- teres femoris, 156

- hepatis, 503

- uteri, 458, 547

thyreo-epiglotticum, 314

tibiona viculare, 186

176

- - atlantis, 288

- cruris. 157

- genu. 181

- pelvis, 442, 449

- scapulae inferius, 71

- trapezoideum, 71

triangulare dextrum (hepatis), 473, 503

- sinistrum (hepatis), 473,503

- tuberculi costae, 429

- umbilicale laterale, 529

- vaginalia digitorum (manus), 102

- venosum [Arantii], 503

- ventriculare, 312

vocale, 312

Iimbic lobe, $\mathbf{3 3 6}$

Iimbus corneae. 378

- fossae ovalis [Vieussenii], 417

- membranae tympani. 387

- palpebralis (anterior, posterior ), 247

Limen insulae, 337

- nasi. 301

Linea, Lineae

- alba, 451, 455

- axillaris, 42

costo-articularis, 42

mammiliaris, 42

mediana anterior, 42

- posterior, 42

obliqua (cartilaginis

thyreoideae), 314

parasternalis. 42

- scapularis. 42

semicircularis [Doug-

lasi ]. 454

- semilunaris [Spigeli], 454

sternalis, 42

- visus, 376

I.ine, anterior median, 42

- axillary, 42

- costo-articular, 42
Line, mammillary, 42

- parasternal, 42

- posterior median, 42

- scapular. 42

- sternal, 42

Lingua, 315

Lingual papiliae, 316

Lingula pulmonis. 408

- (cerebelli). 328

Lips, 248, 288

Liver, 465, 502

Lobules of testicie, 460

Lobulus auriculae, 258

- biventer, 330

- centralis, 329

- paracentralis. 336

- parietalis (inferior. superior), 335

- quadrangularis, 330

- semilunaris inferior, superior, 330

Lobuli corticales (renis), 508

— epididymidis, 460

- hepatis. 505

- pulmonis, 428

- testis, 460

Lobus, Lobi :

- caudatus [Spigeli], 503

- centralis, 329

- frontalis, 333, 334

- hepatis (dexter, siniser ), 503

- occipitalis, 333,336

- olfactorius, 337

- parietalis, 333, 334

- pulmonum, 408

- quadratus (hepatis), 503

- renales, 503

- temporalis, 333, 335

- thymi (dexter, sinister), 411

Locus caeruleus, 331, 367

- perforatus posticus, 322

- anticus, 337

Longitudinal fissure of liver, 504

Lower llmb, 121

I umbar puncture, 238

- fascia, 231

Lumbus, 433

Iungs, 407

- lobes of, 408

- roots of, 408

Isunula unguis. 121

Lunulae valvularum semilunarium aortae, 418 , 423

418

Lymphoglandulae auriculares anteriores, 197

- posteriores. 197

- axillares, 57, 61

cervicales profundae. 213

- - - superiores, 222

- superficiales, 207

- coeliacae. 514 $7^{\text {cubitales superficiales, }}$

- cystica, 490

- hepaticae. 491

- Hiacae, 5i4

- inguinales, 140

- lumbales. 514

- mesentericae. 477

- mesocolica, 477

- occipitales, 197

- pectorales, 57 61

- popliteae. 135

- sternales, 57

- subinguinales profundae, 141
Lymphogiandulae superficiales, 122,140

- submaxillares, 218

- subscapulares, 63

- venae portae, 491

Macula, Maculae :

- acustica sacculi, 394

- cribrosae, 392 Hova (plicae vocalis),

- lutea, 381

Mamma, 52, 57

- accessoria, 52

Manubrium mallei sterni, 52

Manus, 41

Margo. Cf. also text on structure concerned.

- ciliaris iridis, 380

- falciformis. 140, 142

- liber (ovarii), \$46

- mesovaricus. 546

- pupillaris iridis, 380

- radialis, 41

- unguis (lateralis. liber, occultus), 121

Nassa intermedia, 347

Meatus acusticus externus, 385

- - internus, 393

- nasì communis, 302

- - inferior, 294, 302

- - medius, 294, 302

- - superior, 302

- suprenus, 302

nasopharyngeus. 294 $30 \%$

Meckel's ganglion, 301 . 303. 307

Mediastinum testis, 460

Medulla oblongata, 322, $324,325,326$

- spinalis, 242

Medullary pyramids of kidney, 508

- vela, 328, 331

Nembrana, IIembranae.

- atlanto-occipitalis anterior, 287

- posterior. 287

- elastica interna, 312

- hyaloidea, 382

- hyothyreoidea, 222,310 interossea antibrachii,

114,115

- cruris, 184,185

- mucosa nasi. 302

- obturatoria, 5.52

- - (stapedis). 390

- pupiliaris. 331

- sterni, 429

- tectoria, 287

- tympani, 387

- secundaria, 388 vestibularis [Reissneri], 394

Meninges encephali, 317

- spinales, 238

Meniscus lateralis, 181

- medialis. 181

Mentum, 192

Meridiani. 376

Mesencephalon, 324, 325, 326,328

Mesenteric lacteals, 477

Mesenteriolum processus rermiformis. 471,476

Mesenterium, $46 \mathrm{~S}$

- anterius, $\mathbf{4 6 3}$

- commune, 462

- posterius, 463

Mesentery proper. 468

Meso-appendix, $4 \tau 1,476$ 
Mesocolon, 468

Mesometrium, 541

Mesorectum, 46 !

Mesosalpiux, 541

Mesovarium, 5+1

Metacarpus, 41

Metathalamus, 325, 346

Metencephalon, 324, 325

iliddle ear, $305,384,355$

Modiolus, 393

Ionro, foramina of, 346

Mons pubis, 433, 444

- veneris, 444

Monticulus, 329

Morgagni, columns of, 536 , $5+8$

Musculus, Musculi :

- abdominis, 452,454

- abductor digiti quinti (manus), 100, 103

- - - (pedis). 178

- hallucis, 173,178

- pollicis brevis, 98, 103,107

- Iongus, 105, 107

- adductor brevis, 153 .

- hallucis, 179

- longus, 152

- pollicis, 129, 138

153

$-\frac{1}{153}$ magnus, 129,138 ,

158 minimus, 126,153

pollicis, 103

anconaeus, 84,105

- antitragicus, 252

- articularis genu, 152

- aryepiglotticus, 311

- arytaenoideus obliquns, 311

- - transversus, 311

auricularis anterior,

196. 249

auricularis anterior (profundus), 259

- - posterior, 19t;, 249 superior, 196,249

- biceps brachii, 80,87 - femoris, 129, 130 , 135

- flexor cruris, 129 130,135

- brachialis, $80,82,84$

- brachioradialis, 80,84 93,104

- broncho-oesophageus, 424

- buccinator, 250, 261

- buccopharyngeus, 293

- bulbocavernosus, 447

- caninus. 250

- ceratopharyngeus. 291

- chondroglossus, 316

- chondropharyngeus, 291

- ciliaris, $380^{\circ}$

fiblae circulares [Mue] leri], 380 - meridionales [Brueckei],

- coccygeus, 534, 548

- compressor venae dorsalis penis, 438

- constrictor pharyngis inferior, 291

- - medius, 291 - - superior. 202

- coracobrachialis, 80 corrugator supercilii,
250

- cremaster, 457

- crico-arytaenoideus lateralis, 311

- - posterior, 311

- crureus, 150

- deltoideus, 69
Musculus, Musculi :

- depressor septi, 250

- digastricus, 222, 225

- dilator pupillae, 380

- ejaculator semini, 439

- epicranius, 196, 249

- extensor carpi radialis brevis, 104, 107

- - longus, 80, 84 104,107

- - ulnaris, 105, 107 digiti quinti proprius, 104,107

- digitorum brevis, 163

- _ communis, 104 ,

$10 \mathrm{~T}$

- longus, 158, 168

- hallucis brevis, 163

- — Jongus, 160, 168

105,107 indicis proprius,

107

107

pollicis brevis, 105

- longus, 105, 107

- flexor carpi radialis, 93 . 9

- - ulnaris, 94

- digiti quinti brevis

(manus), 100, 103

179

- digitorum brevis, 178

$\overline{173}, \overline{179}$

- profundus, 96 sublimis, 9 hallucis brevis. 179

- - longus, 168, 170 .

173 103, pollicis brevis, 98 ,

gastrocnemins, 130,169

gemellus inferior, 127

- superior, 127

genioglossus, 266

geniohyoideus, 266

glossopalatinus, 295

- glossopharyngeus, 293

glutaeus maximus 125

- medius, 126. $12 \mathrm{~S}$

- minimus. 128

- gracilis, 153, 165

- helicis (major, minor) 259

- hypoglossus, 266, 267

- Horneri, 250

- iliacus, 153,518

- iliococcygeus, 434,548

- iliocostalis, 233

- - cervicis, 233

- dorsi, 233

- lumborum. 233

- iliopsoas, 518

- incisivus labii inferioris, 251

- superioris, 251

- Infraspinatus, 72

- interfoveolaris, 456

- intercostales externi, 399

- interni, 399

- interossei dorsales (manus), 107, 109

- - (pedis), 163, 180

- plantares. 180

- volares, 109

- interspinales, 234

intertransversarii anteriores. 234, 284

- posteriores, 234. 284

- - - laterales, 234
Musculus, Musculi :

- ischiobulbosus, 439

- ischiocavernosus, 438 , 447

latissimus dorsi, 48 , 230

- levator ani, 534, 548 249,276 230 scapulae, 51, 229,

- veli palatini, 295

levatores costarum breves, 234

- - - longi, 234

- lingualis inferior, 316

- - superior, 316

- longissimus, 233

- - capitis, 233

- cervicis, 233

- - dorsi, 233

-- longitudinalis inferior (linguae), 316

$3 \overline{16}$

- longus capitis, 212, 284

- colli, 212, 284

- lumbricales (manus)

$99,102,107,109$ (pedis), 179

- masseter, 260

- mentalis, 250

- multifidus, 234

- mylohyoideus, 222, 264

- mylopharyngeus, 293

- nasalis, 250

- par's alaris, 250

- - transversa, 250

- obliquus auriculae, 259

- - capitis inferior, 234

- - superior, 234

- - inferior (oculi), 280

- superior, 277

- obturator externus, 127, 153

548 internus, 127,535 ,

occipitalis, 196

- occipitofrontalis, 196 , 249

- oculi, 279

- omolivoideus, 51, 211,

217,225

opponens digiti quinti (manus), 103

- - - - (pedis), 179

- pollicis, 98, 103

- orbicularis oculi. 249

- pars lacrimalis [M.

Horneri], 250

- - - orbitalis, 250 palpebralis, 249

- orbicularis oris, 251

- palpebrarum, 249

- palmaris brevis. 97, 100

- - longus, 93, 94, 170

- papillares, 417,421

- pectineus, 152, 153

- pectoralis major. 58, 64 59 - pars abdominalis,

- - clavicularis, 59

- - sternocastalis, 59

- pectoralis minor, 61,65

- perinei, 437

- peronaeus brevis. 163

- - longus, 163, 180 - tertius, 160, 168

- pharyngopalatinus, 295

- piriformis, 125, 126. 535,548

- plantaris, 130, 168, 169

platysma myoides, $\mathbf{5 3}$

$207,222,249$

- pleuro-oesophageus, 424 
Musculus, Musculi :

- popliteus, 170

procerus, 196,249

pronator quadratus, 96

- teres, 93,94

prostaticus, 537, 539

psoas magnus, 153,518

major, 153, 518

- minor, 518

pterygoideus externus, 261

- internus, 261

pterygopharyngeus, 293

pubococcygeus, $534,5.48$

pubovesicalis, 524

pyramidalis, 454

- nasi, 196, 249

quadratus femoris, 127

- labii inferioris, 250 - superioris, 250

lumborum. 518

- plantae, 179

quadriceps femoris, 150

rectococeygeus, 525 ,

536, 548

- rectovesicalis, 537

- rectus abdominis, 454

- capitis anterior, 284

- - lateralis, 272, 28

234

inor, 234

femoris, 150

rectus inferior (oculi), 279

- lateralis, 279

medialis, 279

- superior, 277

rhomboideus major, 51, 231

minor, 51, 231

- risorius, 250

rotatores breves, 234

- longi, 234

sacrococcygeus anterior, 534

- posterior. 534

- sacrospinalis, 233

- salpingopharyngeus, 29 .

- sartorius, 149,165

- scalenus anterior, 210 . 212,225

- medius, 210, 212, 225

- - minimus, 226, 227 posterior, 210, 225

semimembranosus, 129 , 130,135

- semispinalis. 234

- - capitis, 229, 234

- c crvicis. 234

- - dorsi, 234

- semitendinosus, 129

$130,135,165$

- serratus anterior, 61,68 - posterior inferior 231

- superior, 231

- soleus, 169

- sphincter ani externus. 449

$5 \overline{48}-$ internus, 5.36

sphincter pupillae, 380

- pyiori, 500

- urethrae membrana-

ceae, $443,448,537$

spinalis, 233

- cervicis, 233

- dorsi, 233

splenius capitis, 229 231

- - cervicis, 231

- - colli. 231

- stapedius, 391
Musculus, Musculi :

- sternocleidomastoideus, $209,212,230$

sternoliyoideus, 211

217,225

- sternothyreoideus, 217 , 225

- styloglossus, 266, 316

- stylohyoideus, 225

- stylopharyngeus, 267

- subanconaeus, 82

- subclavius, 65, 67

- subcrureus, 152

- subscapularis, 61, 72

- supinator, 82, 105

- brevis, 82,105

- longus, 80, 84, 93, 104

- supraspinatus, 72

- suspensorius duodeni, 493

- tarsalis (superior, inferior), 257

- temporalis, 261

tensor fasciae latae, 129,150

- - tympani, 391

- veli palatini, 295

teres major, 71

- minor, 72

thyreo-arytaenoideus

(externus), 311, 312

- thyreo-epiglotticus. 311

- thyreohyoideus, 211, 225

- thyreopharyngeus. 291

- tibialis anterior, 158 , 168

173,180

tragicus, 259

- transversus abdominis, 454

- auriculae. 259

- linguae, 317

443

$4 \overline{47}-$ supercialis, 438 .

- - thoracis, 402

- trapezius, 48, 209

- triangularis nasi, 250

- - oris, 250

- - sterni, 402

- triceps brachii, 82,84 , 87

- - surae, 168,169

- uvulae, 295

- vastus intermedius, 150

- - lateralis, $\mathbf{1 5 0}$

- - medialis, 150

- ventricularis. 312

- verticalis (linguae), 317

- vocalis, 311

zygomaticus, 250

Myelencephalon. 324,325

Myocardium, 416

Nares, 193

Nasion, 19.

Nasus, 193

Nates, 122

Nervus, Nervi :

- abducens, 201, 275, 280. $324, \quad 367$

- nucleus of, 367

- accessorius, 50,201 , $214,220,228,230,269$, 295,324

- - ramus ext., 50, 220

- - - int., 220

- acusticus, $201,305,307$. 324,367 ,

- nuclel, 367
Nervus, Nervi :

- alveolares superiores, 298

- alveolaris inferior, 264

- anococcygei, 450, 535

- auriculares anteriores, 255,264

auricularis magnus,

196,229

- $\overline{25}$ posterior, 196, 207 .

auriculotemporalis, 195

$255,263,300$

- axillaris, $64,67,68,71$, 216

- buccinatorius, 255, 263

- canalis pterygoidei

[Vidii], 297, 303, 307

cardiacus inferior, 227

272,423

- medius, 227, 272,

423

409

carotici externi, 220,

271

caroticotympanici (sup.

inf.), 271

caroticus internus, 271. 297

- cavernosi clitoridis, $5+1$

- - penis minores, 519

cavernosus penis major. 519

- cerebrales, 322

- cervicales. 50, 214, 241 $269,271,284$ rami cutanei dorsales, 229,234

- - posteriores, 236

ciliares (breves, longi), 277,278

clunium inferiores, 125 , 128,535

- medii. 123

- - superiores, 48, 123

- coccygeus, $48,237,242$

535

cochleae, 368

- communicans fibularis, 166

cutaneus (ei) antibrachii dorsalis, 76,84 - - lateralis, 76, 80 76,78

brachii lateralis, 70 71,216

-- medialis, 64,68 , 76, 78 posterior, 76. 84 - - cervicis (Var.), 207 , 230

- - colli. 207

- femoris lateralis $125,135,140,142,148$ 516

- posterior 125, $128,130,133,136,166$ 535

- pedis dorsalis intermedius, 161

- lateralis, 157 172

- medialis, 160 166

166. $\overline{172}$

- depressor, 270

- digitales dorsales luallucis lateralis et digiti secundi medalis, 160

- - - manus, 89, 9

- - - pedis, 157,161 
Nervus, Nervi : 178

plantares communes,

- proprii. 178,179

volares communes, 99.100

- proprii. 99, 100 $5: 35$

- penis, 440. 535 230

ethmoidalis, 277

- facialis, 195, 201, 209 ,

$248,254,255,260,264$

299, 305, 306, 307, 324

- nucleus, 367

femoralis, 142,144 ,

$148,150,517$

- rami cutanei femoris

anteriores, 135,517

- frontalis. 254. 275

- genitofemoralis, 145.516

- glossopharyngeus. 201.

$260,254,267,268,270$

29), 299, 317, 324, 368 glutaeus inferior, 128 , 534

$-\frac{}{21}$ superior, 127,129 ,

haemorrhoidales inferiores, 450 , 535

- medii. 535

- hypoglossus, 201, 212, $214,218,220,222,228$, 26f, 269, 282, 317,324 iliohypogastricus 123 , 452,506

ramus cutaneus anterior, 516

- lateralis, 516 ilioinguinalis, $140,142$. $452,458,516$

- infraorbitalis, 254, 298

- infraspinatus, 73

- infratrochlearis, 254 , 27

- intercostales, 400,428

- ram. cut. anterior (pector., abdom.). 451 - - lateralis (pector., abdom.), 60,452 - intercostobrachiales, 60 , 74

intermedius, 201. 264, 30.5. 306, 30\%, 31\%, 324, 267

interosseus lantibra(hii), dorsalls, 105, 108 - volaris, 96 cruris, 132,172 ischiadicus, 127,136 , 144. 535

- jugularis, 271

labiales posteriores 447 , $59 \%$

- anteriores, 516

lacrimalls, 275,276

laryngeus inferior, 270

- superior. 220,222 .

. 311

- lamus ext., 220

ling int.. 22

- lingualis, 264, 266, 267 317

- lumbales, 48, 241

- rami posteriores. 237 lumbo-inguinalis. 140 ,

142, $1+5,148,516$

mandibularls, 2.54. 263.

274,299

- massetericus, 263

- masticatorius, 254, 263

maxillaris, 254,274 ,
Nervus, Nervi :

- meatus auditorii externi, 263

medianus, 64, 68, 79,

$82,90,94,98,99,100$

meningeus medius, 275 298

- mentalis, 25j, 264

musculocutaneus, 64 ,

$68,76,79,80$

mylohyoideus, 217. 21s, 264

nasociliaris, 254,275 ,

277,301

- nasopalatinus [Scarpae ]. $300,304,305$

obturatorius, 133. 135 ,

144,516

_- ramus anterior. 516

- - cutaneus, 142

- posterior, 516

occipitalis major, 196, 229. 284

- - minor, 196. 207

— - tertius (Var.), 229

oculomotorius, 200, 275, $280,323,328,366$

- nucleus. 366

- - ramus inf., sup., 275

olfactorii, $199,300,303$, 323.366

- ophthalmicus, 2.54, 274

- opticus, 200, 277, 323 . 366. 375

- nuclei, 366

- palatini (anterior, me

dius, posterior), 296 ,

303. 304, 30:

- perinei, 440, 447, 535

- peronaeus communis.

$130,133,13 i, 16 ;, 165$ 5.3.) 16.5 profundus, 157,160 ,

- superficialis, 157

160, 161, 165, 168 petrosus profundus, $29 \%, 303$

- superficialis major, $275,297,303,307$

- minor, 275, 299 , 306

- phrenicus, 226, 408 , 409

- plantares (medialis, latcralis), 178,179

- pterygoideus (externus, internus) , $26 \%$

- pudendus, 128, 450, 53. radialis, $64.68,76,82$, 89, 90, 92, 97, 105

- ramus prof., 82

- superf. 82,89

- recurrens, $82,222,270$, 311. 312,425

- sacrales, 48,242

- saphenus, 142, 157, 165 518

- rami cutan. cruris

mediales, 157,166

- scrotales anteriores, 516

- posteriores. 440,535

spermaticus ex+elnus,

$140,458,459,516$

- sphenopalatini, 298

- spinales, 241

- spinosus, 263, 300

- splanchnicus major, 428

- imus, 428

- minor, 428, 500

stapedis, 306

- subclavius, 67, 216

- sublingualis, 266,317 suboccipitalis, 235,272
Nervus, Nervi :

subscapulares, 51,67 , 216

supraclaviculares, $\mathbf{5 5}$,

$67,70,207,209,214,216$ supraorbitalis, 254,276 suprascapularis, 73,230

- supraspinatus, 73

- supratrochlearis, 254 , 276

- suralis, 166, 172

- temporales profundi

(ant., post.), 261, 263

tensoris tympani, 300

-

horacales, 48, 123, 241 , 452

- anteriores, 59, 65, 67,216

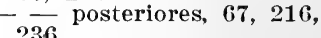

thoracalis longus, 67, 216

- thoracodorsalis, 67,216

tibialis, $132,136,168$,

172,173

trigeminus, 201, 254,

$273,295.317,323,367$ - portio major, minor, 273. 274,323

trochlearis, 201, 275

276,323

- nucleus, 366

- tympanicus, 271

- ulnaris, $64,68,79,84$,

$89,90,94,98,99,100$, 103

— - ram. cut. palmaris, 97

- prof., 100

- - - superf, 100

- ragus, 201. 212. 220, $222,226,228,268,269$, $270,282,324,36 \mathrm{~s}, 408$, $425,497,498$

nuclei 368

vesicales inferiores, 519, 53.5

- - superiores, 519

- vestibuli, 367

- nuclei, 367

zygomaticus, 195, 254 , $260,276,282,298$

Nidus avis, 330

Noduli lymphatici aggregati [Peyeri]. 485, 489

- lienales [Malpighii] 502

494

- valvularum semiluna rium aortae [Arantii]. 423

418

A. pulmonalis,

Nodulus vermis, 329

Nucleus, Nucleí:

- alae cinereae, 348,369

- ambiguus, 348,368

- amygdalae, 342,364

- arcuati, 352

- caudatus, 341

- colliculi inferioris, 358

- corporis geniculati latcralis, 362

- - medialis, 362

- mammillaris, 361 dentatus, 356

dorsalis IStillingi, Clarkii, 246

- emboliformis, 356

- fastigii. 356

- funiculi cuneati, 348

- gracilis, 348

- globosus, 356 
Nucleus, Nuclei :

- habenulae. 362

- hypothalamicus, 360

- laterales. 348

- lemnisci lateralis, 354

- lentiformis, 364

- lentis. 384

- N. abducentis, 3.54

- acustici (dorsalis, ventralis), 354

- - cochleae. 354

- - facialis. 354. 367

- - hypoglossi. 348

- oculomotorii, 357, 363,366

- trigemini motorius. 354,367

- - trochlearis, 357, 360 368

olivares accessorii, 348 , 362

- ollvaris inferior, 348

- - superior, 354

originis Nn. cerebralium, 365-369

- pontis, 354

- pulposus (fibrocartila ginis intervertebralis) 286,430

- radicis descendentis $\mathrm{N}$. trigemini, 357

- ruber, 357

- tegmenti, 357

- terminales Nn. cer., 365-369

- thalami anterior, 362

- - lateralis, 362

- - medialis. 362

tractus solitarii, 348 , 369

- spinalis $N$. trigemini, 348,354

Obex, 331

Occipital lobe, 335

- sinus, 202

Occiput, 193

Occipitotemporal convolution, 335

Oesophageal groove in liver, 504

- opening in diaphragm, 511

Oifactory sulcus, 301,334

- tract. 337

Oliva, 326

Omentum majus. 467, 473

- minus, 468, 489

Operculum, 337

Ora serrata, 351

Orbiculus cillaris, 379,380

Orbita, 194, 275

Organa oculi accessoria, 375

- uropoëtica, 505

Organon auditus, 384

- spirale. 395

- visus, 375

Orificium externum utèri, 544. 549

- internum uteri, 544, 549

- ureteris. 537

- urethrae externum (muliebris), 445. 448

- - (virilis). 435 539

- internum, 537 vaginae, 444

Ossicula auditus, 388

Ostium abdominale tubae uterinae, 547 arteriosum dextrum,

$412,418,421$

- - sinistrum, 412
Ostium pharyngeum tubae audivae, 293, 296

- uterinum tubae nterinae, 550

- venosum dextrum, 417

- - sinistrum, 421

Ovarium, 546

Ovulum, 546

Pacchionian granulations, 198,317

Palatum durum, 288

- molle, 288, 294

Pallium, 325, 332

I'alpebrae, 193, 247

Pancreas, 465, 495

- accessorium, 496

Papilla, Papillae :

- conicae (linguae), 316

- duodeni [Santorini], 494

- filiformes, 316

- foliatae. 316

- fungiformes, 316

- incisiva 290

- lacrimalis, 248

- lenticulares. 316

- mammae, 42

- nervi optici, 381

- renales, 508

- vallatae. 316

Paradidymis, 462

Parametrium, 549

Paries mastoideus, 386

- caroticus, 386

- (cavi tympani) jugu-

laris, 386

- - - labyrinthicus, 386

- - membranaceus 387

- tegmentalis, 385

Paroophoron, 546

Pars basilaris pontis, 354

- dorsalis pontis, 359

- flaccida (membranae tympani), 387

- grisea hypothalami, 360

- libera columnae fornicis, 343

mammillaris hypothalami, 325, 331, 338

- marginalis (sulci cinguli), 336

- membranacea septi atriorum, 417

opercularis (gyri frontalis inferioris), $\mathbf{3 3 4}$

optica hypothalami, 325 , 331, 332, 333

- orbitalis (gyri frontalis inferloris) $\mathbf{3 3 4}$

subfrontalis (gyri cinguli), 336

- tecta columnae fornicis. 343

- tensa (membranae tympani), 387

- triangularis (gyri frontalis inferioris) 334

Partes genitales externae (muliébres), 444

Path. Paths:

- of brachium pontis, 369

- - conjunctivum, 372

- of corpus restiforme, 369

- of diencephalon. 372

- of telencephalon, 372

- secondary. of $\mathrm{Nn}$. cerebrales, 366-369

- tertiary. of Nn. cerebrales, $366-369$

Pedunculus cerebri. $\mathbf{3 2 2}$, $323,326,328,357$

- corporis callosi. 337
Pedunculus flocculi, 330

- thalami inferior, 362

Pelvis renalis, 509

Pericranium, 410

l'erilympha, 394

I'erimetrium, 549

Perineal body, 447

Perineum, 436

Peritonaeum, 45.5

- parietale, 467

- viscerale, 467

Pes cavum, 177

P'etiolus epiglottidis, 313

P'ia mater encephali, 198 , 317,344

- spinalis, 240

Pilus, 194

P'ineal body, 346

Pitre's method, 358

Pituitary body, 203, 322, 338

Platysma, 53, 207, 222, 249

Pleura, 403, 404, 406

Plexus cardiacus, 422

- cavernosi concharum, 302

- chorioidens ventricul

lateralis, $341,343,345$

- - tertii, 345

- gangliosus ciliaris, 380

- gulae, 426

- (lymphaticus) aorticus, 516

- - coeliacus, 516

- - iliacus externus, 514

- lumbalis, 516

(nervorum) aorticus abdominalis, 479, 500

- alveolaris inferior. 271

- brachialis, 66, 67, $215,216,228$

- pars infraclavicularis, 68

laris, 67

lardiacus, 409,429

- caroticus communis 272

- - externus, 220, 271

- - internus, 271, 297

- cavernosus (in sinus cavern ), 275

- - - penis, 519

- cervicales, 66, 228 ,

290

- coeliacus, 492 , 500

- coronarius cordis an-

terior, $41 \overline{5}$

- - - posterior. 415

- deferentialis, 458 ,

- - dentalis inferior, 264

- - superior, 29s

- gastricus anterior.

498

- - posterior, 498

- - - superior, 500

- haemorrhoidalis me-

dius. 519

- - hepatlcus, 490, 500

- hypogastricus, 519 , 541

- - lienalis, 500

- - lumbalis. 516

- maxillaris externus, 220,271

- - internus, 271

mesentericus infe-

rior, 479

- - - superior. 476

- oesophageus. 425

- parotideus, 254

- - pharyngeus, 272 
Plexus (nervorum) phreni('us, 500

( plostaticus, 51!

_ pudendus, 535

- pulmonalis anter:or. $40 \mathrm{~s}, 409,425$

$4 \overline{409}, \overline{425}$

- renalis, 500

- - sacralis, 534

- subclavius, 227, 272

- suplarenalis, 500

- thyreoideus inferior, $220,2 \% 1$

soni ]

- - verteblalis, 272

- (venosus) basilaris, 203

- caroticus internus.

297

- haemorrhoidalis ex ternus, 533

- - internus, 533

- pampiniformis, 458 .

513

- pharyngeus, 269 ,

- pterygoideus, 262

pudendalis, 533

- pudendalis, 533

- thyreoideus major,

226

- uterovaginalis, 533 ,

542

- vesicalis, 533

I'lica, Plicae:

adiposae (pleurae), 406

- alares, 181

- aryepiglottica, 294, 308

- axillaris anterior, 41

- posterior, 41

cillares, 379

circulares [Kerkringi],

484, 494

duodenojejunalis, 470

fimbriata, 315

- gastropancreatica, 472

glossoepiglottica lateralis, 308

- - mediana, 307

- ileo-appendicularis, 471

- ileocaecalis, 471

- incudis. 388

- iridls, 380

- longitudinalis duodeni, 494

malleolaris anterior, 387,388

- posterior, 387, 388

- mesenteriococaecalis. 471

mesentericoparietalis, 471

- palatinae transversae, 290

- palmatae, 549

- parietocaecalis, 471

294

pubovesicalis, 518

recto-uterina [Doug lasi ], 473, 541

- salpingopalatina, 291

- salpingopharyngea, 29?

- semilunares coll. 487

- semilunaris conjunctivae, 247

- sublinguales, 290

- patellaris. 181 transversales recti, 525, 536, 548

tubariae (ampullares), 1'lica, Plicae:

- umbilicalis lateralis, 456

- - media, 456

ureterica, 537

ventricularis (laryn-

gis ), 309

vesicalis transvelsa,

473,537

- vocalis, 309

I'ollex, 41

I'olus anterior bulbi, 376

- lentis, 383

— frontalis, 334

- occipitalis. 335

- posterior bulbi, 376

- - lentis, 383

- temporalis, 322,335

I'ons Varolii, 322, 324, 325,327

I'opliteal space, 122

I'ori sudoriferi, 194

I'ortio dura, 273

- mollis, 273

- supravaginalis (cervi(is) 544,549

- vaginalis, $5 \pm 4,549$

l'orus acusticus externus, 385

- internus, 393

P'raecuneus, 536

Praeputium clitoridis, 444

- penis, 435

Traevertebral region, 282

I'lepuce of the penis, 435

Irocessus caudatus (lobl Spigeli), 503

- ciliaris, $3 \pi^{\prime}$

- coracoideus scapulae, 52

- cochlearis, 387

- falciformis, 552

- lenticularis, 389

- mallei anterior [Folii] 390

- lateralis, 390

- muscularis (cartil. ary taenoideae). 315

- papillaris (lobi Spigeli). 503

- uncinatus (pancreatis) 495

- vaginalis fasciae transversalls, 457

- - peritonaei, 457

- vermiformis, 486

- vocalis (cartil. arytaenoideae ), 315

- xiphoideus, 52, 433

I'rojection fibre systems, 372

Prominentia canalis facialis, 386

- semicircularis lateralis, 386

- laryngea, 194

- malleolaris, 387

- spiralis, 394

- styloidea, 386

Promontorium (cavl tympani), 386

Prosencephalon, 324, 325, 331

Prostata, 525

Prostatic urethra, 539

I'ubes, 433

Puboprostatic ligaments, $523,524,542$

I'udendum muliebre, 444

'ulmo (dexter', sinister), 407

I'ulpa lienis, 502

I'ulvinar, 346

Punctum lacilimale, 248

I'upilla, 380

Putamen, 364

Pyloric sphincter, 500
Pyloric valve, 500

Pylorus, 498

I'yramid, 326

Pylamidal tracts, $\mathbf{3 6 5}$

Pyramides renales, 508

P'yramis medullae oblongatae, 326

_ vestibuli, 392

Radiatio corporis callosi, 338 , 339, 364

- - striati, 364

occipitothalamica [Gratioleti ], 364

Radix, Radices :

- cochlearis, 307,324

- descendens (mesencephalica) N. trigemini, 357

- linguae, 315

- mesencephalica N. trigemini, 354

- mesenterii, 468

- N. acustici, 201

- - facialis, 354

- spinalum, 242

- pulmonis, 408

- unguis, 121

- vestibularis, 307,324

Ramus, Rami :

- alveolares (superiores) 298

- anastomoticus. Cf. nerves involved.

- anterior. Cf. structures involved.

auricularis (N. vagi), $270,271,307$ - bronchiales anteriores, 425

- posteriores, 425 buccales ( $N$. facialis), 254

calcanei mediales, laterales, 172,176

- inferior (N. vagi), 409

27

coeliacus (of chorda

oesoph. post.), 498

- colli (N. facialis), 209

communicans, 516 cutanei (arteriosi)

Cf. parent artery.

- dentales, 264, 298

- descendens N. hypoglossi,

- of Ramón y Cajal,

$212,214,217,220,222$, 269,372

- digastricus, 254

- externus. Cf. structure involved.

- frontalis, 254, 276

- gingivales, 264, 298

- hepatici (of. chorda

oesoph. ant.), 498

- internus. Cf. structure involved.

- labiales inferiores, 255. 264

- superiores, 254

- laryngopharyngel, 272

- lienales (arteriae lienalis). 502

- linguales (N. glossopharyngei), 266,269 , 271,317

- - (N. lingualis), 266

marginalis mandibulae, 254

- meningeus, 242, 270

- mentales, 255, 264 
Ramus, Rami :

- musculares. See parent nerve.

nasales anteriores, 252 , 278,303

- - externi, 254, 303

- interni (mediales), 301,303

- - (laterales). 303

- posteriores, 300, 303, 304

(N. auricularis posterioris). 196

- - oesophagei, 425

- palmaris N. mediani, 96 , 97

palpebrales inferiores,

254,278

- superiores, 254, 276,

- parotidei. 255, 264

- pericardiacus, 409

pharyngeus, 268, 270 ,

$271,29.7$

phrenico-abdominales, 409

profundus. See parent nerve.

stylohyoideus, 254

- stylopharyngeus, 271

- submaxillares (N. lingualis), 267

superficialis. See parent nerve.

superior Cf. parent nerve.

temporales ( $N$. facialis), 195, 254, 255, 260 thyreohyoideus, 220, 269

- tonsillares. 271

- volarls. See parent nerve.

-zygomatici (N. facialis), 254

zygomaticofacialis, 254 , 260,282

zygomaticotemporalis, $195,254,260,282$

Raphe medullae oblongatae, 348

of perineum, 436

palati, 288

palpebralis lateralis, 255

- penis, 435

- perinel, 436

- pontis, 354

- scroti, 435

Recessus cochlearis, 392

- - inferior, 471

- ellipticus, 392

- epitympanlcus, 386

- (fossae interpeduncularis) anterior. 322

- - _ posterior, 322

- ileocaecalis inferior, 471

- - superior, 471

- Inferior omentalis, 472

- Infundibull, 347

- intersigmoideus. 472

- lateralis ventriculi

quarti fossae rhomboidel, 331

- lienalis (bursae omentalis), 472

membranae tympan!, 388

- opticus, 347

- paracolici, 472

- phrenicohepaticus, 472

- pinealis, 346

- piriformis 294

- retrocaecales, 471
Recessus sacciformis, 111, 114,115

- spliaericus, 392

- sphenoethmoidalis, 302

- superior omentalis, 472

- suprapinealis, 346

- triangularis, 347

Rectum, 465, 536, 542, 548

Regions, 43, 44

Regio olfactoria. 302

- perinealis. 434

- respiratoria, 302

Reil, island of. $\mathbf{3 3 7}$

Removal of Brain, 199

Ren, 505, 506

Rete (arteriosum) acromiale, 65

105

- - genu, 134

- calcaneum. 172

- carpi dorsale. 106

- - volare. 104

- - dorsale pedis. 160

- malleolare laterale, 160

- - mediale, 160

- patellae, 134

- testis [Halleri], 460$$
172
$$

17 calcaneum, 172

- dorsale manus, 89

- - pedis, 156

- plantare, 176

Retina, bipolar cells of, 366

pars ciliaris, pars optica, 381

Retinacula cutis of peroneal muscles, 163

Retinaculum Lig. arcuati. 181

- IIm. peronaeorum inferius, 157,164

- - - superius 164 patellae laterale, 152

- - mediale, 152

Rhinencephalon, 325,332 , 337

Rhombencephalon, 324, 325,326

Right auricle, 412, 415 , 416

- auriculoventricular ralve, 417

- lymphatic duct, 226 ventricle, 413. 417

Rima cornealis. 378

- glottidis, 310

- - pars intercartilagi. nea, 310

- - intermembranacea, 310

- oris, 193, 248, 288

- palpebrarum, 193, 247

- pudendi. 444

vestlbuli. 309

Rivini, ducts of, 267,290

Rlvus lacrimalis, 248

Rolando, fissure of. 334

- substantla gelatinosa of, 246

Root of mesentery, 468

Rostrum corporis callosi, 339

Rugae vaginales, 545, 549

Sacculus. 394

Saccus lacrimalis, 257

Sacral plexus. 534

Sacrococcrgeal plexus, 534

Santorini, cartilages of, 314
Saphenous opening, 140

Scala tympani, 393

- vestibuli, 393

Scalp. 194

- dissection of, 194

- nerves of. 195

- surgical anatomy of 196

- vessels of, 195

Scarpa, fascia of, 451

Sclera, 376, 378

Schlemm, canal of, 378

Sclerotic coat. 378

Scrobiculus cordis, 42,433

Scrotum, 435

Secondary paths of Nn. cerebrales. $366-369$

Sections of brain. 347

- of cerebellum, 354

- of diencephalon, 358

- of hypothalamus. 360

- of medulla oblongata, 347

- of pons, 353

- of prosencephalon, 358

- of rhom., isth., corp. quad. and cer ped. 356

of spinal cord, 243

- of telencephalon, 358 , 362

- of thalamencephalon, 362

Semilunar cartilages, 418 , 423

- fascia, 77, 80,90

Septula testis, 460

Septum atriorum, 416

- bulbi urethrae, 440

- cartilagineum nasi. 300

- corporum cavernosorum, 448

- femorale [Cloqueti].

146, 511

- glandis. 435

- intermusculare, 142 150

- femoris mediale, 142.

150

- - posterius, 158 163 80

- mediale, 78

linguae. 317

mediastinale, 406

- membranaceum nasi, 300

- ventriculorum, 422

musculare ventriculorum, 422

nasi, 193, 294, 300

- cartilagineum, 300

- orbitale. 255

- pellucidium. 343

- subarachnoideale posterius, 240

- vaginae vasorum (femoralium ), 145

ventriculorum, 491

Sheath of the rectus, 454

Sigmoid flexure of colon, 486

Sinciput, 193

Simus, 42

aortae [Valsalvae]. 422. 423

- cavernosus, 202, 273

- circularis, 202

coronarius, 414, 416, 417

costomedlastinalis, 406

- durae matris, 199, 202 
Sinus frontales, 303

- intercavernosus anterior, 202

- posterior, 202

- lactiferi. 57

- maxillaris [Highmori], 303

- occipitalis, 202

- paranasales, 30 .

- of Morgagni, 309

petrosus inferior, 202, 269

- - superior, 202

phrenicocostalis, 406 posterior, 386

rectales, 536

- rectus, 199, 202

- renalis, 508

- reuniens, 199, 202

- sagittalis superior, 198 , 199,202

- - inferior. 199, 202

- sphenoidalis 303

splenoparietalis, 202 ,

273

- tonsillaris, 296

- transversus. 202

venarum (cavarum), 416

venosus sclerae. 278

Sinuses of Valsalva, 422 , 4:23

Small intestine, 465. 479

- omentum, 468,489

Socia parotidis, 251,290

Soft palate, 288, 294

Spatium, Spatia :

- anguli iridis, 380

- interfasciale [Tenoni]. 277,282

interossea metacarpi, 116

- - metatarsi, 190

- perichorioideale, 379

- perilymphaticum, 394

- praeperitonaeale [Retzii]. 473

- zonularia, 383

Spermatic cord, $45 \pi$

Sphenoparietal sinus, 202, 273

Spina helicis, 359

Splenium corporis callosi, 338, 3399

Spongy urethra, 539

Stapes, 388

Stenson's duct, 250, 290

Stomach, 465,498

Straight sinus, 199, 202

Stratum album-profundum, 358

- cinereum, 355

- cutaneum (membr. tympani), 387

- gangliosum, 35.5

- granulosum, 35.

- griseum centrale. 357

- colliculi superioris 358

- interolivare lemnisci. 3.12

- mucosum (membr. tym pani). 358

- nucleare (medullae oblongatae), 348

pigmenti corporis cillaris, 381

- - iridis, 376,381

- - retinae. 351,376

zonale (thalamis), 358

Stria. Striae :

- albicantes, 433

- longitudinalis (later-

alis medialis), 387
Stria, Striae :

- malleolaris, 387 $\mathrm{m}_{331}$

- medullaris thalami, 346

lateralis, medialis ), 337

terminalis, $341,3+2$

- transversae, 339

- vascularis, 394

Stroma iridis, 380

- ovarii, 546

vitreum, 382

Subarachnoid space, 240 317

Subdural space, 198, 238, 240

substantia corticalis. Cf. organ concerned.

- cerebelli, 35. - cerebri, 362, 338 lentis, 383

- - [Rolandi], 246

- grisea, 243

- centralis, 244

- lentis. 383

- medullaris.

concerned.

nigra, 358

- perforata anterior, 337

- posterior, 322

propria corneae, 378

reticularis alba, $3 \overline{5}$

- _ - [Arnoldi], 336

- - grisea, 352

Succus pancreaticus 495

Subdivisions of brain, 324

Sulcus, Sulci :

- ampullaris, 394

anthelicis transversus, 260

basilaris, 327

bicipitalis lateralis. 41

- medialis, 41

centralis [Rolandi], 334

- cerebelli, 328

- cerebri. 332

- cingulf, 334, 336

circularis [Reili], 334

corporis callosi, 336

coronarius. 413

- cruris helicis, 260

frontalis inferior, 334

- superior, 334

horizontalis cerebelli, 328

hypothalamicus [Monroi]. 347

- infrapalpebralis, 193

- inguinalis, 122

- insulae, 337

intermedius anterior 243

- posterior. 243

- interparietalis. 335

- lateralis anterior (med. spin.), 243, 326

- posterior (med. spin. ), 243, 326 - mesencephali. 328

limitans fossae rhom boideae. 331

- longitudinalis anterior. $413,417,419,420$ $-\frac{1}{420}$ posterior, 413, 419,

- medianus (linguae), 316

- posterior (med.

spin.) 243

- mentolabialis, 193

- nasolabialis, 193 335

— - superiores, 335

- of Reil, 334
Sulcus, Sulci :

occipitalis transversus, 335

- olfactorius, 301, 334

- orbitales, 334

paraglenoidalis (praeauricularis),

- paroccipitalis, 335

parolfactorius (ant. post.), 337

praecentralis, 334

- promontorii, 387

- sclerae, 378

- spiralis, 395

- subclavius (pulmonis), 408

subparietalis, 336

temporales transversi, 335

temporalis inferior, 335

- medius, 335

- superior, 335

- terminalis atrii dextri, 416

- (linguae), 316

Suprasternal fossa, 204

Surface anatomy of back, 4.3

- of axillary fossa, 51 of face, 247

121

- of thorax (ant.), 51

Supercilium, 193

Superficial laver of triangular ligament, 448, 450 , 520,542

Suprarenal capsules, 505 , 509

Suspensory ligament of ovary, 473,541

- - of penis, 461 muscle of duodenum and mesentery, 493

Sustentaculum lienis, 473

Sylvius, aqueduct of, 328

- fissure of, $322,333,334$

Symphysis ossium pubis, 5.3

- sacrococcygea, 553

Syndesmosis arycornicnlata, 314

- tibiofibularis, 184

- tympanostapedia, 390

Systema nervorum sympathicum, 271

- pars abdominalis,

- - pelvina, 535

- - thoracalis, 428

Taenia, Taeniae :

- chorioidea, 341

- fimbriae, 343

- fornicis, 343

- libera, $486^{\circ}$

- mesocolica, 486

- omentalis, 486

- ventriculi quarti. 331

Tapetum, 338, 341, 342, 364

Tarsi, 255

Tegmen tympani, 385

- ventriculi quarti, 331

Tegmentum, 357

Tela chorocidea ventriculi tertii, 343

- submucosa, 483, 487 494,500

Telencephalon, 324, 325 , 331, 332

Tempora, 193

Tenon, capsule of, 375

Tentorium cerebelli, 202

Testis, 459 
Thalamencephalon, 325 , 331,346

Thalamus, $325,341,346$

Thebesius, foramina of, 417

- valve of, 417

Third ventricle, 346

Thoracic cavity, $39 \overline{7}, 403$

- duct, 226, 4\%6

Thorax, 41

- inlet of, 399

- outlet of, 399

- wall of 399,428

Thymus gland, 411

Thyreohyoid membrane, 222,310

Thyreoid cartilage, 314

Tongue, 315

Tonsilla cerebelli, 330

- lingualis, 316

- palatina, 291, 296

- pharyngea, 293

Torcular Iterophili, 202

Torus tubarius, 293

Trabeculae carneae, 421

Trachea, 424

Tractus olfactorius, 337

- opticus. $322,338,366$

- solitarius 348 spinalis $\mathrm{N}$. trigemini, 348,354

- spiralis foraminosus, 343

Tragi, 258

Transverse colon, 465, 467, 486

- fissure of liver, 504

- mesocolon. 468

Trochlea. $27 \%$

Tuba auditiva [Eustachii], 296, 384

- pars uterina, 550

- uterina [Falloppii], 546, 550

Tuber cinereum, 322. 338

- ischiadicum, 434 omentale hepatis. 504 - pancreatis, 496

Tuberculum anterius thalami, 346

- aurlculae [Darwinl], 259

- cinereum, 327

- corniculatum [Santorini], 308

cuneiforme [Wrisbergi], 308

- epiglotticum. 314

- intervenosum [Loweri], 416

- pubicum. 434

- thyreoideum inferius, 314

- superius. 314

Tunica albuginea (lienis), 502

- testis, 460

conjunctiva, 247

fibrosa oculi, 376

- (renis), 508

mucosa, 484, 494, 500 . 505

- - tympanica, 388

muscularis, 486,494

500,505

- intestini ilei, 482

serosa. $465,483,486$, $494,500,502,505$

vaginalis communis (tes-

tis et fun. sperm.), 451

- propria (testis). 460 vasculosa oculi, 376 . 370

Turbinated bones, 294

Trigona fibrosa, 424
Trigone of bladder, 537

Trigonum collaterale, 342

- corporis callosi, 338

- femorale, 122

- habenulae, 346

- lemnisci, 327

- lumbale [Petiti], 48

- N. hypoglossi, 331

- olfactorium, 337

- vesicae [Lieutaudi] 5.37

Truncus costocervicalis, 226

- intestinalis, 514

- lumbales, 514

- lumbosacralis, 518

- srmpathicus, 222, 226 , 282

- pars cervicalis, 220 . 222,271

- - thoracalis, 428

- thyreocervicalis, 226

Tmbilicus, 433

Umbo (membranae tympani), 387

Uncus (gyri hippocampi), 336

Ungues, 121

Upper limb, 41

Trachus, 456

Lreter. 509

- pars abdominalis, 509

- pelvina. 525, 543

Urethra muliebris, 548

- virilis, 539

- pars cavernosa, 539

- - membranacea, 444, 539

- - prostatica, 539

female, 548

- male, 539

Urethrai orifice (female), 548

Tterus, 543,549

Utriculus prostaticus. 539

Uvula (palatina), 291

- (vermis), 329

- vesicae, 537

Vagina, 545, 549

Vagina, Vaginae

- mucosa intertubercularis, 80,87

- musculi recti abdominis, 4.54

- tendinis Mr extensoris carpl ulnaris, 107

107

- hallucis longi,

160

107

flexoris digitorum (pedis) longi, 170

- - hallucis longi, 170

101

- peronael longi

plantaris. 180

- tibialis anterioris 160

$\overline{170}-$ posterioris

tendinum digitales (manus), 101

- Mm. abductoris longi et extensoris brevis pol licis, 107 radialíum. 107
Vagina, Vaginae:

- tendinum $\mathrm{N1m}$. extensoris digitorum communis et extensoris indicis, 107 - flexorum communium, 92, 101

- peronaeorum communis, 16 it

Vallecula cerebelli, $3: 8$

- epiglottica, 308

Valvula bicuspldalis ( $\mathrm{ml}$ tralis), 421

- cuspis anterior. 421

- posterior, 421 coli, 487

- foraminis ovalis, 419

- processus vermiformis, $4 \mathrm{SS}$

- pylori, 500

- semilunares aortae, 421 , 423

- A. pulmonalis, 418 sinus coronarii. [Thebesii]. 417

- spiralis [Heisteri], 505

- tricuspidalis. 417

Vas afferens (glomeruli renis), 508

- deferens, 448. 52

- prominens, 396

Vasa afferentia, 508

- auris internae. 39.

- - posterior. 417

venae cavae (inferiores. (Eustachii), 417

Vasa aberlantia hepatis, 489

- sanguinea retinae, 381

Velum medullare anterius, 328

- posterius, 331

- palatinum. 291

Vena, Venae:

- alveolaris inferior, 262

- angularis, 195

- anonymae, 55

- - dextra, 226, 411

- - sinistra, 224, 226.411

- aquaeductus vestibuli, 396

- arciformes (renis), $\mathbf{5 0 8}$

- articulares genu, 134

- mandibulae ofe

- auditivae internae 202 auriculares anteriores, 252

auricularis posterior.

196,207

axillaris, 64, 65, 66, 78

azygos, 408, 412, 428 , $429, .16$

- basalis [Rosenthali], $3 \%$

- basilica, 73, 77, 89

- brachiales. 64.78

- bronchiales posterior. 429

- bulbi vestibuli (vaginae), 533

- bulbo-urethralis, 532

- canaliculi cochleae. 396 - cava inferior, 412,415 . 416,513

- radices parietales et viscerales, 513 - superior, 408, 411. 415,416

- centralis (hepatis), 505 - - retinae. 279

cephalica. 58, 64, 65, 70, 73, 7 7. 78,89

- cerebelil inferiores, 322

- cerebri Inferiores, 202 322

- $\stackrel{322}{ }$ interna, 344 
Vena, Venae :

- cerebri magna [Galeni], 345

- - media, 322

— - superiores, 322

cervicalis profunda. 226, 235

chorioldea. 344

279

posteriores,

circum flexae femoris

laterales, 148

- - mediales, 148

- ilium profunda, 514

- superficialis, 140 ,

148, 451

colica dextra. 476

- media, 476

- sinistra, 477

comitans A. lingualis, 267

267

N. hypoglossi, 218.

conjunctivales (ante-

riores, posteriores ), "219

cordis anteriores, $\mathbf{4 1 4}$

- - magna, 414

- media, 414

- minimae, 414

- parva. 414

- coronaria ventriculi. 423, 489

- costo-axillares. 64

- cutaneae pectoris, 55

- cystica, 490

- digitales communes pedis, 156

- pedis dorsales, 1.56

- plantares, 176

- propriae, 89

diploica frontalis, 203

dorsalis penis. 443

duodenales, 476

epigastricae inferiores.

514,527

- superficialis, 140 ,

148,451

- - superior, 412

- episclerales, 279

- ethmoidales. anterior et posterior, 279

facialis anterior, 217

$218,220,252$

- communis, 217, 220 , 269

252,262

- femoralis, $140,145,148$ 1.54

femoris profunda, 166 . 20.3

femoropoplitea, $\mathbf{1 3 0}$

137. $166 \%$

- frontalis, 252

- gastricae breves, 490

gastro-epiploicae, dex-

tra et sinistra, 476,490

glutaeae inferiores, 127 - superioles, 127

haemolrhoidales inferioles, 533

- superior, 477

- hemiazygos, 429, 516

- accessorla, 420

hepaticae, 513

- hypogastrica, 514, 533

- ileocolica, 476

- iliaca communis, 514

- - externa, 514,527

- lliolumbalis, 514

- intercapitulares (pedis), 156, 176

- intercostales, 55, 400,

$402,428,429$

— - ramus dorsalls, 235
Vena, Venae:

- intercostalis suprema, 412

- interlobulares hepatis,

505

- renis, 508

- interosseae volares, 96

- intervertebrales, 243

- intestinales, 476

- jugularis anterior. 207

- externa, 207, 215, 216

- interna, 212, 218, 220, 226, 268, 282

labiales posteriores, 533

labialis inferior, 252

labialis superior, 252

lacrimalis, 279

laryngea inferior, 226

- superior, 220, 310

lienalis, 490

linguales, 218, 220, 267. 269

lumbales, 236

- rami dorsales, 236

lumbalis ascendens, 516

mammaria interna, 55. 411

55 - rami perforantes.

marginalis pedis lateralis, 166

- medialis, $\mathbf{1 6 6}$

massetericae, 252, 262

mediana antibrachii, 77

- basilica, 77

cephalica, 77,78

cubiti, 77

meningeae mediae, 202 . 262

mesenterica inferior 477,48 ?

- - superior, 476, 489

metatarseae dorsales, S?

nasales externae, 252

- nasofiontalis, 252,279

obliqua atrii sinistri

[Marshalli], 414

occipitalis, 196, 203,

214. 235

- oesophageae, 429

- ophthalmicae inferior et superior, $262,273,279$ ophthalmomeningea, 202

- ovarica, 458, 513

- palatina, 218, 295

palpebrales, inferiores

et superiores, 27 ?

- pancreaticae, 47

- pancreaticoduodenalis, 476

- parotideae anteriores, 252

parumbilicales [Sappeyi], 451, 490

- perforans prima, 137

- - secunda. 137

- pharyngeae, 269, 295

- phrenica superior, 411

- poplitea, 166

- popliteae. 134

- portae, 489

- posterior ventriculi sinistri. 414

- profunda clitoridis. 533

- femoris, 148,152 penls, 513

pudendae externae, 140 148,451

- internae, 448, 450

puimonales dextrae $409,417,418,423$
Vena, Venate :

- pulmonales sinistrae, 409

- radiales, $78,90,106$

renales, 513

sacralis media, 514, 533

- saphena accessoria, 140

- magna, 140, 142 ,

$145,148,157,166$

$-\frac{1}{160}$ parva, $130,134,157$, 166

scrotales posteriores, 533

- septi pellucidi, 344

- sigmoideae, 477

- spermatica externa, 458,513

- spinales externae, anteriores, 243

spiralis modioli, 396

- stellatae (renis), 508

- sternocleidomastoidea, 220

- stylomastoidea, 262

- subclavia, 214, 215, 226

- subcutaneae abdominis, 412

- sublingualis, 267

- submentalis, 218

- supraorbitalis, 252

- suprarenalis, 513

- temporales, 202, 203

- profundae, 262

temporalis superficialis, $195,25 \%$

- media, 252

terminalis, $341, \quad 344$

testicularis, 513

thoracalis iateralis, $\mathbf{5 5}$ $6+$

thoraco-acromialis

rami pectorales, 64, 65, 226

thoraco-epigastricae,

$55,64,148,451$

thymicae, 411

- thyreoidea ima, 226

- inferior, 224, 226 ,

411

411

transversa colli, 214.

215,226

- faciei, 252, 262

21

ulnares, 78,94

urethralis. 533

vertebralis, 226,284

411

vestibulares, 396

vorticosae, 279

Ventriculus, 465, 498

(cordis), dexter, 413 ,

417

- - sinister, 413

- laryngis [Morgagnii], 309

- lateralis, $3.37,339$

- paries anterior, 498

- - posterior, 498

- pars cardiaca, 498

- - pylorica, 498

- terminaiis, 243

tertius, 346

Venulae rectae (renis), 508

- retinae, 381,382

Vermis, 328

Vertebra prominens. 45

Vertebral canal, 237

Vertex, 193

- corneae, 378

Verumontanum, 539

Vesica fellea, 489 , 505 
Vesica urinaria, 505, 525, $537,543,549$

Vesicula ophthalmica, 376

- seminalis. 527, 539

Vestlbule, 427

Vestibulum labyrinthl, 305 , 392

- laryngis, 309

- nasi, 301

Vlbrissae, 194

Vicq d'Azyr, bundle of, 361, 373

- foramen caecum of 326
Vieussens, valve of, 328

Vincula tendinum (digitorum manus) 101

- - (- pedis), 101

inculum lingulae (cere-

belli), 329

Vocal cords, 309

Vola manus. 42

Volvulus, 269

Vortex cordis, 424

Vortices pilorum, 194

Vulva, 444

Wharton's duct, 267, 290
White line of pelvic fascia, 521,541

Willis, circle of, 320

Winslow, foramen of, 472

Wirsung, canal of, 496

Word-deafness, 368

Wrisberg, ganglion of, 410

Zinn, zonule of, 376, 383

Zona ciliaris, pupillaris (iridis). 380

- orbicularis, 155

Zonula ciliaris [Zinni], 376,383 


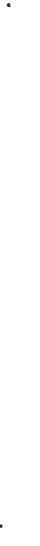



\section{UNIVERSITY OF CALIFORNIA \\ MEDICAL CENTER LIBRARY}

\section{THIS BOOK IS DUE ON THE LAST DATE STAMPED BELOW}

Books not returned on time are subject to a fine of 50c per volume after the third day overdue, increasing to $\$ 1.00$ per volume after the sixth day. Books not in demand may be renewed if application is made before expiration of losn period.

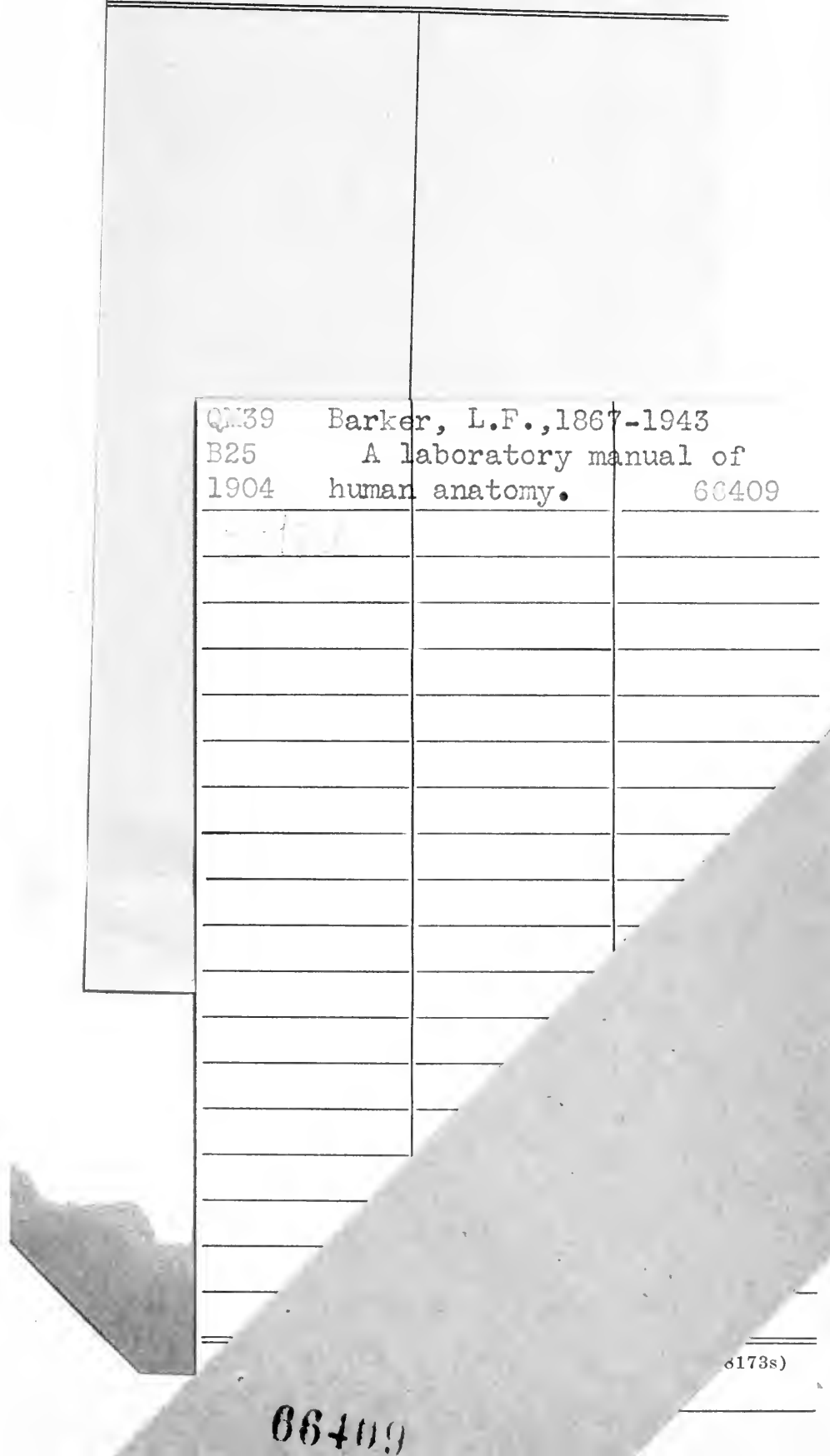


